NASA CONTRACTOR REPOR T

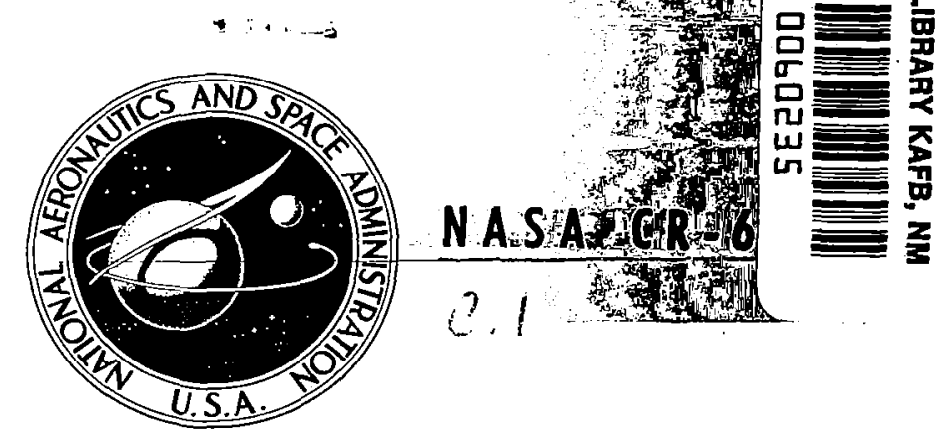

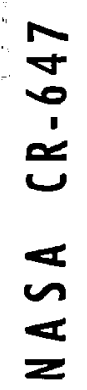

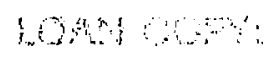

$\therefore \therefore$

खास

\title{
ABSORPTION OF SOUND IN AIR VERSUS HUMIDITY AND TEMPERATURE
}

by Cyril M. Harris

\section{Prepared by}

COLUMBIA UNIVERSITY

New York, N. Y.

for George C. Marshall Space Flight Center

NATIONAL AERONAUTICS AND SPACE ADMINISTRATION - WASHINGTON, D. C. - JANUARY 1967 
NASA CR- 647

ABSORPTION OF SOUND IN AIR VERSUS HUMIDITY AND TEMPERATURE

By Cyril M. Harris

Distribution of this report is provided in the interest of information exchange. Responsibility for the contents resides in the author or organization that prepared it.

Prepared under Contract No. NAS 8-11002 by COLUMBIA UNIVERSITY New York, N.Y.

for George C. Marshall Space Flight Center NATIONAL AERONAUTICS AND SPACE ADMINISTRATION

For sale by the Clearinghouse for Federal Scientific and Technical Information Springfield, Virginia 22151 - Price $\$ 3.75$ 


\section{ACKNOWLEDGMENT}

This research was supported by the George C. Marshall Space Flight Center, National Aeronautics and Space Administration. The author appreciates the assistance of Harry Gong in the measurements and Beth Alberty in the data reduction. 
TABLE OF CONTENTS

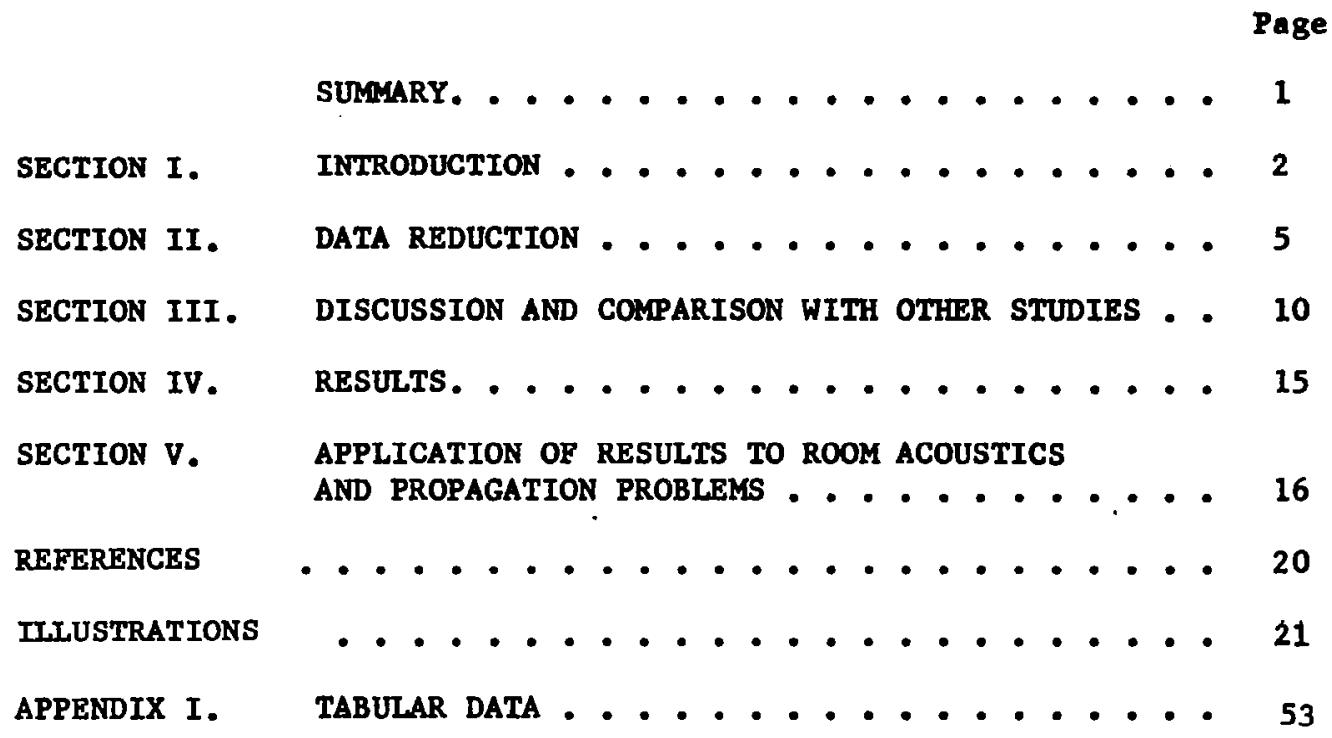




\section{LIST OF ILLUSTRATIONS}

Figure

Title

Page

1. Example of a plot of experimental data of the molecular

attenuation coefficient per wavelength in air as a function

of humidity. These data are for a temperature of $20^{\circ} \mathrm{C}$ and a

frequency of $6400 \mathrm{~Hz}$. . . . . . . . . . . . . . . . . . . . .

2. Relaxation frequency as a function of the concentration of water vapor in air expressed in $\mathrm{gm} / \mathrm{m}^{3}$. The relationship used in the results presented in this paper is shown as the solid curve.

3. Plot of experimental data of the molecular attenuation coefficient in air versus humidity. These data are presented in the normalized form, $\mathrm{m} / \mathrm{m}_{\max } \mathrm{vs} \mathrm{h} / \mathrm{h}_{\max }$ for comparison with the theoretical relationship of Kneser shoin by the dashed line. . . . . . . . . . . . . . . .

4. Simplified schematic diagram of the air-circulation system. Air is recirculated continuously through the spherical chamber. The saturator either takes away moisture from the air or adds moisture to it -- depending on the relative temperatures of the spherical chamber and the saturator. . . . . . . . . . . . . . . . . . . .

5. Values of the total attenuation coefficient $m$ versus percent $R . H$. for air at $20^{\circ} \mathrm{C}$ and normal atmospheric pressure for frequencies between 2.0 and $12.5 \mathrm{kHz}$ at third-octave intervals . . . . . . . . .

6. The attenuation of sound in air versus temperature for various values of relative humidity. The attenuation in $\mathrm{dB} / 100$ meters is shown as the ordinate at the left of the graph . . . . . . . . . . . . 26

7. The attenuation of sound in air versus temperature for various values of relative humidity. The attenuation in $\mathrm{dB} / 1000$ feet is shown as the ordinate at the left of the graph . . . . . . . . . . . . 38

8. Values of total attenuation coefficient m versus humidity for a frequency of $4000 \mathrm{~Hz}$ at temperatures of $-10^{\circ} \mathrm{C}, 0^{\circ} \mathrm{C}, 10^{\circ} \mathrm{C}, 20{ }^{\circ} \mathrm{C}$

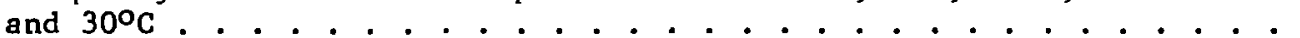

9. Values of total attenuation coefficient m versus temperature for constant values of frequency. All data are for a relative humidity of $50 \%$. . . . . . . . . . . . . . . . . . . . . . . . . 


\author{
ABSORPTION OF SOUND IN AIR \\ VERSUS HUMIDITY AND TEMPERATURE \\ Cyril M. Harris \\ Department of Electrical Engineering \\ Columbia Univeraity, New York, N.Y. 10027
}

\title{
SUMYARY
}

Measurements have been made of the absorption of sound in air at thirdoctave frequency intervals from $2000 \mathrm{~Hz}$ to $12500 \mathrm{~Hz}$, as a function of humidity, at six temperatures in the range from $-0.5^{\circ} \mathrm{C}$ to $25.1^{\circ} \mathrm{C}$ at normal atmospheric pressure. The results of the new study are presented and compared with those of past investigations. Then a "best fit" is obtained to existing data. The resulting information is presented in both tabular and graphical form, useful for the solutions to problems of the calculation of attenuation of sound propagated in the atmosphere, and the computation of the effects of air absorption in problems of room acoustics. These results have been extrapolated downward in frequency to $125 \mathrm{~Hz}$ and extrapolated to cover the temperature range from $-10^{\circ} \mathrm{C}$ to $30^{\circ} \mathrm{C}$. 


\section{ABSORPTION OF SOUND IN AIR VERSUS HUMTDITY AND TEMPERATURE}

\section{SECTION I. INTRODUCTION}

In a paper by the author in $1963^{1}$, measured values of the absorption of sound in air were given as a function of humidity in the frequency range between 2000 and $12500 \mathrm{~Hz}$ (cps), at normal atmospheric pressure and at a temperature of $20^{\circ} \mathrm{C}$. The data presented here greatly extend the previous work over a wide temperature range and provide measured values having greater accuracy through improved methods of data reduction. A total of 2800 measurements were made at third-octave frequency intervals from $2000 \mathrm{~Hz}$ to $12500 \mathrm{~Hz}$ at each of six temperatures in the range from $-0.5^{\circ} \mathrm{C}$ to $25.1^{\circ} \mathrm{C}$ at normal atmospheric pressure. The new data a re compared with those of past investigations. Then a "best fit" is obtained to existing data. These results make it possible to evaluate the effects of air absorption over a range of environmental conditions encountered in practical problems in acoustical engineering, for example: (1) the calculation of the attenuation of sound in air due to air absorption; (2) the calculation of the contribution of alr absorption to the total absorption in a large room; (3) the correction for air absorption in reverberation time measurements in large auditoriums; and (4) the correction for air absorption in the measurement of the sound absorption coefficients of materials tested in a reverberation chamber. These results have been extrapolated downward in frequency to $125 \mathrm{~Hz}$ and extrapolated to cover the temperature range from $-10^{\circ} \mathrm{C}$ to $30^{\circ} \mathrm{C}$.

The experimental setup and measurement techniques used here are the same as those employed in the earlier study and are described in detail in that paper. 1 Therefore they are outlined only briefly here. Suffice it to say that the attenuation coefficient of sound in air was evaluated from measurements of the 
decay rate of diffuse sound in a spherical chamber 1.68 meters in diameter. During measurements, the temperature of the chamber was held constant to within $0.1^{\circ} \mathrm{C}$. Precautions were taken to rid the chamber of contamination by evacuating it by means of vacuum pump; then the spherical chamber was filled with dry air having a carbon dioxide content of 300 parts per million, which is a usual value. Random noise from a source in the chamber was picked up by a microphone, amplified, and fed through a third-octave filter to a bigh-speed level recorder. When the random noise source was turned off, a curve of the decay of sound in the chamber was obtained. The slope of the curve determined the decay rate in $\mathrm{dB} / \mathrm{sec}$ at the frequency of the center of the band to which the third-octave analyzer is set. For a single test condition, three decay curves were superposed; then the average slope of three such sets of superposed curves was obtained to determine the decay rate for this condition. This decay rate, when corrected for losses at the boundaries of the chamber, is a measure of the total absorption of sound in the chamber.

In determining the magnitude of the absorption of sound in air from measurements of the rate of decay of sound in a chamber, it is necessary to evaluate the contribution to decay rate that may be attributed to losses in acoustic energy at the boundaries of the chamber. The technique used here is to evaluate these wall losses directly, by means of decay rate data obtained when the chamber is filled with pre-purified dry nitrogen (which exhibits no anomalous absorption in the frequency range of measurement). The difference between the measured value of the rate of decay of sound in the nitrogenfilled chamber and the value computed from absorption data on nitrogen (the nitrogen data of Parbrook and Tempest ${ }^{2}$ are used in this calculation) represents the contribution to the decay rate due to wall losses. This can be shown as follows: the decay rate of sound in the chamber that one measures, $R_{\text {measured }}\left(\mathrm{N}_{2}\right)$, when it is filled with nitrogen is given by: 


$$
R_{\text {measured }\left(\mathrm{N}_{2}\right)}=\mathrm{R}_{\mathrm{N}_{2}}+\mathbf{R}_{\text {wall }} \mathrm{dB} / \mathrm{sec}
$$

where $R_{\text {wa11 }}$ is the decay rate due to absorption at the boundaries of the chamber, and where $R_{\mathrm{N}_{2}}$ is the decay rate due only to the nitrogen gas. Similarly, when the chamber is filled with air, the measured decay rate is

$$
R_{\text {measured (air) }}=R_{a i r}+R_{\text {wall }} d B / \sec
$$

where $\mathrm{R}_{\text {wa11 }}$ is the decay rate contributed by the wall losses, and $\mathrm{R}_{\mathrm{a} \text { Ir }}$ is the calculated decay rate due only to air absorption. The wall loss term in these equations is approximately the same because both gases are closely similar in molecular weight and characteristic acoustic impedance. By combining Eqs. (1) and (2), the rate of decay of sound in the chamber due only to the absorption of sound in air is given by:

$$
R_{a i r}=R_{\text {measured }}(\text { air })-\left[R_{\text {measured }}\left(N_{2}\right)-R_{N_{2}}\right] d B / \sec \text { (3) }
$$

where the quantity within the brackets represents the contribution to the rate of decay of sound in a chamber that is due to wall losses. Consideration has been given to possible variation in the boundary losses with changes in the humidity within the sphere. As pointed out by Evans and Bazley ${ }^{3}$ in discussing this possibility, the work of Knudsen, Wilson and Anderson ${ }^{4}$ indicates that such an effect is not significant; their data show that there is no appreciable change in wall absorption even when moisture condenses on the wall surface.

The value of $R_{a i r}$ in $d B / \sec$ given by Eq. (3), which represents the contribution to the rate of decay of sound in a chamber or room caused by air absorption, may be converted to the attenuation coefficient m per meter as expressed in the equation $I=I_{0} e^{-m x}$ by the relation:

$$
\mathrm{m}=\mathrm{R}_{\mathrm{air}} /(4.343 \mathrm{c}) \quad \text { meters }{ }^{-1}
$$

where $c$ is the velocity of sound in $\mathrm{m} / \mathrm{sec}$. 
The methods of data reduction that were employed to determine the values of $m$ for various conditions in this study are described in detail. In the next Section. A comparison of the new data for air with earlier studies is given in section III. Then a best fit is obtained. The new results are presented in Section IV. Then they are applied to problems in the propagation of sound in air and room acoustics in Section $V$.

\section{SECTION II. DATA REDUCTION}

For a given condition of temperature and humidity, the decay rate of sound in air was measured for the various frequencies. The result of each decay-rate measurement was punched on an IBM card along with the following Information: (1) temperature; (2) frequency; (3) value of classical absorption, $m_{c}$, for these conditions; (4) humidity; (5) wavelength, $\lambda$, of sound for these conditions, computed from sound velocity data given in tables compiled by the National Bureau of Standards; ${ }^{5}$ and (6) the value of wall losses for these conditions.

From the above input data, a computer program provided for the printout of the following results: (1) total attenuation coefficient $m=m_{m}+m_{c}$ (i.e., the sum of the attenuation coefficients due to molecular and classical attenuation) corrected for wall losses; (2) molecular attenuation coefficient, $m_{m}$; and (3) molecular attenuation coefficient per wavelength, $\mu=m_{m} \lambda$. The results provided by this computer program were analyzed as indicated in this Section. Then a computational procedure was developed for evaluating the total attenuation coefficient for any temperature, frequency, or humidity within the range over which the experimental data may be extrapolated with confidence. Values of $h_{\max }$. For a given temperature and frequency, the molecular absorption $m_{m}$ varies with the moisture content of the air. For a given frequency, the value of humidity at which the molecular absorption is maximum is defined as $h_{\max }$. 
The measured value of $h_{\max }$ may be determined from plots of the molecular attenuation per wavelength versus the logarithm of the relative humidity, such as the one shown in Fig. 1. [Here, because an automatic plotting device was employed, it was convenient to plot $\mu$ against $333 \log (10$ R.H.) rather than against $\log$ (R.H.) directly.) For example, the original data points for sound having a frequency of $6400 \mathrm{~Hz}$ in air having a temperature of $20^{\circ} \mathrm{C}$ are plotted in Fig. 1. Here the resulting value of $h_{\max }$ is $11.7 \%$. A total of 54 such plots were made, i.e., one for each of six different values of temperature $\left(-0.5^{\circ} \mathrm{C}\right.$, $5.6^{\circ} \mathrm{C}, 10.2^{\circ} \mathrm{C}, 14.8^{\circ} \mathrm{C}, 20.0^{\circ} \mathrm{C}$ and $\left.25.1^{\circ} \mathrm{C}\right)$ at nine different frequencies $(2.0$, $2.5,3.2,4.0,5.0,6.4,8.0,10.0$ and $12.5 \mathrm{kHz})$. From these plots an experimentally determined value of $h_{\max }$ for each frequency, and at each temperature, was obtained. These data are shown in Fig. 2 , where the values of $h_{\max }$ are expressed in $\mathrm{gm} / \mathrm{m}^{3}$, along with similar data from other studies. The solid curve represents a best fit to the data at $20^{\circ} \mathrm{C}$ from this and previous data obtained with the same experimental setup, ${ }^{1,6}$ as well as data obtained by other experimenters as weighted by the author. This solid curve, in the frequency range between 125 and $12,500 \mathrm{~Hz}$, may be expressed by the equation:

$$
f_{\max }=0.79 h^{2}+1.47 \mathrm{~h}-0.15
$$

where $f_{\max }$ is the frequency in $\mathrm{kHz}$ corresponding to absorption peak at $\mathrm{h}$ (expressed in $\mathrm{gm} / \mathrm{cm}^{3}$ ). The values of $\mathrm{h}$ given by this solid curve were used in calculating the absorption curves presented in the the next Section. There is a marked disagreement between this curve and the theoretical relationship of Kneser ${ }^{7}$, which predicts that $f_{\max }$ varies as $h^{2}$, as indicated by the dashed curve. In contrast, there is reasonably good agreement with the more recent theoretical relationship given by Henderson and Herzfeld ${ }^{8}$ for a temperature of $20^{\circ} \mathrm{C}$.

In considering the relationship between $f_{\max }$ and $h$, the water vapor content, $h$, is usually expressed either in terms of mol fractions (or percent 
molar concentration) of water or in terms of the weight of the water in $\mathrm{gm} / \mathrm{m}^{3}$ (as in Fig. 2). The temperature dependency of the relationship between $f_{\max }$ and $h$ depends on the units in which $h$ is expressed. According to the experimental data of $k_{n o t z e}{ }^{9}$ the relationship between $f_{\max }$ and $h$ is relatively independent of temperature if the water vapor is expressed in $\mathrm{gm} / \mathrm{m}^{3}$. If we make this assumption and now obtain an equivalent relationship in which the water vapor is expressed in mol fractions, then $f_{\max }$ increases as the temperature decreases! Such an "inverse" relationship has been observed experimentally for other gases containing water vapor. 10 According to data of Ref. 9, the relationship, where $h$ is expressed in $\mathrm{gm} / \mathrm{m}^{3}$, is not actually constant; instead, at high concentrations of water vapor, as the temperature decreases the value of $f_{\text {max }}$ increases, thereby accentuating the "inversion" effect. The data obtained in this study at different temperatures shows an even more pronounced trend in this direction, which would accentuate the inversion effect. This point bears further investigation and may result in a modification of the above Eq. (4) at lower temperatures. But preliminary indications are that such a change with temperature of $f_{\max }$ versus $h_{\max }$ would have relatively small effect on the resulting values of the absorption of sound in air in the ranges of parameter values used most frequently in engineering problems.

Maximum Value of Molecular Absorption Versus Frequency. For a given temperature and frequency, the maximum value of molecular attenuation coefficient $m_{m}$ is represented by $m_{\max }$. According to theory, the maximum values of molecular absorption increase linearly with frequency. 7,10 since $m_{\max }=\mu_{\max } / \lambda$, we may obtain values of both $\mu_{\max }$ and $m_{\max }$ from plots of $\mu$ versus humidity, such as the one shown in Fig. 1. For example, in this illustration, $\mu_{\max }=0.00214$; dividing by the wavelength for a frequency of $6400 \mathrm{~Hz}$ at $20^{\circ} \mathrm{C}$ we obtain the maximum value of molecular absorption at this frequency and temperature, i.e., 0.0399 meters $^{-1}$. The values of $\mu_{\max }$ measured at the various frequencies were 
averaged, obtaining the following values of $\mu_{\max }$ at the temperatures Indicated: $-0.5^{\circ} \mathrm{C}, 0.00152 ; 5.6^{\circ} \mathrm{C}, 0.00170 ; 10.2^{\circ} \mathrm{C}, 0.00187 ; 14.8^{\circ} \mathrm{C}, 0.00205$; $20.0^{\circ} \mathrm{C}, 0.00214 ; 25.1^{\circ} \mathrm{C}, 0.00247$. These values, which are higher than similar data obtained in past studies with this experimental setup, were averaged with the earlier data. ${ }^{1,6}$ A smooth curve drawn through the resulting values of $\mu_{\max }$ yields values that are close to those predicted by theory.

In the calculation presented in Section IV, the following values of $\mu_{\max }$ were employed, at the temperatures indicated, in the computer program for determining the values of $\mathrm{m}_{\max }:-10^{\circ} \mathrm{C}, 0.00114 ;-5^{\circ} \mathrm{C}, 0.00128 ; 0^{\circ} \mathrm{C}, 0.00143$; $5^{\circ} \mathrm{C}, 0.00159 ; 10^{\circ} \mathrm{C}, 0.00176 ; 15^{\circ} \mathrm{C}, 0.00194 ; 20^{\circ} \mathrm{C}, 0.00214 ; 21^{\circ} \mathrm{C}, 0.00218 ; 22^{\circ} \mathrm{C}$, $0.00223 ; 23^{\circ} \mathrm{C}, 0.00227 ; 24^{\circ} \mathrm{C}, 0.00231 ; 25^{\circ} \mathrm{C}, 0.00235 ; 30^{\circ} \mathrm{C}, 0.00258$. Thus, at a given temperature, $T$, the maximum value of the molecular absorption $m_{m}$ for a frequency $f$ was obtained by dividing the value of $\mu_{\max }$ for the temperature by the wavelength corresponding to that frequency at temperature $T$. For example, for a temperature of $30^{\circ} \mathrm{C}$, the maximum absorption at $10,000 \mathrm{~Hz}$ was determined by dividing the value of $\mu_{\max }$ for $30^{\circ} \mathrm{C},(0.00258)$, by $\lambda$ for this frequency and temperature, obtaining a value of $\mathrm{m}_{\max }$ equal to $0.0740 \mathrm{~m}^{-1}$. The corresponding value at $1000 \mathrm{~Hz}$ is equal to $0.0074 \mathrm{~m}^{-1}$, etc.

Normalized Curves of Absorption Versus Humidity. The data points for each curve of molecular absorption versus humidity at constant frequency were normalized in the following way. At a given temperature the value of the coefficient $m_{m}$ for each data point was divided by the maximum value of the molecular attenuation coefficient $\mathrm{m}_{\max }$ for that temperature; similarly, the corresponding value of relative humidity, $h$, for the data point was divided by $h_{\max } \cdot$ According to the theory of Kneser, absorption versus humidity data for vartous frequencles which are so normalized should all fall along a single curve which is shown by dashed lines in Fig. 3. This study shows that such a normalization procedure results In a single curve. For example, the data points for $20^{\circ} \mathrm{C}$ are shown in Fig. 3 
and the best fit through these data, obtained at different frequencies, is represented by the solid curve. This solid curve Indicates that theory does not fit the measured values of absorption below and above the maximum value of absorption -- a result that has been observed in the past when the data of earlier investigators were presented in normalized form. ${ }^{11}$ This is because the above theory assumed that the angular relaxation frequency varies with $h^{2}$, as indicated by the dashed curve of Fig. 2, whereas the experimental data show that the relationship is more complex. The present study indicates that the value of $\mathrm{m} / \mathrm{m}_{\max }$ is slightly below 0.2 at high values of humidity. Knudsen's data 12 show much greater discrepancies between measured and theoretical values of $\mathrm{m} / \mathrm{m}_{\max }$ at high values of humidity. In contrast, the study of Delsasso and Leonard ${ }^{13}$ shows much closer agreement with the present data shown in Fig. 2 . Although the above theory does not fit the experimental data, it is Important to note that a single curve is obtained by rationalizing absorption data at all frequencles and temperatures as indicated above, and that this solid curve represents a best fit through the experimental data, and that the data collapse to a single curve within the limits of experimental measurement. Thus one may conclude that it is possible to measure the value of $h_{\max }$ and $m_{\max }$ for various frequencies and for various conditions of temperature. Then one can use the solid curve shown in Fig. 3 in combination with these measured values, to compute curves of molecular absorption versus humidity for various frequencies and temperatures. This procedure was followed, employing a high-speed digital computer which had been programmed to calculate the curves of molecular absorption verous humidity, using the data from experimental measurements as input information. In the computer, the classical absorption was added to the molecular absorption to yield the total value of the attenuation coefficient. The advantage of this evaluation procedure is that it provides a convenient means of determining sound absorption at frequencies and temperatures other than the exact values at 
which the measurements were made, by interpolation or extrapolation. This procedure was followed to obtain the results presented in Section IV.

\section{SECTION III. DISCUSSION AND COMPARISON WITH OTHER STUDIES}

In this Section a comparison is made between the present results and those obtained in other investigations. In most of these studies the reverberation chamber method has been employed, in which the rate of decay of sound in air has been measured for various humidity conditions at a constant value of frequency. Laboratory measurements of the absorption of sound in air are subject to several major sources of error (systematic or random) resulting from: (1) an inability to evaluate with precision losses in acoustic energy introduced by the walls of the test chamber; (2) the lack of adequate control of the test chamber environment, including temperature and gas composition; and (3) the lack of precision in humidity measurement, either as a result of instrumentation error or as a result of absorption of moisture by the walls of the test chamber. These sources of error are considered below in comparing the results of the various studies.

Evaluation of Wall Losses. In order to achieve higher accuracy in the measurement of the absorption of sound in air by the reverberation method (or the "intensity" method); it is necessary to evaluate wa11 losses with precision. The greater the wall losses, the more important is this source of error. Since the decay rate which is measured depends upon the sum of the absorptions by the walls and the air, the wall losses should be small compared with the air losses. Therefore, the losses of acoustic energy at the walls of the test chamber should be reduced to a bare minimum.

For the above reasons, a spherical chamber was employed in this study, since it presents the smallest wall surface area for a given volume and since its shape makes it possible to achieve a very high value of acoustical impedance at the boundaries. By using very heavy walls in the present experimental setup, 
it was possible to achieve a reverberation time in dry air of 43 seconds at $1000 \mathrm{~Hz}$. This is very many times longer than the comparable values in earlier studies and the associated boundary losses are significantly lower. In determining the attenuation coefficients for air the effects of wall losses were subtracted out as indicated by Eq. (3).

In the "two-chamber" method employed by Knudsen, 12 it was assumed that the absorption coefficients of both chambers were the same. Although his chamber walls were of steel and were reinforced with spot-welded angle irons, it is unlikely that the boundary conditions in the chambers were Identical because the walls were only $4.8 \mathrm{~mm}$ thick. Intercomparisons between three or more similarly fabricated model chambers have shown that it is difficult to achieve identical boundary conditions in chambers of different sizes. In contrast, the steel walls of the sphere used in the present study were $16 \mathrm{~mm}$ thick, and the boundary losses were evaluated in the same chamber in which the measurements were carried out.

In the study by Evans and Bazley, a single chamber was employed, but because of physical limitations it was impractical to seal the chamber and fill it with a gas, such as nitrogen, for the purpose of calibrating wall losses. Hence, they used the following indirect evaluation procedure which afforded greater opportunity for error. They computed their wall losses and curves of absorption vs humidity from the following equation and only a single set of experimental measurements (i.e. measurements of the reverberation time of an empty reverberation chamber for various humidity conditions):

$$
A=\underbrace{\frac{137.2 f \times 10^{-4}}{\frac{k}{2 \pi f}+\frac{2 \pi f}{k}}}_{\text {molecular }}+\underbrace{4.6 \sqrt{f} \times 10^{-2}}_{\text {ideal surface }}+\underbrace{4.1 f^{2} \times 10^{-8}}_{\text {classical }}+\delta
$$

where $A$ is the total absorption of sound in the chamber in square meters, $f$ is the frequency in Hertz, $k$ is the angular relaxation frequency $\left(2 \pi f_{\max }\right), \delta$ 
represents the difference between the actual wall losses and the values predicted by theory, and the numerical constants are dependent on the physical characteristics of the room. The first term had a maximum value of $68.6 \mathrm{f} \times 10^{-4}$. The single set of data, obtained over a period of two years, was corrected to a temperature of $20^{\circ} \mathrm{C}$ and the above equation was used: (1) to evaluate their wall 10sses; (2) to determine the angular relaxation frequency, $k$; (3) to determine the magnitude of the molecular absorption; (4) to compute the total absorption, 1.e., molecular plus classical absorption; and (5) to check their results. Since physical limitations of the measurement setup made it impossible to provide any independent means of measuring the above parameters, including the effects of wall losses, the check was one of self-consistency. Since present theory is not adequate for predicting the value of molecular absorption accurately for various temperatures and humidities, the first term in Eq. (5), which includes the term "k" $\left(k=2 \pi f_{\text {max }}\right)$, is open to question. Further, it has not been established that the room absorption; in a non-ideal situation such as their reverberation chamber, can be represented by the second term plus a constant in the form shown in $\mathrm{Eq} .(5)$.

Control of Environment of Test Chamber. In the present study, the test chamber was completely surrounded by one to two feet of fiberglass to provide thermal insulation. A refrigeration system maintained the temperature of the chamber to within $0.1^{\circ} \mathrm{C}$. In contrast to this and the controls employed in other studies, no temperature control was employed in the chamber used by Bvans and Bazley. As a result, the standard deviation of the room temperature at which measurements were made was $2.1^{\circ} \mathrm{C}$. It was necessary for them to rely on theory to correct their results to $20^{\circ} \mathrm{C}$ and an adequate theoretical treatment of how absorption varies with temperature has yet to be established.

The measurements described in this paper were made using a closed air circulation system, shown in Fig. 4 and described in Ref. 1. To lower the 
humidity, moisture was taken from the test chamber and deposited in the "saturator"; to raise the humidity, the process was reversed. Thus all measurements were made on the same air, of known chemfcal content. This system avoids the possibility of contamination of the air under test, either by smog or by chemical drying agents such as those employed by Evans and Bazley to reduce the value of humidity in their test chamber. Furthermore, it avoids the addition of acoustic absorption tinto the measurement system which such a drying technique Introduces.

Humidity Measurementa; $f_{\max }$ versus $h_{\max }$. Using the present experimental setup the author tried several different methods of humidity measurement that had been employed by earlier investigators. One method was to weight the amount of water that was evaporated in the test chamber prior to the establishment of a given humidity condition. It was demonstrated that this technique can lead to serious error since a significant fraction of the water that enters the chamber may be absorbed by the walls of the chamber. Similarly, if the humidity of the air that enters or leaves the test chamber is measured (which 18 another technique which has been employed by some investigators in the past) the measurement value does not represent the humidity within the chamber because of absorption or emission of molsture by the walls of the test chamber -- unless a steady-state humidity condition is achleved. To avold these errors the humidity control and measurement system shown in Fig. 4 was constructed. Air was recirculated until a steady-8tate condition of humidity was achieved; often, this required as much as $1 / 2$ hour of recirculation. Then the humidity was determined by electric hygrometer sensing elements as described in Ref. 1 .

A determination of the relationship between relaxation frequency and humidity requires highly accurate measurement of humidity, particularly at low concentrations of water vapor where the accuracy of measurement is relatively poor. Although a humidity measurement error may have a small effect on the peak 
value of the absorption curve, a small error in measuring humidity can have a significant effect in shifting the position of the peak along the humidity scale and hence in determining the value of $h_{\max }$. For this reason there has been considerable discrepancy between such data measured by various investigators. Comparisons are shown in Fig. 2 of the results of several air absorption studies in terms of the relaxation frequency, $f_{\max }$, versus concentration of water vapor ( $h$, expressed in $g \mathrm{~m} / \mathrm{cm}^{3}$ ) at which the maximum absorption is achieved for the frequency.

Knudsen's data, represented by the three experimental points at $20^{\circ} \mathrm{C}$ do not provide sufficient data for an explicit relationship between the relaxation frequency and concentration of water vapor. Evans and Bazley reported that the relaxation frequency varies as $h^{1.3}$. This is shown as a dashed curve in Fig. 2. The $20^{\circ} \mathrm{C}$ data of the present study is within the measurement accuracy of the earlier data of Refs. 1 and 6 . Likewise, the data of Knotzel also could be represented by the solid curve of Eq. (4).

Comparison of Data for Oxygen Containing Water Vapor. One means of providing an independent check on the overall measurement system for the absorption of sound in air is to use the system to measure the absorption of sound in oxygen and then to compare these data with results obtained by a different measurement technique, which is not subject to the same types of errors. Such an independent check has been made at $20^{\circ} \mathrm{C}$. The experimental setup used for sound absorption data presented here was used to measure the absorption of sound in oxygen as a function of water vapor content. 14 The results so obtained agree very closely with recent data for oxygen obtained by Henderson 15,16 who employed a resonant pressure chamber measurement system, 17 using a measurement technique that avoids 
the use of a hygrometer, which may introduce one of the principle sources of error: Also, these results are in close agreement with the oxygen data of Knotzel and Knotze ${ }^{18}$ as indicated by the comparison shown in Fig. 2 of Ref. 14 (also see Fig. 3 of Ref. 16). In contrast, the results of Knudsen and Obert ${ }^{19}$ for oxygen are in substantial disagreement with these more recent data. A comparison of this type could not be made by Evans and Bazley ${ }^{3}$ since their experimental setup did not permit them to make sound absorption measurements in gases other than air.

\section{SECTION IV. RESULTS}

Employing the above data reduction procedures, the attenuation coefficients of sound in air at normal pressure have been evaluated for the following conditions: (1) at the temperatures of $-10^{\circ},-5^{\circ}, 0^{\circ}, 5^{\circ}, 10^{\circ}, 15^{\circ}, 20^{\circ}, 21^{\circ}, 22^{\circ}, 23^{\circ}, 24^{\circ}$, $25^{\circ}$ and $30^{\circ}$ Centigrade; (2) at relative humidities of $5 \%$ to $100 \%$ in increments of $5 \%$ R.H. [except in the range from 45 to $55 \%$ R.H. where the increments are 1\%]; and (3) for frequenctes of $125,250,500,1000,2000,2500,3200,4000,5000,5940$, $6300,8000,10000$ and $12500 \mathrm{~Hz}$. Values in the frequency range from 125 to $1000 \mathrm{~Hz}$ were determined by extrapolation from the data at higher frequencies by the procedure indicated in Section II. Data for the full temperature and humidity ranges listed above is tabulated in both the metric and English systems of units in Appendix I. Figure 5 shows the results of this study of total attenuation coefficient versus humidity for sound in air at one of the above temperatures -$20^{\circ} \mathrm{C}$. This information is presented in another form for other values of temperature and humidity in Figs. 6 and 7 .

The effect of temperature 'on curves of sound absorption versus humidity at constant frequency is illustrated in Fig. 8, which presents absorption data for a frequency of $4000 \mathrm{~Hz}$. Here the total attenuation coefficient $\mathrm{m}$ is plotted as a function of humidity for different values of temperature. Note (1) that 
the maximum value of absorption increases with increasing temperature, and (2) that the peak in the curves shifts to a lower value of relative humidity as the temperature increases. Figure 9 shows curves of absorption versus temperature for constant values of frequency -- all for a relative humidity of $50 \%$.

SECTION V. APPLICATION OF RESULTS TO ROOM ACOUSTICS AND PROPAGATION PROBLEMS

Propagation of Sound in Air. For problems concerned with the propagation of sound in air it is convenient to express attenuation of sound in terms of decibels per unit distance. Values of attenuation in $\mathrm{dB} / \mathrm{m}$ are obtained by multiplying the total attenuation coefficient by 4.343. This information has been tabulated in full in Appendix I both in the metric and English systems of units. Some of the data are plotted in Fig. 6, which gives the attenuation in $\mathrm{AB} / 100$ meters as a function of temperature for various values of relative humidity. This represents the attenuation of a plane wave in a homogeneous medium due only to air absorption. This is the attenuation in excess of the loss due to spherical divergence, which is $6 \mathrm{~dB}$ for each doubling of the distance from the source, so that it is sometimes referred to as "excess attenuation." It does not include losses due to other factors such as scattering.

Calculation of Reverberation Time. In calculating the reverberation time of a room, the total absorption in the room is required. This total is the sum of the absorptions due to the losses at the boundaries, to the furnishings in the room and to air absorption. The absorption due to losses in air usually is important only in large rooms and/or at high frequencies, and is given by 4mV. This term represents the area of a perfectly absorptive surface that is equivalent to the absorption of sound in air in a room of volume $V$. The product $4 \mathrm{~m}$ is given in the tabular data of Appendix $I$. These values need only be 
multiplied by the volume of the room to determine the values of air absorption. Examp1e: Suppose a room has a volume of $1,000,000 \mathrm{ft}^{3}\left(28 ; 320 \mathrm{~m}^{3}\right)$ and is at a temperature of $20^{\circ} \mathrm{C}$ and a relative humidity of $47 \%$. Find the absorption In the room due to air at a frequency of $4000 \mathrm{~Hz}$. From Appendix I the product $4 \mathrm{~m}$ for these conditions equals $0.0077 \mathrm{ft}^{-1}$. Multiplying by the volume $1,000,000 \mathrm{ft}^{3}$, we obtain a total absorption of 7700 Sabins. An equivalent result is obtained in the metric system if we use a value of $4 \mathrm{~m}$ equal to $0.0253 \mathrm{~m}^{-1}$ and multiply by a volume of $28,320 \mathrm{~m}^{3}$ to obtain the total absorption in metric Sabins.

Corrections to Reverberation Time Measurements. As indicated by the data of Appendix I, the contribution to the rate of decay of sound in a large room due to air absorption is significant at higher frequencies and it varies with humidity and temperature. One source of discrepancy in the measured values of reverberation time of the same auditorium by different investigators may be attributed to differences, during the measurements, of temperature and humidity conditions. The following procedure is suggested to correct this possible source of discrepancy, and to provide an improved basis for the comparison of reverberation time data for various large halls at highex frequencies: In presenting reverberation time data for large auditoriums, irrespective of the humidity and temperature at which measurements are made, correct the reverberation time so that it represents the reverberation time that would have been obtained if the measurements had been made at a relative humidity of $50 \%$ and a temperature of $20^{\circ} \mathrm{C}$-- "standard conditions." This correction may be made as follows. Subtract the decay rate due to air absorption for the actual measurement conditions from the rate of decay at $20^{\circ} \mathrm{C}$ and $50 \% \mathrm{R} . \mathrm{H}$. This difference is to be added to the measured value of the rate of decay of sound in the room. From this corrected decay rate, compute the corrected reverberation time. 
Example: Suppose that reverberation time measurements, are made in an auditorium at a temperature of $25^{\circ} \mathrm{C}$ and a relative humidity of $80 \%$. At $4000 \mathrm{~Hz}$ the measured value of the reverberation time is 2.10 seconds. What would the reverberation time be under "standard conditions" $\left(20^{\circ} \mathrm{C}, 50 \%\right.$ R.H.)?

Step (1): Calculate the measured decay rate

$$
\mathrm{R}_{\text {measured }}=\frac{60 \mathrm{~dB}}{2.10 \mathrm{sec}}=28.57 \mathrm{~dB} / \mathrm{sec}
$$

Step (2): Subtract the decay rate due to air absorption at a relative humidity of $50 \%$ and a temperature of $20^{\circ} \mathrm{C}$ from the decay rate due to air absorption under measurement conditions. For a frequency of $4000 \mathrm{~Hz}$, the rate of decay due to air absorption at $25^{\circ} \mathrm{C}$ and $80 \%$ relative humidity is $7.57 \mathrm{~dB} / \mathrm{sec}$; at "standard conditions" of $20^{\circ} \mathrm{C}, 50 \% \mathrm{R} . \mathrm{H}$. the corresponding value is $9.11 \mathrm{~dB} / \mathrm{sec}$. Subtracting, the difference, $R_{D}$, is $-1.54 \mathrm{~dB} / \mathrm{sec}$.

Step (3): Add the value of $R_{D}$ obtained in Step (2) to the measured rate of decay. This represents the decay rate corrected to normal conditions.

$$
R_{\text {corrected }}=R_{\text {measured }}+R_{D}=28.57 \mathrm{~dB} / \mathrm{sec}-1.54 \mathrm{~dB} / \mathrm{sec}=27.03 \mathrm{~dB} / \mathrm{sec}
$$

Therefore, the reverberation time under standard conditions is:

$$
t_{60}=\frac{60 \mathrm{~dB}}{27.03 \mathrm{~dB} / \mathrm{sec}}=2.22 \mathrm{sec}
$$

\section{Corrections to Measurements of Sound Absorption Coefficients. According to}

Eq. (2) the rate of decay of sound in a test chamber (or a room) may be represented as the sum of two terms: (1) the rate of decay of sound due to air absorption, and (2) the rate of decay of sound resulting from absorption at the boundaries. Thus if measurements of the absorption coefficients of an acoustical material are made in a reverberation chamber, at higher frequencies -where air absorption is significant -- an error in evaluating the absorption 
coefficients will result unless the contribution to the total decay caused by air absorption is subtracted out. If this correction is made, then one should obtain the same value of absorption coefficient for the material under test even if measured under different conditions of humidity and temperature. Such a correction can be made conveniently by the use of Appendix I. For example, suppose measurements at $4000 \mathrm{~Hz}$ are made in a reverberation chamber at a temperature of $25^{\circ} \mathrm{C}$ and a relative humidity of $80 \%$. According to Appendix I the rate of decay due to sound absorption in air, $R_{a i r}$, is $7.57 \mathrm{~dB} / \mathrm{sec}$. According to Eq. (2), the measured decay rate is equal to the sum of the decay rate due to absorption at the boundaries of the room and due to air absorption. Thus, if the measured value of the rate of decay is $22.10 \mathrm{~dB} / \mathrm{sec}$ the actual decay rate corrected for air absorption is $(22.10-7.57) \mathrm{dB} / \mathrm{sec} \simeq 13.5 \mathrm{~dB} / \mathrm{sec}$. Thus it is possible to correct for air absorption in measuring the absorption coefficient of a material in a reverberation chamber. Such a procedure has been proposed in Comnittee C-20 of the ASTM. 20 
1. C. M. Harris, J. Acoust. Soc. Am., 35, 11-17 (1963).

2. H.D. Parbrook and W. Tempest, Acustica, \&, 345-350 (1958).

3. E. J. Evans and E. N. Bazley, Acustica, 6, 238-245 (1956).

4. V. O. Knudsen, V. Wilson and N. S. Anderson, J. Acoust. Soc. Am., 20, 849-857 (1948).

5. J. Hillsenrath et. a1., Table of Thermal Properties of Gases, National Bureau of Standards Circular 564, November 1, 1955.

6. C. M. Harris and W. Tempest, J. Acoust. Soc. Am., 36, 2390-2394 (1964).

7. H. 0. Kneser, J. Acoust. Soc. Am., 5, 122-126 (1933). Also Akust. Z., 5, 256-257 (1940); Ergeb. exakt. Naturw., 22, 121-185 (1949).

8. M. C. Henderson and K. F. Herzfeld, J. Acoust. Soc. Am., 37, 986-988 (1965).

9. H. Knotzel, Akust. Z., 2, 245-256 (1940).

10. K. F. Herzfeld and T. A. Litovitz, Absorption and Dispersion of Ultrasonic Waves, New York: Academic Press (1959).

11. W. L. Nyborg and D. Mintzer, Sect. 1.2.4., WADC Tech. Rept. 54-602, Wright Air Development Center, Wright Patterson A.F.B., Ohio, May 1955.

12. V. 0. Knudsen, J. Acoust. Soc. Am., 5, 112-121 (1933).

13. L. P. Delsasso and R. W. Leonard, "The Attenuation of Sound in the Atmosphere," Report under U.S.A.F. Contract W-28-099-AC-228, February 25, 1953.

14. C. M. Harris and W. Tempest, J. Acoust. Soc. Am., 36, 2416-2417 (1964).

15. M. C. Henderson, J. Acoust. Soc. Am., 34, 349-350 (1962).

16. M. C. Henderson, A. V. Clark and P. R. Lintz, J. Acoust. Soc. Am., 37, 456-463 (1965).

17. M. C. Henderson and J. G. Donne11y, J. Acoust. Soc. Am., 34, 779-784 (1962).

18. H. Knotzel and L. Knotzel, Ann. Phys., 2, 393-403 (1948).

19. V. O. Knudsen and L. Obert, J. Acoust. Soc. Am., Z, 249-253 (1936).

20. Ralph Huntley, personal communication. 


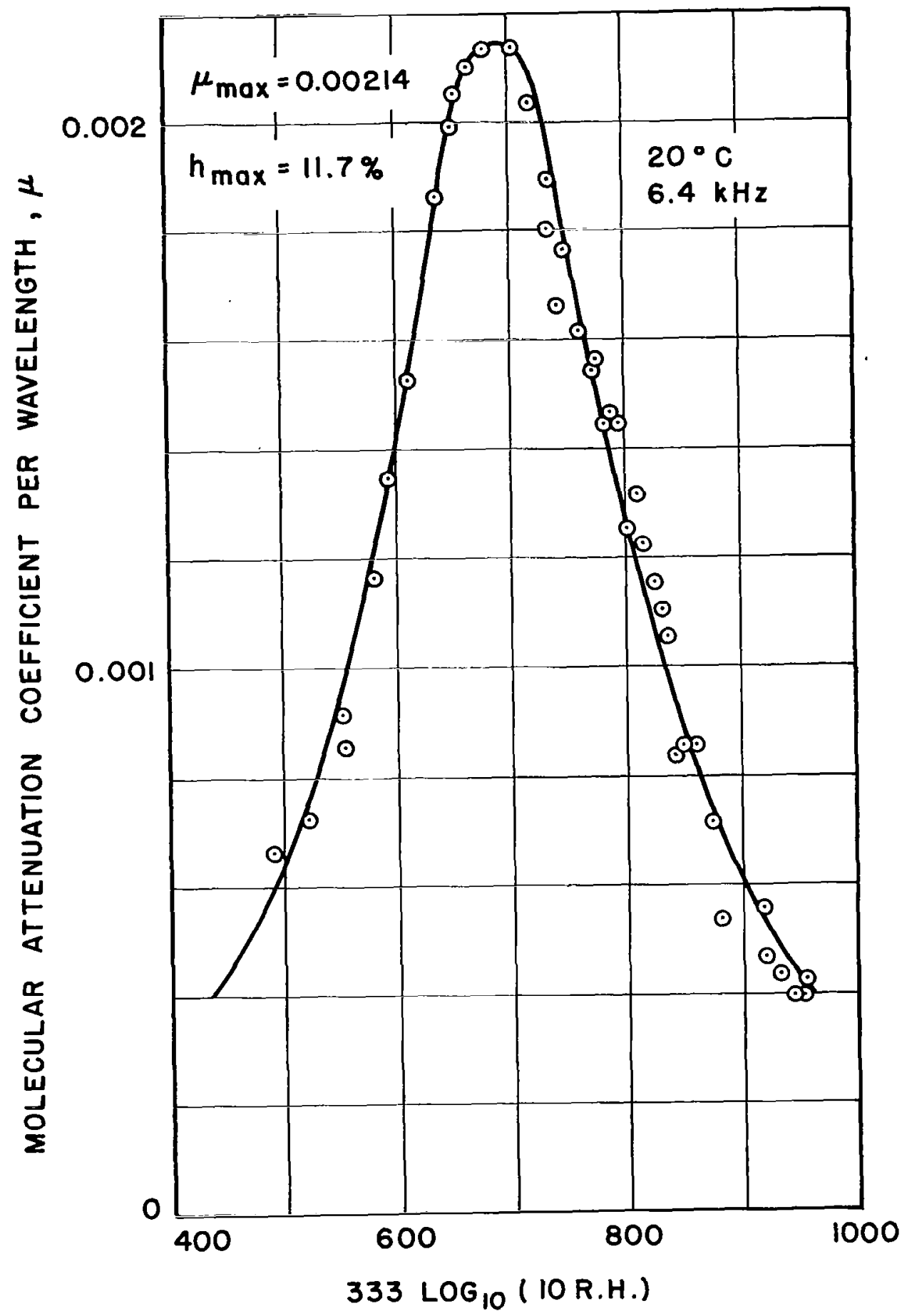

FIGURE 1. FXAMPLE OF A PLOT OF EXPERIMENTAL DATA OF THE MOLECULAR ATTENUATION COEFFICIENT PER WAVELENGTH IN AIR AS A FUNCTION OF HUMIDITY. THESE DATA ARE FOR A TEMPERATURE OF $20^{\circ} \mathrm{C}$ AND A FREQUENCY OF $6400 \mathrm{~Hz}$ 


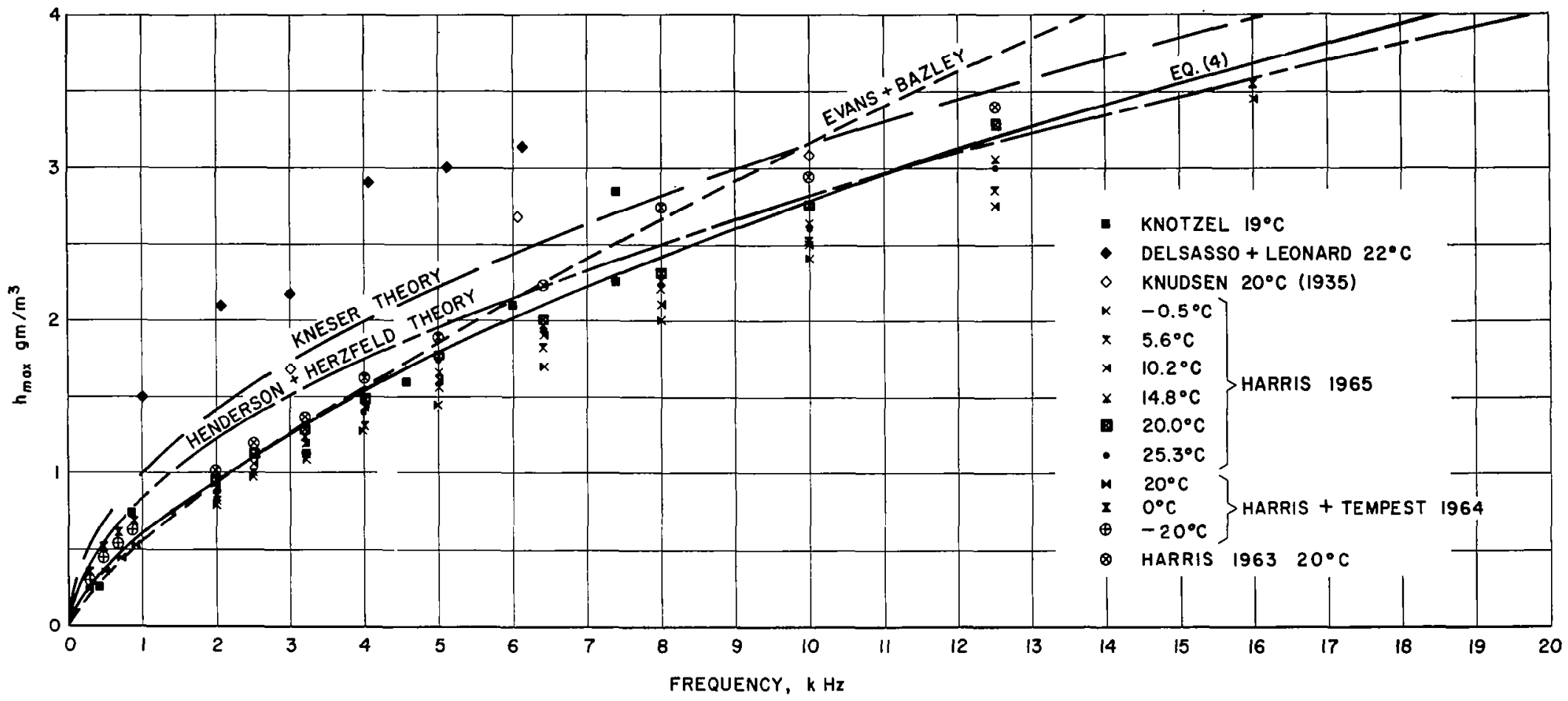

FIGURE 2. RELAXATION FREQUENCY AS A FUNCTION OF THE CONCENTRATION OF WATER VAPOR IN AIR EXPRESSED IN GM/M ${ }^{3}$. THE RELATIONSHIP USED IN THE RESULTS PRESENTED IN THIS PAPER IS SHOWN AS THE SOLID CURVE 


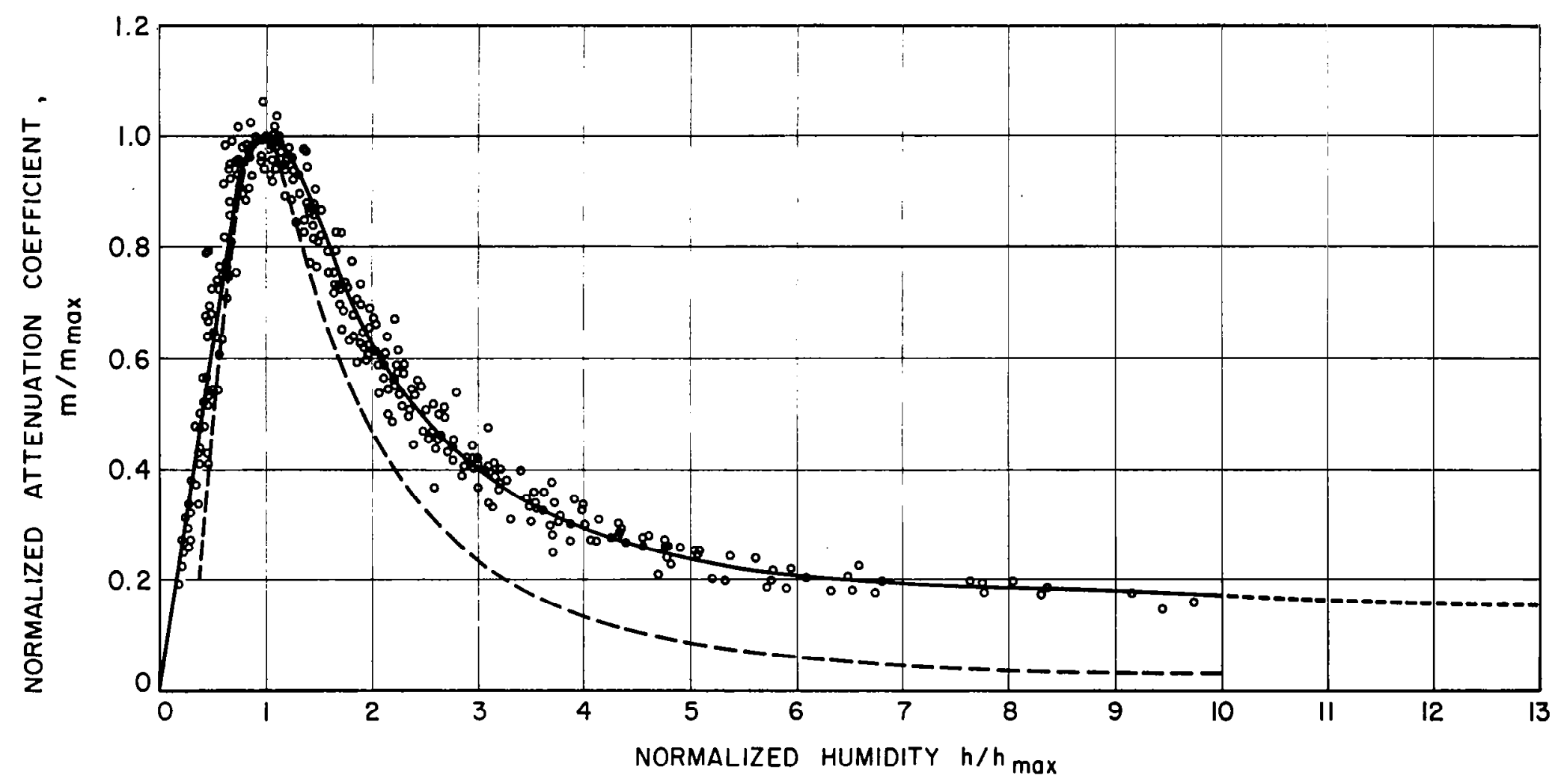

FIGURE 3. PLOT OF EXPERIMENTAL DATA OF THE MOLECULAR ATTENTUATION COEFFICIENT IN AIR VERSUS HUMIDITY. THESE DATA ARE PRESENTED IN THE NORMALIZED FORM, M/M MAX VS H/H ${ }_{\text {MAX }}$ FOR COMPARISON WITH THE THEORETICAL RELATIONSHIP OF KNESER SHOWN BY THE DASHED LINE 


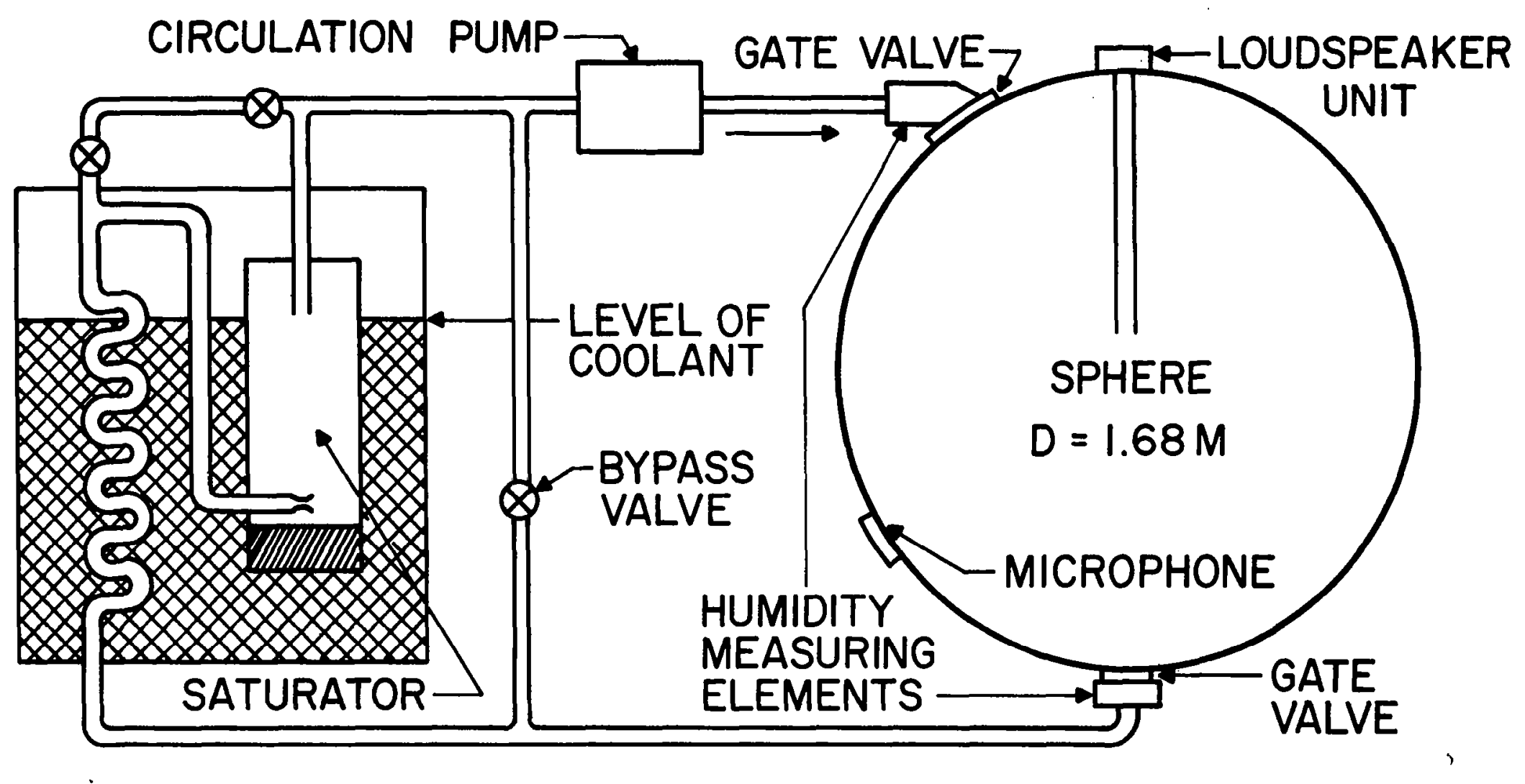

FIGURE 4. SIMPLIFIED SCHEMATIC DIAGRAM OF THE AIR-CIRCULATION SYSTEM. AIR IS RECIRCULATED CONTINUOUSLY THROUGH THE SPHERICAL CHAMBER. THE SATURATOR EITHER TAKES AWAY MOISTURE FROM THE AIR OR ADDS MOISTURE TO IT -- DEPENDING ON THE RELATIVE TEMPERATURES OF THE SPHERICAL CHAMBER AND THE SATURATOR 


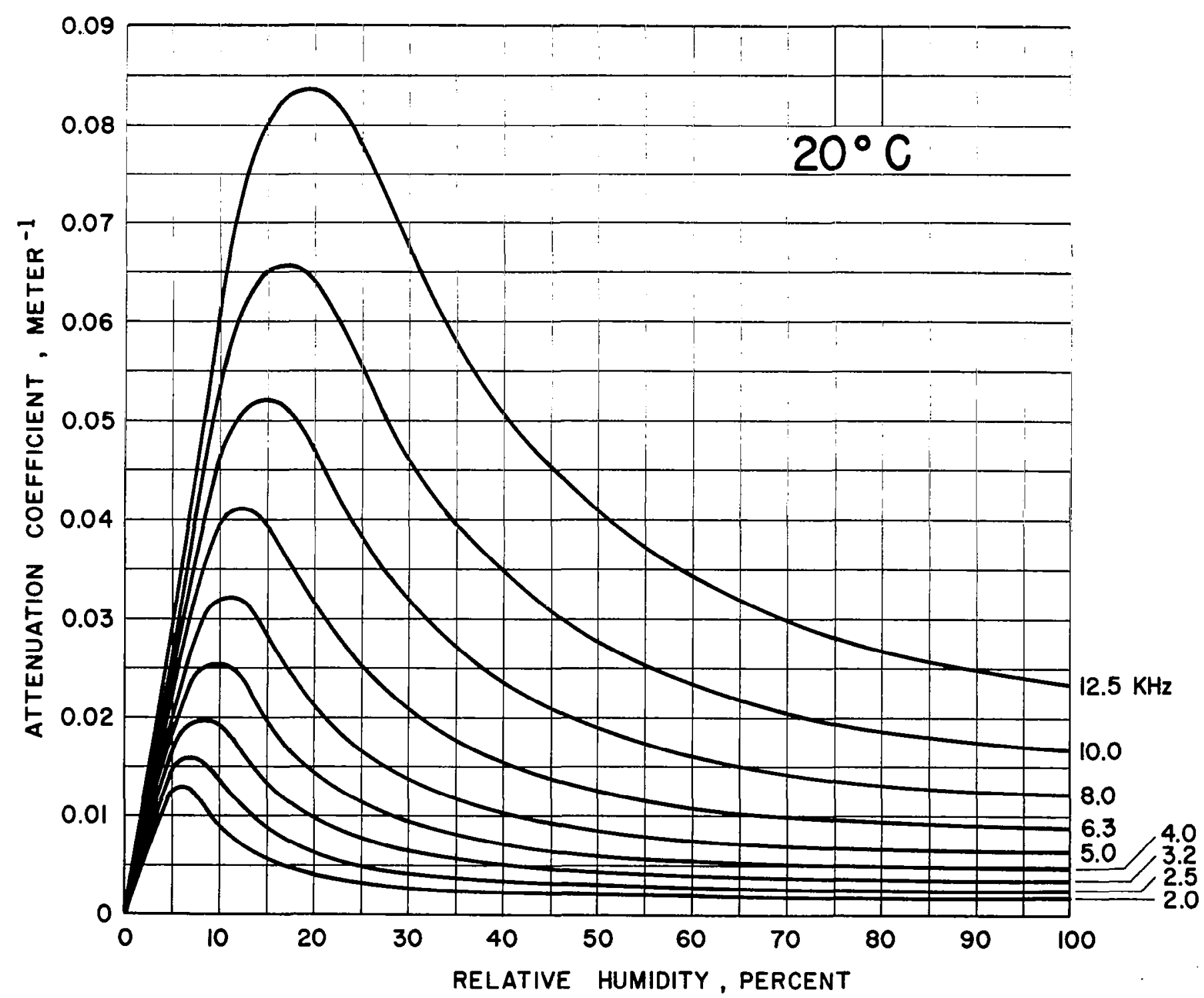

FIGURE 5. VALUES OF THE TOTAL ATTENUATION COEFFICIENT M. VERSUS PERCENT R. H. FOR AIR AT $20^{\circ} \mathrm{C}$ AND NORMAL ATMOSPHERIC PRESSURE FOR FREQUENCIES 


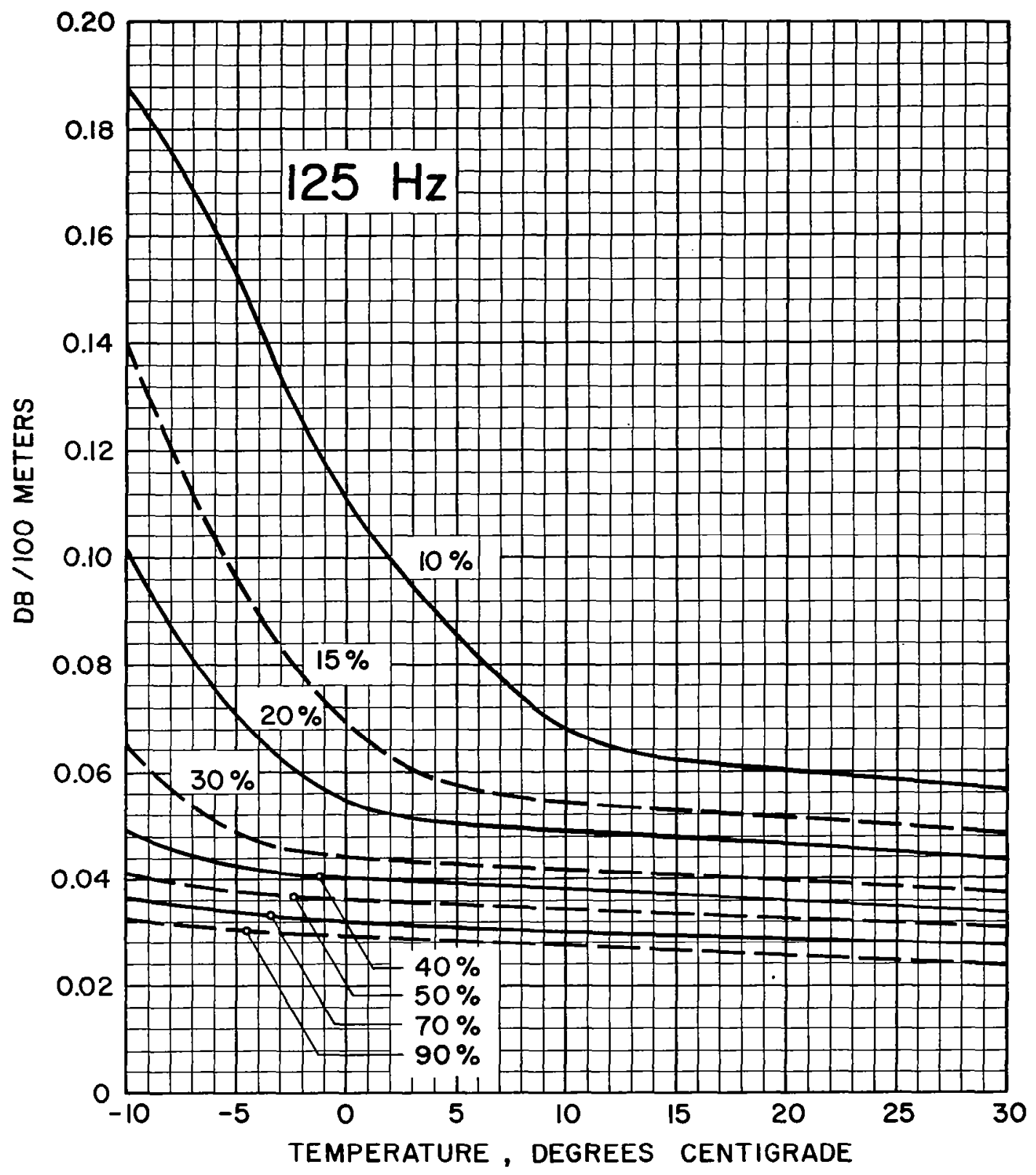

FIGURE 6a. THE ATTENUATION OF SOUND IN AIR VERSUS TEMPERATURE FOR VARIOUS VALUES OF RELATIVE HUMIDITY. THE ATTENUATION OF DB/100 METERS IS SHOWN AS THE ORDINATE AT THE LEFT OF THE GRAPH 


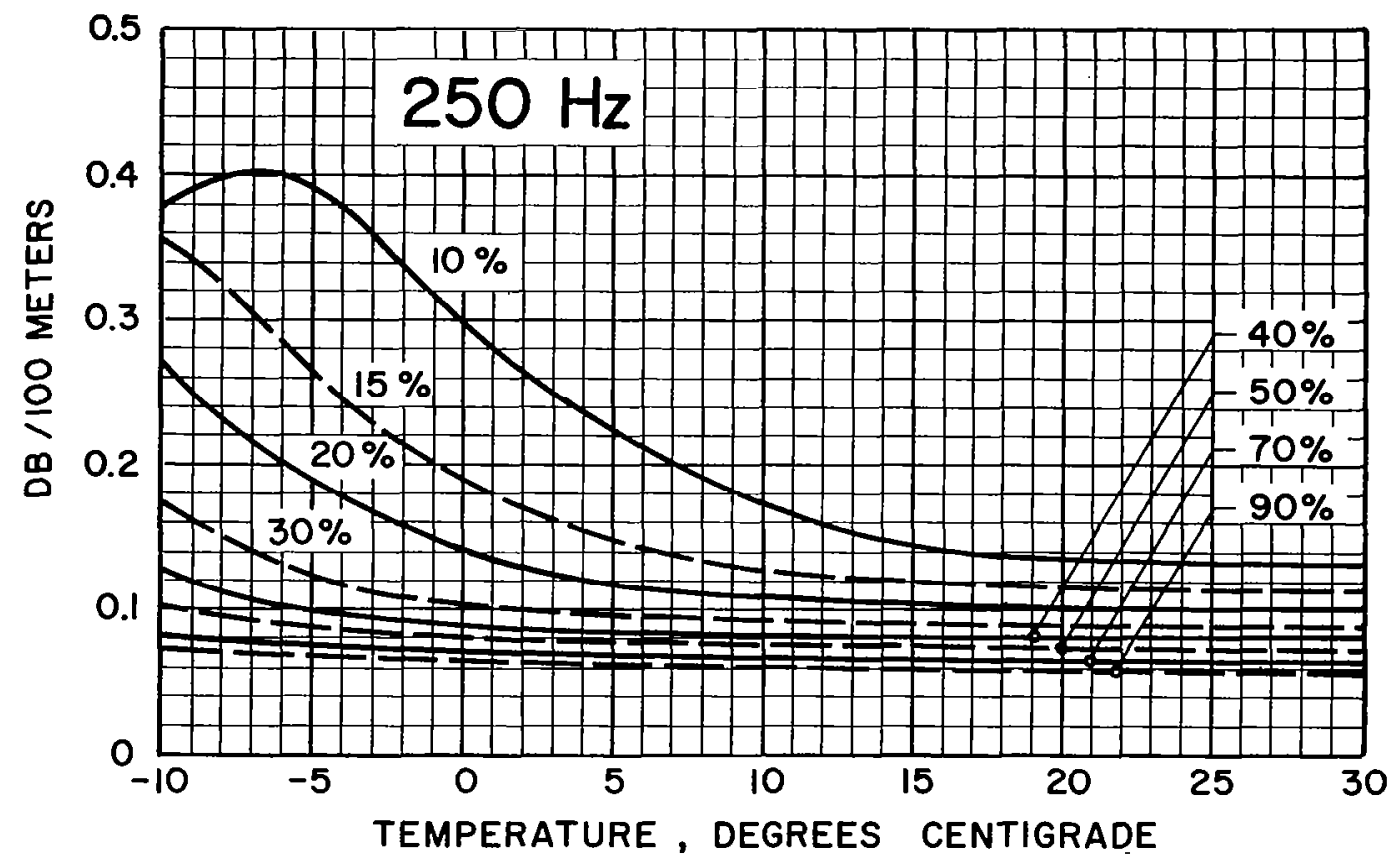

FIGURE 6b. (CONTINUED) 


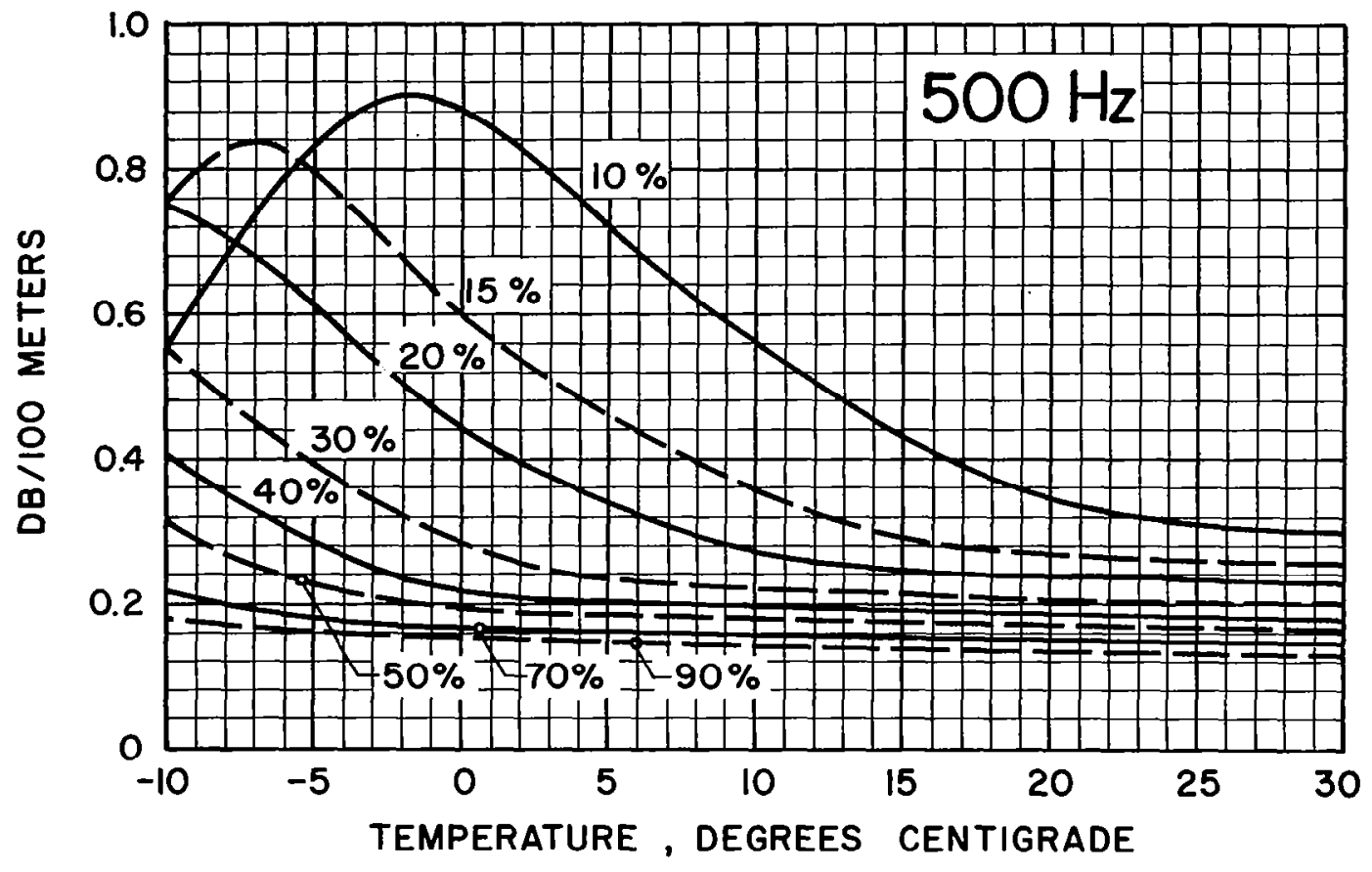

FIGURE 6c. (CONTINUED) 


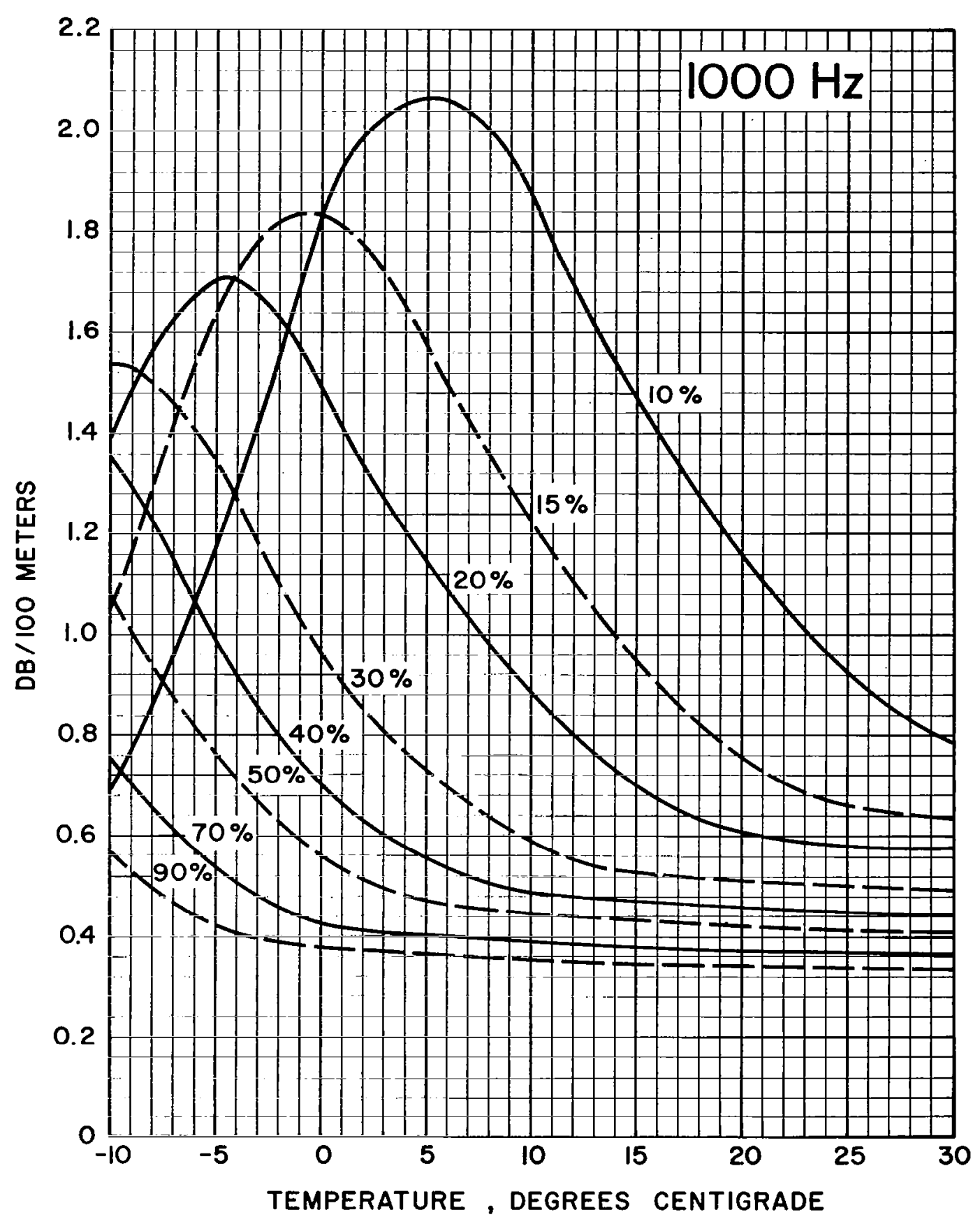

FIGURE 6d. (CONTINUED) 


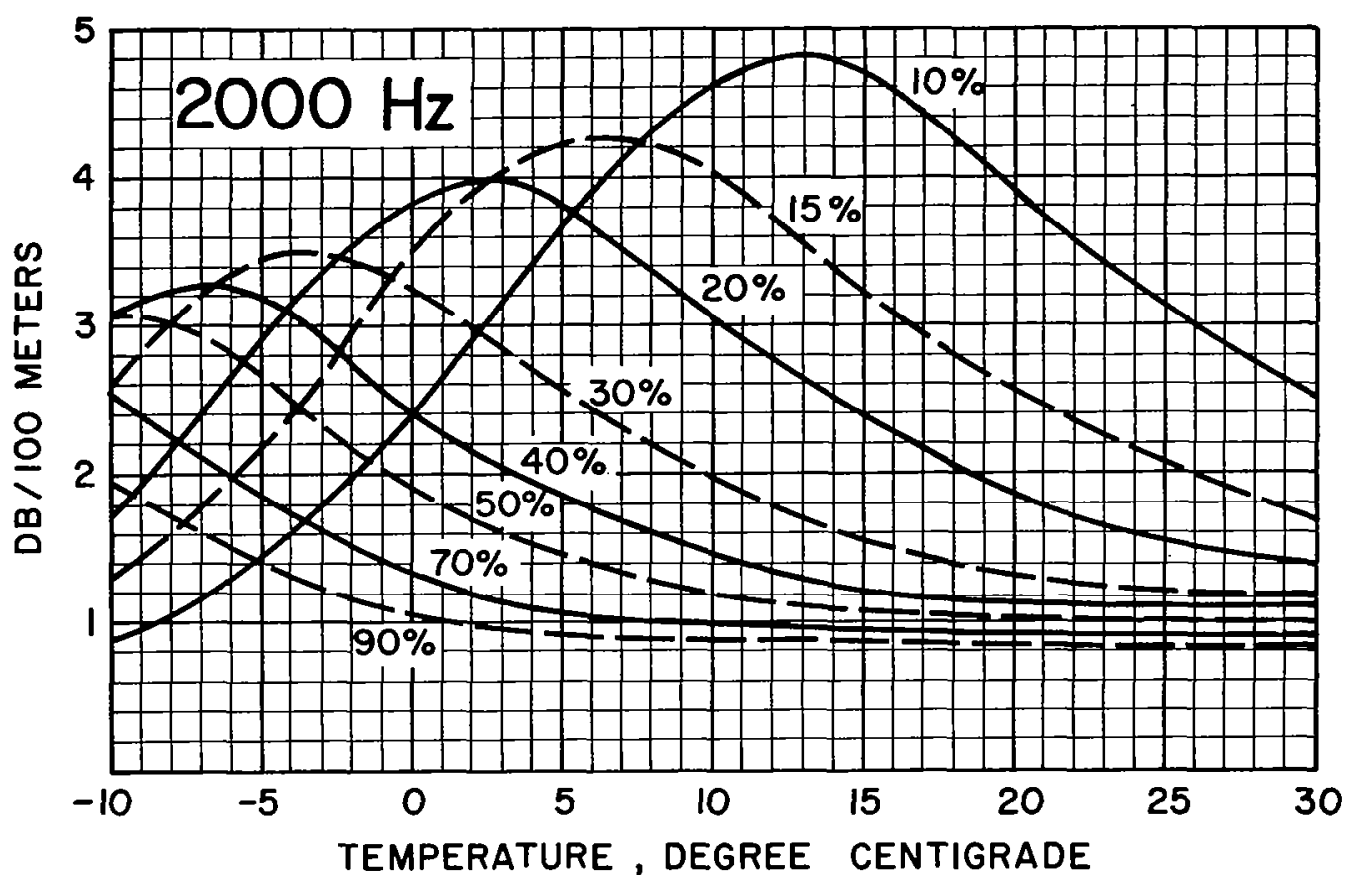

FIGURE 6e. (CONTINUED) 


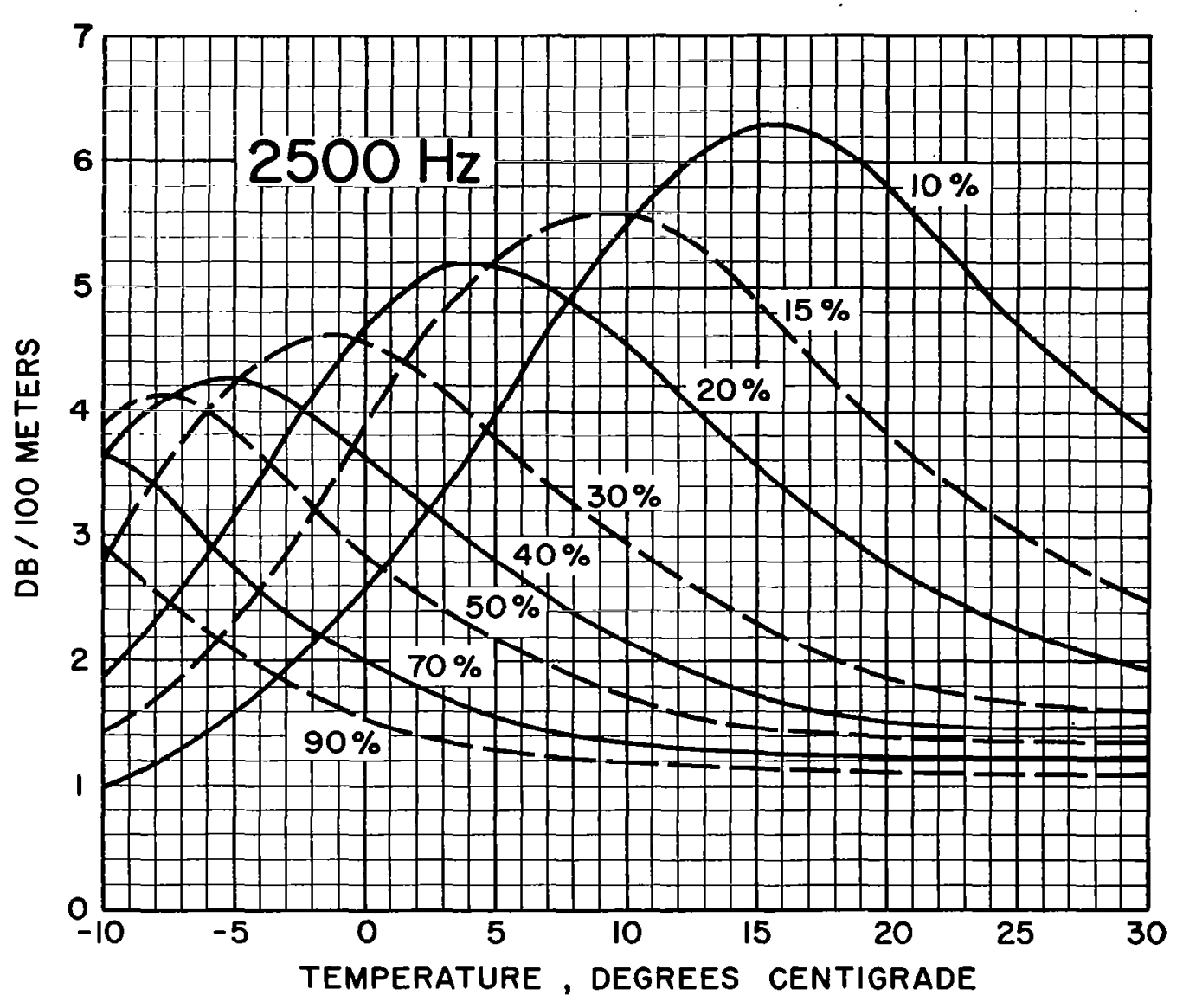

FIGURE 6f. (CONTINUED) 


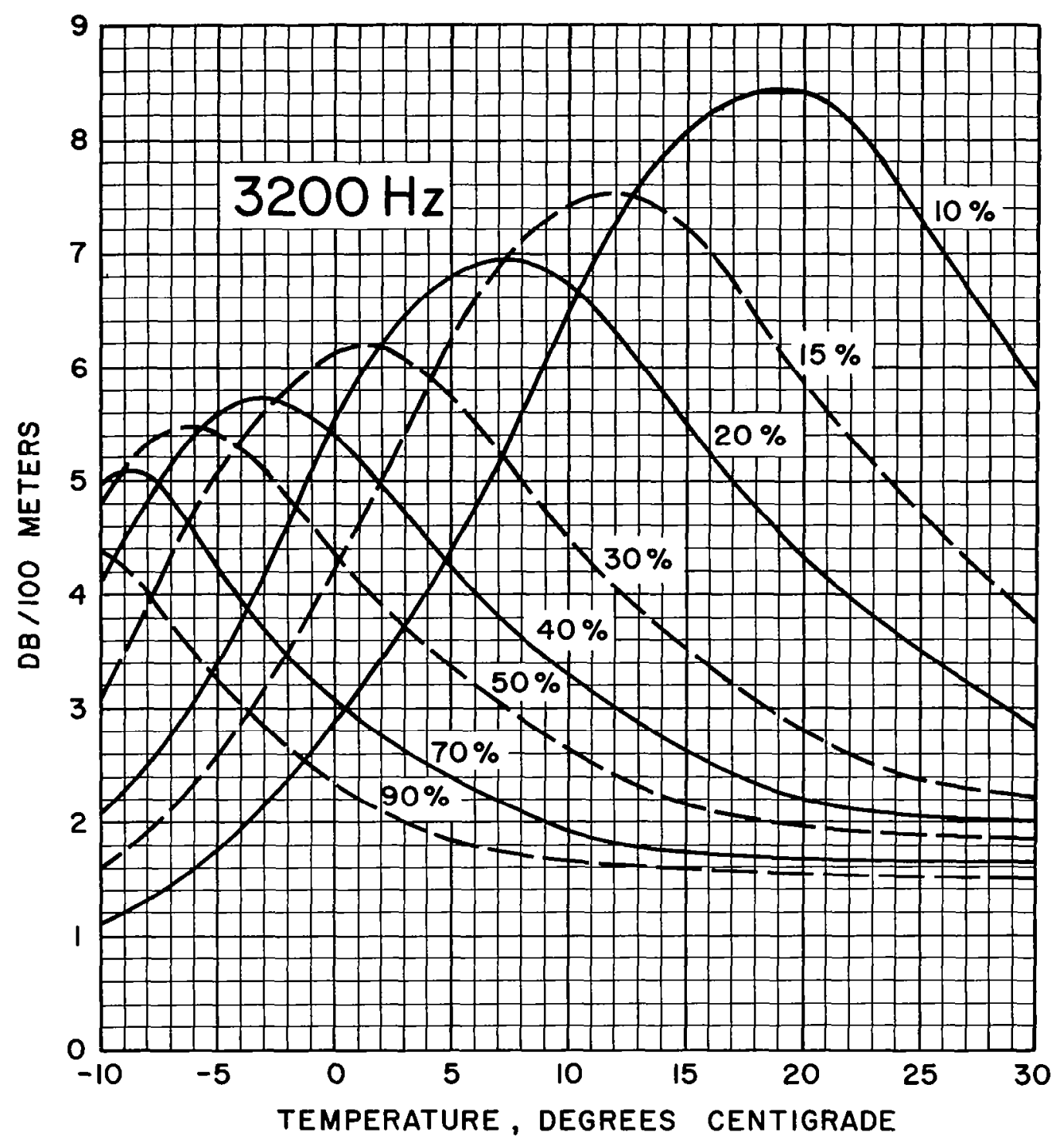

FIGURE 6g. (CONTINUED) 


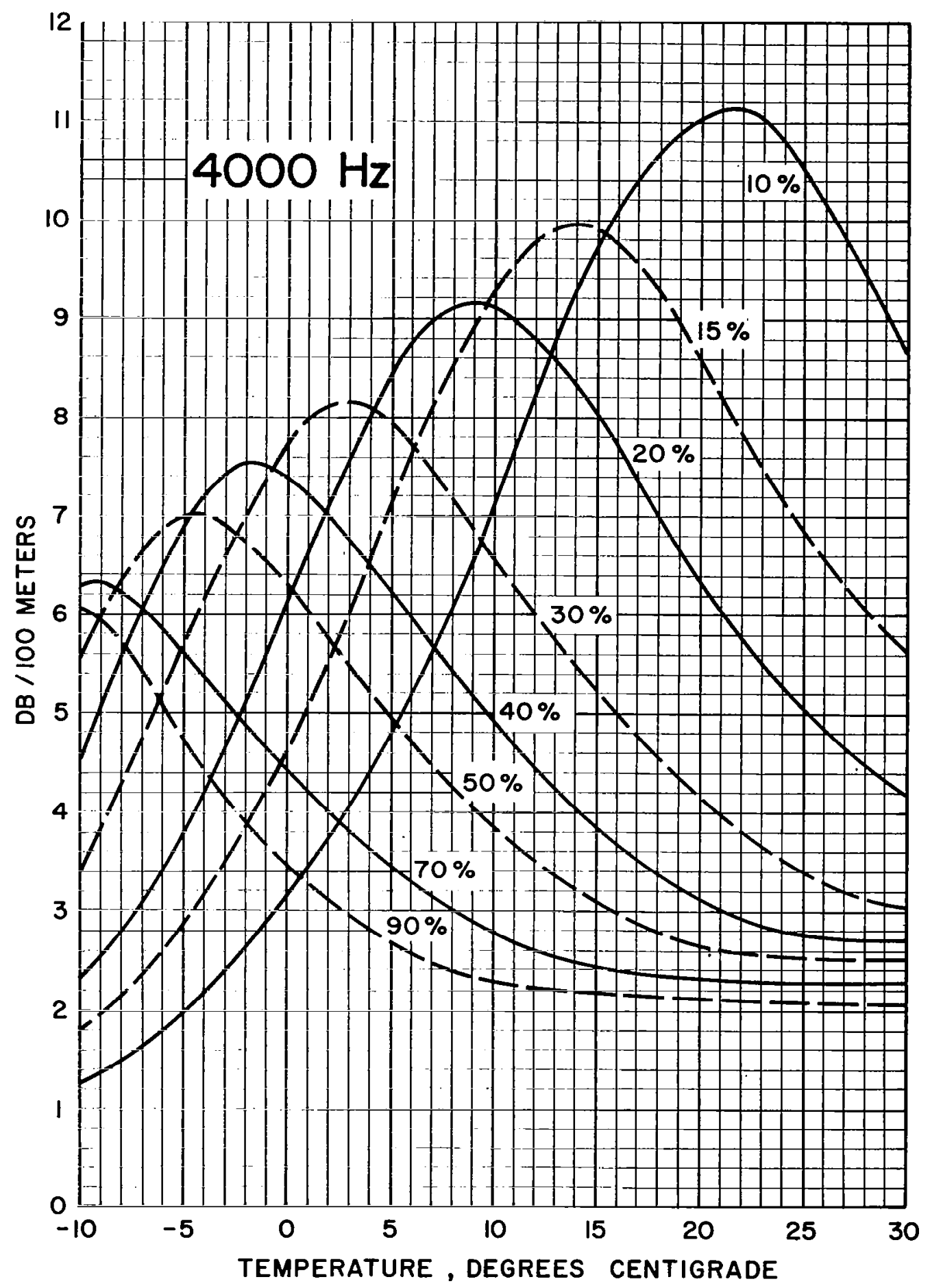

FIGURE 6h. (CONTINUED) 


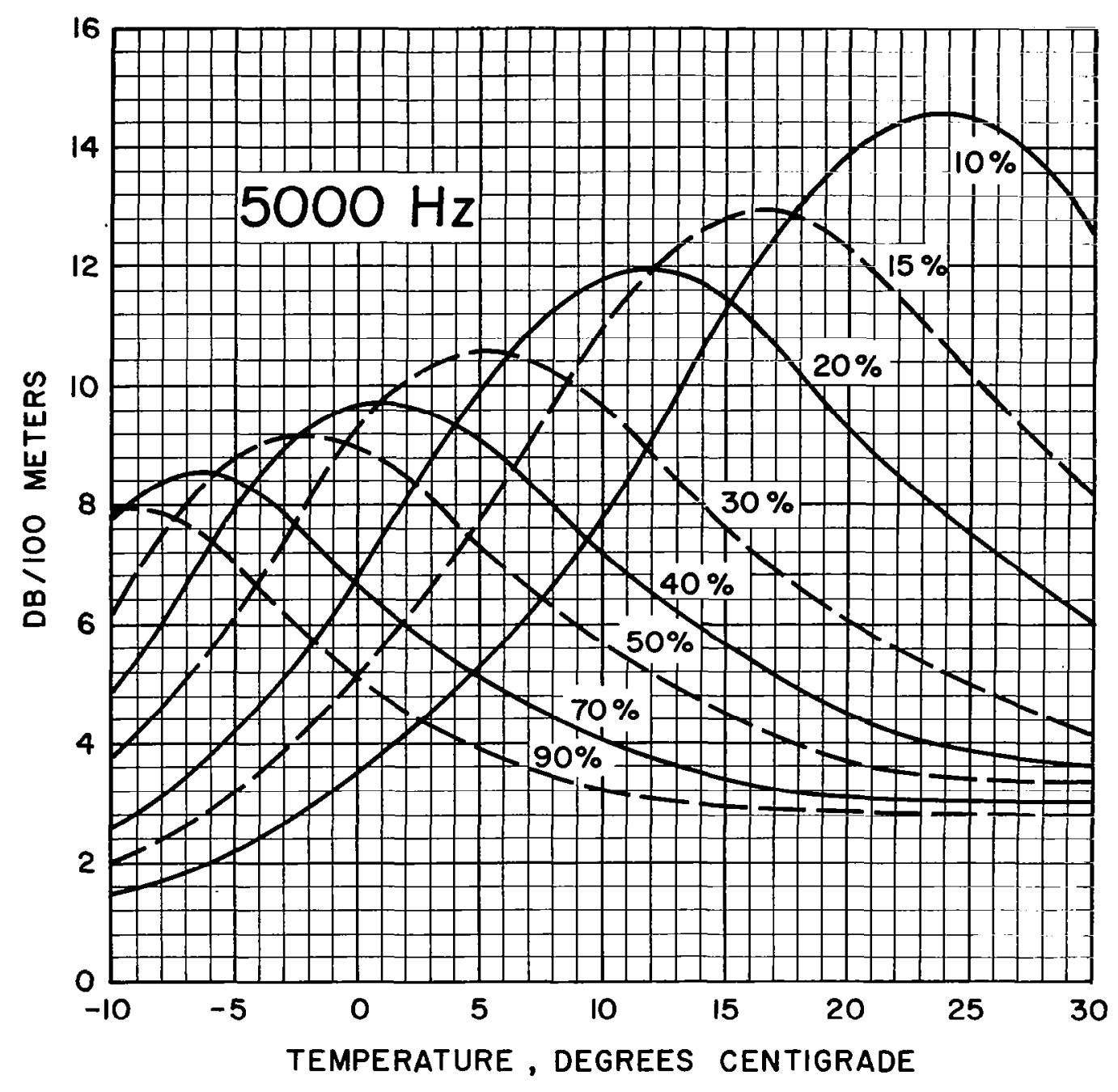

FIGURE 6i. (CONTINUED) 


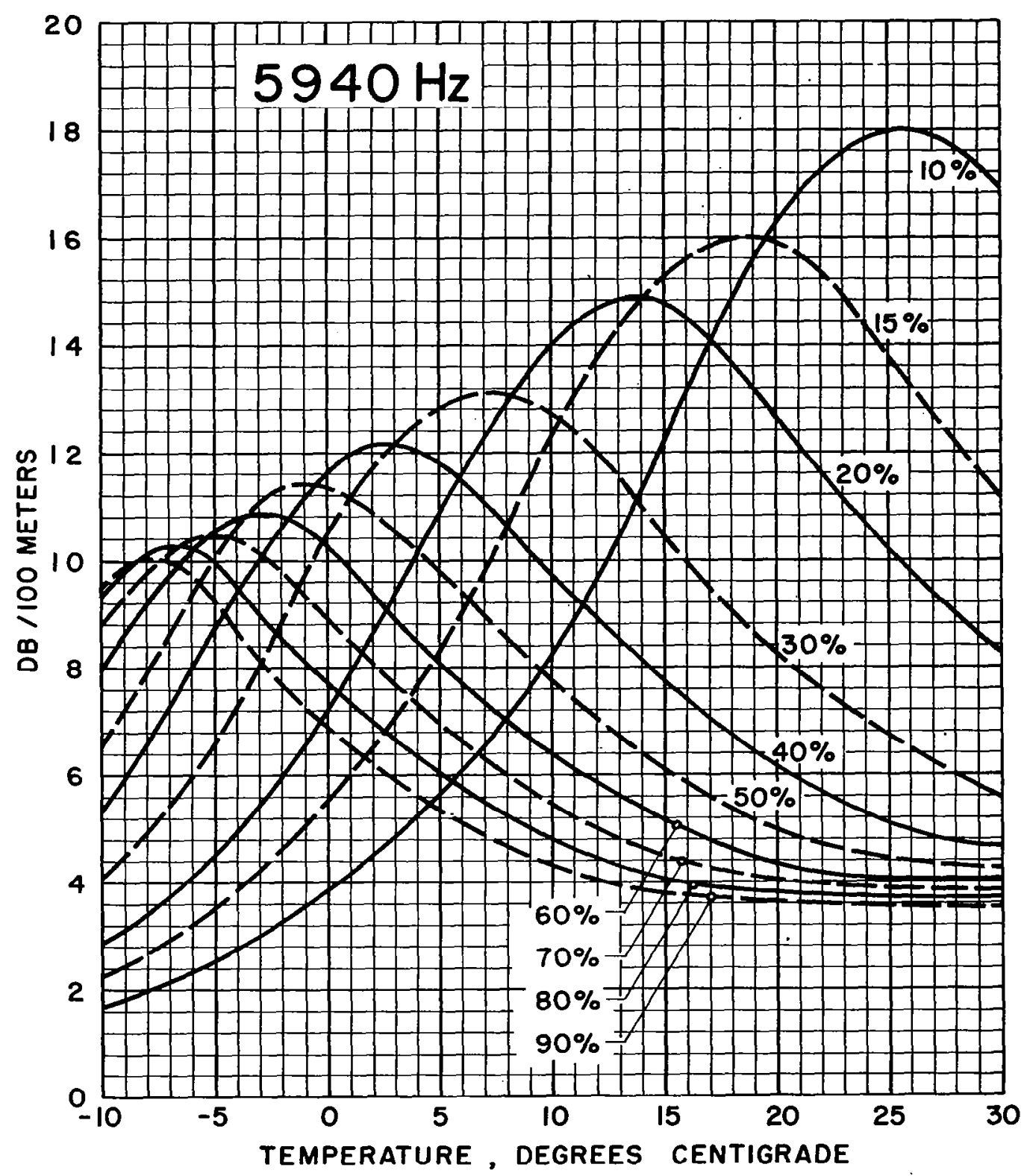

FIGURE 6j. (CONTINUED) 


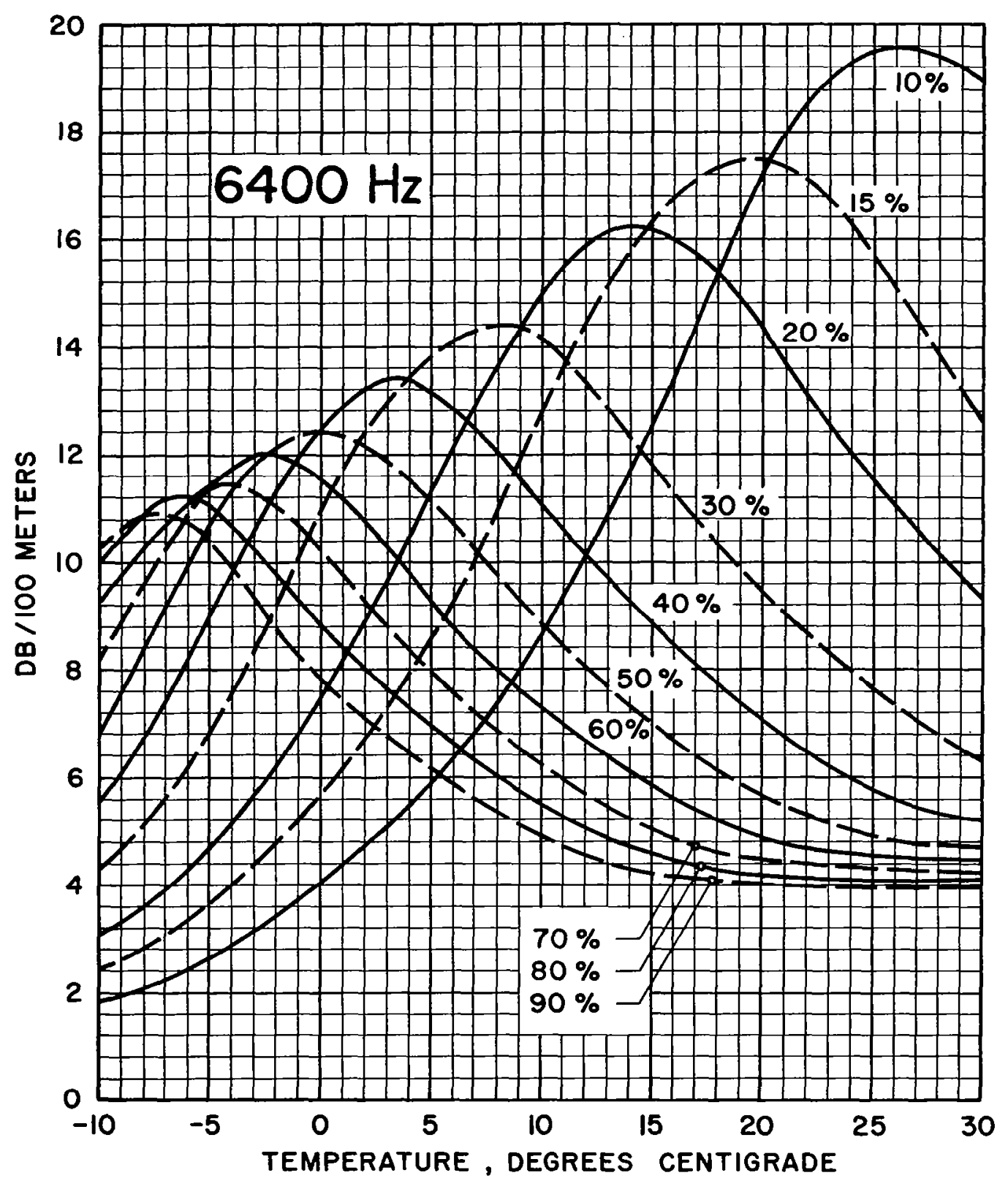

FIGURE 6k. (CONTINUED) 


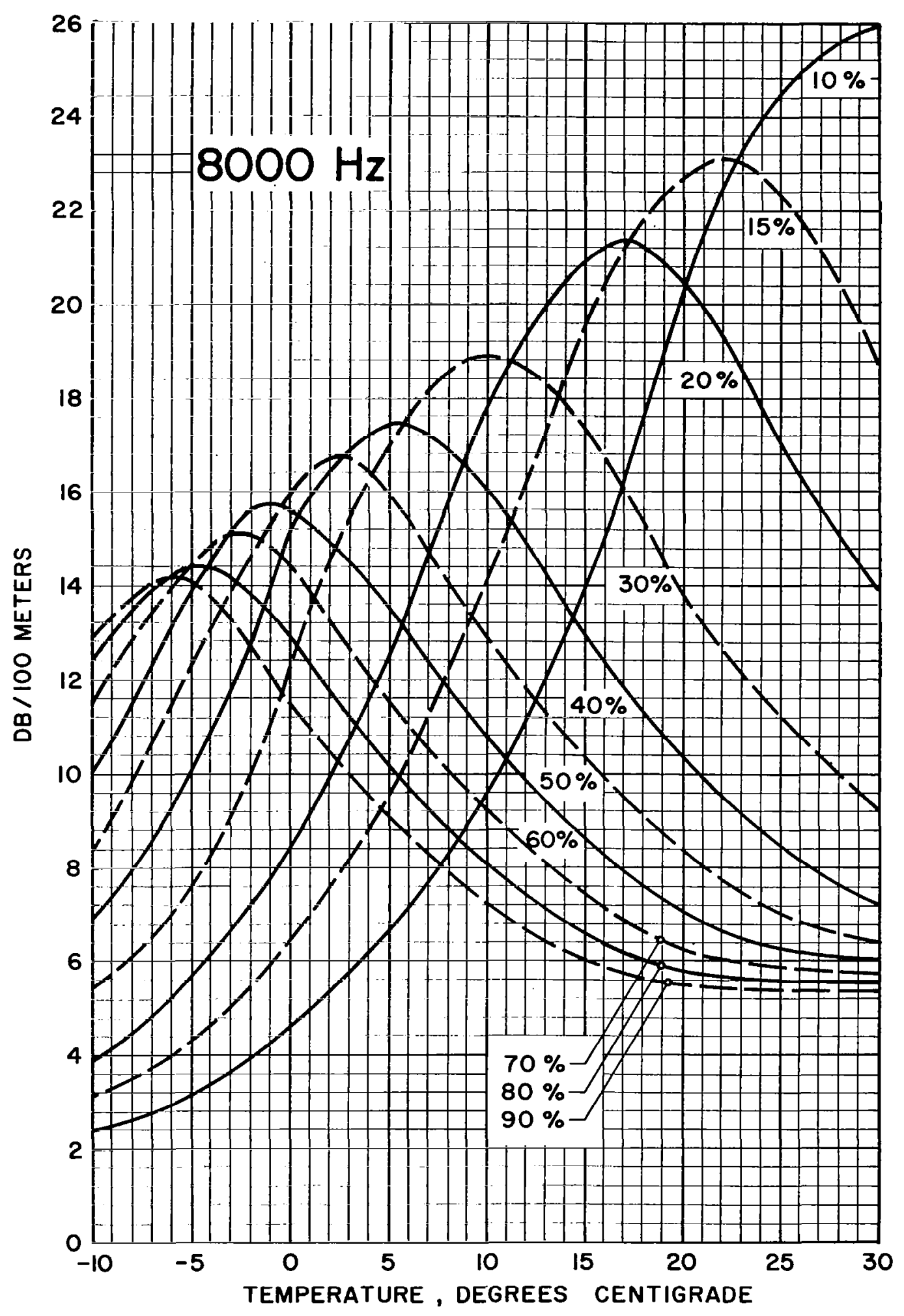

FIGURE 61. (CONCLUDED) 


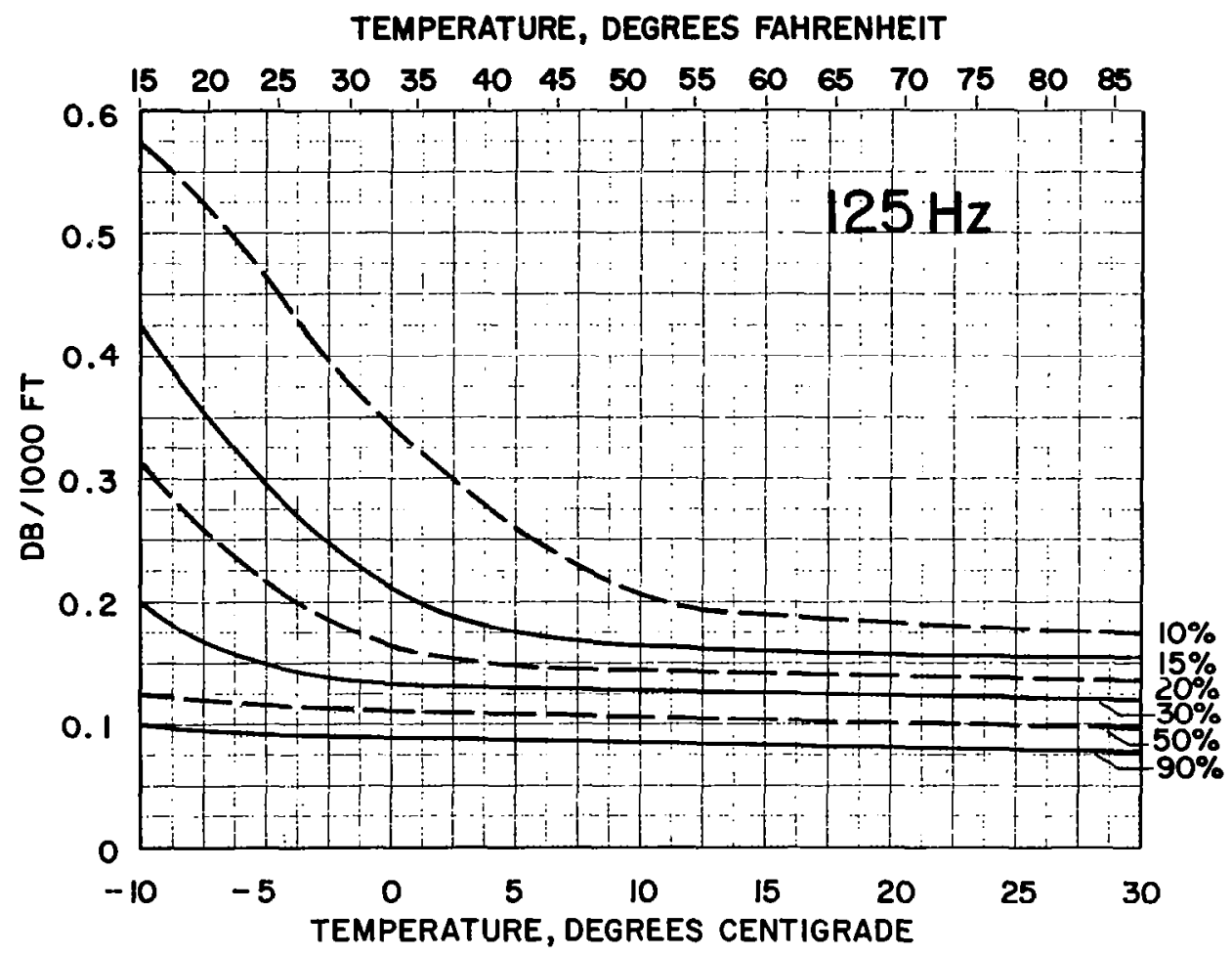

FIGURE 7a. THE ATTENUATION OF SOUND IN AIR VERSUS TEMPERATURE FOR VARIOUS VALUES OF RELATIVE HUMIDITY. THE ATTENUATION OF DB/1000 FEET IS SHOWN AS THE ORDINATE AT THE LEFT OF THE GRAPH 


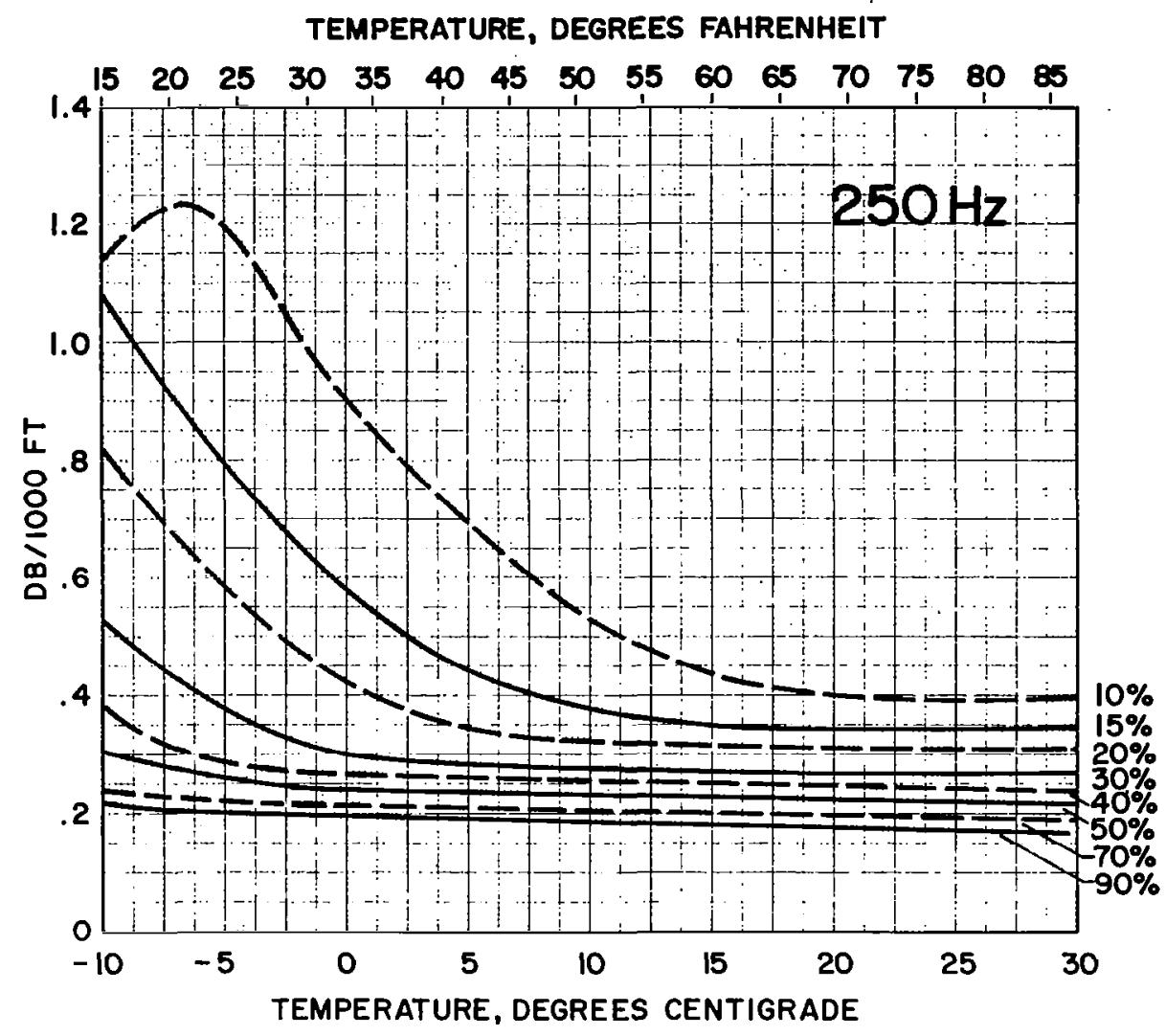

FIGURE 7b. (CONTINUED) 
TEMPERATURE, DEGREES FAHRENHEIT

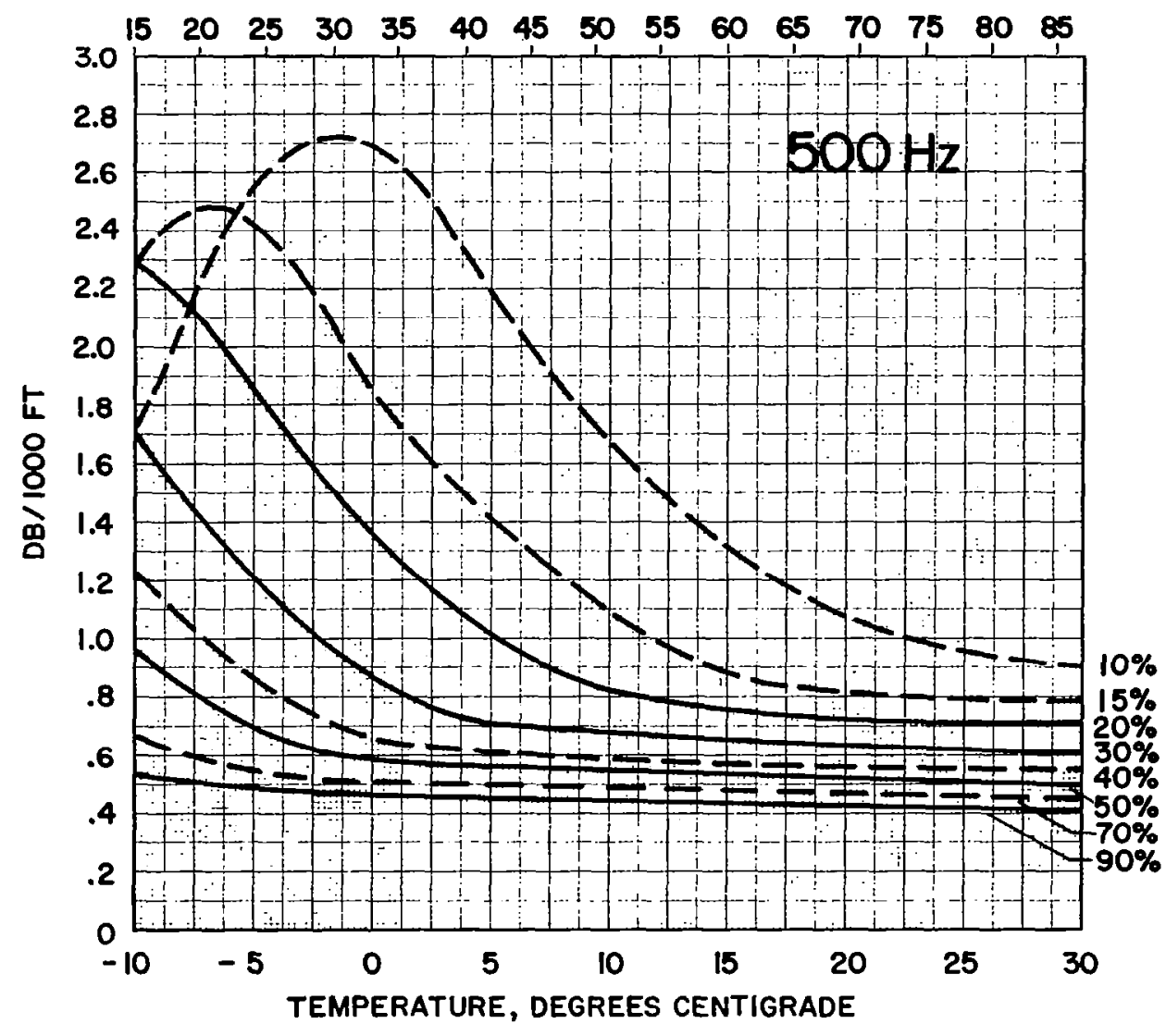

FIGURE 7c. (CONTINUED) 


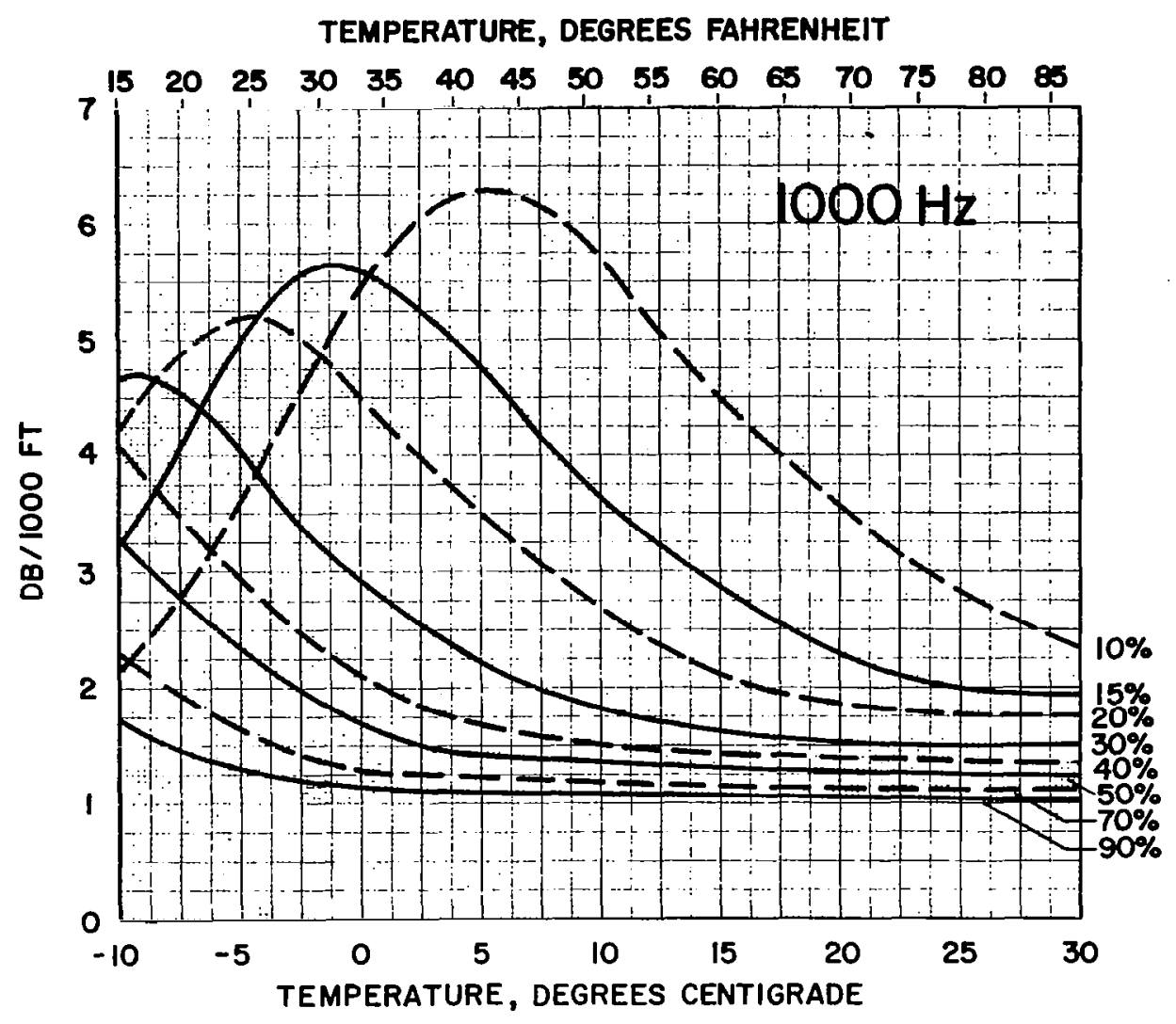

FIGURE 7d. (CONTINUED) 


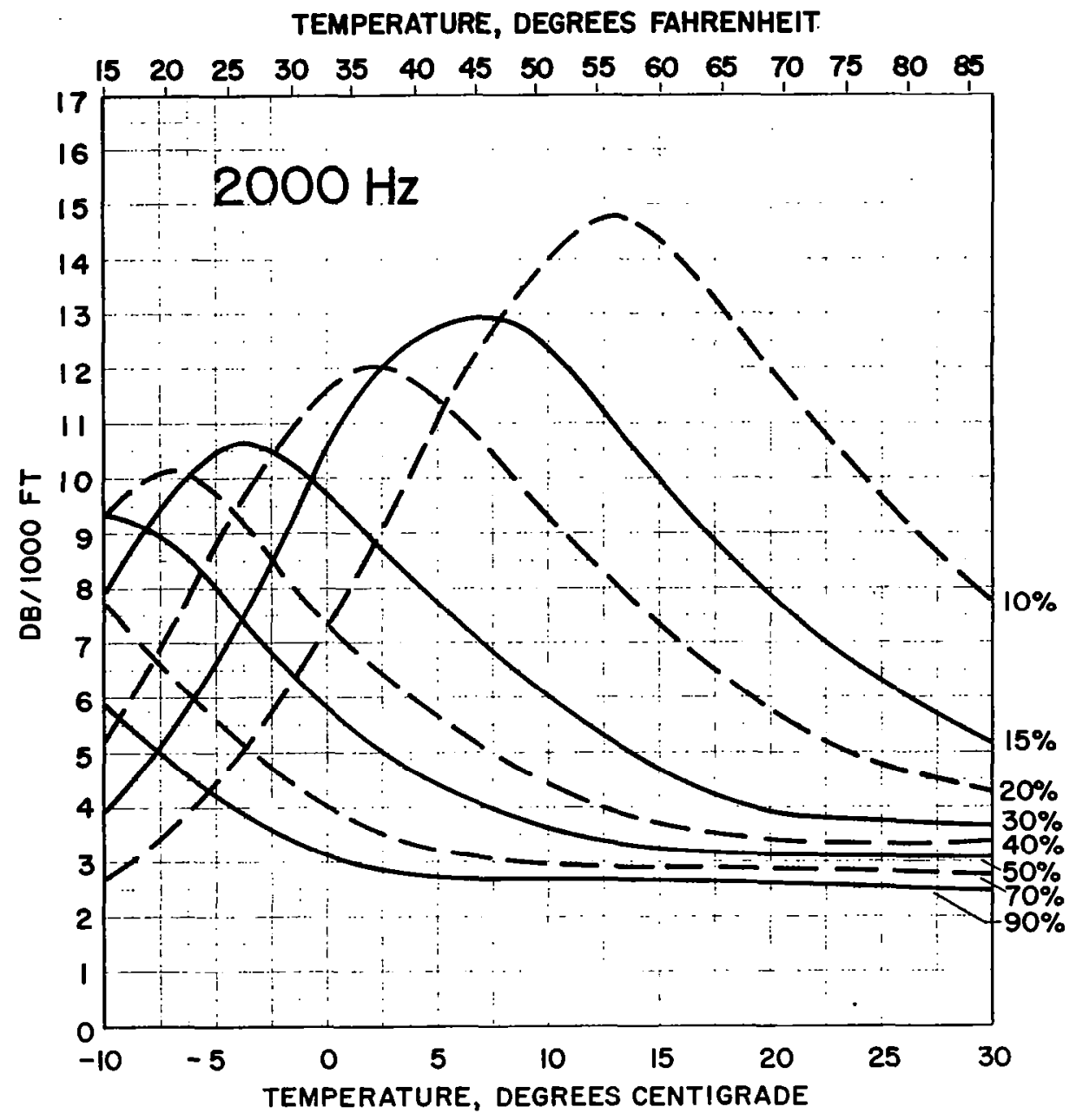

FIGURE 7e. (CONTINUED) 
TEMPERATURE, DEGREES FAHRENHEIT

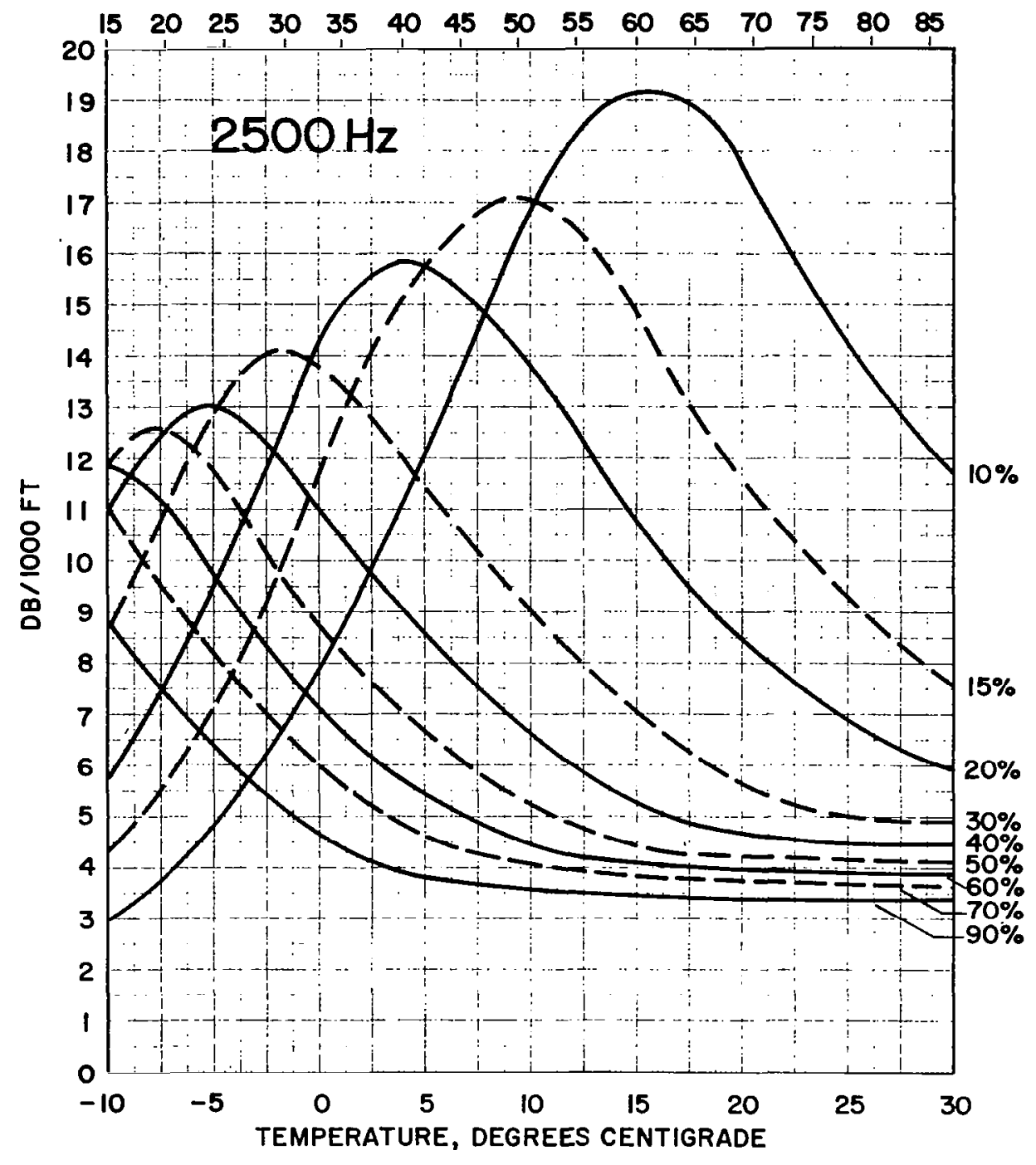

FIGURE 7f. (CONTINUED) 


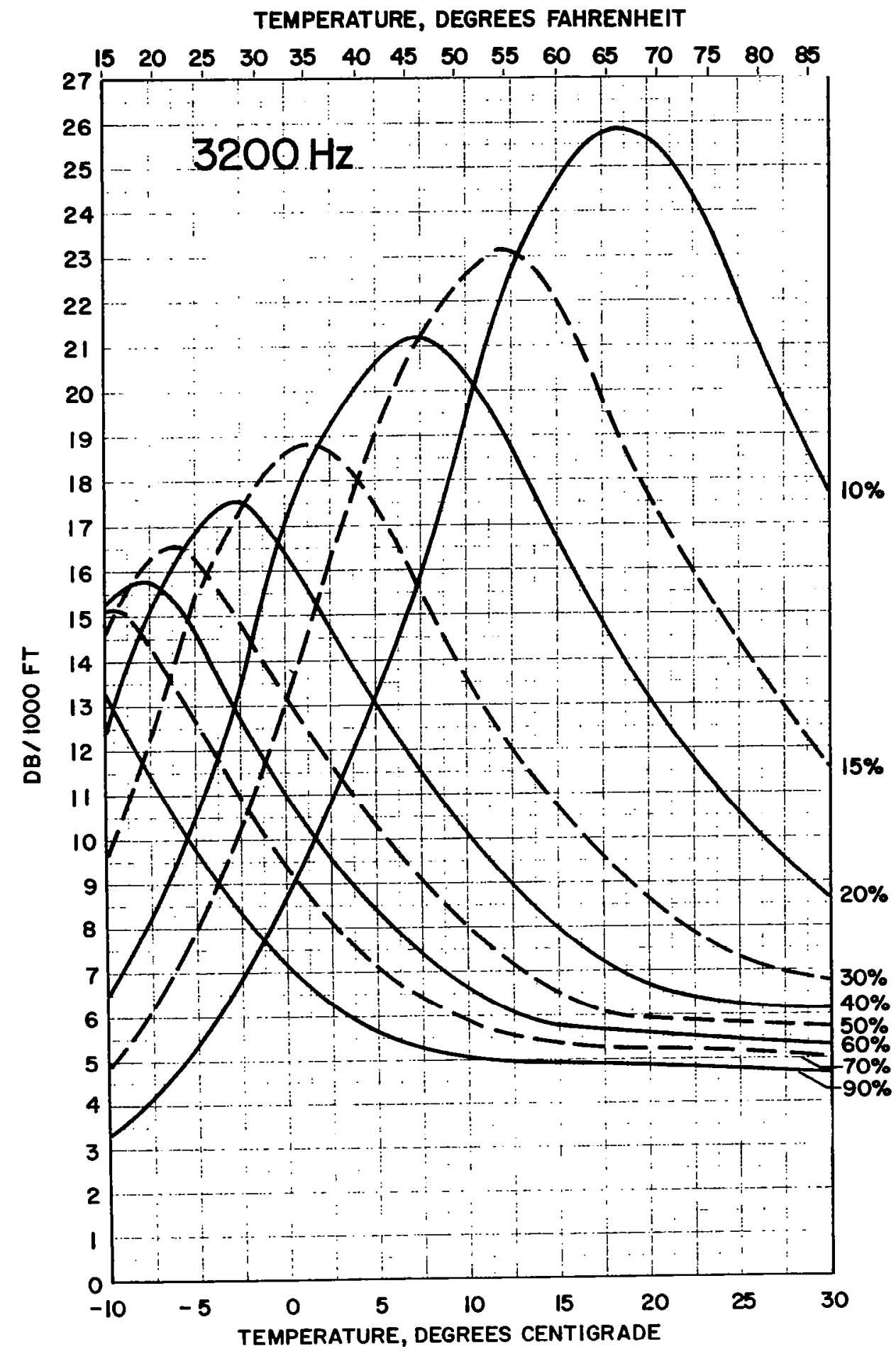

FIGURE 7g. (CONTINUED) 


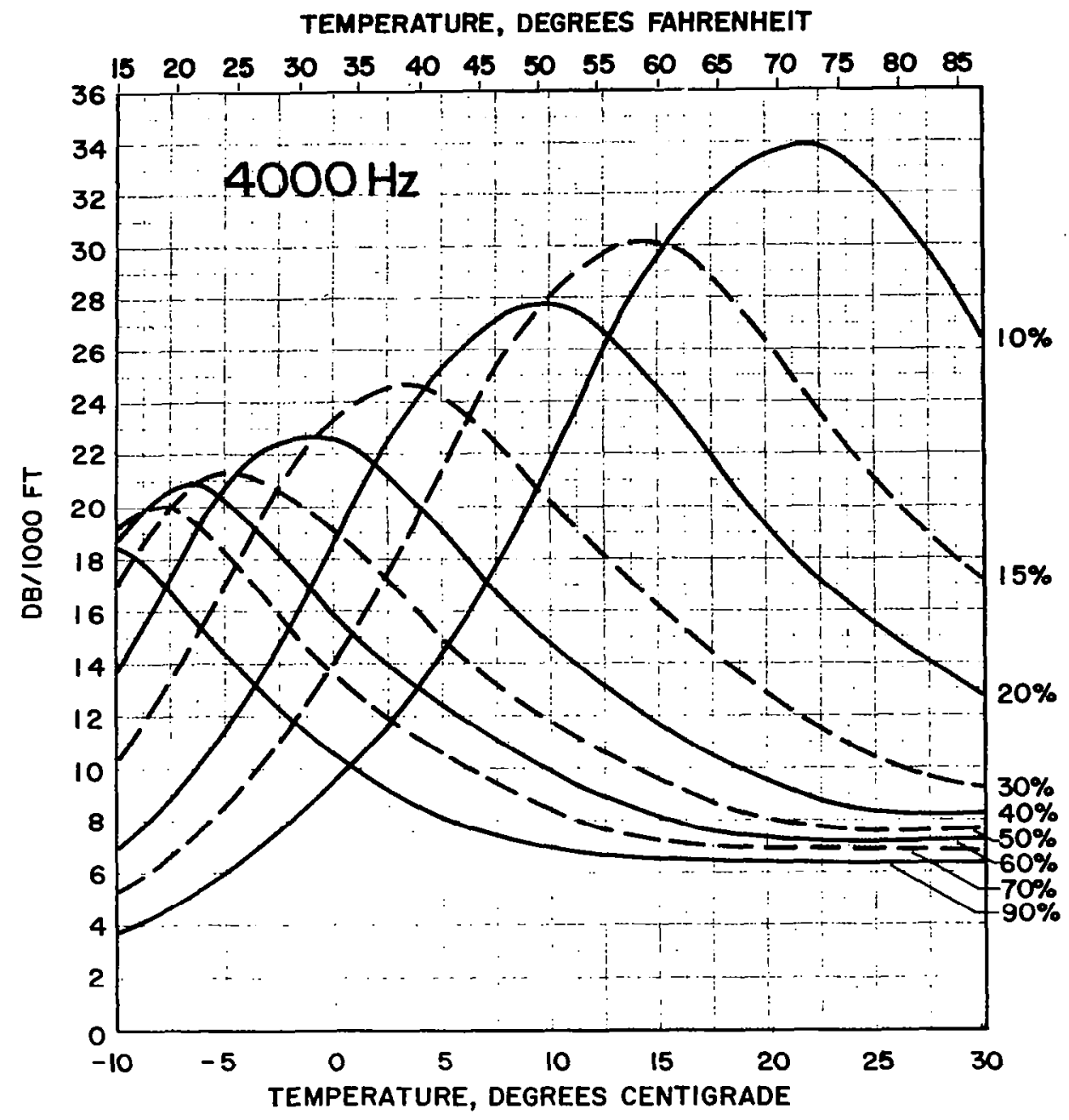

FIGURE 7h. (CONTINUED) 


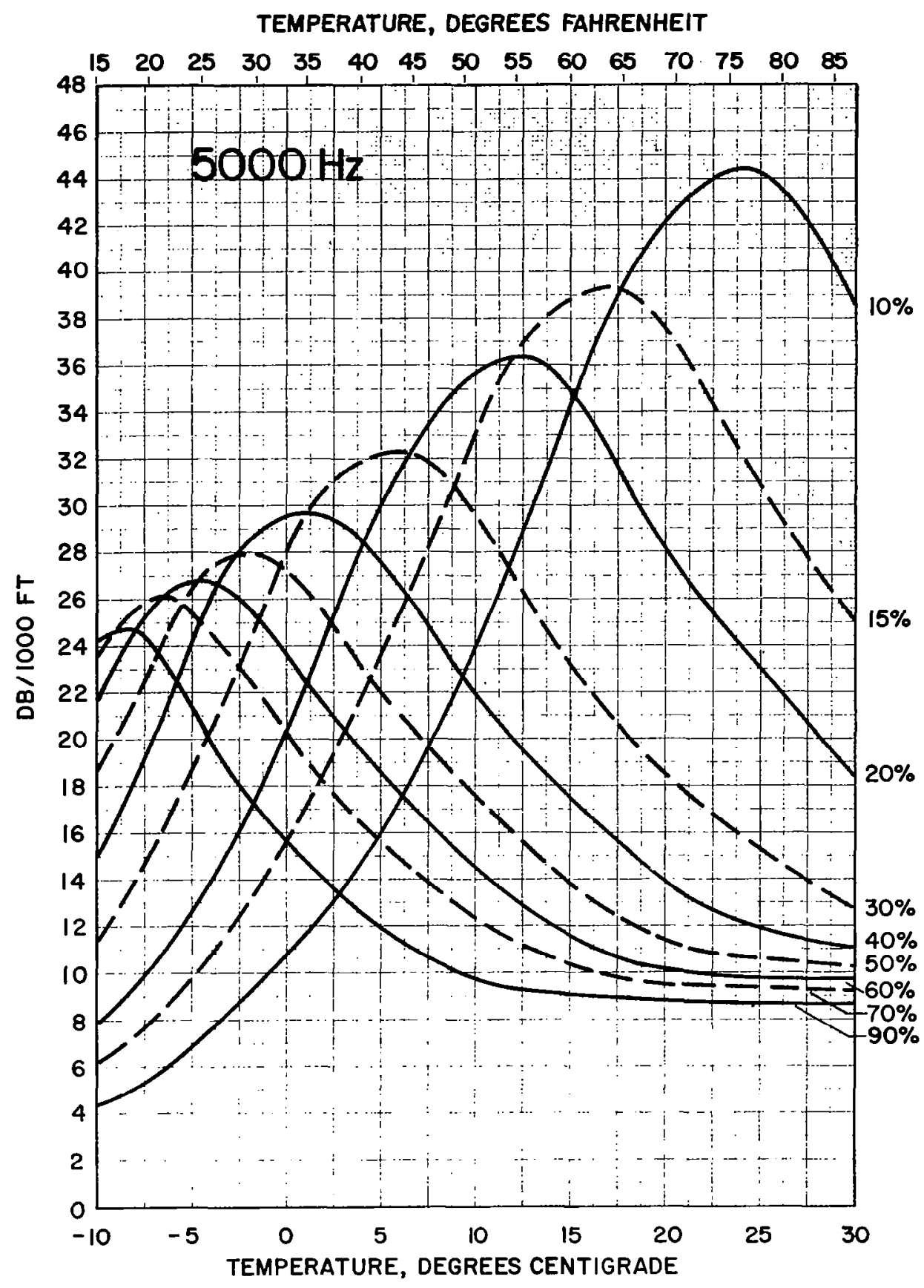

FIGURE 7i. (CONTINUED) 


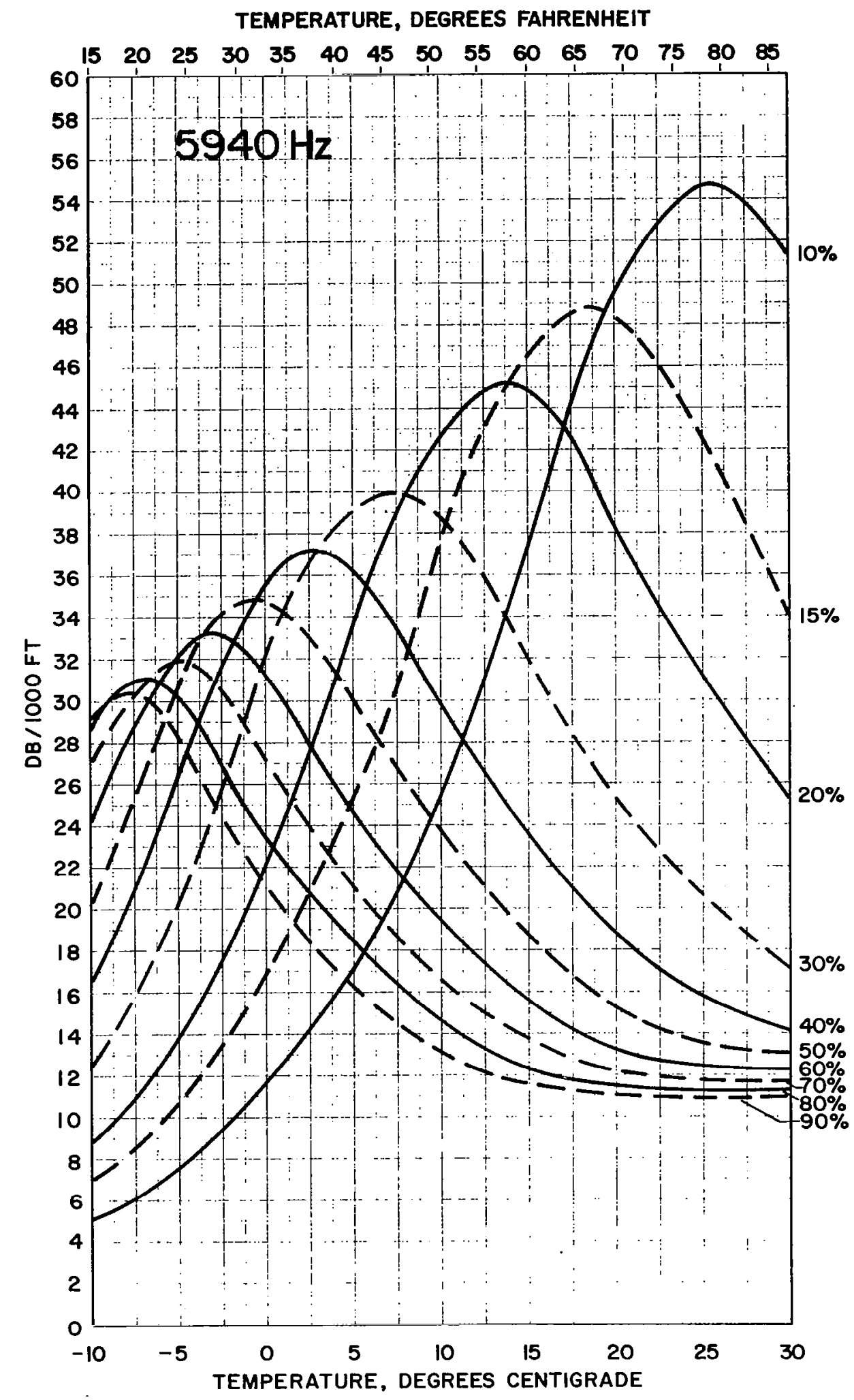

FIGURE 7j. (CONTINUED) 


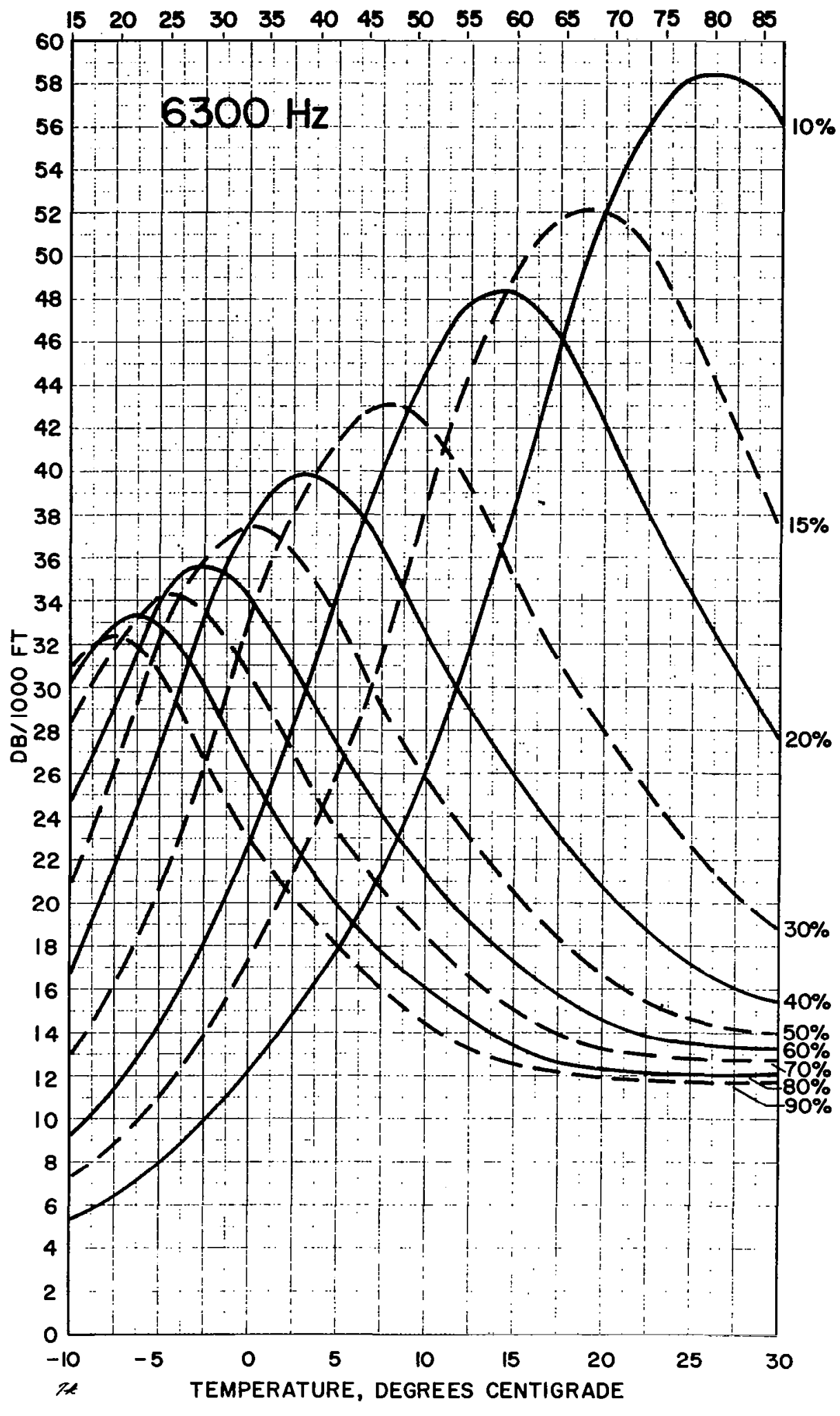

FIGURE 7k. (CONTINUED) 


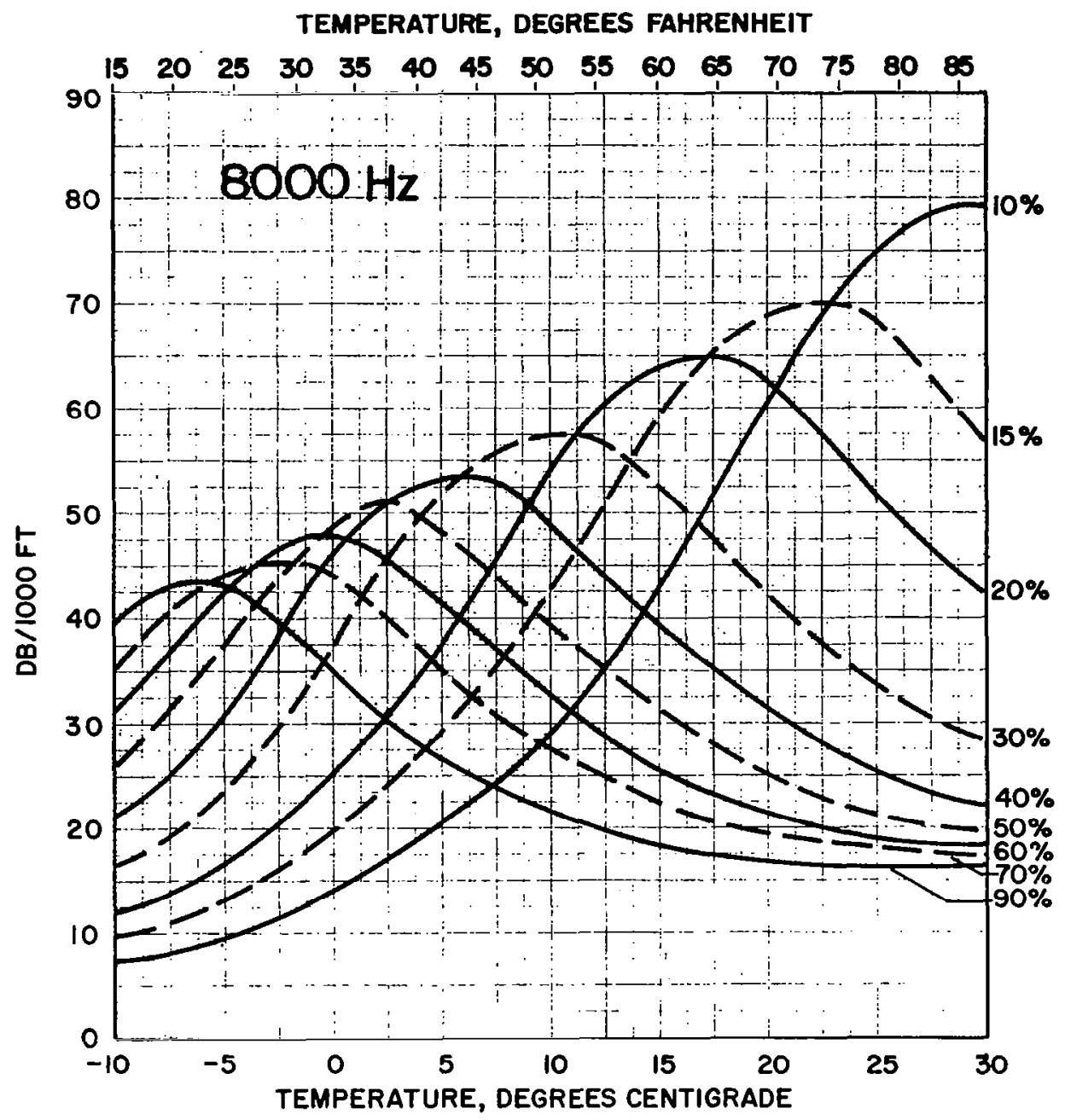

FIGURE 71. (CONCLUDED) 


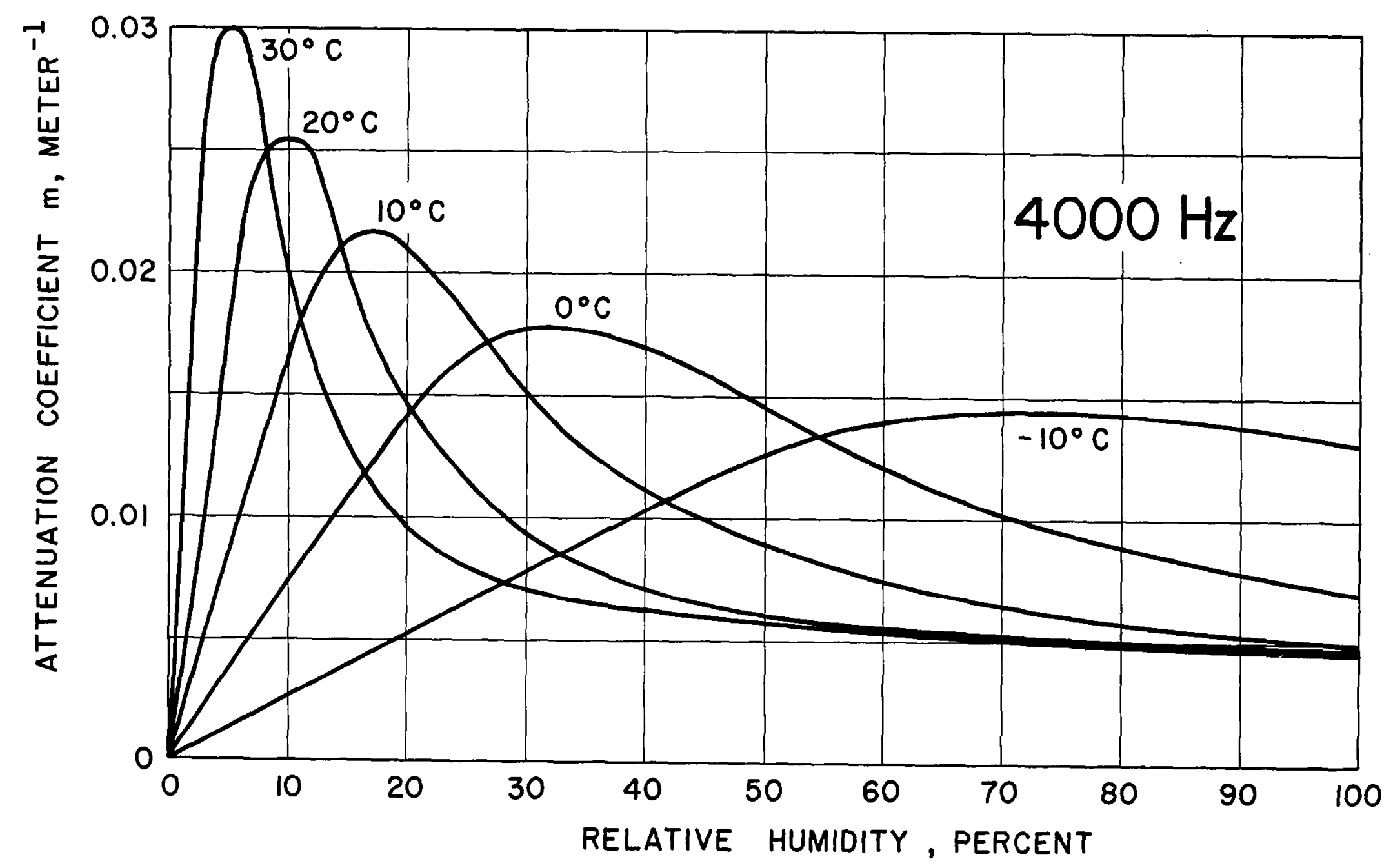

FIGURE 8. VALUES OF TOTAL ATTENUATION COEFFICIENT M VERSUS HUMIDITY FOR A FREQUENCY OF $4000 \mathrm{HZ}$ AT TEMPERATURES OF $-10^{\circ} \mathrm{C}, 0^{\circ} \mathrm{C}, 10^{\circ} \mathrm{C}, 20^{\circ} \mathrm{C}$ and $30^{\circ} \mathrm{C}$ 


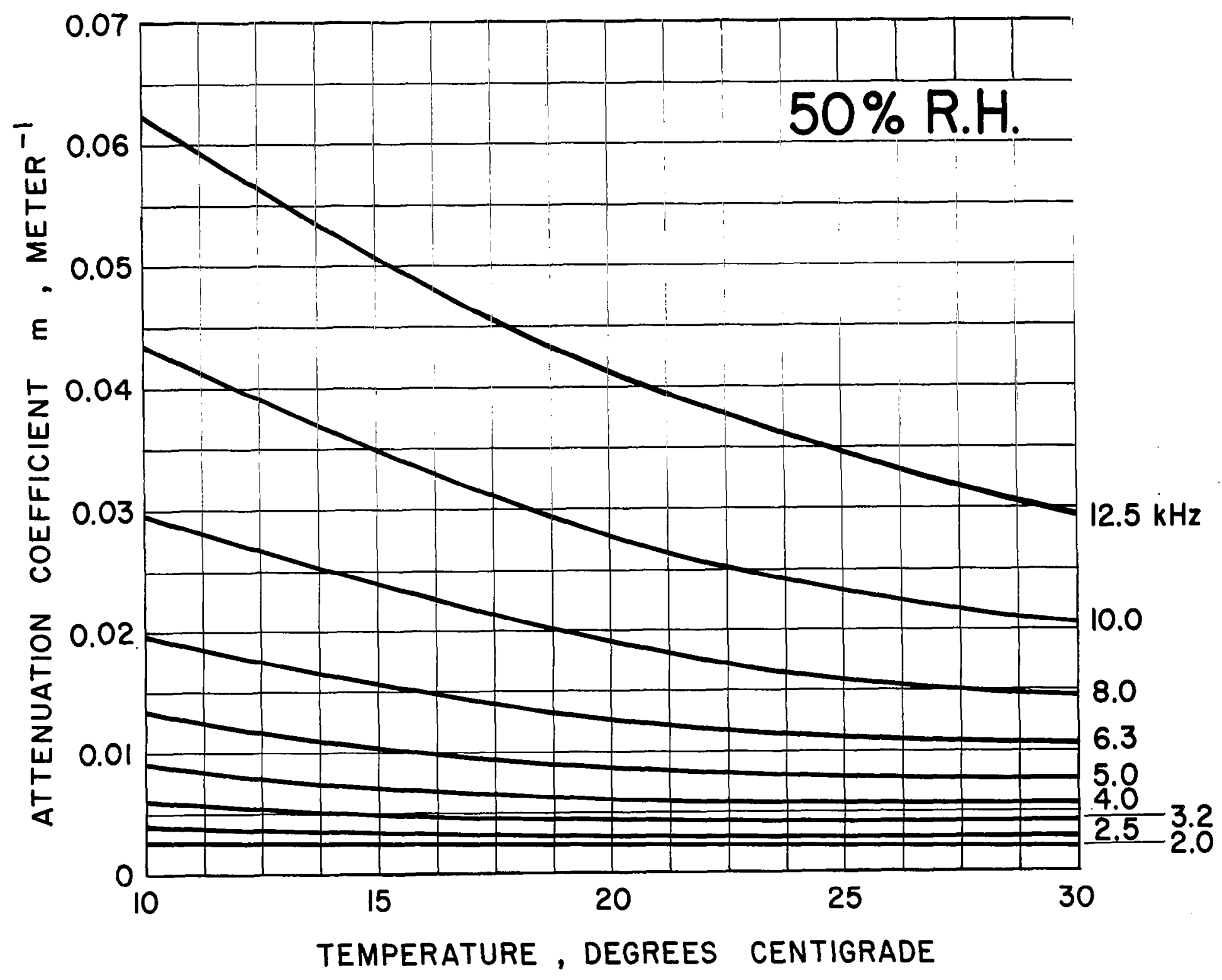


TABULAR DATA ARE GIVEN IN THIS REPORT FOR THE. ABSORPTION OF SOUND IN AIR AT NORMAL ATMOSPHERIC PRESSURE, $760 \mathrm{MM}$

THE ATTENUATION COEFFICIENT, M, WHICH IS TABULATED REPRESENTS THE TOTAL ATTENUATION DUE TO MOLECULAR AND CLASSICAL ABSORPTION OF SOUND. AS EXPRESSED IN THE EQUATION $I=I_{0} e^{-i x}$. 
THE FOLLOWING INFORMATION IS GIVEN AS A FUNCTION OF TEMPERATURE, FREQUENCY AND RELATIVE HUMIDITY.

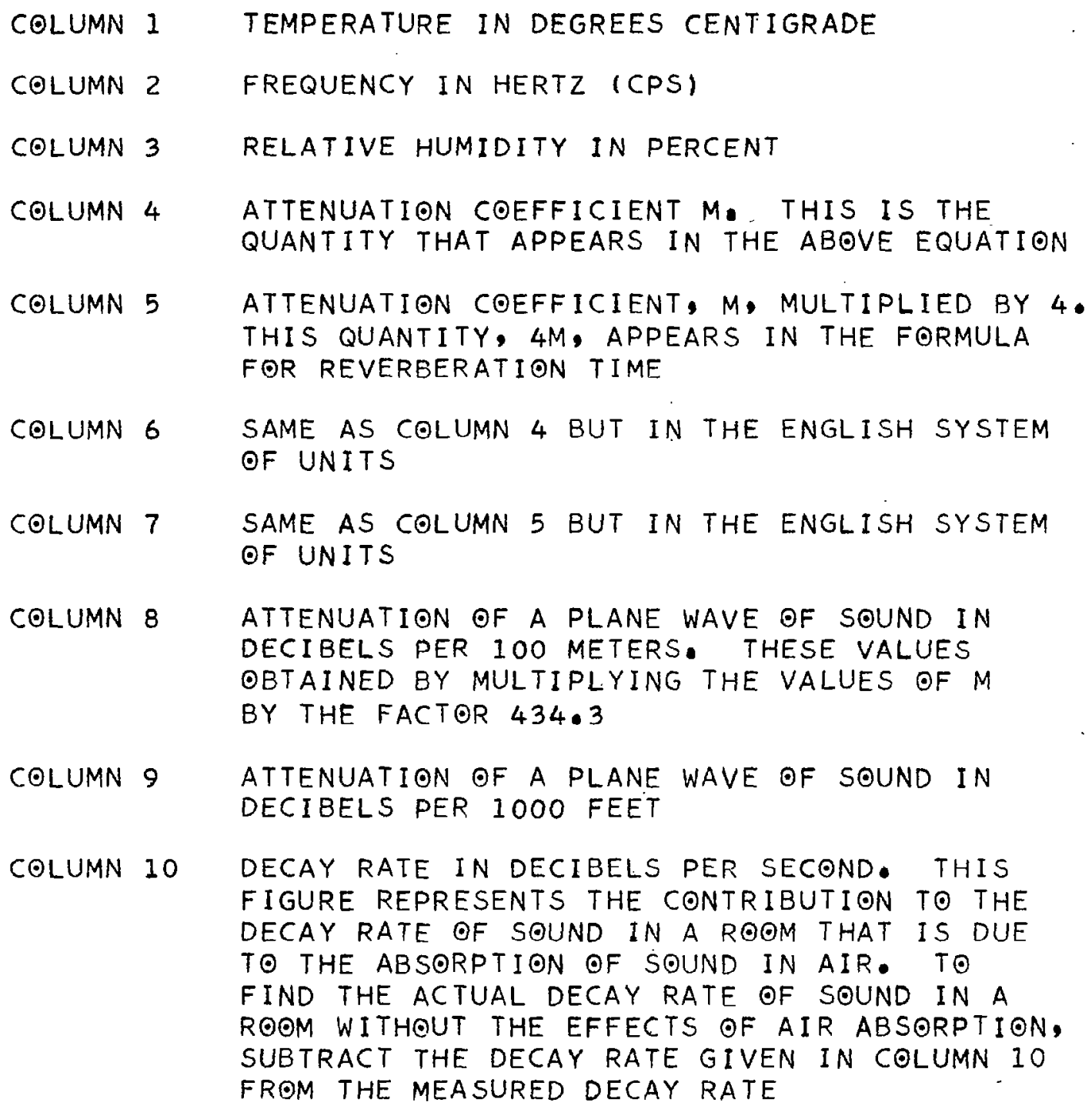


TABULATION OF DATA FOR

ABSORPTION OF SOUND IN AIR VERSUS PERCENT RELATIVE HUMIDITY AT-10 DEGREES CENTIGRADE FOR A FREQUENCY OF 125 HERTZ

(1) (2) (3)

TEMP FREQ. REL

HUM

DEGR - PER

CENT HERTZ CENT

$\begin{array}{lll}-10 & 125 & 5.0\end{array}$

$\begin{array}{lll}-10 & 125 & 10.0\end{array}$

$-10 \quad 125 \quad 15.0$

$\begin{array}{lll}-10 & 125 & 20 \cdot 0\end{array}$

$\begin{array}{lll}-10 & 125 & 25.0\end{array}$

$\begin{array}{lll}-10 & 125 \quad 30.0\end{array}$

$\begin{array}{lll}-10 & 125 & 35.0\end{array}$

$\begin{array}{lll}-10 & 125 & 40.0\end{array}$

$\begin{array}{lll}-10 & 125 & 45.0\end{array}$

$\begin{array}{lll}-10 & 125 & 46.0\end{array}$

$\begin{array}{lll}-10 & 125 & 47.0\end{array}$

$\begin{array}{lll}-10 & 125 & 48.0\end{array}$

$\begin{array}{lll}-10 & 125 & 49.0\end{array}$

$\begin{array}{lll}-10 & 125 & 50.0\end{array}$

$\begin{array}{lll}-10 & 125 & 51.0\end{array}$

$\begin{array}{lll}-10 & 125 & 52.0\end{array}$

$\begin{array}{lll}-10 & 125 & 53.0\end{array}$

$\begin{array}{lll}-10 & 125 & 54.0\end{array}$

$\begin{array}{lll}-10 & 125 & 55.0\end{array}$

125

$-10 \quad 125$

60.0

$65 \cdot 0$

$\begin{array}{lll}-10 & 125 & 70.0\end{array}$

$\begin{array}{lll}-10 & 125 & 75.0\end{array}$

$\begin{array}{lll}-10 & 125 & 80.0\end{array}$

$\begin{array}{lll}-10 & 125 & 85.0\end{array}$

$\begin{array}{lll}-10 & 125 & 90.0\end{array}$

$\begin{array}{lll}-10 & 125 & 95.0\end{array}$

$-10.125 \quad 100 \cdot 0$
(4)

(5)

(6)

(7)

$\begin{array}{ll}\text { ATTEN } & 4 M \\ \text { COEF } & \\ \text { PER } & \text { PER }\end{array}$

COEF

PER

METER

.00031

.00043

.00032

.00023

.00018

.00015

.00012

.00011

.00010

.00010

.00009

.00009

.00009

.00009

.00009

.00009

.00009

.00009

.00009

.00008

.00008

.00008

.00008

.00007

.00032

.00031

.00007

.00030

.00007

.00030

.00002

.00002

.00002

.00007

.00007

FOOT
(8)

(9)

(10)

ATTEN OB PER 100 METER

.00038

.00052

.00039

.00028

.00022

.00018

.00015

.00013

.00012

.00012

. 00012

.00011

.00011

.00011

.00011

.00011

.00011

.00011

.00011

.00010

.00020

.00010

.00009

.00009

.00009

.00009

.00009

DB PER RATE
$.00029 \quad .00002 \quad .00008$

\begin{tabular}{|c|c|c|}
\hline .138 & .420 & .449 \\
\hline $\begin{array}{l}187 \\
.140\end{array}$ & $\begin{array}{r}.57 .1 \\
.428\end{array}$ & $\begin{array}{r}.610 \\
.457\end{array}$ \\
\hline $\begin{array}{l}.102 \\
.079 \\
.065\end{array}$ & $\begin{array}{l}.313 \\
.243 \\
.200\end{array}$ & $\begin{array}{r}.334 \\
.260 \\
.213\end{array}$ \\
\hline $\begin{array}{l}.055 \\
.049 \\
.044\end{array}$ & $\begin{array}{l}1 \\
1 \\
.149 \\
.1134\end{array}$ & $\begin{array}{r}.180 \\
.159 \\
.143\end{array}$ \\
\hline $\begin{array}{l}.043 \\
.043 \\
.042\end{array}$ & $\begin{array}{l}.133 \\
.1131 \\
.1129\end{array}$ & $\begin{array}{r}.142 \\
.140 \\
.1138\end{array}$ \\
\hline $\begin{array}{l}.042 \\
.041 \\
.041\end{array}$ & $\begin{array}{l}.128 \\
.1126 \\
.125\end{array}$ & $\begin{array}{r}.136 \\
.135 \\
.133\end{array}$ \\
\hline $\begin{array}{l}.040 \\
.040 \\
.039\end{array}$ & $\begin{array}{r}.123 \\
.122 \\
.121\end{array}$ & $\begin{array}{r}.132 \\
.130 \\
.129\end{array}$ \\
\hline .039 & .120 & .128 \\
\hline $\begin{array}{l}.038 \\
.037\end{array}$ & $\begin{array}{l}.116 \\
.112\end{array}$ & $\begin{array}{r}.123 \\
.120\end{array}$ \\
\hline $\begin{array}{r}.036 \\
.035 \\
.034\end{array}$ & $\begin{array}{r}.109 \\
.107 \\
.104\end{array}$ & $\begin{array}{r}.117 \\
.114 \\
. \\
.111\end{array}$ \\
\hline $\begin{array}{l}.033 \\
.032 \\
.032\end{array}$ & $\begin{array}{r}.102 \\
.100 \\
.098\end{array}$ & $\begin{array}{r}.109 \\
.106 \\
.104\end{array}$ \\
\hline .031 & .096 & .102 \\
\hline
\end{tabular}

449

410

.334

260

180

159

142

140

136

135

132

(1)

128

123

117

114

109

106

.031

102
ATTEN DECAY

1000 DB PER

FEET SECOND 
TABULATION OF DATA FOR

ABSORPTION OF SOUND IN AIR VERSUS PERCENT RELATIVE HUMIDITY AT-10 DEGREES CENTIGRADE FOR A FREQUENCY OF' 250 HERTZ

\begin{tabular}{|c|c|c|c|c|c|c|c|c|c|}
\hline (1) & (2) & (3) & $(4)$ & (5) & (6) & $(7)$ & (8) & (9) & $(10)$ \\
\hline EMP & FREQ & REL & ATTEN & $4 M$ & ATTEN & $4 M$ & ATTEN & ATTEN & DECAY \\
\hline $\begin{array}{l}\text { EGR } \\
\text { ENT }\end{array}$ & HERTZ & $\begin{array}{l}\text { HUM } \\
\text { PER } \\
\text { CENT }\end{array}$ & $\begin{array}{l}\text { COEF } \\
\text { PER } \\
\text { METER }\end{array}$ & $\begin{array}{l}\text { PER } \\
\text { METER }\end{array}$ & $\begin{array}{l}\text { COEF } \\
\text { PER } \\
\text { FOOT }\end{array}$ & $\begin{array}{l}\text { PER } \\
\text { FOOT }\end{array}$ & $\begin{array}{l}\text { DE PER } \\
100 \\
\text { METER }\end{array}$ & $\begin{array}{l}\text { DB PER } \\
1000 \\
\text { FEET }\end{array}$ & $\begin{array}{l}\text { RATE } \\
\text { DB PER } \\
\text { SECOND }\end{array}$ \\
\hline $\begin{array}{l}10 \\
10 \\
10\end{array}$ & $\begin{array}{l}250 \\
250 \\
250\end{array}$ & $\begin{array}{r}5.0 \\
10.0 \\
15.0\end{array}$ & $\begin{array}{l}.00048 \\
.00086 \\
.00081\end{array}$ & $\begin{array}{l}.00194 \\
.00346 \\
.00325\end{array}$ & $\begin{array}{l}.00014 \\
.00026 \\
.00024\end{array}$ & $\begin{array}{l}.00059 \\
.00105 \\
.00099\end{array}$ & $\begin{array}{r}.211 \\
.375 \\
.353\end{array}$ & $\begin{array}{r}.643 \\
1.145 \\
1.076\end{array}$ & $\begin{array}{r}.687 \\
1.222 \\
1.148\end{array}$ \\
\hline $\begin{array}{l}-10 \\
-10 \\
-10\end{array}$ & $\begin{array}{l}250 \\
250 \\
250\end{array}$ & $\begin{array}{l}20.0 \\
25.0 \\
30.0\end{array}$ & $\begin{array}{r}.00062 \\
.00048 \\
.00040\end{array}$ & $\begin{array}{l}.00248 \\
.00195 \\
.00160\end{array}$ & $\begin{array}{l}.00018 \\
.00014 \\
.00012\end{array}$ & $\begin{array}{r}.00075 \\
.00059 \\
.00048\end{array}$ & $\begin{array}{l}.269 \\
.211 \\
.173\end{array}$ & $\begin{array}{l}.821 \\
.645 \\
.530\end{array}$ & $\begin{array}{r}.877 \\
.689 \\
.565\end{array}$ \\
\hline $\begin{array}{l}10 \\
10 \\
10\end{array}$ & $\begin{array}{l}250 \\
250 \\
250\end{array}$ & $\begin{array}{l}35.0 \\
40.0 \\
45.0\end{array}$ & $\begin{array}{l}.00033 \\
.00029 \\
.00025\end{array}$ & $\begin{array}{l}.00134 \\
.00116 \\
.00102\end{array}$ & $\begin{array}{l}.00010 \\
.00008 \\
.00007\end{array}$ & $\begin{array}{l}.00040 \\
.00035 \\
.00031\end{array}$ & $\begin{array}{r}.145 \\
.126 \\
.111\end{array}$ & $\begin{array}{r}.444 \\
.386 \\
.339\end{array}$ & $\begin{array}{r}.474 \\
.412 \\
.362\end{array}$ \\
\hline $\begin{array}{l}10 \\
10 \\
10\end{array}$ & $\begin{array}{l}250 \\
250 \\
250\end{array}$ & $\begin{array}{l}46.0 \\
47.0 \\
48.0\end{array}$ & $\begin{array}{l}.00025 \\
.00024 \\
.00024\end{array}$ & $\begin{array}{l}.00100 \\
.00098 \\
.00096\end{array}$ & $\begin{array}{l}.00007 \\
.00007 \\
.00007\end{array}$ & $\begin{array}{r}.00030 \\
.00029 \\
.00029\end{array}$ & $\begin{array}{r}.108 \\
.106 \\
.104\end{array}$ & $\begin{array}{r}.332 \\
.324 \\
.318\end{array}$ & $\begin{array}{r}.354 \\
.346 \\
.340\end{array}$ \\
\hline $\begin{array}{l}10 \\
10 \\
10\end{array}$ & $\begin{array}{l}250 \\
250 \\
250\end{array}$ & $\begin{array}{l}49.0 \\
50.0 \\
51.0\end{array}$ & $\begin{array}{l}.00023 \\
.00023 \\
.00022\end{array}$ & $\begin{array}{l}.00094 \\
.00092 \\
.00091\end{array}$ & $\begin{array}{l}.00007 \\
.00007 \\
.00006\end{array}$ & $\begin{array}{l}.00028 \\
.00028 \\
.00027\end{array}$ & $\begin{array}{l}.102 \\
.100 \\
.099\end{array}$ & $\begin{array}{r}.312 \\
.307 \\
.301\end{array}$ & $\begin{array}{l}.333 \\
.327 \\
.322\end{array}$ \\
\hline $\begin{array}{l}10 \\
10 \\
10\end{array}$ & $\begin{array}{l}250 \\
250 \\
250\end{array}$ & $\begin{array}{l}52.0 \\
53.0 \\
54.0\end{array}$ & $\begin{array}{l}.00022 \\
.00021 \\
.00021\end{array}$ & $\begin{array}{r}.00089 \\
.00087 \\
.00086\end{array}$ & $\begin{array}{l}.00006 \\
.00006 \\
.00006\end{array}$ & $\begin{array}{l}.00027 \\
.00026 \\
.00026\end{array}$ & $\begin{array}{l}.097 \\
.095 \\
.093\end{array}$ & $\begin{array}{l}.296 \\
.290 \\
.285\end{array}$ & $\begin{array}{l}.316 \\
.310 \\
.304\end{array}$ \\
\hline $\begin{array}{l}-10 \\
-10\end{array}$ & $\begin{array}{l}250 \\
250 \\
250\end{array}$ & $\begin{array}{l}55.0 \\
60.0 \\
65.0\end{array}$ & $\begin{array}{l}.00021 \\
.00019 \\
.00019\end{array}$ & $\begin{array}{r}.00085 \\
.00079 \\
.00076\end{array}$ & $\begin{array}{l}.00006 \\
.00006 \\
.00005\end{array}$ & $\begin{array}{l}.00 .025 \\
.00024 \\
.00023\end{array}$ & $\begin{array}{l}.092 \\
.086 \\
.082\end{array}$ & $\begin{array}{r}.281 \\
.264 \\
.252\end{array}$ & $\begin{array}{l}.300 \\
.282 \\
.269\end{array}$ \\
\hline $\begin{array}{l}10 \\
10 \\
10\end{array}$ & $\begin{array}{l}250 \\
250 \\
250\end{array}$ & $\begin{array}{l}70.0 \\
75.0 \\
80.0\end{array}$ & $\begin{array}{l}.00018 \\
.00017 \\
.00017\end{array}$ & $\begin{array}{l}.00073 \\
.00071 \\
.00069\end{array}$ & $\begin{array}{l}.00005 \\
.00005 \\
.00005\end{array}$ & $\begin{array}{l}.00022 \\
.00021 \\
.00021\end{array}$ & $\begin{array}{l}.079 \\
.077 \\
.075\end{array}$ & $\begin{array}{r}.241 \\
.235 \\
.229\end{array}$ & $\begin{array}{r}.257 \\
.250 \\
.245\end{array}$ \\
\hline $\begin{array}{l}10 \\
10\end{array}$ & $\begin{array}{l}250 \\
250 \\
250\end{array}$ & $\begin{array}{l}85.0 \\
90.0 \\
95.0\end{array}$ & $\begin{array}{l}.00016 \\
.00016 \\
.00016\end{array}$ & $\begin{array}{l}.00067 \\
.00066 \\
.00065\end{array}$ & $\begin{array}{l}.00005 \\
.00005 \\
.00004\end{array}$ & $\begin{array}{l}.00020 \\
.00020 \\
.00019\end{array}$ & $\begin{array}{l}.073 \\
.072 \\
.070\end{array}$ & $\begin{array}{l}.224 \\
.220 \\
.215\end{array}$ & $\begin{array}{l}.239 \\
.234 \\
.230\end{array}$ \\
\hline 10 & 250 & 0.0 & .00016 & .00064 & .00004 & .00019 & .069 & .211 & .226 \\
\hline
\end{tabular}


TABULATION OF DATA FOR

ABSORPTION OF SOUND IN AIR VERSUS PERCENT RELATIVE HUMIDITY AT-10 DEGREES CENTIGRADE FOR A FREQUENCY OF 500 HERTZ'

\begin{tabular}{|c|c|c|c|c|c|c|c|c|c|}
\hline (1) & $(2)$ & (3) & (4) & (5) & $16 i$ & $(7)$ & $(8)$ & 191 & $(10)$ \\
\hline TEMP & FREQ & $\begin{array}{l}\text { REL } \\
\text { HUM }\end{array}$ & $\begin{array}{l}\text { ATTEN } \\
\text { COEF }\end{array}$ & $4 M$ & $\begin{array}{l}\text { ATTEN } \\
\text { COEF }\end{array}$ & $4 M$ & $\begin{array}{l}\text { ATTEN } \\
\text { DB PER }\end{array}$ & $\begin{array}{l}\text { ATTEN } \\
\text { DB PER }\end{array}$ & $\begin{array}{l}\text { DECAY. } \\
\text { RATE }\end{array}$ \\
\hline $\begin{array}{l}E G R \\
E N T\end{array}$ & HERTZ & $\begin{array}{l}\text { PER } \\
\text { CENT }\end{array}$ & $\begin{array}{l}\text { PER } \\
\text { METER }\end{array}$ & $\begin{array}{l}\text { PER } \\
\text { METER }\end{array}$ & $\begin{array}{l}\text { PER } \\
\text { FOOT }\end{array}$ & $\begin{array}{l}\text { PER } \\
\text { FOOT }\end{array}$ & $\begin{array}{l}100 \\
\text { METER }\end{array}$ & $\begin{array}{l}1000 \\
\text { FEET. }\end{array}$ & $\begin{array}{l}\text { DB PER } \\
\text { SECOND }\end{array}$ \\
\hline $\begin{array}{l}-10 \\
-10 \\
-10\end{array}$ & $\begin{array}{l}500 \\
500 \\
500\end{array}$ & $\begin{array}{r}5.0 \\
10.0 \\
15.0\end{array}$ & $\begin{array}{r}.00063 \\
.00129 \\
.00272\end{array}$ & $\begin{array}{l}.00255 \\
.00517 \\
.00690\end{array}$ & $\begin{array}{r}.00019 \\
.00039 \\
.00052\end{array}$ & $\begin{array}{l}.00077 \\
.00157 \\
.00210\end{array}$ & $\begin{array}{r}.277 \\
.561 \\
.749\end{array}$ & $\begin{array}{r}.845 \\
1.711 \\
2.283\end{array}$ & $\begin{array}{r}.902 \\
1.825 \\
2.436\end{array}$ \\
\hline $\begin{array}{l}-10 \\
-10 \\
-10\end{array}$ & $\begin{array}{l}500 \\
500 \\
500\end{array}$ & $\begin{array}{l}20.0 \\
25.0 \\
30.0\end{array}$ & $\begin{array}{l}.00172 \\
.00153 \\
.00128\end{array}$ & $\begin{array}{l}.00691 \\
.00614 \\
.00513\end{array}$ & $\begin{array}{l}.00052 \\
.00046 \\
.00039\end{array}$ & $\begin{array}{l}.00210 \\
.00187 \\
.00156\end{array}$ & $\begin{array}{l}.750 \\
.667 \\
.557\end{array}$ & $\begin{array}{l}2.288 \\
2.033 \\
1.698\end{array}$ & $\begin{array}{l}2 \cdot 442 \\
2 \cdot 169 \\
1 \cdot 812\end{array}$ \\
\hline $\begin{array}{l}10 \\
10 \\
10\end{array}$ & $\begin{array}{l}500 \\
500 \\
500\end{array}$ & $\begin{array}{l}35.0 \\
40.0 \\
45.0\end{array}$ & $\begin{array}{l}.00107 \\
.00094 \\
.00082\end{array}$ & $\begin{array}{l}.00431 \\
.00376 \\
.00330\end{array}$ & $\begin{array}{l}.00032 \\
.00028 \\
.00025\end{array}$ & $\begin{array}{r}.00131 \\
.00114 \\
.00100\end{array}$ & $\begin{array}{l}.468 \\
.408 \\
.358\end{array}$ & $\begin{array}{l}1.427 \\
1.245 \\
1.094\end{array}$ & $\begin{array}{l}1.523 \\
1.328 \\
1.167\end{array}$ \\
\hline $\begin{array}{l}-10 \\
-10 \\
-10\end{array}$ & $\begin{array}{l}500 \\
500 \\
500\end{array}$ & $\begin{array}{l}46.0 \\
47.0 \\
48.0\end{array}$ & $\begin{array}{l}.00080 \\
.00078 \\
.00076\end{array}$ & $\begin{array}{r}.00322 \\
.00314 \\
.00306\end{array}$ & $\begin{array}{l}.00024 \\
.00023 \\
.00023\end{array}$ & $\begin{array}{l}.00098 \\
.00095 \\
.00093\end{array}$ & $\begin{array}{l}.350 \\
.341 \\
.332\end{array}$ & $\begin{array}{l}1.067 \\
1.040 \\
1.013\end{array}$ & $\begin{array}{l}1.138 \\
1.110 \\
1.081\end{array}$ \\
\hline $\begin{array}{l}-10 \\
-10 \\
-10\end{array}$ & $\begin{array}{l}500 \\
500 \\
500\end{array}$ & $\begin{array}{l}49.0 \\
50.0 \\
51.0\end{array}$ & $\begin{array}{l}.00074 \\
.00073 \\
.00071\end{array}$ & $\begin{array}{l}.00299 \\
.00292 \\
.00286\end{array}$ & $\begin{array}{l}.00022 \\
.00022 \\
.00021\end{array}$ & $\begin{array}{l}.00091 \\
.00089 \\
.00087\end{array}$ & $\begin{array}{r}.325 \\
.318 \\
.310\end{array}$ & $\begin{array}{r}.990 \\
.969 \\
.947\end{array}$ & $\begin{array}{l}1.057 \\
1.034 \\
1.011\end{array}$ \\
\hline $\begin{array}{l}-10 \\
-10 \\
-10\end{array}$ & $\begin{array}{l}500 \\
500 \\
500\end{array}$ & $\begin{array}{l}52.0 \\
53.0 \\
54.0\end{array}$ & $\begin{array}{l}.00070 \\
.00068 \\
.00067\end{array}$ & $\begin{array}{r}.00280 \\
.00274 \\
.00269\end{array}$ & $\begin{array}{l}.00021 \\
.00020 \\
.00020\end{array}$ & $\begin{array}{l}.00085 \\
.00083 \\
.00082\end{array}$ & $\begin{array}{l}.304 \\
.298 \\
.292\end{array}$ & $\begin{array}{r}.927 \\
.908 \\
.890\end{array}$ & $\begin{array}{r}.989 \\
.969 \\
.950\end{array}$ \\
\hline $\begin{array}{l}-10 \\
-10 \\
-10\end{array}$ & $\begin{array}{l}500 \\
500 \\
500\end{array}$ & $\begin{array}{l}55.0 \\
60.0 \\
65.0\end{array}$ & $\begin{array}{l}.00065 \\
.00060 \\
.00055\end{array}$ & $\begin{array}{l}.00263 \\
.00241 \\
.00220\end{array}$ & $\begin{array}{r}.00020 \\
.00018 \\
.00016\end{array}$ & $\begin{array}{r}.00080 \\
.00073 \\
.00067\end{array}$ & $\begin{array}{r}.286 \\
.262 \\
.239\end{array}$ & $\begin{array}{r}.873 \\
.798 \\
.730\end{array}$ & $\begin{array}{l}.931 \\
.852 \\
.779\end{array}$ \\
\hline $\begin{array}{l}-10 \\
-10 \\
-10\end{array}$ & $\begin{array}{l}500 \\
500 \\
500\end{array}$ & $\begin{array}{l}70.0 \\
75.0 \\
80.0\end{array}$ & $\begin{array}{l}.00051 \\
.00047 \\
.00045\end{array}$ & $\begin{array}{l}.00204 \\
.00191 \\
.00180\end{array}$ & $\begin{array}{l}.00015 \\
.00014 \\
.00013\end{array}$ & $\begin{array}{r}.00062 \\
.00058 \\
.00054\end{array}$ & $\begin{array}{l}.221 . \\
.207 \\
.195\end{array}$ & $\begin{array}{r}.675 \\
.632 \\
.597\end{array}$ & $\begin{array}{l}.721 \\
.674 \\
.637\end{array}$ \\
\hline $\begin{array}{l}-10 \\
-10 \\
-10\end{array}$ & $\begin{array}{l}500 \\
500 \\
500\end{array}$ & $\begin{array}{l}85.0 \\
90.0 \\
95.0\end{array}$ & $\begin{array}{l}.00042 \\
.00040 \\
.00039\end{array}$ & $\begin{array}{r}.00170 \\
.00163 \\
.00158\end{array}$ & $\begin{array}{l}.00013 \\
.00012 \\
.00012\end{array}$ & $\begin{array}{r}.00052 \\
.00049 \\
.00048\end{array}$ & $\begin{array}{r}185 \\
.177 \\
.172\end{array}$ & $\begin{array}{r}.565 \\
.541 \\
.524\end{array}$ & $\begin{array}{l}.602 \\
.577 \\
.559\end{array}$ \\
\hline 0 & 500 & $100 \cdot 0$ & .00038 & .00154 & .00011 & .00046 & .167 & .510 & .544 \\
\hline
\end{tabular}


TABULATION OF DATA FOR

ABSORPTION OF SOUND IN AIR VERSUS PERCENT RELATIVE HUMIDITY AT-IO DEGREES. CENTIGRADE FOR A FREQUENCY OF 1000 HERTZ

\begin{tabular}{|c|c|c|c|c|c|c|c|c|c|}
\hline (1) & (2) & $(3)$ & (4) & (5) & (6) & $(7)$ & (8) & (9) & $(10)$ \\
\hline TEMP & FREQ & $\begin{array}{l}\text { REL } \\
\text { HUM }\end{array}$ & $\begin{array}{l}\text { ATTEN } \\
\text { COEF }\end{array}$ & $4 M$ & $\begin{array}{l}\text { AT'TEN } \\
\text { COEF }\end{array}$ & $4 M$ & $\begin{array}{l}\text { ATTEN } \\
\text { DB PER }\end{array}$ & $\begin{array}{l}\text { ATTEN } \\
\text { DB PER }\end{array}$ & $\begin{array}{l}\text { DECAY } \\
\text { RATE }\end{array}$ \\
\hline $\begin{array}{l}\text { DEGR } \\
\text { CENT }\end{array}$ & HERTZ & $\begin{array}{l}\text { PER } \\
\text { CENT }\end{array}$ & $\begin{array}{l}\text { PER } \\
\text { METER }\end{array}$ & $\begin{array}{l}\text { PER } \\
\text { METER }\end{array}$ & $\begin{array}{l}\text { PER } \\
\text { FOOT }\end{array}$ & $\begin{array}{l}\text { PER } \\
\text { FOOT }\end{array}$ & $\begin{array}{l}100 \\
\text { METER }\end{array}$ & $\begin{array}{l}1000 \\
\text { FEET }\end{array}$ & $\begin{array}{l}\text { DB PER } \\
\text { SECOND }\end{array}$ \\
\hline $\begin{array}{l}-10 \\
-10 \\
-10\end{array}$ & $\begin{array}{l}1000 \\
1000 \\
1000\end{array}$ & $\begin{array}{r}5.0 \\
10.0 \\
15.0\end{array}$ & $\begin{array}{l}.00081 \\
.00159 \\
.00241\end{array}$ & $\begin{array}{r}.00325 \\
.00639 \\
.00964\end{array}$ & $\begin{array}{r}.00024 \\
.00048 \\
.00073\end{array}$ & $\begin{array}{r}.00099 \\
.00194 \\
.00294\end{array}$ & $\begin{array}{r}.353 \\
.693 \\
1.047\end{array}$ & $\begin{array}{l}1.078 \\
2.115 \\
3.192\end{array}$ & $\begin{array}{l}1.150 \\
2.256 \\
3.406\end{array}$ \\
\hline $\begin{array}{l}-10 \\
-10 \\
-10\end{array}$ & $\begin{array}{l}1000 \\
1000 \\
1000\end{array}$ & $\begin{array}{l}20.0 \\
25.0 \\
30.0\end{array}$ & $\begin{array}{r}.00317 \\
.00349 \\
.00351\end{array}$ & $\begin{array}{r}.01268 \\
.01397 \\
.01405\end{array}$ & $\begin{array}{r}.00096 \\
.00106 \\
.00107\end{array}$ & $\begin{array}{r}.00386 \\
.00425 \\
.00428\end{array}$ & $\begin{array}{l}1.377 \\
1.517 \\
1.526\end{array}$ & $\begin{array}{l}4.197 \\
4.624 \\
4.652\end{array}$ & $\begin{array}{l}4.478 \\
4.935 \\
4.964\end{array}$ \\
\hline $\begin{array}{l}-10 \\
-10 \\
-10\end{array}$ & $\begin{array}{l}1000 \\
1000 \\
1000\end{array}$ & $\begin{array}{l}35: 0 \\
40.0 \\
45.0\end{array}$ & $\begin{array}{l}.00337 \\
.00310 \\
.00278\end{array}$ & $\begin{array}{r}.01349 \\
.01241 \\
.01115\end{array}$ & $\begin{array}{r}.00102 \\
.00094 \\
.00085\end{array}$ & $\begin{array}{r}.00411 \\
.00378 \\
.00340\end{array}$ & $\begin{array}{l}1.465 \\
1.348 \\
1.211\end{array}$ & $\begin{array}{l}4.467 \\
4.108 \\
3.691\end{array}$ & $\begin{array}{l}4.767 \\
4.384 \\
3.939\end{array}$ \\
\hline $\begin{array}{l}-10 \\
-10 \\
-10\end{array}$ & $\begin{array}{l}1000 \\
1000 \\
1000\end{array}$ & $\begin{array}{l}46.0 \\
47.0 \\
48.0\end{array}$ & $\begin{array}{l}.00272 \\
.00266 \\
.00260\end{array}$ & $\begin{array}{r}.01090 \\
.01056 \\
.01040\end{array}$ & $\begin{array}{r}.00083 \\
.00081 \\
.00079\end{array}$ & $\begin{array}{l}.00332 \\
.00324 \\
.00317\end{array}$ & $\begin{array}{l}1.184 \\
1.157 \\
1.129\end{array}$ & $\begin{array}{l}3.609 \\
3.527 \\
3.442\end{array}$ & $\begin{array}{l}3.852 \\
3.764 \\
3.673\end{array}$ \\
\hline $\begin{array}{l}-10 \\
-10 \\
-10\end{array}$ & $\begin{array}{l}1000 \\
1000 \\
1000\end{array}$ & $\begin{array}{l}49.0 \\
50.0 \\
51.0\end{array}$ & $\begin{array}{r}.00253 \\
.00247 \\
.00242\end{array}$ & $\begin{array}{l}.01014 \\
.00989 \\
.00969\end{array}$ & $\begin{array}{r}.00077 \\
.00075 \\
.00073\end{array}$ & $\begin{array}{l}.00309 \\
.00301 \\
.00295\end{array}$ & $\begin{array}{l}1.101 \\
1.074 \\
1.052\end{array}$ & $\begin{array}{l}3.357 \\
3.274 \\
3.207\end{array}$ & $\begin{array}{l}3.582 \\
3.494 \\
3.423\end{array}$ \\
\hline $\begin{array}{l}-10 \\
-10\end{array}$ & $\begin{array}{l}1000 \\
1000\end{array}$ & $\begin{array}{l}52.0 \\
53.0\end{array}$ & $\begin{array}{l}.00237 \\
.00232\end{array}$ & $\begin{array}{r}.00949 \\
.00929\end{array}$ & $\begin{array}{l}.00072 \\
.00070\end{array}$ & $\begin{array}{l}.00289 \\
.00283\end{array}$ & $\begin{array}{l}1.030 \\
1.009\end{array}$ & $\begin{array}{l}3.141 \\
3.076\end{array}$ & $\begin{array}{l}3.351 \\
3.282\end{array}$ \\
\hline-10 & 1000 & $54 \cdot 0$ & .00227 & .00910 & .00069 & .00277 & .988 & 3.014 & 3.216 \\
\hline $\begin{array}{l}-10 \\
-10\end{array}$ & $\begin{array}{l}1000 \\
1000\end{array}$ & $\begin{array}{l}55.0 \\
60.0\end{array}$ & $\begin{array}{l}.00223 \\
.00203\end{array}$ & $\begin{array}{l}.00892 \\
.00813\end{array}$ & $\begin{array}{l}.00067 \\
.00062\end{array}$ & $\begin{array}{l}.00271 \\
.00248\end{array}$ & $\begin{array}{l}.968 \\
.883\end{array}$ & $\begin{array}{l}2.952 \\
2.693\end{array}$ & $\begin{array}{l}3.150 \\
2.874\end{array}$ \\
\hline-10 & 1000 & 65.0 & .00187 & .00751 & .00057 & .00229 & .815 & 2.486 & 2.653 \\
\hline $\begin{array}{l}-10 \\
-10 \\
-10\end{array}$ & $\begin{array}{l}1000 \\
1000 \\
1000\end{array}$ & $\begin{array}{l}70.0 \\
75.0 \\
80.0\end{array}$ & $\begin{array}{r}.00173 \\
.00160 \\
.00149\end{array}$ & $\begin{array}{r}.00693 \\
.00641 \\
.00596\end{array}$ & $\begin{array}{r}.00052 \\
.00048 \\
.00045\end{array}$ & $\begin{array}{r}.00211 \\
.00195 \\
.00181\end{array}$ & $\begin{array}{l}.752 \\
.696 \\
.647\end{array}$ & $\begin{array}{l}2.294 \\
2.123 \\
1.972\end{array}$ & $\begin{array}{l}2.448 \\
2.266 \\
2.104\end{array}$ \\
\hline $\begin{array}{l}-10 \\
-10 \\
-10\end{array}$ & $\begin{array}{l}1000 \\
1000 \\
1000\end{array}$ & $\begin{array}{l}85.0 \\
90.0 \\
95.0\end{array}$ & $\begin{array}{l}.00139 \\
.00131 \\
.00124\end{array}$ & $\begin{array}{r}.00557 \\
.00524 \\
.00496\end{array}$ & $\begin{array}{r}.00042 \\
.00040 \\
.00037\end{array}$ & $\begin{array}{l}.00169 \\
.00160 \\
.00151\end{array}$ & $\begin{array}{l}.605 \\
.569 \\
.539\end{array}$ & $\begin{array}{l}1.845 \\
1.737 \\
1.644\end{array}$ & $\begin{array}{l}1.968 \\
1.853 \\
1.754\end{array}$ \\
\hline-10 & 1000 & $100 \cdot 0$ & .00117 & .00470 & .00035 & .00143 & .510 & 1.556 & 1.660 \\
\hline
\end{tabular}


TABULATION OF DATA FOR

ABSORPTION OF SOUND IN AIR VERSUS PERCENT RELATIVE HUMIDITY AT-10 DEGREES CENTIGRADE FOR A FREQUENCY OF 2000 HERTZ

\begin{tabular}{|c|c|c|c|c|c|c|c|c|c|}
\hline (1) & (2) & (3) & (4) & (5) & (6) & $(7)$ & $(3)$ & $(9)$ & $110)$ \\
\hline TEMP & FREQ & REL & ATTEN & $4 M$ & ATTEN & $4 M$ & ATTEN & ATTEN & DECAY \\
\hline & & HUM & COEF & & COEF & & DB PER & DB PER & RATE \\
\hline$E G R$ & & PER & PER & PER & PER & PER & 100 & 1000 & DB PER \\
\hline CENT & HER TZ & CENT & METER & .METER & FOOT & $\mathrm{F} \odot \mathrm{T}^{-}$ & METER & FEET & SECOND \\
\hline 10 & 2000 & 5.0 & .00108 & .00435 & .00033 & .00132 & .472 & 1.440 & 1.537 \\
\hline 10 & 2000 & 10.0 & .00205 & .00821 & .00062 & .00250 & .891 & 2.717 & 2.899 \\
\hline 10 & 2000 & 15.0 & .00301 & .01206 & .00091 & .00367 & 1.310 & 3.994 & 4.262 \\
\hline $\begin{array}{l}10 \\
10\end{array}$ & $\begin{array}{l}2000 \\
2000\end{array}$ & $\begin{array}{l}20.0 \\
25.0\end{array}$ & $\begin{array}{l}.00398 \\
.00502\end{array}$ & $\begin{array}{r}.01592 \\
.02008\end{array}$ & $\begin{array}{l}.00121 \\
.00153\end{array}$ & $\begin{array}{l}.00485 \\
.00612\end{array}$ & $\begin{array}{l}1.729 \\
2.180\end{array}$ & & $\begin{array}{l}5.624 \\
7.091\end{array}$ \\
\hline 10 & 2000 & 30.0 & .00601 & .02406 & .00183 & .00733 & 2.612 & 7.963 & 8.497 \\
\hline $\begin{array}{l}10 \\
10\end{array}$ & $\begin{array}{l}2000 \\
2000\end{array}$ & $\begin{array}{l}35.0 \\
40.0\end{array}$ & $\begin{array}{r}.00668 \\
.00702\end{array}$ & $\begin{array}{r}.02673 \\
.02810\end{array}$ & $\begin{array}{l}.00203 \\
.00214\end{array}$ & $\begin{array}{l}.00814 \\
.00856\end{array}$ & $\begin{array}{l}2.903 \\
3.051\end{array}$ & & $\begin{array}{l}9.442 \\
9.923\end{array}$ \\
\hline-10 & 2000 & 45.0 & .00712 & .02849 & .00217 & .00868 & 3.093 & 9.430 & 10.063 \\
\hline $\begin{array}{l}-10 \\
-10\end{array}$ & $\begin{array}{l}2000 \\
2000\end{array}$ & $\begin{array}{l}46.0 \\
47.0\end{array}$ & .00711 & $\begin{array}{r}.02847 \\
.02844\end{array}$ & $\begin{array}{l}.00216 \\
.00216\end{array}$ & $\begin{array}{l}.00867 \\
.00867\end{array}$ & $\begin{array}{l}3.091 \\
3.088\end{array}$ & $\begin{array}{l}9.422 \\
9.414\end{array}$ & $\begin{array}{l}10.054 \\
10.045\end{array}$ \\
\hline-10 & 2000 & 48.0 & .00710 & .02840 & .00216 & .00865 & 3.083 & 9.399 & 10.030 \\
\hline $\begin{array}{l}10 \\
10\end{array}$ & $\begin{array}{l}2000 \\
2000\end{array}$ & $\begin{array}{l}49.0 \\
50.0\end{array}$ & $\begin{array}{l}.00708 \\
.00706\end{array}$ & $\begin{array}{r}.02835 \\
.02827\end{array}$ & $\begin{array}{l}.00216 \\
.00215\end{array}$ & $\begin{array}{l}.00864 \\
.00861\end{array}$ & $\begin{array}{l}3.078 \\
3.070\end{array}$ & $\begin{array}{l}9.383 \\
9.358\end{array}$ & $\begin{array}{r}10.012 \\
9.986\end{array}$ \\
\hline 10 & 2000 & 51.0 & .00704 & .02817 & .00214 & .00858 & 3.059 & 9.325 & 9.951 \\
\hline $\begin{array}{l}10 \\
10\end{array}$ & $\begin{array}{l}2000 \\
2000\end{array}$ & $\begin{array}{l}52.0 \\
53.0\end{array}$ & $\begin{array}{l}.00701 \\
.00698\end{array}$ & $\begin{array}{r}.02807 \\
.02794\end{array}$ & $\begin{array}{l}.00213 \\
.00212\end{array}$ & $\begin{array}{l}.00855 \\
.00851\end{array}$ & $\begin{array}{l}3.048 \\
3.034\end{array}$ & $\begin{array}{l}9.290 \\
9.249\end{array}$ & $\begin{array}{l}9.913 \\
9.869\end{array}$ \\
\hline 10 & 2000 & 54.0 & .00695 & .02782 & .00212 & .00848 & 3.020 & 9.208 & 9.825 \\
\hline $\begin{array}{l}-10 \\
-10\end{array}$ & $\begin{array}{l}2000 \\
2000\end{array}$ & $\begin{array}{l}55.0 \\
60.0\end{array}$ & $\begin{array}{l}.00690 \\
.00663\end{array}$ & $\begin{array}{r}.02762 \\
.02652\end{array}$ & $\begin{array}{l}.00210 \\
.00202\end{array}$ & $\begin{array}{l}.00842 \\
.00808\end{array}$ & $\begin{array}{l}2.999 \\
2.880\end{array}$ & $\begin{array}{l}9.142 \\
8.778\end{array}$ & $\begin{array}{l}9.755 \\
9.367\end{array}$ \\
\hline-10 & 2000 & 65.0 & .00626 & .02507 & .00191 & .00764 & 2.722 & 8.297 & 8.854 \\
\hline 10 & 2000 & 70.0 & .00587 & .02351 & .00179 & .00716 & 2.553 & 7.783 & 8.305 \\
\hline-10 & $\begin{array}{l}2000 \\
2000\end{array}$ & $\begin{array}{l}75.0 \\
80.0\end{array}$ & $\begin{array}{r}.00549 \\
.00510\end{array}$ & $\begin{array}{r}.02198 \\
.02041\end{array}$ & $\begin{array}{r}.00167 \\
.00155\end{array}$ & $\begin{array}{l}.00669 \\
.00622\end{array}$ & $\begin{array}{l}2.386 \\
2.216\end{array}$ & $\begin{array}{l}7.274 \\
6.755\end{array}$ & $\begin{array}{l}7.762 \\
7.208\end{array}$ \\
\hline-10 & 2000 & 85.0 & .00477 & .01910 & .00145 & .00582 & 2.073 & 6.321 & $6 \cdot 745$ \\
\hline-10 & $\begin{array}{l}2000 \\
2000\end{array}$ & $\begin{array}{l}90.0 \\
95.0\end{array}$ & $\begin{array}{l}.00448 \\
.00423\end{array}$ & $\begin{array}{r}.01794 \\
.01695\end{array}$ & $\begin{array}{l}.00136 \\
.00129\end{array}$ & $\begin{array}{l}.00546 \\
.00516\end{array}$ & $\begin{array}{l}1.947 \\
1.841\end{array}$ & $\begin{array}{l}5.937 \\
5.611\end{array}$ & $\begin{array}{r}6.335 \\
5.988\end{array}$ \\
\hline 10 & 2000 & 100.0 & .00402 & .01611 & .00122 & .00491 & 1.749 & 5.331 & 5.689 \\
\hline
\end{tabular}


TABULATION OF DATA FOR

ABSORPTION OF SOUND IN AIR VERSUS PERCENT RELATIVE HUMIDITY AT-10 DEGREES CENTIGRADE FOR A FREQUENCY OF 2500 HERTZ

\begin{tabular}{|c|c|c|c|c|c|c|c|c|c|}
\hline (i) & (2) & (3) & (4) & (5) & $(6)$ & 171 & $(8)$ & $(9)$ & $(10)$ \\
\hline TEMP & FREQ & $\begin{array}{l}\text { REL } \\
\text { HUM }\end{array}$ & $\begin{array}{l}\text { ATTEN } \\
\text { COEF }\end{array}$ & $4 M$ & $\begin{array}{l}\text { ATTEN } \\
\text { COEF }\end{array}$ & $4 M$ & $\begin{array}{l}\text { ATTEN } \\
\text { DB. PER }\end{array}$ & $\begin{array}{l}\text { ATTEN } \\
\text { DB PER }\end{array}$ & $\begin{array}{l}\text { DECAY } \\
\text { RATE }\end{array}$ \\
\hline $\begin{array}{l}\text { DEGR } \\
\text { CENT }\end{array}$ & HERTZ & $\begin{array}{l}\text { PER } \\
\text { CENT }\end{array}$ & $\begin{array}{l}\text { PER } \\
\text { METER }\end{array}$ & $\begin{array}{l}\text { PER } \\
\text { METER }\end{array}$ & $\begin{array}{l}\text { PER } \\
\text { FOOT }\end{array}$ & $\begin{array}{l}\text { PER } \\
\text { FOOT }\end{array}$ & $\begin{array}{l}100 \\
\text { METER }\end{array}$ & $\begin{array}{l}1000 \\
\text { FEET }\end{array}$ & $\begin{array}{l}\text { DB PER } \\
\text { SECOND }\end{array}$ \\
\hline $\begin{array}{l}-10 \\
-10\end{array}$ & $\begin{array}{l}2500 \\
2500\end{array}$ & $\begin{array}{r}5.0 \\
10.0\end{array}$ & $\begin{array}{l}.00122 \\
.00224\end{array}$ & $\begin{array}{l}.00488 \\
.00899\end{array}$ & $\begin{array}{l}.00037 \\
.00068\end{array}$ & $\begin{array}{l}.00148 \\
.00274\end{array}$ & $\begin{array}{r}530 \\
.976\end{array}$ & $\begin{array}{l}1.616 \\
2.977\end{array}$ & $\begin{array}{l}1.725 \\
3.176\end{array}$ \\
\hline-10 & 2500 & 15.0 & .00327 & .01310 & .00099 & .00399 & 1.422 & 4.337 & 4.628 \\
\hline $\begin{array}{l}-10 \\
-10 \\
-10\end{array}$ & $\begin{array}{l}2500 \\
2500 \\
2500\end{array}$ & $\begin{array}{l}20.0 \\
25.0 \\
30.0\end{array}$ & $\begin{array}{l}.00430 \\
.00533 \\
.00646\end{array}$ & $\begin{array}{r}.01721 \\
.02135 \\
.02584\end{array}$ & $\begin{array}{l}.00131 \\
.00162 \\
.00196\end{array}$ & $\begin{array}{l}.00524 \\
.00650 \\
.00787\end{array}$ & $\begin{array}{l}1.869 \\
2.318 \\
2.806\end{array}$ & $\begin{array}{l}5.697 \\
7.068 \\
8.554\end{array}$ & $\begin{array}{l}6.079 \\
7.542 \\
9.128\end{array}$ \\
\hline-10 & 2500 & 35.0 & .00752 & .03008 & .00229 & .00917 & 3.266 & 9.956 & 10.624 \\
\hline $\begin{array}{l}-10 \\
-10\end{array}$ & $\begin{array}{l}2500 \\
2500\end{array}$ & $\begin{array}{l}40.0 \\
45.0\end{array}$ & $\begin{array}{l}.00828 \\
.00873\end{array}$ & $\begin{array}{r}.03314 \\
.03492\end{array}$ & $\begin{array}{r}.00252 \\
.00266\end{array}$ & $\begin{array}{r}.01010 \\
.01064\end{array}$ & $\begin{array}{l}3.598 \\
3.791\end{array}$ & $\begin{array}{l}10.967 \\
11.557\end{array}$ & $\begin{array}{l}11.703 \\
12.332\end{array}$ \\
\hline $\begin{array}{l}-10 \\
-10 \\
-10\end{array}$ & $\begin{array}{l}2500 \\
2500 \\
2500\end{array}$ & $\begin{array}{l}46.0 \\
47.0 \\
48.0\end{array}$ & $\begin{array}{r}.00877 \\
.00882 \\
.00885\end{array}$ & $\begin{array}{r}.03510 \\
.03529 \\
.03543\end{array}$ & $\begin{array}{r}.00267 \\
.00268 \\
.00270\end{array}$ & $\begin{array}{r}.01070 \\
.01075 \\
.01080\end{array}$ & $\begin{array}{l}3.811 \\
3.832 \\
3.847\end{array}$ & $\begin{array}{l}11.618 \\
11.680 \\
11.727\end{array}$ & $\begin{array}{l}12.398 \\
12.463 \\
12.513\end{array}$ \\
\hline $\begin{array}{l}-10 \\
-10 \\
-10\end{array}$ & $\begin{array}{l}2500 \\
2500 \\
2500\end{array}$ & $\begin{array}{l}49.0 \\
50.0 \\
51.0\end{array}$ & $\begin{array}{l}.00888 \\
.00890 \\
.00892\end{array}$ & $\begin{array}{l}.03552 \\
.03562 \\
.03568\end{array}$ & $\begin{array}{l}.00270 \\
.00271 \\
.00271\end{array}$ & $\begin{array}{r}.01082 \\
.01085 \\
.01087\end{array}$ & $\begin{array}{l}3.857 \\
3.867 \\
3.874\end{array}$ & $\begin{array}{l}11.757 \\
11.788 \\
11.808\end{array}$ & $\begin{array}{l}12 \cdot 546 \\
12.579 \\
12.600\end{array}$ \\
\hline $\begin{array}{l}-10 \\
-10 \\
-10\end{array}$ & $\begin{array}{l}2500 \\
2500 \\
2500\end{array}$ & $\begin{array}{l}52.0 \\
53.0 \\
54.0\end{array}$ & $\begin{array}{l}.00893 \\
.00894 \\
.00893\end{array}$ & $\begin{array}{r}.03573 \\
.03577 \\
.03574\end{array}$ & $\begin{array}{l}.00272 \\
.00272 \\
.00272\end{array}$ & $\begin{array}{l}.01089 \\
.01090 \\
.01089\end{array}$ & $\begin{array}{l}3.879 \\
3.883 \\
3.880\end{array}$ & $\begin{array}{l}11.825 \\
11.837 \\
11.829\end{array}$ & $\begin{array}{l}12.618 \\
12.632 \\
12.622\end{array}$ \\
\hline $\begin{array}{l}-10 \\
-10 \\
-10\end{array}$ & $\begin{array}{l}2500 \\
2500 \\
2500\end{array}$ & $\begin{array}{l}55.0 \\
60.0 \\
65.0\end{array}$ & $\begin{array}{l}.00892 \\
.00884 \\
.00864\end{array}$ & $\begin{array}{r}.03571 \\
.03536 \\
.03458\end{array}$ & $\begin{array}{r}.00272 \\
.00269 \\
.00263\end{array}$ & $\begin{array}{r}.01088 \\
.01077 \\
.01054\end{array}$ & $\begin{array}{l}3.878 \\
3.839 \\
3.755\end{array}$ & $\begin{array}{l}11.820 \\
11.702 \\
11.446\end{array}$ & $\begin{array}{l}12.613 \\
12.487 \\
12.214\end{array}$ \\
\hline $\begin{array}{l}-10 \\
-10 \\
-10\end{array}$ & $\begin{array}{l}2500 \\
2500 \\
2500\end{array}$ & $\begin{array}{l}70.0 \\
75.0 \\
80.0\end{array}$ & $\begin{array}{r}.00835 \\
.00797 \\
.00756\end{array}$ & $\begin{array}{r}.03342 \\
.03191 \\
.03026\end{array}$ & $\begin{array}{r}.00254 \\
.00243 \\
.00230\end{array}$ & $\begin{array}{l}.01018 \\
.00972 \\
.00922\end{array}$ & $\begin{array}{l}3.628 \\
3.465 \\
3.285\end{array}$ & $\begin{array}{l}11.060 \\
10.562 \\
10.014\end{array}$ & $\begin{array}{l}11.802 \\
11.271 \\
10.686\end{array}$ \\
\hline $\begin{array}{l}-10 \\
-10 \\
-10\end{array}$ & $\begin{array}{l}2500 \\
2500 \\
2500\end{array}$ & $\begin{array}{l}85.0 \\
90.0 \\
95.0\end{array}$ & $\begin{array}{l}.00715 \\
.00674 \\
.00632\end{array}$ & $\begin{array}{r}.02860 \\
.02697 \\
.02528\end{array}$ & $\begin{array}{l}.00217 \\
.00205 \\
.00192\end{array}$ & $\begin{array}{l}.00871 \\
.00822 \\
.00770\end{array}$ & $\begin{array}{l}3.106 \\
2.929 \\
2.745\end{array}$ & $\begin{array}{l}9.467 \\
8.928 \\
8.369\end{array}$ & $\begin{array}{r}10.102 \\
9.527 \\
8.930\end{array}$ \\
\hline 10 & 25001 & $100 \cdot 0$ & .00599 & .02396 & .00182 & .00730 & 2.601 & 7.929 & 8.461 \\
\hline
\end{tabular}


TABULATION OF DATA FOR

ABSORPTION OF SOUND IN AIR VERSUS. PERCENT RELATIVE HUMIDITY AT-10 DEGREES CENTIGRADE FOR A FREQUENCY OF 3200 HERTZ

\begin{tabular}{|c|c|c|c|c|c|c|c|c|c|}
\hline (1) & $(2)$ & $(3)$ & $(4)$ & (5) & $(6)$ & 171 & $(8)$ & (9) & $(10)$ \\
\hline $\begin{array}{l}\text { DEGR } \\
\text { CENT }\end{array}$ & FREQ & $\begin{array}{l}\text { REL } \\
\text { HUM } \\
\text { PER } \\
\text { CENT }\end{array}$ & $\begin{array}{l}\text { ATTEN } \\
\text { COEF } \\
\text { PER } \\
\text { METER }\end{array}$ & $\begin{array}{l}\text { PER } \\
\text { METER }\end{array}$ & $\begin{array}{l}\text { ATTEN } \\
\text { COEF } \\
\text { PER } \\
\text { FOOT }\end{array}$ & $\begin{array}{l}\text { PER } \\
\text { FOOT }\end{array}$ & $\begin{array}{l}\text { ATTEN } \\
\text { DB PER } \\
100 \\
\text { METER }\end{array}$ & $\begin{array}{l}\text { ATTEN } \\
\text { DB PER } \\
1000 \\
\text { FEET }\end{array}$ & $\begin{array}{l}\text { DECAY } \\
\text { RATE } \\
\text { DB PER } \\
\text { SECOND }\end{array}$ \\
\hline $\begin{array}{l}-10 \\
-10 \\
-10\end{array}$ & $\begin{array}{l}3200 \\
3200 \\
3200\end{array}$ & $\begin{array}{r}5.0 \\
10.0 \\
15.0\end{array}$ & $\begin{array}{l}.00143 \\
.00255 \\
.00367\end{array}$ & $\begin{array}{l}.00574 \\
.01022 \\
.01470\end{array}$ & $\begin{array}{r}.00043 \\
.00077 \\
.00112\end{array}$ & $\begin{array}{l}.00175 \\
.00311 \\
.00448\end{array}$ & $\begin{array}{r}.624 \\
1.110 \\
1.596\end{array}$ & $\begin{array}{l}1.902 \\
3.384 \\
4.867\end{array}$ & $\begin{array}{l}2.029 \\
3.611 \\
5.193\end{array}$ \\
\hline $\begin{array}{l}-10 \\
-10 \\
-10\end{array}$ & $\begin{array}{l}3200 \\
3200 \\
3200\end{array}$ & $\begin{array}{l}20.0 \\
25.0 \\
30.0\end{array}$ & $\begin{array}{l}.00479 \\
.00591 \\
.00705\end{array}$ & $\begin{array}{l}.01918 \\
.02366 \\
.02820\end{array}$ & $\begin{array}{l}.00146 \\
.00180 \\
.00214\end{array}$ & $\begin{array}{l}.00584 \\
.00721 \\
.00859\end{array}$ & & & $\begin{array}{l}6.776 \\
8.358 \\
9.961\end{array}$ \\
\hline-10 & 3200 & 35.0 & .00828 & .03314 & .00252 & .01010 & 3.598 & 10.967 & 11.703 \\
\hline $\begin{array}{l}-10 \\
-10\end{array}$ & $\begin{array}{l}3200 \\
3200\end{array}$ & $\begin{array}{l}40.0 \\
45.0\end{array}$ & $\begin{array}{l}.00946 \\
.01039\end{array}$ & $\begin{array}{l}.03786 \\
.04159\end{array}$ & $\begin{array}{r}.00288 \\
.00316\end{array}$ & $\begin{array}{l}.01154 \\
.01267\end{array}$ & $\begin{array}{l}4.110 \\
4.516\end{array}$ & $\begin{array}{l}12.529 \\
13.765\end{array}$ & $\begin{array}{l}13.370 \\
14.688\end{array}$ \\
\hline $\begin{array}{l}-10 \\
-10 \\
-10\end{array}$ & $\begin{array}{l}3200 \\
3200 \\
3200\end{array}$ & $\begin{array}{l}46.0 \\
47.0 \\
48.0\end{array}$ & $\begin{array}{r}.01054 \\
.01067 \\
.01078\end{array}$ & $\begin{array}{r}.04218 \\
.04270 \\
.04315\end{array}$ & $\begin{array}{l}.00321 \\
.00325 \\
.00328\end{array}$ & $\begin{array}{r}.01285 \\
.01301 \\
.01315\end{array}$ & $\begin{array}{l}4.580 \\
4.637 \\
4.685\end{array}$ & $\begin{array}{l}13.961 \\
14.133 \\
14.282\end{array}$ & $\begin{array}{l}14.898 \\
15.081 \\
15.240\end{array}$ \\
\hline $\begin{array}{l}-10 \\
-10 \\
-10\end{array}$ & $\begin{array}{l}3200 \\
3200 \\
3200\end{array}$ & $\begin{array}{l}49 \cdot 0 \\
50.0 \\
51.0\end{array}$ & $\begin{array}{r}.01090 \\
.01100 \\
.01108\end{array}$ & $\begin{array}{r}.04360 \\
.04401 \\
.04435\end{array}$ & $\begin{array}{r}.00332 \\
.00335 \\
.00338\end{array}$ & $\begin{array}{r}.01329 \\
.01341 \\
.01352\end{array}$ & $\begin{array}{l}4.734 \\
4.778 \\
4.816\end{array}$ & $\begin{array}{l}14.430 \\
14.565 \\
14.680\end{array}$ & $\begin{array}{l}15.398 \\
15.542 \\
15.664\end{array}$ \\
\hline $\begin{array}{l}-10 \\
-10 \\
-10\end{array}$ & $\begin{array}{l}3200 \\
3200 \\
3200\end{array}$ & $\begin{array}{l}52.0 \\
53.0 \\
54.0\end{array}$ & $\begin{array}{r}.01117 \\
.01125 \\
.01130\end{array}$ & $\begin{array}{r}.04470 \\
.04500 \\
.04521\end{array}$ & $\begin{array}{r}.00340 \\
.00342 \\
.00344\end{array}$ & $\begin{array}{l}.01362 \\
.01371 \\
.01378\end{array}$ & $\begin{array}{l}4.853 \\
4.886 \\
4.908\end{array}$ & $\begin{array}{l}14.794 \\
14.895 \\
14.962\end{array}$ & $\begin{array}{l}15.787 \\
15.894 \\
15.965\end{array}$ \\
\hline $\begin{array}{l}-10 \\
-10 \\
-10\end{array}$ & $\begin{array}{l}3200 \\
3200 \\
3200\end{array}$ & $\begin{array}{l}55.0 \\
60.0 \\
65.0\end{array}$ & $\begin{array}{r}.01135 \\
.01148 \\
.01149\end{array}$ & $\begin{array}{r}.04541 \\
.04595 \\
.04598\end{array}$ & $\begin{array}{l}.00346 \\
.00350 \\
.00350\end{array}$ & $\begin{array}{r}.01384 \\
.01400 \\
.01401\end{array}$ & $\begin{array}{l}4.930 \\
4.989 \\
4.992\end{array}$ & $\begin{array}{l}15.029 \\
15.207 \\
15.217\end{array}$ & $\begin{array}{l}16.037 \\
16.227 \\
16.237\end{array}$ \\
\hline $\begin{array}{l}-10 \\
-10 \\
-10\end{array}$ & $\begin{array}{l}3200 \\
3200 \\
3200\end{array}$ & $\begin{array}{l}70.0 \\
75.0 \\
80.0\end{array}$ & $\begin{array}{r}.01139 \\
.01121 \\
.01091\end{array}$ & $\begin{array}{r}.04559 \\
.04485 \\
.04366\end{array}$ & $\begin{array}{l}.00347 \\
.00341 \\
.00332\end{array}$ & $\begin{array}{r}.01389 \\
.01367 \\
.01330\end{array}$ & $\begin{array}{l}4.950 \\
4.870 \\
4.740\end{array}$ & $\begin{array}{l}15.087 \\
14.844 \\
14.449\end{array}$ & $\begin{array}{l}16.100 \\
15.839 \\
15.418\end{array}$ \\
\hline $\begin{array}{l}-10 \\
-10 \\
-10\end{array}$ & $\begin{array}{l}3200 \\
3200 \\
3200\end{array}$ & $\begin{array}{l}85.0 \\
90.0 \\
95.0\end{array}$ & $\begin{array}{r}.01054 \\
.01010 \\
.00965\end{array}$ & $\begin{array}{r}.04218 \\
.04043 \\
.03862\end{array}$ & $\begin{array}{l}.00321 \\
.00308 \\
.00294\end{array}$ & $\begin{array}{r}.01285 \\
.01232 \\
.01177\end{array}$ & $\begin{array}{l}4 \cdot 580 \\
4 \cdot 389 \\
4.193\end{array}$ & $\begin{array}{l}13.961 \\
13.380 \\
12.782\end{array}$ & $\begin{array}{l}14.897 \\
14.278 \\
13.640\end{array}$ \\
\hline-10 & 3200 & $00 \cdot 0$ & .00920 & .03682 & .00280 & .01122 & 3.998 & 12.186 & 13.004 \\
\hline
\end{tabular}


TABULATION OF DATA FOR

ABSORPTION OF SOUND IN AIR VERSUS PERCENT RELATIVE HUMIDITY AT-10 DEGREES CENTIGRADE FOR A FREQUENCY OF 4000 HERTZ

\begin{tabular}{|c|c|c|c|c|c|c|c|c|c|}
\hline (1) & $(2)$ & (3) & (4) & (5) & $(6)$ & 171 & $(8)$ & 191 & $(10)$ \\
\hline TEMP & FREQ & $\begin{array}{l}\text { REL } \\
\text { HUM }\end{array}$ & $\begin{array}{l}\text { ATTEN } \\
\text { COEF }\end{array}$ & $4 M$ & $\begin{array}{l}\text { ATTEN } \\
\text { COEF }\end{array}$ & $4 M$ & $\begin{array}{l}\text { ATTEN } \\
\text { DB PER }\end{array}$ & $\begin{array}{l}\text { ATTEN } \\
\text { DB PER }\end{array}$ & $\begin{array}{l}\text { DECAY } \\
\text { RATE }\end{array}$ \\
\hline $\begin{array}{l}\text { DEGR } \\
\text { CENT }\end{array}$ & HERTZ & $\begin{array}{l}\text { PER } \\
\text { CENT }\end{array}$ & $\begin{array}{l}\text { PER } \\
\text { METER }\end{array}$ & $\begin{array}{l}\text { PER } \\
\text { METER }\end{array}$ & 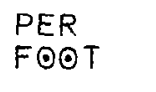 & $\begin{array}{l}\text { PER } \\
\text { FOOT }\end{array}$ & $\begin{array}{l}100 \\
\text { METER }\end{array}$ & $\begin{array}{l}1000 \\
\text { FEET }\end{array}$ & $\begin{array}{l}\text { DB PER } \\
\text { SECOND }\end{array}$ \\
\hline $\begin{array}{l}-10 \\
-10 \\
-10\end{array}$ & $\begin{array}{l}4000 \\
4000 \\
4000\end{array}$ & $\begin{array}{r}5.0 \\
10.0 \\
15.0\end{array}$ & $\begin{array}{r}.00170 \\
.00291 \\
.00411\end{array}$ & $\begin{array}{r}.00681 \\
.01164 \\
.01647\end{array}$ & $\begin{array}{l}.00051 \\
.00088 \\
.00125\end{array}$ & $\begin{array}{l}.00207 \\
.00354 \\
.00502\end{array}$ & $\begin{array}{r}.739 \\
1.264 \\
1.788\end{array}$ & $\begin{array}{l}2.254 \\
3.852 \\
5.450\end{array}$ & $\begin{array}{l}2.405 \\
4.111 \\
5.816\end{array}$ \\
\hline $\begin{array}{l}-10 \\
-10\end{array}$ & $\begin{array}{l}4000 \\
4000\end{array}$ & $\begin{array}{l}20.0 \\
25.0\end{array}$ & $\begin{array}{l}.00532 \\
.00653\end{array}$ & $\begin{array}{r}.02129 \\
.02612\end{array}$ & $\begin{array}{l}.00162 \\
.00199\end{array}$ & $\begin{array}{l}.00649 \\
.00796\end{array}$ & $\begin{array}{l}2.312 \\
2.836\end{array}$ & $\begin{array}{l}7.048 \\
8.647\end{array}$ & $\begin{array}{l}7.521 \\
9.227\end{array}$ \\
\hline-10 & 4000 & 30.0 & .00773 & .03095 & .00235 & .00943 & 3.361 & $10.245^{\prime}$ & 10.932 \\
\hline $\begin{array}{l}-10 \\
-10 \\
-10\end{array}$ & $\begin{array}{l}4000 \\
4000 \\
4000\end{array}$ & $\begin{array}{l}35.0 \\
40.0 \\
45.0\end{array}$ & $\begin{array}{l}.00896 \\
.01030 \\
.01158\end{array}$ & $\begin{array}{l}.03586 \\
.04121 \\
.04634\end{array}$ & $\begin{array}{l}.00273 \\
.00314 \\
.00353\end{array}$ & $\begin{array}{r}.01093 \\
.01256 \\
.01412\end{array}$ & $\begin{array}{l}3.894 \\
4.474 \\
5.031\end{array}$ & $\begin{array}{l}11.870 \\
13.638 \\
15.336\end{array}$ & $\begin{array}{l}12.666 \\
14.553 \\
16.365\end{array}$ \\
\hline $\begin{array}{l}-10 \\
-10\end{array}$ & $\begin{array}{l}4000 \\
4000\end{array}$ & $\begin{array}{l}46.0 \\
47.0\end{array}$ & $\begin{array}{r}.01183 \\
.01207\end{array}$ & $\begin{array}{r}.04733 \\
.04830\end{array}$ & $\begin{array}{l}.00360 \\
.00368\end{array}$ & $\begin{array}{r}.01442 \\
.01472\end{array}$ & $\begin{array}{l}5.139 \\
5.244\end{array}$ & $\begin{array}{l}15.666 \\
15.985\end{array}$ & $\begin{array}{l}16.717 \\
17.057\end{array}$ \\
\hline-10 & 4000 & $48 \cdot 0$ & .01229 & .04917 & .00374 & .01498 & 5.339 & $16 \cdot 274$ & $17 \cdot 365$ \\
\hline $\begin{array}{l}-10 \\
-10\end{array}$ & $\begin{array}{l}4000 \\
4000 \\
4000\end{array}$ & $\begin{array}{l}49.0 \\
50.0 \\
51.0\end{array}$ & $\begin{array}{r}.01251 \\
.01273 \\
.01290\end{array}$ & $\begin{array}{l}.05004 \\
.05092 \\
.05163\end{array}$ & $\begin{array}{r}.00381 \\
.00388 \\
.00393\end{array}$ & $\begin{array}{r}.01525 \\
.01552 \\
.01573\end{array}$ & $\begin{array}{l}5.433 \\
5.528 \\
5.606\end{array}$ & $\begin{array}{l}16.562 \\
16.851 \\
17.088\end{array}$ & $\begin{array}{l}17.673 \\
17.981 \\
18.234\end{array}$ \\
\hline $\begin{array}{l}-10 \\
-10 \\
-10\end{array}$ & $\begin{array}{l}4000 \\
4000 \\
4000\end{array}$ & $\begin{array}{l}52.0 \\
53.0 \\
54.0\end{array}$ & $\begin{array}{r}.01306 \\
.01322 \\
.01338\end{array}$ & $\begin{array}{l}.05227 \\
.05291 \\
.05354\end{array}$ & $\begin{array}{l}.00398 \\
.00403 \\
.00407\end{array}$ & $\begin{array}{r}.01593 \\
.01612 \\
.01631\end{array}$ & $\begin{array}{l}5.675 \\
5.745 \\
5.813\end{array}$ & $\begin{array}{l}17.299 \\
17.511 \\
17.718\end{array}$ & $\begin{array}{l}18.460 \\
18.685 \\
18.907\end{array}$ \\
\hline $\begin{array}{l}-10 \\
-10 \\
-10\end{array}$ & $\begin{array}{l}4000 \\
4000 \\
4000\end{array}$ & $\begin{array}{l}55.0 \\
60.0 \\
65.0\end{array}$ & $\begin{array}{r}.01350 \\
.01404 \\
.01434\end{array}$ & $\begin{array}{r}.05402 \\
.05616 \\
.05739\end{array}$ & $\begin{array}{r}.00411 \\
.00427 \\
.00437\end{array}$ & $\begin{array}{l}.01646 \\
.01711 \\
.01749\end{array}$ & $\begin{array}{l}5.865 \\
6.098 \\
6.232\end{array}$ & $\begin{array}{l}17.878 \\
18.588 \\
18.995\end{array}$ & $\begin{array}{l}19.077 \\
19.834 \\
20.270\end{array}$ \\
\hline $\begin{array}{l}-10 \\
-10 \\
-10\end{array}$ & $\begin{array}{l}4000 \\
4000 \\
4000\end{array}$ & $\begin{array}{l}70.0 \\
75.0 \\
80.0\end{array}$ & $\begin{array}{r}.01446 \\
.01447 \\
.01438\end{array}$ & $\begin{array}{l}.05786 \\
.05788 \\
.05753\end{array}$ & $\begin{array}{r}.00440 \\
.00441 \\
.00438\end{array}$ & $\begin{array}{l}.01763 \\
.01764 \\
.01753\end{array}$ & $\begin{array}{l}6.282 \\
6.285 \\
6.246\end{array}$ & $\begin{array}{l}19.150 \\
19.157 \\
19.040\end{array}$ & $\begin{array}{l}20.434 \\
20.442 \\
20.317\end{array}$ \\
\hline $\begin{array}{l}-10 \\
-10 \\
-10\end{array}$ & $\begin{array}{l}4000 \\
4000 \\
4000\end{array}$ & $\begin{array}{l}85.0 \\
90.0 \\
95.0\end{array}$ & $\begin{array}{r}.01420 \\
.01392 \\
.01357\end{array}$ & $\begin{array}{l}.05683 \\
.05571 \\
.05431\end{array}$ & $\begin{array}{l}.00433 \\
.00424 \\
.00413\end{array}$ & $\begin{array}{r}.01732 \\
.01698 \\
.01655\end{array}$ & $\begin{array}{l}6.171 \\
6.048 \\
5.897\end{array}$ & $\begin{array}{l}18.810 \\
18.437 \\
17.975\end{array}$ & $\begin{array}{l}20.072 \\
19.673 \\
19.180\end{array}$ \\
\hline & 4000 & 00.0 & .01315 & .05262 & .00400 & .01603 & 5.713 & 17.414 & 18.582 \\
\hline
\end{tabular}


TABULATION OF DATA FOR

ABSORPTION OF SOUND IN AIR VERSUS. PERCENT RELATIVE HUMIDITY AT-10 DEGREES CENTIGRADE FOR A FREQUENCY OF 5000 HERTZ

(1) (2) (3)

TEMP FREQ RE

DEGR

CENT HERTZ CENT

$-10.5000 \quad 5.0$

$-10 \quad 5000 \quad 10.0$

$-10 \quad 5000 \quad 15.0$

$\begin{array}{lll}-10 & 5000 \quad 20 \cdot 0\end{array}$

$\begin{array}{lll}-10 & 5000 \quad 25.0\end{array}$

$\begin{array}{lll}-10 & 5000 \quad 30.0\end{array}$

$\begin{array}{lll}-10 & 5000 \quad 35.0\end{array}$

$\begin{array}{lll}-10 & 5000 \quad 40.0\end{array}$

$\begin{array}{lll}-10 & 5000 \quad 45.0\end{array}$

$\begin{array}{lll}-10 & 5000 \quad 46.0\end{array}$

$-10 \quad 5000 \quad 47.0$

$\begin{array}{lll}-10 & 5000 \quad 48 \cdot 0\end{array}$

$-10 \quad 5000 \quad 49 \cdot 0$

$\begin{array}{lll}-10 & 5000 \quad 50 \cdot 0\end{array}$

$-10 \quad 5000 \quad 51.0$

$\begin{array}{lll}-10 & 5000 \quad 52.0\end{array}$

$-10 \quad 5000.53 .0$

$\begin{array}{lll}-10 & 5000 \quad 54 \cdot 0\end{array}$

$-10 \quad 5000 \quad 55.0$

$\begin{array}{lll}-10 & 5000 \quad 60.0\end{array}$

$-10 \quad 5000 \quad 65.0$

$\begin{array}{lll}-10 & 5000 \quad 70.0\end{array}$

$\begin{array}{lll}-10 & 5000 \quad 75.0\end{array}$

$\begin{array}{lll}-10 & 5000 \quad 80.0\end{array}$

$\begin{array}{lll}-10 & 5000 \quad 85.0\end{array}$

$\begin{array}{lll}-10 & 5000 \quad 90.0\end{array}$

$\begin{array}{lll}-10 & 5000 \quad 95.0\end{array}$

$-10 \quad 5000 \quad 100.0$
(4)

(5)

(6)

(7)

$\begin{array}{ll}\text { ATTEN } & 4 M \\ \text { COEF } & \\ \text { PER } & \text { PER }\end{array}$

PER

PER

METER

METER

FOOT

FOOT

- 00208

- 00338

.00469

- 00832

- 01355

. 01878

.00063

-00103

.00143

.00600

.02401

.00731

- 02924

.00182

.00861

.03447

-00222

.00262

.03970

.00302

- 00992

. 01125

.04502

.01269

. 05077

.00343

.00386

. 01298

.05193

.00395

. 01326

.01354

- 05306

.05419

.00404

.00412

- 01383

.01411

.01438

.05532

.00421

.05644

.05752

.00430

.00438

.01465

. 01492

.05860

.00446

.05968

.00454

.00462

.01518

.06075

.01542

.06170

.00470

. 01648

.01723

.06594

.06893

.00502

.00525

. 01778

.07112

01809

.01822

.07236

.0054

.00551

.00555

.02167

.02205

.02221

- 01825

.07303

.00556

.01819

.07276

.00554

.02226

. 01804

.07218

.00550

.02217

.02200

.01782

.0713 .1

.00543

.02173
(8)

191

(10)

$\begin{array}{lll}\text { ATTEN } & \text { ATTEN } & \text { DECAY } \\ \text { DB PER } & \text { DB PER } & \text { RATE } \\ 100 & \text { 1OOO } & \text { DB PER } \\ \text { METER } & \text { FEET } & \text { SECOND }\end{array}$

.904

1.472

2.756

4.486

6.217

$2 \cdot 941$

2.039

7.947

2.607

9.678

$8 \cdot 480$

3.175

3.742

11.408

10.327

4.310

13.139

$12 \cdot 173$

$4 \cdot 888$

14.899

14.020

5.512

16.802

$15 \cdot 898$

$5.638 \quad 17.187$

17.929

5.761

$17 \cdot 187$

18.340

$5 \cdot 884$

17.935

18.739

$6.007 \quad 18.309$

$19 \cdot 138$

6.128

18.680

19.537

6.24

19.037

19.933

6.363

19.394

20.314

6.480

19.752

20.695

6.596

$20 \cdot 107$

21.076

6.699

20.419

$21 \cdot 455$

7.159

$21 \cdot 823$

21.789

7.484

$22 \cdot 814$

23.287

$24 \cdot 344$

$7.722 \quad 23.538 \quad 25.117$

$7.857 \quad 23.948 \quad 25.555$

$7.914 \quad 24.124 \quad 25.743$

$7.929 \quad 24.169 \quad 25.790$

$\begin{array}{lll}7.900 & 24.081 & 25.697\end{array}$

$7.837 .23 .887 \quad 25.489$

$7.742 \quad 23.600 \quad 25.183$ 
TABULATION OF DATA FOR

ABSORPTION OF SOUND IN AIR VERSUS PERCENT RELATIVE HUMIDITY AT-10 DEGREES CENTIGRADE FOR A FREQUENCY OF 5940 HERTZ

\begin{tabular}{|c|c|c|c|c|c|c|c|c|c|}
\hline (1) & $(2)$ & (3) & (4) & (5) & $(6)$ & 171 & $(8)$ & $(9)$ & $(10)$ \\
\hline TEMP & FREQ & $\begin{array}{l}\text { REL } \\
\text { HUM }\end{array}$ & $\begin{array}{l}\text { ATTEN } \\
\text { COEF }\end{array}$ & $4 M$ & $\begin{array}{l}\text { ATTEN } \\
\text { COEF }\end{array}$ & $4 M$ & $\begin{array}{l}\text { ATTEN } \\
\text { DB PER }\end{array}$ & $\begin{array}{l}\text { ATTEN } \\
\text { DB PER }\end{array}$ & $\begin{array}{l}\text { DECAY } \\
\text { RATE }\end{array}$ \\
\hline $\begin{array}{l}\text { DEGR } \\
\text { CENT }\end{array}$ & HERTZ & $\begin{array}{l}\text { PER } \\
\text { CENT }\end{array}$ & $\begin{array}{l}\text { PER } \\
\text { METER }\end{array}$ & $\begin{array}{l}\text { PER } \\
\text { METER }\end{array}$ & $\begin{array}{l}\text { PER } \\
\text { F๑OT }\end{array}$ & $\begin{array}{l}\text { PER } \\
\text { FOOT }\end{array}$ & $\begin{array}{l}100 \\
\text { METER }\end{array}$ & $\begin{array}{l}1000 \\
\text { FEET }\end{array}$ & $\begin{array}{l}\text { DB PER } \\
\text { SECOND }\end{array}$ \\
\hline-10 & 5940 & 5.0 & .00248 & .00994 & .00075 & .00303 & 1.080 & 3.292 & 3.513 \\
\hline $\begin{array}{l}-10 \\
-10\end{array}$ & $\begin{array}{l}5940 \\
5940\end{array}$ & $\begin{array}{l}10.0 \\
15.0\end{array}$ & $\begin{array}{l}.00388 \\
.00527\end{array}$ & $\begin{array}{l}.01552 \\
.02109\end{array}$ & $\begin{array}{l}.00118 \\
.00160\end{array}$ & $\begin{array}{l}.00473 \\
.00643\end{array}$ & $\begin{array}{l}1.685 \\
2.290\end{array}$ & $\begin{array}{l}5.137 \\
6.982\end{array}$ & $\begin{array}{l}5.482 \\
7.450\end{array}$ \\
\hline $\begin{array}{l}-10 \\
-10 \\
-10\end{array}$ & $\begin{array}{l}5940 \\
5940 \\
5940\end{array}$ & $\begin{array}{l}20.0 \\
25.0 \\
30.0\end{array}$ & $\begin{array}{l}.00666 \\
.00806 \\
.00945\end{array}$ & $\begin{array}{l}.02667 \\
.03224 \\
.03781\end{array}$ & $\begin{array}{l}.00203 \\
.00245 \\
.00288\end{array}$ & $\begin{array}{l}.00812 \\
.00982 \\
.01152\end{array}$ & $\begin{array}{l}2.895 \\
3.501 \\
4.106\end{array}$ & $\begin{array}{r}8.826 \\
10.671 \\
12.516\end{array}$ & $\begin{array}{r}9.418 \\
11.387 \\
13.355\end{array}$ \\
\hline $\begin{array}{l}-10 \\
-10 \\
-10\end{array}$ & $\begin{array}{l}5940 \\
5940 \\
5940\end{array}$ & $\begin{array}{l}35.0 \\
40.0 \\
45.0\end{array}$ & $\begin{array}{r}.01084 \\
.01224 \\
.01366\end{array}$ & $\begin{array}{r}.04339 \\
.04896 \\
.05466\end{array}$ & $\begin{array}{l}.00330 \\
.00373 \\
.00416\end{array}$ & $\begin{array}{r}.01322 \\
.01492 \\
.01666\end{array}$ & $\begin{array}{l}4.711 \\
5.316 \\
5.935\end{array}$ & $\begin{array}{l}14 \cdot 360 \\
16 \cdot 205 \\
18.090\end{array}$ & $\begin{array}{l}15 \cdot 324 \\
17.292 \\
19.303\end{array}$ \\
\hline $\begin{array}{l}-10 \\
-10 \\
-10\end{array}$ & $\begin{array}{l}5940 \\
5940 \\
5940\end{array}$ & $\begin{array}{l}46.0 \\
47.0 \\
48.0\end{array}$ & $\begin{array}{r}.01395 \\
.01426 \\
.01457\end{array}$ & $\begin{array}{r}.05581 \\
.05704 \\
.05830\end{array}$ & $\begin{array}{l}.00425 \\
.00434 \\
.00444\end{array}$ & $\begin{array}{l}.01701 \\
.01738 \\
.01777\end{array}$ & $\begin{array}{l}6.059 \\
6.193 \\
6.330\end{array}$ & $\begin{array}{l}18.471 \\
18.878 \\
19.295\end{array}$ & $\begin{array}{l}19.710 \\
20.145 \\
20.589\end{array}$ \\
\hline $\begin{array}{l}-10 \\
-10 \\
-10\end{array}$ & $\begin{array}{l}5940 \\
5940 \\
5940\end{array}$ & $\begin{array}{l}49.0 \\
50.0 \\
51.0\end{array}$ & $\begin{array}{r}.01489 \\
.01520 \\
.01551\end{array}$ & $\begin{array}{r}.05956 \\
.06082 \\
.06207\end{array}$ & $\begin{array}{l}.00453 \\
.00463 \\
.00472\end{array}$ & $\begin{array}{r}.01815 \\
.01853 \\
.01891\end{array}$ & $\begin{array}{l}6.467 \\
6.603 \\
6.739\end{array}$ & $\begin{array}{l}19.711 \\
20.128 \\
20.542\end{array}$ & $\begin{array}{l}21.034 \\
21.478 \\
21.920\end{array}$ \\
\hline $\begin{array}{l}-10 \\
-10\end{array}$ & $\begin{array}{l}5940 \\
5940\end{array}$ & $\begin{array}{l}52.0 \\
53.0\end{array}$ & $\begin{array}{l}.01581 \\
.01612\end{array}$ & $\begin{array}{l}.06327 \\
.06448\end{array}$ & $\begin{array}{r}.00482 \\
.00491\end{array}$ & $\begin{array}{l}.01928 \\
.01965\end{array}$ & $\begin{array}{l}6.870 \\
7.001\end{array}$ & $\begin{array}{l}20.941 \\
21.339\end{array}$ & $\begin{array}{l}22.346 \\
22.771\end{array}$ \\
\hline-10 & 5940 & $54 \cdot 0$ & .01642 & .06568 & .00500 & .02002 & 7.132 & 21.738 & 23.196 \\
\hline $\begin{array}{l}-10 \\
-10 \\
-10\end{array}$ & $\begin{array}{l}5940 \\
5940 \\
5940\end{array}$ & $\begin{array}{l}55.0 \\
60.0 \\
65.0\end{array}$ & $\begin{array}{l}.01672 \\
.01816 \\
.01941\end{array}$ & $\begin{array}{r}.06689 \\
.07267 \\
.07765\end{array}$ & $\begin{array}{l}.00509 \\
.00553 \\
.00591\end{array}$ & $\begin{array}{l}.02038 \\
.02215 \\
.02367\end{array}$ & $\begin{array}{l}7.262 \\
7.890 \\
8.431\end{array}$ & $\begin{array}{l}22.137 \\
24.050 \\
25.700\end{array}$ & $\begin{array}{l}23.622 \\
25.663 \\
27.424\end{array}$ \\
\hline $\begin{array}{l}-10 \\
-10 \\
-10\end{array}$ & $\begin{array}{l}5940 \\
5940 \\
5940\end{array}$ & $\begin{array}{l}70.0 \\
75.0 \\
80.0\end{array}$ & $\begin{array}{l}.02030 \\
.02097 \\
.02145\end{array}$ & $\begin{array}{l}.08123 \\
.08389 \\
.08580\end{array}$ & $\begin{array}{l}.00618 \\
.00639 \\
.00653\end{array}$ & $\begin{array}{l}.02475 \\
.02557 \\
.02615\end{array}$ & $\begin{array}{l}8.819 \\
9.108 \\
9.316\end{array}$ & $\begin{array}{l}26.882 \\
27.763 \\
28.395\end{array}$ & $\begin{array}{l}28.686 \\
29.625 \\
30.300\end{array}$ \\
\hline $\begin{array}{l}-10 \\
-10\end{array}$ & $\begin{array}{l}5940 \\
5940\end{array}$ & $\begin{array}{l}85.0 \\
90.0\end{array}$ & $\begin{array}{l}.02171 \\
.02183\end{array}$ & $\begin{array}{l}.08684 \\
.08735\end{array}$ & $\begin{array}{l}.00661 \\
.00665\end{array}$ & $\begin{array}{l}.02646 \\
.02662\end{array}$ & $\begin{array}{l}9.428 \\
9.484\end{array}$ & $\begin{array}{l}28.739 \\
28.909\end{array}$ & $\begin{array}{l}30.667 \\
30.848\end{array}$ \\
\hline-10 & 5940 & 95.0 & .02186 & .08744 & .00666 & .02665 & 9.494 & 28.939 & 30.880 \\
\hline-10 & 59401 & 100.0 & .02179 & .08716 & 00664 & .02656 & 9.463 & 28.845 & 30.780 \\
\hline
\end{tabular}


TABULATIEN OF DATA FER

ABSENPTIEN OF SOUND IN AIR VERSUS PERCENT RELATIVE HUMIOITY AT-10 DEGREES CENT IGRADE FER A FREQUENCY OF 6300 HERTZ

\begin{tabular}{|c|c|c|c|c|c|c|c|c|c|}
\hline 11 & $(2)$ & (3) & $(4)$ & (5) & $(6)$ & 171 & $(8)$ & $(9)$ & $(10)$ \\
\hline $\begin{array}{l}\text { TEMP } \\
\text { BEGR } \\
\text { CEMT }\end{array}$ & HERTZ & $\begin{array}{l}\text { REL } \\
\text { HUN } \\
\text { PER } \\
\text { CENT }\end{array}$ & $\begin{array}{l}\text { ATTEN } \\
\text { COEF } \\
\text { PER } \\
\text { METER }\end{array}$ & $\begin{array}{l}\text { 4M } \\
\text { PER } \\
\text { METER }\end{array}$ & $\begin{array}{l}\text { ATTEN } \\
\text { COEF } \\
\text { PER } \\
\text { FOOT }\end{array}$ & $\begin{array}{l}4 M \\
\text { PER } \\
\text { FOOT }\end{array}$ & $\begin{array}{l}\text { ATTEN } \\
\text { DB PER } \\
100 \\
\text { METER }\end{array}$ & $\begin{array}{l}\text { ATTEN } \\
\text { DB PER } \\
1000 \\
\text { FEET }\end{array}$ & $\begin{array}{l}\text { OECAY } \\
\text { RATE } \\
\text { OB PER } \\
\text { SECOHD }\end{array}$ \\
\hline $\begin{array}{l}-10 \\
-10 \\
-10\end{array}$ & $\begin{array}{l}6300 \\
6300 \\
6300\end{array}$ & $\begin{array}{r}5.0 \\
10.0 \\
15.0\end{array}$ & $\begin{array}{l}.00265 \\
.00407 \\
.00550\end{array}$ & $\begin{array}{r}.01061 \\
.01631 \\
.02200\end{array}$ & $\begin{array}{l}.00080 \\
.00124 \\
.00167\end{array}$ & $\begin{array}{l}.00323 \\
.00497 \\
.00670\end{array}$ & $\begin{array}{l}1 \cdot 152 \\
1 \cdot 771 \\
2 \cdot 389\end{array}$ & $\begin{array}{l}3 \cdot 513 \\
5.398 \\
7.283\end{array}$ & $\begin{array}{l}3.749 \\
5.760 \\
7.772\end{array}$ \\
\hline $\begin{array}{l}-10 \\
-10 \\
-10\end{array}$ & $\begin{array}{l}6300 \\
6300 \\
6300\end{array}$ & $\begin{array}{l}20.0 \\
25.0 \\
30.0\end{array}$ & $\begin{array}{l}.00692 \\
.00835 \\
.00977\end{array}$ & $\begin{array}{r}.02770 \\
.03340 \\
.03909\end{array}$ & $\begin{array}{l}.00211 \\
.00254 \\
.00297\end{array}$ & $\begin{array}{l}.0 \\
.0 \\
.0\end{array}$ & $\begin{array}{l}28 \\
26 \\
45\end{array}$ & $\begin{array}{r}9 \\
11 \\
12\end{array}$ & $\begin{array}{r}9.783 \\
11.795 \\
13.806\end{array}$ \\
\hline $\begin{array}{l}-10 \\
-10 \\
-10\end{array}$ & $\begin{array}{l}6300 \\
6300 \\
6300\end{array}$ & $\begin{array}{l}35.0 \\
40.0 \\
45.0\end{array}$ & $\begin{array}{r}.01119 \\
.01262 \\
.01406\end{array}$ & $\begin{array}{r}.04479 \\
.05048 \\
.05625\end{array}$ & $\begin{array}{l}.00341 \\
.00384 \\
.00428\end{array}$ & $\begin{array}{l}.01365 \\
.01538 \\
.01714\end{array}$ & $\begin{array}{l}863 \\
481 \\
107\end{array}$ & $\begin{array}{l}14 \cdot 824 \\
16.709 \\
18.616\end{array}$ & $\begin{array}{l}15.818 \\
17.830 \\
19.864\end{array}$ \\
\hline $\begin{array}{l}-10 \\
-10 \\
-10\end{array}$ & $\begin{array}{l}6300 \\
6300 \\
6300\end{array}$ & $\begin{array}{l}46.0 \\
47.0 \\
48.0\end{array}$ & $\begin{array}{r}.01435 \\
.01465 \\
.01494\end{array}$ & $\begin{array}{r}.05742 \\
.05860 \\
.05978\end{array}$ & $\begin{array}{r}.00437 \\
.00446 \\
.00455\end{array}$ & $\begin{array}{r}.01750 \\
.01786 \\
.01822\end{array}$ & & $\begin{array}{l}19.005 \\
19.394 \\
19.783\end{array}$ & $\begin{array}{l}20.280 \\
20.695 \\
21.110\end{array}$ \\
\hline $\begin{array}{l}-10 \\
-10 \\
-10\end{array}$ & $\begin{array}{l}6300 \\
6300 \\
6300\end{array}$ & $\begin{array}{l}49.0 \\
50.0 \\
51.0\end{array}$ & $\begin{array}{l}.01 \\
.01 \\
.01\end{array}$ & $\begin{array}{l}.06106 \\
.06235 \\
.06363\end{array}$ & $\begin{array}{l}.00465 \\
.00475 \\
.00484\end{array}$ & $\begin{array}{l}.01 \\
.01 \\
.01\end{array}$ & & $\begin{array}{l}09 \\
35 \\
60\end{array}$ & $\begin{array}{l}65 \\
19 \\
73\end{array}$ \\
\hline $\begin{array}{l}-10 \\
-10 \\
-10\end{array}$ & $\begin{array}{l}6300 \\
6300 \\
6300\end{array}$ & $\begin{array}{l}52.0 \\
53.0 \\
54.0\end{array}$ & $\begin{array}{r}.01623 \\
.01655 \\
.01685\end{array}$ & $\begin{array}{r}.06492 \\
.06620 \\
.06743\end{array}$ & $\begin{array}{l}.00494 \\
.00504 \\
.00513\end{array}$ & $\begin{array}{l}.01978 \\
.02027 \\
.02055\end{array}$ & & $\begin{array}{l}486 \\
908 \\
315\end{array}$ & $\begin{array}{l}22 \cdot 927 \\
23.378 \\
23.812\end{array}$ \\
\hline $\begin{array}{l}-10 \\
-10 \\
-10\end{array}$ & $\begin{array}{l}6300 \\
6300 \\
6300\end{array}$ & $\begin{array}{l}55.0 \\
60.0 \\
65.0\end{array}$ & $\begin{array}{l}.01716 \\
.01867 \\
.02004\end{array}$ & $\begin{array}{l}.06 \\
.07 \\
.08\end{array}$ & $\begin{array}{l}.00 \\
.00 \\
.00\end{array}$ & $\begin{array}{l}.02092 \\
.02276 \\
.02444\end{array}$ & & $\begin{array}{l}23 \\
16 \\
36\end{array}$ & $\begin{array}{l}24.247 \\
26.374 \\
28.316\end{array}$ \\
\hline $\begin{array}{l}-10 \\
-10 \\
-10\end{array}$ & $\begin{array}{l}6300 \\
6300 \\
6300\end{array}$ & $\begin{array}{l}70.0 \\
75.0 \\
80.0\end{array}$ & $\begin{array}{l}.02113 \\
.02194 \\
.02255\end{array}$ & $\begin{array}{l}.08 \\
.08 \\
.09\end{array}$ & $\begin{array}{l}.00 \\
.00 \\
.00\end{array}$ & $\begin{array}{r}.02577 \\
.02675 \\
.02749\end{array}$ & $\begin{array}{l}0 \\
9 \\
4\end{array}$ & $\begin{array}{l}27 \\
29 \\
29\end{array}$ & $\begin{array}{l}860 \\
996 \\
856\end{array}$ \\
\hline $\begin{array}{l}-10 \\
-10 \\
-10\end{array}$ & $\begin{array}{l}6300 \\
6300 \\
6300\end{array}$ & $\begin{array}{l}85.0 \\
90.0 \\
95.0\end{array}$ & $\begin{array}{l}.02294 \\
.02315 \\
.02326\end{array}$ & $\begin{array}{r}.09178 \\
.09261 \\
.09304\end{array}$ & $\begin{array}{r}.00699 \\
.00705 \\
.00709\end{array}$ & $\begin{array}{r}.02797 \\
.02822 \\
.02836\end{array}$ & $\begin{array}{r}9.966 \\
10.055 \\
10.102\end{array}$ & $\begin{array}{l}30.376 \\
30.649 \\
30.792\end{array}$ & $\begin{array}{l}32.414 \\
32.705 \\
32.858\end{array}$ \\
\hline & & .0 & .02324 & .09297 & .00708 & .02833 & 10.094 & 30.768 & 32.832 \\
\hline
\end{tabular}


TABULATION OF DATA FOR

ABSORPTION OF SOUND IN AIR VERSUS PERCENT RELATIVE HUMIDITY AT-IO DEGREES CENTIGRADE FOR A FREQUENCY OF 8000 HERTZ

\begin{tabular}{|c|c|c|c|c|c|c|c|c|c|}
\hline (1) & $(2)$ & (3) & (4) & (5) & $(6)$ & $(7)$ & $(8)$ & $(9)$ & $(10)$ \\
\hline $\begin{array}{l}\text { TEMP } \\
\text { DEGR } \\
\text { CENT }\end{array}$ & HERTZ & $\begin{array}{l}\text { REL } \\
\text { HUM } \\
\text { PER } \\
\text { CENT }\end{array}$ & $\begin{array}{l}\text { ATTEN } \\
\text { COEF } \\
\text { PER } \\
\text { METER }\end{array}$ & $\begin{array}{l}4 M \\
\text { PER } \\
\text { METER }\end{array}$ & $\begin{array}{l}\text { ATTEN } \\
\text { COEF } \\
\text { PER } \\
\text { FOOT }\end{array}$ & $\begin{array}{l}4 M \\
\text { PER } \\
F \odot O T\end{array}$ & $\begin{array}{l}\text { ATTEN } \\
\text { DB PER } \\
100 \\
\text { METER }\end{array}$ & $\begin{array}{l}\text { ATTEN } \\
\text { DB PER } \\
1000 \\
\text { FEET }\end{array}$ & $\begin{array}{l}\text { DECAY } \\
\text { RATE } \\
\text { DB PER } \\
\text { SECOND }\end{array}$ \\
\hline $\begin{array}{l}-10 \\
-10 \\
-10\end{array}$ & $\begin{array}{l}8000 \\
8000 \\
8000\end{array}$ & $\begin{array}{r}5.0 \\
10.0 \\
15.0\end{array}$ & $\begin{array}{r}.00372 \\
.00545 \\
.00719\end{array}$ & $\begin{array}{r}.01488 \\
.02183 \\
.02878\end{array}$ & $\begin{array}{l}.00113 \\
.00166 \\
.00219\end{array}$ & $\begin{array}{l}.00453 \\
.00665 \\
.00877\end{array}$ & $\begin{array}{l}1.616 \\
2.371 \\
3.125\end{array}$ & $\begin{array}{l}4.926 \\
7.227 \\
9.527\end{array}$ & $\begin{array}{r}5.257 \\
7.711 \\
10.166\end{array}$ \\
\hline $\begin{array}{l}-10 \\
-10 \\
-10\end{array}$ & $\begin{array}{l}8000 \\
8000 \\
8000\end{array}$ & $\begin{array}{l}20.0 \\
25.0 \\
30.0\end{array}$ & $\begin{array}{r}.00893 \\
.01067 \\
.01241\end{array}$ & $\begin{array}{r}.03573 \\
.04269 \\
.04964\end{array}$ & $\begin{array}{l}.00272 \\
.00325 \\
.00378\end{array}$ & $\begin{array}{l}.01089 \\
.01301 \\
.01513\end{array}$ & $\begin{array}{l}3.880 \\
4.635 \\
5.389\end{array}$ & $\begin{array}{l}11.827 \\
14 \cdot 128 \\
16 \cdot 428\end{array}$ & $\begin{array}{l}12.621 \\
15.075 \\
17.530\end{array}$ \\
\hline $\begin{array}{l}-10 \\
-10 \\
-10\end{array}$ & $\begin{array}{l}8000 \\
8000 \\
8000\end{array}$ & $\begin{array}{l}35.0 \\
40.0 \\
45.0\end{array}$ & $\begin{array}{r}.01414 \\
.01588 \\
.01762\end{array}$ & $\begin{array}{r}.05659 \\
.06354 \\
.07049\end{array}$ & $\begin{array}{r}.00431 \\
.00484 \\
.00537\end{array}$ & $\begin{array}{r}.01724 \\
.01936 \\
.02148\end{array}$ & $\begin{array}{l}6.144 \\
6.899 \\
7.654\end{array}$ & $\begin{array}{l}18 \cdot 728 \\
21 \cdot 029 \\
23.330\end{array}$ & $\begin{array}{l}19.985 \\
22.439 \\
24.895\end{array}$ \\
\hline $\begin{array}{l}-10 \\
-10 \\
-10\end{array}$ & $\begin{array}{l}8000 \\
8000 \\
8000\end{array}$ & $\begin{array}{l}46.0 \\
47.0 \\
48.0\end{array}$ & $\begin{array}{r}.01798 \\
.01834 \\
.01870\end{array}$ & $\begin{array}{r}.07193 \\
.07336 \\
.07480\end{array}$ & $\begin{array}{l}.00548 \\
.00559 \\
.00569\end{array}$ & $\begin{array}{l}.02192 \\
.02236 \\
.02279\end{array}$ & $\begin{array}{l}7.809 \\
7.965 \\
8.121\end{array}$ & $\begin{array}{l}23.805 \\
24.280 \\
24.754\end{array}$ & $\begin{array}{l}25.401 \\
25.908 \\
26.415\end{array}$ \\
\hline $\begin{array}{l}-10 \\
-10 \\
-10\end{array}$ & $\begin{array}{l}8000 \\
8000 \\
8000\end{array}$ & $\begin{array}{l}49.0 \\
50.0 \\
51.0\end{array}$ & $\begin{array}{r}.01905 \\
.01941 \\
.01981\end{array}$ & $\begin{array}{r}.07623 \\
.07767 \\
.07924\end{array}$ & $\begin{array}{l}.00580 \\
.00591 \\
.00603\end{array}$ & $\begin{array}{r}.02323 \\
.02357 \\
.02415\end{array}$ & $\begin{array}{l}8.277 \\
8.433 \\
8.604\end{array}$ & $\begin{array}{l}25.229 \\
25.706 \\
26.226\end{array}$ & $\begin{array}{l}26.922 \\
27.431 \\
27.985\end{array}$ \\
\hline $\begin{array}{l}-10 \\
-10 \\
-10\end{array}$ & $\begin{array}{l}8000 \\
8000 \\
8000\end{array}$ & $\begin{array}{l}52.0 \\
53.0 \\
54.0\end{array}$ & $\begin{array}{r}.02020 \\
.02059 \\
.02098\end{array}$ & $\begin{array}{r}.08081 \\
.08238 \\
.08395\end{array}$ & $\begin{array}{l}.00615 \\
.00627 \\
.00639\end{array}$ & $\begin{array}{r}.02463 \\
.02511 \\
.02559\end{array}$ & $\begin{array}{l}8.774 \\
8.945 \\
9.115\end{array}$ & $\begin{array}{l}26.745 \\
27.265 \\
27.784\end{array}$ & $\begin{array}{l}28.539 \\
29.094 \\
29.648\end{array}$ \\
\hline $\begin{array}{l}-10 \\
-10\end{array}$ & $\begin{array}{l}8000 \\
8000\end{array}$ & $\begin{array}{l}55.0 \\
60.0\end{array}$ & $\begin{array}{l}.02138 \\
.02325\end{array}$ & $\begin{array}{l}.08552 \\
.09303\end{array}$ & $\begin{array}{l}.00651 \\
.00708\end{array}$ & $\begin{array}{l}.02606 \\
.02835\end{array}$ & $\begin{array}{r}9.285 \\
10.100\end{array}$ & $\begin{array}{l}28.302 \\
30.787\end{array}$ & $\begin{array}{l}30.201 \\
32.852\end{array}$ \\
\hline-10 & 8000 & 65.0 & .02504 & .10019 & .00763 & .03053 & 10.878 & 33.158 & 35.382 \\
\hline $\begin{array}{l}-10 \\
-10\end{array}$ & $\begin{array}{l}8000 \\
8000\end{array}$ & $\begin{array}{l}70.0 \\
75.0\end{array}$ & $\begin{array}{r}.02661 \\
.02775\end{array}$ & $\begin{array}{r}.10644 \\
.11102\end{array}$ & $\begin{array}{r}.00811 \\
.00846\end{array}$ & $\begin{array}{l}.03244 \\
.03384\end{array}$ & $\begin{array}{l}11.557 \\
12.054\end{array}$ & $\begin{array}{l}35 \cdot 228 \\
36 \cdot 743\end{array}$ & $\begin{array}{l}37.591 \\
39.208\end{array}$ \\
\hline 10 & 8000 & 80.0 & .02862 & .11449 & .00872 & .03489 & 12.430 & 37.889 & 40.431 \\
\hline $\begin{array}{l}-10 \\
-10 \\
-10\end{array}$ & $\begin{array}{l}8000 \\
8000 \\
8000\end{array}$ & $\begin{array}{l}85.0 \\
90.0 \\
95.0\end{array}$ & $\begin{array}{l}.02929 \\
.02967 \\
.02987\end{array}$ & $\begin{array}{l}.11716 \\
.11871 \\
.11949\end{array}$ & $\begin{array}{l}.00892 \\
.00904 \\
.00910\end{array}$ & $\begin{array}{l}.03571 \\
.03618 \\
.03642\end{array}$ & $\begin{array}{l}12.720 \\
12.889 \\
12.974\end{array}$ & $\begin{array}{l}38.773 \\
39.288 \\
39.546\end{array}$ & $\begin{array}{l}41.374 \\
41.924 \\
42.199\end{array}$ \\
\hline-10 & 8000 & 100.0 & .02998 & .11993 & .00913 & .03655 & 13.021 & 39.690 & 42.352 \\
\hline
\end{tabular}


TABULATION OF DATA FOR

ABSORPTION OF SOUND IN AIR VERSUS PERCENT RELATIVE HUMIDITY AT-10 DEGREES CENTIGRADE FOR A FREQUENCY OF10000 HERTZ

(1) (2) (3)

TEMP FREQ

DEGR

CENT HERTZ CENT

$-1010000$

$-1010000$

$-1010000$

$-1010000$

$-1010000$

$-1010000$

$-10 \quad 10000$

$-1010000$

$-1010000$

$-1010000$

$-1010000$

$-1010000$

$-1010000$

$-1010000$

$-10 \quad 10000$

$-10 \quad 10000$

$-1010000$

$-1010000$

$-1010000$

$-10 \quad 10000$

$-1010000$

$-1010000$

$-1010000$

$-1010000$

$-10 \quad 10000$

$-10 \quad 10000$

$-1010000$

$-10 \quad 10000 \quad 100 \cdot 0$

20.0

25.0

30.0

35.0

40.0

45.0

46.0

47.0

48.0

49.0

50.0

51.0

52.0

53.0

54.0

55.0

60.0

65.0

$70 \cdot 0$

75.0

80.0

85.0

90.0

95.0
(4)

(5)

(6)

ATTEN $4 M$

COEF

PER

PER

METER

PER

METER

$F \odot \odot T$

.00527

.02108

10.0

15.0

.00744

.00961

.02977

.03846

.01178

.01396

.04715

.05584

.01613

.06453

.01830

.07322

.02047

.02265

.08190

.09060

.02309

.02354

.02399

.09239

.09418

.09598

.02444

.02489

.09777

.09957

.02538

. 10154

.02587

.02636

- 10350

.02685

- 10546

.10742

.02734

. 02969

.03193

- 10938

- 11876

. 12772

.03388

.13554

.03531

- 14126

. 14559

.03723 .14893

.03771 .0087

.03796

.15185

.03809

. 15239
.01134

.01149

.01157

$.00160 \quad .00642$

$.00226 \quad .00907$

$.00293 \quad .01172$

.00359

.00425

.01437

.01702

.01966

.00557

.02231

.02496

.02761

.02816

.02870

.02925

.02980

.03035

.03094

.03154

.03214

.03274

.03334

.03620

.03892

.04131

.04305

.04437

.04539

.04598

.04628

.01161

.04644
(7)

(8)

$(9)$

$(10)$

$\begin{array}{lll}\text { ATTEN } & \text { ATTEN } & \text { DECAY } \\ \text { DB PER } & \text { DB PER } & \text { RATE } \\ 100^{\circ} & 1000 & \text { DB PER } \\ \text { METER } & \text { FEET } & \text { SECOND }\end{array}$

2.289

6.979

$7 \cdot 447$

3.233

4.176

9.854

12.729

10.515

13.583

5.119

6.063

15.605

16.652

7.006

18.480

19.720

22.788

7.949

8.893

24.231

25.857

9.836

27.107

29.983

28.925

31.994

10.031

30.577

31.170

32.628

10.226

31.764

33.261

33.895

10.616

10.811

$32 \cdot 358$

32.954

34.528

11.024

33.603

35.165

35.857

11.237

34.253

36.550

11.450

34.902

37.243

11.663

35.551

37.936

11.876

12.895

36.199

38.627

13.867

39.305

41.941

42.268

45.103

14.716

44.855

47.864

15.337

46.750

49.886

15.807

48.182

51.415

16.170

$49 \cdot 288$

52.594

16.381

49.931

53.280

16.487

50.253

53.624

16.546

$50.433 \quad 53.816$ 
TABULATION OF DATA FOR

ABSORPTION OF SOUND IN AIR VERSUS PERCENT RELATIVE HUMIDITY AT-10 DEGREES CENTIGRADE FOR A FREQUENCY OFI2500 HERTZ

\begin{tabular}{|c|c|c|c|c|c|c|c|c|c|}
\hline (1) & $(2)$ & (3) & $(4)$ & (5) & $(6)$ & $(7)$ & $(8)$ & $(9)$ & $(10)$ \\
\hline $\begin{array}{l}\text { TEMP } \\
\text { DEGR } \\
\text { CENT }\end{array}$ & HERTZ & $\begin{array}{l}\text { REL } \\
\text { HUM } \\
\text { PER } \\
\text { CENT }\end{array}$ & $\begin{array}{l}\text { ATTEN } \\
\text { COEF } \\
\text { PER } \\
\text { METER }\end{array}$ & $\begin{array}{l}4 M \\
\text { PER } \\
\text { METER }\end{array}$ & $\begin{array}{l}\text { ATTEN } \\
\text { COEF } \\
\text { PER } \\
\text { FOOT }\end{array}$ & $\begin{array}{l}4 M \\
\text { PER } \\
\text { FOOT }\end{array}$ & $\begin{array}{l}\text { ATTEN } \\
\text { DB PER } \\
100 \\
\text { METER }\end{array}$ & $\begin{array}{l}\text { ATTEN } \\
\text { DB PER } \\
1000 \\
\text { FEET }\end{array}$ & $\begin{array}{l}\text { DECAY } \\
\text { RATE } \\
\text { DB PER } \\
\text { SECOND }\end{array}$ \\
\hline $\begin{array}{l}-10 \\
-10 \\
-10\end{array}$ & $\begin{array}{l}12500 \\
12500 \\
12500\end{array}$ & $\begin{array}{r}5.0 \\
10.0 \\
15.0\end{array}$ & $\begin{array}{r}.00755 \\
.01027 \\
.01298\end{array}$ & $\begin{array}{l}.03023 \\
.04109 \\
.05195\end{array}$ & $\begin{array}{l}.00230 \\
.00313 \\
.00395\end{array}$ & $\begin{array}{l}.00921 \\
.01252 \\
.01583\end{array}$ & $\begin{array}{l}3.282 \\
4.462 \\
5.641\end{array}$ & $\begin{array}{l}10.006 \\
13.600 \\
17.195\end{array}$ & $\begin{array}{l}10.677 \\
14.513 \\
18.348\end{array}$ \\
\hline $\begin{array}{l}-10 \\
-10 \\
-10\end{array}$ & $\begin{array}{l}12500 \\
12500 \\
12500\end{array}$ & $\begin{array}{l}20.0 \\
25.0 \\
30.0\end{array}$ & $\begin{array}{r}.01570 \\
.01842 \\
.02113\end{array}$ & $\begin{array}{r}.06281 \\
.07368 \\
.08454\end{array}$ & $\begin{array}{l}.00478 \\
.00561 \\
.00644\end{array}$ & $\begin{array}{r}.01914 \\
.02245 \\
.02576\end{array}$ & $\begin{array}{l}6.820 \\
7.999 \\
9.179\end{array}$ & $\begin{array}{l}20.789 \\
24.383 \\
27.978\end{array}$ & $\begin{array}{l}22.184 \\
26.019 \\
29.854\end{array}$ \\
\hline $\begin{array}{l}-10 \\
-10 \\
-10\end{array}$ & $\begin{array}{l}12500 \\
12500 \\
12500\end{array}$ & $\begin{array}{l}35.0 \\
40.0 \\
45.0\end{array}$ & $\begin{array}{r}.02385 \\
.02656 \\
.02928\end{array}$ & $\begin{array}{r}.09540 \\
.10626 \\
.11712\end{array}$ & $\begin{array}{l}.00726 \\
.00809 \\
.00892\end{array}$ & $\begin{array}{l}.02907 \\
.03238 \\
.03570\end{array}$ & $\begin{array}{l}10.358 \\
11.537 \\
12.717\end{array}$ & $\begin{array}{l}31.572 \\
35.166 \\
38.762\end{array}$ & $\begin{array}{l}33.690 \\
37.525 \\
41.362\end{array}$ \\
\hline $\begin{array}{l}-10 \\
-10 \\
-10\end{array}$ & $\begin{array}{l}12500 \\
12500 \\
12500\end{array}$ & $\begin{array}{l}46.0 \\
47.0 \\
48.0\end{array}$ & $\begin{array}{r}.02984 \\
.03040 \\
.03096\end{array}$ & $\begin{array}{r}.11936 \\
.12161 \\
.12385\end{array}$ & $\begin{array}{l}.00909 \\
.00926 \\
.00943\end{array}$ & $\begin{array}{l}.03638 \\
.03706 \\
.03775\end{array}$ & $\begin{array}{l}12.960 \\
13.203 \\
13.447\end{array}$ & $\begin{array}{l}39.504 \\
40.246 \\
40.988\end{array}$ & $\begin{array}{l}42.154 \\
42.945 \\
43.737\end{array}$ \\
\hline $\begin{array}{l}-10 \\
-10\end{array}$ & $\begin{array}{l}12500 \\
12500\end{array}$ & $\begin{array}{l}49.0 \\
50.0\end{array}$ & $\begin{array}{r}.03152 \\
.03208\end{array}$ & $\begin{array}{r}12609 \\
.12834\end{array}$ & $\begin{array}{l}.00960 \\
.00978\end{array}$ & $\begin{array}{r}.03843 \\
.03912\end{array}$ & $\begin{array}{l}13.690 \\
13.935\end{array}$ & $\begin{array}{l}41.730 \\
42.475\end{array}$ & $\begin{array}{l}44.529 \\
45.325\end{array}$ \\
\hline-10 & 12500 & 51.0 & .03270 & .13080 & .00996 & .03986 & 14.201 & 43.287 & 46.191 \\
\hline $\begin{array}{l}-10 \\
-10 \\
-10\end{array}$ & $\begin{array}{l}12500 \\
12500 \\
12500\end{array}$ & $\begin{array}{l}52.0 \\
53.0 \\
54.0\end{array}$ & $\begin{array}{r}.03331 \\
.03392 \\
.03453\end{array}$ & $\begin{array}{r}.13325 \\
.13570 \\
.13815\end{array}$ & $\begin{array}{r}.01015 \\
.01034 \\
.01052\end{array}$ & $\begin{array}{l}.04061 \\
.04136 \\
.04211\end{array}$ & $\begin{array}{l}14.468 \\
14 \cdot 734 \\
15.000\end{array}$ & $\begin{array}{l}44.099 \\
44.910 \\
45.722\end{array}$ & $\begin{array}{l}47.057 \\
47.923 \\
48.789\end{array}$ \\
\hline $\begin{array}{l}-10 \\
-10 \\
-10\end{array}$ & $\begin{array}{l}12500 \\
12500 \\
12500\end{array}$ & $\begin{array}{l}55.0 \\
60.0 \\
65.0\end{array}$ & $\begin{array}{l}.03515 \\
.03808 \\
.04088\end{array}$ & $\begin{array}{r}.14060 \\
.15233 \\
.16352\end{array}$ & $\begin{array}{l}.01071 \\
.01160 \\
.01246\end{array}$ & $\begin{array}{l}.04285 \\
.04643 \\
.04984\end{array}$ & $\begin{array}{l}15.266 \\
16.539 \\
17.755\end{array}$ & $\begin{array}{l}46.531 \\
50.414 \\
54.118\end{array}$ & $\begin{array}{l}49.653 \\
53.795 \\
57.748\end{array}$ \\
\hline $\begin{array}{l}-10 \\
-10 \\
-10\end{array}$ & $\begin{array}{l}12500 \\
12500 \\
12500\end{array}$ & $\begin{array}{l}70.0 \\
75.0 \\
80.0\end{array}$ & $\begin{array}{l}.04332 \\
.04511 \\
.04646\end{array}$ & $\begin{array}{r}.17330 \\
.18045 \\
.18586\end{array}$ & $\begin{array}{r}.01320 \\
.01375 \\
.01416\end{array}$ & $\begin{array}{l}.05282 \\
.05500 \\
.05665\end{array}$ & $\begin{array}{l}18.816 \\
19.593 \\
20.180\end{array}$ & $\begin{array}{l}57.352 \\
59.720 \\
61.511\end{array}$ & $\begin{array}{l}61.199 \\
63.726 \\
65.637\end{array}$ \\
\hline $\begin{array}{l}-10 \\
-10 \\
-10\end{array}$ & $\begin{array}{l}12500 \\
12500 \\
12500\end{array}$ & $\begin{array}{l}85.0 \\
90.0 \\
95.0\end{array}$ & $\begin{array}{r}.04751 \\
.04811 \\
.04842\end{array}$ & $\begin{array}{r}.19004 \\
.19247 \\
.19368\end{array}$ & $\begin{array}{l}.01448 \\
.01466 \\
.01475\end{array}$ & $\begin{array}{l}.05792 \\
.05866 \\
.05903\end{array}$ & $\begin{array}{l}20.633 \\
20.897 \\
21.029\end{array}$ & $\begin{array}{l}62.892 \\
63.697 \\
64.099\end{array}$ & $\begin{array}{l}67.111 \\
67.969 \\
68.399\end{array}$ \\
\hline 10 & 12 & .0 & .04859 & .19436 & .01481 & .05924 & $21 \cdot 103$ & $64 \cdot 324$ & 68.639 \\
\hline
\end{tabular}


TABULATION OF DATA FOR

ABSORPTION OF SOUND IN AIR VERSUS PERCENT RELATIVE HUMIDITY AT -5 DEGREES CENTIGRADE FOR A FREQUENCY OF 125 HERTZ

\begin{tabular}{|c|c|c|c|c|c|c|c|c|c|}
\hline 1 & $(2)$ & (3) & (4) & (5) & $(6)$ & $(7)$ & $(8)$ & $(9)$ & 1101 \\
\hline$E M P$ & FREQ & $\begin{array}{l}\text { REL } \\
\text { HUM }\end{array}$ & $\begin{array}{l}\text { ATTEN } \\
\text { COEF }\end{array}$ & $4 M$ & $\begin{array}{l}\text { ATTEN } \\
\text { COEF }\end{array}$ & $4 M$ & $\begin{array}{l}\text { ATTEN } \\
\text { DB PER }\end{array}$ & $\begin{array}{l}\text { ATTEN } \\
\text { DB PER }\end{array}$ & $\begin{array}{l}\text { DECAY } \\
\text { RATE }\end{array}$ \\
\hline $\begin{array}{l}E G R \\
\text { ENT }\end{array}$ & HERTZ & $\begin{array}{l}\text { PER } \\
\text { CENT }\end{array}$ & $\begin{array}{l}\text { PER } \\
\text { METER }\end{array}$ & $\begin{array}{l}\text { PER } \\
\text { METER }\end{array}$ & $\begin{array}{l}\text { PER } \\
\text { FOOT }\end{array}$ & $\begin{array}{l}\text { PER } \\
\text { FOOT }\end{array}$ & $\begin{array}{l}100 \\
\text { METER }\end{array}$ & $\begin{array}{l}1000 \\
\text { FEET }\end{array}$ & $\begin{array}{l}\text { DB PER } \\
\text { SECOND }\end{array}$ \\
\hline $\begin{array}{l}-5 \\
-5 \\
-5\end{array}$ & $\begin{array}{l}125 \\
125 \\
125\end{array}$ & $\begin{array}{l}5.0 \\
10.0\end{array}$ & $\begin{array}{r}.00047 \\
.00035\end{array}$ & .00191 & .00014 & $\begin{array}{r}.00058 \\
.00043\end{array}$ & $\begin{array}{r}.208 \\
.153\end{array}$ & $\begin{array}{r}.634 \\
.467\end{array}$ & $\begin{array}{r}.683 \\
.503\end{array}$ \\
\hline-5 & 125 & 15.0 & .00022 & .00090 & .00006 & .00027 & .098 & .300 & .324 \\
\hline $\begin{array}{l}-5 \\
-5 \\
-5\end{array}$ & $\begin{array}{l}125 \\
125 \\
125\end{array}$ & $\begin{array}{l}20.0 \\
25.0 \\
30.0\end{array}$ & $\begin{array}{l}.00016 \\
.00013 \\
.00011\end{array}$ & $\begin{array}{l}.00066 \\
.00052 \\
.00044\end{array}$ & $\begin{array}{l}.00005 \\
.00003 \\
.00003\end{array}$ & $\begin{array}{l}.00020 \\
.00015 \\
.00013\end{array}$ & $\begin{array}{l}.071 \\
.056 \\
.048\end{array}$ & $\begin{array}{r}.219 \\
.173 \\
.148\end{array}$ & $\begin{array}{l}.236 \\
.186 \\
.160\end{array}$ \\
\hline $\begin{array}{l}-5 \\
-5 \\
-5\end{array}$ & $\begin{array}{l}125 \\
125 \\
125\end{array}$ & $\begin{array}{l}35.0 \\
40.0 \\
45.0\end{array}$ & $\begin{array}{l}.00010 \\
.00009 \\
.00009\end{array}$ & $\begin{array}{l}.00040 \\
.00038 \\
.00037\end{array}$ & $\begin{array}{l}.00003 \\
.00002 \\
.00002\end{array}$ & $\begin{array}{l}.00012 \\
.00011 \\
.00011\end{array}$ & $\begin{array}{l}.044 \\
.042 \\
.040\end{array}$ & $\begin{array}{r}.135 \\
.128 \\
.123\end{array}$ & $\begin{array}{r}.146 \\
.138 \\
.132\end{array}$ \\
\hline $\begin{array}{l}-5 \\
-5 \\
-5\end{array}$ & $\begin{array}{l}125 \\
125 \\
125\end{array}$ & $\begin{array}{l}46.0 \\
47.0 \\
48.0\end{array}$ & $\begin{array}{l}.00009 \\
.00009 \\
.00009\end{array}$ & $\begin{array}{l}.00036 \\
.00036 \\
.00036\end{array}$ & $\begin{array}{l}.00002 \\
.00002 \\
.00002\end{array}$ & $\begin{array}{l}.00011 \\
.00011 \\
.00011\end{array}$ & $\begin{array}{l}.040 \\
.039 \\
.039\end{array}$ & $\begin{array}{l}.122 \\
.121 \\
.120\end{array}$ & $\begin{array}{l}.131 \\
.130 \\
.129\end{array}$ \\
\hline $\begin{array}{l}-5 \\
-5 \\
-5\end{array}$ & $\begin{array}{l}125 \\
125 \\
125\end{array}$ & $\begin{array}{l}49.0 \\
50.0 \\
51.0\end{array}$ & $\begin{array}{r}.00009 \\
.00008 \\
.00008\end{array}$ & $\begin{array}{r}.00036 \\
.00035 \\
.00035\end{array}$ & $\begin{array}{l}.00002 \\
.00002 \\
.00002\end{array}$ & $\begin{array}{l}.00011 \\
.00010 \\
.00010\end{array}$ & $\begin{array}{l}.039 \\
.038 \\
.038\end{array}$ & $\begin{array}{r}119 \\
1118 \\
1 \\
1117\end{array}$ & $\begin{array}{r}.128 \\
.127 \\
.126\end{array}$ \\
\hline $\begin{array}{l}-5 \\
-5 \\
-5\end{array}$ & $\begin{array}{l}125 \\
125 \\
125\end{array}$ & $\begin{array}{l}52 \cdot 0 \\
53.0 \\
54 \cdot 0\end{array}$ & $\begin{array}{l}.00008 \\
.00008 \\
.00008\end{array}$ & $\begin{array}{l}.00035 \\
.00035 \\
.00034\end{array}$ & $\begin{array}{l}.00002 \\
.00002 \\
.00002\end{array}$ & $\begin{array}{r}.00010 \\
.00010 \\
.00010\end{array}$ & $\begin{array}{l}.038 \\
.038 \\
.037\end{array}$ & $\begin{array}{l}.116 \\
.116 \\
.115\end{array}$ & $\begin{array}{r}.125 \\
.125 \\
.124\end{array}$ \\
\hline $\begin{array}{l}-5 \\
-5 \\
-5\end{array}$ & $\begin{array}{l}125 \\
125 \\
125\end{array}$ & $\begin{array}{l}55.0 \\
60.0 \\
65.0\end{array}$ & $\begin{array}{l}.00008 \\
.00008 \\
.00008\end{array}$ & $\begin{array}{l}.00034 \\
.00033 \\
.00032\end{array}$ & $\begin{array}{l}.00002 \\
.00002 \\
.00002\end{array}$ & $\begin{array}{l}.00010 \\
.00010 \\
.00009\end{array}$ & $\begin{array}{l}.037 \\
.036 \\
.035\end{array}$ & $\begin{array}{r}.114 \\
.110 \\
.1107\end{array}$ & $\begin{array}{r}.123 \\
.119 \\
.115\end{array}$ \\
\hline-5 & 125 & 70.0 & .00007 & .00031 & .00002 & .00009 & .034 & .104 & .112 \\
\hline $\begin{array}{l}-5 \\
-5\end{array}$ & $\begin{array}{l}125 \\
125\end{array}$ & $\begin{array}{l}75.0 \\
80.0\end{array}$ & $\begin{array}{l}.00007 \\
.00007\end{array}$ & $\begin{array}{l}.00030 \\
.00029\end{array}$ & $\begin{array}{l}.00002 \\
.00002\end{array}$ & $\begin{array}{l}.00009 \\
.00009\end{array}$ & $\begin{array}{l}.033 \\
.032\end{array}$ & $\begin{array}{l}.101 \\
.098\end{array}$ & $\begin{array}{l}.109 \\
.106\end{array}$ \\
\hline $\begin{array}{l}-5 \\
-5\end{array}$ & $\begin{array}{l}125 \\
125\end{array}$ & $\begin{array}{l}85.0 \\
90.0\end{array}$ & $\begin{array}{l}.00007 \\
.00007\end{array}$ & $\begin{array}{l}.00029 \\
.00028\end{array}$ & $\begin{array}{l}.00002 \\
.00002\end{array}$ & $\begin{array}{l}.00008 \\
.00008\end{array}$ & $\begin{array}{l}.031 \\
.031\end{array}$ & $\begin{array}{l}.096 \\
.094\end{array}$ & $\begin{array}{r}.104 \\
.101\end{array}$ \\
\hline .5 & 125 & 95.0 & .00007 & .00028 & .00002 & .00008 & .030 & .092 & .100 \\
\hline-5 & 125 & 100.0 & .00006 & .00027 & .00002 & .00008 & .029 & .091 & .098 \\
\hline
\end{tabular}


TABULATION OF DATA FOR

ABSORPTION OF SOUND IN AIR VERSUS PERCENT RELATIVE HUMIDITY AT -5 DEGREES CENTIGRADE FOR A FREQUENCY OF 250 HERTZ

\begin{tabular}{|c|c|c|c|c|c|c|c|c|c|}
\hline (1) & (2) & $(3)$ & $(4)$ & (5) & $(6)$ & $(7)$ & (8) & (9) & $(10)$ \\
\hline TEMP & FREQ & $\begin{array}{l}\text { REL } \\
\text { HUM }\end{array}$ & $\begin{array}{l}\text { ATTEN } \\
\text { COEF }\end{array}$ & $4 M$ & $\begin{array}{l}\text { ATTEN } \\
\text { COEF }\end{array}$ & $4 M$ & $\begin{array}{l}\text { ATTEN } \\
\text { DB PER }\end{array}$ & $\begin{array}{l}\text { ATTEN } \\
\text { DB PER }\end{array}$ & $\begin{array}{l}\text { DECAY } \\
\text { RATE }\end{array}$ \\
\hline $\begin{array}{l}\text { DEGR } \\
\text { CENT }\end{array}$ & HERTZ & $\begin{array}{l}\text { PER } \\
\text { CENT }\end{array}$ & $\begin{array}{l}\text { PER } \\
\text { METER }\end{array}$ & $\begin{array}{l}\text { PER } \\
\text { METER }\end{array}$ & $\begin{array}{l}\text { PER } \\
\text { FOOT }\end{array}$ & $\begin{array}{l}\text { PER } \\
\text { FOOT }\end{array}$ & $\begin{array}{l}100 \\
\text { METER }\end{array}$ & $\begin{array}{l}1000 \\
\text { FEET }\end{array}$ & $\begin{array}{l}\text { DB PER } \\
\text { SECOND }\end{array}$ \\
\hline $\begin{array}{l}-5 \\
-5\end{array}$ & $\begin{array}{l}250 \\
250\end{array}$ & $\begin{array}{r}5.0 \\
10.0\end{array}$ & $\begin{array}{l}.00083 \\
.00090\end{array}$ & $\begin{array}{l}.00332 \\
.00360\end{array}$ & $\begin{array}{l}.00025 \\
.00027\end{array}$ & $\begin{array}{l}.00101 \\
.00109\end{array}$ & $\begin{array}{r}.361 \\
.391\end{array}$ & $\begin{array}{l}1.101 \\
1.193\end{array}$ & $\begin{array}{l}1.187 \\
1.285\end{array}$ \\
\hline-5 & 250 & 15.0 & .00060 & .00240 & .00018 & .00073 & .261 & .795 & .856 \\
\hline $\begin{array}{l}-5 \\
-5 \\
-5\end{array}$ & $\begin{array}{l}250 \\
250 \\
250\end{array}$ & $\begin{array}{l}20.0 \\
25.0 \\
30.0\end{array}$ & $\begin{array}{l}.00044 \\
.00034 \\
.00028\end{array}$ & $\begin{array}{l}.00176 \\
.00137 \\
.00113\end{array}$ & $\begin{array}{l}.00013 \\
.00010 \\
.00008\end{array}$ & $\begin{array}{l}.00053 \\
.00042 \\
.00034\end{array}$ & $\begin{array}{r}.191 \\
.149 \\
.122\end{array}$ & $\begin{array}{r}.584 \\
.456 \\
.374\end{array}$ & $\begin{array}{l}.629 \\
.491 \\
.403\end{array}$ \\
\hline $\begin{array}{l}-5 \\
-5 \\
-5\end{array}$ & $\begin{array}{l}250 \\
250 \\
250\end{array}$ & $\begin{array}{l}35.0 \\
40.0 \\
45.0\end{array}$ & $\begin{array}{l}.00024 \\
.00022 \\
.00020\end{array}$ & $\begin{array}{l}.00097 \\
.00088 \\
.00082\end{array}$ & $\begin{array}{l}.00007 \\
.00006 \\
.00006\end{array}$ & $\begin{array}{l}.00029 \\
.00026 \\
.00025\end{array}$ & $\begin{array}{l}.106 \\
.096 \\
.089\end{array}$ & $\begin{array}{l}.323 \\
.292 \\
.272\end{array}$ & $\begin{array}{r}.348 \\
.315 \\
.293\end{array}$ \\
\hline $\begin{array}{l}-5 \\
-5 \\
-5\end{array}$ & $\begin{array}{l}250 \\
250 \\
250\end{array}$ & $\begin{array}{l}46.0 \\
47.0 \\
48.0\end{array}$ & $\begin{array}{l}.00020 \\
.00020 \\
.00019\end{array}$ & $\begin{array}{l}.00081 \\
.00080 \\
.00079\end{array}$ & $\begin{array}{l}.00006 \\
.00006 \\
.00006\end{array}$ & $\begin{array}{l}.00024 \\
.00024 \\
.00024\end{array}$ & $\begin{array}{l}.088 \\
.087 \\
.086\end{array}$ & $\begin{array}{l}.269 \\
.266 \\
.264\end{array}$ & $\begin{array}{l}.290 \\
.287 \\
.285\end{array}$ \\
\hline $\begin{array}{l}-5 \\
-5 \\
-5\end{array}$ & $\begin{array}{l}250 \\
250 \\
250\end{array}$ & $\begin{array}{l}49.0 \\
50.0 \\
51.0\end{array}$ & $\begin{array}{l}.00019 \\
.00019 \\
.00019\end{array}$ & $\begin{array}{l}.00079 \\
.00078 \\
.00078\end{array}$ & $\begin{array}{l}.00006 \\
.00006 \\
.00005\end{array}$ & $\begin{array}{l}.00024 \\
.00024 \\
.00023\end{array}$ & $\begin{array}{l}.086 \\
.085 \\
.084\end{array}$ & $\begin{array}{l}.262 \\
.260 \\
.258\end{array}$ & $\begin{array}{l}.282 \\
.280 \\
.278\end{array}$ \\
\hline-5 & 250 & 52.0 & .00019 & .00077 & .00005 & .00023 & .084 & .257 & .277 \\
\hline $\begin{array}{l}-5 \\
-5\end{array}$ & $\begin{array}{l}250 \\
250\end{array}$ & $\begin{array}{l}53.0 \\
54.0\end{array}$ & $\begin{array}{l}.00019 \\
.00019\end{array}$ & $\begin{array}{l}.00077 \\
.00076\end{array}$ & $\begin{array}{l}.00005 \\
.00005\end{array}$ & $\begin{array}{l}.00023 \\
.00023\end{array}$ & $\begin{array}{l}.083 \\
.083\end{array}$ & $\begin{array}{r}.255 \\
.253\end{array}$ & $\begin{array}{l}.275 \\
.273\end{array}$ \\
\hline-5 & 250 & 55.0 & .00019 & .00076 & .00005 & .00023 & .082 & .251 & .271 \\
\hline $\begin{array}{l}-5 \\
-5\end{array}$ & $\begin{array}{l}250 \\
250\end{array}$ & $\begin{array}{l}60.0 \\
65.0\end{array}$ & $\begin{array}{l}.00018 \\
.00017\end{array}$ & $\begin{array}{l}.00073 \\
.00071\end{array}$ & $\begin{array}{l}.00005 \\
.00005\end{array}$ & $\begin{array}{l}.00022 \\
.00021\end{array}$ & $\begin{array}{l}.080 \\
.077\end{array}$ & $\begin{array}{l}.244 \\
.237\end{array}$ & $\begin{array}{l}.263 \\
.255\end{array}$ \\
\hline $\begin{array}{l}-5 \\
-5 \\
-5\end{array}$ & $\begin{array}{l}250 \\
250 \\
250\end{array}$ & $\begin{array}{l}70.0 \\
75.0 \\
80.0\end{array}$ & $\begin{array}{l}.00017 \\
.00017 \\
.00016\end{array}$ & $\begin{array}{l}.00069 \\
.00068 \\
.00066\end{array}$ & $\begin{array}{l}.00005 \\
.00005 \\
.00005\end{array}$ & $\begin{array}{l}.00021 \\
.00020 \\
.00020\end{array}$ & $\begin{array}{l}.075 \\
.073 \\
.072\end{array}$ & $\begin{array}{r}.231 \\
.225 \\
.219\end{array}$ & $\begin{array}{l}.248 \\
.242 \\
.236\end{array}$ \\
\hline $\begin{array}{l}-5 \\
-5 \\
-5\end{array}$ & $\begin{array}{l}250 \\
250 \\
250\end{array}$ & $\begin{array}{l}85.0 \\
90.0 \\
95.0\end{array}$ & $\begin{array}{r}.00016 \\
.00015 \\
.00015\end{array}$ & $\begin{array}{l}.00064 \\
.00063 \\
.00062\end{array}$ & $\begin{array}{l}.00004 \\
.00004 \\
.00004\end{array}$ & $\begin{array}{l}.00019 \\
.00019 \\
.00018\end{array}$ & $\begin{array}{l}.070 \\
.068 \\
.067\end{array}$ & $\begin{array}{l}.214 \\
.209 \\
.205\end{array}$ & $\begin{array}{l}.231 \\
.225 \\
.221\end{array}$ \\
\hline-5 & 2501 & $100 \cdot 0$ & .00015 & .00060 & .00004 & .00018 & .065 & .201 & .216 \\
\hline
\end{tabular}


TABULATION OF DATA FOR

ABSORPTION OF SOUND IN AIR VERSUS PERCENT RELATIVE HUMIDITY AT - 5 DEGREES CENTIGRADE FOR A FREQUENCY OF 500 HERTZ

\begin{tabular}{|c|c|c|c|c|c|c|c|c|c|}
\hline (1) & (2) & $(3)$ & (4) & (5) & 161 & $(7)$ & $(8)$ & 191 & $(10)$ \\
\hline $\begin{array}{l}\text { TEMP } \\
\text { DEGR } \\
\text { CENT }\end{array}$ & HERTZ & $\begin{array}{l}\text { REL } \\
\text { HUM } \\
\text { PER } \\
\text { CENT }\end{array}$ & $\begin{array}{l}\text { ATTEN } \\
\text { COEF } \\
\text { PER } \\
\text { METER }\end{array}$ & $\begin{array}{l}4 M \\
\text { PER } \\
\text { METER }\end{array}$ & $\begin{array}{l}\text { ATTEN } \\
\text { COEF } \\
\text { PER } \\
\text { FOOT }\end{array}$ & $\begin{array}{l}\text { PER } \\
\text { FOOT }\end{array}$ & $\begin{array}{l}\text { ATTEN } \\
\text { DB PER } \\
100 \\
\text { MET.ER }\end{array}$ & $\begin{array}{l}\text { ATTEN } \\
\text { DB PER } \\
1000 \\
\text { FEET }\end{array}$ & $\begin{array}{l}\text { DECAY } \\
\text { RATE } \\
\text { DB PER } \\
\text { SECOND }\end{array}$ \\
\hline $\begin{array}{l}-5 \\
-5 \\
-5\end{array}$ & $\begin{array}{l}500 \\
500 \\
500\end{array}$ & $\begin{array}{r}5.0 \\
10.0 \\
15.0\end{array}$ & $\begin{array}{l}.00106 \\
.00192 \\
.00183\end{array}$ & $\begin{array}{r}.00427 \\
.00769 \\
.00733\end{array}$ & $\begin{array}{l}.00032 \\
.00058 \\
.00055\end{array}$ & $\begin{array}{r}.00130 \\
.00234 \\
.00223\end{array}$ & $\begin{array}{l}.464 \\
.835 \\
.796\end{array}$ & $\begin{array}{l}1.414 \\
2.547 \\
2.428\end{array}$ & $\begin{array}{l}1.523 \\
2.743 \\
2.615\end{array}$ \\
\hline $\begin{array}{l}-5 \\
-5 \\
-5\end{array}$ & $\begin{array}{l}500 \\
500 \\
500\end{array}$ & $\begin{array}{l}20.0 \\
25.0 \\
30.0\end{array}$ & $\begin{array}{l}.00141 \\
.00110 \\
.00091\end{array}$ & $\begin{array}{l}.00567 \\
.00443 \\
.00365\end{array}$ & $\begin{array}{l}.00043 \\
.00033 \\
.00027\end{array}$ & $\begin{array}{l}.00172 \\
.00135 \\
.00111\end{array}$ & $\begin{array}{l}.615 \\
.481 \\
.397\end{array}$ & $\begin{array}{l}1.877 \\
1.468 \\
1.210\end{array}$ & $\begin{array}{l}2.022 \\
1.582 \\
1.303\end{array}$ \\
\hline $\begin{array}{l}-5 \\
-5 \\
-5\end{array}$ & $\begin{array}{l}500 \\
500 \\
500\end{array}$ & $\begin{array}{l}35.0 \\
40.0 \\
45.0\end{array}$ & $\begin{array}{l}.00076 \\
.00066 \\
.00058\end{array}$ & $\begin{array}{l}.00306 \\
.00266 \\
.00234\end{array}$ & $\begin{array}{l}.00023 \\
.00020 \\
.00017\end{array}$ & $\begin{array}{l}.00093 \\
.00081 \\
.00071\end{array}$ & $\begin{array}{r}.333 \\
.289 \\
.254\end{array}$ & $\begin{array}{r}1.015 \\
.883 \\
.775\end{array}$ & $\begin{array}{r}1.093 \\
.951 \\
.835\end{array}$ \\
\hline $\begin{array}{l}-5 \\
-5 \\
-5\end{array}$ & $\begin{array}{l}500 \\
500 \\
500\end{array}$ & $\begin{array}{l}46.0 \\
47.0 \\
48.0\end{array}$ & $\begin{array}{l}.00057 \\
.00056 \\
.00054\end{array}$ & $\begin{array}{l}.00229 \\
.00224 \\
.00219\end{array}$ & $\begin{array}{r}.00017 \\
.00017 \\
.00016\end{array}$ & $\begin{array}{l}.00069 \\
.00068 \\
.00066\end{array}$ & $\begin{array}{r}.248 \\
.243 \\
.238\end{array}$ & $\begin{array}{l}.758 \\
.742 \\
.726\end{array}$ & $\begin{array}{l}.816 \\
.799 \\
.782\end{array}$ \\
\hline $\begin{array}{l}-5 \\
-5 \\
-5\end{array}$ & $\begin{array}{l}500 \\
500 \\
500\end{array}$ & $\begin{array}{l}49.0 \\
50.0 \\
51.0\end{array}$ & $\begin{array}{l}.00053 \\
.00052 \\
.00051\end{array}$ & $\begin{array}{l}.00215 \\
.00211 \\
.00207\end{array}$ & $\begin{array}{l}.00016 \\
.00016 \\
.00015\end{array}$ & $\begin{array}{l}.00065 \\
.00064 \\
.00063\end{array}$ & $\begin{array}{l}.233 \\
.229 \\
.225\end{array}$ & $\begin{array}{l}.712 \\
.699 \\
.687\end{array}$ & $\begin{array}{r}.768 \\
.753 \\
.740\end{array}$ \\
\hline $\begin{array}{l}-5 \\
-5 \\
-5\end{array}$ & $\begin{array}{l}500 \\
500 \\
500\end{array}$ & $\begin{array}{l}52.0 \\
53.0 \\
54.0\end{array}$ & $\begin{array}{l}.00051 \\
.00050 \\
.00049\end{array}$ & $\begin{array}{r}.00204 \\
.00200 \\
.00197\end{array}$ & $\begin{array}{r}.00015 \\
.00015 \\
.00015\end{array}$ & $\begin{array}{l}.00062 \\
.00061 \\
.00060\end{array}$ & $\begin{array}{r}.221 \\
.217 \\
.213\end{array}$ & $\begin{array}{l}.676 \\
.664 \\
.651\end{array}$ & $\begin{array}{r}.728 \\
.715 \\
.702\end{array}$ \\
\hline $\begin{array}{l}-5 \\
-5 \\
-5\end{array}$ & $\begin{array}{l}500 \\
500 \\
500\end{array}$ & $\begin{array}{l}55.0 \\
60.0 \\
65.0\end{array}$ & $\begin{array}{l}.00048 \\
.00045 \\
.00043\end{array}$ & $\begin{array}{r}.00193 \\
.00181 \\
.00173\end{array}$ & $\begin{array}{l}.00014 \\
.00013 \\
.00013\end{array}$ & $\begin{array}{l}.00058 \\
.00055 \\
.00052\end{array}$ & $\begin{array}{r}.210 \\
.196 \\
.188\end{array}$ & $\begin{array}{l}.640 \\
.600 \\
.573\end{array}$ & $\begin{array}{l}.689 \\
.646 \\
.617\end{array}$ \\
\hline $\begin{array}{l}-5 \\
-5 \\
-5\end{array}$ & $\begin{array}{l}500 \\
500 \\
500\end{array}$ & $\begin{array}{l}70.0 \\
75.0 \\
80.0\end{array}$ & $\begin{array}{l}.00041 \\
.00040 \\
.00039\end{array}$ & $\begin{array}{r}.00165 \\
.00160 \\
.00157\end{array}$ & $\begin{array}{l}.00012 \\
.00012 \\
.00011\end{array}$ & $\begin{array}{r}.00050 \\
.00048 \\
.00047\end{array}$ & $\begin{array}{r}.179 \\
.174 \\
.170\end{array}$ & $\begin{array}{r}.548 \\
.531 \\
.519\end{array}$ & $\begin{array}{l}.590 \\
.572 \\
.560\end{array}$ \\
\hline $\begin{array}{l}-5 \\
-5 \\
-5\end{array}$ & $\begin{array}{l}500 \\
500 \\
500\end{array}$ & $\begin{array}{l}85.0 \\
90.0 \\
95.0\end{array}$ & $\begin{array}{r}.00038 \\
.00037 \\
.00036\end{array}$ & $\begin{array}{r}.00153 \\
.00150 \\
.00147\end{array}$ & $\begin{array}{l}.00011 \\
.00011 \\
.00011\end{array}$ & $\begin{array}{r}.00046 \\
.00045 \\
.00045\end{array}$ & $\begin{array}{l}.166 \\
.163 \\
.160\end{array}$ & $\begin{array}{r}.508 \\
.498 \\
.488\end{array}$ & $\begin{array}{l}.547 \\
.536 \\
.526\end{array}$ \\
\hline-5 & 500 & $100 \cdot 0$ & .00036 & .00145 & .00011 & .00044 & .257 & .479 & .516 \\
\hline
\end{tabular}


TABULATION OF DATA FOR

ABSORPTION OF SOUND IN AIR VERSUS PERCENT RELATIVE HUMIDITY AT -5 DEGREES GENTIGRADE FOR A FREQUENCY OF 1000 HERTZ

\begin{tabular}{|c|c|c|c|c|c|c|c|c|c|}
\hline (1) & (2) & (3) & (4) & (5) & $(6)$ & $(7)$ & $(8)$ & (9) & $(10)$ \\
\hline $\begin{array}{l}\text { TEMP } \\
\text { DEGR } \\
\text { CENT }\end{array}$ & HERTZ & $\begin{array}{l}\text { REL } \\
\text { HUM } \\
\text { PER } \\
\text { CENT }\end{array}$ & $\begin{array}{l}\text { ATTEN } \\
\text { COEF } \\
\text { PER } \\
\text { METER }\end{array}$ & $\begin{array}{l}\text { PER } \\
\text { METER }\end{array}$ & $\begin{array}{l}\text { ATTEN } \\
\text { COEF } \\
\text { PER } \\
\text { FOOT }\end{array}$ & $\begin{array}{l}\text { PER } \\
\text { FOOT }\end{array}$ & $\begin{array}{l}\text { ATTEN } \\
\text { DB PER } \\
100 \\
\text { METER }\end{array}$ & $\begin{array}{l}\text { ATTEN } \\
\text { DB. PER } \\
1000 \\
\text { FEET }\end{array}$ & $\begin{array}{l}\text { DECAY } \\
\text { RATE } \\
\text { DB PER } \\
\text { SECOND }\end{array}$ \\
\hline $\begin{array}{l}-5 \\
-5 \\
-5\end{array}$ & $\begin{array}{l}1000 \\
1000 \\
1000\end{array}$ & $\begin{array}{r}5.0 \\
10.0 \\
15.0\end{array}$ & $\begin{array}{l}.00135 \\
.00271 \\
.00377\end{array}$ & $\begin{array}{r}.00541 \\
.01084 \\
.01511\end{array}$ & $\begin{array}{r}.00041 \\
.00082 \\
.00115\end{array}$ & $\begin{array}{l}.00164 \\
.00330 \\
.00460\end{array}$ & $\begin{array}{r}.587 \\
1.177 \\
1.640\end{array}$ & $\begin{array}{l}1.791 \\
3.589 \\
5.000\end{array}$ & $\begin{array}{l}1.929 \\
3.866 \\
5.386\end{array}$ \\
\hline $\begin{array}{l}-5 \\
-5 \\
-5\end{array}$ & $\begin{array}{l}1000 \\
1000 \\
1000\end{array}$ & $\begin{array}{l}20.0 \\
25.0 \\
30.0\end{array}$ & $\begin{array}{l}.00391 \\
.00360 \\
.00307\end{array}$ & $\begin{array}{r}.01564 \\
.01441 \\
.01230\end{array}$ & $\begin{array}{l}.00119 \\
.00109 \\
.00093\end{array}$ & $\begin{array}{l}.00476 \\
.00439 \\
.00375\end{array}$ & $\begin{array}{l}1.698 \\
1.564 \\
1.336\end{array}$ & $\begin{array}{l}5.176 \\
4 \bullet 769 \\
4.072\end{array}$ & $\begin{array}{l}5.576 \\
5.137 \\
4.387\end{array}$ \\
\hline $\begin{array}{l}-5 \\
-5 \\
-5\end{array}$ & $\begin{array}{l}1000 \\
1000 \\
1000\end{array}$ & $\begin{array}{l}35.0 \\
40.0 \\
45.0\end{array}$ & $\begin{array}{l}.00258 \\
.00224 \\
.00198\end{array}$ & $\begin{array}{l}.01035 \\
.00897 \\
.00795\end{array}$ & $\begin{array}{l}.00078 \\
.00068 \\
.00060\end{array}$ & $\begin{array}{l}.00315 \\
.00273 \\
.00242\end{array}$ & $\begin{array}{r}1.123 \\
.974 \\
.863\end{array}$ & $\begin{array}{l}3.425 \\
2.969 \\
2.631\end{array}$ & $\begin{array}{l}3.690 \\
3.198 \\
2.834\end{array}$ \\
\hline $\begin{array}{l}-5 \\
-5 \\
-5\end{array}$ & $\begin{array}{l}1000 \\
1000 \\
1000\end{array}$ & $\begin{array}{l}46.0 \\
47.0 \\
48.0\end{array}$ & $\begin{array}{r}.00193 \\
.00189 \\
.00185\end{array}$ & $\begin{array}{r}.00775 \\
.00757 \\
.00740\end{array}$ & $\begin{array}{l}.00059 \\
.00057 \\
.00056\end{array}$ & $\begin{array}{l}.00236 \\
.00231 \\
.00225\end{array}$ & $\begin{array}{r}.842 \\
.822 \\
.803\end{array}$ & $\begin{array}{l}2.567 \\
2.508 \\
2.450\end{array}$ & $\begin{array}{l}2.765 \\
2.701 \\
2.639\end{array}$ \\
\hline $\begin{array}{l}-5 \\
-5 \\
-5\end{array}$ & $\begin{array}{l}1000 \\
1000 \\
1000\end{array}$ & $\begin{array}{l}49.0 \\
50.0 \\
51.0\end{array}$ & $\begin{array}{l}.00180 \\
.00176 \\
.00172\end{array}$ & $\begin{array}{r}.00723 \\
.00706 \\
.00689\end{array}$ & $\begin{array}{l}.00055 \\
.00053 \\
.00052\end{array}$ & $\begin{array}{l}.00220 \\
.00215 \\
.00210\end{array}$ & $\begin{array}{l}.785 \\
.766 \\
.748\end{array}$ & $\begin{array}{l}2.393 \\
2.337 \\
2.281\end{array}$ & $\begin{array}{l}2.578 \\
2.517 \\
2.457\end{array}$ \\
\hline-5 & 1000 & 52.0 & .00168 & .00674 & .00051 & .00205 & .732 & $2 \cdot 233$ & 2.405 \\
\hline $\begin{array}{l}-5 \\
-5\end{array}$ & $\begin{array}{l}1000 \\
1000\end{array}$ & $\begin{array}{l}53.0 \\
54.0\end{array}$ & $\begin{array}{l}.00165 \\
.00161\end{array}$ & $\begin{array}{l}.00661 \\
.00647\end{array}$ & $\begin{array}{r}.00050 \\
.00049\end{array}$ & .00201 & $\begin{array}{r}.717 \\
.703\end{array}$ & $\begin{array}{l}2.188 \\
2.142\end{array}$ & $\begin{array}{l}2.356 \\
2.308\end{array}$ \\
\hline $\begin{array}{l}-5 \\
-5 \\
-5\end{array}$ & $\begin{array}{l}1000 \\
1000 \\
1000\end{array}$ & $\begin{array}{l}55.0 \\
60.0 \\
65.0\end{array}$ & $\begin{array}{r}.00158 \\
.00144 \\
.00133\end{array}$ & $\begin{array}{r}.00634 \\
.00578 \\
.00532\end{array}$ & $\begin{array}{r}.00048 \\
.00044 \\
.00040\end{array}$ & $\begin{array}{l}.00193 \\
.00176 \\
.00162\end{array}$ & $\begin{array}{r}.688 \\
.627 \\
.578\end{array}$ & $\begin{array}{l}2.098 \\
1.914 \\
1.763\end{array}$ & $\begin{array}{l}2.260 \\
2 \cdot 0.61 \\
1.899\end{array}$ \\
\hline $\begin{array}{l}-5 \\
-5 \\
-5\end{array}$ & $\begin{array}{l}1000 \\
1000 \\
1000\end{array}$ & $\begin{array}{l}70.0 \\
75.0 \\
80.0\end{array}$ & $\begin{array}{r}.00122 \\
.00114 \\
.00107\end{array}$ & $\begin{array}{r}.00491 \\
.00457 \\
.00430\end{array}$ & $\begin{array}{l}.00037 \\
.00034 \\
.00032\end{array}$ & $\begin{array}{l}.00149 \\
.00139 \\
.00131\end{array}$ & $\begin{array}{l}.533 \\
.496 \\
.467\end{array}$ & $\begin{array}{l}1.625 \\
1.514 \\
1.424\end{array}$ & $\begin{array}{l}1.751 \\
1.631 \\
1.534\end{array}$ \\
\hline $\begin{array}{l}-5 \\
-5 \\
-5\end{array}$ & $\begin{array}{l}1000 \\
1000 \\
1000\end{array}$ & $\begin{array}{l}85.0 \\
90.0 \\
95.0\end{array}$ & $\begin{array}{r}.00102 \\
.00096 \\
.00093\end{array}$ & $\begin{array}{r}.00408 \\
.00387 \\
.00372\end{array}$ & $\begin{array}{l}.00031 \\
.00029 \\
.00028\end{array}$ & $\begin{array}{l}.00124 \\
.00118 \\
.00113\end{array}$ & $\begin{array}{l}.443 \\
.420 \\
.403\end{array}$ & $\begin{array}{l}1.351 \\
1.282 \\
1.231\end{array}$ & $\begin{array}{l}1.455 \\
1.381 \\
1.326\end{array}$ \\
\hline-5 & 1000 & 100.0 & .00090 & .00361 & .00027 & .00110 & .392 & 1.195 & 1.287 \\
\hline
\end{tabular}


TABULATION OF DATA FOR

ABSORPTION OF SOUND IN AIR VERSUS PERCENT RELATIVE HUMIDITY AT -5 DEGREES CENTIGRADE. FOR A FREQUENCY OF 2000 HERTZ

\begin{tabular}{|c|c|c|c|c|c|c|c|c|c|}
\hline$(1)$ & (2) & (3) & (4) & (5) & $(6)$ & $(7)$ & $(8)$ & 191 & $(10)$ \\
\hline $\begin{array}{l}\text { TEMP } \\
\text { DEGR } \\
\text { CENT }\end{array}$ & HERTZ & $\begin{array}{l}\text { REL } \\
\text { HUM } \\
\text { PER } \\
\text { CENT }\end{array}$ & $\begin{array}{l}\text { ATTEN } \\
\text { COEF } \\
\text { PER } \\
\text { METER }\end{array}$ & $\begin{array}{l}4 M \\
\text { PER } \\
\text { METER }\end{array}$ & $\begin{array}{l}\text { ATTEN } \\
\text { COEF } \\
\text { PER } \\
\text { FOOT }\end{array}$ & $\begin{array}{l}4 M \\
\text { PER } \\
\text { FOOT }\end{array}$ & $\begin{array}{l}\text { ATTEN } \\
\text { DB PER } \\
\text { IOO } \\
\text { METER }\end{array}$ & $\begin{array}{l}\text { ATTEN } \\
\text { DB PER } \\
1000 \\
\text { FEET }\end{array}$ & $\begin{array}{l}\text { DECAY } \\
\text { RATE } \\
\text { DB PER } \\
\text { SECOND }\end{array}$ \\
\hline-5 & 2000 & 5.0 & .00174 & .00698 & .00053 & .00212 & .758 & 2.310 & 2.488 \\
\hline $\begin{array}{l}-5 \\
-5\end{array}$ & $\begin{array}{l}2000 \\
2000\end{array}$ & $\begin{array}{l}10.0 \\
15.0\end{array}$ & $\begin{array}{r}.00336 \\
.00499\end{array}$ & $\begin{array}{r}.01345 \\
.01999\end{array}$ & $\begin{array}{r}.00102 \\
.00152\end{array}$ & $\begin{array}{r}.00409 \\
.00609\end{array}$ & $\begin{array}{l}1.460 \\
2.170\end{array}$ & $\begin{array}{l}4.451 \\
6.615\end{array}$ & $\begin{array}{l}4.795 \\
7.126\end{array}$ \\
\hline $\begin{array}{l}-5 \\
-5 \\
-5\end{array}$ & $\begin{array}{l}2000 \\
2000 \\
2000\end{array}$ & $\begin{array}{l}20.0 \\
25.0 \\
30.0\end{array}$ & $\begin{array}{l}.00671 \\
.00768 \\
.00792\end{array}$ & $\begin{array}{r}.02684 \\
.03072 \\
.03170\end{array}$ & $\begin{array}{l}.00204 \\
.00234 \\
.00241\end{array}$ & $\begin{array}{l}.00818 \\
.00936 \\
.00966\end{array}$ & $\begin{array}{l}2.915 \\
3.335 \\
3.442\end{array}$ & $\begin{array}{r}8.885 \\
10.167 \\
10.493\end{array}$ & $\begin{array}{r}9.571 \\
10.952 \\
11.303\end{array}$ \\
\hline $\begin{array}{l}-5 \\
-5 \\
-5\end{array}$ & $\begin{array}{l}2000 \\
2000 \\
2000\end{array}$ & $\begin{array}{l}35.0 \\
40.0 \\
45.0\end{array}$ & $\begin{array}{r}.00778 \\
.00736 \\
.00673\end{array}$ & $\begin{array}{l}.03114 \\
.02946 \\
.02695\end{array}$ & $\begin{array}{l}.00237 \\
.00224 \\
.00205\end{array}$ & $\begin{array}{l}.00949 \\
.00898 \\
.00821\end{array}$ & $\begin{array}{l}3.381 \\
3.198 \\
2.926\end{array}$ & $\begin{array}{r}10.307 \\
9.750 \\
8.920\end{array}$ & $\begin{array}{r}11.103 \\
10.502 \\
9.608\end{array}$ \\
\hline $\begin{array}{l}-5 \\
-5 \\
-5\end{array}$ & $\begin{array}{l}2000 \\
2000 \\
2000\end{array}$ & $\begin{array}{l}46.0 \\
47.0 \\
48.0\end{array}$ & $\begin{array}{r}.00660 \\
.00647 \\
.00634\end{array}$ & $\begin{array}{r}.02643 \\
.02591 \\
.02539\end{array}$ & $\begin{array}{r}.00201 \\
.00197 \\
.00193\end{array}$ & $\begin{array}{r}.00805 \\
.00789 \\
.00773\end{array}$ & $\begin{array}{l}2.869 \\
2.813 \\
2.756\end{array}$ & $\begin{array}{l}8.747 \\
8.574 \\
8.402\end{array}$ & $\begin{array}{l}9.422 \\
9.236 \\
9.051\end{array}$ \\
\hline $\begin{array}{l}-5 \\
-5 \\
-5\end{array}$ & $\begin{array}{l}2000 \\
2000 \\
2000\end{array}$ & $\begin{array}{l}49.0 \\
50.0 \\
51.0\end{array}$ & $\begin{array}{l}.00621 \\
.00609 \\
.00596\end{array}$ & $\begin{array}{r}.02487 \\
.02436 \\
.02385\end{array}$ & $\begin{array}{r}.00189 \\
.00185 \\
.00181\end{array}$ & $\begin{array}{l}.00758 \\
.00742 \\
.00727\end{array}$ & $\begin{array}{l}2.701 \\
2.645 \\
2.589\end{array}$ & $\begin{array}{l}8.233 \\
8.064 \\
7.893\end{array}$ & $\begin{array}{l}8.868 \\
8.686 \\
8.503\end{array}$ \\
\hline $\begin{array}{l}-5 \\
-5\end{array}$ & $\begin{array}{l}2000 \\
2000\end{array}$ & $\begin{array}{l}52.0 \\
53.0\end{array}$ & $\begin{array}{r}.00583 \\
.00569\end{array}$ & $\begin{array}{r}.02332 \\
.02278\end{array}$ & $\begin{array}{r}.00177 \\
.00173\end{array}$ & $\begin{array}{l}.00710 \\
.00694\end{array}$ & $\begin{array}{l}2.532 \\
2.474\end{array}$ & $\begin{array}{l}7.717 \\
7.541\end{array}$ & $\begin{array}{l}8.313 \\
8.123\end{array}$ \\
\hline-5 & 2000 & $54 \cdot 0$ & .00556 & .02227 & .00169 & .00678 & 2.418 & 7.372 & 7.941 \\
\hline $\begin{array}{l}-5 \\
-5\end{array}$ & $\begin{array}{l}2000 \\
2000\end{array}$ & $\begin{array}{l}55.0 \\
60.0\end{array}$ & $\begin{array}{r}.00546 \\
.00497\end{array}$ & $\begin{array}{r}.02185 \\
.01988\end{array}$ & $\begin{array}{l}.00166 \\
.00151\end{array}$ & $\begin{array}{l}.00666 \\
.00606\end{array}$ & $\begin{array}{l}2.373 \\
2.158\end{array}$ & $\begin{array}{l}7.234 \\
6.580\end{array}$ & $\begin{array}{l}7.792 \\
7.087\end{array}$ \\
\hline-5 & 2000 & 65.0 & .00457 & .01830 & .00139 & .00557 & 1.987 & 6.058 & 6.525 \\
\hline $\begin{array}{l}-5 \\
-5\end{array}$ & $\begin{array}{l}2000 \\
2000\end{array}$ & $\begin{array}{l}70.0 \\
75.0\end{array}$ & $\begin{array}{r}.00425 \\
.00395\end{array}$ & $\begin{array}{r}.01702 \\
.01580\end{array}$ & $\begin{array}{l}.00129 \\
.00120\end{array}$ & $\begin{array}{r}.00518 \\
.00481\end{array}$ & $\begin{array}{l}1.848 \\
2.716\end{array}$ & $\begin{array}{l}5.632 \\
5.231\end{array}$ & $\begin{array}{l}6.067 \\
5.635\end{array}$ \\
\hline-5 & 2000 & 80.0 & .00368 & .01473 & .00112 & .00449 & 1.599 & 4.876 & $5 \cdot 252$ \\
\hline $\begin{array}{l}-5 \\
-5\end{array}$ & $\begin{array}{l}2000 \\
2000\end{array}$ & $\begin{array}{l}85.0 \\
90.0\end{array}$ & $\begin{array}{r}.00343 \\
.00322\end{array}$ & $\begin{array}{r}.01374 \\
.01291\end{array}$ & $\begin{array}{l}.00104 \\
.00098\end{array}$ & $\begin{array}{l}.00418 \\
.00393\end{array}$ & $\begin{array}{l}1.492 \\
1.402\end{array}$ & $\begin{array}{l}4 \cdot 548 \\
4 \cdot 274\end{array}$ & $\begin{array}{l}4.900 \\
4.604\end{array}$ \\
\hline-5 & 20.00 & 95.0 & .00305 & .01221 & .00093 & .00372 & 1.325 & 4.041 & $4 \cdot 352$ \\
\hline & 2000 & $100 \cdot 0$ & .00289 & 01159 & .00088 & .00353 & 1.258 & 3.836 & $4 \cdot 132$ \\
\hline
\end{tabular}


TABULATION OF DATA FOR

ABSORPTION OF SOUND IN AIR VERSUS PERCENT RELATIVE HUMIDITY AT -5 DEGREES CENTIGRADE FOR A FREQUENCY OF 2500 HERTZ.

\begin{tabular}{|c|c|c|c|c|c|c|c|c|c|}
\hline (1) & $(2)$ & (3) & (4) & (5) & $(6)$ & 171 & $(8)$ & $(9)$ & $(10)$ \\
\hline $\begin{array}{l}\text { DEGR } \\
\text { CENT }\end{array}$ & HERTZ & $\begin{array}{l}\text { REL } \\
\text { HUM } \\
\text { PER } \\
\text { CENT }\end{array}$ & $\begin{array}{l}\text { ATTEN } \\
\text { COEF } \\
\text { PER } \\
\text { METER }\end{array}$ & $\begin{array}{l}4 M \\
\text { PER } \\
\text { METER }\end{array}$ & $\begin{array}{l}\text { ATTEN } \\
\text { COEF } \\
\text { PER } \\
\text { FOOT }\end{array}$ & $\begin{array}{l}4 M \\
\text { PER } \\
\text { FOOT }\end{array}$ & $\begin{array}{l}\text { ATTEN } \\
\text { DB PER } \\
100 \\
\text { METER }\end{array}$ & $\begin{array}{l}\text { ATTEN } \\
\text { DB PER } \\
1000 \\
\text { FEET }\end{array}$ & $\begin{array}{l}\text { DECAY } \\
\text { RATE } \\
\text { DB PER } \\
\text { SECOND }\end{array}$ \\
\hline $\begin{array}{l}-5 \\
-5 \\
-5\end{array}$ & $\begin{array}{l}2500 \\
2500 \\
2500\end{array}$ & $\begin{array}{r}5.0 \\
10.0 \\
15.0\end{array}$ & $\begin{array}{l}.00192 \\
.00365 \\
.00538\end{array}$ & $\begin{array}{r}.00770 \\
.01461 \\
.02152\end{array}$ & $\begin{array}{l}.00058 \\
.00111 \\
.00164\end{array}$ & $\begin{array}{l}.00234 \\
.00445 \\
.00656\end{array}$ & $\begin{array}{r}.836 \\
1.587 \\
2.337\end{array}$ & $\begin{array}{l}2.551 \\
4.837 \\
7.123\end{array}$ & $\begin{array}{l}2.747 \\
5.210 \\
7.673\end{array}$ \\
\hline $\begin{array}{l}-5 \\
-5 \\
-5\end{array}$ & $\begin{array}{l}2500 \\
2500 \\
2500\end{array}$ & $\begin{array}{l}20.0 \\
25.0 \\
30.0\end{array}$ & $\begin{array}{l}.00722 \\
.00888 \\
.00972\end{array}$ & $\begin{array}{r}.02890 \\
.03553 \\
.03890\end{array}$ & $\begin{array}{l}.00220 \\
.00270 \\
.00296\end{array}$ & $\begin{array}{l}.00881 \\
.01083 \\
.01185\end{array}$ & $\begin{array}{l}3.138 \\
3.858 \\
4.223\end{array}$ & $\begin{array}{r}9.566 \\
11.760 \\
12.874\end{array}$ & $\begin{array}{l}10.305 \\
12.668 \\
13.868\end{array}$ \\
\hline $\begin{array}{l}-5 \\
-5 \\
-5\end{array}$ & $\begin{array}{l}2500 \\
2500 \\
2500\end{array}$ & $\begin{array}{l}35.0 \\
40.0 \\
45.0\end{array}$ & $\begin{array}{l}.00995 \\
.00982 \\
.00944\end{array}$ & $\begin{array}{r}.03980 \\
.03929 \\
.03776\end{array}$ & $\begin{array}{l}.00303 \\
.00299 \\
.00287\end{array}$ & $\begin{array}{r}.011213 \\
.01197 \\
.01151\end{array}$ & $\begin{array}{l}4.321 \\
4.266 \\
4.100\end{array}$ & $\begin{array}{l}13.171 \\
13.005 \\
12.498\end{array}$ & $\begin{array}{l}14.188 \\
14.009 \\
13.463\end{array}$ \\
\hline $\begin{array}{l}-5 \\
-5 \\
-5\end{array}$ & $\begin{array}{l}2500 \\
2500 \\
2500\end{array}$ & $\begin{array}{l}46.0 \\
47.0 \\
48.0\end{array}$ & $\begin{array}{l}.00933 \\
.00922 \\
.00910\end{array}$ & $\begin{array}{l}.03735 \\
.03690 \\
.03641\end{array}$ & $\begin{array}{l}.00284 \\
.00281 \\
.00277\end{array}$ & $\begin{array}{r}.01138 \\
.01124 \\
.01109\end{array}$ & $\begin{array}{l}4.055 \\
4.007 \\
3.953\end{array}$ & $\begin{array}{l}12.362 \\
12.214 \\
12.050\end{array}$ & $\begin{array}{l}13.316 \\
13.157 \\
12.980\end{array}$ \\
\hline $\begin{array}{l}-5 \\
-5 \\
-5\end{array}$ & $\begin{array}{l}2500 \\
2500 \\
2500\end{array}$ & $\begin{array}{l}49.0 \\
50.0 \\
51.0\end{array}$ & $\begin{array}{l}.00897 \\
.00883 \\
.00869\end{array}$ & $\begin{array}{r}.03590 \\
.03534 \\
.03478\end{array}$ & $\begin{array}{l}.00273 \\
.00269 \\
.00265\end{array}$ & $\begin{array}{r}.01094 \\
.01077 \\
.01060\end{array}$ & $\begin{array}{l}3.897 \\
3.837 \\
3.776\end{array}$ & $\begin{array}{l}11.880 \\
11.696 \\
11.512\end{array}$ & $\begin{array}{l}12.797 \\
12.599 \\
12.400\end{array}$ \\
\hline $\begin{array}{l}-5 \\
-5 \\
-5\end{array}$ & $\begin{array}{l}2500 \\
2500 \\
2500\end{array}$ & $\begin{array}{l}52.0 \\
53.0 \\
54.0\end{array}$ & $\begin{array}{l}.00855 \\
.00841 \\
.00827\end{array}$ & $\begin{array}{r}.03422 \\
.03367 \\
.03311\end{array}$ & $\begin{array}{l}.00260 \\
.00256 \\
.00252\end{array}$ & $\begin{array}{r}.01043 \\
.01026 \\
.01009\end{array}$ & $\begin{array}{l}3.716 \\
3.655 \\
3.595\end{array}$ & $\begin{array}{l}11.327 \\
11.143 \\
10.958\end{array}$ & $\begin{array}{l}12.201 \\
12.003 \\
11.804\end{array}$ \\
\hline $\begin{array}{l}-5 \\
-5 \\
-5\end{array}$ & $\begin{array}{l}2500 \\
2500 \\
2500\end{array}$ & $\begin{array}{l}55.0 \\
60.0 \\
65.0\end{array}$ & $\begin{array}{l}.00813 \\
.00745 \\
.00680\end{array}$ & $\begin{array}{r}.03255 \\
.02980 \\
.02720\end{array}$ & $\begin{array}{l}.00248 \\
.00227 \\
.00207\end{array}$ & $\begin{array}{l}.00992 \\
.00908 \\
.00829\end{array}$ & $\begin{array}{l}3.534 \\
3.236 \\
2.954\end{array}$ & $\begin{array}{r}10.774 \\
9.863 \\
9.004\end{array}$ & $\begin{array}{r}11.606 \\
10.624 \\
9.699\end{array}$ \\
\hline $\begin{array}{l}-5 \\
-5 \\
-5\end{array}$ & $\begin{array}{l}2500 \\
2500 \\
2500\end{array}$ & $\begin{array}{l}70.0 \\
75.0 \\
80.0\end{array}$ & $\begin{array}{l}.00627 \\
.00584 \\
.00548\end{array}$ & $\begin{array}{r}.02509 \\
.02338 \\
.02195\end{array}$ & $\begin{array}{l}.00191 \\
.00178 \\
.00167\end{array}$ & $\begin{array}{l}.00764 \\
.00712 \\
.00669\end{array}$ & $\begin{array}{l}2.724 \\
2.538 \\
2.383\end{array}$ & $\begin{array}{l}8.305 \\
7.737 \\
7.265\end{array}$ & $\begin{array}{l}8.946 \\
8.334 \\
7.826\end{array}$ \\
\hline $\begin{array}{l}-5 \\
-5 \\
-5\end{array}$ & $\begin{array}{l}2500 \\
2500 \\
2500\end{array}$ & $\begin{array}{l}85.0 \\
90.0 \\
95.0\end{array}$ & $\begin{array}{l}.00515 \\
.00485 \\
.00456\end{array}$ & $\begin{array}{l}.02062 \\
.01940 \\
.01827\end{array}$ & $\begin{array}{l}.00157 \\
.00147 \\
.00139\end{array}$ & $\begin{array}{l}.00628 \\
.00591 \\
.00557\end{array}$ & $\begin{array}{l}2.239 \\
2.106 \\
1.984\end{array}$ & $\begin{array}{l}6.826 \\
6.421 \\
6.048\end{array}$ & $\begin{array}{l}7.353 \\
6.916 \\
6.515\end{array}$ \\
\hline-5 & 2500 & 100.0 & .00431 & .01725 & .00131 & .00525 & 1.873 & 5.709 & 6.150 \\
\hline
\end{tabular}


TABULATION OF DATA FOR

ABSORPTION OF SOUND IN AIR VERSUS PERCENT RELATIVE HUMIDITY AT -5 DEGREES CENTIGRADE FOR A FREQUENCY OF 3200 HERTZ

\begin{tabular}{|c|c|c|c|c|c|c|c|c|c|}
\hline 1 & (2) & (3) & $(4)$ & (5) & (6) & $(7)$ & $(8)$ & $(9)$ & $(10)$ \\
\hline $\begin{array}{l}E G R \\
\text { ENT }\end{array}$ & HERTZ & $\begin{array}{l}\text { REL } \\
\text { HUM } \\
\text { PER } \\
\text { CENT }\end{array}$ & $\begin{array}{l}\text { ATTEN } \\
\text { COEF } \\
\text { PER } \\
\text { METER }\end{array}$ & $\begin{array}{l}\text { PER } \\
\text { METER }\end{array}$ & $\begin{array}{l}\text { ATTEN } \\
\text { COEF } \\
\text { PER } \\
\text { FOOT }\end{array}$ & $\begin{array}{l}\text { PER } \\
\text { FOOT }\end{array}$ & $\begin{array}{l}\text { ATTEN } \\
\text { DB PER } \\
100 \\
\text { METER }\end{array}$ & $\begin{array}{l}\text { ATTEN } \\
\text { DB PER } \\
1000 \\
\text { FEET }\end{array}$ & $\begin{array}{l}\text { DECAY } \\
\text { RATE } \\
\text { DB PER } \\
\text { SECOND }\end{array}$ \\
\hline $\begin{array}{l}-5 \\
-5 \\
-5\end{array}$ & $\begin{array}{l}3200 \\
3200 \\
3200\end{array}$ & $\begin{array}{r}5.0 \\
10.0 \\
15.0\end{array}$ & $\begin{array}{l}.00221 \\
.00410 \\
.00598\end{array}$ & $\begin{array}{r}.00886 \\
.01640 \\
.02395\end{array}$ & $\begin{array}{l}.00067 \\
.00125 \\
.00182\end{array}$ & $\begin{array}{l}.00270 \\
.00500 \\
.00730\end{array}$ & $\begin{array}{r}.962 \\
1.781 \\
2.601\end{array}$ & $\begin{array}{l}2.932 \\
5.430 \\
7.928\end{array}$ & $\begin{array}{l}3.158 \\
5.849 \\
8.540\end{array}$ \\
\hline $\begin{array}{l}-5 \\
-5 \\
-5\end{array}$ & $\begin{array}{l}3200 \\
3200 \\
3200\end{array}$ & $\begin{array}{l}20.0 \\
25.0 \\
30.0\end{array}$ & $\begin{array}{r}.00789 \\
.00995 \\
.01162\end{array}$ & $\begin{array}{r}.03158 \\
.03982 \\
.04648\end{array}$ & $\begin{array}{r}.00240 \\
.00303 \\
.00354\end{array}$ & $\begin{array}{r}.00962 \\
.01213 \\
.01417\end{array}$ & $\begin{array}{l}3.429 \\
4.323 \\
5.047\end{array}$ & $\begin{array}{l}10.452 \\
13.179 \\
15.385\end{array}$ & $\begin{array}{l}11.258 \\
14.196 \\
16.572\end{array}$ \\
\hline-5 & $\begin{array}{l}3200 \\
3200 \\
3200\end{array}$ & $\begin{array}{l}35.0 \\
40.0 \\
45.0\end{array}$ & $\begin{array}{r}.01250 \\
.01278 \\
.01273\end{array}$ & $\begin{array}{r}.05003 \\
.05113 \\
.05094\end{array}$ & $\begin{array}{r}.00381 \\
.00389 \\
.00388\end{array}$ & $\begin{array}{r}.01525 \\
.01558 \\
.01552\end{array}$ & $\begin{array}{l}5.432 \\
5.551 \\
5.531\end{array}$ & $\begin{array}{l}16.558 \\
16.922 \\
16.858\end{array}$ & $\begin{array}{l}17.836 \\
18.228 \\
18.160\end{array}$ \\
\hline-5 & $\begin{array}{l}3200 \\
3200 \\
3200\end{array}$ & $\begin{array}{l}46.0 \\
47.0 \\
48.0\end{array}$ & $\begin{array}{r}.01268 \\
.01264 \\
.01258\end{array}$ & $\begin{array}{l}.05075 \\
.05056 \\
.05032\end{array}$ & $\begin{array}{r}.00386 \\
.00385 \\
.00383\end{array}$ & $\begin{array}{r}.01547 \\
.01541 \\
.01533\end{array}$ & $\begin{array}{l}5.510 \\
5.489 \\
5.464\end{array}$ & $\begin{array}{l}16.797 \\
16.733 \\
16.654\end{array}$ & $\begin{array}{l}18.094 \\
18.024 \\
17.940\end{array}$ \\
\hline-5 & $\begin{array}{l}3200 \\
3200 \\
3200\end{array}$ & $\begin{array}{l}49.0 \\
50.0 \\
51.0\end{array}$ & $\begin{array}{r}.01252 \\
.01243 \\
.01233\end{array}$ & $\begin{array}{r}.05008 \\
.04972 \\
.04933\end{array}$ & $\begin{array}{l}.00381 \\
.00378 \\
.00375\end{array}$ & $\begin{array}{r}.01526 \\
.01515 \\
.01503\end{array}$ & $\begin{array}{l}5.437 \\
5.398 \\
5.356\end{array}$ & $\begin{array}{l}16.574 \\
16.455 \\
16.326\end{array}$ & $\begin{array}{l}17.853 \\
17.725 \\
17.586\end{array}$ \\
\hline-5 & $\begin{array}{l}3200 \\
3200 \\
3200\end{array}$ & $\begin{array}{l}52.0 \\
53.0 \\
54.0\end{array}$ & $\begin{array}{l}.01223 \\
.01212 \\
.01201\end{array}$ & $\begin{array}{l}.04892 \\
.04851 \\
.04804\end{array}$ & $\begin{array}{l}.00372 \\
.00369 \\
.00366\end{array}$ & $\begin{array}{r}.01491 \\
.01478 \\
.01464\end{array}$ & $\begin{array}{l}5.312 \\
5.267 \\
5.216\end{array}$ & $\begin{array}{l}16.191 \\
16.054 \\
15.900 .\end{array}$ & $\begin{array}{l}17.440 \\
17 \cdot 293 \\
17.127\end{array}$ \\
\hline $\begin{array}{l}-5 \\
-5 \\
-5\end{array}$ & $\begin{array}{l}3200 \\
3200 \\
3200\end{array}$ & $\begin{array}{l}55.0 \\
60.0 \\
65.0\end{array}$ & $\begin{array}{r}.01188 \\
.01116 \\
.01040\end{array}$ & $\begin{array}{r}.04755 \\
.04465 \\
.04161\end{array}$ & $\begin{array}{l}.00362 \\
.00340 \\
.00317\end{array}$ & $\begin{array}{r}.01449 \\
.01361 \\
.01268\end{array}$ & $\begin{array}{l}5.163 \\
4.848 \\
4.518\end{array}$ & $\begin{array}{l}15.739 \\
14.778 \\
13.771\end{array}$ & $\begin{array}{l}16.953 \\
15.919 \\
14.834\end{array}$ \\
\hline $\begin{array}{l}-5 \\
-5 \\
-5\end{array}$ & $\begin{array}{l}3200 \\
3200 \\
3200\end{array}$ & $\begin{array}{l}70.0 \\
75.0 \\
80.0\end{array}$ & $\begin{array}{r}.00965 \\
.00891 \\
.00832\end{array}$ & $\begin{array}{r}.03861 \\
.03567 \\
.03331\end{array}$ & $\begin{array}{l}.00294 \\
.00271 \\
.00253\end{array}$ & $\begin{array}{r}.01177 \\
.01087 \\
.01015\end{array}$ & $\begin{array}{l}4.192 \\
3.873 \\
3.616\end{array}$ & $\begin{array}{l}12.779 \\
11.805 \\
11.024\end{array}$ & $\begin{array}{l}13.765 \\
12.716 \\
11.875\end{array}$ \\
\hline $\begin{array}{l}-5 \\
-5 \\
-5\end{array}$ & $\begin{array}{l}3200 \\
3200 \\
3200\end{array}$ & $\begin{array}{l}85.0 \\
90.0 \\
95.0\end{array}$ & $\begin{array}{l}.00781 \\
.00737 \\
.00700\end{array}$ & $\begin{array}{l}.03124 \\
.02948 \\
.02801\end{array}$ & $\begin{array}{l}.00238 \\
.00224 \\
.00213\end{array}$ & $\begin{array}{l}.00952 \\
.00898 \\
.00853\end{array}$ & $\begin{array}{l}3.392 \\
3.201 \\
3.041\end{array}$ & $\begin{array}{r}10.339 \\
9.757 \\
9.271\end{array}$ & $\begin{array}{r}11.137 \\
10.510 \\
9.986\end{array}$ \\
\hline-5 & 3200 & 0.0 & 00664 & 02657 & 00202 & .00809 & 2.885 & 8.794 & 9.472 \\
\hline
\end{tabular}


TABULATION OF DATA FOR

ABSORPTION OF SOUND IN AIR VERSUS PERCENT RELATIVE HUMIDITY AT -5 DEGREES CENTI IGRADE FOR A FREQUENCY OF 4000 HERTZ

\begin{tabular}{|c|c|c|c|c|c|c|c|c|c|}
\hline (1) & (2) & (3) & (4) & (5) & (6) & 171 & 181 & 191 & $(10)$ \\
\hline TEMP & FREQ & REL & ATTEN & $4 M$ & ATTEN & $4 M$ & ATTEN & ATTEN & DECAY \\
\hline & & HUM & COEF & & COEF & & DB PER & DB PER & RATE \\
\hline $\begin{array}{l}\text { EGR } \\
\text { ENT }\end{array}$ & HERTZ & $\begin{array}{l}\text { PER } \\
\text { CENT }\end{array}$ & $\begin{array}{l}\text { PER } \\
\text { METER }\end{array}$ & $\begin{array}{l}\text { PER } \\
\text { METER }\end{array}$ & $\begin{array}{l}\text { PER } \\
\text { FOOT }\end{array}$ & $\begin{array}{l}\text { PER } \\
\text { FOOT }\end{array}$ & $\begin{array}{l}100 \\
\text { METER }\end{array}$ & $\begin{array}{l}1000 \\
\text { FEET }\end{array}$ & $\begin{array}{l}\text { DB PER } \\
\text { SECOND }\end{array}$ \\
\hline $\begin{array}{l}-5 \\
-5 \\
-5\end{array}$ & $\begin{array}{l}4000 \\
4000 \\
4000\end{array}$ & $\begin{array}{r}5.0 \\
10.0 \\
15.0\end{array}$ & $\begin{array}{l}.00254 \\
.00457 \\
.00660\end{array}$ & $\begin{array}{r}.01017 \\
.01830 \\
.02643\end{array}$ & $\begin{array}{l}.00077 \\
.00139 \\
.00201\end{array}$ & $\begin{array}{l}.00310 \\
.00557 \\
.00805\end{array}$ & $\begin{array}{l}1.104 \\
1.987 \\
2.869\end{array}$ & $\begin{array}{l}3.367 \\
6.057 \\
8.747\end{array}$ & $\begin{array}{l}3.627 \\
6.524 \\
9.422\end{array}$ \\
\hline $\begin{array}{l}-5 \\
-5 \\
-5\end{array}$ & $\begin{array}{l}4000 \\
4000 \\
4000\end{array}$ & $\begin{array}{l}20.0 \\
25.0 \\
30.0\end{array}$ & $\begin{array}{l}.00863 \\
.01076 \\
.01295\end{array}$ & $\begin{array}{l}.03455 \\
.04306 \\
.05182\end{array}$ & $\begin{array}{l}.00263 \\
.00328 \\
.00394\end{array}$ & $\begin{array}{l}.01053 \\
.01312 \\
.01579\end{array}$ & $\begin{array}{l}3.752 \\
4.675 \\
5.626\end{array}$ & $\begin{array}{l}11.436 \\
14.251 \\
17.151\end{array}$ & $\begin{array}{l}12 \cdot 3 \\
15 \cdot 3 \\
18.4\end{array}$ \\
\hline $\begin{array}{l}-5 \\
-5 \\
-5\end{array}$ & $\begin{array}{l}4000 \\
4000 \\
4000\end{array}$ & $\begin{array}{l}35.0 \\
40.0 \\
45.0\end{array}$ & $\begin{array}{r}.01467 \\
.01564 \\
.01603\end{array}$ & $\begin{array}{l}.05870 \\
.06259 \\
.06415\end{array}$ & $\begin{array}{r}.00447 \\
.00477 \\
.00488\end{array}$ & $\begin{array}{r}.01789 \\
.01908 \\
.01955\end{array}$ & $\begin{array}{l}6.373 \\
6.796 \\
6.965\end{array}$ & $\begin{array}{l}19.427 \\
20.716 \\
21.232\end{array}$ & $\begin{array}{l}20.926 \\
22.315 \\
22.871\end{array}$ \\
\hline $\begin{array}{l}-5 \\
-5 \\
-5\end{array}$ & $\begin{array}{l}4000 \\
4000 \\
4000\end{array}$ & $\begin{array}{l}46.0 \\
47.0 \\
48.0\end{array}$ & $\begin{array}{r}.01607 \\
.01609 \\
.01610\end{array}$ & $\begin{array}{l}.06428 \\
.06438 \\
.06442\end{array}$ & $\begin{array}{r}.00489 \\
.00490 \\
.00490\end{array}$ & $\begin{array}{r}.01959 \\
.01962 \\
.01963\end{array}$ & $\begin{array}{l}6.979 \\
6.990 \\
6.995\end{array}$ & $\begin{array}{l}21 \cdot 272 \\
21.307 \\
21.321\end{array}$ & $\begin{array}{l}22.914 \\
22.952 \\
22.967\end{array}$ \\
\hline $\begin{array}{l}-5 \\
-5 \\
-5\end{array}$ & $\begin{array}{l}4000 \\
4000 \\
4000\end{array}$ & $\begin{array}{l}49.0 \\
50.0 \\
51.0\end{array}$ & $\begin{array}{l}.01609 \\
.01608 \\
.01605\end{array}$ & $\begin{array}{l}.06437 \\
.06432 \\
.06421\end{array}$ & $\begin{array}{l}.00490 \\
.00490 \\
.00489\end{array}$ & $\begin{array}{l}.019 \\
.019 \\
.019\end{array}$ & & $\begin{array}{l}21 \cdot 304 \\
21 \cdot 286 \\
21 \cdot 251\end{array}$ & $\begin{array}{l}22.948 \\
22.929 \\
22.892\end{array}$ \\
\hline $\begin{array}{l}-5 \\
-5 \\
-5\end{array}$ & $\begin{array}{l}4000 \\
4000 \\
4000\end{array}$ & $\begin{array}{l}52.0 \\
53.0 \\
54.0\end{array}$ & $\begin{array}{l}.01602 \\
.01598 \\
.01593\end{array}$ & $\begin{array}{l}.06411 \\
.06393 \\
.06372\end{array}$ & $\begin{array}{r}.00488 \\
.00487 \\
.00485\end{array}$ & $\begin{array}{l}.01954 \\
.01948 \\
.01942\end{array}$ & $\begin{array}{l}60 \\
42 \\
19\end{array}$ & $\begin{array}{l}217 \\
160 \\
090\end{array}$ & $\begin{array}{l}22.854 \\
22.793 \\
22.718\end{array}$ \\
\hline $\begin{array}{l}-5 \\
-5 \\
-5\end{array}$ & $\begin{array}{l}4000 \\
4000 \\
4000\end{array}$ & $\begin{array}{l}55.0 \\
60.0 \\
65.0\end{array}$ & $\begin{array}{r}.01587 \\
.01543 \\
.01479\end{array}$ & $\begin{array}{l}.06350 \\
.06172 \\
.05919\end{array}$ & $\begin{array}{l}.00483 \\
.00470 \\
.00451\end{array}$ & $\begin{array}{r}.01935 \\
.01881 \\
.01804\end{array}$ & $\begin{array}{l}6.895 \\
6.702 \\
6.427\end{array}$ & $\begin{array}{l}21.016 \\
20.428 \\
19.590\end{array}$ & $\begin{array}{l}22.638 \\
22.005 \\
21.102\end{array}$ \\
\hline-2 & 4000 & $70 \cdot 0$ & .01400 & .05 & .004 & .017 & 80 & $18 \cdot 534$ & 19.965 \\
\hline-5 & $\begin{array}{l}4000 \\
4000\end{array}$ & $\begin{array}{l}75.0 \\
80.0\end{array}$ & $\begin{array}{r}.01318 \\
.01237\end{array}$ & $\begin{array}{r}.05272 \\
.04950\end{array}$ & $\begin{array}{l}.00401 \\
.00377\end{array}$ & $\begin{array}{l}.01 \\
.01\end{array}$ & $\begin{array}{l}5.724 \\
5.374\end{array}$ & $\begin{array}{l}17 \cdot 449 \\
16 \cdot 382\end{array}$ & $\begin{array}{l}18.796 \\
17.646\end{array}$ \\
\hline $\begin{array}{l}-5 \\
-5\end{array}$ & $\begin{array}{l}4000 \\
4000 \\
4000\end{array}$ & $\begin{array}{l}85.0 \\
90.0 \\
95.0\end{array}$ & $\begin{array}{l}.01154 \\
.01086 \\
.01025\end{array}$ & $\begin{array}{l}.04618 \\
.04346 \\
.04102\end{array}$ & $\begin{array}{l}.00351 \\
.00331 \\
.00312\end{array}$ & $\begin{array}{r}.01407 \\
.01324 \\
.01250\end{array}$ & $\begin{array}{l}5.014 \\
4.718 \\
4.453\end{array}$ & $\begin{array}{l}15.283 \\
14.383 \\
13.575\end{array}$ & $\begin{array}{l}16.463 \\
15.493 \\
14.623\end{array}$ \\
\hline-5 & 4000 & 00.0 & .00972 & 03891 & .00296 & .01186 & $4 \cdot 224$ & 12.877 & 13.871 \\
\hline
\end{tabular}


TABULATION OF DATA FOR

ABSORPTION OF SOUND IN AIR VERSUS PERCENT RELATIVE HUMIDITY AT -5 DEGREES CENTIGRADE FOR A FREQUENCY OF 5000 HERTZ
(1)
(2)
(3)
(4)
(5)
(6)
(8)
(9)
(10)

TEMP FREQ REL

ATTEN 4M ATTEN 4M

HUM COEF

DEGR

PER

PER

CENT HERTZ CENT

METER

PER

COEF

PER

$\begin{array}{lll}-5 & 5000 \quad 5.0\end{array}$

.00299

METER

FO०T

PER

$\begin{array}{lll}-5 & 5000 \quad 10 \cdot 0\end{array}$

.00299

.01199

.00091

FOOT

$\begin{array}{lll}-5 & 5000 \quad 15.0\end{array}$

.00739

.02078

.00158

.00365

.00633

.00225 .00901

$\begin{array}{lll}-5 & 5000 & 20.0\end{array}$

.00959

.03837

.00292

.01169

.01179

.04717

.00359

.01437

.01416

.05667

.00431

.01727

.01650

.06603

.00503 .02012

.01837

.07350

.00560

.02240

.01951

.07805

.00594 .02379

$\begin{array}{lll}\text { ATTEN } & \text { ATTEN } & \text { DECAY } \\ \text { DB PER } & \text { DB PER } & \text { RATE } \\ 100 & 1000 & \text { DB PER } \\ \text { METER } & \text { FEET } & \text { SECOND }\end{array}$

I. 302

3.968

4.275

2.256

3.211

6.878

9.788

7.409

4.166

12.698

5.122

15.613

6.153

18.757

10.544

7.169

21.852

7.980

$24 \cdot 326$

8.475

25.832

13.678

16.818

20.205

\section{$\begin{array}{lll}-5 & 5000 \quad 46.0\end{array}$}

.01968

.07873

$.00599 \quad .02399$

.01983

.07934

.00604

.02418

.01993

.07974

.00607

.02430

8.549

26.058

8.615

26.259

23.539

$-5500048.0$

.02003

.08014

.00610

.02442

.02011

.08044

.00612

.02451

.02015

.08063

.00614 .02457

8.658

26.391

26.203

8.701

8.733

26.522

26.621

8.755

$26 \cdot 687$

$27 \cdot 826$

$\begin{array}{lll}-5 & 5000 \quad 52.0\end{array}$

.02020

.08083

$.00615 \quad .02463$

8.777

26.752

$8.791 \quad 26.797$

28.069

$.02024 \quad .08097$

$.00617 \quad .02468$

8.803

26.834

28.286

$-55000$

54.0

.08108

.00617 .02471

8.816

26.872

.00618 .02474

$.00615 \quad .02462$

.02019

.08078

.00606 .02426

8.771

26.736

28.428

$\begin{array}{lll}-5 & 5000 \quad 65.0\end{array}$

.01990

.07961

8.643

26.346

28.569

28.676

28.746

\section{$\begin{array}{lll}-5 & 5000 \quad 70.0\end{array}$}

.01936

.07747

.00590

.02361

.01867

.07470

.00569

.02277

8.41

25.640

28.817

-5
$-5000 \quad 80.0$

.01781

.07127

.00543 .02172

8.111

24.722

7.738

23.587

28.865

28.906

$$
\begin{aligned}
& -5 \\
& -5 \\
& -5 \\
& -5
\end{aligned}
$$

.01693

.01605

.06772

.00516

.02064

.06421

.00489

.01957

.01517

.06069

.00462

.01849

7.353

22.413

6.971

21.249

28.946

28.799

28.380

.01430

$.05723 \quad .00436 \quad .01744$

6.589

20.085

27.619

26.631

25.407

$6.214 \quad 18.940 \quad 20.402$ 
TABULATION OF DATA FOR

ABSORPTION OF SOUND IN AIR VERSUS PERCENT RELATIVE HUMIDITY AT -5 DEGREES CENTIGRADE FOR A FREQUENCY OF 5940 HERTZ

\begin{tabular}{|c|c|c|c|c|c|c|c|c|c|}
\hline (1) & (2) & $(3)$ & $(4)$ & (5) & (6) & $(7)$ & (8) & (9) & $(10)$ \\
\hline TEMP & FREQ & $\begin{array}{l}\text { REL } \\
\text { HUM }\end{array}$ & $\begin{array}{l}\text { ATTEN } \\
\text { COEF }\end{array}$ & $4 M$ & $\begin{array}{l}\text { ATTEN } \\
\text { COEF }\end{array}$ & $4 M$ & $\begin{array}{l}\text { ATTEN } \\
\text { DB PER }\end{array}$ & $\begin{array}{l}\text { ATTEN } \\
\text { DB PER }\end{array}$ & $\begin{array}{l}\text { DECAY } \\
\text { RATE }\end{array}$ \\
\hline $\begin{array}{l}\text { DEGR } \\
\text { CENT }\end{array}$ & HERTZ & $\begin{array}{l}\text { PER } \\
\text { CENT }\end{array}$ & $\begin{array}{l}\text { PER } \\
\text { METER }\end{array}$ & $\begin{array}{l}\text { PER } \\
\text { METER }\end{array}$ & $\begin{array}{l}\text { PER } \\
\text { FOOT }\end{array}$ & $\begin{array}{l}\text { PER } \\
\text { FOOT }\end{array}$ & $\begin{array}{l}100 \\
\text { METER }\end{array}$ & $\begin{array}{l}1000 \\
\text { FEET }\end{array}$ & $\begin{array}{l}\text { DB PER } \\
\text { SECOND }\end{array}$ \\
\hline $\begin{array}{l}-5 \\
-5 \\
-5\end{array}$ & $\begin{array}{l}5940 \\
5940 \\
5940\end{array}$ & $\begin{array}{r}5.0 \\
10.0 \\
15.0\end{array}$ & $\begin{array}{r}.00344 \\
.00576 \\
.00807\end{array}$ & $\begin{array}{l}.01378 \\
.02304 \\
.03231\end{array}$ & $\begin{array}{l}.00105 \\
.00175 \\
.00246\end{array}$ & $\begin{array}{l}.00420 \\
.00702 \\
.00984\end{array}$ & $\begin{array}{l}1.496 \\
2.502 \\
3.508\end{array}$ & $\begin{array}{r}4.561 \\
7.627 \\
10.694\end{array}$ & $\begin{array}{r}4.913 \\
8.216 \\
11.519\end{array}$ \\
\hline $\begin{array}{l}-5 \\
-5\end{array}$ & $\begin{array}{l}5940 \\
5940\end{array}$ & $\begin{array}{l}20.0 \\
25.0\end{array}$ & $\begin{array}{l}.01039 \\
.01271\end{array}$ & $\begin{array}{r}.04158 \\
.05084\end{array}$ & $\begin{array}{r}.00316 \\
.00387\end{array}$ & $\begin{array}{r}.01267 \\
.01549\end{array}$ & $\begin{array}{l}4.514 \\
5.520\end{array}$ & $\begin{array}{l}13.761 \\
16.827\end{array}$ & $\begin{array}{l}14.823 \\
18.126\end{array}$ \\
\hline-5 & 5940 & 30.0 & .01505 & .06023 & .00459 & .01836 & 6.540 & 19.935 & 21.474 \\
\hline $\begin{array}{l}-5 \\
-5 \\
-5\end{array}$ & $\begin{array}{l}5940 \\
5940 \\
5940\end{array}$ & $\begin{array}{l}35.0 \\
40.0 \\
45.0\end{array}$ & $\begin{array}{r}.01761 \\
.02005 \\
.02198\end{array}$ & $\begin{array}{l}.07044 \\
.08020 \\
.08792\end{array}$ & $\begin{array}{l}.00536 \\
.00611 \\
.00670\end{array}$ & $\begin{array}{l}.02147 \\
.02444 \\
.02680\end{array}$ & $\begin{array}{l}7.648 \\
8.708 \\
9.546\end{array}$ & $\begin{array}{l}23.312 \\
26.542 \\
29.099\end{array}$ & $\begin{array}{l}25 \cdot 111 \\
28.591 \\
31.345\end{array}$ \\
\hline $\begin{array}{l}-5 \\
-5 \\
-5\end{array}$ & $\begin{array}{l}5940 \\
5940 \\
5940\end{array}$ & $\begin{array}{l}46.0 \\
47.0 \\
48.0\end{array}$ & $\begin{array}{l}.02228 \\
.02255 \\
.02278\end{array}$ & $\begin{array}{l}.08915 \\
.09023 \\
.09115\end{array}$ & $\begin{array}{l}.00679 \\
.00687 \\
.00694\end{array}$ & $\begin{array}{l}.02717 \\
.02750 \\
.02778\end{array}$ & $\begin{array}{l}9.679 \\
9.796 \\
9.897\end{array}$ & $\begin{array}{l}29.504 \\
29.860 \\
30.167\end{array}$ & $\begin{array}{l}31.782 \\
32.165 \\
32.495\end{array}$ \\
\hline $\begin{array}{l}-5 \\
-5\end{array}$ & $\begin{array}{l}5940 \\
5940\end{array}$ & $\begin{array}{l}49.0 \\
50.0\end{array}$ & $\begin{array}{l}.02302 \\
.02323\end{array}$ & $\begin{array}{l}.09208 \\
.09292\end{array}$ & $\begin{array}{l}.00701 \\
.00708\end{array}$ & $\begin{array}{l}.02806 \\
.02832\end{array}$ & $\begin{array}{r}9.997 \\
10.089\end{array}$ & $\begin{array}{l}30.474 \\
30.753\end{array}$ & $\begin{array}{l}32.826 \\
33.126\end{array}$ \\
\hline-5 & 5940 & 51.0 & .02341 & .09364 & .00713 & .02854 & 10.167 & 30.990 & $33 \cdot 382$ \\
\hline $\begin{array}{l}-5 \\
-5 \\
-5\end{array}$ & $\begin{array}{l}5940 \\
5940 \\
5940\end{array}$ & $\begin{array}{l}52.0 \\
53.0 \\
54.0\end{array}$ & $\begin{array}{r}.02359 \\
.02374 \\
.02385\end{array}$ & $\begin{array}{r}.09436 \\
.09498 \\
.09540\end{array}$ & $\begin{array}{r}.00719 \\
.00723 \\
.00727\end{array}$ & $\begin{array}{r}.02876 \\
.02895 \\
.02908\end{array}$ & $\begin{array}{l}10.245 \\
10.313 \\
10.358\end{array}$ & $\begin{array}{l}31.227 \\
31.435 \\
31.574\end{array}$ & $\begin{array}{l}33.638 \\
33.862 \\
34.011\end{array}$ \\
\hline $\begin{array}{l}-5 \\
-5 \\
-5\end{array}$ & $\begin{array}{l}5940 \\
5940 \\
5940\end{array}$ & $\begin{array}{l}55.0 \\
60.0 \\
65.0\end{array}$ & $\begin{array}{r}.02395 \\
.02423 \\
.02425\end{array}$ & $\begin{array}{l}.09582 \\
.09594 \\
.09700\end{array}$ & $\begin{array}{r}.00730 \\
.00738 \\
.00739\end{array}$ & $\begin{array}{r}.02920 \\
.02954 \\
.02956\end{array}$ & $\begin{array}{l}10.404 \\
10.525 \\
10.531\end{array}$ & $\begin{array}{l}31.712 \\
32.081 \\
32.101\end{array}$ & $\begin{array}{l}34.160 \\
34.557 \\
34.579\end{array}$ \\
\hline $\begin{array}{l}-5 \\
-5 \\
-5\end{array}$ & $\begin{array}{l}5940 \\
5940 \\
5940\end{array}$ & $\begin{array}{l}70.0 \\
75.0 \\
80.0\end{array}$ & $\begin{array}{l}.02404 \\
.02366 \\
.02305\end{array}$ & $\begin{array}{l}.09619 \\
.09466 \\
.09220\end{array}$ & $\begin{array}{l}.00732 \\
.00721 \\
.00702\end{array}$ & $\begin{array}{l}.02931 \\
.02885 \\
.02810\end{array}$ & $\begin{array}{l}10.444 \\
10.278 \\
10.010\end{array}$ & $\begin{array}{l}31.834 \\
31.329 \\
30.513\end{array}$ & $\begin{array}{l}34.291 \\
33.748 \\
32.868\end{array}$ \\
\hline $\begin{array}{l}-5 \\
-5 \\
-5\end{array}$ & $\begin{array}{l}5940 \\
5940 \\
5940\end{array}$ & $\begin{array}{l}85.0 \\
90.0 \\
95.0\end{array}$ & $\begin{array}{r}.02228 \\
.02138 \\
.02044\end{array}$ & $\begin{array}{l}.08915 \\
.08552 \\
.08178\end{array}$ & $\begin{array}{l}.00679 \\
.00651 \\
.00623\end{array}$ & $\begin{array}{l}.02717 \\
.02606 \\
.02492\end{array}$ & $\begin{array}{l}9.679 \\
9.285 \\
8.879\end{array}$ & $\begin{array}{l}29.503 \\
28.302 \\
27.066\end{array}$ & $\begin{array}{l}31.780 \\
30.487 \\
29.155\end{array}$ \\
\hline-5 & 5940 & 100.0 & .01951 & .07806 & .00594 & .02379 & 8.475 & 25.833 & 27.827 \\
\hline
\end{tabular}


TABULATION OF DATA FOR

ABSERPTION OF SOUND IN AIR VERSUS PERCENT RELATIVE HUMIDITY AT -5 DEGREES CENTIGRADE FOR A FREQUENCY OF 6300 HERTZ

\begin{tabular}{|c|c|c|c|c|c|c|c|c|c|}
\hline$(1)$ & $(2)$ & (3) & (4) & (5) & (6) & 171 & $(8)$ & $(9)$ & $(10)$ \\
\hline $\begin{array}{l}\text { EMP } \\
\text { EGR } \\
\text { ENT }\end{array}$ & HERTZ & $\begin{array}{l}\text { REL } \\
\text { HUM } \\
\text { PER } \\
\text { CENT }\end{array}$ & $\begin{array}{l}\text { ATTEN } \\
\text { COEF } \\
\text { PER } \\
\text { METER }\end{array}$ & $\begin{array}{l}\text { 4M } \\
\text { PER } \\
\text { METER }\end{array}$ & $\begin{array}{l}\text { ATTEN } \\
\text { COEF } \\
\text { PER } \\
\text { FOOT }\end{array}$ & $\begin{array}{l}4 M \\
\text { PER } \\
\text { FGOT }\end{array}$ & $\begin{array}{l}\text { ATTEN } \\
\text { DB PER } \\
100 \\
\text { METER }\end{array}$ & $\begin{array}{l}\text { ATTEN } \\
\text { DB PER } \\
1000 \\
\text { FEET }\end{array}$ & $\begin{array}{l}\text { DECAY } \\
\text { RATE } \\
\text { DB PER } \\
\text { SECOND }\end{array}$ \\
\hline $\begin{array}{l}-5 \\
-5 \\
-5\end{array}$ & $\begin{array}{l}6300 \\
6300 \\
6300\end{array}$ & $\begin{array}{r}5.0 \\
1000 \\
15.0\end{array}$ & $\begin{array}{l}.00365 \\
.00603 \\
.00841\end{array}$ & $\begin{array}{r}.01460 \\
.02412 \\
.03364\end{array}$ & $\begin{array}{l}.00 \\
.00 \\
.00\end{array}$ & $\begin{array}{r}.00445 \\
.00735 \\
.01025\end{array}$ & $\begin{array}{l}1.585 \\
2.619 \\
3.652\end{array}$ & $\begin{array}{r}4.832 \\
7.982 \\
11.133\end{array}$ & $\begin{array}{r}5.204 \\
8.599 \\
11.993\end{array}$ \\
\hline $\begin{array}{l}-5 \\
-5 \\
-5\end{array}$ & $\begin{array}{l}6300 \\
6300 \\
6300\end{array}$ & $\begin{array}{l}20.0 \\
25.0 \\
30.0\end{array}$ & $\begin{array}{r}.01079 \\
.01317 \\
.01556\end{array}$ & $\begin{array}{l}.04316 \\
.05268 \\
.06227\end{array}$ & $\begin{array}{l}.00328 \\
.00401 \\
.00474\end{array}$ & $\begin{array}{l}315 \\
605 \\
898\end{array}$ & $\begin{array}{l}4.6 \\
5.7 \\
6.7\end{array}$ & $\begin{array}{l}284 \\
435 \\
510\end{array}$ & $\begin{array}{l}387 \\
781 \\
201\end{array}$ \\
\hline $\begin{array}{l}-5 \\
-5 \\
-5\end{array}$ & $\begin{array}{l}6300 \\
6300 \\
6300\end{array}$ & $\begin{array}{l}35.0 \\
40.0 \\
45.0\end{array}$ & $\begin{array}{l}.01816 \\
.02070 \\
.02292\end{array}$ & $\begin{array}{r}.07265 \\
.08281 \\
.09171\end{array}$ & $\begin{array}{l}.00 \\
.00 \\
.00\end{array}$ & $\begin{array}{l}.0 \\
.0 \\
.0\end{array}$ & & & $\begin{array}{l}902 \\
523 \\
696\end{array}$ \\
\hline $\begin{array}{l}-5 \\
-5 \\
-5\end{array}$ & $\begin{array}{l}6300 \\
6300 \\
6300\end{array}$ & $\begin{array}{l}46.0 \\
47.0 \\
48.0\end{array}$ & $\begin{array}{l}.02 \\
.02 \\
.02\end{array}$ & $\begin{array}{l}.0 \\
.0 \\
.0\end{array}$ & $\begin{array}{l}.08 \\
.08 \\
.08\end{array}$ & & & & $\begin{array}{l}145 \\
594 \\
343\end{array}$ \\
\hline $\begin{array}{l}-5 \\
-5 \\
-5\end{array}$ & $\begin{array}{l}6300 \\
6300 \\
6300\end{array}$ & $\begin{array}{l}50 \\
5\end{array}$ & $\begin{array}{l}.02 \\
.02 \\
.02\end{array}$ & $\begin{array}{l}.0 \\
.0 \\
.0\end{array}$ & $\begin{array}{l}.08 \\
.08 \\
.08\end{array}$ & & & & $\begin{array}{l}382 \\
722 \\
061\end{array}$ \\
\hline $\begin{array}{l}-5 \\
-5 \\
-5\end{array}$ & $\begin{array}{l}6300 \\
6300 \\
6300\end{array}$ & $\begin{array}{l}52.0 \\
53.0 \\
54.0\end{array}$ & $\begin{array}{r}.02478 \\
.02496 \\
.02515\end{array}$ & $\begin{array}{r}.09913 \\
.09987 \\
.10060\end{array}$ & $\begin{array}{r}.00755 \\
.00761 \\
.00766\end{array}$ & $\begin{array}{l}.03021 \\
.03044 \\
.03066\end{array}$ & & & $\begin{array}{l}339 \\
502 \\
865\end{array}$ \\
\hline $\begin{array}{l}-5 \\
-5 \\
-5\end{array}$ & $\begin{array}{l}6300 \\
6300 \\
6300\end{array}$ & $\begin{array}{l}55.0 \\
60.0 \\
65.0\end{array}$ & $\begin{array}{l}.02 \\
.02 \\
.02\end{array}$ & $\begin{array}{r}.10116 \\
.10279 \\
.10329\end{array}$ & $\begin{array}{r}.00770 \\
.00783 \\
.00787\end{array}$ & & $\begin{array}{l}10 \cdot 983 \\
11 \cdot 160 \\
11 \cdot 215\end{array}$ & & $\begin{array}{l}36.062 \\
36.644 \\
36.824\end{array}$ \\
\hline $\begin{array}{l}-5 \\
-5 \\
-5\end{array}$ & $\begin{array}{l}6300 \\
6300 \\
6300\end{array}$ & $\begin{array}{l}70.0 \\
75.0 \\
80.0\end{array}$ & $\begin{array}{r}.02570 \\
.02538 \\
.02485\end{array}$ & $\begin{array}{r}10281 \\
.10155 \\
.09942\end{array}$ & $\begin{array}{r}.00783 \\
.00773 \\
.00757\end{array}$ & $\begin{array}{l}.0 \\
.0 \\
.0\end{array}$ & $\begin{array}{l}11 \cdot 163 \\
11 \cdot 026 \\
10.795\end{array}$ & $\begin{array}{l}34.027 \\
33.601 \\
32.905\end{array}$ & $\begin{array}{l}36.653 \\
36.202 \\
35.444\end{array}$ \\
\hline $\begin{array}{l}-5 \\
-5 \\
-5\end{array}$ & $\begin{array}{l}6300 \\
6300 \\
6300\end{array}$ & $\begin{array}{l}85.0 \\
90 \cdot 0 \\
95.0\end{array}$ & $\begin{array}{l}.02416 \\
.02330 \\
.02234\end{array}$ & $\begin{array}{r}.09665 \\
.09322 \\
.08938\end{array}$ & $\begin{array}{r}.00736 \\
.00710 \\
.00681\end{array}$ & $\begin{array}{l}.02945 \\
.02841 \\
.02724\end{array}$ & $\begin{array}{r}10.494 \\
10.121 \\
9.705\end{array}$ & $\begin{array}{l}31.986 \\
30.851 \\
29.581\end{array}$ & $\begin{array}{l}34.455 \\
33.233 \\
31.864\end{array}$ \\
\hline- & 6300 & $00 \bullet 0$ & 02138 & .08554 & .00651 & .02607 & $9 \cdot 288$ & $28 \cdot 310$ & 30.496 \\
\hline
\end{tabular}


TABULATION OF DATA FOR

ABSORPTION OF SOUND IN AIR VERSUS PERCENT RELATIVE HUMIDITY AT -5 DEGREES CENTIGRADE FOR A FREQUENCY OF 8000 HERTZ

\begin{tabular}{|c|c|c|c|c|c|c|c|c|c|}
\hline (i) & (2) & $(3)$ & (4) & (5) & (6) & (7) & $(8)$ & $(9)$ & $(10)$ \\
\hline TEMP & FREQ & REL & ATTEN & $4 M$ & ATTEN & $4 M$ & ATTEN & ATTEN & DECAY \\
\hline $\begin{array}{l}\text { DEGR } \\
\text { CENT }\end{array}$ & HERTZ & $\begin{array}{l}\text { HUM } \\
\text { PER } \\
\text { CENT }\end{array}$ & $\begin{array}{l}\text { COEF } \\
\text { PER } \\
\text { METER }\end{array}$ & $\begin{array}{l}\text { PER } \\
\text { METER }\end{array}$ & 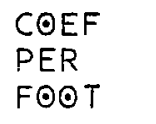 & $\begin{array}{l}\text { PER. } \\
\text { FOOT }\end{array}$ & $\begin{array}{l}\text { DB PER } \\
100 \\
\text { METER }\end{array}$ & $\begin{array}{l}\text { DB PER } \\
1000 \\
\text { FEET }\end{array}$ & $\begin{array}{l}\text { RATE } \\
\text { DB PER } \\
\text { SECOND }\end{array}$ \\
\hline-5 & 8000 & 5.0 & .00463 & .01854 & .00141 & .00565 & 2.013 & 6.137 & 6.611 \\
\hline $\begin{array}{l}-5 \\
-5\end{array}$ & $\begin{array}{l}8000 \\
8000\end{array}$ & $\begin{array}{l}10.0 \\
15.0\end{array}$ & $\begin{array}{r}.00723 \\
.00982\end{array}$ & $\begin{array}{l}.02893 \\
.03931\end{array}$ & $\begin{array}{l}.00220 \\
.00299\end{array}$ & $\begin{array}{l}.00881 \\
.01198\end{array}$ & $\begin{array}{l}3 \cdot 141 \\
4 \cdot 268\end{array}$ & $\begin{array}{r}9.574 \\
13.011\end{array}$ & $\begin{array}{l}10.313 \\
14.016\end{array}$ \\
\hline $\begin{array}{l}-5 \\
-5 \\
-5\end{array}$ & $\begin{array}{l}8000 \\
8000 \\
8000\end{array}$ & $\begin{array}{l}20.0 \\
25.0 \\
30.0\end{array}$ & $\begin{array}{r}.01242 \\
.01502 \\
.01761\end{array}$ & $\begin{array}{l}.04970 \\
.06009 \\
.07047\end{array}$ & $\begin{array}{r}.00378 \\
.00457 \\
.00537\end{array}$ & $\begin{array}{r}.01514 \\
.01831 \\
.02148\end{array}$ & $\begin{array}{l}5.396 \\
6.524 \\
7.651\end{array}$ & $\begin{array}{l}16.449 \\
19.886 \\
23.323\end{array}$ & $\begin{array}{l}17.718 \\
21.421 \\
25.123\end{array}$ \\
\hline $\begin{array}{l}-5 \\
-5 \\
-5\end{array}$ & $\begin{array}{l}8000 \\
8000 \\
8000\end{array}$ & $\begin{array}{l}35.0 \\
40.0 \\
45.0\end{array}$ & $\begin{array}{r}.02024 \\
.02305 \\
.02588\end{array}$ & $\begin{array}{l}.08096 \\
.09223 \\
.10352\end{array}$ & $\begin{array}{l}.00616 \\
.00702 \\
.00788\end{array}$ & $\begin{array}{l}.02467 \\
.02811 \\
.03155\end{array}$ & $\begin{array}{r}8.790 \\
10.014 \\
11.240\end{array}$ & $\begin{array}{l}26.793 \\
30.524 \\
34.261\end{array}$ & $\begin{array}{l}28.861 \\
32.880 \\
36.905\end{array}$ \\
\hline $\begin{array}{l}-5 \\
-5 \\
-5\end{array}$ & $\begin{array}{l}8000 \\
8000 \\
8000\end{array}$ & $\begin{array}{l}46.0 \\
47.0 \\
48.0\end{array}$ & $\begin{array}{r}.02641 \\
.02695 \\
.02748\end{array}$ & $\begin{array}{r}.10567 \\
.10781 \\
.10995\end{array}$ & $\begin{array}{l}.00805 \\
.00821 \\
.00837\end{array}$ & $\begin{array}{l}.03220 \\
.03286 \\
.03351\end{array}$ & $\begin{array}{l}11.473 \\
11.706 \\
11.938\end{array}$ & $\begin{array}{l}34.970 \\
35.680 \\
36.389\end{array}$ & $\begin{array}{l}37.669 \\
38.434 \\
39.198\end{array}$ \\
\hline $\begin{array}{l}-5 \\
-5 \\
-5\end{array}$ & $\begin{array}{l}8000 \\
8000 \\
8000\end{array}$ & $\begin{array}{l}49.0 \\
50.0 \\
51.0\end{array}$ & $\begin{array}{r}.02798 \\
.02845 \\
.02892\end{array}$ & $\begin{array}{l}.11194 \\
.11382 \\
.11570\end{array}$ & $\begin{array}{l}.00853 \\
.00867 \\
.00881\end{array}$ & $\begin{array}{l}.03412 \\
.03469 \\
.03526\end{array}$ & $\begin{array}{l}12.154 \\
12.358 \\
12.562\end{array}$ & $\begin{array}{l}37.048 \\
37.669 \\
38.290\end{array}$ & $\begin{array}{l}39.908 \\
40.576 \\
41.245\end{array}$ \\
\hline $\begin{array}{l}-5 \\
-5 \\
-5\end{array}$ & $\begin{array}{l}8000 \\
8000 \\
8000\end{array}$ & $\begin{array}{l}52.0 \\
53.0 \\
54.0\end{array}$ & $\begin{array}{l}.02939 \\
.02975 \\
.03010\end{array}$ & $\begin{array}{l}.11757 \\
.11902 \\
.12040\end{array}$ & $\begin{array}{l}.00895 \\
.00906 \\
.00917\end{array}$ & $\begin{array}{l}.03583 \\
.03627 \\
.03669\end{array}$ & $\begin{array}{l}12.766 \\
12.923 \\
13.072\end{array}$ & $\begin{array}{l}38.911 \\
39.390 \\
39.845\end{array}$ & $\begin{array}{l}41.914 \\
42.431 \\
42.920\end{array}$ \\
\hline $\begin{array}{l}-5 \\
-5 \\
-5\end{array}$ & $\begin{array}{l}8000 \\
8000 \\
8000\end{array}$ & $\begin{array}{l}55.0 \\
60.0 \\
65.0\end{array}$ & $\begin{array}{r}.03044 \\
.03179 \\
.03265\end{array}$ & $\begin{array}{r}.12177 \\
.12716 \\
.13062\end{array}$ & $\begin{array}{l}.00927 \\
.00969 \\
.00995\end{array}$ & $\begin{array}{r}.037 .11 \\
.03876 \\
.03981\end{array}$ & $\begin{array}{l}13.221 \\
13.807 \\
14.182\end{array}$ & $\begin{array}{l}40.300 \\
42.084 \\
43.229\end{array}$ & $\begin{array}{l}43.410 \\
45.332 \\
46.565\end{array}$ \\
\hline $\begin{array}{l}-5 \\
-5 \\
-5\end{array}$ & $\begin{array}{l}8000 \\
8000 \\
8000\end{array}$ & $\begin{array}{l}70.0 \\
75.0 \\
80.0\end{array}$ & $\begin{array}{l}.03306 \\
.03323 \\
.03311\end{array}$ & $\begin{array}{r}.13227 \\
.13292 \\
.13247\end{array}$ & $\begin{array}{l}.01007 \\
.01012 \\
.01009\end{array}$ & $\begin{array}{r}.04031 \\
.04051 \\
.04037\end{array}$ & $\begin{array}{l}14 \cdot 362 \\
14 \cdot 432 \\
14 \cdot 383\end{array}$ & $\begin{array}{l}43.776 \\
43.990 \\
43.840\end{array}$ & $\begin{array}{l}47 \cdot 155 \\
47 \cdot 386 \\
47 \cdot 224\end{array}$ \\
\hline $\begin{array}{l}-5 \\
-5 \\
-5\end{array}$ & $\begin{array}{l}8000 \\
8000 \\
8000\end{array}$ & $\begin{array}{l}85.0 \\
90.0 \\
95.0\end{array}$ & $\begin{array}{l}.03284 \\
.03241 \\
.03172\end{array}$ & $\begin{array}{r}.13139 \\
.12964 \\
.12690\end{array}$ & $\begin{array}{l}.01001 \\
.00987 \\
.00966\end{array}$ & $\begin{array}{l}.04004 \\
.03951 \\
.03867\end{array}$ & $\begin{array}{l}14.266 \\
14.076 \\
13.778\end{array}$ & $\begin{array}{l}43.483 \\
42.904 \\
41.996\end{array}$ & $\begin{array}{l}46.839 \\
46.215 \\
45.237\end{array}$ \\
\hline - & 8000 & 100.0 & .03093 & .12373 & .00942 & .03771 & 13.434 & 40.949 & 44.109 \\
\hline
\end{tabular}


TABULATION OF DATA FOR

ABSORPTION OF SOUND IN AIR VERSUS PERCENT RELATIVE HUMIDITY AT -5 DEGREES CENTIGRADE FOR A FREQUENCY OF 10000 HERTZ

\begin{tabular}{|c|c|c|c|c|c|c|c|c|c|}
\hline$(1)$ & $(2)$ & (3) & $(4)$ & (5) & (6) & $(7)$ & $(8)$ & 191 & $(10)$ \\
\hline $\begin{array}{l}\text { TEMP } \\
\text { DEGR } \\
\text { CENT }\end{array}$ & HERTZ & $\begin{array}{l}\text { REL } \\
\text { HUM } \\
\text { PER } \\
\text { CENT }\end{array}$ & $\begin{array}{l}\text { ATTEN } \\
\text { COEF } \\
\text { PER } \\
\text { METER }\end{array}$ & $\begin{array}{l}4 M \\
\text { PER } \\
\text { METER }\end{array}$ & $\begin{array}{l}\text { ATTEN } \\
\text { COEF } \\
\text { PER } \\
\text { FOOT }\end{array}$ & $\begin{array}{l}4 M \\
\text { PER } \\
\text { FOO.T }\end{array}$ & $\begin{array}{l}\text { AT TEN } \\
\text { DB PER } \\
100 \\
\text { METER }\end{array}$ & $\begin{array}{l}\text { ATTEN } \\
\text { DB PER } \\
1000 \\
\text { FEET }\end{array}$ & $\begin{array}{l}\text { DECAY } \\
\text { RATE } \\
\text { DB PER } \\
\text { SECOND }\end{array}$ \\
\hline $\begin{array}{l}-5 \\
-5 \\
-5\end{array}$ & $\begin{array}{l}10000 \\
10000 \\
10000\end{array}$ & $\begin{array}{r}5.0 \\
10.0 \\
15.0\end{array}$ & $\begin{array}{r}.00602 \\
.00884 \\
.01166\end{array}$ & $\begin{array}{r}.02408 \\
.03537 \\
.04665\end{array}$ & $\begin{array}{l}.00183 \\
.00269 \\
.00355\end{array}$ & $\begin{array}{r}.00734 \\
.01078 \\
.01422\end{array}$ & $\begin{array}{l}2.615 \\
3.840 \\
5.065\end{array}$ & $\begin{array}{r}7.971 \\
11.705 \\
15.440\end{array}$ & $\begin{array}{r}8.586 \\
12.609 \\
16.632\end{array}$ \\
\hline $\begin{array}{l}-5 \\
-5 \\
-5\end{array}$ & $\begin{array}{l}10000 \\
10000 \\
10000\end{array}$ & $\begin{array}{l}20.0 \\
25.0 \\
30.0\end{array}$ & $\begin{array}{r}.01448 \\
.01730 \\
.02012\end{array}$ & $\begin{array}{r}.05794 \\
.06922 \\
.08051\end{array}$ & $\begin{array}{l}.00441 \\
.00527 \\
.00613\end{array}$ & $\begin{array}{r}.01766 \\
.02110 \\
.02454\end{array}$ & $\begin{array}{l}6.291 \\
7.516 \\
8.741\end{array}$ & $\begin{array}{l}19.175 \\
22.910 \\
26.645\end{array}$ & $\begin{array}{l}20.655 \\
24.679 \\
28.702\end{array}$ \\
\hline-5 & 10000 & 35.0 & .02295 & .09180 & .00699 & .02798 & 9.967 & $30 \cdot 380$ & 32.725 \\
\hline $\begin{array}{l}-5 \\
-5\end{array}$ & $\begin{array}{l}10000 \\
10000\end{array}$ & $\begin{array}{l}40.0 \\
45.0\end{array}$ & $\begin{array}{l}.02 \\
.02\end{array}$ & $\begin{array}{r}.10319 \\
.11531\end{array}$ & $\begin{array}{l}.00786 \\
.00878\end{array}$ & $\begin{array}{r}.03145 \\
.03514\end{array}$ & $\begin{array}{l}11.204 \\
12.519\end{array}$ & $\begin{array}{l}34 \\
38\end{array}$ & $\begin{array}{l}36.786 \\
41.106\end{array}$ \\
\hline $\begin{array}{l}-5 \\
-5 \\
-5\end{array}$ & $\begin{array}{l}10000 \\
10000 \\
10000\end{array}$ & $\begin{array}{l}46.0 \\
47.0 \\
48.0\end{array}$ & $\begin{array}{l}.02 \\
.03 \\
.03\end{array}$ & $\begin{array}{r}.11785 \\
.12040 \\
.12286\end{array}$ & $\begin{array}{l}.00898 \\
.00917 \\
.00936\end{array}$ & $\begin{array}{r}.03592 \\
.03670 \\
.03744\end{array}$ & $\begin{array}{l}13 \\
13\end{array}$ & & $\begin{array}{l}42.015 \\
42.923 \\
43.798\end{array}$ \\
\hline $\begin{array}{l}-5 \\
-5 \\
-5\end{array}$ & $\begin{array}{l}10000 \\
10000 \\
10000\end{array}$ & $\begin{array}{l}49.0 \\
50.0 \\
51.0\end{array}$ & $\begin{array}{l}.03 \\
.03 \\
.03\end{array}$ & $\begin{array}{r}.12530 \\
.12774 \\
.13017\end{array}$ & $\begin{array}{r}.00954 \\
.00973 \\
.00991\end{array}$ & $\begin{array}{r}.03819 \\
.03893 \\
.03967\end{array}$ & $\begin{array}{l}13.604 \\
13.869 \\
14.134\end{array}$ & $\begin{array}{l}41.467 \\
42.274 \\
43.081\end{array}$ & $\begin{array}{l}44.667 \\
45.537 \\
46.406\end{array}$ \\
\hline $\begin{array}{l}-5 \\
-5 \\
-5\end{array}$ & $\begin{array}{l}10000 \\
10000 \\
10000\end{array}$ & $\begin{array}{l}52.0 \\
53.0 \\
54.0\end{array}$ & $\begin{array}{l}.03 \\
.03 \\
.03\end{array}$ & $\begin{array}{r}.13255 \\
.13488 \\
.13721\end{array}$ & $\begin{array}{r}.01010 \\
.01027 \\
.01045\end{array}$ & $\begin{array}{r}.04040 \\
.04111 \\
.04182\end{array}$ & $\begin{array}{l}14.392 \\
14.645 \\
14.898\end{array}$ & $\begin{array}{l}43.868 \\
44.639 \\
45.410\end{array}$ & $\begin{array}{l}253 \\
084 \\
915\end{array}$ \\
\hline $\begin{array}{l}-5 \\
-5 \\
-5\end{array}$ & $\begin{array}{l}10000 \\
10000 \\
10000\end{array}$ & $\begin{array}{l}55.0 \\
60.0 \\
65.0\end{array}$ & $\begin{array}{l}.03 \\
.03 \\
.03\end{array}$ & $\begin{array}{r}.13954 \\
.14993 \\
.15713\end{array}$ & $\begin{array}{l}.01 \\
.0 \\
.01\end{array}$ & $\begin{array}{l}.0 \\
.0 \\
.0\end{array}$ & $\begin{array}{l}15 \\
16 \\
17\end{array}$ & $\begin{array}{l}46 \\
49 \\
52\end{array}$ & $\begin{array}{r}745 \\
+50 \\
17\end{array}$ \\
\hline $\begin{array}{l}-5 \\
-5 \\
-5\end{array}$ & $\begin{array}{l}10000 \\
10000 \\
10000\end{array}$ & $\begin{array}{l}70.0 \\
75.0 \\
80.0\end{array}$ & $\begin{array}{r}.04060 \\
.04149 \\
.04195\end{array}$ & $\begin{array}{r}.16241 \\
.16599 \\
.16781\end{array}$ & $\begin{array}{r}.01237 \\
.01264 \\
.01278\end{array}$ & $\begin{array}{l}.04950 \\
.05059 \\
.05115\end{array}$ & $\begin{array}{l}17 \\
18 \\
18\end{array}$ & $\begin{array}{l}53.749 \\
54.935 \\
55.536\end{array}$ & $\begin{array}{l}57.898 \\
59.175 \\
59.823\end{array}$ \\
\hline $\begin{array}{l}-5 \\
-5 \\
-5\end{array}$ & $\begin{array}{l}10000 \\
10000 \\
10000\end{array}$ & $\begin{array}{l}85.0 \\
90.0 \\
95.0\end{array}$ & $\begin{array}{r}.04217 \\
.04212 \\
.04191\end{array}$ & $\begin{array}{r}.16869 \\
.16848 \\
.16765\end{array}$ & $\begin{array}{r}.01285 \\
.01283 \\
.01277\end{array}$ & $\begin{array}{l}.05141 \\
.05135 \\
.05110\end{array}$ & $\begin{array}{l}18.316 \\
18.293 \\
18.202\end{array}$ & $\begin{array}{l}55.829 \\
55.758 \\
55.482\end{array}$ & $\begin{array}{l}60.138 \\
60.062 \\
59.765\end{array}$ \\
\hline - & OC & .0 & .04152 & 16608 & 01265 & .05062 & 18.033 & $54 \cdot 965$ & 59.208 \\
\hline
\end{tabular}


TABULATION OF DATA FOR ABSORPTION OF SOUND IN AIR VERSUS PERCENT RELATIVE HUMIDITY AT -5 DEGREES CENTIGRADE FOR A FREQUENCY OF 12500 HERTZ

\begin{tabular}{|c|c|c|c|c|c|c|c|c|c|}
\hline$(1)$ & (2) & (3) & (4) & (5) & (6) & $(7)$ & $(8)$ & $(9)$ & $(10)$ \\
\hline $\begin{array}{l}\text { TEMP } \\
\text { DEGR } \\
\text { CENT }\end{array}$ & FREQ & $\begin{array}{l}\text { REL } \\
\text { HUM } \\
\text { PER } \\
\text { CENT }\end{array}$ & $\begin{array}{l}\text { ATTEN } \\
\text { COEF } \\
\text { PER } \\
\text { METER }\end{array}$ & $\begin{array}{l}\text { PER } \\
\text { METER }\end{array}$ & $\begin{array}{l}\text { ATTEN } \\
\text { COEF } \\
\text { PER } \\
\text { FOOT }\end{array}$ & $\begin{array}{l}\text { PER } \\
\text { FOOT }\end{array}$ & $\begin{array}{l}\text { ATTEN } \\
\text { DB PER } \\
\text { IOO } \\
\text { METER }\end{array}$ & $\begin{array}{l}\text { ATTEN } \\
\text { DB PER } \\
\text { IOOO } \\
\text { FEET }\end{array}$ & $\begin{array}{l}\text { DECAY } \\
\text { RATE } \\
\text { DB PER } \\
\text { SECOND }\end{array}$ \\
\hline $\begin{array}{l}-5 \\
-5 \\
-5\end{array}$ & $\begin{array}{l}12500 \\
12500 \\
12500\end{array}$ & $\begin{array}{r}5.0 \\
10.0 \\
15.0\end{array}$ & $\begin{array}{l}.00809 \\
.01118 \\
.01427\end{array}$ & $\begin{array}{r}.03236 \\
.04472 \\
.05708\end{array}$ & $\begin{array}{l}.00246 \\
.00340 \\
.00434\end{array}$ & $\begin{array}{l}.00986 \\
.01363 \\
.01739\end{array}$ & $\begin{array}{l}3.513 \\
4.855 \\
6.198\end{array}$ & $\begin{array}{l}10.709 \\
14.800 \\
18.892\end{array}$ & $\begin{array}{l}11.536 \\
15.943 \\
20.350\end{array}$ \\
\hline $\begin{array}{l}-5 \\
-5 \\
-5\end{array}$ & $\begin{array}{l}12500 \\
12500 \\
12500\end{array}$ & $\begin{array}{l}20.0 \\
25.0 \\
30.0\end{array}$ & $\begin{array}{r}.01736 \\
.02045 \\
.02354\end{array}$ & $\begin{array}{l}.06944 \\
.08180 \\
.09417\end{array}$ & $\begin{array}{l}.00529 \\
.00623 \\
.00717\end{array}$ & $\begin{array}{l}.02116 \\
.02493 \\
.02870\end{array}$ & $\begin{array}{r}7.540 \\
8.882 \\
10.224\end{array}$ & $\begin{array}{l}22.983 \\
27.074 \\
31.165\end{array}$ & $\begin{array}{l}24.757 \\
29.163 \\
33.570\end{array}$ \\
\hline $\begin{array}{l}-5 \\
-5 \\
-5\end{array}$ & $\begin{array}{l}12500 \\
12500 \\
12500\end{array}$ & $\begin{array}{l}35.0 \\
40.0 \\
45.0\end{array}$ & $\begin{array}{r}.02663 \\
.02972 \\
.03283\end{array}$ & $\begin{array}{r}.10653 \\
.11889 \\
.13133\end{array}$ & $\begin{array}{l}.00811 \\
.00905 \\
.01000\end{array}$ & $\begin{array}{l}.03247 \\
.03623 \\
.04003\end{array}$ & $\begin{array}{l}11.566 \\
12.909 \\
14.259\end{array}$ & $\begin{array}{l}35.256 \\
39.347 \\
43.464\end{array}$ & $\begin{array}{l}37.977 \\
42.384 \\
46.819\end{array}$ \\
\hline $\begin{array}{l}-5 \\
-5 \\
-5\end{array}$ & $\begin{array}{l}12500 \\
12500 \\
12500\end{array}$ & $\begin{array}{l}46.0 \\
47.0 \\
48.0\end{array}$ & $\begin{array}{r}.03347 \\
.03411 \\
.03474\end{array}$ & $\begin{array}{r}. \\
.13388 \\
.13644 \\
.13899\end{array}$ & $\begin{array}{r}.01020 \\
.01039 \\
.01059\end{array}$ & $\begin{array}{r}.04080 \\
.04158 \\
.04236\end{array}$ & $\begin{array}{l}14.53 .6 \\
14.814 \\
15.091\end{array}$ & $\begin{array}{l}44 \cdot 309 \\
45 \cdot 153 \\
45.998\end{array}$ & $\begin{array}{l}47.729 \\
48.639 \\
49.548\end{array}$ \\
\hline $\begin{array}{l}-5 \\
-5 \\
-5\end{array}$ & $\begin{array}{l}12500 \\
12500 \\
12500\end{array}$ & $\begin{array}{l}49.0 \\
50.0 \\
51.0\end{array}$ & $\begin{array}{r}.03539 \\
.03609 \\
.03678\end{array}$ & $\begin{array}{r}.14156 \\
.14436 \\
.14715\end{array}$ & $\begin{array}{l}.01078 \\
.01100 \\
.01121\end{array}$ & $\begin{array}{r}.04315 \\
.04400 \\
.04485\end{array}$ & $\begin{array}{l}15.370 \\
15.673 \\
15.977\end{array}$ & $\begin{array}{l}46.851 \\
47.774 \\
48.698\end{array}$ & $\begin{array}{l}50.467 \\
51.462 \\
52.457\end{array}$ \\
\hline $\begin{array}{ll}-5 & 1 \\
-5 & 1\end{array}$ & $\begin{array}{l}12500 \\
12500\end{array}$ & $\begin{array}{l}52.0 \\
53.0\end{array}$ & $\begin{array}{r}.03748 \\
.03818\end{array}$ & $\begin{array}{l}.14994 \\
.15273\end{array}$ & $\begin{array}{r}.01142 \\
.01163\end{array}$ & $\begin{array}{l}.04570 \\
.04655\end{array}$ & $\begin{array}{l}16 \cdot 280 \\
16.583\end{array}$ & $\begin{array}{l}49.622 \\
50.546\end{array}$ & $\begin{array}{l}53.452 \\
54.447\end{array}$ \\
\hline$-5:$ & 12500 & 54.0 & .03887 & .15550 & .01184 & .04739 & 16.883 & 51.461 & 55.433 \\
\hline $\begin{array}{l}-5 \\
-5\end{array}$ & $\begin{array}{l}12500 \\
12500\end{array}$ & $\begin{array}{l}55.0 \\
60.0\end{array}$ & $\begin{array}{r}.03954 \\
.04284\end{array}$ & $\begin{array}{r}15817 \\
.17137\end{array}$ & $\begin{array}{r}.01205 \\
.01305\end{array}$ & $\begin{array}{l}.04821 \\
.05223\end{array}$ & $\begin{array}{l}17.173 \\
18.606\end{array}$ & $\begin{array}{l}52.345 \\
56.714\end{array}$ & $\begin{array}{l}56.386 \\
61.092\end{array}$ \\
\hline-5 & 12500 & 65.0 & .04591 & .18367 & .01399 & .05598 & 19.942 & 60.786 & 65.478 , \\
\hline $\begin{array}{l}-5 \\
-5 \\
-5\end{array}$ & $\begin{array}{l}12500 \\
12500 \\
12500\end{array}$ & $\begin{array}{l}70.0 \\
75.0 \\
80.0\end{array}$ & $\begin{array}{l}.04848 \\
.05035 \\
.05178\end{array}$ & $\begin{array}{l}.19392 \\
.20143 \\
.20712\end{array}$ & $\begin{array}{r}.01477 \\
.01534 \\
.01578\end{array}$ & $\begin{array}{l}.05910 \\
.06139 \\
.06313\end{array}$ & $\begin{array}{l}21.055 \\
21.871 \\
22.489\end{array}$ & $\begin{array}{l}64.177 \\
66.664 \\
68.547\end{array}$ & $\begin{array}{l}69.130 \\
71.810 \\
73.838\end{array}$ \\
\hline $\begin{array}{l}-5 \\
-5 \\
-5\end{array}$ & $\begin{array}{l}12500 \\
12500 \\
12500\end{array}$ & $\begin{array}{l}85.0 \\
90.0 \\
95.0\end{array}$ & $\begin{array}{r}.05279 \\
.05335 \\
.05363\end{array}$ & $\begin{array}{l}.21116 \\
.21340 \\
.21455\end{array}$ & $\begin{array}{r}.01609 \\
.01626 \\
.01634\end{array}$ & $\begin{array}{l}.06436 \\
.06504 \\
.06539\end{array}$ & $\begin{array}{l}22.927 \\
23.170 \\
23.295\end{array}$ & $\begin{array}{l}69.884 \\
70.625 \\
71.004\end{array}$ & $\begin{array}{l}75.278 \\
76.076 \\
76.484\end{array}$ \\
\hline- & 125 & 00.0 & .05370 & .21482 & .01636 & .06547 & $23 \cdot 324$ & 71.094 & 76.581 \\
\hline
\end{tabular}


TABULATION OF DATA FOR

ABSORPTION OF SOUND IN AIR VERSUS PERCENT RELATIVE HUMIDITY AT O DEGREES CENTIGRADE FOR A FREQUENCY OF' 125 HERTZ

\begin{tabular}{|c|c|c|c|c|c|c|c|c|c|}
\hline (1) & (2) & $(3)$ & 141 & (5) & $(6)$ & $(7)$ & $(8)$ & $(9)$ & $(10)$ \\
\hline $\begin{array}{l}\text { TEMP } \\
\text { DEGR } \\
\text { CENT }\end{array}$ & HERTZ & $\begin{array}{l}\text { REL } \\
\text { HUM } \\
\text { PER } \\
\text { CENT }\end{array}$ & $\begin{array}{l}\text { ATTEN } \\
\text { COEF } \\
\text { PER } \\
\text { METER }\end{array}$ & $\begin{array}{l}4 M \\
\text { PER } \\
\text { METER }\end{array}$ & $\begin{array}{l}\text { ATTEN } \\
\text { COEF } \\
\text { PER } \\
\text { F○OT }\end{array}$ & $\begin{array}{l}4 M \\
\text { PER } \\
F O O T\end{array}$ & $\begin{array}{l}\text { ATTEN } \\
\text { DB PER } \\
100 \\
\text { METER }\end{array}$ & $\begin{array}{l}\text { ATTEN } \\
\text { DB PER } \\
1000 \\
\text { FEET }\end{array}$ & $\begin{array}{l}\text { DECAY } \\
\text { RATE } \\
\text { DB PER } \\
\text { SECOND }\end{array}$ \\
\hline $\begin{array}{l}0 \\
0 \\
0\end{array}$ & $\begin{array}{l}125 \\
125 \\
125\end{array}$ & $\begin{array}{r}5.0 \\
10.0 \\
15.0\end{array}$ & $\begin{array}{l}.00050 \\
.00025 \\
.00016\end{array}$ & $\begin{array}{l}.00202 \\
.00100 \\
.00064\end{array}$ & $\begin{array}{l}.00015 \\
.00007 \\
.00004\end{array}$ & $\begin{array}{l}.00061 \\
.00030 \\
.00019\end{array}$ & $\begin{array}{l}.220 \\
.109 \\
.069\end{array}$ & $\begin{array}{r}.670 \\
.333 \\
.213\end{array}$ & $\begin{array}{l}.729 \\
.362 \\
.231\end{array}$ \\
\hline $\begin{array}{l}0 \\
0 \\
0\end{array}$ & $\begin{array}{l}125 \\
125 \\
125\end{array}$ & $\begin{array}{l}20.0 \\
25.0 \\
30.0\end{array}$ & $\begin{array}{l}.00012 \\
.00011 \\
.00010\end{array}$ & $\begin{array}{l}.00049 \\
.00044 \\
.00041\end{array}$ & $\begin{array}{l}.00003 \\
.00003 \\
.00003\end{array}$ & $\begin{array}{l}.00015 \\
.00013 \\
.00012\end{array}$ & $\begin{array}{l}.054 \\
.047 \\
.044\end{array}$ & $\begin{array}{r}.164 \\
.145 \\
.136\end{array}$ & $\begin{array}{r}.178 \\
.158 \\
.148\end{array}$ \\
\hline $\begin{array}{l}0 \\
0 \\
0\end{array}$ & $\begin{array}{l}125 \\
125 \\
125\end{array}$ & $\begin{array}{l}35.0 \\
40.0 \\
45.0\end{array}$ & $\begin{array}{l}.00009 \\
.00009 \\
.00008\end{array}$ & $\begin{array}{l}.00038 \\
.00037 \\
.00035\end{array}$ & $\begin{array}{l}.00002 \\
.00002 \\
.00002\end{array}$ & $\begin{array}{l}.00011 \\
.00011 \\
.00010\end{array}$ & $\begin{array}{l}.042 \\
.040 \\
.038\end{array}$ & $\begin{array}{r}.128 \\
.122 \\
.117\end{array}$ & $\begin{array}{r}.140 \\
.133 \\
.127\end{array}$ \\
\hline $\begin{array}{l}0 \\
0 \\
0\end{array}$ & $\begin{array}{l}125 \\
125 \\
125\end{array}$ & $\begin{array}{l}46.0 \\
47.0 \\
48.0\end{array}$ & $\begin{array}{l}.00008 \\
.00008 \\
.00008\end{array}$ & $\begin{array}{r}.00035 \\
.00034 \\
.00034\end{array}$ & $\begin{array}{l}.00002 \\
.00002 \\
.00002\end{array}$ & $\begin{array}{l}.00010 \\
.00010 \\
.00010\end{array}$ & $\begin{array}{l}.038 \\
.037 \\
.037\end{array}$ & $\begin{array}{l}.116 \\
.115 \\
.114\end{array}$ & $\begin{array}{l}.126 \\
.125 \\
.124\end{array}$ \\
\hline $\begin{array}{l}0 \\
0 \\
0\end{array}$ & $\begin{array}{l}125 \\
125 \\
125\end{array}$ & $\begin{array}{l}49.0 \\
50.0 \\
51.0\end{array}$ & $\begin{array}{l}.00008 \\
.00008 \\
.00008\end{array}$ & $\begin{array}{l}.00034 \\
.00033 \\
.00033\end{array}$ & $\begin{array}{l}.00002 \\
.00002 \\
.00002\end{array}$ & $\begin{array}{l}.00010 \\
.00010 \\
.00010\end{array}$ & $\begin{array}{l}.037 \\
.036 \\
.036\end{array}$ & $\begin{array}{l}.113 \\
011 \\
.112 \\
0\end{array}$ & $\begin{array}{r}.123 \\
.122 \\
.121\end{array}$ \\
\hline $\begin{array}{l}0 \\
0 \\
0\end{array}$ & $\begin{array}{l}125 \\
125 \\
125\end{array}$ & $\begin{array}{l}52.0 \\
53.0 \\
54.0\end{array}$ & $\begin{array}{l}.00008 \\
.00008 \\
.00008\end{array}$ & $\begin{array}{l}.00033 \\
.00033 \\
.00032\end{array}$ & $\begin{array}{l}.00002 \\
.00002 \\
.00002\end{array}$ & $\begin{array}{l}.00010 \\
.00010 \\
.00010\end{array}$ & $\begin{array}{l}.036 \\
.035 \\
.035\end{array}$ & $\begin{array}{l}.110 \\
.109 \\
.108\end{array}$ & $\begin{array}{r}.120 \\
.119 \\
.118\end{array}$ \\
\hline $\begin{array}{l}0 \\
0 \\
0\end{array}$ & $\begin{array}{l}125 \\
125 \\
125\end{array}$ & $\begin{array}{l}55.0 \\
60.0 \\
65.0\end{array}$ & $\begin{array}{l}.00008 \\
.00007 \\
.00007\end{array}$ & $\begin{array}{l}.00032 \\
.00031 \\
.00030\end{array}$ & $\begin{array}{l}.00002 \\
.00002 \\
.00002\end{array}$ & $\begin{array}{l}.00009 \\
.00009 \\
.00009\end{array}$ & $\begin{array}{l}.035 \\
.034 \\
.033\end{array}$ & $\begin{array}{l}.108 \\
.104 \\
.101\end{array}$ & $\begin{array}{r}.117 \\
.113 \\
.110\end{array}$ \\
\hline $\begin{array}{l}0 \\
0 \\
0\end{array}$ & $\begin{array}{l}125 \\
125 \\
125\end{array}$ & $\begin{array}{l}70.0 \\
75.0 \\
80.0\end{array}$ & $\begin{array}{l}.00007 \\
.00007 \\
.00007\end{array}$ & $\begin{array}{r}.00029 \\
.00029 \\
.00028\end{array}$ & $\begin{array}{l}.00002 \\
.00002 \\
.00002\end{array}$ & $\begin{array}{l}.00009 \\
.00008 \\
.00008\end{array}$ & $\begin{array}{l}.032 \\
.031 \\
.031\end{array}$ & $\begin{array}{r}.099 \\
.096 \\
.094\end{array}$ & $\begin{array}{l}.107 \\
.105 \\
.102\end{array}$ \\
\hline $\begin{array}{l}0 \\
0 \\
0\end{array}$ & $\begin{array}{l}125 \\
125 \\
125\end{array}$ & $\begin{array}{l}85.0 \\
90.0 \\
95.0\end{array}$ & $\begin{array}{l}.00006 \\
.00006 \\
.00006\end{array}$ & $\begin{array}{l}.00027 \\
.00027 \\
.00026\end{array}$ & $\begin{array}{l}.00002 \\
.00002 \\
.00002\end{array}$ & $\begin{array}{l}.00008 \\
.00008 \\
.00008\end{array}$ & $\begin{array}{l}.030 \\
.029 \\
.029\end{array}$ & $\begin{array}{l}.092 \\
.090 \\
.088\end{array}$ & $\begin{array}{l}.100 \\
.098 \\
.096\end{array}$ \\
\hline 0 & 125 & 100.0 & .00006 & .00026 & .00002 & .00008 & .028 & .087 & .094 \\
\hline
\end{tabular}


TABULATION OF DATA FOR

ABSORPTION OF SOUND IN AIR VERSUS PERCENT RELATIVE HUMIDITY AT O DEGREES CENTIGRADE FOR A FREQUENCY OF 250 HERTZ

\begin{tabular}{|c|c|c|c|c|c|c|c|c|c|}
\hline (1) & $(2)$ & (3) & (4) & (5) & (6). & 171 & $(8)$ & $(9)$ & $(10)$ \\
\hline TEMP & FREQ & REL & ATTEN & $4 M$ & ATTEN & $4 M$ & ATTEN & ATTEN & DECAY \\
\hline $\begin{array}{l}\text { DEGR } \\
\text { CENT }\end{array}$ & HERTZ & $\begin{array}{l}\text { HUM } \\
\text { PER } \\
\text { CENT }\end{array}$ & $\begin{array}{l}\text { C๑EF } \\
\text { PER } \\
\text { METER }\end{array}$ & $\begin{array}{l}\text { PER } \\
\text { METER }\end{array}$ & $\begin{array}{l}\text { COEF } \\
\text { PER } \\
\text { FOOT. }\end{array}$ & $\begin{array}{l}\text { PER } \\
\text { FOOT }\end{array}$ & $\begin{array}{l}\text { DE PER } \\
100 \\
\text { METER }\end{array}$ & $\begin{array}{l}\text { DB PER } \\
1000 \\
\text { FEET }\end{array}$ & $\begin{array}{l}\text { RAंTE } \\
\text { DB PER } \\
\text { SFCOND }\end{array}$ \\
\hline 0 & 250 & 5.0 & .00108 & .00432 & .00032 & .00131 & .469 & 1.432 & 1.557 \\
\hline $\begin{array}{l}0 \\
0\end{array}$ & $\begin{array}{l}250 \\
250\end{array}$ & $\begin{array}{l}10.0 \\
15.0\end{array}$ & $\begin{array}{l}.00067 \\
.00043\end{array}$ & $\begin{array}{l}.00269 \\
.00173\end{array}$ & $\begin{array}{l}.00020 \\
.00013\end{array}$ & $\begin{array}{l}.00082 \\
.00052\end{array}$ & $\begin{array}{r}.293 \\
.188\end{array}$ & $\begin{array}{l}.893 \\
.573\end{array}$ & $\begin{array}{l}.971 \\
.622\end{array}$ \\
\hline $\begin{array}{l}0 \\
0 \\
0\end{array}$ & $\begin{array}{l}250 \\
250 \\
250\end{array}$ & $\begin{array}{l}20.0 \\
25.0 \\
30.0\end{array}$ & $\begin{array}{l}.00031 \\
.00025 \\
.00022\end{array}$ & $\begin{array}{l}.00126 \\
.00103 \\
.00091\end{array}$ & $\begin{array}{l}.00009 \\
.00007 \\
.00007\end{array}$ & $\begin{array}{l}.00038 \\
.00031 \\
.00028\end{array}$ & $\begin{array}{r}.137 \\
.112 \\
.099\end{array}$ & $\begin{array}{r}.420 \\
.341 \\
.304\end{array}$ & $\begin{array}{r}.456 \\
.371 \\
.330\end{array}$ \\
\hline $\begin{array}{l}0 \\
0 \\
0\end{array}$ & $\begin{array}{l}250 \\
250 \\
250\end{array}$ & $\begin{array}{l}35.0 \\
40.0 \\
45.0\end{array}$ & $\begin{array}{l}.00021 \\
.00020 \\
.00019\end{array}$ & $\begin{array}{l}.00086 \\
.00082 \\
.00078\end{array}$ & $\begin{array}{l}.00006 \\
.00006 \\
.00005\end{array}$ & $\begin{array}{l}.00026 \\
.00024 \\
.00023\end{array}$ & $\begin{array}{l}.093 \\
.089 \\
.085\end{array}$ & $\begin{array}{l}.285 \\
.271 \\
.259\end{array}$ & $\begin{array}{l}.309 \\
.295 \\
.282\end{array}$ \\
\hline $\begin{array}{l}0 \\
0 \\
0\end{array}$ & $\begin{array}{l}250 \\
250 \\
250\end{array}$ & $\begin{array}{l}46.0 \\
47.0 \\
48.0\end{array}$ & $\begin{array}{l}.00019 \\
.00019 \\
.00019\end{array}$ & $\begin{array}{l}.00077 \\
.00077 \\
.00076\end{array}$ & $\begin{array}{l}.00005 \\
.00005 \\
.00005\end{array}$ & $\begin{array}{l}.00023 \\
.00023 \\
.00023\end{array}$ & $\begin{array}{l}.084 \\
.084 \\
.083\end{array}$ & $\begin{array}{r}.258 \\
.256 \\
.254\end{array}$ & $\begin{array}{r}.280 \\
.278 \\
.276\end{array}$ \\
\hline $\begin{array}{l}0 \\
0 \\
0\end{array}$ & $\begin{array}{l}250 \\
250 \\
250\end{array}$ & $\begin{array}{l}49.0 \\
50.0 \\
51.0\end{array}$ & $\begin{array}{l}.00019 \\
.00018 \\
.00018\end{array}$ & $\begin{array}{r}.00076 \\
.00075 \\
.00075\end{array}$ & $\begin{array}{l}.00005 \\
.00005 \\
.00005\end{array}$ & $\begin{array}{l}.00023 \\
.00023 \\
.00022\end{array}$ & $\begin{array}{l}.082 \\
.082 \\
.081\end{array}$ & $\begin{array}{l}.252 \\
.250 \\
.248\end{array}$ & $\begin{array}{r}.274 \\
.272 \\
.270\end{array}$ \\
\hline $\begin{array}{l}0 \\
0 \\
0\end{array}$ & $\begin{array}{l}250 \\
250 \\
250\end{array}$ & $\begin{array}{l}52.0 \\
53.0 \\
54.0\end{array}$ & $\begin{array}{l}.00018 \\
.00018 \\
.00018\end{array}$ & $\begin{array}{l}.00074 \\
.00073 \\
.00073\end{array}$ & $\begin{array}{l}.000 p 5 \\
.00005 \\
.00005\end{array}$ & $\begin{array}{l}.00022 \\
.00022 \\
.00022\end{array}$ & $\begin{array}{l}.080 \\
.080 \\
.079\end{array}$ & $\begin{array}{l}.246 \\
.244 \\
.243\end{array}$ & $\begin{array}{l}.268 \\
.266 \\
.264\end{array}$ \\
\hline $\begin{array}{l}0 \\
0 \\
0\end{array}$ & $\begin{array}{l}250 \\
250 \\
250\end{array}$ & $\begin{array}{l}55.0 \\
60.0 \\
65.0\end{array}$ & $\begin{array}{r}.00018 \\
.00017 \\
.00017\end{array}$ & $\begin{array}{r}.00073 \\
.00070 \\
.00068\end{array}$ & $\begin{array}{l}.00005 \\
.00005 \\
.00005\end{array}$ & $\begin{array}{l}.00022 \\
.00021 \\
.00020\end{array}$ & $\begin{array}{l}.079 \\
.076 \\
.074\end{array}$ & $\begin{array}{l}.241 \\
.233 \\
.226\end{array}$ & $\begin{array}{l}.262 \\
.253 \\
.245\end{array}$ \\
\hline $\begin{array}{l}0 \\
0 \\
0\end{array}$ & $\begin{array}{l}250 \\
250 \\
250\end{array}$ & $\begin{array}{l}70.0 \\
75.0 \\
80.0\end{array}$ & $\begin{array}{l}.00016 \\
.00016 \\
.00015\end{array}$ & $\begin{array}{l}.00066 \\
.00064 \\
.00063\end{array}$ & $\begin{array}{l}.00005 \\
.00004 \\
.00004\end{array}$ & $\begin{array}{l}.00020 \\
.00019 \\
.00019\end{array}$ & $\begin{array}{l}.071 \\
.070 \\
.068\end{array}$ & $\begin{array}{l}.218 \\
.213 \\
.208\end{array}$ & $\begin{array}{l}.237 \\
.232 \\
.226\end{array}$ \\
\hline 0 & 250 & 85.0 & .00015 & .00061 & .00004 & .00018 & .067 & .204 & .222 \\
\hline $\begin{array}{l}0 \\
0\end{array}$ & $\begin{array}{l}250 \\
250\end{array}$ & $\begin{array}{l}90.0 \\
95.0\end{array}$ & $\begin{array}{l}.00015 \\
.00014\end{array}$ & $\begin{array}{l}.00060 \\
.00059\end{array}$ & $\begin{array}{l}.00004 \\
.00004\end{array}$ & $\begin{array}{l}.00018 \\
.00018\end{array}$ & $\begin{array}{l}.065 \\
.064\end{array}$ & $\begin{array}{l}.199 \\
.196\end{array}$ & $\begin{array}{r}.217 \\
.213\end{array}$ \\
\hline 0 & $250]$ & 100.0 & .00014 & .00058 & .00004 & .00017 & .063 & .192 & .209 \\
\hline
\end{tabular}


TABULATION OF DATA FOR

ABSORPTION OF SOUND IN AIR VERSUS PERCENT RELATIVE HUMIDITY AT O DEGREES CENTIGRADE FOR A FREQUFAIY OF 500 HERTZ

\begin{tabular}{|c|c|c|c|c|c|c|c|c|c|}
\hline$(1)$ & (2) & $(3)$ & $(4)$ & (5) & (6) & 171 & (8) & 191 & $(10)$ \\
\hline $\begin{array}{l}\text { TEMP } \\
\text { DEGR } \\
\text { CENT }\end{array}$ & HERTZ & $\begin{array}{l}\text { REL } \\
\text { HUM } \\
\text { PER } \\
\text { CENT }\end{array}$ & $\begin{array}{l}\text { ATTEN } \\
\text { COEF } \\
\text { PER } \\
\text { METER }\end{array}$ & $\begin{array}{l}4 M \\
\text { PER } \\
\text { METER }\end{array}$ & $\begin{array}{l}\text { ATTEN } \\
\text { COEF } \\
\text { PER } \\
\text { FOOT }\end{array}$ & $\begin{array}{l}4 M \\
\text { PER } \\
\text { FOOT }\end{array}$ & $\begin{array}{l}\text { ATTEN } \\
\text { DB PER } \\
100 \\
\text { METER }\end{array}$ & $\begin{array}{l}\text { ATTEN } \\
\text { DB PER } \\
1000 \\
\text { FEET }\end{array}$ & $\begin{array}{l}\text { DECAY } \\
\text { RATE } \\
\text { DB PER } \\
\text { SECOND }\end{array}$ \\
\hline $\begin{array}{l}0 \\
0 \\
0\end{array}$ & $\begin{array}{l}500 \\
500 \\
500\end{array}$ & $\begin{array}{r}5.0 \\
10.0 \\
15.0\end{array}$ & $\begin{array}{l}.00180 \\
.00203 \\
.00137\end{array}$ & $\begin{array}{l}.00721 \\
.00812 \\
.00549\end{array}$ & $\begin{array}{l}.00055 \\
.00061 \\
.00041\end{array}$ & $\begin{array}{l}.00220 \\
.00247 \\
.00167\end{array}$ & $\begin{array}{r}.783 \\
.882 \\
.597\end{array}$ & $\begin{array}{l}2.389 \\
2.688 \\
1.819\end{array}$ & $\begin{array}{l}2.597 \\
2.923 \\
1.978\end{array}$ \\
\hline & 500 & 15.0 & .00137 & .00549 & .00041 & .00167 & & & \\
\hline 0 & 500 & $20 \cdot 0$ & .00101 & .00404 & .00030 & .00123 & .439 & 1.339 & 1.455 \\
\hline $\begin{array}{l}0 \\
0\end{array}$ & $\begin{array}{l}500 \\
500\end{array}$ & $\begin{array}{l}25.0 \\
30.0\end{array}$ & $\begin{array}{l}.00078 \\
.00064\end{array}$ & $\begin{array}{l}.00315 \\
.00259\end{array}$ & $\begin{array}{l}.00024 \\
.00019\end{array}$ & $\begin{array}{l}.00096 \\
.00078\end{array}$ & $\begin{array}{l}.342 \\
.281\end{array}$ & $\begin{array}{r}1.043 \\
.857\end{array}$ & $\begin{array}{r}1.134 \\
.932\end{array}$ \\
\hline 0 & 500 & 35.0 & .00056 & .00224 & .00017 & .00068 & .243 & .741 & .806 \\
\hline $\begin{array}{l}0 \\
0\end{array}$ & $\begin{array}{l}500 \\
500\end{array}$ & $\begin{array}{l}40.0 \\
45.0\end{array}$ & $\begin{array}{l}.00050 \\
.00046\end{array}$ & $\begin{array}{r}.00200 \\
.00187\end{array}$ & $\begin{array}{l}.00015 \\
.00014\end{array}$ & $\begin{array}{l}.00061 \\
.00057\end{array}$ & $\begin{array}{l}.217 \\
.203\end{array}$ & $\begin{array}{r}.663 \\
.619\end{array}$ & $\begin{array}{l}.721 \\
.673\end{array}$ \\
\hline $\begin{array}{l}0 \\
0 \\
0\end{array}$ & $\begin{array}{l}500 \\
500 \\
500\end{array}$ & $\begin{array}{l}46.0 \\
47.0 \\
48.0\end{array}$ & $\begin{array}{l}.00046 \\
.00045 \\
.00045\end{array}$ & $\begin{array}{l}.00184 \\
.00182 \\
.00180\end{array}$ & $\begin{array}{l}.00014 \\
.00013 \\
.00013\end{array}$ & $\begin{array}{l}.00056 \\
.00055 \\
.00055\end{array}$ & $\begin{array}{r}.200 \\
.198 \\
.196\end{array}$ & $\begin{array}{r}.610 \\
.603 \\
.597\end{array}$ & $\begin{array}{l}.664 \\
.656 \\
.649\end{array}$ \\
\hline 0 & 500 & 49.0 & .00044 & .00178 & .00013 & .00054 & .194 & .592 & .643 \\
\hline $\begin{array}{l}0 \\
0\end{array}$ & $\begin{array}{l}500 \\
500\end{array}$ & $\begin{array}{l}50.0 \\
51.0\end{array}$ & $\begin{array}{l}.00044 \\
.00044\end{array}$ & $\begin{array}{r}.00177 \\
.00176\end{array}$ & $\begin{array}{r}.00013 \\
.00013\end{array}$ & $\begin{array}{l}.00054 \\
.00053\end{array}$ & $\begin{array}{r}192 \\
.191\end{array}$ & $\begin{array}{r}.588 \\
.583\end{array}$ & $\begin{array}{l}.639 \\
.634\end{array}$ \\
\hline 0 & 500 & 52.0 & .00043 & .00175 & .00013 & .00053 & .190 & .579 & .630 \\
\hline $\begin{array}{l}0 \\
0\end{array}$ & $\begin{array}{l}500 \\
500\end{array}$ & $\begin{array}{l}53 \cdot 0 \\
54 \cdot 0\end{array}$ & $\begin{array}{r}.00043 \\
.00043\end{array}$ & $\begin{array}{r}.00174 \\
.00172\end{array}$ & $\begin{array}{r}.00013 \\
.00013\end{array}$ & $\begin{array}{l}.00053 \\
.00052\end{array}$ & $\begin{array}{r}.188 \\
.187\end{array}$ & $\begin{array}{l}.576 \\
.572\end{array}$ & $\begin{array}{l}.626 \\
.622\end{array}$ \\
\hline $\begin{array}{l}0 \\
0 \\
0\end{array}$ & $\begin{array}{l}500 \\
500 \\
500\end{array}$ & $\begin{array}{l}55.0 \\
60.0 \\
65.0\end{array}$ & $\begin{array}{l}.00042 \\
.00041 \\
.00040\end{array}$ & $\begin{array}{l}.00171 \\
.00166 \\
.00161\end{array}$ & $\begin{array}{l}.00013 \\
.00012 \\
.00012\end{array}$ & $\begin{array}{l}.00052 \\
.00050 \\
.00049\end{array}$ & $\begin{array}{r}.186 \\
.180 \\
.175\end{array}$ & $\begin{array}{l}.568 \\
.550 \\
.535\end{array}$ & $\begin{array}{r}.618 \\
.598 \\
.582\end{array}$ \\
\hline $\begin{array}{l}0 \\
0 \\
0\end{array}$ & $\begin{array}{l}500 \\
500 \\
500\end{array}$ & $\begin{array}{l}70.0 \\
75.0 \\
80.0\end{array}$ & $\begin{array}{l}.00039 \\
.00038 \\
.00037\end{array}$ & $\begin{array}{l}.00157 \\
.00153 \\
.00149\end{array}$ & $\begin{array}{l}.00012 \\
.00011 \\
.00011\end{array}$ & $\begin{array}{r}.00048 \\
.00046 \\
.00045\end{array}$ & $\begin{array}{r}.171 \\
.166 \\
.162\end{array}$ & $\begin{array}{l}.521 \\
.508 \\
.496\end{array}$ & $\begin{array}{l}.566 \\
.553 \\
.539\end{array}$ \\
\hline $\begin{array}{l}0 \\
0 \\
0\end{array}$ & $\begin{array}{l}500 \\
500 \\
500\end{array}$ & $\begin{array}{l}85.0 \\
90.0 \\
95.0\end{array}$ & $\begin{array}{l}.00036 \\
.00035 \\
.00035\end{array}$ & $\begin{array}{l}.00146 \\
.00143 \\
.00140\end{array}$ & $\begin{array}{l}.00011 \\
.0 .0010 \\
.00010\end{array}$ & $\begin{array}{l}.00044 \\
.00043 \\
.00042\end{array}$ & $\begin{array}{r}.159 \\
.155 \\
.152\end{array}$ & $\begin{array}{r}.485 \\
.474 \\
.464\end{array}$ & $\begin{array}{r}.527 \\
.516 \\
.504\end{array}$ \\
\hline 0 & 500 & 100.0 & .00034 & .00137 & .00010 & .00041 & .149 & .455 & .49 \\
\hline
\end{tabular}


TABULATION OF DATA FOR

ABSORPTION OF SOUND IN AIR VERSUS PERCENT RELATIVE HUMIDITY AT $O$ DEGREES CENTIGRADE FOR A FREQUENCY OF 1000 HERTZ

\begin{tabular}{|c|c|c|c|c|c|c|c|c|c|}
\hline (1) & (2) & $(3)$ & $(4)$ & (5) & $(6)$ & $(7)$ & $(8)$ & 191 & $(10)$ \\
\hline EMP & FREQ & REL & ATTEN & $4 M$ & ATTEN & $4 M$ & ATTEN & ATTEN & DECAY \\
\hline $\begin{array}{l}\text { DEGR } \\
\text { CENT }\end{array}$ & HERTZ & $\begin{array}{l}\text { HUM } \\
\text { PER } \\
\text { CENT }\end{array}$ & $\begin{array}{l}\text { COEF } \\
\text { PER } \\
\text { METER }\end{array}$ & $\begin{array}{l}\text { PER } \\
\text { METER }\end{array}$ & $\begin{array}{l}\text { COEF } \\
\text { PER } \\
F \odot \odot T\end{array}$ & $\begin{array}{l}\text { PER } \\
\text { FOOT }\end{array}$ & $\begin{array}{l}\text { DB PER } \\
100 \\
\text { METER }\end{array}$ & $\begin{array}{l}\text { DB PER } \\
1000 \\
\text { FEET }\end{array}$ & $\begin{array}{l}\text { RATE } \\
\text { DB PER } \\
\text { SECOND }\end{array}$ \\
\hline 0 & 1000 & 5.0 & .00222 & .00891 & .00067 & .00271 & .967 & 2.949 & 3.206 \\
\hline 0 & 1000 & 10.0 & .00418 & .01672 & .00127 & .00509 & 1.816 & 5.536 & .018 \\
\hline 0 & 1000 & 15.0 & .00420 & .01683 & .00128 & .00513 & 1.827 & 5.571 & 6.056 \\
\hline $\begin{array}{l}0 \\
0\end{array}$ & $\begin{array}{l}1000 \\
1000\end{array}$ & $\begin{array}{l}20.0 \\
25.0\end{array}$ & $\begin{array}{l}.00340 \\
.00265\end{array}$ & $\begin{array}{r}.01362 \\
.01062\end{array}$ & $\begin{array}{l}.00103 \\
.00080\end{array}$ & $\begin{array}{r}.00415 \\
.00323\end{array}$ & $\begin{array}{l}1.479 \\
1.153\end{array}$ & $\begin{array}{l}4.508 \\
3.516\end{array}$ & $\begin{array}{l}4.901 \\
3.822\end{array}$ \\
\hline 0 & 1000 & 30.0 & .00219 & .00879 & .00067 & .00268 & .955 & 2.911 & 3.164 \\
\hline 0 & 1000 & 35.0 & .00184 & .00738 & .00056 & .00225 & .802 & 2.445 & 2.658 \\
\hline 0 & 1000 & $40 \cdot 0$ & .00 & .00639 & .00048 & .00194 & .694 & 2.117 & 2.301 \\
\hline 0 & 1000 & 45.0 & .00141 & .00564 & .00043 & .00172 & .613 & 1.868 & 2.031 \\
\hline 0 & 1000 & 46.0 & .00137 & .00551 & .00042 & .00168 & .599 & 1.825 & 1.984 \\
\hline 0 & 1000 & 47.0 & .00134 & .00538 & .00041 & .00164 & .585 & 1.783 & 1.939 \\
\hline 0 & 1000 & 48.0 & .00131 & .00526 & .00040 & .00160 & .572 & 1.743 & 1.895 \\
\hline 0 & 100 & 49.0 & .00128 & .00515 & .00039 & .00157 & .560 & 1.706 & 1.855 \\
\hline $\begin{array}{l}0 \\
0\end{array}$ & $\begin{array}{l}1000 \\
1000\end{array}$ & $\begin{array}{l}50.0 \\
51.0\end{array}$ & $\begin{array}{l}.00126 \\
.00123\end{array}$ & $\begin{array}{l}.00505 \\
.00495\end{array}$ & $\begin{array}{l}.00038 \\
.00037\end{array}$ & $\begin{array}{l}.00154 \\
.00151\end{array}$ & $\begin{array}{l}.549 \\
.538\end{array}$ & $\begin{array}{l}1.674 \\
1.641\end{array}$ & $\begin{array}{l}1.819 \\
1.784\end{array}$ \\
\hline 0 & 1000 & 52.0 & .00121 & .00487 & .00037 & .00148 & .528 & 1.612 & 1.752 \\
\hline 0 & 100 & 53.0 & .00 & .00478 & .0003 & .001 & .519 & 83 & 1.721 \\
\hline 0 & 100 & 54.0 & .00117 & .00470 & .000 & .00 & .510 & 57 & 92 \\
\hline 0 & 100 & 55. & .00115 & .00463 & .00035 & .00141 & .503 & 1.533 & 1.667 \\
\hline 0 & 100 & 60 & .00 & .00428 & .00032 & .06 & .465 & 417 & 41 \\
\hline 0 & 1000 & 65.0 & .00101 & .00405 & .00030 & .00123 & .439 & 1.340 & 1.457 \\
\hline 0 & 1000 & 70. & .00097 & .00388 & .00029 & .00118 & .421 & $1 \cdot 286$ & 1.398 \\
\hline 0 & 100 & 75 & .00 & .00372 & .000 & .00 & .404 & 233 & 1.340 \\
\hline 0 & 100 & 8 & .00 & .00362 & .0002 & .0011 & .393 & 1.200 & 1.30 \\
\hline 0 & 1000 & 85 & .00088 & .00355 & .00027 & .001 & .385 & 1.174 & 1.27 \\
\hline 0 & 1000 & & .00086 & .00347 & .00026 & .001 & .377. & & \\
\hline 0 & 100 & & .00 & .003 & .000 & .0010 & .370 & 1.128 & 6 \\
\hline ( & 1000 & .0 & .00083 & .00335 & 00025 & .00102 & .364 & 1.109 & 1.206 \\
\hline
\end{tabular}


TABULATION OF DATA FOR

ABSORPTION OF SOUND IN AIR VERSUS PERCENT RELATIVE HUMIDITY AT O DEGREES CENTIGRADE FOR A FREQUENCY OF 2000 HERTZ

\begin{tabular}{|c|c|c|c|c|c|c|c|c|c|}
\hline (1) & $(2)$ & (3) & (4) & (5) & $(6)$ & (7) & $(3)$ & $(9)$ & $110)$ \\
\hline EMP & FREQ & $\begin{array}{l}\text { REL } \\
\text { HUM }\end{array}$ & $\begin{array}{l}\text { ATTEN } \\
\text { COEF }\end{array}$ & $4 M$ & $\begin{array}{l}\text { ATTEN } \\
\text { COEF }\end{array}$ & $4 M$ & $\begin{array}{l}\text { ATTEN } \\
\text { DB PER }\end{array}$ & $\begin{array}{l}\text { ATTEN } \\
\text { DB PER }\end{array}$ & $\begin{array}{l}\text { DECAY } \\
\text { RATE }\end{array}$ \\
\hline $\begin{array}{l}\text { EGR } \\
\text { ENT }\end{array}$ & HERTZ & $\begin{array}{l}\text { PER } \\
\text { CENT }\end{array}$ & $\begin{array}{l}\text { PER } \\
\text { METER }\end{array}$ & $\begin{array}{l}\text { PER } \\
\text { METER }\end{array}$ & 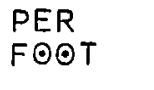 & $\begin{array}{l}\text { PER } \\
\text { FOOT }\end{array}$ & $\begin{array}{l}100 \\
\text { METER }\end{array}$ & $\begin{array}{l}1000 \\
\text { FEET }\end{array}$ & $\begin{array}{l}\text { DB PER } \\
\text { SECOND }\end{array}$ \\
\hline $\begin{array}{l}0 \\
0 \\
0\end{array}$ & $\begin{array}{l}2000 \\
2000 \\
2000\end{array}$ & $\begin{array}{r}5.0 \\
10.0 \\
15.0\end{array}$ & $\begin{array}{l}.00282 \\
.00553 \\
.00810\end{array}$ & $\begin{array}{r}.01129 \\
.02214 \\
.03240\end{array}$ & $\begin{array}{r}.00086 \\
.00168 \\
.00246\end{array}$ & $\begin{array}{l}.00344 \\
.00675 \\
.00987\end{array}$ & $\begin{array}{l}1.226 \\
2.404 \\
3.518\end{array}$ & $\begin{array}{r}3.738 \\
7.328 \\
10.723\end{array}$ & $\begin{array}{r}4.063 \\
7.967 \\
11.657\end{array}$ \\
\hline $\begin{array}{l}0 \\
0 \\
0\end{array}$ & $\begin{array}{l}2000 \\
2000 \\
2000\end{array}$ & $\begin{array}{l}20.0 \\
25.0 \\
30.0\end{array}$ & $\begin{array}{r}.00877 \\
.00840 \\
.00744\end{array}$ & $\begin{array}{r}.03508 \\
.03363 \\
.02977\end{array}$ & $\begin{array}{l}.00267 \\
.00256 \\
.00226\end{array}$ & $\begin{array}{r}.01069 \\
.01025 \\
.00907\end{array}$ & $\begin{array}{l}3.808 \\
3.651 \\
3.232\end{array}$ & $\begin{array}{r}11.609 \\
11.130 \\
9.853\end{array}$ & $\begin{array}{l}12.620 \\
12.099 \\
10.711\end{array}$ \\
\hline $\begin{array}{l}0 \\
0 \\
0\end{array}$ & $\begin{array}{l}2000 \\
2000 \\
2000\end{array}$ & $\begin{array}{l}35.0 \\
40.0 \\
45.0\end{array}$ & $\begin{array}{r}.00636 \\
.00548 \\
.00486\end{array}$ & $\begin{array}{r}.02544 \\
.02194 \\
.01947\end{array}$ & $\begin{array}{l}.00193 \\
.00167 \\
.00148\end{array}$ & $\begin{array}{l}.00775 \\
.00668 \\
.00593\end{array}$ & $\begin{array}{l}2.762 \\
2.382 \\
2.114\end{array}$ & $\begin{array}{l}8.421 \\
7.263 \\
6.445\end{array}$ & $\begin{array}{l}9.154 \\
7.895 \\
7.007\end{array}$ \\
\hline $\begin{array}{l}0 \\
0 \\
0\end{array}$ & $\begin{array}{l}2000 \\
2000 \\
2000\end{array}$ & $\begin{array}{l}46.0 \\
47.0 \\
48.0\end{array}$ & $\begin{array}{r}.00476 \\
.00466 \\
.00455\end{array}$ & $\begin{array}{r}.01905 \\
.01864 \\
.01823\end{array}$ & $\begin{array}{r}.00145 \\
.00142 \\
.00138\end{array}$ & $\begin{array}{l}.00580 \\
.00568 \\
.00555\end{array}$ & $\begin{array}{l}2.069 \\
2.024 \\
1.979\end{array}$ & $\begin{array}{l}6.307 \\
6.169 \\
6.033\end{array}$ & $\begin{array}{l}6.857 \\
6.707 \\
6.558\end{array}$ \\
\hline 0 & 2000 & $49: 0$ & .00445 & .01783 & .00135 & .00543 & 1.936 & 5.901 & 6.414 \\
\hline $\begin{array}{l}0 \\
0\end{array}$ & $\begin{array}{l}2000 \\
2000\end{array}$ & $\begin{array}{l}50.0 \\
51.0\end{array}$ & $\begin{array}{l}.00436 \\
.00426\end{array}$ & $\begin{array}{l}.01744 \\
.01707\end{array}$ & $\begin{array}{l}.00132 \\
.00130\end{array}$ & $\begin{array}{l}.00531 \\
.00520\end{array}$ & $\begin{array}{l}1.893 \\
1.853\end{array}$ & $\begin{array}{l}5.771 \\
5.651\end{array}$ & $\begin{array}{l}6.274 \\
6.143\end{array}$ \\
\hline $\begin{array}{l}0 \\
0 \\
0\end{array}$ & $\begin{array}{l}2000 \\
2000 \\
2000\end{array}$ & $\begin{array}{l}52.0 \\
53.0 \\
54.0\end{array}$ & $\begin{array}{r}.00417 \\
.00409 \\
.00400\end{array}$ & $\begin{array}{r}.01671 \\
.01636 \\
.01602\end{array}$ & $\begin{array}{r}.00127 \\
.00124 \\
.00122\end{array}$ & $\begin{array}{r}.00509 \\
.00498 \\
.00488\end{array}$ & $\begin{array}{l}1.814 \\
1.777 \\
1.739\end{array}$ & $\begin{array}{l}5.531 \\
5.416 \\
5.301\end{array}$ & $\begin{array}{l}6.013 \\
5.888 \\
5.763\end{array}$ \\
\hline $\begin{array}{l}0 \\
0 \\
0\end{array}$ & $\begin{array}{l}2000 \\
2000 \\
2000\end{array}$ & $\begin{array}{l}55.0 \\
60.0 \\
65.0\end{array}$ & $\begin{array}{l}.00391 \\
.00356 \\
.00327\end{array}$ & $\begin{array}{r}.01567 \\
.01424 \\
.01310\end{array}$ & $\begin{array}{l}.00119 \\
.00108 \\
.00099\end{array}$ & $\begin{array}{l}.00477 \\
.00434 \\
.00399\end{array}$ & $\begin{array}{l}1.701 \\
1.546 \\
1.423\end{array}$ & $\begin{array}{l}5.186 \\
4.714 \\
4.338\end{array}$ & $\begin{array}{l}5.638 \\
5.124 \\
4.716\end{array}$ \\
\hline $\begin{array}{l}0 \\
0 \\
0\end{array}$ & $\begin{array}{l}2000 \\
2000 \\
2000\end{array}$ & $\begin{array}{l}70.0 \\
75.0 \\
80.0\end{array}$ & $\begin{array}{l}.00304 \\
.00282 \\
.00264\end{array}$ & $\begin{array}{r}.01217 \\
.01130 \\
.01057\end{array}$ & $\begin{array}{l}.00092 \\
.00086 \\
.00080\end{array}$ & $\begin{array}{l}.00371 \\
.00344 \\
.00322\end{array}$ & $\begin{array}{l}1.322 \\
1.227 \\
1.147\end{array}$ & $\begin{array}{l}4.029 \\
3.740 \\
3.498\end{array}$ & $\begin{array}{l}4.380 \\
4.066 \\
3.803\end{array}$ \\
\hline $\begin{array}{l}0 \\
0 \\
0\end{array}$ & $\begin{array}{l}2000 \\
2000 \\
2000\end{array}$ & $\begin{array}{l}85.0 \\
90.0 \\
95.0\end{array}$ & $\begin{array}{l}.00249 \\
.00237 \\
.00226\end{array}$ & $\begin{array}{l}.00998 \\
.00950 \\
.00904\end{array}$ & $\begin{array}{l}.00076 \\
.00072 \\
.00068\end{array}$ & $\begin{array}{l}.00304 \\
.00289 \\
.00275\end{array}$ & $\begin{array}{r}1.084 \\
1.032 \\
.982\end{array}$ & $\begin{array}{l}3.305 \\
3.146 \\
2.993\end{array}$ & $\begin{array}{l}3.593 \\
3.421 \\
3.254\end{array}$ \\
\hline 0 & 2000 & $100 \cdot 0$ & กo2 17 & .00868 & .00066 & .00264 & .943 & 2.875 & 3.125 \\
\hline
\end{tabular}


TABULATION OF DATA FOR

ABSORPTION OF SOUND IN AIR VERSUS PERCENT RELATIVE HUMIDITY AT O DEGREES CENTIGRADE FOR A FREQUENCY OF 2500 HERTZ

\begin{tabular}{|c|c|c|c|c|c|c|c|c|c|}
\hline (1) & (2) & $(3)$ & $(4)$ & (5) & (6) & $(7)$ & $(8)$ & 191 & $(10)$ \\
\hline TEMP & FREQ & $\begin{array}{l}\text { REL } \\
\text { HUM }\end{array}$ & $\begin{array}{l}\text { ATTEN } \\
\text { COEF }\end{array}$ & $4 M$ & $\begin{array}{l}\text { ATTEN } \\
\text { COEF }\end{array}$ & $4 M$ & $\begin{array}{l}\text { ATTEN } \\
\text { DB PER }\end{array}$ & $\begin{array}{l}\text { ATTEN } \\
\text { DB PER }\end{array}$ & $\begin{array}{l}\text { DECAY } \\
\text { RATE }\end{array}$ \\
\hline $\begin{array}{l}\text { DEGR } \\
\text { CENT }\end{array}$ & HERTZ & $\begin{array}{l}\text { PER } \\
\text { CENT }\end{array}$ & $\begin{array}{l}\text { PER } \\
\text { METER }\end{array}$ & $\begin{array}{l}\text { PER } \\
\text { METER }\end{array}$ & 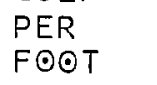 & 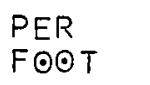 & $\begin{array}{l}100 \\
\text { METER }\end{array}$ & $\begin{array}{l}1000 \\
\text { FEET }\end{array}$ & $\begin{array}{l}\text { DB PER } \\
\text { SECOND }\end{array}$ \\
\hline $\begin{array}{l}0 \\
0\end{array}$ & $\begin{array}{l}2500 \\
2500\end{array}$ & 5.0 & $\begin{array}{l}.00306 \\
.00592\end{array}$ & $\begin{array}{r}.01227 \\
.02371\end{array}$ & $\begin{array}{l}.00093 \\
.00180\end{array}$ & $\begin{array}{r}.00373 \\
.00722\end{array}$ & $\begin{array}{l}1.332 \\
2.574\end{array}$ & 4.060 & 4.414 \\
\hline 0 & 2500 & 15.0 & .00897 & .03588 & .00273 & .01093 & 3.895 & 11.874 & 12.908 \\
\hline $\begin{array}{l}0 \\
0\end{array}$ & $\begin{array}{l}2500 \\
2500\end{array}$ & $\begin{array}{l}20.0 \\
25.0\end{array}$ & $\begin{array}{r}.01075 \\
.01096\end{array}$ & $\begin{array}{r}.04300 \\
.04387\end{array}$ & $\begin{array}{l}.00327 \\
.00334\end{array}$ & $\begin{array}{r}.01310 \\
.01337\end{array}$ & $\begin{array}{l}4.668 \\
4.763\end{array}$ & $\begin{array}{l}14.230 \\
14.519\end{array}$ & $\begin{array}{l}15.470 \\
15.784\end{array}$ \\
\hline 0 & 2500 & 30.0 & .01045 & .04182 & .00318 & .01274 & 4.541 & 13.841 & 15.047 \\
\hline $\begin{array}{l}0 \\
0 \\
0\end{array}$ & $\begin{array}{l}2500 \\
2500 \\
2500\end{array}$ & $\begin{array}{l}35.0 \\
40.0 \\
45.0\end{array}$ & $\begin{array}{l}.00940 \\
.00826 \\
.00724\end{array}$ & $\begin{array}{r}.03763 \\
.03306 \\
.02896\end{array}$ & $\begin{array}{l}.00286 \\
.00251 \\
.00220\end{array}$ & $\begin{array}{r}.01147 \\
.01007 \\
.00882\end{array}$ & $\begin{array}{l}4.086 \\
3.589 \\
3.145\end{array}$ & $\begin{array}{r}12.455 \\
10.940 \\
9.586\end{array}$ & $\begin{array}{l}13.540 \\
11.893 \\
10.421\end{array}$ \\
\hline 0 & 2500 & 46.0 & .00707 & .02828 & .00 .215 & .00862 & 3.070 & 9.360 & 10.175 \\
\hline $\begin{array}{l}0 \\
0\end{array}$ & $\begin{array}{l}2500 \\
2500\end{array}$ & $\begin{array}{l}47.0 \\
48.0\end{array}$ & $\begin{array}{l}.00690 \\
.00675\end{array}$ & $\begin{array}{l}.02761 \\
.02702\end{array}$ & $\begin{array}{r}.00210 \\
.00205\end{array}$ & $\begin{array}{l}.00841 \\
.00823\end{array}$ & $\begin{array}{l}2.998 \\
2.934\end{array}$ & $\begin{array}{l}9.140 \\
8.944\end{array}$ & $\begin{array}{l}9.936 \\
9.723\end{array}$ \\
\hline $\begin{array}{l}0 \\
0 \\
0\end{array}$ & $\begin{array}{l}2500 \\
2500 \\
2500\end{array}$ & $\begin{array}{l}49.0 \\
50.0 \\
51.0\end{array}$ & $\begin{array}{l}.00660 \\
.00647 \\
.00634\end{array}$ & $\begin{array}{r}.02643 \\
.02591 \\
.02539\end{array}$ & $\begin{array}{l}.00201 \\
.00197 \\
.00193\end{array}$ & $\begin{array}{r}.00805 \\
.00789 \\
.00774\end{array}$ & $\begin{array}{l}2.870 \\
2.813 \\
2.757\end{array}$ & $\begin{array}{l}8.749 \\
8.574 \\
8.403\end{array}$ & $\begin{array}{l}9.511 \\
9.321 \\
9.135\end{array}$ \\
\hline $\begin{array}{l}0 \\
0 \\
0\end{array}$ & $\begin{array}{l}2500 \\
2500 \\
2500\end{array}$ & $\begin{array}{l}52.0 \\
53.0 \\
54.0\end{array}$ & $\begin{array}{l}.00622 \\
.00611 \\
.00600\end{array}$ & $\begin{array}{r}.02491 \\
.02447 \\
.02402\end{array}$ & $\begin{array}{l}.00189 \\
.00186 \\
.00183\end{array}$ & $\begin{array}{l}.00759 \\
.00745 \\
.00732\end{array}$ & $\begin{array}{l}2.705 \\
2.657 \\
2.608\end{array}$ & $\begin{array}{l}8.245 \\
8.098 \\
7.952\end{array}$ & $\begin{array}{l}8.963 \\
8.804 \\
8.644\end{array}$ \\
\hline $\begin{array}{l}0 \\
0 \\
0\end{array}$ & $\begin{array}{l}2500 \\
2500 \\
2500\end{array}$ & $\begin{array}{l}55.0 \\
60.0 \\
65.0\end{array}$ & $\begin{array}{l}.00589 \\
.00537 \\
.00490\end{array}$ & $\begin{array}{r}.02358 \\
.02149 \\
.01963\end{array}$ & $\begin{array}{l}.00179 \\
.00163 \\
.00149\end{array}$ & $\begin{array}{l}.00718 \\
.00655 \\
.00598\end{array}$ & $\begin{array}{l}2.560 \\
2.334 \\
2.131\end{array}$ & $\begin{array}{l}7.805 \\
7.114 \\
6.498\end{array}$ & $\begin{array}{l}8.485 \\
7.733 \\
7.064\end{array}$ \\
\hline $\begin{array}{l}0 \\
0 \\
0\end{array}$ & $\begin{array}{l}2500 \\
2500 \\
2500\end{array}$ & $\begin{array}{l}70.0 \\
75.0 \\
80.0\end{array}$ & $\begin{array}{l}.00452 \\
.00421 \\
.00395\end{array}$ & $\begin{array}{r}.01811 \\
.01686 \\
.01581\end{array}$ & $\begin{array}{l}.00138 \\
.00128 \\
.00120\end{array}$ & $\begin{array}{l}.00552 \\
.00514 \\
.00482\end{array}$ & $\begin{array}{l}1.967 \\
1.831 \\
1.717\end{array}$ & $\begin{array}{l}5.996 \\
5.582 \\
5.235\end{array}$ & $\begin{array}{l}6.518 \\
6.068 \\
5.691\end{array}$ \\
\hline $\begin{array}{l}0 \\
0 \\
0\end{array}$ & $\begin{array}{l}2500 \\
2500 \\
2500\end{array}$ & $\begin{array}{l}85.0 \\
90.0 \\
95.0\end{array}$ & $\begin{array}{l}.00371 \\
.00349 \\
.00331\end{array}$ & $\begin{array}{r}.01485 \\
.01399 \\
.01325\end{array}$ & $\begin{array}{l}.00113 \\
.00106 \\
.00101\end{array}$ & $\begin{array}{l}.00452 \\
.00426 \\
.00404\end{array}$ & $\begin{array}{l}1.612 \\
1.519 \\
1.439\end{array}$ & $\begin{array}{l}4.915 \\
4.630 \\
4.387\end{array}$ & $\begin{array}{l}5.343 \\
5.034 \\
4.769\end{array}$ \\
\hline 0 & 2500 & $100 \cdot 0$ & .00316 & .01264 & .00096 & .00385 &. .372 & $4 \cdot 184$ & 4.548 \\
\hline
\end{tabular}


TABULATION OF DATA FOR

ABSORPTION OF SOUND IN AIR VERSUS PERCENT RELATIVE HUMIDITY AT O DEGREES CENTIGRADE FOR A FREQUENCY OF 3200 HERTZ

\begin{tabular}{|c|c|c|c|c|c|c|c|c|c|}
\hline$(1)$ & (2) & $(3)$ & $(4)$ & $(5)$ & (6) & $(7)$ & $(8)$ & 191 & $(10)$ \\
\hline $\begin{array}{l}\text { TEMP } \\
\text { DEGR } \\
\text { CENT }\end{array}$ & FREQ & $\begin{array}{l}\text { REL } \\
\text { HUM } \\
\text { PER } \\
\text { CENT }\end{array}$ & $\begin{array}{l}\text { ATTEN } \\
\text { COEF } \\
\text { PER } \\
\text { METER }\end{array}$ & $\begin{array}{l}4 M \\
\text { PER } \\
\text { METER }\end{array}$ & $\begin{array}{l}\text { ATTEN } \\
\text { COEF } \\
\text { PER } \\
\text { FOOT }\end{array}$ & $\begin{array}{l}4 M \\
\text { PER } \\
\text { FOOT }\end{array}$ & $\begin{array}{l}\text { ATTEN } \\
\text { DB PER } \\
1 O O \\
\text { METER }\end{array}$ & $\begin{array}{l}\text { ATTEN } \\
\text { DB PER } \\
1000 \\
\text { FEET }\end{array}$ & $\begin{array}{l}\text { DECAY } \\
\text { RATE } \\
\text { DB PER } \\
\text { SECOND }\end{array}$ \\
\hline $\begin{array}{l}0 \\
0 \\
0\end{array}$ & $\begin{array}{l}3200 \\
3200 \\
3200\end{array}$ & $\begin{array}{r}5.0 \\
10.0 \\
15.0\end{array}$ & $\begin{array}{l}.00345 \\
.00657 \\
.00981\end{array}$ & $\begin{array}{r}.01381 \\
.02628 \\
.03926\end{array}$ & $\begin{array}{l}.00105 \\
.00200 \\
.00299\end{array}$ & $\begin{array}{l}.00421 \\
.00801 \\
.01196\end{array}$ & $\begin{array}{l}1.500 \\
2.853 \\
4.263\end{array}$ & $\begin{array}{r}4.573 \\
8.698 \\
12.994\end{array}$ & $\begin{array}{r}4.971 \\
9.456 \\
14.126\end{array}$ \\
\hline $\begin{array}{l}0 \\
0 \\
0\end{array}$ & $\begin{array}{l}3200 \\
3200 \\
3200\end{array}$ & $\begin{array}{l}20.0 \\
25.0 \\
30.0\end{array}$ & $\begin{array}{r}.01279 \\
.01402 \\
.01408\end{array}$ & $\begin{array}{l}.05119 \\
.05611 \\
.05635\end{array}$ & $\begin{array}{r}.00390 \\
.00427 \\
.00429\end{array}$ & $\begin{array}{l}.01560 \\
.01710 \\
.01717\end{array}$ & $\begin{array}{l}5.558 \\
6.092 \\
6.118\end{array}$ & $\begin{array}{l}16.942 \\
18.569 \\
18.650\end{array}$ & $\begin{array}{l}18.417 \\
20.186 \\
20.274\end{array}$ \\
\hline $\begin{array}{l}0 \\
0 \\
0\end{array}$ & $\begin{array}{l}3200 \\
3200 \\
3200\end{array}$ & $\begin{array}{l}35.0 \\
40.0 \\
45.0\end{array}$ & $\begin{array}{r}.01350 \\
.01240 \\
.01115\end{array}$ & $\begin{array}{l}.05400 \\
.04961 \\
.04460\end{array}$ & $\begin{array}{l}.00411 \\
.00378 \\
.00339\end{array}$ & $\begin{array}{l}.01646 \\
.01512 \\
.01359\end{array}$ & $\begin{array}{l}5.864 \\
5.386 \\
4.842\end{array}$ & $\begin{array}{l}17.873 \\
16.418 \\
14.761\end{array}$ & $\begin{array}{l}19.430 \\
17.847 \\
16.046\end{array}$ \\
\hline $\begin{array}{l}0 \\
0 \\
0\end{array}$ & $\begin{array}{l}3200 \\
3200 \\
3200\end{array}$ & $\begin{array}{l}46.0 \\
47.0 \\
48.0\end{array}$ & $\begin{array}{r}.01090 \\
.01065 \\
.01039\end{array}$ & $\begin{array}{r}.04361 \\
.04262 \\
.04159\end{array}$ & $\begin{array}{l}.00332 \\
.00324 \\
.00316\end{array}$ & $\begin{array}{r}.01329 \\
.01299 \\
.01267\end{array}$ & $\begin{array}{l}4.735 \\
4.627 \\
4.516\end{array}$ & $\begin{array}{l}14.435 \\
14.105 \\
13.766\end{array}$ & $\begin{array}{l}15.692 \\
15.334 \\
14.965\end{array}$ \\
\hline $\begin{array}{l}0 \\
0 \\
0\end{array}$ & $\begin{array}{l}3200 \\
3200 \\
3200\end{array}$ & $\begin{array}{l}49.0 \\
50.0 \\
51.0\end{array}$ & $\begin{array}{l}.01014 \\
.00991 \\
.00971\end{array}$ & $\begin{array}{l}.04057 \\
.03965 \\
.03885\end{array}$ & $\begin{array}{l}.00309 \\
.00302 \\
.00296\end{array}$ & $\begin{array}{l}.01236 \\
.01208 \\
.01184\end{array}$ & $\begin{array}{r}4.405 \\
-4.305 \\
4.218\end{array}$ & $\begin{array}{l}13.426 \\
13.124 \\
12.857\end{array}$ & $\begin{array}{l}14.596 \\
14.267 \\
13.977\end{array}$ \\
\hline $\begin{array}{l}0 \\
0 \\
0\end{array}$ & $\begin{array}{l}3200 \\
3200 \\
3200\end{array}$ & $\begin{array}{l}52.0 \\
53.0 \\
54.0\end{array}$ & $\begin{array}{l}.00951 \\
.00932 \\
.00913\end{array}$ & $\begin{array}{l}.03804 \\
.03728 \\
.03654\end{array}$ & $\begin{array}{l}.00289 \\
.00284 \\
.00278\end{array}$ & $\begin{array}{l}.01159 \\
.01136 \\
.01113\end{array}$ & $\begin{array}{l}4.131 \\
4.048 \\
3.967\end{array}$ & $\begin{array}{l}12.591 \\
12.340 \\
12.094\end{array}$ & $\begin{array}{l}13.688 \\
13.415 \\
13.147\end{array}$ \\
\hline 0 & 3200 & 55.0 & .00895 & .03580 & .00272 & .01091 & 87 & 11. & 880 \\
\hline $\begin{array}{l}0 \\
0\end{array}$ & $\begin{array}{l}3200 \\
3200\end{array}$ & $\begin{array}{l}60.0 \\
65.0\end{array}$ & $\begin{array}{r}.00819 \\
.00757\end{array}$ & $\begin{array}{r}.03276 \\
.03031\end{array}$ & $\begin{array}{r}.00249 \\
.00230\end{array}$ & $\begin{array}{l}.00998 \\
.00923\end{array}$ & $\begin{array}{l}3.557 \\
3.291\end{array}$ & $\begin{array}{l}10.843 \\
10.031\end{array}$ & $\begin{array}{l}11.787 \\
10.905\end{array}$ \\
\hline $\begin{array}{l}0 \\
0 \\
0\end{array}$ & $\begin{array}{l}3200 \\
3200 \\
3200\end{array}$ & $\begin{array}{l}70.0 \\
75.0 \\
80.0\end{array}$ & $\begin{array}{l}.00700 \\
.00649 \\
.00605\end{array}$ & $\begin{array}{r}.02802 \\
.02598 \\
.02421\end{array}$ & $\begin{array}{r}.00213 \\
.00198 \\
.0 .0184\end{array}$ & $\begin{array}{l}.00854 \\
.00792 \\
.00738\end{array}$ & $\begin{array}{l}3.043 \\
2.821 \\
2.629\end{array}$ & $\begin{array}{l}9.276 \\
8.600 \\
8.015\end{array}$ & $\begin{array}{r}10.084 \\
9.349 \\
8.713\end{array}$ \\
\hline $\begin{array}{l}0 \\
0 \\
0\end{array}$ & $\begin{array}{l}3200 \\
3200 \\
3200\end{array}$ & $\begin{array}{l}85.0 \\
90.0 \\
95.0\end{array}$ & $\begin{array}{l}.00567 \\
.00535 \\
.00508\end{array}$ & $\begin{array}{l}.02271 \\
.02143 \\
.02034\end{array}$ & $\begin{array}{l}.00173 \\
.00163 \\
.00155\end{array}$ & $\begin{array}{l}.00692 \\
.00653 \\
.00620\end{array}$ & $\begin{array}{l}2.465 \\
2.327 \\
2.209\end{array}$ & $\begin{array}{l}7.516 \\
7.095 \\
6.734\end{array}$ & $\begin{array}{l}8.170 \\
7.713 \\
7.320\end{array}$ \\
\hline 0 & 3200 & 100.0 & .00482 & .01928 & .00146 & .00587 & 2.094 & .383 & 6.939 \\
\hline
\end{tabular}


TABULATION OF DATA FOR

ABSORPTION OF SOUND IN AIR VERSUS PERCENT RELATIVE HUMIDITY AT $O$ DEGREES CENTIGRADE FOR A FREQUENCY OF 4000 HERTZ

\begin{tabular}{|c|c|c|c|c|c|c|c|c|c|}
\hline (1) & (2) & (3) & (.4) & (5) & $(6)$ & $(7)$ & $(8)$ & 191 & $(10)$ \\
\hline TEMP & FREQ & $\begin{array}{l}\text { REL } \\
\text { HUM }\end{array}$ & $\begin{array}{l}\text { ATTEN } \\
\text { COEF }\end{array}$ & $4 M$ & $\begin{array}{l}\text { ATTEN } \\
\text { COEF }\end{array}$ & $4 M$ & $\begin{array}{l}\text { ATTEN } \\
\text { DB PER }\end{array}$ & $\begin{array}{l}\text { ATTEN } \\
\text { DB PER }\end{array}$ & $\begin{array}{l}\text { DECAY } \\
\text { RATE }\end{array}$ \\
\hline $\begin{array}{l}\text { EGR } \\
\text { CENT }\end{array}$ & HERTZ & $\begin{array}{l}\text { PER } \\
\text { CENT }\end{array}$ & $\begin{array}{l}\text { PER } \\
\text { METER }\end{array}$ & $\begin{array}{l}\text { PER } \\
\text { METER }\end{array}$ & 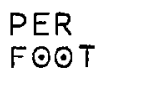 & 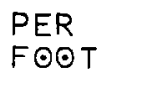 & $\begin{array}{l}100 \\
\text { METER }\end{array}$ & $\begin{array}{l}1000 \\
\text { FEET }\end{array}$ & $\begin{array}{l}\text { DB PER } \\
\text { SECOND }\end{array}$ \\
\hline $\begin{array}{l}0 \\
0\end{array}$ & $\begin{array}{l}4000 \\
4000\end{array}$ & $\begin{array}{r}5.0 \\
10.0\end{array}$ & $\begin{array}{r}.00389 \\
.00726\end{array}$ & $\begin{array}{r}.01558 \\
.02906\end{array}$ & $\begin{array}{r}.00118 \\
.00221\end{array}$ & $\begin{array}{l}.00475 \\
.00885\end{array}$ & $\begin{array}{l}1.692 \\
3.155\end{array}$ & $\begin{array}{l}5.158 \\
9.618\end{array}$ & $\begin{array}{r}5.60 \\
10.45\end{array}$ \\
\hline 0 & 4000 & 15.0 & .01065 & .04260 & .00324 & .01298 & $4 \cdot 625$ & 14.098 & $15 \cdot 326$ \\
\hline $\begin{array}{l}0 \\
0 \\
0\end{array}$ & $\begin{array}{l}4000 \\
4000 \\
4000\end{array}$ & $\begin{array}{l}20.0 \\
25.0 \\
30.0\end{array}$ & $\begin{array}{r}.01428 \\
.01681 \\
.01772\end{array}$ & $\begin{array}{r}.05713 \\
.06725 \\
.07089\end{array}$ & $\begin{array}{r}.00435 \\
.00512 \\
.00540\end{array}$ & $\begin{array}{r}.01741 \\
.02049 \\
.02160\end{array}$ & $\begin{array}{l}6.203 \\
7.301 \\
7.697\end{array}$ & $\begin{array}{l}18.907 \\
22.256 \\
23.460\end{array}$ & $\begin{array}{l}20.554 \\
24.194 \\
25.504\end{array}$ \\
\hline $\begin{array}{l}0 \\
0\end{array}$ & $\begin{array}{l}4000 \\
4000\end{array}$ & $\begin{array}{l}35.0 \\
40.0\end{array}$ & $\begin{array}{r}.01770 \\
.01707\end{array}$ & $\begin{array}{l}.07081 \\
.06828\end{array}$ & $\begin{array}{l}.00539 \\
.00520\end{array}$ & $\begin{array}{l}.02158 \\
.02081\end{array}$ & $\begin{array}{l}7.688 \\
7.413\end{array}$ & $\begin{array}{l}23.434 \\
22.597\end{array}$ & $\begin{array}{l}25.474 \\
24.565\end{array}$ \\
\hline 0 & 4000 & 45.0 & .01594 & .06379 & .00486 & .01944 & 6.926 & $21 \cdot 113$ & 22.952 \\
\hline $\begin{array}{l}0 \\
0 \\
0\end{array}$ & $\begin{array}{l}4000 \\
4000 \\
4000\end{array}$ & $\begin{array}{l}46.0 \\
47.0 \\
48.0\end{array}$ & $\begin{array}{r}.01567 \\
.01540 \\
.01513\end{array}$ & $\begin{array}{l}.06271 \\
.06162 \\
.06053\end{array}$ & $\begin{array}{l}.00477 \\
.00469 \\
.00461\end{array}$ & $\begin{array}{r}.01911 \\
.01878 \\
.01845\end{array}$ & $\begin{array}{l}6.808 \\
6.690 \\
6.572\end{array}$ & $\begin{array}{l}20.753 \\
20.394 \\
20.034\end{array}$ & $\begin{array}{l}22.561 \\
22.170 \\
21.779\end{array}$ \\
\hline $\begin{array}{l}0 \\
0 \\
0\end{array}$ & $\begin{array}{l}4000 \\
4000 \\
4000\end{array}$ & $\begin{array}{l}49.0 \\
50.0 \\
51.0\end{array}$ & $\begin{array}{r}.01486 \\
.01459 \\
.01432\end{array}$ & $\begin{array}{l}.05945 \\
.05836 \\
.05728\end{array}$ & $\begin{array}{l}.00453 \\
.00444 \\
.00436\end{array}$ & $\begin{array}{r}.01812 \\
.01778 \\
.01745\end{array}$ & $\begin{array}{l}6.454 \\
6.336 \\
6.219\end{array}$ & $\begin{array}{l}19.674 \\
19.315 \\
18.956\end{array}$ & $\begin{array}{l}21.388 \\
20.997 \\
20.607\end{array}$ \\
\hline $\begin{array}{l}0 \\
0\end{array}$ & $\begin{array}{l}4000 \\
4000\end{array}$ & $\begin{array}{l}52.0 \\
53.0\end{array}$ & $\begin{array}{r}.01405 \\
.01378\end{array}$ & $\begin{array}{l}.05621 \\
.05515\end{array}$ & $\begin{array}{l}.00428 \\
.00420\end{array}$ & $\begin{array}{l}.01713 \\
.01680\end{array}$ & $\begin{array}{l}6.103 \\
5.987\end{array}$ & $\begin{array}{l}18.603 \\
18.251\end{array}$ & $\begin{array}{l}20.224 \\
19.840\end{array}$ \\
\hline 0 & 4000 & 54.0 & .01352 & .05408 & .00412 & .01648 & 5.872 & 17.898 & 19.45 \\
\hline $\begin{array}{l}0 \\
0 \\
0\end{array}$ & $\begin{array}{l}4000 \\
4000 \\
4000\end{array}$ & $\begin{array}{l}55.0 \\
60.0 \\
65.0\end{array}$ & $\begin{array}{l}.01324 \\
.01202 \\
.01104\end{array}$ & $\begin{array}{r}.05297 \\
.04809 \\
.04419\end{array}$ & $\begin{array}{r}.00403 \\
.00366 \\
.00336\end{array}$ & $\begin{array}{r}.01614 \\
.01465 \\
.01347\end{array}$ & $\begin{array}{l}5.752 \\
5.222 \\
4.799\end{array}$ & $\begin{array}{l}17.532 \\
15.917 \\
14.627\end{array}$ & $\begin{array}{l}19.059 \\
17.303 \\
15.901\end{array}$ \\
\hline 0 & 4000 & 70.0 & .01025 & .04100 & .00312 & .01249 & $4 \cdot 452$ & 13.570 & 14.752 \\
\hline $\begin{array}{l}0 \\
0\end{array}$ & $\begin{array}{l}4000 \\
4000\end{array}$ & $\begin{array}{l}75 \cdot 0 \\
80.0\end{array}$ & $\begin{array}{r}.00959 \\
.00897\end{array}$ & $\begin{array}{l}.03839 \\
.03589\end{array}$ & $\begin{array}{l}.00292 \\
.00273\end{array}$ & $\begin{array}{r}.01170 \\
.01093\end{array}$ & $\begin{array}{l}4.168 \\
3.896\end{array}$ & $\begin{array}{l}12.707 \\
11.877\end{array}$ & $\begin{array}{l}13.813 \\
12.912\end{array}$ \\
\hline 0 & 4000 & 85.0 & .00841 & .03365 & .00256 & .01025 & 3.654 & $11 \cdot 139$ & 12.109 \\
\hline $\begin{array}{l}0 \\
0\end{array}$ & $\begin{array}{l}4000 \\
4000\end{array}$ & $\begin{array}{l}90.0 \\
95.0\end{array}$ & $\begin{array}{l}.00789 \\
.00745\end{array}$ & $\begin{array}{r}.03156 \\
.02982\end{array}$ & $\begin{array}{l}.00240 \\
.00227\end{array}$ & $\begin{array}{l}.00962 \\
.00909\end{array}$ & $\begin{array}{l}3.427 \\
3.238\end{array}$ & $\begin{array}{r}10.447 \\
9.871\end{array}$ & $\begin{array}{l}11.357 \\
10.731\end{array}$ \\
\hline 0 & 4000 & $100 \cdot 0$ & .00708 & .02832 & .00215 & .00863 & 3.075 & 9.372 & 10.189 \\
\hline
\end{tabular}


TABULATION OF DATA FOR

ABSORPTION OF SOUND IN AIR VERSUS PERCENT RELATIVE HUMIDITY AT O DEGREES CENTIGRADE FOR A FREQUENCY OF 5000 HERTZ

\begin{tabular}{|c|c|c|c|c|c|c|c|c|c|}
\hline 11 & $(2)$ & (3) & (4) & (5) & $(6)$ & 171 & $(8)$ & $(9)$ & $(10)$ \\
\hline $\begin{array}{l}\text { EMP } \\
\text { EGR } \\
\text { ENT }\end{array}$ & FREQ & $\begin{array}{l}\text { REL } \\
\text { HUM } \\
\text { PER } \\
\text { CENT }\end{array}$ & $\begin{array}{l}\text { ATTEN } \\
\text { COEF } \\
\text { PER } \\
\text { METER }\end{array}$ & $\begin{array}{l}4 M \\
\text { PER } \\
\text { METER }\end{array}$ & $\begin{array}{l}\text { ATTEN. } \\
\text { COEF } \\
\text { PER. } \\
\text { FOOT }\end{array}$ & $\begin{array}{l}4 M \\
\text { PER } \\
\text { FOOT }\end{array}$ & $\begin{array}{l}\text { ATTEN } \\
\text { DB PER } \\
100 \\
\text { METER }\end{array}$ & $\begin{array}{l}\text { ATTEN. } \\
\text { DB PER } \\
1000 \\
\text { FEET }\end{array}$ & $\begin{array}{l}\text { DECAY } \\
\text { RATE } \\
\text { DB PER } \\
\text { SECOND }\end{array}$ \\
\hline $\begin{array}{l}0 \\
0 \\
0\end{array}$ & $\begin{array}{l}5000 \\
5000 \\
5000\end{array}$ & $\begin{array}{r}5.0 \\
10.0 \\
15.0\end{array}$ & $\begin{array}{r}.00446 \\
.00810 \\
.01174\end{array}$ & $\begin{array}{l}.01785 \\
.03241 \\
.04696\end{array}$ & $\begin{array}{r}.00136 \\
.00246 \\
.00357\end{array}$ & $\begin{array}{r}.00544 \\
.00987 \\
.01431\end{array}$ & $\begin{array}{l}1.938 \\
3.519 \\
5.099\end{array}$ & $\begin{array}{r}5.909 \\
10.726 \\
15.544\end{array}$ & $\begin{array}{r}6.424 \\
11.661 \\
16.897\end{array}$ \\
\hline $\begin{array}{l}0 \\
0 \\
0\end{array}$ & $\begin{array}{l}5000 \\
5000 \\
5000\end{array}$ & $\begin{array}{l}20.0 \\
25.0 \\
30.0\end{array}$ & $\begin{array}{r}.01557 \\
.01931 \\
.02152\end{array}$ & $\begin{array}{r}.06229 \\
.07724 \\
.08609\end{array}$ & $\begin{array}{r}.00474 \\
.00588 \\
.00656\end{array}$ & $\begin{array}{l}.01898 \\
.02354 \\
.02624\end{array}$ & $\begin{array}{l}6.764 \\
8.387 \\
9.348\end{array}$ & $\begin{array}{l}20.617 \\
25.564 \\
28.493\end{array}$ & $\begin{array}{l}22.413 \\
27.790 \\
30.974\end{array}$ \\
\hline $\begin{array}{l}0 \\
0 \\
0\end{array}$ & $\begin{array}{l}5000 \\
5000 \\
5000\end{array}$ & $\begin{array}{l}35.0 \\
40.0 \\
45.0\end{array}$ & $\begin{array}{l}.02234 \\
.02231 \\
.02174\end{array}$ & $\begin{array}{r}.08936 \\
.08927 \\
.08699\end{array}$ & $\begin{array}{r}.00680 \\
.00680 \\
.00662\end{array}$ & $\begin{array}{r}.02723 \\
.02721 \\
.02651\end{array}$ & $\begin{array}{l}9.702 \\
9.692 \\
9.445\end{array}$ & $\begin{array}{l}29.573 \\
29.543 \\
28.790\end{array}$ & $\begin{array}{l}32.149 \\
32.116 \\
31.297\end{array}$ \\
\hline $\begin{array}{l}0 \\
0 \\
0\end{array}$ & $\begin{array}{l}5000 \\
5000 \\
5000\end{array}$ & $\begin{array}{l}46.0 \\
47.0 \\
48.0\end{array}$ & $\begin{array}{l}.02156 \\
.02136 \\
.02115\end{array}$ & $\begin{array}{r}.08624 \\
.08544 \\
.08462\end{array}$ & $\begin{array}{l}.00657 \\
.00651 \\
.00644\end{array}$ & $\begin{array}{r}.02628 \\
.02604 \\
.02579\end{array}$ & $\begin{array}{l}9.363 \\
9.277 \\
9.188\end{array}$ & $\begin{array}{l}28.541 \\
28.277 \\
28.005\end{array}$ & $\begin{array}{l}31.02 .7 \\
30.740 \\
30.444\end{array}$ \\
\hline $\begin{array}{l}0 \\
0\end{array}$ & $\begin{array}{l}5000 \\
5000\end{array}$ & $\begin{array}{l}49.0 \\
50.0\end{array}$ & $\begin{array}{l}.02092 \\
.02067\end{array}$ & $\begin{array}{l}.08368 \\
.08270\end{array}$ & $\begin{array}{l}.00637 \\
.00630\end{array}$ & $\begin{array}{l}.02550 \\
.02520\end{array}$ & $\begin{array}{l}9.086 \\
8.979\end{array}$ & $\begin{array}{l}27 \cdot 695 \\
27 \cdot 369\end{array}$ & $\begin{array}{l}30.107 \\
29.752\end{array}$ \\
\hline 0 & 5000 & 51.0 & .02040 & .08162 & .00621 & .02487 & 8.862 & 27.011 & $29 \cdot 364$ \\
\hline $\begin{array}{l}0 \\
0 \\
0\end{array}$ & $\begin{array}{l}5000 \\
5000 \\
5000\end{array}$ & $\begin{array}{l}52.0 \\
53.0 \\
54.0\end{array}$ & $\begin{array}{r}.02012 \\
.01983 \\
.01953\end{array}$ & $\begin{array}{r}.08049 \\
.07932 \\
.07814\end{array}$ & $\begin{array}{l}.00613 \\
.00604 \\
.00595\end{array}$ & $\begin{array}{l}.02453 \\
.02417 \\
.02381\end{array}$ & $\begin{array}{l}8.739 \\
8.612 \\
8.484\end{array}$ & $\begin{array}{l}26.639 \\
26.251 \\
25.862\end{array}$ & $\begin{array}{l}28.959 \\
28.537 \\
28.115\end{array}$ \\
\hline $\begin{array}{l}0 \\
0\end{array}$ & $\begin{array}{l}5000 \\
5000\end{array}$ & $\begin{array}{l}55.0 \\
60.0\end{array}$ & $\begin{array}{r}01924 \\
.01778\end{array}$ & $\begin{array}{l}.07697 \\
.07113\end{array}$ & $\begin{array}{l}.00586 \\
.00542\end{array}$ & $\begin{array}{l}.02346 \\
.02168\end{array}$ & $\begin{array}{l}8.357 \\
7.723\end{array}$ & $\begin{array}{l}25.474 \\
23.540\end{array}$ & $\begin{array}{l}27.692 \\
25.590\end{array}$ \\
\hline 0 & 5000 & 65.0 & .01631 & .06526 & .00497 & .01989 & 7.086 & 21.598 & 23.479 \\
\hline $\begin{array}{l}0 \\
0\end{array}$ & $\begin{array}{l}5000 \\
5000\end{array}$ & $\begin{array}{l}70.0 \\
75.0\end{array}$ & $\begin{array}{r}.01506 \\
.01401\end{array}$ & $\begin{array}{l}.06025 \\
.05607\end{array}$ & $\begin{array}{l}.00459 \\
.00427\end{array}$ & $\begin{array}{r}.01836 \\
.01709\end{array}$ & & $\begin{array}{l}19 \\
18\end{array}$ & $\begin{array}{l}21.677 \\
20.173\end{array}$ \\
\hline 0. & 5000 & 80.0 & .01314 & .05257 & .00400 & .01602 & 5.708 & 17.398 & 18.914 \\
\hline $\begin{array}{l}0 \\
0\end{array}$ & $\begin{array}{l}5000 \\
5000\end{array}$ & $\begin{array}{l}85.0 \\
90.0\end{array}$ & $\begin{array}{l}.01241 \\
.01172\end{array}$ & $\begin{array}{l}.04966 \\
.04688\end{array}$ & $\begin{array}{l}.00378 \\
.00357\end{array}$ & $\begin{array}{r}.01513 \\
.01429\end{array}$ & $\begin{array}{l}5.392 \\
5.090\end{array}$ & $\begin{array}{l}16.436 \\
15.517\end{array}$ & $\begin{array}{l}17.868 \\
16.868\end{array}$ \\
\hline 0 & 5000 & 95.0 & .01108 & .04432 & .00337 & .01351 & 4.813 & 14.670 & 15.948 \\
\hline 0 & 0 & .0 & 01049 & 04196 & 00319 & .01279 & 4.556 & 13.888 & 15.097 \\
\hline
\end{tabular}


TABULATION OF DATA FOR

ABSORPTION OF SOUND IN AIR VERSUS. PERCENT RELATIVE HUMIDITY AT O DEGREES CENTIGRADE FOR A FREQUENCY OF 5940 HERTZ

\begin{tabular}{|c|c|c|c|c|c|c|c|c|c|}
\hline (1) & (2) & (3) & (4) & (5) & $(6)$ & (7) & $(8)$ & $(9)$ & $(10)$ \\
\hline $\begin{array}{l}\text { DEGR } \\
\text { CENT }\end{array}$ & HERTZ & $\begin{array}{l}\text { REL } \\
\text { HUM } \\
\text { PER } \\
\text { CENT }\end{array}$ & $\begin{array}{l}\text { ATTEN } \\
\text { COEF } \\
\text { PER } \\
\text { METER }\end{array}$ & $\begin{array}{l}4 M \\
\text { PER } \\
\text { METER }\end{array}$ & $\begin{array}{l}\text { ATTEN } \\
\text { COEF } \\
\text { PER } \\
\text { FOOT }\end{array}$ & $\begin{array}{l}4 M \\
\text { PER } \\
\text { FOOT }\end{array}$ & $\begin{array}{l}\text { ATTEN } \\
\text { DB PER } \\
100 \\
\text { METER }\end{array}$ & $\begin{array}{l}\text { ATTEN } \\
\text { DB PER } \\
1000 \\
\text { FEET }\end{array}$ & $\begin{array}{l}\text { DECAY } \\
\text { RATE } \\
\text { DB PER } \\
\text { SECOND }\end{array}$ \\
\hline $\begin{array}{l}0 \\
0 \\
0\end{array}$ & $\begin{array}{l}5940 \\
5940 \\
5940\end{array}$ & $\begin{array}{r}5.0 \\
10.0 \\
15.0\end{array}$ & $\begin{array}{l}.00501 \\
.00886 \\
.01272\end{array}$ & $\begin{array}{l}.02006 \\
.03547 \\
.05088\end{array}$ & $\begin{array}{r}.00152 \\
.00270 \\
.00387\end{array}$ & $\begin{array}{l}.00611 \\
.01081 \\
.01550\end{array}$ & $\begin{array}{l}2 \cdot 178 \\
3 \bullet 851 \\
5.524\end{array}$ & $\begin{array}{r}6.640 \\
11.739 \\
16.838\end{array}$ & $\begin{array}{r}7 \cdot 218 \\
12 \cdot 761 \\
18 \cdot 305\end{array}$ \\
\hline $\begin{array}{l}0 \\
0 \\
0\end{array}$ & $\begin{array}{l}5940 \\
5940 \\
5940\end{array}$ & $\begin{array}{l}20.0 \\
25.0 \\
30.0\end{array}$ & $\begin{array}{l}.01660 \\
.02081 \\
.02427\end{array}$ & $\begin{array}{l}.06643 \\
.08325 \\
.09709\end{array}$ & $\begin{array}{l}.00506 \\
.00634 \\
.00739\end{array}$ & $\begin{array}{l}.02024 \\
.02537 \\
.02959\end{array}$ & $\begin{array}{r}7.212 \\
9.039 \\
10.542\end{array}$ & $\begin{array}{l}21.984 \\
27 \cdot 554 \\
32.133\end{array}$ & $\begin{array}{l}23.899 \\
29.953 \\
34.932\end{array}$ \\
\hline $\begin{array}{l}0 \\
0 \\
0\end{array}$ & $\begin{array}{l}5940 \\
5940 \\
5940\end{array}$ & $\begin{array}{l}35.0 \\
40.0 \\
45.0\end{array}$ & $\begin{array}{l}.02615 \\
.02675 \\
.02669\end{array}$ & $\begin{array}{l}.10460 \\
.10703 \\
.10676\end{array}$ & $\begin{array}{l}.00797 \\
.00815 \\
.00813\end{array}$ & $\begin{array}{l}.03188 \\
.03262 \\
.03254\end{array}$ & $\begin{array}{l}11.357 \\
11.621 \\
11.592\end{array}$ & $\begin{array}{l}34.617 \\
35.423 \\
35.333\end{array}$ & $\begin{array}{l}37.632 \\
38.508 \\
38.410\end{array}$ \\
\hline $\begin{array}{l}0 \\
0 \\
0\end{array}$ & $\begin{array}{l}5940 \\
5940 \\
5940\end{array}$ & $\begin{array}{l}46.0 \\
47.0 \\
48.0\end{array}$ & $\begin{array}{l}.02661 \\
.02651 \\
.02640\end{array}$ & $\begin{array}{l}.10645 \\
.10605 \\
.10560\end{array}$ & $\begin{array}{l}.00811 \\
.00808 \\
.00804\end{array}$ & $\begin{array}{l}.03244 \\
.03232 \\
.03218\end{array}$ & $\begin{array}{l}11.558 \\
11.515 \\
11.466\end{array}$ & $\begin{array}{l}35.229 \\
35.098 \\
34.950\end{array}$ & $\begin{array}{l}38.298 \\
38.155 \\
37.993\end{array}$ \\
\hline $\begin{array}{l}0 \\
0 \\
0\end{array}$ & $\begin{array}{l}5940 \\
5940 \\
5940\end{array}$ & $\begin{array}{l}49.0 \\
50.0 \\
51.00\end{array}$ & $\begin{array}{l}.02627 \\
.02612 \\
.02592\end{array}$ & $\begin{array}{l}10511 \\
.10448 \\
.10368\end{array}$ & $\begin{array}{l}.00800 \\
.00796 \\
.00790\end{array}$ & $\begin{array}{l}.03203 \\
.03184 \\
.03160\end{array}$ & $\begin{array}{l}11 \cdot 412 \\
11 \cdot 344 \\
11 \cdot 257\end{array}$ & $\begin{array}{l}34 \cdot 785 \\
34.577 \\
34 \cdot 314\end{array}$ & $\begin{array}{l}37.815 \\
37.589 \\
37.302\end{array}$ \\
\hline $\begin{array}{l}0 \\
0 \\
0\end{array}$ & $\begin{array}{l}5940 \\
5940 \\
5940\end{array}$ & $\begin{array}{l}52.0 \\
53.0 \\
54.0\end{array}$ & $\begin{array}{l}.02571 \\
.02550 \\
.02528\end{array}$ & $\begin{array}{l}10287 \\
.10202 \\
.10113\end{array}$ & $\begin{array}{l}.00783 \\
.00777 \\
.00770\end{array}$ & $\begin{array}{l}.03135 \\
.03109 \\
.03082\end{array}$ & $\begin{array}{l}11.169 \\
11.077 \\
10.980\end{array}$ & $\begin{array}{l}34.045 \\
33.765 \\
33.470\end{array}$ & $\begin{array}{l}37.010 \\
36.706 \\
36.385\end{array}$ \\
\hline $\begin{array}{l}0 \\
0 \\
0\end{array}$ & $\begin{array}{l}5940 \\
5940 \\
5940\end{array}$ & $\begin{array}{l}55.0 \\
60.0 \\
65.0\end{array}$ & $\begin{array}{l}.02503 \\
.02358 \\
.02202\end{array}$ & $\begin{array}{l}.10014 \\
.09432 \\
.08811\end{array}$ & $\begin{array}{l}.00763 \\
.00718 \\
.00671\end{array}$ & $\begin{array}{l}.03052 \\
.02874 \\
.02685\end{array}$ & $\begin{array}{r}10.873 \\
10.241 \\
0.566\end{array}$ & $\begin{array}{l}33.141 \\
31.215 \\
29.159\end{array}$ & $\begin{array}{l}36.027 \\
33.933 \\
31.698\end{array}$ \\
\hline $\begin{array}{l}0 \\
0 \\
0\end{array}$ & $\begin{array}{l}5940 \\
5940 \\
5940\end{array}$ & $\begin{array}{l}70.0 \\
75.0 \\
80.0\end{array}$ & $\begin{array}{r}.02049 \\
.01896 \\
.01774\end{array}$ & $\begin{array}{r}08199 \\
.07584 \\
.07099\end{array}$ & $\begin{array}{l}.00624 \\
.00577 \\
.00540\end{array}$ & $\begin{array}{l}.02499 \\
.02311 \\
.02163\end{array}$ & $\begin{array}{l}8.902 \\
8.235 \\
7.707\end{array}$ & $\begin{array}{l}27.135 \\
25.101 \\
23.493\end{array}$ & $\begin{array}{l}29.498 \\
27.287 \\
25.540\end{array}$ \\
\hline $\begin{array}{l}0 \\
0 \\
0\end{array}$ & $\begin{array}{l}5940 \\
5940 \\
5940\end{array}$ & $\begin{array}{l}85.0 \\
90.0 \\
95.0\end{array}$ & $\begin{array}{r}.01667 \\
.01576 \\
.01499\end{array}$ & $\begin{array}{l}.06669 \\
.06304 \\
.05997\end{array}$ & $\begin{array}{l}.00508 \\
.00480 \\
.00456\end{array}$ & $\begin{array}{l}.02032 \\
.01921 \\
.01827\end{array}$ & $\begin{array}{l}7.241 \\
6.844 \\
6.511\end{array}$ & $\begin{array}{l}22.071 \\
20.862 \\
19.847\end{array}$ & $\begin{array}{l}23.993 \\
22.679 \\
21.576\end{array}$ \\
\hline 0 & 5940 & 100.0 & .01425 & .05701 & .00434 & .01737 & 6.190 & 18.867 & 20.511 \\
\hline
\end{tabular}


TABULATION OF DATA FOR

ABSORPTION OF SOUND IN AIR VERSUS PERCENT RELATIVE HUMIDITY AT O DEGREES CENTIGRADE FOR A FREQUENCY OF 6300 HERTZ

\begin{tabular}{|c|c|c|c|c|c|c|c|c|c|}
\hline 1) & $(2)$ & (3) & $(4)$ & (5) & $(6)$ & $(7)$ & $(8)$ & $(9)$ & $(10)$ \\
\hline $\begin{array}{l}\text { TEMP } \\
\text { DEGR } \\
\text { CENT }\end{array}$ & FREQ & $\begin{array}{l}\text { REL } \\
\text { HUM } \\
\text { PER } \\
\text { CENT }\end{array}$ & $\begin{array}{l}\text { ATTEN } \\
\text { COEF } \\
\text { PER } \\
\text { METER }\end{array}$ & $\begin{array}{l}\text { 4M } \\
\text { PER } \\
\text { METER }\end{array}$ & $\begin{array}{l}\text { ATTEN } \\
\text { CEEF } \\
\text { PER } \\
\text { FQOT }\end{array}$ & $\begin{array}{l}4 M \\
\text { PER } \\
\text { FGOT }\end{array}$ & $\begin{array}{l}\text { ATTEN } \\
\text { DB PER } \\
100 \\
\text { METER }\end{array}$ & $\begin{array}{l}\text { ATTEN } \\
\text { DB PER } \\
1000 \\
\text { FEET }\end{array}$ & $\begin{array}{l}\text { DECAY } \\
\text { RATE } \\
\text { DB PER } \\
\text { SECOND }\end{array}$ \\
\hline $\begin{array}{l}0 \\
0 \\
0\end{array}$ & $\begin{array}{l}6300 \\
6300 \\
6300\end{array}$ & $\begin{array}{r}5.0 \\
10.0 \\
15.0\end{array}$ & $\begin{array}{l}.00523 \\
.00915 \\
.01308\end{array}$ & $\begin{array}{r}.02093 \\
.03663 \\
.05233\end{array}$ & $\begin{array}{r}.00159 \\
.00279 \\
.00398\end{array}$ & $\begin{array}{l}.00638 \\
.01116 \\
.01595\end{array}$ & $\begin{array}{l}2.273 \\
3.977 \\
5.681\end{array}$ & $\begin{array}{r}6.928 \\
12 \cdot 123 \\
17.328\end{array}$ & $\begin{array}{r}7.532 \\
13.179 \\
18.826\end{array}$ \\
\hline $\begin{array}{l}0 \\
0 \\
0\end{array}$ & $\begin{array}{l}6300 \\
6300 \\
6300\end{array}$ & $\begin{array}{l}20.0 \\
25.0 \\
30.0\end{array}$ & $\begin{array}{r}.01702 \\
.02128 \\
.02516\end{array}$ & $\begin{array}{r}.06809 \\
.08515 \\
.10064\end{array}$ & $\begin{array}{l}.00518 \\
.00648 \\
.00766\end{array}$ & $\begin{array}{l}.02075 \\
.02595 \\
.03067\end{array}$ & $\begin{array}{r}7.393 \\
9.245 \\
10.927\end{array}$ & $\begin{array}{l}22.534 \\
28.180 \\
33.308\end{array}$ & $\begin{array}{l}24.497 \\
30.635 \\
36.209\end{array}$ \\
\hline $\begin{array}{l}0 \\
0 \\
0\end{array}$ & $\begin{array}{l}6300 \\
6300 \\
6300\end{array}$ & $\begin{array}{l}35.0 \\
40.0 \\
45.0\end{array}$ & $\begin{array}{l}.02737 \\
.02834 \\
.02847\end{array}$ & $\begin{array}{r}.10949 \\
.11336 \\
.11390\end{array}$ & $\begin{array}{l}.00834 \\
.00863 \\
.00867\end{array}$ & $\begin{array}{l}.03337 \\
.03455 \\
.03471\end{array}$ & $\begin{array}{l}11 \cdot 888 \\
12.308 \\
12.366\end{array}$ & $\begin{array}{l}36.237 \\
37.517 \\
37.694\end{array}$ & $\begin{array}{l}39.393 \\
40.785 \\
40.977\end{array}$ \\
\hline $\begin{array}{l}0 \\
0 \\
0\end{array}$ & $\begin{array}{l}6300 \\
6300 \\
6300\end{array}$ & $\begin{array}{l}46.0 \\
47.0 \\
48.0\end{array}$ & $\begin{array}{l}.02842 \\
.02837 \\
.02829\end{array}$ & $\begin{array}{r}11371 \\
-11351 \\
-11316\end{array}$ & $\begin{array}{l}.00866 \\
.00864 \\
.00862\end{array}$ & $\begin{array}{l}.03466 \\
.03459 \\
.03449\end{array}$ & $\begin{array}{l}12 \cdot 346 \\
12 \cdot 324 \\
12 \cdot 287\end{array}$ & $\begin{array}{l}37.632 \\
37.565 \\
37.451\end{array}$ & $\begin{array}{r}40.910 \\
40.837 \\
40.713\end{array}$ \\
\hline $\begin{array}{l}0 \\
0 \\
0\end{array}$ & $\begin{array}{l}6300 \\
6300 \\
6300\end{array}$ & $\begin{array}{l}49.0 \\
50.0 \\
51.0\end{array}$ & $\begin{array}{l}.02819 \\
.02807 \\
.02794\end{array}$ & $\begin{array}{r}11276 \\
.11230 \\
.11179\end{array}$ & $\begin{array}{l}.00859 \\
.00855 \\
.00851\end{array}$ & $\begin{array}{l}.03436 \\
.03422 \\
.03407\end{array}$ & $\begin{array}{l}12 \cdot 243 \\
12: 193 \\
12 \cdot 138\end{array}$ & $\begin{array}{l}97 \cdot 317 \\
37.165 \\
36.997\end{array}$ & $\begin{array}{l}40.567 \\
40.401 \\
40.210\end{array}$ \\
\hline $\begin{array}{l}0 \\
0 \\
0\end{array}$ & $\begin{array}{l}6300 \\
6300 \\
6300\end{array}$ & $\begin{array}{l}52.0 \\
53.0 \\
54.0\end{array}$ & $\begin{array}{r}.02779 \\
.02758 \\
.02738\end{array}$ & $\begin{array}{r}11216 \\
.11035 \\
.10953\end{array}$ & $\begin{array}{l}.00847 \\
.00840 \\
.00834\end{array}$ & $\begin{array}{l}.0 \\
.0 \\
.03\end{array}$ & $\begin{array}{l}12.069 \\
11.982 \\
11.892\end{array}$ & $\begin{array}{l}36.789 \\
36.521 \\
36.249\end{array}$ & $\begin{array}{l}993 \\
702 \\
406\end{array}$ \\
\hline $\begin{array}{l}0 \\
0 \\
0\end{array}$ & $\begin{array}{l}6390 \\
6300 \\
6300\end{array}$ & $\begin{array}{l}55.0 \\
60.0 \\
65.0\end{array}$ & $\begin{array}{r}.02716 \\
.02586 \\
.02428\end{array}$ & $\begin{array}{r}.10867 \\
.10345 \\
.09714\end{array}$ & $\begin{array}{l}.00828 \\
.00788 \\
.00740\end{array}$ & $\begin{array}{l}.03312 \\
.03153 \\
.02960\end{array}$ & $\begin{array}{l}11.799 \\
11.232 \\
10.547\end{array}$ & $\begin{array}{l}35.964 \\
34.236 \\
32.148\end{array}$ & $\begin{array}{l}39.096 \\
37.217 \\
34.947\end{array}$ \\
\hline $\begin{array}{l}0 \\
0 \\
0\end{array}$ & $\begin{array}{l}6300 \\
6300 \\
6300\end{array}$ & $\begin{array}{l}70.0 \\
75.0 \\
80.0\end{array}$ & $\begin{array}{l}.02271 \\
.02113 \\
.01970\end{array}$ & $\begin{array}{l}.09084 \\
.08454 \\
.07881\end{array}$ & $\begin{array}{l}.00692 \\
.00644 \\
.00600\end{array}$ & $\begin{array}{l}.02768 \\
.02576 \\
.02402\end{array}$ & $\begin{array}{l}9.863 \\
9.179 \\
8.556\end{array}$ & $\begin{array}{l}30.063 \\
27.978 \\
26.082\end{array}$ & $\begin{array}{l}32.681 \\
30.415 \\
28.353\end{array}$ \\
\hline $\begin{array}{l}0 \\
0 \\
0\end{array}$ & $\begin{array}{l}6300 \\
6300 \\
6300\end{array}$ & $\begin{array}{l}85.0 \\
90.0 \\
95.0\end{array}$ & $\begin{array}{r}.01849 \\
.01745 \\
.01657\end{array}$ & $\begin{array}{l}.07399 \\
.06982 \\
.06628\end{array}$ & $\begin{array}{l}.00563 \\
.00532 \\
.00505\end{array}$ & $\begin{array}{l}.02255 \\
.02128 \\
.02020\end{array}$ & $\begin{array}{l}8.034 \\
7.580 \\
7.197\end{array}$ & $\begin{array}{l}24 \cdot 489 \\
23 \cdot 106 \\
21.937\end{array}$ & $\begin{array}{l}26.622 \\
25.119 \\
23.847\end{array}$ \\
\hline 0 & 6300 & 100.0 & .0158 & 06324 & 100481 & 01927 & 80867 & 10.931 & $22 \cdot 754$ \\
\hline
\end{tabular}


TABULATION OF DATA FOR

ABSORPTION OF SOUND IN AIR VERSUS PERGENT RELATIVE HUMIDITY AT O DEGREES CENTIGRADE FOR A FREQUENCY OF 8000 HERTZ

\begin{tabular}{|c|c|c|c|c|c|c|c|c|c|}
\hline 1 & (2) & 131 & (4) & (5) & 161. & $(7)$. & $(8)$ & 191 & $(10)$ \\
\hline $\begin{array}{l}\text { EMP } \\
\text { EGR } \\
\text { ENT }\end{array}$ & HFRTZ. & $\begin{array}{l}\text { REL } \\
\text { HUM } \\
\text { PER } \\
\text { CENT }\end{array}$ & $\begin{array}{l}\text { ATTEN } \\
\text { COEF } \\
\text { PER } \\
\text { METER }\end{array}$ & $\begin{array}{l}\text { PER. } \\
\text { METER }\end{array}$ & $\begin{array}{l}\text { ATTEN } \\
\text { COEF } \\
\text { PER } \\
\text { FOOT. }\end{array}$ & $\begin{array}{l}4 M \\
\text { PER } \\
\text { FOOT }\end{array}$ & $\begin{array}{l}\text { ATTEN } \\
\text { OB PER } \\
100 \\
\text { METER }\end{array}$ & $\begin{array}{l}\text { ATTEN } \\
\text { DB PER } \\
1000 \\
\text { FEET }\end{array}$ & $\begin{array}{l}\text { DECAY } \\
\text { RATE } \\
\text { DB PER } \\
\text { SECOND }\end{array}$ \\
\hline $\begin{array}{l}0 \\
0 \\
0\end{array}$ & $\begin{array}{l}8000 \\
8000 \\
8000\end{array}$ & $\begin{array}{r}5.0 \\
10.0 \\
15.0\end{array}$ & $\begin{array}{r}.00640 \\
.01069 \\
.01499\end{array}$ & $\begin{array}{l}.0256 i \\
.04279 \\
.05996\end{array}$ & $\begin{array}{l}.00195 \\
.00326 \\
.00456\end{array}$ & $\begin{array}{r}.00780 \\
.01304 \\
.01827\end{array}$ & $\begin{array}{l}2.781 \\
4.646 \\
6.511\end{array}$ & $\begin{array}{r}8.47 .7 \\
14 \cdot 161 \\
19.845\end{array}$ & $\begin{array}{r}9.215 \\
25.395 \\
21.574\end{array}$ \\
\hline $\begin{array}{l}0 \\
0 \\
0\end{array}$ & $\begin{array}{l}8000 \\
8000 \\
8000\end{array}$ & $\begin{array}{l}20.0 \\
25.0 \\
30.0\end{array}$ & $\begin{array}{l}.01928 \\
.02365 \\
.02839\end{array}$ & $\begin{array}{l}.077 \\
.094 \\
.113\end{array}$ & $\begin{array}{l}.00587 \\
.00720 \\
.00865\end{array}$ & $\begin{array}{l}.02351 \\
.02883 \\
.03461\end{array}$ & $\begin{array}{l}8 . \\
10 . \\
12 .\end{array}$ & & $\begin{array}{l}27.753 \\
34.040 \\
40.860\end{array}$ \\
\hline $\begin{array}{l}0 \\
0 \\
0\end{array}$ & $\begin{array}{l}8000 \\
8000 \\
8000\end{array}$ & $\begin{array}{l}35.0 \\
40.0 \\
45.0\end{array}$ & $\begin{array}{r}.03252 \\
.03500 \\
.03629\end{array}$ & $\begin{array}{r}.13011 \\
\cdot 14001 \\
\cdot 14519\end{array}$ & $\begin{array}{r}.00991 \\
.01066 \\
.01106\end{array}$ & $\begin{array}{r}.03965 \\
.04267 \\
.04425\end{array}$ & & & \\
\hline $\begin{array}{l}0 \\
0 \\
0\end{array}$ & $\begin{array}{l}8000 \\
8000 \\
8000\end{array}$ & $\begin{array}{l}46.0 \\
47.0 \\
48.0\end{array}$ & $\begin{array}{l}.03 \\
.03 \\
.03\end{array}$ & $\begin{array}{r}.14 \\
.14 \\
.14\end{array}$ & $\begin{array}{r}.01109 \\
.01112 \\
.01114\end{array}$ & $\begin{array}{l}.04437 \\
.04449 \\
.04457\end{array}$ & $\begin{array}{l}15 \\
15 \\
15\end{array}$ & $\begin{array}{l}48.179 \\
48.307 \\
48.403\end{array}$ & $\begin{array}{l}52 \cdot 375 \\
52 \cdot 5.14 \\
52 \cdot 618\end{array}$ \\
\hline $\begin{array}{l}0 \\
0 \\
0\end{array}$ & $\begin{array}{l}8000 \\
8000 \\
8000\end{array}$ & $\begin{array}{l}49.0 \\
50.0 \\
51.0\end{array}$ & $\begin{array}{r}.03662 \\
.03666 \\
.03663\end{array}$ & $\begin{array}{r}.14648 \\
.14666 \\
.14655\end{array}$ & $\begin{array}{l}.01116 \\
.01117 \\
.01116\end{array}$ & $\begin{array}{r}.04464 \\
.04470 \\
.04467\end{array}$ & $\begin{array}{l}15.904 \\
15.924 \\
15.912\end{array}$ & $\begin{array}{l}48.476 \\
48: 538 \\
48.502\end{array}$ & $\begin{array}{l}52 \\
52 \\
52\end{array}$ \\
\hline $\begin{array}{l}0 \\
0 \\
0\end{array}$ & $\begin{array}{l}8000 \\
8000 \\
8000\end{array}$ & $\begin{array}{l}52 \cdot 0 \\
53.0 \\
54 \cdot 0\end{array}$ & $\begin{array}{l}.03 \\
.03 \\
.03\end{array}$ & $\begin{array}{r}.14644 \\
.14626 \\
.14604\end{array}$ & $\begin{array}{l}.01115 \\
.011114 \\
.01112\end{array}$ & $\begin{array}{r}.04463 \\
.04458 \\
.04451\end{array}$ & & & $\begin{array}{l}52 \\
52 \\
52\end{array}$ \\
\hline $\begin{array}{l}0 \\
0 \\
0\end{array}$ & $\begin{array}{l}8000 \\
8000 \\
8000\end{array}$ & $\begin{array}{l}55.0 \\
60.0 \\
65.0\end{array}$ & $\begin{array}{l}.03 \\
.03 \\
.03\end{array}$ & $\begin{array}{l}.14 \\
.14 \\
.13\end{array}$ & $\begin{array}{r}.01111 \\
.01091 \\
.01056\end{array}$ & $\begin{array}{r}.04444 \\
.04366 \\
.04226\end{array}$ & $\begin{array}{l}15 \\
15 \\
15\end{array}$ & $\begin{array}{l}48.251 \\
47.409 \\
45.887\end{array}$ & $\begin{array}{l}454 \\
538 \\
883\end{array}$ \\
\hline $\begin{array}{l}0 \\
0 \\
0\end{array}$ & $\begin{array}{l}8000 \\
8000 \\
8000\end{array}$ & $\begin{array}{l}70.0 \\
75.0 \\
80.0\end{array}$ & $\begin{array}{l}.03 \\
.03 \\
.02\end{array}$ & $\begin{array}{l}.13266 \\
.12573 \\
.11881\end{array}$ & $\begin{array}{l}.01010 \\
.00958 \\
.00905\end{array}$ & $\begin{array}{r}.04043 \\
.03832 \\
.03621\end{array}$ & $\begin{array}{l}14.4 \\
13.6 \\
12.9\end{array}$ & $\begin{array}{l}43.003 \\
41.611 \\
39.320\end{array}$ & $\begin{array}{l}47.726 \\
45.235 \\
42.745\end{array}$ \\
\hline $\begin{array}{l}0 \\
0 \\
0\end{array}$ & $\begin{array}{l}8000 \\
8000 \\
8000\end{array}$ & $\begin{array}{l}85.0 \\
90.0 \\
95.0\end{array}$ & $\begin{array}{r}.02800 \\
.02625 \\
.02487\end{array}$ & $\begin{array}{l}\text { - } 11201 \\
\text { - } 10500 \\
.09948\end{array}$ & $\begin{array}{l}.00853 \\
.00800 \\
.00758\end{array}$ & $\begin{array}{l}.03414 \\
.03200 \\
.03032\end{array}$ & $\begin{array}{l}12.162 \\
11.401 \\
10.801\end{array}$ & $\begin{array}{l}37.071 \\
34.751 \\
32.923\end{array}$ & $\begin{array}{l}40.299 \\
37.778 \\
35.790\end{array}$ \\
\hline 0 & 8000 & 1 & 02359 & 09438 & 9 & $0<810$ & 10.247 & 31.236 & 33.956 \\
\hline
\end{tabular}


TABULATION OF DATA FOR

ABSORPTION OF SOUND IN AIR VERSUS PERCENT RELATIVE HUMIDITY AT O DEGREES CENTIGRADE FOR A FREQUENCY OF 10000 HERTZ

\begin{tabular}{|c|c|c|c|c|c|c|c|c|c|}
\hline 11 & $(2)$ & (3) & (4) & (5) & $(6)$ & 171 & $(8)$ & $(9)$ & $(10)$ \\
\hline EMP & FREQ & $\begin{array}{l}\text { REL } \\
\text { HUM }\end{array}$ & $\begin{array}{l}\text { ATTEN } \\
\text { COEF }\end{array}$ & $4 M$ & $\begin{array}{l}\text { ATTEN } \\
\text { COEF }\end{array}$ & $4 M$ & $\begin{array}{l}\text { ATTTEN } \\
\text { DB } \cdot \text { PER }\end{array}$ & $\begin{array}{l}\text { ATTEN } \\
\text { DB PER }\end{array}$ & $\begin{array}{l}\text { DECAY } \\
\text { RATE }\end{array}$ \\
\hline $\begin{array}{l}\text { ER } \\
\text { NT }\end{array}$ & HERTZ & $\begin{array}{l}\text { PER } \\
\text { CENT }\end{array}$ & $\begin{array}{l}\text { PER } \\
\text { METER }\end{array}$ & $\begin{array}{l}\text { PER } \\
\text { METER }\end{array}$ & $\begin{array}{l}\text { PER } \\
\text { FOOT }\end{array}$ & $\begin{array}{l}\text { PER } \\
\text { FOOT }\end{array}$ & $\begin{array}{l}100 \\
\text { METER }\end{array}$ & $\begin{array}{l}1000 \\
\text { FEET }\end{array}$ & $\begin{array}{l}\text { DB PER } \\
\text { SECOND }\end{array}$ \\
\hline $\begin{array}{l}0 \\
0\end{array}$ & $\begin{array}{l}10000 \\
10000\end{array}$ & $\begin{array}{r}5.0 \\
10.0\end{array}$ & $\begin{array}{r}.00795 \\
.01261\end{array}$ & $\begin{array}{r}.03183 \\
.05046\end{array}$ & $\begin{array}{r}.00242 \\
.00384\end{array}$ & $\begin{array}{r}.00970 \\
.01538\end{array}$ & $\begin{array}{l}3.456 \\
5.479\end{array}$ & $\begin{array}{l}10.534 \\
16.700\end{array}$ & $\begin{array}{l}11.452 \\
18.155\end{array}$ \\
\hline $0:$ & 10000 & 15.0 & .01727 & .06909 & .00526 & .02106 & 7.502 & 22.867 & 24.858 \\
\hline $\begin{array}{l}0 \\
0\end{array}$ & $\begin{array}{l}10000 \\
10000\end{array}$ & $\begin{array}{l}20.0 \\
25.0\end{array}$ & $\begin{array}{r}.02193 \\
.02659\end{array}$ & $\begin{array}{l}.08772 \\
.10636\end{array}$ & $\begin{array}{l}.00668 \\
.00810\end{array}$ & $\begin{array}{l}.02673 \\
.03241\end{array}$ & $\begin{array}{r}9.525 \\
11.548\end{array}$ & $\begin{array}{l}29.033 \\
35.199\end{array}$ & $\begin{array}{l}31.561 \\
38.265\end{array}$ \\
\hline 0 & 10000 & 30.0 & .03148 & .12594 & .00959 & .03838 & 13.674 & 41.678 & $45 \cdot 308$ \\
\hline $\begin{array}{l}0 \\
0\end{array}$ & $\begin{array}{l}10000 \\
10000\end{array}$ & $\begin{array}{l}35.0 \\
40.0\end{array}$ & $\begin{array}{l}.03656 \\
.04106\end{array}$ & $\begin{array}{r}.14627 \\
.16424\end{array}$ & $\begin{array}{r}.01114 \\
.011251\end{array}$ & $\begin{array}{l}.04458 \\
.05006\end{array}$ & $\begin{array}{l}15.882 \\
17.833\end{array}$ & & $\begin{array}{l}52.625 \\
59.091\end{array}$ \\
\hline 0 & 10000 & $45 \cdot 0$ & .04391 & .17566 & .01338 & .05354 & 19.073 & $58 \cdot 136$ & 63.199 \\
\hline $\begin{array}{l}0 \\
0 \\
0\end{array}$ & $\begin{array}{l}10000 \\
10000 \\
10000\end{array}$ & $\begin{array}{l}46.0 \\
47.0 \\
48.0\end{array}$ & $\begin{array}{l}.04438 \\
.04474 \\
.04510\end{array}$ & $\begin{array}{r}.17753 \\
.17897 \\
.18041\end{array}$ & $\begin{array}{r}.01352 \\
.01363 \\
.01374\end{array}$ & $\begin{array}{l}.05411 \\
.05455 \\
.05499\end{array}$ & $\begin{array}{l}19.275 \\
19.432 \\
19.588\end{array}$ & $\begin{array}{l}58.752 \\
59.230 \\
59.707\end{array}$ & $\begin{array}{l}63.869 \\
64.388 \\
64.907\end{array}$ \\
\hline $\begin{array}{l}0 \\
0 \\
0\end{array}$ & $\begin{array}{l}10000 \\
10000 \\
10000\end{array}$ & $\begin{array}{l}49.0 \\
50.0 \\
51.0\end{array}$ & $\begin{array}{l}.04544 \\
.04565 \\
.04586\end{array}$ & $\begin{array}{r}.18178 \\
.18262 \\
.18346\end{array}$ & $\begin{array}{r}.01385 \\
.01391 \\
.01398\end{array}$ & $\begin{array}{r}.05540 \\
.05566 \\
.05592\end{array}$ & $\begin{array}{l}19.737 \\
19.828 \\
19.920\end{array}$ & $\begin{array}{l}60.160 \\
60.438 \\
60.717\end{array}$ & $\begin{array}{l}65.399 \\
65.702 \\
66.005\end{array}$ \\
\hline $\begin{array}{l}0 \\
0 \\
0\end{array}$ & $\begin{array}{l}10000 \\
10000 \\
10000\end{array}$ & $\begin{array}{l}52.0 \\
53.0 \\
54.0\end{array}$ & $\begin{array}{r}.04605 \\
.04615 \\
.04626\end{array}$ & $\begin{array}{r}.18420 \\
.18462 \\
.18504\end{array}$ & $\begin{array}{r}.01403 \\
.01406 \\
.01410\end{array}$ & $\begin{array}{l}.05614 \\
.05627 \\
.05640\end{array}$ & $\begin{array}{l}19.999 \\
20.045 \\
20.091\end{array}$ & $\begin{array}{l}60.960 \\
61 \cdot 100 \\
61.239\end{array}$ & $\begin{array}{l}66.269 \\
66: 421 \\
66.572\end{array}$ \\
\hline $\begin{array}{l}0 \\
0 \\
0\end{array}$ & $\begin{array}{l}10000 \\
10000 \\
10000\end{array}$ & $\begin{array}{l}55.0 \\
60.0 \\
65.0\end{array}$ & $\begin{array}{l}.04634 \\
.04642 \\
.04603\end{array}$ & $\begin{array}{r}.18539 \\
.18569 \\
.18412\end{array}$ & $\begin{array}{r}.01412 \\
.01415 \\
.01403\end{array}$ & $\begin{array}{l}.05 \\
.05 \\
.05\end{array}$ & $\begin{array}{l}20 \\
20 \\
19\end{array}$ & $\begin{array}{l}61 \\
61 \\
60\end{array}$ & $\begin{array}{l}599 \\
307 \\
240\end{array}$ \\
\hline $\begin{array}{l}0 \\
0\end{array}$ & $\begin{array}{l}10000 \\
10000\end{array}$ & $\begin{array}{l}70.0 \\
75.0\end{array}$ & $\begin{array}{l}.04522 \\
.04396\end{array}$ & $\begin{array}{r}18089 \\
-17584\end{array}$ & $\begin{array}{r}.01378 \\
.01339\end{array}$ & $\begin{array}{r}.05513 \\
.05359\end{array}$ & $\begin{array}{l}19 \\
19\end{array}$ & $\begin{array}{l}59 \\
58\end{array}$ & $\begin{array}{l}65.077 \\
63.264\end{array}$ \\
\hline 0 & 10000 & 80.0 & .04235 & .16941 & .01290 & .05163 & 18.393 & 56.065 & 60.947 \\
\hline $\begin{array}{l}0 \\
0 \\
0\end{array}$ & $\begin{array}{l}10000 \\
10000 \\
10000\end{array}$ & $\begin{array}{l}85.0 \\
90.0 \\
95.0\end{array}$ & $\begin{array}{r}.04048 \\
.03861 \\
.03675\end{array}$ & $\begin{array}{r}.16195 \\
.15444 \\
.14702\end{array}$ & $\begin{array}{l}.01234 \\
.01176 \\
.01120\end{array}$ & $\begin{array}{l}.04936 \\
.04707 \\
.04481\end{array}$ & $\begin{array}{l}17.584 \\
16.768 \\
15.962\end{array}$ & $\begin{array}{l}53.598 \\
51.112 \\
48.655\end{array}$ & $\begin{array}{l}58.266 \\
55.563 \\
52.893\end{array}$ \\
\hline 0 & 10000 & 100.0 & .03488 & 13952 & 01063 & .04252 & 15.148 & 6.174 & 50.195 \\
\hline
\end{tabular}


TABULATION OF DATA FOR

ABSORPTION OF SOUND IN AIR VERSUS PERCENT RELATIVE HUMIDITY AT O DEGREES CENTIGRADE FOR A FREQUENCY OF 12500 HERTZ

\begin{tabular}{|c|c|c|c|c|c|c|c|c|c|}
\hline$(1)$ & $(2)$ & $(3)$ & (4) & (5) & (6) & (7) & $(8)$ & (9) & $(10)$ \\
\hline $\begin{array}{l}\text { TEMP } \\
\text { DEGR } \\
\text { CENT }\end{array}$ & HERTZ & $\begin{array}{l}\text { REL } \\
\text { HUM } \\
\text { PER } \\
\text { CENT }\end{array}$ & $\begin{array}{l}\text { ATTEN } \\
\text { COEF } \\
\text { PER } \\
\text { METER }\end{array}$ & $\begin{array}{l}4 M \\
\text { PER } \\
\text { METER }\end{array}$ & $\begin{array}{l}\text { ATTEN } \\
\text { COEF } \\
\text { PER } \\
\text { FOOT }\end{array}$ & $\begin{array}{l}4 M \\
\text { PER } \\
\text { FOOT }\end{array}$ & $\begin{array}{l}\text { AT TEN } \\
\text { DB PER } \\
100 \\
\text { METER }\end{array}$ & $\begin{array}{l}\text { ATTEN } \\
\text { DB PER } \\
\text { 1OOO } \\
\text { FEET }\end{array}$ & $\begin{array}{l}\text { DECAY } \\
\text { RATE } \\
\text { DB PER } \\
\text { SECOND }\end{array}$ \\
\hline $\begin{array}{l}0 \\
0 \\
0\end{array}$ & $\begin{array}{l}12500 \\
12500 \\
12500\end{array}$ & $\begin{array}{r}5.0 \\
10.0 \\
15.0\end{array}$ & $\begin{array}{r}.01009 \\
.01503 \\
.01997\end{array}$ & $\begin{array}{l}.04039 \\
.06014 \\
.07989\end{array}$ & $\begin{array}{l}.00307 \\
.00458 \\
.00608\end{array}$ & $\begin{array}{r}.01231 \\
.01833 \\
.02435\end{array}$ & $\begin{array}{l}4.385 \\
6.530 \\
8.674\end{array}$ & $\begin{array}{l}13.367 \\
19.904 \\
26.441\end{array}$ & $\begin{array}{l}14.531 \\
21.637 \\
28.743\end{array}$ \\
\hline $\begin{array}{l}0 \\
0 \\
0\end{array}$ & $\begin{array}{l}12500 \\
12500 \\
12500\end{array}$ & $\begin{array}{l}20.0 \\
25.0 \\
30.0\end{array}$ & $\begin{array}{r}.02491 \\
.02985 \\
.03478\end{array}$ & $\begin{array}{r}.09964 \\
.11940 \\
.13915\end{array}$ & $\begin{array}{l}.00759 \\
.00909 \\
.01060\end{array}$ & $\begin{array}{l}.03037 \\
.03639 \\
.04241\end{array}$ & $\begin{array}{l}10.819 \\
12.963 \\
15.108\end{array}$ & $\begin{array}{l}32.977 \\
39.514 \\
46.051\end{array}$ & $\begin{array}{l}35.849 \\
42.956 \\
50.062\end{array}$ \\
\hline 0 & 12500 & 35.0 & .03997 & .15989 & .01218 & .04873 & 17.360 & 52.916 & $57 \cdot 525$ \\
\hline $\begin{array}{l}0 \\
0\end{array}$ & $\begin{array}{l}12500 \\
12500\end{array}$ & $\begin{array}{l}40.0 \\
45.0\end{array}$ & $\begin{array}{l}.04542 \\
.05043\end{array}$ & $\begin{array}{r}.18168 \\
.20172\end{array}$ & $\begin{array}{r}.01384 \\
.01537\end{array}$ & $\begin{array}{r}.05537 \\
.06148\end{array}$ & $\begin{array}{l}19.726 \\
21.902\end{array}$ & $\begin{array}{l}60.125 \\
66.759\end{array}$ & $\begin{array}{r}65.362 \\
.72 .573\end{array}$ \\
\hline 0 & 12500 & 46.0 & .05132 & .20529 & .01564 & .06257 & 22.289 & $67 \cdot 940$ & 73.857 \\
\hline $\begin{array}{l}0 \\
0\end{array}$ & $\begin{array}{l}12500 \\
12500\end{array}$ & $\begin{array}{l}47.0 \\
48.0\end{array}$ & $\begin{array}{l}.05221 \\
.05297\end{array}$ & $\begin{array}{r}.20886 \\
.21191\end{array}$ & $\begin{array}{l}.01591 \\
.01614\end{array}$ & $\begin{array}{l}.06366 \\
.06459\end{array}$ & $\begin{array}{l}22.677 \\
23.008\end{array}$ & $\begin{array}{l}69 \cdot 121 \\
70 \cdot 131\end{array}$ & $\begin{array}{l}75.141 \\
76.239\end{array}$ \\
\hline $\begin{array}{l}0 \\
0 \\
0\end{array}$ & $\begin{array}{l}12500 \\
12500 \\
12500\end{array}$ & $\begin{array}{l}49.0 \\
50.0 \\
51.0\end{array}$ & $\begin{array}{l}.05363 \\
.05428 \\
.05491\end{array}$ & $\begin{array}{r}.21452 \\
.21713 \\
.21965\end{array}$ & $\begin{array}{r}.01634 \\
.01654 \\
.01673\end{array}$ & $\begin{array}{l}.06538 \\
.06618 \\
.06695\end{array}$ & $\begin{array}{l}23.292 \\
23.575 \\
23.849\end{array}$ & $\begin{array}{l}70.995 . \\
71.860 \\
72.693\end{array}$ & $\begin{array}{l}77.178 \\
78.118 \\
79.024\end{array}$ \\
\hline $\begin{array}{l}0 \\
0 \\
0\end{array}$ & $\begin{array}{l}12500 \\
12500 \\
12500\end{array}$ & $\begin{array}{l}52.0 \\
53.0 \\
54.0\end{array}$ & $\begin{array}{r}.05540 \\
.05590 \\
.05639\end{array}$ & $\begin{array}{l}.22163 \\
.22360 \\
.22558\end{array}$ & $\begin{array}{l}.01688 \\
.01703 \\
.01718\end{array}$ & $\begin{array}{l}.06755 \\
.06815 \\
.06875\end{array}$ & $\begin{array}{l}24 \cdot 063 \\
24.278 \\
24.492\end{array}$ & $\begin{array}{l}73 \cdot 346 \\
74 \cdot 000 \\
74.654\end{array}$ & $\begin{array}{l}79 \cdot 734 \\
80.445 \\
81 \cdot 156\end{array}$ \\
\hline $\begin{array}{l}0 \\
0 \\
0\end{array}$ & $\begin{array}{l}12500 \\
12500 \\
12500\end{array}$ & $\begin{array}{l}55.0 \\
60.0 \\
65.0\end{array}$ & $\begin{array}{l}.05680 \\
.05833 \\
.05898\end{array}$ & $\begin{array}{r}.22721 \\
.23335 \\
.23592\end{array}$ & $\begin{array}{r}.01731 \\
.01778 \\
.01797\end{array}$ & $\begin{array}{l}.06925 \\
.07112 \\
.07191\end{array}$ & $\begin{array}{l}24.670 \\
25.336 \\
25.615\end{array}$ & $\begin{array}{l}75.195 \\
77.226 \\
78.077\end{array}$ & $\begin{array}{l}81.744 \\
83.952 \\
84.877\end{array}$ \\
\hline $\begin{array}{l}0 \\
0 \\
0\end{array}$ & $\begin{array}{l}12500 \\
12500 \\
12500\end{array}$ & $\begin{array}{l}70.0 \\
75.0 \\
80.0\end{array}$ & $\begin{array}{r}.05908 \\
.05878 \\
.05808\end{array}$ & $\begin{array}{l}.23635 \\
.23512 \\
.23232\end{array}$ & $\begin{array}{l}.01801 \\
.01791 \\
.01770\end{array}$ & $\begin{array}{l}.07204 \\
.07166 \\
.07081\end{array}$ & $\begin{array}{l}25.662 \\
25.529 \\
25.224\end{array}$ & $\begin{array}{l}78.221 \\
77.814 \\
76.885\end{array}$ & $\begin{array}{l}85.033 \\
84.591 \\
83.581\end{array}$ \\
\hline $\begin{array}{l}0 \\
0 \\
0\end{array}$ & $\begin{array}{l}12500 \\
12500 \\
12500\end{array}$ & $\begin{array}{l}85.0 \\
90.0 \\
95.0\end{array}$ & $\begin{array}{r}.05693 \\
.05548 \\
.05372\end{array}$ & $\begin{array}{r}.22772 \\
.22195 \\
.21491\end{array}$ & $\begin{array}{l}.01735 \\
.01691 \\
.01637\end{array}$ & $\begin{array}{l}.06941 \\
.06765 \\
.06550\end{array}$ & $\begin{array}{l}24 \cdot 725 \\
24 \cdot 099 \\
23.334\end{array}$ & $\begin{array}{l}75 \cdot 364 \\
73 \cdot 454 \\
71 \cdot 123\end{array}$ & $\begin{array}{l}81.928 \\
79.852 \\
77.317\end{array}$ \\
\hline 0 & 12500 & 100.0 & .05173 & 20694 & .01576 & 06307 & 22.469 & 8.487 & 74.452 \\
\hline
\end{tabular}


TABULATION OF DATA FOR

ABSORPTION OF SOUND IN AIR VERSUS PERCENT RELATIVE HUMIDITY AT 5 DEGREES CENTIGRADE FOR A FREQUENCY OF $125 \mathrm{HERTZ}$

\begin{tabular}{|c|c|c|c|c|c|c|c|c|c|}
\hline (1) & (2) & $(3)$ & 148 & $(5)$ & $(6)$ & (7) & (8) & $(9)$ & $(10)$ \\
\hline $\begin{array}{l}\text { TEMP } \\
\text { DEGR } \\
\text { CENT }\end{array}$ & HERTZ & $\begin{array}{l}\text { REL } \\
\text { HUM } \\
\text { PER } \\
\text { CENT }\end{array}$ & $\begin{array}{l}\text { ATTEN } \\
\text { COEF } \\
\text { PER } \\
\text { METER }\end{array}$ & $\begin{array}{l}4 M \\
\text { PER } \\
\text { METER }\end{array}$ & $\begin{array}{l}\text { ATTEN } \\
\text { COEF } \\
\text { PER } \\
\text { FOOT }\end{array}$ & $\begin{array}{l}4 M \\
\text { PER } \\
\text { FOOT }\end{array}$ & $\begin{array}{l}\text { ATTEN } \\
\text { DB PER } \\
\text { IOO } \\
\text { METER }\end{array}$ & $\begin{array}{l}\text { ATTEN } \\
\text { DB PER } \\
1000 \\
\text { FEET }\end{array}$ & $\begin{array}{l}\text { DECAY } \\
\text { RATE } \\
\text { DB PER } \\
\text { SECOND }\end{array}$ \\
\hline $\begin{array}{l}5 \\
5 \\
5\end{array}$ & $\begin{array}{l}125 \\
125 \\
125\end{array}$ & $\begin{array}{r}5.0 \\
10.0 \\
15.0\end{array}$ & $\begin{array}{l}.00042 \\
.00019 \\
.00013\end{array}$ & $\begin{array}{l}.00168 \\
.00079 \\
.00054\end{array}$ & $\begin{array}{l}.00012 \\
.00006 \\
.00004\end{array}$ & $\begin{array}{l}.00051 \\
.00024 \\
.00016\end{array}$ & $\begin{array}{r}.183 \\
.086 \\
.058\end{array}$ & $\begin{array}{l}.558 \\
.262 \\
.179\end{array}$ & $\begin{array}{l}.612 \\
.287 \\
.196\end{array}$ \\
\hline $\begin{array}{l}5 \\
5 \\
5\end{array}$ & $\begin{array}{l}125 \\
125 \\
125\end{array}$ & $\begin{array}{l}20 \cdot 0 \\
25.0 \\
30.0\end{array}$ & $\begin{array}{l}.00011 \\
.00010 \\
.00010\end{array}$ & $\begin{array}{l}.00047 \\
.00043 \\
.00040\end{array}$ & $\begin{array}{l}.00003 \\
.00003 \\
.00003\end{array}$ & $\begin{array}{l}.00014 \\
.00013 \\
.000112\end{array}$ & $\begin{array}{l}.051 \\
.047 \\
.044\end{array}$ & $\begin{array}{l}.155 \\
.143 \\
.134\end{array}$ & $\begin{array}{r}.170 \\
.157 \\
.147\end{array}$ \\
\hline $\begin{array}{l}5 \\
5 \\
5\end{array}$ & $\begin{array}{l}125 \\
125 \\
125\end{array}$ & $\begin{array}{l}35.0 \\
40.0 \\
45.0\end{array}$ & $\begin{array}{l}.00009 \\
.00009 \\
.00008\end{array}$ & $\begin{array}{l}.00038 \\
.00036 \\
.00034\end{array}$ & $\begin{array}{l}.00002 \\
.00002 \\
.00002\end{array}$ & $\begin{array}{l}.00011 \\
.00010 \\
.00010\end{array}$ & $\begin{array}{l}.041 \\
.039 \\
.037\end{array}$ & $\begin{array}{l}.126 \\
.119 \\
.114\end{array}$ & $\begin{array}{r}.138 \\
.130 \\
+125\end{array}$ \\
\hline $\begin{array}{l}5 \\
5 \\
5\end{array}$ & $\begin{array}{l}125 \\
125 \\
125\end{array}$ & $\begin{array}{l}46.0 \\
47.0 \\
48.0\end{array}$ & $\begin{array}{l}.00008 \\
.00008 \\
.00008\end{array}$ & $\begin{array}{r}.00034 \\
.00034 \\
.00033\end{array}$ & $\begin{array}{l}.00002 \\
.00002 \\
.00002\end{array}$ & $\begin{array}{l}.00010 \\
.00010 \\
.00010\end{array}$ & $\begin{array}{l}.037 \\
.037 \\
.036\end{array}$ & $\begin{array}{l}.113 \\
.112 \\
.112\end{array}$ & $\begin{array}{r}.124 \\
.123 \\
.122\end{array}$ \\
\hline $\begin{array}{l}5 \\
5 \\
5\end{array}$ & $\begin{array}{l}125 \\
125 \\
125\end{array}$ & $\begin{array}{l}49 \cdot 0 \\
50.0 \\
51.0\end{array}$ & $\begin{array}{l}.00008 \\
.00008 \\
.00008\end{array}$ & $\begin{array}{l}.00033 \\
.00033 \\
.00033\end{array}$ & $\begin{array}{l}.00002 \\
.00002 \\
.00002\end{array}$ & $\begin{array}{l}.00010 \\
.00010 \\
.00010\end{array}$ & $\begin{array}{l}.036 \\
.036 \\
.035\end{array}$ & $\begin{array}{l}.111 \\
.110 \\
.109\end{array}$ & $\begin{array}{l}.121 \\
.121 \\
.120\end{array}$ \\
\hline 5 & 125 & $52 \cdot 0$ & .00008 & .00032 & .00002 & .00010 & .035 & .108 & .114 \\
\hline $\begin{array}{l}5 \\
5\end{array}$ & $\begin{array}{l}125 \\
125\end{array}$ & $\begin{array}{l}53 \cdot 0 \\
54.0\end{array}$ & $\begin{array}{l}.00008 \\
.00008\end{array}$ & $\begin{array}{l}.00032 \\
.00032\end{array}$ & $\begin{array}{l}.00002 \\
.00002\end{array}$ & $\begin{array}{l}.00009 \\
.00009\end{array}$ & $\begin{array}{l}.035 \\
.035\end{array}$ & $\begin{array}{l}.108 \\
.107\end{array}$ & $\begin{array}{r}.118 \\
.117\end{array}$ \\
\hline $\begin{array}{l}5 \\
5 \\
5\end{array}$ & $\begin{array}{l}125 \\
125 \\
125\end{array}$ & $\begin{array}{l}55.0 \\
60.0 \\
65.0\end{array}$ & $\begin{array}{l}.00008 \\
.00007 \\
.00007\end{array}$ & $\begin{array}{l}.00032 \\
.00031 \\
.00030\end{array}$ & $\begin{array}{l}.00002 \\
.00002 \\
.00002\end{array}$ & $\begin{array}{l}.00009 \\
.00009 \\
.00009\end{array}$ & $\begin{array}{l}.035 \\
.033 \\
.032\end{array}$ & $\begin{array}{l}.106 \\
.103 \\
.100\end{array}$ & $\begin{array}{r}.117 \\
.113 \\
.109\end{array}$ \\
\hline $\begin{array}{l}5 \\
5 \\
5\end{array}$ & $\begin{array}{l}125 \\
125 \\
125\end{array}$ & $\begin{array}{l}70.0 \\
75.0 \\
80.0\end{array}$ & $\begin{array}{l}.00007 \\
.00007 \\
.00007\end{array}$ & $\begin{array}{l}.00029 \\
.00028 \\
.00028\end{array}$ & $\begin{array}{l}.00002 \\
.00002 \\
.00002\end{array}$ & $\begin{array}{l}.00008 \\
.00008 \\
.00008\end{array}$ & $\begin{array}{l}.031 \\
.031 \\
.030\end{array}$ & $\begin{array}{l}.097 \\
.095 \\
.092\end{array}$ & $\begin{array}{l}.106 \\
.104 \\
.101\end{array}$ \\
\hline $\begin{array}{l}5 \\
5 \\
5\end{array}$ & $\begin{array}{l}125 \\
125 \\
125\end{array}$ & $\begin{array}{l}85.0 \\
90.0 \\
95.0\end{array}$ & $\begin{array}{l}.00006 \\
.00006 \\
.00006\end{array}$ & $\begin{array}{l}.00027 \\
.00026 \\
.00026\end{array}$ & $\begin{array}{l}.00002 \\
.00002 \\
.00002\end{array}$ & $\begin{array}{l}.00008 \\
.00008 \\
.00008\end{array}$ & $\begin{array}{l}.029 \\
.029 \\
.028\end{array}$ & $\begin{array}{l}.090 \\
.089 \\
.087\end{array}$ & $\begin{array}{l}.099 \\
.097 \\
.095\end{array}$ \\
\hline 5 & 125 & 100.0 & .00006 & .00025 & .00001 & .00007 & .028 & .085 & .094 \\
\hline
\end{tabular}


TABULATION OF DATA FOR

ABSORPTION OF SOUND. IN AIR VERSUS PERCENT RELATIVE HUMIDITY AT 5 DEGREES CENTIGRADE FOR A FREQUENCY, OF 250 HERTZ

\begin{tabular}{|c|c|c|c|c|c|c|c|c|c|}
\hline (1) & (2) & (3) & (4) & $(5)$ & (6) & (7) & $(8)$ & $(9)$ & $(10)$ \\
\hline TEMP & FREQ & $\begin{array}{l}\text { REL } \\
\text { HUM }\end{array}$ & $\begin{array}{l}\text { ATTEN } \\
\text { COEF }\end{array}$ & $4 M$ & $\begin{array}{l}\text { ATTEN } \\
\text { COEF }\end{array}$ & $4 M$ & $\begin{array}{l}\text { ATTEN } \\
\text { DB PER }\end{array}$ & $\begin{array}{l}\text { ATTEN } \\
\text { DB PER }\end{array}$ & $\begin{array}{l}\text { DECAY } \\
\text { RATE }\end{array}$ \\
\hline $\begin{array}{l}\text { DEGR } \\
\text { CENT }\end{array}$ & HERTZ & $\begin{array}{l}\text { PER } \\
\text { CENT }\end{array}$ & $\begin{array}{l}\text { PER } \\
\text { METER }\end{array}$ & $\begin{array}{l}\text { PER } \\
\text { METER }\end{array}$ & $\begin{array}{l}\text { PER } \\
\text { FOOT }\end{array}$ & $\begin{array}{l}\text { PER } \\
\text { FOOT }\end{array}$ & $\begin{array}{l}100 \\
\text { METER }\end{array}$ & $\begin{array}{l}1000 \\
\text { FEET }\end{array}$ & $\begin{array}{l}\text { DB PER } \\
\text { SECOND }\end{array}$ \\
\hline $\begin{array}{l}5 \\
5 \\
5\end{array}$ & $\begin{array}{l}250 \\
250 \\
250\end{array}$ & $\begin{array}{r}5.0 \\
10.0\end{array}$ & $\begin{array}{l}.00107 \\
.00052\end{array}$ & $\begin{array}{r}.00431 \\
.00208\end{array}$ & $\begin{array}{l}.00032 \\
.00015\end{array}$ & $\begin{array}{r}.00131 \\
.00063\end{array}$ & $\begin{array}{r}468 \\
.225 \\
.145\end{array}$ & $\begin{array}{r}1.428 \\
.688 \\
.444\end{array}$ & $\begin{array}{r}1.567 \\
.759\end{array}$ \\
\hline & 250 & $15 \cdot 0$ & .00033 & .00134 & .00010 & .00040 & .145 & .444 & .487. \\
\hline $\begin{array}{l}5 \\
5 \\
5\end{array}$ & $\begin{array}{l}250 \\
250 \\
250\end{array}$ & $\begin{array}{l}20.0 \\
25.0 \\
30.0\end{array}$ & $\begin{array}{l}.00026 \\
.00023 \\
.00022\end{array}$ & $\begin{array}{l}.00106 \\
.00095 \\
.00089\end{array}$ & $\begin{array}{l}.00008 \\
.00007 \\
.00006\end{array}$ & $\begin{array}{l}.00032 \\
.00028 \\
.00027\end{array}$ & $\begin{array}{l}.115 \\
.103 \\
.096\end{array}$ & $\begin{array}{l}.350 \\
.314 \\
.294\end{array}$ & $\begin{array}{r}.385 \\
.345 \\
.323\end{array}$ \\
\hline $\begin{array}{l}5 \\
5\end{array}$ & $\begin{array}{l}250 \\
250\end{array}$ & $\begin{array}{l}35.0 \\
40.0\end{array}$ & $\begin{array}{l}.00021 \\
.00020\end{array}$ & $\begin{array}{l}.00084 \\
.00080\end{array}$ & $\begin{array}{l}.00006 \\
.00006\end{array}$ & $\begin{array}{l}.00025 \\
.00024\end{array}$ & $\begin{array}{l}.091 \\
.086\end{array}$ & $\begin{array}{r}.278 \\
.264\end{array}$ & $\begin{array}{r}305 \\
.290\end{array}$ \\
\hline 5 & 250 & 45.0 & .00019 & .00076 & .00005 & .00023 & .082 & .252 & .277 \\
\hline $\begin{array}{l}5 \\
5 \\
5\end{array}$ & $\begin{array}{l}250 \\
250 \\
250\end{array}$ & $\begin{array}{l}46.0 \\
47.0 \\
48.0\end{array}$ & $\begin{array}{r}.00018 \\
.00018 \\
.00018\end{array}$ & $\begin{array}{r}.00075 \\
.00075 \\
.00074\end{array}$ & $\begin{array}{l}.00005 \\
.00005 \\
.00005\end{array}$ & $\begin{array}{l}.00023 \\
.00022 \\
.00022\end{array}$ & $\begin{array}{l}.082 \\
.081 \\
.080\end{array}$ & $\begin{array}{l}.250 \\
.248 \\
.246\end{array}$ & $\begin{array}{r}.274 \\
.272 \\
.270\end{array}$ \\
\hline $\begin{array}{l}5 \\
5 \\
5\end{array}$ & $\begin{array}{l}250 \\
250 \\
250\end{array}$ & $\begin{array}{l}49.0 \\
50.0 \\
51.0\end{array}$ & $\begin{array}{r}.00018 \\
.00018 \\
.00018\end{array}$ & $\begin{array}{l}.00073 \\
.00073 \\
.00072\end{array}$ & $\begin{array}{l}.00005 \\
.00005 \\
.00005\end{array}$ & $\begin{array}{l}.00022 \\
.00022 \\
.00022\end{array}$ & $\begin{array}{l}.080 \\
.079 \\
.078\end{array}$ & $\begin{array}{l}.243 \\
.241 \\
.239\end{array}$ & $\begin{array}{l}.267 \\
.265 \\
.262\end{array}$ \\
\hline $\begin{array}{l}5 \\
5 \\
5\end{array}$ & $\begin{array}{l}250 \\
250 \\
250\end{array}$ & $\begin{array}{l}52.0 \\
53.0 \\
54.0\end{array}$ & $\begin{array}{r}.00017 \\
.00017 \\
.00017\end{array}$ & $\begin{array}{l}.00071 \\
.00071 \\
.00070\end{array}$ & $\begin{array}{l}.00005 \\
.00005 \\
.00005\end{array}$ & $\begin{array}{l}.00021 \\
.00021 \\
.00021\end{array}$ & $\begin{array}{l}.078 \\
.077 \\
.077\end{array}$ & $\begin{array}{r}.237 \\
.236 \\
.234\end{array}$ & $\begin{array}{l}.260 \\
.259 \\
.257\end{array}$ \\
\hline $\begin{array}{l}5 \\
5 \\
5\end{array}$ & $\begin{array}{l}250 \\
250 \\
250\end{array}$ & $\begin{array}{l}55.0 \\
60.0 \\
65.0\end{array}$ & $\begin{array}{l}.00017 \\
.00017 \\
.00016\end{array}$ & $\begin{array}{l}.00070 \\
.00068 \\
.00066\end{array}$ & $\begin{array}{l}.00005 \\
.00005 \\
.00005\end{array}$ & $\begin{array}{l}.00021 \\
.00020 \\
.00020\end{array}$ & $\begin{array}{l}.076 \\
.074 \\
.072\end{array}$ & $\begin{array}{r}.233 \\
.226 \\
.219\end{array}$ & $\begin{array}{l}.256 \\
.248 \\
.241\end{array}$ \\
\hline $\begin{array}{l}5 \\
5 \\
5\end{array}$ & $\begin{array}{l}250 \\
250 \\
250\end{array}$ & $\begin{array}{l}70.0 \\
75.0 \\
80.0\end{array}$ & $\begin{array}{l}.00016 \\
.00015 \\
.00015\end{array}$ & $\begin{array}{l}.00064 \\
.00063 \\
.00061\end{array}$ & $\begin{array}{l}.00004 \\
.00004 \\
.00004\end{array}$ & $\begin{array}{l}.00019 \\
.00019 \\
.00018\end{array}$ & $\begin{array}{l}.070 \\
.068 \\
.066\end{array}$ & $\begin{array}{r}.214 \\
.209 \\
.203\end{array}$ & $\begin{array}{r}.235 \\
.229 \\
.223\end{array}$ \\
\hline $\begin{array}{l}5 \\
5\end{array}$ & $\begin{array}{l}250 \\
250\end{array}$ & $\begin{array}{l}85.0 \\
90.0\end{array}$ & $\begin{array}{r}.00015 \\
.00014\end{array}$ & $\begin{array}{l}.00060 \\
.00058\end{array}$ & $\begin{array}{l}.00004 \\
.00004\end{array}$ & $\begin{array}{l}.00018 \\
.00017\end{array}$ & $\begin{array}{l}.065 \\
.063\end{array}$ & $\begin{array}{r}199 \\
.194\end{array}$ & $\begin{array}{r}.218 \\
.213\end{array}$ \\
\hline 5 & 250 & 95.00 & .00014 & .00057 & .00004 & .00017 & .062 & - 191 & .209 \\
\hline 5 & 250 & $100 \cdot 0$ & .00014 & .00056 & .00004 & .00017 & .061 & .187 & $.206^{\circ}$ \\
\hline
\end{tabular}


TABULATION OF DATA FOR

ABSORPTION OF SOUND IN AIR VERSUS PERCENT RELATIVE HUMIDITY AT 5 DEGREES CENTIGRADE FOR A FREQUENCY OF 500 HERTZ

\begin{tabular}{|c|c|c|c|c|c|c|c|c|c|}
\hline 11 & $(2)$ & (3) & $(4)$ & (5) & $(6)$ & $(7)$ & $(8)$ & 191 & $(10)$ \\
\hline TEMP & FREQ & $\begin{array}{l}\text { REL } \\
\text { HUM }\end{array}$ & $\begin{array}{l}\text { ATTEN } \\
\text { COEF }\end{array}$ & $4 M$ & $\begin{array}{l}\text { ATTEN } \\
\text { COEF }\end{array}$ & $4 M$ & $\begin{array}{l}\text { ATTEN } \\
\text { DB PER }\end{array}$ & $\begin{array}{l}\text { ATTEN } \\
\text { DB PER }\end{array}$ & $\begin{array}{l}\text { DECAY } \\
\text { RATE }\end{array}$ \\
\hline $\begin{array}{l}E G R \\
E N T\end{array}$ & HERTZ & $\begin{array}{l}\text { PER } \\
\text { CENT }\end{array}$ & $\begin{array}{l}\text { PER } \\
\text { METER }\end{array}$ & $\begin{array}{l}\text { PER } \\
\text { METER }\end{array}$ & $\begin{array}{l}\text { PER } \\
\text { FOOT }\end{array}$ & $\begin{array}{l}\text { PER } \\
\text { F๑๐T }\end{array}$ & $\begin{array}{l}100 \\
\text { METER }\end{array}$ & $\begin{array}{l}1000 \\
\text { FEET }\end{array}$ & $\begin{array}{l}\text { DB PER } \\
\text { SECOND }\end{array}$ \\
\hline $\begin{array}{l}5 \\
5 \\
5\end{array}$ & $\begin{array}{l}500 \\
500 \\
500\end{array}$ & $\begin{array}{l}5.0 \\
10.0 \\
15.0\end{array}$ & $\begin{array}{r}.00236 \\
.00165 \\
.00106\end{array}$ & $\begin{array}{r}.00946 \\
.00663 \\
.00427\end{array}$ & $\begin{array}{l}.00072 \\
.00050 \\
.00032\end{array}$ & $\begin{array}{l}.00288 \\
.00202 \\
.00130\end{array}$ & $\begin{array}{r}1.027 \\
.719 \\
.464\end{array}$ & $\begin{array}{l}3.131 \\
2.194 \\
.1 .415\end{array}$ & $\begin{array}{l}3.435 \\
2.407 \\
1.552\end{array}$ \\
\hline $\begin{array}{l}5 \\
5 \\
5\end{array}$ & $\begin{array}{l}500 \\
500 \\
500\end{array}$ & $\begin{array}{l}20.0 \\
25.0 \\
30.0\end{array}$ & $\begin{array}{l}.00078 \\
.00062 \\
.00054\end{array}$ & $\begin{array}{r}.00312 \\
.00249 \\
.00216\end{array}$ & $\begin{array}{l}.00023 \\
.00019 \\
.00016\end{array}$ & $\begin{array}{l}.00095 \\
.00076 \\
.00065\end{array}$ & $\begin{array}{l}.339 \\
.271 \\
.235\end{array}$ & $\begin{array}{r}1.033 \\
.827 \\
.716\end{array}$ & $\begin{array}{r}1.134 \\
.907 \\
.785\end{array}$ \\
\hline $\begin{array}{l}5 \\
5 \\
5\end{array}$ & $\begin{array}{l}500 \\
500 \\
500\end{array}$ & $\begin{array}{l}3.5 .0 \\
40.0 \\
45.0\end{array}$ & $\begin{array}{l}.00049 \\
.00047 \\
.00045\end{array}$ & $\begin{array}{l}.00198 \\
.00189 \\
.00181\end{array}$ & $\begin{array}{l}.00015 \\
.00014 \\
.00013\end{array}$ & $\begin{array}{l}.00060 \\
.00057 \\
.00055\end{array}$ & $\begin{array}{r}.215 \\
.205 \\
.196\end{array}$ & $\begin{array}{l}.656 \\
.625 \\
.599\end{array}$ & $\begin{array}{l}.720 \\
.686 \\
.657\end{array}$ \\
\hline $\begin{array}{l}5 \\
5 \\
5\end{array}$ & $\begin{array}{l}500 \\
500 \\
500\end{array}$ & $\begin{array}{l}46.0 \\
47.0 \\
48.0\end{array}$ & $\begin{array}{r}.00044 \\
.00044 \\
.00044\end{array}$ & $\begin{array}{r}.00179 \\
.00178 \\
.00177\end{array}$ & $\begin{array}{l}.00013 \\
.00013 \\
.00013\end{array}$ & $\begin{array}{l}.00054 \\
.00054 \\
.00053\end{array}$ & $\begin{array}{r}195 \\
.193 \\
.192\end{array}$ & $\begin{array}{l}.595 \\
.590 \\
.585\end{array}$ & $\begin{array}{l}.652 \\
.647 \\
.642\end{array}$ \\
\hline $\begin{array}{l}5 \\
5 \\
5\end{array}$ & $\begin{array}{l}500 \\
500 \\
500\end{array}$ & $\begin{array}{l}49.0 \\
50.0 \\
51.0\end{array}$ & $\begin{array}{l}.00043 \\
.00043 \\
.00043\end{array}$ & $\begin{array}{r}.00175 \\
.00174 \\
.00173\end{array}$ & $\begin{array}{l}.00013 \\
.00013 \\
.00013\end{array}$ & $\begin{array}{l}.00053 \\
.00053 \\
.00052\end{array}$ & $\begin{array}{r}190 \\
1189 \\
.188\end{array}$ & $\begin{array}{l}.581 \\
.577 \\
.573\end{array}$ & $\begin{array}{l}.637 \\
.633 \\
.628\end{array}$ \\
\hline 5 & 500 & 52.0 & .00043 & .00172 & .00013 & .00052 & .186 & .569 & .624 \\
\hline $\begin{array}{l}5 \\
5\end{array}$ & $\begin{array}{l}500 \\
500\end{array}$ & $\begin{array}{l}53.0 \\
54.0\end{array}$ & $\begin{array}{r}.00042 \\
.00042\end{array}$ & $\begin{array}{r}.00170 \\
.00169\end{array}$ & $\begin{array}{r}.00013 \\
.00012\end{array}$ & $\begin{array}{l}.00052 \\
.00051\end{array}$ & $\begin{array}{l}.185 \\
.184\end{array}$ & $\begin{array}{l}.565 \\
.561\end{array}$ & $\begin{array}{l}.620 \\
.616\end{array}$ \\
\hline $\begin{array}{l}5 \\
5 \\
5\end{array}$ & $\begin{array}{l}500 \\
500 \\
500\end{array}$ & $\begin{array}{l}55.0 \\
60.0 \\
65.0\end{array}$ & $\begin{array}{l}.00042 \\
.00040 \\
.00039\end{array}$ & $\begin{array}{l}.00168 \\
.00163 \\
.00158\end{array}$ & $\begin{array}{l}.00012 \\
.00012 \\
.00012\end{array}$ & $\begin{array}{l}.00051 \\
.00049 \\
.00048\end{array}$ & $\begin{array}{r}.183 \\
.177 \\
.171\end{array}$ & $\begin{array}{l}.558 \\
.540 \\
.523\end{array}$ & $\begin{array}{l}.612 \\
.592 \\
.574\end{array}$ \\
\hline $\begin{array}{l}5 \\
5 \\
5\end{array}$ & $\begin{array}{l}500 \\
500 \\
500\end{array}$ & $\begin{array}{l}70.0 \\
75.0 \\
80.0\end{array}$ & $\begin{array}{l}.00038 \\
.00037 \\
.00036\end{array}$ & $\begin{array}{r}.00153 \\
.00149 \\
.00145\end{array}$ & $\begin{array}{l}.00011 \\
.00011 \\
.00011\end{array}$ & $\begin{array}{l}.00046 \\
.00045 \\
.00044\end{array}$ & $\begin{array}{l}.166 \\
.162 \\
.157\end{array}$ & $\begin{array}{l}.508 \\
.494 \\
.481\end{array}$ & $\begin{array}{l}.557 \\
.542 \\
.527\end{array}$ \\
\hline $\begin{array}{l}5 \\
5 \\
5\end{array}$ & $\begin{array}{l}500 \\
500 \\
500\end{array}$ & $\begin{array}{l}85.0 \\
90.0 \\
95.0\end{array}$ & $\begin{array}{l}.00035 \\
.00034 \\
.00034\end{array}$ & $\begin{array}{r}.00142 \\
.00139 \\
.00137\end{array}$ & $\begin{array}{l}.00010 \\
.00010 \\
.00010\end{array}$ & $\begin{array}{l}.00043 \\
.00042 \\
.00041\end{array}$ & $\begin{array}{r}.154 \\
.151 \\
.148\end{array}$ & $\begin{array}{r}.471 \\
.462 \\
.453\end{array}$ & $\begin{array}{r}.517 \\
.506 \\
.497\end{array}$ \\
\hline 5 & 00 & 100.0 & .00033 & .00134 & .00010 & .00041 & .146 & .445 & .488 \\
\hline
\end{tabular}


TABULATION OF DATA FOR

ABSORPTION OF SOUND IN AIR VERSUS PERCENT RELATIVE HUMIDITY AT 5 DEGREES CENTIGRADE FOR A FREQUENCY OF 1000 HERTZ

\begin{tabular}{|c|c|c|c|c|c|c|c|c|c|}
\hline (1) & $(2)$ & (3) & $(4)$ & $(5)$ & 161 & 171 & $(8)$ & 191 & $(10)$ \\
\hline $\begin{array}{l}\text { TEMP } \\
\text { DEGR } \\
\text { CENT }\end{array}$ & HERTZ & $\begin{array}{l}\text { REL } \\
\text { HUM } \\
\text { PER-- } \\
\text { CENT }\end{array}$ & $\begin{array}{l}\text { ATTEN } \\
\text { COEF } \\
\text { PER } \\
\text { METER. }\end{array}$ & $\begin{array}{l}4 M \\
\text { PER } \\
\text { METER }\end{array}$ & $\begin{array}{l}\text { ATTEN } \\
\text { COEF } \\
\text { PER } \\
\text { FOOT }\end{array}$ & $\begin{array}{l}4 M \\
\text { PER } \\
\text { FOOT }\end{array}$ & $\begin{array}{l}\text { ATTEN } \\
\text { DB PER } \\
100 \\
\text { METER }\end{array}$ & $\begin{array}{l}\text { ATTEN } \\
\text { DB PER } \\
1000 \\
\text { FEET }\end{array}$ & $\begin{array}{l}\text { DECAY } \\
\text { RATE } \\
\text { DB PER } \\
\text { SECOND }\end{array}$ \\
\hline $\begin{array}{l}5 \\
5 \\
5\end{array}$ & $\begin{array}{l}1000 \\
1000 \\
1000\end{array}$ & $\begin{array}{r}5.0 \\
10.0 \\
15.0\end{array}$ & $\begin{array}{l}.00344 \\
.00473 \\
.00359\end{array}$ & $\begin{array}{r}.01377 \\
.01895 \\
.01439\end{array}$ & $\begin{array}{l}.00104 \\
.00144 \\
.00109\end{array}$ & $\begin{array}{l}.00419 \\
.00577 \\
.00438\end{array}$ & $\begin{array}{l}1.495 \\
2.057 \\
1.562\end{array}$ & $\begin{array}{l}4 \cdot 558 \\
6 \cdot 271 \\
4 \cdot 762\end{array}$ & $\begin{array}{l}5.001 \\
6.880 \\
5.224\end{array}$ \\
\hline $\begin{array}{l}5 \\
5 \\
5\end{array}$ & $\begin{array}{l}1000 \\
1000 \\
1000\end{array}$ & $\begin{array}{l}20.0 \\
25.0 \\
30.0\end{array}$ & $\begin{array}{l}.00263 \\
.00205 \\
.00169\end{array}$ & $\begin{array}{l}.01052 \\
.00821 \\
.00676\end{array}$ & $\begin{array}{l}.00080 \\
.00062 \\
.00051\end{array}$ & $\begin{array}{l}.00320 \\
.00250 \\
.00206\end{array}$ & $\begin{array}{r}1.142 \\
.892 \\
.734\end{array}$ & $\begin{array}{l}3.482 \\
2.719 \\
2.238\end{array}$ & $\begin{array}{l}3.820 \\
2.983 \\
2.455\end{array}$ \\
\hline $\begin{array}{l}5 \\
5 \\
5\end{array}$ & $\begin{array}{l}1000 \\
1000 \\
1000\end{array}$ & $\begin{array}{l}35.0 \\
40.0 \\
45.0\end{array}$ & $\begin{array}{l}.00143 \\
.00126 \\
.00114\end{array}$ & $\begin{array}{l}.00573 \\
.00506 \\
.00457\end{array}$ & $\begin{array}{l}.00043 \\
.00038 \\
.00034\end{array}$ & $\begin{array}{r}.00174 \\
.00154 \\
.00139\end{array}$ & $\begin{array}{l}.622 \\
.549 \\
.496\end{array}$ & $\begin{array}{l}1.896 \\
1.675 \\
1.513\end{array}$ & $\begin{array}{l}2.080 \\
1.837 \\
1.660\end{array}$ \\
\hline $\begin{array}{l}5 \\
5 \\
5\end{array}$ & $\begin{array}{l}1000 \\
1000 \\
1000\end{array}$ & $\begin{array}{l}46.0 \\
47.0 \\
48.0\end{array}$ & $\begin{array}{l}.001112 \\
.00111 \\
.00109\end{array}$ & $\begin{array}{l}.00450 \\
.00444 \\
.00439\end{array}$ & $\begin{array}{l}.00034 \\
.00033 \\
.00033\end{array}$ & $\begin{array}{l}.00137 \\
.00135 \\
.00133\end{array}$ & $\begin{array}{l}.488 \\
.482 \\
.477\end{array}$ & $\begin{array}{l}1.489 \\
1.472 \\
1.454\end{array}$ & $\begin{array}{l}1.634 \\
1.614 \\
1.595\end{array}$ \\
\hline $\begin{array}{l}5 \\
5 \\
5\end{array}$ & $\begin{array}{l}1000 \\
1000 \\
1000\end{array}$ & $\begin{array}{l}49.0 \\
50.0 \\
51.0\end{array}$ & $\begin{array}{l}.00108 \\
.00107 \\
.00106\end{array}$ & $\begin{array}{r}.00433 \\
.00429 \\
.00425\end{array}$ & $\begin{array}{l}.00033 \\
.00032 \\
.00032\end{array}$ & $\begin{array}{l}.00132 \\
.00130 \\
.00129\end{array}$ & $\begin{array}{l}.471 \\
.466 \\
.461\end{array}$ & $\begin{array}{l}1.436 \\
1.420 \\
1.406\end{array}$ & $\begin{array}{l}1.575 \\
1.558 \\
1.543\end{array}$ \\
\hline $\begin{array}{l}5 \\
5 \\
5\end{array}$ & $\begin{array}{l}1000 \\
1000 \\
1000\end{array}$ & $\begin{array}{l}52.0 \\
53.0 \\
54.0\end{array}$ & $\begin{array}{r}.00104 \\
.00103 \\
.00102\end{array}$ & $\begin{array}{l}.00419 \\
.00414 \\
.00410\end{array}$ & $\begin{array}{l}.00031 \\
.00031 \\
.00031\end{array}$ & $\begin{array}{l}.00127 \\
.00126 \\
.00125\end{array}$ & $\begin{array}{l}.455 \\
.450 \\
.445\end{array}$ & $\begin{array}{l}1.388 \\
1.371 \\
1.357\end{array}$ & $\begin{array}{l}1.523 \\
1.504 \\
1.489\end{array}$ \\
\hline $\begin{array}{l}5 \\
5 \\
5\end{array}$ & $\begin{array}{l}1000 \\
1000 \\
1000\end{array}$ & $\begin{array}{l}55 \cdot 0 \\
60.0 \\
65.0\end{array}$ & $\begin{array}{l}.00101 \\
.00098 \\
.00095\end{array}$ & $\begin{array}{l}.00406 \\
.00393 \\
.00382\end{array}$ & $\begin{array}{l}.00030 \\
.00029 \\
.00029\end{array}$ & $\begin{array}{l}.00123 \\
.00119 \\
.00116\end{array}$ & $\begin{array}{l}.441 \\
.426 \\
.415\end{array}$ & $\begin{array}{l}1.345 \\
1.300 \\
1.265\end{array}$ & $\begin{array}{l}1.476 \\
1.427 \\
1.387\end{array}$ \\
\hline $\begin{array}{l}5 \\
5 \\
5\end{array}$ & $\begin{array}{l}1000 \\
1000 \\
1000\end{array}$ & $\begin{array}{l}70.0 \\
75.0 \\
80.0\end{array}$ & $\begin{array}{l}.00093 \\
.00090 \\
.00088\end{array}$ & $\begin{array}{r}.00372 \\
.00363 \\
.00355\end{array}$ & $\begin{array}{l}.00028 \\
.00027 \\
.00027\end{array}$ & $\begin{array}{l}.00113 \\
.00110 \\
.00108\end{array}$ & $\begin{array}{r}.404 \\
.394 \\
.385\end{array}$ & $\begin{array}{l}1 \cdot 231 \\
1 \cdot 203 \\
1 \cdot 175\end{array}$ & $\begin{array}{l}1.351 \\
1.319 \\
1.289\end{array}$ \\
\hline $\begin{array}{l}5 \\
5 \\
5\end{array}$ & $\begin{array}{l}1000 \\
1000 \\
1000\end{array}$ & $\begin{array}{l}85.0 \\
90.0 \\
95.0\end{array}$ & $\begin{array}{l}.00086 \\
.00085 \\
.00083\end{array}$ & $\begin{array}{r}.00347 \\
.00340 \\
.00334\end{array}$ & $\begin{array}{l}.00026 \\
.00025 \\
.00025\end{array}$ & $\begin{array}{l}.00106 \\
.00103 \\
.00101\end{array}$ & $\begin{array}{l}.377 \\
.369 \\
.362\end{array}$ & $\begin{array}{l}1 \cdot 151 \\
1 \cdot 127 \\
1 \cdot 105\end{array}$ & $\begin{array}{l}1.263 \\
1.237 \\
1.212\end{array}$ \\
\hline 5 & 1000 & $100 \cdot 0$ & .00081 & .00327 & .00024 & .00099 & .355 & 1.085 & 1.190 \\
\hline
\end{tabular}


TABULATION OF DATA. FOR

ABSORPTION OF SOUND IN AIR VERSUS PERCENT RELATIVE HUMIDITY AT 5 DEGREES CENTIGRADE FOR A FREQUENCY OF 2000 HERTZ

\begin{tabular}{|c|c|c|c|c|c|c|c|c|c|}
\hline (1) & $(2)$ & (3) & (4) & (5) & $(6)$ & $(7)$ & (3) & 191 & $(10)$ \\
\hline $\begin{array}{l}\text { TEMP } \\
\text { DEGR } \\
\text { CENT }\end{array}$ & HERTZ & $\begin{array}{l}\text { REL } \\
\text { HUM } \\
\text { PER } \\
\text { CENT. }\end{array}$ & $\begin{array}{l}\text { ATTEN } \\
\text { COEF } \\
\text { PER } \\
\text { METER }\end{array}$ & $\begin{array}{l}4 M \\
\text { PER } \\
\text { METER }\end{array}$ & $\begin{array}{l}\text { ATTEN } \\
\text { COEF } \\
\text { PER } \\
\text { FOOT }\end{array}$ & $\begin{array}{l}4 M \\
\text { PER } \\
\text { FOOT }\end{array}$ & $\begin{array}{l}\text { ATTEN } \\
\text { DB PER } \\
100 \\
\text { METER }\end{array}$ & $\begin{array}{l}\text { ATTEN } \\
\text { DB PER } \\
1000 \\
\text { FEET }\end{array}$ & $\begin{array}{l}\text { DECAY } \\
\text { RATE } \\
\text { DB PER } \\
\text { SECOND }\end{array}$ \\
\hline $\begin{array}{l}5 \\
5 \\
5\end{array}$ & $\begin{array}{l}2000 \\
2000 \\
2000\end{array}$ & $\begin{array}{r}5.0 \\
10.0 \\
15.0\end{array}$ & $\begin{array}{l}.00423 \\
.00844 \\
.00964\end{array}$ & $\begin{array}{l}.01693 \\
.03377 \\
.03856\end{array}$ & $\begin{array}{r}.00129 \\
.00257 \\
.00293\end{array}$ & $\begin{array}{l}.00516 \\
.01029 \\
.01175\end{array}$ & $\begin{array}{l}1.839 \\
3.667 \\
4.186\end{array}$ & $\begin{array}{r}5.605 \\
11.178 \\
12.761\end{array}$ & $\begin{array}{r}6.149 \\
12.263 \\
13.999\end{array}$ \\
\hline $\begin{array}{l}5 \\
5 \\
5\end{array}$ & $\begin{array}{l}2000 \\
2000 \\
2000\end{array}$ & $\begin{array}{l}20.0 \\
25.0 \\
30.0\end{array}$ & $\begin{array}{l}.00875 \\
.00711 \\
.00581\end{array}$ & $\begin{array}{l}.03501 \\
.02844 \\
.02325\end{array}$ & $\begin{array}{l}.00266 \\
.00216 \\
.00177\end{array}$ & $\begin{array}{l}.01067 \\
.00866 \\
.00708\end{array}$ & $\begin{array}{l}3.801 \\
3.087 \\
2.524\end{array}$ & $\begin{array}{r}21.586 \\
9.412 \\
7.695\end{array}$ & $\begin{array}{r}12.710 \\
10.325 \\
8.441\end{array}$ \\
\hline $\begin{array}{l}5 \\
5 \\
5\end{array}$ & $\begin{array}{l}2000 \\
2000 \\
2000\end{array}$ & $\begin{array}{l}35.0 \\
40.0 \\
45.0\end{array}$ & $\begin{array}{r}.00497 \\
.00428 \\
.00377\end{array}$ & $\begin{array}{r}.01988 \\
.01712 \\
.01508\end{array}$ & $\begin{array}{l}.00151 \\
.00130 \\
.00114\end{array}$ & $\begin{array}{l}.00606 \\
.00521 \\
.00459\end{array}$ & $\begin{array}{l}2.159 \\
1.859 \\
1.637\end{array}$ & $\begin{array}{l}6.581 \\
5.667 \\
4.990\end{array}$ & $\begin{array}{l}7.220 \\
6.217 \\
5.475\end{array}$ \\
\hline $\begin{array}{l}5 \\
5 \\
5\end{array}$ & $\begin{array}{l}2000 \\
2000 \\
2000\end{array}$ & $\begin{array}{l}46.0 \\
47.0 \\
48.0\end{array}$ & $\begin{array}{l}.00368 \\
.00360 \\
.00352\end{array}$ & $\begin{array}{l}.01473 \\
.01441 \\
.01411\end{array}$ & $\begin{array}{l}.00112 \\
.00109 \\
.00107\end{array}$ & $\begin{array}{r}.00449 \\
.00439 \\
.00430\end{array}$ & $\begin{array}{l}1.600 \\
1.565 \\
1.532\end{array}$ & $\begin{array}{l}4 \cdot 877 \\
4 \cdot 771 \\
4.670\end{array}$ & $\begin{array}{l}5.350 \\
5.234 \\
5.123\end{array}$ \\
\hline $\begin{array}{l}5 \\
5 \\
5\end{array}$ & $\begin{array}{l}2000 \\
2000 \\
2000\end{array}$ & $\begin{array}{l}49.0 \\
50.0 \\
51.0\end{array}$ & $\begin{array}{l}.00345 \\
.00338 \\
.00331\end{array}$ & $\begin{array}{l}.01382 \\
.01355 \\
.01327\end{array}$ & $\begin{array}{l}.00105 \\
.00103 \\
.00101\end{array}$ & $\begin{array}{l}.00421 \\
.00413 \\
.00404\end{array}$ & $\begin{array}{l}1.500 \\
1.472 \\
1.441\end{array}$ & $\begin{array}{l}4.574 \\
4.487 \\
4.394\end{array}$ & $\begin{array}{l}5.018 \\
4.922 \\
4.820\end{array}$ \\
\hline $\begin{array}{l}5 \\
5 \\
5\end{array}$ & $\begin{array}{l}2000 \\
2000 \\
2000\end{array}$ & $\begin{array}{l}52.0 \\
53.0 \\
54.0\end{array}$ & $\begin{array}{l}.00324 \\
.00318 \\
.00312\end{array}$ & $\begin{array}{l}.01299 \\
.01272 \\
.01248\end{array}$ & $\begin{array}{l}.00098 \\
.00096 \\
.00095\end{array}$ & $\begin{array}{l}.00395 \\
.00387 \\
.00380\end{array}$ & $\begin{array}{l}1.410 \\
1.381 \\
1.355\end{array}$ & $\begin{array}{l}4 \cdot 299 \\
4 \cdot 212 \\
4 \cdot 130\end{array}$ & $\begin{array}{l}4.716 \\
4.620 \\
4.531\end{array}$ \\
\hline $\begin{array}{l}5 \\
5 \\
5\end{array}$ & $\begin{array}{l}2000 \\
2000 \\
2000\end{array}$ & $\begin{array}{l}55.0 \\
60.0 \\
65.0\end{array}$ & $\begin{array}{l}.00306 \\
.00280 \\
.00261\end{array}$ & $\begin{array}{l}.01224 \\
.01121 \\
.01045\end{array}$ & $\begin{array}{l}.00093 \\
.00085 \\
.00079\end{array}$ & $\begin{array}{l}.00373 \\
.00341 \\
.00318\end{array}$ & $\begin{array}{l}1.329 \\
1.218 \\
1.135\end{array}$ & $\begin{array}{l}4.052 \\
3.712 \\
3.460\end{array}$ & $\begin{array}{l}4.445 \\
4.073 \\
3.796\end{array}$ \\
\hline $\begin{array}{l}5 \\
5 \\
5\end{array}$ & $\begin{array}{l}2000 \\
2000 \\
2000\end{array}$ & $\begin{array}{l}70.0 \\
75.0 \\
80.0\end{array}$ & $\begin{array}{l}.00244 \\
.00232 \\
.00224\end{array}$ & $\begin{array}{l}.00978 \\
.00929 \\
.00896\end{array}$ & $\begin{array}{l}.00074 \\
.00070 \\
.00068\end{array}$ & $\begin{array}{l}.00298 \\
.00283 \\
.00273\end{array}$ & $\begin{array}{r}1.061 \\
1.009 \\
.973\end{array}$ & $\begin{array}{l}3.236 \\
3.075 \\
2.966\end{array}$ & $\begin{array}{l}3.550 \\
3.374 \\
3.254\end{array}$ \\
\hline $\begin{array}{l}5 \\
5 \\
5\end{array}$ & $\begin{array}{l}2000 \\
2000 \\
2000\end{array}$ & $\begin{array}{l}85.0 \\
90.0 \\
95.0\end{array}$ & $\begin{array}{l}.00216 \\
.00210 \\
.00205\end{array}$ & $\begin{array}{l}.00867 \\
.00840 \\
.00822\end{array}$ & $\begin{array}{l}.00066 \\
.00064 \\
.00062\end{array}$ & $\begin{array}{l}.00264 \\
.00256 \\
.00250\end{array}$ & $\begin{array}{r}.941 \\
.912 \\
.893\end{array}$ & $\begin{array}{l}2.869 \\
2.781 \\
2.722\end{array}$ & $\begin{array}{l}3.148 \\
3.050 \\
2.986\end{array}$ \\
\hline 5 & 2000 & $100 \cdot 0$ & .00202 & .00808 & 00061 & .00246 & .877 & 2.675 & 2.935 \\
\hline
\end{tabular}


TABULATION OF DATA FOR

ABSORPTION OF SOUND IN AIR VERSUS PERCENT RELATIVE HUMIDITY AT 5 DEGREES CENTIGRADE FOR A FREQUENCY OF 2500 HERTZ

\begin{tabular}{|c|c|c|c|c|c|c|c|c|c|}
\hline$(1)$ & (2) & (3) & $(4)$ & (5) & (6) & 171 & $(8)$ & $(9)$ & 1101 \\
\hline TEMP & FREQ & $\begin{array}{l}\text { REL } \\
\text { HUM }\end{array}$ & $\begin{array}{l}\text { ATTEN } \\
\text { COEF }\end{array}$ & $4 M$ & $\begin{array}{l}\text { ATTEN } \\
\text { COEF }\end{array}$ & $4 M$ & $\begin{array}{l}\text { ATTEN } \\
\text { DB PER }\end{array}$ & $\begin{array}{l}\text { ATTEN } \\
D B\end{array}$ & DECAY \\
\hline $\begin{array}{l}\text { DEGR } \\
\text { CENT }\end{array}$ & HERTZ & $\begin{array}{l}\text { PER } \\
\text { CENT }\end{array}$ & $\begin{array}{l}\text { PER } \\
\text { METER }\end{array}$ & $\begin{array}{l}\text { PER } \\
\text { METER }\end{array}$ & $\begin{array}{l}\text { PER } \\
\text { FOOT }\end{array}$ & $\begin{array}{l}\text { PEER } \\
\text { FOOT }\end{array}$ & $\begin{array}{l}100 \\
\text { ME TER }\end{array}$ & $\begin{array}{l}1000 \\
\text { FEET }\end{array}$ & $\begin{array}{l}\text { D.B PER } \\
\text { SECOND }\end{array}$ \\
\hline $\begin{array}{l}5 \\
5\end{array}$ & $\begin{array}{l}2500 \\
2500\end{array}$ & $\begin{array}{r}5.0 \\
10.0\end{array}$ & $\begin{array}{l}.00460 \\
.00916\end{array}$ & $\begin{array}{l}.01841 \\
.03667\end{array}$ & $\begin{array}{r}.00140 \\
.00279\end{array}$ & $\begin{array}{r}.00561 \\
.01117\end{array}$ & $\begin{array}{l}1.999 \\
3.982\end{array}$ & $\begin{array}{r}6.095 \\
12.137\end{array}$ & $\begin{array}{r}6.686 \\
13.315\end{array}$ \\
\hline 5 & 2500 & 15.0 & .01195 & .04783 & .00364 & .01457 & $5 \cdot 193$ & 15.829 & 17.365 \\
\hline $\begin{array}{l}5 \\
5 \\
5\end{array}$ & $\begin{array}{l}2500 \\
2500 \\
2500\end{array}$ & $\begin{array}{l}20.0 \\
25.0 \\
30.0\end{array}$ & $\begin{array}{l}.01185 \\
.01039 \\
.00862\end{array}$ & $\begin{array}{l}.04740 \\
.04159 \\
.03451\end{array}$ & $\begin{array}{l}.00361 \\
.00316 \\
.00263\end{array}$ & $\begin{array}{r}.01444 \\
.01267 \\
.01052\end{array}$ & $\begin{array}{l}5.146 \\
4.516 \\
3.747\end{array}$ & $\begin{array}{l}15.686 \\
13.765 \\
11.423\end{array}$ & $\begin{array}{l}17.208 \\
15.100 \\
12.531\end{array}$ \\
\hline $\begin{array}{l}5 \\
5 \\
5\end{array}$ & $\begin{array}{l}2500 \\
2500 \\
2500\end{array}$ & $\begin{array}{l}35.0 \\
40.0 \\
45.0\end{array}$ & $\begin{array}{l}.00730 \\
.00639 \\
.00563\end{array}$ & $\begin{array}{l}.02923 \\
.02558 \\
.02252\end{array}$ & $\begin{array}{r}.00222 \\
.00194 \\
.00171\end{array}$ & $\begin{array}{l}.00891 \\
.00779 \\
.00686\end{array}$ & $\begin{array}{r}3.174 \\
2.777 \\
1.445\end{array}$ & $\begin{array}{l}9.675 \\
8.466 \\
7.453\end{array}$ & $\begin{array}{r}10.614 \\
9.287 \\
8.177\end{array}$ \\
\hline $\begin{array}{l}5 \\
5 \\
5\end{array}$ & $\begin{array}{l}2500 \\
2500 \\
2500\end{array}$ & $\begin{array}{l}46.0 \\
47.0\end{array}$ & $\begin{array}{r}.00548 \\
.00534\end{array}$ & $\begin{array}{r}.02195 \\
.02139\end{array}$ & $\begin{array}{r}00167 \\
.00162\end{array}$ & $\begin{array}{r}.00669 \\
.00651\end{array}$ & $\begin{array}{l}2.383 \\
2.322 \\
2.372\end{array}$ & $\begin{array}{l}7.266 \\
7.078 \\
6.077\end{array}$ & $\begin{array}{l}7.971 \\
7.765 \\
7.500\end{array}$ \\
\hline $\begin{array}{l}5 \\
5 \\
5\end{array}$ & $\begin{array}{l}2500 \\
2500 \\
2500\end{array}$ & $\begin{array}{l}49.0 \\
50.0 \\
51.0\end{array}$ & $\begin{array}{l}.00511 \\
.00500 \\
.00490\end{array}$ & $\begin{array}{l}.02047 \\
.02002 \\
.01960\end{array}$ & $\begin{array}{l}.00156 \\
.00152 \\
.00149\end{array}$ & $\begin{array}{l}.00624 \\
.00610 \\
.00597\end{array}$ & $\begin{array}{l}2.223 \\
2.174 \\
2.128\end{array}$ & $\begin{array}{l}6.777 \\
6.627 \\
6.488\end{array}$ & $\begin{array}{l}7.434 \\
7.270 \\
7.117\end{array}$ \\
\hline $\begin{array}{l}5 \\
5 \\
5\end{array}$ & $\begin{array}{l}2500 \\
2500 \\
2500\end{array}$ & $\begin{array}{l}52.0 \\
53.0 \\
54.0\end{array}$ & $\begin{array}{l}.00480 \\
.00470 \\
.00461\end{array}$ & $\begin{array}{l}.01920 \\
.01883 \\
.01847\end{array}$ & $\begin{array}{r}.00146 \\
.00143 \\
.00140\end{array}$ & $\begin{array}{l}.00585 \\
.00574 \\
.00563\end{array}$ & $\begin{array}{l}2.085 \\
2.045 \\
2.006\end{array}$ & $\begin{array}{l}6.357 \\
6.234 \\
6.114\end{array}$ & $\begin{array}{l}6.973 \\
6.839 \\
6.708\end{array}$ \\
\hline $\begin{array}{l}5 \\
5 \\
5\end{array}$ & $\begin{array}{l}2500 \\
2500 \\
2500\end{array}$ & $\begin{array}{l}55.0 \\
60.0 \\
65.0\end{array}$ & $\begin{array}{l}.00453 \\
.00415 \\
.00381\end{array}$ & $\begin{array}{l}.01813 \\
.01661 \\
.01526\end{array}$ & $\begin{array}{l}.00138 \\
.00126 \\
.00116\end{array}$ & $\begin{array}{l}.00552 \\
.00506 \\
.00465\end{array}$ & $\begin{array}{l}1.969 \\
1.803 \\
1.657\end{array}$ & $\begin{array}{l}6.002 \\
5.498 \\
5.051\end{array}$ & $\begin{array}{l}6.584 \\
6.031 \\
5.541\end{array}$ \\
\hline $\begin{array}{l}5 \\
5 \\
5\end{array}$ & $\begin{array}{l}2500 \\
2500 \\
2500\end{array}$ & $\begin{array}{l}70.0 \\
75.0 \\
80.0\end{array}$ & $\begin{array}{l}.00354 \\
.00334 \\
.00315\end{array}$ & $\begin{array}{l}.01419 \\
.01336 \\
.01261\end{array}$ & $\begin{array}{l}.00108 \\
.00101 \\
.00096\end{array}$ & $\begin{array}{r}.00432 \\
.00407 \\
.00384\end{array}$ & $\begin{array}{l}1.541 \\
1.450 \\
1.369\end{array}$ & $\begin{array}{l}4.697 \\
4.421 \\
4.174\end{array}$ & $\begin{array}{l}5.153 \\
4.850 \\
4.579\end{array}$ \\
\hline $\begin{array}{l}5 \\
5\end{array}$ & $\begin{array}{l}2500 \\
2500\end{array}$ & $\begin{array}{l}85.0 \\
90.0\end{array}$ & $\begin{array}{l}.00300 \\
.00290\end{array}$ & $\begin{array}{r}.01203 \\
.01161\end{array}$ & $\begin{array}{r}.00091 \\
.00088\end{array}$ & $\begin{array}{r}.00366 \\
.00353\end{array}$ & $\begin{array}{l}1.306 \\
1.260\end{array}$ & $\begin{array}{l}3.982 \\
3.843\end{array}$ & $\begin{array}{l}4.368 \\
4.216\end{array}$ \\
\hline 5 & 2500 & 95.0 & .00281 & .01127 & .00085 & .00343 & 23 & 3.730 & 4.092 \\
\hline 5 & 2500 & $100 \cdot 0$ & .00273 & .01095 & .00083 & .00333 & .189 & 3.624 & 3.976 \\
\hline
\end{tabular}


TABULATION OF DATA FOR

ABSORPTION OF SOUND IN AIR VERSUS PERCENT RELATIVE .UMIDITY AT 5 DEGREES GENTIGRADE FOR A FREQUENCY OF 3200 HERTZ

\begin{tabular}{|c|c|c|c|c|c|c|c|c|c|}
\hline (1) & (2) & (3) & (4) & (5) & (6) & 171 & (8) & $(9)$ & $(10)$ \\
\hline $\begin{array}{l}\text { TEMP } \\
\text { DEGR } \\
\text { EENT }\end{array}$ & HERTZ & $\begin{array}{l}\text { REL } \\
\text { HUM } \\
\text { PER } \\
\text { CENT }\end{array}$ & $\begin{array}{l}\text { ATTEN } \\
\text { COEF } \\
\text { PER : } \\
\text { METER }\end{array}$ & $\begin{array}{l}4 M \\
\text { PER } \\
\text { METER }\end{array}$ & $\begin{array}{l}\text { ATTEN } \\
\text { COEF } \\
\text { PER } \\
\text { FOOT }\end{array}$ & $\begin{array}{l}4 M \\
\text { PER } \\
\text { FOOT }\end{array}$ & $\begin{array}{l}\text { ATTEN } \\
\text { DB PER } \\
100^{\circ} \\
\text { METER }\end{array}$ & $\begin{array}{l}\text { ATTEN } \\
\text { DB PER } \\
\text { IOOO } \\
\text { FEET }\end{array}$ & $\begin{array}{l}\text { DECAY } \\
\text { RATE } \\
\text { DB PER } \\
\text { SECOND }\end{array}$ \\
\hline $\begin{array}{l}5 \\
5 \\
5\end{array}$ & $\begin{array}{l}3200 \\
3.200 \\
3200\end{array}$ & $\begin{array}{r}5.0 \\
10.0 \\
15.0\end{array}$ & $\begin{array}{l}.00511 \\
.00993 \\
.01443\end{array}$ & $\begin{array}{r}.02047 \\
.03972 \\
.05773\end{array}$ & $\begin{array}{l}.00155 \\
.00302 \\
.00439\end{array}$ & $\begin{array}{l}.00623 \\
.01210 \\
.01759\end{array}$ & $\begin{array}{l}2 \cdot 222 \\
4 \cdot 312 \\
6 \cdot 268\end{array}$ & $\begin{array}{r}6.774 \\
13 \cdot 144 \\
19.1 .05\end{array}$ & $\begin{array}{r}7.431 \\
14.420 \\
20.959\end{array}$ \\
\hline $\begin{array}{l}5 \\
5 \\
5\end{array}$ & $\begin{array}{l}3200 \\
3200 \\
3200\end{array}$ & $\begin{array}{l}20.0 \\
25.0 \\
30.0\end{array}$ & $\begin{array}{r}.01557 \\
.01490 \\
.01317\end{array}$ & $\begin{array}{l}.06229 \\
.05962 \\
.05271\end{array}$ & $\begin{array}{l}.00474 \\
.00454 \\
.00401\end{array}$ & $\begin{array}{r}.01898 \\
.01817 \\
.01606\end{array}$ & $\begin{array}{l}6.763 \\
6.473 \\
5.723\end{array}$ & $\begin{array}{l}20.616 \\
19.731 \\
17.446\end{array}$ & $\begin{array}{l}22.615 \\
21.645 \\
19.139\end{array}$ \\
\hline $\begin{array}{l}5 \\
5 \\
5\end{array}$ & $\begin{array}{l}3200 \\
3200 \\
3200\end{array}$ & $\begin{array}{l}35.0 \\
40.0 \\
45.0\end{array}$ & $\begin{array}{l}.01126 \\
.00973 \\
.00865\end{array}$ & $\begin{array}{r}.04504 \\
.03895 \\
.03463\end{array}$ & $\begin{array}{l}.00343 \\
.00296 \\
.00263\end{array}$ & $\begin{array}{r}.01373 \\
.01187 \\
.01055\end{array}$ & $\begin{array}{l}4.890 \\
4.229 \\
3.760\end{array}$ & $\begin{array}{l}14.907 \\
12.892 \\
11.460\end{array}$ & $\begin{array}{l}16.353 \\
14.142 \\
12.572\end{array}$ \\
\hline $\begin{array}{l}5 \\
5 \\
5\end{array}$ & $\begin{array}{l}3200 \\
3200 \\
3200\end{array}$ & $\begin{array}{l}46.0 \\
47.0 \\
48.0\end{array}$ & $\begin{array}{l}.00847 \\
.00828 \\
.00810\end{array}$ & $\begin{array}{r}.03389 \\
.03315 \\
.03243\end{array}$ & $\begin{array}{l}.00258 \\
.00252 \\
.00247\end{array}$ & $\begin{array}{l}.01033 \\
.01010 \\
.00988\end{array}$ & $\begin{array}{l}3.679 \\
3.599 \\
3.521\end{array}$ & $\begin{array}{l}11.216 \\
10.972 \\
10.732\end{array}$ & $\begin{array}{l}12.304 . \\
12.036 \\
11.773\end{array}$ \\
\hline $\begin{array}{l}5 \\
5\end{array}$ & $\begin{array}{l}3200 \\
3200\end{array}$ & $\begin{array}{l}49.0 \\
50.0\end{array}$ & $\begin{array}{l}.00793 \\
.00776\end{array}$ & $\begin{array}{l}.03172 \\
.03104\end{array}$ & $\begin{array}{l}.00241 \\
.00236\end{array}$ & $\begin{array}{l}.00966 \\
.00946\end{array}$ & $\begin{array}{l}3.444 \\
3.370\end{array}$ & $\begin{array}{l}10.498 \\
10.274\end{array}$ & $\begin{array}{l}11.516 \\
11.270\end{array}$ \\
\hline 5 & 3200 & 51.0 & .00759 & .03039 & .00231 & .00926 & 3.300 & 10.060 & 11.036 \\
\hline $\begin{array}{l}5 \\
5\end{array}$ & $\begin{array}{l}3200 \\
3200\end{array}$ & $\begin{array}{l}52.0 \\
53.0\end{array}$ & $\begin{array}{l}.00744 \\
.00728\end{array}$ & $\begin{array}{r}.02976 \\
.02915\end{array}$ & $\begin{array}{r}.00226 \\
.00222\end{array}$ & $\begin{array}{l}.00907 \\
.00888\end{array}$ & $\begin{array}{l}3.232 \\
3.165\end{array}$ & $\begin{array}{l}9.851 \\
9.648\end{array}$ & $\begin{array}{l}10.807 \\
10.583\end{array}$ \\
\hline 5 & 3200 & 54.0 & .00713 & .02853 & .00217 & .00869 & 3.098 & 9.444 & 10.360 \\
\hline $\begin{array}{l}5 \\
5 \\
5\end{array}$ & $\begin{array}{l}3200 \\
3200 \\
3200\end{array}$ & $\begin{array}{l}55.0 \\
60.0 \\
65.0\end{array}$ & $\begin{array}{l}.00698 \\
.00636 \\
.00586\end{array}$ & $\begin{array}{r}.02792 \\
.02544 \\
.02345\end{array}$ & $\begin{array}{l}.00212 \\
.00193 \\
.00178\end{array}$ & $\begin{array}{l}.00851 \\
.00775 \\
.00714\end{array}$ & $\begin{array}{l}3.031 \\
2.762 \\
2.546\end{array}$ & $\begin{array}{l}9.240 \\
8.420 \\
7.761\end{array}$ & $\begin{array}{r}10.137 \\
9.237 \\
8.513\end{array}$ \\
\hline $\begin{array}{l}5 \\
5\end{array}$ & $\begin{array}{l}3200 \\
3200\end{array}$ & $\begin{array}{l}70.0 \\
75.0\end{array}$ & $\begin{array}{l}.00545 \\
.00507\end{array}$ & $\begin{array}{r}.02180 \\
.02028\end{array}$ & $\begin{array}{r}.00166 \\
.00154\end{array}$ & $\begin{array}{l}.00664 \\
.00618\end{array}$ & $\begin{array}{l}2 \cdot 367 \\
2 \cdot 202\end{array}$ & $\begin{array}{l}7.217 \\
6.713\end{array}$ & $\begin{array}{l}7.917 \\
7.364\end{array}$ \\
\hline 5 & 3200 & 80.0 & .00475 & .01901 & .00144 & .00579 & 2.064 & 6.291 & 6.901 \\
\hline $\begin{array}{l}5 \\
5 \\
5\end{array}$ & $\begin{array}{l}3200 \\
3200 \\
3200\end{array}$ & $\begin{array}{l}85.0 \\
90.0 \\
95.0\end{array}$ & $\begin{array}{l}.00449 \\
.00428 \\
.00408\end{array}$ & $\begin{array}{r}.01799 \\
.01715 \\
.01632\end{array}$ & $\begin{array}{l}.00137 \\
.00130 \\
.00124\end{array}$ & $\begin{array}{l}.00548 \\
.00522 \\
.00497\end{array}$ & $\begin{array}{l}1.953 \\
1.862 \\
1.772\end{array}$ & $\begin{array}{l}5.953 \\
5.677 \\
5.402\end{array}$ & $\begin{array}{l}6.531 \\
6.228 \\
5.927\end{array}$ \\
\hline 5 & 3200 & $100 \cdot 0$ & .00393 & .01572 & .00119 & .00479 & 1.707 & 5.203 & 5.708 \\
\hline
\end{tabular}


TABULATION OF DATA FOR

ABSORPTION OF SOUND . IN AIR VERSUS PERGENT RELATIVE HUMIDITY AT 5 DEGREES GENTIGRADE FOR A FREQUENCY OF 4000 HERTZ

\begin{tabular}{|c|c|c|c|c|c|c|c|c|c|}
\hline 11 & $(2)$ & (3) & $(4)$ & (5) & $(6)$ & $(7)$ & $(8)$ & $|9|$ & $(10)$ \\
\hline TEMP & FREQ & $\begin{array}{l}\text { REL } \\
\text { HUM }\end{array}$ & $\begin{array}{l}\text { ATTEN } \\
\text { COEF }\end{array}$ & $4 M$ & $\begin{array}{l}\text { ATTEN } \\
\text { COEF }\end{array}$ & $4 M$ & $\begin{array}{l}\text { ATTEN } \\
\text { DB PER }\end{array}$ & $\begin{array}{l}\text { ATTEN } \\
\text { DB PER }\end{array}$ & $\begin{array}{l}\text { DECAY } \\
\text { RATE }\end{array}$ \\
\hline $\begin{array}{l}E G R \\
\text { ENT }\end{array}$ & HER TZ & $\begin{array}{l}\text { PER } \\
\text { CENT }\end{array}$ & $\begin{array}{l}\text { PER } \\
\text { METER }\end{array}$ & $\begin{array}{l}\text { PER } \\
\text { METER }\end{array}$ & $\begin{array}{l}\text { PER } \\
\text { FOOT }\end{array}$ & $\begin{array}{l}\text { PER } \\
\text { FOOT }\end{array}$ & $\begin{array}{l}100 \\
\text { METER }\end{array}$ & $\begin{array}{l}1.000 \\
\text { FEET }\end{array}$ & $\begin{array}{l}\text { DB PER } \\
\text { SECOND }\end{array}$ \\
\hline $\begin{array}{l}5 \\
5 \\
5\end{array}$ & $\begin{array}{l}4000 \\
4000 \\
4000\end{array}$ & $\begin{array}{r}5.0 \\
10.0 \\
15.0\end{array}$ & $\begin{array}{r}.00569 \\
.01085 \\
.01632\end{array}$ & $\begin{array}{l}.02279 \\
.04341 \\
.06528\end{array}$ & $\begin{array}{l}.00173 \\
.00330 \\
.00497\end{array}$ & $\begin{array}{r}.00694 \\
.01323 \\
.01989\end{array}$ & $\begin{array}{l}2.475 \\
4.713 \\
7.088\end{array}$ & $\begin{array}{r}7.544 \\
14.367 \\
21.605\end{array}$ & $\begin{array}{r}8.275 \\
15.761 \\
23.700\end{array}$ \\
\hline $\begin{array}{l}5 \\
5 \\
5\end{array}$ & $\begin{array}{l}4000 \\
4000 \\
4000\end{array}$ & $\begin{array}{l}20.0 \\
25.0 \\
30.0\end{array}$ & $\begin{array}{r}.01923 \\
.01948 \\
.01842\end{array}$ & $\begin{array}{l}.07693 \\
.07792 \\
.07368\end{array}$ & $\begin{array}{l}.00586 \\
.00593 \\
.00561\end{array}$ & $\begin{array}{l}.02344 \\
.02375 \\
.02246\end{array}$ & $\begin{array}{l}8.352 \\
8.461 \\
8.000\end{array}$ & $\begin{array}{l}25.459 \\
25.790 \\
24.386\end{array}$ & $\begin{array}{l}27.928 \\
28 \cdot 291 \\
26.752\end{array}$ \\
\hline $\begin{array}{l}5 \\
5 \\
5\end{array}$ & $\begin{array}{l}4000 \\
4000 \\
4000\end{array}$ & $\begin{array}{l}35.0 \\
40.0 \\
45.0\end{array}$ & $\begin{array}{r}.01645 \\
.01438 \\
.01265\end{array}$ & $\begin{array}{l}.06583 \\
.05755 \\
.05060\end{array}$ & $\begin{array}{l}.00501 \\
.00438 \\
.00385\end{array}$ & $\begin{array}{r}.02006 \\
.01754 \\
.01542\end{array}$ & $\begin{array}{l}7.147 \\
6.249 \\
5.494\end{array}$ & $\begin{array}{l}21.786 \\
19.048 \\
16.748\end{array}$ & $\begin{array}{l}23.899 \\
20.895 \\
18.372\end{array}$ \\
\hline $\begin{array}{l}5 \\
5 \\
5\end{array}$ & $\begin{array}{l}4000 \\
4000 \\
4000\end{array}$ & $\begin{array}{l}46.0 \\
47.0 \\
48.0\end{array}$ & $\begin{array}{r}.01235 \\
.01208 \\
.01182\end{array}$ & $\begin{array}{r}.04941 \\
.04834 \\
.04728\end{array}$ & $\begin{array}{l}.00376 \\
.00368 \\
.00360\end{array}$ & $\begin{array}{r}.01506 \\
.01473 \\
.01441\end{array}$ & $\begin{array}{l}5.364 \\
5.249 \\
5.133\end{array}$ & $\begin{array}{l}16.352 \\
15.999 \\
15.647\end{array}$ & $\begin{array}{l}17.938 \\
17.551 \\
17.165\end{array}$ \\
\hline $\begin{array}{l}5 \\
5 \\
5\end{array}$ & $\begin{array}{l}4000 \\
4000 \\
4000\end{array}$ & $\begin{array}{l}49.0 \\
50.0 \\
51.0\end{array}$ & $\begin{array}{r}.01158 \\
.01135 \\
.01113\end{array}$ & $\begin{array}{l}.04633 \\
.04540 \\
.04455\end{array}$ & $\begin{array}{r}.00353 \\
.00346 \\
.00339\end{array}$ & $\begin{array}{r}.01412 \\
.01384 \\
.01358\end{array}$ & $\begin{array}{l}5.031 \\
4.930 \\
4.838\end{array}$ & $\begin{array}{l}15.335 \\
15.027 \\
14.746\end{array}$ & $\begin{array}{l}16.823 \\
16.485 \\
16.176\end{array}$ \\
\hline $\begin{array}{l}5 \\
5\end{array}$ & $\begin{array}{l}4000 \\
4000\end{array}$ & $\begin{array}{l}52.0 \\
53.0\end{array}$ & $\begin{array}{r}.01094 \\
.01074\end{array}$ & $\begin{array}{r}.04376 \\
.04296\end{array}$ & $\begin{array}{l}.00333 \\
.00327\end{array}$ & $\begin{array}{r}.01333 \\
.01309\end{array}$ & $\begin{array}{l}4.751 \\
4.664\end{array}$ & $\begin{array}{l}14 \cdot 482 \\
14 \cdot 218\end{array}$ & $\begin{array}{l}15.887 \\
15.597\end{array}$ \\
\hline 5 & 4000 & 54.0 & .01054 & .04216 & .00321 & .01285 & 4.578 & 13.954 & 15.307 \\
\hline $\begin{array}{l}5 \\
5\end{array}$ & $\begin{array}{l}4000 \\
4000\end{array}$ & $\begin{array}{l}55.0 \\
60.0\end{array}$ & $\begin{array}{r}.01034 \\
.00943\end{array}$ & $\begin{array}{r}.04136 \\
.03774\end{array}$ & $\begin{array}{r}.00315 \\
.00287\end{array}$ & $\begin{array}{r}.01260 \\
.01150\end{array}$ & $\begin{array}{l}4.491 \\
4.097\end{array}$ & $\begin{array}{l}13.690 \\
12.490\end{array}$ & $\begin{array}{l}15.018 \\
13.701\end{array}$ \\
\hline 5 & 4000 & 65.0 & .00863 & .03453 & .00263 & .01052 & 3.749 & 11.428 & 12.536 \\
\hline $\begin{array}{l}5 \\
5\end{array}$ & $\begin{array}{l}4000 \\
4000\end{array}$ & $\begin{array}{l}70.0 \\
75.0\end{array}$ & $\begin{array}{r}.00798 \\
.00745\end{array}$ & $\begin{array}{r}03195 \\
.02982\end{array}$ & $\begin{array}{l}.00243 \\
.00227\end{array}$ & $\begin{array}{l}.00974 \\
.00908\end{array}$ & $\begin{array}{l}3.469 \\
3.237\end{array}$ & $\begin{array}{r}10.576 \\
9.869\end{array}$ & $\begin{array}{l}11.602 \\
10.826\end{array}$ \\
\hline 5 & 4000 & 80.0 & .00701 & .02804 & .00213 & .00854 & 3.044 & 9.280 & 10.181 \\
\hline $\begin{array}{l}5 \\
5 \\
5\end{array}$ & $\begin{array}{l}4000 \\
4000 \\
4000\end{array}$ & $\begin{array}{l}85.0 \\
90.0 \\
95.0\end{array}$ & $\begin{array}{l}.00657 \\
.00621 \\
.00590\end{array}$ & $\begin{array}{r}.02631 \\
.02484 \\
.02362\end{array}$ & $\begin{array}{l}.00200 \\
.00189 \\
.00180\end{array}$ & $\begin{array}{r}.00802 \\
.00757 \\
.00720\end{array}$ & $\begin{array}{l}2.857 \\
2.697 \\
2.564\end{array}$ & $\begin{array}{l}8.708 \\
8.220 \\
7.817\end{array}$ & $\begin{array}{l}9.553 \\
9.018 \\
8.576\end{array}$ \\
\hline 5 & 4000 & $100 \cdot 0$ & .00564 & .02258 & .00172 & .00688 & 2.452 & $7 \cdot 474$ & $8.199^{\circ}$ \\
\hline
\end{tabular}


TABULATION OF DATA FOR

ABSORPTION OF SOUND IN AIR VERSUS PERCENT RELATIVE HUMIDITY AT 5 DEGREES CENTIGRADE FOR A FREQUENCY OF 5000 HERTZ

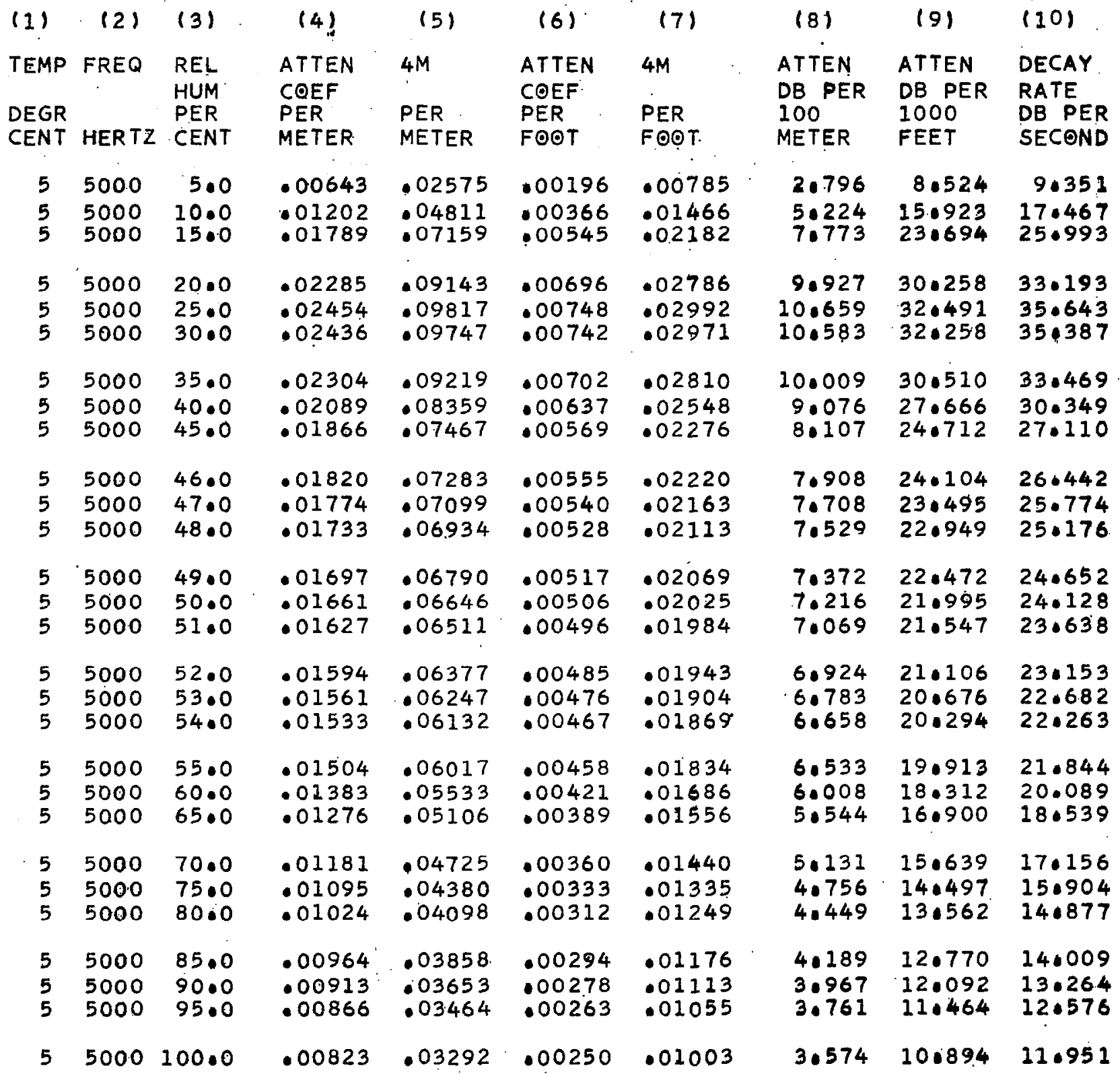


TABULATION OF DATA FOR

ABSORPTION OF SOUND IN AIR VERSUS PERCENT RELATIVE HUMIDITY AT 5 DEGREES CENTIGRADE FOR A FREQUENCY OF 5940 HERTZ

\begin{tabular}{|c|c|c|c|c|c|c|c|c|c|}
\hline$(1)$ & $(2)$ & $(3)$ & (4) & (5) & $(6)$ & 171 & (8) & $(9)$ & $(10)$ \\
\hline TEMP & FREQ & $\begin{array}{l}\text { REL } \\
\text { HUM }\end{array}$ & $\begin{array}{l}\text { ATTEN } \\
\text { COEF }\end{array}$ & $4 M$ & $\begin{array}{l}\text { ATTEN } \\
\text { COEF }\end{array}$ & $4 M$ & $\begin{array}{l}\text { ATTEN } \\
\text { DB PER }\end{array}$ & $\begin{array}{l}\text { ATTEN } \\
\text { DB PER }\end{array}$ & $\begin{array}{l}\text { DECAY } \\
\text { RATE }\end{array}$ \\
\hline $\begin{array}{l}\text { DEGR } \\
\text { CENT }\end{array}$ & HERTZ & $\begin{array}{l}\text { PER } \\
\text { CENT }\end{array}$ & $\begin{array}{l}\text { PER } \\
\text { METER }\end{array}$ & $\begin{array}{l}\text { PER } \\
\text { METER }\end{array}$ & $\begin{array}{l}\text { PER } \\
\text { FOOT }\end{array}$ & $\begin{array}{l}\text { PER } \\
\text { FOOT }\end{array}$ & $\begin{array}{l}100 \\
\text { METER }\end{array}$ & $\begin{array}{l}1000 \\
\text { FEET }\end{array}$ & $\begin{array}{l}\text { DB PER } \\
\text { SECOND }\end{array}$ \\
\hline $\begin{array}{l}5 \\
5 \\
5\end{array}$ & $\begin{array}{l}5940 \\
5940 \\
5940\end{array}$ & $\begin{array}{r}5.0 \\
10.0 \\
15.0\end{array}$ & $\begin{array}{r}.00712 \\
.01304 \\
.01905\end{array}$ & $\begin{array}{l}.02848 \\
.05217 \\
.07621\end{array}$ & $\begin{array}{l}.00217 \\
.00397 \\
.00580\end{array}$ & $\begin{array}{l}.00868 \\
.01590 \\
.02323\end{array}$ & $\begin{array}{l}3.093 \\
5.665 \\
8.275\end{array}$ & $\begin{array}{r}9.428 \\
17 \cdot 267 \\
25.222\end{array}$ & $\begin{array}{l}10.342 \\
18.942 \\
27.669\end{array}$ \\
\hline 5 & 5940 & 20.0 & .02528 & .10113 & .00770 & .03082 & 10.980 & 33.468 & 36.714 \\
\hline $\begin{array}{l}5 \\
5\end{array}$ & $\begin{array}{l}5940 \\
5940\end{array}$ & $\begin{array}{l}25.0 \\
30.0\end{array}$ & $\begin{array}{r}.02869 \\
.02945\end{array}$ & $\begin{array}{r}.11477 \\
.11783\end{array}$ & $\begin{array}{l}.00874 \\
.00897\end{array}$ & $\begin{array}{r}.03498 \\
.03591\end{array}$ & $\begin{array}{l}12.461 \\
12.793\end{array}$ & $\begin{array}{l}37.983 \\
38.996\end{array}$ & $\begin{array}{l}41.668 \\
42.779\end{array}$ \\
\hline $\begin{array}{l}5 \\
5 \\
5\end{array}$ & $\begin{array}{l}5940 \\
5940 \\
5940\end{array}$ & $\begin{array}{l}35.0 \\
40.0 \\
45.0\end{array}$ & $\begin{array}{r}.02889 \\
.02727 \\
.02494\end{array}$ & $\begin{array}{l}11557 \\
.10911 \\
.09978\end{array}$ & $\begin{array}{l}.00880 \\
.00831 \\
.00760\end{array}$ & $\begin{array}{l}.03522 \\
.03325 \\
.03041\end{array}$ & $\begin{array}{l}12.548 \\
11.847 \\
10.834\end{array}$ & $\begin{array}{l}38.249 \\
36.111 \\
33.023\end{array}$ & $\begin{array}{l}41.960 \\
39.614 \\
36.226\end{array}$ \\
\hline $\begin{array}{l}5 \\
5 \\
5\end{array}$ & $\begin{array}{l}5940 \\
5940 \\
5940\end{array}$ & $\begin{array}{l}46.0 \\
47.0 \\
48.0\end{array}$ & $\begin{array}{r}.02446 \\
.02399 \\
.02351\end{array}$ & $\begin{array}{l}.09787 \\
.09596 \\
.09407\end{array}$ & $\begin{array}{r}.00745 \\
.00731 \\
.00716\end{array}$ & $\begin{array}{r}.02983 \\
.02924 \\
.02867\end{array}$ & $\begin{array}{l}10.626 \\
10.419 \\
10.214\end{array}$ & $\begin{array}{l}32.390 \\
31.758 \\
31.134\end{array}$ & $\begin{array}{l}35.532 \\
34.839 \\
34.154\end{array}$ \\
\hline $\begin{array}{l}5 \\
5 \\
5\end{array}$ & $\begin{array}{l}5940 \\
5940 \\
5940\end{array}$ & $\begin{array}{l}49.0 \\
50.0 \\
51.0\end{array}$ & $\begin{array}{l}.02305 \\
.02258 \\
.02210\end{array}$ & $\begin{array}{l}.09220 \\
.09033 \\
.0884 .1\end{array}$ & $\begin{array}{l}.00702 \\
.00688 \\
.00673\end{array}$ & $\begin{array}{l}.02810 \\
.02753 \\
.02694\end{array}$ & $\begin{array}{r}10.011 \\
9.808 \\
9.599\end{array}$ & $\begin{array}{l}30.514 \\
29.895 \\
29.258\end{array}$ & $\begin{array}{l}33.474 \\
32.795 \\
32.096\end{array}$ \\
\hline $\begin{array}{l}5 \\
5 \\
5\end{array}$ & $\begin{array}{l}5940 \\
5940 \\
5940\end{array}$ & $\begin{array}{l}52.0 \\
53.0 \\
54.0\end{array}$ & $\begin{array}{l}.02161 \\
.02112 \\
.02071\end{array}$ & $\begin{array}{l}.08646 \\
.08451 \\
.08286\end{array}$ & $\begin{array}{l}.00658 \\
.00643 \\
.00631\end{array}$ & $\begin{array}{l}.02635 \\
.02575 \\
.02525\end{array}$ & $\begin{array}{l}9.387 \\
9.175 \\
8.997\end{array}$ & $\begin{array}{l}28.613 \\
27.968 \\
27.424\end{array}$ & $\begin{array}{l}31.389 \\
30.681 \\
30.084\end{array}$ \\
\hline $\begin{array}{l}5 \\
5 \\
5\end{array}$ & $\begin{array}{l}5940 \\
5940 \\
.5940\end{array}$ & $\begin{array}{l}55.0 \\
60.0 \\
65.0\end{array}$ & $\begin{array}{r}.02033 \\
.01857 \\
.01715\end{array}$ & $\begin{array}{l}.08133 \\
.07428 \\
.06860\end{array}$ & $\begin{array}{l}.00619 \\
.00566 \\
.00522\end{array}$ & $\begin{array}{l}.02479 \\
.02264 \\
.02090\end{array}$ & $\begin{array}{l}8.831 \\
8.065 \\
7.448\end{array}$ & $\begin{array}{l}26.918 \\
24.582 \\
22.703\end{array}$ & $\begin{array}{l}29.529 \\
26.967 \\
24.905\end{array}$ \\
\hline 5 & 5940 & 70.0 & .01599 & .06399 & .00487 & .01950 & 948 & 79 & 234 \\
\hline $\begin{array}{l}5 \\
5\end{array}$ & $\begin{array}{l}5940 \\
5940\end{array}$ & $\begin{array}{l}75.0 \\
80.0\end{array}$ & $\begin{array}{l}.01491 \\
.01393\end{array}$ & $\begin{array}{l}.05964 \\
.05574\end{array}$ & $\begin{array}{l}.00454 \\
.00424\end{array}$ & $\begin{array}{r}.01817 \\
.01699\end{array}$ & $\begin{array}{l}6.475 \\
6.052\end{array}$ & $\begin{array}{l}19.737 \\
18.448\end{array}$ & $\begin{array}{l}21.652 \\
20.237\end{array}$ \\
\hline $\begin{array}{l}5 \\
5 \\
5\end{array}$ & $\begin{array}{l}5940 \\
5940 \\
5940\end{array}$ & $\begin{array}{l}85.0 \\
90.0 \\
95.0\end{array}$ & $\begin{array}{r}.01306 \\
.01232 \\
.01168\end{array}$ & $\begin{array}{l}.05224 \\
.04928 \\
.04674\end{array}$ & $\begin{array}{l}.00398 \\
.00375 \\
.00356\end{array}$ & $\begin{array}{l}.01592 \\
.01502 \\
.01424\end{array}$ & $\begin{array}{l}5.672 \\
5.350 \\
5.075\end{array}$ & $\begin{array}{l}17 \cdot 291 \\
16.309 \\
15.469\end{array}$ & $\begin{array}{l}18.968 \\
17.891 \\
16.969\end{array}$ \\
\hline 5 & 59401 & 100.0 & .01113 & .04453 & .00339 & .01357 & 4.835 & $14 \cdot 739$ & $16 \cdot 169$ \\
\hline
\end{tabular}


TABULATIEN OF DATA FER

ABSERPTIEN OF SOUND IN AIR VERSUS PERCENT RELATIVE HUMIDITY AT 5 DEGREES CENTIGRADE FGR A FREQUENCY OF 6300 HERTZ

\begin{tabular}{|c|c|c|c|c|c|c|c|c|c|}
\hline 1 & $(2)$ & (3) & $(4)$ & $(5)$ & (6) & $(7)$ & (8) & $(9)$ & $(10)$ \\
\hline $\begin{array}{l}\text { EMP } \\
\text { EGR } \\
\text { ENT }\end{array}$ & HERTZ & $\begin{array}{l}\text { REL } \\
\text { HUM } \\
\text { PER } \\
\text { CENT }\end{array}$ & $\begin{array}{l}\text { ATTEN } \\
\text { COEF } \\
\text { PER } \\
\text { METER }\end{array}$ & $\begin{array}{l}\text { 4M } \\
\text { PER } \\
\text { METER }\end{array}$ & $\begin{array}{l}\text { ATTEN } \\
\text { COEF } \\
\text { PER } \\
\text { FOQT }\end{array}$ & $\begin{array}{l}4 M \\
\text { PER } \\
\text { FOOT }\end{array}$ & $\begin{array}{l}\text { ATTEN } \\
\text { DB PER } \\
100 \\
\text { METER }\end{array}$ & $\begin{array}{l}\text { ATTEN } \\
\text { DB PER } \\
1000 \\
\text { FEET }\end{array}$ & $\begin{array}{l}\text { DECAY } \\
\text { RATE } \\
\text { DB' PER } \\
\text { SECOND }\end{array}$ \\
\hline $\begin{array}{l}5 \\
5 \\
5\end{array}$ & $\begin{array}{l}6300 \\
6300 \\
6300\end{array}$ & $\begin{array}{r}5.0 \\
10.0 \\
15.0\end{array}$ & $\begin{array}{l}.00738 \\
.01342 \\
.01950\end{array}$ & $\begin{array}{l}.02954 \\
.05369 \\
.07801\end{array}$ & $\begin{array}{l}.00225 \\
.00409 \\
.00594\end{array}$ & $\begin{array}{l}.00900 \\
.01636 \\
.02377\end{array}$ & $\begin{array}{l}3.207 \\
5.829 \\
8.470\end{array}$ & $\begin{array}{r}9.778 \\
17.769 \\
25.828\end{array}$ & $\begin{array}{l}10 \cdot 721 \\
19 \cdot 492 \\
28.323\end{array}$ \\
\hline $\begin{array}{l}5 \\
5 \\
5\end{array}$ & $\begin{array}{l}6300 \\
6300 \\
6300\end{array}$ & $\begin{array}{l}20 \bullet 0 \\
25.0 \\
30.0\end{array}$ & $\begin{array}{l}.02600 \\
.03003 \\
.03127\end{array}$ & $\begin{array}{r}.10402 \\
.12014 \\
.12510\end{array}$ & $\begin{array}{l}.00792 \\
.00915 \\
.00953\end{array}$ & $\begin{array}{l}.03170 \\
.03662 \\
.03813\end{array}$ & $\begin{array}{l}11.294 \\
13.044 \\
13.583\end{array}$ & $\begin{array}{l}34 \cdot 424 \\
39 \cdot 761 \\
41.401\end{array}$ & $\begin{array}{l}37.763 \\
43.618 \\
45.417\end{array}$ \\
\hline $\begin{array}{l}5 \\
5 \\
5\end{array}$ & $\begin{array}{l}6300 \\
6300 \\
6300\end{array}$ & $\begin{array}{l}35.0 \\
40.0 \\
45.0\end{array}$ & $\begin{array}{l}.03098 \\
.02964 \\
.02742\end{array}$ & $\begin{array}{r}.12393 \\
.11656 \\
.10969\end{array}$ & $\begin{array}{l}.00944 \\
.00903 \\
.00835\end{array}$ & $\begin{array}{l}.03777 \\
.03613 \\
.03343\end{array}$ & $\begin{array}{l}13.456 \\
12.873 \\
11.910\end{array}$ & $\begin{array}{l}41.014 \\
39.238 \\
36.302\end{array}$ & $\begin{array}{l}993 \\
045 \\
824\end{array}$ \\
\hline $\begin{array}{l}3 \\
5 \\
5\end{array}$ & $\begin{array}{l}6300 \\
6300 \\
6300\end{array}$ & $\begin{array}{l}46.0 \\
47.0 \\
48.0\end{array}$ & $\begin{array}{l}.02693 \\
.02645 \\
.02596\end{array}$ & $\begin{array}{l}.10774 \\
.10580 \\
.10385\end{array}$ & $\begin{array}{l}.00821 \\
.00806 \\
.00791\end{array}$ & $\begin{array}{l}.03284 \\
.03224 \\
.03165\end{array}$ & $\begin{array}{l}11.698 \\
11.487 \\
11.275\end{array}$ & $\begin{array}{l}35.658 \\
35.014 \\
34.369\end{array}$ & $\begin{array}{l}39.117 \\
38.410 \\
37.703\end{array}$ \\
\hline $\begin{array}{l}5 \\
5 \\
5\end{array}$ & $\begin{array}{l}6300 \\
6300 \\
6300\end{array}$ & $\begin{array}{l}49.0 \\
50.0 \\
51.0\end{array}$ & $\begin{array}{r}.02547 \\
.02499 \\
.02451\end{array}$ & $\begin{array}{r}.10190 \\
.09998 \\
.09807\end{array}$ & $\begin{array}{l}.00776 \\
.00761 \\
.00747\end{array}$ & $\begin{array}{l}.03106 \\
.03047 \\
.02989\end{array}$ & $\begin{array}{l}11.064 \\
10.856 \\
10.648\end{array}$ & $\begin{array}{l}33.725 \\
33.089 \\
32.458\end{array}$ & $\begin{array}{l}36 \cdot 996 \\
36.299 \\
35.606\end{array}$ \\
\hline $\begin{array}{l}5 \\
5 \\
5\end{array}$ & $\begin{array}{l}6300 \\
6300 \\
6300\end{array}$ & $\begin{array}{l}52.0 \\
53.0 \\
54.0\end{array}$ & $\begin{array}{l}.02404 \\
.02355 \\
.02305\end{array}$ & $\begin{array}{l}.09617 \\
.09421 \\
.09222\end{array}$ & $\begin{array}{l}.00732 \\
.00717 \\
.00702\end{array}$ & $\begin{array}{l}.02931 \\
.02871 \\
.02811\end{array}$ & $\begin{array}{l}10.441 \\
10.229 \\
10.013\end{array}$ & $\begin{array}{l}31.826 \\
31.178 \\
30.521\end{array}$ & $\begin{array}{l}34.914 \\
34 \cdot 203 \\
33 \cdot 482\end{array}$ \\
\hline $\begin{array}{l}5 \\
5 \\
5\end{array}$ & $\begin{array}{l}6300 \\
6300 \\
6300\end{array}$ & $\begin{array}{l}55.0 \\
60.0 \\
65.0\end{array}$ & $\begin{array}{l}.02256 \\
.02060 \\
.01898\end{array}$ & $\begin{array}{r}.09024 \\
.08243 \\
.07594\end{array}$ & $\begin{array}{l}.00687 \\
.00628 \\
.00578\end{array}$ & $\begin{array}{l}.02750 \\
.02512 \\
.02314\end{array}$ & $\begin{array}{l}9.797 \\
8.950 \\
8.245\end{array}$ & $\begin{array}{l}29.864 \\
27.281 \\
25.132\end{array}$ & $\begin{array}{l}32.761 \\
29.927 \\
27.570\end{array}$ \\
\hline $\begin{array}{l}5 \\
5 \\
5\end{array}$ & $\begin{array}{l}6300 \\
6300 \\
6300\end{array}$ & $\begin{array}{l}70.0 \\
75.0 \\
80.0\end{array}$ & $\begin{array}{r}.01770 \\
.01655 \\
.01549\end{array}$ & $\begin{array}{l}.07083 \\
.06620 \\
.06196\end{array}$ & $\begin{array}{l}.00539 \\
.00504 \\
.00472\end{array}$ & $\begin{array}{l}.02159 \\
.02018 \\
.01888\end{array}$ & $\begin{array}{l}7.691 \\
7.188 \\
6.727\end{array}$ & $\begin{array}{l}+43 \\
10 \\
506\end{array}$ & $\begin{array}{l}25 \cdot 717 \\
24 \cdot 036 \\
22.495\end{array}$ \\
\hline $\begin{array}{l}3 \\
5 \\
5\end{array}$ & $\begin{array}{l}6300 \\
6300 \\
6300\end{array}$ & $\begin{array}{l}85.0 \\
90.0 \\
95.0\end{array}$ & $\begin{array}{r}.01451 \\
.01368 \\
.01296\end{array}$ & $\begin{array}{l}.05806 \\
.05475 \\
.05184\end{array}$ & $\begin{array}{l}.00442 \\
.00417 \\
.00395\end{array}$ & $\begin{array}{l}.017 \\
.016 \\
.015\end{array}$ & $\begin{array}{l}6.304 \\
5.945 \\
5.628\end{array}$ & $\begin{array}{l}19 \cdot 216 \\
18 \cdot 121 \\
17.157\end{array}$ & $\begin{array}{l}21.080 \\
19.878 \\
18.021\end{array}$ \\
\hline 5 & 6300 & 00.0 & .01233 & 04934 & .00376 & .01504 & 5.358 & 16.331 & 17.915 \\
\hline
\end{tabular}


TABULATION OF DATA FOR

ABSORPTION OF SOUND IN AIR VERSUS PERCENT RELATIVE HUMIDITY AT 5 DEGREES CENTIGRADE FOR A FREQUENCY OF 8000 HERTZ

\begin{tabular}{|c|c|c|c|c|c|c|c|c|c|}
\hline$(1)$ & $(2)$ & (3) & 14.1. & $(5)$ & (6) & 171 & 181 & $(9)$ & $(10)$ \\
\hline $\begin{array}{l}\text { TEMP } \\
\text { DEGR } \\
\text { CENT }\end{array}$ & FREQ & $\begin{array}{l}\text { REL } \\
\text { HUM } \\
\text { PER } \\
\text { CENT }\end{array}$ & $\begin{array}{l}\text { ATTEN } \\
\text { COEF } \\
\text { PER } \\
\text { METER }\end{array}$ & $\begin{array}{l}4 M \\
\text { PER } \\
\text { METER }\end{array}$ & $\begin{array}{l}\text { ATTEN } \\
\text { COEF } \\
\text { PER } \\
\text { FOOT }\end{array}$ & $\begin{array}{l}4 M \\
\text { PER } \\
\text { FOOT }\end{array}$ & $\begin{array}{l}\text { ATTEN } \\
\text { DB PER } \\
100 \\
\text { METER }\end{array}$ & $\begin{array}{l}\text { ATTEN } \\
\text { DB PER } \\
1000 \\
\text { FEET }\end{array}$ & $\begin{array}{l}\text { DECAY } \\
\text { RATE } \\
\text { DB PER } \\
\text { SECOND }\end{array}$ \\
\hline $\begin{array}{l}5 \\
5 \\
5\end{array}$ & $\begin{array}{l}8000 \\
8000 \\
8000\end{array}$ & $\begin{array}{r}5.0 \\
10.0 \\
15.0\end{array}$ & $\begin{array}{r}.00875 \\
.01533 \\
.02190\end{array}$ & $\begin{array}{l}.03502 \\
.06133 \\
.08763\end{array}$ & $\begin{array}{l}.00266 \\
.00467 \\
.00667\end{array}$ & $\begin{array}{l}.01067 \\
.01869 \\
.02671\end{array}$ & $\begin{array}{l}3.802 \\
6.659 \\
9.515\end{array}$ & $\begin{array}{l}11 \cdot 591 \\
20.297 \\
29.002\end{array}$ & $\begin{array}{l}12.715 \\
22.266 \\
31.816\end{array}$ \\
\hline $\begin{array}{l}5 \\
5 \\
5\end{array}$ & $\begin{array}{l}8000 \\
8000 \\
8000\end{array}$ & $\begin{array}{l}20.0 \\
25.0 \\
30.0\end{array}$ & $\begin{array}{l}.02889 \\
.03549 \\
.03904\end{array}$ & $\begin{array}{r}11557 \\
.14199 \\
\cdot 15618\end{array}$ & $\begin{array}{l}.00880 \\
.01081 \\
.01190\end{array}$ & $\begin{array}{r}.03522 \\
.04327 \\
.04760\end{array}$ & $\begin{array}{l}12.548 \\
15.416 \\
16.957\end{array}$ & $\begin{array}{l}38 \cdot 248 \\
46.990 \\
51.687\end{array}$ & $\begin{array}{l}41 \cdot 958 \\
51.548 \\
56 \cdot 701\end{array}$ \\
\hline $\begin{array}{l}5 \\
5 \\
5\end{array}$ & $\begin{array}{l}8000 \\
8000 \\
8000\end{array}$ & $\begin{array}{l}35.0 \\
40.0 \\
45.0\end{array}$ & $\begin{array}{l}.04018 \\
.03994 \\
.03869\end{array}$ & $\begin{array}{r}.16073 \\
.15977 \\
.15476\end{array}$ & $\begin{array}{r}.01224 \\
.01217 \\
.01179\end{array}$ & $\begin{array}{r}.04899 \\
.04870 \\
.04717\end{array}$ & $\begin{array}{l}17.451 \\
17.348 \\
16.803\end{array}$ & $\begin{array}{l}53 \cdot 193 \\
52.877 \\
51.218\end{array}$ & $\begin{array}{l}58.353 \\
58.006 \\
56.186\end{array}$ \\
\hline $\begin{array}{l}5 \\
5 \\
5\end{array}$ & $\begin{array}{l}8000 \\
8000 \\
8000\end{array}$ & $\begin{array}{l}46.0 \\
47.0 \\
48.0\end{array}$ & $\begin{array}{r}.03833 \\
.03794 \\
.03752\end{array}$ & $\begin{array}{r}15332 \\
.15179 \\
.15010\end{array}$ & $\begin{array}{r}.01168 \\
.01156 \\
.01143\end{array}$ & $\begin{array}{r}.04673 \\
.04626 \\
.04575\end{array}$ & $\begin{array}{l}16.647 \\
16.481 \\
16.297\end{array}$ & $\begin{array}{l}50.741 \\
50.236 \\
49.674\end{array}$ & $\begin{array}{l}55.663 \\
55.108 \\
54.492\end{array}$ \\
\hline $\begin{array}{l}5 \\
5 \\
5\end{array}$ & $\begin{array}{l}8000 \\
8000 \\
8000\end{array}$ & $\begin{array}{l}49.0 \\
50.0 \\
51.0\end{array}$ & $\begin{array}{r}.03706 \\
.03657 \\
.03605\end{array}$ & $\begin{array}{r}14826 \\
.14631 \\
.14423\end{array}$ & $\begin{array}{l}.01129 \\
.01114 \\
.01099\end{array}$ & $\begin{array}{l}.04519 \\
.04459 \\
.04396\end{array}$ & $\begin{array}{l}16.098 \\
15.886 \\
15.660\end{array}$ & $\begin{array}{l}49.067 \\
48.421 \\
47.734\end{array}$ & $\begin{array}{l}53.827 \\
53.118 \\
52.364\end{array}$ \\
\hline $\begin{array}{l}5 \\
5 \\
5\end{array}$ & $\begin{array}{l}8000 \\
8000 \\
8000\end{array}$ & $\begin{array}{l}52.0 \\
53.0 \\
54.0\end{array}$ & $\begin{array}{r}.03552 \\
.03499 \\
.03446\end{array}$ & $\begin{array}{r}14211 \\
.13999 \\
.13787\end{array}$ & $\begin{array}{r}.01082 \\
.01066 \\
.01050\end{array}$ & $\begin{array}{r}.04331 \\
.04267 \\
.04202\end{array}$ & $\begin{array}{l}15.430 \\
15.199 \\
14.969\end{array}$ & $\begin{array}{l}47.032 \\
46.330 \\
45.628\end{array}$ & $\begin{array}{l}51.594 \\
50.824 \\
50.054\end{array}$ \\
\hline $\begin{array}{l}5 \\
5 \\
5\end{array}$ & $\begin{array}{l}8000 \\
8000 \\
8000\end{array}$ & $\begin{array}{l}55.0 \\
60.0 \\
65.0\end{array}$ & $\begin{array}{r}.03393 \\
.03131 \\
.02867\end{array}$ & $\begin{array}{r}.13575 \\
.12525 \\
.11469\end{array}$ & $\begin{array}{l}.01034 \\
.00954 \\
.00874\end{array}$ & $\begin{array}{r}.04137 \\
.03817 \\
.03496\end{array}$ & $\begin{array}{l}14.739 \\
13.599 \\
12.453\end{array}$ & $\begin{array}{l}44: 926 \\
41.451 \\
27.958\end{array}$ & $\begin{array}{l}49.283 \\
45.472 \\
41.640\end{array}$ \\
\hline $\begin{array}{l}5 \\
5 \\
5\end{array}$ & $\begin{array}{l}8000 \\
8000 \\
8000\end{array}$ & $\begin{array}{l}70.0 \\
75.0 \\
80.0\end{array}$ & $\begin{array}{l}.02661 \\
.02481 \\
.02335\end{array}$ & $\begin{array}{r}.10644 \\
.09927 \\
.09343\end{array}$ & $\begin{array}{l}.00811 \\
.00756 \\
.00711\end{array}$ & $\begin{array}{l}.03244 \\
.03025 \\
.02847\end{array}$ & $\begin{array}{l}11.556 \\
10.778 \\
10.144\end{array}$ & $\begin{array}{l}35.225 \\
32.852 \\
30.922\end{array}$ & $\begin{array}{l}38.642 \\
36.039 \\
33.921\end{array}$ \\
\hline $\begin{array}{l}5 \\
5 \\
5\end{array}$ & $\begin{array}{l}8000 \\
8000 \\
8000\end{array}$ & $\begin{array}{l}85.0 \\
90.0 \\
95.0\end{array}$ & $\begin{array}{l}.02208 \\
.02085 \\
.02976\end{array}$ & $\begin{array}{r}.08834 \\
.08343 \\
.07905\end{array}$ & $\begin{array}{l}.00673 \\
.00635 \\
.00602\end{array}$ & $\begin{array}{r}.02692 \\
.02543 \\
.02409\end{array}$ & $\begin{array}{l}9.592 \\
9.059 \\
8.583\end{array}$ & $\begin{array}{l}29.237 \\
27.613 \\
26.162\end{array}$ & $\begin{array}{l}32.073 \\
30.291 \\
28.699\end{array}$ \\
\hline 5 & 8000 & $100 \cdot 0$ & .01870 & .0748 .1 & .00570 & .02280 & 8.122 & $24 \cdot 758$ & $27 \cdot 159$ \\
\hline
\end{tabular}


TABULATION OF DATA FOR

ABSORPTION OF SOUND IN AIR VERSUS PERCENT RELATIVE HUMIOITY AT 5 DEGREES CENTIGRADE FOR A FREQUENCY OF 10000 HERTZ

\begin{tabular}{|c|c|c|c|c|c|c|c|c|c|}
\hline (1) & $(2)$ & $(3)$ & $(4)$ & $(5)$ & $(6)$ & $(7)$ & $(8)$ & $(9)$ & $(10)$ \\
\hline $\begin{array}{l}\text { EGR } \\
\text { ENT }\end{array}$ & HERTZ & $\begin{array}{l}\text { REL } \\
\text { HUM } \\
\text { PER } \\
\text { CENT }\end{array}$ & $\begin{array}{l}\text { ATTEN } \\
\text { COEF } \\
\text { PER } \\
\text { METER }\end{array}$ & $\begin{array}{l}\text { PER } \\
\text { METER }\end{array}$ & $\begin{array}{l}\text { ATTEN } \\
\text { COEF } \\
\text { PER } \\
\text { FOOT }\end{array}$ & $\begin{array}{l}4 M \\
\text { PER } \\
\text { FOOT }\end{array}$ & $\begin{array}{l}\text { ATTEN } \\
\text { DB PER } \\
100 \\
\text { METER }\end{array}$ & $\begin{array}{l}\text { ATTEN } \\
\text { DB PER } \\
1000 \\
\text { FEET }\end{array}$ & $\begin{array}{l}\text { DECAY } \\
\text { RATE } \\
\text { DB PER } \\
\text { SECOND }\end{array}$ \\
\hline $\begin{array}{ll}5 & 1 \\
5 & 1 \\
5 & 1\end{array}$ & $\begin{array}{l}10000 \\
10000 \\
10000\end{array}$ & $\begin{array}{r}5.0 . \\
10.0 \\
15.0\end{array}$ & $\begin{array}{r}.01056 \\
.01772 \\
.02488\end{array}$ & $\begin{array}{r}.04225 \\
.07090 \\
.09955\end{array}$ & $\begin{array}{r}.00322 \\
.00540 \\
.00758\end{array}$ & $\begin{array}{r}.01287 \\
.02161 \\
.03034\end{array}$ & $\begin{array}{r}4.587 \\
7.698 \\
10.809\end{array}$ & $\begin{array}{l}13.983 \\
23.465 \\
32.947\end{array}$ & $\begin{array}{l}15 \cdot 339 \\
25.741 \\
36.143\end{array}$ \\
\hline $\begin{array}{l}5 \\
5 \\
5\end{array}$ & $\begin{array}{l}10000 \\
10000 \\
10000\end{array}$ & $\begin{array}{l}20.0 \\
25.0 \\
30.0\end{array}$ & $\begin{array}{l}.03 \\
.03 \\
.04\end{array}$ & $\begin{array}{l}.12 \\
.12 \\
.18\end{array}$ & $\begin{array}{l}.00979 \\
.01217 \\
.01412\end{array}$ & $\begin{array}{l}.03916 \\
.04869 \\
.05649\end{array}$ & $\begin{array}{l}13.949 \\
17.347 \\
20.125\end{array}$ & $\begin{array}{l}42.519 \\
52.874 \\
61.342\end{array}$ & $\begin{array}{l}46.643 \\
58.003 \\
67.292\end{array}$ \\
\hline $\begin{array}{l}5 \\
5 \\
5\end{array}$ & $\begin{array}{l}10000 \\
10000 \\
10000\end{array}$ & $\begin{array}{l}35.0 \\
40.0 \\
45.0\end{array}$ & $\begin{array}{r}.04979 \\
.05088 \\
.05074\end{array}$ & $\begin{array}{r}.19919 \\
.20355 \\
.20297\end{array}$ & $\begin{array}{l}.01517 \\
.01551 \\
.01546\end{array}$ & $\begin{array}{l}.06071 \\
.06204 \\
.06186\end{array}$ & $\begin{array}{l}21.627 \\
22.101 \\
22.038\end{array}$ & $\begin{array}{l}65.921 \\
67.365 \\
67.173\end{array}$ & $\begin{array}{l}72.316 \\
73.899 \\
73.688\end{array}$ \\
\hline $\begin{array}{l}5 \\
5 \\
5\end{array}$ & $\begin{array}{l}10000 \\
10000 \\
10000\end{array}$ & $\begin{array}{l}46.0 \\
47.0 \\
48.0\end{array}$ & $\begin{array}{r}.05058 \\
.05040 \\
.05019\end{array}$ & $\begin{array}{l}.20235 \\
.20161 \\
\cdot 20076\end{array}$ & $\begin{array}{r}.01541 \\
.01536 \\
.01529\end{array}$ & $\begin{array}{l}.06167 \\
.06145 \\
.06119\end{array}$ & $\begin{array}{l}21.970 \\
21.890 \\
21.797\end{array}$ & $\begin{array}{l}66.967 \\
66.722 \\
66.440\end{array}$ & $\begin{array}{l}73.463 \\
73.194 \\
72.884\end{array}$ \\
\hline $\begin{array}{l}5 \\
5 \\
5\end{array}$ & $\begin{array}{l}10000 \\
10000 \\
10000\end{array}$ & $\begin{array}{l}49.0 \\
50.0 \\
51.0\end{array}$ & $\begin{array}{r}.04995 \\
.04965 \\
.04928\end{array}$ & $\begin{array}{r}.19983 \\
.19860 \\
.19712\end{array}$ & $\begin{array}{l}.01522 \\
.01513 \\
.01502\end{array}$ & $\begin{array}{l}.06091 \\
.06053 \\
.06008\end{array}$ & $\begin{array}{l}21.697 \\
21.563 \\
21.402\end{array}$ & $\begin{array}{l}66.134 \\
65.725 \\
65.236\end{array}$ & $\begin{array}{l}72.549 \\
72.101 \\
71.564\end{array}$ \\
\hline $\begin{array}{l}5 \\
5 \\
5\end{array}$ & $\begin{array}{l}10000 \\
10000 \\
10000\end{array}$ & $\begin{array}{l}52.0 \\
53.0 \\
54.0\end{array}$ & $\begin{array}{r}.04889 \\
.04850 \\
.04808\end{array}$ & $\begin{array}{r}.19559 \\
\cdot 19402 \\
.19233\end{array}$ & $\begin{array}{l}.01490 \\
.01478 \\
.01465\end{array}$ & $\begin{array}{l}.05961 \\
.05913 \\
.05862\end{array}$ & $\begin{array}{l}21.237 \\
21.066 \\
20.882\end{array}$ & & $\begin{array}{l}71.010 \\
70.440 \\
69.825\end{array}$ \\
\hline $\begin{array}{l}5 \\
5 \\
5\end{array}$ & $\begin{array}{l}10000 \\
10000 \\
10000\end{array}$ & $\begin{array}{l}55.0 \\
60.0 \\
65.0\end{array}$ & $\begin{array}{r}.04762 \\
.04489 \\
.04201\end{array}$ & $\begin{array}{r}.19048 \\
\cdot 17959 \\
.16804\end{array}$ & $\begin{array}{l}.01451 \\
.01368 \\
.01280\end{array}$ & $\begin{array}{l}.05805 \\
.05474 \\
.05122\end{array}$ & $\begin{array}{l}20.681 \\
18.499 \\
18.245\end{array}$ & $\begin{array}{l}63.039 \\
59.436 \\
55.613\end{array}$ & $\begin{array}{l}154 \\
202 \\
207\end{array}$ \\
\hline $\begin{array}{l}5 \\
5 \\
5\end{array}$ & $\begin{array}{l}10000 \\
10000 \\
10000\end{array}$ & $\begin{array}{l}70.0 \\
75.0 \\
80.0\end{array}$ & $\begin{array}{r}.03917 \\
.03633 \\
.03408\end{array}$ & $\begin{array}{l}.15 \\
.14 \\
.13\end{array}$ & $\begin{array}{r}.01293 \\
.01107 \\
.01038\end{array}$ & $\begin{array}{r}.04775 \\
.04429 \\
.04155\end{array}$ & $\begin{array}{l}011 \\
778 \\
801\end{array}$ & $\begin{array}{l}852 \\
094 \\
114\end{array}$ & $\begin{array}{l}881 \\
759 \\
490\end{array}$ \\
\hline $\begin{array}{l}5 \\
5 \\
5\end{array}$ & $\begin{array}{l}10000 \\
10000 \\
10000\end{array}$ & $\begin{array}{l}85.0 \\
90.0 \\
95.0\end{array}$ & $\begin{array}{r}.03209 \\
.03040 \\
.02898\end{array}$ & $\begin{array}{r}12837 \\
.12 .162 \\
-11595\end{array}$ & $\begin{array}{l}.00978 \\
.00926 \\
.00883\end{array}$ & $\begin{array}{l}.03912 \\
.03707 \\
.03534\end{array}$ & $\begin{array}{l}13.938 \\
13.205 \\
12.590\end{array}$ & $\begin{array}{l}42.484 \\
40.250 \\
38.375\end{array}$ & $\begin{array}{l}46.605 \\
44.154 \\
42.098\end{array}$ \\
\hline 51 & 10000 & 100.0 & .02761 & .11046 & .00841 & .03366 & 11.993 & 36.557 & 40.103 \\
\hline
\end{tabular}


TABULATION OF DATA FOR

ABSORPTION OF SOUND IN AIR VERSUS PERCENT RELATIVE HUMIDITY AT 5 DEGREES CENTIGRADE FOR A FREQUENCY OF 12500 HERTZ

\begin{tabular}{|c|c|c|c|c|c|c|c|c|c|}
\hline (1) & (2) & (3) & $(4)$ & (5) & (6) & 171 & $(8)$ & 191 & $(10)$ \\
\hline $\begin{array}{l}\text { EMP } \\
\text { EGR } \\
\text { ENT }\end{array}$ & HERTZ & $\begin{array}{l}\text { REL. } \\
\text { HUM } \\
\text { PER } \\
\text { CENT }\end{array}$ & $\begin{array}{l}\text { ATTEN } \\
\text { COEF } \\
\text { PER } \\
\text { METER }\end{array}$ & $\begin{array}{l}4 M \\
\text { PER } \\
\text { METER }\end{array}$ & $\begin{array}{l}\text { ATTEN } \\
\text { COEF } \\
\text { PER } \\
\text { FOOT }\end{array}$ & $\begin{array}{l}4 M \\
\text { PER } \\
\text { FOOT }\end{array}$ & $\begin{array}{l}\text { ATTEN } \\
\text { DB PER } \\
1 \text { OO } \\
\text { METER }\end{array}$ & $\begin{array}{l}\text { ATTEN } \\
\text { DB PER } \\
1000 \\
\text { FEET }\end{array}$ & $\begin{array}{l}\text { DECAY. } \\
\text { RATE } \\
\text { DB PER } \\
\text { SECOND }\end{array}$ \\
\hline $\begin{array}{l}5 \\
5 \\
5\end{array}$ & $\begin{array}{l}12500 \\
12500 \\
12500\end{array}$ & $\begin{array}{r}5.0 \\
10.0 \\
15.0\end{array}$ & $\begin{array}{r}.01312 \\
.02094 \\
.02875\end{array}$ & $\begin{array}{r}.05250 \\
.08376 \\
.11502\end{array}$ & $\begin{array}{l}.00400 \\
.00638 \\
.00876\end{array}$ & $\begin{array}{r}.01600 \\
.02553 \\
.03506\end{array}$ & $\begin{array}{r}5.700 \\
9.094 \\
12.489\end{array}$ & $\begin{array}{l}17.375 \\
27.721 \\
38.067\end{array}$ & $\begin{array}{l}19 . \\
30 . \\
41 .\end{array}$ \\
\hline $\begin{array}{l}5 \\
5 \\
5\end{array}$ & $\begin{array}{l}12500 \\
12500 \\
12500\end{array}$ & $\begin{array}{l}20.0 \\
25.0 \\
30.0\end{array}$ & $\begin{array}{r}.03657 \\
.04478 \\
.05318\end{array}$ & $\begin{array}{r}14629 \\
.17915 \\
.21272\end{array}$ & $\begin{array}{r}.01114 \\
.01365 \\
.01620\end{array}$ & $\begin{array}{r}.04458 \\
.05460 \\
.06483\end{array}$ & $\begin{array}{l}15.883 \\
19.452 \\
23.096\end{array}$ & $\begin{array}{l}48.413 \\
59.291 \\
70.399\end{array}$ & $\begin{array}{l}53 \cdot 10 \\
65.04 \\
77.22\end{array}$ \\
\hline $\begin{array}{l}5 \\
5 \\
5\end{array}$ & $\begin{array}{l}12500 \\
12500 \\
12500\end{array}$ & $\begin{array}{l}35.0 \\
40.0 \\
45.0\end{array}$ & $\begin{array}{l}.05963 \\
.06324 \\
.06458\end{array}$ & $\begin{array}{r}.23854 \\
.25299 \\
.25835\end{array}$ & $\begin{array}{r}.01817 \\
.01927 \\
.01968\end{array}$ & $\begin{array}{l}.07270 \\
.07711 \\
.07874\end{array}$ & $\begin{array}{l}25.900 \\
27.469 \\
28.050\end{array}$ & $\begin{array}{l}78.944 \\
83.727 \\
85.499\end{array}$ & 9 \\
\hline $\begin{array}{l}5 \\
5\end{array}$ & $\begin{array}{l}12500 \\
12500\end{array}$ & $\begin{array}{l}46.0 \\
47.0\end{array}$ & $\begin{array}{l}.06468 \\
.06478\end{array}$ & $\begin{array}{r}.25875 \\
.25915\end{array}$ & $\begin{array}{l}.01971 \\
.01974\end{array}$ & $\begin{array}{l}.07886 \\
.07899\end{array}$ & $\begin{array}{l}28.094 \\
28.138\end{array}$ & $\begin{array}{l}85.632 \\
85.766\end{array}$ & \\
\hline 5 & 12500 & 48.0 & .06476 & .25907 & .01974 & .07896 & $28 \cdot 129$ & 85.739 & 94.05 \\
\hline $\begin{array}{l}5 \\
5\end{array}$ & $\begin{array}{l}12500 \\
12500\end{array}$ & $\begin{array}{l}49.0 \\
50.0\end{array}$ & $\begin{array}{l}.06471 \\
.06464\end{array}$ & $\begin{array}{r}.25887 \\
.25858\end{array}$ & $\begin{array}{l}.01972 \\
.01970\end{array}$ & $\begin{array}{l}.07 \\
.07\end{array}$ & $\begin{array}{l}28.107 \\
28.076\end{array}$ & $\begin{array}{l}85.672 \\
85.576\end{array}$ & \\
\hline 5 & 12500 & 51.0 & .06454 & .25818 & .01967 & .07869 & $28 \cdot 032$ & $85 \cdot 443$ & 93.73 \\
\hline $\begin{array}{l}5 \\
5 \\
5\end{array}$ & $\begin{array}{l}12500 \\
12500 \\
12500\end{array}$ & $\begin{array}{l}52.0 \\
53.0 \\
54.0\end{array}$ & $\begin{array}{l}.06 \\
.06 \\
.06\end{array}$ & $\begin{array}{r}.25774 \\
.25694 \\
.25613\end{array}$ & $\begin{array}{l}.01 \\
.01 \\
.01\end{array}$ & $\begin{array}{l}.0 \\
.0 \\
.0 \\
.0\end{array}$ & $\begin{array}{l}27 \\
27 \\
27\end{array}$ & $\begin{array}{l}85.299 \\
85.032 \\
84.765\end{array}$ & $\begin{array}{l}9 \\
9\end{array}$ \\
\hline $\begin{array}{l}5 \\
5 \\
5\end{array}$ & $\begin{array}{l}12500 \\
12500 \\
12500\end{array}$ & $\begin{array}{l}55 \\
60 \\
65\end{array}$ & $\begin{array}{l}.06 \\
.06 \\
.05\end{array}$ & $\begin{array}{r}.25518 \\
.24800 \\
.23794\end{array}$ & $\begin{array}{r}.01944 \\
.01889 \\
.01813\end{array}$ & $\begin{array}{l}.07 \\
.07 \\
.07\end{array}$ & $\begin{array}{l}27 \\
26 \\
25\end{array}$ & $\begin{array}{l}84.450 \\
82.075 \\
78.745\end{array}$ & $\begin{array}{l}7 \\
9 \\
8\end{array}$ \\
\hline $\begin{array}{l}5 \\
5 \\
5\end{array}$ & $\begin{array}{l}12500 \\
12500 \\
12500\end{array}$ & $\begin{array}{l}70 \cdot 0 \\
75 \cdot 0 \\
80 \cdot 0 .\end{array}$ & $\begin{array}{l}.05638 \\
.05323 \\
.05014\end{array}$ & $\begin{array}{r}.22555 \\
.21295 \\
.20057\end{array}$ & $\begin{array}{l}.01718 \\
.01622 \\
.01528\end{array}$ & $\begin{array}{l}.06874 \\
.06490 \\
.06113\end{array}$ & $\begin{array}{l}24 \cdot 489 \\
23.121 \\
21.776\end{array}$ & $\begin{array}{l}74 \cdot 646 \\
70 \cdot 474 \\
66 \cdot 377\end{array}$ & $\begin{array}{l}81 \cdot 88 \\
77 \cdot 31 \\
72 \cdot 81\end{array}$ \\
\hline $\begin{array}{l}5 \\
5 \\
5\end{array}$ & $\begin{array}{l}12500 \\
12500 \\
12500\end{array}$ & $\begin{array}{l}85.0 \\
90.0 \\
95.0\end{array}$ & $\begin{array}{r}.04693 \\
.04443 \\
.04213\end{array}$ & $\begin{array}{r}1 \\
.18775 \\
.17772 \\
.16854\end{array}$ & $\begin{array}{l}.01430 \\
.01354 \\
.01284\end{array}$ & $\begin{array}{l}.05722 \\
.05416 \\
.05137\end{array}$ & $\begin{array}{l}20.385 \\
19.296 \\
18.299\end{array}$ & $\begin{array}{r}62.137 \\
-58.815 \\
55.778\end{array}$ & $\begin{array}{l}68 \cdot 16 \\
64.52 \\
61.18\end{array}$ \\
\hline 5 & $500:$ & $00 \cdot 0$ & 04016 & 16065 & .01224 & .04896 & 17.443 & 53.167 & $58 \cdot 32$ \\
\hline
\end{tabular}


TABULATION OF DATA FOR

ABSORPTION OF SOUND IN AIR VERSUS PERCENT RELATIVE HUMIDITY AT 10 DEGREES CENTIGRADE FOR A FREQUENCY OF 125 HERTZ

\begin{tabular}{|c|c|c|c|c|c|c|c|c|c|}
\hline 111 & (2) & $(3)$ & $(4)$ & (5) & $(6)$ & $(7)$ & $(8)$ & $(9)$ & $(10)$ \\
\hline $\begin{array}{l}\text { TEMP } \\
\text { DEGR } \\
\text { CENT }\end{array}$ & HER TZ & $\begin{array}{l}\text { REL } \\
\text { HUM } \\
\text { PER } \\
\text { CENT }\end{array}$ & $\begin{array}{l}\text { ATTEN } \\
\text { COEF } \\
\text { PER } \\
\text { METER }\end{array}$ & $\begin{array}{l}\text { 4M } \\
\text { PER } \\
\text { METER }\end{array}$ & $\begin{array}{l}\text { ATTEN } \\
\text { COEF } \\
\text { PER } \\
\text { FOOT }\end{array}$ & $\begin{array}{l}4 M \\
\text { PER } \\
\text { FOOT }\end{array}$ & $\begin{array}{l}\text { ATTEN } \\
\text { DB PER } \\
100 \\
\text { METER }\end{array}$ & $\begin{array}{l}\text { ATTEN } \\
\text { DB PER } \\
1000 \\
\text { FEET }\end{array}$ & $\begin{array}{l}\text { DECAY } \\
\text { RATE } \\
\text { DB PER } \\
\text { SECOND }\end{array}$ \\
\hline $\begin{array}{l}10 \\
10 \\
10\end{array}$ & $\begin{array}{l}125 \\
125 \\
125\end{array}$ & $\begin{array}{l}5.0 \\
10.0 \\
15.0\end{array}$ & $\begin{array}{l}.00032 \\
.00015 \\
.00012\end{array}$ & $\begin{array}{l}.00129 \\
.00062 \\
.00050\end{array}$ & $\begin{array}{l}.00009 \\
.00004 \\
.00003\end{array}$ & $\begin{array}{l}.00039 \\
.00019 \\
.00015\end{array}$ & $\begin{array}{l}.140 \\
.067 \\
.055\end{array}$ & $\begin{array}{r}.427 \\
.206 \\
.167\end{array}$ & $\begin{array}{r}.472 \\
.228 \\
.285\end{array}$ \\
\hline $\begin{array}{l}10 \\
10 \\
10\end{array}$ & $\begin{array}{l}125 \\
125 \\
125\end{array}$ & $\begin{array}{l}20.0 \\
25.0 \\
30.0\end{array}$ & $\begin{array}{l}.00011 \\
.00010 \\
.00009\end{array}$ & $\begin{array}{l}.00045 \\
.00041 \\
.00038\end{array}$ & $\begin{array}{l}.00003 \\
.00003 \\
.00002\end{array}$ & $\begin{array}{l}.00013 \\
.00012 \\
.00011\end{array}$ & $\begin{array}{l}.049 \\
.045 \\
.042\end{array}$ & $\begin{array}{l}.151 \\
.138 \\
.128\end{array}$ & $\begin{array}{r}.167 \\
.153 \\
.142\end{array}$ \\
\hline $\begin{array}{l}10 \\
10 \\
10\end{array}$ & $\begin{array}{l}125 \\
125 \\
125\end{array}$ & $\begin{array}{l}35.0 \\
40.0 \\
45.0\end{array}$ & $\begin{array}{l}.00009 \\
.00008 \\
.00008\end{array}$ & $\begin{array}{l}.00036 \\
.00035 \\
.00033\end{array}$ & $\begin{array}{r}.00002 \\
.00002 \\
.00002\end{array}$ & $\begin{array}{l}.00011 \\
.00010 \\
.00010\end{array}$ & $\begin{array}{l}.040 \\
.038 \\
.036\end{array}$ & $\begin{array}{l}.122 \\
.116 \\
.112\end{array}$ & $\begin{array}{l}.135 \\
.128 \\
.123\end{array}$ \\
\hline $\begin{array}{l}10 \\
10 \\
10\end{array}$ & $\begin{array}{l}125 \\
125 \\
125\end{array}$ & $\begin{array}{l}46.0 \\
47.0 \\
48.0\end{array}$ & $\begin{array}{l}.00008 \\
.00008 \\
.00008\end{array}$ & $\begin{array}{l}.00033 \\
.00033 \\
.00032\end{array}$ & $\begin{array}{l}.00002 \\
.00002 \\
.00002\end{array}$ & $\begin{array}{l}.00010 \\
.00010 \\
.00010\end{array}$ & $\begin{array}{l}.036 \\
.035 \\
.035\end{array}$ & $\begin{array}{r}.110 \\
.109 \\
.108\end{array}$ & $\begin{array}{r}.122 \\
.121 \\
-120\end{array}$ \\
\hline $\begin{array}{l}10 \\
10 \\
10\end{array}$ & $\begin{array}{l}125 \\
125 \\
125\end{array}$ & $\begin{array}{l}49.0 \\
50.0 \\
51.0\end{array}$ & $\begin{array}{l}.00008 \\
.00008 \\
.00008\end{array}$ & $\begin{array}{l}.00032 \\
.00032 \\
.00032\end{array}$ & $\begin{array}{l}.00002 \\
.00002 \\
.00002\end{array}$ & $\begin{array}{l}.00009 \\
.00009 \\
.00009\end{array}$ & $\begin{array}{l}.035 \\
.035 \\
.034\end{array}$ & $\begin{array}{l}.107 \\
.106 \\
.106\end{array}$ & $\begin{array}{l}.119 \\
.118 \\
.117\end{array}$ \\
\hline 10 & 125 & 52.0 & .00 & .00031 & .0 & .00009 & .034 & & .116 \\
\hline $\begin{array}{l}10 \\
10\end{array}$ & $\begin{array}{l}125 \\
125\end{array}$ & $\begin{array}{l}53.0 \\
54.0\end{array}$ & $\begin{array}{l}.00007 \\
.00007\end{array}$ & $\begin{array}{l}.00031 \\
.00031\end{array}$ & $\begin{array}{l}.00002 \\
.00002\end{array}$ & $\begin{array}{l}.00009 \\
.00009\end{array}$ & $\begin{array}{l}.034 \\
.034\end{array}$ & & $\begin{array}{l}.116 \\
.115\end{array}$ \\
\hline $\begin{array}{l}10 \\
10 \\
10\end{array}$ & $\begin{array}{l}125 \\
125 \\
125\end{array}$ & $\begin{array}{l}55.0 \\
60.0 \\
65.0\end{array}$ & $\begin{array}{l}.00007 \\
.00007 \\
.00007\end{array}$ & $\begin{array}{l}.00031 \\
.00030 \\
.00029\end{array}$ & $\begin{array}{l}.00002 \\
.00002 \\
.00002\end{array}$ & $\begin{array}{l}.00009 \\
.00009 \\
.00008\end{array}$ & $\begin{array}{l}.033 \\
.032 \\
.031\end{array}$ & $\begin{array}{l}.103 \\
.100 \\
.097\end{array}$ & $\begin{array}{l}.1114 \\
11110 \\
.107\end{array}$ \\
\hline $\begin{array}{l}10 \\
10 \\
10\end{array}$ & $\begin{array}{l}125 \\
125 \\
125\end{array}$ & $\begin{array}{l}70.0 \\
75.0 \\
80.0\end{array}$ & $\begin{array}{l}.00007 \\
.00006 \\
.00006\end{array}$ & $\begin{array}{r}.00028 \\
.00027 \\
.00027\end{array}$ & $\begin{array}{l}.00002 \\
.00002 \\
.00002\end{array}$ & $\begin{array}{l}.00008 \\
.00008 \\
.00008\end{array}$ & $\begin{array}{l}.031 \\
.030 \\
.029\end{array}$ & $\begin{array}{l}.094 \\
.092 \\
.090\end{array}$ & $\begin{array}{l}.104 \\
.102 \\
.099\end{array}$ \\
\hline $\begin{array}{l}10 \\
10\end{array}$ & $\begin{array}{l}125 \\
125\end{array}$ & $\begin{array}{l}85.0 \\
90.0\end{array}$ & $\begin{array}{l}.00006 \\
.00006\end{array}$ & $\begin{array}{l}.00026 \\
.00026\end{array}$ & .00002 & $\begin{array}{l}.00008 \\
.00007\end{array}$ & $\begin{array}{r}.028 \\
.028\end{array}$ & $\begin{array}{l}.087 \\
.086\end{array}$ & $\begin{array}{r}.097 \\
.095\end{array}$ \\
\hline 10 & 125 & 95.0 & .00006 & .00025 & .00001 & .00007 & .027 & .084 & .093 \\
\hline 1 & -125 & $100 \cdot 0$ & .00006 & .00025 & .00001 & .00007 & .027 & .083 & .092 \\
\hline
\end{tabular}


TABULATION OF DATA FOR

ABSORPTION OF SOUND IN AIR. VERSUS PERCENT RELATIVE HUMIDITY AT 10 DEGREES CENTIGRADE FOR A FREQUENCY OF 250 HERTZ

\begin{tabular}{|c|c|c|c|c|c|c|c|c|c|}
\hline (1) & $(2)$ & $(3)$ & $\therefore(4)$ & (5) & (6) & (7.) & $(8)$ & 191 & $(10)$ \\
\hline TEMP & FREQ & $\begin{array}{l}\text { REL } \\
\text { HUM }\end{array}$ & $\begin{array}{l}\text { ATTEN } \\
\text { COEF }\end{array}$ & $4 M$ & $\begin{array}{l}\text { AT TEN } \\
\text { COEF }\end{array}$ & $4 M$ & $\begin{array}{l}\text { ATTEN } \\
\text { DB PER }\end{array}$ & $\begin{array}{l}\text { ATTEN } \\
\text { DB PER }\end{array}$ & $\begin{array}{l}\text { DECAY } \\
\text { RATE }\end{array}$ \\
\hline $\begin{array}{l}\text { DEGR } \\
\text { CENT }\end{array}$ & HERTZ & $\begin{array}{l}\text { PER } \\
\text { CENT }\end{array}$ & $\begin{array}{l}\text { PER } \\
\text { METER }\end{array}$ & $\begin{array}{l}\text { PER } \\
\text { METER }\end{array}$ & $\begin{array}{l}\text { PER } \\
\text { FOOT }\end{array}$ & $\begin{array}{l}\text { PER } \\
\text { FOOT }\end{array}$ & $\begin{array}{l}100 \\
\text { METER }\end{array}$ & $\begin{array}{l}1000 \\
\text { FEET }\end{array}$ & $\begin{array}{l}\text { DB PER } \\
\text { SECOND }\end{array}$ \\
\hline $\begin{array}{l}10 \\
10 \\
10\end{array}$ & $\begin{array}{l}250 \\
250 \\
250\end{array}$ & $\begin{array}{r}5.0 \\
10.0 \\
15.0\end{array}$ & $\begin{array}{r}.00085 \\
.00040 \\
.00028\end{array}$ & $\begin{array}{l}.00341 \\
.00160 \\
.00114\end{array}$ & $\begin{array}{l}.00026 \\
.00012 \\
.00008\end{array}$ & $\begin{array}{l}.00104 \\
.00048 \\
.00034\end{array}$ & $\begin{array}{r}.370 \\
.173 \\
.123\end{array}$ & $\begin{array}{r}1.129 \\
.530 \\
.377\end{array}$ & $\begin{array}{r}1.250 \\
.586 \\
.417\end{array}$ \\
\hline $\begin{array}{l}10 \\
10 \\
10\end{array}$ & $\begin{array}{l}250 \\
250 \\
250\end{array}$ & $\begin{array}{l}20.0 \\
25.0 \\
30.0\end{array}$ & $\begin{array}{l}.00025 \\
.00023 \\
.00021\end{array}$ & $\begin{array}{l}.00100 \\
.00092 \\
.00086\end{array}$ & $\begin{array}{l}.00007 \\
.00007 \\
.00006\end{array}$ & $\begin{array}{l}.00030 \\
.00028 \\
.00026\end{array}$ & $\begin{array}{l}.109 \\
.100 \\
.093\end{array}$ & $\begin{array}{l}.332 \\
.306 \\
.286\end{array}$ & $\begin{array}{r}.367 \\
.339 \\
.317\end{array}$ \\
\hline $\begin{array}{l}10 \\
10 \\
10\end{array}$ & $\begin{array}{l}250 \\
250 \\
250\end{array}$ & $\begin{array}{l}35.0 \\
40.0 \\
45.0\end{array}$ & $\begin{array}{l}.00020 \\
.00019 \\
.00018\end{array}$ & $\begin{array}{l}.00081 \\
.00077 \\
.00074\end{array}$ & $\begin{array}{l}.00006 \\
.00005 \\
.00005\end{array}$ & $\begin{array}{l}.00024 \\
.00023 \\
.00022\end{array}$ & $\begin{array}{l}.088 \\
.083 \\
.080\end{array}$ & $\begin{array}{l}.268 \\
.255 \\
.245\end{array}$ & $\begin{array}{l}.297 \\
.282 \\
.271\end{array}$ \\
\hline $\begin{array}{l}10 \\
10 \\
10\end{array}$ & $\begin{array}{l}250 \\
250 \\
250\end{array}$ & $\begin{array}{l}46.0 \\
47.0 \\
48.0\end{array}$ & $\begin{array}{l}.00018 \\
.00018 \\
.00018\end{array}$ & $\begin{array}{l}.00073 \\
.00072 \\
.00072\end{array}$ & $\begin{array}{l}.00005 \\
.00005 \\
.00005\end{array}$ & $\begin{array}{l}.00022 \\
.00022 \\
.00022\end{array}$ & $\begin{array}{r}.079 \\
.079 \\
.078\end{array}$ & $\begin{array}{l}.243 \\
.241 \\
.239\end{array}$ & $\begin{array}{r}.268 \\
.266 \\
.264\end{array}$ \\
\hline $\begin{array}{l}10 \\
10 \\
10\end{array}$ & $\begin{array}{l}250 \\
250 \\
250\end{array}$ & $\begin{array}{l}49.0 \\
50.0 \\
51.0\end{array}$ & $\begin{array}{l}.00017 \\
.00017 \\
.00017\end{array}$ & $\begin{array}{l}.00071 \\
.00071 \\
.00070\end{array}$ & $\begin{array}{l}.00005 \\
.00005 \\
.00005\end{array}$ & $\begin{array}{l}.00021 \\
.00021 \\
.00021\end{array}$ & $\begin{array}{l}.078 \\
.077 \\
.076\end{array}$ & $\begin{array}{l}.237 \\
.236 \\
.234\end{array}$ & $\begin{array}{l}.263 \\
.261 \\
.259\end{array}$ \\
\hline $\begin{array}{l}10 \\
10 \\
10\end{array}$ & $\begin{array}{l}250 \\
250 \\
250\end{array}$ & $\begin{array}{l}52.0 \\
53.0 \\
54.0\end{array}$ & $\begin{array}{l}.00017 \\
.00017 \\
.00017\end{array}$ & $\begin{array}{l}.00070 \\
.00069 \\
.00069\end{array}$ & $\begin{array}{l}.00005 \\
.00005 \\
.00005\end{array}$ & $\begin{array}{l}.00021 \\
.00021 \\
.00021\end{array}$ & $\begin{array}{l}.076 \\
.075 \\
.075\end{array}$ & $\begin{array}{r}.232 \\
.231 \\
.229\end{array}$ & $\begin{array}{r}.257 \\
.255 \\
.254\end{array}$ \\
\hline $\begin{array}{l}10 \\
10 \\
10\end{array}$ & $\begin{array}{l}250 \\
250 \\
250\end{array}$ & $\begin{array}{l}55.0 \\
60.0 \\
65.0\end{array}$ & $\begin{array}{l}.00017 \\
.00016 \\
.00016\end{array}$ & $\begin{array}{l}.00068 \\
.00066 \\
.00064\end{array}$ & $\begin{array}{l}.00005 \\
.00005 \\
.00004\end{array}$ & $\begin{array}{l}.00020 \\
.00020 \\
.00019\end{array}$ & $\begin{array}{l}.074 \\
.072 \\
.070\end{array}$ & $\begin{array}{l}.227 \\
.220 \\
.213\end{array}$ & $\begin{array}{r}.252 \\
.243 \\
.236\end{array}$ \\
\hline $\begin{array}{l}10 \\
10\end{array}$ & $\begin{array}{l}250 \\
250\end{array}$ & $\begin{array}{l}70.0 \\
75.0\end{array}$ & $\begin{array}{l}.00015 \\
.00015\end{array}$ & $\begin{array}{l}.00062 \\
.00061\end{array}$ & $\begin{array}{l}.00004 \\
.00004\end{array}$ & $\begin{array}{l}.00019 \\
.00018\end{array}$ & $\begin{array}{l}.068 \\
.066\end{array}$ & $\begin{array}{l}.208 \\
.203\end{array}$ & $\begin{array}{l}.230 \\
.224\end{array}$ \\
\hline 10 & 250 & 80.0 & .00014 & .00059 & .00004 & .00018 & .065 & .198 & .219 \\
\hline $\begin{array}{l}10 \\
10\end{array}$ & $\begin{array}{l}250 \\
250\end{array}$ & $\begin{array}{l}85.0 \\
90.0\end{array}$ & $\begin{array}{l}.00014 \\
.00014\end{array}$ & $\begin{array}{l}.00058 \\
.00057\end{array}$ & $\begin{array}{l}.00004 \\
.00004\end{array}$ & .00017 & $\begin{array}{l}.063 \\
.062\end{array}$ & $\begin{array}{l}.194 \\
.190\end{array}$ & $\begin{array}{l}.215 \\
.210\end{array}$ \\
\hline 10 & 250 & 95.0 & .00014 & .00056 & .00004 & .00017 & .061 & .186 & .206 \\
\hline 0 & 250 & 100.0. & .00013 & .00055 & .00004 & .00016 & .059 & .182 & .202 \\
\hline
\end{tabular}


TABULATION OF DATA FOR

ABSORPTION OF SOUND IN AIR VERSUS PERCENT RELATIVE HUMIDITY AT 10 DEGREES CENTIGRADE FOR A FREQUENCY OF $500 \mathrm{HERTZ}$

\begin{tabular}{|c|c|c|c|c|c|c|c|c|c|}
\hline (1) & $(2)$ & (3). & $(4)$ & $(5)$ & 161 & $(7)$ & $(8)$. & 191 & 1101 \\
\hline $\begin{array}{l}\text { EMP } \\
\text { EGR } \\
\text { ENT }\end{array}$ & HERTZ & $\begin{array}{l}\text { REL } \\
\text { HUM } \\
\text { PER } \\
\text { CENT }\end{array}$ & $\begin{array}{l}\text { ATTEN } \\
\text { COEF } \\
\text { PER } \\
\text { METER }\end{array}$ & $\begin{array}{l}4 M \\
\text { PER } \\
\text { METER }\end{array}$ & $\begin{array}{l}\text { ATTEN } \\
\text { COEF } \\
\text { PER } \\
\text { FOOT }\end{array}$ & $\begin{array}{l}4 M \\
\text { PER } \\
\text { FOOT }\end{array}$ & $\begin{array}{l}\text { ATTEN } \\
\text { OB PER } \\
100 \\
\text { METER }\end{array}$ & $\begin{array}{l}\text { ATTEN } \\
\text { DB PER } \\
1000 \\
\text { FEET }\end{array}$ & $\begin{array}{l}\text { DECAY } \\
\text { RATE } \\
\text { DB PER } \\
\text { SECOND }\end{array}$ \\
\hline $\begin{array}{l}10 \\
10 \\
10\end{array}$ & $\begin{array}{l}500 \\
500 \\
500\end{array}$ & $\begin{array}{r}5.0 \\
10.0 \\
15.0\end{array}$ & $\begin{array}{l}.00251 \\
.00129 \\
.00082\end{array}$ & $\begin{array}{r}.01007 \\
.00518 \\
.00331\end{array}$ & $\begin{array}{l}.00076 \\
.00039 \\
.00025\end{array}$ & $\begin{array}{l}.00307 \\
.00157 \\
.00101\end{array}$ & $\begin{array}{r}1.094 \\
.562 \\
.359\end{array}$ & $\begin{array}{l}3.335 \\
1.715 \\
1.097\end{array}$ & $\begin{array}{l}3.691 \\
1.898 \\
1.214\end{array}$ \\
\hline $\begin{array}{l}10 \\
10 \\
10\end{array}$ & $\begin{array}{l}500 \\
500 \\
500\end{array}$ & $\begin{array}{l}20.0 \\
25.0 \\
30.0\end{array}$ & $\begin{array}{l}.00062 \\
.00054 \\
.00051\end{array}$ & $\begin{array}{l}.00251 \\
.00219 \\
.00204\end{array}$ & $\begin{array}{l}.00019 \\
.00016 \\
.00015\end{array}$ & $\begin{array}{l}.00076 \\
.00066 \\
.00062\end{array}$ & $\begin{array}{r}.273 \\
.238 \\
.222\end{array}$ & $\begin{array}{l}.832 \\
.726 \\
.678\end{array}$ & $\begin{array}{r}.921 \\
.803 \\
.750\end{array}$ \\
\hline $\begin{array}{l}10 \\
10 \\
10\end{array}$ & $\begin{array}{l}500 \\
500 \\
500\end{array}$ & $\begin{array}{l}3.5 .0 \\
40.0 \\
45.0\end{array}$ & $\begin{array}{r}.00048 \\
.00046 \\
.00044\end{array}$ & $\begin{array}{r}.00193 \\
.00184 \\
.00176\end{array}$ & $\begin{array}{l}.00014 \\
.00014 \\
.00013\end{array}$ & $\begin{array}{l}.00059 \\
.00056 \\
.00053\end{array}$ & $\begin{array}{l}.210 \\
.200 \\
.292\end{array}$ & $\begin{array}{l}.641 \\
.611 \\
.585\end{array}$ & $\begin{array}{l}.710 \\
.677 \\
.648\end{array}$ \\
\hline 10 & 500 & 46.0 & .00043 & .00175 & .00013 & .00053 & .190 & .580 & .642 \\
\hline $\begin{array}{l}10 \\
10\end{array}$ & $\begin{array}{l}500 \\
500\end{array}$ & $\begin{array}{l}47.0 \\
48.0\end{array}$ & $\begin{array}{l}.00043 \\
.00043\end{array}$ & $\begin{array}{r}.00173 \\
.00172\end{array}$ & $\begin{array}{l}.00013 \\
.00013\end{array}$ & $\begin{array}{l}.00053 \\
.00052\end{array}$ & $\begin{array}{r}188 \\
.187\end{array}$ & $\begin{array}{l}.575 \\
.570\end{array}$ & $\begin{array}{l}.637 \\
.631\end{array}$ \\
\hline $\begin{array}{l}10 \\
10 \\
10\end{array}$ & $\begin{array}{l}500 \\
500 \\
500\end{array}$ & $\begin{array}{l}49.0 \\
50.0 \\
51.0\end{array}$ & $\begin{array}{r}.00042 \\
.00042 \\
.00042\end{array}$ & $\begin{array}{l}.00170 \\
.00169 \\
.00168\end{array}$ & $\begin{array}{l}.00013 \\
.00012 \\
.00012\end{array}$ & $\begin{array}{l}.00052 \\
.00051 \\
.00051\end{array}$ & $\begin{array}{r}.185 \\
.184 \\
.182\end{array}$ & $\begin{array}{l}.565 \\
.561 \\
.557\end{array}$ & $\begin{array}{l}.626 \\
.621 \\
.616\end{array}$ \\
\hline $\begin{array}{l}10 \\
10 \\
10\end{array}$ & $\begin{array}{l}500 \\
500 \\
500\end{array}$ & $\begin{array}{l}52.0 \\
53.0 \\
54.0\end{array}$ & $\begin{array}{l}.00041 \\
.00041 \\
.00041\end{array}$ & $\begin{array}{r}.00167 \\
.00165 \\
.00164\end{array}$ & $\begin{array}{l}.00012 \\
.00012 \\
.00012\end{array}$ & $\begin{array}{l}.00050 \\
.00050 \\
.00050\end{array}$ & $\begin{array}{r}.181 \\
.179 \\
.178\end{array}$ & $\begin{array}{r}.552 \\
.548 \\
.544\end{array}$ & $\begin{array}{l}.611 \\
.607 \\
.602\end{array}$ \\
\hline $\begin{array}{l}10 \\
10 \\
10\end{array}$ & $\begin{array}{l}500 \\
500 \\
500\end{array}$ & $\begin{array}{l}55.0 \\
60.0 \\
65.0\end{array}$ & $\begin{array}{r}.00040 \\
.00039 \\
.00038\end{array}$ & $\begin{array}{l}.00163 \\
.00157 \\
.00153\end{array}$ & $\begin{array}{l}.00012 \\
.00012 \\
.00011\end{array}$ & $\begin{array}{l}.00049 \\
.00048 \\
.00046\end{array}$ & $\begin{array}{r}177 \\
.171 \\
.166\end{array}$ & $\begin{array}{r}.539 \\
.522 \\
.507\end{array}$ & $\begin{array}{r}.597 \\
.578 \\
.562\end{array}$ \\
\hline $\begin{array}{l}10 \\
10 \\
10\end{array}$ & $\begin{array}{l}500 \\
500 \\
500\end{array}$ & $\begin{array}{l}70.0 \\
75.0 \\
80.0\end{array}$ & $\begin{array}{l}.00037 \\
.00036 \\
.00035\end{array}$ & $\begin{array}{l}.00149 \\
.00145 \\
.00142\end{array}$ & $\begin{array}{l}.00011 \\
.00011 \\
.00010\end{array}$ & $\begin{array}{l}.00045 \\
.00044 \\
.00043\end{array}$ & $\begin{array}{r}.162 \\
.158 \\
.154\end{array}$ & $\begin{array}{r}.494 \\
.483 \\
.472\end{array}$ & $\begin{array}{l}.547 \\
.534 \\
.522\end{array}$ \\
\hline $\begin{array}{l}10 \\
10 \\
10\end{array}$ & $\begin{array}{l}500 \\
500 \\
500\end{array}$ & $\begin{array}{l}85.0 \\
90.0 \\
95.0\end{array}$ & $\begin{array}{l}.00034 \\
.00034 \\
.00033\end{array}$ & $\begin{array}{l}.00139 \\
.00136 \\
.00133\end{array}$ & $\begin{array}{l}.00010 \\
.00010 \\
.00010\end{array}$ & $\begin{array}{l}.00042 \\
.00041 \\
.00040\end{array}$ & $\begin{array}{r}151 \\
.248 \\
.145\end{array}$ & $\begin{array}{l}.461 \\
.451 \\
.442\end{array}$ & $\begin{array}{r}.511 \\
.499 \\
.490\end{array}$ \\
\hline 10 & $500:$ & 100.0 & .00032 & .00131 & .00009 & .00039 & .142 & .434 & .480 \\
\hline
\end{tabular}


TABULATION OF DATA FOR

ABSORPTION OF SOUND IN AIR VERSUS PERCENT RELATIVE HUMIDITY AT 10 DEGREES CENTIGRADE FOR A FREQUENCY OF 1000 HERTZ

\begin{tabular}{|c|c|c|c|c|c|c|c|c|c|}
\hline (1) & $(2)$ & (3) &..$(4)$ & (5) & $(6)$ & 171 & $(8)$ & 191 & $(10)$ \\
\hline $\begin{array}{l}\text { TEMP } \\
\text { DEGR } \\
\text { CENT }\end{array}$ & HERTZ & $\begin{array}{l}\text { REL } \\
\text { HUM } \\
\text { PER } \\
\text { CENT }\end{array}$ & $\begin{array}{l}\text { ATTEN } \\
\text { COEF } \\
\text { PER } \\
\text { METER }\end{array}$ & $\begin{array}{l}4 M \\
\text { PER } \\
\text { METER }\end{array}$ & $\begin{array}{l}\text { ATTEN } \\
\text { COEF } \\
\text { PER } \\
\text { FOOT }\end{array}$ & $\begin{array}{l}4 M \\
\text { PER } \\
\text { FOOT }\end{array}$ & $\begin{array}{l}\text { ATTEN } \\
\text { DB :PER } \\
100 \\
\text { METER }\end{array}$ & $\begin{array}{l}\text { ATTEN } \\
\text { DB PER } \\
1000 \\
\text { FEET }\end{array}$ & $\begin{array}{l}\text { DECAY } \\
\text { RATE } \\
\text { DB PER } \\
\text { SECOND }\end{array}$ \\
\hline $\begin{array}{l}10 \\
10 \\
10\end{array}$ & $\begin{array}{l}1000 \\
1000 \\
1000\end{array}$ & $\begin{array}{r}5.0 \\
10.0 \\
15.0\end{array}$ & $\begin{array}{r}.00494 \\
.00431 \\
.00279\end{array}$ & $\begin{array}{r}.01979 \\
.01726 \\
.01119\end{array}$ & $\begin{array}{l}.00150 \\
.00131 \\
.00085\end{array}$ & $\begin{array}{l}.00603 \\
.00526 \\
.00341\end{array}$ & $\begin{array}{l}2.148 \\
2.874 \\
2.215\end{array}$ & $\begin{array}{l}6.549 \\
5.714 \\
3.705\end{array}$ & $\begin{array}{l}7.249 \\
6.324 \\
4.100\end{array}$ \\
\hline $\begin{array}{l}10 \\
10 \\
10\end{array}$ & $\begin{array}{l}1000 \\
1000 \\
1000\end{array}$ & $\begin{array}{l}20.0 \\
25.0 \\
30.0\end{array}$ & $\begin{array}{r}.00203 \\
.00160 \\
.00134\end{array}$ & $\begin{array}{l}.00812 \\
.00640 \\
.00539\end{array}$ & $\begin{array}{l}.00061 \\
.00048 \\
.00041\end{array}$ & $\begin{array}{l}.00247 \\
.00195 \\
.00164\end{array}$ & $\begin{array}{r}.882 \\
.695 \\
.585\end{array}$ & $\begin{array}{l}2.689 \\
2.119 \\
1.785\end{array}$ & $\begin{array}{l}2.976 \\
2.346 \\
1.976\end{array}$ \\
\hline $\begin{array}{l}10 \\
10 \\
10\end{array}$ & $\begin{array}{l}1000 \\
1000 \\
1000\end{array}$ & $\begin{array}{l}35.0 \\
40.0 \\
45.0\end{array}$ & $\begin{array}{l}.00120 \\
.00111 \\
.00106\end{array}$ & $\begin{array}{l}.00481 \\
.00445 \\
.00426\end{array}$ & $\begin{array}{r}.00036 \\
.00033 \\
.00032\end{array}$ & $\begin{array}{l}.00146 \\
.00135 \\
.00130\end{array}$ & $\begin{array}{r}.522 \\
.484 \\
.463\end{array}$ & $\begin{array}{l}1.592 \\
1.475 \\
1.411\end{array}$ & $\begin{array}{l}1.762 \\
1.632 \\
1.562\end{array}$ \\
\hline $\begin{array}{l}10 \\
10 \\
10\end{array}$ & $\begin{array}{l}1000 \\
1000 \\
1000\end{array}$ & $\begin{array}{l}46.0 \\
47.0 \\
48.0\end{array}$ & $\begin{array}{l}.00105 \\
.00104 \\
.001 .04\end{array}$ & $\begin{array}{r}.00423 \\
.00419 \\
.00416\end{array}$ & $\begin{array}{l}.00032 \\
.00032 \\
.00031\end{array}$ & $\begin{array}{l}.00129 \\
.00128 \\
.00127\end{array}$ & $\begin{array}{r}.459 \\
.455 \\
.452\end{array}$ & $\begin{array}{l}1.400 \\
1.389 \\
1.379\end{array}$ & $\begin{array}{l}1.550 \\
1.538 \\
1.526\end{array}$ \\
\hline $\begin{array}{l}10 \\
10 \\
10\end{array}$ & $\begin{array}{l}1000 \\
1000 \\
1000\end{array}$ & $\begin{array}{l}49.0 \\
50.0 \\
51.0\end{array}$ & $\begin{array}{l}.00103 \\
.00102 \\
.00101\end{array}$ & $\begin{array}{r}.00413 \\
.00410 \\
.00407\end{array}$ & $\begin{array}{l}.00031 \\
.0 .0031 \\
.00031\end{array}$ & $\begin{array}{l}.00126 \\
.00125 \\
.00124\end{array}$ & $\begin{array}{l}.448 \\
.445 \\
.442\end{array}$ & $\begin{array}{l}1.368 \\
1.358 \\
1.349\end{array}$ & $\begin{array}{l}1.514 \\
1.503 \\
1.493\end{array}$ \\
\hline 10 & 1000 & 52.0 & .00101 & .00405 & .00030 & .00123 & .439 & 1.340 & 1.484 \\
\hline $\begin{array}{l}10 \\
10\end{array}$ & $\begin{array}{l}1000 \\
1000\end{array}$ & $\begin{array}{l}53 \cdot 0 \\
54.0\end{array}$ & $\begin{array}{l}.00100 \\
.00099\end{array}$ & $\begin{array}{r}.00402 \\
.00399\end{array}$ & $\begin{array}{l}.00030 \\
.00030\end{array}$ & $\begin{array}{l}.00122 \\
.00121\end{array}$ & $\begin{array}{r}.437 \\
.434\end{array}$ & $\begin{array}{l}1.332 \\
1.323\end{array}$ & $\begin{array}{l}1.474 \\
1.465\end{array}$ \\
\hline $\begin{array}{l}10 \\
10 \\
10\end{array}$ & $\begin{array}{l}1000 \\
1000 \\
1000\end{array}$ & $\begin{array}{l}55.0 \\
60.0 \\
65.0\end{array}$ & $\begin{array}{l}.00099 \\
.00096 \\
.00093\end{array}$ & $\begin{array}{r}.00397 \\
.00385 \\
.00374\end{array}$ & $\begin{array}{l}.00030 \\
.00029 \\
.00028\end{array}$ & $\begin{array}{l}.00121 \\
.00117 \\
.00114\end{array}$ & $\begin{array}{r}.431 \\
.418 \\
.406\end{array}$ & $\begin{array}{l}1.315 \\
1.275 \\
1.239\end{array}$ & $\begin{array}{l}1.455 \\
1.411 \\
1.371\end{array}$ \\
\hline $\begin{array}{l}10 \\
10 \\
10\end{array}$ & $\begin{array}{l}1000 \\
1000 \\
1000\end{array}$ & $\begin{array}{l}70.0 \\
75.0 \\
80.0\end{array}$ & $\begin{array}{l}.00091 \\
.00088 \\
.00086\end{array}$ & $\begin{array}{l}.00364 \\
.00355 \\
.00346\end{array}$ & $\begin{array}{l}.00027 \\
.00027 \\
.00026\end{array}$ & $\begin{array}{l}.00111 \\
.00108 \\
.00105\end{array}$ & $\begin{array}{r}.395 \\
.385 \\
.375\end{array}$ & $\begin{array}{l}1.205 \\
1.175 \\
1.145\end{array}$ & $\begin{array}{l}1.334 \\
1.300 \\
1.267\end{array}$ \\
\hline $\begin{array}{l}10 \\
10 \\
10\end{array}$ & $\begin{array}{l}1000 \\
1000 \\
1000\end{array}$ & $\begin{array}{l}85.0 \\
90.0 \\
95.0\end{array}$ & $\begin{array}{r}.00084 \\
.00082 \\
.00080\end{array}$ & $\begin{array}{l}.00337 \\
.00329 \\
.00323\end{array}$ & $\begin{array}{r}.00025 \\
.00025 \\
.00024\end{array}$ & $\begin{array}{l}.00103 \\
.00100 \\
.00098\end{array}$ & $\begin{array}{r}.366 \\
.358 \\
.351\end{array}$ & $\begin{array}{l}1.118 \\
1.091 \\
1.070\end{array}$ & $\begin{array}{l}1.237 \\
1.207 \\
1.185\end{array}$ \\
\hline 10 & 1000 & 100.0 & .00079 & .00317 & .00024 & .00096 & .345 & 1.052 & 1.164 \\
\hline
\end{tabular}


TABULATION OF DATA FOR

ABSORPTION OF SOUND IN AIR VERSUS PERCENT RELA'TIVE HUMIDITY AT 10 DEGREES CENTIGRADE FOR A FREQUENCY OF 2000 HERTZ
(1) (2) (3)
(4)
(5)
(6)
(7)
(8)
(9)
$(10)$

TEMP FREQ RE

\section{ATTEN $\quad 4 M$}

COEF

PER

PER

CENT HERTZ CENT

$102000 \quad 5.0$

$102000 \quad 10.0$

$102000 \quad 15.0$

$10 \quad 2000 \quad 20.0$

$102000 \quad 25.0$

$102000 \quad 30.0$

$10 \quad 2000 \quad 35.0$

$10 \quad 2000 \quad 40.0$

$102000 \quad 45.0$

$102000 \quad 46.0$

$10.2000 \quad 47.0$

$102000 \quad 48.0$

$10 \quad 2000 \quad 49.0$

$102000 \quad 50.0$

$102000^{\circ} 51.0$

$102000 \quad 52.0$

$102000 \quad 53.0$

$102000 \quad 54.0$

$10 \quad 2000 \quad 55.0$

$102000 \quad 60.0$

$102000 \quad 65.0$

$\begin{array}{lll}10 & 2000 \quad 70.0\end{array}$

$10 \quad 2000 \quad 75.0$

$102000 \quad 80.0$

$102000 \quad 85.0$

$102000 \quad 90.0$

$102000 \quad 95.0$

$102000 \quad 100.0$

METER

.00638

.01056

.00933

.00695

.00551

.00450

.00383

.00332

.00296

.00291

.00286

.00280

.00275

.00269

.00265

.00261

.00258

.00252

.00240

.00223

.00213

.00209

.00205

.00201
PER

METER

.02555

.04227

.03733

$\begin{array}{ll}\text { ATTEN } & 4 \mathrm{M} . \\ \text { COEF } & \\ \text { PER } & \text { PER }\end{array}$

FOOT

FOOT

.00194

.00322

.00284

.00778

.01288

.01137

.02781

.00211

.00847

.02207

.00168

.00137

.00672

.00549

.01532

.00116

.00466

- 01331

100101

.00405

.00361

.01164

.00088

.00354

.01144

.01123

.00087

.00085

.00348

.00342

.01101

.00083

.01079

.00082

.00335

.00329

.01063

.00081

.00324

.01047

.00079

.00329

.01033

.00078

.00315

.00310

.01009

.00076

.00307

.00962

.00919

.00073

.00293

.00280

.00893

.00070

.00272

.00873

.00068

.00266

.00853

.00065 .00260

.00836

.00820

.00063 .00255

.00062

.00250

.00805

.00061

.00245

.00241

$\begin{array}{lll}\text { ATTEN } & \text { ATTEN } & \text { DECAY } \\ \text { DB. PER } & \text { DB PER } & \text { RATE } \\ \text { 1OO } & 1000 & \text { DB P.ER } \\ \text { METER } & \text { FEET } & \text { SECOND }\end{array}$

$2.774 \quad 8.457 \quad 9.360$

$4.589 \quad 13.989 \quad 15.483$

$4.053 \quad 12.354 \quad 13.673$

$3.020 \quad 9.205 \quad 10.188$

$2.396 \quad 7.304 \quad 8.083$

$1.957 \quad 5.965 \quad 6.602$

$1.663 \quad 5.070 \quad 5.611$

$1.445 \quad 4.406 \quad 4.876$

$1.289 \quad 3.929 \quad 4.348$

$1.263 \quad 3.852 \quad 4.263$

$1.242 \quad 3.785 \quad 4.190$

$1.219 \quad 3.718$

$4 \cdot 115$

$2.195 \quad 3.645 \quad 4.034$

$1.172 \quad 3.573 \quad 3.955$

$1.154 \quad 3.520 \quad 3.896$

$\begin{array}{lll}1.137 & 3.466 & 3.837 \\ 1.122 & 3.420 & 3.785 \\ 1.106 & 3.373 & 3.734\end{array}$

$1.095 \quad 3.339 \quad 3.695$

$1.044 \quad 3.184 \quad 3.524$

$.998 \quad 3.043 \quad 3.368$

$.970 \quad 2.957 \quad 3.273$

$.948 \quad 2.890 \quad 3.199$

$.926 \quad 2.824 \quad 3.125$

$\begin{array}{lll}.908 & 2.769 \quad 3.065\end{array}$

$.891 \quad 2.716 \quad 3.006$

$.874 \quad 2.665 \quad 2.949$

$.859 \quad 2.620 \quad 2.900$ 
TABULATION OF DATA FOR.

ABSORPTION OF SOUND IN AIR VERSUS PERCENT RELATIVE HUMIDITY AT 10 DEGREES CENTIGRADE FOR A FREQUENCY OF 2500 HERTZ

\begin{tabular}{|c|c|c|c|c|c|c|c|c|c|}
\hline (1) & $(2)$ & (3) & $(4)$ & (5) & $(6)$ & $(7)$ & $(8)$ & 191 & $|10|$ \\
\hline TEMP & FREQ & $\begin{array}{l}\text { REL } \\
\text { HUM }\end{array}$ & $\begin{array}{l}\text { ATTEN } \\
\text { COEF }\end{array}$ & $4 M$ & $\begin{array}{l}\text { ATTEN } \\
\text { COEF }\end{array}$ & $4 M^{\prime}$ & $\begin{array}{l}\text { ATTEN } \\
\text { DB PER }\end{array}$ & $\begin{array}{l}\text { ATTEN } \\
\text { DB PER }\end{array}$ & $\begin{array}{l}\text { DECAY } \\
\text { RATE. }\end{array}$ \\
\hline $\begin{array}{l}\text { DEGR } \\
\text { CENT }\end{array}$ & HERTZ & $\begin{array}{l}\text { PER } \\
\text { CENT }\end{array}$ & $\begin{array}{l}\text { PER } \\
\text { METER }\end{array}$ & $\begin{array}{l}\text { PER } \\
\text { METER }\end{array}$ & $\begin{array}{l}\text { PER } \\
\text { FOOT }\end{array}$ & $\begin{array}{l}\text { PER } \\
\text { FOOT }\end{array}$ & $\begin{array}{l}100 \\
\text { METER }\end{array}$ & $\begin{array}{l}1000 \\
\text { FEET }\end{array}$ & $\begin{array}{l}\text { DB PER } \\
\text { SECOND }\end{array}$ \\
\hline $\begin{array}{l}10 \\
10 \\
10\end{array}$ & $\begin{array}{l}2500 \\
2500 \\
2500\end{array}$ & $\begin{array}{r}5.0 \\
20.0 \\
15.0\end{array}$ & $\begin{array}{l}.00686 \\
.01277 \\
.01285\end{array}$ & $\begin{array}{r}.02745 \\
.05110 \\
.05142\end{array}$ & $\begin{array}{l}.00209 \\
.00389 \\
.00391\end{array}$ & $\begin{array}{r}.00836 \\
.01557 \\
.01567\end{array}$ & $\begin{array}{l}2.980 \\
5.548 \\
5.583\end{array}$ & $\begin{array}{r}9.085 \\
16.9 .13 \\
17.019\end{array}$ & $\begin{array}{l}10.056 \\
18.719 \\
18.836\end{array}$ \\
\hline $\begin{array}{l}10 \\
10\end{array}$ & $\begin{array}{l}2500 \\
2500\end{array}$ & $\begin{array}{l}20.0 \\
25.0\end{array}$ & $\begin{array}{l}.01042 \\
.00815\end{array}$ & $\begin{array}{l}.04170 \\
.03263\end{array}$ & $\begin{array}{r}.00317 \\
.00248\end{array}$ & $\begin{array}{l}.01271 \\
.00994\end{array}$ & $\begin{array}{l}4.528 \\
3.543\end{array}$ & $\begin{array}{l}13.802 \\
10.800\end{array}$ & $\begin{array}{l}15.276 \\
11.953\end{array}$ \\
\hline 10 & 2500 & 30.0 & .00677 & .02710 & .00206 & .00826 & 2.942 & 8.968 & 9.926 \\
\hline 10 & 2500 & 35.0 & .00571 & .02284 & .00174 & .00696 & 2.479 & 7.559 & 8.366 \\
\hline $\begin{array}{l}10 \\
10\end{array}$ & $\begin{array}{l}2500 \\
2500\end{array}$ & $\begin{array}{l}40.0 \\
45.0\end{array}$ & $\begin{array}{r}.00495 \\
.00439\end{array}$ & $\begin{array}{r}.01983 \\
.01756\end{array}$ & $\begin{array}{l}.00151 \\
.00133\end{array}$ & $\begin{array}{l}.00604 \\
.00535\end{array}$ & $\begin{array}{l}2.153 \\
1.907\end{array}$ & $\begin{array}{l}6.565 \\
5.813\end{array}$ & $\begin{array}{l}7.266 \\
6.434\end{array}$ \\
\hline 10 & 2500 & 46.0 & .00429 & .01717 & .00130 & .00523 & 1.864 & 5.684 & 6.291 \\
\hline $\begin{array}{l}10 \\
10\end{array}$ & $\begin{array}{l}2500 \\
2500\end{array}$ & $\begin{array}{l}47.0 \\
48.0\end{array}$ & $\begin{array}{r}.00419 \\
.00410\end{array}$ & $\begin{array}{r}.01678 \\
.01642\end{array}$ & $\begin{array}{l}.00127 \\
.00125\end{array}$ & $\begin{array}{l}.00511 \\
.00500\end{array}$ & $\begin{array}{l}1.822 \\
1.783\end{array}$ & $\begin{array}{l}5.556 \\
5.434\end{array}$ & $\begin{array}{l}6.149 \\
6.014\end{array}$ \\
\hline $\begin{array}{l}10 \\
10 \\
10\end{array}$ & $\begin{array}{l}2500 \\
2500 \\
2500\end{array}$ & $\begin{array}{l}49.0 \\
50.0 \\
51.0\end{array}$ & $\begin{array}{l}.00402 \\
.00394 \\
.00387\end{array}$ & $\begin{array}{r}.01608 \\
.01578 \\
.01548\end{array}$ & $\begin{array}{l}.00122 \\
.00120 \\
.00118\end{array}$ & $\begin{array}{l}.00490 \\
.00481 \\
.00472\end{array}$ & $\begin{array}{l}1.746 \\
1.714 \\
1.681\end{array}$ & $\begin{array}{l}5.324 \\
5.224 \\
5.125\end{array}$ & $\begin{array}{l}5.892 \\
5.782 \\
5.672\end{array}$ \\
\hline $\begin{array}{l}10 \\
10 \\
10\end{array}$ & $\begin{array}{l}2500 \\
2500 \\
2500\end{array}$ & $\begin{array}{l}52.0 \\
53.0 \\
54.0\end{array}$ & $\begin{array}{r}.00380 \\
.00374 \\
.00367\end{array}$ & $\begin{array}{r}.01521 \\
.01496 \\
.01471\end{array}$ & $\begin{array}{l}.00115 \\
.00114 \\
.00112\end{array}$ & $\begin{array}{l}.00463 \\
.00456 \\
.00448\end{array}$ & $\begin{array}{l}1.652 \\
1.624 \\
1.598\end{array}$ & $\begin{array}{l}5.036 \\
4.951 \\
4.871\end{array}$ & $\begin{array}{l}5.574 \\
5.480 \\
5.391\end{array}$ \\
\hline $\begin{array}{l}10 \\
10 \\
10\end{array}$ & $\begin{array}{l}2500 \\
2500 \\
2500\end{array}$ & $\begin{array}{l}55.0 \\
60.0 \\
65.0\end{array}$ & $\begin{array}{l}.00362 \\
.00336 \\
.00318\end{array}$ & $\begin{array}{r}.01450 \\
.01344 \\
.01273\end{array}$ & $\begin{array}{l}.00110 \\
.00102 \\
.00097\end{array}$ & $\begin{array}{r}.00442 \\
.00409 \\
.00388\end{array}$ & $\begin{array}{l}1.574 \\
1.459 \\
1.383\end{array}$ & $\begin{array}{l}4.800 \\
4.448 \\
4.215\end{array}$ & $\begin{array}{l}5.312 \\
4.923 \\
4.665\end{array}$ \\
\hline $\begin{array}{l}10 \\
10 \\
10\end{array}$ & $\begin{array}{l}2500 \\
2500 \\
2500\end{array}$ & $\begin{array}{l}70.0 \\
75.0 \\
80.0\end{array}$ & $\begin{array}{l}.00305 \\
.00293 \\
.00286\end{array}$ & $\begin{array}{r}.01223 \\
.01175 \\
.01145\end{array}$ & $\begin{array}{l}.00093 \\
.00089 \\
.00087\end{array}$ & $\begin{array}{r}.00373 \\
.00358 \\
.00349\end{array}$ & $\begin{array}{l}1.328 \\
1.276 \\
1.243\end{array}$ & $\begin{array}{l}4.050 \\
3.890 \\
3.789\end{array}$ & $\begin{array}{l}4.482 \\
4.306 \\
4.194\end{array}$ \\
\hline $\begin{array}{l}10 \\
10 \\
10\end{array}$ & $\begin{array}{l}2500 \\
2500 \\
2500\end{array}$ & $\begin{array}{l}85.0 \\
90.0 \\
95.0\end{array}$ & $\begin{array}{l}.00280 \\
.00275 \\
.00269\end{array}$ & $\begin{array}{l}.01122 \\
.01100 \\
.01079\end{array}$ & $\begin{array}{l}.00085 \\
.00083 \\
.00082\end{array}$ & $\begin{array}{l}.00342 \\
.00335 \\
.00329\end{array}$ & $\begin{array}{l}1.218 \\
1 \bullet 195 \\
1.172\end{array}$ & $\begin{array}{l}3.713 \\
3.643 \\
3.573\end{array}$ & $\begin{array}{l}4.110 \\
4.032 \\
3.954\end{array}$ \\
\hline 10 & 2500 & $100 \cdot 0$ & .00265 & .01062 & .00080 & .00323 & 1.153 & 3.516 & 3.892 \\
\hline
\end{tabular}


TABULATION OF DATA FOR

ABSORPTION OF SOUND IN AIR VERSUS PERCENT RELATIVE HUMIDITY AT 10 DEGREES CENTIGRADE FOR A FREQUENCY OF 3200 HERTZ

\begin{tabular}{|c|c|c|c|c|c|c|c|c|c|}
\hline (1) & $(2)$ & $(3)$ & $(4)$ & $(5)$ & $(6)$ & $(7)$ & $(8)$ & $(9)$ & $(10)$ \\
\hline $\begin{array}{l}\text { TEMP } \\
\text { DEGR } \\
\text { CENT }\end{array}$ & HER TZ & $\begin{array}{l}\text { REL } \\
\text { HUM } \\
\text { PER } \\
\text { CENT }\end{array}$ & $\begin{array}{l}\text { ATTEN } \\
\text { COEF } \\
\text { PER } \\
\text { METER }\end{array}$ & $\begin{array}{l}4 M \\
\text { PER } \\
\text { METER }\end{array}$ & $\begin{array}{l}\text { ATTEN } \\
\text { COEF } \\
\text { PER } \\
\text { FOOT }\end{array}$ & $\begin{array}{l}4 M \\
\text { PER } \\
\text { FOOT }\end{array}$ & $\begin{array}{l}\text { ATTEN } \\
\text { DB PER } \\
100 \\
\text { METER }\end{array}$ & $\begin{array}{l}\text { ATTEN } \\
\text { DB PER } \\
1000 \\
\text { FEET }\end{array}$ & $\begin{array}{l}\text { DECAY } \\
\text { RATE } \\
\text { DB PER } \\
\text { SECOND }\end{array}$ \\
\hline $\begin{array}{l}10 \\
10 \\
10\end{array}$ & $\begin{array}{l}3200 \\
3200 \\
3200\end{array}$ & $\begin{array}{r}5.0 \\
10.0 \\
15.0\end{array}$ & $\begin{array}{l}.00761 \\
.01505 \\
.01706\end{array}$ & $\begin{array}{l}.03045 \\
.06022 \\
.06824\end{array}$ & $\begin{array}{l}.00232 \\
.00458 \\
.00520\end{array}$ & $\begin{array}{r}.00928 \\
.01835 \\
.02080\end{array}$ & $\begin{array}{l}3.306 \\
6.538 \\
7.409\end{array}$ & $\begin{array}{l}10.079 \\
19.930 \\
22.585\end{array}$ & $\begin{array}{l}11.155 \\
22.058 \\
24.996\end{array}$ \\
\hline $\begin{array}{l}10 \\
10 \\
10\end{array}$ & $\begin{array}{l}3200 \\
3200 \\
3200\end{array}$ & $\begin{array}{l}20.0 \\
25.0 \\
30.0\end{array}$ & $\begin{array}{r}.01543 \\
.01251 \\
.01026\end{array}$ & $\begin{array}{l}.06172 \\
.05005 \\
.04104\end{array}$ & $\begin{array}{l}.00470 \\
.00381 \\
.00312\end{array}$ & $\begin{array}{l}.01881 \\
.01525 \\
.01250\end{array}$ & $\begin{array}{l}6.701 \\
5.435 \\
4.456\end{array}$ & $\begin{array}{l}20.427 \\
16.566 \\
13.582\end{array}$ & $\begin{array}{l}22.608 \\
18.335 \\
15.032\end{array}$ \\
\hline $\begin{array}{l}10 \\
10 \\
10\end{array}$ & $\begin{array}{l}3200 \\
3200 \\
3200\end{array}$ & $\begin{array}{l}35.0 \\
40.0 \\
45.0\end{array}$ & $\begin{array}{r}.00879 \\
.00758 \\
.00669\end{array}$ & $\begin{array}{r}.03577 \\
.03032 \\
.02679\end{array}$ & $\begin{array}{l}.00268 \\
.00231 \\
.00204\end{array}$ & $\begin{array}{l}.01072 \\
.00924 \\
.00816\end{array}$ & $\begin{array}{l}3.819 \\
3.292 \\
2.909\end{array}$ & $\begin{array}{r}11.641 \\
10.034 \\
8.868\end{array}$ & $\begin{array}{r}12.884 \\
11.106 \\
9.815\end{array}$ \\
\hline $\begin{array}{l}10 \\
10 \\
10\end{array}$ & $\begin{array}{l}3200 \\
3200 \\
3200\end{array}$ & $\begin{array}{l}46.0 \\
47.0 \\
48.0\end{array}$ & $\begin{array}{l}.00655 \\
.00640 \\
.00627\end{array}$ & $\begin{array}{l}.02620 \\
.02563 \\
.02511\end{array}$ & $\begin{array}{l}.00199 \\
.00195 \\
.00191\end{array}$ & $\begin{array}{l}.00798 \\
.00781 \\
.00765\end{array}$ & $\begin{array}{l}2.844 \\
2.783 \\
2.726\end{array}$ & $\begin{array}{l}8.670 \\
8.484 \\
8.311\end{array}$ & $\begin{array}{l}9.596 \\
9.390 \\
9.198\end{array}$ \\
\hline $\begin{array}{l}10 \\
10 \\
10\end{array}$ & $\begin{array}{l}3200 \\
3200 \\
3200\end{array}$ & $\begin{array}{l}49.0 \\
50.0 \\
51.0\end{array}$ & $\begin{array}{l}.00615 \\
.00603 \\
.00590\end{array}$ & $\begin{array}{l}.02461 \\
.02414 \\
.02363\end{array}$ & $\begin{array}{l}.00187 \\
.00184 \\
.00180\end{array}$ & $\begin{array}{l}.00750 \\
.00736 \\
.00720\end{array}$ & $\begin{array}{l}2.672 \\
2.622 \\
2.566\end{array}$ & $\begin{array}{l}8.146 \\
7.892 \\
7.822\end{array}$ & $\begin{array}{l}9.016 \\
8.845 \\
8.657\end{array}$ \\
\hline $\begin{array}{l}10 \\
10 \\
10\end{array}$ & $\begin{array}{l}3200 \\
3200 \\
3200\end{array}$ & $\begin{array}{l}52.0 \\
53.0 \\
54.0\end{array}$ & $\begin{array}{l}.00578 \\
.00567 \\
.00556\end{array}$ & $\begin{array}{l}.02314 \\
.02268 \\
.02226\end{array}$ & $\begin{array}{l}.00176 \\
.00172 \\
.00169\end{array}$ & $\begin{array}{l}.00705 \\
.00691 \\
.00678\end{array}$ & $\begin{array}{l}2.513 \\
2.462 \\
2.416\end{array}$ & $\begin{array}{l}7.660 \\
7.506 \\
7.367\end{array}$ & $\begin{array}{l}8.477 \\
8.308 \\
8.153\end{array}$ \\
\hline $\begin{array}{l}10 \\
10 \\
10\end{array}$ & $\begin{array}{l}3200 \\
3200 \\
3200\end{array}$ & $\begin{array}{l}55.0 \\
60.0 \\
65.0\end{array}$ & $\begin{array}{l}.00545 \\
.00501 \\
.00468\end{array}$ & $\begin{array}{l}.02183 \\
.02006 \\
.01875\end{array}$ & $\begin{array}{l}.00166 \\
.00152 \\
.00142\end{array}$ & $\begin{array}{l}.00665 \\
.00611 \\
.00571\end{array}$ & $\begin{array}{l}2.371 \\
2.178 \\
2.036\end{array}$ & $\begin{array}{l}7.227 \\
6.639 \\
6.205\end{array}$ & $\begin{array}{l}7.999 \\
7.348 \\
6.868\end{array}$ \\
\hline $\begin{array}{l}10 \\
10 \\
10\end{array}$ & $\begin{array}{l}3200 \\
3200 \\
3200\end{array}$ & $\begin{array}{l}70.0 \\
75.0 \\
80.0\end{array}$ & $\begin{array}{r}.00439 \\
.00418 \\
.00404\end{array}$ & $\begin{array}{r}.01757 \\
.01675 \\
.01616\end{array}$ & $\begin{array}{l}.00133 \\
.00127 \\
.00123\end{array}$ & $\begin{array}{l}.00535 \\
.00510 \\
.00492\end{array}$ & $\begin{array}{l}1.908 \\
1.819 \\
1.755\end{array}$ & $\begin{array}{l}5.817 \\
5.544 \\
5.350\end{array}$ & $\begin{array}{l}6.438 \\
6.136 \\
5.922\end{array}$ \\
\hline $\begin{array}{l}10 \\
10 \\
10\end{array}$ & $\begin{array}{l}3200 \\
3200 \\
3200\end{array}$ & $\begin{array}{l}85.0 \\
90.0 \\
95.0\end{array}$ & $\begin{array}{l}.00391 \\
.00380 \\
.00372\end{array}$ & $\begin{array}{r}.01565 \\
.01520 \\
.01490\end{array}$ & $\begin{array}{l}.00119 \\
.00115 \\
.00113\end{array}$ & $\begin{array}{l}.00477 \\
.00463 \\
.00454\end{array}$ & $\begin{array}{l}1.699 \\
1.650 \\
1.618\end{array}$ & $\begin{array}{l}5.179 \\
5.031 \\
4.931\end{array}$ & $\begin{array}{l}5.732 \\
5.568 \\
5.458\end{array}$ \\
\hline 0 & 3200 & .0 & .00366 & 01465 & .00111 & .00446 & 1.591 & 4.850 & 5.368 \\
\hline
\end{tabular}


TABULATION OF DATA FOR.

ABSORPTION OF SOUND IN AIR VERSUS PERCENT RELATIVE HUMIDITY AT 10 DEGREES CENTIGRADE FOR A FREQUENCY OF 4000 HERTZ

\begin{tabular}{|c|c|c|c|c|c|c|c|c|c|}
\hline$(1)$ & (2) & $(3)$ & (4) & $(5)$ & $(6)$. & $(7)$ & 1.81 & $(9)$ & 1201 \\
\hline $\begin{array}{l}\text { TEMP } \\
\text { DEGR } \\
\text { CENT }\end{array}$ & HERTZ & $\begin{array}{l}\text { REL } \\
\text { HUM } \\
\text { PER } \\
\text { CENT }\end{array}$ & $\begin{array}{l}\text { ATTEN } \\
\text { COEF. } \\
\text { PER } \\
\text { METER }\end{array}$ & $\begin{array}{l}4 M \\
\text { PER } \\
\text { METER }\end{array}$ & $\begin{array}{l}\text { ATTEN } \\
\text { COEF } \\
\text { PER } \\
\text { FOOT. }\end{array}$ & $\begin{array}{l}4 M \\
\text { PER } \\
\text { FOOT }\end{array}$ & $\begin{array}{l}\text { ATTEN } \\
\text { DB PER } \\
100 \\
\text { METER }\end{array}$ & $\begin{array}{l}\text { ATTEN } \\
\text { DB PER } \\
1000 \\
\text { FEET }\end{array}$ & $\begin{array}{l}\text { DECAY } \\
\text { RATE } \\
\text { DB PER } \\
\text { SECOND }\end{array}$ \\
\hline $\begin{array}{l}10 \\
10 \\
10\end{array}$ & $\begin{array}{l}4000 \\
4000 \\
4000\end{array}$ & $\begin{array}{r}5.0 \\
10.0 \\
15.0\end{array}$ & $\begin{array}{r}.00837 \\
.01650 \\
.02126\end{array}$ & $\begin{array}{l}.03349 \\
.06601 \\
.08504\end{array}$ & $\begin{array}{l}.00255 \\
.00503 \\
.00648\end{array}$ & $\begin{array}{r}.01020 \\
.02012 \\
.02592\end{array}$ & $\begin{array}{l}3.636 \\
7.167 \\
9.233\end{array}$ & $\begin{array}{l}11.084 \\
21.845 \\
28.143\end{array}$ & $\begin{array}{l}12.268 \\
24.178 \\
31.148\end{array}$ \\
\hline $\begin{array}{l}10 \\
10 \\
10\end{array}$ & $\begin{array}{l}4000 \\
4000 \\
4000\end{array}$ & $\begin{array}{l}20.0 \\
25.0 \\
30.0\end{array}$ & $\begin{array}{r}.02094 \\
.01827 \\
.01514\end{array}$ & $\begin{array}{l}.08378 \\
.07311 \\
.06057\end{array}$ & $\begin{array}{l}.00638 \\
.00557 \\
.00461\end{array}$ & $\begin{array}{l}.02553 \\
.02228 \\
.01846\end{array}$ & $\begin{array}{l}9.096 \\
7.938 \\
6.576\end{array}$ & $\begin{array}{l}27.727 \\
24.197 \\
20.046\end{array}$ & $\begin{array}{l}30.687 \\
26.780 \\
22.186\end{array}$ \\
\hline $\begin{array}{l}10 \\
10 \\
10\end{array}$ & $\begin{array}{l}4000 \\
4000 \\
4000\end{array}$ & $\begin{array}{l}35.0 \\
40.0 \\
45.0\end{array}$ & $\begin{array}{r}.01287 \\
.01128 \\
.00994\end{array}$ & $\begin{array}{r}.05149 \\
.04514 \\
.03979\end{array}$ & $\begin{array}{r}.00392 \\
.00344 \\
.00303\end{array}$ & $\begin{array}{r}.01569 \\
.01376 \\
.01213\end{array}$ & $\begin{array}{l}5.591 \\
4.902 \\
4.321\end{array}$ & $\begin{array}{l}17.042 \\
14.941 \\
13.170\end{array}$ & $\begin{array}{l}18.862 \\
16.537 \\
14.577\end{array}$ \\
\hline $\begin{array}{l}10 \\
10 \\
10\end{array}$ & $\begin{array}{l}4000 \\
4000 \\
4000\end{array}$ & $\begin{array}{l}46.0 \\
47.0 \\
48.0\end{array}$ & $\begin{array}{r}.00969 \\
.00947 \\
.00927\end{array}$ & $\begin{array}{r}.03879 \\
.03788 \\
.03708\end{array}$ & $\begin{array}{l}.00295 \\
.00288 \\
.00282\end{array}$ & $\begin{array}{l}.01182 \\
.01154 \\
.01130\end{array}$ & $\begin{array}{l}4.211 \\
4.113 \\
4.025\end{array}$ & $\begin{array}{l}12.837 \\
12.538 \\
12.271\end{array}$ & $\begin{array}{l}14.207 \\
13.877 \\
13.581\end{array}$ \\
\hline $\begin{array}{l}10 \\
10 \\
10\end{array}$ & $\begin{array}{l}4000 \\
4000 \\
4000\end{array}$ & $\begin{array}{l}49.0 \\
50.0 \\
51.0\end{array}$ & $\begin{array}{l}.00906 \\
.00887 \\
.00869\end{array}$ & $\begin{array}{r}.03627 \\
.03548 \\
.03478\end{array}$ & $\begin{array}{l}.00276 \\
.00270 \\
.00265\end{array}$ & $\begin{array}{r}.01105 \\
.01081 \\
.01060\end{array}$ & $\begin{array}{l}3.938 \\
3.853 \\
3.776\end{array}$ & $\begin{array}{l}12.004 \\
11.744 \\
11.510\end{array}$ & $\begin{array}{l}13.286 \\
12.998 \\
12.739\end{array}$ \\
\hline $\begin{array}{l}10 \\
10\end{array}$ & $\begin{array}{l}4000 \\
4000\end{array}$ & $\begin{array}{l}52.0 \\
53.0\end{array}$ & $\begin{array}{l}.00852 \\
.00836\end{array}$ & $\begin{array}{r}.03410 \\
.03344\end{array}$ & $\begin{array}{l}.00259 \\
.00254\end{array}$ & $\begin{array}{l}.01039 \\
.01019\end{array}$ & $\begin{array}{l}3.702 \\
3.631\end{array}$ & $\begin{array}{l}11.286 \\
11.069\end{array}$ & $\begin{array}{l}12.491 \\
12.251\end{array}$ \\
\hline 10 & 4000 & 54.0 & .00820 & .03283 & .00250 & .01000 & 3.565 & 10.866 & 12.027 \\
\hline $\begin{array}{l}10 \\
10 \\
10\end{array}$ & $\begin{array}{l}4000 \\
4000 \\
4000\end{array}$ & $\begin{array}{l}55.0 \\
60.0 \\
65.0\end{array}$ & $\begin{array}{r}.00806 \\
.00739 \\
.00680\end{array}$ & $\begin{array}{r}.03224 \\
.02956 \\
.02723\end{array}$ & $\begin{array}{l}.00245 \\
.00225 \\
.00207\end{array}$ & $\begin{array}{l}.00982 \\
.00901 \\
.00830\end{array}$ & $\begin{array}{l}3.500 \\
3.210 \\
2.957\end{array}$ & $\begin{array}{r}10.670 \\
9.784 \\
9.014\end{array}$ & $\begin{array}{r}11.809 \\
10.829 \\
9.977\end{array}$ \\
\hline $\begin{array}{l}10 \\
10 \\
10\end{array}$ & $\begin{array}{l}4000 \\
4000 \\
4000\end{array}$ & $\begin{array}{l}70.0 \\
75.0 \\
80.0\end{array}$ & $\begin{array}{l}.00635 \\
.00600 \\
.00566\end{array}$ & $\begin{array}{r}.02541 \\
.02400 \\
.02267\end{array}$ & $\begin{array}{l}.00193 \\
.00182 \\
.00172\end{array}$ & $\begin{array}{l}.00774 \\
.00731 \\
.00691\end{array}$ & $\begin{array}{l}2.759 \\
2.606 \\
2.462\end{array}$ & $\begin{array}{l}8.411 \\
7.944 \\
7.504\end{array}$ & $\begin{array}{l}9.310 \\
8.792 \\
8.306\end{array}$ \\
\hline $\begin{array}{l}10 \\
10\end{array}$ & $\begin{array}{l}4000 \\
4000\end{array}$ & $\begin{array}{l}85.0 \\
90.0\end{array}$ & $\begin{array}{l}.00542 \\
.00525\end{array}$ & .02171 & $\begin{array}{l}.00165 \\
.00160\end{array}$ & $\begin{array}{l}.00662 \\
.00640\end{array}$ & $\begin{array}{l}2.358 \\
2.282\end{array}$ & $\begin{array}{l}7.187 \\
6.957\end{array}$ & $\begin{array}{l}7.955 \\
7.700\end{array}$ \\
\hline 10 & 4000 & 95.0 & .00511 & .02044 & .00155 & .00623 & 2.219 & 6.765 & 7.488 \\
\hline 10 & 4000 & .0 & .00496 & .01985 & $: 00151$ & .00605 & 2.155 & 6.571 & 7.272 \\
\hline
\end{tabular}


TABULATION OF DATA FOR

ABSORPTION OF SOUND IN AIR VERSUS PERCENT RELATIVE HUMIDITY AT 10 DEGREES CENTIGRADE FOR A FREQUENCY OF 5000 HERTZ

\begin{tabular}{|c|c|c|c|c|c|c|c|c|c|}
\hline (i) & $(2)$ & (3) & $(4)$ & $(5)$ & $(6)$ & 171 & $|8|$ & 191 & $(10)$ \\
\hline $\begin{array}{l}\text { EMP } \\
\text { EGR } \\
\text { ENT }\end{array}$ & HERTZ & $\begin{array}{l}\text { REL } \\
\text { HUM } \\
\text { PER } \\
\text { CENT }\end{array}$ & $\begin{array}{l}\text { A.TTEN } \\
\text { COEF } \\
\text { PER } \\
\text { METER }\end{array}$ & $\begin{array}{l}4 M \\
\text { PER } \\
\text { METER }\end{array}$ & $\begin{array}{l}\text { ATTEN } \\
\text { COEF } \\
\text { PER } \\
\text { FOOT }\end{array}$ & $\begin{array}{l}\text { PER } \\
\text { FOOT }\end{array}$ & $\begin{array}{l}\text { ATTEN } \\
\text { DB PER } \\
\text { IOO } \\
\text { METER }\end{array}$ & $\begin{array}{l}\text { ATTEN } \\
\text { DB PER } \\
1000 \\
\text { FEET }\end{array}$ & $\begin{array}{l}\text { DECAY } \\
\text { RATE } \\
\text { DB PER } \\
\text { SECOND }\end{array}$ \\
\hline $\begin{array}{l}10 \\
10 \\
10\end{array}$ & $\begin{array}{l}5000 \\
5000 \\
5000\end{array}$ & $\begin{array}{r}5.0 \\
10.0 \\
15.0\end{array}$ & $\begin{array}{r}.00931 \\
.01790 \\
.02543\end{array}$ & $\begin{array}{l}.03727 \\
.07161 \\
.10175\end{array}$ & $\begin{array}{r}.00284 \\
.00545 \\
.00775\end{array}$ & $\begin{array}{l}.01136 \\
.02182 \\
.03101\end{array}$ & $\begin{array}{r}4.047 \\
7.775 \\
11.048\end{array}$ & $\begin{array}{l}12.336 \\
23.699 \\
33.676\end{array}$ & $\begin{array}{l}13.653 \\
26.229 \\
37.271\end{array}$ \\
\hline $\begin{array}{l}10 \\
10 \\
10\end{array}$ & $\begin{array}{l}5000 \\
5000 \\
5000\end{array}$ & $\begin{array}{l}20.0 \\
25.0 \\
30.0\end{array}$ & $\begin{array}{l}.02698 \\
.02551 \\
.02228\end{array}$ & $\begin{array}{l}.10792 \\
.10205 \\
.08914\end{array}$ & $\begin{array}{l}.00822 \\
.00777 \\
.00679\end{array}$ & $\begin{array}{l}.03289 \\
.03110 \\
.02717\end{array}$ & $\begin{array}{r}11.718 \\
11.080 \\
9.678\end{array}$ & $\begin{array}{l}35.717 \\
33.772 \\
29.500\end{array}$ & $\begin{array}{l}39.530 \\
37.378 \\
32.650\end{array}$ \\
\hline $\begin{array}{l}10 \\
10 \\
10\end{array}$ & $\begin{array}{l}5000 \\
5000 \\
5000\end{array}$ & $\begin{array}{l}35.0 \\
40.0 \\
45.0\end{array}$ & $\begin{array}{r}.01894 \\
.01646 \\
.01469\end{array}$ & $\begin{array}{r}.07576 \\
.06587 \\
.05878\end{array}$ & $\begin{array}{l}.00577 \\
.00501 \\
.00447\end{array}$ & $\begin{array}{l}.02309 \\
.02007 \\
.01791\end{array}$ & $\begin{array}{l}8.225 \\
7.152 \\
6.382\end{array}$ & $\begin{array}{l}25.072 \\
21.801 \\
19.453\end{array}$ & $\begin{array}{l}27.749 \\
24.129 \\
21.530\end{array}$ \\
\hline $\begin{array}{l}10 \\
10 \\
10\end{array}$ & $\begin{array}{l}5000 \\
5000 \\
5000\end{array}$ & $\begin{array}{l}46.0 \\
47.0 \\
48.0\end{array}$ & $\begin{array}{l}.01 \\
.01 \\
.01\end{array}$ & $\begin{array}{l}.05747 \\
.05621 \\
.05496\end{array}$ & $\begin{array}{l}.00437 \\
.00428 \\
.00418\end{array}$ & $\begin{array}{l}.01751 \\
.01713 \\
.01675\end{array}$ & $\begin{array}{l}240 \\
103 \\
967\end{array}$ & $\begin{array}{l}20 \\
604 \\
189\end{array}$ & $\begin{array}{l}511 \\
590 \\
131\end{array}$ \\
\hline $\begin{array}{l}10 \\
10 \\
10\end{array}$ & $\begin{array}{l}5000 \\
5000 \\
5000\end{array}$ & $\begin{array}{l}49.0 \\
50.0 \\
51.0\end{array}$ & $\begin{array}{r}.01345 \\
.01316 \\
.01289\end{array}$ & $\begin{array}{l}.05381 \\
.05267 \\
.05158\end{array}$ & $\begin{array}{l}.00410 \\
.00401 \\
.00393\end{array}$ & $\begin{array}{r}.01640 \\
.01605 \\
.01572\end{array}$ & $\begin{array}{l}5.843 \\
5.719 \\
5.601\end{array}$ & $\begin{array}{l}17.810 \\
17.433 \\
17.073\end{array}$ & $\begin{array}{l}19.712 \\
19.295 \\
18.896\end{array}$ \\
\hline $\begin{array}{l}10 \\
10 \\
10\end{array}$ & $\begin{array}{l}5000 \\
5000 \\
5000\end{array}$ & $\begin{array}{l}52.0 \\
53.0 \\
54.0\end{array}$ & $\begin{array}{l}.01262 \\
.01235 \\
.01209\end{array}$ & $\begin{array}{l}.05050 \\
.04941 \\
.04837\end{array}$ & $\begin{array}{l}.00384 \\
.00376 \\
.00368\end{array}$ & $\begin{array}{l}.01539 \\
.01506 \\
.011474\end{array}$ & $\begin{array}{l}5.483 \\
5.364 \\
5.252\end{array}$ & $\begin{array}{l}16.712 \\
16.352 \\
16.008\end{array}$ & $\begin{array}{l}18.497 \\
18.098 \\
17.718\end{array}$ \\
\hline $\begin{array}{l}10 \\
10 \\
10\end{array}$ & $\begin{array}{l}5000 \\
5000 \\
5000\end{array}$ & $\begin{array}{l}55.0 \\
60.0 \\
65.0\end{array}$ & $\begin{array}{l}.01187 \\
.01085 \\
.01004\end{array}$ & $\begin{array}{l}.04750 \\
.04343 \\
.04017\end{array}$ & $\begin{array}{l}.00361 \\
.00330 \\
.00306\end{array}$ & $\begin{array}{r}.01447 \\
.01323 \\
.011224\end{array}$ & $\begin{array}{l}5.157 \\
4.715 \\
4.361\end{array}$ & $\begin{array}{l}15.720 \\
14.373 \\
13.294\end{array}$ & $\begin{array}{l}17.398 \\
15.907 \\
14.714\end{array}$ \\
\hline $\begin{array}{l}10 \\
10 \\
10\end{array}$ & $\begin{array}{l}5000 \\
5000 \\
5000\end{array}$ & $\begin{array}{l}70.0 \\
75.0 \\
80.0\end{array}$ & $\begin{array}{l}.00933 \\
.00870 \\
.00820\end{array}$ & $\begin{array}{r}.03732 \\
.03482 \\
.03282\end{array}$ & $\begin{array}{l}.00284 \\
.00265 \\
.00250\end{array}$ & $\begin{array}{l}.01137 \\
.01061 \\
.01000\end{array}$ & $\begin{array}{l}53 \\
81 \\
63\end{array}$ & $\begin{array}{l}353 \\
525 \\
862\end{array}$ & $\begin{array}{l}13.672 \\
12.756 \\
12.022\end{array}$ \\
\hline $\begin{array}{l}10 \\
10\end{array}$ & $\begin{array}{l}5000 \\
5000 \\
5000\end{array}$ & $\begin{array}{l}85.0 \\
90.0 \\
95.0\end{array}$ & $\begin{array}{l}.00779 \\
.00744 \\
.00712\end{array}$ & $\begin{array}{r}.03118 \\
.02977 \\
.02850\end{array}$ & $\begin{array}{l}.00237 \\
.00226 \\
.00217\end{array}$ & $\begin{array}{l}.00950 \\
.00907 \\
.00868\end{array}$ & $\begin{array}{l}3.385 \\
3.232 \\
3.095\end{array}$ & $\begin{array}{r}10.319 \\
9.853 \\
9.434\end{array}$ & $\begin{array}{l}11.421 \\
10.905 \\
10.441\end{array}$ \\
\hline 10 & 5000 & .0 & .00688 & .02753 & .00209 & .00839 & 2.989 & 9.112 & 10.085 \\
\hline
\end{tabular}


TABULATION OF DATA F.OR

ABSORPTION OF SOUND IN AIR VERSUS PERCENT RELATIVE. HUMIDITY AT 10 DEGREES CENTIGRADE. FOR A FREQUENCY OF 5.9.40 HERTZ

\begin{tabular}{|c|c|c|c|c|c|c|c|c|c|}
\hline (1) & (2) & $(3)$ & (4) & $(5)$ & 161 & 171 & $(8)$ & 191 & 1101 \\
\hline TEMP & FREQ & $\begin{array}{l}\text { REL } \\
\text { HUM }\end{array}$ & $\begin{array}{l}\text { ATTEN } \\
\text { COEF }\end{array}$ & $4 M$ & $\begin{array}{l}\text { ATTEN } \\
\text { COEF }\end{array}$ & $4 M$ & $\begin{array}{l}\text { ATTEN } \\
\text { DB PER }\end{array}$ & $\begin{array}{l}\text { ATTEN } \\
\text { DB PER }\end{array}$ & $\begin{array}{l}\text { DECAY } \\
\text { RATE }\end{array}$ \\
\hline $\begin{array}{l}\text { DEGR } \\
\text { CENT }\end{array}$ & HERTZ & $\begin{array}{l}\text { PER } \\
\text { CENT }\end{array}$ & $\begin{array}{l}\text { PER } \\
\text { METER }\end{array}$ & $\begin{array}{l}\text { PER } \\
\text { ME TER }\end{array}$ & $\begin{array}{l}\text { PER } \\
\text { FOOT }\end{array}$ & $\begin{array}{l}\text { PER } \\
\text { FOOT }\end{array}$ & $\begin{array}{l}100 \\
\text { METER }\end{array}$ & $\begin{array}{l}1000 \\
\text { FEET }\end{array}$ & $\begin{array}{l}\text { DB PER } \\
\text { SECOND }\end{array}$ \\
\hline $\begin{array}{l}10 \\
10 \\
10\end{array}$ & $\begin{array}{l}5940 \\
5940 \\
5940\end{array}$ & $\begin{array}{r}5.0 \\
10.0 \\
15.0\end{array}$ & $\begin{array}{r}.01019 \\
.01917 \\
.02846\end{array}$ & $\begin{array}{r}.04077 \\
.07668 \\
.11384\end{array}$ & $\begin{array}{l}.00320 \\
.00584 \\
.00867\end{array}$ & $\begin{array}{l}.01242 \\
.02337 \\
.03469\end{array}$ & $\begin{array}{r}4.427 \\
8.325 \\
12.360\end{array}$ & $\begin{array}{l}13.493 \\
25.377 \\
37.675\end{array}$ & $\begin{array}{l}14.934 \\
28.087 \\
41.698\end{array}$ \\
\hline $\begin{array}{l}10 \\
10 \\
10\end{array}$ & $\begin{array}{l}5940 \\
5940 \\
5940\end{array}$ & $\begin{array}{l}20.0 \\
25.0 \\
30.0\end{array}$ & $\begin{array}{r}.03209 \\
.03178 \\
.02926\end{array}$ & $\begin{array}{r}.12836 \\
.12714 \\
.11704\end{array}$ & $\begin{array}{l}.00978 \\
.00968 \\
.00891\end{array}$ & $\begin{array}{l}.03912 \\
.03875 \\
.03567\end{array}$ & $\begin{array}{l}13.937 \\
13.804 \\
12.707\end{array}$ & $\begin{array}{l}42.480 \\
42.077 \\
38.734\end{array}$ & $\begin{array}{l}47.016 \\
46.570 \\
42.870\end{array}$ \\
\hline $\begin{array}{l}10 \\
10 \\
10\end{array}$ & $\begin{array}{l}5940 \\
5940 \\
5940\end{array}$ & $\begin{array}{l}35.0 \\
40.0 \\
45.0\end{array}$ & $\begin{array}{l}.02566 \\
.02222 \\
.01966\end{array}$ & $\begin{array}{r}.10265 \\
.08891 \\
.07865\end{array}$ & $\begin{array}{l}.00782 \\
.00677 \\
.00599\end{array}$ & $\begin{array}{r}.03128 \\
.02710 \\
.02397\end{array}$ & $\begin{array}{r}11.145 \\
9.654 \\
8.540\end{array}$ & $\begin{array}{l}33.971 \\
29.427 \\
26.030\end{array}$ & $\begin{array}{l}37.599 \\
32.569 \\
28.810\end{array}$ \\
\hline $\begin{array}{l}10 \\
10\end{array}$ & $\begin{array}{l}5940 \\
5940\end{array}$ & $\begin{array}{l}46.0 \\
47.0\end{array}$ & $\begin{array}{r}.01925 \\
.01884\end{array}$ & $\begin{array}{l}.07700 \\
.07538\end{array}$ & $\begin{array}{l}.00586 \\
.00574\end{array}$ & $\begin{array}{l}.02347 \\
.02297\end{array}$ & $\begin{array}{l}8.360 \\
8.185\end{array}$ & $\begin{array}{l}25 \cdot 483 \\
24 \cdot 948\end{array}$ & $\begin{array}{l}28.205 \\
27.612\end{array}$ \\
\hline 10 & 5940 & 48.0 & .01848 & .07392 & .00563 & .02253 & 8.026 & $24 \cdot 466$ & 27.078 \\
\hline $\begin{array}{l}10 \\
10 \\
10\end{array}$ & $\begin{array}{l}5940 \\
5940 \\
5940\end{array}$ & $\begin{array}{l}49.0 \\
50.0 \\
51.0\end{array}$ & $\begin{array}{r}.01813 \\
.01778 \\
.01744\end{array}$ & $\begin{array}{l}.07254 \\
.07115 \\
.06976\end{array}$ & $\begin{array}{l}.00552 \\
.00542 \\
.00531\end{array}$ & $\begin{array}{r}.02211 \\
.02168 \\
.02126\end{array}$ & $\begin{array}{l}7.876 \\
7.725 \\
7.575\end{array}$ & $\begin{array}{l}24.007 \\
23.548 \\
23.089\end{array}$ & $\begin{array}{l}26.570 \\
26.062 \\
25.554\end{array}$ \\
\hline $\begin{array}{l}10 \\
10 \\
10\end{array}$ & $\begin{array}{l}5940 \\
5940 \\
5940\end{array}$ & $\begin{array}{l}52.0 \\
53.0 \\
54.0\end{array}$ & $\begin{array}{r}.01710 \\
.01676 \\
.01644\end{array}$ & $\begin{array}{l}.06840 \\
.06707 \\
.06577\end{array}$ & $\begin{array}{l}.00521 \\
.00511 \\
.00501\end{array}$ & $\begin{array}{l}.02084 \\
.02044 \\
.02004\end{array}$ & $\begin{array}{l}7.426 \\
7.282 \\
7.141\end{array}$ & $\begin{array}{l}22.637 \\
22.197 \\
21.767\end{array}$ & $\begin{array}{l}25.054 \\
24.568 \\
24.091\end{array}$ \\
\hline $\begin{array}{l}10 \\
10 \\
10\end{array}$ & $\begin{array}{l}5940 \\
5940 \\
5940\end{array}$ & $\begin{array}{l}55.0 \\
60.0 \\
65.0\end{array}$ & $\begin{array}{r}.01614 \\
.01468 \\
.01352\end{array}$ & $\begin{array}{l}.06456 \\
.05872 \\
.05411\end{array}$ & $\begin{array}{l}.00491 \\
.00447 \\
.00412\end{array}$ & $\begin{array}{r}.01967 \\
.01790 \\
.01649\end{array}$ & $\begin{array}{l}7.009 \\
6.376 \\
5.875\end{array}$ & $\begin{array}{l}21.365 \\
19.435 \\
17.909\end{array}$ & $\begin{array}{l}23.647 \\
21.511 \\
19.821\end{array}$ \\
\hline $\begin{array}{l}10 \\
10 \\
10\end{array}$ & $\begin{array}{l}5940 \\
5940 \\
5940\end{array}$ & $\begin{array}{l}70.0 \\
75.0 \\
80.0\end{array}$ & $\begin{array}{r}.01258 \\
.01180 \\
.01105\end{array}$ & $\begin{array}{r}.05033 \\
.04720 \\
.04422\end{array}$ & $\begin{array}{l}.00383 \\
.00359 \\
.00336\end{array}$ & $\begin{array}{r}.01534 \\
.01438 \\
.01347\end{array}$ & $\begin{array}{l}5.465 \\
5.125 \\
4.801\end{array}$ & $\begin{array}{l}16.657 \\
15.623 \\
14.634\end{array}$ & $\begin{array}{l}18 \cdot 436 \\
17 \cdot 291 \\
16.197\end{array}$ \\
\hline $\begin{array}{l}10 \\
10 \\
10\end{array}$ & $\begin{array}{l}5940 \\
5940 \\
5940^{\prime}\end{array}$ & $\begin{array}{l}85.0 \\
90.0 \\
95.0\end{array}$ & $\begin{array}{r}.01042 \\
.00990 \\
.00946\end{array}$ & $\begin{array}{r}.04168 \\
.03960 \\
.03787\end{array}$ & $\begin{array}{l}.00317 \\
.00301 \\
.00288\end{array}$ & $\begin{array}{l}.01270 \\
.01207 \\
.01154\end{array}$ & $\begin{array}{l}4.526 \\
4 \cdot 300 \\
4.112\end{array}$ & $\begin{array}{l}13.796 \\
13.107 \\
12.535\end{array}$ & $\begin{array}{l}15.269 \\
14.507 \\
13.874\end{array}$ \\
\hline 10 & 59401 & 100.0 & .00910 & .03640 & .00277 & .01109 & 3.952 & 12.046 & $13 \cdot 333$ \\
\hline
\end{tabular}


TABULATION OF DATA FOR

ABSERPTIEN OF SOUND IN AIR VERSUS PERCENT RELATIVE HUMIOITY AT 10 DEGREES CENTIGRADE FOR A FREQUENCY OF 6300 HERTZ

\begin{tabular}{|c|c|c|c|c|c|c|c|c|c|}
\hline 11 & (2) & (3) & $(4)$ & (5) & $(6)$ & $(7)$ & $(8)$ & $(9)$ & 1101 \\
\hline $\begin{array}{l}\text { TEMP } \\
\text { DEGR } \\
\text { CENT }\end{array}$ & HERTZ & $\begin{array}{l}\text { REL } \\
\text { HUM } \\
\text { PER } \\
\text { CENT }\end{array}$ & $\begin{array}{l}\text { ATTEN } \\
\text { CEEF } \\
\text { PER } \\
\text { METER }\end{array}$ & $\begin{array}{l}\text { 4M } \\
\text { PER } \\
\text { METER }\end{array}$ & $\begin{array}{l}\text { ATTEN } \\
\text { COEF } \\
\text { PER } \\
\text { FOOT }\end{array}$ & $\begin{array}{l}\text { 4M } \\
\text { PER } \\
\text { FOOT }\end{array}$ & $\begin{array}{l}\text { ATTEN } \\
\text { DB PER } \\
1 \text { OO } \\
\text { METER }\end{array}$ & $\begin{array}{l}\text { ATTEN } \\
\text { DB PER } \\
1000 \\
\text { FEET }\end{array}$ & $\begin{array}{l}\text { DECAY } \\
\text { RATE } \\
\text { OB PER } \\
\text { SECOND }\end{array}$ \\
\hline $\begin{array}{l}10 \\
10 \\
10\end{array}$ & $\begin{array}{l}6300 \\
6300 \\
6300\end{array}$ & $\begin{array}{r}5.0 \\
10.0 \\
15.0\end{array}$ & $\begin{array}{l}.01054 \\
.02971 \\
.02934\end{array}$ & $\begin{array}{r}.04219 \\
.07884 \\
.11736\end{array}$ & $\begin{array}{l}.00321 \\
.00600 \\
.00894\end{array}$ & $\begin{array}{l}.01286 \\
.02403 \\
.03577\end{array}$ & $\begin{array}{r}4.581 \\
. .560 \\
12.743\end{array}$ & $\begin{array}{l}13.965 \\
26.091 \\
38.841\end{array}$ & $\begin{array}{l}15.456 \\
28.877 \\
42.988\end{array}$ \\
\hline $\begin{array}{l}10 \\
10 \\
10\end{array}$ & $\begin{array}{l}6300 \\
6300 \\
6300\end{array}$ & $\begin{array}{l}20.0 \\
25.0 \\
30.0\end{array}$ & $\begin{array}{l}.03394 \\
.03402 \\
.03186\end{array}$ & $\begin{array}{l}-13578 \\
-13611 \\
-12745\end{array}$ & $\begin{array}{r}.01034 \\
.01037 \\
.00971\end{array}$ & $\begin{array}{l}.04138 \\
.04148 \\
.03884\end{array}$ & $\begin{array}{l}14.743 \\
14.778 \\
13.838\end{array}$ & $\begin{array}{l}44 . \\
45 . \\
42 .\end{array}$ & $\begin{array}{l}35 \\
56 \\
82\end{array}$ \\
\hline $\begin{array}{l}10 \\
10 \\
10\end{array}$ & $\begin{array}{l}6300 \\
6300 \\
6300\end{array}$ & $\begin{array}{l}35.0 \\
40.0 \\
45.0\end{array}$ & $\begin{array}{l}.02825 \\
.02455 \\
.02172\end{array}$ & $\begin{array}{l}.11300 \\
.09823 \\
.08690\end{array}$ & $\begin{array}{l}.00861 \\
.00748 \\
.00662\end{array}$ & $\begin{array}{l}.03444 \\
.02994 \\
.02648\end{array}$ & $\begin{array}{r}12.269 \\
10.665 \\
9.436\end{array}$ & $\begin{array}{l}37 \\
32 . \\
28 .\end{array}$ & $\begin{array}{l}4 ! \\
3 ! \\
3\end{array}$ \\
\hline $\begin{array}{l}10 \\
10 \\
10\end{array}$ & $\begin{array}{l}6300 \\
6300 \\
6300\end{array}$ & $\begin{array}{l}46.0 \\
47.0 \\
48.0\end{array}$ & $\begin{array}{r}.02125 \\
.02079 \\
.02037\end{array}$ & $\begin{array}{l}.08501 \\
.08316 \\
.08151\end{array}$ & $\begin{array}{l}.00647 \\
.00633 \\
.00621\end{array}$ & $\begin{array}{l}.02591 \\
.02534 \\
.02484\end{array}$ & $\begin{array}{l}30 \\
29 \\
50\end{array}$ & $\begin{array}{l}135 \\
823 \\
975\end{array}$ & $\begin{array}{l}139 \\
461 \\
055\end{array}$ \\
\hline $\begin{array}{l}10 \\
10 \\
10\end{array}$ & $\begin{array}{l}6300 \\
6300 \\
6300\end{array}$ & $\begin{array}{l}49.0 \\
50.0 \\
51.0\end{array}$ & $\begin{array}{l}.01996 \\
.01960 \\
.01925\end{array}$ & $\begin{array}{l}.07985 \\
.07842 \\
.07700\end{array}$ & $\begin{array}{l}.00608 \\
.00597 \\
.00586\end{array}$ & $\begin{array}{l}.0 \\
.0 \\
.0\end{array}$ & & & $\begin{array}{l}249 \\
724 \\
205\end{array}$ \\
\hline $\begin{array}{l}10 \\
10 \\
10\end{array}$ & $\begin{array}{l}6300 \\
6300 \\
6300\end{array}$ & $\begin{array}{l}52.0 \\
53.0 \\
54.0\end{array}$ & $\begin{array}{r}.01889 \\
.01854 \\
.01819\end{array}$ & $\begin{array}{r}.07558 \\
.07416 \\
.07277\end{array}$ & $\begin{array}{l}.00 \\
.00 \\
.00\end{array}$ & $\begin{array}{r}.02303 \\
.02260 \\
.02218\end{array}$ & & & $\begin{array}{l}585 \\
66 \\
557\end{array}$ \\
\hline $\begin{array}{l}10 \\
10 \\
10\end{array}$ & $\begin{array}{l}6300 \\
6300 \\
6300\end{array}$ & $\begin{array}{l}5 \\
6 \\
6\end{array}$ & $\begin{array}{l}.01785 \\
.01631 \\
.01498\end{array}$ & $\begin{array}{r}.07141 \\
.06524 \\
.05993\end{array}$ & $\begin{array}{r}.00544 \\
.00497 \\
.00456\end{array}$ & $\begin{array}{r}.02176 \\
.01988 \\
.01826\end{array}$ & & $\begin{array}{l}35 \\
91 \\
36\end{array}$ & $\begin{array}{l}26.159 \\
23.897 \\
21.954\end{array}$ \\
\hline $\begin{array}{l}10 \\
10 \\
10\end{array}$ & $\begin{array}{l}6300 \\
6300 \\
6300\end{array}$ & $\begin{array}{l}70.0 \\
75.0 \\
80.0\end{array}$ & $\begin{array}{r}.01390 \\
.01302 \\
.01224\end{array}$ & $\begin{array}{r}.05562 \\
.05208 \\
.04896\end{array}$ & $\begin{array}{l}.00423 \\
.00396 \\
.00373\end{array}$ & $\begin{array}{r}.01695 \\
.01587 \\
.01492\end{array}$ & $\begin{array}{l}9 \\
3 \\
6\end{array}$ & $\begin{array}{l}09 \\
37 \\
05\end{array}$ & $\begin{array}{l}375 \\
077 \\
935\end{array}$ \\
\hline $\begin{array}{l}10 \\
10 \\
10\end{array}$ & $\begin{array}{l}6300 \\
6300 \\
6300\end{array}$ & $\begin{array}{l}85.0 \\
90.0 \\
95.0\end{array}$ & $\begin{array}{r}.01133 \\
.01092 \\
.01043\end{array}$ & $\begin{array}{l}.04614 \\
.04370 \\
.04172\end{array}$ & $\begin{array}{r}.00 \\
.00 \\
00\end{array}$ & $\begin{array}{r}.01406 \\
.01332 \\
.01271\end{array}$ & $\begin{array}{l}9 \\
5 \\
9\end{array}$ & $\begin{array}{l}70 \\
55 \\
06\end{array}$ & $\begin{array}{l}16.901 \\
16.009 \\
15.201\end{array}$ \\
\hline 10 & 6300 & 100 & .01001 & .04005 & .00305 & .01220 & 4.349 & 13.256 & 14.672 \\
\hline
\end{tabular}


TABULATION OF DATA FOR

ABSORPTION OF SOUND IN AIR VERSUS PERCENT RELATIVE HUMIDITY AT 10 DEGREES GENTIGRADE FOR A FREQUENCY OP 8000 HERTZ

\begin{tabular}{|c|c|c|c|c|c|c|c|c|c|}
\hline$(1)$ & $(2)$ & (3) & 141 & (5) & $(6)$ & 171 & $(8)$ & 191 & 1101 \\
\hline EMP & FREQ & REL & ATTEN & $4 M$ & ATTEN & $4 M$ & ATTEN & ATTEN & DECAY \\
\hline $\begin{array}{l}\text { DEGR } \\
\text { CENT }\end{array}$ & HERTZ & $\begin{array}{l}\text { HUM } \\
\text { PER } \\
\text { CENT }\end{array}$ & $\begin{array}{l}\text { COEF } \\
\text { PER } \\
\text { METER }\end{array}$ & $\begin{array}{l}\text { PER } \\
\text { METER }\end{array}$ & $\begin{array}{l}\text { COEF } \\
\text { PER } \\
\text { FOOT }\end{array}$ & $\begin{array}{l}\text { PER } \\
\text { FOOT }\end{array}$ & $\begin{array}{l}\text { DB PER } \\
\text { IOO } \\
\text { METER }\end{array}$ & $\begin{array}{l}\text { DB PER } \\
1000 \\
\text { FEET }\end{array}$ & $\begin{array}{l}\text { RATE } \\
\text { DB PEI } \\
\text { SECON }\end{array}$ \\
\hline 10 & 8000 & 5.0 & .01221 & .04886 & .00372 & .01489 & 5.305 & 16.172 & 7.89 \\
\hline $\begin{array}{l}10 \\
10\end{array}$ & $\begin{array}{l}8000 \\
8000\end{array}$ & $\begin{array}{l}10.0 \\
15.0\end{array}$ & $\begin{array}{l}.02219 \\
.03270\end{array}$ & $\begin{array}{r}.08877 \\
.13081\end{array}$ & $\begin{array}{r}.00676 \\
.00996\end{array}$ & $\begin{array}{l}.02705 \\
.03987\end{array}$ & $\begin{array}{r}9.639 \\
14.202\end{array}$ & $\begin{array}{l}29.380 \\
43.290\end{array}$ & ? \\
\hline $\begin{array}{l}10 \\
10 \\
10\end{array}$ & $\begin{array}{l}8000 \\
8000 \\
8000\end{array}$ & $\begin{array}{l}20.0 \\
25.0 \\
30.0\end{array}$ & $\begin{array}{r}.04123 \\
.04395 \\
.04346\end{array}$ & $\begin{array}{r}.16492 \\
.17580 \\
.17384\end{array}$ & $\begin{array}{r}.01256 \\
.01339 \\
.01324\end{array}$ & $\begin{array}{r}.05026 \\
.05358 \\
.05298\end{array}$ & $\begin{array}{l}17.906 \\
19.088 \\
18.875\end{array}$ & $\begin{array}{l}54.580 \\
58.181 \\
57.533\end{array}$ & $\begin{array}{l}60.408 \\
64.393 \\
63.676\end{array}$ \\
\hline $\begin{array}{l}10 \\
10 \\
10\end{array}$ & $\begin{array}{l}8000 \\
8000 \\
8000\end{array}$ & $\begin{array}{l}35.0 \\
40.0 \\
45.0\end{array}$ & $\begin{array}{r}.04092 \\
.03699 \\
.03300\end{array}$ & $\begin{array}{r}.16369 \\
.14799 \\
.13202\end{array}$ & $\begin{array}{r}.01247 \\
.01127 \\
.01006\end{array}$ & $\begin{array}{r}.04989 \\
.04510 \\
.04024\end{array}$ & $\begin{array}{l}17.772 \\
16.068 \\
14.334\end{array}$ & $\begin{array}{l}54.171 \\
48.978 \\
43.691\end{array}$ & $\begin{array}{l}59.956 \\
54.208 \\
48.356\end{array}$ \\
\hline $\begin{array}{l}10 \\
10 \\
10\end{array}$ & $\begin{array}{l}8000 \\
8000 \\
8000\end{array}$ & $\begin{array}{l}46.0 \\
47.0 \\
48.0\end{array}$ & $\begin{array}{r}.03218 \\
.03139 \\
.03075\end{array}$ & $\begin{array}{r}.12873 \\
.12559 \\
.12302\end{array}$ & $\begin{array}{l}.00980 \\
.00957 \\
.00937\end{array}$ & $\begin{array}{l}.03923 \\
.03828 \\
.03749\end{array}$ & $\begin{array}{l}13.977 \\
13.636 \\
13.357\end{array}$ & $\begin{array}{l}42.604 \\
41.565 \\
40.713\end{array}$ & $\begin{array}{l}47 . \\
46 \\
45\end{array}$ \\
\hline $\begin{array}{l}10 \\
10 \\
10\end{array}$ & $\begin{array}{l}8000 \\
8000 \\
8000\end{array}$ & $\begin{array}{l}49.0 \\
50.0 \\
51.0\end{array}$ & $\begin{array}{r}.03011 \\
.02949 \\
.02890\end{array}$ & $\begin{array}{r}.12044 \\
\cdot 11798 \\
\cdot 11560\end{array}$ & $\begin{array}{l}.00917 \\
.00899 \\
.00880\end{array}$ & $\begin{array}{r}.03671 \\
.03596 \\
.03523\end{array}$ & $\begin{array}{l}13.077 \\
12.810 \\
12.552\end{array}$ & $\begin{array}{l}39.861 \\
39.047 \\
38.259\end{array}$ & $\begin{array}{l}44.117 \\
43.216 \\
42.344\end{array}$ \\
\hline 10 & 8000 & 52.0 & .02 & .11 & .0 & .03 & & 37 & 47 \\
\hline $\begin{array}{l}10 \\
10\end{array}$ & $\begin{array}{l}8000 \\
8000\end{array}$ & $\begin{array}{l}53.0 \\
54.0\end{array}$ & $\begin{array}{l}.02 \\
.02\end{array}$ & $\begin{array}{l}.11 \\
.10\end{array}$ & .08 & $\begin{array}{r}.03388 \\
.03325\end{array}$ & $\begin{array}{l}12 \\
11\end{array}$ & $\begin{array}{l}36.789 \\
36.107\end{array}$ & $\begin{array}{l}40.71 \\
39.96\end{array}$ \\
\hline $\begin{array}{l}10 \\
10 \\
10\end{array}$ & $\begin{array}{l}8000 \\
8000 \\
8000\end{array}$ & $\begin{array}{l}55.0 \\
60.0 \\
65.0\end{array}$ & $\begin{array}{r}.02678 \\
.02471 \\
.02282\end{array}$ & $\begin{array}{r}.10714 \\
.09885 \\
.09130\end{array}$ & $\begin{array}{l}.00816 \\
.00753 \\
.00695\end{array}$ & $\begin{array}{l}.03265 \\
.03013 \\
.02782\end{array}$ & $\begin{array}{r}11.633 \\
10.733 \\
9.913\end{array}$ & $\begin{array}{l}35.459 \\
32.716 \\
30.215\end{array}$ & $\begin{array}{l}39.246 \\
36.209 \\
33.441\end{array}$ \\
\hline $\begin{array}{l}10 \\
10 \\
10\end{array}$ & $\begin{array}{l}8000 \\
8000 \\
8000\end{array}$ & $\begin{array}{l}70.0 \\
75.0 \\
80.0\end{array}$ & $\begin{array}{l}.02117 \\
.01970 \\
.01848\end{array}$ & $\begin{array}{r}.08469 \\
.07882 \\
.07393\end{array}$ & $\begin{array}{l}.00645 \\
.00600 \\
.00563\end{array}$ & $\begin{array}{l}.02581 \\
.02402 \\
.02253\end{array}$ & $\begin{array}{l}9.195 \\
8.558 \\
8.027\end{array}$ & $\begin{array}{l}28.029 \\
26.086 \\
24.467\end{array}$ & $\begin{array}{l}31.022 \\
28.872 \\
27.080\end{array}$ \\
\hline $\begin{array}{l}10 \\
10 \\
10\end{array}$ & $\begin{array}{l}8000 \\
8000 \\
8000\end{array}$ & $\begin{array}{l}85.0 \\
90.0 \\
95.0\end{array}$ & $\begin{array}{r}.01745 \\
.01658 \\
.01573\end{array}$ & $\begin{array}{l}.06982 \\
.06633 \\
.06295\end{array}$ & $\begin{array}{r}.00532 \\
.00505 \\
.00479\end{array}$ & $\begin{array}{r}.02128 \\
.02022 \\
.01918\end{array}$ & $\begin{array}{l}7.581 \\
7.202 \\
6.835\end{array}$ & $\begin{array}{l}23.108 \\
21.954 \\
20.833\end{array}$ & $\begin{array}{l}25.57 \\
24.29 \\
23.05\end{array}$ \\
\hline 10 & 8000 & 100.0 & .01499 & .05997 & .00457 & .01828 & 6.512 & 19.849 & 1.96 \\
\hline
\end{tabular}


TABULATION OF DATA FOR

ABSORPTION OF SOUND IN AIR VERSUS PERCENT RELATIVE HUMIDITY AT 10 DEGREES CENTIGRADE FOR A FREQUENCY OF 10000 HERTZ

\begin{tabular}{|c|c|c|c|c|c|c|c|c|c|}
\hline 11 & (2) & (3) & 141 & (5) & (6) & 171 & $(8)$ & 191 & 1101 \\
\hline EMP & FREQ & $\begin{array}{l}\text { REL } \\
\text { HUM }\end{array}$ & $\begin{array}{l}\text { ATTEN } \\
\text { COEF }\end{array}$ & $4 M$ & $\begin{array}{l}\text { ATTEN } \\
\text { COEF }\end{array}$ & $4 M$ & $\begin{array}{l}\text { ATTEN } \\
\text { DB PER }\end{array}$ & $\begin{array}{l}\text { ATTEN } \\
\text { DB PER }\end{array}$ & $\begin{array}{l}\text { DECAY } \\
\text { RATE }\end{array}$ \\
\hline $\begin{array}{l}E G R \\
\text { ENT }\end{array}$ & HERTZ & $\begin{array}{l}\text { PER } \\
\text { CENT }\end{array}$ & $\begin{array}{l}\text { PER } \\
\text { METER }\end{array}$ & $\begin{array}{l}\text { PER } \\
\text { METER }\end{array}$ & $\begin{array}{l}\text { PER. } \\
\text { FOOT }\end{array}$ & $\begin{array}{l}\text { PER } \\
\text { FOOT }\end{array}$ & $\begin{array}{l}100 \\
\text { METER }\end{array}$ & $\begin{array}{l}1000 \\
\text { FEET }\end{array}$ & $\begin{array}{l}\text { DB PER } \\
\text { SECOND }\end{array}$ \\
\hline $\begin{array}{l}10 \\
10 \\
10\end{array}$ & $\begin{array}{l}10000 \\
10000 \\
10000\end{array}$ & $\begin{array}{r}5.0 \\
10.0 \\
15.0\end{array}$ & $\begin{array}{l}.01434 \\
.02518 \\
.03615\end{array}$ & $\begin{array}{r}.05737 \\
.10075 \\
.14461\end{array}$ & $\begin{array}{r}.00437 \\
.00767 \\
.01101\end{array}$ & $\begin{array}{l}.017 .48 \\
.03071 \\
.04407\end{array}$ & $\begin{array}{r}6.229 \\
10.939 \\
15.701\end{array}$ & $\begin{array}{l}18.989 \\
33.345 \\
47.858\end{array}$ & $\begin{array}{l}21.016 \\
36.905 \\
52.968\end{array}$ \\
\hline $\begin{array}{l}10 \\
10 \\
10\end{array}$ & $\begin{array}{l}10000 \\
10000 \\
10000\end{array}$ & $\begin{array}{l}20.0 \\
25.0 \\
30.0\end{array}$ & $\begin{array}{l}.04764 \\
.05414 \\
.05579\end{array}$ & $\begin{array}{r}19059 \\
.21657 \\
.22317\end{array}$ & $\begin{array}{l}.01452 \\
.01650 \\
.01700\end{array}$ & $\begin{array}{l}.05809 \\
.06601 \\
.06802\end{array}$ & $\begin{array}{l}20.694 \\
23.514 \\
24.230\end{array}$ & $\begin{array}{l}63.077 \\
71.673 \\
73.856\end{array}$ & $\begin{array}{l}69.812 \\
79.327 \\
81.742\end{array}$ \\
\hline $\begin{array}{l}10 \\
10 \\
10\end{array}$ & $\begin{array}{l}10000 \\
10000 \\
10000\end{array}$ & $\begin{array}{l}35.0 \\
40.0 \\
45.0\end{array}$ & $\begin{array}{r}.05485 \\
.05202 \\
.04782\end{array}$ & $\begin{array}{r}.21941 \\
.20811 \\
.19129\end{array}$ & $\begin{array}{l}.01671 \\
.01585 \\
.01457\end{array}$ & $\begin{array}{l}.06687 \\
.06343 \\
.05830\end{array}$ & $\begin{array}{l}23.822 \\
22.595 \\
20.769\end{array}$ & $\begin{array}{l}72.6 \\
68.8 \\
63.3\end{array}$ & $\begin{array}{l}80.365 \\
76.227 \\
70.067\end{array}$ \\
\hline $\begin{array}{l}10 \\
10 \\
10\end{array}$ & $\begin{array}{l}10000 \\
10000 \\
10000\end{array}$ & $\begin{array}{l}46.0 \\
47.0 \\
48.0\end{array}$ & $\begin{array}{l}.04694 \\
.04607 \\
.04520\end{array}$ & $\begin{array}{r}.18779 \\
.18429 \\
.18081\end{array}$ & $\begin{array}{r}.01431 \\
.01404 \\
.01377\end{array}$ & $\begin{array}{l}.05724 \\
.05617 \\
.05511\end{array}$ & $\begin{array}{l}20.390 \\
20.010 \\
19.631\end{array}$ & & $\begin{array}{l}68.785 \\
67.504 \\
66.227\end{array}$ \\
\hline 10 & 10000 & 49.0 & .04434 & .17738 & .01351 & .05406 & 19.259 & $58 \cdot 703$ & 64.971 \\
\hline $\begin{array}{l}10 \\
10\end{array}$ & $\begin{array}{l}10000 \\
10000\end{array}$ & $\begin{array}{l}50 \cdot 0 \\
51.0\end{array}$ & $\begin{array}{l}.04348 \\
.04262\end{array}$ & $\begin{array}{r}.17395 \\
.17050\end{array}$ & $\begin{array}{r}.01325 \\
.01299\end{array}$ & & $\begin{array}{l}887 \\
512\end{array}$ & & $\begin{array}{l}63.715 \\
62.451\end{array}$ \\
\hline $\begin{array}{l}10 \\
10 \\
10\end{array}$ & $\begin{array}{l}10000 \\
10000 \\
10000\end{array}$ & $\begin{array}{l}52.0 \\
53.0 \\
54.0\end{array}$ & $\begin{array}{l}.04173 \\
.04084 \\
.03998\end{array}$ & $\begin{array}{r}16693 \\
.16336 \\
.15993\end{array}$ & $\begin{array}{r}.01272 \\
.01244 \\
.01218\end{array}$ & $\begin{array}{l}.05088 \\
.04979 \\
.04874\end{array}$ & $\begin{array}{l}18.124 \\
17.737 \\
17.365\end{array}$ & $\begin{array}{l}55.245 \\
54 \cdot 064 \\
52.929\end{array}$ & $\begin{array}{l}61.144 \\
59.837 \\
58.581\end{array}$ \\
\hline $\begin{array}{l}10 \\
10 \\
10\end{array}$ & $\begin{array}{l}10000 \\
10000 \\
10000\end{array}$ & $\begin{array}{l}55.0 \\
60.0 \\
65.0\end{array}$ & $\begin{array}{r}.03928 \\
.03597 \\
.03332\end{array}$ & $\begin{array}{l}.15713 \\
.14388 \\
\cdot 13330\end{array}$ & $\begin{array}{r}.01197 \\
.01096 \\
.01015\end{array}$ & $\begin{array}{r}.04789 \\
.04385 \\
.04063\end{array}$ & $\begin{array}{l}17.061 \\
15.622 \\
14.473\end{array}$ & $\begin{array}{l}52.003 \\
47.617 \\
44.116\end{array}$ & $\begin{array}{l}57.555 \\
52.701 \\
48.827\end{array}$ \\
\hline $\begin{array}{l}10 \\
10 \\
10\end{array}$ & $\begin{array}{l}10000 \\
10000 \\
10000\end{array}$ & $\begin{array}{l}70.0 \\
75.0 \\
80.0\end{array}$ & $\begin{array}{l}.03117 \\
.02913 \\
.02734\end{array}$ & $\begin{array}{r}.12469 \\
.11655 \\
.10937\end{array}$ & $\begin{array}{l}.00950 \\
.00888 \\
.00833\end{array}$ & $\begin{array}{r}.03800 \\
.03552 \\
.03333\end{array}$ & $\begin{array}{r}13.538 \\
12.655 \\
11.874\end{array}$ & $\begin{array}{l}41.266 \\
38.574 \\
36.195\end{array}$ & $\begin{array}{l}45.672 \\
42.692 \\
40.060\end{array}$ \\
\hline $\begin{array}{l}10 \\
10 \\
10\end{array}$ & $\begin{array}{l}10000 \\
10000 \\
10000\end{array}$ & $\begin{array}{l}85.0 \\
90.0 \\
95.0\end{array}$ & $\begin{array}{r}.02568 \\
.02429 \\
.02311\end{array}$ & $\begin{array}{r}.10273 \\
.09717 \\
.09244\end{array}$ & $\begin{array}{l}.00782 \\
.00740 \\
.00704\end{array}$ & $\begin{array}{l}.03131 \\
.02961 \\
.02817\end{array}$ & $\begin{array}{l}11.154 \\
10.550 \\
10.036\end{array}$ & $\begin{array}{l}33.998 \\
32.159 \\
30.592\end{array}$ & $\begin{array}{l}37.628 \\
35.593 \\
33.859\end{array}$ \\
\hline 10 & 10000 & 10 & .02207 & .08828 & .00672 & .02691 & 9.585 & $29 \cdot 218$ & $32 \cdot 338$ \\
\hline
\end{tabular}


TABULATION OF DATA FOR

ABSORPTION OF SOUND IN AIR VERSUS PERCENT RELATIVE HUMIDITY AT 10 DEGREES CENTIGRADE FOR A FREQUENCY. OF 12500 HERTZ

\begin{tabular}{|c|c|c|c|c|c|c|c|c|c|}
\hline (1) & $(2)$ & (3) & 141 & (5) & (6) & 171 & $(8)$ & $(9)$ & $(10)$ \\
\hline $\begin{array}{l}\text { TEMP } \\
\text { DEGR } \\
\text { CENT }\end{array}$ & HERTZ & $\begin{array}{l}\text { REL } \\
\text { HUM } \\
\text { PER } \\
\text { CENT }\end{array}$ & $\begin{array}{l}\text { ATTEN } \\
\text { COEF } \\
\text { PER } \\
\text { METER }\end{array}$ & $\begin{array}{l}4 M \\
\cdot \\
\text { PER } \\
\text { METER }\end{array}$ & $\begin{array}{l}\text { ATTEN } \\
\text { COEF } \\
\text { PER } \\
\text { FOOT }\end{array}$ & $\begin{array}{l}4 M \\
\text { PER } \\
\text { FOOT }\end{array}$ & $\begin{array}{l}\text { ATTEN } \\
\text { DB PER } \\
100 \\
\text { METER }\end{array}$ & $\begin{array}{l}\text { ATTEN } \\
\text { DB PER } \\
1000 \\
\text { FEET }\end{array}$ & $\begin{array}{l}\text { DECAY } \\
\text { RATE } \\
\text { DB PER } \\
\text { SECOND }\end{array}$ \\
\hline $\begin{array}{l}10 \\
10 \\
10\end{array}$ & $\begin{array}{l}12500 \\
12500 \\
12500\end{array}$ & $\begin{array}{r}5.0 \\
10.0 \\
15.0\end{array}$ & $\begin{array}{r}.01727 \\
.02909 \\
.04091\end{array}$ & $\begin{array}{l}.06910 \\
.11637 \\
.16364\end{array}$ & $\begin{array}{l}.00526 \\
.00886 \\
.01246\end{array}$ & $\begin{array}{l}.02106 \\
.03547 \\
.04987\end{array}$ & $\begin{array}{r}7.503 \\
12.635 \\
17.767\end{array}$ & $\begin{array}{l}22.870 \\
38.513 \\
54.156\end{array}$ & $\begin{array}{l}25.312 \\
42.626 \\
59.939\end{array}$ \\
\hline $\begin{array}{l}10 \\
10 \\
10\end{array}$ & $\begin{array}{l}12500 \\
12500 \\
12500\end{array}$ & $\begin{array}{l}20.0 \\
25.0 \\
30.0\end{array}$ & $\begin{array}{l}.05359 \\
.06447 \\
.06965\end{array}$ & $\begin{array}{r}.21439 \\
.25788 \\
.27860\end{array}$ & $\begin{array}{l}.01633 \\
.01965 \\
.02122\end{array}$ & $\begin{array}{l}.06534 \\
.07860 \\
.08491\end{array}$ & $\begin{array}{l}23.277 \\
28.000 \\
30.249\end{array}$ & $\begin{array}{l}70.951 \\
85.345 \\
92.202\end{array}$ & $\begin{array}{r}78.527 \\
94.458 \\
102.047\end{array}$ \\
\hline $\begin{array}{l}10 \\
10 \\
10\end{array}$ & $\begin{array}{l}12500 \\
12500 \\
12500\end{array}$ & $\begin{array}{l}35.0 \\
40.0 \\
45.0\end{array}$ & $\begin{array}{l}.07078 \\
.06970 \\
.06679\end{array}$ & $\begin{array}{l}.28312 \\
.27883 \\
.26718\end{array}$ & $\begin{array}{l}.02157 \\
.02124 \\
.02035\end{array}$ & $\begin{array}{l}.08629 \\
.08498 \\
.08143\end{array}$ & $\begin{array}{l}30.740 \\
30.274 \\
29.009\end{array}$ & $\begin{array}{l}93.698 \\
92.277 \\
88.422\end{array}$ & $\begin{array}{r}103.702 \\
102.130 \\
97.863\end{array}$ \\
\hline $\begin{array}{l}10 \\
10 \\
10\end{array}$ & $\begin{array}{l}12500 \\
12500 \\
12500\end{array}$ & $\begin{array}{l}46.0 \\
47.0 \\
48.0\end{array}$ & $\begin{array}{l}.06603 \\
.06519 \\
.06431\end{array}$ & $\begin{array}{r}.26413 \\
.26076 \\
.25726\end{array}$ & $\begin{array}{r}.02012 \\
.01987 \\
.01960\end{array}$ & $\begin{array}{l}.08050 \\
.07948 \\
.07841\end{array}$ & $\begin{array}{l}28.678 \\
28.312 \\
27.932\end{array}$ & $\begin{array}{l}87.412 \\
86.298 \\
85.137\end{array}$ & $\begin{array}{l}96.746 \\
95.513 \\
94.228\end{array}$ \\
\hline $\begin{array}{l}10 \\
10 \\
10:\end{array}$ & $\begin{array}{l}12500 \\
12500 \\
12500\end{array}$ & $\begin{array}{l}49.0 \\
50.0 \\
51.0\end{array}$ & $\begin{array}{l}.06336 \\
.06241 \\
.06145\end{array}$ & $\begin{array}{r}.25345 \\
.24964 \\
.24583\end{array}$ & $\begin{array}{l}.01931 \\
.01902 \\
.01873\end{array}$ & $\begin{array}{l}.07725 \\
.07609 \\
.07492\end{array}$ & $\begin{array}{l}27.518 \\
27.104 \\
26.691\end{array}$ & $\begin{array}{l}83.878 \\
82.616 \\
81.355\end{array}$ & $\begin{array}{l}92.834 \\
91.438 \\
90.042\end{array}$ \\
\hline $\begin{array}{l}10 \\
10 \\
10\end{array}$ & $\begin{array}{l}12500 \\
12500 \\
12500\end{array}$ & $\begin{array}{l}52 \cdot 0 \\
53 \cdot 0 \\
54 \cdot 0\end{array}$ & $\begin{array}{r}.06050 \\
.05955 \\
.05859\end{array}$ & $\begin{array}{l}.24201 \\
.23820 \\
.23439\end{array}$ & $\begin{array}{l}.01844 \\
.01815 \\
.01786\end{array}$ & $\begin{array}{l}.07376 \\
.07260 \\
.07144\end{array}$ & $\begin{array}{l}26.277 \\
25.863 \\
25.449\end{array}$ & $\begin{array}{l}80.093 \\
78.832 \\
77.570\end{array}$ & $\begin{array}{l}88.64 .5 \\
87.249 \\
85.853\end{array}$ \\
\hline $\begin{array}{l}10 \\
10 \\
10:\end{array}$ & $\begin{array}{l}12500 \\
12500 \\
12500\end{array}$ & $\begin{array}{l}55.0 \\
60.0 \\
65.0\end{array}$ & $\begin{array}{l}.05764 \\
.05291 \\
.04873\end{array}$ & $\begin{array}{r}.23059 \\
.21165 \\
.19494\end{array}$ & $\begin{array}{l}.01757 \\
.01612 \\
.01485\end{array}$ & $\begin{array}{l}.07028 \\
.06451 \\
.05942\end{array}$ & $\begin{array}{l}25.036 \\
22.980 \\
21.166\end{array}$ & $\begin{array}{l}76.312 \\
70.045 \\
64.516\end{array}$ & $\begin{array}{l}84.460 \\
77.524 \\
71.405\end{array}$ \\
\hline $\begin{array}{l}10 \\
1.0 \\
10\end{array}$ & $\begin{array}{l}12500 \\
12500 \\
12500\end{array}$ & $\begin{array}{l}70.0 \\
75.0 \\
80.0\end{array}$ & $\begin{array}{l}.04533 \\
.04251 \\
.04018\end{array}$ & $\begin{array}{r}18133 \\
.17004 \\
.16075\end{array}$ & $\begin{array}{l}.01381 \\
.01295 \\
.01224\end{array}$ & $\begin{array}{l}.05527 \\
.05183 \\
.04899\end{array}$ & $\begin{array}{l}19.688 \\
18.462 \\
17.454\end{array}$ & $\begin{array}{l}60.012 \\
56.275 \\
53.201\end{array}$ & $\begin{array}{l}66.420 \\
62.284 \\
58.881\end{array}$ \\
\hline $\begin{array}{l}10 \\
10 \\
10\end{array}$ & $\begin{array}{l}12500 \\
12500 \\
12500\end{array}$ & $\begin{array}{l}85.0 \\
90.0 \\
95.0\end{array}$ & $\begin{array}{r}.03795 \\
.03593 \\
.03403\end{array}$ & $\begin{array}{r}.15181 \\
.14375 \\
.13613\end{array}$ & $\begin{array}{l}.01156 \\
.01095 \\
.01037\end{array}$ & $\begin{array}{r}.04627 \\
.04381 \\
.04149\end{array}$ & $\begin{array}{l}16.483 \\
15.608 \\
14.780\end{array}$ & $\begin{array}{l}50.241 \\
47.574 \\
45.051\end{array}$ & $\begin{array}{l}55.605 \\
52.654 \\
49.862\end{array}$ \\
\hline 10 & 500 & 100.0 & .03242 & .12971 & .00988 & .03953 & 14.083 & 42.928 & 47.512 \\
\hline
\end{tabular}


TABULATION OF DATA FOR

ABSORPTION OF SOUND IN AIR VERSUS PERCENT RELATIVE HUMIDITY AT 15 DEGREES CENTIGRADE FOR A FREQUENCY OF 125 HERTZ

\begin{tabular}{|c|c|c|c|c|c|c|c|c|c|}
\hline (1) & $(2)$ & $(3)$ & $(4)$ & (5) & $(6)$ & 171 & 181 & 191 & $(10\}$ \\
\hline $\begin{array}{l}\text { TEMP } \\
\text { DEGR } \\
\text { CENT }\end{array}$ & HERTZ & $\begin{array}{l}\text { REL } \\
\text { HUM } \\
\text { PER } \\
\text { CENT }\end{array}$ & $\begin{array}{l}\text { ATTEN } \\
\text { COEF } \\
\text { PER } \\
\text { METER }\end{array}$ & $\begin{array}{l}4 M \\
\text { PER } \\
\text { METER }\end{array}$ & $\begin{array}{l}\text { ATTEN } \\
\text { COEF } \\
\text { PER } \\
\text { FOOT }\end{array}$ & $\begin{array}{l}4 M \\
\text { PER } \\
\text { FOOT. }\end{array}$ & $\begin{array}{l}\text { ATTEN } \\
\text { DB PER } \\
100 \\
\text { METER }\end{array}$ & $\begin{array}{l}\text { ATTEN } \\
\text { DB PER } \\
1000 \\
\text { FEET }\end{array}$ & $\begin{array}{l}\text { DECAY } \\
\text { RATE } \\
\text { DB PER } \\
\text { SECOND }\end{array}$ \\
\hline $\begin{array}{l}15 \\
15 \\
15\end{array}$ & $\begin{array}{l}125 \\
125 \\
125\end{array}$ & $\begin{array}{r}5.0 \\
10.0 \\
15.0\end{array}$ & $\begin{array}{l}.00025 \\
.00014 \\
.00012\end{array}$ & $\begin{array}{l}.00102 \\
.00057 \\
.00049\end{array}$ & $\begin{array}{l}.00007 \\
.00004 \\
.00003\end{array}$ & $\begin{array}{l}.00031 \\
.00017 \\
.00015\end{array}$ & $\begin{array}{r}.111 \\
.062 \\
.054\end{array}$ & $\begin{array}{l}.338 \\
.191 \\
.165\end{array}$ & $\begin{array}{l}.378 \\
.213 \\
.184\end{array}$ \\
\hline $\begin{array}{l}15 \\
15\end{array}$ & $\begin{array}{l}125 \\
125\end{array}$ & $\begin{array}{l}20.0 \\
25.0\end{array}$ & $\begin{array}{l}.00011 \\
.00010\end{array}$ & $\begin{array}{l}.00044 \\
.00041\end{array}$ & $\begin{array}{l}.00003 \\
.00003\end{array}$ & $\begin{array}{l}.00013 \\
.00012\end{array}$ & $\begin{array}{l}.048 \\
.044\end{array}$ & $\begin{array}{l}147 \\
.135\end{array}$ & $\begin{array}{r}164 \\
.151\end{array}$ \\
\hline 15 & 125 & 30.0 & .00009 & .00038 & .00002 & .00011 & .041 & .127 & .142 \\
\hline $\begin{array}{l}15 \\
15 \\
15\end{array}$ & $\begin{array}{l}125 \\
125 \\
125\end{array}$ & $\begin{array}{l}35.0 \\
40.0 \\
45.0\end{array}$ & $\begin{array}{l}.00009 \\
.00008 \\
.00008\end{array}$ & $\begin{array}{l}.00036 \\
.00034 \\
.00033\end{array}$ & $\begin{array}{l}.00002 \\
.00002 \\
.00002\end{array}$ & $\begin{array}{l}.00011 \\
.00010 \\
.00010\end{array}$ & $\begin{array}{l}.039 \\
.037 \\
.035\end{array}$ & $\begin{array}{r}.119 \\
.114 \\
.109\end{array}$ & $\begin{array}{r}.133 \\
.127 \\
.121\end{array}$ \\
\hline $\begin{array}{l}15 \\
15\end{array}$ & $\begin{array}{l}125 \\
125\end{array}$ & $\begin{array}{l}46.0 \\
47.0\end{array}$ & $\begin{array}{l}.00008 \\
.00008\end{array}$ & $\begin{array}{l}.00032 \\
.00032\end{array}$ & $\begin{array}{l}.00002 \\
.00002\end{array}$ & $\begin{array}{l}.00009 \\
.00009\end{array}$ & $\begin{array}{r}.035 \\
.035\end{array}$ & $\begin{array}{r}-108 \\
.107\end{array}$ & $\begin{array}{l}.121 \\
.120\end{array}$ \\
\hline 15 & 125 & $48 \cdot 0$ & .00008 & .00032 & .00002 & .00009 & .035 & .106 & .119 \\
\hline $\begin{array}{l}15 \\
15 \\
15\end{array}$ & $\begin{array}{l}125 \\
125 \\
125\end{array}$ & $\begin{array}{l}49.0 \\
50.0 \\
51.0\end{array}$ & $\begin{array}{l}.00008 \\
.00007 \\
.00007\end{array}$ & $\begin{array}{r}.00032 \\
.00031 \\
.00031\end{array}$ & $\begin{array}{l}.00002 \\
.00002 \\
.00002\end{array}$ & $\begin{array}{l}.00009 \\
.00009 \\
.00009\end{array}$ & $\begin{array}{l}.034 \\
.034 \\
.034\end{array}$ & $\begin{array}{l}.106 \\
.105 \\
.104\end{array}$ & $\begin{array}{l}.118 \\
.117 \\
.116\end{array}$ \\
\hline $\begin{array}{l}15 \\
15\end{array}$ & $\begin{array}{l}125 \\
125\end{array}$ & $\begin{array}{l}52.0 \\
53.0\end{array}$ & $\begin{array}{r}.00007 \\
.00007\end{array}$ & $\begin{array}{l}.00031 \\
.00031\end{array}$ & $\begin{array}{l}.00002 \\
.00002\end{array}$ & $\begin{array}{l}.00009 \\
.00009\end{array}$ & $\begin{array}{l}.034 \\
.033\end{array}$ & .103 & $\begin{array}{r}116 \\
.115\end{array}$ \\
\hline 15. & 125 & $54 \cdot 0$ & .00007 & $: 00030$ & .00002 & .00009 & .033 & .102 & .114 \\
\hline $\begin{array}{l}15 \\
15 \\
15\end{array}$ & $\begin{array}{l}125 \\
125 \\
125\end{array}$ & $\begin{array}{l}55.0 \\
60.0 \\
65.0\end{array}$ & $\begin{array}{l}.00007 \\
.00007 \\
.00007\end{array}$ & $\begin{array}{l}.00030 \\
.00029 \\
.00028\end{array}$ & $\begin{array}{l}.00002 \\
.00002 \\
.00002\end{array}$ & $\begin{array}{r}.00009 \\
.00009 \\
.00008\end{array}$ & $\begin{array}{l}.033 \\
.032 \\
.031\end{array}$ & $\begin{array}{l}.101 \\
.098 \\
.095\end{array}$ & $\begin{array}{l}.113 \\
.109 \\
.106\end{array}$ \\
\hline $\begin{array}{l}15 \\
15 . \\
15\end{array}$ & $\begin{array}{l}125 \\
125 \\
125\end{array}$ & $\begin{array}{l}70.0 \\
75.0 \\
80.0\end{array}$ & $\begin{array}{l}.00007 \\
.00006 \\
.00006\end{array}$ & $\begin{array}{l}.00028 \\
.00027 \\
.00026\end{array}$ & $\begin{array}{l}.00002 \\
.00002 \\
.00002\end{array}$ & $\begin{array}{l}.00008 \\
.00008 \\
.00008\end{array}$ & $\begin{array}{l}.030 \\
.029 \\
.029\end{array}$ & $\begin{array}{l}.092 \\
.090 \\
.088\end{array}$ & $\begin{array}{l}.103 \\
.101 \\
.098\end{array}$ \\
\hline $\begin{array}{l}15 \\
15 \\
15\end{array}$ & $\begin{array}{l}125 \\
125 \\
125\end{array}$ & $\begin{array}{l}85.0 \\
90.0 \\
95.0\end{array}$ & $\begin{array}{l}.00006 \\
.00006 \\
.00006\end{array}$ & $\begin{array}{l}.00026 \\
.00025 \\
.00025\end{array}$ & $\begin{array}{l}.00001 \\
.00001 \\
.00001\end{array}$ & $\begin{array}{l}.00007 \\
.00007 \\
.00007\end{array}$ & $\begin{array}{l}.028 \\
.027 \\
.027\end{array}$ & $\begin{array}{l}.086 \\
.084 \\
.083\end{array}$ & $\begin{array}{l}.096 \\
.094 \\
.092\end{array}$ \\
\hline 15 & 125 & 100.0 & .00006 & .00024 & .00001 & .00007 & .026 & .081 & .090 \\
\hline
\end{tabular}


TABULATION OF DATA FOR

ABSORPTION OF SOUND IN AIR VERSUS PERCENT RELATIVE HUMIDITY AT 15 DEGREES CENTIGRADE FOR A FREQUENCY OF 250 HERTZ

\begin{tabular}{|c|c|c|c|c|c|c|c|c|c|}
\hline (1) & (2) & (3) & 141 & (5) & (6) & 171 & $(8)$ & 191 & $(10)$ \\
\hline $\begin{array}{l}\text { TEMP } \\
\text { DEGR } \\
\text { CENT }\end{array}$ & HERTZ & $\begin{array}{l}\text { REL } \\
\text { HUM } \\
\text { PER } \\
\text { CENT }\end{array}$ & $\begin{array}{l}\text { ATTEN" } \\
\text { COEF } \\
\text { PER } \\
\text { METER }\end{array}$ & $\begin{array}{l}4 M \\
\text { PER } \\
\text { METER }\end{array}$ & $\begin{array}{l}\text { ATTEN } \\
\text { COEF } \\
\text { PER } \\
\text { FOOT }\end{array}$ & $\begin{array}{l}4 M \\
\text { PER } \\
\text { FOOT }\end{array}$ & $\begin{array}{l}\text { ATTEN } \\
\text { DB PER } \\
100 \\
\text { METER }\end{array}$ & $\begin{array}{l}\text { ATTEN } \\
\text { DB PER } \\
1000 \\
\text { FEET }\end{array}$ & $\begin{array}{l}\text { DECAY } \\
\text { RATE } \\
\text { DB PER } \\
\text { SECOND }\end{array}$ \\
\hline $\begin{array}{l}15 \\
15 \\
15\end{array}$ & $\begin{array}{l}250 \\
250 \\
250\end{array}$ & $\begin{array}{r}5.0 \\
10.0 \\
15.0\end{array}$ & $\begin{array}{l}.00066 \\
.00032 \\
.00027\end{array}$ & $\begin{array}{l}.00266 \\
.00131 \\
.00108\end{array}$ & $\begin{array}{l}.00020 \\
.00010 \\
.00008\end{array}$ & $\begin{array}{l}.00081 \\
.00040 \\
.00033\end{array}$ & $\begin{array}{r}.289 \\
.142 \\
.118\end{array}$ & $\begin{array}{l}.882 \\
.435 \\
.360\end{array}$ & $\begin{array}{r}.985 \\
.485 \\
.402\end{array}$ \\
\hline $\begin{array}{l}15 \\
15 \\
15\end{array}$ & $\begin{array}{l}250 \\
250 \\
250\end{array}$ & $\begin{array}{l}20.0 \\
25.0 \\
30.0\end{array}$ & $\begin{array}{l}.00024 \\
.00022 \\
.00020\end{array}$ & $\begin{array}{l}.00097 \\
.00089 \\
.00083\end{array}$ & $\begin{array}{l}.00007 \\
.00006 \\
.00006\end{array}$ & $\begin{array}{l}.00029 \\
.00027 \\
.00025\end{array}$ & $\begin{array}{l}.106 \\
.097 \\
.090\end{array}$ & $\begin{array}{l}.324 \\
.296 \\
.276\end{array}$ & $\begin{array}{l}.361 \\
.331 \\
.308\end{array}$ \\
\hline $\begin{array}{l}15 \\
15 \\
15\end{array}$ & $\begin{array}{l}250 \\
250 \\
250\end{array}$ & $\begin{array}{l}35.0 \\
40.0 \\
45.0\end{array}$ & $\begin{array}{l}.00019 \\
.00018 \\
.00018\end{array}$ & $\begin{array}{r}.00079 \\
.00075 \\
.00072\end{array}$ & $\begin{array}{l}.00006 \\
.00005 \\
.00005\end{array}$ & $\begin{array}{l}.00024 \\
.00022 \\
.00021\end{array}$ & $\begin{array}{l}.085 \\
.081 \\
.078\end{array}$ & $\begin{array}{l}.261 \\
.249 \\
.238\end{array}$ & $\begin{array}{l}.292 \\
.278 \\
.266\end{array}$ \\
\hline $\begin{array}{l}15 \\
15 \\
15\end{array}$ & $\begin{array}{l}250 \\
250 \\
250\end{array}$ & $\begin{array}{l}46.0 \\
47.0 \\
48.0\end{array}$ & $\begin{array}{l}.00017 \\
.00017 \\
.00017\end{array}$ & $\begin{array}{l}.00071 \\
.00070 \\
.00070\end{array}$ & $\begin{array}{l}.00005 \\
.00005 \\
.00005\end{array}$ & $\begin{array}{l}.00021 \\
.00021 \\
.00021\end{array}$ & $\begin{array}{l}.077 \\
.076 \\
.076\end{array}$ & $\begin{array}{l}.236 \\
.234 \\
.232\end{array}$ & $\begin{array}{l}.264 \\
.261 \\
.259\end{array}$ \\
\hline $\begin{array}{l}15 \\
15\end{array}$ & $\begin{array}{l}250 \\
250\end{array}$ & $\begin{array}{l}49.0 \\
50.0\end{array}$ & $\begin{array}{l}.00017 \\
.00017\end{array}$ & .00069 & $\begin{array}{l}.00005 \\
.00005\end{array}$ & $\begin{array}{l}.00021 \\
.00021\end{array}$ & $\begin{array}{l}.075 \\
.075\end{array}$ & $\begin{array}{r}231 \\
.229\end{array}$ & $\begin{array}{l}.258 \\
.256\end{array}$ \\
\hline 15 & 250 & 51.0 & .00017 & .00068 & .00005 & .00021 & .074 & .228 & .254 \\
\hline $\begin{array}{l}15 \\
15 \\
15\end{array}$ & $\begin{array}{l}250 \\
250 \\
25.0\end{array}$ & $\begin{array}{l}52.0 \\
53.0 \\
54.0\end{array}$ & $\begin{array}{l}.00017 \\
.00016 \\
.00016\end{array}$ & $\begin{array}{l}.00068 \\
.00067 \\
.00067\end{array}$ & $\begin{array}{l}.00005 \\
.00005 \\
.00005\end{array}$ & $\begin{array}{l}.00020 \\
.00020 \\
.00020\end{array}$ & $\begin{array}{l}.074 \\
.073 \\
.073\end{array}$ & $\begin{array}{l}.226 \\
.224 \\
.223\end{array}$ & $\begin{array}{l}.252 \\
.251 \\
.249\end{array}$ \\
\hline $\begin{array}{l}15 \\
15 \\
15\end{array}$ & $\begin{array}{l}250 \\
250 \\
250\end{array}$ & $\begin{array}{l}55.0 \\
60.0 \\
65.0\end{array}$ & $\begin{array}{l}.00016 \\
.00016 \\
.00015\end{array}$ & $\begin{array}{l}.00066 \\
.00064 \\
.00063\end{array}$ & $\begin{array}{l}.00005 \\
.00004 \\
.00004\end{array}$ & $\begin{array}{l}.00020 \\
.00019 \\
.00019\end{array}$ & $\begin{array}{l}.072 \\
.070 \\
.068\end{array}$ & $\begin{array}{l}.221 \\
.214 \\
.208\end{array}$ & $\begin{array}{l}.247 \\
.239 \\
.233\end{array}$ \\
\hline $\begin{array}{l}15 \\
15 \\
15\end{array}$ & $\begin{array}{l}250 \\
250 \\
250\end{array}$ & $\begin{array}{l}70.0 \\
75.0 \\
80.0\end{array}$ & $\begin{array}{l}.00015 \\
.00014 \\
.00014\end{array}$ & $\begin{array}{l}.00061 \\
.00059 \\
.00058\end{array}$ & $\begin{array}{l}.00004 \\
.00004 \\
.00004\end{array}$ & $\begin{array}{l}.00018 \\
.00018 \\
.00017\end{array}$ & $\begin{array}{l}.066 \\
.064 \\
.063\end{array}$ & $\begin{array}{l}.202 \\
.197 \\
.192\end{array}$ & $\begin{array}{l}.226 \\
.220 \\
.215\end{array}$ \\
\hline $\begin{array}{l}15 \\
15 \\
15\end{array}$ & $\begin{array}{l}250 \\
250 \\
250\end{array}$ & $\begin{array}{l}85.0 \\
90.0 \\
95.0\end{array}$ & $\begin{array}{l}.00014 \\
.00013 \\
.00013\end{array}$ & $\begin{array}{l}.00056 \\
.00055 \\
.00054\end{array}$ & $\begin{array}{l}.00004 \\
.00004 \\
.00004\end{array}$ & $\begin{array}{l}.00017 \\
.00017 \\
.00016\end{array}$ & $\begin{array}{l}.061 \\
.060 \\
.059\end{array}$ & $\begin{array}{r}188 \\
.185 \\
.181\end{array}$ & $\begin{array}{l}.210 \\
.206 \\
.202\end{array}$ \\
\hline 15 & 250 & 100.0 & .00013 & .00053 & .00004 & .00016 & .058 & .178 & .198 \\
\hline
\end{tabular}


TABULATION OF DATA FOR

ABSORPTION OF SOUND IN AIR VERSUS PERCENT RELATIVE HUMIDITY AT 15 DEGREES CENTIGRADE FOR A FREQUENCY OF 500 HERTZ

\begin{tabular}{|c|c|c|c|c|c|c|c|c|c|}
\hline (1) & 121 & (3) & (4) & (5) & $(6)$ & 171 & $(8)$ & $(9)$ & $(10)$ \\
\hline $\begin{array}{l}\text { TEMP } \\
\text { DEGR } \\
\text { CENT. }\end{array}$ & HERTZ & $\begin{array}{l}\text { REL } \\
\text { HUM } \\
\text { PER } \\
\text { CENT }\end{array}$ & $\begin{array}{l}\text { ATTEN } \\
\text { COEF } \\
\text { PER } \\
\text { METER }\end{array}$ & $\begin{array}{l}4 M \\
\text { PER } \\
\text { METER }\end{array}$ & $\begin{array}{l}\text { ATTEN } \\
\text { COEF } \\
\text { PER } \\
\text { FOOT. }\end{array}$ & $\begin{array}{l}4 M \\
\text { PER } \\
\text { FOOT }\end{array}$ & $\begin{array}{l}\text { ATTEN } \\
\text { DB PER } \\
\text { IOO } \\
\text { METER }\end{array}$ & $\begin{array}{l}\text { ATTEN } \\
\text { DB PER } \\
1000 \\
\text { FEET }\end{array}$ & $\begin{array}{l}\text { DECAY } \\
\text { RATE } \\
\text { DB PER } \\
\text { SECOND }\end{array}$ \\
\hline $\begin{array}{l}15 . \\
15 \\
15\end{array}$ & $\begin{array}{l}500 \\
500 \\
500\end{array}$ & $\begin{array}{r}5.0 \\
10.0 \\
15.0\end{array}$ & $\begin{array}{l}.00212 \\
.00099 \\
.00066\end{array}$ & $\begin{array}{l}.00848 \\
.00397 \\
.00267\end{array}$ & $\begin{array}{l}.00064 \\
.00030 \\
.00020\end{array}$ & $\begin{array}{l}.00258 \\
.00121 \\
.00081\end{array}$ & $\begin{array}{r}.921 \\
.431 \\
.290\end{array}$ & $\begin{array}{r}2.808 \\
1.314 \\
\cdot 886\end{array}$ & $\begin{array}{r}3.135 \\
1.468 \\
.989\end{array}$ \\
\hline $\begin{array}{l}15 \\
15 \\
15\end{array}$ & $\begin{array}{l}500 \\
500 \\
500\end{array}$ & $\begin{array}{l}20.0 \\
25.0 \\
30.0\end{array}$ & $\begin{array}{r}.00057 \\
.00053 \\
.00049\end{array}$ & $\begin{array}{l}.00230 \\
.00212 \\
.00199\end{array}$ & $\begin{array}{l}.00017 \\
.00016 \\
.00015\end{array}$ & $\begin{array}{l}.00070 \\
.00064 \\
.00060\end{array}$ & $\begin{array}{l}.250 \\
.231 \\
.216\end{array}$ & $\begin{array}{l}.763 \\
.704 \\
.659\end{array}$ & $\begin{array}{l}.852 \\
.786 \\
.736\end{array}$ \\
\hline $\begin{array}{l}15 \\
15 \\
15\end{array}$ & $\begin{array}{l}500 \\
500 \\
500\end{array}$ & $\begin{array}{l}35.0 \\
40.0 \\
45.0\end{array}$ & $\begin{array}{l}.00046 \\
.00044 \\
.00042\end{array}$ & $\begin{array}{l}.00187 \\
.00177 \\
.00170\end{array}$ & $\begin{array}{l}.00014 \\
.00013 \\
.00012\end{array}$ & $\begin{array}{l}.00057 \\
.00054 \\
.00051\end{array}$ & $\begin{array}{r}.203 \\
.193 \\
.184\end{array}$ & $\begin{array}{l}.621 \\
.588 \\
.563\end{array}$ & $\begin{array}{l}.693 \\
.656 \\
.628\end{array}$ \\
\hline $\begin{array}{l}15 \\
15 \\
15\end{array}$ & $\begin{array}{l}500 \\
500 \\
500\end{array}$ & $\begin{array}{l}46.0 \\
47.0 \\
48.0\end{array}$ & $\begin{array}{l}.00042 \\
.00041 \\
.00041\end{array}$ & $\begin{array}{l}.00168 \\
.00167 \\
.00166\end{array}$ & $\begin{array}{l}.00012 \\
.00012 \\
.00012\end{array}$ & $\begin{array}{l}.00051 \\
.00051 \\
.00050\end{array}$ & $\begin{array}{r}183 \\
.181 \\
.180\end{array}$ & $\begin{array}{r}.558 \\
.554 \\
.550\end{array}$ & $\begin{array}{r}.623 \\
.619 \\
.614\end{array}$ \\
\hline $\begin{array}{l}15 \\
15 \\
15\end{array}$ & $\begin{array}{l}500 \\
500 \\
500\end{array}$ & $\begin{array}{l}49.0 \\
50.0 \\
51.0\end{array}$ & $\begin{array}{l}.00041 \\
.00041 \\
.00040\end{array}$ & $\begin{array}{l}.00165 \\
.00164 \\
.00162\end{array}$ & $\begin{array}{l}.00012 \\
.00012 \\
.00012\end{array}$ & $\begin{array}{l}.00050 \\
.00050 \\
.00049\end{array}$ & $\begin{array}{r}179 \\
-178 \\
.176\end{array}$ & $\begin{array}{l}.546 \\
.542 \\
.539\end{array}$ & $\begin{array}{l}.610 \\
.606 \\
.601\end{array}$ \\
\hline $\begin{array}{l}15 \\
15 \\
15\end{array}$ & $\begin{array}{l}500 \\
500 \\
500\end{array}$ & $\begin{array}{l}52.0 \\
53.0 \\
54.0\end{array}$ & $\begin{array}{l}.00040 \\
.00040 \\
.00039\end{array}$ & $\begin{array}{l}.00161 \\
.00160 \\
.00159\end{array}$ & $\begin{array}{l}.00012 \\
.00012 \\
.00012\end{array}$ & $\begin{array}{l}.00049 \\
.00048 \\
.00048\end{array}$ & $\begin{array}{r}.175 \\
.174 \\
.173\end{array}$ & $\begin{array}{l}.535 \\
.531 \\
.528\end{array}$ & $\begin{array}{r}.597 \\
.593 \\
.590\end{array}$ \\
\hline $\begin{array}{l}15 \\
15 \\
15\end{array}$ & $\begin{array}{l}500 \\
500 \\
500\end{array}$ & $\begin{array}{l}55.0 \\
60.0 \\
65.0\end{array}$ & $\begin{array}{l}.00039 \\
.00038 \\
.00037\end{array}$ & $\begin{array}{r}.00158 \\
.00153 \\
.00149\end{array}$ & $\begin{array}{l}.00012 \\
.00011 \\
.00011\end{array}$ & $\begin{array}{l}.00048 \\
.00046 \\
.00045\end{array}$ & $\begin{array}{r}.172 \\
.167 \\
.161\end{array}$ & $\begin{array}{l}.525 \\
.509 \\
.493\end{array}$ & $\begin{array}{r}.586 \\
.568 \\
.550\end{array}$ \\
\hline $\begin{array}{l}15 \\
15 \\
15\end{array}$ & $\begin{array}{l}500 \\
500 \\
500\end{array}$ & $\begin{array}{l}70.0 \\
75.0 \\
80.0\end{array}$ & $\begin{array}{l}.00036 \\
.00035 \\
.00034\end{array}$ & $\begin{array}{l}.00145 \\
.00141 \\
.00138\end{array}$ & $\begin{array}{l}.00011 \\
.000110 \\
.00010\end{array}$ & $\begin{array}{l}.00044 \\
.00043 \\
.00042\end{array}$ & $\begin{array}{l}.157 \\
.153 \\
.150\end{array}$ & $\begin{array}{r}.480 \\
.468 \\
.458\end{array}$ & $\begin{array}{r}.536 \\
.523 \\
.511\end{array}$ \\
\hline $\begin{array}{l}15 \\
15 \\
15\end{array}$ & $\begin{array}{l}500 \\
500 \\
500\end{array}$ & $\begin{array}{l}85.0 \\
90.0 \\
95.0\end{array}$ & $\begin{array}{l}.00033 \\
.00033 \\
.00032\end{array}$ & $\begin{array}{l}.00135 \\
.00132 \\
.00130\end{array}$ & $\begin{array}{l}.00010 \\
.00010 \\
.00009\end{array}$ & $\begin{array}{r}.00041 \\
.00040 \\
.00039\end{array}$ & $\begin{array}{r}.146 \\
.143 \\
.141\end{array}$ & $\begin{array}{r}.447 \\
.438 \\
.430\end{array}$ & $\begin{array}{r}.499 \\
.489 \\
.481\end{array}$ \\
\hline 15 & 500 & 100.0 & .00031 & .00127 & .00009 & .00038 & .138 & .423 & .472 \\
\hline
\end{tabular}


TABULATION OF DATA FOR

ABSORPTION OF SOUND IN AIR VERSUS PERCENT RELATIVE HUMIDITY AT 15 DEGREES CENTIGRADE FOR A FREQUENCY OF 1000 HERTZ

\begin{tabular}{|c|c|c|c|c|c|c|c|c|c|}
\hline 1.) & (2) & $|3|$ & (4) & (5) & $(6)$ & 171 & $(8)$ & 191 & 1101 \\
\hline $\begin{array}{l}\text { EMP } \\
\text { EGR } \\
\text { ENT }\end{array}$ & FREQ & $\begin{array}{l}\text { REL } \\
\text { HUM } \\
\text { PER } \\
\text { CENT }\end{array}$ & $\begin{array}{l}\text { ATTEN } \\
\text { COEF } \\
\text { PER } \\
\text { METER }\end{array}$ & $\begin{array}{l}4 M \\
\text { PER } \\
\text { METER }\end{array}$ & $\begin{array}{l}\text { ATTEN } \\
\text { COEF } \\
\text { PER } \\
\text { FOOT }\end{array}$ & $\begin{array}{l}4 M \\
\text { PER } \\
\text { FOOT }\end{array}$ & $\begin{array}{l}\text { ATTEN } \\
\text { DB PER } \\
100 \\
\text { METER }\end{array}$ & $\begin{array}{l}\text { ATTEN } \\
\text { DB PER } \\
1000 \\
\text { FEET }\end{array}$ & $\begin{array}{l}\text { DECAY. } \\
\text { RATE } \\
\text { DB PER } \\
\text { SECOND }\end{array}$ \\
\hline $\begin{array}{l}15 \\
15 \\
15\end{array}$ & $\begin{array}{l}1000 \\
1000 \\
1000\end{array}$ & $\begin{array}{r}5.0 \\
10.0 \\
15.0\end{array}$ & $\begin{array}{l}.00573 \\
.00337 \\
.00217\end{array}$ & $\begin{array}{r}.02295 \\
.01348 \\
.00868\end{array}$ & $\begin{array}{l}.00174 \\
.00102 \\
.00066\end{array}$ & $\begin{array}{l}.00699 \\
.00411 \\
.00264\end{array}$ & $\begin{array}{r}2.492 \\
1.464 \\
.942\end{array}$ & $\begin{array}{l}7.596 \\
4.464 \\
2.872\end{array}$ & $\begin{array}{l}8.480 \\
4.983 \\
3.207\end{array}$ \\
\hline $\begin{array}{l}15 \\
15 \\
15\end{array}$ & $\begin{array}{l}1000 \\
1000 \\
1000\end{array}$ & $\begin{array}{l}20.0 \\
25.0 \\
30.0\end{array}$ & $\begin{array}{l}.00160 \\
.00133 \\
.00120\end{array}$ & $\begin{array}{l}.00642 \\
.00533 \\
.00481\end{array}$ & $\begin{array}{r}.00048 \\
.00040 \\
.00036\end{array}$ & $\begin{array}{l}.00195 \\
.00162 \\
.00146\end{array}$ & $\begin{array}{r}.697 \\
.579 \\
.523\end{array}$ & $\begin{array}{l}2.127 \\
1.766 \\
1.594\end{array}$ & $\begin{array}{l}2.375 \\
1.972 \\
1.780\end{array}$ \\
\hline $\begin{array}{l}15 \\
15 \\
15\end{array}$ & $\begin{array}{l}1000 \\
1000 \\
1000\end{array}$ & $\begin{array}{l}35.0 \\
40.0 \\
45.0\end{array}$ & $\begin{array}{l}.00114 \\
.00108 \\
.00104\end{array}$ & $\begin{array}{l}.00456 \\
.00435 \\
.00417\end{array}$ & $\begin{array}{l}.00034 \\
.00033 \\
.00031\end{array}$ & $\begin{array}{l}.00139 \\
.00132 \\
.00127\end{array}$ & $\begin{array}{l}.495 \\
.472 \\
.453\end{array}$ & $\begin{array}{l}1.509 \\
1.440 \\
1.381\end{array}$ & $\begin{array}{l}1.685 \\
1.607 \\
1.542\end{array}$ \\
\hline $\begin{array}{l}15 \\
15 \\
15\end{array}$ & $\begin{array}{l}1000 \\
1000 \\
1000\end{array}$ & $\begin{array}{l}46.0 \\
47 \cdot 0 \\
48 \cdot 0\end{array}$ & $\begin{array}{l}.00103 \\
.00102 \\
.00101\end{array}$ & $\begin{array}{l}.00414 \\
.00410 \\
.00407\end{array}$ & $\begin{array}{l}.00031 \\
.00031 \\
.00031\end{array}$ & $\begin{array}{l}.00126 \\
.00125 \\
.00124\end{array}$ & $\begin{array}{l}.449 \\
.446 \\
.442\end{array}$ & $\begin{array}{l}1 \cdot 370 \\
1 \cdot 360 \\
1.349\end{array}$ & $\begin{array}{l}1.530 \\
1.518 \\
1.506\end{array}$ \\
\hline $\begin{array}{l}15 \\
15 \\
15\end{array}$ & $\begin{array}{l}1000 \\
1000 \\
1000\end{array}$ & $\begin{array}{l}49.0 \\
50.0 \\
51.0\end{array}$ & $\begin{array}{l}.00101 \\
.00100 \\
.00099\end{array}$ & $\begin{array}{l}.00404 \\
.00401 \\
.00398\end{array}$ & $\begin{array}{l}.00030 \\
.00030 \\
.00030\end{array}$ & $\begin{array}{l}.00123 \\
.00122 \\
.00121\end{array}$ & $\begin{array}{r}.439 \\
.435 \\
.432\end{array}$ & $\begin{array}{l}1.338 \\
1.328 \\
1.319\end{array}$ & $\begin{array}{l}1.494 \\
1.483 \\
1.473\end{array}$ \\
\hline $\begin{array}{l}15 \\
15 \\
15\end{array}$ & $\begin{array}{l}1000 \\
1000 \\
1000\end{array}$ & $\begin{array}{l}52.0 \\
53.0 \\
54.0\end{array}$ & $\begin{array}{l}.00098 \\
.00098 \\
.00097\end{array}$ & $\begin{array}{l}.00395 \\
.00393 \\
.00390\end{array}$ & $\begin{array}{l}.00030 \\
.00029 \\
.00029\end{array}$ & $\begin{array}{l}.00120 \\
.00119 \\
.00119\end{array}$ & $\begin{array}{r}.429 \\
.426 \\
.423\end{array}$ & $\begin{array}{l}1 \cdot 310 \\
1 \cdot 30.1 \\
1.292\end{array}$ & $\begin{array}{l}1.463 \\
1.452 \\
1.442\end{array}$ \\
\hline $\begin{array}{l}15 \\
15 \\
15\end{array}$ & $\begin{array}{l}1000 \\
1000 \\
1000\end{array}$ & $\begin{array}{l}55.0 \\
60.0 \\
65.0\end{array}$ & $\begin{array}{l}.00096 \\
.00093 \\
.00090\end{array}$ & $\begin{array}{l}.00387 \\
.00374 \\
.00362\end{array}$ & $\begin{array}{l}.00029 \\
.00028 \\
.00027\end{array}$ & $\begin{array}{l}.00118 \\
.00114 \\
.00110\end{array}$ & $\begin{array}{l}.420 \\
.406 \\
.393\end{array}$ & $\begin{array}{l}1.282 \\
1.239 \\
1.199\end{array}$ & $\begin{array}{l}1.432 \\
1.383 \\
1.339\end{array}$ \\
\hline $\begin{array}{l}15 \\
15 \\
15\end{array}$ & $\begin{array}{l}1000 \\
1000 \\
1000\end{array}$ & $\begin{array}{l}70.0 \\
75.0 \\
80.0\end{array}$ & $\begin{array}{l}.00088 \\
.00086 \\
.00084\end{array}$ & $\begin{array}{l}.00352 \\
.00344 \\
.00336\end{array}$ & $\begin{array}{l}.00026 \\
.00026 \\
.00025\end{array}$ & $\begin{array}{l}.00107 \\
.00104 \\
.00102\end{array}$ & $\begin{array}{l}.382 \\
.373 \\
.365\end{array}$ & $\begin{array}{l}1.166 \\
1.139 \\
1.114\end{array}$ & $\begin{array}{l}1.302 \\
1.271 \\
1.244\end{array}$ \\
\hline $\begin{array}{l}15 \\
15 \\
15\end{array}$ & $\begin{array}{l}1000 \\
1000 \\
1000\end{array}$ & $\begin{array}{l}85.0 \\
90.0 \\
95.0\end{array}$ & $\begin{array}{l}.00082 \\
.00080 \\
.00079\end{array}$ & $\begin{array}{l}.00329 \\
.00323 \\
.00317\end{array}$ & $\begin{array}{l}.00025 \\
.00024 \\
.00024\end{array}$ & $\begin{array}{l}.00100 \\
.00098 \\
.00096\end{array}$ & $\begin{array}{l}.357 \\
.351 \\
.344\end{array}$ & $\begin{array}{l}1.090 \\
1.070 \\
1.050\end{array}$ & $\begin{array}{l}1.218 \\
1.195 \\
1.173\end{array}$ \\
\hline 5 & 000 & 10 & .00077 & 00311 & 00023 & 00095 & .338 & 1.031 & 1.15 \\
\hline
\end{tabular}


TABULATION OF DATA FOR.

ABSORPT ION OF SOUND IN AIR VERSUS PERCENT RELATIVE HUMIDITY AT. 15 DEGREES CENTIGRADE FOR A FREQUENCY OF 2000 HERTZ

\begin{tabular}{|c|c|c|c|c|c|c|c|c|c|}
\hline 11 & $(2)$ & (3) & (4) & (5) & (6) & 171 & (8) & $(9)$ & $(10)$ \\
\hline $\begin{array}{l}\text { EMP } \\
\text { EGR } \\
\text { ENT }\end{array}$ & HERTZ & $\begin{array}{l}\text { REL } \\
\text { HUM } \\
\text { PER } \\
\text { CENT }\end{array}$ & $\begin{array}{l}\text { ATTEN } \\
\text { COEF } \\
\text { PER } \\
\text { METER }\end{array}$ & $\begin{array}{l}\text { 4M } \\
\text { PER } \\
\text { METER. }\end{array}$ & $\begin{array}{l}\text { ATTEN } \\
\text { COEF } \\
\text { PER } \\
\text { FOOT }\end{array}$ & $\begin{array}{l}4 M \\
\text { PER } \\
\text { FOOT }\end{array}$ & $\begin{array}{l}\text { ATTEN } \\
\text { DB PER } \\
\text { IOO } \\
\text { METER }\end{array}$ & $\begin{array}{l}\text { ATTEN } \\
\text { DB PER } \\
1000 \\
\text { FEET. }\end{array}$ & $\begin{array}{l}\text { DECAY } \\
\text { RATE } \\
\text { DB PER } \\
\text { SECOND }\end{array}$ \\
\hline $\begin{array}{l}15 \\
15 \\
15\end{array}$ & $\begin{array}{l}2000 \\
2000 \\
2000\end{array}$ & $\begin{array}{r}5.0 \\
10.0 \\
15.0\end{array}$ & $\begin{array}{r}.00954 \\
.01093 \\
.00749\end{array}$ & $\begin{array}{l}.03819 \\
.04374 \\
.02996\end{array}$ & $\begin{array}{l}.00291 \\
.00333 \\
.00228\end{array}$ & $\begin{array}{r}.01164 \\
.01333 \\
.00913\end{array}$ & $\begin{array}{l}4.147 \\
4.749 \\
3.253\end{array}$ & $\begin{array}{r}12.641 \\
14.477 \\
9.916\end{array}$ & $\begin{array}{l}14 \cdot 113 \\
16 \cdot 164 \\
11 \cdot 071\end{array}$ \\
\hline $\begin{array}{l}15 \\
1.5 \\
15\end{array}$ & $\begin{array}{l}2000 \\
2000 \\
2000\end{array}$ & $\begin{array}{l}20.0 \\
25.0 \\
30.0\end{array}$ & $\begin{array}{l}.00553 \\
.00433 \\
.00358\end{array}$ & $\begin{array}{l}.02215 \\
.01734 \\
.01432\end{array}$ & $\begin{array}{l}.00168 \\
.00132 \\
.00109\end{array}$ & $\begin{array}{l}.00675 \\
.00528 \\
.00436\end{array}$ & $\begin{array}{l}2.405 \\
1.882 \\
1.554\end{array}$ & $\begin{array}{l}7.331 \\
5.739 \\
4.739\end{array}$ & $\begin{array}{l}8.185 \\
6.407 \\
5.290\end{array}$ \\
\hline $\begin{array}{l}15 \\
15\end{array}$ & $\begin{array}{l}2000 \\
2000\end{array}$ & $\begin{array}{l}35.0 \\
40.0\end{array}$ & $\begin{array}{l}.00310 \\
.00277\end{array}$ & $\begin{array}{l}.01241 \\
.01110\end{array}$ & $\begin{array}{l}.00094 \\
.00084\end{array}$ & $\begin{array}{l}.00378 \\
.00338\end{array}$ & $\begin{array}{l}1.347 \\
1.206\end{array}$ & $\begin{array}{l}4 \cdot 108 \\
3.676\end{array}$ & $\begin{array}{l}4 \cdot 587 \\
4 \cdot 104\end{array}$ \\
\hline 15 & 2000 & 45.0 & .00260 & .01040 & .00079 & .00317 & 1.130 & 3.444 & 3.846 \\
\hline $\begin{array}{l}15 \\
15 \\
15\end{array}$ & $\begin{array}{l}2000 \\
2000 \\
2000\end{array}$ & $\begin{array}{l}46.0 \\
47.0 \\
48.0\end{array}$ & $\begin{array}{l}.00256 \\
.00253 \\
.00250\end{array}$ & $\begin{array}{l}.01026 \\
.01013 \\
.01003\end{array}$ & $\begin{array}{l}.00078 \\
.00077 \\
.00076\end{array}$ & $\begin{array}{l}.00312 \\
.00308 \\
.00305\end{array}$ & $\begin{array}{l}1.114 \\
1.100 \\
1.089\end{array}$ & $\begin{array}{l}3.395 \\
3.354 \\
3.320\end{array}$ & $\begin{array}{l}3.791 \\
3.745 \\
3.706\end{array}$ \\
\hline $\begin{array}{l}15 \\
15 \\
15\end{array}$ & $\begin{array}{l}2000 \\
2000 \\
2000\end{array}$ & $\begin{array}{l}49.0 \\
50.0 \\
51.0\end{array}$ & $\begin{array}{l}.00248 \\
.00246 \\
.00244\end{array}$ & $\begin{array}{l}.00993 \\
.00986 \\
.00979\end{array}$ & $\begin{array}{l}.00075 \\
.00075 \\
.00074\end{array}$ & $\begin{array}{l}.00302 \\
.00300 \\
.00298\end{array}$ & $\begin{array}{l}1.078 \\
1.070 \\
1.063\end{array}$ & $\begin{array}{l}3.288 \\
3.263 \\
3.241\end{array}$ & $\begin{array}{l}3.671 \\
3.643 \\
3.618\end{array}$ \\
\hline $\begin{array}{l}15 \\
15 \\
15\end{array}$ & $\begin{array}{l}2000 \\
2000 \\
2000\end{array}$ & $\begin{array}{l}52.0 \\
53.0 \\
54.0\end{array}$ & $\begin{array}{l}.00243 \\
.00241 \\
.00240\end{array}$ & $\begin{array}{r}.00972 \\
.00966 \\
.00960\end{array}$ & $\begin{array}{l}.00074 \\
.00073 \\
.00073\end{array}$ & $\begin{array}{l}.00296 \\
.00294 \\
.00292\end{array}$ & $\begin{array}{l}1.056 \\
1.049 \\
1.043\end{array}$ & $\begin{array}{l}3.218 \\
3.199 \\
3.179\end{array}$ & $\begin{array}{l}3.593 \\
3.571 \\
3.549\end{array}$ \\
\hline $\begin{array}{l}15 \\
15 \\
15\end{array}$ & $\begin{array}{l}2000 \\
2000 \\
2000\end{array}$ & $\begin{array}{l}55.0 \\
60.0 \\
65.0\end{array}$ & $\begin{array}{l}.00238 \\
.00231 \\
.00225\end{array}$ & $\begin{array}{l}.00954 \\
.00925 \\
.00901\end{array}$ & $\begin{array}{l}.00072 \\
.00070 \\
.00068\end{array}$ & $\begin{array}{l}.00291 \\
.00282 \\
.00274\end{array}$ & $\begin{array}{r}1.036 \\
1.004 \\
.979\end{array}$ & $\begin{array}{l}3.159 \\
3.062 \\
2.984\end{array}$ & $\begin{array}{l}3.527 \\
3.419 \\
3.331\end{array}$ \\
\hline $\begin{array}{l}15 \\
15 \\
15\end{array}$ & $\begin{array}{l}2000 \\
2000 \\
2000\end{array}$ & $\begin{array}{l}70.0 \\
75.0 \\
80.0\end{array}$ & $\begin{array}{l}.00219 \\
.00214 \\
.00209\end{array}$ & $\begin{array}{l}.00878 \\
.00858 \\
.00838\end{array}$ & $\begin{array}{l}.00066 \\
.00065 \\
.00063\end{array}$ & $\begin{array}{l}.00267 \\
.00261 \\
.00255\end{array}$ & $\begin{array}{r}.953 \\
.932 \\
.910\end{array}$ & $\begin{array}{l}2.907 \\
2.841 \\
2.776\end{array}$ & $\begin{array}{l}3.245 \\
3.172 \\
3.099\end{array}$ \\
\hline $\begin{array}{l}15 \\
15 \\
15\end{array}$ & $\begin{array}{l}2000 \\
2000 \\
2000\end{array}$ & $\begin{array}{l}85.0 \\
90.0 \\
95.0\end{array}$ & $\begin{array}{l}.00205 \\
.00201 \\
.00196\end{array}$ & $\begin{array}{r}.00821 \\
.00804 \\
.00787\end{array}$ & $\begin{array}{l}.00062 \\
.00061 \\
.00060\end{array}$ & $\begin{array}{l}.00250 \\
.00245 \\
.00240\end{array}$ & $\begin{array}{l}.891 \\
.873 \\
.855\end{array}$ & $\begin{array}{l}2.718 \\
2.662 \\
2.607\end{array}$ & $\begin{array}{l}3.035 \\
2.972 \\
2.910\end{array}$ \\
\hline 15 & 2000 & 100.0 & .00193 & .00772 & .00058 & .00235 & .839 & .557 & 2.855 \\
\hline
\end{tabular}


TABULATION OF DATA FOR

ABSORPTION OF SOUND IN AIR VERSUS PERCENT RELATIVE HUMIDITY AT 15 DEGREES CENTIGRADE FOR A, FREQUENCY OF 2500 HERTZ

\begin{tabular}{|c|c|c|c|c|c|c|c|c|c|}
\hline (1.) & (2) & $(3)$ & $(4)$ & $(5)$ & (6) & 171 & $(8)$ & (9) & $(10)$ \\
\hline $\begin{array}{l}\text { EMP } \\
\text { EGR } \\
\text { ENT }\end{array}$ & HERTZ & $\begin{array}{l}\text { REL } \\
\text { HUM } \\
\text { PER } \\
\text { CENT }\end{array}$ & $\begin{array}{l}\text { ATTEN } \\
\text { COEF } \\
\text { PER } \\
\text { METER }\end{array}$ & $\begin{array}{l}4 M \\
\text { PER } \\
\text { METER }\end{array}$ & $\begin{array}{l}\text { ATTEN } \\
\text { COEF } \\
\text { PER } \\
\text { FOOT }\end{array}$ & $\begin{array}{l}4 M \\
\text { PER } \\
\text { FOOT }\end{array}$ & $\begin{array}{l}\text { ATTEN } \\
\text { DB PER } \\
100 \\
\text { METER }\end{array}$ & $\begin{array}{l}\text { ATTEN } \\
\text { DB PER } \\
1000 \\
\text { FEET }\end{array}$ & $\begin{array}{l}\text { DECAY } \\
\text { RATE } \\
\text { DB PER } \\
\text { SECOND }\end{array}$ \\
\hline $\begin{array}{l}15 \\
15 \\
15\end{array}$ & $\begin{array}{l}2500 \\
2500 \\
2500\end{array}$ & $\begin{array}{r}5.0 \\
10.0 \\
15.0\end{array}$ & $\begin{array}{r}.01022 \\
.01441 \\
.01119\end{array}$ & $\begin{array}{l}.04090 \\
.05765 \\
.04479\end{array}$ & $\begin{array}{l}.00311 \\
.00439 \\
.00341\end{array}$ & $\begin{array}{l}.01246 \\
.01757 \\
.01365\end{array}$ & $\begin{array}{l}4.441 \\
6.259 \\
4.863\end{array}$ & $\begin{array}{l}13.538 \\
19.080 \\
14.823\end{array}$ & $\begin{array}{l}15.114 \\
21.302 \\
16.549\end{array}$ \\
\hline $\begin{array}{l}15 \\
15 \\
15\end{array}$ & $\begin{array}{l}2500 \\
2500 \\
2500\end{array}$ & $\begin{array}{l}20.0 \\
25.0 \\
30.0\end{array}$ & $\begin{array}{l}.00819 \\
.00645 \\
.00532\end{array}$ & $\begin{array}{l}.03279 \\
.02580 \\
.02128\end{array}$ & $\begin{array}{l}.00249 \\
.00196 \\
.00162\end{array}$ & $\begin{array}{l}.00999 \\
.00786 \\
.00648\end{array}$ & $\begin{array}{l}3.561 \\
2.802 \\
2.310\end{array}$ & $\begin{array}{r}10.854 \\
8.540 \\
7.043\end{array}$ & $\begin{array}{r}12.118 \\
9.535 \\
7.863\end{array}$ \\
\hline $\begin{array}{l}15 \\
15 \\
15\end{array}$ & $\begin{array}{l}2500 \\
2500 \\
2500\end{array}$ & $\begin{array}{l}35.0 \\
40.0 \\
45.0\end{array}$ & $\begin{array}{r}.00452 \\
.00399 \\
.00361\end{array}$ & $\begin{array}{r}.01811 \\
.01598 \\
.01445\end{array}$ & $\begin{array}{l}.00138 \\
.00121 \\
.00110\end{array}$ & $\begin{array}{l}.00552 \\
.00487 \\
.00440\end{array}$ & $\begin{array}{l}1.967 \\
1.735 \\
1.569\end{array}$ & $\begin{array}{l}5.995 \\
5.291 \\
4.784\end{array}$ & $\begin{array}{l}6.693 \\
5.907 \\
5.341\end{array}$ \\
\hline $\begin{array}{l}15 \\
15 \\
15\end{array}$ & $\begin{array}{l}2500 \\
2500 \\
2500\end{array}$ & $\begin{array}{l}46.0 \\
47.0 \\
48.0\end{array}$ & $\begin{array}{l}.00355 \\
.00350 \\
.00346\end{array}$ & $\begin{array}{r}.01423 \\
.01402 \\
.01386\end{array}$ & $\begin{array}{r}.00108 \\
.00106 \\
.00105\end{array}$ & $\begin{array}{l}.00433 \\
.00427 \\
.00422\end{array}$ & $\begin{array}{l}1.545 \\
1.522 \\
1.505\end{array}$ & $\begin{array}{l}4.710 \\
4.641 \\
4.588\end{array}$ & $\begin{array}{l}5.259 \\
5.181 \\
5.122\end{array}$ \\
\hline $\begin{array}{l}15 \\
15\end{array}$ & $\begin{array}{l}2500 \\
2500\end{array}$ & $\begin{array}{l}49.0 \\
50.0\end{array}$ & $\begin{array}{l}.00342 \\
.00338\end{array}$ & $\begin{array}{r}.01370 \\
.01354\end{array}$ & $\begin{array}{l}.00104 \\
.00103\end{array}$ & $\begin{array}{l}.00417 \\
.00412\end{array}$ & $\begin{array}{l}1.488 \\
1.470\end{array}$ & $\begin{array}{l}4.536 \\
4.483\end{array}$ & $\begin{array}{l}5.064 \\
5.005\end{array}$ \\
\hline 15 & 2500 & $51 \cdot 0$ & .00335 & .01340 & .00102 & .00408 & 1.455 & $4 \cdot 437$ & 4.953 \\
\hline $\begin{array}{l}15 \\
15 \\
15\end{array}$ & $\begin{array}{l}2500 \\
2500 \\
2500\end{array}$ & $\begin{array}{l}52 \cdot 0 \\
53 \cdot 0 \\
54 \cdot 0\end{array}$ & $\begin{array}{l}.00332 \\
.00328 \\
.00324\end{array}$ & $\begin{array}{r}.01328 \\
.01312 \\
.01296\end{array}$ & $\begin{array}{l}.00101 \\
.00100 \\
.00098\end{array}$ & $\begin{array}{l}.00404 \\
.00400 \\
.00395\end{array}$ & $\begin{array}{l}1.441 \\
1.425 \\
1.408\end{array}$ & $\begin{array}{l}4 \cdot 395 \\
4 \cdot 344 \\
4 \cdot 292\end{array}$ & $\begin{array}{l}4 \cdot 90 \\
4 \cdot 85 \\
4 \cdot 79\end{array}$ \\
\hline $\begin{array}{l}15 \\
15 \\
15\end{array}$ & $\begin{array}{l}2500 \\
2500 \\
2500\end{array}$ & $\begin{array}{l}55.0 \\
60.0 \\
65.0\end{array}$ & $\begin{array}{l}.00321 \\
.00309 \\
.00301\end{array}$ & $\begin{array}{r}.01284 \\
.01239 \\
.01206\end{array}$ & $\begin{array}{l}.00097 \\
.00094 \\
.00091\end{array}$ & $\begin{array}{r}.00391 \\
.00377 \\
.00367\end{array}$ & $\begin{array}{l}1.394 \\
1.345 \\
1.310\end{array}$ & $\begin{array}{l}4.249 \\
4.101 \\
3.994\end{array}$ & $\begin{array}{l}4.744 \\
4.579 \\
4.459\end{array}$ \\
\hline $\begin{array}{l}15 \\
15 \\
15\end{array}$ & $\begin{array}{l}2500 \\
2500 \\
2500\end{array}$ & $\begin{array}{l}70.0 \\
75.0 \\
80.0\end{array}$ & $\begin{array}{r}.00293 \\
.00287 \\
.00281\end{array}$ & $\begin{array}{r}.01175 \\
.01150 \\
.01124\end{array}$ & $\begin{array}{l}.00089 \\
.00087 \\
.00085\end{array}$ & $\begin{array}{l}.00358 \\
.00350 \\
.00342\end{array}$ & $\begin{array}{l}1.276 \\
1.248 \\
1.221\end{array}$ & $\begin{array}{l}3.890 \\
3.806 \\
3.722\end{array}$ & $\begin{array}{l}4 \cdot 343 \\
4 \cdot 249 \\
4.155\end{array}$ \\
\hline $\begin{array}{l}15 \\
15 \\
15\end{array}$ & $\begin{array}{l}2500 \\
2500 \\
2500\end{array}$ & $\begin{array}{l}85.0 \\
90.0 \\
95.0\end{array}$ & $\begin{array}{l}.00275 \\
.00270 \\
.00265\end{array}$ & $\begin{array}{r}.01102 \\
.01081 \\
.01060\end{array}$ & $\begin{array}{l}.00084 \\
.00082 \\
.00080\end{array}$ & $\begin{array}{l}.00336 \\
.00329 \\
.00323\end{array}$ & $\begin{array}{l}1.197 \\
1.174 \\
1.151\end{array}$ & $\begin{array}{l}3.649 \\
3.579 \\
3.509\end{array}$ & $\begin{array}{l}4.074 \\
3.995 \\
3.918\end{array}$ \\
\hline 5 & 2 & J & 00260 & 042 & .00079 & 0031.7 & 31 & 3.449 & .85 \\
\hline
\end{tabular}


TABULATION OF DATA FOR

ABSORPTION OF SOUND IN AIR VERSUS PERCENT RELATIVE HUMIDITY AT 15 DEGREES CENTIGRADE FOR A FREQUENCY OF 3200 HERTZ

\begin{tabular}{|c|c|c|c|c|c|c|c|c|c|}
\hline (I) & $(2)$ & (3) & (4) & (5) & $(6)$ & (7) & 181 & $(9)$ &.$(10)$ \\
\hline $\begin{array}{l}\text { TEMP } \\
\text { DEGR } \\
\text { CENT }\end{array}$ & FREQ & $\begin{array}{l}\text { REL } \\
\text { HUM } \\
\text { PER } \\
\text { CENT }\end{array}$ & $\begin{array}{l}\text { ATTEN } \\
\text { COEF } \\
\text { PER } \\
\text { METER }\end{array}$ & $\begin{array}{l}\text { PER } \\
\text { METER }\end{array}$ & $\begin{array}{l}\text { ATTEN } \\
\text { COEF } \\
\text { PER } \\
\text { FOOT. }\end{array}$ & $\begin{array}{l}\text { PER } \\
\text { FOOT }\end{array}$ & $\begin{array}{l}\text { ATTEN } \\
\text { DB PER } \\
100 \\
\text { METER }\end{array}$ & $\begin{array}{l}\text { ATTEN } \\
\text { DB PER } \\
\text { IOOO } \\
\text { FEET }\end{array}$ & $\begin{array}{l}\text { DECAY } \\
\text { RATE } \\
\text { DB PER } \\
\text { SECOND }\end{array}$ \\
\hline $\begin{array}{l}15 \\
15 \\
15\end{array}$ & $\begin{array}{l}3200 \\
3200 \\
3200\end{array}$ & $\begin{array}{r}5.0 \\
10.0 \\
15.0\end{array}$ & $\begin{array}{r}.01108 \\
.01858 \\
.01670\end{array}$ & $\begin{array}{r}.04435 \\
.07432 \\
.06681\end{array}$ & $\begin{array}{l}.00338 \\
.00566 \\
.00509\end{array}$ & $\begin{array}{l}.01352 \\
.02265 \\
.02036\end{array}$ & $\begin{array}{l}4.816 \\
8.070 \\
7.254\end{array}$ & $\begin{array}{l}14.680 \\
24: 597 \\
22.111\end{array}$ & $\begin{array}{l}16.390 \\
27.462 \\
24.686\end{array}$ \\
\hline $\begin{array}{l}15 \\
15 \\
15\end{array}$ & $\begin{array}{l}3200 \\
3200 \\
3200\end{array}$ & $\begin{array}{l}20.0 \\
25.0 \\
30.0\end{array}$ & $\begin{array}{r}.01254 \\
.00997 \\
.00816\end{array}$ & $\begin{array}{r}.05018 \\
.03990 \\
.03267\end{array}$ & $\begin{array}{r}.00382 \\
.00304 \\
.00248\end{array}$ & $\begin{array}{l}.01529 \\
.01216 \\
.00995\end{array}$ & $\begin{array}{l}5.448 \\
4.332 \\
3.547\end{array}$ & $\begin{array}{l}16.607 \\
13.207 \\
10.813\end{array}$ & $\begin{array}{l}18.541 \\
14.745 \\
12.072\end{array}$ \\
\hline $\begin{array}{l}15 \\
15 \\
15\end{array}$ & $\begin{array}{l}3200 \\
3200 \\
3200\end{array}$ & $\begin{array}{l}35.0 \\
40.0 \\
45.0\end{array}$ & $\begin{array}{l}.00695 \\
.00606 \\
.00540\end{array}$ & $\begin{array}{r}.02781 \\
.02426 \\
.02162\end{array}$ & $\begin{array}{l}.00211 \\
.00184 \\
.00164\end{array}$ & $\begin{array}{l}.00847 \\
.00739 \\
.00659\end{array}$ & $\begin{array}{l}3.019 \\
2.635 \\
2.348\end{array}$ & $\begin{array}{l}9.204 \\
8.031 \\
7.157\end{array}$ & $\begin{array}{r}10.276 \\
8.967 \\
7.990\end{array}$ \\
\hline $\begin{array}{l}15 \\
15 \\
15\end{array}$ & $\begin{array}{l}3200 \\
3200 \\
3200\end{array}$ & $\begin{array}{l}46.0 \\
47.0 \\
48.0\end{array}$ & $\begin{array}{l}.00530 \\
.00520 \\
.00511\end{array}$ & $\begin{array}{r}.02121 \\
.02082 \\
.02047\end{array}$ & $\begin{array}{r}.00161 \\
.00158 \\
.00156\end{array}$ & $\begin{array}{l}.006646 \\
.00634 \\
.00624\end{array}$ & $\begin{array}{l}2.303 \\
2.260 \\
2.223\end{array}$ & $\begin{array}{l}7.020 \\
6.891 \\
6.776\end{array}$ & $\begin{array}{l}7.837 \\
7.693 \\
7.566\end{array}$ \\
\hline $\begin{array}{l}15 \\
15 \\
15\end{array}$ & $\begin{array}{l}3200 \\
3200 \\
3200\end{array}$ & $\begin{array}{l}49.0 \\
50.0 \\
51.0\end{array}$ & $\begin{array}{r}.00503 \\
.00493 \\
.00484\end{array}$ & $\begin{array}{r}.02012 \\
.01974 \\
.01937\end{array}$ & $\begin{array}{l}.00153 \\
.00150 \\
.00147\end{array}$ & $\begin{array}{l}.00613 \\
.00601 \\
.00590\end{array}$ & $\begin{array}{l}2 \cdot 184 \\
2 \cdot 143 \\
2 \cdot 103\end{array}$ & $\begin{array}{l}6.659 \\
6.534 \\
6.412\end{array}$ & $\begin{array}{l}7.435 \\
7.295 \\
7.159\end{array}$ \\
\hline $\begin{array}{l}15 \\
15 \\
15 .\end{array}$ & $\begin{array}{l}3200 \\
3200 \\
3200\end{array}$ & $\begin{array}{l}52.0 \\
53.0 \\
54.0\end{array}$ & $\begin{array}{r}.00477 \\
.00470 \\
.00464\end{array}$ & $\begin{array}{r}.01910 \\
.01882 \\
.01858\end{array}$ & $\begin{array}{l}.00145 \\
.00143 \\
.00141\end{array}$ & $\begin{array}{l}.00582 \\
.00573 \\
.00566\end{array}$ & $\begin{array}{l}2.073 \\
2.043 \\
2.017\end{array}$ & $\begin{array}{l}6.321 \\
6.229 \\
6.149\end{array}$ & $\begin{array}{l}7.057 \\
6.955 \\
6.865\end{array}$ \\
\hline $\begin{array}{l}15 \\
15 \\
15\end{array}$ & $\begin{array}{l}3200 \\
3200 \\
3200\end{array}$ & $\begin{array}{l}55.0 \\
60.0 \\
65.0\end{array}$ & $\begin{array}{r}.00458 \\
.00437 \\
.00418\end{array}$ & $\begin{array}{r}.01834 \\
.01749 \\
.01672\end{array}$ & $\begin{array}{l}.00139 \\
.00133 \\
.00127\end{array}$ & $\begin{array}{r}.00559 \\
.00533 \\
.00509\end{array}$ & $\begin{array}{l}1.991 \\
1.899 \\
1.816\end{array}$ & $\begin{array}{l}6.069 \\
5.788 \\
5.535\end{array}$ & $\begin{array}{l}6.776 \\
6.462 \\
6.180\end{array}$ \\
\hline $\begin{array}{l}15 \\
15 \\
15\end{array}$ & $\begin{array}{l}3200 \\
3200 \\
3200\end{array}$ & $\begin{array}{l}70.0 \\
75.0 \\
80.0\end{array}$ & $\begin{array}{l}.00405 \\
.00396 \\
.00388\end{array}$ & $\begin{array}{r}.01623 \\
.01587 \\
.01553\end{array}$ & $\begin{array}{l}.00123 \\
.00120 \\
.00118\end{array}$ & $\begin{array}{l}.00494 \\
.00483 \\
.00473\end{array}$ & $\begin{array}{l}1.762 \\
1.723 \\
1.686\end{array}$ & $\begin{array}{l}\$ .373 \\
5.254 \\
5.140\end{array}$ & $\begin{array}{l}5.999 \\
5.866 \\
5.738\end{array}$ \\
\hline $\begin{array}{l}15 \\
15 \\
15\end{array}$ & $\begin{array}{l}3200 \\
3200 \\
3200\end{array}$ & $\begin{array}{l}85.0 \\
90.0 \\
95.0\end{array}$ & $\begin{array}{l}.00380 \\
.00373 \\
.00366\end{array}$ & $\begin{array}{r}.01522 \\
.01494 \\
.01467\end{array}$ & $\begin{array}{l}.00116 \\
.00113 \\
.00111\end{array}$ & $\begin{array}{l}.00464 \\
.00455 \\
.00447\end{array}$ & $\begin{array}{l}1.653 \\
1.623 \\
1.593\end{array}$ & $\begin{array}{l}5.038 \\
4.947 \\
4.855\end{array}$ & $\begin{array}{l}5.625 \\
5.523 \\
5.421\end{array}$ \\
\hline 15 & 0 & 100.0 & .00360 & .01443 & .00110 & .00440 & 1.567 & 4.778 & 5.334 \\
\hline
\end{tabular}


TABULATION OF DATA FOR

ABSORPTION OF SOUND IN AIR VERSUS PERCENT RELATIVE HUMIDITY AT 15 DEGREES GENTIGRADE FOR A FREQUENCY OF 4000 HERTZ

\begin{tabular}{|c|c|c|c|c|c|c|c|c|c|}
\hline (1) & $(2)$ & (3) & $(4)$ & (5) & $(6)$ & 171 & 181 & 191 & $(10)$ \\
\hline EMP & FREQ & $\begin{array}{l}\text { REL } \\
\text { HUM }\end{array}$ & $\begin{array}{l}\text { ATTEN. } \\
\text { COEF }\end{array}$ & $4 M$ & $\begin{array}{l}\text { ATTEN } \\
\text { COEF }\end{array}$ & $4 M$ & $\begin{array}{l}\text { ATTEN } \\
\text { DB PER }\end{array}$ & $\begin{array}{l}\text { ATTEN } \\
\text { DB PER }\end{array}$ & $\begin{array}{l}\text { DECAY } \\
\text { RATE }\end{array}$ \\
\hline $\begin{array}{l}E G R \\
\text { ENT }\end{array}$ & HERTZ & $\begin{array}{l}\text { PER } \\
\text { CENT }\end{array}$ & $\begin{array}{l}\text { PEER } \\
\text { METER }\end{array}$ & $\begin{array}{l}\text { PER } \\
\text { METER }\end{array}$ & $\begin{array}{l}\text { PER } \\
\text { FOOT }\end{array}$ & $\begin{array}{l}\text { PER } \\
\text { FOOT }\end{array}$ & $\begin{array}{l}100 \\
\text { METER }\end{array}$ & $\begin{array}{l}1000 \\
\text { FEET }\end{array}$ & $\begin{array}{l}\text { DB PER } \\
\text { SECOND }\end{array}$ \\
\hline $\begin{array}{l}15 \\
15 \\
15\end{array}$ & $\begin{array}{l}4000 \\
4000 \\
4000\end{array}$ & $\begin{array}{r}5.0 \\
10.0 \\
15.0\end{array}$ & $\begin{array}{l}.01210 \\
.02247 \\
.02276\end{array}$ & $\begin{array}{r}.04843 \\
.08991 \\
.09105\end{array}$ & $\begin{array}{l}.00369 \\
.00685 \\
.00693\end{array}$ & $\begin{array}{r}.01476 \\
.02740 \\
.02775\end{array}$ & $\begin{array}{l}5.258 \\
9.762 \\
9.886\end{array}$ & $\begin{array}{l}16.029 \\
29.755 \\
30.134\end{array}$ & $\begin{array}{l}17.896 \\
33.220 \\
33.643\end{array}$ \\
\hline $\begin{array}{l}15 \\
15 \\
15\end{array}$ & $\begin{array}{l}4000 \\
4000 \\
4000\end{array}$ & $\begin{array}{l}20.0 \\
25.0 \\
30.0\end{array}$ & $\begin{array}{r}.01858 \\
.01459 \\
.01215\end{array}$ & $\begin{array}{r}.07435 \\
.05836 \\
.04861\end{array}$ & $\begin{array}{l}.00566 \\
.00444 \\
.00370\end{array}$ & $\begin{array}{r}.02266 \\
.01779 \\
.01481\end{array}$ & $\begin{array}{l}8.072 \\
6.337 \\
5.278\end{array}$ & $\begin{array}{l}24.606 \\
19.316 \\
16.088\end{array}$ & $\begin{array}{l}27.472 \\
21.566 \\
17.962\end{array}$ \\
\hline $\begin{array}{l}15 \\
15 \\
15\end{array}$ & $\begin{array}{l}4000 \\
4000 \\
4000\end{array}$ & $\begin{array}{l}35.0 \\
40.0 \\
45.0\end{array}$ & $\begin{array}{r}.01027 \\
.00894 \\
.00794\end{array}$ & $\begin{array}{r}.04108 \\
.03577 \\
.03178\end{array}$ & $\begin{array}{l}.00313 \\
.00272 \\
.00242\end{array}$ & $\begin{array}{r}.01252 \\
.01090 \\
.00968\end{array}$ & $\begin{array}{l}4.460 \\
3.884 \\
3.451\end{array}$ & $\begin{array}{l}13.595 \\
11.840 \\
10.519\end{array}$ & $\begin{array}{l}15.179 \\
13.219 \\
11.744\end{array}$ \\
\hline 15 & 4000 & 46.0 & .00776 & .03107 & .00236 & .00947 & 3.374 & 10.285 & $11 \cdot 483$ \\
\hline $\begin{array}{l}15 \\
15\end{array}$ & $\begin{array}{l}4000 \\
4000\end{array}$ & $\begin{array}{l}47.0 \\
48.0\end{array}$ & $\begin{array}{l}.00760 \\
.00743\end{array}$ & $\begin{array}{l}.03040 \\
.02974\end{array}$ & $\begin{array}{l}.00231 \\
.00226\end{array}$ & $\begin{array}{l}.00926 \\
.00906\end{array}$ & $\begin{array}{l}3.301 \\
3.229\end{array}$ & $\begin{array}{r}10.063 \\
9.842\end{array}$ & $\begin{array}{l}11.235 \\
10.989\end{array}$ \\
\hline $\begin{array}{l}15 \\
15 \\
15\end{array}$ & $\begin{array}{l}4000 \\
4000 \\
4000\end{array}$ & $\begin{array}{l}49.0 \\
50.0 \\
51.0\end{array}$ & $\begin{array}{r}.00728 \\
.00715 \\
.00702\end{array}$ & $\begin{array}{l}.02914 \\
.02861 \\
.02809\end{array}$ & $\begin{array}{l}.00222 \\
.00218 \\
.00214\end{array}$ & $\begin{array}{l}.00888 \\
.00872 \\
.00856\end{array}$ & $\begin{array}{l}3.164 \\
3.106 \\
3.049\end{array}$ & $\begin{array}{l}9.645 \\
9.468 \\
9.296\end{array}$ & $\begin{array}{l}10.769 \\
10.571 \\
10.378\end{array}$ \\
\hline 15 & 4000 & 52.0 & .00689 & .02759 & .00210 & .0084 & 996 & 9.132 & 195 \\
\hline 15 & $\begin{array}{l}4000 \\
4000\end{array}$ & $\begin{array}{l}53.0 \\
54.0\end{array}$ & $\begin{array}{l}.00678 \\
.00667\end{array}$ & $\begin{array}{l}.02714 \\
.02670\end{array}$ & $\begin{array}{l}.00206 \\
.00203\end{array}$ & $\begin{array}{l}.00827 \\
.00813\end{array}$ & $\begin{array}{l}2.947 \\
2.899\end{array}$ & $\begin{array}{l}8.984 \\
8.836\end{array}$ & $\begin{array}{r}10.030 \\
9.865\end{array}$ \\
\hline $\begin{array}{l}15 \\
15 \\
15\end{array}$ & $\begin{array}{l}4000 \\
4000 \\
4000\end{array}$ & $\begin{array}{l}55.0 \\
60.0 \\
65.0\end{array}$ & $\begin{array}{l}.00658 \\
.00611 \\
.00579\end{array}$ & $\begin{array}{l}.02632 \\
.02444 \\
.02316\end{array}$ & $\begin{array}{l}.00200 \\
.00186 \\
.00176\end{array}$ & $\begin{array}{l}.00802 \\
.00744 \\
.00705\end{array}$ & $\begin{array}{l}2.857 \\
2.653 \\
2.514\end{array}$ & $\begin{array}{l}8.710 \\
8.088 \\
7.664\end{array}$ & $\begin{array}{l}9.725 \\
9.030 \\
8.557 .\end{array}$ \\
\hline $\begin{array}{l}15 \\
15 \\
15\end{array}$ & $\begin{array}{l}4000 \\
4000 \\
4000\end{array}$ & $\begin{array}{l}70.0 \\
75.0 \\
80.0\end{array}$ & $\begin{array}{l}.00556 \\
.00535 \\
.00521\end{array}$ & $\begin{array}{l}.02227 \\
.02143 \\
.02086\end{array}$ & $\begin{array}{l}.00169 \\
.00163 \\
.00158\end{array}$ & $\begin{array}{l}.00678 \\
.00653 \\
.00635\end{array}$ & $\begin{array}{l}2.418 \\
2.327 \\
2.265\end{array}$ & $\begin{array}{l}7.370 \\
7.094 \\
6.905\end{array}$ & $\begin{array}{l}8.228 \\
7.920 \\
7.709\end{array}$ \\
\hline $\begin{array}{l}15 \\
15 \\
15\end{array}$ & $\begin{array}{l}4000 \\
4000 \\
4000\end{array}$ & $\begin{array}{l}85.0 \\
90.0 \\
95.0\end{array}$ & $\begin{array}{r}.00511 \\
.00502 \\
.00492\end{array}$ & $\begin{array}{l}.02046 \\
.02008 \\
.01971\end{array}$ & $\begin{array}{l}.00155 \\
.00153 \\
.00150\end{array}$ & $\begin{array}{l}.00623 \\
.00612 \\
.00600\end{array}$ & $\begin{array}{l}2.221 \\
2.181 \\
2.140\end{array}$ & $\begin{array}{l}6.771 \\
6.648 \\
6.525\end{array}$ & $\begin{array}{l}7.559 \\
7.422 \\
7.285\end{array}$ \\
\hline 15 & 4000 & 0.0 & 00485 & 01941 & 00147 & .00591 & 2.107 & 6.425 & 7.173 \\
\hline
\end{tabular}


TABULATION OF DATA FOR

ABSORPTION OF SOUND IN AIR VERSUS PERCENT RELATIVE HUMIDITY AT 15 DEGREES CENTIGRADE FOR A.FREQUENCY OF 5000 HERTZ

\begin{tabular}{|c|c|c|c|c|c|c|c|c|c|}
\hline 111 & (2) & (3) & (4) & (5) & (6) & $(7)$ & $(8)$ & $(9)$ & $(10)$ \\
\hline EMP & FREQ & $\begin{array}{l}\text { REL } \\
\text { HUM }\end{array}$ & $\begin{array}{l}\text { ATTEN } \\
\text { COEF }\end{array}$ & $4 M$ & $\begin{array}{l}\text { ATTEN } \\
\text { COEF }\end{array}$ & $4 M$ & $\begin{array}{l}\text { ATTEN } \\
\text { DB , PER }\end{array}$ & $\begin{array}{l}\text { ATTEN } \\
\text { DB PER }\end{array}$ & $\begin{array}{l}\text { DECAY } \\
\text { RATE }\end{array}$ \\
\hline $\begin{array}{l}\text { DEGR } \\
\text { CENT }\end{array}$ & HERTZ & $\begin{array}{l}\text { PER } \\
\text { CENT }\end{array}$ & $\begin{array}{l}\text { PER } \\
\text { METER }\end{array}$ & $\begin{array}{l}\text { PER } \\
\text { METER }\end{array}$ & $\begin{array}{l}\text { PER } \\
\text { FOOT }\end{array}$ & $\begin{array}{l}\text { PER } \\
\text { FOOT }\end{array}$ & $\begin{array}{l}100 \\
\text { METER }\end{array}$ & $\begin{array}{l}1000 \\
\text { FEET. }\end{array}$ & $\begin{array}{l}\text { DB PER PER } \\
\text { SECOND }\end{array}$ \\
\hline $\begin{array}{l}15 \\
15 \\
15\end{array}$ & $\begin{array}{l}5000 \\
5000 \\
5000\end{array}$ & $\begin{array}{r}5.0 \\
10.0 \\
15.0\end{array}$ & $\begin{array}{r}.01338 \\
.02613 \\
.02942\end{array}$ & $\begin{array}{r}.05354 \\
.10455 \\
.11771\end{array}$ & $\begin{array}{l}.00408 \\
.00796 \\
.00896\end{array}$ & $\begin{array}{r}.01632 \\
.03186 \\
.03587\end{array}$ & $\begin{array}{r}5.814 \\
11.351 \\
12.780\end{array}$ & $\begin{array}{l}17.721 \\
34.601 \\
38.956\end{array}$ & $\begin{array}{l}19.785 \\
38.630 \\
43.492\end{array}$ \\
\hline $\begin{array}{l}15 \\
15\end{array}$ & $\begin{array}{l}5000 \\
5000\end{array}$ & $\begin{array}{l}20.0 \\
25.0\end{array}$ & $\begin{array}{r}.02651 \\
.02149\end{array}$ & $\begin{array}{r}.10607 \\
.08598\end{array}$ & $\begin{array}{l}.00808 \\
.00655\end{array}$ & $\begin{array}{l}.03233 \\
.02620\end{array}$ & $\begin{array}{r}11.517 \\
9.335\end{array}$ & $\begin{array}{l}35.105 \\
28.454\end{array}$ & $\begin{array}{l}39.193 \\
31.768\end{array}$ \\
\hline 15 & 5000 & 30.0 & .01770 & .07080 & .00539 & .02158 & 7.687 & $23 \cdot 432$ & 26.160 \\
\hline $\begin{array}{l}15 \\
15\end{array}$ & $\begin{array}{l}5000 \\
5000\end{array}$ & $\begin{array}{l}35.0 \\
40.0\end{array}$ & $\begin{array}{r}.01520 \\
.01314\end{array}$ & $\begin{array}{l}.06081 \\
.05259\end{array}$ & $\begin{array}{l}.00463 \\
.00400\end{array}$ & $\begin{array}{r}.01853 \\
.01603\end{array}$ & & $\begin{array}{l}20.124 \\
17.406 .\end{array}$ & $\begin{array}{l}22.468 \\
19.433\end{array}$ \\
\hline 15 & 5000 & 45.0 & .01165 & .04661 & .00355 & .01420 & 5.061 & $15 \cdot 427$ & $17 \cdot 224$ \\
\hline $\begin{array}{l}15 \\
15\end{array}$ & $\begin{array}{l}5000 \\
5000\end{array}$ & $\begin{array}{l}46.0 \\
47.0\end{array}$ & $\begin{array}{l}.01140 \\
.01116\end{array}$ & $\begin{array}{l}.04561 \\
.04465\end{array}$ & $\begin{array}{l}.00347 \\
.00340\end{array}$ & & & $\begin{array}{l}15.096 \\
14.779\end{array}$ & $\begin{array}{l}16.854 \\
16.500\end{array}$ \\
\hline 15 & 5000 & 48.0 & .01094 & .04377 & .00333 & .01334 & $4 \cdot 752$ & $14 \cdot 486$ & 16.173 \\
\hline $\begin{array}{l}15 \\
15\end{array}$ & $\begin{array}{l}5000 \\
5000\end{array}$ & $\begin{array}{l}49.0 \\
50.0\end{array}$ & $\begin{array}{l}.01073 \\
.01052\end{array}$ & $\begin{array}{r}.04294 \\
.04211\end{array}$ & $\begin{array}{l}.00327 \\
.00320\end{array}$ & $\begin{array}{r}.01308 \\
.01283\end{array}$ & & $\begin{array}{l}14.212 \\
13.937\end{array}$ & $\begin{array}{l}15.867 \\
15.560\end{array}$ \\
\hline 15 & 5000 & 51.0 & .01030 & .04122 & .00314 & .01256 & 4.476 & $13 \cdot 644$ & 15.233 \\
\hline $\begin{array}{l}15 \\
15\end{array}$ & $\begin{array}{l}5000 \\
5000\end{array}$ & $\begin{array}{l}52.0 \\
53.0\end{array}$ & $\begin{array}{l}.01010 \\
.00991\end{array}$ & $\begin{array}{l}.04041 \\
.03964\end{array}$ & $\begin{array}{l}.00307 \\
.00302\end{array}$ & & & $\begin{array}{l}13.374 \\
13.120\end{array}$ & $\begin{array}{l}14.932 \\
14.648\end{array}$ \\
\hline 15 & 5000 & 54.0 & .00973 & .03892 & .00296 & .01186 & $4 \cdot 225$ & 12.880 & 14.380 \\
\hline $\begin{array}{l}15 \\
15 \\
15\end{array}$ & $\begin{array}{l}5000 \\
5000 \\
5000\end{array}$ & $\begin{array}{l}55.0 \\
60.0 \\
65.0\end{array}$ & $\begin{array}{l}.00954 \\
.00880 \\
.00825\end{array}$ & $\begin{array}{l}.03819 \\
.03522 \\
.03301\end{array}$ & $\begin{array}{l}.00291 \\
.00268 \\
.00251\end{array}$ & $\begin{array}{l}.01164 \\
.01073 \\
.01006\end{array}$ & & $\begin{array}{l}12.640 \\
11.658 \\
10.926\end{array}$ & $\begin{array}{l}14 \cdot 112 \\
13.016 \\
12.198\end{array}$ \\
\hline $\begin{array}{l}15 \\
15 \\
15\end{array}$ & $\begin{array}{l}5000 \\
5000 \\
5000\end{array}$ & $\begin{array}{l}70.0 \\
75.0 \\
80.0\end{array}$ & $\begin{array}{l}.00775 \\
.00741 \\
.00717\end{array}$ & $\begin{array}{l}.03103 \\
.02967 \\
.02869\end{array}$ & $\begin{array}{l}.00236 \\
.00226 \\
.00218\end{array}$ & $\begin{array}{r}.00945 \\
.00904 \\
.00874\end{array}$ & & $\begin{array}{r}10.270 \\
9.821 \\
9.494\end{array}$ & $\begin{array}{l}11.466 \\
10.965 \\
10.600\end{array}$ \\
\hline $\begin{array}{l}15 \\
15 \\
15\end{array}$ & $\begin{array}{l}5000 \\
5000 \\
5000\end{array}$ & $\begin{array}{l}85.0 \\
90.0 \\
95.0\end{array}$ & $\begin{array}{l}.00694 \\
.00676 \\
.00663\end{array}$ & $\begin{array}{l}.02777 \\
.02705 \\
.02655\end{array}$ & $\begin{array}{l}.00211 \\
.00206 \\
.00202\end{array}$ & $\begin{array}{r}.00846 \\
.00824 \\
.00809\end{array}$ & $\begin{array}{l}6 \\
7 \\
3\end{array}$ & $\begin{array}{l}9.193 \\
8.952 \\
8.789\end{array}$ & $\begin{array}{r}10.263 \\
9.994 \\
9.812\end{array}$ \\
\hline 15 & 5000 & . & .00653 & 02614 & .00199 & .00796 & 2.838 & 8.652 & 9.66 \\
\hline
\end{tabular}


TABULATION OF DATA FOR

ABSORPTION OF SOUND IN AIR VERSUS PERCENT RELATIVE HUMIDITY AT 15 DEGREES CENTIGRADE FOR A FREQUENCY OF 5940 HERTZ

\begin{tabular}{|c|c|c|c|c|c|c|c|c|c|}
\hline (1) & (2) & (3) & $(4)$ & (5) & (6) & $(7)$ & (8) & $(9)$ & $(20)$ \\
\hline $\begin{array}{l}\text { TEMP } \\
\text { DEGR } \\
\text { CENT }\end{array}$ & HER T & $\begin{array}{l}\text { REL } \\
\text { HUM } \\
\text { PER } \\
\text { CENT }\end{array}$ & $\begin{array}{l}\text { ATTEN } \\
\text { COEF } \\
\text { PER } \\
\text { METER }\end{array}$ & $\begin{array}{l}4 M \\
\text { PER } \\
\text { METER }\end{array}$ & $\begin{array}{l}\text { ATTEN } \\
\text { COEF } \\
\text { PER } \\
\text { FOOT }\end{array}$ & $\begin{array}{l}4 M \\
\text { PER } \\
\text { FOOT }\end{array}$ & $\begin{array}{l}\text { ATTEN } \\
\text { DB PER } \\
100 \\
\text { METER }\end{array}$ & $\begin{array}{l}\text { ATTEN } \\
\text { DB PER } \\
1000 \\
\text { FEET }\end{array}$ & $\begin{array}{l}\text { DECAY } \\
\text { RATE } \\
\text { DB PER } \\
\text { SECOND }\end{array}$ \\
\hline $\begin{array}{l}15 \\
15 \\
15\end{array}$ & $\begin{array}{l}5940 \\
5940 \\
5940\end{array}$ & $\begin{array}{r}5.0 \\
10.0 \\
15.0\end{array}$ & $\begin{array}{r}.01443 \\
.02814 \\
.03505\end{array}$ & $\begin{array}{r}.05774 \\
.11258 \\
.14020\end{array}$ & $\begin{array}{l}.00440 \\
.00857 \\
.01068\end{array}$ & $\begin{array}{r}.01760 \\
.03431 \\
.04273\end{array}$ & $\begin{array}{r}6.269 \\
12.223 \\
15.222\end{array}$ & $\begin{array}{l}19.109 \\
37.257 \\
46.398\end{array}$ & $\begin{array}{l}21.334 \\
41.596 \\
51.802\end{array}$ \\
\hline $\begin{array}{l}15 \\
15 \\
15\end{array}$ & $\begin{array}{l}5940 \\
5940 \\
5940\end{array}$ & $\begin{array}{l}20.0 \\
25.0 \\
30.0\end{array}$ & $\begin{array}{r}.03388 \\
.02908 \\
.02403\end{array}$ & $\begin{array}{r}.13554 \\
.11635 \\
.09613\end{array}$ & $\begin{array}{r}.01032 \\
.00886 \\
.00732\end{array}$ & $\begin{array}{r}.04131 \\
.03546 \\
.02930\end{array}$ & $\begin{array}{l}14.717 \\
12.632 \\
10.437\end{array}$ & $\begin{array}{l}44 \cdot 858 \\
38.505 \\
31.815\end{array}$ & $\begin{array}{l}50.082 \\
42.989 \\
35.520\end{array}$ \\
\hline $\begin{array}{l}15 \\
15 \\
15\end{array}$ & $\begin{array}{l}5940 \\
5940 \\
5940\end{array}$ & $\begin{array}{l}35.0 \\
40.0 \\
45.0\end{array}$ & $\begin{array}{r}.02051 \\
.01798 \\
.01584\end{array}$ & $\begin{array}{l}.08206 \\
.07194 \\
.06338\end{array}$ & $\begin{array}{r}.00625 \\
.00548 \\
.00482\end{array}$ & $\begin{array}{l}.02501 \\
.02192 \\
.01931\end{array}$ & $\begin{array}{l}8.910 \\
7.811 \\
6.881\end{array}$ & $\begin{array}{l}27 \cdot 160 \\
23.809 \\
20.975\end{array}$ & $\begin{array}{l}30.323 \\
26.582 \\
23.418\end{array}$ \\
\hline $\begin{array}{l}15 \\
15 \\
15\end{array}$ & $\begin{array}{l}5940 \\
5940 \\
5940\end{array}$ & $\begin{array}{l}46.0 \\
47.0 \\
48.0\end{array}$ & $\begin{array}{r}.01550 \\
.01516 \\
.01482\end{array}$ & $\begin{array}{l}.06202 \\
.06066 \\
.05930\end{array}$ & $\begin{array}{l}.00472 \\
.00462 \\
.00451\end{array}$ & $\begin{array}{r}.01890 \\
.01849 \\
.01807\end{array}$ & $\begin{array}{l}6.734 \\
6.586 \\
6.439\end{array}$ & $\begin{array}{l}20.526 \\
20.076 \\
19.626\end{array}$ & $\begin{array}{l}22.916 \\
22.414 \\
21.912\end{array}$ \\
\hline $\begin{array}{l}15 \\
15 \\
15\end{array}$ & $\begin{array}{l}5940 \\
5940 \\
5940\end{array}$ & $\begin{array}{l}49.0 \\
50.0 \\
51.0\end{array}$ & $\begin{array}{r}.01452 \\
.01424 \\
.01396\end{array}$ & $\begin{array}{l}.05811 \\
.05696 \\
.05585\end{array}$ & $\begin{array}{l}.00442 \\
.00434 \\
.00425\end{array}$ & $\begin{array}{r}.01771 \\
.01736 \\
.01702\end{array}$ & $\begin{array}{l}6.310 \\
6.184 \\
6.064\end{array}$ & $\begin{array}{l}19.233 \\
18.850 \\
18.485\end{array}$ & $\begin{array}{l}21.472 \\
21.046 \\
20.638\end{array}$ \\
\hline $\begin{array}{l}15 \\
15 \\
15\end{array}$ & $\begin{array}{l}5940 \\
5940 \\
5940\end{array}$ & $\begin{array}{l}52.0 \\
53.0 \\
54.0\end{array}$ & $\begin{array}{r}.01370 \\
.01345 \\
.01322\end{array}$ & $\begin{array}{r}.05482 \\
.05381 \\
.05288\end{array}$ & $\begin{array}{l}.00417 \\
.00410 \\
.00402\end{array}$ & $\begin{array}{l}.01670 \\
.01640 \\
.01611\end{array}$ & $\begin{array}{l}52 \\
43 \\
41\end{array}$ & $\begin{array}{l}18.142 \\
17 \cdot 810 \\
17.501\end{array}$ & $\begin{array}{l}20.255 \\
19.884 \\
19.539\end{array}$ \\
\hline $\begin{array}{l}15 \\
15 \\
15\end{array}$ & $\begin{array}{l}5940 \\
5940 \\
5940\end{array}$ & $\begin{array}{l}55.0 \\
60.0 \\
65.0\end{array}$ & $\begin{array}{l}.01300 \\
.01192 \\
.01104\end{array}$ & $\begin{array}{r}.05200 \\
.04768 \\
.04416\end{array}$ & $\begin{array}{l}.00396 \\
.00363 \\
.00336\end{array}$ & $\begin{array}{r}.01584 \\
.01453 \\
.01346\end{array}$ & $\begin{array}{l}5.645 \\
5.177 \\
4.795\end{array}$ & $\begin{array}{l}17.209 \\
15.781 \\
14.617\end{array}$ & $\begin{array}{l}19.213 \\
17.619 \\
16.319\end{array}$ \\
\hline $\begin{array}{l}15 \\
15 \\
15\end{array}$ & $\begin{array}{l}5940 \\
5940 \\
5940\end{array}$ & $\begin{array}{l}70.0 \\
75.0 \\
80.0\end{array}$ & $\begin{array}{r}.01035 \\
.00980 \\
.00932\end{array}$ & $\begin{array}{l}.04143 \\
.03921 \\
.03728\end{array}$ & $\begin{array}{l}.00315 \\
.00298 \\
.00284\end{array}$ & $\begin{array}{l}.01262 \\
.01195 \\
.01136\end{array}$ & $\begin{array}{l}4 \cdot 498 \\
4 \cdot 258 \\
4 \cdot 048\end{array}$ & $\begin{array}{l}13.712 \\
12.978 \\
12.340\end{array}$ & $\begin{array}{l}15.309 \\
14.490 \\
13.777\end{array}$ \\
\hline $\begin{array}{l}15 \\
15 \\
15\end{array}$ & $\begin{array}{l}5940 \\
5940 \\
5940\end{array}$ & $\begin{array}{l}85.0 \\
90.0 \\
95.0\end{array}$ & $\begin{array}{l}.00898 \\
.00872 \\
.00849\end{array}$ & $\begin{array}{l}.03595 \\
.03491 \\
.03396\end{array}$ & $\begin{array}{l}.00273 \\
.00266 \\
.00258\end{array}$ & $\begin{array}{l}.01095 \\
.01064 \\
.01035\end{array}$ & $\begin{array}{l}3.903 \\
3.790 \\
3.688\end{array}$ & $\begin{array}{l}11.899 \\
11.553 \\
11.241\end{array}$ & $\begin{array}{l}13.284 \\
12.898 \\
12.550\end{array}$ \\
\hline 5 & 5940 & 100.0 & .00828 & 03313 & .00252 & .01009 & 3.597 & 10.964 & $12 \cdot 241$ \\
\hline
\end{tabular}


TABULATION OF DATA FOR

ABSORPTIEN OF SOUND IN AIR VERSUS PERCENT RELATIVE HUMIDITY AT 15 DEGREES CENTIGRADE FER A FREQUENCY OF 6300 HERTZ

\begin{tabular}{|c|c|c|c|c|c|c|c|c|c|}
\hline 111 & $(2)$ & $(3)$ & $(4)$ & (5) & (6) & $(7)$ & (8) & $(9)$ & $(10)$ \\
\hline $\begin{array}{l}\text { EMP } \\
\text { EGR } \\
\text { ENT }\end{array}$ & FREQ & $\begin{array}{l}\text { REL } \\
\text { HUM } \\
\text { PER } \\
\text { CENT }\end{array}$ & $\begin{array}{l}\text { ATTEN } \\
\text { COEF } \\
\text { PER } \\
\text { METER }\end{array}$ & $\begin{array}{l}\text { 4M } \\
\text { PER } \\
\text { METER }\end{array}$ & $\begin{array}{l}\text { ATTEN } \\
\text { COEF } \\
\text { PER } \\
\text { FOOT }\end{array}$ & $\begin{array}{l}4 M \\
\text { PER } \\
\text { FOOT }\end{array}$ & $\begin{array}{l}\text { ATTEN } \\
\text { DB PER } \\
100 \\
\text { METER }\end{array}$ & $\begin{array}{l}\text { ATTEN } \\
\text { DB PER } \\
1000 \\
\text { FEET }\end{array}$ & $\begin{array}{l}\text { DECAY } \\
\text { RATE } \\
\text { OB PER } \\
\text { SECONO }\end{array}$ \\
\hline $\begin{array}{l}15 \\
15 \\
15\end{array}$ & $\begin{array}{l}6300 \\
6300 \\
6300\end{array}$ & $\begin{array}{r}5.0 \\
10.0 \\
15.0\end{array}$ & $\begin{array}{r}.01496 \\
.02905 \\
.03711\end{array}$ & $\begin{array}{r}.05987 \\
.11623 \\
.14845\end{array}$ & $\begin{array}{r}.00456 \\
.00885 \\
.01131\end{array}$ & $\begin{array}{r}.01825 \\
.03542 \\
.04524\end{array}$ & $\begin{array}{r}6.501 \\
12.620 \\
16.118\end{array}$ & $\begin{array}{l}19.816 \\
38.468 \\
49.130\end{array}$ & $\begin{array}{l}22 \cdot 124 \\
42.948 \\
54 \cdot 852\end{array}$ \\
\hline $\begin{array}{l}15 \\
15 \\
15\end{array}$ & $\begin{array}{l}6300 \\
6300 \\
6300\end{array}$ & $\begin{array}{l}20.0 \\
25.0 \\
30.0\end{array}$ & $\begin{array}{l}.03646 \\
.03178 \\
.02639\end{array}$ & $\begin{array}{l}14584 \\
.12714 \\
.10556\end{array}$ & $\begin{array}{r}.01111 \\
.00968 \\
.00804\end{array}$ & $\begin{array}{l}.04445 \\
.03875 \\
.03217\end{array}$ & $\begin{array}{l}15.834 \\
13.804 \\
11.462\end{array}$ & $\begin{array}{l}48.264 \\
42.077 \\
34.936\end{array}$ & $\begin{array}{l}53.885 \\
46.978 \\
39.005\end{array}$ \\
\hline $\begin{array}{l}15 \\
15 \\
15\end{array}$ & $\begin{array}{l}6300 \\
6300 \\
6300\end{array}$ & $\begin{array}{l}35.0 \\
40.0 \\
45.0\end{array}$ & $\begin{array}{r}.02251 \\
.01978 \\
.01748\end{array}$ & $\begin{array}{l}.09004 \\
.07914 \\
.06993\end{array}$ & $\begin{array}{l}.00686 \\
.00603 \\
.00532\end{array}$ & $\begin{array}{l}.02744 \\
.02412 \\
.02131\end{array}$ & $\begin{array}{l}77 \\
93 \\
93\end{array}$ & $\begin{array}{l}29.800 \\
26.192 \\
23.145\end{array}$ & $\begin{array}{l}33.271 \\
29.243 \\
25.841\end{array}$ \\
\hline $\begin{array}{l}15 \\
15 \\
15\end{array}$ & $\begin{array}{l}6300 \\
6300 \\
6300\end{array}$ & $\begin{array}{l}46.0 \\
47.0 \\
48.0\end{array}$ & $\begin{array}{r}.01704 \\
.01668 \\
.01633\end{array}$ & $\begin{array}{l}.06819 \\
.06672 \\
.06532\end{array}$ & $\begin{array}{l}.00519 \\
.00508 \\
.00497\end{array}$ & $\begin{array}{r}.02078 \\
.02033 \\
.01991\end{array}$ & $\begin{array}{l}7.403 \\
7.244 \\
7.092\end{array}$ & $\begin{array}{l}22.567 \\
22.081 \\
21.619\end{array}$ & $\begin{array}{l}25 \cdot 195 \\
24 \cdot 653 \\
24 \cdot 136\end{array}$ \\
\hline $\begin{array}{l}15 \\
15 \\
15\end{array}$ & $\begin{array}{l}6300 \\
6300 \\
6300\end{array}$ & $\begin{array}{l}49.0 \\
50.0 \\
51.0\end{array}$ & $\begin{array}{r}.01598 \\
.01565 \\
.01534\end{array}$ & $\begin{array}{l}.06392 \\
.06261 \\
.06139\end{array}$ & $\begin{array}{l}.00487 \\
.00477 \\
.00467\end{array}$ & $\begin{array}{r}.01948 \\
.01908 \\
.01871\end{array}$ & $\begin{array}{l}41 \\
98 \\
65\end{array}$ & $\begin{array}{l}21 \cdot 156 \\
20 \cdot 722 \\
20 \cdot 318\end{array}$ & $\begin{array}{l}23.620 \\
23.136 \\
22.634\end{array}$ \\
\hline $\begin{array}{l}15 \\
15 \\
1.5\end{array}$ & $\begin{array}{l}6300 \\
6300 \\
6300\end{array}$ & $\begin{array}{l}52.0 \\
53.0 \\
54.0\end{array}$ & $\begin{array}{l}.01506 \\
.01478 \\
.01451\end{array}$ & $\begin{array}{l}.06024 \\
.05912 \\
.05807\end{array}$ & $\begin{array}{l}.00459 \\
.00450 \\
.00442\end{array}$ & $\begin{array}{r}.01836 \\
.01802 \\
.01770\end{array}$ & $\begin{array}{l}6.541 \\
6.419 \\
6.305\end{array}$ & $\begin{array}{l}19.937 \\
19.567 \\
19.220\end{array}$ & $\begin{array}{l}22.259 \\
21.846 \\
21.459\end{array}$ \\
\hline $\begin{array}{l}15 \\
15 \\
15\end{array}$ & $\begin{array}{l}6300 \\
6300 \\
6300\end{array}$ & $\begin{array}{l}55.0 \\
60.0 \\
65.0\end{array}$ & $\begin{array}{r}.01426 \\
.01311 \\
.01212\end{array}$ & $\begin{array}{r}.05707 \\
.05247 \\
.04850\end{array}$ & $\begin{array}{l}.00434 \\
.00399 \\
.00369\end{array}$ & $\begin{array}{r}.01739 \\
.01599 \\
.01478\end{array}$ & $\begin{array}{l}6.197 \\
5.697 \\
5.266\end{array}$ & $\begin{array}{l}18.889 \\
17.364 \\
16.052\end{array}$ & $\begin{array}{l}21.089 \\
19.387 \\
17.921\end{array}$ \\
\hline $\begin{array}{l}15 \\
15 \\
15\end{array}$ & $\begin{array}{l}6300 \\
6300 \\
6300\end{array}$ & $\begin{array}{l}70.0 \\
75.0 \\
80.0\end{array}$ & $\begin{array}{r}.01135 \\
.01075 \\
.01018\end{array}$ & $\begin{array}{r}.04542 \\
.04300 \\
.04075\end{array}$ & $\begin{array}{l}.00346 \\
.00327 \\
.00310\end{array}$ & $\begin{array}{r}.01384 \\
.01310 \\
.01242\end{array}$ & $\begin{array}{r}4.931 \\
4.669 \\
4.424\end{array}$ & $\begin{array}{l}15.031 \\
14.233 \\
13.486\end{array}$ & $\begin{array}{l}16.782 \\
15.891 \\
15.056\end{array}$ \\
\hline $\begin{array}{l}15 \\
15 \\
15\end{array}$ & $\begin{array}{l}6300 \\
6300 \\
6300\end{array}$ & $\begin{array}{l}85.0 \\
90.0 \\
95.0\end{array}$ & $\begin{array}{l}.00977 \\
.00948 \\
.00924\end{array}$ & $\begin{array}{l}.03911 \\
.03795 \\
.03697\end{array}$ & $\begin{array}{l}.00298 \\
.00289 \\
.00281\end{array}$ & $\begin{array}{r}.01192 \\
.01156 \\
.01126\end{array}$ & $\begin{array}{r}4.246 \\
4.120 \\
4.014\end{array}$ & $\begin{array}{l}12 \cdot 9: 44 \\
12 \cdot 559 \\
12 \cdot 236\end{array}$ & $\begin{array}{l}14.451 \\
14.022 \\
13.660\end{array}$ \\
\hline 5 & 6300 & .0 & .00898 & 03595 & .00273 & 01095 & 3.903 & 1.899 & 3.20 .4 \\
\hline
\end{tabular}


TABULLATION OF DATA FOR

ABSORPTION OF SOUND IN AIR VERSUS PERCENT RELATIVE HUMIDITY AT 15 DEGREES CENTIGRADE FOR A FREQUENCY OF 8000. HERTZ

\begin{tabular}{|c|c|c|c|c|c|c|c|c|c|}
\hline (1) & (2) & $(3)$ & (4) & (5) & (6) & $(7)$ & $(8)$ & 191 & $(10)$ \\
\hline $\begin{array}{l}\text { EMP } \\
\text { EGR } \\
\text { ENT }\end{array}$ & HERTZ & $\begin{array}{l}\text { REL } \\
\text { HUM } \\
\text { PER } \\
\text { CENT }\end{array}$ & $\begin{array}{l}\text { ATTEN } \\
\text { COEF } \\
\text { PER } \\
\text { METER }\end{array}$ & $\begin{array}{l}4 M \\
\text { PER } \\
\text { METER }\end{array}$ & $\begin{array}{l}\text { ATTEN } \\
\text { COEF } \\
\text { PER } \\
\text { FOOT }\end{array}$ & $\begin{array}{l}4 M \\
\text { PER } \\
\text { FOOT }\end{array}$ & $\begin{array}{l}\text { ATTEN } \\
\text { DB PER } \\
100 \\
\text { METER }\end{array}$ & $\begin{array}{l}\text { ATTEN } \\
\text { DB PER } \\
1000 \\
\text { FEET }\end{array}$ & $\begin{array}{l}\text { DECAY } \\
\text { RATE } \\
\text { DB PER } \\
\text { SECOND }\end{array}$ \\
\hline $\begin{array}{l}15 \\
15 \\
15\end{array}$ & $\begin{array}{l}8000 \\
8000 \\
8000\end{array}$ & $\begin{array}{r}5.0 \\
10.0 \\
15.0\end{array}$ & $\begin{array}{r}.01700 \\
.03192 \\
.04517\end{array}$ & $\begin{array}{r}.06800 \\
.12769 \\
.18068\end{array}$ & $\begin{array}{r}.00518 \\
.00973 \\
.01376\end{array}$ & $\begin{array}{r}.02072 \\
.03892 \\
.05507\end{array}$ & $\begin{array}{r}7.383 \\
13.864 \\
19.617\end{array}$ & $\begin{array}{l}22.504 \\
42.260 \\
59.795\end{array}$ & $\begin{array}{l}25 \cdot 124 \\
47 \cdot 181 \\
66 \cdot 759\end{array}$ \\
\hline $\begin{array}{l}15 \\
15 \\
15\end{array}$ & $\begin{array}{l}8000 \\
8000 \\
8000\end{array}$ & $\begin{array}{l}20.0 \\
25.0 \\
30.0\end{array}$ & $\begin{array}{r}.04799 \\
.04552 \\
.03995\end{array}$ & $\begin{array}{r}.19197 \\
.18209 \\
.15980\end{array}$ & $\begin{array}{r}.01462 \\
.01387 \\
.01217\end{array}$ & $\begin{array}{l}.05851 \\
.05550 \\
.04870\end{array}$ & $\begin{array}{l}20.843 \\
19.771 \\
17.350\end{array}$ & $\begin{array}{l}63.532 \\
60.263 \\
52.885\end{array}$ & $\begin{array}{l}70.930 \\
67.281 \\
59.044\end{array}$ \\
\hline $\begin{array}{l}15 \\
15\end{array}$ & $\begin{array}{l}8000 \\
8000 \\
8000\end{array}$ & $\begin{array}{l}35.0 \\
40.0 \\
45.0\end{array}$ & $\begin{array}{r}.03408 \\
.02974 \\
.02661\end{array}$ & $\begin{array}{r}. \\
13634 \\
.11898 \\
.10647\end{array}$ & $\begin{array}{r}.01038 \\
.00906 \\
.00811\end{array}$ & $\begin{array}{l}.04 \\
.03 \\
.03\end{array}$ & $\begin{array}{l}14.803 \\
12.918 \\
11.559\end{array}$ & $\begin{array}{l}45 \\
39 \\
35\end{array}$ & $\begin{array}{l}50.375 \\
43.962 \\
39.338\end{array}$ \\
\hline $\begin{array}{l}15 \\
15\end{array}$ & $\begin{array}{l}8000 \\
8000\end{array}$ & $\begin{array}{l}46.0 \\
47.0\end{array}$ & $\begin{array}{r}.02604 \\
.02549\end{array}$ & $\begin{array}{l}.10419 \\
.10198\end{array}$ & $\begin{array}{l}.00793 \\
.00777\end{array}$ & $\begin{array}{r}.03175 \\
.03108\end{array}$ & $\begin{array}{l}11 \cdot 312 \\
11.072\end{array}$ & $\begin{array}{l}34.482 \\
33.750\end{array}$ & $\begin{array}{l}38.497 \\
37.68\end{array}$ \\
\hline 15 & 8000 & 48.0 & .02495 & .09980 & .00760 & .03041 & 10.835 & 33.028 & 36.87 \\
\hline $\begin{array}{l}15 \\
15 \\
15 .\end{array}$ & $\begin{array}{l}8000 \\
8000 \\
8000\end{array}$ & $\begin{array}{l}49.0 \\
50.0 \\
51.0\end{array}$ & $\begin{array}{l}.02444 \\
.02394 \\
.02346\end{array}$ & $\begin{array}{l}.09776 \\
.09577 \\
.09385\end{array}$ & $\begin{array}{l}.00744 \\
.00729 \\
.00715\end{array}$ & $\begin{array}{l}.02979 \\
.02919 \\
.02860\end{array}$ & $\begin{array}{l}10.614 \\
10.398 \\
10.190\end{array}$ & $\begin{array}{l}32.353 \\
31.694 \\
31.060\end{array}$ & $\begin{array}{l}36.121 \\
35.385 \\
34.678\end{array}$ \\
\hline $\begin{array}{l}15 \\
15 \\
15\end{array}$ & $\begin{array}{l}8000 \\
8000 \\
8000\end{array}$ & $\begin{array}{l}52.0 \\
53.0 \\
54.0\end{array}$ & $\begin{array}{r}.02298 \\
.02251 \\
.02204\end{array}$ & $\begin{array}{l}.09195 \\
.09006 \\
.08816\end{array}$ & $\begin{array}{r}.00700 \\
.00686 \\
.00671\end{array}$ & $\begin{array}{r}.02802 \\
.02745 \\
.02687\end{array}$ & $\begin{array}{l}9.984 \\
9.778 \\
9.572\end{array}$ & $\begin{array}{l}30.433 \\
29.805 \\
29.177\end{array}$ & $\begin{array}{l}33.977 \\
33.276 \\
32.575\end{array}$ \\
\hline $\begin{array}{l}15 \\
15 \\
15\end{array}$ & $\begin{array}{l}8000 \\
8000 \\
8000\end{array}$ & $\begin{array}{l}55.0 \\
60.0 \\
65.0\end{array}$ & $\begin{array}{l}.02165 \\
.01986 \\
.01843\end{array}$ & $\begin{array}{l}.08663 \\
.07946 \\
.07372\end{array}$ & $\begin{array}{l}.00660 \\
.00605 \\
.00561\end{array}$ & $\begin{array}{l}.02640 \\
.02421 \\
.02247\end{array}$ & $\begin{array}{l}9.406 \\
8.627 \\
8.004\end{array}$ & $\begin{array}{l}28.670 \\
26.296 \\
24.399\end{array}$ & $\begin{array}{l}32.009 \\
29.359 \\
27.240\end{array}$ \\
\hline $\begin{array}{l}15 \\
15 \\
15\end{array}$ & $\begin{array}{l}8000 \\
8000 \\
8000\end{array}$ & $\begin{array}{l}70.0 \\
75.0 \\
80.0\end{array}$ & $\begin{array}{r}.01718 \\
.01608 \\
.01519\end{array}$ & $\begin{array}{r}.06874 \\
.06434 \\
.06078\end{array}$ & $\begin{array}{l}.00523 \\
.00490 \\
.00463\end{array}$ & $\begin{array}{l}.02095 \\
.01961 \\
.01852\end{array}$ & $\begin{array}{l}7.463 \\
6.985 \\
6.600\end{array}$ & $\begin{array}{l}22.750 \\
21.293 \\
20.117\end{array}$ & $\begin{array}{l}25.399 \\
23.773 \\
22.460\end{array}$ \\
\hline $\begin{array}{l}15 \\
15\end{array}$ & $\begin{array}{l}8000 \\
8000 \\
8000\end{array}$ & $\begin{array}{l}85.0 \\
90.0 \\
95.0\end{array}$ & $\begin{array}{r}.01446 \\
.01385 \\
.01328\end{array}$ & $\begin{array}{l}.05785 \\
.05542 \\
.05314\end{array}$ & $\begin{array}{l}.00440 \\
.00422 \\
.00404\end{array}$ & $\begin{array}{r}.01763 \\
.01689 \\
.01619\end{array}$ & $\begin{array}{l}6.281 \\
6.017 \\
5.770\end{array}$ & $\begin{array}{l}19.145 \\
18.341 \\
17.589\end{array}$ & $\begin{array}{l}21.374 \\
20.477 \\
19.637\end{array}$ \\
\hline 1 & 8000 & .0 & 01285 & .05141 & .00391 & .01567 & 5.582 & 7.016 & 8.99 \\
\hline
\end{tabular}


TABULATION OF DATA FOR

ABSORPTION OF SOUND IN AIR VERSUS PERCENT RELATIVE HUMIDITY AT 15 DEGREES CENTIGRADE FOR A.FREQUENCY OFI0000 HERT.Z

\begin{tabular}{|c|c|c|c|c|c|c|c|c|c|}
\hline (1) & (2) & $(3)$ & (4) & (5) & $(6)$ & $(7)$ & $(8)$ & 191 & $(10)$ \\
\hline $\begin{array}{l}\text { TEMP } \\
\text { DEGR } \\
\text { CENT }\end{array}$ & HERTZ & $\begin{array}{l}\text { REL } \\
\text { HUM } \\
\text { PER } \\
\text { CENT }\end{array}$ & $\begin{array}{l}\text { ATTEN } \\
\text { COEF } \\
\text { PER } \\
\text { METER }\end{array}$ & $\begin{array}{l}4 M \\
\text { PER } \\
\text { METER }\end{array}$ & $\begin{array}{l}\text { ATTEN } \\
\text { COEF } \\
\text { PER } \\
\text { FOOT }\end{array}$ & $\begin{array}{l}4 M \\
\text { PER } \\
\text { FOOT }\end{array}$ & $\begin{array}{l}\text { ATTEN } \\
\text { OB PER } \\
\text { IOO } \\
\text { METER }\end{array}$ & $\begin{array}{l}\text { ATTEN } \\
\text { DB PER } \\
1000 \\
\text { FEET }\end{array}$ & $\begin{array}{l}\text { DECAY } \\
\text { RATE } \\
\text { DB PER } \\
\text { SECOND }\end{array}$ \\
\hline $\begin{array}{l}15 \\
15 \\
15\end{array}$ & $\begin{array}{l}10000 \\
10000 \\
10000\end{array}$ & $\begin{array}{r}5.0 \\
10.0 \\
15.0\end{array}$ & $\begin{array}{r}.01964 \\
.03569 \\
.05251\end{array}$ & $\begin{array}{r}.07858 \\
.14279 \\
.21006\end{array}$ & $\begin{array}{l}.00598 \\
.01088 \\
.01600\end{array}$ & $\begin{array}{l}.02395 \\
.04352 \\
.06402\end{array}$ & $\begin{array}{r}8.532 \\
15.504 \\
22.807\end{array}$ & $\begin{array}{l}26.008 \\
47.258 \\
69.519\end{array}$ & $\begin{array}{l}29.036 \\
52.761 \\
77.615\end{array}$ \\
\hline $\begin{array}{l}15 \\
15 \\
15\end{array}$ & $\begin{array}{l}10000 \\
10000 \\
10000\end{array}$ & $\begin{array}{l}20.0 \\
25.0 \\
30.0\end{array}$ & $\begin{array}{l}.06021 \\
.06017 \\
.05619\end{array}$ & $\begin{array}{r}.24084 \\
.24068 \\
.22479\end{array}$ & $\begin{array}{l}.01835 \\
.01834 \\
.01712\end{array}$ & $\begin{array}{l}.07340 \\
.07336 \\
.06851\end{array}$ & $\begin{array}{l}26.149 \\
26.132 \\
24.407\end{array}$ & $\begin{array}{l}79.704 \\
79.652 \\
74.394\end{array}$ & $\begin{array}{l}88.986 \\
88.928 \\
83.058\end{array}$ \\
\hline 15 & 10000 & 35.0 & .04982 & .19930 & .01518 & .06074 & 21.639 & 65.957 & 73.638 \\
\hline $\begin{array}{l}15 \\
15\end{array}$ & $\begin{array}{l}10000 \\
10000\end{array}$ & $\begin{array}{l}40.0 \\
45.0\end{array}$ & $\begin{array}{r}.04341 \\
.03857\end{array}$ & $\begin{array}{r}.17364 \\
.15429\end{array}$ & $\begin{array}{r}.01323 \\
.01175\end{array}$ & .0 & $\begin{array}{l}18.853 \\
16.752\end{array}$ & $\begin{array}{l}57.467 \\
51.061\end{array}$ & $\begin{array}{l}64.159 \\
57.007\end{array}$ \\
\hline 15 & 10000 & 46.0 & .03774 & .15097 & .01150 & .04601 & 16.392 & 49.965 & 55.783 \\
\hline $\begin{array}{l}15 \\
15\end{array}$ & $\begin{array}{l}10000 \\
10000\end{array}$ & $\begin{array}{l}47.0 \\
48.0\end{array}$ & $\begin{array}{r}.03697 \\
.03625\end{array}$ & $\begin{array}{r}.14791 \\
.14501\end{array}$ & $\begin{array}{l}.01127 \\
.01104\end{array}$ & $\begin{array}{r}.04508 \\
.04419\end{array}$ & $\begin{array}{l}16.059 \\
15.744\end{array}$ & $\begin{array}{l}48 \cdot 949 \\
47 \cdot 990\end{array}$ & $\begin{array}{l}54.650 \\
53.579\end{array}$ \\
\hline 15 & 10000 & 49.0 & .03556 & .14227 & .01084 & .04336 & $15 \cdot 446$ & 47.083 & 566 \\
\hline $\begin{array}{l}15 \\
15\end{array}$ & $\begin{array}{l}10000 \\
10000\end{array}$ & $\begin{array}{l}50.0 \\
51.0\end{array}$ & $\begin{array}{r}.03494 \\
.03432\end{array}$ & $\begin{array}{r}.13978 \\
.13730\end{array}$ & $\begin{array}{r}.01065 \\
.01046\end{array}$ & .0 & $\begin{array}{l}15.177 \\
14.907\end{array}$ & $\begin{array}{l}46 \cdot 260 \\
45 \cdot 438\end{array}$ & $\begin{array}{l}51.648 \\
50.729\end{array}$ \\
\hline $\begin{array}{l}15 \\
15 \\
15\end{array}$ & $\begin{array}{l}10000 \\
10000 \\
10000\end{array}$ & $\begin{array}{l}52.0 \\
53.0 \\
54.0\end{array}$ & $\begin{array}{r}.03370 \\
.03308 \\
.03248\end{array}$ & $\begin{array}{r}.13481 \\
.13233 \\
.12994\end{array}$ & $\begin{array}{r}.01027 \\
.01008 \\
.00990\end{array}$ & $\begin{array}{l}.04109 \\
.04033^{\circ} \\
.03960\end{array}$ & $\begin{array}{l}14 \cdot 637 \\
14 \cdot 367 \\
14 \cdot 108\end{array}$ & $\begin{array}{l}44.616 \\
43.793 \\
43.004\end{array}$ & $\begin{array}{l}49.811 \\
48.893 \\
48.012\end{array}$ \\
\hline $\begin{array}{l}15 \\
15 \\
15\end{array}$ & $\begin{array}{l}10000 \\
10000 \\
10000\end{array}$ & $\begin{array}{l}55.0 \\
60.0 \\
65.0\end{array}$ & $\begin{array}{r}.03189 \\
.02922 \\
.02696\end{array}$ & $\begin{array}{r}12756 \\
.11690 \\
.10784\end{array}$ & $\begin{array}{l}.00972 \\
.00890 \\
.00821\end{array}$ & $\begin{array}{l}.03888 \\
.03563 \\
.03287\end{array}$ & $\begin{array}{l}13.850 \\
12.693 \\
11.709\end{array}$ & $\begin{array}{l}42.216 \\
38.688 \\
35.690\end{array}$ & $\begin{array}{l}47.132 \\
43.194 \\
39.846\end{array}$ \\
\hline $\begin{array}{l}15 \\
15 \\
15\end{array}$ & $\begin{array}{l}10000 \\
10000 \\
10000\end{array}$ & $\begin{array}{l}70.0 \\
75.0 \\
80.0\end{array}$ & $\begin{array}{r}.02512 \\
.02360 \\
.02224\end{array}$ & $\begin{array}{r}.10048 \\
.09441 \\
.08899\end{array}$ & $\begin{array}{l}.00765 \\
.00719 \\
.00678\end{array}$ & $\begin{array}{l}.03 \\
.02 \\
.02\end{array}$ & $\begin{array}{r}10.909 \\
10.250 \\
9.662\end{array}$ & $\begin{array}{l}33.253 \\
31.244 \\
29.450\end{array}$ & $\begin{array}{l}37.126 \\
34.882 \\
32.880\end{array}$ \\
\hline $\begin{array}{l}15 \\
15 \\
15\end{array}$ & $\begin{array}{l}10000 \\
10000 \\
10000\end{array}$ & $\begin{array}{l}85.0 \\
90.0 \\
95.0\end{array}$ & $\begin{array}{r}.02103 \\
.02001 \\
.01916\end{array}$ & $\begin{array}{r}.08414 \\
.08005 \\
.07665\end{array}$ & $\begin{array}{r}.00641 \\
.00609 \\
.00584\end{array}$ & $\begin{array}{l}.02564 \\
.02439 \\
.02336\end{array}$ & $\begin{array}{l}9.136 \\
8.691 \\
8.322\end{array}$ & $\begin{array}{l}27 \cdot 848 \\
26 \cdot 491 \\
25 \cdot 367\end{array}$ & $\begin{array}{l}31.091 \\
29.576 \\
28.321\end{array}$ \\
\hline 5 & 10000 & $\bullet$ & 01845 & 07383 & .00562 & .02250 & 8.016 & $4 \cdot 434$ & $27 \cdot 279$ \\
\hline
\end{tabular}


TABULATION OF DATA FOR

ABSORPTION OF SOUND IN AIR VERSUS PERCENT RELATIVE HUMIDITY AT 15 DEGREES CENTIGRADE FOR A FREQUENCY OF 12500 HERTZ.

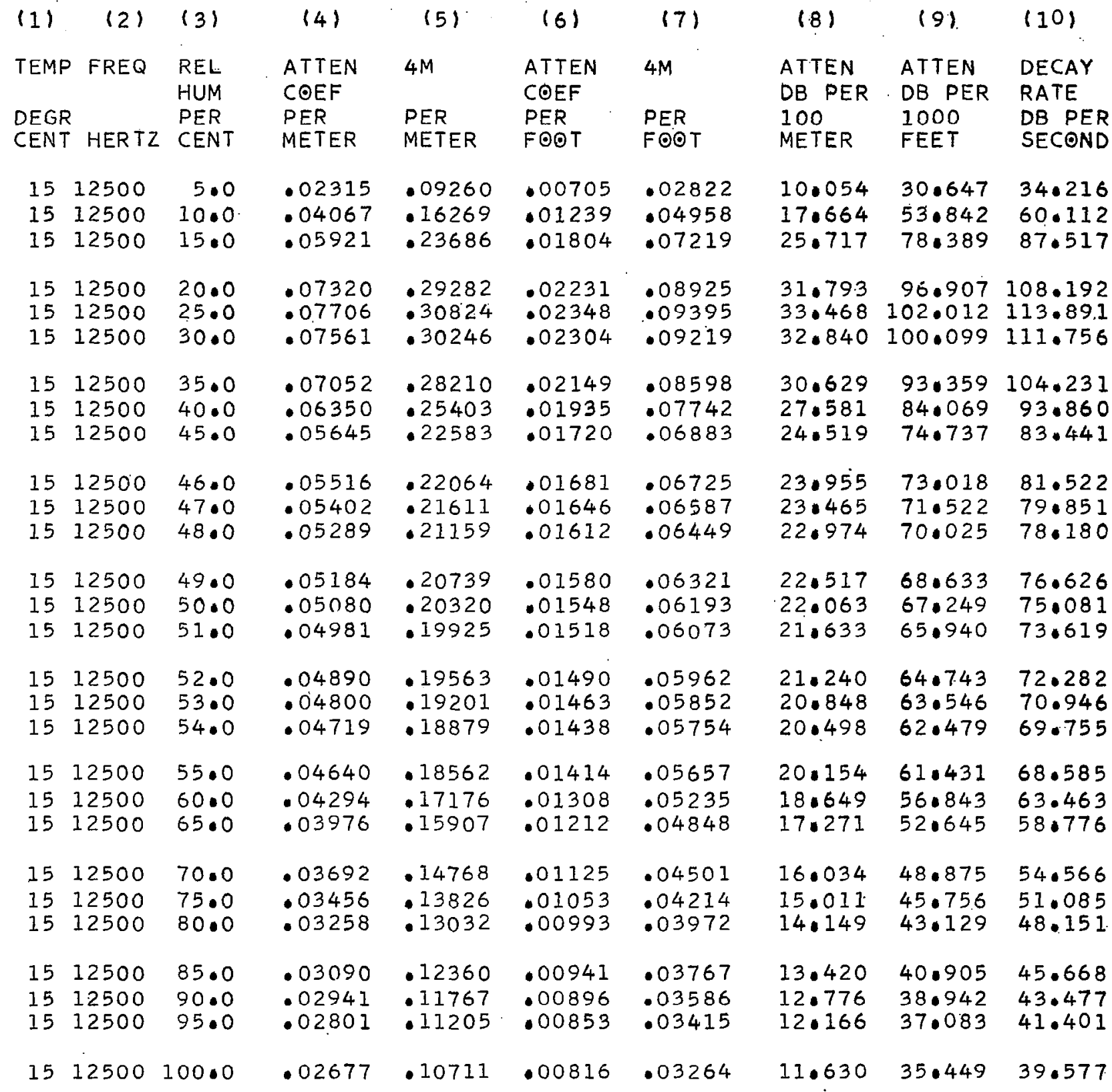


TABULATION OF DATA FOR

ABSORPTION OF SOUND IN AIR VERSUS PERCENT RELATIVE HUMIDITY AT 20 DEGREES CENTIGRADE FOR A FREQUENCY OF 125 HERTZ

\begin{tabular}{|c|c|c|c|c|c|c|c|c|c|}
\hline 11$)$ & $(2)$ & (3) & (4) & (5) & $(6)$ & $(7)$ & $(8)$ & $(9)$ & $(10)$ \\
\hline EMP & FREQ & $\begin{array}{l}\text { REL } \\
\text { HUM }\end{array}$ & $\begin{array}{l}\text { ATTEN } \\
\text { COEF }\end{array}$ & $4 M$ & $\begin{array}{l}\text { ATTEN } \\
\text { COEF }\end{array}$ & $4 M$ & $\begin{array}{l}\text { ATTEN } \\
\text { DB PER }\end{array}$ & $\begin{array}{l}\text { ATTEN } \\
\text { DB PER }\end{array}$ & $\begin{array}{l}\text { DECAY } \\
\text { RATE }\end{array}$ \\
\hline $\begin{array}{l}2 E G R \\
\text { CENT }\end{array}$ & HER TZ & $\begin{array}{l}\text { PER } \\
\text { CENT }\end{array}$ & $\begin{array}{l}\text { PER } \\
\text { METER }\end{array}$ & $\begin{array}{l}\text { PER } \\
\text { METER }\end{array}$ & $\begin{array}{l}\text { PER } \\
\text { FOOT }\end{array}$ & $\begin{array}{l}\text { PER } \\
\text { FOOT }\end{array}$ & $\begin{array}{l}100 \\
\text { METER }\end{array}$ & $\begin{array}{l}1000 \\
\text { FEET }\end{array}$ & $\begin{array}{l}\text { DB PER } \\
\text { SECOND }\end{array}$ \\
\hline $\begin{array}{l}20 \\
20 \\
20\end{array}$ & $\begin{array}{l}125 \\
125 \\
125\end{array}$ & $\begin{array}{r}5.0 \\
10.0 \\
15.0\end{array}$ & $\begin{array}{l}.00020 \\
.00014 \\
.00012\end{array}$ & $\begin{array}{l}.00081 \\
.00056 \\
.00048\end{array}$ & $\begin{array}{l}.00006 \\
.00004 \\
.00003\end{array}$ & $\begin{array}{l}.00024 \\
.00017 \\
.00014\end{array}$ & $\begin{array}{l}.088 \\
.061 \\
.052\end{array}$ & $\begin{array}{r}.268 \\
.186 \\
.159\end{array}$ & $\begin{array}{r}.302 \\
.210 \\
.179\end{array}$ \\
\hline $\begin{array}{l}20 \\
20 \\
20\end{array}$ & $\begin{array}{l}125 \\
125 \\
125\end{array}$ & $\begin{array}{l}20.0 \\
25.0 \\
30.0\end{array}$ & $\begin{array}{l}.00010 \\
.00009 \\
.00009\end{array}$ & $\begin{array}{l}.00043 \\
.00039 \\
.00037\end{array}$ & $\begin{array}{l}.00003 \\
.00003 \\
.00002\end{array}$ & $\begin{array}{l}.00013 \\
.00012 \\
.00011\end{array}$ & $\begin{array}{l}.047 \\
.043 \\
.040\end{array}$ & $\begin{array}{r}.143 \\
.131 \\
.123\end{array}$ & $\begin{array}{r}.161 \\
.148 \\
.139\end{array}$ \\
\hline $\begin{array}{l}20 \\
20 \\
20\end{array}$ & $\begin{array}{l}125 \\
125 \\
125\end{array}$ & $\begin{array}{l}35.0 \\
40.0 \\
45.0\end{array}$ & $\begin{array}{l}.00008 \\
.00008 \\
.00008\end{array}$ & $\begin{array}{l}.00035 \\
.00033 \\
.00032\end{array}$ & $\begin{array}{l}.00002 \\
.00002 \\
.00002\end{array}$ & $\begin{array}{l}.00010 \\
.00010 \\
.00009\end{array}$ & $\begin{array}{l}.038 \\
.036 \\
.034\end{array}$ & $\begin{array}{l}.116 \\
.111 \\
.106\end{array}$ & $\begin{array}{r}.131 \\
.125 \\
.119\end{array}$ \\
\hline $\begin{array}{l}20 \\
20 \\
20\end{array}$ & $\begin{array}{l}125 \\
125 \\
125\end{array}$ & $\begin{array}{l}46.0 \\
47.0 \\
48.0\end{array}$ & $\begin{array}{l}.00007 \\
.00007 \\
.00007\end{array}$ & $\begin{array}{l}.00031 \\
.00031 \\
.00031\end{array}$ & $\begin{array}{l}.00002 \\
.00002 \\
.00002\end{array}$ & $\begin{array}{l}.00009 \\
.00009 \\
.00009\end{array}$ & $\begin{array}{l}.034 \\
.034 \\
.034\end{array}$ & $\begin{array}{l}.105 \\
.104 \\
.103\end{array}$ & $\begin{array}{r}.118 \\
.117 \\
.116\end{array}$ \\
\hline $\begin{array}{l}20 \\
20 \\
20\end{array}$ & $\begin{array}{l}125 \\
125 \\
125\end{array}$ & $\begin{array}{l}49.0 \\
50.0 \\
51.0\end{array}$ & $\begin{array}{l}.00007 \\
.00007 \\
.00007\end{array}$ & $\begin{array}{l}.00031 \\
.00030 \\
.00030\end{array}$ & $\begin{array}{l}.00002 \\
.00002 \\
.00002\end{array}$ & $\begin{array}{l}.00009 \\
.00009 \\
.00009\end{array}$ & $\begin{array}{l}.033 \\
.033 \\
.033\end{array}$ & $\begin{array}{l}.102 \\
.102 \\
.101\end{array}$ & $\begin{array}{l}.115 \\
.115 \\
.114\end{array}$ \\
\hline $\begin{array}{l}20 \\
20 \\
20\end{array}$ & $\begin{array}{l}125 \\
125 \\
125\end{array}$ & $\begin{array}{l}52.0 \\
53.0 \\
54.0\end{array}$ & $\begin{array}{l}.00007 \\
.00007 \\
.00007\end{array}$ & $\begin{array}{r}.00030 \\
.00030 \\
.00030\end{array}$ & $\begin{array}{l}.00002 \\
.00002 \\
.00002\end{array}$ & $\begin{array}{l}.00009 \\
.00009 \\
.00009\end{array}$ & $\begin{array}{l}.033 \\
.032 \\
.032\end{array}$ & $\begin{array}{l}.100 \\
.100 \\
.099\end{array}$ & $\begin{array}{r}.113 \\
.112 \\
.112\end{array}$ \\
\hline 20 & 125 & 55.0 & .00007 & .00029 & .00002 & .00009 & .032 & .098 & .111 \\
\hline $\begin{array}{l}20 \\
20\end{array}$ & $\begin{array}{l}125 \\
125\end{array}$ & $\begin{array}{l}60.0 \\
65.0\end{array}$ & $\begin{array}{r}.00007 \\
.00007\end{array}$ & $\begin{array}{r}.00028 \\
.00028\end{array}$ & $\begin{array}{l}.00002 \\
.00002\end{array}$ & $\begin{array}{l}.00008 \\
.00008\end{array}$ & $\begin{array}{l}.031 \\
.030\end{array}$ & $\begin{array}{r}.095 \\
.092\end{array}$ & $\begin{array}{r}.107 \\
.104\end{array}$ \\
\hline 20 & 125 & 70.0 & .00006 & .00027 & .00002 & .00008 & .029 & .090 & .101 \\
\hline $\begin{array}{l}20 \\
20\end{array}$ & $\begin{array}{l}125 \\
125\end{array}$ & $\begin{array}{l}75.0 \\
80.0\end{array}$ & $\begin{array}{l}.00006 \\
.00006\end{array}$ & $\begin{array}{l}.00026 \\
.00025\end{array}$ & $\begin{array}{l}.00002 \\
.00001\end{array}$ & $\begin{array}{l}.00008 \\
.00007\end{array}$ & $\begin{array}{l}.028 \\
.028\end{array}$ & $\begin{array}{r}.087 \\
.085\end{array}$ & $\begin{array}{l}.099 \\
.096\end{array}$ \\
\hline $\begin{array}{l}20 \\
20 \\
20\end{array}$ & $\begin{array}{r}125 \\
125 \\
125\end{array}$ & $\begin{array}{l}85.0 \\
90.0 \\
95.0\end{array}$ & $\begin{array}{l}.00006 \\
.00006 \\
.00005\end{array}$ & $\begin{array}{l}.00025 \\
.00024 \\
.00023\end{array}$ & $\begin{array}{l}.00001 \\
.00001 \\
.00001\end{array}$ & $\begin{array}{l}.00007 \\
.00007 \\
.00007\end{array}$ & $\begin{array}{l}.027 \\
.026 \\
.025\end{array}$ & $\begin{array}{r}.083 \\
.080 \\
.078\end{array}$ & $\begin{array}{l}.093 \\
.091 \\
.088\end{array}$ \\
\hline 20 & .125 & $100 \cdot 0$ & .00005 & .00023 & .00001 & .00007 & .025 & .076 & .085 \\
\hline
\end{tabular}


TABULATION OF DATA FOR

ABSORPTION OF SOUND IN AIR VERSUS PERCENT RELATIVE HUMIDITY AT 20 DEGREES CENTIGRADE FOR A FREQUENCY OF 250 HERTZ

\begin{tabular}{|c|c|c|c|c|c|c|c|c|c|}
\hline (1) & $(2)$ & (3) & (4) & (5) & $(6)$ & $(7)$ & $(8)$ & $(9)$ & $(10)$ \\
\hline TEMP & FREQ & $\begin{array}{l}\text { REL } \\
\text { HUM }\end{array}$ & $\begin{array}{l}\text { ATTEN } \\
\text { COEF }\end{array}$ & $4 M$ & $\begin{array}{l}\text { ATTEN } \\
\text { COEF }\end{array}$ & $4 M$ & $\begin{array}{l}\text { ATTEN } \\
\text { DB PER }\end{array}$ & $\begin{array}{l}\text { ATTEN } \\
\text { DB PER }\end{array}$ & $\begin{array}{l}\text { DECAY } \\
\text { RATE }\end{array}$ \\
\hline $\begin{array}{l}\text { DEGR } \\
\text { CENT. }\end{array}$ & HERTZ & $\begin{array}{l}\text { PER } \\
\text { CENT }\end{array}$ & $\begin{array}{l}\text { PER } \\
\text { METER }\end{array}$ & $\begin{array}{l}\text { PER } \\
\text { METER }\end{array}$ & $\begin{array}{l}\text { PER } \\
\text { FOOT }\end{array}$ & $\begin{array}{l}\text { PER } \\
\text { FOOT }\end{array}$ & $\begin{array}{l}100 \\
\text { METER }\end{array}$ & $\begin{array}{l}1000 \\
\text { FEET }\end{array}$ & $\begin{array}{l}\text { DB PER } \\
\text { SECOND }\end{array}$ \\
\hline $\begin{array}{l}20 \\
20\end{array}$ & $\begin{array}{l}250 \\
250\end{array}$ & $\begin{array}{r}5.0 \\
10.0\end{array}$ & $\begin{array}{l}.00051 \\
.00030\end{array}$ & $\begin{array}{l}.00207 \\
.00123\end{array}$ & $\begin{array}{l}.00015 \\
.00009\end{array}$ & $\begin{array}{l}.00063 \\
.00037\end{array}$ & $\begin{array}{r}225 \\
133\end{array}$ & $\begin{array}{l}.686 \\
.407\end{array}$ & $\begin{array}{r}.773 \\
.458\end{array}$ \\
\hline 20 & 250 & 15.0 & .00026 & .00106 & .00008 & .00032 & .115 & .351 & .395 \\
\hline $\begin{array}{l}20 \\
20 \\
20\end{array}$ & $\begin{array}{l}250 \\
250 \\
250\end{array}$ & $\begin{array}{l}20 \cdot 0 \\
25 \cdot 0 \\
30 \cdot 0\end{array}$ & $\begin{array}{l}.00023 \\
.00021 \\
.00020\end{array}$ & $\begin{array}{l}.00094 \\
.00087 \\
.00081\end{array}$ & $\begin{array}{l}.00007 \\
.00006 \\
.00006\end{array}$ & $\begin{array}{l}.00028 \\
.00026 \\
.00024\end{array}$ & $\begin{array}{l}.102 \\
.094 \\
.088\end{array}$ & $\begin{array}{r}.312 \\
.288 \\
.270\end{array}$ & $\begin{array}{l}.351 \\
.324 \\
.304\end{array}$ \\
\hline $\begin{array}{l}20 \\
20\end{array}$ & $\begin{array}{l}250 \\
250\end{array}$ & $\begin{array}{l}35.0 \\
40.0\end{array}$ & $\begin{array}{l}.00019 \\
.00018\end{array}$ & $\begin{array}{l}.00076 \\
.00073 .\end{array}$ & $\begin{array}{l}.00005 \\
.00005\end{array}$ & $\begin{array}{l}.00023 \\
.00022\end{array}$ & $\begin{array}{r}.083 \\
.079\end{array}$ & $\begin{array}{r}254 \\
.242\end{array}$ & $\begin{array}{r}.286 \\
.273\end{array}$ \\
\hline 20 & 250 & 45.0 & .00017 & .00070 & .00005 & .00021 & .076 & .232 & .262 \\
\hline $\begin{array}{l}20 \\
20\end{array}$ & $\begin{array}{l}250 \\
250\end{array}$ & $\begin{array}{l}46.0 \\
47.0\end{array}$ & $\begin{array}{l}.00017 \\
.00017\end{array}$ & $\begin{array}{l}.00069 \\
.00069\end{array}$ & $\begin{array}{l}.00005 \\
.00005\end{array}$ & $\begin{array}{l}.00021 \\
.00021\end{array}$ & $\begin{array}{l}.075 \\
.075\end{array}$ & $\begin{array}{l}.230 \\
.229\end{array}$ & $\begin{array}{r}.260 \\
.258\end{array}$ \\
\hline 20 & 250 & $48 \cdot 0$ & .00017 & .00068 & .00005 & .00020 & .074 & .227 & .256 \\
\hline $\begin{array}{l}20 \\
20\end{array}$ & $\begin{array}{l}250 \\
250\end{array}$ & $\begin{array}{l}49.0 \\
50.0\end{array}$ & $\begin{array}{l}.00017 \\
.00016\end{array}$ & $\begin{array}{l}.00068 \\
.00067\end{array}$ & $\begin{array}{l}.00005 \\
.00005\end{array}$ & $\begin{array}{l}.00020 \\
.00020\end{array}$ & $\begin{array}{l}.074 \\
.073\end{array}$ & $\begin{array}{r}.225 \\
.223\end{array}$ & $\begin{array}{l}.254 \\
.252\end{array}$ \\
\hline 20 & 250 & 51.0 & .00016 & .00067 & .00005 & .00020 & .072 & .222 & .250 \\
\hline $\begin{array}{l}20 \\
20 \\
20\end{array}$ & $\begin{array}{l}250 \\
250 \\
250\end{array}$ & $\begin{array}{l}52.0 \\
53.0 \\
54.0\end{array}$ & $\begin{array}{l}.00016 \\
.00016 \\
.00016\end{array}$ & $\begin{array}{l}.00066 \\
.00066 \\
.00065\end{array}$ & $\begin{array}{l}.00005 \\
.00005 \\
.00005\end{array}$ & $\begin{array}{l}.00020 \\
.00020 \\
.00020\end{array}$ & $\begin{array}{l}.072 \\
.071 \\
.071\end{array}$ & $\begin{array}{l}.220 \\
.218 \\
.217\end{array}$ & $\begin{array}{l}.248 \\
.246 \\
.244\end{array}$ \\
\hline $\begin{array}{l}20 \\
20 \\
20\end{array}$ & $\begin{array}{l}250 \\
250 \\
250\end{array}$ & $\begin{array}{l}55.0 \\
60.0 \\
65.0\end{array}$ & $\begin{array}{l}.00016 \\
.00015 \\
.00015\end{array}$ & $\begin{array}{l}.00065 \\
.00063 \\
.00061\end{array}$ & $\begin{array}{l}.00004 \\
.00004 \\
.00004\end{array}$ & $\begin{array}{l}.00019 \\
.00019 \\
.00018\end{array}$ & $\begin{array}{l}.070 \\
.068 \\
.066\end{array}$ & $\begin{array}{r}.215 \\
.208 \\
.202\end{array}$ & $\begin{array}{r}.243 \\
.234 \\
.228\end{array}$ \\
\hline $\begin{array}{l}20 \\
20 \\
20\end{array}$ & $\begin{array}{l}250 \\
250 \\
250\end{array}$ & $\begin{array}{l}70.0 \\
75.0 \\
80.0\end{array}$ & $\begin{array}{l}.00014 \\
.00014 \\
.00014\end{array}$ & $\begin{array}{l}.00059 \\
.00058 \\
.00056\end{array}$ & $\begin{array}{l}.00004 \\
.00004 \\
.00004\end{array}$ & $\begin{array}{l}.00018 \\
.00017 \\
.00017\end{array}$ & $\begin{array}{l}.064 \\
.063 \\
.061\end{array}$ & $\begin{array}{r}.197 \\
.192 \\
.187\end{array}$ & $\begin{array}{r}.222 \\
.216 \\
.211\end{array}$ \\
\hline $\begin{array}{l}20 \\
20 \\
20\end{array}$ & $\begin{array}{l}250 \\
250 \\
250\end{array}$ & $\begin{array}{l}85.0 \\
90.0 \\
95.0\end{array}$ & $\begin{array}{l}.00013 \\
.00013 \\
.00013\end{array}$ & $\begin{array}{l}.00055 \\
.00054 \\
.00053\end{array}$ & $\begin{array}{l}.00004 \\
.00004 \\
.00004\end{array}$ & $\begin{array}{l}.00016 \\
.00016 \\
.00016\end{array}$ & $\begin{array}{l}.060 \\
.059 \\
.057\end{array}$ & $\begin{array}{r}.183 \\
.179 \\
.176\end{array}$ & $\begin{array}{l}.206 \\
.202 \\
.198\end{array}$ \\
\hline 10 & 250 & $100 \cdot 0$ & .00013 & .00052 & .00003 & .00015 & .056 & .172 & .194 \\
\hline
\end{tabular}


TABULATION OF DATA FOR

ABSORPTION OF SOUND IN AIR VERSUS PERCENT RELATIVE HUMIDITY AT 20 DEGREES CENTIGRADE F.OR A FREQUENCY OF 500 HERTZ

\begin{tabular}{|c|c|c|c|c|c|c|c|c|c|}
\hline (1) & (2) & $(3)$ & $(4)$ & (5) & 161 & $(7)$ & $(8)$ & $(9)$ & $(10)$ \\
\hline $\begin{array}{l}\text { TEMP } \\
\text { DEGR } \\
\text { CENT }\end{array}$ & HER TZ & $\begin{array}{l}\text { REL } \\
\text { HUM } \\
\text { PER } \\
\text { CENT }\end{array}$ & $\begin{array}{l}\text { ATTEN } \\
\text { COEF } \\
\text { PER } \\
\text { METER }\end{array}$ & $\begin{array}{l}4 M \\
\text { PER } \\
\text { METER }\end{array}$ & $\begin{array}{l}\text { ATTEN } \\
\text { COEF } \\
\text { PER } \\
\text { FOOT }\end{array}$ & $\begin{array}{l}4 M \\
P E R \\
F \odot O T\end{array}$ & $\begin{array}{l}\text { ATTEN } \\
\text { DB PER } \\
100 \\
\text { METER }\end{array}$ & $\begin{array}{l}\text { ATTEN } \\
\text { DB PER } \\
\text { IOOO } \\
\text { FEET }\end{array}$ & $\begin{array}{l}\text { DECAY } \\
\text { RATE } \\
\text { DB PER } \\
\text { SECOND }\end{array}$ \\
\hline $\begin{array}{l}20 \\
20 \\
20\end{array}$ & $\begin{array}{l}500 \\
500 \\
500\end{array}$ & $\begin{array}{r}5.0 \\
10.0 \\
15.0\end{array}$ & $\begin{array}{l}.00171 \\
.00081 \\
.00063\end{array}$ & $\begin{array}{l}.00684 \\
.00326 \\
.00252\end{array}$ & $\begin{array}{l}.00052 \\
.00024 \\
.00019\end{array}$ & $\begin{array}{l}.00208 \\
.00099 \\
.00076\end{array}$ & $\begin{array}{r}.743 \\
.354 \\
.273\end{array}$ & $\begin{array}{r}2.265 \\
1.080 \\
.835\end{array}$ & $\begin{array}{r}2.550 \\
1.216 \\
.940\end{array}$ \\
\hline $\begin{array}{l}20 \\
20 \\
20\end{array}$ & $\begin{array}{l}500 \\
500 \\
500\end{array}$ & $\begin{array}{l}20.0 \\
25.0 \\
30.0\end{array}$ & $\begin{array}{l}.00056 \\
.00052 \\
.00048\end{array}$ & $\begin{array}{l}.00227 \\
.00209 \\
.00194\end{array}$ & $\begin{array}{l}.00017 \\
.00015 \\
.00014\end{array}$ & $\begin{array}{l}.00069 \\
.00063 \\
.00059\end{array}$ & $\begin{array}{r}.246 \\
.227 \\
.211\end{array}$ & $\begin{array}{l}.752 \\
.693 \\
.644\end{array}$ & $\begin{array}{l}.847 \\
.781 \\
.725\end{array}$ \\
\hline $\begin{array}{l}20 \\
20 \\
20\end{array}$ & $\begin{array}{l}500 \\
500 \\
500\end{array}$ & $\begin{array}{l}35.0 \\
40.0 \\
45.0\end{array}$ & $\begin{array}{l}.00045 \\
.00043 \\
.00042\end{array}$ & $\begin{array}{r}.00183 \\
.00175 \\
.00168\end{array}$ & $\begin{array}{l}.00013 \\
.00013 \\
.00012\end{array}$ & $\begin{array}{l}.00055 \\
.00053 \\
.00051\end{array}$ & $\begin{array}{r}199 \\
.190 \\
.182\end{array}$ & $\begin{array}{l}.607 \\
.579 \\
.556\end{array}$ & $\begin{array}{l}.684 \\
.653 \\
.626\end{array}$ \\
\hline $\begin{array}{l}20 \\
20\end{array}$ & $\begin{array}{l}500 \\
500\end{array}$ & $\begin{array}{l}46.0 \\
47.0\end{array}$ & $\begin{array}{l}.00041 \\
.00041\end{array}$ & $\begin{array}{l}.00166 \\
.00165\end{array}$ & $\begin{array}{l}.00012 \\
.00012\end{array}$ & $\begin{array}{l}.00050 \\
.00050\end{array}$ & .181 & $\begin{array}{l}.552 \\
.547\end{array}$ & $\begin{array}{l}.621 \\
.616\end{array}$ \\
\hline 20 & 500 & $48 \cdot 0$ & .00041 & .00164 & .00012 & .00050 & .178 & .543 & .611 \\
\hline $\begin{array}{l}20 \\
20\end{array}$ & $\begin{array}{l}500 \\
500\end{array}$ & $\begin{array}{l}49.0 \\
50.0\end{array}$ & .00040 & $\begin{array}{l}.00162 \\
.00161\end{array}$ & $\begin{array}{l}.00012 \\
.00012\end{array}$ & $\begin{array}{r}.00049 \\
.00049\end{array}$ & $\begin{array}{r}176 \\
.175\end{array}$ & $\begin{array}{l}.538 \\
.534\end{array}$ & $\begin{array}{l}.606 \\
.601\end{array}$ \\
\hline 20 & 500 & 51.0 & .00040 & .00160 & .00012 & .00048 & .174 & .530 & .597 \\
\hline $\begin{array}{l}20 \\
20 \\
20\end{array}$ & $\begin{array}{l}500 \\
500 \\
500\end{array}$ & $\begin{array}{l}52.0 \\
53.0 \\
54.0\end{array}$ & $\begin{array}{l}.00039 \\
.00039 \\
.00039\end{array}$ & $\begin{array}{l}.00159 \\
.00158 \\
.00156\end{array}$ & $\begin{array}{l}.00012 \\
.00012 \\
.00011\end{array}$ & $\begin{array}{l}.00048 \\
.00048 \\
.00047\end{array}$ & $\begin{array}{r}172 \\
.171 \\
.170\end{array}$ & $\begin{array}{l}.527 \\
.523 \\
.519\end{array}$ & $\begin{array}{l}.593 \\
.589 \\
.585\end{array}$ \\
\hline $\begin{array}{l}20 \\
20\end{array}$ & $\begin{array}{l}500 \\
500\end{array}$ & $\begin{array}{l}55.0 \\
60.0\end{array}$ & $\begin{array}{r}.00038 \\
.00037\end{array}$ & $\begin{array}{l}.00155 \\
.00151\end{array}$ & $\begin{array}{l}.00011 \\
.00011\end{array}$ & $\begin{array}{l}.00047 \\
.00046\end{array}$ & $\begin{array}{r}169 \\
.164\end{array}$ & $\begin{array}{r}.515 \\
.500\end{array}$ & $\begin{array}{l}.580 \\
.563\end{array}$ \\
\hline 20 & 500 & 65.0 & .00036 & .00146 & .00011 & .00044 & .159 & .485 & .547 \\
\hline $\begin{array}{l}20 \\
20 \\
20\end{array}$ & $\begin{array}{l}500 \\
500 \\
500\end{array}$ & $\begin{array}{l}70.0 \\
75.0 \\
80.0\end{array}$ & $\begin{array}{l}.00035 \\
.00034 \\
.00034\end{array}$ & $\begin{array}{r}.00143 \\
.00139 \\
.00136\end{array}$ & $\begin{array}{l}.00010 \\
.00010 \\
.00010\end{array}$ & $\begin{array}{l}.00043 \\
.00042 \\
.00041\end{array}$ & $\begin{array}{l}.155 \\
.151 \\
.148\end{array}$ & $\begin{array}{l}.473 \\
.462 \\
.451\end{array}$ & $\begin{array}{l}.533 \\
.520 \\
.508\end{array}$ \\
\hline $\begin{array}{l}20 \\
20 \\
20\end{array}$ & $\begin{array}{l}500 \\
500 \\
500\end{array}$ & $\begin{array}{l}85.0 \\
90.0 \\
95.0\end{array}$ & $\begin{array}{l}.00033 \\
.00032 \\
.00031\end{array}$ & $\begin{array}{l}.00133 \\
.00130 \\
.00127\end{array}$ & $\begin{array}{l}.00010 \\
.00009 \\
.00009\end{array}$ & $\begin{array}{l}.00040 \\
.00039 \\
.00038\end{array}$ & $\begin{array}{r}.144 \\
.141 \\
.138\end{array}$ & $\begin{array}{r}.440 \\
.431 \\
.422\end{array}$ & $\begin{array}{l}.496 \\
.485 \\
.475\end{array}$ \\
\hline 20 & 500 & $100 \cdot 0$ & .00031 & .00125 & .00009 & .00038 & .136 & .415 & .467 \\
\hline
\end{tabular}


TABULATION OF DATA FOR

ABSORPTION OF SOUND IN AIR VERSUS PERCENT RELATIVE HUMIDITY AT 20 DEGREES CENTIGRADE FOR A FREQUENCY OF 1000 HERTZ

\begin{tabular}{|c|c|c|c|c|c|c|c|c|c|}
\hline (1) & (2) & $(3)$ & (4) & (5) & $(6)$ & $(7)$ & 181 & $|9|$ & $(10)$ \\
\hline $\begin{array}{l}\text { TEMP } \\
\text { DEGR } \\
\text { CENT }\end{array}$ & HERTZ & $\begin{array}{l}\text { REL } \\
\text { HUM } \\
\text { PER } \\
\text { CENT }\end{array}$ & $\begin{array}{l}\text { ATTEN } \\
\text { COEF } \\
\text { PER } \\
\text { METER }\end{array}$ & $\begin{array}{l}4 M \\
\text { PER } \\
\text { METER }\end{array}$ & $\begin{array}{l}\text { ATTEN } \\
\text { COEF } \\
\text { PER. } \\
\text { FOOT }\end{array}$ & $\begin{array}{l}4 M \\
\text { PER } \\
\text { FOOT }\end{array}$ & $\begin{array}{l}\text { ATTEN } \\
\text { DB PER } \\
\text { IOO } \\
\text { METER }\end{array}$ & $\begin{array}{l}\text { ATTEN } \\
\text { DB PER } \\
\text { IOOO } \\
\text { FEET }\end{array}$ & $\begin{array}{l}\text { DECAY } \\
\text { RATE } \\
\text { DB PER } \\
\text { SECOND }\end{array}$ \\
\hline $\begin{array}{l}20 \\
20 \\
20\end{array}$ & $\begin{array}{l}1000 \\
1000 \\
1000\end{array}$ & $\begin{array}{l}5.0 \\
10.0 \\
15.0\end{array}$ & $\begin{array}{l}.00556 \\
.00266 \\
.00173\end{array}$ & $\begin{array}{r}.02225 \\
.01067 \\
.00695\end{array}$ & $\begin{array}{l}.00169 \\
.00081 \\
.00053\end{array}$ & $\begin{array}{l}.00678 \\
.00325 \\
.00212\end{array}$ & $\begin{array}{r}2.416 \\
1.159 \\
.755\end{array}$ & $\begin{array}{l}7.365 \\
3.533 \\
2.302\end{array}$ & $\begin{array}{l}8.294 \\
3.979 \\
.2 .593\end{array}$ \\
\hline $\begin{array}{l}20 \\
20 \\
20\end{array}$ & $\begin{array}{l}1000 \\
1000 \\
1000\end{array}$ & $\begin{array}{l}20.0 \\
25.0 \\
30.0\end{array}$ & $\begin{array}{r}.00139 \\
.00126 \\
.00118\end{array}$ & $\begin{array}{r}.00558 \\
.00504 \\
.00472\end{array}$ & $\begin{array}{l}.00042 \\
.00038 \\
.00036\end{array}$ & $\begin{array}{l}.00170 \\
.00153 \\
.00144\end{array}$ & $\begin{array}{l}.506 \\
.547 \\
.513\end{array}$ & $\begin{array}{l}1.847 \\
1.668 \\
1.564\end{array}$ & $\begin{array}{l}2.080 \\
1.879 \\
1.761\end{array}$ \\
\hline $\begin{array}{l}20 \\
20 \\
20\end{array}$ & $\begin{array}{l}1000 \\
1000 \\
1000\end{array}$ & $\begin{array}{l}35.0 \\
40.0 \\
45.0\end{array}$ & $\begin{array}{l}.00111 \\
.00106 \\
.00101\end{array}$ & $\begin{array}{r}.00447 \\
.00425 \\
.00406\end{array}$ & $\begin{array}{l}.00034 \\
.00032 \\
.00030\end{array}$ & $\begin{array}{l}.00136 \\
.00129 \\
.00123\end{array}$ & $\begin{array}{r}.485 \\
.462 \\
.441\end{array}$ & $\begin{array}{l}1.480 \\
1.409 \\
1.345\end{array}$ & $\begin{array}{l}1.667 \\
1.587 \\
1.515\end{array}$ \\
\hline $\begin{array}{l}20 \\
20 \\
20\end{array}$ & $\begin{array}{l}1000 \\
1000 \\
1000\end{array}$ & $\begin{array}{l}46 \cdot 0 \\
47 \cdot 0 \\
48 \cdot 0\end{array}$ & $\begin{array}{r}.00100 \\
.00099 \\
.00098\end{array}$ & $\begin{array}{r}.00403 \\
.00399 \\
.00395\end{array}$ & $\begin{array}{l}.00030 \\
.00030 \\
.00030\end{array}$ & $\begin{array}{l}.00122 \\
.00121 \\
.00120\end{array}$ & $\begin{array}{l}.437 \\
.433 \\
.429\end{array}$ & $\begin{array}{l}1.333 \\
1.321 \\
1.310\end{array}$ & $\begin{array}{l}1.501 \\
1.488 \\
1.475\end{array}$ \\
\hline $\begin{array}{l}20 \\
20 \\
20\end{array}$ & $\begin{array}{l}1000 \\
1000 \\
1000\end{array}$ & $\begin{array}{l}49.0 \\
50.0 \\
51.0\end{array}$ & $\begin{array}{l}.00098 \\
.00097 \\
.00096\end{array}$ & $\begin{array}{l}.00392 \\
.00388 \\
.00386\end{array}$ & $\begin{array}{l}.00029 \\
.00029 \\
.00029\end{array}$ & $\begin{array}{l}.00119 \\
.00118 \\
.00117\end{array}$ & $\begin{array}{r}.425 \\
.422 \\
.419\end{array}$ & $\begin{array}{l}1.298 \\
1.287 \\
1.278\end{array}$ & $\begin{array}{l}1.462 \\
1.449 \\
1.440\end{array}$ \\
\hline $\begin{array}{l}2.0 \\
20 \\
20\end{array}$ & $\begin{array}{l}1000 \\
1000 \\
1000\end{array}$ & $\begin{array}{l}52.0 \\
53.0 \\
54.0\end{array}$ & $\begin{array}{l}.00096 \\
.00095 \\
.00094\end{array}$ & $\begin{array}{l}.00384 \\
.00381 \\
.00379\end{array}$ & $\begin{array}{l}.00029 \\
.00029 \\
.00028\end{array}$ & $\begin{array}{l}.00117 \\
.00116 \\
.00115\end{array}$ & $\begin{array}{r}.416 \\
.414 \\
.411\end{array}$ & $\begin{array}{l}1.270 \\
1.262 \\
1.254\end{array}$ & $\begin{array}{l}1.431 \\
1.422 \\
1.412\end{array}$ \\
\hline $\begin{array}{l}20 \\
20 \\
20\end{array}$ & $\begin{array}{l}1000 \\
1000 \\
1000\end{array}$ & $\begin{array}{l}55.0 \\
60.0 \\
65.0\end{array}$ & $\begin{array}{l}.00094 \\
.00091 \\
.00088\end{array}$ & $\begin{array}{l}.00376 \\
.00365 \\
.00355\end{array}$ & $\begin{array}{l}.00028 \\
.00027 \\
.00027\end{array}$ & $\begin{array}{l}.00114 \\
.00111 \\
.00108\end{array}$ & $\begin{array}{r}.408 \\
.397 \\
.386\end{array}$ & $\begin{array}{l}1.246 \\
1.210 \\
1.177\end{array}$ & $\begin{array}{l}1.403 \\
1.362 \\
1.325\end{array}$ \\
\hline $\begin{array}{l}20 \\
20 \\
20\end{array}$ & $\begin{array}{l}1000 \\
1000 \\
1000\end{array}$ & $\begin{array}{l}70.0 \\
75.0 \\
80.0\end{array}$ & $\begin{array}{l}.00086 \\
.00084 \\
.00082\end{array}$ & $\begin{array}{r}.00346 \\
.00338 \\
.00330\end{array}$ & $\begin{array}{l}.00026 \\
.00025 \\
.00025\end{array}$ & $\begin{array}{l}.00105 \\
.00103 \\
.00100\end{array}$ & $\begin{array}{r}.376 \\
.367 \\
.358\end{array}$ & $\begin{array}{l}1.147 \\
1.119 \\
1.092\end{array}$ & $\begin{array}{l}1.292 \\
1.260 \\
1.229\end{array}$ \\
\hline $\begin{array}{l}20 \\
20 \\
20\end{array}$ & $\begin{array}{l}1000 \\
1000 \\
1000\end{array}$ & $\begin{array}{l}85.0 \\
90.0 \\
95.0\end{array}$ & $\begin{array}{l}.00080 \\
.00079 \\
.00077\end{array}$ & $\begin{array}{r}.00322 \\
.00316 \\
.00310\end{array}$ & $\begin{array}{l}.00024 \\
.00024 \\
.00023\end{array}$ & $\begin{array}{l}.00098 \\
.00096 \\
.00094\end{array}$ & $\begin{array}{l}.350 \\
.343 \\
.337\end{array}$ & $\begin{array}{l}1.068 \\
1.047 \\
1.028\end{array}$ & $\begin{array}{l}1.203 \\
1.179 \\
1.158\end{array}$ \\
\hline 20 & 1000 & $100 \cdot 0$ & .00076 & .00305 & .00023 & .00092 & .331 & 1.009 & 1.136 \\
\hline
\end{tabular}


TABULATION OF DATA FOR

ABSORPTION OF SOUND IN AIR VERSUS PERCENT RELATIVE HUMIDITY AT 20 DEGREES CENTIGRADE FOR A FREQUENCY OF 2000 HERTZ

\begin{tabular}{|c|c|c|c|c|c|c|c|c|c|}
\hline (1) & $(2)$ & (3) & (4) & (5) & $(6)^{\circ}$ & 171 & $(8)$ & 191 & $(10)$ \\
\hline EMP & FREQ & $\begin{array}{l}\text { REL } \\
\text { HUM }\end{array}$ & $\begin{array}{l}\text { ATTEN } \\
\text { COEF }\end{array}$ & $4 M$ & $\begin{array}{l}\text { ATTEN } \\
\text { COEF }\end{array}$ & $4 M$ & $\begin{array}{l}\text { ATTEN } \\
\text { DB PER }\end{array}$ & $\begin{array}{l}\text { ATTEN } \\
\text { DB PER }\end{array}$ & $\begin{array}{l}\text { DECAY } \\
\text { RATE }\end{array}$ \\
\hline $\begin{array}{l}\text { EGR } \\
\text { ENT }\end{array}$ & HERTZ & $\begin{array}{l}\text { PER } \\
\text { CENT }\end{array}$ & $\begin{array}{l}\text { PER } \\
\text { METER }\end{array}$ & $\begin{array}{l}\text { PER } \\
\text { METER }\end{array}$ & $\begin{array}{l}\text { PER } \\
\text { FOOT }\end{array}$ & $\begin{array}{l}\text { PER } \\
\text { FOOT }\end{array}$ & $\begin{array}{l}100 \\
\text { METER }\end{array}$ & $\begin{array}{l}1000 \\
\text { FEET }\end{array}$ & $\begin{array}{l}\text { DB PER } \\
\text { SECOND }\end{array}$ \\
\hline $\begin{array}{l}20 \\
20 \\
20\end{array}$ & $\begin{array}{l}2000 \\
2000 \\
2000\end{array}$ & $\begin{array}{r}5.0 \\
10.0 \\
15.0\end{array}$ & $\begin{array}{c}.01246 \\
.00897 \\
.00582\end{array}$ & $\begin{array}{r}.04986 \\
.03589 \\
.02331\end{array}$ & $\begin{array}{l}.00379 \\
.00273 \\
.00177\end{array}$ & $\begin{array}{l}.01519 \\
.01094 \\
.00710\end{array}$ & $\begin{array}{l}5.413 \\
3.897 \\
2.531\end{array}$ & $\begin{array}{r}16.501 \\
11.880 \\
7.717\end{array}$ & $\begin{array}{r}18.583 \\
13.379 \\
8.690\end{array}$ \\
\hline $\begin{array}{l}20 \\
20\end{array}$ & $\begin{array}{l}2000 \\
2000\end{array}$ & $\begin{array}{l}20 \cdot 0 \\
25: 0\end{array}$ & $\begin{array}{l}.00428 \\
.00342\end{array}$ & $\begin{array}{l}.01712 \\
.01371\end{array}$ & $\begin{array}{l}.00130 \\
.00104\end{array}$ & $\begin{array}{l}.00521 \\
.00417\end{array}$ & $\begin{array}{l}1.859 \\
1.488\end{array}$ & $\begin{array}{l}5.666 \\
4.537\end{array}$ & $\begin{array}{l}6.381 \\
5.109\end{array}$ \\
\hline 20 & 2000 & 30.0 & .00296 & .01187 & .00090 & .00362 & 1.289 & 3.930 & 4.426 \\
\hline $\begin{array}{l}20 \\
20 \\
20\end{array}$ & $\begin{array}{l}2000 \\
2000 \\
2000\end{array}$ & $\begin{array}{l}35.0 \\
40.0 \\
45.0\end{array}$ & $\begin{array}{l}.00272 \\
.00259 \\
.00249\end{array}$ & $\begin{array}{r}.01089 \\
.01037 \\
.00996\end{array}$ & $\begin{array}{l}.00083 \\
.00079 \\
.00075\end{array}$ & $\begin{array}{l}.00332 \\
.00316 \\
.00303\end{array}$ & $\begin{array}{l}1.182 \\
1.126 \\
1.081\end{array}$ & $\begin{array}{l}3.605 \\
3.435 \\
3.297\end{array}$ & $\begin{array}{l}4.060 \\
3.868 \\
3.712\end{array}$ \\
\hline $\begin{array}{l}20 \\
20 \\
20\end{array}$ & $\begin{array}{l}2000 \\
2000 \\
2000\end{array}$ & $\begin{array}{l}46.0 \\
47.0 \\
48.0\end{array}$ & $\begin{array}{l}.00247 \\
.00245 \\
.00243\end{array}$ & $\begin{array}{r}.00989 \\
.00982 \\
.00974\end{array}$ & $\begin{array}{l}.00075 \\
.00074 \\
.00074\end{array}$ & $\begin{array}{l}.00301 \\
.00299 \\
.00297\end{array}$ & $\begin{array}{l}1.073 \\
1.066 \\
1.058\end{array}$ & $\begin{array}{l}3.273 \\
3.249 \\
3.226\end{array}$ & $\begin{array}{l}3.686 \\
3.659 \\
3.633\end{array}$ \\
\hline $\begin{array}{l}20 \\
20 \\
20\end{array}$ & $\begin{array}{l}2000 \\
2000 \\
2000\end{array}$ & $\begin{array}{l}49.0 \\
50.0 \\
51.0\end{array}$ & $\begin{array}{l}.00241 \\
.00240 \\
.00238\end{array}$ & $\begin{array}{l}.00967 \\
.00960 \\
.00954\end{array}$ & $\begin{array}{l}.00073 \\
.00073 \\
.00072\end{array}$ & $\begin{array}{l}.00294 \\
.00292 \\
.00290\end{array}$ & $\begin{array}{l}1.050 \\
1.042 \\
1.036\end{array}$ & $\begin{array}{l}3.202 \\
3.179 \\
3.157\end{array}$ & $\begin{array}{l}3.606 \\
3.580 \\
3.556\end{array}$ \\
\hline $\begin{array}{l}20 \\
20 \\
20\end{array}$ & $\begin{array}{l}2000 \\
2000 \\
2000\end{array}$ & $\begin{array}{l}52.0 \\
53.0 \\
54.0\end{array}$ & $\begin{array}{r}.00237 \\
.00235 \\
.00234\end{array}$ & $\begin{array}{l}.00948 \\
.00942 \\
.00936\end{array}$ & $\begin{array}{l}.00072 \\
.00071 \\
.00071\end{array}$ & $\begin{array}{l}.00289 \\
.00287 \\
.00285\end{array}$ & $\begin{array}{l}1.029 \\
1.023 \\
1.016\end{array}$ & $\begin{array}{l}3.138 \\
3.118 \\
3.098\end{array}$ & $\begin{array}{l}3.533 \\
3.511 \\
3.489\end{array}$ \\
\hline $\begin{array}{l}20 \\
20 \\
20\end{array}$ & $\begin{array}{l}2000 \\
2000 \\
2000\end{array}$ & $\begin{array}{l}55.0 \\
60.0 \\
65.0\end{array}$ & $\begin{array}{l}.00232 \\
.00225 \\
.00219\end{array}$ & $\begin{array}{l}.00930 \\
.00901 \\
.00876\end{array}$ & $\begin{array}{l}.00070 \\
.00068 \\
.00066\end{array}$ & $\begin{array}{l}.00283 \\
.00274 \\
.00267\end{array}$ & $\begin{array}{r}1.010 \\
.979 \\
.951\end{array}$ & $\begin{array}{l}3.079 \\
2.984 \\
2.899\end{array}$ & $\begin{array}{l}3.467 \\
3.360 \\
3.265\end{array}$ \\
\hline $\begin{array}{l}20 \\
20 \\
20\end{array}$ & $\begin{array}{l}2000 \\
2000 \\
2000\end{array}$ & $\begin{array}{l}70.0 \\
75.0 \\
80.0\end{array}$ & $\begin{array}{l}.00212 \\
.00207 \\
.00201\end{array}$ & $\begin{array}{l}.00851 \\
.00829 \\
.00807\end{array}$ & $\begin{array}{l}.00064 \\
.00063 \\
.00061\end{array}$ & $\begin{array}{l}.00259 \\
.00252 \\
.00246\end{array}$ & $\begin{array}{l}.924 \\
.900 \\
.876\end{array}$ & $\begin{array}{r}2.818 \\
2.744 \\
\cdot 2.671\end{array}$ & $\begin{array}{l}3.174 \\
3.091 \\
3.009\end{array}$ \\
\hline $\begin{array}{l}20 \\
20 \\
20\end{array}$ & $\begin{array}{l}2000 \\
2000 \\
2000\end{array}$ & $\begin{array}{l}85.0 \\
90.0 \\
95.0\end{array}$ & $\begin{array}{l}.00198 \\
.00194 \\
.00190\end{array}$ & $\begin{array}{l}.00792 \\
.00776 \\
.00763\end{array}$ & $\begin{array}{l}.00060 \\
.00059 \\
.00058\end{array}$ & $\begin{array}{l}.00241 \\
.00236 \\
.00232\end{array}$ & $\begin{array}{r}.860 \\
.843 \\
.829\end{array}$ & $\begin{array}{l}2.621 \\
2.571 \\
2.527\end{array}$ & $\begin{array}{l}2.952 \\
2.895 \\
2.845\end{array}$ \\
\hline 20 & 2000 & $100 \cdot 0$ & .00187 & 00750 & .00057 & .00228 & .814 & 2.482 & 2.796 \\
\hline
\end{tabular}


TABULATION OF DATA FOR

ABSORPTION OF SOUND IN AIR VERSUS PERCENT RELATIVE HUMIDITY AT 20 DEGREES CENTIGRADE FOR A FREQUENCY OF 2500 HERTZ

\begin{tabular}{|c|c|c|c|c|c|c|c|c|c|}
\hline (1) & (2) & $(3)$ & (4). & $(5)$ & $(6)$ & $17)$ & $(8)$ & $(9)$ & $(10)$ \\
\hline $\begin{array}{l}\text { TEMP } \\
\text { DEGR } \\
\text { CENT }\end{array}$ & HERTZ & $\begin{array}{l}\text { REL } \\
\text { HUM } \\
\text { PER } \\
\text { CENT }\end{array}$ & $\begin{array}{l}\text { ATTEN } \\
\text { COEF } \\
\text { PER } \\
\text { METER }\end{array}$ & $\begin{array}{l}4 M \\
\text { PER } \\
\text { METER }\end{array}$ & $\begin{array}{l}\text { ATTEN } \\
\text { COEF } \\
\text { PER } \\
\text { FOOT }\end{array}$ & $\begin{array}{l}4 M \\
\text { PER } \\
\text { FOOT }\end{array}$ & $\begin{array}{l}\text { ATTEN } \\
\text { DB PER } \\
100 \\
\text { METER }\end{array}$ & $\begin{array}{l}\text { ATTEN } \\
\text { DB PER } \\
\text { IOOO } \\
\text { FEET }\end{array}$ & $\begin{array}{l}\text { DECAY } \\
\text { RATE } \\
\text { DB PER } \\
\text { SECOND }\end{array}$ \\
\hline $\begin{array}{l}20 \\
20 \\
20\end{array}$ & $\begin{array}{l}2500 \\
2500 \\
2500\end{array}$ & $\begin{array}{r}5.0 \\
10.0 \\
15.0\end{array}$ & $\begin{array}{r}.01465 \\
.01337 \\
.00874\end{array}$ & $\begin{array}{r}.05862 \\
.05349 \\
.03496\end{array}$ & $\begin{array}{r}.00446 \\
.00407 \\
.00266\end{array}$ & $\begin{array}{r}.01786 \\
.01630 \\
.01065\end{array}$ & $\begin{array}{l}6.365 \\
5.807 \\
3.796\end{array}$ & $\begin{array}{l}19.401 \\
17.702 \\
11.571\end{array}$ & $\begin{array}{l}21.848 \\
19.935 \\
13.031\end{array}$ \\
\hline $\begin{array}{l}20 \\
20 \\
20\end{array}$ & $\begin{array}{l}2500 \\
2500 \\
2500\end{array}$ & $\begin{array}{l}20.0 \\
25.0 \\
30.0\end{array}$ & $\begin{array}{l}.00638 \\
.00506 \\
.00426\end{array}$ & $\begin{array}{r}.02555 \\
.02027 \\
.01706\end{array}$ & $\begin{array}{l}.00194 \\
.00154 \\
.00130\end{array}$ & $\begin{array}{l}.00778 \\
.00617 \\
.00520\end{array}$ & $\begin{array}{l}2.774 \\
2.201 \\
1.853\end{array}$ & $\begin{array}{l}8.457 \\
6.709 \\
5.648\end{array}$ & $\begin{array}{l}9.524 \\
7.555 \\
6.361\end{array}$ \\
\hline $\begin{array}{l}20 \\
20 \\
20\end{array}$ & $\begin{array}{l}2500 \\
2500 \\
2500\end{array}$ & $\begin{array}{l}35.0 \\
40.0 \\
45.0\end{array}$ & $\begin{array}{r}.00377 \\
.00350 \\
.00334\end{array}$ & $\begin{array}{r}.01511 \\
.01401 \\
.01336\end{array}$ & $\begin{array}{l}.00115 \\
.00106 \\
.00101\end{array}$ & $\begin{array}{l}.00460 \\
.00427 \\
.00407\end{array}$ & $\begin{array}{l}1.641 \\
1.521 \\
1.450\end{array}$ & $\begin{array}{l}5.002 \\
4.638 \\
4.421\end{array}$ & $\begin{array}{l}5.633 \\
5.223 \\
4.979\end{array}$ \\
\hline $\begin{array}{l}20 \\
20\end{array}$ & $\begin{array}{l}2500 \\
2500\end{array}$ & $\begin{array}{l}46.0 \\
47.0\end{array}$ & $\begin{array}{r}.00331 \\
.00329\end{array}$ & $\begin{array}{r}.01326 \\
.01317\end{array}$ & $\begin{array}{l}.00101 \\
.00100\end{array}$ & $\begin{array}{r}.00404 \\
.00401\end{array}$ & $\begin{array}{l}1.440 \\
1.430\end{array}$ & $\begin{array}{l}4 \cdot 390 \\
4 \cdot 359\end{array}$ & $\begin{array}{l}4.944 \\
4.909\end{array}$ \\
\hline 20 & 2500 & 48.0 & .00326 & .01307 & .00099 & .00398 & 1.419 & $4 \cdot 327$ & 4.873 \\
\hline $\begin{array}{l}20 \\
20\end{array}$ & $\begin{array}{l}2500 \\
2500\end{array}$ & $\begin{array}{l}49.0 \\
50.0\end{array}$ & $\begin{array}{l}.00324 \\
.00322\end{array}$ & $\begin{array}{r}.01298 \\
.01288\end{array}$ & $\begin{array}{l}.00098 \\
00098\end{array}$ & $\begin{array}{r}.00395 \\
.00392\end{array}$ & $\begin{array}{l}1.409 \\
1.399\end{array}$ & $\begin{array}{l}4 \cdot 296 \\
4 \cdot 265\end{array}$ & $\begin{array}{l}4.838 \\
4.803\end{array}$ \\
\hline 20 & 2500 & 51.0 & .00319 & .01279 & .00097 & .00389 & 1.389 & $4 \cdot 234$ & 4.768 \\
\hline $\begin{array}{l}20 \\
20\end{array}$ & $\begin{array}{l}2500 \\
2500\end{array}$ & $\begin{array}{l}52.0 \\
53.0\end{array}$ & .00317 & $\begin{array}{r}01271 \\
.01264\end{array}$ & .00096 & $\begin{array}{l}.00387 \\
.00385\end{array}$ & $\begin{array}{l}1.380 \\
1.372\end{array}$ & $\begin{array}{l}4 \cdot 208 \\
4 \cdot 183\end{array}$ & $\begin{array}{l}4.738 \\
4.710\end{array}$ \\
\hline $\begin{array}{l}20 \\
20 \\
20\end{array}$ & $\begin{array}{l}2500 \\
2500 \\
2500\end{array}$ & $\begin{array}{l}55.0 \\
60.0 \\
65.0\end{array}$ & $\begin{array}{r}.00312 \\
.00302 \\
.00295\end{array}$ & $\begin{array}{r}.01248 \\
.01211 \\
.01180\end{array}$ & $\begin{array}{l}.00095 \\
.00092 \\
.00089\end{array}$ & $\begin{array}{l}.00380 \\
.00369 \\
.00359\end{array}$ & $\begin{array}{l}1.355 \\
1.315 \\
1.281\end{array}$ & $\begin{array}{l}4.133 \\
4.010 \\
3.906\end{array}$ & $\begin{array}{l}4.654 \\
4.516 \\
4.398\end{array}$ \\
\hline $\begin{array}{l}20 \\
20 \\
20\end{array}$ & $\begin{array}{l}2500 \\
2500 \\
2500\end{array}$ & $\begin{array}{l}70.0 \\
75.0 \\
80.0\end{array}$ & $\begin{array}{r}.00287 \\
.00280 \\
.00273\end{array}$ & $\begin{array}{r}.01149 \\
.01122 \\
.01095\end{array}$ & $\begin{array}{l}.00087 \\
.00085 \\
.00083\end{array}$ & $\begin{array}{l}.00350 \\
.00342 \\
.00333\end{array}$ & $\begin{array}{l}1.247 \\
1.218 \\
1.189\end{array}$ & $\begin{array}{l}3.803 \\
3.714 \\
3.625\end{array}$ & $\begin{array}{l}4.283 \\
4.183 \\
4.082\end{array}$ \\
\hline $\begin{array}{l}20 \\
20 \\
20\end{array}$ & $\begin{array}{l}2500 \\
2500 \\
2500\end{array}$ & $\begin{array}{l}85.0 \\
90.0 \\
95.0\end{array}$ & $\begin{array}{r}.00267 \\
.00261 \\
.00256\end{array}$ & $\begin{array}{r}.01071 \\
.01047 \\
.01025\end{array}$ & $\begin{array}{r}.00081 \\
.00079 \\
.00078\end{array}$ & $\begin{array}{l}.00326 \\
.00319 \\
.00312\end{array}$ & $\begin{array}{l}1 \cdot 163 \\
1.137 \\
1.113\end{array}$ & $\begin{array}{l}3.544 \\
3.466 \\
3.393\end{array}$ & $\begin{array}{l}3.992 \\
3.904 \\
3.821\end{array}$ \\
\hline 20 & 2500 & 100.0 & .00252 & .01009 & .00076 & 00307 & 1.095 & 3.339 & 3.760 \\
\hline
\end{tabular}


TABULATION OF DATA FOR

ABSORPTION OF SOUND IN AIR VERSUS PERCENT RELATIVE HUMIDITY AT 20 DEGREES CENTIGRADE FOR A FREQUENCY OF 3200 HERTZ

\begin{tabular}{|c|c|c|c|c|c|c|c|c|c|}
\hline (1) & (2) & $(3)$ &.$(4)$ & $15)$ & 161 & $(7)$ & $(8)$ & 191 & $(10)$ \\
\hline $\begin{array}{l}\text { TEMP } \\
\text { DEGR } \\
\text { CENT }\end{array}$ & HERTZ & $\begin{array}{l}\text { REL } \\
\text { HUM } \\
\text { PER } \\
\text { CENT }\end{array}$ & $\begin{array}{l}\text { ATTEN } \\
\text { COEF } \\
\text { PER } \\
\text { METER }\end{array}$ & $\begin{array}{l}4 M \\
\text { PER } \\
\text { METER }\end{array}$ & $\begin{array}{l}\text { ATTEN } \\
\text { COEF } \\
\text { PER } \\
\text { FOOT }\end{array}$ & $\begin{array}{l}4 M \\
\text { PER } \\
\text { FOOT }\end{array}$ & $\begin{array}{l}\text { AT'TEN } \\
\text { DB PER } \\
100 \\
\text { METER }\end{array}$ & $\begin{array}{l}\text { ATTEN } \\
\text { DB PER } \\
1000 \\
\text { FEET }\end{array}$ & $\begin{array}{l}\text { DECAY } \\
\text { RATE } \\
\text { DB PER } \\
\text { SECOND }\end{array}$ \\
\hline $\begin{array}{l}20 \\
20 \\
20\end{array}$ & $\begin{array}{l}3200 \\
3200 \\
3200\end{array}$ & $\begin{array}{r}5.0 \\
10.0 \\
15.0\end{array}$ & $\begin{array}{r}.01658 \\
.01933 \\
.01338\end{array}$ & $\begin{array}{l}.06633 \\
.07732 \\
.05354\end{array}$ & $\begin{array}{l}.00505 \\
.00589 \\
.00408\end{array}$ & $\begin{array}{l}.02021 \\
.02356 \\
.01632\end{array}$ & $\begin{array}{l}7.202 \\
8.395 \\
5.814\end{array}$ & $\begin{array}{l}21.952 \\
25.589 \\
17.721\end{array}$ & $\begin{array}{l}24.721 \\
28.816 \\
19.957\end{array}$ \\
\hline $\begin{array}{l}20 \\
20 \\
20\end{array}$ & $\begin{array}{l}3200 \\
3200 \\
3200\end{array}$ & $\begin{array}{l}20.0 \\
25.0 \\
30.0\end{array}$ & $\begin{array}{l}.00993 \\
.00779 \\
.00646\end{array}$ & $\begin{array}{l}.03973 \\
.03117 \\
.02586\end{array}$ & $\begin{array}{l}.00302 \\
.00237 \\
.00197\end{array}$ & $\begin{array}{l}.01211 \\
.00950 \\
.00788\end{array}$ & $\begin{array}{l}4.314 \\
3.385 \\
2.808\end{array}$ & $\begin{array}{r}13.150 \\
10.318 \\
8.559\end{array}$ & $\begin{array}{r}14.809 \\
11.619 \\
9.639\end{array}$ \\
\hline $\begin{array}{l}20 \\
20 \\
20\end{array}$ & $\begin{array}{l}3200 \\
3200 \\
3200\end{array}$ & $\begin{array}{l}35.0 \\
40.0 \\
45.0\end{array}$ & $\begin{array}{l}.00560 \\
.00502 \\
.00470\end{array}$ & $\begin{array}{r}.02241 \\
.02008 \\
.01881\end{array}$ & $\begin{array}{l}.00170 \\
.00153 \\
.00143\end{array}$ & $\begin{array}{l}.00683 \\
.00612 \\
.00573\end{array}$ & $\begin{array}{l}2.433 \\
2.180 \\
2.043\end{array}$ & $\begin{array}{l}7.417 \\
6.647 \\
6.227\end{array}$ & $\begin{array}{l}8.353 \\
7.486 \\
7.012\end{array}$ \\
\hline $\begin{array}{l}20 \\
20 \\
20\end{array}$ & $\begin{array}{l}3200 \\
3200 \\
3200\end{array}$ & $\begin{array}{l}46.0 \\
47.0 \\
48.0\end{array}$ & $\begin{array}{l}.00464 \\
.00458 \\
.00453\end{array}$ & $\begin{array}{l}.01857 \\
.01832 \\
.01812\end{array}$ & $\begin{array}{l}.00141 \\
.00139 \\
.00138\end{array}$ & $\begin{array}{l}.00566 \\
.00558 \\
.00552\end{array}$ & $\begin{array}{l}2.016 \\
1.989 \\
1.967\end{array}$ & $\begin{array}{l}6.146 \\
6.065 \\
5.998\end{array}$ & $\begin{array}{l}6.922 \\
6.830 \\
6.754\end{array}$ \\
\hline $\begin{array}{l}20 \\
20 \\
20\end{array}$ & $\begin{array}{l}3200 \\
3200 \\
3200\end{array}$ & $\begin{array}{l}49.0 \\
50.0 \\
51.0\end{array}$ & $\begin{array}{l}.00449 \\
.00445 \\
.00442\end{array}$ & $\begin{array}{r}.01796 \\
.01780 \\
.01768\end{array}$ & $\begin{array}{l}.00136 \\
.00135 \\
.00134\end{array}$ & $\begin{array}{l}.00547 \\
.00542 \\
.00538\end{array}$ & $\begin{array}{l}1.950 \\
1.932 \\
1.919\end{array}$ & $\begin{array}{l}5.943 \\
5.890 \\
5.852\end{array}$ & $\begin{array}{l}6.693 \\
6.633 \\
6.590\end{array}$ \\
\hline $\begin{array}{l}20 \\
20 \\
20\end{array}$ & $\begin{array}{l}3200 \\
3200 \\
3200\end{array}$ & $\begin{array}{l}52.0 \\
53.0 \\
54.0\end{array}$ & $\begin{array}{l}.00439 \\
.00436 \\
.00433\end{array}$ & $\begin{array}{r}.01756 \\
.01745 \\
.01735\end{array}$ & $\begin{array}{l}.00133 \\
.00133 \\
.00132\end{array}$ & $\begin{array}{l}.00535 \\
.00532 \\
.00528\end{array}$ & & $\begin{array}{l}5.813 \\
5.776 \\
5.742\end{array}$ & $\begin{array}{l}6.546 \\
6.505 \\
6.466\end{array}$ \\
\hline $\begin{array}{l}20 \\
20 \\
20\end{array}$ & $\begin{array}{l}3200 \\
3200 \\
3200\end{array}$ & $\begin{array}{l}55.0 \\
60.0 \\
65.0\end{array}$ & $\begin{array}{l}.00431 \\
.00418 \\
.00407\end{array}$ & $\begin{array}{r}.01724 \\
.01673 \\
.01631\end{array}$ & $\begin{array}{l}.00131 \\
.00127 \\
.00124\end{array}$ & $\begin{array}{l}.00525 \\
.00510 \\
.00497\end{array}$ & $\begin{array}{l}1.872 \\
1.817 \\
1.771\end{array}$ & $\begin{array}{l}5.708 \\
5.539 \\
5.400\end{array}$ & $\begin{array}{l}6.428 \\
6.237 \\
6.081\end{array}$ \\
\hline $\begin{array}{l}20 \\
20 \\
20\end{array}$ & $\begin{array}{l}3200 \\
3200 \\
3200\end{array}$ & $\begin{array}{l}70.0 \\
75.0 \\
80.0\end{array}$ & $\begin{array}{l}.00397 \\
.00389 \\
.00380\end{array}$ & $\begin{array}{r}.01590 \\
.01556 \\
.01522\end{array}$ & $\begin{array}{l}.00121 \\
.00118 \\
.00116\end{array}$ & $\begin{array}{l}.00484 \\
.00474 \\
.00464\end{array}$ & $\begin{array}{l}1.727 \\
1.690 \\
1.652\end{array}$ & $\begin{array}{l}5.265 \\
5.151 \\
5.038\end{array}$ & $\begin{array}{l}5.929 \\
5.800 \\
5.673\end{array}$ \\
\hline $\begin{array}{l}20 \\
20 \\
20\end{array}$ & $\begin{array}{l}3200 \\
3200 \\
3200\end{array}$ & $\begin{array}{l}85.0 \\
90.0 \\
95.0\end{array}$ & $\begin{array}{r}.00372 \\
.00365 \\
.00358\end{array}$ & $\begin{array}{r}.01491 \\
.01462 \\
.01432\end{array}$ & $\begin{array}{l}.00113 \\
.00111 \\
.00109\end{array}$ & $\begin{array}{l}.00454 \\
.00445 \\
.00436\end{array}$ & $\begin{array}{l}1.619 \\
1.587 \\
1.555\end{array}$ & $\begin{array}{l}4.935 \\
4.838 \\
4.741\end{array}$ & $\begin{array}{l}5.557 \\
5.448 \\
5.339\end{array}$ \\
\hline 20 & 3200 & $100 \cdot 0$ & 00351 & .01406 & .00107 & .00428 & 1.527 & $4 \cdot 655$ & 5.242 \\
\hline
\end{tabular}


TABULATION OF DATA FOR

ABSORPTION OF SOUND IN AIR VERSUS PERCENT RELATIVE HUMIDITY AT 20 DEGREES CENTIGRADE FOR A FREQUENCY OF 4000 HERTZ

\begin{tabular}{|c|c|c|c|c|c|c|c|c|c|}
\hline 1) & (2) & (3) & $(4)$ & (5) & (6). & $(7)$ & 181 & 191 & $(10)$ \\
\hline $\begin{array}{l}\text { EMP } \\
\text { EGR } \\
\text { ENT }\end{array}$ & HERTZ & $\begin{array}{l}\text { REL } \\
\text { HUM } \\
\text { PER } \\
\text { CENT }\end{array}$ & $\begin{array}{l}\text { ATTEN } \\
\text { COEF } \\
\text { PER } \\
\text { METER }\end{array}$ & $\begin{array}{l}4 M \\
\text { PER } \\
\text { METER }\end{array}$ & $\begin{array}{l}\text { ATTEN } \\
\text { COEF } \\
\text { PER } \\
\text { FOOT }\end{array}$ & $\begin{array}{l}4 M \\
\text { PER } \\
\text { FOOT }\end{array}$ & $\begin{array}{l}\text { ATTEN } \\
\text { OB PER } \\
100 \\
\text { METER }\end{array}$ & $\begin{array}{l}\text { ATTEN } \\
\text { DB PER } \\
1000 \\
\text { FEET. }\end{array}$ & $\begin{array}{l}\text { DECAY } \\
\text { RATE } \\
\text { DB PER } \\
\text { SECOND }\end{array}$ \\
\hline $\begin{array}{l}20 \\
20 \\
20\end{array}$ & $\begin{array}{l}4000 \\
4000 \\
4000\end{array}$ & $\begin{array}{r}5.0 \\
10.0 \\
15.0\end{array}$ & $\begin{array}{r}.01805 \\
.02535 \\
.01974\end{array}$ & $\begin{array}{r}.07220 \\
.10143 \\
.07897\end{array}$ & $\begin{array}{l}.00550 \\
.00772 \\
.00601\end{array}$ & $\begin{array}{l}.02200 \\
.03091 \\
.02407\end{array}$ & $\begin{array}{r}7.839 \\
11.012 \\
8.575\end{array}$ & $\begin{array}{l}23.894 \\
33.567 \\
26.137\end{array}$ & $\begin{array}{l}26.907 \\
37.801 \\
29.434\end{array}$ \\
\hline $\begin{array}{l}20 \\
20 \\
20\end{array}$ & $\begin{array}{l}4000 \\
4000 \\
4000\end{array}$ & $\begin{array}{l}20.0 \\
25.0 \\
30.0\end{array}$ & $\begin{array}{l}.01451 \\
.01146 \\
.00948\end{array}$ & $\begin{array}{r}.05804 \\
.04584 \\
.03794\end{array}$ & $\begin{array}{r}.00442 \\
.00349 \\
.00289\end{array}$ & $\begin{array}{r}.01769 \\
.01397 \\
.01156\end{array}$ & $\begin{array}{l}6.302 \\
4.977 \\
4.119\end{array}$ & $\begin{array}{l}19.209 \\
15.171 \\
12.557\end{array}$ & $\begin{array}{l}21.632 \\
17.084 \\
14.141\end{array}$ \\
\hline $\begin{array}{l}20 \\
20 \\
20\end{array}$ & $\begin{array}{l}4000 \\
4000 \\
4000\end{array}$ & $\begin{array}{l}35.0 \\
40.0 \\
45.0\end{array}$ & $\begin{array}{l}.00810 \\
.00717 \\
.00650\end{array}$ & $\begin{array}{r}.03241 \\
.02870 \\
.02603\end{array}$ & $\begin{array}{r}.00247 \\
.00218 \\
.00198\end{array}$ & $\begin{array}{l}.00988 \\
.00874 \\
.00793\end{array}$ & $\begin{array}{l}3.519 \\
3.116 \\
2.826\end{array}$ & $\begin{array}{r}10.729 \\
9.499 \\
8.614\end{array}$ & $\begin{array}{r}12.082 \\
10.697 \\
9.700\end{array}$ \\
\hline $\begin{array}{l}20 \\
20 \\
20\end{array}$ & $\begin{array}{l}4000 \\
4000 \\
4000\end{array}$ & $\begin{array}{l}46.0 \\
47.0 \\
48.0\end{array}$ & $\begin{array}{l}.00641 \\
.00631 \\
.00624\end{array}$ & $\begin{array}{l}.02564 \\
.02527 \\
.02499\end{array}$ & $\begin{array}{r}.00195 \\
.00192 \\
.00190\end{array}$ & $\begin{array}{l}.00781 \\
.00770 \\
.00761\end{array}$ & $\begin{array}{l}2.784 \\
2.744 \\
2.714\end{array}$ & $\begin{array}{l}8.485 \\
8.364 \\
8.272\end{array}$ & $\begin{array}{l}9.556 \\
9.419 \\
9.316\end{array}$ \\
\hline $\begin{array}{l}20 \\
20 \\
20\end{array}$ & $\begin{array}{l}4000 \\
4000 \\
4000\end{array}$ & $\begin{array}{l}49.0 \\
50.0 \\
51.0\end{array}$ & $\begin{array}{l}.00618 \\
.00611 \\
.00604\end{array}$ & $\begin{array}{r}.02472 \\
.02444 \\
.02419\end{array}$ & $\begin{array}{r}.00188 \\
.00186 \\
.00184\end{array}$ & $\begin{array}{l}.00753 \\
.00745 \\
.00737\end{array}$ & $\begin{array}{l}2.684 \\
2.653 \\
2.627\end{array}$ & $\begin{array}{l}8.181 \\
8.089 \\
8.008\end{array}$ & $\begin{array}{l}9.213 \\
9.109 \\
9.018\end{array}$ \\
\hline $\begin{array}{l}20 \\
20 \\
20\end{array}$ & $\begin{array}{l}4000 \\
4000 \\
4000\end{array}$ & $\begin{array}{l}52.0 \\
53.0 \\
54.0\end{array}$ & $\begin{array}{l}.00599 \\
.00592 \\
.00585\end{array}$ & $\begin{array}{l}.02397 \\
.02371 \\
.02343\end{array}$ & $\begin{array}{l}.00182 \\
.00180 \\
.00178\end{array}$ & $\begin{array}{l}.00730 \\
.00722 \\
.00714\end{array}$ & $\begin{array}{l}2.603 \\
2.574 \\
2.544\end{array}$ & $\begin{array}{l}7 \cdot 935 \\
7 \cdot 847 \\
7.755\end{array}$ & $\begin{array}{l}8.936 \\
8.836 \\
8.733\end{array}$ \\
\hline $\begin{array}{l}20 \\
20 \\
20\end{array}$ & $\begin{array}{l}4000 \\
4000 \\
4000\end{array}$ & $\begin{array}{l}55.0 \\
60.0 \\
65.0\end{array}$ & $\begin{array}{l}.00580 \\
.00560 \\
.00546\end{array}$ & $\begin{array}{r}.02321 \\
.02243 \\
.02186\end{array}$ & $\begin{array}{r}.00176 \\
.00170 \\
.00166\end{array}$ & $\begin{array}{r}.00707 \\
.00683 \\
.00666\end{array}$ & $\begin{array}{l}2.520 \\
2.435 \\
2.373\end{array}$ & $\begin{array}{l}7.681 \\
7.423 \\
7.235\end{array}$ & $\begin{array}{l}8.650 \\
8.359 \\
8.148\end{array}$ \\
\hline $\begin{array}{l}20 \\
20 \\
20\end{array}$ & $\begin{array}{l}4000 \\
4000 \\
4000\end{array}$ & $\begin{array}{l}70.0 \\
75.0 \\
80.0\end{array}$ & $\begin{array}{l}.00532 \\
.00521 \\
.00510\end{array}$ & $\begin{array}{r}.02131 \\
.02087 \\
.02042\end{array}$ & $\begin{array}{l}.00162 \\
.00159 \\
.00155\end{array}$ & $\begin{array}{l}.00649 \\
.00636 \\
.00622\end{array}$ & $\begin{array}{l}2.314 \\
2.266 \\
2.217\end{array}$ & $\begin{array}{l}7.054 \\
6.907 \\
6.760\end{array}$ & $\begin{array}{l}7.943 \\
7.778 \\
7.613\end{array}$ \\
\hline $\begin{array}{l}20 \\
20 \\
20\end{array}$ & $\begin{array}{l}4000 \\
4000 \\
4000\end{array}$ & $\begin{array}{l}85 \cdot 0 \\
90.0 \\
95.0\end{array}$ & $\begin{array}{l}.00501 \\
.00491 \\
.00482\end{array}$ & $\begin{array}{l}.02004 \\
.01967 \\
.01930\end{array}$ & $\begin{array}{r}.00152 \\
.00149 \\
.00147\end{array}$ & $\begin{array}{l}.00610 \\
.00599 \\
.00588\end{array}$ & $\begin{array}{l}2.176 \\
2.136 \\
2.096\end{array}$ & $\begin{array}{l}6.633 \\
6.510 \\
6.390\end{array}$ & $\begin{array}{l}7.469 \\
7.332 \\
7.196\end{array}$ \\
\hline & 4000 & 1.0 & 00474 & 01899 & 0144 & 005 & 2.062 & $6 \cdot 285$ & 7.078 \\
\hline
\end{tabular}


TABULATION OF DATA FOR

ABSORPTION OF SOUND IN AIR VERSUS PERCENT RELATIVE HUMIDITY AT 20 DEGREES CENTIGRADE FOR A FREQUENCY OF 5000 HERTZ

\begin{tabular}{|c|c|c|c|c|c|c|c|c|c|}
\hline (1) & $(2)$ & (3) & $(4)$ & $(5)$ & 161 & 171 & 181 & 191 & $(10)$ \\
\hline $\begin{array}{l}\text { EMP } \\
\text { EGR } \\
\text { ENT }\end{array}$ & HERTZ & $\begin{array}{l}\text { REL } \\
\text { HUM } \\
\text { PER } \\
\text { CENT }\end{array}$ & $\begin{array}{l}\text { ATTEN } \\
\text { COEF } \\
\text { PER } \\
\text { METER }\end{array}$ & $\begin{array}{l}4 M \\
\text { PER } \\
\text { METER }\end{array}$ & $\begin{array}{l}\text { ATTEN } \\
\text { COEF } \\
\text { PER } \\
\text { FOOT }\end{array}$ & $\begin{array}{l}4 M \\
P \dot{R} R \\
F O O T\end{array}$ & $\begin{array}{l}\text { ATTEN } \\
\text { DB PER } \\
\text { IOO } \\
\text { METER }\end{array}$ & $\begin{array}{l}\text { ATTEN } \\
\text { DB PER } \\
1000 \\
\text { FEET }\end{array}$ & $\begin{array}{l}\text { DECAY } \\
\text { RATE } \\
\text { DB PER } \\
\text { SECOND }\end{array}$ \\
\hline $\begin{array}{l}20 \\
20 \\
20\end{array}$ & $\begin{array}{l}5000 \\
5000 \\
5000\end{array}$ & $\begin{array}{r}5.0 \\
10.0 \\
15.0\end{array}$ & $\begin{array}{r}.01956 \\
.03202 \\
.02834\end{array}$ & $\begin{array}{r}.07825 \\
.12811 \\
.11338\end{array}$ & $\begin{array}{l}.00596 \\
.00976 \\
.00863\end{array}$ & $\begin{array}{r}.02385 \\
.03904 \\
.03455\end{array}$ & $\begin{array}{r}8.496 \\
13.910 \\
12.310\end{array}$ & $\begin{array}{l}25.897 \\
42 \cdot 398 \\
37.522\end{array}$ & $\begin{array}{l}29.163 \\
47.746 \\
42.254\end{array}$ \\
\hline $\begin{array}{l}20 \\
20 \\
20\end{array}$ & $\begin{array}{l}5000 \\
5000 \\
5000\end{array}$ & $\begin{array}{l}20.0 \\
25.0 \\
30.0\end{array}$ & $\begin{array}{r}.02125 \\
.01696 \\
.01394\end{array}$ & $\begin{array}{l}.08500 \\
.06786 \\
.05579\end{array}$ & $\begin{array}{r}.00647 \\
.00517 \\
.00425\end{array}$ & $\begin{array}{l}.02590 \\
.02068 \\
.01700\end{array}$ & $\begin{array}{l}9.229 \\
7.368 \\
6.058\end{array}$ & $\begin{array}{l}28.130 \\
22.458 \\
18.466\end{array}$ & $\begin{array}{l}31.679 \\
25 \cdot 290 \\
20.79 .5\end{array}$ \\
\hline $\begin{array}{l}20 \\
20 \\
20\end{array}$ & $\begin{array}{l}5000 \\
5000 \\
5000\end{array}$ & $\begin{array}{l}35.0 \\
40.0 \\
45.0\end{array}$ & $\begin{array}{r}.01193 \\
.01043 \\
.00936\end{array}$ & $\begin{array}{r}.04773 \\
.04174 \\
.03744\end{array}$ & $\begin{array}{r}.00363 \\
.00318 \\
.00285\end{array}$ & $\begin{array}{r}.01454 \\
.01272 \\
.01141\end{array}$ & $\begin{array}{l}5.182 \\
4.532 \\
4.065\end{array}$ & $\begin{array}{l}15.795 \\
13.815 \\
12.391 .\end{array}$ & $\begin{array}{l}17.788 \\
15.557 \\
13.954\end{array}$ \\
\hline $\begin{array}{l}20 \\
20 \\
20\end{array}$ & $\begin{array}{l}5000 \\
5000 \\
5000\end{array}$ & $\begin{array}{l}46.0 \\
47.0 \\
48.0\end{array}$ & $\begin{array}{l}.00918 \\
.00903 \\
.00888\end{array}$ & $\begin{array}{r}.03675 \\
.03615 \\
.03554\end{array}$ & $\begin{array}{l}.00280 \\
.00275 \\
.00270\end{array}$ & $\begin{array}{r}.01120 \\
.01101 \\
.01083\end{array}$ & $\begin{array}{l}3.990 \\
3.925 \\
3.859\end{array}$ & $\begin{array}{l}12.163 \\
11.964 \\
11.762\end{array}$ & $\begin{array}{l}13.697 \\
13.473 \\
13.246\end{array}$ \\
\hline $\begin{array}{l}20 \\
20 \\
20\end{array}$ & $\begin{array}{l}5000 \\
5000 \\
5000\end{array}$ & $\begin{array}{l}49.0 \\
50.0 \\
51.0\end{array}$ & $\begin{array}{l}.00872 \\
.00855 \\
.00843\end{array}$ & $\begin{array}{r}.03488 \\
.03423 \\
.03375\end{array}$ & $\begin{array}{l}.00265 \\
.00260 \\
.00257\end{array}$ & $\begin{array}{r}.01063 \\
.01043 \\
.01028\end{array}$ & $\begin{array}{l}3.787 \\
3.717 \\
3.665\end{array}$ & $\begin{array}{l}11 \cdot 544 \\
11 \cdot 330 \\
11.171\end{array}$ & $\begin{array}{l}13.000 \\
12.759 \\
12.580\end{array}$ \\
\hline $\begin{array}{l}20 \\
20 \\
20\end{array}$ & $\begin{array}{l}5000 \\
5000 \\
5000\end{array}$ & $\begin{array}{l}52.0 \\
53.0 \\
54.0\end{array}$ & $\begin{array}{l}.00831 \\
.00821 \\
.00810\end{array}$ & $\begin{array}{l}.03327 \\
.03285 \\
.03243\end{array}$ & $\begin{array}{l}.00253 \\
.00250 \\
.00247\end{array}$ & $\begin{array}{r}.0101 .4 \\
.01001 \\
.00988\end{array}$ & $\begin{array}{l}3.613 \\
3.567 \\
3.521\end{array}$ & $\begin{array}{l}11.012 \\
10.873 \\
10.734\end{array}$ & $\begin{array}{l}12.402 \\
12.245 \\
12.088\end{array}$ \\
\hline $\begin{array}{l}20 \\
20\end{array}$ & $\begin{array}{l}5000 \\
5000\end{array}$ & $\begin{array}{l}55.0 \\
60.0\end{array}$ & $\begin{array}{l}.00803 \\
.00768\end{array}$ & $\begin{array}{l}.03212 \\
.03073\end{array}$ & $\begin{array}{l}.00244 \\
.00234\end{array}$ & $\begin{array}{l}.00979 \\
.00936\end{array}$ & $\begin{array}{l}3.488 \\
3.336\end{array}$ & $\begin{array}{l}10.632 \\
10.171\end{array}$ & $\begin{array}{l}11.973 \\
11.453\end{array}$ \\
\hline 20 & 5000 & 65.0 & .00736 & .02945 & .00224 & .00897 & 3.198 & 9.749 & 10.978 \\
\hline $\begin{array}{l}20 \\
20\end{array}$ & $\begin{array}{l}5000 \\
5000\end{array}$ & $\begin{array}{l}70.0 \\
75.0\end{array}$ & $\begin{array}{l}.00717 \\
.00702\end{array}$ & $\begin{array}{r}.02868 \\
.02808\end{array}$ & $\begin{array}{l}.00218 \\
.00214\end{array}$ & $\begin{array}{l}.00874 \\
.00856\end{array}$ & $\begin{array}{l}3.114 \\
3.049\end{array}$ & $\begin{array}{l}9.493 \\
9.294\end{array}$ & $\begin{array}{l}10.691 \\
10.466\end{array}$ \\
\hline 20 & 5000 & 80.0 & .00687 & .02748 & .00209 & .00837 & 2.984 & 9.095 & 10.242 \\
\hline $\begin{array}{l}20 \\
20 \\
20\end{array}$ & $\begin{array}{l}5000 \\
5000 \\
5000\end{array}$ & $\begin{array}{l}85.0 \\
90.0 \\
95.0\end{array}$ & $\begin{array}{l}.00674 \\
.00662 \\
.00651\end{array}$ & $\begin{array}{r}.02698 \\
.02650 \\
.02605\end{array}$ & $\begin{array}{l}.00205 \\
.00202 \\
.00198\end{array}$ & $\begin{array}{l}.00822 \\
.00808 \\
.00794\end{array}$ & $\begin{array}{l}2.930 \\
2.878 \\
2.828\end{array}$ & $\begin{array}{l}8.931 \\
8.773 \\
8.621\end{array}$ & $\begin{array}{r}10.058 \\
9.879 \\
9.709\end{array}$ \\
\hline 2 & 5000 & $100 \cdot 0$ & .00641 & .02565 & .00195 & .00781 & 2.785 & 8.489 & 9.56 \\
\hline
\end{tabular}


TABULATION OF DATA FOR

ABSORPTION OF SOUND IN AIR VERSUS PERCENT RELATIVE HUMIDITY AT 20 DEGREES CENTIGRADE FOR A FREQUENCY OF: 5940 HERTZ

\begin{tabular}{|c|c|c|c|c|c|c|c|c|c|}
\hline (1) & $(2)$ & (3) & $(4)$ & $(5)$ & $(6)$ & 171 & 181 & 191 & $(10)$ \\
\hline EMP & FREQ & $\begin{array}{l}\text { REL } \\
\text { HUM }\end{array}$ & $\begin{array}{l}\text { ATTEN } \\
\text { COEF }\end{array}$ & $4 M$ & $\begin{array}{l}\text { ATTEN } \\
\text { COEF }\end{array}$ & $4 M$ & $\begin{array}{l}\text { ATTEN } \\
\text { DB PER }\end{array}$ & $\begin{array}{l}\text { ATTEN } \\
\text { DB PER }\end{array}$ & $\begin{array}{l}\text { DECAY } \\
\text { RATE }\end{array}$ \\
\hline $\begin{array}{l}\text { GR } \\
\text { NT }\end{array}$ & HERTZ & $\begin{array}{l}\text { PER } \\
\text { CENT }\end{array}$ & $\begin{array}{l}\text { PER } \\
\text { METER }\end{array}$ & $\begin{array}{l}\text { PERR } \\
\text { METTER }\end{array}$ & $\begin{array}{l}\text { PER } \\
\text { FOOT }\end{array}$ & $\begin{array}{l}\text { PER } \\
\text { FOOT }\end{array}$ & $\begin{array}{l}100 \\
\text { METER }\end{array}$ & $\begin{array}{l}1000 \\
\text { FEET. }\end{array}$ & $\begin{array}{l}\text { DB PER } \\
\text { SECOND }\end{array}$ \\
\hline $\begin{array}{l}20 \\
20 \\
20\end{array}$ & $\begin{array}{l}5940 \\
5940 \\
5940\end{array}$ & $\begin{array}{r}5.0 \\
10.0 \\
15.0\end{array}$ & $\begin{array}{r}.02094 \\
.03749 \\
.03648\end{array}$ & $\begin{array}{r}.08378 \\
.14996 \\
.14593\end{array}$ & $\begin{array}{r}.00638 \\
.01142 \\
.01112\end{array}$ & $\begin{array}{r}.02553 \\
.04571 \\
.04448\end{array}$ & $\begin{array}{r}9.096 \\
16.282 \\
15.844\end{array}$ & $\begin{array}{l}27.727 \\
49.630 \\
48.295\end{array}$ & $\begin{array}{l}31.225 \\
55.890 \\
54.387\end{array}$ \\
\hline $\begin{array}{l}20 \\
20 \\
20\end{array}$ & $\begin{array}{l}5940 \\
5940 \\
5940\end{array}$ & $\begin{array}{l}20.0 \\
25.0 \\
30.0\end{array}$ & $\begin{array}{r}.02896 \\
.02282 \\
.01904\end{array}$ & $\begin{array}{r}.11585 \\
.09131 \\
.07616\end{array}$ & $\begin{array}{l}.00882 \\
.00695 \\
.00580\end{array}$ & $\begin{array}{l}.03531 \\
.02783 \\
.02321\end{array}$ & $\begin{array}{r}12.578 \\
9.914 \\
8.269\end{array}$ & $\begin{array}{l}38 \cdot 340 \\
30 \cdot 220 \\
25 \cdot 207\end{array}$ & $\begin{array}{l}43.176 \\
34.031 \\
28.386\end{array}$ \\
\hline $\begin{array}{l}20 \\
20 \\
20\end{array}$ & $\begin{array}{l}5940 \\
5940 \\
5940\end{array}$ & $\begin{array}{l}35.0 \\
40.0 \\
45.0\end{array}$ & $\begin{array}{r}.01614 \\
.01417 \\
.01260\end{array}$ & $\begin{array}{l}.06457 \\
.05668 \\
.05041\end{array}$ & $\begin{array}{r}.00492 \\
.00431 \\
.00384\end{array}$ & $\begin{array}{r}.01968 \\
.01727 \\
.01536\end{array}$ & $\begin{array}{l}7.011 \\
6.154 \\
5.473\end{array}$ & $\begin{array}{l}21 \cdot 371 \\
18 \cdot 759 \\
16 \cdot 684\end{array}$ & $\begin{array}{l}24.067 \\
21.125 \\
18.789\end{array}$ \\
\hline $\begin{array}{l}20 \\
20 \\
20\end{array}$ & $\begin{array}{l}5940 \\
5940 \\
5940\end{array}$ & $\begin{array}{l}46.0 \\
47.0 \\
48.0\end{array}$ & $\begin{array}{l}.01233 \\
.01208 \\
.01186\end{array}$ & $\begin{array}{r}.04932 \\
.04833 \\
.04744\end{array}$ & $\begin{array}{l}.00375 \\
.00368 \\
.00361\end{array}$ & $\begin{array}{r}.01503 \\
.01473 \\
.01446\end{array}$ & $\begin{array}{l}5.355 \\
5.247 \\
5.151\end{array}$ & $\begin{array}{l}16 \cdot 322 \\
15.995 \\
15.702\end{array}$ & $\begin{array}{l}18.381 \\
18.013 \\
17.682\end{array}$ \\
\hline $\begin{array}{l}20 \\
20 \\
20\end{array}$ & $\begin{array}{l}5940 \\
5940 \\
5940\end{array}$ & $\begin{array}{l}49.0 \\
50.0 \\
51.0\end{array}$ & $\begin{array}{r}.01164 \\
.01144 \\
.01125\end{array}$ & $\begin{array}{r}.04656 \\
.04578 \\
.04502\end{array}$ & $\begin{array}{l}.00354 \\
.00348 \\
.00343\end{array}$ & $\begin{array}{r}.01419 \\
.01395 \\
.01372\end{array}$ & $\begin{array}{l}5.055 \\
4.970 \\
4.888\end{array}$ & $\begin{array}{l}15.408 \\
15.151 \\
14.899\end{array}$ & $\begin{array}{l}17.352 \\
17.062 \\
16.779\end{array}$ \\
\hline $\begin{array}{l}20 \\
20 \\
20\end{array}$ & $\begin{array}{l}5940 \\
5940 \\
5940\end{array}$ & $\begin{array}{l}52.0 \\
53.0 \\
54.0\end{array}$ & $\begin{array}{r}.01108 \\
.01092 \\
.01076\end{array}$ & $\begin{array}{l}.04432 \\
.04369 \\
.04304\end{array}$ & $\begin{array}{l}.00337 \\
.00332 \\
.00328\end{array}$ & $\begin{array}{l}.01351 \\
.01331 \\
.01312\end{array}$ & $\begin{array}{l}40 \\
40 \\
4 .\end{array}$ & $\begin{array}{l}14.669 \\
14.460 \\
14.246\end{array}$ & $\begin{array}{l}16.520 \\
16.284 \\
16.043\end{array}$ \\
\hline $\begin{array}{l}20 \\
20 \\
20\end{array}$ & $\begin{array}{l}5940 \\
5940 \\
5940\end{array}$ & $\begin{array}{l}55.0 \\
60.0 \\
65.0\end{array}$ & $\begin{array}{l}.01058 \\
.00992 \\
.00950\end{array}$ & $\begin{array}{l}.04235 \\
.03969 \\
.03800\end{array}$ & $\begin{array}{r}.00322 \\
.00302 \\
.00289\end{array}$ & $\begin{array}{l}.01290 \\
.01209 \\
.01158\end{array}$ & $\begin{array}{l}4.598 \\
4.309 \\
4.126\end{array}$ & $\begin{array}{l}016 \\
135 \\
576\end{array}$ & $\begin{array}{l}15.783 \\
14.792 \\
14.162\end{array}$ \\
\hline $\begin{array}{l}20 \\
20\end{array}$ & $\begin{array}{l}5940 \\
5940\end{array}$ & $\begin{array}{l}70.0 \\
75.0\end{array}$ & $\begin{array}{l}.005 \\
.008\end{array}$ & $\begin{array}{l}.03656 \\
.03544\end{array}$ & $\begin{array}{l}.00278 \\
.00270\end{array}$ & $\begin{array}{l}.01114 \\
.01080\end{array}$ & $\begin{array}{l}3.9 \\
3.8\end{array}$ & & $\begin{array}{l}13.627 \\
13.210\end{array}$ \\
\hline 20 & 5940 & 80.0 & .00868 & .03474 & .00264 & .01058 & 3.772 & 11.497 & 12.947 \\
\hline $\begin{array}{l}20 \\
20\end{array}$ & $\begin{array}{l}5940 \\
5940 \\
5940\end{array}$ & $\begin{array}{l}85.0 \\
90.0 \\
95.0\end{array}$ & $\begin{array}{l}.00852 \\
.00836 \\
.00823\end{array}$ & $\begin{array}{l}.03410 \\
.03347 \\
.03295\end{array}$ & $\begin{array}{l}.00259 \\
.00255\end{array}$ & $\begin{array}{r}.01039 \\
.01020 \\
.01004\end{array}$ & $\begin{array}{l}3.703 \\
3.634 \\
3.577\end{array}$ & $\begin{array}{l}287 \\
078 \\
904\end{array}$ & $\begin{array}{l}12.711 \\
12.475 \\
12.280\end{array}$ \\
\hline & 5940 & 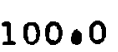 & 00811 & 3244 & 00247 & .00988 & 6.522 & 0.737 & 0 \\
\hline
\end{tabular}


TABULATIEN OF DATA FOR

ABSEAPTION OF SEUND IN ATR VERSUS PERCENT RELATIVE HUMIOITY AT 20 DEGREES CENTIGRADE FOR A FREQUENCY OF 6300 HERTZ

\begin{tabular}{|c|c|c|c|c|c|c|c|c|c|}
\hline$(1)$ & $(2)$ & (3) & (4) & (5) & (6) & (7) & $(8)$ & (9) & $110 \mid$ \\
\hline $\begin{array}{l}\text { EMP } \\
\text { EGR } \\
\text { ENT }\end{array}$ & FREQ & $\begin{array}{l}\text { REL } \\
\text { HUM } \\
\text { PER } \\
\text { CENT }\end{array}$ & $\begin{array}{l}\text { ATTEN } \\
\text { COEF } \\
\text { PER } \\
\text { METER }\end{array}$ & $\begin{array}{l}\text { 4M } \\
\text { PER } \\
\text { METER }\end{array}$ & $\begin{array}{l}\text { ATTEN } \\
\text { COEF } \\
\text { PER } \\
\text { FOOT }\end{array}$ & $\begin{array}{l}4 M \\
\text { PER } \\
\text { FOOT }\end{array}$ & $\begin{array}{l}\text { ATTEN } \\
\text { DB PER } \\
100 \\
\text { METER }\end{array}$ & $\begin{array}{l}\text { ATTEN } \\
\text { OB PER } \\
\text { IOOO } \\
\text { FEET }\end{array}$ & $\begin{array}{l}\text { DECAY } \\
\text { RATE } \\
\text { DB PER } \\
\text { SECONO }\end{array}$ \\
\hline $\begin{array}{l}20 \\
20 \\
20\end{array}$ & $\begin{array}{l}6300 \\
6300 \\
6300\end{array}$ & $\begin{array}{l}5.0 \\
10.0 \\
15.0\end{array}$ & $\begin{array}{l}.02261 \\
.03935 \\
.03933\end{array}$ & $\begin{array}{l}.08644 \\
.15741 \\
.15735\end{array}$ & $\begin{array}{l}.00 \\
.01 \\
.01\end{array}$ & $\begin{array}{l}.02634 \\
.04798 \\
.04796\end{array}$ & $\begin{array}{r}9.386 \\
17.091 \\
17.084\end{array}$ & $\begin{array}{l}28.609 \\
52.095 \\
52.074\end{array}$ & $\begin{array}{l}32.218 \\
58.665 \\
58.643\end{array}$ \\
\hline $\begin{array}{l}20 \\
20 \\
20\end{array}$ & $\begin{array}{l}6300 \\
6300 \\
6300\end{array}$ & $\begin{array}{l}20.0 \\
25.0 \\
30.0\end{array}$ & $\begin{array}{r}.03189 \\
.02512 \\
.02099\end{array}$ & $\begin{array}{l}.12759 \\
.10051 \\
.08398\end{array}$ & $\begin{array}{l}.00 \\
.00 \\
.00\end{array}$ & $\begin{array}{r}.03889 \\
.03063 \\
.02559\end{array}$ & $\begin{array}{r}13.854 \\
10.913 \\
9.119\end{array}$ & $\begin{array}{l}42.227 \\
33.264 \\
27.795\end{array}$ & $\begin{array}{l}47.554 \\
37.460 \\
31.301\end{array}$ \\
\hline $\begin{array}{l}20 \\
20 \\
20\end{array}$ & $\begin{array}{l}6300 \\
6300 \\
6300\end{array}$ & $\begin{array}{l}35.0 \\
40.0 \\
45.0\end{array}$ & $\begin{array}{r}.01783 \\
.01559 \\
.01390\end{array}$ & $\begin{array}{l}.07132 \\
.06238 \\
.05561\end{array}$ & $\begin{array}{l}.00543 \\
.00475 \\
.00423\end{array}$ & $\begin{array}{l}.02173 \\
.01901 \\
.01695\end{array}$ & & $\begin{array}{l}23 \cdot 603 \\
20.645 \\
18.404\end{array}$ & $\begin{array}{l}26.580 \\
23.249 \\
20.725\end{array}$ \\
\hline $\begin{array}{l}20 \\
20 \\
20\end{array}$ & $\begin{array}{l}6300 \\
6300 \\
6300\end{array}$ & $\begin{array}{l}46.0 \\
47.0 \\
48.0\end{array}$ & $\begin{array}{r}.01361 \\
.01331 \\
.01305\end{array}$ & $\begin{array}{l}.05444 \\
.05327 \\
.05220\end{array}$ & $\begin{array}{l}.00414 \\
.00405 \\
.00397\end{array}$ & $\begin{array}{r}.01659 \\
.01623 \\
.01591\end{array}$ & & $\begin{array}{l}18.017 \\
17.630 \\
17.277\end{array}$ & $\begin{array}{l}20.289 \\
19.853 \\
19.457\end{array}$ \\
\hline $\begin{array}{l}20 \\
20 \\
20\end{array}$ & $\begin{array}{l}6300 \\
6360 \\
6300\end{array}$ & $\begin{array}{l}49.0 \\
50.0 \\
51.0\end{array}$ & $\begin{array}{l}.01281 \\
.01298 \\
.01236\end{array}$ & $\begin{array}{l}.05124 \\
.05033 \\
.04945\end{array}$ & $\begin{array}{l}.00390 \\
.00383 \\
.00376\end{array}$ & $\begin{array}{l}.01561 \\
.01534 \\
.01507\end{array}$ & $\begin{array}{l}5 . \\
50 \\
50\end{array}$ & $\begin{array}{l}16 \cdot 959 \\
16.658 \\
16.365\end{array}$ & $\begin{array}{l}19.098 \\
18.759 \\
18.429\end{array}$ \\
\hline $\begin{array}{l}20 \\
20 \\
20\end{array}$ & $\begin{array}{l}6300 \\
6300 \\
6300\end{array}$ & $\begin{array}{l}52.0 \\
53.0 \\
54.0\end{array}$ & $\begin{array}{r}.01216 \\
.01197 \\
.01180\end{array}$ & $\begin{array}{l}.04867 \\
.04789 \\
.04721\end{array}$ & $\begin{array}{l}.00370 \\
.00364 \\
.00359\end{array}$ & $\begin{array}{r}.01483 \\
.01459 \\
.01438\end{array}$ & & $\begin{array}{l}16.107 \\
15.849 \\
15.623\end{array}$ & $\begin{array}{l}18 \cdot 138 \\
17.848 \\
17.594\end{array}$ \\
\hline $\begin{array}{l}20 \\
20 \\
20\end{array}$ & $\begin{array}{l}6300 \\
6300 \\
6300\end{array}$ & $\begin{array}{l}55.0 \\
60.0 \\
65.0\end{array}$ & $\begin{array}{l}.01164 \\
.01085 \\
.01034\end{array}$ & $\begin{array}{l}.04656 \\
.04340 \\
.04136\end{array}$ & $\begin{array}{l}.00354 \\
.00330 \\
.00315\end{array}$ & & & $\begin{array}{l}15.408 \\
14.364 \\
13.690\end{array}$ & $\begin{array}{l}17 \cdot 352 \\
16.175 \\
15.416\end{array}$ \\
\hline $\begin{array}{l}20 \\
20 \\
20\end{array}$ & $\begin{array}{l}6300 \\
6300 \\
6300\end{array}$ & $\begin{array}{l}70.0 \\
75.0 \\
80.0\end{array}$ & $\begin{array}{l}.00997 \\
.00961 \\
.00939\end{array}$ & $\begin{array}{r}.03988 \\
.03846 \\
.03757\end{array}$ & $\begin{array}{l}.00303 \\
.00293 \\
.00286\end{array}$ & $\begin{array}{l}.01215 \\
.01172 \\
.01145\end{array}$ & $\begin{array}{l}40 \\
4 .\end{array}$ & $\begin{array}{l}13 \cdot 200 \\
12.728 \\
12.434\end{array}$ & $\begin{array}{l}14.865 \\
14.334 \\
14.003\end{array}$ \\
\hline $\begin{array}{l}20 \\
20\end{array}$ & $\begin{array}{l}6300 \\
6300 \\
6300\end{array}$ & $\begin{array}{l}85.0 \\
90.0 \\
95.0\end{array}$ & $\begin{array}{r}.00922 \\
.00906 \\
.00890\end{array}$ & $\begin{array}{l}.03689 \\
.03624 \\
.03562\end{array}$ & $\begin{array}{l}.00281 \\
.00276 \\
.00271\end{array}$ & $\begin{array}{l}.01124 \\
.01104 \\
.01085\end{array}$ & $\begin{array}{l}4.005 \\
3.935 \\
3.868\end{array}$ & $\begin{array}{l}12 \cdot 209 \\
11.994 \\
11.790\end{array}$ & $\begin{array}{l}13.749 \\
13.507 \\
13.277\end{array}$ \\
\hline & 63 & 100 & .00877 & 03510 & 00267 & .01070 & 3.811 & 1.617 & 3.083 \\
\hline
\end{tabular}


TABULATION OF DATA FOR ABSORPTION OF SOUND IN AIR VERSUS PERCENT RELATIVE HUMIDITY AT 20 DEGREES CENTIGRADE FOR A FREQUENCY OF $8000 \mathrm{HERTZ}$

\begin{tabular}{|c|c|c|c|c|c|c|c|c|c|}
\hline (1) & (2) & (3) & (4) & (5) & $(6)$ & (7) & $(8)$ & (9) & $(10)$ \\
\hline $\begin{array}{l}\text { TEMP } \\
\text { DEGR } \\
\text { CENT }\end{array}$ & HERTZ & $\begin{array}{l}\text { REL } \\
\text { HUM } \\
\text { PER } \\
\text { CENT }\end{array}$ & $\begin{array}{l}\text { ATTEN } \\
\text { COEF } \\
\text { PER } \\
\text { METER }\end{array}$ & $\begin{array}{l}4 M \\
\text { PER } \\
\text { METER }\end{array}$ & $\begin{array}{l}\text { ATTEN } \\
\text { COEF } \\
\text { PER } \\
\text { FOOT }\end{array}$ & $\begin{array}{l}4 M \\
\text { PER } \\
\text { FOOT }\end{array}$ & $\begin{array}{l}\text { ATTEN } \\
\text { DB PER } \\
100 \\
\text { METER }\end{array}$ & $\begin{array}{l}\text { ATTEN } \\
\text { DB PER } \\
\text { IOOO } \\
\text { FEET }\end{array}$ & $\begin{array}{l}\text { DECAY } \\
\text { RATE } \\
\text { DB PER } \\
\text { SECOND }\end{array}$ \\
\hline $\begin{array}{l}20 \\
20 \\
20\end{array}$ & $\begin{array}{l}8000 \\
8000 \\
8000\end{array}$ & $\begin{array}{r}5.0 \\
10.0 \\
15.0\end{array}$ & $\begin{array}{l}.02415 \\
.04641 \\
.05215\end{array}$ & $\begin{array}{r}.09662 \\
.18565 \\
.20861\end{array}$ & $\begin{array}{r}.00736 \\
.01414 \\
.01589\end{array}$ & $\begin{array}{r}.02945 \\
.05658 \\
.06358\end{array}$ & $\begin{array}{l}10.490 \\
20.157 \\
22.650\end{array}$ & $\begin{array}{l}31.976 \\
61.439 \\
69.040\end{array}$ & $\begin{array}{l}36.010 \\
69.188 \\
77.749\end{array}$ \\
\hline $\begin{array}{l}20 \\
20 \\
20\end{array}$ & $\begin{array}{l}8000 \\
8000 \\
8000\end{array}$ & $\begin{array}{l}20.0 \\
25.0 \\
30.0\end{array}$ & $\begin{array}{r}.04707 \\
.03830 \\
.03168\end{array}$ & $\begin{array}{r}.18830 \\
.15323 \\
.12674\end{array}$ & $\begin{array}{r}.01434 \\
.01167 \\
.00965\end{array}$ & $\begin{array}{r}.05739 \\
.04670 \\
.03863\end{array}$ & $\begin{array}{l}20.445 \\
16.637 \\
13.761\end{array}$ & $\begin{array}{l}62.318 \\
50.710 \\
41.944\end{array}$ & $\begin{array}{l}70.179 \\
57.107 \\
47.234\end{array}$ \\
\hline $\begin{array}{l}20 \\
20 \\
20\end{array}$ & $\begin{array}{l}8000 \\
8000 \\
8000\end{array}$ & $\begin{array}{l}35.0 \\
40.0 \\
45.0\end{array}$ & $\begin{array}{r}.02732 \\
.02374 \\
.02113\end{array}$ & $\begin{array}{l}.10929 \\
.09496 \\
.08452\end{array}$ & $\begin{array}{r}.00832 \\
.00723 \\
.00644\end{array}$ & $\begin{array}{r}.03331 \\
.02894 \\
.02576\end{array}$ & $\begin{array}{r}11.867 \\
10.310 \\
9.177\end{array}$ & $\begin{array}{l}36.171 \\
31.426 \\
27.972\end{array}$ & $\begin{array}{l}40.734 \\
35.390 \\
31.501\end{array}$ \\
\hline $\begin{array}{l}20 \\
20 \\
20\end{array}$ & $\begin{array}{l}8000 \\
8000 \\
8000\end{array}$ & $\begin{array}{l}46.0 \\
47.0 \\
48.0\end{array}$ & $\begin{array}{l}.02069 \\
.02027 \\
.01989\end{array}$ & $\begin{array}{r}.08277 \\
.08110 \\
.07956\end{array}$ & $\begin{array}{l}.00630 \\
.00618 \\
.00606\end{array}$ & $\begin{array}{l}.02523 \\
.02472 \\
.02425\end{array}$ & $\begin{array}{l}8.987 \\
8.806 \\
8.638\end{array}$ & $\begin{array}{l}27 \cdot 393 \\
26 \cdot 842 \\
26 \cdot 330\end{array}$ & $\begin{array}{l}30.849 \\
30.227 \\
29.651\end{array}$ \\
\hline $\begin{array}{l}20 \\
20 \\
20\end{array}$ & $\begin{array}{l}8000 \\
8000 \\
8000\end{array}$ & $\begin{array}{l}49.0 \\
50.0 \\
51.0\end{array}$ & $\begin{array}{r}.01952 \\
.01916 \\
.01878\end{array}$ & $\begin{array}{l}.07811 \\
.07666 \\
.07512\end{array}$ & $\begin{array}{r}.00595 \\
.00584 \\
.00572\end{array}$ & $\begin{array}{l}.02380 \\
.02336 \\
.02289\end{array}$ & $\begin{array}{l}8.481 \\
8.324 \\
8.156\end{array}$ & $\begin{array}{l}25.851 \\
25.372 \\
24.860\end{array}$ & $\begin{array}{l}29.112 \\
28.572 \\
27.996\end{array}$ \\
\hline $\begin{array}{l}20 \\
20 \\
20\end{array}$ & $\begin{array}{l}8000 \\
8000 \\
8000\end{array}$ & $\begin{array}{l}52.0 \\
53.0 \\
54.0\end{array}$ & $\begin{array}{r}.01842 \\
.01808 \\
.01777\end{array}$ & $\begin{array}{r}.07369 \\
.07235 \\
.07109\end{array}$ & $\begin{array}{l}.00561 \\
.00551 \\
.00541\end{array}$ & $\begin{array}{r}.02246 \\
.02205 \\
.02166\end{array}$ & $\begin{array}{l}8.001 \\
7.856 \\
7.718\end{array}$ & $\begin{array}{l}24 \cdot 389 \\
23.945 \\
23.526\end{array}$ & $\begin{array}{l}27.465 \\
26.965 \\
26.494\end{array}$ \\
\hline $\begin{array}{l}20 \\
20 \\
20\end{array}$ & $\begin{array}{l}8000 \\
8000 \\
8000\end{array}$ & $\begin{array}{l}55.0 \\
60.0 \\
65.0\end{array}$ & $\begin{array}{r}.01745 \\
.01616 \\
.01519\end{array}$ & $\begin{array}{r}.06982 \\
.06464 \\
.06078\end{array}$ & $\begin{array}{l}.00532 \\
.00492 \\
.00463\end{array}$ & $\begin{array}{r}.02128 \\
.01970 \\
.01852\end{array}$ & $\begin{array}{l}7.581 \\
7.019 \\
6.599\end{array}$ & $\begin{array}{l}23 \cdot 107 \\
21 \cdot 394 \\
20 \cdot 115\end{array}$ & $\begin{array}{l}26.022 \\
24.092 \\
22.652\end{array}$ \\
\hline $\begin{array}{l}20 \\
20\end{array}$ & $\begin{array}{l}8000 \\
8000\end{array}$ & $\begin{array}{l}70.0 \\
75.0\end{array}$ & $\begin{array}{r}.01433 \\
.01373\end{array}$ & $\begin{array}{r}.05732 \\
.05495\end{array}$ & $\begin{array}{r}.00436 \\
.00418\end{array}$ & $\begin{array}{r}.01747 \\
.01675\end{array}$ & $\begin{array}{l}6.224 \\
5.967\end{array}$ & $\begin{array}{l}18.971 \\
18.188\end{array}$ & $\begin{array}{l}21 \cdot 364 \\
20.482\end{array}$ \\
\hline 20 & 8000 & 80.0 & .01330 & .05323 & .00405 & .01622 & 5.779 & 17.617 & 19.839 \\
\hline $\begin{array}{l}20 \\
20 \\
20\end{array}$ & $\begin{array}{l}8000 \\
8000 \\
8000^{-}\end{array}$ & $\begin{array}{l}85.0 \\
90.0 \\
95.0\end{array}$ & $\begin{array}{l}.01291 \\
.01259 \\
.01237\end{array}$ & $\begin{array}{l}.05154 \\
.05037 \\
.04951\end{array}$ & $\begin{array}{l}.00393 \\
.00383 \\
.00377\end{array}$ & $\begin{array}{l}.01574 \\
.01535 \\
.01509\end{array}$ & $\begin{array}{l}5.607 \\
5.469 \\
5.375\end{array}$ & $\begin{array}{l}17.090 \\
16.670 \\
16.385\end{array}$ & $\begin{array}{l}19.246 \\
18.773 \\
18.452\end{array}$ \\
\hline 20 & 80001 & 100.0 & .01219 & .04879 & .00371 & .01487 & 5.297 & $16 \cdot 147$ & 18.184 \\
\hline
\end{tabular}


TABULATION OF DATA FOR

ABSORPTION OF SOUND IN AIR VERSUS PERCENT RELATIVE HUMIDITY AT 20 DEGREES CENTIGRADE FOR A FREQUENCY OF 10000 HERTZ

\begin{tabular}{|c|c|c|c|c|c|c|c|c|c|}
\hline (1) & $(2)$ & (3) & $(4)$ & (5) & $(6)$ & (7) & $(8)$ & $(9)$ & $(10)$ \\
\hline $\begin{array}{l}\text { TEMP } \\
\text { DEGR } \\
\text { CENT }\end{array}$ & HERTZ & $\begin{array}{l}\text { REL } \\
\text { HUM } \\
\text { PER } \\
\text { CENT }\end{array}$ & $\begin{array}{l}\text { ATTEN } \\
\text { COEF } \\
\text { PER } \\
\text { METER }\end{array}$ & $\begin{array}{l}4 M \\
\text { PER } \\
\text { METER }\end{array}$ & $\begin{array}{l}\text { ATTEN } \\
\text { COEF } \\
\text { PER } \\
\text { FOOT }\end{array}$ & $\begin{array}{l}4 M \\
\text { PER } \\
\text { FOOT }\end{array}$ & $\begin{array}{l}\text { ATTEN } \\
\text { DB PER } \\
100 \\
\text { METER }\end{array}$ & $\begin{array}{l}\text { ATTEN } \\
\text { DB PER } \\
1000 \\
\text { FEET }\end{array}$ & $\begin{array}{l}\text { DECAY } \\
\text { RATE } \\
\text { DB PER } \\
\text { SECOND }\end{array}$ \\
\hline $\begin{array}{l}20 \\
20 \\
20\end{array}$ & $\begin{array}{l}10000 \\
10000 \\
10000\end{array}$ & $\begin{array}{r}5.0 \\
10.0 \\
15.0\end{array}$ & $\begin{array}{r}.02758 \\
.05244 \\
.06564\end{array}$ & $\begin{array}{r}.11032 \\
.20976 \\
.26256\end{array}$ & $\begin{array}{r}.00840 \\
.01598 \\
.02000\end{array}$ & $\begin{array}{r}.03362 \\
.06393 \\
.08002\end{array}$ & $\begin{array}{l}11.978 \\
22.775 \\
28.507\end{array}$ & $\begin{array}{l}36.511 \\
69.420 \\
86.891\end{array}$ & $\begin{array}{l}41.116 \\
78.177 \\
97.851\end{array}$ \\
\hline $\begin{array}{l}20 \\
20 \\
20\end{array}$ & $\begin{array}{l}10000 \\
10000 \\
10000\end{array}$ & $\begin{array}{l}20.0 \\
25.0 \\
30.0\end{array}$ & $\begin{array}{r}.06391 \\
.05539 \\
.04608\end{array}$ & $\begin{array}{r}.25564 \\
.22156 \\
.18434\end{array}$ & $\begin{array}{r}.01948 \\
.01688 \\
.01404\end{array}$ & $\begin{array}{l}.07792 \\
.06753 \\
.05618\end{array}$ & $\begin{array}{l}27 \\
24 \\
20\end{array}$ & $\begin{array}{l}84.604 \\
73.325 \\
61.006\end{array}$ & $\begin{array}{l}95.276 \\
82.574 \\
68.701\end{array}$ \\
\hline $\begin{array}{l}20 \\
20 \\
20\end{array}$ & $\begin{array}{l}10000 \\
10000 \\
10000\end{array}$ & $\begin{array}{l}35.0 \\
40.0 \\
45.0\end{array}$ & $\begin{array}{r}.03952 \\
.03484 \\
.03087\end{array}$ & $\begin{array}{r}.15808 \\
\cdot 13937 \\
\cdot 12348\end{array}$ & $\begin{array}{l}.01204 \\
.01062 \\
.00940\end{array}$ & $\begin{array}{r}.04818 \\
.04248 \\
.03763\end{array}$ & & $\begin{array}{l}52.317 \\
46.124 \\
40.866\end{array}$ & $\begin{array}{l}58.916 \\
51.942 \\
46.021\end{array}$ \\
\hline $\begin{array}{l}20 \\
20 \\
20\end{array}$ & $\begin{array}{l}10000 \\
10000 \\
10000\end{array}$ & $\begin{array}{l}46.0 \\
47.0 \\
48.0\end{array}$ & $\begin{array}{l}.03019 \\
.02958 \\
.02896\end{array}$ & $\begin{array}{r}.12079 \\
\cdot 11833 \\
.11586\end{array}$ & $\begin{array}{l}.00920 \\
.00901 \\
.00882\end{array}$ & $\begin{array}{l}.03681 \\
.03606 \\
.03531\end{array}$ & & $\begin{array}{l}39.977 \\
39.161 \\
38.345\end{array}$ & $\begin{array}{l}45.020 \\
44.101 \\
43.182\end{array}$ \\
\hline $\begin{array}{l}20 \\
20 \\
20\end{array}$ & $\begin{array}{l}10000 \\
10000 \\
10000\end{array}$ & $\begin{array}{l}49.0 \\
50.0 \\
51.0\end{array}$ & $\begin{array}{r}.02838 \\
.02784 \\
.02733\end{array}$ & $\begin{array}{r}.11352 \\
.11137 \\
.10933\end{array}$ & $\begin{array}{l}.00865 \\
.00848 \\
.00833\end{array}$ & $\begin{array}{r}.03460 \\
.03394 \\
.03332\end{array}$ & & $\begin{array}{l}37.570 \\
36.857 \\
36.182\end{array}$ & $\begin{array}{l}42.309 \\
41.505 \\
40.746\end{array}$ \\
\hline $\begin{array}{l}20 \\
20\end{array}$ & $\begin{array}{l}10000 \\
10000\end{array}$ & $\begin{array}{l}52.0 \\
53.0\end{array}$ & $\begin{array}{l}.026 \\
.026\end{array}$ & .107 & $\begin{array}{l}.00818 \\
.00803\end{array}$ & $\begin{array}{l}.03272 \\
.03215\end{array}$ & & $\begin{array}{l}35.528 \\
34.916\end{array}$ & $\begin{array}{l}40.009 \\
39.320\end{array}$ \\
\hline 20 & 10000 & $54 \cdot 0$ & .02593 & .10374 & .00790 & .03162 & $11 \cdot 263$ & 34.332 & 38.662 \\
\hline $\begin{array}{l}20 \\
20 \\
20\end{array}$ & $\begin{array}{l}10000 \\
10000 \\
10000\end{array}$ & $\begin{array}{l}55.0 \\
60.0 \\
65.0\end{array}$ & $\begin{array}{r}.02551 \\
.02352 \\
.02186\end{array}$ & $\begin{array}{r}.10204 \\
.09408 \\
.08746\end{array}$ & $\begin{array}{l}.00777 \\
.00716 \\
.00666\end{array}$ & $\begin{array}{r}.03110 \\
.02867 \\
.02666\end{array}$ & & $\begin{array}{l}33.771 \\
31.138 \\
28.946\end{array}$ & $\begin{array}{l}38.030 \\
35.065 \\
32.597\end{array}$ \\
\hline $\begin{array}{l}20 \\
20 \\
20\end{array}$ & $\begin{array}{l}10000 \\
10000 \\
10000\end{array}$ & $\begin{array}{l}70.0 \\
75.0 \\
80.0\end{array}$ & $\begin{array}{r}.02059 \\
.01957 \\
.01864\end{array}$ & $\begin{array}{r}.08237 \\
.07829 \\
.07457\end{array}$ & $\begin{array}{l}.00627 \\
.00596 \\
.00568\end{array}$ & $\begin{array}{l}.02510 \\
.02386 \\
.02272\end{array}$ & $\begin{array}{l}8.944 \\
8.500 \\
8.096\end{array}$ & $\begin{array}{l}27.261 \\
25.909 \\
24.678\end{array}$ & $\begin{array}{l}30.700 \\
29.177 \\
27.791\end{array}$ \\
\hline $\begin{array}{l}20 \\
20 \\
20\end{array}$ & $\begin{array}{l}10000 \\
10000 \\
10000\end{array}$ & $\begin{array}{l}85.0 \\
90.0 \\
95.0\end{array}$ & $\begin{array}{r}.01797 \\
.01749 \\
.01707\end{array}$ & $\begin{array}{r}.07191 \\
.06998 \\
.06831\end{array}$ & $\begin{array}{l}.00547 \\
.00533 \\
.00520\end{array}$ & $\begin{array}{r}.02191 \\
.02133 \\
.02082\end{array}$ & $\begin{array}{l}7.808 \\
7.599 \\
7.417\end{array}$ & $\begin{array}{l}23.799 \\
23.162 \\
22.607\end{array}$ & $\begin{array}{l}26.801 \\
26.083 \\
25.459\end{array}$ \\
\hline 20 & 10000 & $100 \cdot 0$ & .01665 & .06662 & .00507 & .02030 & $7 \cdot 233$ & 22.049 & 24.830 \\
\hline
\end{tabular}


TABULATION OF DATA FOR

ABSORPTION OF SOUND IN AIR VERSUS PERCENT RELATIVE HUMIDITY AT 20 DEGREES CENTIGRADE FOR A FREQUENCY OF 12500 HERTZ

\begin{tabular}{|c|c|c|c|c|c|c|c|c|c|}
\hline (1) & $(2)$ & $(3)$ & (4) & (5) & $(6)$ & 171 & $(8)$ & 191 & 1101 \\
\hline $\begin{array}{l}\text { TEMP } \\
\text { SEGR } \\
\text { CENT }\end{array}$ & $\begin{array}{l}\text { FREQ } \\
\text { HERTZ }\end{array}$ & $\begin{array}{l}\text { REL } \\
\text { HUM } \\
\text { PER } \\
\text { CENT }\end{array}$ & $\begin{array}{l}\text { ATTEN } \\
\text { COEF } \\
\text { PER } \\
\text { METER }\end{array}$ & $\begin{array}{l}4 M \\
\text { PER } \\
\text { METER }\end{array}$ & $\begin{array}{l}\text { ATTEN } \\
\text { COEF } \\
\text { PER } \\
\text { FOOT. }\end{array}$ & $\begin{array}{l}4 M \\
\text { PER } \\
\text { FOOT }\end{array}$ & $\begin{array}{l}\text { ATTEN } \\
\text { DB PER } \\
100 \\
\text { METER }\end{array}$ & $\begin{array}{l}\text { ATTEN } \\
\text { DB PER } \\
1000 \\
\text { FEET }\end{array}$ & $\begin{array}{l}\text { DECAY } \\
\text { RATE } \\
\text { DB PER } \\
\text { SECOND }\end{array}$ \\
\hline $\begin{array}{l}20 \\
20 \\
20\end{array}$ & $\begin{array}{l}12500 \\
12500 \\
12500\end{array}$ & $\begin{array}{r}5.0 \\
10.0 \\
15.0\end{array}$ & $\begin{array}{r}.03177 \\
.05840 \\
.08019\end{array}$ & $\begin{array}{l}.12711 \\
.23362 \\
.32079\end{array}$ & $\begin{array}{l}.00968 \\
.01780 \\
.02444\end{array}$ & $\begin{array}{r}.03874 \\
.07120 \\
.09777\end{array}$ & $\begin{array}{l}13.802 \\
25.365 \\
34.830\end{array}$ & $\begin{array}{r}42.069 \\
77.315 \\
106.165\end{array}$ & $\begin{array}{r}47.375 \\
87.067 \\
119.556\end{array}$ \\
\hline $\begin{array}{l}20 \\
20 \\
20\end{array}$ & $\begin{array}{l}12500 \\
12500 \\
12500\end{array}$ & $\begin{array}{l}20.0 \\
25.0 \\
30.0\end{array}$ & $\begin{array}{r}.08346 \\
.07795 \\
.06768\end{array}$ & $\begin{array}{r}.33386 \\
.31183 \\
.27074\end{array}$ & $\begin{array}{l}.02544 \\
.02376 \\
.02063\end{array}$ & $\begin{array}{r}.10176 \\
.09504 \\
.08252\end{array}$ & $\begin{array}{l}36.249 \\
33.857 \\
29.396\end{array}$ & $\begin{array}{r}110.489 \\
103.198 \\
89.601\end{array}$ & $\begin{array}{l}124.426 \\
116.215 \\
100.903\end{array}$ \\
\hline $\begin{array}{l}20 \\
20 \\
20\end{array}$ & $\begin{array}{l}12500 \\
12500 \\
12500\end{array}$ & $\begin{array}{l}35.0 \\
40.0 \\
45.0\end{array}$ & $\begin{array}{l}.05783 \\
.05077 \\
.04560\end{array}$ & $\begin{array}{r}.23132 \\
.20309 \\
.18241\end{array}$ & $\begin{array}{r}.01762 \\
.01547 \\
.01389\end{array}$ & $\begin{array}{l}.07050 \\
.06190 \\
.05559\end{array}$ & $\begin{array}{l}25.115 \\
22.051 \\
19.805\end{array}$ & $\begin{array}{l}76.553 \\
67.213 \\
60.367\end{array}$ & $\begin{array}{l}86.209 \\
75.690 \\
67.981\end{array}$ \\
\hline $\begin{array}{l}20 \\
20 \\
20\end{array}$ & $\begin{array}{l}12500 \\
12500 \\
12500\end{array}$ & $\begin{array}{l}46.0 \\
47.0 \\
48.0\end{array}$ & $\begin{array}{r}.04463 \\
.04371 \\
.04283\end{array}$ & $\begin{array}{r}.17855 \\
.17486 \\
.17134\end{array}$ & $\begin{array}{r}.01360 \\
.01332 \\
.01305\end{array}$ & $\begin{array}{r}.05442 \\
.05329 \\
.05222\end{array}$ & $\begin{array}{l}19.386 \\
18.985 \\
18.603\end{array}$ & $\begin{array}{l}59.090 \\
57.869 \\
56.703\end{array}$ & $\begin{array}{l}66.543 \\
65.168 \\
63.855\end{array}$ \\
\hline $\begin{array}{l}20 \\
20 \\
20\end{array}$ & $\begin{array}{l}12500 \\
12500 \\
12500\end{array}$ & $\begin{array}{l}49.0 \\
50.0 \\
51.0\end{array}$ & $\begin{array}{r}.04198 \\
.04114 \\
.04030\end{array}$ & $\begin{array}{l}.16792 \\
.16457 \\
.16121\end{array}$ & $\begin{array}{r}.01279 \\
.01254 \\
.01228\end{array}$ & $\begin{array}{l}.05118 \\
.05016 \\
.04913\end{array}$ & $\begin{array}{l}18.232 \\
17.868 \\
17.503\end{array}$ & $\begin{array}{l}55.573 \\
54.462 \\
53.352\end{array}$ & $\begin{array}{l}62.582 \\
61.332 \\
60.082\end{array}$ \\
\hline $\begin{array}{ll}20 & 1 \\
20 & 1 \\
20 & 1\end{array}$ & $\begin{array}{l}12500 \\
12500 \\
12500\end{array}$ & $\begin{array}{l}52.0 \\
53.0 \\
54.0\end{array}$ & $\begin{array}{r}.03946 \\
.03878 \\
.03810\end{array}$ & $\begin{array}{l}.15786 \\
.15512 \\
.15243\end{array}$ & $\begin{array}{l}.01202 \\
.01182 \\
.01161\end{array}$ & $\begin{array}{r}.04811 \\
.04728 \\
.04646\end{array}$ & $\begin{array}{l}17.139 \\
16.842 \\
16.551\end{array}$ & $\begin{array}{l}52.242 \\
51.336 \\
50.448\end{array}$ & $\begin{array}{l}58.831 \\
57.811 \\
56.811\end{array}$ \\
\hline $\begin{array}{l}20 \\
20 \\
20\end{array}$ & $\begin{array}{l}12500 \\
12500 \\
12500\end{array}$ & $\begin{array}{l}55.0 \\
60.0 \\
65.0\end{array}$ & $\begin{array}{r}.03743 \\
.03455 \\
.03226\end{array}$ & $\begin{array}{r}14975 \\
.13821 \\
.12905\end{array}$ & $\begin{array}{r}.01141 \\
.01053 \\
.00983\end{array}$ & $\begin{array}{r}.04564 \\
.04212 \\
.03933\end{array}$ & $\begin{array}{l}16.259 \\
15.006 \\
14.012\end{array}$ & $\begin{array}{l}49.560 \\
45.741 \\
42.709\end{array}$ & $\begin{array}{l}55.811 \\
51.511 \\
48.096\end{array}$ \\
\hline $\begin{array}{l}201 \\
201\end{array}$ & $\begin{array}{l}12500 \\
12500\end{array}$ & $\begin{array}{l}70.0 \\
75.0\end{array}$ & $\begin{array}{r}.03013 \\
.02837\end{array}$ & $\begin{array}{r}12055 \\
.11351\end{array}$ & $\begin{array}{l}.00918 \\
.00865\end{array}$ & $\begin{array}{l}.03674 \\
.03460\end{array}$ & $\begin{array}{l}13.089 \\
12.325\end{array}$ & $\begin{array}{l}39.896 \\
37.568\end{array}$ & $\begin{array}{l}44 \cdot 928 \\
42 \cdot 306\end{array}$ \\
\hline 201 & 12500 & 80.0 & .02699 & .10796 & .00822 & .03290 & $11 \cdot 722$ & $35 \cdot 729$ & 40.236 \\
\hline $\begin{array}{l}201 \\
201\end{array}$ & $\begin{array}{l}12500 \\
12500\end{array}$ & $\begin{array}{l}85.0 \\
90.0\end{array}$ & $\begin{array}{l}.02586 \\
.02476\end{array}$ & $\begin{array}{r}10346 \\
.09907\end{array}$ & $\begin{array}{l}.00788 \\
.00754\end{array}$ & $\begin{array}{r}.03153 \\
.03019\end{array}$ & $\begin{array}{l}11.233 \\
10.757\end{array}$ & $\begin{array}{l}34.239 \\
32.788\end{array}$ & $\begin{array}{l}38.558 \\
36.924\end{array}$ \\
\hline 201 & 12500 & 95.0 & .02397 & .09588 & .00730 & .02922 & $10 \cdot 411$ & 31.733 & 35.736 \\
\hline 1 & 1250 & & .02337 & .09350 & .00712 & .02850 & 10.152 & 30.944 & 34.847 \\
\hline
\end{tabular}


TABULATION OF DATA FOR

ABSORPTION OF SOUND IN AIR VERSUS PERCENT RELATIVE HUMIDITY AT 21 DEGREES CENTIGRADE FOR A FREQUENCY OF 125 HERTZ

\begin{tabular}{|c|c|c|c|c|c|c|c|c|c|}
\hline$(1)$ & $(2)$ & (3) & (4) & (5) & $(6)$ & $(7)$ & $(8)$ & $(9)$ & $(10)$ \\
\hline $\begin{array}{l}\text { TEMP } \\
\text { DEGR } \\
\text { CENT }\end{array}$ & FREQ & $\begin{array}{l}\text { REL } \\
\text { HUM } \\
\text { PER } \\
\text { CENT }\end{array}$ & $\begin{array}{l}\text { ATTEN } \\
\text { COEF } \\
\text { PER } \\
\text { METER }\end{array}$ & $\begin{array}{l}\text { PER } \\
\text { METER }\end{array}$ & $\begin{array}{l}\text { ATTEN } \\
\text { COEF } \\
\text { PER } \\
\text { FOOT }\end{array}$ & $\begin{array}{l}\text { PER } \\
\text { FOO'ं }\end{array}$ & $\begin{array}{l}\text { ATTEN } \\
\text { DE PER } \\
\text { IOO } \\
\text { METER }\end{array}$ & $\begin{array}{l}\text { ATTEN } \\
\text { DB PER } \\
1000 \\
\text { FEET }\end{array}$ & $\begin{array}{l}\text { DECAY } \\
\text { RATE } \\
\text { DB PER } \\
\text { SECOND }\end{array}$ \\
\hline $\begin{array}{l}21 \\
21 \\
21\end{array}$ & $\begin{array}{l}125 \\
125 \\
125\end{array}$ & $\begin{array}{r}5.0 \\
10.0 \\
15.0\end{array}$ & $\begin{array}{l}.00018 \\
.00013 \\
.00011\end{array}$ & $\begin{array}{l}.00075 \\
.00055 \\
.00047\end{array}$ & $\begin{array}{l}.00005 \\
.00004 \\
.00003\end{array}$ & $\begin{array}{l}.00023 \\
.00016 \\
.00014\end{array}$ & $\begin{array}{l}.082 \\
.060 \\
.051\end{array}$ & $\begin{array}{r}.250 \\
.183 \\
.156\end{array}$ & $\begin{array}{l}.282 \\
.206 \\
.176\end{array}$ \\
\hline $\begin{array}{l}21 \\
21 \\
21\end{array}$ & $\begin{array}{l}125 \\
125 \\
125\end{array}$ & $\begin{array}{l}20.0 \\
25.0 \\
30.0\end{array}$ & $\begin{array}{l}.00010 \\
.00009 \\
.00009\end{array}$ & $\begin{array}{l}.00042 \\
.00039 \\
.00036\end{array}$ & $\begin{array}{l}.00003 \\
.00002 \\
.00002\end{array}$ & $\begin{array}{l}.00012 \\
.00011 \\
.00011\end{array}$ & $\begin{array}{l}.046 \\
.042 \\
.039\end{array}$ & $\begin{array}{r}140 \\
.129 \\
.120\end{array}$ & $\begin{array}{r}.158 \\
.145 \\
.136\end{array}$ \\
\hline $\begin{array}{l}21 \\
21 \\
21\end{array}$ & $\begin{array}{l}125 \\
125 \\
125\end{array}$ & $\begin{array}{l}35.0 \\
40.0 \\
45.0\end{array}$ & $\begin{array}{l}.00008 \\
.00008 \\
.00007\end{array}$ & $\begin{array}{l}.00034 \\
.00032 \\
.00031\end{array}$ & $\begin{array}{l}.00002 \\
.00002 \\
.00002\end{array}$ & $\begin{array}{l}.00010 \\
.00010 \\
.00009\end{array}$ & $\begin{array}{l}.037 \\
.035 \\
.034\end{array}$ & $\begin{array}{l}.114 \\
.1108 \\
.1 \\
104\end{array}$ & $\begin{array}{l}.129 \\
.122 \\
.117\end{array}$ \\
\hline $\begin{array}{l}21 \\
21 \\
21\end{array}$ & $\begin{array}{l}125 \\
125 \\
125\end{array}$ & $\begin{array}{l}46.0 \\
47.0 \\
48.0\end{array}$ & $\begin{array}{l}.00007 \\
.00007 \\
.00007\end{array}$ & $\begin{array}{l}.00031 \\
.00031 \\
.00030\end{array}$ & $\begin{array}{l}.00002 \\
.00002 \\
.00002\end{array}$ & $\begin{array}{l}.00009 \\
.00009 \\
.00009\end{array}$ & $\begin{array}{l}.033 \\
.033 \\
.033\end{array}$ & $\begin{array}{l}.103 \\
.102 \\
.101\end{array}$ & $\begin{array}{r}.116 \\
.115 \\
.115\end{array}$ \\
\hline $\begin{array}{l}21 \\
21 \\
21\end{array}$ & $\begin{array}{l}125 \\
125 \\
125\end{array}$ & $\begin{array}{l}49.0 \\
50.0 \\
51.0\end{array}$ & $\begin{array}{r}.00007 \\
.00007 \\
.00007\end{array}$ & $\begin{array}{l}.00030 \\
.00030 \\
.00030\end{array}$ & $\begin{array}{l}.00002 \\
.00002 \\
.00002\end{array}$ & $\begin{array}{l}.00009 \\
.00009 \\
.00009\end{array}$ & $\begin{array}{l}.033 \\
.032 \\
.032\end{array}$ & $\begin{array}{l}.101 \\
.100 \\
.099\end{array}$ & $\begin{array}{r}.114 \\
.113 \\
.112\end{array}$ \\
\hline $\begin{array}{ll}21 \\
21\end{array}$ & $\begin{array}{l}125 \\
125\end{array}$ & $\begin{array}{l}52.0 \\
53.0\end{array}$ & $\begin{array}{l}.00007 \\
.00007\end{array}$ & $\begin{array}{l}.00029 \\
.00029\end{array}$ & $\begin{array}{l}.00002 \\
.00002\end{array}$ & $\begin{array}{l}.00009 \\
.00009\end{array}$ & $\begin{array}{r}.032 \\
.032\end{array}$ & $\begin{array}{l}.099 \\
.098\end{array}$ & .111 \\
\hline $21^{\circ}$ & 125 & 54.0 & .00007 & .00029 & .00002 & .00008 & .032 & .097 & .110 \\
\hline $\begin{array}{l}21 \\
21\end{array}$ & $\begin{array}{l}125 \\
125\end{array}$ & $\begin{array}{l}55.0 \\
60.0\end{array}$ & $\begin{array}{l}.00007 \\
.00007\end{array}$ & $\begin{array}{r}.00029 \\
.00028\end{array}$ & $\begin{array}{l}.00002 \\
.00002\end{array}$ & $\begin{array}{l}.00008 \\
.00008\end{array}$ & $\begin{array}{r}.031 \\
.030\end{array}$ & $\begin{array}{l}.096 \\
.093\end{array}$ & $\begin{array}{r}.109 \\
.105\end{array}$ \\
\hline 21 & 125 & 65.0 & .00006 & .00027 & .00002 & .00008 & .029 & .091 & .102 \\
\hline $\begin{array}{l}21 \\
21\end{array}$ & $\begin{array}{l}125 \\
125\end{array}$ & $\begin{array}{l}70.0 \\
75.0\end{array}$ & $\begin{array}{l}.00006 \\
.00006\end{array}$ & $\begin{array}{l}.00026 \\
.00025\end{array}$ & $\begin{array}{l}.00002 \\
.00001\end{array}$ & $\begin{array}{l}.00008 \\
.00007\end{array}$ & $\begin{array}{r}.029 \\
.028\end{array}$ & $\begin{array}{l}.088 \\
.085\end{array}$ & $\begin{array}{l}.099 \\
.096\end{array}$ \\
\hline 21 & 125 & 80.0 & .00006 & .00025 & .00001 & .00007 & .027 & .083 & .093 \\
\hline $\begin{array}{l}21 \\
21\end{array}$ & $\begin{array}{l}125 \\
125\end{array}$ & $\begin{array}{l}85.0 \\
90.0\end{array}$ & $\begin{array}{l}.00006 \\
.00005\end{array}$ & $\begin{array}{l}.00024 \\
.00023\end{array}$ & $\begin{array}{l}.00001 \\
.00001\end{array}$ & $\begin{array}{l}.00007 \\
.00007\end{array}$ & $\begin{array}{l}.026 \\
.025\end{array}$ & $\begin{array}{r}.080 \\
.077\end{array}$ & $\begin{array}{l}.090 \\
.087\end{array}$ \\
\hline 21 & 125 & 95.0 & .00005 & .00022 & .00001 & .00006 & .024 & .075 & .085 \\
\hline 21 & 125 & $100 \cdot 0$ & .00005 & .00022 & .00001 & .00006 & .024 & .073 & .083 \\
\hline
\end{tabular}


TABULATION OF DATA FOR

ABSORPTION OF SOUND IN AIR VERSUS PERCENT RELATIVE HUMIDITY AT 21 DEGREES CENTIGRADE FOR A FREQUENCY OF. 250 HERTZ

\begin{tabular}{|c|c|c|c|c|c|c|c|c|c|}
\hline 111 & $(2)$ & (3) & (4) & (5) & $(6)$ & 171 & $(8)$ & 191 & $(10)$ \\
\hline EMP & FREQ & REL & ATTEN & $4 M$ & ATTEN & $4 M$ & ATTEN & ATTEN & DECAY \\
\hline $\begin{array}{l}\text { DEGR } \\
\text { CENT }\end{array}$ & HERTZ & $\begin{array}{l}\text { HUM } \\
\text { PER } \\
\text { CENT }\end{array}$ & $\begin{array}{l}\text { COEF } \\
\text { PER } \\
\text { METER }\end{array}$ & $\begin{array}{l}\text { PER } \\
\text { METER }\end{array}$ & $\begin{array}{l}\text { COEF } \\
\text { PER } \\
\text { FOOT }\end{array}$ & $\begin{array}{l}\text { PER } \\
\text { FOOT }\end{array}$ & $\begin{array}{l}\text { DB PER } \\
100 \cdot \\
\text { METER }\end{array}$ & $\begin{array}{l}\text { DB PER } \\
1000 \\
\text { FEET }\end{array}$ & $\begin{array}{l}\text { RATE } \\
\text { DB PER } \\
\text { SECOND }\end{array}$ \\
\hline $\begin{array}{l}21 \\
21\end{array}$ & $\begin{array}{l}250 \\
250\end{array}$ & $\begin{array}{r}5.0 \\
10.0\end{array}$ & $\begin{array}{l}.00048 \\
.00030\end{array}$ & $\begin{array}{l}.00194 \\
.00121\end{array}$ & $\begin{array}{l}.00014 \\
.00009\end{array}$ & $\begin{array}{l}.00059 \\
.00037\end{array}$ & $\begin{array}{l}.210 \\
.132\end{array}$ & $\begin{array}{l}643 \\
.403\end{array}$ & $\begin{array}{r}.725 \\
.454\end{array}$ \\
\hline 21 & 250 & 15.0 & .00026 & .00104 & .00007 & .00031 & .113 & .347 & .391 \\
\hline $\begin{array}{l}21 \\
21\end{array}$ & $\begin{array}{l}250 \\
250\end{array}$ & $\begin{array}{l}20.0 \\
25.0\end{array}$ & $\begin{array}{l}.00023 \\
.00021\end{array}$ & $\begin{array}{l}.00093 \\
.00086\end{array}$ & $\begin{array}{l}.00007 \\
.00006\end{array}$ & $\begin{array}{l}.00028 \\
.00026\end{array}$ & $\begin{array}{l}.101 \\
.093\end{array}$ & $\begin{array}{l}.309 \\
.286\end{array}$ & $\begin{array}{r}.349 \\
.322\end{array}$ \\
\hline 21 & 250 & $30 \cdot 0$ & .00020 & .00080 & .00006 & .00024 & .087 & .267 & .301 \\
\hline $\begin{array}{l}21 \\
21\end{array}$ & $\begin{array}{l}250 \\
250\end{array}$ & $\begin{array}{l}35.0 \\
40.0\end{array}$ & $\begin{array}{l}.00019 \\
.00018\end{array}$ & $\begin{array}{l}.00076 \\
.00072\end{array}$ & $\begin{array}{r}.00005 \\
.00005\end{array}$ & $\begin{array}{l}.00023 \\
.00022\end{array}$ & $\begin{array}{l}.082 \\
.078\end{array}$ & $\begin{array}{l}.252 \\
.240\end{array}$ & $\begin{array}{r}.284 \\
.270\end{array}$ \\
\hline 21 & 250 & 45.0 & .00017 & .00069 & .00005 & .00021 & .075 & .230 & .260 \\
\hline $\begin{array}{l}21 \\
21\end{array}$ & $\begin{array}{l}250 \\
250\end{array}$ & $\begin{array}{l}46.0 \\
47.0\end{array}$ & $\begin{array}{l}.00017 \\
.00017\end{array}$ & $\begin{array}{l}.00069 \\
.00068\end{array}$ & $\begin{array}{l}.00005 \\
.00005\end{array}$ & $\begin{array}{l}.00021 \\
.00020\end{array}$ & $\begin{array}{l}.074 \\
.074\end{array}$ & $\begin{array}{r}.228 \\
.226\end{array}$ & $\begin{array}{l}.257 \\
.255\end{array}$ \\
\hline 21 & 250 & 48.0 & .00016 & $.00067:$ & .00005 & .00020 & .073 & .224 & .253 \\
\hline $\begin{array}{l}21 \\
21 \\
21\end{array}$ & $\begin{array}{l}250 \\
250 \\
250\end{array}$ & $\begin{array}{l}49.0 \\
50.0 \\
51.0\end{array}$ & $\begin{array}{r}.00016 \\
.00016 \\
.00016\end{array}$ & $\begin{array}{l}.00067 \\
.00066 \\
.00066\end{array}$ & $\begin{array}{l}.00005 \\
.00005 \\
.00005\end{array}$ & $\begin{array}{l}.00020 \\
.00020 \\
.00020\end{array}$ & $\begin{array}{l}.073 \\
.072 \\
.072\end{array}$ & $\begin{array}{l}.222 \\
.221 \\
.219\end{array}$ & $\begin{array}{l}.251 \\
.249 \\
.247\end{array}$ \\
\hline $\begin{array}{l}21 \\
21 \\
21\end{array}$ & $\begin{array}{l}250 \\
250 \\
250\end{array}$ & $\begin{array}{l}52 \cdot 0 \\
53 \cdot 0 \\
54 \cdot 0\end{array}$ & $\begin{array}{l}.00016 \\
.00016 \\
.00016\end{array}$ & $\begin{array}{l}.00065 \\
.00065 \\
.00064\end{array}$ & $\begin{array}{l}.00005 \\
.00004 \\
.00004\end{array}$ & $\begin{array}{l}.00020 \\
.00019 \\
.00019\end{array}$ & $\begin{array}{l}.071 \\
.070 \\
.070\end{array}$ & $\begin{array}{r}.217 \\
.216 \\
.214\end{array}$ & $\begin{array}{l}.245 \\
.244 \\
.242\end{array}$ \\
\hline $\begin{array}{l}21 \\
21 \\
21\end{array}$ & $\begin{array}{l}250 \\
250 \\
250\end{array}$ & $\begin{array}{l}55.0 \\
60.0 \\
65.0\end{array}$ & $\begin{array}{l}.00016 \\
.00015 \\
.00015\end{array}$ & $\begin{array}{l}.00064 \\
.00062 \\
.00060\end{array}$ & $\begin{array}{r}.00004 \\
.00004 \\
.00004\end{array}$ & $\begin{array}{r}.00019 \\
.00019 \\
.00018\end{array}$ & $\begin{array}{l}.069 \\
.067 \\
.066\end{array}$ & $\begin{array}{l}.213 \\
.206 \\
.201\end{array}$ & $\begin{array}{l}.240 \\
.233 \\
.226\end{array}$ \\
\hline $\begin{array}{l}21 \\
21 \\
21\end{array}$ & $\begin{array}{l}250 \\
250 \\
250\end{array}$ & $\begin{array}{l}70.0 \\
75.0 \\
80.0\end{array}$ & $\begin{array}{l}.00014 \\
.00014 \\
.00014\end{array}$ & $\begin{array}{l}.00059 \\
.00057 \\
.00056\end{array}$ & $\begin{array}{l}.00004 \\
.00004 \\
.00004\end{array}$ & $\begin{array}{l}.00018 \\
.00017 \\
.00017\end{array}$ & $\begin{array}{l}.064 \\
.062 \\
.060\end{array}$ & $\begin{array}{r}.195 \\
.190 \\
.185\end{array}$ & $\begin{array}{l}.220 \\
.214 \\
.209\end{array}$ \\
\hline $\begin{array}{l}21 \\
21 \\
21\end{array}$ & $\begin{array}{l}250 \\
250 \\
250\end{array}$ & $\begin{array}{l}85.0 \\
90.0 \\
95.0\end{array}$ & $\begin{array}{l}.00013 \\
.00013 \\
.00013\end{array}$ & $\begin{array}{l}.00054 \\
.00053 \\
.00052\end{array}$ & $\begin{array}{l}.00004 \\
.00004 \\
.00004\end{array}$ & $\begin{array}{l}.00016 \\
.00016 \\
.00016\end{array}$ & $\begin{array}{l}.059 \\
.058 \\
.057\end{array}$ & $\begin{array}{r}.181 \\
.177 \\
.173\end{array}$ & $\begin{array}{l}.205 \\
.200 \\
.196\end{array}$ \\
\hline 21 & 50 & $100 \cdot 0$ & .00012 & .00051 & .00003 & .00015 & .055 & .169 & .191 \\
\hline
\end{tabular}


TABULATION OF DATA FOR

ABSORPTION OF SOUND IN AIR VERSUS PERCENT RELATIVE HUMIDITY AT 21 DEGREES CENTIGRADE FOR A FREQUENCY OF 500 HERTZ

(1) (2) (3)

TEMP FREQ

DEGR CENT HERTZ CENT

21

21

21

$21500 \quad 20 \cdot 0$

$21 \quad 500 \quad 25.0$

$21500 \quad 30 \cdot 0$

$21500 \quad 35.0$

$21 \quad 500 \quad 40.0$

$21500 \quad 45.0$

$21500 \quad 46.0$

$21500 \quad 47.0$

$21500 \quad 48.0$

$21500 \quad 49.0$

$21 \quad 500 \quad 50.0$

$21500 \quad 51.0$

$21500 \quad 52.0$

$21500 \quad 53.0$

$21500 \quad 54.0$

21

21

21

21

21

21

2

21

21

21

$500 \quad 60.0$

65.0

$500 \quad 70.0$

$500 \quad 75.0$

$500 \quad 80.0$
(4)

(5)

ATTEN $4 M$

COEF

PER

METER

.00157

.00076

.00062

.00055

.00051

.00047

.00045

.00043

.00041

.00040

.00040

.00040

.00039

.00039

.00039

.00039

.00038

.00038

.00038

.00037

.00036

.00153

.00148

.00035

.00034

.00033

00140

.00136

.00133

.00032

.00032

.00130

.00128

.00125

.00123
(6)

(7)

$\begin{array}{ll}\text { ATTEN } & 4 M \\ \text { COEF } & \\ \text { PER } & \text { PER }\end{array}$

FOOT FOOT

.00047

.00023

.00018

.00092

.00075

$.00017 \quad .00068$

$.00015 \quad .00062$

$.00014 \quad .00058$

.00013 .00055

.00013 .00052

.00012 .00050

$.00012 \quad .00049$

$.00012 \quad .00049$

$.00012 \quad .00049$

$.00012 \quad .00048$

$.00012 \quad .00048$

.00012 .00048

$.00011 \quad .00047$

$.00011 \quad .00047$

$.00011 \quad .00047$

$.00011 \quad .00046$

$.00011 \quad .00045$

.00011 .00044

$.00010 \quad .00042$

.00010 .00041

.00010 .00040

$.00009 \quad .00039$

.00009 .00039

$.00009 \quad .00038$

$.00009 \quad .00037$
(8)

(9)

(10)

$\begin{array}{lll}\text { ATTEN } & \text { ATTEN } & \text { DECAY } \\ \text { DB PER } & \text { DB PER } & \text { RATE } \\ 100 & 1000 & \text { DB PER } \\ \text { METER } & \text { FEET } & \text { SECOND }\end{array}$

$\begin{array}{rrr}.682 & 2.079 & 2.345 \\ .330 & 1.008 & 1.137 \\ .269 & .820 & .926 \\ .242 & .740 & .835 \\ .222 & .679 & .766 \\ .207 & .631 & .712 \\ .196 & .598 & .674 \\ .187 & .570 & .643 \\ .179 & .545 & .615 \\ .177 & .541 & .610 \\ .176 & .537 & .606 \\ .174 & .533 & .601 \\ .173 & .528 & .596 \\ .172 & .524 & .591 \\ .171 & .521 & .588\end{array}$

$\begin{array}{lll}.169 & .517 & .584\end{array}$

$.168 \quad .514 \quad .580$

$.167 .0511 \quad .576$

$\begin{array}{lll}.166 & .507 & .572\end{array}$

$.161 \quad .491 \quad .553$

$.156 \quad .478 \quad .539$

$.152 .465 \quad .525$

$.148 \quad .453 \quad .511$

$.145 \quad .442 \quad .499$

$.141 .432 \quad .487$

$.139 \quad .423 \quad .478$

$.136 \quad .416 \quad .469$

$.134 \quad .409 \quad .461$ 
TABULATION OF DATA FOR

ABSORPTION OF SOUND IN AIR VERSUS PERCENT RELATIVE HUMIDITY AT 21 DEGREES CENTIGRADE FOR A FREQUENCY OF 1000 HERTZ

\begin{tabular}{|c|c|c|c|c|c|c|c|c|c|}
\hline (1) & $(2)$ & $(3)$ & (4) & (5) & (6) & 171 & (3) & 191 & $(10)$ \\
\hline TEMP & FREQ & $\begin{array}{l}\text { REL } \\
\text { HUM }\end{array}$ & $\begin{array}{l}\text { ATTEN } \\
\text { COEF }\end{array}$ & $4 M$ & $\begin{array}{l}\text { ATTEN } \\
\text { COEF }\end{array}$ & $4 M$ & $\begin{array}{l}\text { ATTEN } \\
\text { DB PER }\end{array}$ & $\begin{array}{l}\text { ATTEN } \\
\text { DB P.ER }\end{array}$ & $\begin{array}{l}\text { DECAY } \\
\text { RATE. }\end{array}$ \\
\hline $\begin{array}{l}\text { DEGR } \\
\text { CENT }\end{array}$ & HERTZ & $\begin{array}{l}\text { PER } \\
\text { CENT }\end{array}$ & $\begin{array}{l}\text { PER } \\
\text { METER }\end{array}$ & $\begin{array}{l}\text { PER } \\
\text { METER }\end{array}$ & $\begin{array}{l}\text { PER } \\
\text { FOOT }\end{array}$ & $\begin{array}{l}\text { PER } \\
\text { FOOT }\end{array}$ & $\begin{array}{l}\text { I00 } \\
\text { METER }\end{array}$ & $\begin{array}{l}1000 \\
\text { FEET }\end{array}$ & $\begin{array}{l}\text { DB PER } \\
\text { SECOND }\end{array}$ \\
\hline $\begin{array}{l}21 \\
21 \\
21\end{array}$ & $\begin{array}{l}1000 \\
1000 \\
1000\end{array}$ & $\begin{array}{r}5.0 \\
10.0 \\
15.0\end{array}$ & $\begin{array}{l}.00538 \\
.00254 \\
.00167\end{array}$ & $\begin{array}{l}.02154 \\
.01017 \\
.00671\end{array}$ & $\begin{array}{r}.00164 \\
.00077 \\
.00051\end{array}$ & $\begin{array}{l}.00656 \\
.00310 \\
.00204\end{array}$ & $\begin{array}{r}2.339 \\
1.104 \\
.729\end{array}$ & $\begin{array}{l}7.130 \\
3.367 \\
2.223\end{array}$ & $\begin{array}{l}8.042 \\
3.798 \\
2.508\end{array}$ \\
\hline $\begin{array}{l}21 \\
21 \\
21\end{array}$ & $\begin{array}{l}1000 \\
1000 \\
1000\end{array}$ & $\begin{array}{l}20.0 \\
25.0 \\
30.0\end{array}$ & $\begin{array}{l}.00136 \\
.00125 \\
.00117\end{array}$ & $\begin{array}{l}.00547 \\
.00501 \\
.00470\end{array}$ & $\begin{array}{l}.00041 \\
.00038 \\
.00035\end{array}$ & $\begin{array}{l}.00166 \\
.00152 \\
.00143\end{array}$ & $\begin{array}{l}.594 \\
.544 \\
.510\end{array}$ & $\begin{array}{l}1.812 \\
1.660 \\
1.556\end{array}$ & $\begin{array}{l}2.044 \\
1.873 \\
1.756\end{array}$ \\
\hline $\begin{array}{l}21 \\
21 \\
21\end{array}$ & $\begin{array}{l}1000 \\
1000 \\
1000\end{array}$ & $\begin{array}{l}35.0 \\
40.0 \\
45.0\end{array}$ & $\begin{array}{l}.00111 \\
.00105 \\
.00100\end{array}$ & $\begin{array}{l}.00445 \\
.00423 \\
.00403\end{array}$ & $\begin{array}{l}.00033 \\
.00032 \\
.00030\end{array}$ & $\begin{array}{l}.00135 \\
.00128 \\
.00123\end{array}$ & $\begin{array}{l}.483 \\
.459 \\
.438\end{array}$ & $\begin{array}{l}1.472 \\
1.400 \\
1.335\end{array}$ & $\begin{array}{l}1.661 \\
1.579 \\
1.506\end{array}$ \\
\hline $\begin{array}{l}21 \\
21 \\
21\end{array}$ & $\begin{array}{l}1000 \\
1000 \\
1000\end{array}$ & $\begin{array}{l}46.0 \\
47.0 \\
48.0\end{array}$ & $\begin{array}{l}.00099 \\
.00098 \\
.00098\end{array}$ & $\begin{array}{l}.00399 \\
.00395 \\
.00393\end{array}$ & $\begin{array}{l}.00030 \\
.00030 \\
.00029\end{array}$ & $\begin{array}{l}.00121 \\
.00120 \\
.00119\end{array}$ & $\begin{array}{l}.434 \\
.429 \\
.426\end{array}$ & $\begin{array}{l}1.322 \\
1.310 \\
1.301\end{array}$ & $\begin{array}{l}1.492 \\
1.477 \\
1.467\end{array}$ \\
\hline $\begin{array}{ll}21 \\
21 \\
21\end{array}$ & $\begin{array}{l}1000 \\
1000 \\
1000\end{array}$ & $\begin{array}{l}49.0 \\
50.0 \\
51.0\end{array}$ & $\begin{array}{l}.00097 \\
.00096 \\
.00096\end{array}$ & $\begin{array}{l}.00390 \\
.00387 \\
.00385\end{array}$ & $\begin{array}{r}.00029 \\
.00029 \\
.00029\end{array}$ & $\begin{array}{l}.00119 \\
.00118 \\
.00117\end{array}$ & $\begin{array}{l}.424 \\
.421 \\
.418\end{array}$ & $\begin{array}{l}1.292 \\
1.283 \\
1.275\end{array}$ & $\begin{array}{l}1.458 \\
1.448 \\
1.438\end{array}$ \\
\hline $\begin{array}{ll}21 \\
21 \\
21\end{array}$ & $\begin{array}{l}1000 \\
1000 \\
1000\end{array}$ & $\begin{array}{l}52.0 \\
53.0 \\
54.0\end{array}$ & $\begin{array}{l}.00095 \\
.00095 \\
.00094\end{array}$ & $\begin{array}{l}.00382 \\
.00380 \\
.00377\end{array}$ & $\begin{array}{l}.00029 \\
.00028 \\
.00028\end{array}$ & $\begin{array}{l}.00116 \\
.00115 \\
.00115\end{array}$ & $\begin{array}{l}.415 \\
.412 \\
.410\end{array}$ & $\begin{array}{l}1.266 \\
1.257 \\
1.250\end{array}$ & $\begin{array}{l}1.428 \\
1.418 \\
1.410\end{array}$ \\
\hline $\begin{array}{l}21 \\
21 \\
21\end{array}$ & $\begin{array}{l}1000 \\
1000 \\
1000\end{array}$ & $\begin{array}{l}55.0 \\
60.0 \\
65.0\end{array}$ & $\begin{array}{l}.00093 \\
.00091 \\
.00088\end{array}$ & $\begin{array}{l}.00375 \\
.00364 \\
.00354\end{array}$ & $\begin{array}{l}.00028 \\
.00027 \\
.00027\end{array}$ & $\begin{array}{l}.00114 \\
.00110 \\
.00108\end{array}$ & $\begin{array}{r}.407 \\
.395 \\
.384\end{array}$ & $\begin{array}{l}1.242 \\
1.205 \\
1.173\end{array}$ & $\begin{array}{l}1.401 \\
1.359 \\
1.323\end{array}$ \\
\hline 21 & 1000 & $70 \cdot 0$ & .00086 & .00345 & .00026 & .00105 & .374 & 1.142 & 1.288 \\
\hline $\begin{array}{l}21 \\
21\end{array}$ & $\begin{array}{l}1000 \\
1000\end{array}$ & $\begin{array}{l}75.0 \\
80.0\end{array}$ & $\begin{array}{l}.00084 \\
.00082\end{array}$ & $\begin{array}{l}.00336 \\
.00328\end{array}$ & $\begin{array}{l}.00025 \\
.00025\end{array}$ & $\begin{array}{l}.00102 \\
.00100\end{array}$ & $\begin{array}{l}.365 \\
.356\end{array}$ & $\begin{array}{l}1.112 \\
1.087\end{array}$ & $\begin{array}{l}1.255 \\
1.226\end{array}$ \\
\hline 21 & 1000 & 85.0 & .00080 & .00321 & .00024 & .00098 & .349 & 1. & 1.200 \\
\hline $\begin{array}{l}21 \\
21\end{array}$ & $\begin{array}{l}1000 \\
1000\end{array}$ & $\begin{array}{l}90.0 \\
95.0\end{array}$ & $\begin{array}{l}.00078 \\
.00077\end{array}$ & $\begin{array}{l}.00315 \\
.00309\end{array}$ & $\begin{array}{l}.00024 \\
.00023\end{array}$ & $\begin{array}{l}.00096 \\
.00094\end{array}$ & $\begin{array}{l}.342 \\
.335\end{array}$ & $\begin{array}{l}1.043 \\
1.023\end{array}$ & $\begin{array}{l}1.177 \\
1.154\end{array}$ \\
\hline 2 & 1000 & 100.0 & .00075 & .00303 & .00023 & .00092 & .329 & 1.004 & 1.132 \\
\hline
\end{tabular}


TABULATION OF DATA FOR

ABSORPTION OF SOUND IN AIR VERSUS PERCENT RELATIVE HUMIDITY AT 21 DEGREES CENTIGRADE FOR A FREQUENCY OF 2000 HERTZ

\begin{tabular}{|c|c|c|c|c|c|c|c|c|c|}
\hline (1) & (2) & (3) & $(4)$ & (5) & (6) & 171 & $(8)$ & 191 & $(10)$ \\
\hline TEMP & FREQ & $\begin{array}{l}\text { REL } \\
\text { HUM }\end{array}$ & $\begin{array}{l}\text { ATTEN } \\
\text { COEF }\end{array}$ & $4 M$ & $\begin{array}{l}\text { ATTEN } \\
\text { COEF }\end{array}$ & $4 M$ & $\begin{array}{l}\text { ATTEN } \\
\text { DB PER }\end{array}$ & $\begin{array}{l}\text { ATTEN } \\
\text { DB. PER }\end{array}$ & $\begin{array}{l}\text { DECAY } \\
\text { RATE }\end{array}$ \\
\hline $\begin{array}{l}\text { DEGR } \\
\text { CENT }\end{array}$ & HERTZ & $\begin{array}{l}\text { PER } \\
\text { CENT }\end{array}$ & $\begin{array}{l}\text { PER } \\
\text { METER }\end{array}$ & $\begin{array}{l}\text { PER } \\
\text { METER }\end{array}$ & $\begin{array}{l}\text { PER } \\
\text { FOOT }\end{array}$ & $\begin{array}{l}\text { PER } \\
\text { FOOT }\end{array}$ & $\begin{array}{l}100 \\
\text { METER }\end{array}$ & $\begin{array}{l}1000 \\
\text { FEET }\end{array}$ & $\begin{array}{l}\text { DB PER } \\
\text { SECOND }\end{array}$ \\
\hline $\begin{array}{l}21 \\
21\end{array}$ & $\begin{array}{l}2000 \\
2000\end{array}$ & $\begin{array}{r}5.0 \\
10.0\end{array}$ & $\begin{array}{r}.01275 \\
.00877\end{array}$ & $\begin{array}{l}.05102 \\
.03510\end{array}$ & $\begin{array}{l}.00388 \\
.00267\end{array}$ & $\begin{array}{l}.01555 \\
.01069\end{array}$ & $\begin{array}{l}5.539 \\
3.811\end{array}$ & $\begin{array}{l}16.885 \\
11.616\end{array}$ & $\begin{array}{l}19.045 \\
13.103\end{array}$ \\
\hline 21 & 2000 & 15.0 & .00567 & .02271 & .00173 & .00692 & 2.466 & 7.517 & 8.479 \\
\hline $\begin{array}{l}21 \\
21\end{array}$ & $\begin{array}{l}2000 \\
2000\end{array}$ & $\begin{array}{l}20.0 \\
25.0\end{array}$ & $\begin{array}{l}.00418 \\
.00337\end{array}$ & $\begin{array}{r}.01673 \\
.01351\end{array}$ & $\begin{array}{l}.00127 \\
.00103\end{array}$ & $\begin{array}{l}.00510 \\
.00412\end{array}$ & $\begin{array}{l}1: 816 \\
1: 467\end{array}$ & $\begin{array}{l}5.537 \\
4.473\end{array}$ & $\begin{array}{l}6.246 \\
5.046\end{array}$ \\
\hline 21 & 2000 & 30.0 & .00295 & .01182 & .00090 & .00360 & 1.284 & 3.914 & 4.415 \\
\hline $\begin{array}{l}21 \\
21\end{array}$ & $\begin{array}{l}2000 \\
2000\end{array}$ & $\begin{array}{l}35.0 \\
40.0\end{array}$ & $\begin{array}{l}.00272 \\
.00260\end{array}$ & $\begin{array}{r}.01091 \\
.01042\end{array}$ & $\begin{array}{r}.00083 \\
.00079\end{array}$ & $\begin{array}{l}.00332 \\
.00317\end{array}$ & $\begin{array}{l}1 \cdot 184 \\
1 \cdot 131\end{array}$ & $\begin{array}{l}3.611 \\
3.449\end{array}$ & $\begin{array}{l}4.073 \\
3.890\end{array}$ \\
\hline 21 & 2000 & 45.0 & .00250 & .01001 & .00076 & .00305 & 2.086 & 3.313 & 3.736 \\
\hline $\begin{array}{l}21 \\
21\end{array}$ & $\begin{array}{l}2000 \\
2000\end{array}$ & $\begin{array}{l}46.0 \\
47.0\end{array}$ & $\begin{array}{l}.00248 \\
.00246\end{array}$ & $\begin{array}{r}.00993 \\
.00986\end{array}$ & $\begin{array}{r}.00075 \\
.00075\end{array}$ & $\begin{array}{l}.00302 \\
.00300\end{array}$ & $\begin{array}{l}1.078 \\
1.070\end{array}$ & $\begin{array}{l}3.288 \\
3.263\end{array}$ & $\begin{array}{l}3.708 \\
3.680\end{array}$ \\
\hline 21 & 2000 & 48.0 & .00244 & .00978 & .00074 & .00298 & 1.062 & $3 \cdot 238$ & 3.652 \\
\hline $\begin{array}{ll}21 \\
21\end{array}$ & $\begin{array}{l}2000 \\
2000\end{array}$ & $\begin{array}{l}49.0 \\
50.0\end{array}$ & $\begin{array}{r}.00242 \\
.00241\end{array}$ & $\begin{array}{r}00971 \\
.00965\end{array}$ & $\begin{array}{l}.00074 \\
.00073\end{array}$ & $\begin{array}{r}.00296 \\
.00294\end{array}$ & $\begin{array}{l}1.054 \\
1.047\end{array}$ & $\begin{array}{l}3.215 \\
3.194\end{array}$ & $\begin{array}{l}3.626 \\
3.603\end{array}$ \\
\hline 21 & 2000 & 51.0 & .00239 & .00958 & .00073 & .00292 & 1.041 & $3 \cdot 173$ & 3.579 \\
\hline $\begin{array}{l}21 \\
21 \\
21\end{array}$ & $\begin{array}{l}2000 \\
2000 \\
2000\end{array}$ & $\begin{array}{l}52.0 \\
53.0 \\
54.0\end{array}$ & $\begin{array}{l}.00238 \\
.00236 \\
.00235\end{array}$ & $\begin{array}{r}.00952 \\
.00946 \\
.00940\end{array}$ & $\begin{array}{r}.00072 \\
.00072 \\
.00071\end{array}$ & $\begin{array}{l}.00290 \\
.00288 \\
.00286\end{array}$ & $\begin{array}{l}1.034 \\
1.027 \\
1.020\end{array}$ & $\begin{array}{l}3.152 \\
3.132 \\
3.111\end{array}$ & $\begin{array}{l}3.556 \\
3.532 \\
3.509\end{array}$ \\
\hline $\begin{array}{l}21 \\
21 \\
21\end{array}$ & $\begin{array}{l}2000 \\
2000 \\
2000\end{array}$ & $\begin{array}{l}55.0 \\
60.0 \\
65.0\end{array}$ & $\begin{array}{l}.00233 \\
.00226 \\
.00219\end{array}$ & $\begin{array}{l}.00933 \\
.00905 \\
.00878\end{array}$ & $\begin{array}{l}.00071 \\
.00068 \\
.00066\end{array}$ & $\begin{array}{l}.00284 \\
.00275 \\
.00267\end{array}$ & $\begin{array}{r}1.013 \\
.983 \\
.953\end{array}$ & $\begin{array}{l}3.090 \\
2.996 \\
2.907\end{array}$ & $\begin{array}{l}3.486 \\
3.380 \\
3.279\end{array}$ \\
\hline $\begin{array}{l}21 \\
21 \\
21\end{array}$ & $\begin{array}{l}2000 \\
2000 \\
2000\end{array}$ & $\begin{array}{l}70.0 \\
75.0 \\
80.0\end{array}$ & $\begin{array}{l}.00213 \\
.00207 \\
.00202\end{array}$ & $\begin{array}{l}.00854 \\
.00830 \\
.00811\end{array}$ & $\begin{array}{l}.00065 \\
.00063 \\
.00061\end{array}$ & $\begin{array}{l}.00260 \\
.00253 \\
.00247\end{array}$ & $\begin{array}{r}.927 \\
.902 \\
.881\end{array}$ & $\begin{array}{l}2.827 \\
2.749 \\
2.686\end{array}$ & $\begin{array}{l}3.188 \\
3.101 \\
3.030\end{array}$ \\
\hline $\begin{array}{l}21 \\
21 \\
21\end{array}$ & $\begin{array}{l}2000 \\
2000 \\
2000\end{array}$ & $\begin{array}{l}85.0 \\
90.0 \\
95.0\end{array}$ & $\begin{array}{l}.00198 \\
.00195 \\
.00191\end{array}$ & $\begin{array}{r}.00795 \\
.00780 \\
.00766\end{array}$ & $\begin{array}{r}.00060 \\
.00059 \\
.00058\end{array}$ & $\begin{array}{l}.00242 \\
.00238 \\
.00233\end{array}$ & $\begin{array}{l}.863 \\
.847 \\
.832\end{array}$ & $\begin{array}{l}2.633 \\
2.584 \\
2.537\end{array}$ & $\begin{array}{l}2.970 \\
2.915 \\
2.862\end{array}$ \\
\hline 21 & 2000 & $100 \cdot 0$ & .00188 & .00754 & .00057 & .00229 & .818 & $2 \cdot 495$ & 2.814 \\
\hline
\end{tabular}


TABULATIION OF DATA FOR

ABSORPTION OF SOUND IN AIR VERSUS PERCENT RELATIVE HUMIDITY AT 21 DEGREES CENTIGRADE FOR A FREQUENCY OF 2500 HERTZ

\begin{tabular}{|c|c|c|c|c|c|c|c|c|c|}
\hline (1) & (2) & (3) & 141 & (5) & $(6)$ & 171 & $(8)$ & $(9)$ & $(10)$ \\
\hline $\begin{array}{l}\text { EMP } \\
\text { EGR } \\
\text { ENT }\end{array}$ & FREQ & $\begin{array}{l}\text { REL } \\
\text { HUM } \\
\text { PER } \\
\text { CENT }\end{array}$ & $\begin{array}{l}\text { ATTEN } \\
\text { COEF } \\
\text { PER } \\
\text { METER }\end{array}$ & $\begin{array}{l}4 M \\
\text { PER } \\
\text { METER }\end{array}$ & $\begin{array}{l}\text { ATTEN } \\
\text { COEF } \\
\text { PER } \\
\text { FOOT }\end{array}$ & $\begin{array}{l}4 M \\
\text { PER } \\
\text { FOOT }\end{array}$ & $\begin{array}{l}\text { ATTEN } \\
\text { DB PER } \\
100 \\
\text { METER }\end{array}$ & $\begin{array}{l}\text { ATTEN } \\
\text { DB PER } \\
\text { IOOO } \\
\text { FEET }\end{array}$ & $\begin{array}{l}\text { DECAY } \\
\text { RATE } \\
\text { DB PER } \\
\text { SECOND }\end{array}$ \\
\hline $\begin{array}{l}21 \\
21 \\
21\end{array}$ & $\begin{array}{l}2500 \\
2500 \\
2500\end{array}$ & $\begin{array}{r}5.0 \\
10.0 \\
15.0\end{array}$ & $\begin{array}{r}.01537 \\
.01283 \\
.00833\end{array}$ & $\begin{array}{l}.06151 \\
.05132 \\
.03334\end{array}$ & $\begin{array}{r}.00468 \\
.00391 \\
.00254\end{array}$ & $\begin{array}{r}.01874 \\
.01564 \\
.01016\end{array}$ & $\begin{array}{l}6.678 \\
5.572 \\
3.620\end{array}$ & $\begin{array}{l}20.357 \\
16.986 \\
11.036\end{array}$ & $\begin{array}{l}22.962 \\
19.159 \\
12.448\end{array}$ \\
\hline $\begin{array}{l}21 \\
21 \\
21\end{array}$ & $\begin{array}{l}2500 \\
2500 \\
2500\end{array}$ & $\begin{array}{l}20.0 \\
25.0 \\
30.0\end{array}$ & $\begin{array}{r}.00608 \\
.00483 \\
.00409\end{array}$ & $\begin{array}{r}.02435 \\
.01932 \\
.01638\end{array}$ & $\begin{array}{l}.00185 \\
.00147 \\
.00124\end{array}$ & $\begin{array}{l}.00742 \\
.00589 \\
.00499\end{array}$ & $\begin{array}{l}2.644 \\
2.098 \\
1.779\end{array}$ & $\begin{array}{l}8.059 \\
6.395 \\
5.423\end{array}$ & 117 \\
\hline $\begin{array}{l}21 \\
21 \\
21\end{array}$ & $\begin{array}{l}2500 \\
2500 \\
2500\end{array}$ & $\begin{array}{l}35.0 \\
40.0 \\
45.0\end{array}$ & $\begin{array}{l}.00371 \\
.00346 \\
.00332\end{array}$ & $\begin{array}{r}.01484 \\
.01384 \\
.01330\end{array}$ & $\begin{array}{r}.00113 \\
.00105 \\
.00101\end{array}$ & $\begin{array}{l}.00452 \\
.00421 \\
.00405\end{array}$ & $\begin{array}{l}1.611 \\
1.503 \\
1.444\end{array}$ & $\begin{array}{l}4.911 \\
4.581 \\
4.403\end{array}$ & $\begin{array}{l}5.539 \\
5.168 \\
4.966\end{array}$ \\
\hline 21 & 2500 & 46.0 & .00330 & .01320 & .00100 & .00402 & 1.433 & 4.369 & $4 \cdot 9,28$ \\
\hline $\begin{array}{l}21 \\
21\end{array}$ & $\begin{array}{l}2500 \\
2500\end{array}$ & $\begin{array}{l}47.0 \\
48.0\end{array}$ & $\begin{array}{r}.00327 \\
.00324\end{array}$ & $\begin{array}{r}.01310 \\
.01299\end{array}$ & $\begin{array}{l}.00099 \\
.00099\end{array}$ & $\begin{array}{r}.00399 \\
.00396\end{array}$ & $\begin{array}{l}1.422 \\
1.411\end{array}$ & $\begin{array}{l}4.335 \\
4.301\end{array}$ & $\begin{array}{l}4.890 \\
4.852\end{array}$ \\
\hline $\begin{array}{l}21 \\
21 \\
21\end{array}$ & $\begin{array}{l}2500 \\
2500 \\
2500\end{array}$ & $\begin{array}{l}49 \cdot 0 \\
50.0 \\
51.0\end{array}$ & $\begin{array}{l}.00322 \\
.00320 \\
.00318\end{array}$ & $\begin{array}{l}.01291 \\
.01283 \\
.01275\end{array}$ & $\begin{array}{l}.00098 \\
.00097 \\
.00097\end{array}$ & $\begin{array}{l}.00393 \\
.00391 \\
.00388\end{array}$ & $\begin{array}{l}1.402 \\
1.393 \\
1.384\end{array}$ & $\begin{array}{l}4.274 \\
4.247 \\
4.219\end{array}$ & $4 \cdot 759$ \\
\hline $\begin{array}{l}21 \\
21 \\
21\end{array}$ & $\begin{array}{l}2500 \\
2500 \\
2500\end{array}$ & $\begin{array}{l}52.0 \\
53.0 \\
54.0\end{array}$ & $\begin{array}{l}.00316 \\
.00314 \\
.00312\end{array}$ & $\begin{array}{r}.01266 \\
.01258 \\
.01250\end{array}$ & $\begin{array}{l}.00096 \\
.00095 \\
.00095\end{array}$ & $\begin{array}{l}.00386 \\
.00383 \\
.00381\end{array}$ & $\begin{array}{l}1 \cdot 375 \\
1.366 \\
1.357\end{array}$ & $\begin{array}{l}4.192 \\
4 \cdot 165 \\
4.138\end{array}$ & $\begin{array}{l}4.729 \\
4.698 \\
4.668\end{array}$ \\
\hline $\begin{array}{l}21 \\
21 \\
21\end{array}$ & $\begin{array}{l}2500 \\
2500 \\
2500\end{array}$ & $\begin{array}{l}55.0 \\
60.0 \\
65.0\end{array}$ & $\begin{array}{l}.00310 \\
.00301 \\
.00293\end{array}$ & $\begin{array}{r}.01242 \\
.01207 \\
.01172\end{array}$ & $\begin{array}{l}.00094 \\
.00091 \\
.00089\end{array}$ & $\begin{array}{r}.00378 \\
.00367 \\
.00357\end{array}$ & $\begin{array}{l}1.348 \\
1.310 \\
1.273\end{array}$ & $\begin{array}{l}4.111 \\
3.994 \\
3.881\end{array}$ & $\begin{array}{l}4.637 \\
4.506 \\
4.378\end{array}$ \\
\hline $\begin{array}{l}21 \\
21 \\
21\end{array}$ & $\begin{array}{l}2500 \\
2500 \\
2500\end{array}$ & $\begin{array}{l}70.0 \\
75.0 \\
80.0\end{array}$ & $\begin{array}{r}.00285 \\
.00278 \\
.00272\end{array}$ & $\begin{array}{r}.01143 \\
.01114 \\
.01088\end{array}$ & $\begin{array}{r}.00087 \\
.00084 \\
.00082\end{array}$ & $\begin{array}{l}.00348 \\
.00339 \\
.00331\end{array}$ & $\begin{array}{l}1.241 \\
1.209 \\
1.181\end{array}$ & $\begin{array}{l}3.784 \\
3.687 \\
3.600\end{array}$ & $\begin{array}{l}4.269 \\
4.159 \\
4.061\end{array}$ \\
\hline $\begin{array}{l}21 \\
21 \\
21\end{array}$ & $\begin{array}{l}2500 \\
2500 \\
2500\end{array}$ & $\begin{array}{l}85.0 \\
90.0 \\
95.0\end{array}$ & $\begin{array}{l}.00265 \\
.00259 \\
.00255\end{array}$ & $\begin{array}{r}.01062 \\
.01039 \\
.01021\end{array}$ & $\begin{array}{l}.00080 \\
.00079 \\
.00077\end{array}$ & $\begin{array}{l}.00323 \\
.00316 \\
.00311\end{array}$ & $\begin{array}{l}1.153 \\
1.128 \\
1.109\end{array}$ & $\begin{array}{l}3.516 \\
3.440 \\
3.382\end{array}$ & $\begin{array}{l}3.966 \\
3.880 \\
3.814\end{array}$ \\
\hline 21 & 2500 & 00.0 & .00251 & .01004 & .00076 & .00306 & 1.090 & 3.325 & 3.75 \\
\hline
\end{tabular}


TABULATION OF DATA FOR

ABSORPTION OF SOUND IN AIR VERSUS PERCENT RELATIVE HUMIDITY AT 21 DEGREES CENTIGRADE. FOR A FREQUENCY OF 3200 HERTZ

\begin{tabular}{|c|c|c|c|c|c|c|c|c|c|}
\hline$(1)$ & (2) & $(3)$ & $(4)$ & (5) & (6) & $|7|$ & (8) & 191 & $(10)$ \\
\hline TEMP & FREQ & $\begin{array}{l}\text { REL } \\
\text { HUM }\end{array}$ & $\begin{array}{l}\text { ATTEN } \\
\text { COEF }\end{array}$ & $4 M$ & $\begin{array}{l}\text { ATTEN } \\
\text { COEF }\end{array}$ & $4 M$ & $\begin{array}{l}\text { ATTEN } \\
\text { DB PER }\end{array}$ & $\begin{array}{l}\text { ATTEN } \\
\text { DB PER }\end{array}$ & $\begin{array}{l}\text { DECAY } \\
\text { RATE }\end{array}$ \\
\hline $\begin{array}{l}\text { DEGR } \\
\text { CENT }\end{array}$ & HERTZ & $\begin{array}{l}\text { PER } \\
\text { CENT }\end{array}$ & $\begin{array}{l}\text { PER } \\
\text { METER }\end{array}$ & $\begin{array}{l}\text { PER } \\
\text { METER }\end{array}$ & $\begin{array}{l}\text { PER } \\
\text { FO०T }\end{array}$ & $\begin{array}{l}\text { PER } \\
\text { FOOT }\end{array}$ & $\begin{array}{l}100 \\
\text { METER }\end{array}$ & $\begin{array}{l}1000 \\
\text { FEET }\end{array}$ & $\begin{array}{l}\text { DB PER } \\
\text { SECOND }\end{array}$ \\
\hline $\begin{array}{l}21 \\
21 \\
21\end{array}$ & $\begin{array}{l}3200 \\
3200 \\
3200\end{array}$ & $\begin{array}{r}5.0 \\
10.0 \\
15.0\end{array}$ & $\begin{array}{r}.01768 \\
.01912 \\
.01286\end{array}$ & $\begin{array}{l}.07075 \\
.07649 \\
.05145\end{array}$ & $\begin{array}{l}.00539 \\
.00582 \\
.00392\end{array}$ & $\begin{array}{r}.02156 \\
.02331 \\
.01568\end{array}$ & $\begin{array}{l}7.682 \\
8.305 \\
5.587\end{array}$ & $\begin{array}{l}23.415 \\
25.315 \\
17.029\end{array}$ & $\begin{array}{l}26.412 \\
28.554 \\
19.209\end{array}$ \\
\hline $\begin{array}{l}21 \\
21 \\
21\end{array}$ & $\begin{array}{l}3200 \\
3200 \\
3200\end{array}$ & $\begin{array}{l}20.0 \\
25.0 \\
30.0\end{array}$ & $\begin{array}{l}.00953 \\
.00752 \\
.00623\end{array}$ & $\begin{array}{l}.03813 \\
.03008 \\
.02495\end{array}$ & $\begin{array}{l}.00290 \\
.00229 \\
.00190\end{array}$ & $\begin{array}{l}.01162 \\
.00916 \\
.00760\end{array}$ & $\begin{array}{l}4.140 \\
3.266 \\
2.708\end{array}$ & $\begin{array}{r}12.621 \\
9.956 \\
8.257\end{array}$ & $\begin{array}{r}14 \cdot 236 \\
11.230 \\
9.313\end{array}$ \\
\hline $\begin{array}{l}21 \\
21 \\
21\end{array}$ & $\begin{array}{l}3200 \\
3200 \\
3200\end{array}$ & $\begin{array}{l}35.0 \\
40.0 \\
45.0\end{array}$ & $\begin{array}{r}.00543 \\
.00495 \\
.00463\end{array}$ & $\begin{array}{r}.02175 \\
.01980 \\
.01854\end{array}$ & $\begin{array}{r}.00165 \\
.00150 \\
.00141\end{array}$ & $\begin{array}{l}.00662 \\
.00603 \\
.00565\end{array}$ & $\begin{array}{l}2.361 \\
2.149 \\
2: 013\end{array}$ & $\begin{array}{l}7.198 \\
6.552 \\
6.136\end{array}$ & $\begin{array}{l}8.119 \\
7.391 \\
6.921\end{array}$ \\
\hline $\begin{array}{ll}21 \\
21 \\
21\end{array}$ & $\begin{array}{l}3200 \\
3200 \\
3200\end{array}$ & $\begin{array}{l}46.0 \\
47.0 \\
48.0\end{array}$ & $\begin{array}{l}.00458 \\
.00454 \\
.00450\end{array}$ & $\begin{array}{r}.01834 \\
.01816 \\
.01802\end{array}$ & $\begin{array}{l}.00139 \\
.00138 \\
.00137\end{array}$ & $\begin{array}{l}.00559 \\
.00553 \\
.00549\end{array}$ & $\begin{array}{l}1.991 \\
1.972 \\
1.957\end{array}$ & $\begin{array}{l}6.070 \\
6.012 \\
5.965\end{array}$ & $\begin{array}{l}6.847 \\
6.782 \\
6.728\end{array}$ \\
\hline 21 & 3200 & 49.0 & .00447 & .01789 & .00136 & .00545 & 1.943 & 5.923 & 6.681 \\
\hline $\begin{array}{l}21 \\
21\end{array}$ & $\begin{array}{l}3200 \\
3200\end{array}$ & $\begin{array}{l}50.0 \\
51.0\end{array}$ & $\begin{array}{r}.00444 \\
.00441\end{array}$ & $\begin{array}{l}.01777 \\
.01766\end{array}$ & $\begin{array}{l}.00135 \\
.00134\end{array}$ & $\begin{array}{l}.00541 \\
.00538\end{array}$ & $\begin{array}{l}1.929 \\
1.917\end{array}$ & $\begin{array}{l}5.882 \\
5.845\end{array}$ & $\begin{array}{l}6.635 \\
6.594\end{array}$ \\
\hline 21 & 3200 & 52.0 & .00438 & .01755 & .00133 & $.00535^{\circ}$ & 1.906 & 5.809 & 6.553 \\
\hline $\begin{array}{l}21 \\
21\end{array}$ & $\begin{array}{l}3200 \\
3200\end{array}$ & $\begin{array}{l}53.0 \\
54.0\end{array}$ & $\begin{array}{r}.00436 \\
.00433\end{array}$ & $\begin{array}{r}.01744 \\
.01733\end{array}$ & $\begin{array}{l}.00132 \\
.00132\end{array}$ & $\begin{array}{l}.00531 \\
.00528\end{array}$ & $\begin{array}{l}1.894 \\
1.882\end{array}$ & $\begin{array}{l}5.773 \\
5.736\end{array}$ & $\begin{array}{l}6.512 \\
6.471\end{array}$ \\
\hline 21 & 3200 & 55.0 & .00430 & .01722 & .00131 & .00525 & 1.870 & 5.700 & 6.430 \\
\hline $\begin{array}{l}21 \\
21\end{array}$ & $\begin{array}{l}3200 \\
3200\end{array}$ & $\begin{array}{l}60.0 \\
65.0\end{array}$ & $\begin{array}{l}.00418 \\
.00407\end{array}$ & $\begin{array}{l}.01673 \\
.01629\end{array}$ & $\begin{array}{l}.00127 \\
.00124\end{array}$ & $\begin{array}{l}.00510 \\
.00496\end{array}$ & $\begin{array}{l}1.816 \\
1.769\end{array}$ & $\begin{array}{l}5.538 \\
5.392\end{array}$ & $\begin{array}{l}6.246 \\
6.082\end{array}$ \\
\hline 21 & 3200 & 70.0 & .00397 & .01590 & .00121 & .004 & 1. & $5 \cdot 263$ & 5.937 \\
\hline $\begin{array}{l}21 \\
21\end{array}$ & $\begin{array}{l}3200 \\
3200\end{array}$ & $\begin{array}{l}75.0 \\
80.0\end{array}$ & $\begin{array}{r}.00388 \\
.00379\end{array}$ & $\begin{array}{r}.01553 \\
.01519\end{array}$ & $\begin{array}{l}.00118 \\
.00115\end{array}$ & $\begin{array}{l}.004 \\
.004\end{array}$ & 1. & $\begin{array}{l}5.142 \\
5.029\end{array}$ & $\begin{array}{l}5.800 \\
5.673\end{array}$ \\
\hline $\begin{array}{ll}21 \\
21 \\
21\end{array}$ & $\begin{array}{l}3200 \\
3200 \\
3200\end{array}$ & $\begin{array}{l}85.0 \\
90.0 \\
95.0\end{array}$ & $\begin{array}{l}.00372 \\
.00364 \\
.00357\end{array}$ & $\begin{array}{r}.01488 \\
.01456 \\
.01428\end{array}$ & $\begin{array}{r}.00113 \\
.00111 \\
.00108\end{array}$ & $\begin{array}{l}.00453 \\
.00444 \\
.00435\end{array}$ & $\begin{array}{l}1.615 \\
1.581 \\
1.551\end{array}$ & $\begin{array}{l}4.925 \\
4.821 \\
4.729\end{array}$ & $\begin{array}{l}5.555 \\
5.438 \\
5.334\end{array}$ \\
\hline 21 & 3200 & 100.0 & .00350 & .01401 & .00106 & .00427 & 1.521 & 4.638 & $5 \cdot 231$ \\
\hline
\end{tabular}


TABULATION OF DATA FOR

ABSORPTION OF SOUND IN AIR VERSUS PERCENT RELATIVE HUMIDITY AT 21 DEGREES CENTIGRADE FOR A. FREQUENCY OF 4000 HERTZ

\begin{tabular}{|c|c|c|c|c|c|c|c|c|c|}
\hline (1) & (2) & (3.) & (4) & (5) & (6) & $(7)$ & (8) & (9) & $(10)$ \\
\hline $\begin{array}{l}\text { EMP } \\
\text { EGR } \\
\text { ENT }\end{array}$ & HERTZ & $\begin{array}{l}\text { REL } \\
\text { HUM } \\
\text { PER } \\
\text { CENT }\end{array}$ & $\begin{array}{l}\text { ATTEN } \\
\text { COEF } \\
\text { PER } \\
\text { METER }\end{array}$ & $\begin{array}{l}4 M \\
\text { PER } \\
\text { METER }\end{array}$ & $\begin{array}{l}\text { ATTEN } \\
\text { COEF } \\
\text { PER } \\
\text { FOOT }\end{array}$ & $\begin{array}{l}4 M \\
\text { PER } \\
\text { FOOT }\end{array}$ & $\begin{array}{l}\text { AT TEN } \\
\text { DB PER } \\
100 \\
\text { METER }\end{array}$ & $\begin{array}{l}\text { ATTEN } \\
\text { DB PER } \\
1000 \\
\text { FEET }\end{array}$ & $\begin{array}{l}\text { DECAY } \\
\text { RATE } \\
\text { DB PER } \\
\text { SECOND }\end{array}$ \\
\hline $\begin{array}{l}21 \\
21 \\
21\end{array}$ & $\begin{array}{l}4000 \\
4000 \\
4.000\end{array}$ & $\begin{array}{r}5.0 \\
10.0 \\
15.0\end{array}$ & $\begin{array}{r}.01924 \\
.02557 \\
.01909\end{array}$ & $\begin{array}{r}.07696 \\
.10229 \\
.07639\end{array}$ & $\begin{array}{l}.00586 \\
.00779 \\
.00582\end{array}$ & $\begin{array}{l}.02345 \\
.03118 \\
.02328\end{array}$ & $\begin{array}{r}8.356 \\
11.106 \\
8.294\end{array}$ & $\begin{array}{l}25.469 \\
33.854 \\
25.281\end{array}$ & $\begin{array}{l}28.729 \\
38.186 \\
28.516\end{array}$ \\
\hline $\begin{array}{l}21 \\
21 \\
21\end{array}$ & $\begin{array}{l}4000 \\
4000 \\
4000\end{array}$ & $\begin{array}{l}20.0 \\
25.0 \\
30.0\end{array}$ & $\begin{array}{r}.01412 \\
.01110 \\
.00923\end{array}$ & $\begin{array}{r}.05651 \\
.04443 \\
.03694\end{array}$ & $\begin{array}{r}.00430 \\
.00338 \\
.00281\end{array}$ & $\begin{array}{r}.01722 \\
.011354 \\
.01125\end{array}$ & $\begin{array}{l}6.136 \\
4.824 \\
4.010\end{array}$ & $\begin{array}{l}18.704 \\
14.703 \\
12.225\end{array}$ & $\begin{array}{l}21.097 \\
16.585 \\
13.789\end{array}$ \\
\hline $\begin{array}{l}21 \\
21\end{array}$ & $\begin{array}{l}4000 \\
4000\end{array}$ & $\begin{array}{l}35.0 \\
40.0 .\end{array}$ & $\begin{array}{l}.00788 \\
.00702\end{array}$ & $\begin{array}{r}.03155 \\
.02810\end{array}$ & $\begin{array}{l}.00240 \\
.00214\end{array}$ & $\begin{array}{l}.00961 \\
.00856\end{array}$ & $\begin{array}{l}3.425 \\
3.051\end{array}$ & $\begin{array}{r}10.441 \\
9.300\end{array}$ & $\begin{array}{l}11.777 \\
10.490\end{array}$ \\
\hline 21 & 4000 & 45.0 & .00641 & .02566 & .00195 & .00782 & 2.786 & 8.493 & 9.580 \\
\hline $\begin{array}{l}21 \\
21 \\
21\end{array}$ & $\begin{array}{l}4000 \\
4000 \\
4000\end{array}$ & $\begin{array}{l}46.0 \\
47.0 \\
48.0\end{array}$ & $\begin{array}{l}.00634 \\
.00626 \\
.00619\end{array}$ & $\begin{array}{r}.02536 \\
.02507 \\
.02477\end{array}$ & $\begin{array}{l}.00193 \\
.00191 \\
.00188\end{array}$ & $\begin{array}{r}.007773 \\
.00764 \\
.00755\end{array}$ & $\begin{array}{l}2.754 \\
2.722 \\
2.690\end{array}$ & $\begin{array}{l}8.395 \\
8.298 \\
8.200\end{array}$ & $\begin{array}{l}.470 \\
.360 \\
.249\end{array}$ \\
\hline $\begin{array}{l}21 \\
21\end{array}$ & $\begin{array}{l}4000 \\
4000\end{array}$ & $\begin{array}{l}49.0 \\
50.0\end{array}$ & $\begin{array}{r}.00613 \\
.00607\end{array}$ & $\begin{array}{r}.02453 \\
.02429\end{array}$ & $\begin{array}{l}.00186 \\
.00185\end{array}$ & $\begin{array}{l}.00747 \\
.00740\end{array}$ & $\begin{array}{l}2.663 \\
2.637\end{array}$ & $\begin{array}{l}8.119 \\
8.038\end{array}$ & $\begin{array}{l}9.158 \\
9.067\end{array}$ \\
\hline 21 & 4000 & 51.0 & .00599 & .02399 & .00182 & .00731 & 2.605 & 7.940 & 8.957 \\
\hline $\begin{array}{l}21 \\
21 \\
21\end{array}$ & $\begin{array}{l}4000 \\
4000 \\
4000\end{array}$ & $\begin{array}{l}52.0 \\
53.0 \\
54.0\end{array}$ & $\begin{array}{l}.00593 \\
.00587 \\
.00582\end{array}$ & $\begin{array}{l}.02372 \\
.02349 \\
.02330\end{array}$ & $\begin{array}{l}.00180 \\
.00179 \\
.00177\end{array}$ & $\begin{array}{r}.00723 \\
.00716 \\
.00710\end{array}$ & $\begin{array}{l}2.575 \\
2.550 \\
2.530\end{array}$ & $\begin{array}{l}7.850 \\
7.774 \\
7.711\end{array}$ & $\begin{array}{l}8.855 \\
8.769 \\
8.698\end{array}$ \\
\hline $\begin{array}{l}21 \\
21 \\
21\end{array}$ & $\begin{array}{l}4000 \\
4000 \\
4000\end{array}$ & $\begin{array}{l}55.0 \\
60.0 \\
65.0\end{array}$ & $\begin{array}{l}.00577 \\
.00561 \\
.00546\end{array}$ & $\begin{array}{r}.02311 \\
.02246 \\
.02187\end{array}$ & $\begin{array}{l}.00176 \\
.00171 \\
.00166\end{array}$ & $\begin{array}{r}.00704 \\
.00684 \\
.00666\end{array}$ & $\begin{array}{l}2.509 \\
2.439 \\
2.375\end{array}$ & $\begin{array}{l}7.649 \\
7.436 \\
7.240\end{array}$ & $\begin{array}{l}8.628 \\
8.387 \\
8.167\end{array}$ \\
\hline $\begin{array}{l}21 \\
21 \\
21\end{array}$ & $\begin{array}{l}4000 \\
4000 \\
4000\end{array}$ & $\begin{array}{l}70.0 \\
75.0 \\
80.0\end{array}$ & $\begin{array}{l}.00534 \\
.00522 \\
.00511\end{array}$ & $\begin{array}{r}.02136 \\
.02089 \\
.02046\end{array}$ & $\begin{array}{l}.00162 \\
.00159 \\
.00155\end{array}$ & $\begin{array}{l}.00651 \\
.00636 \\
.00623\end{array}$ & $\begin{array}{l}2.319 \\
2.268 \\
2.221\end{array}$ & $\begin{array}{l}7.071 \\
6.914 \\
6.772\end{array}$ & $\begin{array}{l}7.976 \\
7.799 \\
7.638\end{array}$ \\
\hline $\begin{array}{l}21 \\
21 \\
21\end{array}$ & $\begin{array}{l}4000 \\
4000 \\
4000\end{array}$ & $\begin{array}{l}85.0 \\
90.0 \\
95.0\end{array}$ & $\begin{array}{l}.00501 \\
.00491 \\
.00483\end{array}$ & $\begin{array}{r}.02006 \\
.01967 \\
.01933\end{array}$ & $\begin{array}{l}.00152 \\
.00149 \\
.00147\end{array}$ & $\begin{array}{l}.00611 \\
.00599 \\
.00589\end{array}$ & $\begin{array}{l}2.179 \\
2.136 \\
2.099\end{array}$ & $\begin{array}{l}6.641 \\
6.511 \\
6.398\end{array}$ & $\begin{array}{l}7.491 \\
7.344 \\
7.217\end{array}$ \\
\hline & 4 & & .00474 & .01899 & 00144 & .00579 & 2.062 & 6.286 & 7.091 \\
\hline
\end{tabular}


TABULATION OF DATA FOR

ABSORPTION OF SOUND IN AIR VERSUS PERCENT RELATIVE HUMIDITY AT 21 DEGREES CENTIGRADE FOR A FREQUENCY OF 5000 HERTZ

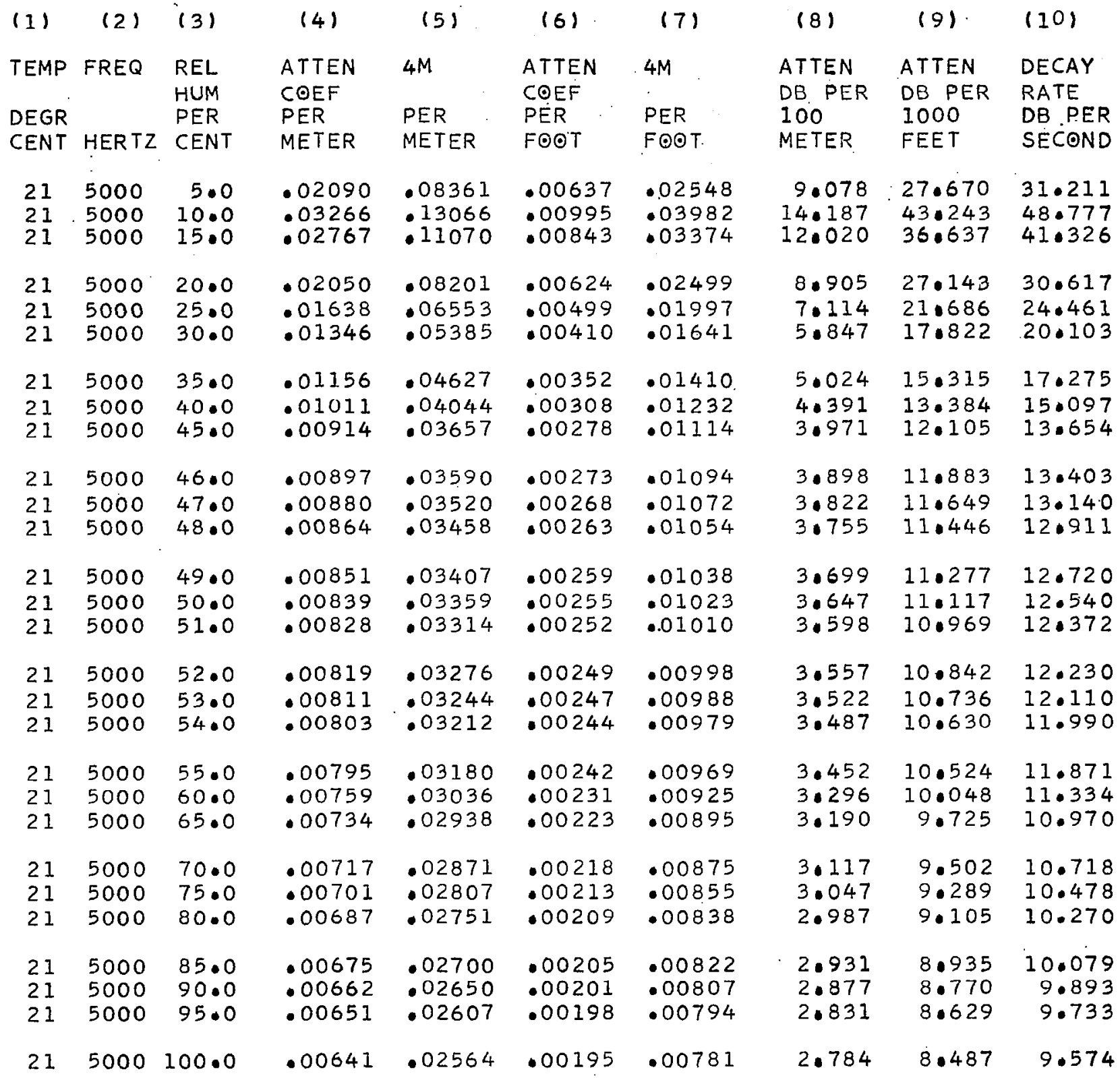


TABULATION OF DATA FOR

ABSORPTION OF SOUND IN AIR VERSUS PERCENT RELATIVE HUMIDITY AT 21 DEGREES CENTIGRADE FOR A FREQUENCY OF 5940 HERTZ

\begin{tabular}{|c|c|c|c|c|c|c|c|c|c|}
\hline (1) & $(2)$ & (3) & (4) & (5) & (6) & 171 & $(8)$ & 191 & $(10)$ \\
\hline TEMP & FREQ & REL & ATTEN & $4 M$ & $\begin{array}{l}\text { ATTEN } \\
\text { COFF }\end{array}$ & $4 M$ & $\begin{array}{l}\text { ATTEN } \\
\text { DR }\end{array}$ & $\begin{array}{l}\text { ATTEN } \\
D R\end{array}$ & DECAY \\
\hline $\begin{array}{l}\text { DEGR } \\
\text { CENT }\end{array}$ & HER TZ & $\begin{array}{l}\text { PER } \\
\text { CENT }\end{array}$ & $\begin{array}{l}\text { PER } \\
\text { METER }\end{array}$ & $\begin{array}{l}\text { PER } \\
\text { METER }\end{array}$ & $\begin{array}{l}\text { PER } \\
\text { FOOT }\end{array}$ & $\begin{array}{l}\text { PER } \\
\text { FOOT }\end{array}$ & $\begin{array}{l}100 \\
\text { METER }\end{array}$ & $\begin{array}{l}1000 \\
\text { FEET }\end{array}$ & $\begin{array}{l}\text { DB PER } \\
\text { SECOND }\end{array}$ \\
\hline $\begin{array}{l}21 \\
21 \\
21\end{array}$ & $\begin{array}{l}5940 \\
5940 \\
5940\end{array}$ & $\begin{array}{r}5.0 \\
10.0 \\
15.0\end{array}$ & $\begin{array}{r}.02238 \\
.03862 \\
.03610\end{array}$ & $\begin{array}{r}08952 \\
.15448 \\
.14441\end{array}$ & $\begin{array}{r}.00682 \\
.01177 \\
.01100\end{array}$ & $\begin{array}{l}.02728 \\
.04708 \\
.04401\end{array}$ & $\begin{array}{r}9.720 \\
16.773 \\
15.680\end{array}$ & $\begin{array}{l}29.628 \\
51.126 \\
47.793\end{array}$ & $\begin{array}{l}33.419 \\
57.668 \\
53.909\end{array}$ \\
\hline $\begin{array}{l}21 \\
21 \\
21\end{array}$ & $\begin{array}{l}5940 \\
5940 \\
5940\end{array}$ & $\begin{array}{l}20.0 \\
25.0 \\
30.0\end{array}$ & $\begin{array}{l}.02768 \\
.02207 \\
.01830\end{array}$ & $\begin{array}{l}.11075 \\
.08830 \\
.07321\end{array}$ & $\begin{array}{l}.00843 \\
.00672 \\
.00557\end{array}$ & $\begin{array}{l}.03375 \\
.02691 \\
.02231\end{array}$ & $\begin{array}{r}12.025 \\
9.587 \\
7.949\end{array}$ & $\begin{array}{l}36.653 \\
29.223 \\
24.230\end{array}$ & $\begin{array}{l}41.344 \\
32.962 \\
27.330\end{array}$ \\
\hline $\begin{array}{l}21 \\
21 \\
21\end{array}$ & $\begin{array}{l}5940 \\
5940 \\
5940\end{array}$ & $\begin{array}{l}35.0 \\
40.0 \\
45.0\end{array}$ & $\begin{array}{r}.01557 \\
.01367 \\
.01218\end{array}$ & $\begin{array}{l}.06229 \\
.05468 \\
.04873\end{array}$ & $\begin{array}{l}.00474 \\
.00416 \\
.00371\end{array}$ & $\begin{array}{r}.01898 \\
.01666 \\
.01485\end{array}$ & $\begin{array}{l}6.763 \\
5.937 \\
5.291\end{array}$ & $\begin{array}{l}20.616 \\
18.097 \\
16.129\end{array}$ & $\begin{array}{l}23.254 \\
20.413 \\
18.193\end{array}$ \\
\hline $\begin{array}{l}21 \\
21\end{array}$ & $\begin{array}{l}5940 \\
5940\end{array}$ & $\begin{array}{l}46.0 \\
47.0\end{array}$ & $\begin{array}{r}.01194 \\
.01172\end{array}$ & $\begin{array}{l}.04778 \\
.04688\end{array}$ & $\begin{array}{r}.00364 \\
.00357\end{array}$ & $\begin{array}{r}.01456 \\
.01429\end{array}$ & $\begin{array}{l}5.188 \\
5.090\end{array}$ & $\begin{array}{l}15.814 \\
15.516\end{array}$ & $\begin{array}{l}17.837 \\
17.502\end{array}$ \\
\hline 21 & 5940 & 48.0 & .01151 & .04607 & .00351 & .01404 & 5.002 & $15 \cdot 246$ & $17 \cdot 197$ \\
\hline $\begin{array}{l}21 \\
21 \\
21\end{array}$ & $\begin{array}{l}5940 \\
5940 \\
5940\end{array}$ & $\begin{array}{l}49.0 \\
50.0 \\
51.0\end{array}$ & $\begin{array}{r}.01131 \\
.01114 \\
.01097\end{array}$ & $\begin{array}{r}.04527 \\
.04459 \\
.04391\end{array}$ & $\begin{array}{l}.00345 \\
.00339 \\
.00334\end{array}$ & $\begin{array}{l}.01380 \\
.01359 \\
.01338\end{array}$ & $\begin{array}{l}4.915 \\
4.842 \\
4.768\end{array}$ & $\begin{array}{l}14.983 \\
14.758 \\
14.533\end{array}$ & $\begin{array}{l}16.901 \\
16.647 \\
16.393\end{array}$ \\
\hline $\begin{array}{l}21 \\
21 \\
21\end{array}$ & $\begin{array}{l}5940 \\
5940 \\
5940\end{array}$ & $\begin{array}{l}52.0 \\
53.0 \\
54.0\end{array}$ & $\begin{array}{l}.01079 \\
.01060 \\
.01045\end{array}$ & $\begin{array}{l}.04317 \\
.04242 \\
.04182\end{array}$ & $\begin{array}{l}.00328 \\
.00323 \\
.00318\end{array}$ & $\begin{array}{l}.01315 \\
.01293 \\
.01274\end{array}$ & $\begin{array}{l}4.687 \\
4.606 \\
4.541\end{array}$ & $\begin{array}{l}14.287 \\
14.040 \\
13.841\end{array}$ & $\begin{array}{l}16.116 \\
15.837 \\
15.612\end{array}$ \\
\hline $\begin{array}{l}21 \\
21 \\
21\end{array}$ & $\begin{array}{l}5940 \\
5940 \\
5940\end{array}$ & $\begin{array}{l}55.0 \\
60.0 \\
65.0\end{array}$ & $\begin{array}{r}.01032 \\
.00979 \\
.00940\end{array}$ & $\begin{array}{l}.04728 \\
.03917 \\
.03763\end{array}$ & $\begin{array}{l}.00314 \\
.00298 \\
.00286\end{array}$ & $\begin{array}{r}.01258 \\
.01194 \\
.01146\end{array}$ & $\begin{array}{l}4.482 \\
4.253 \\
4.085\end{array}$ & $\begin{array}{l}13.661 \\
12.965 \\
12.453\end{array}$ & $\begin{array}{l}15.410 \\
14.625 \\
14.047\end{array}$ \\
\hline $\begin{array}{l}21 \\
21 \\
21\end{array}$ & $\begin{array}{l}5940 \\
5940 \\
5940\end{array}$ & $\begin{array}{l}70.0 \\
75.0 \\
80.0\end{array}$ & $\begin{array}{l}.00906 \\
.00885 \\
.00868\end{array}$ & $\begin{array}{l}.03625 \\
.03541 \\
.03473\end{array}$ & $\begin{array}{r}.00276 \\
.00269 \\
.00264\end{array}$ & $\begin{array}{r}.01105 \\
.01079 \\
.01058\end{array}$ & $\begin{array}{l}3.936 \\
3.845 \\
3.771\end{array}$ & $\begin{array}{l}11.9 .98 \\
11.720 \\
11.496\end{array}$ & $\begin{array}{l}13.534 \\
13.220 \\
12.967\end{array}$ \\
\hline $\begin{array}{l}21 \\
21 \\
21\end{array}$ & $\begin{array}{l}5940 \\
5940 \\
5940\end{array}$ & $\begin{array}{l}85.0 \\
90.0 \\
95.0\end{array}$ & $\begin{array}{l}.00851 \\
.00837 \\
.00823\end{array}$ & $\begin{array}{r}.03405 \\
.03348 \\
.03294\end{array}$ & $\begin{array}{l}.00259 \\
.00255 \\
.00251\end{array}$ & $\begin{array}{l}.01038 \\
.01020 \\
.01004\end{array}$ & $\begin{array}{l}3.697 \\
3.635 \\
3.576\end{array}$ & $\begin{array}{l}11.271 \\
11.081 \\
10.902\end{array}$ & $\begin{array}{l}12.713 \\
12.500 \\
12.297\end{array}$ \\
\hline 2 & 5940 & 100.0 & .00810 & .03240 & .00246 & .00987 & 3.517 & 10.722 & 12.094 \\
\hline
\end{tabular}


TABULATIOM OF DATA FOR

ABSERPTIEN OF SOUND IN AIR VERSUS PERCENT RELATIVE HUMIOITY AT 21 DEGREES CENTIGRAOE FOR A FREQUENCY OF 6300 HERTZ

\begin{tabular}{|c|c|c|c|c|c|c|c|c|c|}
\hline 11 & $(2)$ & (3) & $(4)$ & (5) & $(6)$ & (7) & $(8)$ & $(9)$ & $(10)$ \\
\hline $\begin{array}{l}\text { TEMP } \\
\text { DEGR } \\
\text { CENT }\end{array}$ & HERTZ & $\begin{array}{l}\text { REL } \\
\text { HUM } \\
\text { PER } \\
\text { CENT }\end{array}$ & $\begin{array}{l}\text { ATTEN } \\
\text { CEEF } \\
\text { PER } \\
\text { METER }\end{array}$ & $\begin{array}{l}\text { 4M } \\
\text { PER } \\
\text { METER }\end{array}$ & $\begin{array}{l}\text { ATTEN } \\
\text { COEF } \\
\text { PER } \\
\text { FOOT }\end{array}$ & $\begin{array}{l}4 M \\
\text { PER } \\
\text { FOOT }\end{array}$ & $\begin{array}{l}\text { ATTEN } \\
\text { DB PER } \\
100 \\
\text { METER }\end{array}$ & $\begin{array}{l}\text { ATTEN } \\
\text { DB PER } \\
1000 \\
\text { FEET }\end{array}$ & $\begin{array}{l}\text { DECAY } \\
\text { RATE } \\
\text { DB PER } \\
\text { SECONO }\end{array}$ \\
\hline $\begin{array}{l}21 \\
21 \\
21\end{array}$ & $\begin{array}{l}6300 \\
6300 \\
6300\end{array}$ & $\begin{array}{r}5.0 \\
10.0 \\
15.0\end{array}$ & $\begin{array}{l}.02304 \\
.04070 \\
.03913\end{array}$ & $\begin{array}{r}.09217 \\
.16280 \\
.15652\end{array}$ & $\begin{array}{l}.00702 \\
.01240 \\
.01192\end{array}$ & $\begin{array}{l}.02809 \\
.04962 \\
.04770\end{array}$ & $\begin{array}{l}10.007 \\
17.676 \\
16.994\end{array}$ & $\begin{array}{l}30.504 \\
53.877 \\
51.800\end{array}$ & $\begin{array}{l}34.408 \\
60.771 \\
58 \cdot 429\end{array}$ \\
\hline $\begin{array}{l}21 \\
21 \\
21\end{array}$ & $\begin{array}{l}6300 \\
6300 \\
6300\end{array}$ & $\begin{array}{l}20.0 \\
25.0 \\
30.0\end{array}$ & $\begin{array}{l}.03072 \\
.02429 \\
.02024\end{array}$ & $\begin{array}{l}.22288 \\
.09716 \\
.08099\end{array}$ & $\begin{array}{l}.00936 \\
.00740 \\
.00617\end{array}$ & $\begin{array}{l}.03745 \\
.02961 \\
.02468\end{array}$ & $\begin{array}{r}13.342 \\
10.549 \\
8.794\end{array}$ & $\begin{array}{l}40.667 \\
32.155 \\
26.804\end{array}$ & $\begin{array}{l}45.872 \\
36.270 \\
30.234\end{array}$ \\
\hline $\begin{array}{l}21 \\
21 \\
21\end{array}$ & $\begin{array}{l}6300 \\
6300 \\
6300\end{array}$ & $\begin{array}{l}35.0 \\
40.0 \\
45.0\end{array}$ & $\begin{array}{r}.01719 \\
.01512 \\
.01343\end{array}$ & $\begin{array}{l}.06877 \\
.06049 \\
.05374\end{array}$ & $\begin{array}{l}.00524 \\
.00460 \\
.00409\end{array}$ & $\begin{array}{l}.02096 \\
.01843 \\
.01638\end{array}$ & $\begin{array}{l}7.467 \\
6.568 \\
5.835\end{array}$ & $\begin{array}{l}22.761 \\
20.021 \\
17.785\end{array}$ & $\begin{array}{l}25.674 \\
22.583 \\
20.061\end{array}$ \\
\hline $\begin{array}{l}21 \\
21 \\
21\end{array}$ & $\begin{array}{l}6300 \\
6300 \\
6300\end{array}$ & $\begin{array}{l}46.9 \\
47.0 \\
48.0\end{array}$ & $\begin{array}{r}.01315 \\
.01291 \\
.01267\end{array}$ & $\begin{array}{l}.05262 \\
.05165 \\
.05068\end{array}$ & $\begin{array}{l}.00401 \\
.00393 \\
.003 .86\end{array}$ & $\begin{array}{l}.01 \\
.01 \\
.0\end{array}$ & $\begin{array}{l}14 \\
08 \\
02\end{array}$ & $\begin{array}{l}17 \cdot 417 \\
17 \cdot 095 \\
16 \cdot 772\end{array}$ & $\begin{array}{l}546 \\
282 \\
919\end{array}$ \\
\hline $\begin{array}{l}21 \\
21 \\
21\end{array}$ & $\begin{array}{l}6300 \\
6300 \\
6300\end{array}$ & $\begin{array}{l}49 \cdot 0 \\
50 \cdot 0 \\
51.0\end{array}$ & $\begin{array}{r}.01245 \\
.01224 \\
.01204\end{array}$ & $\begin{array}{r}.04980 \\
.04897 \\
.04819\end{array}$ & $\begin{array}{l}.00379 \\
.00373 \\
.00367\end{array}$ & $\begin{array}{l}.01518 \\
.01492 \\
.01468\end{array}$ & $\begin{array}{l}5.407 \\
5.316 \\
5 \cdot 232\end{array}$ & $\begin{array}{l}16.482 \\
16.206 \\
15.948\end{array}$ & $\begin{array}{l}8.591 \\
8.280 \\
7.989\end{array}$ \\
\hline $\begin{array}{l}21 \\
21 \\
21\end{array}$ & $\begin{array}{l}6300 \\
6300 \\
6300\end{array}$ & $\begin{array}{l}52 \cdot 0 \\
53 \cdot 0 \\
54.0\end{array}$ & $\begin{array}{r}.01167 \\
.01169 \\
.01150\end{array}$ & $\begin{array}{l}.04749 \\
.04679 \\
.04602\end{array}$ & $\begin{array}{l}.00361 \\
.00356 \\
.00350\end{array}$ & $\begin{array}{l}.01447 \\
.01426 \\
.01402\end{array}$ & $\begin{array}{l}5.156 \\
5.080 \\
4.997\end{array}$ & $\begin{array}{l}15 \cdot 718 \\
15 \cdot 485 \\
15 \cdot 232\end{array}$ & $\begin{array}{l}17.729 \\
17.467 \\
17.181\end{array}$ \\
\hline $\begin{array}{l}21 \\
21 \\
21\end{array}$ & $\begin{array}{l}6300 \\
6300 \\
6300\end{array}$ & $\begin{array}{l}55.0 \\
60.0 \\
65.0\end{array}$ & $\begin{array}{r}.01131 \\
.01065 \\
.01023\end{array}$ & $\begin{array}{l}.04526 \\
.04263 \\
.04094\end{array}$ & $\begin{array}{l}.00344 \\
.00324 \\
.00311\end{array}$ & $\begin{array}{l}.01379 \\
.01299 \\
.01247\end{array}$ & $\begin{array}{l}4.914 \\
4.629 \\
4.445\end{array}$ & $\begin{array}{l}979 \\
110 \\
549\end{array}$ & $\begin{array}{l}16.895 \\
15.918 \\
15.283\end{array}$ \\
\hline $\begin{array}{l}21 . \\
21 \\
21\end{array}$ & $\begin{array}{l}6300 \\
6300 \\
6300\end{array}$ & $\begin{array}{l}70 \cdot 0 \\
75.0 \\
80 \cdot 0\end{array}$ & $\begin{array}{l}.00984 \\
.00958 \\
.00939\end{array}$ & $\begin{array}{l}.03938 \\
.03832 \\
.03757\end{array}$ & $\begin{array}{l}.00300 \\
.00292 \\
.00286\end{array}$ & $\begin{array}{l}.01200 \\
.01168 \\
.01145\end{array}$ & $\begin{array}{l}4.276 \\
4.161 \\
4.079\end{array}$ & $\begin{array}{l}13.035 \\
12.683 \\
12.435\end{array}$ & $\begin{array}{l}14 \cdot 703 \\
14.306 \\
14.027\end{array}$ \\
\hline $\begin{array}{l}21 \\
21 \\
21\end{array}$ & $\begin{array}{l}6300 \\
6300 \\
6300\end{array}$ & $\begin{array}{l}85.0 \\
90.0 \\
95.0\end{array}$ & $\begin{array}{l}.00922 \\
.00905 \\
.00892\end{array}$ & $\begin{array}{l}.03688 \\
.03621 \\
.03565\end{array}$ & $\begin{array}{l}.00281 \\
.00275 \\
.00271\end{array}$ & $\begin{array}{l}.01124 \\
.01103 \\
.01086\end{array}$ & $\begin{array}{l}4.0 \\
3.9 \\
3.8\end{array}$ & $\begin{array}{l}05 \\
83 \\
99\end{array}$ & $\begin{array}{l}13.767 \\
13.517 \\
13.309\end{array}$ \\
\hline 21 & 6300 & 100.0 & .00877 & .03509 & .00267 & .01069 & 3.810 & 11.615 & 13.101 \\
\hline
\end{tabular}


TABULATION OF DATA FOR

ABSORPTION OF SOUND IN AIR. VERSUS PERCENT RELATIVE HUMIDITY AT 21. DEGREES CENTIGRADE FOR A FREQUENCY OF 8000 HERTZ

\begin{tabular}{|c|c|c|c|c|c|c|c|c|c|}
\hline (1) & (2) & (3) & (4) & (5) & $(6)$ & (7) & 181 & $(9)$ & $(1.0)$ \\
\hline EMP & FREQ & $\begin{array}{l}\text { REL } \\
\text { HUM. }\end{array}$ & $\begin{array}{l}\text { ATTEN } \\
\text { COEF }\end{array}$ & $4 M$ & $\begin{array}{l}\text { ATTEN } \\
\text { COEF }\end{array}$ & $4 M$ & $\begin{array}{l}A T T E N \\
D B \text { PER }\end{array}$ & $\begin{array}{l}A T T E N \\
D B \text { PER }\end{array}$ & $\begin{array}{l}\text { DECAY } \\
\text { RATE }\end{array}$ \\
\hline $\begin{array}{l}E G R \\
\text { ENT }\end{array}$ & HERTZ & $\begin{array}{l}\text { PER } \\
\text { CENT }\end{array}$ & $\begin{array}{l}\text { PER } \\
\text { METER }\end{array}$ & $\begin{array}{l}\text { PER } \\
\text { METER }\end{array}$ & $\begin{array}{l}\text { PER } \\
\text { FOOT }\end{array}$ & $\begin{array}{l}\text { PER } \\
\text { FO०T }\end{array}$ & $\begin{array}{l}100 \\
\text { METER }\end{array}$ & $\begin{array}{l}1000 \\
\text { FEET }\end{array}$ & $\begin{array}{l}\text { DB PER } \\
\text { SECOND }\end{array}$ \\
\hline $\begin{array}{l}21 \\
21 \\
21\end{array}$ & $\begin{array}{l}8000 \\
8000 \\
8000\end{array}$ & $\begin{array}{r}5.0 \\
10.0 \\
15.0\end{array}$ & $\begin{array}{l}.02587 \\
.04895 \\
.05271\end{array}$ & $\begin{array}{r}.10351 \\
.19581 \\
.21084\end{array}$ & $\begin{array}{r}.00788 \\
.01492 \\
.01606\end{array}$ & $\begin{array}{r}.03155 \\
.05968 \\
.06426\end{array}$ & $\begin{array}{l}11.239 \\
21.260 \\
22.892\end{array}$ & $\begin{array}{l}34.256 \\
64.804 \\
69.777\end{array}$ & $\begin{array}{r}38.640 \\
.73 .097 \\
78.707\end{array}$ \\
\hline $\begin{array}{l}21 \\
21 \\
21\end{array}$ & $\begin{array}{l}8000 \\
8000 \\
8000\end{array}$ & $\begin{array}{l}20.0 \\
25.0 \\
30.0\end{array}$ & $\begin{array}{r}.04574 \\
.03659 \\
.03050\end{array}$ & $\begin{array}{r}.18297 \\
.14639 \\
.12200\end{array}$ & $\begin{array}{l}.01394 \\
.01115 \\
.00929\end{array}$ & $\begin{array}{r}.05577 \\
.04462 \\
.03718\end{array}$ & $\begin{array}{l}19.866 \\
15.895 \\
13.246\end{array}$ & $\begin{array}{l}60.553 \\
48.449 \\
40.376\end{array}$ & $\begin{array}{l}68.302 \\
54.649 \\
45.543\end{array}$ \\
\hline $\begin{array}{l}21 \\
21 \\
21\end{array}$ & $\begin{array}{l}8000 \\
8000 \\
8000\end{array}$ & $\begin{array}{l}35.0 \\
40.0 \\
45.0\end{array}$ & $\begin{array}{r}.02617 \\
.02276 \\
.02033\end{array}$ & $\begin{array}{r}.10471 \\
.09105 \\
.08134\end{array}$ & $\begin{array}{l}.00797 \\
.00693 \\
.00619\end{array}$ & $\begin{array}{r}.03191 \\
.02775 \\
.02479\end{array}$ & $\begin{array}{r}11.369 \\
9.886 \\
8.832\end{array}$ & $\begin{array}{l}34.655 \\
30.134 \\
26.921\end{array}$ & $\begin{array}{l}39.090 \\
33.991 \\
30.366\end{array}$ \\
\hline $\begin{array}{l}21 \\
21 \\
21\end{array}$ & $\begin{array}{l}8000 \\
8000 \\
8000\end{array}$ & $\begin{array}{l}46.0 \\
47.0 \\
48.0\end{array}$ & $\begin{array}{r}.01993 \\
.01955 \\
.01913\end{array}$ & $\begin{array}{r}.07974 \\
.07821 \\
.07654\end{array}$ & $\begin{array}{l}.00607 \\
.00595 \\
.00583\end{array}$ & $\begin{array}{l}.02430 \\
.02383 \\
.02333\end{array}$ & & $\begin{array}{l}26.391 \\
25.884 \\
25.332\end{array}$ & $\begin{array}{l}768 \\
196 \\
573\end{array}$ \\
\hline $\begin{array}{l}21 \\
21 \\
21\end{array}$ & $\begin{array}{l}8000 \\
8000 \\
8000\end{array}$ & $\begin{array}{l}49.0 \\
50.0 \\
51.0\end{array}$ & $\begin{array}{r}.01874 \\
.01838 \\
.01804\end{array}$ & $\begin{array}{r}.07499 \\
.07354 \\
.07217\end{array}$ & $\begin{array}{r}.00571 \\
.00560 \\
.00549\end{array}$ & $\begin{array}{l}.02285 \\
.02241 \\
.02199\end{array}$ & $\begin{array}{l}8.142 \\
7.984 \\
7.836\end{array}$ & $\begin{array}{l}24.818 \\
24.337 \\
23.885\end{array}$ & $\begin{array}{l}27.994 \\
27.452 \\
26.942\end{array}$ \\
\hline $\begin{array}{l}21 \\
21 \\
21\end{array}$ & $\begin{array}{l}8000 \\
8000 \\
8000\end{array}$ & $\begin{array}{l}52.0 \\
53.0 \\
54.0\end{array}$ & $\begin{array}{r}.01770 \\
.01738 \\
.01710\end{array}$ & $\begin{array}{r}.07081 \\
.06955 \\
.06840\end{array}$ & $\begin{array}{l}.00539 \\
.00530 \\
.00521\end{array}$ & $\begin{array}{r}.02158 \\
.02120 \\
.02085\end{array}$ & $\begin{array}{l}7.688 \\
7.552 \\
7.427\end{array}$ & $\begin{array}{l}23.434 \\
23.019 \\
22.638\end{array}$ & $\begin{array}{l}26.433 \\
25.965 \\
25.535\end{array}$ \\
\hline $\begin{array}{l}21 \\
21 \\
21\end{array}$ & $\begin{array}{l}8000 \\
8000 \\
8000\end{array}$ & $\begin{array}{l}55.0 \\
60.0 \\
65.0\end{array}$ & $\begin{array}{r}.01683 \\
.01568 \\
.01471\end{array}$ & $\begin{array}{l}.06734 \\
.06275 \\
.05886\end{array}$ & $\begin{array}{l}.00513 \\
.00478 \\
.00448\end{array}$ & $\begin{array}{r}.02052 \\
.01912 \\
.01794\end{array}$ & $\begin{array}{l}7.311 \\
6.814 \\
6.391\end{array}$ & $\begin{array}{l}22.286 \\
20.769 \\
19.481\end{array}$ & $\begin{array}{l}25.139 \\
23.427 \\
21.974\end{array}$ \\
\hline $\begin{array}{l}21 \\
21 \\
21\end{array}$ & $\begin{array}{l}8000 \\
8000 \\
8000\end{array}$ & $\begin{array}{l}70.0 \\
75.0 \\
80.0\end{array}$ & $\begin{array}{r}.01402 \\
.01355 \\
.01313\end{array}$ & $\begin{array}{r}.05611 \\
.05421 \\
.05252\end{array}$ & $\begin{array}{r}.00427 \\
.00413 \\
.00400\end{array}$ & $\begin{array}{r}.01710 \\
.01652 \\
.01600\end{array}$ & $\begin{array}{l}6.092 \\
5.886 \\
5.702\end{array}$ & $\begin{array}{l}18.569 \\
17.942 \\
17.381\end{array}$ & $\begin{array}{l}20.945 \\
20.238 \\
19.606\end{array}$ \\
\hline $\begin{array}{l}21 \\
21\end{array}$ & $\begin{array}{l}8000 \\
8000 \\
8000\end{array}$ & $\begin{array}{l}85.0 \\
90.0 \\
95.0\end{array}$ & $\begin{array}{r}.01278 \\
.01255 \\
.01236\end{array}$ & $\begin{array}{l}.05113 \\
.05021 \\
.04944\end{array}$ & $\begin{array}{l}.00389 \\
.00382 \\
.00376\end{array}$ & $\begin{array}{r}.01558 \\
.01530 \\
.01507\end{array}$ & $\begin{array}{l}5.551 \\
5.451 \\
5.368\end{array}$ & $\begin{array}{l}16.922 \\
16.617 \\
16.363\end{array}$ & $\begin{array}{l}19.087 \\
18.744 \\
18.457\end{array}$ \\
\hline & 8 & & 01217 & .04868 & 00371 & .01484 & 286 & 112 & .174 \\
\hline
\end{tabular}


TABULATION OF DATA FOR

ABSORPTION OF SOUND IN AIR VERSUS PERCENT RELATIVE HUMIDITY AT $2 I$ DEGREES CENTIGRADE FOR A FREQUENCY OF 10000 HERTZ

\begin{tabular}{|c|c|c|c|c|c|c|c|c|c|}
\hline (1) & (2) & (3) & (4) & (5) & $(6)$ & 171 & (8) & $(9)$ & $(10)$ \\
\hline $\begin{array}{l}\text { TEMP } \\
\text { DEGR } \\
\text { CENT }\end{array}$ & HERTZ & $\begin{array}{l}\text { REL } \\
\text { HUM } \\
\text { PER } \\
\text { CENT }\end{array}$ & $\begin{array}{l}\text { ATTEN } \\
\text { COEF } \\
\text { PER } \\
\text { METER }\end{array}$ & $\begin{array}{l}4 M \\
\text { PER } \\
\text { METER }\end{array}$ & $\begin{array}{l}\text { ATTEN } \\
\text { COEF } \\
\text { PER } \\
\text { FOOT }\end{array}$ & $\begin{array}{l}4 M \\
\text { PER } \\
\text { FOOT }\end{array}$ & $\begin{array}{l}\text { ATTEN } \\
\text { DB PER } \\
100 \\
\text { METER }\end{array}$ & $\begin{array}{l}\text { ATTEN } \\
\text { DB PER } \\
1000 \\
\text { FEET }\end{array}$ & $\begin{array}{l}\text { DECAY } \\
\text { RATE } \\
\text { DB PER } \\
\text { SECOND }\end{array}$ \\
\hline $\begin{array}{l}21 \\
21 \\
21\end{array}$ & $\begin{array}{l}10000 \\
10000 \\
10000\end{array}$ & $\begin{array}{r}5.0 \\
10.0 \\
15.0\end{array}$ & $\begin{array}{r}.02928 \\
.05592 \\
.06708\end{array}$ & $\begin{array}{r}.11713 \\
.22371 \\
.26835\end{array}$ & $\begin{array}{r}.00892 \\
.01704 \\
.02044\end{array}$ & $\begin{array}{l}.03570 \\
.06818 \\
.08179\end{array}$ & $\begin{array}{l}12.718 \\
24.289 \\
29.136\end{array}$ & $\begin{array}{l}38.766 \\
74.035 \\
88.808\end{array}$ & $\begin{array}{r}43.727 \\
83.509 \\
100.173\end{array}$ \\
\hline $\begin{array}{l}21 \\
21 \\
21\end{array}$ & $\begin{array}{l}10000 \\
10000 \\
10000\end{array}$ & $\begin{array}{l}20.0 \\
25.0 \\
30.0\end{array}$ & $\begin{array}{l}.06362 \\
.05379 \\
.04450\end{array}$ & $\begin{array}{r}.25451 \\
.21517 \\
.17800\end{array}$ & $\begin{array}{l}.01939 \\
.01639 \\
.01356\end{array}$ & $\begin{array}{l}.07757 \\
.06558 \\
.05425\end{array}$ & $\begin{array}{l}27.633 \\
23.362 \\
19.326\end{array}$ & $\begin{array}{l}84.229 \\
71.208 \\
58.908\end{array}$ & $\begin{array}{l}95.008 \\
80.321 \\
66.447\end{array}$ \\
\hline $\begin{array}{l}21 \\
21 \\
21\end{array}$ & $\begin{array}{l}10000 \\
10000 \\
10000\end{array}$ & $\begin{array}{l}35.0 \\
40.0 \\
45.0\end{array}$ & $\begin{array}{r}.03836 \\
.03366 \\
.02984\end{array}$ & $\begin{array}{r}.15344 \\
.13465 \\
.11939\end{array}$ & $\begin{array}{l}.01 \\
.01 \\
.00\end{array}$ & $\begin{array}{r}.04677 \\
.04104 \\
.03639\end{array}$ & $\begin{array}{l}16.660 \\
14.619 \\
12.963\end{array}$ & $\begin{array}{l}50.782 \\
44.562 \\
39.511\end{array}$ & $\begin{array}{l}57.281 \\
50.264 \\
44.568\end{array}$ \\
\hline $\begin{array}{l}21 \\
21 \\
21\end{array}$ & $\begin{array}{l}10000 \\
10000 \\
10000\end{array}$ & $\begin{array}{l}46.0 \\
47.0 \\
48.0\end{array}$ & $\begin{array}{l}.02918 \\
.02859 \\
.02802\end{array}$ & $\begin{array}{r}.11675 \\
.11437 \\
.11211\end{array}$ & $\begin{array}{l}.00889 \\
.00871 \\
.00854\end{array}$ & $\begin{array}{l}.03558 \\
.03486 \\
.03417\end{array}$ & $\begin{array}{l}12.676 \\
12.418 \\
12.172\end{array}$ & $\begin{array}{l}38.638 \\
37.852 \\
37.102\end{array}$ & $\begin{array}{l}43.583 \\
42.696 \\
41.850\end{array}$ \\
\hline $\begin{array}{l}21 \\
21 \\
21\end{array}$ & $\begin{array}{l}10000 \\
10000 \\
10000\end{array}$ & $\begin{array}{l}49.0 \\
50.0 \\
51.0\end{array}$ & $\begin{array}{r}.02749 \\
.02698 \\
.02649\end{array}$ & $\begin{array}{r}.10996 \\
.10794 \\
.10599\end{array}$ & $\begin{array}{l}.00837 \\
.00822 \\
.00807\end{array}$ & $\begin{array}{l}.03351 \\
.03290 \\
.03230\end{array}$ & $\begin{array}{l}11.939 \\
11.719 \\
11.508\end{array}$ & $\begin{array}{l}36.393 \\
35.722 \\
35.077\end{array}$ & $\begin{array}{l}41.050 \\
40.294 \\
39.566\end{array}$ \\
\hline $\begin{array}{l}21 \\
21 \\
21\end{array}$ & $\begin{array}{l}10000 \\
10000 \\
10000\end{array}$ & $\begin{array}{l}52.0 \\
53.0 \\
54.0\end{array}$ & $\begin{array}{r}.02604 \\
.02561 \\
.02520\end{array}$ & $\begin{array}{r}.10417 \\
.10247 \\
.10080\end{array}$ & $\begin{array}{l}.00793 \\
.00780 \\
.00768\end{array}$ & $\begin{array}{l}.03175 \\
.03123 \\
.03072\end{array}$ & $\begin{array}{l}11.311 \\
11.125 \\
10.944\end{array}$ & $\begin{array}{l}34.477 \\
33.911 \\
33.360\end{array}$ & $\begin{array}{l}38.889 \\
38.251 \\
37.629\end{array}$ \\
\hline $\begin{array}{l}21 \\
21 \\
21\end{array}$ & $\begin{array}{l}10000 \\
10000 \\
10000\end{array}$ & $\begin{array}{l}55.0 \\
60.0 \\
65.0\end{array}$ & $\begin{array}{l}.02474 \\
.02279 \\
.02129\end{array}$ & $\begin{array}{r}.09899 \\
.09116 \\
.08517\end{array}$ & $\begin{array}{l}.00754 \\
.00694 \\
.00649\end{array}$ & $\begin{array}{r}.03017 \\
.02778 \\
.02596\end{array}$ & $\begin{array}{r}10.747 \\
9.898 \\
9.247\end{array}$ & $\begin{array}{l}32.759 \\
30.171 \\
28.188\end{array}$ & $\begin{array}{l}36.952 \\
34.032 \\
31.795\end{array}$ \\
\hline $\begin{array}{l}21 \\
21 \\
21\end{array}$ & $\begin{array}{l}10000 \\
10000 \\
10000\end{array}$ & $\begin{array}{l}70.0 \\
75.0 \\
80.0\end{array}$ & $\begin{array}{r}.02014 \\
.01909 \\
.01833\end{array}$ & $\begin{array}{r}.08059 \\
.07639 \\
.07334\end{array}$ & $\begin{array}{l}.00614 \\
.00582 \\
.00558\end{array}$ & $\begin{array}{l}.02456 \\
.02328 \\
.02235\end{array}$ & $\begin{array}{l}8.750 \\
8.294 \\
7.963\end{array}$ & $\begin{array}{l}26.672 \\
25.283 \\
24.273\end{array}$ & $\begin{array}{l}30.086 \\
28.518 \\
27.379\end{array}$ \\
\hline $\begin{array}{l}21 \\
21 \\
21\end{array}$ & $\begin{array}{l}10000 \\
10000 \\
10000\end{array}$ & $\begin{array}{l}85.0 \\
90.0 \\
95.0\end{array}$ & $\begin{array}{r}.01781 \\
.01736 \\
.01690\end{array}$ & $\begin{array}{r}.07127 \\
.06946 \\
.06763\end{array}$ & $\begin{array}{l}.00543 \\
.00529 \\
.00515\end{array}$ & $\begin{array}{r}.02172 \\
.02117 \\
.02061\end{array}$ & $\begin{array}{l}7.738 \\
7.542 \\
7.343\end{array}$ & $\begin{array}{l}23.586 \\
22.988 \\
22.383\end{array}$ & $\begin{array}{l}26.604 \\
25.930 \\
25.247\end{array}$ \\
\hline 21 & 10000 & & 01660 & .06642 & 00506 & .02024 & 7.212 & 21.982 & 24.795 \\
\hline
\end{tabular}


TABULATION OF DATA FOR

ABSORPTION OF SOUND IN AIR VERSUS PERCENT RELATIVE -UMIDITY AT 21 DEGREES CENTIGRADE FOR A FREQUENCY. OF12500 HERTZ

\begin{tabular}{|c|c|c|c|c|c|c|c|c|c|}
\hline (1) & (2) & $(3)$ & 141 & (5) & (6) & 171 & $(8)$ & $(9)$ & $(10)$ \\
\hline $\begin{array}{l}\text { EGR } \\
\text { ENT }\end{array}$ & HERTZ & $\begin{array}{l}\text { REL } \\
\text { HUM } \\
\text { PER } \\
\text { CENT }\end{array}$ & $\begin{array}{l}\text { ATTEN } \\
\text { COEF } \\
\text { PER } \\
\text { METER }\end{array}$ & $\begin{array}{l}\text { PER } \\
\text { METER }\end{array}$ & $\begin{array}{l}\text { ATTEN } \\
\text { COEF } \\
\text { PER } \\
\text { FOOT }\end{array}$ & $\begin{array}{l}\text { PER } \\
\text { FOOT }\end{array}$ & $\begin{array}{l}\text { ATTEN } \\
\text { DB PER } \\
100 \\
\text { METER }\end{array}$ & $\begin{array}{l}\text { ATTEN } \\
\text { DB PER } \\
1000 \\
\text { FEET }\end{array}$ & $\begin{array}{l}\text { DECAY } \\
\text { RATE } \\
\text { DB PER } \\
\text { SECOND }\end{array}$ \\
\hline 21 & 12500 & 5.0 & .03361 & .13446 & .01024 & .04098 & 14.599 & $44 \cdot 499$ & 50.193 \\
\hline $\begin{array}{l}21 \\
21\end{array}$ & $\begin{array}{l}12500 \\
12500\end{array}$ & $\begin{array}{l}1.0 .0 \\
15.0\end{array}$ & $\begin{array}{l}.06233 \\
.08310\end{array}$ & $\begin{array}{r}.24932 \\
.33242\end{array}$ & $\begin{array}{r}.01899 \\
.02533\end{array}$ & $\begin{array}{r}.07599 \\
.10132\end{array}$ & $\begin{array}{l}27.070 \\
36.092\end{array}$ & $\begin{array}{r}82.512 \\
110.012\end{array}$ & $\begin{array}{r}93.071 \\
124.090\end{array}$ \\
\hline $\begin{array}{l}21 \\
21 \\
21\end{array}$ & $\begin{array}{l}12500 \\
12500 \\
12500\end{array}$ & $\begin{array}{l}20.0 \\
25.0 \\
30.0\end{array}$ & $\begin{array}{l}.08 \\
.07 \\
.06\end{array}$ & $\begin{array}{r}.33732 \\
.30702 \\
.26248\end{array}$ & $\begin{array}{l}.02570 \\
.02339 \\
.02000\end{array}$ & $\begin{array}{r}.10281 \\
.09358 \\
.08000\end{array}$ & $\begin{array}{l}36.624 \\
33.335 \\
28.498\end{array}$ & $\begin{array}{r}111.633 \\
101.607 \\
86.865\end{array}$ & $\begin{array}{r}125.919 \\
114.609 \\
97.982\end{array}$ \\
\hline 21 & 12500 & 35.0 & .05 & 70 & .01704 & .06818 & 24.288 & 74.033 & 83.507 \\
\hline $\begin{array}{l}21 \\
21\end{array}$ & $\begin{array}{l}12500 \\
12500\end{array}$ & $\begin{array}{l}40.0 \\
45.0\end{array}$ & .02 & $: 1$ & $\begin{array}{l}.01 \\
.01\end{array}$ & $\begin{array}{l}.06 \\
.05\end{array}$ & $\begin{array}{l}21.436 \\
19.177\end{array}$ & $\begin{array}{l}340 \\
+55\end{array}$ & $\begin{array}{l}701 \\
935\end{array}$ \\
\hline 21 & 1250 & 46.0 & .04 & .17286 & .01317 & .05268 & 18.768 & 57 & 529 \\
\hline $\begin{array}{l}21 \\
21\end{array}$ & $\begin{array}{l}12500 \\
12500\end{array}$ & $\begin{array}{l}47.0 \\
48.0\end{array}$ & $\begin{array}{r}.04231 \\
.04142\end{array}$ & $\begin{array}{r}.16927 \\
.16568\end{array}$ & $\begin{array}{r}.01289 \\
.01262\end{array}$ & $\begin{array}{l}.05159 \\
.05050\end{array}$ & $\begin{array}{l}18 \\
17\end{array}$ & & $\begin{array}{l}3.188 \\
1.849\end{array}$ \\
\hline $\begin{array}{l}21 \\
21 \\
21\end{array}$ & $\begin{array}{l}12500 \\
12500 \\
12500\end{array}$ & $\begin{array}{l}49.0 \\
50.0 \\
51.0\end{array}$ & $\begin{array}{r}.04052 \\
.03970 \\
.03898\end{array}$ & $\begin{array}{r}.16209 \\
.15882 \\
.15595\end{array}$ & $\begin{array}{r}.01235 \\
.01210 \\
.01188\end{array}$ & $\begin{array}{l}.04940 \\
.04841 \\
.04753\end{array}$ & $\begin{array}{l}17.599 \\
17.244 \\
16.933\end{array}$ & $\begin{array}{l}53.645 \\
52.562 \\
51.612\end{array}$ & $\begin{array}{l}510 \\
289 \\
217\end{array}$ \\
\hline $\begin{array}{l}21 \\
21 \\
21\end{array}$ & $\begin{array}{l}12500 \\
12500 \\
12500\end{array}$ & $\begin{array}{l}52.0 \\
53.0 \\
54.0\end{array}$ & $\begin{array}{r}.03827 \\
.03755 \\
.03691\end{array}$ & $\begin{array}{r}.15308 \\
.15021 \\
.14767\end{array}$ & $\begin{array}{l}.01166 \\
.01144 \\
.01125\end{array}$ & $\begin{array}{l}.0466 \\
.0457 \\
.0450\end{array}$ & $\begin{array}{l}621 \\
309 \\
033\end{array}$ & $\begin{array}{l}662 \\
713 \\
870\end{array}$ & $\begin{array}{l}57.146 \\
56.074 \\
55.124\end{array}$ \\
\hline $\begin{array}{l}21 \\
21 \\
21\end{array}$ & $\begin{array}{l}12500 \\
12500 \\
12500\end{array}$ & $\begin{array}{l}55.0 \\
60.0 \\
65.0\end{array}$ & $\begin{array}{l}.03629 \\
.03360 \\
.03127\end{array}$ & $\begin{array}{r}.14518 \\
.13441 \\
.12509\end{array}$ & $\begin{array}{l}.01106 \\
.01024 \\
.00953\end{array}$ & $\begin{array}{l}.04425 \\
.04096 \\
.03812\end{array}$ & $\begin{array}{l}15.763 \\
14.593 \\
13.581\end{array}$ & $\begin{array}{l}48 \cdot 046 \\
44.481 \\
41.398\end{array}$ & $\begin{array}{l}54.195 \\
50.174 \\
46.695\end{array}$ \\
\hline $\begin{array}{l}21 \\
21 \\
21\end{array}$ & $\begin{array}{l}12500 \\
12500 \\
12500\end{array}$ & $\begin{array}{l}70.0 \\
75.0 \\
80.0\end{array}$ & $\begin{array}{l}.02927 \\
.02770 \\
.02645\end{array}$ & $\begin{array}{r}.11710 \\
.11080 \\
.10580\end{array}$ & $\begin{array}{l}.00892 \\
.00844 \\
.00806\end{array}$ & $\begin{array}{r}.03569 \\
.03377 \\
.03224\end{array}$ & $\begin{array}{l}12.714 \\
12.030 \\
11.487\end{array}$ & $\begin{array}{l}38.755 \\
36.669 \\
35.014\end{array}$ & $\begin{array}{l}43.714 \\
41.361 \\
39.494\end{array}$ \\
\hline $\begin{array}{l}21 \\
21 \\
21\end{array}$ & $\begin{array}{l}12500 \\
12500 \\
12500\end{array}$ & $\begin{array}{l}85.0 \\
90.0 \\
95.0\end{array}$ & $\begin{array}{l}.02525 \\
.02438 \\
.02373\end{array}$ & $\begin{array}{r}.10100 \\
.09755 \\
.09494\end{array}$ & $\begin{array}{l}.00769 \\
.00743 \\
.00723\end{array}$ & $\begin{array}{r}.03078 \\
.02973 \\
.02893\end{array}$ & $\begin{array}{l}10.966 \\
10.591 \\
10.308\end{array}$ & $\begin{array}{l}33.427 \\
32.284 \\
31.421\end{array}$ & $\begin{array}{l}37.705 \\
36.416 \\
35.442\end{array}$ \\
\hline 2 & 12 & & 0231 & 09278 & 00707 & 02828 & 0.073 & 0.705 & +.63 \\
\hline
\end{tabular}


TABULATION OF DATA FOR

ABSORPTION OF SOUND IN AIR VERSUS PERCENT RELATIVE HUMIDITY AT 22 DEGREES CENTIGRADE FOR. A FREQUENCY OF 125 HERTZ

\begin{tabular}{|c|c|c|c|c|c|c|c|c|c|}
\hline 1) & (2) & (3) & 141 & (5) & 161 & $(7)$ & $(8)$ & $(9)$ & $(10)$ \\
\hline EMP & FREQ & REL & ATTEN & $4 M$ & ATTEN & $4 M$ & ATTEN & ATTEN & DECAY \\
\hline $\begin{array}{l}E G R \\
\text { ENT }\end{array}$ & HERTZ & $\begin{array}{l}\text { HUM } \\
\text { PER } \\
\text { CENT }\end{array}$ & $\begin{array}{l}\text { COEF } \\
\text { PER } \\
\text { METER }\end{array}$ & $\begin{array}{l}\text { PER } \\
\text { METER }\end{array}$ & $\begin{array}{l}\text { COEF } \\
\text { PER } \\
\text { FOOT }\end{array}$ & $\begin{array}{l}\text { PER } \\
\text { FOOT }\end{array}$ & $\begin{array}{l}\text { DE PER } \\
100 \\
\text { METER }\end{array}$ & $\begin{array}{l}\text { DB PER } \\
\text { IOOO } \\
\text { FEET. }\end{array}$ & $\begin{array}{l}\text { RATE } \\
\text { DB PER } \\
\text { SECOND }\end{array}$ \\
\hline $\begin{array}{l}22 \\
22 \\
22\end{array}$ & $\begin{array}{l}125 . \\
125 \\
125\end{array}$ & $\begin{array}{r}5.0 \\
10.0 \\
15.0\end{array}$ & $\begin{array}{l}.00019 \\
.00014 \\
.00012\end{array}$ & $\begin{array}{r}.00076 \\
.00056 \\
.00048\end{array}$ & $\begin{array}{l}.00005 \\
.00004 \\
.00003\end{array}$ & $\begin{array}{l}.00023 \\
.00017 \\
.00014\end{array}$ & $\begin{array}{l}.083 \\
.061 \\
.052\end{array}$ & $\begin{array}{r}.254 \\
.186 \\
.159\end{array}$ & $\begin{array}{r}.287 \\
.210 \\
.179\end{array}$ \\
\hline $\begin{array}{l}22 \\
22 \\
22\end{array}$ & $\begin{array}{l}125 \\
125 \\
125\end{array}$ & $\begin{array}{l}20.0 \\
25.0 \\
30.0\end{array}$ & $\begin{array}{l}.00010 \\
.00009 \\
.00009\end{array}$ & $\begin{array}{l}.00043 \\
.00039 \\
.00037\end{array}$ & $\begin{array}{l}.00003 \\
.00003 \\
.00002\end{array}$ & $\begin{array}{l}.00013 \\
.00012 \\
.00011\end{array}$ & $\begin{array}{l}.047 \\
.043 \\
.040\end{array}$ & $\begin{array}{r}.143 \\
.131 \\
.123\end{array}$ & $\begin{array}{r}.162 \\
.148 \\
.139\end{array}$ \\
\hline $\begin{array}{l}22 \\
22 \\
22\end{array}$ & $\begin{array}{l}125 \\
125 \\
125\end{array}$ & $\begin{array}{l}35.0 \\
40.0 \\
45.0\end{array}$ & $\begin{array}{l}.00008 \\
.00008 \\
.00008\end{array}$ & $\begin{array}{l}.00035 \\
.00033 \\
.00032\end{array}$ & $\begin{array}{l}.00002 \\
.00002 \\
.00002\end{array}$ & $\begin{array}{l}.00010 \\
.00010 \\
.00009\end{array}$ & $\begin{array}{l}.038 \\
.036 \\
.034\end{array}$ & $\begin{array}{l}.116 \\
.110 \\
.106\end{array}$ & $\begin{array}{r}.132 \\
.125 \\
.119\end{array}$ \\
\hline $\begin{array}{l}22 \\
22 \\
22\end{array}$ & $\begin{array}{l}125 \\
125 \\
125\end{array}$ & $\begin{array}{l}48.0 \\
47.0 \\
48.0\end{array}$ & $\begin{array}{l}.00007 \\
.00007 \\
.00007\end{array}$ & $\begin{array}{l}.00031 \\
.00031 \\
.00031\end{array}$ & $\begin{array}{l}.00002 \\
.00002 \\
.00002\end{array}$ & $\begin{array}{l}.00009 \\
.00009 \\
.00009\end{array}$ & $\begin{array}{l}.034 \\
.034 \\
.034\end{array}$ & $\begin{array}{l}.105 \\
.104 \\
.103\end{array}$ & $\begin{array}{r}.119 \\
.118 \\
.117\end{array}$ \\
\hline $\begin{array}{l}22 \\
22 \\
22\end{array}$ & $\begin{array}{l}125 \\
125 \\
125\end{array}$ & $\begin{array}{l}49 \cdot 0 \\
50.0 \\
51.0\end{array}$ & $\begin{array}{l}.00007 \\
.00007 \\
.00007\end{array}$ & $\begin{array}{l}.00031 \\
.00030 \\
.00030\end{array}$ & $\begin{array}{l}.00002 \\
.00002 \\
.00002\end{array}$ & $\begin{array}{l}.00009 \\
.00009 \\
.00009\end{array}$ & $\begin{array}{l}.033 \\
.033 \\
.033\end{array}$ & $\begin{array}{l}.103 \\
.102 \\
.101\end{array}$ & $\begin{array}{r}.116 \\
.115 \\
.114\end{array}$ \\
\hline $\begin{array}{l}22 \\
22 \\
22\end{array}$ & $\begin{array}{l}125 \\
125 \\
125\end{array}$ & $\begin{array}{l}52.0 \\
53.0 \\
54.0\end{array}$ & $\begin{array}{l}.00007 \\
.00007 \\
.00007\end{array}$ & $\begin{array}{l}.00030 \\
.00030 \\
.00030\end{array}$ & $\begin{array}{l}.00002 \\
.00002 \\
.00002\end{array}$ & $\begin{array}{l}.00009 \\
.00009 \\
.00009\end{array}$ & $\begin{array}{l}.033 \\
.032 \\
.032\end{array}$ & $\begin{array}{l}.100 \\
.100 \\
.099\end{array}$ & $\begin{array}{r}.114 \\
.113 \\
.112\end{array}$ \\
\hline $\begin{array}{l}22 \\
22\end{array}$ & $\begin{array}{l}125 \\
125\end{array}$ & $\begin{array}{l}55.0 \\
60.0\end{array}$ & $\begin{array}{l}.00007 \\
.00007\end{array}$ & $\begin{array}{l}.00029 \\
.00028\end{array}$ & $\begin{array}{l}.00002 \\
.00002\end{array}$ & $\begin{array}{l}.00009 \\
.00008\end{array}$ & $\begin{array}{r}.032 \\
.031\end{array}$ & $\begin{array}{l}.098 \\
.095\end{array}$ & .111 \\
\hline 22 & 125 & 65.0 & .00007 & .00028 & .00002 & .00008 & .030 & .092 & .104 \\
\hline $\begin{array}{l}22 \\
22\end{array}$ & $\begin{array}{l}125 \\
125\end{array}$ & $\begin{array}{l}70.0 \\
75.0\end{array}$ & $\begin{array}{l}.00006 \\
.00006\end{array}$ & $\begin{array}{l}.00027 \\
.00026\end{array}$ & $\begin{array}{l}.00002 \\
.00002\end{array}$ & $\begin{array}{l}.00008 \\
.00008\end{array}$ & $\begin{array}{r}.029 \\
.028\end{array}$ & $\begin{array}{l}.090 \\
.087\end{array}$ & $\begin{array}{r}101 \\
.098\end{array}$ \\
\hline 22 & 125 & 80.0 & .00006 & .00025 & .00001 & .00007 & .027 & .084 & .095 \\
\hline $\begin{array}{l}22 \\
22\end{array}$ & $\begin{array}{l}125 \\
125\end{array}$ & $\begin{array}{l}85.0 \\
90.0\end{array}$ & $\begin{array}{l}.00006 \\
.00005\end{array}$ & $\begin{array}{l}.00024 \\
.00023\end{array}$ & $\begin{array}{l}.00001 \\
.00001\end{array}$ & $\begin{array}{l}.00007 \\
.00007\end{array}$ & $\begin{array}{l}.026 \\
.026\end{array}$ & $\begin{array}{r}082 \\
\quad 079\end{array}$ & $\begin{array}{l}.092 \\
.089\end{array}$ \\
\hline 22 & 125 & 95.0 & .00005 & .00023 & .00001 & .00007 & .025 & .077 & .087 \\
\hline 22 & 125 & 100.0 & .0 .0 & .00022 & .00001 & .00006 & .024 & .075 & .084 \\
\hline
\end{tabular}


TABULATION OF DATA FOR

ABSORPTION OF SOUND IN AIR VERSUS PERCENT RELATIVE HUMIDITY AT 22 DEGREES CENTIGRADE FOR A FREQUENCY OF 250 HERTZ

\begin{tabular}{|c|c|c|c|c|c|c|c|c|c|}
\hline (1) & $(2)$ & (3) & $(4)$ & (5) & 161 & 171 & $(8)$ & 191 & $(10)$ \\
\hline $\begin{array}{l}\text { TEMP } \\
\text { DEGR } \\
\text { CENT }\end{array}$ & HERTZ & $\begin{array}{l}\text { REL } \\
\text { HUM } \\
\text { PER } \\
\text { CENT }\end{array}$ & $\begin{array}{l}\text { ATTEN } \\
\text { COEF } \\
\text { PER } \\
\text { METER }\end{array}$ & $\begin{array}{l}\text { PER } \\
\text { METER }\end{array}$ & $\begin{array}{l}\text { ATTEN } \\
\text { COEF } \\
\text { PER } \\
\text { FOOT }\end{array}$ & 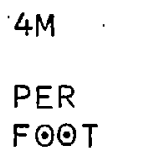 & $\begin{array}{l}\text { ATTEN } \\
\text { DB. PER } \\
100 \\
\text { METER }\end{array}$ & $\begin{array}{l}\text { ATTEN } \\
\text { DB PER } \\
1000 \\
\text { FEET }\end{array}$ & $\begin{array}{l}\text { DECAY } \\
\text { RATE } \\
\text { DB PER } \\
\text { SECOND }\end{array}$ \\
\hline 22 & 250 & 5.0 & .00049 & .00197 & .00015 & .00060 & .214 & .655 & .740 \\
\hline $\begin{array}{l}22 \\
22\end{array}$ & $\begin{array}{l}250 \\
250\end{array}$ & $\begin{array}{l}10.0 \\
15.0\end{array}$ & $\begin{array}{l}.00031 \\
.00026\end{array}$ & $\begin{array}{r}.00124 \\
.00106\end{array}$ & $\begin{array}{l}.00009 \\
.00008\end{array}$ & $\begin{array}{l}.00037 \\
.00032\end{array}$ & $\begin{array}{r}.134 \\
.116\end{array}$ & $\begin{array}{r}.410 \\
.353\end{array}$ & $\begin{array}{l}.463 \\
.399\end{array}$ \\
\hline 22 & 250 & 20.0 & .00023 & .00095 & .00007 & .00029 & .103 & .315 & .356 \\
\hline $\begin{array}{l}22 \\
22\end{array}$ & $\begin{array}{l}250 \\
250\end{array}$ & $\begin{array}{l}25.0 \\
30.0\end{array}$ & $\begin{array}{l}.00022 \\
.00020\end{array}$ & $\begin{array}{l}.00088 \\
.00082\end{array}$ & $\begin{array}{l}.00006 \\
.00006\end{array}$ & $\begin{array}{l}.00026 \\
.00025\end{array}$ & $\begin{array}{l}.095 \\
.089\end{array}$ & $\begin{array}{l}.291 \\
.272\end{array}$ & $\begin{array}{r}.329 \\
.307\end{array}$ \\
\hline $\begin{array}{l}22 \\
22\end{array}$ & $\begin{array}{l}250 \\
250\end{array}$ & $\begin{array}{l}35.0 \\
40.0\end{array}$ & $\begin{array}{l}.00019 \\
.00018\end{array}$ & $\begin{array}{r}.00077 \\
.00073\end{array}$ & $\begin{array}{l}.00005 \\
.00005\end{array}$ & $\begin{array}{l}.00023 \\
.00022\end{array}$ & $\begin{array}{l}.084 \\
.080\end{array}$ & $\begin{array}{r}.257 \\
.244\end{array}$ & $\begin{array}{l}.290 \\
.276\end{array}$ \\
\hline 22 & 250 & 45.0 & .00017 & .00070 & .00005 & .00021 & .077 & .234 & .265 \\
\hline $\begin{array}{l}22 \\
22\end{array}$ & $\begin{array}{l}250 \\
250\end{array}$ & $\begin{array}{l}46.0 \\
47.0\end{array}$ & $\begin{array}{l}.00017 \\
.00017\end{array}$ & $\begin{array}{l}.00070 \\
.00069\end{array}$ & $\begin{array}{l}.00005 \\
.00005\end{array}$ & $\begin{array}{l}.00021 \\
.00021\end{array}$ & $\begin{array}{r}.076 \\
.075\end{array}$ & $\begin{array}{l}.232 \\
.230\end{array}$ & $\begin{array}{l}.263 \\
.260\end{array}$ \\
\hline 22 & 250 & 48.0 & .00017 & .00069 & .00005 & .00021 & .075 & .228 & .258 \\
\hline $\begin{array}{l}22 \\
22 \\
22\end{array}$ & $\begin{array}{l}250 \\
250 \\
250\end{array}$ & $\begin{array}{l}49.0 \\
50.0 \\
51.0\end{array}$ & $\begin{array}{l}.00017 \\
.00017 \\
.00016\end{array}$ & $\begin{array}{r}.00068 \\
.00068 \\
.00067\end{array}$ & $\begin{array}{l}.00005 \\
.00005 \\
.00005\end{array}$ & $\begin{array}{l}.00020 \\
.00020 \\
.00020\end{array}$ & $\begin{array}{l}.074 \\
.073 \\
.073\end{array}$ & $\begin{array}{l}.226 \\
.225 \\
.223\end{array}$ & $\begin{array}{r}.256 \\
.254 \\
.252\end{array}$ \\
\hline $\begin{array}{l}22 \\
22 \\
22\end{array}$ & $\begin{array}{l}250 \\
250 \\
250\end{array}$ & $\begin{array}{l}52.0 \\
53.0 \\
54.0\end{array}$ & $\begin{array}{l}.00016 \\
.00016 \\
.00016\end{array}$ & $\begin{array}{l}.00067 \\
.00066 \\
.00066\end{array}$ & $\begin{array}{l}.00005 \\
.00005 \\
.00005\end{array}$ & $\begin{array}{l}.00020 \\
.00020 \\
.00020\end{array}$ & $\begin{array}{l}.072 \\
.072 \\
.071\end{array}$ & $\begin{array}{l}.222 \\
.220 \\
.218\end{array}$ & $\begin{array}{l}.250 \\
.249 \\
.247\end{array}$ \\
\hline $\begin{array}{l}22 \\
22 \\
22\end{array}$ & $\begin{array}{l}250 \\
250 \\
250\end{array}$ & $\begin{array}{l}55.0 \\
60.0 \\
65.0\end{array}$ & $\begin{array}{l}.00016 \\
.00015 \\
.00015\end{array}$ & $\begin{array}{r}.00065 \\
.00063 \\
.00061\end{array}$ & $\begin{array}{r}.00004 \\
.00004 \\
.00004\end{array}$ & $\begin{array}{l}.00019 \\
.00019 \\
.00018\end{array}$ & $\begin{array}{l}.071 \\
.069 \\
.067\end{array}$ & $\begin{array}{l}.217 \\
.210 \\
.204\end{array}$ & $\begin{array}{r}.245 \\
.238 \\
.231\end{array}$ \\
\hline $\begin{array}{l}22 \\
22 \\
22\end{array}$ & $\begin{array}{l}250 \\
250 \\
250\end{array}$ & $\begin{array}{l}70.0 \\
75.0 \\
80.0\end{array}$ & $\begin{array}{l}.00015 \\
.00014 \\
.00014\end{array}$ & $\begin{array}{l}.00060 \\
.00058 \\
.00057\end{array}$ & $\begin{array}{l}.00004 \\
.00004 \\
.00004\end{array}$ & $\begin{array}{r}.00018 \\
.00017 \\
.00017\end{array}$ & $\begin{array}{l}.065 \\
.063 \\
.062\end{array}$ & $\begin{array}{r}199 \\
.193 \\
.189\end{array}$ & $\begin{array}{r}.225 \\
.219 \\
.213\end{array}$ \\
\hline $\begin{array}{l}22 \\
22\end{array}$ & $\begin{array}{l}250 \\
250\end{array}$ & $\begin{array}{l}85.0 \\
90.0\end{array}$ & $\begin{array}{r}.00013 \\
.00013\end{array}$ & $\begin{array}{l}.00055 \\
.00054\end{array}$ & $\begin{array}{l}.00004 \\
.00004\end{array}$ & $\begin{array}{l}.00017 \\
.00016\end{array}$ & $\begin{array}{l}.060 \\
.059\end{array}$ & $\begin{array}{r}185 \\
.181\end{array}$ & $\begin{array}{l}.209 \\
.204\end{array}$ \\
\hline 22 & 250 & 95.0 & .00013 & .00053 & .00004 & .00016 & .058 & .177 & .200 \\
\hline 22 & 250 & 00.0 & .00013 & .00052 & 0.0003 & .00015 & .056 & .172 & .195 \\
\hline
\end{tabular}


TABULATION OF DATA FOR

ABSORPTION OF SOUND IN AIR VERSUS PERCENT RELATIVE HUMIDITY AT 22 DEGREES CENTIGRADE FOR A FREQUENCY OF 500 HERTZ

\begin{tabular}{|c|c|c|c|c|c|c|c|c|c|}
\hline$(1)$ & $(2)$ & $(3)$ & (4)! & (5) & 161 & (7) & $(8)$ & 191 & $(10)$ \\
\hline $\begin{array}{l}\text { TEMP } \\
\text { DEGR } \\
\text { CENT }\end{array}$ & HERTZ & $\begin{array}{l}\text { REL } \\
\text { HUM } \\
\text { PER } \\
\text { CENT }\end{array}$ & $\begin{array}{l}\text { ATTEN } \\
\text { COEF } \\
\text { PER } \\
\text { METER }\end{array}$ & $\begin{array}{l}4 M \\
\text { PER } \\
\text { METER }\end{array}$ & $\begin{array}{l}\text { ATTEN } \\
\text { COEF } \\
\text { PER } \\
\text { FOOT }\end{array}$ & $\begin{array}{l}4 M \\
\text { PER } \\
\text { FO○T }\end{array}$ & $\begin{array}{l}\text { ATTEN } \\
\text { DB. PER } \\
\text { IOO. } \\
\text { METER }\end{array}$ & $\begin{array}{l}\text { ATTEN } \\
\text { DB PER } \\
\text { IOOO } \\
\text { FEET }\end{array}$ & $\begin{array}{l}\text { DECAY } \\
\text { RATE } \\
\text { DB PER } \\
\text { SECOND }\end{array}$ \\
\hline $\begin{array}{l}22 \\
22 \\
22\end{array}$ & $\begin{array}{l}500 \\
500 \\
500\end{array}$ & $\begin{array}{r}5.0 \\
10.0 \\
15.0\end{array}$ & $\begin{array}{l}.00151 \\
.00074 \\
.00061\end{array}$ & $\begin{array}{l}.00604 \\
.00299 \\
.00247\end{array}$ & $\begin{array}{l}.00046 \\
.00022 \\
.00018\end{array}$ & $\begin{array}{l}.00184 \\
.00091 \\
.00075\end{array}$ & $\begin{array}{l}.656 \\
.324 \\
.269\end{array}$ & $\begin{array}{r}2.001 \\
.990 \\
.820\end{array}$ & $\begin{array}{r}2.261 \\
1.118 \\
.927\end{array}$ \\
\hline $\begin{array}{l}22 \\
22 \\
22\end{array}$ & $\begin{array}{l}500 \\
500 \\
500\end{array}$ & $\begin{array}{l}20.0 \\
25.0 \\
30.0\end{array}$ & $\begin{array}{l}.00055 \\
.00051 \\
.00047\end{array}$ & $\begin{array}{l}.00223 \\
.00204 \\
.00190\end{array}$ & $\begin{array}{r}.00017 \\
.00015 \\
.00014\end{array}$ & $\begin{array}{l}.00068 \\
.00062 \\
.00058\end{array}$ & $\begin{array}{r}.242 \\
.222 \\
.207\end{array}$ & $\begin{array}{l}.739 \\
.677 \\
.631\end{array}$ & $\begin{array}{l}.835 \\
.765 \\
.713\end{array}$ \\
\hline $\begin{array}{l}22 \\
22 \\
22\end{array}$ & $\begin{array}{l}500 \\
500 \\
500\end{array}$ & $\begin{array}{l}35.0 \\
40.0 \\
45.0\end{array}$ & $\begin{array}{l}.00045 \\
.00043 \\
.00041\end{array}$ & $\begin{array}{l}.00180 \\
.00172 \\
.00164\end{array}$ & $\begin{array}{l}.00013 \\
.00013 \\
.00012\end{array}$ & $\begin{array}{l}.00055 \\
.00052 \\
.00050\end{array}$ & $\begin{array}{l}.196 \\
.187 \\
.179\end{array}$ & $\begin{array}{l}.598 \\
.570 \\
.545\end{array}$ & $\begin{array}{l}.676 \\
.644 \\
.616\end{array}$ \\
\hline $\begin{array}{l}22 \\
22\end{array}$ & $\begin{array}{l}500 \\
500\end{array}$ & $\begin{array}{l}46.0 \\
47.0\end{array}$ & $\begin{array}{l}.00040 \\
.00040\end{array}$ & $\begin{array}{l}.00163 \\
.00162\end{array}$ & $\begin{array}{l}.00012 \\
.00012\end{array}$ & $\begin{array}{l}.00049 \\
.00049\end{array}$ & .177 & $\begin{array}{l}.541 \\
.536\end{array}$ & $\begin{array}{l}.611 \\
.606\end{array}$ \\
\hline 22. & 500 & 48.0 & .00040 & .00160 & .00012 & .00049 & .174 & .532 & .601 \\
\hline $\begin{array}{l}22 \\
22\end{array}$ & $\begin{array}{l}500 \\
500\end{array}$ & $\begin{array}{l}49.0 \\
50.0\end{array}$ & $\begin{array}{l}.00039 \\
.00039\end{array}$ & $\begin{array}{l}.00159 \\
.00158\end{array}$ & $\begin{array}{l}.00012 \\
.00012\end{array}$ & $\begin{array}{r}.00048 \\
.00048\end{array}$ & $\begin{array}{r}173 \\
.172\end{array}$ & $\begin{array}{r}.528 \\
.525\end{array}$ & $\begin{array}{l}.597 \\
.593\end{array}$ \\
\hline 22 & 500 & 51.0 & .00039 & .00157 & .00012 & .00048 & .171 & .521 & .589 \\
\hline $\begin{array}{l}22 \\
22 \\
22\end{array}$ & $\begin{array}{l}500 \\
500 \\
500\end{array}$ & $\begin{array}{l}52.0 \\
53.0 \\
54.0\end{array}$ & $\begin{array}{l}.00039 \\
.00038 \\
.00038\end{array}$ & $\begin{array}{l}.00156 \\
.00155 \\
.00154\end{array}$ & $\begin{array}{l}.00011 \\
.00011 \\
.00011\end{array}$ & $\begin{array}{l}.00047 \\
.00047 \\
.00047\end{array}$ & $\begin{array}{l}.169 \\
.168 \\
.167\end{array}$ & $\begin{array}{l}.518 \\
.514 \\
.510\end{array}$ & $\begin{array}{l}.585 \\
.581 \\
.577\end{array}$ \\
\hline $\begin{array}{l}22 \\
22\end{array}$ & $\begin{array}{l}500 \\
500\end{array}$ & $\begin{array}{l}55.0 \\
60.0\end{array}$ & $\begin{array}{l}.00038 \\
.00037\end{array}$ & $\begin{array}{r}.00153 \\
.00148\end{array}$ & $\begin{array}{l}.00011 \\
.00011\end{array}$ & $\begin{array}{l}.00046 \\
.00045\end{array}$ & .166 & $\begin{array}{r}.507 \\
.491\end{array}$ & $\begin{array}{l}.573 \\
.555\end{array}$ \\
\hline 22 & 500 & 65.0 & .00036 & .00144 & .00011 & .00044 & .156 & .478 & .540 \\
\hline $\begin{array}{l}22 \\
22 \\
22\end{array}$ & $\begin{array}{l}500 \\
500 \\
500\end{array}$ & $\begin{array}{l}70.0 \\
75.0 \\
80.0\end{array}$ & $\begin{array}{l}.00035 \\
.00034 \\
.00033\end{array}$ & $\begin{array}{l}.00140 \\
.00136 \\
.00133\end{array}$ & $\begin{array}{l}.00010 \\
.00010 \\
.00010\end{array}$ & $\begin{array}{l}.00042 \\
.00041 \\
.00040\end{array}$ & $\begin{array}{l}.152 \\
.148 \\
.144\end{array}$ & $\begin{array}{r}.464 \\
.453 \\
.441\end{array}$ & $\begin{array}{l}.525 \\
.511 \\
.499\end{array}$ \\
\hline $\begin{array}{l}22 \\
22 \\
22\end{array}$ & $\begin{array}{l}500 \\
500 \\
500\end{array}$ & $\begin{array}{l}85.0 \\
90.0 \\
95.0\end{array}$ & $\begin{array}{l}.00032 \\
.00032 \\
.00031\end{array}$ & $\begin{array}{r}.00130 \\
.00128 \\
.00125\end{array}$ & $\begin{array}{l}.00009 \\
.00009 \\
.00009\end{array}$ & $\begin{array}{l}.00039 \\
.00039 \\
.00038\end{array}$ & $\begin{array}{r}.141 \\
.139 \\
.136\end{array}$ & $\begin{array}{r}.432 \\
.424 \\
.416\end{array}$ & $\begin{array}{l}.488 \\
.479 \\
.470\end{array}$ \\
\hline 22 & 500 & $100 \cdot 0$ & .00030 & .00123 & .00009 & .00037 & .134 & .408 & .461 \\
\hline
\end{tabular}


TABULATION OF: DATA FOR

ABSORPTION OF SOUND IN AIR VERSUS PERCENT RELATIVE HUMMIDITY AT 22 DEGREES CENTIGRADE FOR A FREQUENCY OF 1000 HERTZ

\begin{tabular}{|c|c|c|c|c|c|c|c|c|c|}
\hline (1) & (2) & (3) & (4) & (5) & (6) & 171 & $(3)$ & 191 & $(10)$ \\
\hline $\begin{array}{l}\text { TEMP } \\
\text { DEGR } \\
\text { CENT }\end{array}$ & HER TZ & $\begin{array}{l}\text { REL } \\
\text { HUM } \\
\text { PER } \\
\text { CENT }\end{array}$ & $\begin{array}{l}\text { ATTEN } \\
\text { COEF } \\
\text { PER } \\
\text { METER }\end{array}$ & $\begin{array}{l}4 M \\
\text { PER } \\
\text { METER }\end{array}$ & $\begin{array}{l}\text { ATTEN } \\
\text { COEF } \\
\text { PER } \\
\text { FOOT }\end{array}$ & $\begin{array}{l}4 M \\
\text { PER } \\
F \odot \odot T\end{array}$ & $\begin{array}{l}\text { ATTEN } \\
\text { DB PER } \\
100 \\
\text { METER }\end{array}$ & $\begin{array}{l}\text { ATTEN } \\
\text { DB PER } \\
\text { IOOO } \\
\text { FEET }\end{array}$ & $\begin{array}{l}\text { DECAY } \\
\text { RATE } \\
\text { DB PER } \\
\text { SECOND }\end{array}$ \\
\hline $\begin{array}{l}22 \\
22 \\
22\end{array}$ & $\begin{array}{l}1000 \\
1000 \\
1000\end{array}$ & $\begin{array}{r}5.0 \\
10.0 \\
15.0\end{array}$ & $\begin{array}{l}.00517 \\
.00242 \\
.00161\end{array}$ & $\begin{array}{r}.02068 \\
.00969 \\
.00644\end{array}$ & $\begin{array}{l}.00157 \\
.00073 \\
.00049\end{array}$ & $\begin{array}{l}.00630 \\
.00295 \\
.00196\end{array}$ & $\begin{array}{r}2.246 \\
1.052 \\
.700\end{array}$ & $\begin{array}{l}6.846 \\
3.208 \\
2.134\end{array}$ & $\begin{array}{l}7.736 \\
3.625 \\
2.411\end{array}$ \\
\hline $\begin{array}{l}22 \\
22 \\
22\end{array}$ & $\begin{array}{l}1000 \\
1000 \\
1000\end{array}$ & $\begin{array}{l}20.0 \\
25.0 \\
30.0\end{array}$ & $\begin{array}{r}.00135 \\
.00125 \\
.00117\end{array}$ & $\begin{array}{l}.00541 \\
.00500 \\
.00468\end{array}$ & $\begin{array}{l}.00041 \\
.00038 \\
.00035\end{array}$ & $\begin{array}{l}.00164 \\
.00152 \\
.00142\end{array}$ & $\begin{array}{r}.587 \\
.542 \\
.509\end{array}$ & $\begin{array}{l}1.791 \\
1.654 \\
1.551\end{array}$ & $\begin{array}{l}2.023 \\
1.869 \\
1.753\end{array}$ \\
\hline $\begin{array}{l}22 \\
22 \\
22\end{array}$ & $\begin{array}{l}1000^{\circ} \\
1000 \\
1000\end{array}$ & $\begin{array}{l}35.0 \\
40.0 \\
45.0\end{array}$ & $\begin{array}{l}.00110 \\
.00105 \\
.00100\end{array}$ & $\begin{array}{l}.00443 \\
.00420 \\
.00400\end{array}$ & $\begin{array}{l}.00033 \\
.00032 \\
.00030\end{array}$ & $\begin{array}{l}.00135 \\
.00128 \\
.00122\end{array}$ & $\begin{array}{l}.481 \\
.456 \\
.434\end{array}$ & $\begin{array}{l}1.466 \\
1.391 \\
1.325\end{array}$ & $\begin{array}{l}1.656 \\
1.572 \\
1.498\end{array}$ \\
\hline $\begin{array}{l}22 \\
22 \\
22\end{array}$ & $\begin{array}{l}1000 \\
1000 \\
1000\end{array}$ & $\begin{array}{l}46.0 \\
47.0 \\
48.0\end{array}$ & $\begin{array}{l}.00099 \\
.00098 \\
.00098\end{array}$ & $\begin{array}{r}.00397 \\
.00394 \\
.00392\end{array}$ & $\begin{array}{l}.00030 \\
.00030 \\
.00029\end{array}$ & $\begin{array}{l}.00121 \\
.00120 \\
.00119\end{array}$ & $\begin{array}{l}.431 \\
.428 \\
.425\end{array}$ & $\begin{array}{l}1.316 \\
1.306 \\
1.297\end{array}$ & $\begin{array}{l}1.487 \\
1.476 \\
1.466\end{array}$ \\
\hline $\begin{array}{l}22 \\
22 \\
22\end{array}$ & $\begin{array}{l}1000 \\
1000 \\
1000\end{array}$ & $\begin{array}{l}49.0 \\
50.0 \\
51.0\end{array}$ & $\begin{array}{l}.00097 \\
.00096 \\
.00095\end{array}$ & $\begin{array}{l}.00389 \\
.00386 \\
.00383\end{array}$ & $\begin{array}{l}.00029 \\
.00029 \\
.00029\end{array}$ & $\begin{array}{l}.00118 \\
.00117 \\
.00117\end{array}$ & $\begin{array}{r}.422 \\
.419 \\
.416\end{array}$ & $\begin{array}{l}1 \cdot 287 \\
1 \cdot 278 \\
1 \cdot 270\end{array}$ & $\begin{array}{l}1.455 \\
1.445 \\
1.435\end{array}$ \\
\hline $\begin{array}{l}22 \\
22 \\
22\end{array}$ & $\begin{array}{l}1000 \\
1000 \\
1000\end{array}$ & $\begin{array}{l}52.0 \\
53.0 \\
54.0\end{array}$ & $\begin{array}{l}.00095 \\
.00094 \\
.00094\end{array}$ & $\begin{array}{r}.00381 \\
.00378 \\
.00376\end{array}$ & $\begin{array}{l}.00029 \\
.00028 \\
.00028\end{array}$ & $\begin{array}{l}.00116 \\
.00115 \\
.00114\end{array}$ & $\begin{array}{r}.414 \\
.411 \\
.408\end{array}$ & $\begin{array}{l}1.262 \\
1.254 \\
1.245\end{array}$ & $\begin{array}{l}1.426 \\
1.417 \\
1.407\end{array}$ \\
\hline $\begin{array}{l}22 \\
22 \\
22\end{array}$ & $\begin{array}{l}1000 \\
1000 \\
1000\end{array}$ & $\begin{array}{l}55.0 \\
60.0 \\
65.0\end{array}$ & $\begin{array}{l}.00093 \\
.00090 \\
.00088\end{array}$ & $\begin{array}{r}.00373 \\
.00363 \\
.00352\end{array}$ & $\begin{array}{l}.00028 \\
.00027 \\
.00026\end{array}$ & $\begin{array}{r}.00113 \\
.00110 \\
.00107\end{array}$ & $\begin{array}{r}.406 \\
.394 \\
.383\end{array}$ & $\begin{array}{l}1.237 \\
1.201 \\
1.168\end{array}$ & $\begin{array}{l}1.398 \\
1.358 \\
1.319\end{array}$ \\
\hline 22 & 1000 & 70.0 & .00085 & .00343 & .00026 & .00104 & .372 & 1.135 & 1.282 \\
\hline $\begin{array}{l}22 \\
22\end{array}$ & $\begin{array}{l}1000 \\
1000\end{array}$ & $\begin{array}{l}75.0 \\
80.0\end{array}$ & $\begin{array}{r}.00083 \\
.00081\end{array}$ & $\begin{array}{l}.00334 \\
.00327\end{array}$ & $\begin{array}{l}.00025 \\
.00024\end{array}$ & $\begin{array}{l}.00102 \\
.00099\end{array}$ & $\begin{array}{r}.363 \\
.355\end{array}$ & $\begin{array}{l}1.107 \\
1.082\end{array}$ & $\begin{array}{l}1.251 \\
1.223\end{array}$ \\
\hline $\begin{array}{l}22 \\
22 \\
22\end{array}$ & $\begin{array}{l}1000 \\
1000 \\
1000\end{array}$ & $\begin{array}{l}85.0 \\
90.0 \\
95.0\end{array}$ & $\begin{array}{l}.00080 \\
.00078 \\
.00076\end{array}$ & $\begin{array}{l}.00320 \\
.00313 \\
.00307\end{array}$ & $\begin{array}{l}.00024 \\
.00023 \\
.00023\end{array}$ & $\begin{array}{l}.00097 \\
.00095 \\
.00093\end{array}$ & $\begin{array}{l}.348 \\
.340 \\
.334\end{array}$ & $\begin{array}{l}1.060 \\
1.038 \\
1.018\end{array}$ & $\begin{array}{l}1.198 \\
1.173 \\
1.151\end{array}$ \\
\hline 22 & 1000 & 100.0 & .00075 & .00302 & .00023 & .00092 & .328 & 1.002 & 1.132 \\
\hline
\end{tabular}


TABULATION OF DATA FOR

ABSORPTION OF SOUND IN AIR VERSUS PERCENT RELATIVE HUMIDITY AT 22 DEGREES CENTIGRADE FOR A FREQUENCY OF 2000 HERTZ

\begin{tabular}{|c|c|c|c|c|c|c|c|c|c|}
\hline$(1)$ & (2) & $(3)$ & (4.) & (5) & (6) & (7) & $(8)$ & $(9)$ & $(10)$ \\
\hline TEMP & FREQ & $\begin{array}{l}\text { REL } \\
\text { HUM }\end{array}$ & $\begin{array}{l}\text { ATTEN } \\
\text { COEF }\end{array}$ & $4 M$ & $\begin{array}{l}\text { ATTEN } \\
\text { COEF }\end{array}$ & $4 M$ & $\begin{array}{l}\text { ATTEN } \\
\text { DB PER }\end{array}$ & $\begin{array}{l}\text { ATTEN } \\
\text { DB PER }\end{array}$ & $\begin{array}{l}\text { DECAY } \\
\text { RATE }\end{array}$ \\
\hline $\begin{array}{l}\text { DEGR } \\
\text { CENT }\end{array}$ & HERTZ & $\begin{array}{l}\text { PER } \\
\text { CENT }\end{array}$ & $\begin{array}{l}\text { PER } \\
\text { METER }\end{array}$ & $\begin{array}{l}\text { PER } \\
\text { METER }\end{array}$ & $\begin{array}{l}\text { PER } \\
\text { FOOT }\end{array}$ & $\begin{array}{l}\text { PER } \\
\text { FOOT }\end{array}$ & $\begin{array}{l}100 \\
\text { METER }\end{array}$ & $\begin{array}{l}1000 \\
\text { FEET }\end{array}$ & $\begin{array}{l}\text { DB PER } \\
\text { SECOND }\end{array}$ \\
\hline $\begin{array}{l}22 \\
22\end{array}$ & $\begin{array}{l}2000 \\
2000\end{array}$ & $\begin{array}{r}5.0 \\
10.0\end{array}$ & $\begin{array}{r}.01306 \\
.00839\end{array}$ & $\begin{array}{r}.05227 \\
.03359\end{array}$ & $\begin{array}{r}.00398 \\
.00256\end{array}$ & $\begin{array}{r}.01593 \\
.01024\end{array}$ & $\begin{array}{l}5.676 \\
3.647\end{array}$ & $\begin{array}{l}17 \cdot 301 \\
11.118\end{array}$ & $\begin{array}{l}19.549 \\
12.563\end{array}$ \\
\hline 22 & 2000 & $15 \cdot 0$ & .00543 & .02173 & .00165 & .00662 & 2.360 & .7 .194 & 8.129 \\
\hline $\begin{array}{l}22 \\
22 \\
22\end{array}$ & $\begin{array}{l}2000 \\
2000 \\
2000\end{array}$ & $\begin{array}{l}20.0 \\
25.0 \\
30.0\end{array}$ & $\begin{array}{l}.00400 \\
.00326 \\
.00291\end{array}$ & $\begin{array}{r}.01603 \\
.01307 \\
.01167\end{array}$ & $\begin{array}{l}.00122 \\
.00099 \\
.00088\end{array}$ & $\begin{array}{l}.00488 \\
.00398 \\
.00355\end{array}$ & $\begin{array}{l}1.741 \\
1.419 \\
1.267\end{array}$ & $\begin{array}{l}5.307 \\
4.325 \\
3.863\end{array}$ & $\begin{array}{l}5.997 \\
4.887 \\
4.365\end{array}$ \\
\hline $\begin{array}{l}22 \\
22 \\
22\end{array}$ & $\begin{array}{l}2000 \\
2000 \\
2000\end{array}$ & $\begin{array}{l}35.0 \\
40.0 \\
45.0\end{array}$ & $\begin{array}{l}.00272 \\
.00259 \\
.00249\end{array}$ & $\begin{array}{l}.01089 \\
.01039 \\
.00998\end{array}$ & $\begin{array}{l}.00083 \\
.00079 \\
.00076\end{array}$ & $\begin{array}{l}.00332 \\
.00316 \\
.00304\end{array}$ & $\begin{array}{l}1.182 \\
1.128 \\
1.084\end{array}$ & $\begin{array}{l}3.605 \\
3.440 \\
3.306\end{array}$ & $\begin{array}{l}4.073 \\
3.887 \\
3.735\end{array}$ \\
\hline $\begin{array}{l}22 \\
22 \\
22\end{array}$ & $\begin{array}{l}2000 \\
2000 \\
2000\end{array}$ & $\begin{array}{l}46.0 \\
47.0 \\
48.0\end{array}$ & $\begin{array}{l}.00247 \\
.00246 \\
.00244\end{array}$ & $\begin{array}{l}.00991 \\
.00984 \\
.00977\end{array}$ & $\begin{array}{l}.00075 \\
.00074 \\
.00074\end{array}$ & $\begin{array}{l}.0 .0302 \\
.00299 \\
.00297\end{array}$ & & & $\begin{array}{l}3.705 \\
3.680 \\
3.655\end{array}$ \\
\hline $\begin{array}{l}22 \\
22 \\
22\end{array}$ & $\begin{array}{l}2000 \\
2000 \\
2000\end{array}$ & $\begin{array}{l}49.0 \\
50.0 \\
51.0\end{array}$ & $\begin{array}{l}.00242 \\
.00240 \\
.00239\end{array}$ & $\begin{array}{l}.00970 \\
.00963 \\
.00957\end{array}$ & $\begin{array}{l}.00073 \\
.00073 \\
.00072\end{array}$ & $\begin{array}{l}.00295 \\
.00293 \\
.00291\end{array}$ & $\begin{array}{l}1.053 \\
1.046 \\
1.039\end{array}$ & $\begin{array}{l}3.212 \\
3.190 \\
3.167\end{array}$ & $\begin{array}{l}3.629 \\
3.604 \\
3.579\end{array}$ \\
\hline $\begin{array}{l}22 \\
22 \\
22\end{array}$ & $\begin{array}{l}2000 \\
2000 \\
2000\end{array}$ & $\begin{array}{l}52.0 \\
53.0 \\
54.0\end{array}$ & $\begin{array}{l}.00237 \\
.00235 \\
.00234\end{array}$ & $\begin{array}{l}.00950 \\
.00943 \\
.00937\end{array}$ & $\begin{array}{l}.00072 \\
.00071 \\
.00071\end{array}$ & $\begin{array}{l}.00289 \\
.00287 \\
.00285\end{array}$ & $\begin{array}{l}1.031 \\
1.024 \\
1.017\end{array}$ & $\begin{array}{l}3 \bullet 145 \\
3 \cdot 122 \\
3 \bullet 101\end{array}$ & $\begin{array}{l}3.553 \\
3.528 \\
3.505\end{array}$ \\
\hline $\begin{array}{l}22 \\
22 \\
22\end{array}$ & $\begin{array}{l}2000 \\
2000 \\
2000\end{array}$ & $\begin{array}{l}55.0 \\
60.0 \\
65.0\end{array}$ & $\begin{array}{l}.00232 \\
.00225 \\
.00218\end{array}$ & $\begin{array}{l}.00931 \\
.00902 \\
.00875\end{array}$ & $\begin{array}{l}.00070 \\
.00068 \\
.00066\end{array}$ & $\begin{array}{l}.00283 \\
.00275 \\
.00266\end{array}$ & $\begin{array}{r}1.011 \\
\cdot 979 \\
\cdot 950\end{array}$ & $\begin{array}{l}3.082 \\
2.986 \\
2.896\end{array}$ & $\begin{array}{l}3.483 \\
3.374 \\
3.273\end{array}$ \\
\hline $\begin{array}{l}22 \\
22 \\
22\end{array}$ & $\begin{array}{l}2000 \\
2000 \\
2000\end{array}$ & $\begin{array}{l}70.0 \\
75.0 \\
80.0\end{array}$ & $\begin{array}{l}.00212 \\
.00207 \\
.00202\end{array}$ & $\begin{array}{l}.00849 \\
.00828 \\
.00810\end{array}$ & $\begin{array}{l}.00064 \\
.00063 \\
.00061\end{array}$ & $\begin{array}{l}.00259 \\
.00252 \\
.00247\end{array}$ & $\begin{array}{r}.922 \\
.899 \\
.880\end{array}$ & $\begin{array}{l}2.812 \\
2.741 \\
2.683\end{array}$ & $\begin{array}{l}3.178 \\
3.097 \\
3.032\end{array}$ \\
\hline $\begin{array}{l}22 \\
22 \\
22\end{array}$ & $\begin{array}{l}2000 \\
2000 \\
2000\end{array}$ & $\begin{array}{l}85.0 \\
90.0 \\
95.0\end{array}$ & $\begin{array}{l}.00198 \\
.00194 \\
.00191\end{array}$ & $\begin{array}{r}.00794 \\
.00779 \\
.00766\end{array}$ & $\begin{array}{l}.00060 \\
.00059 \\
.00058\end{array}$ & $\begin{array}{l}.00242 \\
.00237 \\
.00233\end{array}$ & $\begin{array}{r}.863 \\
.846 \\
.831\end{array}$ & $\begin{array}{l}2.630 \\
2.580 \\
2.535\end{array}$ & $\begin{array}{l}2.972 \\
2.915 \\
2.865\end{array}$ \\
\hline 22 & 2000 & 100.0 & .00188 & 00753 & 00057 & .00229 & .818 & 2.493 & 2.817 \\
\hline
\end{tabular}


TABULATION OF DATA FOR

ABSORPTION OF SOUND IN AIR VERSUS PERCENT RELATIVE HUMIDITY AT 22 DEGREES CENTIGRADE FOR A FREQUENCY OF 2500 HERTZ

\begin{tabular}{|c|c|c|c|c|c|c|c|c|c|}
\hline (1) & (2) & (3) & (4) & $(5)$ & $(6)$ & (7) & (8) & (9) & $(10)$ \\
\hline $\begin{array}{l}\text { TEMP } \\
\\
\text { DEGR } \\
\text { CENT }\end{array}$ & $\begin{array}{l}\text { FREQ: } \\
\text { HERTZ }\end{array}$ & $\begin{array}{l}\text { REL } \\
\text { HUM } \\
\text { PER } \\
\text { CENT. }\end{array}$ & $\begin{array}{l}\text { ATTEN } \\
\text { COEF } \\
\text { PER } \\
\text { METER }\end{array}$ & $\begin{array}{l}4 M \\
\text { PER } \\
\text { METER }\end{array}$ & $\begin{array}{l}\text { ATTEN } \\
\text { COEF } \\
\text { PER } \\
\text { FOOT }\end{array}$ & $\begin{array}{l}\text { PER } \\
\text { FO०T }\end{array}$ & $\begin{array}{l}\text { AT.TEN } \\
\text { DB PER } \\
100 \\
\text { METER }\end{array}$ & $\begin{array}{l}\text { ATTEN } \\
\text { DB PER } \\
1000 \\
\text { FEET }\end{array}$ & $\begin{array}{l}\text { DECAY } \\
\text { RATE } \\
\text { DB PER } \\
\text { SECOND }\end{array}$ \\
\hline $\begin{array}{l}22 \\
22 \\
22\end{array}$ & $\begin{array}{l}2500 \\
2500 \\
2500\end{array}$ & $\begin{array}{r}5.0 \\
10 \cdot 0 \\
15.0\end{array}$ & $\begin{array}{r}.01598 \\
.01242 \\
.00804\end{array}$ & $\begin{array}{l}.06393 \\
.04968 \\
.03219\end{array}$ & $\begin{array}{l}.00487 \\
.00378 \\
.00245\end{array}$ & $\begin{array}{r}.01948 \\
.01514 \\
.00981\end{array}$ & $\begin{array}{l}6.942 \\
5.394 \\
3.495\end{array}$ & $\begin{array}{l}21.160 \\
16.443 \\
10.653\end{array}$ & $\begin{array}{l}23.909 \\
18.580 \\
12.038\end{array}$ \\
\hline $\begin{array}{l}22 \\
22\end{array}$ & $\begin{array}{l}2500 \\
2500\end{array}$ & $\begin{array}{l}20.0 \\
25.0\end{array}$ & $\begin{array}{l}.00589 \\
.00469\end{array}$ & $\begin{array}{l}.02358 \\
.01877\end{array}$ & $\begin{array}{l}.00179 \\
.00143\end{array}$ & $\begin{array}{l}.00718 \\
.00572\end{array}$ & $\begin{array}{l}2.561 \\
2.038\end{array}$ & $\begin{array}{l}7.806 \\
6.214\end{array}$ & $\begin{array}{l}8.820 \\
7.021\end{array}$ \\
\hline 22 & 2500 & 30.0 & .00401 & .01607 & .00122 & .00490 & 1.745 & 5.320 & 6.012 \\
\hline $\begin{array}{l}22 \\
22 \\
22\end{array}$ & $\begin{array}{l}2500 \\
2500 \\
2500\end{array}$ & $\begin{array}{l}35.0 \\
40.0 \\
45.0\end{array}$ & $\begin{array}{l}.00367 \\
.00346 \\
.00332\end{array}$ & $\begin{array}{r}.01469 \\
.01385 \\
.01330\end{array}$ & $\begin{array}{l}.00111 \\
.00105 \\
.00101\end{array}$ & $\begin{array}{l}.00447 \\
.00422 \\
.00405\end{array}$ & $\begin{array}{l}1.595 \\
1.504 \\
1.444\end{array}$ & $\begin{array}{l}4 \cdot 863 \\
4.58 .5 \\
4 \cdot 404\end{array}$ & $\begin{array}{l}5.495 \\
5.181 \\
4.976\end{array}$ \\
\hline $\begin{array}{l}22 \\
22\end{array}$ & $\begin{array}{l}2500 \\
2500\end{array}$ & $\begin{array}{l}46.0 \\
47.0\end{array}$ & $\begin{array}{l}.00330 \\
.00327\end{array}$ & $\begin{array}{l}.01320 \\
.01311\end{array}$ & $\begin{array}{l}.00100 \\
.00099\end{array}$ & $\begin{array}{l}.00402 \\
.00399\end{array}$ & $\begin{array}{l}1.433 \\
1.424\end{array}$ & $\begin{array}{l}4 \cdot 370 \\
4 \cdot 341\end{array}$ & $\begin{array}{l}4.937 \\
4.905\end{array}$ \\
\hline 22 & 2500 & 48.0 & .00325 & .01302 & .00099 & .00397 & 1.414 & $4 \cdot 311$ & $4 \cdot 872$ \\
\hline $\begin{array}{l}22 \\
22 \\
22\end{array}$ & $\begin{array}{l}2500 \\
2500 \\
2500\end{array}$ & $\begin{array}{l}49.0 \\
50.0 \\
51.0\end{array}$ & $\begin{array}{l}.00323 \\
.00321 \\
.00319\end{array}$ & $\begin{array}{r}.01294 \\
.01285 \\
.01276\end{array}$ & $\begin{array}{l}.00098 \\
.00097 \\
.00097\end{array}$ & $\begin{array}{l}.00394 \\
.00391 \\
.00380\end{array}$ & $\begin{array}{l}1.405 \\
1.395 \\
1.386\end{array}$ & $\begin{array}{l}4 \cdot 282 \\
4 \cdot 253 \\
4 \cdot 224\end{array}$ & $\begin{array}{l}4.839 \\
4.806 \\
4.773\end{array}$ \\
\hline $\begin{array}{l}22 \\
22 \\
22\end{array}$ & $\begin{array}{l}2500 \\
2500 \\
2500\end{array}$ & $\begin{array}{l}52.0 \\
53.0 \\
54.0\end{array}$ & $\begin{array}{l}.00316 \\
.00314 \\
.00312\end{array}$ & $\begin{array}{l}.01267 \\
.01259 \\
.01251\end{array}$ & $\begin{array}{l}.00096 \\
.0009 j \\
.00095\end{array}$ & $\begin{array}{l}.00386 \\
.00383 \\
.00381\end{array}$ & $\begin{array}{l}1.376 \\
1.367 \\
1.358\end{array}$ & $\begin{array}{l}4 \cdot 195 \\
4 \cdot 166 \\
4 \cdot 142\end{array}$ & $\begin{array}{l}4 \cdot 741 \\
4 \cdot 708 \\
4.680\end{array}$ \\
\hline 22 & 2500 & 55.0 & .00 & .01244 & .00094 & .0 & 51 & $4 \cdot 117$ & 653 \\
\hline $\begin{array}{l}22 \\
22\end{array}$ & $\begin{array}{l}2500 \\
2500\end{array}$ & $\begin{array}{l}60.0 \\
65.0\end{array}$ & $\begin{array}{l}.00301 \\
.00293\end{array}$ & $\begin{array}{r}.01207 \\
.01174\end{array}$ & $\begin{array}{l}.00092 \\
.00089\end{array}$ & $\begin{array}{l}.00368 \\
.00357\end{array}$ & $\begin{array}{l}1.311 \\
1.275\end{array}$ & $\begin{array}{l}3.996 \\
3.886\end{array}$ & $\begin{array}{l}4 \cdot 516 \\
4 \cdot 391\end{array}$ \\
\hline 22 & 2500 & 70.0 & .00285 & .01143 & .00087 & .00348 & 1.241 & 3.782 & $4 \cdot 274$ \\
\hline $\begin{array}{l}22 \\
22\end{array}$ & $\begin{array}{l}2500 \\
2500\end{array}$ & $\begin{array}{l}75.0 \\
80.0\end{array}$ & $\begin{array}{r}.00278 \\
.00271\end{array}$ & $\begin{array}{r}.01113 \\
.01086\end{array}$ & $\begin{array}{l}.00084 \\
.00082\end{array}$ & $\begin{array}{l}.00339 \\
.00331\end{array}$ & $\begin{array}{l}1.209 \\
1.179\end{array}$ & $\begin{array}{l}3.686 \\
3.595\end{array}$ & $\begin{array}{l}4.165 \\
4.062\end{array}$ \\
\hline $\begin{array}{l}22 \\
22 \\
22\end{array}$ & $\begin{array}{l}2500 \\
2500 \\
2500\end{array}$ & $\begin{array}{l}85.0 \\
90.0 \\
95.0\end{array}$ & $\begin{array}{l}.00265 \\
.00260 \\
.00255\end{array}$ & $\begin{array}{r}.01060 \\
.01041 \\
.01023\end{array}$ & $\begin{array}{l}.00080 \\
.00079 \\
.00077\end{array}$ & $\begin{array}{l}.00323 \\
.00317 \\
.00311\end{array}$ & $\begin{array}{l}1 \bullet 151 \\
1 \bullet 131 \\
1.111\end{array}$ & $\begin{array}{l}3.510 \\
3.447 \\
3.386\end{array}$ & $\begin{array}{l}3.966 \\
3.895 \\
3.826\end{array}$ \\
\hline 2 & 500 & $100 \cdot 0$ & 00251 & .01006 & .00076 & 00306 & 1.093 & $3 \cdot 332$ & 3.765 \\
\hline
\end{tabular}


TABULATION OF DATA FOR

ABSORPTION OF SOUND IN AIR VERSUS PERCENT RELATIVE HUMIDITY AT 22 DEGREES CENTIGRADE FOR A FREQUENCY OF 3200 HERTZ

$(1)$

(2) (3)

(4.)

(5)

(6)

(7)

(8)

(9)

1101

TEMP FREQ RE

ATTEN $4 M$

DEGR

CENT HERTZ CENT

$22 \quad 3200 \quad 5.0$

$\begin{array}{lll}22 & 3200 & 10.0\end{array}$

$22 \quad 3200 \quad 15.0$

$223200 \quad 20.0$

$223200 \quad 25.0$

$223200 \quad 30.0$

223200

223200

223200

35.0

40.0

45.0

223200

46.0

22

22

22

22

\section{2}

2

22

\section{2}

22

2

2

22

2

2

22

22

2

ATTEN
COEF

PER'

METER

.01882

.01872

.01237

.00911

.00723

.00603

.00528

.00489

.00459

.00456

.00453

.00450

$48 \cdot 0$

3200

3200

49.0

50.0

$3200 \quad 51.0$

.00447

.00444

.00442

.00438

.00435

.00432

3200

52.0

53.0

54.0

.00429

.00418

.00407

60.0

65.0

3200

$3200 \quad 70.0$

$3200 \quad 80.0$

.00397

.00387

.00379

.00370

.00355
320075.0

.03646

.02895

.02412

.02115

.01956

.01839

.01826

.01812

.01800

.01788

.01776

.01765

.01753

.01741

.01729

.01719

- 01672

.01628

.01588

.01550

.01517

.00121

.00128

.00115

.00472

.00462

.01483

.00113 .00452

.01453

.00120

.00442

.01423

$.00108 \quad .00433$

$.00348 \quad .01394 \quad .00106 \quad .00425$ $\begin{array}{lll}\text { ATTEN } & \text { ATTEN } & \text { DECAY } \\ \text { DB PER } & \text { DB PER } & \text { RATE } \\ 100 & 1000 & \text { DB PER } \\ \text { METER } & \text { FEET } & \text { SECOND }\end{array}$

$8.175 \quad 24.918 \quad 28.156$

$8.130 \quad 24.781 \quad 28.002$

$5.372 \quad 16.375 \quad 18.503$

$\begin{array}{lll}3.959 & 12.068 & 13.637\end{array}$

$3.143 \quad 9.582 \quad 10.827$

$2.619 \quad 7.985 \quad 9.022$

$2.297 \quad 7.002 \quad 7.912$

$2.123 \quad 6.473 \quad 7.315$

$1.997 \quad 6.0896 .880$

$1.983 \quad 6.044 \quad 6.829$

$1.968 \quad 5.999 \quad 6.779$

$1.955 \quad 5.959 \quad 6.733$

$1.942 \quad 5.919 \quad 6.689$

$1.829 \quad 5.880 \quad 6.644$

$1.916 \quad 5.841 \quad 6.600$

$1.903 \quad 5.802 \quad 6.556$

$1.890 \quad 5.763 \quad 6.512$

$1.877 \quad 5.724 \quad 6.467$

$1.867 \quad 5.690 \quad 6.430$

$1.815 \quad 5.534 \quad 6.253$

$1.767 \quad 5.388 \quad 6.088$

$1.724 \quad 5.257 \quad 5.940$

$1.683 \quad 5.132 \quad 5.799$

$1.647 \quad 5.020 \quad 5.673$

$1.610 \quad 4.908 \quad 5.546$

$1.577 \quad 4.808 \quad 5.433$

$1.545 \quad 4.711 \quad 5.323$

$1.513 .4 .614 \quad 5.214$ 
TABULATION OF DATA FOR

ABSORPTION OF SOUND IN AIR VERSUS PERCENT RELATIVE HUMIDITY AT 22 DEGREES CENTIGRADE FOR A FREQUENCY OF 4000 HERTZ

\begin{tabular}{|c|c|c|c|c|c|c|c|c|c|}
\hline (1) & $(2)$ & (3) & (4) & (5) & (6) & 171 & 181 & 191 & $(10)$ \\
\hline EMP. & FREQ & $\begin{array}{l}\text { REL } \\
\text { HUM }\end{array}$ & $\begin{array}{l}\text { ATTEN } \\
\text { COEF }\end{array}$ & $4 M$ & $\begin{array}{l}\text { AT TEN } \\
\text { COEF }\end{array}$ & $4 M$ & $\begin{array}{l}\text { ATTEN } \\
\text { DB PER }\end{array}$ & $\begin{array}{l}\text { ATTEN } \\
\text { DB PER }\end{array}$ & $\begin{array}{l}\text { DECAY } \\
\text { RATE }\end{array}$ \\
\hline $\begin{array}{l}\text { DEGR } \\
\text { CENT }\end{array}$ & HERTZ & $\begin{array}{l}\text { PER } \\
\text { CENT }\end{array}$ & $\begin{array}{l}\text { PER } \\
\text { METER }\end{array}$ & $\begin{array}{l}\text { PER } \\
\text { METER }\end{array}$ & $\begin{array}{l}\text { PER } \\
\text { FOOT }\end{array}$ & $\begin{array}{l}\text { PER } \\
\text { FOOT }\end{array}$ & $\begin{array}{l}100 \\
\text { METER }\end{array}$ & $\begin{array}{l}1000 \\
\text { FEET }\end{array}$ & $\begin{array}{l}\text { DB PER } \\
\text { SECOND }\end{array}$ \\
\hline $\begin{array}{l}22 \\
22\end{array}$ & $\begin{array}{l}4000 \\
4000\end{array}$ & $\begin{array}{r}5.0 \\
10.0\end{array}$ & $\begin{array}{r}.02081 \\
.02557\end{array}$ & $\begin{array}{r}.08325 \\
.10228\end{array}$ & $\begin{array}{r}.00634 \\
.00779\end{array}$ & $\begin{array}{l}.02537 \\
.03117\end{array}$ & $\begin{array}{r}9.039 \\
11.105\end{array}$ & $\begin{array}{l}27.552 \\
33.849\end{array}$ & $\begin{array}{l}31.132 \\
38.247\end{array}$ \\
\hline 22 & 4000 & 15.0 & .01817 & .0727 .0 & .00554 & .02216 & 7.894 & 24.062 & 27.189 \\
\hline $\begin{array}{l}22 \\
22\end{array}$ & $\begin{array}{l}4000 \\
4000\end{array}$ & $\begin{array}{l}20.0 \\
25.0\end{array}$ & $\begin{array}{l}.01351 \\
.01061\end{array}$ & $\begin{array}{l}.05405 \\
.04244\end{array}$ & $\begin{array}{l}.00411 \\
.00323\end{array}$ & $\begin{array}{r}.01647 \\
.01293\end{array}$ & $\begin{array}{l}5.869 \\
4.608\end{array}$ & $\begin{array}{l}17.890 \\
14.046\end{array}$ & $\begin{array}{l}20.214 \\
15.871\end{array}$ \\
\hline 22 & 4000 & 30.0 & .00881 & .03527 & .00268 & .01075 & 3.830 & 11.674 & 13.191 \\
\hline $\begin{array}{l}22 \\
22\end{array}$ & $\begin{array}{l}4000 \\
4000\end{array}$ & $\begin{array}{l}35: 0 \\
40.0\end{array}$ & $\begin{array}{l}.00760 \\
.00679\end{array}$ & $\begin{array}{l}.03041 \\
.02718\end{array}$ & $\begin{array}{l}.00231 \\
.00207\end{array}$ & $\begin{array}{l}.00927 \\
.00828\end{array}$ & $\begin{array}{l}3.302 \\
2.951\end{array}$ & $\begin{array}{r}10.066 \\
8.995\end{array}$ & $\begin{array}{l}11.375 \\
10.164\end{array}$ \\
\hline 22 & 4000 & 45.0 & .00632 & .02528 & .00192 & .00770 & 2.744 & 8.366 & 9.453 \\
\hline $\begin{array}{l}22 \\
22 \\
22\end{array}$ & $\begin{array}{l}4000 \\
4000 \\
4000\end{array}$ & $\begin{array}{l}46.0 \\
47.0 \\
48.0\end{array}$ & $\begin{array}{l}.00625 \\
.00618 \\
.00610\end{array}$ & $\begin{array}{r}.02500 \\
.02474 \\
.02442\end{array}$ & $\begin{array}{l}.00190 \\
.00188 \\
.00186\end{array}$ & $\begin{array}{l}.00762 \\
.00754 \\
.00744\end{array}$ & $\begin{array}{l}2.714 \\
2.686 \\
2.652\end{array}$ & $\begin{array}{l}8.274 \\
8.189 \\
8.083\end{array}$ & $\begin{array}{l}9.349 \\
9.253 \\
9.134\end{array}$ \\
\hline $\begin{array}{l}22 \\
22 \\
22\end{array}$ & $\begin{array}{l}4000 \\
4000 \\
4000\end{array}$ & $\begin{array}{l}49.0 \\
50.0 \\
51.0\end{array}$ & $\begin{array}{l}.00603 \\
.00597 \\
.00592\end{array}$ & $\begin{array}{l}.02413 \\
.02388 \\
.02368\end{array}$ & $\begin{array}{l}.00183 \\
.00182 \\
.00180\end{array}$ & $\begin{array}{l}.00735 \\
.00728 \\
.00721\end{array}$ & $\begin{array}{l}2.620 \\
2.593 \\
2.571\end{array}$ & $\begin{array}{l}7.986 \\
7.904 \\
7.837\end{array}$ & $\begin{array}{l}9.024 \\
8.932 \\
8.855\end{array}$ \\
\hline $\begin{array}{l}22 \\
22 \\
22\end{array}$ & $\begin{array}{l}4000 \\
4000 \\
4000\end{array}$ & $\begin{array}{l}52.0 \\
53.0 \\
54.0\end{array}$ & $\begin{array}{l}.00587 \\
.00583 \\
.00579\end{array}$ & $\begin{array}{l}.02348 \\
.02334 \\
.02319\end{array}$ & $\begin{array}{l}.00178 \\
.00177 \\
.00176\end{array}$ & $\begin{array}{l}.00715 \\
.00711 \\
.00706\end{array}$ & $\begin{array}{l}2.550 \\
2.534 \\
2.518\end{array}$ & $\begin{array}{l}7.772 \\
7.724 \\
7.676\end{array}$ & $\begin{array}{l}8.782 \\
8.728 \\
8.673\end{array}$ \\
\hline $\begin{array}{l}22 \\
22 \\
22\end{array}$ & $\begin{array}{l}4000 \\
4000 \\
4000\end{array}$ & $\begin{array}{l}55.0 \\
60.0 \\
65.0\end{array}$ & $\begin{array}{l}.00576 \\
.00560 \\
.00545\end{array}$ & $\begin{array}{l}.02305 \\
.02241 \\
.02183\end{array}$ & $\begin{array}{l}.00175 \\
.00170 \\
.00166\end{array}$ & $\begin{array}{l}.00702 \\
.00683 \\
.00665\end{array}$ & $\begin{array}{l}2.503 \\
2.433 \\
2.370\end{array}$ & $\begin{array}{l}7.629 \\
7.418 \\
7.225\end{array}$ & $\begin{array}{l}8.621 \\
8.382 \\
8.164\end{array}$ \\
\hline $\begin{array}{l}22 \\
22 \\
22\end{array}$ & $\begin{array}{l}4000 \\
4000 \\
4000\end{array}$ & $\begin{array}{l}70.0 \\
75.0 \\
80.0\end{array}$ & $\begin{array}{l}.00533 \\
.00521 \\
.00510\end{array}$ & $\begin{array}{r}.02132 \\
.02084 \\
.02042\end{array}$ & $\begin{array}{r}.00162 \\
.00158 \\
.00155\end{array}$ & $\begin{array}{r}.00649 \\
.00635 \\
.00622\end{array}$ & $\begin{array}{l}2.315 \\
2.263 \\
2.217\end{array}$ & $\begin{array}{l}7.056 \\
6.899 \\
6.758\end{array}$ & $\begin{array}{l}7.973 \\
7.795 \\
7.636\end{array}$ \\
\hline $\begin{array}{l}22 \\
22 \\
22\end{array}$ & $\begin{array}{l}4000 \\
4000 \\
4000\end{array}$ & $\begin{array}{l}85.0 \\
90.0 \\
95.0\end{array}$ & $\begin{array}{r}.00499 \\
.00490 \\
.00481\end{array}$ & $\begin{array}{r}.01999 \\
.01962 \\
.01926\end{array}$ & $\begin{array}{l}.00152 \\
.00149 \\
.00146\end{array}$ & $\begin{array}{l}.00609 \\
.00598 \\
.00587\end{array}$ & $\begin{array}{l}2.170 \\
2.131 \\
2.091 .\end{array}$ & $\begin{array}{l}6.617 \\
6.496 \\
6.375\end{array}$ & $\begin{array}{l}7.476 \\
7.340 \\
7.203\end{array}$ \\
\hline 22 & 4000 & 100.0 & .00472 & .01890 & .00144 & .00576 & 2.052 & 6.256 & 7.069 \\
\hline
\end{tabular}


TABULATION OF DATA FOR

ABSORPTION OF SOUND IN AIR VERSUS PERCENT RELATIVE HUMIDITY AT 22 DEGREES CENTIGRADE FOR A FREQUENCY OF 5000 HERTZ

\begin{tabular}{|c|c|c|c|c|c|c|c|c|c|}
\hline (1) & (2) & $(3)$ & $(4)$ & $(5)$ & (6) & 171 & $(8)$ & $(9)$ & 1201 \\
\hline $\begin{array}{l}\text { TEMP } \\
\text { CEGR } \\
\text { CENT }\end{array}$ & HERTZ & $\begin{array}{l}\text { REL } \\
\text { HUM } \\
\text { PER } \\
\text { CENT }\end{array}$ & $\begin{array}{l}\text { ATTEN } \\
\text { COEF } \\
\text { PER } \\
\text { METER }\end{array}$ & $\begin{array}{l}4 M \\
\text { PER } \\
\text { METER }\end{array}$ & $\begin{array}{l}\text { ATTEN } \\
\text { COEF } \\
\text { PER } \\
\text { FOOT }\end{array}$ & $\begin{array}{l}4 M \\
\text { PER } \\
\text { FOOT }\end{array}$ & $\begin{array}{l}\text { ATTEN } \\
\text { DB PER } \\
100 \\
\text { METER }\end{array}$ & $\begin{array}{l}\text { ATTEN } \\
\text { DB PER } \\
1000 \\
\text { FEET }\end{array}$ & $\begin{array}{l}\text { DECAY } \\
\text { RATE } \\
\text { DB PER } \\
\text { SECOND }\end{array}$ \\
\hline $\begin{array}{l}22 \\
22 \\
22\end{array}$ & $\begin{array}{l}5000 \\
5000 \\
5000\end{array}$ & $\begin{array}{r}5.0 \\
10.0 \\
15.0\end{array}$ & $\begin{array}{r}.02276 \\
.03315 \\
.02661\end{array}$ & $\begin{array}{r}.09104 \\
.13261 \\
.10644\end{array}$ & $\begin{array}{l}.00693 \\
.01010 \\
.00811\end{array}$ & $\begin{array}{l}.02775 \\
.04042 \\
.03244\end{array}$ & $\begin{array}{r}9.885 \\
14.399 \\
11.556\end{array}$ & $\begin{array}{l}30.130 \\
43.889 \\
35.225\end{array}$ & $\begin{array}{l}34.045 \\
49.592 \\
39.803\end{array}$ \\
\hline $\begin{array}{l}22 \\
22 \\
22\end{array}$ & $\begin{array}{l}5000 \\
5000 \\
5000\end{array}$ & $\begin{array}{l}20.0 \\
25.0 \\
30.0\end{array}$ & $\begin{array}{r}.01959 \\
.01560 \\
.01286\end{array}$ & $\begin{array}{l}.07838 \\
.06240 \\
.05147\end{array}$ & $\begin{array}{l}.00597 \\
.00475 \\
.00392\end{array}$ & $\begin{array}{l}.02389 \\
.01902 \\
.01568\end{array}$ & $\begin{array}{l}8.510 \\
6.775 \\
5.589\end{array}$ & $\begin{array}{l}25.940 \\
20.652 \\
17.035\end{array}$ & $\begin{array}{l}29 \cdot 311 \\
23 \cdot 336 \\
19 \cdot 249\end{array}$ \\
\hline $\begin{array}{l}22 \\
22 \\
22\end{array}$ & $\begin{array}{l}5000 \\
5000 \\
5000\end{array}$ & $\begin{array}{l}35.0 \\
40.0 \\
45.0\end{array}$ & $\begin{array}{l}.01103 \\
.00973 \\
.00881\end{array}$ & $\begin{array}{r}.04415 \\
.03892 \\
.03524\end{array}$ & $\begin{array}{l}.00336 \\
.00296 \\
.00268\end{array}$ & $\begin{array}{l}.01345 \\
.01186 \\
.01074\end{array}$ & $\begin{array}{l}4.793 \\
4.226 \\
3.826\end{array}$ & $\begin{array}{l}14.611 \\
12.883 \\
11.663\end{array}$ & $\begin{array}{l}16.509 \\
14.557 \\
13.178\end{array}$ \\
\hline $\begin{array}{l}22 \\
22 \\
22\end{array}$ & $\begin{array}{l}5000 \\
5000 \\
5000\end{array}$ & $\begin{array}{l}46.0 \\
47.0 \\
48.0\end{array}$ & $\begin{array}{r}.00867 \\
.00854 \\
.00841\end{array}$ & $\begin{array}{r}.03468 \\
.03416 \\
.03367\end{array}$ & $\begin{array}{l}.00264 \\
.00260 \\
.00256\end{array}$ & $\begin{array}{l}.01057 \\
.01041 \\
.01026\end{array}$ & $\begin{array}{l}3.766 \\
3.709 \\
3.656\end{array}$ & $\begin{array}{l}11.479 \\
11.306 \\
11.145\end{array}$ & $\begin{array}{l}12.970 \\
12.775 \\
12.593\end{array}$ \\
\hline $\begin{array}{l}22 \\
22 \\
22\end{array}$ & $\begin{array}{l}5000 \\
5000 \\
5000\end{array}$ & $\begin{array}{l}49.0 \\
50.0 \\
51.0\end{array}$ & $\begin{array}{r}.00831 \\
.00823 \\
.00814\end{array}$ & $\begin{array}{l}.03327 \\
.03293 \\
.03258\end{array}$ & $\begin{array}{r}.00253 \\
.00250 \\
.00248\end{array}$ & $\begin{array}{l}.01014 \\
.01003 \\
.00993\end{array}$ & $\begin{array}{l}3.613 \\
3.575 \\
3.537\end{array}$ & $\begin{array}{l}11.013 \\
10.898 \\
10.783\end{array}$ & $\begin{array}{l}12.444 \\
12.314 \\
12.184\end{array}$ \\
\hline $\begin{array}{l}22 \\
22 \\
22\end{array}$ & $\begin{array}{l}5000 \\
5000 \\
5000\end{array}$ & $\begin{array}{l}52.0 \\
53.0 \\
54.0\end{array}$ & $\begin{array}{l}.00805 \\
.00798 \\
.00791\end{array}$ & $\begin{array}{r}.03223 \\
.03195 \\
.03166\end{array}$ & $\begin{array}{l}.00245 \\
.00243 \\
.00241\end{array}$ & $\begin{array}{l}.00982 \\
.00973 \\
.00965\end{array}$ & $\begin{array}{l}3.500 \\
3.469 \\
3.438\end{array}$ & $\begin{array}{l}10.668 \\
10.574 \\
10.480\end{array}$ & $\begin{array}{l}12.054 \\
11 \cdot 948 \\
11.842\end{array}$ \\
\hline $\begin{array}{l}22 \\
22\end{array}$ & $\begin{array}{l}5000 \\
5000\end{array}$ & $\begin{array}{l}55.0 \\
60.0\end{array}$ & $\begin{array}{r}.00783 \\
.00751\end{array}$ & $\begin{array}{r}.03132 \\
.03006\end{array}$ & $\begin{array}{r}.00238 \\
.00229\end{array}$ & & & $\begin{array}{r}10.365 \\
9.948\end{array}$ & $\begin{array}{l}11.712 \\
11.241\end{array}$ \\
\hline 22 & 5000 & 65.0 & .00732 & .02931 & .00223 & .00893 & 3.182 & 9.700 & 10.960 \\
\hline $\begin{array}{l}22 \\
22\end{array}$ & $\begin{array}{l}5000 \\
5000\end{array}$ & $\begin{array}{l}70.0 \\
75.0\end{array}$ & $\begin{array}{l}.00715 \\
.00700\end{array}$ & $\begin{array}{r}.02861 \\
.02800\end{array}$ & $\begin{array}{r}.00218 \\
.00213\end{array}$ & $\begin{array}{l}.00872 \\
.00853\end{array}$ & $\begin{array}{l}3.106 \\
3.040\end{array}$ & $\begin{array}{l}9.469 \\
9.266\end{array}$ & $\begin{array}{l}10.700 \\
10.470\end{array}$ \\
\hline 22 & 5000 & 80.0 & .00686 & .02744 & .00209 & .00836 & 2.979 & 9.082 & $10 \cdot 262$ \\
\hline $\begin{array}{l}22 \\
22 \\
22\end{array}$ & $\begin{array}{l}5000 \\
5000 \\
5000\end{array}$ & $\begin{array}{l}85.0 \\
90.0 \\
95.0\end{array}$ & $\begin{array}{l}.00672 \\
.00661 \\
.00649\end{array}$ & $\begin{array}{l}.02691 \\
.02644 \\
.02598\end{array}$ & $\begin{array}{l}.00205 \\
.00201 \\
.00197\end{array}$ & $\begin{array}{l}.00820 \\
.00806 \\
.00791\end{array}$ & $\begin{array}{l}2.921 \\
2.871 \\
2.821\end{array}$ & $\begin{array}{l}8.906 \\
8.752 \\
8.599\end{array}$ & $\begin{array}{r}10.063 \\
9.889 \\
9.716\end{array}$ \\
\hline 22 & 000 & 100.0 & 00638 & 02555 & 00194 & .00778 & 2.774 & 8.455 & 9.554 \\
\hline
\end{tabular}


TABULATION OF DATA FOR

ABSORPTION OF SOUND IN AIR VERSUS PERCENT RELATIVE HUMIDITY AT 22 DEGREES CENTIGRADE FOR A FREQUENCY OF 5940 HERTZ

\begin{tabular}{|c|c|c|c|c|c|c|c|c|c|}
\hline (1) & $(2)$ & (3) & $(4)$ & (5) & $(6)$ & (7) & $(8)$ & $(9)$ & $(10)$ \\
\hline $\begin{array}{l}\text { TEMP } \\
\text { DEGR } \\
\text { CENT }\end{array}$ & FREQ & $\begin{array}{l}\text { REL } \\
\text { HUM } \\
\text { PER } \\
\text { CENT }\end{array}$ & $\begin{array}{l}\text { ATTEN } \\
\text { COEF } \\
\text { PER } \\
\text { METER }\end{array}$ & $\begin{array}{l}4 M \\
\text { PER } \\
\text { METER }\end{array}$ & $\begin{array}{l}\text { ATTEN } \\
\text { COEF } \\
\text { PER } \\
\text { FOOT }\end{array}$ & $\begin{array}{l}4 M \\
\text { PER } \\
\text { FOOT }\end{array}$ & $\begin{array}{l}\text { ATTEN } \\
\text { DB PER } \\
100 \\
\text { METER }\end{array}$ & $\begin{array}{l}\text { ATTEN } \\
\text { DB PER } \\
1000 \\
\text { FEET }\end{array}$ & $\begin{array}{l}\text { DECAY } \\
\text { RATE. } \\
\text { DB PER } \\
\text { SECOND. }\end{array}$ \\
\hline $\begin{array}{l}22 \\
22 \\
22\end{array}$ & $\begin{array}{l}5940 \\
5940 \\
5940\end{array}$ & $\begin{array}{r}5.0 \\
10.0 \\
15.0\end{array}$ & $\begin{array}{l}.02405 \\
.03960 \\
.03535\end{array}$ & $\begin{array}{r}.09621 \\
.15841 \\
.14143\end{array}$ & $\begin{array}{r}.00733 \\
.01207 \\
.01077\end{array}$ & $\begin{array}{l}.02932 \\
.04828 \\
.04310\end{array}$ & $\begin{array}{l}10 \cdot 446 \\
17 \cdot 200 \\
15 \cdot 356\end{array}$ & $\begin{array}{l}31.840 \\
52 \cdot 427 \\
46.806\end{array}$ & $\begin{array}{l}35.977 \\
59.239 \\
52.888\end{array}$ \\
\hline $\begin{array}{l}22 \\
22 \\
22\end{array}$ & $\begin{array}{l}5940 \\
5940 \\
5940\end{array}$ & $\begin{array}{l}20.0 \\
25.0 \\
30.0\end{array}$ & $\begin{array}{l}.02661 \\
.02128 \\
.01753\end{array}$ & $\begin{array}{r}.10646 \\
.08515 \\
.07012\end{array}$ & $\begin{array}{l}.00811 \\
.00648 \\
.00534\end{array}$ & $\begin{array}{r}.03245 \\
.02595 \\
.02137\end{array}$ & $\begin{array}{r}11.559 \\
9.245 \\
7.614\end{array}$ & $\begin{array}{l}35 \cdot 234 \\
28.179 \\
23.208\end{array}$ & $\begin{array}{l}39.812 \\
31.841 \\
26.224\end{array}$ \\
\hline $\begin{array}{l}22 \\
22 \\
22\end{array}$ & $\begin{array}{l}5940 \\
5940 \\
5940\end{array}$ & $\begin{array}{l}35.0 \\
40.0 \\
45.0\end{array}$ & $\begin{array}{r}.01501 \\
.01316 \\
.01180\end{array}$ & $\begin{array}{l}.06005 \\
.05264 \\
.04722\end{array}$ & $\begin{array}{l}.00457 \\
.00401 \\
.00359\end{array}$ & $\begin{array}{l}.01830 \\
.01604 \\
.01439\end{array}$ & $\begin{array}{l}6.520 \\
5.716 \\
5.127\end{array}$ & $\begin{array}{l}19.875 \\
17.423 \\
15.628\end{array}$ & $\begin{array}{l}22.457 \\
19.687 \\
17.659\end{array}$ \\
\hline $\begin{array}{l}22 \\
22 \\
22\end{array}$ & $\begin{array}{l}5940 \\
5940 \\
5940\end{array}$ & $\begin{array}{l}46.0 \\
47.0 \\
48.0\end{array}$ & $\begin{array}{r}.01158 \\
.01139 \\
.01121\end{array}$ & $\begin{array}{r}.04634 \\
.04558 \\
.04485\end{array}$ & $\begin{array}{l}.00353 \\
.00347 \\
.00341\end{array}$ & $\begin{array}{r}.01412 \\
.01389 \\
.01367\end{array}$ & $\begin{array}{l}5.032 \\
4.949 \\
4.869\end{array}$ & $\begin{array}{l}15 \cdot 337 \\
15.085 \\
14 \cdot 843\end{array}$ & $\begin{array}{l}17.330 . \\
17.046 \\
16.772\end{array}$ \\
\hline $\begin{array}{l}22 \\
22 \\
22\end{array}$ & $\begin{array}{l}5940 \\
5940 \\
5940\end{array}$ & $\begin{array}{l}49.0 \\
50.0 \\
51.0\end{array}$ & $\begin{array}{l}.01101 \\
.01081 . \\
.01064\end{array}$ & $\begin{array}{r}.04406 \\
.04326 \\
.04259\end{array}$ & $\begin{array}{l}.00335 \\
.00329 \\
.00324\end{array}$ & $\begin{array}{r}.01343 \\
.01318 \\
.01298\end{array}$ & $\begin{array}{l}4.784 \\
4.697 \\
4.624\end{array}$ & $\begin{array}{l}14 \cdot 584 \\
14 \cdot 317 \\
14.095\end{array}$ & $\begin{array}{l}16.479 \\
16.178 \\
15.926\end{array}$ \\
\hline $\begin{array}{l}22 \\
22 \\
22\end{array}$ & $\begin{array}{l}5940 \\
5940 \\
5940\end{array}$ & $\begin{array}{l}52.0 \\
53.0 \\
54.0\end{array}$ & $\begin{array}{l}.01050 \\
.01036 \\
.01023\end{array}$ & $\begin{array}{r}.04200 \\
.04145 \\
.04094\end{array}$ & $\begin{array}{l}.00320 \\
.00315 \\
.00311\end{array}$ & $\begin{array}{r}.01280 \\
.01263 \\
.01247\end{array}$ & $\begin{array}{l}4.560 \\
4.501 \\
4.445\end{array}$ & $\begin{array}{l}13.901 \\
13.719 \\
13.550\end{array}$ & $\begin{array}{l}15.707 \\
15.502 \\
15.310\end{array}$ \\
\hline $\begin{array}{l}22 \\
22 \\
22\end{array}$ & $\begin{array}{l}5940 \\
5940 \\
5940\end{array}$ & $\begin{array}{l}55.0 \\
60.0 \\
65.0\end{array}$ & $\begin{array}{r}.01012 \\
.00968 \\
.00928\end{array}$ & $\begin{array}{r}.04049 \\
.03874 \\
.03715\end{array}$ & $\begin{array}{l}.00308 \\
.00295 \\
.00283\end{array}$ & $\begin{array}{l}.01234 \\
.01181 \\
.01132\end{array}$ & $\begin{array}{l}4.396 \\
4.207 \\
4.034\end{array}$ & $\begin{array}{l}13.399 \\
12.823 \\
12.296\end{array}$ & $\begin{array}{l}15.141 \\
14.489 \\
13.893\end{array}$ \\
\hline $\begin{array}{l}22 \\
22 \\
22\end{array}$ & $\begin{array}{l}5940 \\
5940 \\
5940\end{array}$ & $\begin{array}{l}70.0 \\
75.0 \\
80.0\end{array}$ & $\begin{array}{l}.00904 \\
.00885 \\
.00867\end{array}$ & $\begin{array}{l}.03617 \\
.03542 \\
.03468\end{array}$ & $\begin{array}{l}.00275 \\
.00269 \\
.00264\end{array}$ & $\begin{array}{l}.01102 \\
.01079 \\
.01057\end{array}$ & $\begin{array}{l}3.927 \\
3.845 \\
3.766\end{array}$ & $\begin{array}{l}11.970 \\
11.722 \\
11.479\end{array}$ & $\begin{array}{l}13.525 \\
13.245 \\
12.971\end{array}$ \\
\hline $\begin{array}{l}22 \\
22 \\
22\end{array}$ & $\begin{array}{l}5940 \\
5940 \\
5940\end{array}$ & $\begin{array}{l}85.0 \\
90.0 \\
95.0\end{array}$ & $\begin{array}{l}.00851 \\
.00836 \\
.00822\end{array}$ & $\begin{array}{l}.03406 \\
.03347 \\
.03290\end{array}$ & $\begin{array}{l}.00259 \\
.00255 \\
.00250\end{array}$ & $\begin{array}{l}.01038 \\
.01020 \\
.01002\end{array}$ & $\begin{array}{l}3.698 \\
3.634 \\
3.572\end{array}$ & $\begin{array}{l}11.272 \\
11.079 \\
10.888\end{array}$ & $\begin{array}{l}12.737 \\
12.518 \\
12.303\end{array}$ \\
\hline 22 & 5940 & 100.0 & .00810 & .03241 & .00246 & .00987 & 3.519 & 10.726 & 12.120 \\
\hline
\end{tabular}


TABULATIEN OF DATA FOR

AOSERPTION OF SOUND IN AIR VERSUS PERCENT RELATIVE HUMIDITY AT 22 DEGREES CENTIGRADE FOR A FREQUENCY OF 6300 HERTZ

\begin{tabular}{|c|c|c|c|c|c|c|c|c|c|}
\hline (1) & (2) & (3) & 141 & (5) & $(6)$ & 171 & $(8)$ & (9) & 1101 \\
\hline $\begin{array}{l}\text { TEMP } \\
\text { DEGR } \\
\text { CENT }\end{array}$ & HERTZ & $\begin{array}{l}\text { REL } \\
\text { HUM } \\
\text { PER } \\
\text { CENT }\end{array}$ & $\begin{array}{l}\text { ATTEN } \\
\text { COEF } \\
\text { PER } \\
\text { METER }\end{array}$ & $\begin{array}{l}\text { 4M } \\
\text { PER } \\
\text { METER }\end{array}$ & $\begin{array}{l}\text { ATTEN } \\
\text { COEF } \\
\text { PER } \\
\text { FOOT }\end{array}$ & $\begin{array}{l}4 M \\
\text { PER } \\
\text { FEOT }\end{array}$ & $\begin{array}{l}\text { ATTEN } \\
\text { DB PER } \\
100 \\
\text { METER }\end{array}$ & $\begin{array}{l}\text { ATTEN } \\
\text { DB PER } \\
1 \text { OOO } \\
\text { FEET }\end{array}$ & $\begin{array}{l}\text { DECAY } \\
\text { RATE } \\
\text { DB PER } \\
\text { SECOND }\end{array}$ \\
\hline $\begin{array}{l}22 \\
22 \\
22\end{array}$ & $\begin{array}{l}6300 \\
6300 \\
6300\end{array}$ & $\begin{array}{r}5.0 \\
10.0 \\
15.0\end{array}$ & $\begin{array}{r}.02490 \\
.04194 \\
.03837\end{array}$ & $\begin{array}{r}.09961 \\
.16777 \\
.15350\end{array}$ & $\begin{array}{l}.00759 \\
.01278 \\
.01169\end{array}$ & $\begin{array}{l}.03036 \\
.05113 \\
.04678\end{array}$ & $\begin{array}{l}10.816 \\
18.216 \\
16.667\end{array}$ & $\begin{array}{l}32.968 \\
55.524 \\
50.802\end{array}$ & $\begin{array}{l}37.252 \\
62.739 \\
57.403\end{array}$ \\
\hline $\begin{array}{l}22 \\
22 \\
22\end{array}$ & $\begin{array}{l}6300 \\
6300 \\
6300\end{array}$ & $\begin{array}{l}20.0 \\
25.0 \\
30.0\end{array}$ & $\begin{array}{l}.02917 \\
.02330 \\
.01923\end{array}$ & $\begin{array}{r}.11668 \\
.09323 \\
.07693\end{array}$ & $\begin{array}{l}.00889 \\
.00710 \\
.00586\end{array}$ & $\begin{array}{l}.03556 \\
.02841 \\
.02344\end{array}$ & $\begin{array}{r}12.669 \\
10.123 \\
8.352\end{array}$ & $\begin{array}{l}38.615 \\
30.855 \\
25.460\end{array}$ & $\begin{array}{l}43.633 \\
34.865 \\
28.768\end{array}$ \\
\hline $\begin{array}{l}22 \\
22 \\
22\end{array}$ & $\begin{array}{l}6300 \\
6300 \\
6300\end{array}$ & $\begin{array}{l}35.0 \\
40.0 \\
45.0\end{array}$ & $\begin{array}{r}.01644 \\
.01443 \\
.01291\end{array}$ & $\begin{array}{r}.06579 \\
.05772 \\
.05164\end{array}$ & $\begin{array}{l}.00501 \\
.00439 \\
.00393\end{array}$ & $\begin{array}{l}.02005 \\
.01759 \\
.01574\end{array}$ & $\begin{array}{l}.143 \\
.267 \\
.607\end{array}$ & $\begin{array}{l}773 \\
105 \\
090\end{array}$ & $\begin{array}{l}24.602 \\
21.587 \\
19.311\end{array}$ \\
\hline $\begin{array}{l}22 \\
22 \\
22\end{array}$ & $\begin{array}{l}6300 \\
6300 \\
6300\end{array}$ & $\begin{array}{l}46.0 \\
47.0 \\
48.0\end{array}$ & $\begin{array}{r}.01267 \\
.01244 \\
.01223\end{array}$ & $\begin{array}{r}.05068 \\
.04977 \\
.04894\end{array}$ & $\begin{array}{l}.00386 \\
.00379 \\
.00372\end{array}$ & $\begin{array}{r}.01544 \\
.01517 \\
.01491\end{array}$ & $\begin{array}{l}5.502 \\
5.404 \\
5.314\end{array}$ & $\begin{array}{l}16 \cdot 772 \\
16 \cdot 473 \\
16 \cdot 197\end{array}$ & $\begin{array}{l}18.952 \\
18.613 \\
18.302\end{array}$ \\
\hline $\begin{array}{l}22 \\
22 \\
22\end{array}$ & $\begin{array}{l}6300 \\
6300 \\
6300\end{array}$ & $\begin{array}{l}49.0 \\
50.0 \\
51.0\end{array}$ & $\begin{array}{r}.01204 \\
.01185 \\
.01164\end{array}$ & $\begin{array}{r}.04818 \\
.04740 \\
.04657\end{array}$ & $\begin{array}{l}.00367 \\
.00361 \\
.00354\end{array}$ & $\begin{array}{r}.01468 \\
.01445 \\
.01419\end{array}$ & $\begin{array}{l}5.232 \\
5.147 \\
5.057\end{array}$ & $\begin{array}{l}15.947 \\
15.689 \\
15.414\end{array}$ & $\begin{array}{l}18.019 \\
17.728 \\
17.417\end{array}$ \\
\hline $\begin{array}{l}22 \\
22 \\
22\end{array}$ & $\begin{array}{l}6300 \\
6300 \\
6300\end{array}$ & $\begin{array}{l}52.0 \\
53.0 \\
54.0\end{array}$ & $\begin{array}{l}.01144 \\
.01129 \\
.01114\end{array}$ & $\begin{array}{l}.04578 \\
.04518 \\
.04457\end{array}$ & $\begin{array}{l}.00348 \\
.00344 \\
.00339\end{array}$ & $\begin{array}{l}.01395 \\
.01377 \\
.01358\end{array}$ & & $\begin{array}{l}15.152 \\
14.952 \\
14.752\end{array}$ & $\begin{array}{l}17.121 \\
16.895 \\
16.669\end{array}$ \\
\hline $\begin{array}{l}22 \\
22 \\
22\end{array}$ & $\begin{array}{l}6300 \\
6300 \\
6300\end{array}$ & $\begin{array}{l}55.0 \\
60.0 \\
65.0\end{array}$ & $\begin{array}{r}.01101 \\
.01049 \\
.01006\end{array}$ & $\begin{array}{r}.04404 \\
.04198 \\
.04027\end{array}$ & $\begin{array}{l}.00335 \\
.00319 \\
.00306\end{array}$ & $\begin{array}{r}.01342 \\
.01279 \\
.01227\end{array}$ & $\begin{array}{l}32 \\
8 \\
3\end{array}$ & $\begin{array}{l}77 \\
95 \\
29\end{array}$ & $\begin{array}{l}471 \\
700 \\
061\end{array}$ \\
\hline $\begin{array}{l}22 \\
22 \\
22\end{array}$ & $\begin{array}{l}6300 \\
6300 \\
6300\end{array}$ & $\begin{array}{l}70.0 \\
75.0 \\
80.0\end{array}$ & $\begin{array}{l}.00976 \\
.00955 \\
.00936\end{array}$ & $\begin{array}{l}.03904 \\
.03822 \\
.03747\end{array}$ & $\begin{array}{l}.00297 \\
.00291 \\
.00285\end{array}$ & $\begin{array}{l}.01 \\
.01 \\
.01\end{array}$ & $\begin{array}{l}39 \\
50 \\
68\end{array}$ & $\begin{array}{l}12.921 \\
12.651 \\
12.401\end{array}$ & $\begin{array}{l}600 \\
295 \\
012\end{array}$ \\
\hline $\begin{array}{l}22 \\
22 \\
22\end{array}$ & $\begin{array}{l}6300 \\
6300 \\
6300\end{array}$ & $\begin{array}{l}85.0 \\
90.0 \\
95.0\end{array}$ & $\begin{array}{l}.00918 \\
.00903 \\
.00888\end{array}$ & $\begin{array}{l}.03675 \\
.03615 \\
.03554\end{array}$ & $\begin{array}{l}.00280 \\
.00275 \\
.00270\end{array}$ & $\begin{array}{l}.01120 \\
.01101 \\
.01083\end{array}$ & $\begin{array}{l}3.990 \\
3.925 \\
3.859\end{array}$ & $\begin{array}{l}12.164 \\
11.964 \\
11.764\end{array}$ & $\begin{array}{l}13.745 \\
13.519 \\
13.293\end{array}$ \\
\hline 22 & 63001 & $100 \cdot 0$ & .00875 & .03500 & .00266 & .01066 & 3.800 & 11.583 & 13.088 \\
\hline
\end{tabular}


TABULATION OF DATA FOR

ABSORPTION OF SOUND IN AIR VERSUS PERCENT RELATIVE HUMIDITY AT 22 DEGREES CENTIGRADE FOR A FREQUENCY OF 8000 HERTZ

\begin{tabular}{|c|c|c|c|c|c|c|c|c|c|}
\hline (1) & (2) & (3) & $(4)$ & (5) & (6) & (7) & $(8)$ & 191 & $(10)$ \\
\hline TEMP & FREQ & $\begin{array}{l}\text { REL } \\
\text { HUM }\end{array}$ & $\begin{array}{l}\text { ATTEN } \\
\text { COEF }\end{array}$ & $4 M$ & $\begin{array}{l}\text { ATTEN } \\
\text { COEF }\end{array}$ & $4 M$ & $\begin{array}{l}\text { ATTEN } \\
\text { DB PER }\end{array}$ & $\begin{array}{l}\text { ATTEN } \\
\text { DB PER }\end{array}$ & $\begin{array}{l}\text { DECAY } \\
\text { RATE }\end{array}$ \\
\hline $\begin{array}{l}\text { DEGR } \\
\text { CENT }\end{array}$ & HERT:Z & $\begin{array}{l}\text { PER } \\
\text { CENT }\end{array}$ & $\begin{array}{l}\text { PER } \\
\text { METER }\end{array}$ & $\begin{array}{l}\text { PER } \\
\text { METER }\end{array}$ & $\begin{array}{l}\text { PER } \\
\text { FOOT }\end{array}$ & $\begin{array}{l}\text { PER } \\
\text { FOOT }\end{array}$ & $\begin{array}{l}100 \\
\text { METER }\end{array}$ & $\begin{array}{l}1000 \\
\text { FEET }\end{array}$ & $\begin{array}{l}\text { DB PER } \\
\text { SECOND }\end{array}$ \\
\hline $\begin{array}{l}22 \\
22 \\
22\end{array}$ & $\begin{array}{l}8000 \\
8000 \\
8000\end{array}$ & $\begin{array}{r}5.0 \\
10.0 \\
15.0\end{array}$ & $\begin{array}{l}.02765 \\
.05120 \\
.05310\end{array}$ & $\begin{array}{r}.11062 \\
.20482 \\
.21240\end{array}$ & $\begin{array}{r}.00842 \\
.01560 \\
.01618\end{array}$ & $\begin{array}{l}.03371 \\
.06243 \\
.06474\end{array}$ & $\begin{array}{l}12.011 \\
22.239 \\
23.061\end{array}$ & $\begin{array}{l}36.610 \\
67.786 \\
70.293\end{array}$ & $\begin{array}{l}41.368 \\
76.594 \\
79.427\end{array}$ \\
\hline $\begin{array}{l}22 \\
22 \\
22\end{array}$ & $\begin{array}{l}8000 \\
8000 \\
8000\end{array}$ & $\begin{array}{l}20.0 \\
25.0 \\
30.0\end{array}$ & $\begin{array}{l}.04444 \\
.03523 \\
.02951\end{array}$ & $\begin{array}{r}.17779 \\
.14095 \\
.11805\end{array}$ & $\begin{array}{l}.01354 \\
.01074 \\
.00899\end{array}$ & $\begin{array}{l}.05419 \\
.04296 \\
.03598\end{array}$ & $\begin{array}{l}19.303 \\
15.303 \\
12.817\end{array}$ & $\begin{array}{l}58.838 \\
46.646 \\
39.069\end{array}$ & $\begin{array}{l}66.484 \\
52.707 \\
44.146\end{array}$ \\
\hline $\begin{array}{l}22 \\
22 \\
22\end{array}$ & $\begin{array}{l}8000 \\
8000 \\
8000\end{array}$ & $\begin{array}{l}35.0 \\
40.0 \\
45.0\end{array}$ & $\begin{array}{l}.02515 \\
.02198 \\
.01968\end{array}$ & $\begin{array}{r}.10061 \\
.08792 \\
.07875\end{array}$ & $\begin{array}{l}.00766 \\
.00670 \\
.00600\end{array}$ & $\begin{array}{r}.03066 \\
.02680 \\
.02400\end{array}$ & $\begin{array}{r}10.923 \\
9.546 \\
8.550\end{array}$ & $\begin{array}{l}33.296 \\
29.098 \\
26.062\end{array}$ & $\begin{array}{l}37.623 \\
32.879 \\
29.448\end{array}$ \\
\hline $\begin{array}{l}22 \\
22 \\
22\end{array}$ & $\begin{array}{l}8000 \\
8000 \\
8000\end{array}$ & $\begin{array}{l}46.0 \\
47.0 \\
48.0\end{array}$ & $\begin{array}{r}.01925 \\
.01884 \\
.01847\end{array}$ & $\begin{array}{l}.07700 \\
.07537 \\
.07390\end{array}$ & $\begin{array}{l}.00586 \\
.00574 \\
.00563\end{array}$ & $\begin{array}{l}.02347 \\
.02297 \\
.02252\end{array}$ & $\begin{array}{l}8.360 \\
8.183 \\
8.024\end{array}$ & $\begin{array}{l}25 \cdot 483 \\
24 \cdot 945 \\
24 \cdot 459\end{array}$ & $\begin{array}{l}28.795 \\
28.186 \\
27.637\end{array}$ \\
\hline $\begin{array}{l}22 \\
22 \\
22\end{array}$ & $\begin{array}{l}8000 \\
8000 \\
8000\end{array}$ & $\begin{array}{l}49.0 \\
50.0 \\
51.0\end{array}$ & $\begin{array}{r}.01811 \\
.01776 \\
.01744\end{array}$ & $\begin{array}{l}.07244 \\
.07105 \\
.06977\end{array}$ & $\begin{array}{l}.00552 \\
.00541 \\
.00531\end{array}$ & $\begin{array}{l}.02208 \\
.02165 \\
.02126\end{array}$ & $\begin{array}{l}7.865 \\
7.714 \\
7.576\end{array}$ & $\begin{array}{l}23.973 \\
23.513 \\
23.092\end{array}$ & $\begin{array}{l}27.089 \\
26.569 \\
26.093\end{array}$ \\
\hline $\begin{array}{l}22 \\
22 \\
22\end{array}$ & $\begin{array}{l}8000 \\
8000 \\
8000\end{array}$ & $\begin{array}{l}52.0 \\
53.0 \\
54.0\end{array}$ & $\begin{array}{r}.01715 \\
.01687 \\
.01661\end{array}$ & $\begin{array}{l}.06863 \\
.06749 \\
.06646\end{array}$ & $\begin{array}{l}.00523 \\
.00514 \\
.00506\end{array}$ & $\begin{array}{l}.02092 \\
.02057 \\
.02025\end{array}$ & $\begin{array}{l}7.452 \\
7.328 \\
7.216\end{array}$ & $\begin{array}{l}22.715 \\
22.337 \\
21.995\end{array}$ & $\begin{array}{l}25.666 \\
25.240 \\
24.853\end{array}$ \\
\hline $\begin{array}{l}22 \\
22 \\
22\end{array}$ & $\begin{array}{l}8000 \\
8000 \\
8000\end{array}$ & $\begin{array}{l}55.0 \\
60.0 \\
65.0\end{array}$ & $\begin{array}{r}.01637 \\
.01528 \\
.01444\end{array}$ & $\begin{array}{l}.06548 \\
.06113 \\
.05776\end{array}$ & $\begin{array}{l}.00498 \\
.00465 \\
.00440\end{array}$ & $\begin{array}{l}.01995 \\
.01863 \\
.01760\end{array}$ & $\begin{array}{l}7.110 \\
6.638 \\
6.271\end{array}$ & $\begin{array}{l}21.671 \\
20.232 \\
19.116\end{array}$ & $\begin{array}{l}24.487 \\
22.861 \\
21.600\end{array}$ \\
\hline $\begin{array}{l}22 \\
22 \\
22\end{array}$ & $\begin{array}{l}8000 \\
8000 \\
8000\end{array}$ & $\begin{array}{l}70.0 \\
75.0 \\
80.0\end{array}$ & $\begin{array}{r}.01388 \\
.01343 \\
.01303\end{array}$ & $\begin{array}{l}.05555 \\
.05372 \\
.05212\end{array}$ & $\begin{array}{l}.00423 \\
.00409 \\
.00397\end{array}$ & $\begin{array}{l}.01693 \\
.01637 \\
.01588\end{array}$ & $\begin{array}{l}6.032 \\
5.833 \\
5.659\end{array}$ & $\begin{array}{l}18.386 \\
17.780 \\
17.249\end{array}$ & $\begin{array}{l}20.775 \\
20.090 \\
19.490\end{array}$ \\
\hline $\begin{array}{l}22 \\
22 \\
22\end{array}$ & $\begin{array}{l}8000 \\
8000 \\
8000\end{array}$ & $\begin{array}{l}85.0 \\
90.0 \\
95.0\end{array}$ & $\begin{array}{r}.01277 \\
.01256 \\
.01236\end{array}$ & $\begin{array}{l}.05109 \\
.05026 \\
.04944\end{array}$ & $\begin{array}{l}.00389 \\
.00383 \\
.00376\end{array}$ & $\begin{array}{l}.01557 \\
.01532 \\
.01507\end{array}$ & $\begin{array}{l}5.547 \\
5.457 \\
5.368\end{array}$ & $\begin{array}{l}16.909 \\
16.634 \\
16.364\end{array}$ & $\begin{array}{l}19.106 \\
18.795 \\
18.490\end{array}$ \\
\hline 22 & 8000 & 100.0 & .01217 & .04868 & .00370 & .01483 & 5.285 & 16.111 & $18 \cdot 205$ \\
\hline
\end{tabular}


TABULATION OF DATA FOR

ABSORPTION OF SOUND IN AIR VERSUS PERCENT RELATIVE HUMIDITY AT 22 DEGREES CENTIGRADE FOR A FREQUENCY OF 10000 HERTZ

\begin{tabular}{|c|c|c|c|c|c|c|c|c|c|}
\hline 11 & (2) & (3) & (4) & (5) & (6) & $(7)$ & $(8)$ & 191 & $(10)$ \\
\hline $\begin{array}{l}\text { TEMP } \\
\text { DEGR } \\
\text { CENT }\end{array}$ & HERTZ & $\begin{array}{l}\text { REL } \\
\text { HUM } \\
\text { PER } \\
\text { CENT }\end{array}$ & $\begin{array}{l}\text { ATTEN } \\
\text { COEF } \\
\text { PER } \\
\text { METER }\end{array}$ & $\begin{array}{l}4 M \\
\text { PER } \\
\text { METER }\end{array}$ & $\begin{array}{l}\text { ATTEN } \\
\text { COEF } \\
\text { PER } \\
\text { FOOT }\end{array}$ & $\begin{array}{l}4 M \\
\text { PER } \\
\text { FOOT }\end{array}$ & $\begin{array}{l}\text { ATTEN } \\
\text { DB PER } \\
100 \\
\text { METER. }\end{array}$ & $\begin{array}{l}\text { ATTEN } \\
\text { DB PER } \\
\text { IOOO } \\
\text { FEET }\end{array}$ & $\begin{array}{l}\text { DECAY } \\
\text { RATE } \\
\text { DB PER } \\
\text { SECOND }\end{array}$ \\
\hline $\begin{array}{l}22 \\
22 \\
22\end{array}$ & $\begin{array}{l}10000 \\
10000 \\
10000\end{array}$ & $\begin{array}{r}5.0 \\
10.0 \\
15.0\end{array}$ & $\begin{array}{l}.03140 \\
.05986 \\
.06835\end{array}$ & $\begin{array}{r}.12561 \\
.23944 \\
.27340\end{array}$ & $\begin{array}{l}.00957 \\
.01824 \\
.02083\end{array}$ & $\begin{array}{l}.03828 \\
.07298 \\
.08333\end{array}$ & $\begin{array}{l}13.639 \\
25.997 \\
29.684\end{array}$ & $\begin{array}{l}41.572 \\
79.242 \\
90.480\end{array}$ & $\begin{array}{r}46.974 \\
89.539 \\
102.237\end{array}$ \\
\hline $\begin{array}{l}22 \\
22 \\
22\end{array}$ & $\begin{array}{l}10000 \\
10000 \\
10000\end{array}$ & $\begin{array}{l}20.0 \\
25.0 \\
30.0\end{array}$ & $\begin{array}{l}.06258 \\
.05153 \\
.04262\end{array}$ & $\begin{array}{l}.25034 \\
.20613 \\
.17048\end{array}$ & $\begin{array}{l}.01907 \\
.01570 \\
.01299\end{array}$ & $\begin{array}{l}.07630 \\
.06282 \\
.05196\end{array}$ & $\begin{array}{l}27.180 \\
22.380 \\
18.509\end{array}$ & $\begin{array}{l}82.848 \\
68.217 \\
56.419\end{array}$ & $\begin{array}{l}93.614 \\
77.081 \\
63.750\end{array}$ \\
\hline $\begin{array}{ll}22 & 3 \\
22 & 1 \\
22 & 3\end{array}$ & $\begin{array}{l}10000 \\
10000 \\
10000\end{array}$ & $\begin{array}{l}35.0 \\
40.0 \\
45.0\end{array}$ & $\begin{array}{l}.03685 \\
.03216 \\
.02862\end{array}$ & $\begin{array}{r}.14742 \\
.12864 \\
.11448\end{array}$ & $\begin{array}{l}.01123 \\
.00980 \\
.00872\end{array}$ & $\begin{array}{l}.04493 \\
.03921 \\
.03489\end{array}$ & $\begin{array}{l}16.006 \\
13.967 \\
12.429\end{array}$ & $\begin{array}{l}48.787 \\
42.573 \\
37.886\end{array}$ & $\begin{array}{l}55 \cdot 127 \\
48 \cdot 105 \\
42.809\end{array}$ \\
\hline $\begin{array}{l}22 \\
22 \\
22\end{array}$ & $\begin{array}{l}10000 \\
10000 \\
10000\end{array}$ & $\begin{array}{l}46.0 \\
47.0 \\
48.0\end{array}$ & $\begin{array}{l}.02804 \\
.02748 \\
.02695\end{array}$ & $\begin{array}{l}.11216 \\
.10994 \\
.10783\end{array}$ & $\begin{array}{l}.00854 \\
.00837 \\
.00821\end{array}$ & $\begin{array}{l}.03418 \\
.03351 \\
.03286\end{array}$ & $\begin{array}{l}12.177 \\
11.937 \\
11.708\end{array}$ & $\begin{array}{l}37.118 \\
36.386 \\
35.686\end{array}$ & $\begin{array}{l}41.942 \\
41 \cdot 114 \\
40.323\end{array}$ \\
\hline $\begin{array}{l}22 \\
22 \\
22\end{array}$ & $\begin{array}{l}10000 \\
10000 \\
10000\end{array}$ & $\begin{array}{l}49.0 \\
50.0 \\
51.0\end{array}$ & $\begin{array}{l}.02646 \\
.02600 \\
.02555\end{array}$ & $\begin{array}{r}.10587 \\
.10403 \\
.10220\end{array}$ & $\begin{array}{l}.00806 \\
.00792 \\
.00778\end{array}$ & $\begin{array}{r}.03226 \\
.03170 \\
.03115\end{array}$ & $\begin{array}{l}11.494 \\
11.295 \\
11.096\end{array}$ & $\begin{array}{l}35.036 \\
34.428 \\
33.822\end{array}$ & $\begin{array}{l}39.589 \\
38.901 \\
38.217\end{array}$ \\
\hline $\begin{array}{l}22 \\
22\end{array}$ & $\begin{array}{l}10000 \\
10000\end{array}$ & $\begin{array}{l}52.0 \\
53.0\end{array}$ & $\begin{array}{l}.02505 \\
.02460\end{array}$ & $\begin{array}{r}10023 \\
.09841\end{array}$ & $\begin{array}{l}.00763 \\
.00749\end{array}$ & $\begin{array}{l}.03055 \\
.02999\end{array}$ & $\begin{array}{l}10.883 \\
10.685\end{array}$ & $\begin{array}{l}33.173 \\
32.570\end{array}$ & $\begin{array}{l}37.483 \\
36.802\end{array}$ \\
\hline 22 & 10000 & 54.0 & .02417 & .09669 & .00736 & .02947 & 10.498 & 32.000 & 36.158 \\
\hline $\begin{array}{l}22 \\
22 \\
22\end{array}$ & $\begin{array}{l}10000 \\
10000 \\
10000\end{array}$ & $\begin{array}{l}55.0 \\
60.0 \\
65.0\end{array}$ & $\begin{array}{l}.02377 \\
.02200 \\
.02068\end{array}$ & $\begin{array}{l}.09508 \\
.08803 \\
.08272\end{array}$ & $\begin{array}{l}.00724 \\
.00670 \\
.00630\end{array}$ & $\begin{array}{l}.02898 \\
.02683 \\
.02521\end{array}$ & $\begin{array}{r}10.324 \\
9.557 \\
8.982\end{array}$ & $\begin{array}{l}31.469 \\
29.133 \\
27.378\end{array}$ & $\begin{array}{l}35.558 \\
32.918 \\
30.936\end{array}$ \\
\hline $\begin{array}{l}22 \\
22 \\
22\end{array}$ & $\begin{array}{l}10000 \\
10000 \\
10000\end{array}$ & $\begin{array}{l}70.0 \\
75.0 \\
80.0\end{array}$ & $\begin{array}{r}.01952 \\
.01868 \\
.01810\end{array}$ & $\begin{array}{l}.07808 \\
.07473 \\
.07243\end{array}$ & $\begin{array}{l}.00594 \\
.00569 \\
.00551\end{array}$ & $\begin{array}{l}.02379 \\
.02277 \\
.02207\end{array}$ & $\begin{array}{l}8.477 \\
8.114 \\
7.864\end{array}$ & $\begin{array}{l}25.840 \\
24.732 \\
23.970\end{array}$ & $\begin{array}{l}29.197 \\
27.946 \\
27.085\end{array}$ \\
\hline $\begin{array}{l}22 \\
22 \\
22\end{array}$ & $\begin{array}{l}10000 \\
10000 \\
10000\end{array}$ & $\begin{array}{l}85.0 \\
90.0 \\
95.0\end{array}$ & $\begin{array}{r}.01761 \\
.01713 \\
.01682\end{array}$ & $\begin{array}{r}.07045 \\
.06855 \\
.06730\end{array}$ & $\begin{array}{l}.00536 \\
.00522 \\
.00512\end{array}$ & $\begin{array}{l}.02147 \\
.02089 \\
.02051\end{array}$ & $\begin{array}{l}7.650 \\
7.443 \\
7.307\end{array}$ & $\begin{array}{l}23.317 \\
22.687 \\
22.274\end{array}$ & $\begin{array}{l}26.347 \\
25.635 \\
25.168\end{array}$ \\
\hline 22 & 10 & & .01658 & .06634 & .00505 & .02022 & $7 \cdot 202$ & 1.954 & 24.807 \\
\hline
\end{tabular}


TABULATION OF DATA FOR

ABSORPTION OF SOUND IN AIR VERSUS PERCENT RELATIVE HUMIDITY AT 22 DEGREES CENTIGRADE FOR A FREQUENCY OF 12500 HERTZ

\begin{tabular}{|c|c|c|c|c|c|c|c|c|c|}
\hline 111 & (2) & (3) & (4) & (5) & (6) & 171 & $(8)$ & (9) & $(10)$ \\
\hline $\begin{array}{l}\text { TEMP } \\
\text { DEGR } \\
\text { CENT }\end{array}$ & $\begin{array}{l}\text { FREQ } \\
\text { HERTZ }\end{array}$ & $\begin{array}{l}\text { REL } \\
\text { HUM } \\
\text { PER } \\
\text { CENT }\end{array}$ & $\begin{array}{l}\text { ATTEN } \\
\text { COEF } \\
\text { PER } \\
\text { METER }\end{array}$ & $\begin{array}{l}4 M \\
\text { PER } \\
\text { METER }\end{array}$ & $\begin{array}{l}\text { ATTEN } \\
\text { COEF } \\
\text { PER } \\
\text { FOOT }\end{array}$ & $\begin{array}{l}4 M \\
\cdot \\
\text { PER } \\
\text { FOOT }\end{array}$ & $\begin{array}{l}\text { ATTEN } \\
\text { DB PER } \\
100 \\
\text { METER }\end{array}$ & $\begin{array}{l}\text { ATTEN } \\
\text { DB PER } \\
1000 \\
\text { FEET }\end{array}$ & $\begin{array}{l}\text { DECAY } \\
\text { RATE } \\
\text { DB PER } \\
\text { SECOND }\end{array}$ \\
\hline $\begin{array}{l}22 \\
22 \\
22\end{array}$ & $\begin{array}{l}12500 \\
12500 \\
12500\end{array}$ & $\begin{array}{r}5.0 \\
10.0 \\
15.0\end{array}$ & $\begin{array}{r}.03586 \\
.06709 \\
.08578\end{array}$ & $\begin{array}{r}.14347 \\
.26839 \\
.34315\end{array}$ & $\begin{array}{l}.01093 \\
.02045 \\
.02614\end{array}$ & $\begin{array}{r}.04373 \\
.08180 \\
.10459\end{array}$ & $\begin{array}{l}15.578 \\
29.140 \\
37.258\end{array}$ & $\begin{array}{r}47.482 \\
88.822 \\
113.563\end{array}$ & $\begin{array}{r}53 \\
100 \\
128\end{array}$ \\
\hline $\begin{array}{l}22 \\
22 \\
22\end{array}$ & $\begin{array}{l}12500 \\
12500 \\
12500\end{array}$ & $\begin{array}{l}20.0 \\
25.0 \\
30.0\end{array}$ & $\begin{array}{l}.08481 \\
.07470 \\
.06260\end{array}$ & $\begin{array}{l}.33926 \\
.29883 \\
.25041\end{array}$ & $\begin{array}{l}.02585 \\
.02277 \\
.01908\end{array}$ & $\begin{array}{l}.10340 \\
.09108 \\
.07632\end{array}$ & $\begin{array}{l}36.83 .5 \\
32.446 \\
27.189\end{array}$ & $\begin{array}{r}112.277 \\
98.896 \\
82.873\end{array}$ & $\begin{array}{l}.866 \\
.747 \\
.642\end{array}$ \\
\hline $\begin{array}{l}22 \\
22 \\
22\end{array}$ & $\begin{array}{l}12500 \\
12500 \\
12500\end{array}$ & $\begin{array}{l}35.0 \\
40.0 \\
45.0\end{array}$ & $\begin{array}{r}.05374 \\
.04758 \\
.04240\end{array}$ & $\begin{array}{r}.21499 \\
.19035 \\
.16962\end{array}$ & $\begin{array}{l}.01638 \\
.01450 \\
.01292\end{array}$ & $\begin{array}{l}.06553 \\
.05801 \\
.05170\end{array}$ & & $\begin{array}{l}71.151 \\
62.994 \\
56.135\end{array}$ & $\begin{array}{l}80.397 \\
71.180 \\
63.429\end{array}$ \\
\hline $\begin{array}{l}22 \\
22 \\
22\end{array}$ & $\begin{array}{l}12500 \\
12500 \\
12500\end{array}$ & $\begin{array}{l}46.0 \\
47.0 \\
48.0\end{array}$ & $\begin{array}{l}.04143 \\
.04051 \\
.03974\end{array}$ & $\begin{array}{l}.16574 \\
.16206 \\
.15896\end{array}$ & $\begin{array}{l}.01263 \\
.01234 \\
.01211\end{array}$ & $\begin{array}{l}.05052 \\
.04939 \\
.04845\end{array}$ & $\begin{array}{l}17.996 \\
17.595 \\
17.259\end{array}$ & $\begin{array}{l}54.853 \\
53.632 \\
52.606\end{array}$ & $\begin{array}{l}61.980 \\
60.601 \\
59.442\end{array}$ \\
\hline $\begin{array}{l}22 \\
22 \\
22\end{array}$ & $\begin{array}{l}12500 \\
12500 \\
12500\end{array}$ & $\begin{array}{l}49.0 \\
50.0 \\
51.0\end{array}$ & $\begin{array}{r}.03896 \\
.03819 \\
.03750\end{array}$ & $\begin{array}{r}15586 \\
\cdot 15276 \\
.15001\end{array}$ & $\begin{array}{r}.01187 \\
.01164 \\
.01143\end{array}$ & $\begin{array}{r}.04750 \\
.04656 \\
.04572\end{array}$ & $\begin{array}{l}16.922 \\
16.586 \\
16.287\end{array}$ & $\begin{array}{l}51.581 \\
50.555 \\
49.645\end{array}$ & $\begin{array}{l}58.283 \\
57.124 \\
56.095\end{array}$ \\
\hline $\begin{array}{l}22 \\
22 \\
22\end{array}$ & $\begin{array}{l}12500 \\
12500 \\
12500\end{array}$ & $\begin{array}{l}52 . \\
53 . \\
54 .\end{array}$ & $\begin{array}{l}.03683 \\
.03620 \\
.03560\end{array}$ & $\begin{array}{r}.14734 \\
.14482 \\
.14241\end{array}$ & $\begin{array}{l}.01 \\
.01 \\
.01\end{array}$ & $\begin{array}{r}.04491 \\
.04414 \\
.04340\end{array}$ & $\begin{array}{l}97 \\
724 \\
+62\end{array}$ & & $\begin{array}{l}098 \\
156 \\
255\end{array}$ \\
\hline $\begin{array}{l}22 \\
22 \\
22\end{array}$ & $\begin{array}{l}12500 \\
12500 \\
12500\end{array}$ & $\begin{array}{l}55.0 \\
60.0 \\
65.0\end{array}$ & $\begin{array}{r}.03502 \\
.03243 \\
.03016\end{array}$ & $\begin{array}{r}.14009 \\
.12973 \\
.12065\end{array}$ & $\begin{array}{l}.01067 \\
.00988 \\
.00919\end{array}$ & $\begin{array}{r}.04269 \\
.03954 \\
.03677\end{array}$ & $\begin{array}{l}15.210 \\
14.086 \\
13.099\end{array}$ & $\begin{array}{l}46.36 .2 \\
42.936 \\
39.928\end{array}$ & $\begin{array}{l}52.386 \\
48.515 \\
45.117\end{array}$ \\
\hline $\begin{array}{l}22 \\
22\end{array}$ & $\begin{array}{l}12500 \\
12500\end{array}$ & $\begin{array}{l}70.0 \\
75.0\end{array}$ & $\begin{array}{l}.02837 \\
.02698\end{array}$ & $\begin{array}{l}11349 \\
.10795\end{array}$ & $\begin{array}{l}.00864 \\
.00822\end{array}$ & $\begin{array}{l}.03459 \\
.03290\end{array}$ & $\begin{array}{l}12 \cdot 323 \\
.11 \cdot 721\end{array}$ & $\begin{array}{l}37.561 \\
35.726\end{array}$ & $\begin{array}{l}42.442 \\
40.368\end{array}$ \\
\hline 22 & 12500 & 80.0 & .02570 & .10280 & .00783 & .03133 & $11 \cdot 161$ & 34.022 & $38 \cdot 442$ \\
\hline $\begin{array}{l}22 \\
22 \\
22\end{array}$ & $\begin{array}{l}12500 \\
12500 \\
12500\end{array}$ & $\begin{array}{l}85.0 \\
90.0 \\
95.0\end{array}$ & $\begin{array}{l}.02475 \\
.02406 \\
.02349\end{array}$ & $\begin{array}{l}.09902 \\
.09625 \\
.09397\end{array}$ & $\begin{array}{l}.00754 \\
.00733 \\
.00716\end{array}$ & $\begin{array}{l}.03018 \\
.02933 \\
.02864\end{array}$ & $\begin{array}{l}10.752 \\
10.450 \\
10.203\end{array}$ & $\begin{array}{l}32.772 \\
31.853 \\
31.099\end{array}$ & $\begin{array}{l}37.031 \\
35.992 \\
35.140\end{array}$ \\
\hline$c$ & 12500 & $100 \cdot 0$ & .02293 & .09173 & .00698 & .02795 & 9.959 & $30 \cdot 357$ & $34 \cdot 302$ \\
\hline
\end{tabular}


TABULATION OF DATA FOR

ABSORPTION OF SOUND IN AIR VERSUS PERCENT RELATIVE HUMIDITY

AT 23 DEGREES CENTIGRADE FOR A FREQUENCY OF I2.5 HERTZ

\begin{tabular}{|c|c|c|c|c|c|c|c|c|c|}
\hline 1) & (2) & $(3)$ & (4) & (5) & $(6)$ & 171 & (8) & 191 & 1101 \\
\hline $\begin{array}{l}\text { TEMP. } \\
\text { DEGR } \\
\text { CENT. }\end{array}$ & FREQ & $\begin{array}{l}\text { REL } \\
\text { HUM } \\
\text { PER } \\
\text { CENT }\end{array}$ & $\begin{array}{l}\text { ATTEN } \\
\text { COEF } \\
\text { PER } \\
\text { METER }\end{array}$ & $\begin{array}{l}4 M \\
\text { PER } \\
\text { METER }\end{array}$ & $\begin{array}{l}\text { ATTEN } \\
\text { COEF } \\
\text { PER } \\
\text { FOOT }\end{array}$ & $\begin{array}{l}4 M \\
\text { PER } \\
\text { FOOT }\end{array}$ & $\begin{array}{l}\text { ATTEN } \\
\text { DB PER } \\
100 \\
\text { METER. }\end{array}$ & $\begin{array}{l}\text { ATTEN. } \\
\text { DB PER } \\
\text { IOOO } \\
\text { FEET }\end{array}$ & $\begin{array}{l}\text { DECAY } \\
\text { RATE } \\
\text { DB PER } \\
\text { SECOND }\end{array}$ \\
\hline $\begin{array}{l}23 \\
23 \\
23\end{array}$ & $\begin{array}{l}125 \\
125 \\
125\end{array}$ & $\begin{array}{r}5.0 \\
10.0 \\
15.0\end{array}$ & $\begin{array}{l}.00018 \\
.00013 \\
.00011\end{array}$ & $\begin{array}{l}.00073 \\
.00055 \\
.00047\end{array}$ & $\begin{array}{l}.00005 \\
.00004 \\
.00003\end{array}$ & $\begin{array}{l}.00022 \\
.00016 \\
.00014\end{array}$ & $\begin{array}{l}.079 \\
.059 \\
.051\end{array}$ & $\begin{array}{l}.241 \\
.182 \\
.155\end{array}$ & $\begin{array}{r}.273 \\
.206 \\
.176\end{array}$ \\
\hline $\begin{array}{l}23 \\
23 \\
23\end{array}$ & $\begin{array}{l}125 \\
125 \\
125\end{array}$ & $\begin{array}{l}20.0 \\
25.0 \\
30.0\end{array}$ & $\begin{array}{l}.00010 \\
.00009 \\
.00009\end{array}$ & $\begin{array}{l}.00042 \\
.00039 \\
.00036\end{array}$ & $\begin{array}{l}.00003 \\
.00002 \\
.00002\end{array}$ & $\begin{array}{l}.00012 \\
.00011 \\
.00011\end{array}$ & $\begin{array}{l}.046 \\
.042 \\
.039\end{array}$ & $\begin{array}{l}.140 \\
.129 \\
.121\end{array}$ & $\begin{array}{r}.158 \\
.146 \\
.136\end{array}$ \\
\hline $\begin{array}{l}23 \\
23 \\
23\end{array}$ & $\begin{array}{l}125 \\
125 \\
125\end{array}$ & $\begin{array}{l}35.0 \\
40.0 \\
45.0\end{array}$ & $\begin{array}{l}.00008 \\
.00008 \\
.00007\end{array}$ & $\begin{array}{l}.00034 \\
.00032 \\
.00031\end{array}$ & $\begin{array}{l}.00002 \\
.00002 \\
.00002\end{array}$ & $\begin{array}{l}.00010 \\
.00009 \\
.00009\end{array}$ & $\begin{array}{l}.037 \\
.035 \\
.034\end{array}$ & $\begin{array}{l}.114 \\
.108 \\
.104\end{array}$ & $\begin{array}{l}.129 \\
.122 \\
.117\end{array}$ \\
\hline $\begin{array}{l}23 \\
23\end{array}$ & $\begin{array}{l}125 \\
125\end{array}$ & $\begin{array}{l}46.0 \\
47.0\end{array}$ & $\begin{array}{l}.00007 \\
.00007\end{array}$ & $\begin{array}{l}.00031 \\
.00030\end{array}$ & $\begin{array}{l}.00002 \\
.00002\end{array}$ & $\begin{array}{l}.00009 \\
.00009\end{array}$ & $\begin{array}{l}.033 \\
.033\end{array}$ & $\begin{array}{r}.103 \\
.102\end{array}$ & $\begin{array}{r}1116 \\
.115\end{array}$ \\
\hline 23 & 125 & 48.0 & .00007 & .00030 & .00002 & .00009 & .033 & .101 & .115 \\
\hline $\begin{array}{l}23 \\
23 \\
23\end{array}$ & $\begin{array}{l}125 \\
125 \\
125\end{array}$ & $\begin{array}{l}49.0 \\
50.0 \\
51.0\end{array}$ & $\begin{array}{l}.00007 \\
.00007 \\
.00007\end{array}$ & $\begin{array}{l}.00030 \\
.00030 \\
.00029\end{array}$ & $\begin{array}{l}.00002 \\
.00002 \\
.00002\end{array}$ & $\begin{array}{l}.00009 \\
.00009 \\
.00009\end{array}$ & $\begin{array}{l}.033 \\
.032 \\
.032\end{array}$ & $\begin{array}{l}.100 \\
.099 \\
.099\end{array}$ & $\begin{array}{l}.1114 \\
.1113 \\
.112\end{array}$ \\
\hline $\begin{array}{l}23 \\
23\end{array}$ & $\begin{array}{l}125 \\
125\end{array}$ & $\begin{array}{l}52.0 \\
53.0\end{array}$ & $\begin{array}{l}.00007 \\
.00007\end{array}$ & $\begin{array}{l}.00029 \\
.00029\end{array}$ & $\begin{array}{l}.00002 \\
.00002\end{array}$ & $\begin{array}{l}.00009 \\
.00009\end{array}$ & $\begin{array}{r}.032 \\
.032\end{array}$ & $\begin{array}{l}.098 \\
.097\end{array}$ & . 111 \\
\hline 23 & 125 & 54.0 & .00007 & .00029 & .00002 & .00008 & .031 & .097 & .109 \\
\hline $\begin{array}{l}23 \\
23 \\
23\end{array}$ & $\begin{array}{l}125 \\
125 \\
125\end{array}$ & $\begin{array}{l}55.0 \\
60.0 \\
65.0\end{array}$ & $\begin{array}{l}.00007 \\
.00007 \\
.00006\end{array}$ & $\begin{array}{l}.00029 \\
.00028 \\
.00027\end{array}$ & $\begin{array}{l}.00002 \\
.00002 \\
.00002\end{array}$ & $\begin{array}{l}.00008 \\
.00008 \\
.00008\end{array}$ & $\begin{array}{l}.031 \\
.030 \\
.029\end{array}$ & $\begin{array}{l}.096 \\
.093 \\
.090\end{array}$ & $\begin{array}{l}.109 \\
.105 \\
.102\end{array}$ \\
\hline $\begin{array}{l}23 \\
23 \\
23\end{array}$ & $\begin{array}{l}125 \\
125 \\
125\end{array}$ & $\begin{array}{l}70.0 \\
75.0 \\
80.0\end{array}$ & $\begin{array}{l}.00006 \\
.00006 \\
.00006\end{array}$ & $\begin{array}{l}.00026 \\
.00025 \\
.00024\end{array}$ & $\begin{array}{l}.00002 \\
.00001 \\
.00001\end{array}$ & $\begin{array}{l}.00008 \\
.00007 \\
.00007\end{array}$ & $\begin{array}{l}.028 \\
.027 \\
.026\end{array}$ & $\begin{array}{l}.087 \\
.0884 \\
.081\end{array}$ & $\begin{array}{l}.098 \\
.095 \\
.092\end{array}$ \\
\hline $\begin{array}{l}23 \\
23 \\
23\end{array}$ & $\begin{array}{l}125 \\
125 \\
125\end{array}$ & $\begin{array}{l}85.0 \\
90.0 \\
95.0\end{array}$ & $\begin{array}{l}.00005 \\
.00005 \\
.00005\end{array}$ & $\begin{array}{l}.00023 \\
.00023 \\
.00022\end{array}$ & $\begin{array}{l}.00001 \\
.00001 \\
.00001\end{array}$ & $\begin{array}{l}.00007 \\
.00007 \\
.00006\end{array}$ & $\begin{array}{l}.025 \\
.025 \\
.024\end{array}$ & $\begin{array}{r}.078 \\
.076 \\
.074\end{array}$ & $\begin{array}{l}.089 \\
.086 \\
.084\end{array}$ \\
\hline 23 & 125 & $100 \cdot 0$ & .00005 & .00021 & .00001 & .00006 & .023 & .072 & .082 \\
\hline
\end{tabular}


TABULATION OF DATA FOR

ABSORPTION OF SOUND IN AIR VERSUS PERCENT RELATIVE HUMIDITY AT 23 DEGREES CENTIGRADE FOR A FREQUENCY OF 250 HERTZ

\begin{tabular}{|c|c|c|c|c|c|c|c|c|c|}
\hline (1) & $(2)$ & $(3)$ & $(4)$ & 151 & $(6)$ & (7) & 181 & 191 & $(10)$ \\
\hline $\begin{array}{l}\text { TEMP } \\
\text { DEGR } \\
\text { CENT }\end{array}$ & HERTZ & $\begin{array}{l}\text { REL } \\
\text { HUM } \\
\text { PER } \\
\text { CENT }\end{array}$ & $\begin{array}{l}\text { ATTEN } \\
\text { COEF } \\
\text { PER } \\
\text { METER }\end{array}$ & $\begin{array}{l}4 M \\
\text { PER } \\
\text { METER }\end{array}$ & $\begin{array}{l}\text { ATTEN } \\
\text { COEF } \\
\text { PER } \\
\text { FOOT }\end{array}$ & $\begin{array}{l}4 M \\
\text { PER } \\
\text { FOOT }\end{array}$ & $\begin{array}{l}\text { ATTEN } \\
\text { DB PER } \\
100 \\
\text { METER }\end{array}$ & $\begin{array}{l}\text { ATTEN } \\
\text { DB PER } \\
1 \text { OOO } \\
\text { FEET }\end{array}$ & $\begin{array}{l}\text { DECAY } \\
\text { RATE } \\
\text { DB PER } \\
\text { SECOND }\end{array}$ \\
\hline $\begin{array}{l}23 \\
23\end{array}$ & $\begin{array}{l}250 \\
250\end{array}$ & $\begin{array}{r}5.0 \\
10.0\end{array}$ & $\begin{array}{l}.00046 \\
.00030\end{array}$ & $\begin{array}{l}.00185 \\
.00122\end{array}$ & $\begin{array}{l}.00014 \\
.00009\end{array}$ & $\begin{array}{l}.00056 \\
.00037\end{array}$ & $\begin{array}{r}.201 \\
.133\end{array}$ & .613 & $\begin{array}{r}.693 \\
.459\end{array}$ \\
\hline 23 & 250 & 15.0 & .00026 & .00105 & .00008 & .00032 & .114 & .348 & .394 \\
\hline $\begin{array}{l}23 \\
23\end{array}$ & $\begin{array}{l}250 \\
250\end{array}$ & $\begin{array}{l}20.0 \\
25.0\end{array}$ & $\begin{array}{l}.00023 \\
.00021\end{array}$ & $\begin{array}{l}.00094 \\
.00086\end{array}$ & $\begin{array}{l}.00007 \\
.00006\end{array}$ & $\begin{array}{l}.00028 \\
.00026\end{array}$ & $\begin{array}{l}.102 \\
.094\end{array}$ & $\begin{array}{l}.311 \\
.287\end{array}$ & $\begin{array}{r}.353 \\
.325\end{array}$ \\
\hline 23 & 250 & 30.0 & .00020 & .00081 & .00006 & .00024 & .088 & .268 & .303 \\
\hline $\begin{array}{l}23 \\
23\end{array}$ & $\begin{array}{l}250 \\
250\end{array}$ & $\begin{array}{l}35.0 \\
40.0\end{array}$ & $\begin{array}{l}.00019 \\
.00018\end{array}$ & $\begin{array}{l}.00076 \\
.00073\end{array}$ & $\begin{array}{l}.00005 \\
.00005\end{array}$ & $\begin{array}{l}.00023 \\
.00022\end{array}$ & $\begin{array}{l}.083 \\
.079\end{array}$ & $\begin{array}{l}.253 \\
.242\end{array}$ & $\begin{array}{l}.287 \\
.274\end{array}$ \\
\hline 23 & 250 & $45 \cdot 0$ & .00017 & .00069 & .00005 & .00021 & .075 & .231 & .261 \\
\hline $\begin{array}{l}23 \\
23\end{array}$ & $\begin{array}{l}250 \\
250\end{array}$ & $\begin{array}{l}46.0 \\
47.0\end{array}$ & $\begin{array}{l}.00017 \\
.00017\end{array}$ & $\begin{array}{l}.00069 \\
.00068\end{array}$ & $\begin{array}{l}.00005 \\
.00005\end{array}$ & $\begin{array}{l}.00021 \\
.00020\end{array}$ & $\begin{array}{l}.075 \\
.074\end{array}$ & $\begin{array}{r}.229 \\
.227\end{array}$ & $\begin{array}{r}.259 \\
.257\end{array}$ \\
\hline 23 & 250 & 48.0 & .00017 & .00068 & .00005 & .00020 & .074 & .225 & .255 \\
\hline $\begin{array}{l}23 \\
23 \\
23\end{array}$ & $\begin{array}{l}250 \\
250 \\
250\end{array}$ & $\begin{array}{l}49.0 \\
50.0 \\
51.0\end{array}$ & $\begin{array}{l}.00016 \\
.00016 \\
.00016\end{array}$ & $\begin{array}{l}.00067 \\
.00067 \\
.00066\end{array}$ & $\begin{array}{l}.00005 \\
.00005 \\
.00005\end{array}$ & $\begin{array}{l}.00020 \\
.00020 \\
.00020\end{array}$ & $\begin{array}{l}.073 \\
.072 \\
.072\end{array}$ & $\begin{array}{r}.224 \\
.222 \\
.220\end{array}$ & $\begin{array}{l}.253 \\
.251 \\
.249\end{array}$ \\
\hline $\begin{array}{l}23 \\
23 \\
23\end{array}$ & $\begin{array}{l}250 \\
250 \\
250\end{array}$ & $\begin{array}{l}52.0 \\
53.0 \\
54.0\end{array}$ & $\begin{array}{l}.00016 \\
.00016 \\
.00016\end{array}$ & $\begin{array}{l}.00066 \\
.00065 \\
.00065\end{array}$ & $\begin{array}{l}.00005 \\
.00005 \\
.00004\end{array}$ & $\begin{array}{l}.00020 \\
.00020 \\
.00019\end{array}$ & $\begin{array}{l}.071 \\
.071 \\
.070\end{array}$ & $\begin{array}{r}.218 \\
.217 \\
.216\end{array}$ & $\begin{array}{l}.247 \\
.246 \\
.244\end{array}$ \\
\hline $\begin{array}{l}23 \\
23 \\
23\end{array}$ & $\begin{array}{l}250 \\
250 \\
250\end{array}$ & $\begin{array}{l}55.0 \\
60.0 \\
65.0\end{array}$ & $\begin{array}{l}.00016 \\
.00015 \\
.00015\end{array}$ & $\begin{array}{l}.00064 \\
.00062 \\
.00061\end{array}$ & $\begin{array}{l}.00004 \\
.00004 \\
.00004\end{array}$ & $\begin{array}{l}.00019 \\
.00019 \\
.00018\end{array}$ & $\begin{array}{l}.070 \\
.068 \\
.066\end{array}$ & $\begin{array}{l}.214 \\
.208 \\
.202\end{array}$ & $\begin{array}{r}.243 \\
.235 \\
.228\end{array}$ \\
\hline $\begin{array}{l}23 \\
23 \\
23\end{array}$ & $\begin{array}{l}250 \\
250 \\
250\end{array}$ & $\begin{array}{l}70.0 \\
75.0 \\
80.0\end{array}$ & $\begin{array}{l}.00014 \\
.00014 \\
.00014\end{array}$ & $\begin{array}{l}.00059 \\
.00057 \\
.00056\end{array}$ & $\begin{array}{l}.00004 \\
.00004 \\
.00004\end{array}$ & $\begin{array}{l}.00018 \\
.00017 \\
.00017\end{array}$ & $\begin{array}{l}.064 \\
.062 \\
.061\end{array}$ & $\begin{array}{r}.196 \\
.191 \\
.187\end{array}$ & $\begin{array}{r}.222 \\
.216 \\
.211\end{array}$ \\
\hline $\begin{array}{l}23 \\
23 \\
23\end{array}$ & $\begin{array}{l}250 \\
250 \\
250\end{array}$ & $\begin{array}{l}85.0 \\
90 \cdot 0 \\
95.0\end{array}$ & $\begin{array}{r}.00013 \\
.00013 \\
.00013\end{array}$ & $\begin{array}{r}.00055 \\
.00053 \\
.00052\end{array}$ & $\begin{array}{l}.00004 \\
.00004 \\
.00003\end{array}$ & $\begin{array}{l}.00016 \\
.00016 \\
.00015\end{array}$ & $\begin{array}{l}.059 \\
.058 \\
.056\end{array}$ & $\begin{array}{r}.182 \\
.177 \\
.173\end{array}$ & $\begin{array}{l}.206 \\
.201 \\
.196\end{array}$ \\
\hline 23 & 2501 & 100.0 & .00012 & $.0005 i$ & .00003 & .00015 & .055 & .168 & .191 \\
\hline
\end{tabular}


TABULATION OF DATA FOR

ABSORPTION OF SOUND IN AIR VERSUS PERCENT RELATIVE HUMIDITY AT 23 .DEGREES CENTIGRADE FOR A FREQUENCY OF 500 HERTZ

\begin{tabular}{|c|c|c|c|c|c|c|c|c|c|}
\hline (1) & $(2)$ & $(3)$ & (4) & (5) & $(6)$ & 171 & (8) & (9) & $(10)$ \\
\hline $\begin{array}{l}\text { TEMP } \\
\text { DEGR } \\
\text { CENT }\end{array}$ & HERTZ & $\begin{array}{l}\text { REL } \\
\text { HUM } \\
\text { PER } \\
\text { CENT }\end{array}$ & $\begin{array}{l}\text { ATTEN } \\
\text { COEF } \\
\text { PER } \\
\text { METER }\end{array}$ & $\begin{array}{l}4 M \\
\text { PER } \\
\text { METER }\end{array}$ & $\begin{array}{l}\text { ATTEN } \\
\text { COEF } \\
\text { PER } \\
\text { FOOT }\end{array}$ & $\begin{array}{l}4 M \\
\text { PER } \\
\text { FOOT }\end{array}$ & $\begin{array}{l}\text { ATTEN } \\
\text { DB PER } \\
100 \\
\text { METER }\end{array}$ & $\begin{array}{l}\text { ATTEN } \\
\text { DB PER } \\
1000 \\
\text { FEET }\end{array}$ & $\begin{array}{l}\text { DECAY } \\
\text { RATE } \\
\text { DB PER } \\
\text { SECOND }\end{array}$ \\
\hline $\begin{array}{l}23 \\
23 \\
23\end{array}$ & $\begin{array}{l}500 \\
500 \\
500\end{array}$ & $\begin{array}{r}5.0 \\
10.0 \\
15.0\end{array}$ & $\begin{array}{l}.00144 \\
.00073 \\
.00061\end{array}$ & $\begin{array}{l}.00576 \\
.00294 \\
.00247\end{array}$ & $\begin{array}{l}.00043 \\
.00022 \\
.00018\end{array}$ & $\begin{array}{l}.00175 \\
.00089 \\
.00075\end{array}$ & $\begin{array}{r}.626 \\
.319 \\
.268\end{array}$ & $\begin{array}{r}1.908 \\
.974 \\
.819\end{array}$ & $\begin{array}{r}2.160 \\
1.103 \\
.927\end{array}$ \\
\hline $\begin{array}{l}23 \\
23 \\
23\end{array}$ & $\begin{array}{l}500 \\
500 \\
500\end{array}$ & $\begin{array}{l}20.0 \\
25.0 \\
30.0\end{array}$ & $\begin{array}{l}.00055 \\
.00050 \\
.00047\end{array}$ & $\begin{array}{l}.00222 \\
.00203 \\
.00190\end{array}$ & $\begin{array}{l}.00016 \\
.00015 \\
.00014\end{array}$ & $\begin{array}{l}.00067 \\
.00061 \\
.00058\end{array}$ & $\begin{array}{l}.241 \\
.220 \\
.206\end{array}$ & $\begin{array}{l}.737 \\
.673 \\
.630\end{array}$ & $\begin{array}{r}.834 \\
.761 \\
.713\end{array}$ \\
\hline $\begin{array}{l}23 \\
23 \\
23\end{array}$ & $\begin{array}{l}500 \\
500 \\
500\end{array}$ & $\begin{array}{l}35.0 \\
40.0 \\
45.0\end{array}$ & $\begin{array}{l}.00045 \\
.00042 \\
.00041\end{array}$ & $\begin{array}{l}.00180 \\
.00171 \\
.00164\end{array}$ & $\begin{array}{l}.00013 \\
.00013 \\
.00012\end{array}$ & $\begin{array}{l}.00055 \\
.00052 \\
.00050\end{array}$ & $\begin{array}{r}.195 \\
.186 \\
.178\end{array}$ & $\begin{array}{r}.597 \\
.567 \\
.543\end{array}$ & $\begin{array}{l}.675 \\
.642 \\
.614\end{array}$ \\
\hline $\begin{array}{l}23 \\
23 \\
23\end{array}$ & $\begin{array}{l}500 \\
500 \\
500\end{array}$ & $\begin{array}{l}46.0 \\
47.0 \\
48.0\end{array}$ & $\begin{array}{l}.00040 \\
.00040 \\
.00040\end{array}$ & $\begin{array}{l}.00162 \\
.00161 \\
.00160\end{array}$ & $\begin{array}{l}.00012 \\
.00012 \\
.00012\end{array}$ & $\begin{array}{l}.00049 \\
.00049 \\
.00048\end{array}$ & $\begin{array}{r}176 \\
.175 \\
.174\end{array}$ & $\begin{array}{r}.539 \\
-.535 \\
.531\end{array}$ & $\begin{array}{l}.610 \\
.606 \\
.601\end{array}$ \\
\hline $\begin{array}{l}23 \\
23 \\
23\end{array}$ & $\begin{array}{l}500 \\
500 \\
500\end{array}$ & $\begin{array}{l}49.0 \\
50.0 \\
51.0\end{array}$ & $\begin{array}{l}.00039 \\
.00039 \\
.00039\end{array}$ & $\begin{array}{l}.00159 \\
.00158 \\
.00157\end{array}$ & $\begin{array}{l}.00012 \\
.00012 \\
.00011\end{array}$ & $\begin{array}{r}.00048 \\
.00048 \\
.00047\end{array}$ & $\begin{array}{r}.173 \\
.171 \\
.170\end{array}$ & $\begin{array}{l}.527 \\
.523 \\
.520\end{array}$ & $\begin{array}{r}.597 \\
.592 \\
.588\end{array}$ \\
\hline $\begin{array}{l}23 \\
23 \\
23\end{array}$ & $\begin{array}{l}500 \\
500 \\
500\end{array}$ & $\begin{array}{l}52.0 \\
53.0 \\
54.0\end{array}$ & $\begin{array}{l}.00038 \\
.00038 \\
.00038\end{array}$ & $\begin{array}{r}.00155 \\
.00154 \\
.00153\end{array}$ & $\begin{array}{l}.00011 \\
.00011 \\
.00011\end{array}$ & $\begin{array}{r}.00047 \\
.00047 \\
.00046\end{array}$ & $\begin{array}{l}169 \\
.168 \\
1166\end{array}$ & $\begin{array}{r}.516 \\
.512 \\
.508\end{array}$ & $\begin{array}{r}.584 \\
.579 \\
.575\end{array}$ \\
\hline $\begin{array}{l}23 \\
23 \\
23\end{array}$ & $\begin{array}{l}500 \\
500 \\
500\end{array}$ & $\begin{array}{l}55.0 \\
60.0 \\
65.0\end{array}$ & $\begin{array}{l}.00038 \\
.00037 \\
.00035\end{array}$ & $\begin{array}{r}.00152 \\
.00148 \\
.00143\end{array}$ & $\begin{array}{l}.00011 \\
.00011 \\
.00010\end{array}$ & $\begin{array}{l}.00046 \\
.00045 \\
.00043\end{array}$ & $\begin{array}{l}.165 \\
.161 \\
.156\end{array}$ & $\begin{array}{l}.505 \\
.490 \\
.476\end{array}$ & $\begin{array}{r}.572 \\
.555 \\
.539\end{array}$ \\
\hline $\begin{array}{l}23 \\
23 \\
23\end{array}$ & $\begin{array}{l}500 \\
500 \\
500\end{array}$ & $\begin{array}{l}70.0 \\
75.0 \\
80.0\end{array}$ & $\begin{array}{l}.00034 \\
.00034 \\
.00033\end{array}$ & $\begin{array}{l}.00139 \\
.00136 \\
.00133\end{array}$ & $\begin{array}{l}.00010 \\
.00010 \\
.00010\end{array}$ & $\begin{array}{l}.00042 \\
.00041 \\
.00040\end{array}$ & $\begin{array}{r}.151 \\
.147 \\
.144\end{array}$ & $\begin{array}{l}.463 \\
.451 \\
.440\end{array}$ & $\begin{array}{r}.524 \\
.510 \\
.498\end{array}$ \\
\hline $\begin{array}{l}23 \\
23\end{array}$ & $\begin{array}{l}500 \\
500\end{array}$ & $\begin{array}{l}85.0 \\
90.0\end{array}$ & $\begin{array}{l}.00032 \\
.00031\end{array}$ & $\begin{array}{l}.00130 \\
.00127\end{array}$ & $\begin{array}{l}.00009 \\
.00009\end{array}$ & $\begin{array}{l}.00039 \\
.00039\end{array}$ & $\begin{array}{r}141 \\
.138\end{array}$ & $\begin{array}{r}431 \\
.423\end{array}$ & $\begin{array}{r}.488 \\
.479\end{array}$ \\
\hline 23 & 500 & 95.0 & $.0,0031$ & .00125 & .00009 & .00038 & .136 & .415 & .469 \\
\hline 23 & 500 & $100 \cdot 0$ & .00030 & .00122 & .00009 & .00037 & .133 & .406 & .460 \\
\hline
\end{tabular}


TABULATION OF DATA FOR

ABSORPTION OF SOUND IN AIR VERSUS PERCENT RELATIVE HUMIDITY AT 23 DEGREES CENTIGRADE FOR A FREQUENCY OF 1000 HERTZ

\begin{tabular}{|c|c|c|c|c|c|c|c|c|c|}
\hline (I) & (2) & (3) & (4) & (5) & $(6)$ & $\cdot(7)$ & $(3)$ & $(9)$ & $(10)$ \\
\hline $\begin{array}{l}\text { TEMP } \\
\text { DEGR } \\
\text { CENT }\end{array}$ & HERTZ & $\begin{array}{l}\text { REL } \\
\text { HUM } \\
\text { PER } \\
\text { CENT }\end{array}$ & $\begin{array}{l}\text { ATTEN } \\
\text { COEF } \\
\text { PER } \\
\text { METER }\end{array}$ & $\begin{array}{l}4 M \\
\text { PER } \\
\text { METER }\end{array}$ & $\begin{array}{l}\text { ATTEN } \\
\text { COEF } \\
\text { PER } \\
\text { FOOT }\end{array}$ & $\begin{array}{l}4 . M \\
\text { PER } \\
\text { FOOT }\end{array}$ & $\begin{array}{l}\text { ATTEN } \\
\text { DB PER } \\
100 \\
\text { METER }\end{array}$ & $\begin{array}{l}\text { ATTEN } \\
\text { DB PER } \\
1000 \\
\text { FEET }\end{array}$ & $\begin{array}{l}\text { DECAY } \\
\text { RATE } \\
\text { DB PER } \\
\text { SECOND }\end{array}$ \\
\hline 23 & 1000 & 5.0 & .00489 & .01959 & .00149 & .00597 & $2 \cdot 127$ & 6.484 & 7.339 \\
\hline $\begin{array}{l}23 \\
23\end{array}$ & $\begin{array}{l}1000 \\
1000\end{array}$ & $\begin{array}{l}10.0 \\
15.0\end{array}$ & $\begin{array}{r}.00230 \\
.00155\end{array}$ & $\begin{array}{l}.00921 \\
.00623\end{array}$ & $\begin{array}{r}.00070 \\
.00047\end{array}$ & $\begin{array}{l}.00280 \\
.00189\end{array}$ & $\begin{array}{r}1.000 \\
.676\end{array}$ & $\begin{array}{l}3.048 \\
2.062\end{array}$ & $\begin{array}{l}3.450 \\
2.334\end{array}$ \\
\hline $\begin{array}{l}23 \\
23 \\
23\end{array}$ & $\begin{array}{l}1000 \\
1000 \\
1000\end{array}$ & $\begin{array}{l}20.0 \\
25.0 \\
30.0\end{array}$ & $\begin{array}{l}.00134 \\
.00124 \\
.00116\end{array}$ & $\begin{array}{r}.00537 \\
.00496 \\
.00465\end{array}$ & $\begin{array}{l}.00040 \\
.00037 \\
.00035\end{array}$ & $\begin{array}{l}.00163 \\
.00151 \\
.00141\end{array}$ & $\begin{array}{l}.583 \\
.539 \\
.505\end{array}$ & $\begin{array}{l}1.779 \\
1.644 \\
1.540\end{array}$ & $\begin{array}{l}2.014 \\
1.861 \\
1.743\end{array}$ \\
\hline $\begin{array}{l}23 \\
23 \\
23\end{array}$ & $\begin{array}{l}1000 \\
1000 \\
1000\end{array}$ & $\begin{array}{l}35.0 \\
40.0 \\
45.0\end{array}$ & $\begin{array}{r}.00109 \\
.00104 \\
.00099\end{array}$ & $\begin{array}{l}.00439 \\
.00416 \\
.00398\end{array}$ & $\begin{array}{l}.00033 \\
.00031 \\
.00030\end{array}$ & $\begin{array}{l}.00133 \\
.00126 \\
.00121\end{array}$ & $\begin{array}{l}.476 \\
.451 \\
.432\end{array}$ & $\begin{array}{l}1.452 \\
1.376 \\
1.319\end{array}$ & $\begin{array}{l}1.644 \\
1.558 \\
1.493\end{array}$ \\
\hline $\begin{array}{l}23 \\
23 \\
23\end{array}$ & $\begin{array}{l}1000 \\
1000 \\
1000\end{array}$ & $\begin{array}{l}46.0 \\
47.0 \\
48.0\end{array}$ & $\begin{array}{l}.00098 \\
.00098 \\
.00097\end{array}$ & $\begin{array}{l}.00395 \\
.00392 \\
.00389\end{array}$ & $\begin{array}{l}.00030 \\
.00029 \\
.00029\end{array}$ & $\begin{array}{l}.001120 \\
.00119 \\
.00118\end{array}$ & $\begin{array}{r}.429 \\
.426 \\
.423\end{array}$ & $\begin{array}{l}1.308 \\
1.299 \\
1.290\end{array}$ & $\begin{array}{l}1.481 \\
1.470 \\
1.460\end{array}$ \\
\hline $\begin{array}{l}23 \\
23 \\
23\end{array}$ & $\begin{array}{l}1000 \\
1000 \\
1000\end{array}$ & $\begin{array}{l}49.0 \\
50.0 \\
51.0\end{array}$ & $\begin{array}{l}.00096 \\
.00096 \\
.00095\end{array}$ & $\begin{array}{l}.00387 \\
.00384 \\
.00381\end{array}$ & $\begin{array}{l}.00029 \\
.00029 \\
.00029\end{array}$ & $\begin{array}{l}.00117 \\
.00117 \\
.00116\end{array}$ & $\begin{array}{r}.420 \\
.417 \\
.414\end{array}$ & $\begin{array}{l}1 \cdot 281 \\
1.272 \\
1.263\end{array}$ & $\begin{array}{l}1.450 \\
1.439 \\
1.429\end{array}$ \\
\hline $\begin{array}{l}23 \\
23 \\
23\end{array}$ & $\begin{array}{l}1000 \\
1000 \\
1000\end{array}$ & $\begin{array}{l}52.0 \\
53.0 \\
54.0\end{array}$ & $\begin{array}{l}.00094 \\
.00094 \\
.00093\end{array}$ & $\begin{array}{r}.00378 \\
.00376 \\
.00374\end{array}$ & $\begin{array}{l}.00028 \\
.00028 \\
.00028\end{array}$ & $\begin{array}{l}.001115 \\
.001114 \\
.00114\end{array}$ & $\begin{array}{l}.411 \\
.408 \\
.406\end{array}$ & $\begin{array}{l}1.254 \\
1.246 \\
1.238\end{array}$ & $\begin{array}{l}1.419 \\
1.410 \\
1.402\end{array}$ \\
\hline $\begin{array}{l}23 \\
23\end{array}$ & $\begin{array}{l}1000 \\
1000\end{array}$ & $\begin{array}{l}55.0 \\
60.0\end{array}$ & $\begin{array}{l}.00093 \\
.00090\end{array}$ & $\begin{array}{l}.00372 \\
.00360\end{array}$ & $\begin{array}{l}.00028 \\
.00027\end{array}$ & $\begin{array}{l}.00113 \\
.00110\end{array}$ & $\begin{array}{r}.403 \\
.391\end{array}$ & $\begin{array}{l}1.231 \\
1.194\end{array}$ & $\begin{array}{l}1.393 \\
1.351\end{array}$ \\
\hline 23 & 1000 & 65.0 & .00087 & .00350 & .00026 & .00106 & .380 & 1.158 & 1.311 \\
\hline $\begin{array}{l}23 \\
23 \\
23\end{array}$ & $\begin{array}{l}1000 \\
1000 \\
1000\end{array}$ & $\begin{array}{l}70.0 \\
75.0 \\
80.0\end{array}$ & $\begin{array}{r}.00085 \\
.00083 \\
.00081\end{array}$ & $\begin{array}{r}.00340 \\
.00332 \\
.00325\end{array}$ & $\begin{array}{l}.00025 \\
.00025 \\
.00024\end{array}$ & $\begin{array}{l}.00103 \\
.00101 \\
.00099\end{array}$ & $\begin{array}{l}.370 \\
.361 \\
.353\end{array}$ & $\begin{array}{l}1.127 \\
1.100 \\
1.076\end{array}$ & $\begin{array}{l}1.276 \\
1.246 \\
1.218\end{array}$ \\
\hline 23 & 1000 & 85.0 & .00079 & .00318 & .00024 & .00096 & .345 & 1.052 & 1.191 \\
\hline $\begin{array}{l}23 \\
23\end{array}$ & $\begin{array}{l}1000 \\
1000\end{array}$ & $\begin{array}{l}90.0 \\
95.0\end{array}$ & $\begin{array}{l}.00077 \\
.00076\end{array}$ & $\begin{array}{l}.00311 \\
.00306\end{array}$ & $\begin{array}{l}.00023 \\
.00023\end{array}$ & $\begin{array}{l}.00095 \\
.00093\end{array}$ & $\begin{array}{r}.338 \\
.332\end{array}$ & $\begin{array}{l}1.032 \\
1.014\end{array}$ & $\begin{array}{l}1.168 \\
1.148\end{array}$ \\
\hline 23 & 1000 & $100 \cdot 0$ & .00075 & .00301 & .00022 & .00091 & .326 & .996 & 1.127 \\
\hline
\end{tabular}


TABULATION OF DATA FOR

ABSORPTION OF SOUND IN AIR VERSUS PERCENT RELATIVE HUMIDITY AT 23. DEGREES CENTIGRADE FOR A FREQUENCY OF 2000 HERTZ

\begin{tabular}{|c|c|c|c|c|c|c|c|c|c|}
\hline (1) & (2) & $(3)$ & (4) & (5) & (6) & $(7)$ & $(8)$ & $(9)$ & $(1.0)$ \\
\hline TEMP & FREQ & $\begin{array}{l}\text { REL } \\
\text { HUM }\end{array}$ & $\begin{array}{l}\text { ATTEN } \\
\text { COEF }\end{array}$ & $4 M$ & $\begin{array}{l}\text { ATTEN } \\
\text { COEF }\end{array}$ & $4 M$ & $\begin{array}{l}\text { A'TEN } \\
\text { DB PER }\end{array}$ & $\begin{array}{l}\text { ATTEN } \\
\text { DB PER }\end{array}$ & $\begin{array}{l}\text { DECAY } \\
\text { RATE }\end{array}$ \\
\hline $\begin{array}{l}\text { DEGR } \\
\text { CENT }\end{array}$ & HERTZ & $\begin{array}{l}\text { PER } \\
\text { CENT }\end{array}$ & $\begin{array}{l}\text { PER } \\
\text { METER }\end{array}$ & $\begin{array}{l}\text { PER } \\
\text { METER }\end{array}$ & $\begin{array}{l}\text { PER } \\
\text { FOOT }\end{array}$ & $\begin{array}{l}\text { PER } \\
\text { FOOT }\end{array}$ & $\begin{array}{l}100 \\
\text { METER }\end{array}$ & $\begin{array}{l}1000 \\
\text { FEET }\end{array}$ & $\begin{array}{l}\text { DB PER } \\
\text { SECOND }\end{array}$ \\
\hline $\begin{array}{l}23 \\
23\end{array}$ & $\begin{array}{l}2000 \\
2000\end{array}$ & $\begin{array}{r}5.0 \\
10.0\end{array}$ & $\begin{array}{l}.01328 \\
.00799\end{array}$ & $\begin{array}{r}.05315 \\
.03199\end{array}$ & $\begin{array}{r}.00405 \\
.00243\end{array}$ & $\begin{array}{l}.01620 \\
.00975\end{array}$ & $\begin{array}{l}5.771 \\
3.473\end{array}$ & $\begin{array}{l}17.591 \\
10.588\end{array}$ & $\begin{array}{l}19.912 \\
11.985\end{array}$ \\
\hline 23 & 2000 & 15.0 & .00517 & .02069 & .00157 & .00630 & $2 \cdot 247$ & 6.850 & 7.753 \\
\hline $\begin{array}{l}23 \\
23 \\
23\end{array}$ & $\begin{array}{l}2000 \\
2000 \\
2000\end{array}$ & $\begin{array}{l}20.0 \\
25.0 \\
30.0\end{array}$ & $\begin{array}{l}.00384 \\
.00317 \\
.00286\end{array}$ & $\begin{array}{r}.01536 \\
.01270 \\
.01146\end{array}$ & $\begin{array}{l}.00117 \\
.00096 \\
.00087\end{array}$ & $\begin{array}{r}.00468 \\
.00387 \\
.00349\end{array}$ & $\begin{array}{l}1.667 \\
1.378 \\
1.245\end{array}$ & $\begin{array}{l}5.083 \\
4.203 \\
3.795\end{array}$ & $\begin{array}{l}5.754 \\
4 \cdot 757 \\
4.296\end{array}$ \\
\hline 23 & 2000 & 35.0 & .00271 & .01084 & .00082 & .00330 & 1.177 & 3.589 & 4.063 \\
\hline $\begin{array}{l}23 \\
23\end{array}$ & $\begin{array}{l}2000 \\
2000\end{array}$ & $\begin{array}{l}40.0 \\
45.0\end{array}$ & $\begin{array}{l}.00258 \\
.00248\end{array}$ & $\begin{array}{r}.01035 \\
.00994\end{array}$ & $\begin{array}{l}.00078 \\
.00075\end{array}$ & $\begin{array}{l}.00315 \\
.00303\end{array}$ & $\begin{array}{l}1.124 \\
1.080\end{array}$ & $\begin{array}{l}3.428 \\
3.292\end{array}$ & $\begin{array}{l}3.880 \\
3.726\end{array}$ \\
\hline 23 & 2000 & 46.0 & .00246 & .00987 & .00075 & .00300 & 1.072 & 3.267 & 3.698 \\
\hline $\begin{array}{l}23 \\
23\end{array}$ & $\begin{array}{l}2000 \\
2000\end{array}$ & $\begin{array}{l}47 \cdot 0 \\
48.0\end{array}$ & $\begin{array}{l}.00245 \\
.00243\end{array}$ & $\begin{array}{r}.00980 \\
.00972\end{array}$ & $\begin{array}{r}.00074 \\
.00074\end{array}$ & $\begin{array}{l}.00298 \\
.00296\end{array}$ & $\begin{array}{l}1.064 \\
1.056\end{array}$ & $\begin{array}{l}3 \cdot 243 \\
3 \cdot 219\end{array}$ & $\begin{array}{l}3.671 \\
3.644\end{array}$ \\
\hline $\begin{array}{l}23 \\
23 \\
23\end{array}$ & $\begin{array}{l}2000 \\
2000 \\
2000\end{array}$ & $\begin{array}{l}49.0 \\
50.0 \\
51.0\end{array}$ & $\begin{array}{r}.00241 \\
.00239 \\
.00237\end{array}$ & $\begin{array}{l}.00965 \\
.00958 \\
.00951\end{array}$ & $\begin{array}{l}.00073 \\
.00073 \\
.00072\end{array}$ & $\begin{array}{r}.00294 \\
.00292 \\
.00290\end{array}$ & $\begin{array}{l}1.048 \\
1.040 \\
1.033\end{array}$ & $\begin{array}{l}3.195 \\
3.171 \\
3.148\end{array}$ & $\begin{array}{l}3.616 \\
3.589 \\
3.564\end{array}$ \\
\hline $\begin{array}{l}23 \\
23\end{array}$ & $\begin{array}{l}2000 \\
2000\end{array}$ & $\begin{array}{l}52.0 \\
53.0\end{array}$ & $\begin{array}{l}.00236 \\
.00234\end{array}$ & $\begin{array}{r}.00945 \\
.00938\end{array}$ & $\begin{array}{l}.00072 \\
.00071\end{array}$ & $\begin{array}{r}.00288 \\
.00286\end{array}$ & $\begin{array}{l}1.026 \\
1.019\end{array}$ & $\begin{array}{l}3.128 \\
3.107\end{array}$ & $\begin{array}{l}3.540 \\
3.517\end{array}$ \\
\hline 23 & 2000 & $54 \cdot 0$ & .00233 & .00932 & .00071 & .00284 & 1.012 & 3.086 & 3.493 \\
\hline $\begin{array}{l}23 \\
23 \\
23\end{array}$ & $\begin{array}{l}2000 \\
2000\end{array}$ & $\begin{array}{l}55.0 \\
60.0\end{array}$ & $\begin{array}{r}.00231 \\
.00224\end{array}$ & .00926 & .00070 & .00282 & $\begin{array}{r}1.005 \\
.972\end{array}$ & $\begin{array}{l}3.066 \\
2.965\end{array}$ & $\begin{array}{l}3.470 \\
3.356\end{array}$ \\
\hline 23 & 2000 & $65 \cdot 0$ & .00217 & .00868 & .00066 & .00264 & .943 & 2.874 & 3.253 \\
\hline $\begin{array}{l}23 \\
23\end{array}$ & $\begin{array}{l}2000 \\
2000\end{array}$ & $\begin{array}{l}70.0 \\
75.0\end{array}$ & $\begin{array}{l}.00210 \\
.00206\end{array}$ & $\begin{array}{l}.00843 \\
.00825\end{array}$ & $\begin{array}{l}.00064 \\
.00062\end{array}$ & $\begin{array}{l}.00257 \\
.00251\end{array}$ & $\begin{array}{r}.916 \\
.895\end{array}$ & $\begin{array}{l}2.793 \\
2.730\end{array}$ & $\begin{array}{l}3.161 \\
3.091\end{array}$ \\
\hline 23 & 2000 & 80.0 & .00201 & .00807 & .00061 & .00246 & .877 & 2.673 & 3.026 \\
\hline $\begin{array}{l}23 \\
23 \\
23\end{array}$ & $\begin{array}{l}2000 \\
2000 \\
2000\end{array}$ & $\begin{array}{l}85.0 \\
90.0 \\
95.0\end{array}$ & $\begin{array}{l}.00197 \\
.00194 \\
.00190\end{array}$ & $\begin{array}{l}.00791 \\
.00776 \\
.00763\end{array}$ & $\begin{array}{l}.00060 \\
.00059 \\
.00058\end{array}$ & $\begin{array}{l}.00241 \\
.00236 \\
.00232\end{array}$ & $\begin{array}{r}.859 \\
.843 \\
.828\end{array}$ & $\begin{array}{l}2.619 \\
2.571 \\
2.525\end{array}$ & $\begin{array}{l}2.964 \\
2.910 \\
2.859\end{array}$ \\
\hline 23 & 2000 & 100.0 & .00187 & .00749 & .00057 & .00228 & .814 & 2.482 & 2.809 \\
\hline
\end{tabular}


TABULATION OF DATA FOR

ABSORPTION OF SOUND IN AIR VERSUS PERCENT RELATIVE HUMIDITY AT 23 DEGREES CENTIGRADE FOR A FREQUENCY OF 2500 HERTZ

\begin{tabular}{|c|c|c|c|c|c|c|c|c|c|}
\hline 1) & (2) & (3) & $(4)$ & (5) & $(6)$ & (7) & (8) & (9) & $(10)$ \\
\hline $\begin{array}{l}\text { EMP } \\
\text { EGR } \\
\text { ENT }\end{array}$ & HERTZ & $\begin{array}{l}\text { REL } \\
\text { HUM } \\
\text { PER } \\
\text { CENT }\end{array}$ & $\begin{array}{l}\text { ATTEN } \\
\text { COEF } \\
\text { PER } \\
\text { METER }\end{array}$ & $\begin{array}{l}4 M \\
\text { PER } \\
\text { METER }\end{array}$ & $\begin{array}{l}\text { ATTEN } \\
\text { COEF } \\
\text { PER } \\
\text { FOOT }\end{array}$ & $\begin{array}{l}4 M . \\
\text { PER } \\
\text { FOOT. }\end{array}$ & $\begin{array}{l}\text { AT TEN } \\
\text { DB PER } \\
100 \\
\text { METER }\end{array}$ & $\begin{array}{l}\text { ATTEN } \\
\text { DB PER } \\
1000 \\
\text { FEET }\end{array}$ & $\begin{array}{l}\text { DECAY } \\
\text { RATE } \\
\text { DB PER } \\
\text { SECOND }\end{array}$ \\
\hline $\begin{array}{l}23 \\
23 \\
23\end{array}$ & $\begin{array}{l}2500 \\
2500 \\
2500\end{array}$ & $\begin{array}{r}5.0 \\
10.0 \\
15.0\end{array}$ & $\begin{array}{r}.01647 \\
.01186 \\
.00772\end{array}$ & $\begin{array}{r}.06588 \\
.04747 \\
.03089\end{array}$ & $\begin{array}{l}.00502 \\
.00361 \\
.00235\end{array}$ & $\begin{array}{r}.02008 \\
.01447 \\
.00941\end{array}$ & $\begin{array}{l}7.153 \\
5.155 \\
3.354\end{array}$ & $\begin{array}{l}21.803 \\
15.712 \\
10.225\end{array}$ & $\begin{array}{l}24.680 \\
17.785 \\
11.574\end{array}$ \\
\hline $\begin{array}{l}23 \\
23 \\
23\end{array}$ & $\begin{array}{l}2500 \\
2500 \\
2500\end{array}$ & $\begin{array}{l}20.0 \\
25.0 \\
30.0\end{array}$ & $\begin{array}{r}.00568 \\
.00455 \\
.00395\end{array}$ & $\begin{array}{r}.02272 \\
.01823 \\
.01581\end{array}$ & $\begin{array}{l}.00173 \\
.00138 \\
.00120\end{array}$ & $\begin{array}{l}.00692 \\
.00555 \\
.00482\end{array}$ & $\begin{array}{l}2.467 \\
1.979 \\
1.717\end{array}$ & $\begin{array}{l}7.522 \\
6.033 \\
5.233\end{array}$ & $\begin{array}{l}6.829 \\
5.924\end{array}$ \\
\hline $\begin{array}{l}23 \\
23 \\
23\end{array}$ & $\begin{array}{l}2500 \\
2500 \\
2500\end{array}$ & $\begin{array}{l}35.0 \\
40.0 \\
45.0\end{array}$ & $\begin{array}{l}.00363 \\
.00346 \\
.00332\end{array}$ & $\begin{array}{l}.01452 \\
.01384 \\
.01329\end{array}$ & $\begin{array}{l}.00110 \\
.00105 \\
.00101\end{array}$ & $\begin{array}{l}.00442 \\
.00421 \\
.00405\end{array}$ & $\begin{array}{l}1.576 \\
1.502 \\
1.443\end{array}$ & $\begin{array}{l}4.805 \\
4.580 \\
4.398\end{array}$ & $\begin{array}{l}5.439 \\
5 \cdot 184 \\
4.978\end{array}$ \\
\hline $\begin{array}{l}23 \\
23\end{array}$ & $\begin{array}{l}2500 \\
2500\end{array}$ & $\begin{array}{l}46.0 \\
47.0\end{array}$ & $\begin{array}{l}.00329 \\
.00327\end{array}$ & $\begin{array}{l}.01319 \\
.01310 !\end{array}$ & $\begin{array}{l}.00100 \\
.00099\end{array}$ & $\begin{array}{l}.00402 \\
.00399\end{array}$ & $\begin{array}{l}1.432 \\
1.422\end{array}$ & $\begin{array}{l}4 \cdot 367 \\
4 \cdot 336\end{array}$ & $\begin{array}{l}4.943 \\
4.908\end{array}$ \\
\hline 23 & 2500 & 48.0 & .00325 & .01300 & .00099 & .00396 & 1.412 & 4.305 & $4 \cdot 87.3$ \\
\hline $\begin{array}{l}23 \\
23\end{array}$ & $\begin{array}{l}2500 \\
2500\end{array}$ & $\begin{array}{l}49.0 \\
50.0\end{array}$ & $\begin{array}{l}.00322 \\
.00320\end{array}$ & $\begin{array}{r}.01291 \\
.01282\end{array}$ & $\begin{array}{l}.00098 \\
.00097\end{array}$ & $\begin{array}{l}.00393 \\
.00390\end{array}$ & $\begin{array}{l}1.402 \\
1.392\end{array}$ & $\begin{array}{l}4.274 \\
4.243\end{array}$ & $\begin{array}{l}4.838 \\
4.802\end{array}$ \\
\hline 23 & 2500 & 51.0 & .00318 & .01273 & .00097 & .00388 & $2 \cdot 382$ & $4 \cdot 215$ & 4.771 \\
\hline $\begin{array}{l}23 \\
23 \\
23\end{array}$ & $\begin{array}{l}2500 \\
2500 \\
2500\end{array}$ & $\begin{array}{l}52.0 \\
53.0 \\
54.0\end{array}$ & $\begin{array}{l}.00316 \\
.00314 \\
.00312\end{array}$ & $\begin{array}{l}.01265 \\
.01258 \\
.01250\end{array}$ & $\begin{array}{l}.00096 \\
.00095 \\
.00095\end{array}$ & $\begin{array}{l}.00385 \\
.00383 \\
.0038 .1\end{array}$ & $\begin{array}{l}1.374 \\
1.365 \\
1.357\end{array}$ & $\begin{array}{l}4 \cdot 189 \\
4 \cdot 163 \\
4 \cdot 137\end{array}$ & $\begin{array}{l}4.741 \\
4 \cdot 712 \\
4.683\end{array}$ \\
\hline $\begin{array}{l}23 \\
23 \\
23\end{array}$ & $\begin{array}{l}2500 \\
2500 \\
2500\end{array}$ & $\begin{array}{l}55.0 \\
60.0 \\
65.0\end{array}$ & $\begin{array}{r}.00310 \\
.00301 \\
.00292\end{array}$ & $\begin{array}{r}.01242 \\
.01204 \\
.01170\end{array}$ & $\begin{array}{l}.00094 \\
.00091 \\
.00089\end{array}$ & $\begin{array}{r}.00378 \\
.00367 \\
.00356\end{array}$ & $\begin{array}{l}1 \cdot 348 \\
1.307 \\
1.271\end{array}$ & $\begin{array}{l}4.111 \\
3.986 \\
3.875\end{array}$ & $\begin{array}{l}4.653 \\
4.512 \\
4.386\end{array}$ \\
\hline $\begin{array}{l}23 \\
23 \\
23\end{array}$ & $\begin{array}{l}2500 \\
2500 \\
2500\end{array}$ & $\begin{array}{l}70.0 \\
75.0 \\
80.0\end{array}$ & $\begin{array}{r}.00284 \\
.00277 \\
.00270\end{array}$ & $\begin{array}{r}.01138 \\
.01109 \\
.01080\end{array}$ & $\begin{array}{r}.00086 \\
.00084 \\
.00082\end{array}$ & $\begin{array}{l}.00347 \\
.00338 \\
.00329\end{array}$ & $\begin{array}{l}1.236 \\
1.204 \\
1.172\end{array}$ & $\begin{array}{l}68 \\
70 \\
74\end{array}$ & $\begin{array}{l}4 \cdot 265 \\
4 \cdot 155 \\
4.046\end{array}$ \\
\hline $\begin{array}{l}23 \\
23\end{array}$ & $\begin{array}{l}2500 \\
2500 \\
2500\end{array}$ & $\begin{array}{l}85.0 \\
90.0 \\
95.0\end{array}$ & $\begin{array}{l}.00265 \\
.00260 \\
.00255\end{array}$ & $\begin{array}{r}.01060 \\
.01040 \\
.01022\end{array}$ & $\begin{array}{l}.00080 \\
.00079 \\
.00077\end{array}$ & $\begin{array}{l}.00323 \\
.00317 \\
.00311\end{array}$ & $\begin{array}{l}1.150 \\
1.129 \\
1.110\end{array}$ & $\begin{array}{l}3.508 \\
3.442 \\
3.383\end{array}$ & $\begin{array}{l}3.970 \\
3.896 \\
3.830\end{array}$ \\
\hline 23 & 2500 & 100.0 & .00251 & .01004 & .000 .76 & .00306 & 1.091 & $3 \cdot 325$ & 3.764 \\
\hline
\end{tabular}


TABULATION OF DATA FOR

ABSORPTION OF SOUND IN AIR VERSUS PERCENT RELATIVE HUMIDITY AT 23 DEGREES CENTIGRADE FOR A FREQUENCY OF 3200 HERTZ

\begin{tabular}{|c|c|c|c|c|c|c|c|c|c|}
\hline (1) & $(2)$ & (3) & (4) & (5) & (6) & 171 & $(8)$ & 191 & $(10)$ \\
\hline EMP & FREQ & $\begin{array}{l}\text { REL } \\
\text { HUM }\end{array}$ & $\begin{array}{l}\text { ATTEN } \\
\text { COEF }\end{array}$ & $4 M$ & $\begin{array}{l}\text { ATTEN } \\
\text { COEF }\end{array}$ & $4 M$ & $\begin{array}{l}\text { A.TTEN } \\
\text { DB PER }\end{array}$ & $\begin{array}{l}\text { ATTEN } \\
\text { DB PER }\end{array}$ & $\begin{array}{l}\text { DECAY } \\
\text { RATE }\end{array}$ \\
\hline $\begin{array}{l}\text { EGR } \\
\text { ENT }\end{array}$ & HERTZ & $\begin{array}{l}\text { PER } \\
\text { CENT }\end{array}$ & $\begin{array}{l}\text { PER } \\
\text { METER }\end{array}$ & $\begin{array}{l}\text { PER } \\
\text { METER }\end{array}$ & $\begin{array}{l}\text { PER } \\
\text { FOOT }\end{array}$ & $\begin{array}{l}\text { PER } \\
\text { FOOT }\end{array}$ & $\begin{array}{l}100 \\
\text { METER }\end{array}$ & $\begin{array}{l}1000 \\
\text { FEET }\end{array}$ & $\begin{array}{l}\text { DB PER } \\
\text { SECOND }\end{array}$ \\
\hline $\begin{array}{l}23 \\
23 \\
23\end{array}$ & $\begin{array}{l}3200 \\
3200 \\
3200\end{array}$ & $\begin{array}{r}5.0 \\
10.0 \\
15.0\end{array}$ & $\begin{array}{l}.01985 \\
.01812 \\
.01187\end{array}$ & $\begin{array}{r}.07942 \\
.07249 \\
.04748\end{array}$ & $\begin{array}{l}.00605 \\
.00552 \\
.00361\end{array}$ & $\begin{array}{r}.02420 \\
.02209 \\
.01447\end{array}$ & $\begin{array}{l}8.623 \\
7.87 .0 \\
5.156\end{array}$ & $\begin{array}{l}26.283 \\
23.990 \\
15.715\end{array}$ & $\begin{array}{l}29.750 \\
27.155 \\
17.788\end{array}$ \\
\hline $\begin{array}{l}23 \\
23 \\
23\end{array}$ & $\begin{array}{l}3200 \\
3200 \\
3200\end{array}$ & $\begin{array}{l}20.0 \\
25.0 \\
30.0\end{array}$ & $\begin{array}{l}.00869 \\
.00691 \\
.00583\end{array}$ & $\begin{array}{r}.03478 \\
.02765 \\
.02333\end{array}$ & $\begin{array}{l}.00265 \\
.00210 \\
.00177\end{array}$ & $\begin{array}{l}.01060 \\
.00842 \\
.00711\end{array}$ & $\begin{array}{l}3.777 \\
3.002 \\
2.533\end{array}$ & $\begin{array}{r}11.512 \\
9.152 \\
7.721\end{array}$ & $\begin{array}{r}13.031 \\
10.360 \\
8.739\end{array}$ \\
\hline $\begin{array}{l}23 \\
23 \\
23\end{array}$ & $\begin{array}{l}3200 \\
3200 \\
3200\end{array}$ & $\begin{array}{l}35.0 \\
40.0 \\
45.0\end{array}$ & $\begin{array}{l}.00517 \\
.00480 \\
.00458\end{array}$ & $\begin{array}{l}.02069 \\
.01920 \\
.01832\end{array}$ & $\begin{array}{r}.00157 \\
.00146 \\
.00139\end{array}$ & $\begin{array}{l}.00630 \\
.00585 \\
.00558\end{array}$ & $\begin{array}{l}2.246 \\
2.085 \\
1.989\end{array}$ & $\begin{array}{l}6.848 \\
6.357 \\
6.065\end{array}$ & $\begin{array}{l}7.752 \\
7.195 \\
6.865\end{array}$ \\
\hline $\begin{array}{l}23 \\
23 \\
23\end{array}$ & $\begin{array}{l}3200 \\
3200 . \\
3200\end{array}$ & $\begin{array}{l}46.0 \\
47.0 \\
48.0\end{array}$ & $\begin{array}{r}.00454 \\
.00451 \\
.00448\end{array}$ & $\begin{array}{r}.01819 \\
.01807 \\
.01794\end{array}$ & $\begin{array}{l}.00138 \\
.00137 \\
.00136\end{array}$ & $\begin{array}{r}.00554 \\
.00550 \\
.00546\end{array}$ & $\begin{array}{l}1.976 \\
1.962 \\
1.948\end{array}$ & $\begin{array}{l}6.023 \\
5.980 \\
5.938\end{array}$ & $\begin{array}{l}6.817 \\
6.769 \\
6.721\end{array}$ \\
\hline $\begin{array}{l}23 \\
23 \\
23\end{array}$ & $\begin{array}{l}3200 \\
3200 \\
3200\end{array}$ & $\begin{array}{l}49.0 \\
50.0 \\
51.0\end{array}$ & $\begin{array}{l}.00445 \\
.00442 \\
.00439\end{array}$ & $\begin{array}{r}.01781 \\
.01768 \\
.01756\end{array}$ & $\begin{array}{l}.00135 \\
.00134 \\
.00133\end{array}$ & $\begin{array}{l}.00543 \\
.00539 \\
.00535\end{array}$ & $\begin{array}{l}1.934 \\
1.920 \\
1.906\end{array}$ & $\begin{array}{l}5.896 \\
5.854 \\
5.811\end{array}$ & $\begin{array}{l}6.674 \\
6.626 \\
6.578\end{array}$ \\
\hline $\begin{array}{l}23 \\
23 \\
23\end{array}$ & $\begin{array}{l}3200 \\
3200 \\
3200\end{array}$ & $\begin{array}{l}52.0 \\
53.0 \\
54.0\end{array}$ & $\begin{array}{l}.00436 \\
.00433 \\
.00431\end{array}$ & $\begin{array}{r}.01745 \\
.01735 \\
.01725\end{array}$ & $\begin{array}{l}.001 \\
.001 \\
.001\end{array}$ & $\begin{array}{l}.00532 \\
.00528 \\
.00525\end{array}$ & & & $\begin{array}{l}6.538 \\
6.500 \\
6.462\end{array}$ \\
\hline $\begin{array}{l}23 \\
23 \\
23\end{array}$ & $\begin{array}{l}3200 \\
3200 \\
3200\end{array}$ & $\begin{array}{l}55.0 \\
60.0 \\
65.0\end{array}$ & $\begin{array}{l}.00428 \\
.00416 \\
.00405\end{array}$ & $\begin{array}{r}.01714 \\
.01664 \\
.01622\end{array}$ & $\begin{array}{l}.00130 \\
.00126 \\
.00123\end{array}$ & $\begin{array}{l}.00522 \\
.00507 \\
.00494\end{array}$ & $\begin{array}{l}1.862 \\
1.807 \\
1.761\end{array}$ & $\begin{array}{l}5.675 \\
5.509 \\
5.369\end{array}$ & $\begin{array}{l}6.424 \\
6.236 \\
6.077\end{array}$ \\
\hline $\begin{array}{l}23 \\
23 \\
23\end{array}$ & $\begin{array}{l}3200 \\
3200 \\
3200\end{array}$ & $\begin{array}{l}70.0 \\
75.0 \\
80.0\end{array}$ & $\begin{array}{r}.00395 \\
.00386 \\
.00376\end{array}$ & $\begin{array}{r}.01580 \\
.01544 \\
.01507\end{array}$ & $\begin{array}{l}.00120 \\
.00117 \\
.00114\end{array}$ & $\begin{array}{r}.00481 \\
.00470 \\
.00459\end{array}$ & $\begin{array}{l}1.716 \\
1.676 \\
1.637\end{array}$ & $\begin{array}{l}5 \cdot 231 \\
5 \cdot 110 \\
4 \cdot 989\end{array}$ & $\begin{array}{l}5.921 \\
5.784 \\
5.648\end{array}$ \\
\hline $\begin{array}{l}23 \\
23 \\
23\end{array}$ & $\begin{array}{l}3200 \\
3200 \\
3200\end{array}$ & $\begin{array}{l}85.0 \\
90.0 \\
95.0\end{array}$ & $\begin{array}{l}.00368 \\
.00360 \\
.00353\end{array}$ & $\begin{array}{l}.01475 \\
.01443 \\
.01413\end{array}$ & $\begin{array}{l}.00112 \\
.00109 \\
.00107\end{array}$ & $\begin{array}{l}.00449 \\
.00439 \\
.00430\end{array}$ & $\begin{array}{l}1.601 \\
1.566 \\
1.534\end{array}$ & $\begin{array}{l}4.881 \\
4.776 \\
4.676\end{array}$ & $\begin{array}{l}5.529 \\
5.406 \\
5.293\end{array}$ \\
\hline 23 & 3200 & 100.0 & .00347 & .01391 & .00106 & .00424 & 1.510 & 4.604 & 5.211 \\
\hline
\end{tabular}


TABULATION OF DATA FOR

ABSORPTION OF SOUND IN AIR VERSUS PERCENT RELATIVE HUMIDITY AT 23 DEGREES CENTIGRADE FOR A FREQUENCY OF 4000 HERTZ

\begin{tabular}{|c|c|c|c|c|c|c|c|c|c|}
\hline (1) & (2) & (3) & (4) & (5) & $(6)$ & (7) & $(8)$ & $(9)$ & $(10)$ \\
\hline EMP & FREQ & $\begin{array}{l}\text { REL } \\
\text { HUM }\end{array}$ & $\begin{array}{l}\text { ATTEN } \\
\text { COEF }\end{array}$ & $4 M$ & $\begin{array}{l}\text { ATTEN } \\
\text { COEF }\end{array}$ & $4 M$ & $\begin{array}{l}\text { ATTEN } \\
\text { DB PER }\end{array}$ & $\begin{array}{l}\text { ATTEN } \\
\text { DB PER }\end{array}$ & $\begin{array}{l}\text { DECAY } \\
\text { RATE }\end{array}$ \\
\hline $\begin{array}{l}\text { EGR } \\
\text { ENT }\end{array}$ & HERTZ & $\begin{array}{l}\text { PER } \\
\text { CENT }\end{array}$ & $\begin{array}{l}\text { PER } \\
\text { METER }\end{array}$ & $\begin{array}{l}\text { PER } \\
\text { METER }\end{array}$ & $\begin{array}{l}\text { PER } \\
\text { FOOT }\end{array}$ & $\begin{array}{l}\text { PER } \\
\text { FOOT }\end{array}$ & $\begin{array}{l}100 \\
\text { METER }\end{array}$ & $\begin{array}{l}1000 \\
\text { FEET }\end{array}$ & $\begin{array}{l}\text { DB PER } \\
\text { SECOND }\end{array}$ \\
\hline $\begin{array}{l}23 \\
23\end{array}$ & $\begin{array}{l}4000 \\
4000\end{array}$ & $\begin{array}{r}5.0 \\
10.0\end{array}$ & $\begin{array}{l}.02224 \\
.02543\end{array}$ & $\begin{array}{l}.08896 \\
.10172\end{array}$ & $\begin{array}{l}.00677 \\
.00775\end{array}$ & $\begin{array}{l}.02711 \\
.03100\end{array}$ & $\begin{array}{r}9.659 \\
11.045\end{array}$ & $\begin{array}{l}29.441 \\
33.666\end{array}$ & $\begin{array}{l}33.324 \\
38.107\end{array}$ \\
\hline 23 & 4000 & $15 \cdot 0$ & .01750 & .07001 & .00533 & .02134 & 7.602 & 23.171 & $26 \cdot 227$ \\
\hline $\begin{array}{l}23 \\
23\end{array}$ & $\begin{array}{l}4000 \\
4000\end{array}$ & $\begin{array}{l}20.0 \\
25.0\end{array}$ & $\begin{array}{r}.01301 \\
.01024\end{array}$ & $\begin{array}{r}.05204 \\
.04097\end{array}$ & $\begin{array}{r}.00396 \\
.00312\end{array}$ & $\begin{array}{r}.01586 \\
.01248\end{array}$ & $\begin{array}{l}5.651 \\
4.449\end{array}$ & $\begin{array}{l}17.224 \\
13.561\end{array}$ & $\begin{array}{l}19.497 \\
15.349\end{array}$ \\
\hline 23 & 4000 & 30.0 & .00850 & .03402 & .00259 & .01037 & 3.694 & 11.260 & 12.745 \\
\hline $\begin{array}{l}23 \\
23 \\
23\end{array}$ & $\begin{array}{c}4000 \\
4000 \\
4000\end{array}$ & $\begin{array}{l}35.0 \\
40.0 \\
45.0\end{array}$ & $\begin{array}{l}.00741 \\
.00665 \\
.00625\end{array}$ & $\begin{array}{l}.02964 \\
.02663 \\
.02502\end{array}$ & $\begin{array}{l}.00225 \\
.00202 \\
.00190\end{array}$ & $\begin{array}{l}.00903 \\
.00811 \\
.00762\end{array}$ & $\begin{array}{l}3.218 \\
2.892 \\
2.717\end{array}$ & $\begin{array}{l}9.810 \\
8.815 \\
8.282\end{array}$ & $\begin{array}{r}11.104 \\
9.978 \\
9.375\end{array}$ \\
\hline $\begin{array}{l}23 \\
23 \\
23\end{array}$ & $\begin{array}{l}4000 \\
4000 \\
4000\end{array}$ & $\begin{array}{l}46.0 \\
47.0 \\
48.0\end{array}$ & $\begin{array}{l}.00617 \\
.00609 \\
.00604\end{array}$ & $\begin{array}{r}.02468 \\
.02439 \\
.02416\end{array}$ & $\begin{array}{l}.00188 \\
.00185 \\
.00184\end{array}$ & $\begin{array}{l}.00752 \\
.00743 \\
.00736\end{array}$ & $\begin{array}{l}2.680 \\
2.649 \\
2.623\end{array}$ & & $\begin{array}{l}9.247 \\
9.139 \\
9.050\end{array}$ \\
\hline $\begin{array}{l}23 \\
23 \\
23\end{array}$ & $\begin{array}{l}4000 \\
4000 \\
4000\end{array}$ & $\begin{array}{l}49.0 \\
50.0 \\
51.0\end{array}$ & $\begin{array}{l}.00598 \\
.00594 \\
.00590\end{array}$ & $\begin{array}{l}.02394 \\
.02376 \\
.02361\end{array}$ & $\begin{array}{l}.00182 \\
.00181 \\
.00179\end{array}$ & $\begin{array}{l}.00729 \\
.00724 \\
.00719\end{array}$ & $\begin{array}{l}2.599 \\
2.580 \\
2.563\end{array}$ & & $\begin{array}{l}8.968 \\
8.903 \\
8.845\end{array}$ \\
\hline $\begin{array}{l}23 \\
23 \\
23\end{array}$ & $\begin{array}{l}4000 \\
4000 \\
4000\end{array}$ & $\begin{array}{l}52.0 \\
53.0 \\
54.0\end{array}$ & $\begin{array}{l}.00586 \\
.00582 \\
.00579\end{array}$ & $\begin{array}{l}.02345 \\
.02331 \\
.02318\end{array}$ & $\begin{array}{l}.00178 \\
.00177 \\
.00176\end{array}$ & $\begin{array}{l}.00714 \\
.00710 \\
.00706\end{array}$ & $\begin{array}{l}2.546 \\
2.531 \\
2.517\end{array}$ & $\begin{array}{l}7.762 \\
7.717 \\
7.672\end{array}$ & $\begin{array}{l}8.786 \\
8.735 \\
8.684\end{array}$ \\
\hline $\begin{array}{l}23 \\
23 \\
23\end{array}$ & $\begin{array}{l}4000 \\
4000 \\
4000\end{array}$ & $\begin{array}{l}55.0 \\
60.0 \\
65.0\end{array}$ & $\begin{array}{r}.00576 \\
.00559 \\
.00545\end{array}$ & $\begin{array}{l}.02304 \\
.02237 \\
.02182\end{array}$ & $\begin{array}{l}.00175 \\
.00170 \\
.00166\end{array}$ & $\begin{array}{l}.00702 \\
.00681 \\
.00665\end{array}$ & $\begin{array}{l}2.502 \\
2.429 \\
2.369\end{array}$ & $\begin{array}{l}7.626 \\
7.403 \\
7.222\end{array}$ & $\begin{array}{l}8.633 \\
8.380 \\
8.175\end{array}$ \\
\hline $\begin{array}{l}23 \\
23 \\
23\end{array}$ & $\begin{array}{l}4000 \\
4000 \\
4000\end{array}$ & $\begin{array}{l}70.0 \\
75.0 \\
80.0\end{array}$ & $\begin{array}{l}.00532 \\
.00520 \\
.00509\end{array}$ & $\begin{array}{l}.02129 \\
.02083 \\
.02037\end{array}$ & $\begin{array}{l}.00162 \\
.00158 \\
.00155\end{array}$ & $\begin{array}{l}.00648 \\
.00635 \\
.00621\end{array}$ & $\begin{array}{l}311 \\
262 \\
212\end{array}$ & & $\begin{array}{l}7.975 \\
7.804 \\
7.633\end{array}$ \\
\hline $\begin{array}{l}23 \\
23 \\
23\end{array}$ & $\begin{array}{l}4000 \\
4000 \\
4000\end{array}$ & $\begin{array}{l}85.0 \\
90.0 \\
95.0\end{array}$ & $\begin{array}{l}.00499 \\
.00489 \\
.00480\end{array}$ & $\begin{array}{r}.01997 \\
.01958 \\
.01920\end{array}$ & $\begin{array}{l}.00152 \\
.00149 \\
.00146\end{array}$ & $\begin{array}{l}.00608 \\
.00597 \\
.00585\end{array}$ & $\begin{array}{l}2.169 \\
2.126 \\
2.084\end{array}$ & $\begin{array}{l}6.611 \\
6.482 \\
6.354\end{array}$ & $\begin{array}{l}7.483 \\
7.337 \\
7.193\end{array}$ \\
\hline 23 & 4000 & 100.0 & .00471 & .01886 & .00143 & .00574 & 2.047 & $6 \cdot 241$ & 7.065 \\
\hline
\end{tabular}


TABULATION OF DATA FOR

ABSORPTION OF SOUND IN AIR VERSUS PERCENT RELATIVE HUMIDITY AT 23. DEGREES CENTIGRADE FOR A FREQUENCY OF 5000 HERTZ

\begin{tabular}{|c|c|c|c|c|c|c|c|c|c|}
\hline$(1)$ & $(2)$ & (3) & (4) & (5) & 161 & (7.) & (8) & $(9)$ & $(10)$ \\
\hline $\begin{array}{l}\text { TEMP } \\
\text { DEGR } \\
\text { CENT }\end{array}$ & HERTZ & $\begin{array}{l}\text { REL } \\
\text { HUM } \\
\text { PER } \\
\text { CENT }\end{array}$ & $\begin{array}{l}\text { ATTEN } \\
\text { COEF } \\
\text { PER } \\
\text { METER }\end{array}$ & $\begin{array}{l}4 M \\
\text { PER } \\
\text { METER. }\end{array}$ & $\begin{array}{l}\text { ATTEN } \\
\text { COEF } \\
\text { PER } \\
\text { FOOT }\end{array}$ & $\begin{array}{l}4 M \\
\text { PER } \\
\text { FOOT }\end{array}$ & $\begin{array}{l}\text { ATTEN } \\
\text { DB PER } \\
100 \\
\text { METER }\end{array}$ & $\begin{array}{l}\text { ATTEN } \\
\text { DB PER } \\
1000 \\
\text { FEET }\end{array}$ & $\begin{array}{l}\text { DECAY } \\
\text { RATE } \\
\text { DB PER } \\
\text { SECOND }\end{array}$ \\
\hline $\begin{array}{l}23 \\
23 \\
23\end{array}$ & $\begin{array}{l}5000 \\
5000 \\
5000\end{array}$ & $\begin{array}{r}5.0 \\
10.0 \\
15.0\end{array}$ & $\begin{array}{l}.02422 \\
.03351 \\
.02588\end{array}$ & $\begin{array}{r}.09690 \\
.13407 \\
.10353\end{array}$ & $\begin{array}{l}.00738 \\
.01021 \\
.00788\end{array}$ & $\begin{array}{l}.02953 \\
.04086 \\
.03155\end{array}$ & $\begin{array}{l}10.521 \\
14.557 \\
11.2 .41\end{array}$ & $\begin{array}{l}32.070 \\
44.370 \\
34.263\end{array}$ & $\begin{array}{l}36.301 \\
50.223 \\
38.783\end{array}$ \\
\hline $\begin{array}{l}23 \\
23 \\
23\end{array}$ & $\begin{array}{l}5000 \\
5000 \\
5000\end{array}$ & $\begin{array}{l}20.0 \\
25.0 \\
30.0\end{array}$ & $\begin{array}{r}.01909 \\
.01507 \\
.01252\end{array}$ & $\begin{array}{l}.07636 \\
.06029 \\
.05011\end{array}$ & $\begin{array}{l}.00581 \\
.00459 \\
.00381\end{array}$ & $\begin{array}{r}.02327 \\
.01837 \\
.01527\end{array}$ & $\begin{array}{l}8.290 \\
6.546 \\
5.440\end{array}$ & $\begin{array}{l}25.270 \\
19.952 \\
16.583\end{array}$ & $\begin{array}{l}28.604 \\
22.584 \\
18.771\end{array}$ \\
\hline $\begin{array}{l}23 \\
23 \\
23\end{array}$ & $\begin{array}{l}5000 \\
5000 \\
5000\end{array}$ & $\begin{array}{l}35.0 \\
40.0 \\
45.0\end{array}$ & $\begin{array}{l}.01072 \\
.00953 \\
.00867\end{array}$ & $\begin{array}{r}.04289 \\
.03814 \\
.03469\end{array}$ & $\begin{array}{l}.00326 \\
.00290 \\
.00264\end{array}$ & $\begin{array}{l}.01307 \\
.01162 \\
.011057\end{array}$ & $\begin{array}{l}4.657 \\
4.141 \\
3.766\end{array}$ & $\begin{array}{l}14.196 \\
12.623 \\
11.480\end{array}$ & $\begin{array}{l}16.068 \\
14.288 \\
12.994\end{array}$ \\
\hline $\begin{array}{l}23 \\
23 \\
23\end{array}$ & $\begin{array}{l}5000 \\
5000 \\
5000\end{array}$ & $\begin{array}{l}46.0 \\
47.0 \\
48.0\end{array}$ & $\begin{array}{l}.00854 \\
.00844 \\
.00834\end{array}$ & $\begin{array}{r}.03417 \\
.03376 \\
.03339\end{array}$ & $\begin{array}{l}.00260 \\
.00257 \\
.00254\end{array}$ & $\begin{array}{r}.01041 \\
.01029 \\
.01017\end{array}$ & $\begin{array}{l}3.710 \\
3.665 \\
3.625\end{array}$ & $\begin{array}{l}11.309 \\
11.173 \\
11.050\end{array}$ & $\begin{array}{l}12.800 \\
12.647 \\
12.508\end{array}$ \\
\hline $\begin{array}{l}23 \\
23 \\
23\end{array}$ & $\begin{array}{l}5000 \\
5000 \\
5000\end{array}$ & $\begin{array}{l}49.0 \\
50.0 \\
51.0\end{array}$ & $\begin{array}{l}.00825 \\
.00816 \\
.00809\end{array}$ & $\begin{array}{r}.03302 \\
.03266 \\
.03236\end{array}$ & $\begin{array}{l}.00251 \\
.00248 \\
.00246\end{array}$ & $\begin{array}{l}.01006 \\
.00995 \\
.00986\end{array}$ & & $\begin{array}{l}10.928 \\
10.810 \\
10.712\end{array}$ & $\begin{array}{l}12.370 \\
12.236 \\
12.125\end{array}$ \\
\hline $\begin{array}{l}23 \\
23 \\
23\end{array}$ & $\begin{array}{l}5000 \\
5000 \\
5000\end{array}$ & $\begin{array}{l}52.0 \\
53.0 \\
54.0\end{array}$ & $\begin{array}{r}.00801 \\
.00791 \\
.00783\end{array}$ & $\begin{array}{r}.03204 \\
.03167 \\
.03135\end{array}$ & $\begin{array}{l}.00244 \\
.00241 \\
.00238\end{array}$ & $\begin{array}{l}.00976 \\
.00965 \\
.00955\end{array}$ & $\begin{array}{l}3.479 \\
3.439 \\
3.403\end{array}$ & $\begin{array}{l}10.605 \\
10.482 \\
10.375\end{array}$ & $\begin{array}{l}12.003 \\
11.865 \\
11.743\end{array}$ \\
\hline $\begin{array}{l}23 \\
23 \\
23\end{array}$ & $\begin{array}{l}5000 \\
5000 \\
5000\end{array}$ & $\begin{array}{l}55.0 \\
60.0 \\
65.0\end{array}$ & $\begin{array}{l}.00776 \\
.00752 \\
.00733\end{array}$ & $\begin{array}{r}.03107 \\
.03009 \\
.02935\end{array}$ & $\begin{array}{l}.00236 \\
.00229 \\
.00223\end{array}$ & $\begin{array}{l}.00947 \\
.00917 \\
.00894\end{array}$ & $\begin{array}{l}3.373 \\
3.267 \\
3.186\end{array}$ & $\begin{array}{r}10.282 \\
9.958 \\
9.713\end{array}$ & $\begin{array}{l}11.638 \\
11.272 \\
10.995\end{array}$ \\
\hline $\begin{array}{l}23 \\
23 \\
23\end{array}$ & $\begin{array}{l}5000 \\
5000 \\
5000\end{array}$ & $\begin{array}{l}70.0 \\
75.0 \\
80.0\end{array}$ & $\begin{array}{r}.00716 \\
.00701 \\
.00686\end{array}$ & $\begin{array}{r}.02864 \\
.02805 \\
.02745\end{array}$ & $\begin{array}{l}.00218 \\
.00213 \\
.00209\end{array}$ & $\begin{array}{r}.00873 \\
.00855 \\
.00836\end{array}$ & $\begin{array}{l}3.109 \\
3.045 \\
2.981\end{array}$ & $\begin{array}{l}9.479 \\
9.283 \\
9.087\end{array}$ & $\begin{array}{l}10.729 \\
10.507 \\
10.286\end{array}$ \\
\hline $\begin{array}{l}23 \\
23 \\
23\end{array}$ & $\begin{array}{l}5000 \\
5000 \\
5000\end{array}$ & $\begin{array}{l}85.0 \\
90.0 \\
95.0\end{array}$ & $\begin{array}{l}.00674 \\
.00661 \\
.00650\end{array}$ & $\begin{array}{r}.02696 \\
.02647 \\
.02600\end{array}$ & $\begin{array}{l}.00205 \\
.00201 \\
.00198\end{array}$ & $\begin{array}{l}.00821 \\
.00806 \\
.00792\end{array}$ & $\begin{array}{l}2.927 \\
2.874 \\
2.823\end{array}$ & $\begin{array}{l}8.923 \\
8.760 \\
8.604\end{array}$ & $\begin{array}{r}10.100 \\
9.916 \\
9.739\end{array}$ \\
\hline 23 & 000 & 00.0 & .00639 & .02557 & .00194 & .00779 & 2 & $8 \cdot 464$ & 9.581 \\
\hline
\end{tabular}


TABULATION OF DATA FOR

ABSORPTION OF SOUND IN AIR VERSUS PERCENT RELATIVE HUMIDITY AT 23 DEGREES CENTIGRADE FOR A FREQUENCY OF $5940 \mathrm{HERTZ}$

\begin{tabular}{|c|c|c|c|c|c|c|c|c|c|}
\hline (1) & $(2)$ & (3) & $(4)$ & (5) & (6) & 171 & $(8)$ & 191 & 1201 \\
\hline EMP & FREQ & $\begin{array}{l}\text { REL } \\
\text { HUM }\end{array}$ & $\begin{array}{l}\text { ATTEN } \\
\text { COEF }\end{array}$ & $4 M$ & $\begin{array}{l}\text { ATTEN } \\
\text { COEF }\end{array}$ & $4 M$ & $\begin{array}{l}\text { ATTEN } \\
\text { DB PER }\end{array}$ & $\begin{array}{l}\text { ATTEN } \\
\text { DB PER }\end{array}$ & $\begin{array}{l}\text { DECAY } \\
\text { RATE }\end{array}$ \\
\hline $\begin{array}{l}\text { DEGR } \\
\text { CENT }\end{array}$ & HERTZ & $\begin{array}{l}\text { PER } \\
\text { CENT }\end{array}$ & $\begin{array}{l}\text { PER } \\
\text { METER }\end{array}$ & $\begin{array}{l}\text { PER } \\
\text { METER }\end{array}$ & $\begin{array}{l}\text { PER } \\
\text { FOOT }\end{array}$ & $\begin{array}{l}\text { PER } \\
\text { FOOT }\end{array}$ & $\begin{array}{l}100 \\
\text { METER }\end{array}$ & $\begin{array}{l}1000 \\
\text { FEET }\end{array}$ & $\begin{array}{l}\text { DB PER } \\
\text { SECOND }\end{array}$ \\
\hline $\begin{array}{l}23 \\
23 \\
23\end{array}$ & $\begin{array}{l}5940 \\
5940 \\
5940\end{array}$ & $\begin{array}{r}5.0 \\
10.0 \\
15.0\end{array}$ & $\begin{array}{l}.02591 \\
.04039 \\
.03425\end{array}$ & $\begin{array}{r}10365 \\
.16158 \\
.13701\end{array}$ & $\begin{array}{r}.00789 \\
.01231 \\
.01044\end{array}$ & $\begin{array}{r}.03159 \\
.04925 \\
.04176\end{array}$ & $\begin{array}{l}11 \cdot 254 \\
17 \cdot 544 \\
14 \cdot 876\end{array}$ & $\begin{array}{l}34 \cdot 304 \\
53 \cdot 475 \\
45 \cdot 343\end{array}$ & $\begin{array}{l}38.829 \\
60.528 \\
51.324\end{array}$ \\
\hline $\begin{array}{l}23 \\
23 \\
23\end{array}$ & $\begin{array}{l}5940 \\
5940 \\
5940\end{array}$ & $\begin{array}{l}20.0 \\
25.0 \\
30.0\end{array}$ & $\begin{array}{r}.02542 \\
.02034 \\
.01675\end{array}$ & $\begin{array}{r}.10169 \\
.08139 \\
.06702\end{array}$ & $\begin{array}{r}.00774 \\
.00620 \\
.00510\end{array}$ & $\begin{array}{r}.03099 \\
.02481 \\
.02042\end{array}$ & $\begin{array}{r}11.041 \\
8.837 \\
7.277\end{array}$ & $\begin{array}{l}33 \cdot 655 \\
26.938 \\
22.181\end{array}$ & $\begin{array}{l}38.095 \\
30.491 \\
25.106\end{array}$ \\
\hline $\begin{array}{l}23 \\
23 \\
23\end{array}$ & $\begin{array}{l}5940 \\
5940 \\
5940\end{array}$ & $\begin{array}{l}35.0 \\
40.0 \\
45.0\end{array}$ & $\begin{array}{r}.01442 \\
.01262 \\
.01143\end{array}$ & $\begin{array}{r}.05769 \\
.05051 \\
.04575\end{array}$ & $\begin{array}{r}.00439 \\
.00384 \\
.00348\end{array}$ & $\begin{array}{l}.01758 \\
.01539 \\
.01394\end{array}$ & $\begin{array}{l}6.264 \\
5.484 \\
4.968\end{array}$ & $\begin{array}{l}19.095 \\
16.718 \\
15.143\end{array}$ & $\begin{array}{l}21.613 \\
18.923 \\
17.140\end{array}$ \\
\hline $\begin{array}{l}23 \\
23 \\
23\end{array}$ & $\begin{array}{l}5940 \\
5940 \\
5940\end{array}$ & $\begin{array}{l}46.0 \\
47.0 \\
48.0\end{array}$ & $\begin{array}{r}.01123 \\
.01101 \\
.01082\end{array}$ & $\begin{array}{r}.04493 \\
.04406 \\
.04330\end{array}$ & $\begin{array}{l}.00342 \\
.00335 \\
.00330\end{array}$ & $\begin{array}{r}.01369 \\
.01343 \\
.01320\end{array}$ & $\begin{array}{r}4.878 \\
4.784 \\
4.702\end{array}$ & $\begin{array}{l}14 \cdot 869 \\
14 \cdot 582 \\
14 \cdot 332\end{array}$ & $\begin{array}{l}16.831 \\
16.505 \\
16.222\end{array}$ \\
\hline $\begin{array}{l}23 \\
23 \\
23\end{array}$ & $\begin{array}{l}5940 \\
5940 \\
5940\end{array}$ & $\begin{array}{l}49.0 \\
50.0 \\
51.0\end{array}$ & $\begin{array}{r}.01066 \\
.01052 \\
.01038\end{array}$ & $\begin{array}{r}.04267 \\
.04208 \\
.04153\end{array}$ & $\begin{array}{r}.00325 \\
.00320 \\
.00316\end{array}$ & $\begin{array}{r}.01300 \\
.01282 \\
.01265\end{array}$ & $\begin{array}{l}4.633 \\
4.569 \\
4.509\end{array}$ & $\begin{array}{l}14.123 \\
13.927 \\
13.744\end{array}$ & $\begin{array}{l}15.986 \\
15.764 \\
15.557\end{array}$ \\
\hline $\begin{array}{l}23 \\
23 \\
23\end{array}$ & $\begin{array}{l}5940 \\
5940 \\
5940\end{array}$ & $\begin{array}{l}52.0 \\
53.0 \\
54.0\end{array}$ & $\begin{array}{r}.01026 \\
.01016 \\
.01006\end{array}$ & $\begin{array}{r}.04106 \\
.04066 \\
.04027\end{array}$ & $\begin{array}{r}.00312 \\
.00309 \\
.00306\end{array}$ & $\begin{array}{r}.01251 \\
.01239 \\
.01227\end{array}$ & $\begin{array}{l}4.458 \\
4.415 \\
4.372\end{array}$ & $\begin{array}{l}13.588 \\
13.458 \\
13.327\end{array}$ & $\begin{array}{l}15.381 \\
15.233 \\
15.085\end{array}$ \\
\hline $\begin{array}{l}23 \\
23 \\
23\end{array}$ & $\begin{array}{l}5940 \\
5940 \\
5940\end{array}$ & $\begin{array}{l}55.0 \\
60.0 \\
65.0\end{array}$ & $\begin{array}{l}.00996 \\
.00952 \\
.00922\end{array}$ & $\begin{array}{r}.03987 \\
.03810 \\
.03690\end{array}$ & $\begin{array}{l}.00303 \\
.00290 \\
.00281\end{array}$ & $\begin{array}{l}.01215 \\
.01161 \\
.01124\end{array}$ & $\begin{array}{r}4.329 \\
4.137 \\
4.007\end{array}$ & $\begin{array}{l}13.196 \\
12.610 \\
12.213\end{array}$ & $\begin{array}{l}14.937 \\
14.274 \\
13.824\end{array}$ \\
\hline $\begin{array}{l}23 \\
23 \\
23\end{array}$ & $\begin{array}{l}5940 \\
5940 \\
5940\end{array}$ & $\begin{array}{l}70.0 \\
7.5 .0 \\
80.0\end{array}$ & $\begin{array}{r}.00901 \\
.00882 \\
.00864\end{array}$ & $\begin{array}{r}.03607 \\
.03528 \\
.03459\end{array}$ & $\begin{array}{r}.00274 \\
.00268 \\
.00263\end{array}$ & $\begin{array}{r}.01099 \\
.01075 \\
.01054\end{array}$ & $\begin{array}{l}3.916 \\
3.831 \\
3.756\end{array}$ & $\begin{array}{l}11.938 \\
11.677 \\
11.450\end{array}$ & $\begin{array}{l}13.513 \\
13.217 \\
12.960\end{array}$ \\
\hline $\begin{array}{l}23 \\
23 \\
23\end{array}$ & $\begin{array}{l}5940 \\
5940 \\
5940\end{array}$ & $\begin{array}{l}85.0 \\
90.0 \\
95.0\end{array}$ & $\begin{array}{l}.00849 \\
.00833 \\
.00820\end{array}$ & $\begin{array}{r}.03396 \\
.03335 \\
.03282\end{array}$ & $\begin{array}{r}.00258 \\
.00254 \\
.00250\end{array}$ & $\begin{array}{r}.01035 \\
.01016 \\
.01000\end{array}$ & $\begin{array}{l}3.687 \\
3.621 \\
3.564\end{array}$ & $\begin{array}{l}11.241 \\
11.038 \\
10.864\end{array}$ & $\begin{array}{l}12.723 \\
12.494 \\
12.297\end{array}$ \\
\hline 2 & 5940 & $00 \cdot 0$ & .00807 & .03230 & .0 .0246 & .00984 & 3.507 & 10.689 & $12 \cdot 100$ \\
\hline
\end{tabular}


TABULATION OF DATA FOR

ABSORPTION OF SOUND IN AIR VERSUS PERCENT RELATIVE HUMIDITY AT 23 DEGREES CENTIGRADE FOR A FREQUENCY OF 6300 HERTZ

\begin{tabular}{|c|c|c|c|c|c|c|c|c|c|}
\hline (1) & $(2)$ & (3) & $(4)$ & (5) & $(6)$ & $(7)$ & $(8)$ & $(9)$ & 1101 \\
\hline $\begin{array}{l}\text { TEMP } \\
\text { DEGR } \\
\text { CENT }\end{array}$ & HERTZ & $\begin{array}{l}\text { REL } \\
\text { HUM } \\
\text { PER } \\
\text { CENT }\end{array}$ & $\begin{array}{l}\text { ATTEN } \\
\text { COEF } \\
\text { PER } \\
\text { METER }\end{array}$ & $\begin{array}{l}\text { 4M } \\
\text { PER } \\
\text { METER }\end{array}$ & $\begin{array}{l}\text { ATTEN } \\
\text { COEF } \\
\text { PER } \\
\text { FOOT }\end{array}$ & $\begin{array}{l}4 M \\
\text { PER } \\
\text { FOOT }\end{array}$ & $\begin{array}{l}\text { ATTEN } \\
\text { DB PER } \\
100 \\
\text { METER }\end{array}$ & $\begin{array}{l}\text { ATTEA } \\
\text { OB PER } \\
1000 \\
\text { FEET }\end{array}$ & $\begin{array}{l}\text { DECAY } \\
\text { RATE } \\
\text { DB PER } \\
\text { SECEND }\end{array}$ \\
\hline $\begin{array}{l}23 \\
23 \\
23\end{array}$ & $\begin{array}{l}6300 \\
6300 \\
6300\end{array}$ & $\begin{array}{r}5.0 \\
10.0 \\
15.0\end{array}$ & $\begin{array}{r}.02650 \\
.04284 \\
.03763\end{array}$ & $\begin{array}{r}10601 \\
.17139 \\
.15053\end{array}$ & $\begin{array}{r}.00807 \\
.01306 \\
.01147\end{array}$ & $\begin{array}{r}.03231 \\
.05224 \\
.04588\end{array}$ & $\begin{array}{l}11.510 \\
18.609 \\
16.344\end{array}$ & $\begin{array}{l}35.084 \\
56.722 \\
49.817\end{array}$ & $\begin{array}{l}39.712 \\
64.204 \\
56.389\end{array}$ \\
\hline $\begin{array}{l}23 \\
23 \\
23\end{array}$ & $\begin{array}{l}6300 \\
6300 \\
6300\end{array}$ & $\begin{array}{l}20.0 \\
25.0 \\
30.0\end{array}$ & $\begin{array}{r}.02821 \\
.02258 \\
.01861\end{array}$ & $\begin{array}{r}.11284 \\
.09032 \\
.07444\end{array}$ & $\begin{array}{l}.00859 \\
.00688 \\
.00567\end{array}$ & $\begin{array}{l}.03439 \\
.02753 \\
.02269\end{array}$ & $\begin{array}{r}12.252 \\
9.807 \\
8.083\end{array}$ & $\begin{array}{l}37 \cdot 345 \\
29 \cdot 892 \\
24 \cdot 637\end{array}$ & $\begin{array}{l}42.271 \\
33.835 \\
27.847\end{array}$ \\
\hline $\begin{array}{l}23 \\
23 \\
23\end{array}$ & $\begin{array}{l}6300 \\
6300 \\
6300\end{array}$ & $\begin{array}{l}35 \cdot 0 \\
40 \cdot 0 \\
45 \cdot 0\end{array}$ & $\begin{array}{r}.01596 \\
.01398 \\
.01259\end{array}$ & $\begin{array}{l}.06387 \\
.05595 \\
.05038\end{array}$ & $\begin{array}{l}.00486 \\
.00426 \\
.00383\end{array}$ & $\begin{array}{l}.01946 \\
.01705 \\
.01535\end{array}$ & $\begin{array}{l}35 \\
75 \\
70\end{array}$ & $\begin{array}{l}21.138 \\
18.519 \\
16.674\end{array}$ & $\begin{array}{l}23.926 \\
20.961 \\
18.873\end{array}$ \\
\hline $\begin{array}{l}23 \\
23 \\
23\end{array}$ & $\begin{array}{l}6300 \\
6300 \\
6300\end{array}$ & $\begin{array}{l}46.0 \\
47.0 \\
48.0\end{array}$ & $\begin{array}{r}.01238 \\
.01218 \\
.01196\end{array}$ & $\begin{array}{r}.04952 \\
.04872 \\
.04786\end{array}$ & $\begin{array}{l}.0 \\
.0 \\
.0\end{array}$ & $\begin{array}{l}.01509 \\
.01485 \\
.01459\end{array}$ & & $\begin{array}{l}90 \\
24 \\
41\end{array}$ & $\begin{array}{l}552 \\
251 \\
931\end{array}$ \\
\hline $\begin{array}{l}23 \\
23 \\
23\end{array}$ & $\begin{array}{l}6300 \\
6300 \\
6300\end{array}$ & $\begin{array}{l}49.0 \\
50.0 \\
51.0\end{array}$ & $\begin{array}{r}.01174 \\
.01155 \\
.01139\end{array}$ & $\begin{array}{r}.04698 \\
.04623 \\
.04558\end{array}$ & $\begin{array}{l}.00358 \\
.00352 \\
.00347\end{array}$ & $\begin{array}{r}.01432 \\
.01409 \\
.01389\end{array}$ & & $\begin{array}{l}548 \\
300 \\
087\end{array}$ & $\begin{array}{l}17.599 \\
17.318 \\
17.077\end{array}$ \\
\hline $\begin{array}{l}23 \\
23 \\
23\end{array}$ & $\begin{array}{l}6300 \\
6300 \\
6300\end{array}$ & $\begin{array}{l}52 \cdot 0 \\
53.0 \\
54.0\end{array}$ & $\begin{array}{r}.01124 \\
.01110 \\
.01098\end{array}$ & $\begin{array}{r}.04498 \\
.04442 \\
.04392\end{array}$ & $\begin{array}{l}.00342 \\
.00338 \\
.00334\end{array}$ & $\begin{array}{l}.01371 \\
.01353 \\
.01338\end{array}$ & $\begin{array}{l}4.884 \\
4.823 \\
4.769\end{array}$ & $\begin{array}{l}14.887 \\
14.700 \\
14.537\end{array}$ & $\begin{array}{l}16.851 \\
16.639 \\
16.455\end{array}$ \\
\hline $\begin{array}{l}23 \\
23 \\
23\end{array}$ & $\begin{array}{l}6300 \\
6300 \\
6300\end{array}$ & $\begin{array}{l}55 \\
60 \\
65\end{array}$ & $\begin{array}{r}.01088 \\
.01041 \\
.01000\end{array}$ & $\begin{array}{r}.04352 \\
.04167 \\
.04003\end{array}$ & $\begin{array}{l}.0 \\
.0 \\
.01\end{array}$ & $\begin{array}{l}.01326 \\
.01270 \\
.01220\end{array}$ & & $\begin{array}{l}04 \\
93 \\
48\end{array}$ & $\begin{array}{l}304 \\
612 \\
995\end{array}$ \\
\hline $\begin{array}{l}23 \\
23 \\
23\end{array}$ & $\begin{array}{l}6300 \\
6300 \\
6300\end{array}$ & $\begin{array}{l}70.0 \\
75.0 \\
80.0\end{array}$ & $\begin{array}{r}.00976 \\
.00956 \\
.00936\end{array}$ & $\begin{array}{r}.03905 \\
.03824 \\
.03745\end{array}$ & $\begin{array}{l}.0 \\
.0 \\
.0\end{array}$ & $\begin{array}{l}.01190 \\
.01165 \\
.01141\end{array}$ & $\begin{array}{l}4.240 \\
4.152 \\
4.066\end{array}$ & $\begin{array}{l}24 \\
58 \\
93\end{array}$ & $\begin{array}{l}14.629 \\
14.328 \\
14.028\end{array}$ \\
\hline $\begin{array}{l}23 \\
23 \\
23\end{array}$ & $\begin{array}{l}6300 \\
6300 \\
6300\end{array}$ & $\begin{array}{l}85 \bullet 0 \\
90 \cdot 0 \\
95.0\end{array}$ & $\begin{array}{l}.00920 \\
.00904 \\
.00889\end{array}$ & $\begin{array}{r}.03680 \\
.03616 \\
.03556\end{array}$ & $\begin{array}{l}.00280 \\
.00275 \\
.00271\end{array}$ & $\begin{array}{l}.01121 \\
.01102 \\
.01084\end{array}$ & $\begin{array}{l}3.996 \\
3.926 \\
3.861\end{array}$ & $\begin{array}{l}12.180 \\
11.967 \\
11.770\end{array}$ & $\begin{array}{l}13 \cdot 787 \\
13 \cdot 546 \\
13.323\end{array}$ \\
\hline 23 & 6300 & $100 \cdot 0$ & 00875 & .03503 & .00266 & .01067 & 3.803 & 1.593 & 3.122 \\
\hline
\end{tabular}


TABULATION OF DATA FOR

ABSORPTION OF SOUND IN AIR VERSUS PERCENT RELATIVE HUMIDITY AT 23 DEGREES GENTIGRADE FOR A FREQUENCY OF 8000 HERTZ

\begin{tabular}{|c|c|c|c|c|c|c|c|c|c|}
\hline (1) & (2) & (3) & $(4)$ & (5) & (6) & 171 & $(8)$ & (9) & $(10)$ \\
\hline TEMP & FREQ & $\begin{array}{l}\text { REL } \\
\text { HUM }\end{array}$ & $\begin{array}{l}\text { ATTEN } \\
\text { COEF }\end{array}$ & $4 M$ & $\begin{array}{l}\text { ATTEN } \\
\text { COEF }\end{array}$ & $4 M$ & $\begin{array}{l}\text { ATTEN } \\
\text { DB PER. }\end{array}$ & $\begin{array}{l}\text { ATTEN } \\
\text { DB PER }\end{array}$ & $\begin{array}{l}\text { DECAY } \\
\text { RATE }\end{array}$ \\
\hline $\begin{array}{l}\text { DEGR } \\
\text { CENT }\end{array}$ & HERTZ & $\begin{array}{l}\text { PER } \\
\text { CENT }\end{array}$ & $\begin{array}{l}\text { PER } \\
\text { METER }\end{array}$ & $\begin{array}{l}\text { PER } \\
\text { METER }\end{array}$ & $\begin{array}{l}\text { PER } \\
\text { FOOT }\end{array}$ & $\begin{array}{l}\text { PER } \\
\text { FOOT }\end{array}$ & $\begin{array}{l}100 \\
\text { METER }\end{array}$ & $\begin{array}{l}1000 \\
\text { FEET }\end{array}$ & $\begin{array}{l}\text { DB PER } \\
\text { SECOND }\end{array}$ \\
\hline $\begin{array}{l}23 \\
23 \\
23\end{array}$ & $\begin{array}{l}8000 \\
8000 \\
8000\end{array}$ & $\begin{array}{r}5.0 \\
10.0 \\
15.0\end{array}$ & $\begin{array}{l}.02959 \\
.05330 \\
.05293\end{array}$ & $\begin{array}{r}11839 \\
.21321 \\
.21174\end{array}$ & $\begin{array}{r}.00902 \\
.01624 \\
.01613\end{array}$ & $\begin{array}{r}.03608 \\
.06498 \\
.06454\end{array}$ & $\begin{array}{l}12.854 \\
23.150 \\
22.990\end{array}$ & $\begin{array}{l}39.182 \\
70.563 \\
70.075\end{array}$ & $\begin{array}{l}44 \cdot 350 \\
79.870 \\
79.318\end{array}$ \\
\hline $\begin{array}{l}23 \\
23 \\
23\end{array}$ & $\begin{array}{l}8000 \\
8000 \\
8000\end{array}$ & $\begin{array}{l}20.0 \\
25.0 \\
30.0\end{array}$ & $\begin{array}{r}.04279 \\
.03379 \\
.02830\end{array}$ & $\begin{array}{r}.17117 \\
.13517 \\
\cdot 11320\end{array}$ & $\begin{array}{r}.01304 \\
.01030 \\
.00862\end{array}$ & $\begin{array}{l}.05217 \\
.04120 \\
.03450\end{array}$ & $\begin{array}{l}18.585 \\
14.676 \\
12.291\end{array}$ & $\begin{array}{l}56.649 \\
44 \cdot 733 \\
37.463\end{array}$ & $\begin{array}{l}64 \cdot 122 \\
50.634 \\
42.405\end{array}$ \\
\hline $\begin{array}{l}23 \\
23 \\
23\end{array}$ & $\begin{array}{l}8000 \\
8000 \\
8000\end{array}$ & $\begin{array}{l}35.0 \\
40.0 \\
45.0\end{array}$ & $\begin{array}{l}.02410 \\
.02115 \\
.01890\end{array}$ & $\begin{array}{l}.09642 \\
.08463 \\
.07561\end{array}$ & $\begin{array}{l}.00734 \\
.00644 \\
.00576\end{array}$ & $\begin{array}{l}.02939 \\
.02579 \\
.02304\end{array}$ & $\begin{array}{r}10.469 \\
9.189 \\
8.210\end{array}$ & $\begin{array}{l}31.912 \\
28.010 \\
25.025\end{array}$ & $\begin{array}{l}36.121 \\
31.704 \\
28.326\end{array}$ \\
\hline 23 & 8000 & 46.0 & .01850 & .07403 & .00564 & .02256 & 8.038 & 24.502 & 27.734 \\
\hline $\begin{array}{l}23 \\
23\end{array}$ & $\begin{array}{l}8000 \\
8000\end{array}$ & $\begin{array}{l}47.0 \\
48.0\end{array}$ & $\begin{array}{r}.01812 \\
.01777\end{array}$ & $\begin{array}{r}.07249 \\
.07109\end{array}$ & $\begin{array}{r}.00552 \\
.00541\end{array}$ & $\begin{array}{r}.02209 \\
.02166\end{array}$ & $\begin{array}{l}7.871 \\
7.718\end{array}$ & $\begin{array}{l}23.991 \\
23.527\end{array}$ & $\begin{array}{l}27.156 \\
26.630\end{array}$ \\
\hline 23 & 8000 & 49.0 & .01746 & .06986 & .00532 & .02129 & $7 \cdot 585$ & $23 \cdot 120$ & 26.170 \\
\hline $\begin{array}{l}23 \\
23\end{array}$ & $\begin{array}{l}8000 \\
8000\end{array}$ & $\begin{array}{l}50.0 \\
51.0\end{array}$ & $\begin{array}{l}.01715 \\
.01687\end{array}$ & $\begin{array}{l}.06863 \\
.06751\end{array}$ & $\begin{array}{l}.00522 \\
.00514\end{array}$ & $\begin{array}{r}.02091 \\
.02057\end{array}$ & $\begin{array}{l}7.452 \\
7.330\end{array}$ & $\begin{array}{l}22.714 \\
22.342\end{array}$ & $\begin{array}{l}25.71 .0 \\
25.289\end{array}$ \\
\hline $\begin{array}{l}23 \\
23 \\
23\end{array}$ & $\begin{array}{l}8000 \\
8000 \\
8000\end{array}$ & $\begin{array}{l}52.0 \\
53.0 \\
54.0\end{array}$ & $\begin{array}{r}.01661 \\
.01636 \\
.01614\end{array}$ & $\begin{array}{l}.06645 \\
.06544 \\
.06456\end{array}$ & $\begin{array}{l}.00506 \\
.00498 \\
.00491\end{array}$ & $\begin{array}{l}.02025 \\
.01994 \\
.01967\end{array}$ & $\begin{array}{l}7.215 \\
7.105 \\
7.010\end{array}$ & $\begin{array}{l}21.994 \\
21.657 \\
21.366\end{array}$ & $\begin{array}{l}24 \cdot 895 \\
24 \cdot 514 \\
24 \cdot 185\end{array}$ \\
\hline $\begin{array}{l}23 \\
23 \\
23\end{array}$ & $\begin{array}{l}8000 \\
8000 \\
8000\end{array}$ & $\begin{array}{l}55.0 \\
60.0 \\
65.0\end{array}$ & $\begin{array}{r}.01592 \\
.01487 \\
.01422\end{array}$ & $\begin{array}{l}.06368 \\
.05951 \\
.05689\end{array}$ & $\begin{array}{l}.00485 \\
.00453 \\
.00433\end{array}$ & $\begin{array}{r}.01941 \\
.01813 \\
.01734\end{array}$ & $\begin{array}{l}6.914 \\
6.461 \\
6.177\end{array}$ & $\begin{array}{l}21.076 \\
19.694 \\
18.829\end{array}$ & $\begin{array}{l}23.856 \\
22 \cdot 292 \\
21 \cdot 313\end{array}$ \\
\hline $\begin{array}{l}23 \\
23 \\
23\end{array}$ & $\begin{array}{l}8000 \\
8000 \\
8000\end{array}$ & $\begin{array}{l}70.0 \\
75.0 \\
80.0\end{array}$ & $\begin{array}{r}.01372 \\
.01326 \\
.01298\end{array}$ & $\begin{array}{l}.05491 \\
.05306 \\
.05192\end{array}$ & $\begin{array}{l}.00418 \\
.00404 \\
.00395\end{array}$ & $\begin{array}{l}.01673 \\
.01617 \\
.01582\end{array}$ & $\begin{array}{l}5.962 \\
5.761 \\
5.637\end{array}$ & $\begin{array}{l}18.173 \\
17.562 \\
17.182\end{array}$ & $\begin{array}{l}20.570 \\
19.878 \\
19.449\end{array}$ \\
\hline $\begin{array}{l}23 \\
23 \\
23\end{array}$ & $\begin{array}{l}8000 \\
8000 \\
8000\end{array}$ & $\begin{array}{l}85.0 \\
90.0 \\
95.0\end{array}$ & $\begin{array}{l}.01275 \\
.01253 \\
.01233\end{array}$ & $\begin{array}{r}.05101 \\
.05014 \\
.04933\end{array}$ & $\begin{array}{l}.00388 \\
.00382 \\
.00375\end{array}$ & $\begin{array}{r}.01555 \\
.01528 \\
.01503\end{array}$ & $\begin{array}{l}5.539 \\
5.444 \\
5.356\end{array}$ & $\begin{array}{l}16.883 \\
16.593 \\
16.326\end{array}$ & $\begin{array}{l}19.111 \\
18.782 \\
18.480\end{array}$ \\
\hline 23 & 8000 & 100.0 & .01215 & .04863 & .00370 & .01482 & $5.280^{\circ}$ & 6.094 & 18.217 \\
\hline
\end{tabular}


TABULATION OF DATA FOR

ABSORPTION OF SOUND IN AIR VERSUS PERCENT RELATIVE HUMIDITY AT 23 DEGREES CENTIGRADE FOR A FREQUENCY OF 10000 HERTZ

\begin{tabular}{|c|c|c|c|c|c|c|c|c|c|}
\hline (1) & (2) & (3) & (4) & (5) & (6) & $(7)$ & $(8)$ & $\cdot 191$ & $(10)$ \\
\hline $\begin{array}{l}\text { EMP } \\
\text { EGR } \\
\text { ENT }\end{array}$ & HERTZ & $\begin{array}{l}\text { REL } \\
\text { HUM } \\
\text { PER } \\
\text { CENT }\end{array}$ & $\begin{array}{l}\text { ATTEN } \\
\text { COEF } \\
\text { PER } \\
\text { METER }\end{array}$ & $\begin{array}{l}4 M \\
\text { PER } \\
\text { METER }\end{array}$ & $\begin{array}{l}\text { ATTEN } \\
\text { COEF } \\
\text { PER } \\
\text { FOOT }\end{array}$ & $\begin{array}{l}4 M \\
\text { PER } \\
\text { FOOT }\end{array}$ & $\begin{array}{l}\text { ATTEN } \\
\text { DB PER } \\
1 \text { OO } \\
\text { METER }\end{array}$ & $\begin{array}{l}\text { ATTEN } \\
\text { DB PER } \\
1000 \\
\text { FEET }\end{array}$ & $\begin{array}{l}\text { DECAY } \\
\text { RATE } \\
\text { DB PER } \\
\text { SECOND }\end{array}$ \\
\hline $\begin{array}{l}23 \\
23 \\
23\end{array}$ & $\begin{array}{l}10000 \\
10000 \\
10000\end{array}$ & $\begin{array}{r}5.0 \\
10.0 \\
15.0\end{array}$ & $\begin{array}{l}.03332 \\
.06292 \\
.06923\end{array}$ & $\begin{array}{r}.13328 \\
.25171 \\
.27693\end{array}$ & $\begin{array}{l}.01015 \\
.01918 \\
.02110\end{array}$ & $\begin{array}{l}.04062 \\
.07672 \\
.08440\end{array}$ & $\begin{array}{l}14.471 \\
27.329 \\
30.068\end{array}$ & $\begin{array}{l}44.110 \\
83.301 \\
91.649\end{array}$ & $\begin{array}{r}49.929 \\
94.289 \\
103.738\end{array}$ \\
\hline $\begin{array}{l}23 \\
23 \\
23\end{array}$ & $\begin{array}{l}10000 \\
10000 \\
10000\end{array}$ & $\begin{array}{l}20.0 \\
25.0 \\
30.0\end{array}$ & $\begin{array}{l}.06135 \\
.04951 \\
.04128\end{array}$ & $\begin{array}{r}24543 \\
1 \\
.19805 \\
16514\end{array}$ & $\begin{array}{r}.01870 \\
.01509 \\
.01258\end{array}$ & $\begin{array}{l}.07480 \\
.06036 \\
.05033\end{array}$ & $\begin{array}{l}26.648 \\
21.504 \\
17.930\end{array}$ & $\begin{array}{l}81.224 \\
65.545 \\
54.652\end{array}$ & $\begin{array}{l}91.938 \\
74.191 \\
61.862\end{array}$ \\
\hline $\begin{array}{l}23 \\
23 \\
23\end{array}$ & $\begin{array}{l}10000 \\
10000 \\
10000\end{array}$ & $\begin{array}{l}35.0 \\
40.0 \\
45.0\end{array}$ & $\begin{array}{r}.03563 \\
.03108 \\
.02775\end{array}$ & $\begin{array}{r}.14253 \\
.12432 \\
\cdot 11102\end{array}$ & $\begin{array}{l}.01086 \\
.00947 \\
.00846\end{array}$ & $\begin{array}{r}.04344 \\
.03789 \\
.03384\end{array}$ & $\begin{array}{l}15.475 \\
13.499 \\
12.054\end{array}$ & $\begin{array}{l}47 \cdot 169 \\
41 \cdot 145 \\
36 \cdot 743\end{array}$ & $\begin{array}{l}53.391 \\
46.572 \\
41.590\end{array}$ \\
\hline $\begin{array}{l}23 \\
23 \\
23\end{array}$ & $\begin{array}{l}10000 \\
10000 \\
10000\end{array}$ & $\begin{array}{l}46.0 \\
47.0 \\
48.0\end{array}$ & $\begin{array}{l}.02720 \\
.02668 \\
.02620\end{array}$ & $\begin{array}{l}.10882 \\
.10674 \\
.10483\end{array}$ & $\begin{array}{l}.00829 \\
.00813 \\
.00798\end{array}$ & $\begin{array}{l}.03317 \\
.03253 \\
.03195\end{array}$ & $\begin{array}{l}11.815 \\
11.589 \\
11.382\end{array}$ & $\begin{array}{l}36.015 \\
35.326 \\
34.694\end{array}$ & $\begin{array}{l}40.765 \\
39.985 \\
39.271\end{array}$ \\
\hline $\begin{array}{l}23 \\
23 \\
23\end{array}$ & $\begin{array}{l}10000 \\
10000 \\
10000\end{array}$ & $\begin{array}{l}49.0 \\
50.0 \\
51.0\end{array}$ & $\begin{array}{l}.02569 \\
.02518 \\
.02471\end{array}$ & $\begin{array}{r}.10279 \\
.10075 \\
.09885\end{array}$ & $\begin{array}{l}.00783 \\
.00767 \\
.00753\end{array}$ & $\begin{array}{l}.03133 \\
.03071 \\
.03013\end{array}$ & $\begin{array}{l}11.161 \\
10.939 \\
10.732\end{array}$ & $\begin{array}{l}34 \cdot 019 \\
33 \cdot 345 \\
32.714\end{array}$ & $\begin{array}{l}38.506 \\
37.743 \\
37.029\end{array}$ \\
\hline $\begin{array}{l}23 \\
23 \\
23\end{array}$ & $\begin{array}{l}10000 \\
10000 \\
10000\end{array}$ & $\begin{array}{l}52.0 \\
53.0 \\
54.0\end{array}$ & $\begin{array}{l}.02 \\
.02 \\
.02\end{array}$ & $\begin{array}{l}.09 \\
.09 \\
.09\end{array}$ & $\begin{array}{l}.0 \\
.0 \\
.0\end{array}$ & $\begin{array}{l}.0 \\
.0 \\
.0\end{array}$ & $\begin{array}{l}10.545 \\
10.358 \\
10.176\end{array}$ & $\begin{array}{l}32 \cdot 142 \\
31.574 \\
31.017\end{array}$ & $\begin{array}{l}36.382 \\
35.739 \\
35.109\end{array}$ \\
\hline $\begin{array}{l}23 \\
23 \\
23\end{array}$ & $\begin{array}{l}10000 \\
10000 \\
10000\end{array}$ & $\begin{array}{l}55.0 \\
60.0 \\
65.0\end{array}$ & $\begin{array}{r}.02304 \\
.02146 \\
.02020\end{array}$ & $\begin{array}{l}.09219 \\
.08587 \\
.08082\end{array}$ & $\begin{array}{l}.00702 \\
.00654 \\
.00615\end{array}$ & $\begin{array}{l}.02810 \\
.02617 \\
.02463\end{array}$ & $\begin{array}{r}10.010 \\
9.323 \\
8.775\end{array}$ & $\begin{array}{l}30.512 \\
28.419 \\
26.748\end{array}$ & $\begin{array}{l}34.537 \\
32.168 \\
30.276\end{array}$ \\
\hline $\begin{array}{l}23 \\
23 \\
23\end{array}$ & $\begin{array}{l}10000 \\
10000 \\
10000\end{array}$ & $\begin{array}{l}70.0 \\
75.0 \\
80.0\end{array}$ & $\begin{array}{r}.01918 \\
.01850 \\
.01797\end{array}$ & $\begin{array}{r}.07675 \\
.07403 \\
.07190\end{array}$ & $\begin{array}{l}.00584 \\
.00564 \\
.00547\end{array}$ & $\begin{array}{l}.02339 \\
.02256 \\
.02191\end{array}$ & $\begin{array}{l}8.333 \\
8.037 \\
7.807\end{array}$ & $\begin{array}{l}25 \cdot 400 \\
24 \cdot 499 \\
23.796\end{array}$ & $\begin{array}{l}28.750 \\
27.731 \\
26.935\end{array}$ \\
\hline $\begin{array}{l}23 \\
23 \\
23\end{array}$ & $\begin{array}{l}10000 \\
10000 \\
10000\end{array}$ & $\begin{array}{l}85.0 \\
90.0 \\
95.0\end{array}$ & $\begin{array}{r}.01743 \\
.01709 \\
.01683\end{array}$ & $\begin{array}{l}.06974 \\
.06836 \\
.06732\end{array}$ & $\begin{array}{l}.00531 \\
.00520 \\
.00513\end{array}$ & $\begin{array}{l}.02125 \\
.02083 \\
.02052\end{array}$ & $\begin{array}{l}7.572 \\
7.422 \\
7.310\end{array}$ & $\begin{array}{l}23.081 \\
22.625 \\
22.281\end{array}$ & $\begin{array}{l}26.126 \\
25.609 \\
25.220\end{array}$ \\
\hline 3 & 10000 & 1.0 & 01659 & .06637 & .00505 & .02023 & 7.206 & $21 \cdot 965$ & 24.863 \\
\hline
\end{tabular}


TABULATION OF DATA FOR

ABSORPTION OF SOUND IN AIR VERSUS PERCENT RELATIVE HUMIDITY AT 23 DEGREES CENTIGRADE FOR A FREQUENCY OFI 12500 HERTZ

\begin{tabular}{|c|c|c|c|c|c|c|c|c|c|}
\hline (1) & (2) & (3) & 141 & (5) & (6) & (7) & $(8)$ & 191 & $(10)$ \\
\hline $\begin{array}{l}\text { EMP } \\
\text { EGR } \\
\text { ENT }\end{array}$ & T HERTZ & $\begin{array}{l}\text { REL } \\
\text { HUM } \\
\text { PER } \\
\text { CENT }\end{array}$ & $\begin{array}{l}\text { ATTEN } \\
\text { COEF } \\
\text { PER } \\
\text { METER }\end{array}$ & $\begin{array}{l}4 M \\
\text { PER } \\
\text { METER }\end{array}$ & $\begin{array}{l}\text { ATTEN } \\
\text { COEF } \\
\text { PER } \\
\text { FOOT }\end{array}$ & $\begin{array}{l}4 M \\
\text { PER } \\
\text { FOOT }\end{array}$ & $\begin{array}{l}\text { ATTEN } \\
\text { DB PER } \\
100 \\
\text { METER }\end{array}$ & $\begin{array}{l}\text { ATTEN } \\
\text { DB PER } \\
1000 \\
\text { FEET }\end{array}$ & $\begin{array}{l}\text { DECAY } \\
\text { RATE } \\
\text { DB PER } \\
\text { SECOND. }\end{array}$ \\
\hline $\begin{array}{l}23 \\
23 \\
23\end{array}$ & $\begin{array}{l}12500 \\
12500 \\
12500\end{array}$ & $\begin{array}{r}5.0 \\
10.0 \\
15.0\end{array}$ & $\begin{array}{r}.03815 \\
.07176 \\
.08778\end{array}$ & $\begin{array}{r}15260 \\
.28707 \\
.35115\end{array}$ & $\begin{array}{r}.01162 \\
.02187 \\
.02675\end{array}$ & $\begin{array}{l}.04651 \\
.08750 \\
.10703\end{array}$ & $\begin{array}{l}16.569 \\
31.169 \\
38.127\end{array}$ & $\begin{array}{r}50.503 \\
95.004 \\
116.212\end{array}$ & $\begin{array}{r}57 \\
107 \\
131\end{array}$ \\
\hline $\begin{array}{l}23 \\
23 \\
23\end{array}$ & $\begin{array}{l}12500 \\
12500 \\
12500\end{array}$ & $\begin{array}{l}20.0 \\
25.0 \\
30.0\end{array}$ & $\begin{array}{l}.08439 \\
.07240 \\
.06020\end{array}$ & $\begin{array}{r}.33757 \\
.28961 \\
.24081\end{array}$ & $\begin{array}{l}.02572 \\
.02206 \\
.01834\end{array}$ & $\begin{array}{l}.10289 \\
.08827 \\
.07339\end{array}$ & $\begin{array}{l}36.652 \\
31.445 \\
26.146\end{array}$ & $\begin{array}{r}111.717 \\
95.846 \\
79.694\end{array}$ & $\begin{array}{r}12 \\
10 \\
9\end{array}$ \\
\hline $\begin{array}{l}23 \\
23 \\
23\end{array}$ & $\begin{array}{l}12500 \\
12500 \\
12500\end{array}$ & $\begin{array}{l}35.0 \\
40.0 \\
45.0\end{array}$ & $\begin{array}{r}.05188 \\
.04579 \\
.04069\end{array}$ & $\begin{array}{r}.20753 \\
.18318 \\
.16277\end{array}$ & $\begin{array}{l}.01581 \\
.01395 \\
.01240\end{array}$ & $\begin{array}{l}.06325 \\
.05583 \\
.04961\end{array}$ & $\begin{array}{l}22.532 \\
19.889 \\
17.672\end{array}$ & $\begin{array}{l}68.680 \\
60.623 \\
53.867\end{array}$ & $\begin{array}{l}77.74 \\
68.62 \\
60.97\end{array}$ \\
\hline $\begin{array}{l}23 \\
23 \\
23\end{array}$ & $\begin{array}{l}12500 \\
12500 \\
12500\end{array}$ & $\begin{array}{l}46 \cdot 0 \\
47 \cdot 0 \\
48.0\end{array}$ & $\begin{array}{r}.03985 \\
.03902 \\
.03825\end{array}$ & $\begin{array}{r}.15943 \\
.15610 \\
.15302\end{array}$ & $\begin{array}{r}.01214 \\
.01189 \\
.01166\end{array}$ & $\begin{array}{r}.04859 \\
.04758 \\
.04664\end{array}$ & $\begin{array}{l}17.311 \\
16.949 \\
16.614\end{array}$ & $\begin{array}{l}52.764 \\
51.662 \\
50.642\end{array}$ & $\begin{array}{l}59.725 \\
58.477 \\
57.322\end{array}$ \\
\hline $\begin{array}{l}23 \\
23 \\
23\end{array}$ & $\begin{array}{l}12500 \\
12500 \\
12500\end{array}$ & $\begin{array}{l}49.0 \\
50.0 \\
51.0\end{array}$ & $\begin{array}{r}.03752 \\
.03685 \\
.03619\end{array}$ & $\begin{array}{l}.1501 \\
.1474 \\
.1447\end{array}$ & $\begin{array}{l}.01143 \\
.01123 \\
.01103\end{array}$ & $\begin{array}{r}.04575 \\
.04492 \\
.04413\end{array}$ & $\begin{array}{l}98 \\
04 \\
20\end{array}$ & & $\begin{array}{l}56 \\
55 \\
52\end{array}$ \\
\hline $\begin{array}{l}23 \\
23 \\
23\end{array}$ & $\begin{array}{l}12500 \\
12500 \\
12500\end{array}$ & $\begin{array}{l}52.0 \\
53.0 \\
54.0\end{array}$ & $\begin{array}{r}.03557 \\
.03499 \\
.03443\end{array}$ & $\begin{array}{r}.14229 \\
.13997 \\
.13774\end{array}$ & $\begin{array}{r}.01084 \\
.01066 \\
.01049\end{array}$ & $\begin{array}{r}.04337 \\
.04266 \\
.04198\end{array}$ & $\begin{array}{l}15 \cdot 449 \\
15.197 \\
14.955\end{array}$ & $\begin{array}{l}47.090 \\
46.323 \\
45.584\end{array}$ & $\begin{array}{l}53.302 \\
52.433 \\
51.597\end{array}$ \\
\hline $\begin{array}{l}23 \\
23 \\
23\end{array}$ & $\begin{array}{l}12500 \\
12500 \\
12500\end{array}$ & $\begin{array}{l}55.0 \\
60.0 \\
65.0\end{array}$ & $\begin{array}{r}.03391 \\
.03130 \\
.02923\end{array}$ & $\begin{array}{r}.13565 \\
.12523 \\
.11694\end{array}$ & $\begin{array}{l}.01033 \\
.00954 \\
.00891\end{array}$ & $\begin{array}{r}.04134 \\
.03817 \\
.03564\end{array}$ & $\begin{array}{l}14.729 \\
13.597 \\
12.697\end{array}$ & $\begin{array}{l}44 \cdot 895 \\
41 \cdot 447 \\
38 \cdot 702\end{array}$ & $\begin{array}{l}5 \\
4 \\
4\end{array}$ \\
\hline $\begin{array}{l}23 \\
23 \\
23\end{array}$ & $\begin{array}{l}12500 \\
12500 \\
12500\end{array}$ & $\begin{array}{l}70.0 \\
75.0 \\
80.0\end{array}$ & $\begin{array}{r}.02762 \\
.02626 \\
.02518\end{array}$ & $\begin{array}{l}.1105 \\
.1050 \\
.1007\end{array}$ & $\begin{array}{l}.00842 \\
.00800 \\
.00767\end{array}$ & $\begin{array}{l}.03 \\
.03 \\
.03\end{array}$ & $\begin{array}{l}99 \\
08 \\
38\end{array}$ & $\begin{array}{l}36.575 \\
34.772 \\
33.341\end{array}$ & $\begin{array}{l}99 \\
59 \\
39\end{array}$ \\
\hline $\begin{array}{l}23 \\
23 \\
23\end{array}$ & $\begin{array}{l}12500 \\
12500 \\
12500\end{array}$ & $\begin{array}{l}85.0 \\
90.0 \\
95.0\end{array}$ & $\begin{array}{l}.02442 \\
.02381 \\
.02320\end{array}$ & $\begin{array}{r}.09769 \\
.09525 \\
.09283\end{array}$ & $\begin{array}{l}.00744 \\
.00725 \\
.00707\end{array}$ & $\begin{array}{r}.02977 \\
.02903 \\
.02829\end{array}$ & $\begin{array}{l}10.607 \\
10.341 \\
10.080\end{array}$ & $\begin{array}{l}32.331 \\
31.522 \\
30.724\end{array}$ & $\begin{array}{l}36.596 \\
35.680 \\
34.777\end{array}$ \\
\hline 2 & & .0 & 02274 & 09099 & .00693 & $0<113$. & 379 & 13 & 085 \\
\hline
\end{tabular}


TABULATION OF DATA FOR

ABSORPTION OF SOUND IN AIR VERSUS PERCENT RELATIVE HUMIDITY AT 24 DEGREES CENTIGRADE FOR A FREQUENCY OF 125 HERTZ

\begin{tabular}{|c|c|c|c|c|c|c|c|c|c|}
\hline (1) & (2) & (3) & (4) & (5) & $(6)$ & 171 & $(8)$ & $(9)$ & 110)$. \\
\hline $\begin{array}{l}\text { TEMP } \\
\text { DEGR } \\
\text { CENT }\end{array}$ & HERTZ & $\begin{array}{l}\text { REL } \\
\text { HUM } \\
\text { PER } \\
\text { CENT }\end{array}$ & $\begin{array}{l}\text { ATTEN } \\
\text { COEF } \\
\text { PER } \\
\text { METER }\end{array}$ & $\begin{array}{l}4 M \\
\text { PER } \\
\text { METER }\end{array}$ & $\begin{array}{l}\text { ATTEN } \\
\text { COEF } \\
\text { PER } \\
\text { FOOT }\end{array}$ & $\begin{array}{l}4 M \\
\text { PER } \\
\text { FOOT }\end{array}$ & $\begin{array}{l}\text { ATTEN } \\
\text { DE PER } \\
100 \\
\text { METER }\end{array}$ & $\begin{array}{l}\text { ATTEN. } \\
\text { DB PER } \\
1000 \\
\text { FEET }\end{array}$ & $\begin{array}{l}\text { DECAY } \\
\text { RATE } \\
\text { DB PER } \\
\text { SECOND }\end{array}$ \\
\hline $\begin{array}{l}24 \\
24 \\
24\end{array}$ & $\begin{array}{l}125 \\
125 \\
125\end{array}$ & $\begin{array}{r}5.0 \\
10.0 \\
15.0\end{array}$ & $\begin{array}{l}.00018 \\
.00014 \\
.00011\end{array}$ & $\begin{array}{l}.00074 \\
.00056 \\
.00047\end{array}$ & $\begin{array}{l}.00005 \\
.00004 \\
.00003\end{array}$ & $\begin{array}{l}.00022 \\
.00017 \\
.00014\end{array}$ & $\begin{array}{l}.080 \\
.060 \\
.051\end{array}$ & $\begin{array}{r}.246 \\
.185 \\
.158\end{array}$ & $\begin{array}{r}.278 \\
.210 \\
.179\end{array}$ \\
\hline $\begin{array}{l}24 \\
24 \\
24\end{array}$ & $\begin{array}{l}125 \\
125 \\
125\end{array}$ & $\begin{array}{l}20.0 \\
25.0 \\
30.0\end{array}$ & $\begin{array}{l}.00010 \\
.00009 \\
.00009\end{array}$ & $\begin{array}{l}.00043 \\
.00039 \\
.00037\end{array}$ & $\begin{array}{l}.00003 \\
.00003 \\
.00002\end{array}$ & $\begin{array}{l}.00013 \\
.00012 \\
.00011\end{array}$ & $\begin{array}{l}.046 \\
.043 \\
.040\end{array}$ & $\begin{array}{r}.142 \\
.131 \\
.123\end{array}$ & $\begin{array}{r}.161 \\
.148 \\
.139\end{array}$ \\
\hline $\begin{array}{l}24 \\
24\end{array}$ & $\begin{array}{l}125 \\
125\end{array}$ & $\begin{array}{l}35.0 \\
40.0\end{array}$ & $\begin{array}{l}.00008 \\
.00008\end{array}$ & $\begin{array}{l}.00035 \\
.00033\end{array}$ & $\begin{array}{l}.00002 \\
.00002\end{array}$ & $\begin{array}{l}.00010 \\
.00010\end{array}$ & $\begin{array}{l}.038 \\
.036\end{array}$ & 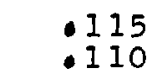 & $\begin{array}{r}131 \\
.125\end{array}$ \\
\hline 24 & 125 & $45 \cdot 0$ & .00008 & .00032 & .00002 & .00009 & .034 & .105 & .120 \\
\hline $\begin{array}{l}24 \\
24\end{array}$ & $\begin{array}{l}125 \\
125\end{array}$ & $\begin{array}{l}46.0 \\
47.0\end{array}$ & $\begin{array}{l}.00007 \\
.00007\end{array}$ & .00031 & $\begin{array}{l}.00002 \\
.00002\end{array}$ & $\begin{array}{l}.00009 \\
.00009\end{array}$ & $\begin{array}{l}.034 \\
.034\end{array}$ & $\begin{array}{l}.105 \\
.104\end{array}$ & $\begin{array}{r}.119 \\
.118\end{array}$ \\
\hline 24 & 125 & $48 \cdot 0$ & .00007 & .00031 & .00002 & .00009 & .033 & .103 & .117 \\
\hline $\begin{array}{l}24 \\
24 \\
24\end{array}$ & $\begin{array}{l}125 \\
125 \\
125\end{array}$ & $\begin{array}{l}49.0 \\
50.0 \\
51.0\end{array}$ & $\begin{array}{l}.00007 \\
.00007 \\
.00007\end{array}$ & $\begin{array}{l}.00030 \\
.00030 \\
.00030\end{array}$ & $\begin{array}{l}.00002 \\
.00002 \\
.00002\end{array}$ & $\begin{array}{l}.00009 \\
.00009 \\
.00009\end{array}$ & $\begin{array}{l}.033 \\
.033 \\
.033\end{array}$ & $\begin{array}{l}.102 \\
.101 \\
.100\end{array}$ & $\begin{array}{r}.116 \\
.115 \\
.114\end{array}$ \\
\hline $\begin{array}{l}24 \\
24 \\
24\end{array}$ & $\begin{array}{l}125 \\
125 \\
125\end{array}$ & $\begin{array}{l}52.0 \\
53.0 \\
54.0\end{array}$ & $\begin{array}{r}.00007 \\
.00007 \\
.00007\end{array}$ & $\begin{array}{l}.00030 \\
.00030 \\
.00029\end{array}$ & $\begin{array}{l}.00002 \\
.00002 \\
.00002\end{array}$ & $\begin{array}{l}.00009 \\
.00009 \\
.00009\end{array}$ & $\begin{array}{l}.032 \\
.032 \\
.032\end{array}$ & $\begin{array}{l}.100 \\
.099 \\
.098\end{array}$ & $\begin{array}{l}.113 \\
.112 \\
.111\end{array}$ \\
\hline $\begin{array}{l}24 \\
24 \\
24\end{array}$ & $\begin{array}{l}125 \\
125 \\
125\end{array}$ & $\begin{array}{l}55.0 \\
60.0 \\
65.0\end{array}$ & $\begin{array}{l}.00007 \\
.00007 \\
.00006\end{array}$ & $\begin{array}{l}.00029 \\
.00028 \\
.00027\end{array}$ & $\begin{array}{l}.00002 \\
.00002 \\
.00002\end{array}$ & $\begin{array}{l}.00009 \\
.00008 \\
.00008\end{array}$ & $\begin{array}{l}.032 \\
.031 \\
.030\end{array}$ & $\begin{array}{l}.098 \\
.095 \\
.091\end{array}$ & $\begin{array}{l}.111 \\
.107 \\
.104\end{array}$ \\
\hline $\begin{array}{l}24 \\
24 \\
24\end{array}$ & $\begin{array}{l}125 \\
125 \\
125\end{array}$ & $\begin{array}{l}70.0 \\
75.0 \\
80.0\end{array}$ & $\begin{array}{l}.00006 \\
.00006 \\
.00006\end{array}$ & $\begin{array}{l}.00026 \\
.00025 \\
.00024\end{array}$ & $\begin{array}{l}.00002 \\
.00001 \\
.00001\end{array}$ & $\begin{array}{l}.00008 \\
.00007 \\
.00007\end{array}$ & $\begin{array}{l}.029 \\
.028 \\
.027\end{array}$ & $\begin{array}{l}.088 \\
.085 \\
.082\end{array}$ & $\begin{array}{l}.100 \\
.097 \\
.093\end{array}$ \\
\hline $\begin{array}{l}24 \\
24 \\
24\end{array}$ & $\begin{array}{l}125 \\
125 \\
125\end{array}$ & $\begin{array}{l}85.0 \\
90.0 \\
95.0\end{array}$ & $\begin{array}{l}.00006 \\
.00005 \\
.00005\end{array}$ & $\begin{array}{l}.00024 \\
.00023 \\
.00022\end{array}$ & $\begin{array}{l}.00001 \\
.00001 \\
.00001\end{array}$ & $\begin{array}{l}.00007 \\
.00007 \\
.00006\end{array}$ & $\begin{array}{l}.026 \\
.025 \\
.024\end{array}$ & $\begin{array}{l}.080 \\
.077 \\
.075\end{array}$ & $\begin{array}{l}.090 \\
.088 \\
.085\end{array}$ \\
\hline 24 & 125 & $100 \cdot 0$ & .00005 & 00022 & .00001 & .00006 & .024 & .073 & .083 \\
\hline
\end{tabular}


TABULATION OF DATA FOR

ABSORPTION OF SOUND IN AIR VERSUS PERCENT RELATIVE HUMIDITY AT 24 DEGREES CENTIGRADE FOR A FREQUENCY OF 250 HERTZ

\begin{tabular}{|c|c|c|c|c|c|c|c|c|c|}
\hline (1) & (2) & (3) & (4) & (5) & (6) & 171 & 181 & 191 & $(10)$ \\
\hline TEMP & FREQ & $\begin{array}{l}\text { REL } \\
\text { HUM }\end{array}$ & $\begin{array}{l}\text { ATTEN } \\
\text { COEF }\end{array}$ & $4 M$ & $\begin{array}{l}\text { ATTEN } \\
\text { COEF }\end{array}$ & $4 M$ & $\begin{array}{l}\text { ATTEN } \\
\text { DB PER }\end{array}$ & $\begin{array}{l}\text { ATTEN } \\
\text { DB PER }\end{array}$ & $\begin{array}{l}\text { DECAY' } \\
\text { RATE }\end{array}$ \\
\hline $\begin{array}{l}\text { DEGR } \\
\text { CENT }\end{array}$ & HERTZ & $\begin{array}{l}\text { PER } \\
\text { CENT }\end{array}$ & $\begin{array}{l}\text { PER } \\
\text { METER }\end{array}$ & $\begin{array}{l}\text { PER } \\
\text { METER }\end{array}$ & $\begin{array}{l}\text { PER } \\
\text { FOOT }\end{array}$ & 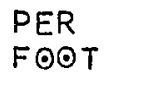 & $\begin{array}{l}100 \\
\text { METER }\end{array}$ & $\begin{array}{l}1000 \\
\text { FEET }\end{array}$ & $\begin{array}{l}\text { DB PER } \\
\text { SECOND }\end{array}$ \\
\hline $\begin{array}{l}24 \\
24\end{array}$ & $\begin{array}{l}250 \\
250\end{array}$ & $\begin{array}{r}5.0 \\
10.0\end{array}$ & $\begin{array}{l}.00043 \\
.00030\end{array}$ & $\begin{array}{l}.00173 \\
.00120\end{array}$ & $\begin{array}{l}.00013 \\
.00009\end{array}$ & $\begin{array}{l}.00052 \\
.00036\end{array}$ & $\begin{array}{l}188 \\
.131\end{array}$ & $\begin{array}{r}.575 \\
.399\end{array}$ & $\begin{array}{r}.652 \\
.453\end{array}$ \\
\hline 24 & 250 & 15.0 & .00025 & .00103 & .00007 & .00031 & .112 & . 341 & .387 \\
\hline $\begin{array}{l}24 \\
24 \\
24\end{array}$ & $\begin{array}{l}250 \\
250 \\
250\end{array}$ & $\begin{array}{l}20.0 \\
25 \cdot 0 \\
30.0\end{array}$ & $\begin{array}{l}.00023 . \\
.00021 \\
.00019\end{array}$ & $\begin{array}{l}.00092 \\
.00085 \\
.00079\end{array}$ & $\begin{array}{l}.00007 \\
.00006 \\
.00006\end{array}$ & $\begin{array}{l}.00028 \\
.00026 \\
.00024\end{array}$ & $\begin{array}{l}.100 \\
.092 \\
.086\end{array}$ & $\begin{array}{l}.307 \\
.282 \\
.264\end{array}$ & $\begin{array}{l}.348 \\
.320 \\
.300\end{array}$ \\
\hline $\begin{array}{l}24 \\
24\end{array}$ & $\begin{array}{l}250 \\
250\end{array}$ & $\begin{array}{l}35.0 \\
40.0\end{array}$ & $\begin{array}{l}.00018 \\
.00017\end{array}$ & $\begin{array}{l}.00075 \\
.00071\end{array}$ & $\begin{array}{l}.00005 \\
.00005\end{array}$ & $\begin{array}{l}.00023 \\
.00021\end{array}$ & $\begin{array}{l}.082 \\
.078\end{array}$ & $\begin{array}{l}.250 \\
.238\end{array}$ & $\begin{array}{r}283 \\
.269\end{array}$ \\
\hline 24 & 250 & 45.0 & .00017 & .00068 & .00005 & .00020 & .074 & .227 & .257 \\
\hline $\begin{array}{l}24 \\
24\end{array}$ & $\begin{array}{l}250 \\
250\end{array}$ & $\begin{array}{l}46.0 \\
47.0\end{array}$ & $\begin{array}{l}.00017 \\
.00016\end{array}$ & $\begin{array}{l}.00068 \\
.00067\end{array}$ & $\begin{array}{l}.00005 \\
.00005\end{array}$ & $\begin{array}{l}.00020 \\
.00020\end{array}$ & $\begin{array}{l}.073 \\
.073\end{array}$ & $\begin{array}{l}.225 \\
.223\end{array}$ & $\begin{array}{l}.255 \\
.253\end{array}$ \\
\hline 24 & 250 & $48 \cdot 0$ & .00016 & .00067 & .00005 & .00020 & .072 & .221 & .251 \\
\hline $\begin{array}{l}24 \\
24\end{array}$ & $\begin{array}{l}250 \\
250\end{array}$ & $\begin{array}{l}49.0 \\
50.0\end{array}$ & $\begin{array}{l}.00016 \\
.00016\end{array}$ & $\begin{array}{l}.00066 \\
.00066\end{array}$ & $\begin{array}{l}.00005 \\
.00005\end{array}$ & $\begin{array}{l}.00020 \\
.00020\end{array}$ & $\begin{array}{l}.072 \\
.071\end{array}$ & $\begin{array}{r}.220 \\
.218\end{array}$ & $\begin{array}{r}.249 \\
.248\end{array}$ \\
\hline 24 & 250 & 51.0 & .00016 & .00065 & .00005 & .00020 & .071 & .217 & .246 \\
\hline $\begin{array}{l}24 \\
24\end{array}$ & $\begin{array}{l}250 \\
250\end{array}$ & $\begin{array}{l}52.0 \\
53.0\end{array}$ & $\begin{array}{l}.00016 \\
.00016\end{array}$ & $\begin{array}{r}.00065 \\
.00064\end{array}$ & $\begin{array}{l}.00004 \\
.00004\end{array}$ & $\begin{array}{l}.00019 \\
.00019\end{array}$ & $\begin{array}{l}.070 \\
.070\end{array}$ & $\begin{array}{r}.216 \\
.214\end{array}$ & $\begin{array}{l}.244 \\
.243\end{array}$ \\
\hline 24 & 250 & $54 \cdot 0$ & .00016 & .00064 & .00004 & .00019 & .069 & .213 & .241 \\
\hline $\begin{array}{l}24 \\
24\end{array}$ & $\begin{array}{l}250 \\
250\end{array}$ & $\begin{array}{l}55.0 \\
60.0\end{array}$ & $\begin{array}{l}.00016 \\
.00015\end{array}$ & $\begin{array}{l}.00064 \\
.00061\end{array}$ & $\begin{array}{l}.00004 \\
.00004\end{array}$ & $\begin{array}{l}.00019 \\
.00018\end{array}$ & $\begin{array}{l}.069 \\
.067\end{array}$ & $\begin{array}{l}.211 \\
.204\end{array}$ & $\begin{array}{l}.240 \\
.232\end{array}$ \\
\hline 24 & 250 & 65.0 & .00015 & .00060 & .00004 & .00018 & .065 & .198 & .225 \\
\hline $\begin{array}{l}24 \\
24\end{array}$ & $\begin{array}{l}250 \\
250\end{array}$ & $\begin{array}{l}70.0 \\
75.0\end{array}$ & $\begin{array}{l}.00014 \\
.00014\end{array}$ & $\begin{array}{r}.00058 \\
.00056\end{array}$ & $\begin{array}{l}.00004 \\
.00004\end{array}$ & $\begin{array}{r}.00017 \\
.00017\end{array}$ & $\begin{array}{l}.063 \\
.061\end{array}$ & $\begin{array}{r}193 \\
-188\end{array}$ & $\begin{array}{r}219 \\
.213\end{array}$ \\
\hline 24 & 250 & 80.0 & .00013 & .00055 & .00004 & .00016 & .060 & .183 & .207 \\
\hline $\begin{array}{l}24 \\
24 \\
24\end{array}$ & $\begin{array}{l}250 \\
250 \\
250\end{array}$ & $\begin{array}{l}85.0 \\
90.0 \\
95.0\end{array}$ & $\begin{array}{l}.00013 \\
.00013 \\
.00012\end{array}$ & $\begin{array}{l}.00053 \\
.00052 \\
.00050\end{array}$ & $\begin{array}{l}.00004 \\
.00003 \\
.00003\end{array}$ & $\begin{array}{l}.00016 \\
.00015 \\
.00015\end{array}$ & $\begin{array}{l}.058 \\
.056 \\
.055\end{array}$ & $\begin{array}{r}.178 \\
.173 \\
.168\end{array}$ & $\begin{array}{l}.202 \\
.196 \\
.190\end{array}$ \\
\hline 24 & 2501 & 100.0 & .00012 & .00049 & .00003 & .00015 & .053 & .163 & .185 \\
\hline
\end{tabular}


TABULATION ÖF DATA FOR

ABSORPTION OF SOUND IN AIR VERSUS PERCENT RELATIVE HUMIDITY AT 24 DEGREES CENTIGRADE FOR A.FREQUENCY OF 500 HERTZ

\begin{tabular}{|c|c|c|c|c|c|c|c|c|c|}
\hline (1) & (2) & $(3)$ & 141 & (5) & $(6)$ & 171 & (8) & (9) & $(10)$ \\
\hline $\begin{array}{l}\text { TEMP } \\
\text { DEGR } \\
\text { CENT }\end{array}$ & HERTZ & $\begin{array}{l}\text { REL } \\
\text { HUM } \\
\text { PER } \\
\text { CENT }\end{array}$ & $\begin{array}{l}\text { ATTEN } \\
\text { COEF } \\
\text { PER } \\
\text { METER }\end{array}$ & $\begin{array}{l}4 M \\
\text { PER } \\
\text { METER }\end{array}$ & 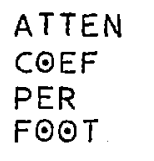 & $\begin{array}{l}4 M \\
P E R \\
F \odot \odot T\end{array}$ & $\begin{array}{l}\text { ATTEN } \\
\text { DB PER } \\
1 O O \\
\text { METER }\end{array}$ & $\begin{array}{l}\text { ATTEN } \\
\text { DB PER } \\
1000 \\
\text { FEET }\end{array}$ & $\begin{array}{l}\text { DECAY } \\
\text { RATE } \\
\text { DB PER } \\
\text { SECOND }\end{array}$ \\
\hline $\begin{array}{l}24 \\
24 \\
24\end{array}$ & $\begin{array}{l}500 \\
500 \\
500\end{array}$ & $\begin{array}{r}5.0 \\
10.0 \\
15.0\end{array}$ & $\begin{array}{l}.00137 \\
.00072 \\
.00061\end{array}$ & $\begin{array}{l}.00550 \\
.00289 \\
.00246\end{array}$ & $\begin{array}{l}.00041 \\
.00022 \\
.00018\end{array}$ & $\begin{array}{l}.00167 \\
.00088 \\
.00075\end{array}$ & $\begin{array}{r}.597 \\
.314 \\
.267\end{array}$ & $\begin{array}{r}1.820 \\
.959 \\
.815\end{array}$ & $\begin{array}{r}2.063 \\
1.087 \\
.924\end{array}$ \\
\hline $\begin{array}{l}24 \\
24 \\
24\end{array}$ & $\begin{array}{l}500 \\
500 \\
500\end{array}$ & $\begin{array}{l}20.0 \\
25.0 \\
30.0\end{array}$ & $\begin{array}{l}.00055 \\
.00050 \\
.00047\end{array}$ & $\begin{array}{l}.00221 \\
.00202 \\
.00189\end{array}$ & $\begin{array}{l}.00016 \\
.00015 \\
.00014\end{array}$ & $\begin{array}{l}.00067 \\
.00061 \\
.00057\end{array}$ & $\begin{array}{r}.240 \\
.219 \\
.205\end{array}$ & $\begin{array}{l}.732 \\
.669 \\
.627\end{array}$ & $\begin{array}{r}.830 \\
.758 \\
.711\end{array}$ \\
\hline $\begin{array}{l}24 \\
24 \\
24\end{array}$ & $\begin{array}{l}500 \\
500 \\
500\end{array}$ & $\begin{array}{l}35.0 \\
40.0 \\
45.0\end{array}$ & $\begin{array}{r}.00044 \\
.00042 \\
.00040\end{array}$ & $\begin{array}{l}.00179 \\
.00170 \\
.00163\end{array}$ & $\begin{array}{l}.00013 \\
.00013 \\
.00012\end{array}$ & $\begin{array}{l}.00054 \\
.00052 \\
.00049\end{array}$ & $\begin{array}{r}195 \\
1185 \\
177\end{array}$ & $\begin{array}{l}.594 \\
.565 \\
.541\end{array}$ & $\begin{array}{r}.674 \\
.640 \\
.614\end{array}$ \\
\hline $\begin{array}{l}24 \\
24 \\
24\end{array}$ & $\begin{array}{l}500 \\
500 \\
500\end{array}$ & $\begin{array}{l}46.0 \\
47.0 \\
48.0\end{array}$ & $\begin{array}{l}.00040 \\
.00040 \\
.00039\end{array}$ & $\begin{array}{l}.00162 \\
.00161 \\
.00159\end{array}$ & $\begin{array}{l}.00012 \\
.00012 \\
.00012\end{array}$ & $\begin{array}{r}.00049 \\
.00049 \\
.00048\end{array}$ & $\begin{array}{r}.176 \\
.174 \\
.173\end{array}$ & $\begin{array}{l}.537 \\
.533 \\
.529\end{array}$ & $\begin{array}{l}.609 \\
.604 \\
.599\end{array}$ \\
\hline $\begin{array}{l}24 \\
24 \\
24\end{array}$ & $\begin{array}{l}500 \\
500 \\
500\end{array}$ & $\begin{array}{l}49.0 \\
50.0 \\
51.0\end{array}$ & $\begin{array}{l}.00039 \\
.00039 \\
.00039\end{array}$ & $\begin{array}{r}.00158 \\
.00157 \\
.00156\end{array}$ & $\begin{array}{l}.00012 \\
.00011 \\
.00011\end{array}$ & $\begin{array}{l}.00048 \\
.00047 \\
.00047\end{array}$ & $\begin{array}{r}172 \\
.170 \\
.169\end{array}$ & $\begin{array}{l}.525 \\
.520 \\
.516\end{array}$ & $\begin{array}{l}.595 \\
.590 \\
.585\end{array}$ \\
\hline $\begin{array}{l}24 \\
24 \\
24\end{array}$ & $\begin{array}{l}500 \\
500 \\
500\end{array}$ & $\begin{array}{l}52.0 \\
53.0 \\
54.0\end{array}$ & $\begin{array}{l}.00038 \\
.00038 \\
.00038\end{array}$ & $\begin{array}{r}.00155 \\
.00154 \\
.00153\end{array}$ & $\begin{array}{l}.00011 \\
.00011 \\
.00011\end{array}$ & $\begin{array}{r}.00047 \\
.00047 \\
.00046\end{array}$ & $\begin{array}{r}.168 \\
.167 \\
.166\end{array}$ & $\begin{array}{l}.513 \\
.510 \\
.507\end{array}$ & $\begin{array}{r}.582 \\
.578 \\
.575\end{array}$ \\
\hline $\begin{array}{l}24 \\
24\end{array}$ & $\begin{array}{l}500 \\
500\end{array}$ & $\begin{array}{l}55.0 \\
60.0\end{array}$ & $\begin{array}{l}.00038 \\
.00036\end{array}$ & $\begin{array}{l}.00152 \\
.00147\end{array}$ & $\begin{array}{l}.00011 \\
.00011\end{array}$ & $\begin{array}{r}.00046 \\
.00044\end{array}$ & .165 & $\begin{array}{l}.504 \\
.488\end{array}$ & $\begin{array}{l}.571 \\
.553\end{array}$ \\
\hline 24 & 500 & 65.0 & .00035 & .00143 & .00010 & .00043 & .155 & .473 & .536 \\
\hline $\begin{array}{l}24 \\
24 \\
24\end{array}$ & $\begin{array}{l}500 \\
500 \\
500\end{array}$ & $\begin{array}{l}70.0 \\
75.0 \\
80.0\end{array}$ & $\begin{array}{r}.00034 \\
.00033 \\
.00033\end{array}$ & $\begin{array}{l}.00139 \\
.00135 \\
.00132\end{array}$ & $\begin{array}{l}.00010 \\
.00010 \\
.00010\end{array}$ & $\begin{array}{l}.00042 \\
.00041 \\
.00040\end{array}$ & $\begin{array}{r}.151 \\
.147 \\
.144\end{array}$ & $\begin{array}{r}.460 \\
.448 \\
.439\end{array}$ & $\begin{array}{l}.522 \\
.509 \\
.498\end{array}$ \\
\hline $\begin{array}{l}24 \\
24 \\
24\end{array}$ & $\begin{array}{l}500 \\
500 \\
500\end{array}$ & $\begin{array}{l}85.0 \\
90.0 \\
95.0\end{array}$ & $\begin{array}{l}.00032 \\
.00031 \\
.00031\end{array}$ & $\begin{array}{l}.00130 \\
.00127 \\
.00124\end{array}$ & $\begin{array}{l}.00009 \\
.00009 \\
.00009\end{array}$ & $\begin{array}{r}.00039 \\
.00038 \\
.00037\end{array}$ & $\begin{array}{r}.141 \\
.138 \\
.135\end{array}$ & $\begin{array}{l}.430 \\
.421 \\
.412\end{array}$ & $\begin{array}{r}.487 \\
.477 \\
.467\end{array}$ \\
\hline 24 & 500 & 100.0 & .00030 & 00122 & .00009 & .00037 & .132 & .404 & .458 \\
\hline
\end{tabular}


TABULATION OF DATA FOR

ABSORPTION OF SOUND IN AIR VERSUS PERCENT RELATIVE HUMMIDITY AT 24 DEGREES CENTIGRADE FOR A FREQUENCY OF 1000 HERTZ

\begin{tabular}{|c|c|c|c|c|c|c|c|c|c|}
\hline (1) & $(2)$ & (3) & (4) & (5) & (6)! & 171 & (3) & 191 & $\mid 10\}$ \\
\hline TEMP & FREQ & $\begin{array}{l}\text { REL } \\
\text { HUM }\end{array}$ & $\begin{array}{l}\text { ATTEN } \\
\text { COEF }\end{array}$ & $4 M$ & $\begin{array}{l}\text { ATTEN } \\
\text { COEF }\end{array}$ & $4 M$ & $\begin{array}{l}\text { ATTEN } \\
\text { DB PER }\end{array}$ & $\begin{array}{l}\text { ATTEN } \\
\text { DB PER }\end{array}$ & $\begin{array}{l}\text { DECAY } \\
\text { RATE }\end{array}$ \\
\hline $\begin{array}{l}\text { DEGR } \\
\text { CENT }\end{array}$ & HERTZ & $\begin{array}{l}\text { PER } \\
\text { CENT }\end{array}$ & $\begin{array}{l}\text { PER } \\
\text { METER }\end{array}$ & $\begin{array}{l}\text { PER } \\
\text { METER }\end{array}$ & 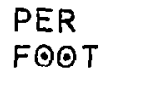 & $\begin{array}{l}\text { PER } \\
\text { FOOT }\end{array}$ & $\begin{array}{l}100 \\
\text { METER }\end{array}$ & $\begin{array}{l}1000 \\
\text { FEET }\end{array}$ & $\begin{array}{l}\text { DB PER } \\
\text { SECOND }\end{array}$ \\
\hline $\begin{array}{l}24 \\
24\end{array}$ & $\begin{array}{l}1000 \\
1000\end{array}$ & $\begin{array}{r}5.0 \\
10.0\end{array}$ & $\begin{array}{l}.00476 \\
.00225\end{array}$ & $\begin{array}{l}.01907 \\
.00901\end{array}$ & $\begin{array}{l}.00145 \\
.00068\end{array}$ & $\begin{array}{l}.00581 \\
.00274\end{array}$ & $\begin{array}{r}2.071 \\
.978\end{array}$ & $\begin{array}{l}6.314 \\
2.982\end{array}$ & $\begin{array}{l}7.158 \\
3.381\end{array}$ \\
\hline 24 & 1000 & $15 \cdot 0$ & .00155 & .00620 & .00047 & .00189 & .673 & 2.052 & 2.326 \\
\hline $\begin{array}{l}24 \\
24 \\
24\end{array}$ & $\begin{array}{l}1000 \\
1000 \\
1000\end{array}$ & $\begin{array}{l}20.0 \\
25.0 \\
30.0\end{array}$ & $\begin{array}{r}.00134 \\
.00124 \\
.00116\end{array}$ & $\begin{array}{r}.00539 \\
.00498 \\
.00466\end{array}$ & $\begin{array}{r}.00041 \\
.00037 \\
.00035\end{array}$ & $\begin{array}{l}.00164 \\
.00151 \\
.00142\end{array}$ & $\begin{array}{l}.586 \\
.541 \\
.506\end{array}$ & $\begin{array}{l}1.786 \\
1.649 \\
1.544\end{array}$ & $\begin{array}{l}2.025 \\
1.870 \\
1.751\end{array}$ \\
\hline $\begin{array}{l}24 \\
24 \\
24\end{array}$ & $\begin{array}{l}1000 \\
1000 \\
1000\end{array}$ & $\begin{array}{l}35.0 \\
40.0 \\
45.0\end{array}$ & $\begin{array}{l}.00110 \\
.00104 \\
.00099\end{array}$ & $\begin{array}{r}.00440 \\
.00416 \\
.00399\end{array}$ & $\begin{array}{l}.00033 \\
.00031 \\
.00030\end{array}$ & $\begin{array}{l}.00134 \\
.00126 \\
.00121\end{array}$ & $\begin{array}{r}.477 \\
.451 \\
.434\end{array}$ & $\begin{array}{l}1 \cdot 456 \\
1 \cdot 377 \\
1 \cdot 323\end{array}$ & $\begin{array}{l}1.650 \\
1.561 \\
1.500\end{array}$ \\
\hline $\begin{array}{l}24 \\
24 \\
24\end{array}$ & $\begin{array}{l}1000 \\
1000 \\
1000\end{array}$ & $\begin{array}{l}46 \cdot 0 \\
47 \cdot 0 \\
48.0\end{array}$ & $\begin{array}{r}.00099 \\
.00098 \\
.00097\end{array}$ & $\begin{array}{l}.00397 \\
.00394 \\
.00391\end{array}$ & $\begin{array}{l}.00030 \\
.00030 \\
.00029\end{array}$ & $\begin{array}{l}.00121 \\
.00120 \\
.00119\end{array}$ & $\begin{array}{r}.431 \\
.428 \\
.424\end{array}$ & $\begin{array}{l}1.314 \\
1.304 \\
1.295\end{array}$ & $\begin{array}{l}1.489 \\
1.479 \\
1.468\end{array}$ \\
\hline $\begin{array}{l}24 \\
24 \\
24\end{array}$ & $\begin{array}{l}1000 \\
1000 \\
1000\end{array}$ & $\begin{array}{l}49 \cdot 0 \\
50.0 \\
51.0\end{array}$ & $\begin{array}{l}.00097 \\
.00096 \\
.00095\end{array}$ & $\begin{array}{l}.00388 \\
.00385 \\
.00383\end{array}$ & $\begin{array}{l}.00029 \\
.00029 \\
.00029\end{array}$ & $\begin{array}{l}.00118 \\
.00117 \\
.00116\end{array}$ & $\begin{array}{r}.421 \\
.418 \\
.415\end{array}$ & $\begin{array}{l}1.285 \\
1.276 \\
1.267\end{array}$ & $\begin{array}{l}1.457 \\
1.446 \\
1.437\end{array}$ \\
\hline $\begin{array}{l}24 \\
24 \\
24\end{array}$ & $\begin{array}{l}1000 \\
1000 \\
1000\end{array}$ & $\begin{array}{l}52 \cdot 0 \\
53 \cdot 0 \\
54 \cdot 0\end{array}$ & $\begin{array}{r}.00095 \\
.00094 \\
.00093\end{array}$ & $\begin{array}{l}.00380 \\
.00378 \\
.00375\end{array}$ & $\begin{array}{l}.00029 \\
.00028 \\
.00028\end{array}$ & $\begin{array}{l}.00116 \\
.00115 \\
.00114\end{array}$ & $\begin{array}{l}.413 \\
.410 \\
.408\end{array}$ & $\begin{array}{l}1.259 \\
1.251 \\
1.243\end{array}$ & $\begin{array}{l}1.428 \\
1.419 \\
1.410\end{array}$ \\
\hline $\begin{array}{l}24 \\
24\end{array}$ & $\begin{array}{l}1000 \\
1000\end{array}$ & $\begin{array}{l}55.0 \\
60.0\end{array}$ & $\begin{array}{l}.00093 \\
.00090\end{array}$ & $\begin{array}{l}.00373 \\
.00361\end{array}$ & $\begin{array}{l}.00028 \\
.00027\end{array}$ & $\begin{array}{l}.00113 \\
.00110\end{array}$ & $\begin{array}{l}.405 \\
.392\end{array}$ & $\begin{array}{l}1.236 \\
1.197\end{array}$ & $\begin{array}{l}1.401 \\
1.357\end{array}$ \\
\hline 24 & 1000 & 65.0 & .00087 & .00351 & .00026 & .00107 & .381 & 1.162 & 1.317 \\
\hline $\begin{array}{l}24 \\
24\end{array}$ & $\begin{array}{l}1000 \\
1000\end{array}$ & $\begin{array}{l}70.0 \\
75.0\end{array}$ & $\begin{array}{l}.00085 \\
.00083\end{array}$ & $\begin{array}{l}.00341 \\
.00334\end{array}$ & $\begin{array}{l}.00026 \\
.00025\end{array}$ & $\begin{array}{l}.00104 \\
.00101\end{array}$ & $\begin{array}{l}.370 \\
.362\end{array}$ & $\begin{array}{l}1.130 \\
1.105\end{array}$ & $\begin{array}{l}1.281 \\
1.253\end{array}$ \\
\hline 24 & 1000 & $80 \cdot 0$ & .00081 & .00326 & .00024 & .00099 & .354 & 1.080 & 1.224 \\
\hline $\begin{array}{l}24 \\
24\end{array}$ & $\begin{array}{l}1000 \\
1000\end{array}$ & $\begin{array}{l}85.0 \\
90.0\end{array}$ & $\begin{array}{l}.00079 \\
.00078\end{array}$ & $\begin{array}{l}.00319 \\
.00313\end{array}$ & $\begin{array}{l}.00024 \\
.00023\end{array}$ & $\begin{array}{l}.00097 \\
.00095\end{array}$ & $\begin{array}{r}346 \\
.340\end{array}$ & $\begin{array}{l}1.056 \\
1.037\end{array}$ & $\begin{array}{l}1.197 \\
1.175\end{array}$ \\
\hline 24 & 1000 & 95.0 & .00076 & .00307 & .00023 & .00093 & .334 & 1.018 & 1.154 \\
\hline 24 & 10001 & 100.0 & .00075 & .00301 & .00023 & .00092 & .327 & .999 & 1.132 \\
\hline
\end{tabular}


TABULATION OF DATA FOR

ABSORPTION OF SOUND IN AIR VERSUS PERCENT RELATIVE HUMIDITY AT 24 DEGREES CENTIGRADE FOR A FREQUENCY OF 2000 HERTZ

\begin{tabular}{|c|c|c|c|c|c|c|c|c|c|}
\hline (1) & $(2)$ & (3) & $(4)$ & (5) & $(6)$ & $(7)$ & $(8)$ & 191 & $(10)$ \\
\hline $\begin{array}{l}\text { TEMP } \\
\text { DEGR } \\
\text { CENT }\end{array}$ & HER TZ & $\begin{array}{l}\text { REL } \\
\text { HUM } \\
\text { PER } \\
\text { CENT }\end{array}$ & $\begin{array}{l}\text { ATTEN } \\
\text { COEF } \\
\text { PER } \\
\text { METER }\end{array}$ & $\begin{array}{l}4 M \\
\text { PER } \\
\text { METER }\end{array}$ & $\begin{array}{l}\text { ATTEN } \\
\text { COEF } \\
\text { PER } \\
\text { FOOT }\end{array}$ & $\begin{array}{l}4 M \\
\text { PER } \\
\text { FOOT }\end{array}$ & $\begin{array}{l}\text { AT.TEN } \\
\text { DB PER } \\
100 \\
\text { METER }\end{array}$ & $\begin{array}{l}\text { ATTEN } \\
\text { DB PER } \\
\text { IOOO. } \\
\text { FEET }\end{array}$ & $\begin{array}{l}\text { DECAY } \\
\text { RATE } \\
\text { DB PER } \\
\text { SECOND }\end{array}$ \\
\hline $\begin{array}{l}24 \\
24 \\
24\end{array}$ & $\begin{array}{l}2000 \\
2000 \\
2000\end{array}$ & $\begin{array}{r}5.0 \\
10.0 \\
15.0\end{array}$ & $\begin{array}{l}.01342 \\
.00761 \\
.00491\end{array}$ & $\begin{array}{r}.05371 \\
.03045 \\
.01967\end{array}$ & $\begin{array}{l}.00409 \\
.00232 \\
.00149\end{array}$ & $\begin{array}{l}.01637 \\
.00928 \\
.00599\end{array}$ & $\begin{array}{l}5.831 \\
3.306 \\
2.136\end{array}$ & $\begin{array}{r}17.775 \\
10.078 \\
6.512\end{array}$ & $\begin{array}{r}20.152 \\
11.426 \\
7.383\end{array}$ \\
\hline $\begin{array}{l}24 \\
24 \\
24\end{array}$ & $\begin{array}{l}2000 \\
2000 \\
2000\end{array}$ & $\begin{array}{l}20.0 \\
25.0 \\
30.0\end{array}$ & $\begin{array}{r}.00368 \\
.00311 \\
.00284\end{array}$ & $\begin{array}{r}.01472 \\
.01244 \\
.01136\end{array}$ & $\begin{array}{l}.00112 \\
.00094 \\
.00086\end{array}$ & $\begin{array}{r}.00448 \\
.00379 \\
.00346\end{array}$ & $\begin{array}{l}1.598 \\
1.350 \\
1.233\end{array}$ & $\begin{array}{l}4.873 \\
4.117 \\
3.760\end{array}$ & $\begin{array}{l}5.525 \\
4.668 \\
4.263\end{array}$ \\
\hline $\begin{array}{l}24 \\
24 \\
24\end{array}$ & $\begin{array}{l}2000 \\
2000 \\
2000\end{array}$ & $\begin{array}{l}35.0 \\
40.0 \\
45.0\end{array}$ & $\begin{array}{l}.00269 \\
.00257 \\
.00247\end{array}$ & $\begin{array}{r}.01076 \\
.01028 \\
.00988\end{array}$ & $\begin{array}{l}.00082 \\
.00078 \\
.00075\end{array}$ & $\begin{array}{l}.00328 \\
.00313 \\
.00301\end{array}$ & $\begin{array}{l}1.168 \\
1.117 \\
1.073\end{array}$ & $\begin{array}{l}3.562 \\
3.405 \\
3.271\end{array}$ & $\begin{array}{l}4.039 \\
3.850 \\
3.708\end{array}$ \\
\hline $\begin{array}{l}24 \\
24 \\
24\end{array}$ & $\begin{array}{l}2000 \\
2000 \\
2000\end{array}$ & $\begin{array}{l}46.0 \\
47.0 \\
48.0\end{array}$ & $\begin{array}{l}.00245 \\
.00243 \\
.00241\end{array}$ & $\begin{array}{l}.00980 \\
.00972 \\
.00965\end{array}$ & $\begin{array}{l}.00074 \\
.00074 \\
.00073\end{array}$ & $\begin{array}{l}.00298 \\
.00296 \\
.00294\end{array}$ & $\begin{array}{l}1.064 \\
1.056 \\
1.048\end{array}$ & $\begin{array}{l}3.245 \\
3.218 \\
3.195\end{array}$ & $\begin{array}{l}3.679 \\
3.649 \\
3.622\end{array}$ \\
\hline $\begin{array}{l}24 \\
24 \\
24\end{array}$ & $\begin{array}{l}2000 \\
2000 \\
2000\end{array}$ & $\begin{array}{l}49.0 \\
50.0 \\
51.0\end{array}$ & $\begin{array}{l}.00239 \\
.00237 \\
.00236\end{array}$ & $\begin{array}{r}.00958 \\
.00951 \\
.00945\end{array}$ & $\begin{array}{r}.00073 \\
.00072 \\
.00072\end{array}$ & $\begin{array}{l}.00292 \\
.00290 \\
.00288\end{array}$ & $\begin{array}{l}1.040 \\
1.033 \\
1.026\end{array}$ & $\begin{array}{l}3.172 \\
3.150 \\
3.127\end{array}$ & $\begin{array}{l}3.597 \\
3.571 \\
3.546\end{array}$ \\
\hline $\begin{array}{l}24 \\
24 \\
24\end{array}$ & $\begin{array}{l}2000 \\
2000 \\
2000\end{array}$ & $\begin{array}{l}52.0 \\
53.0 \\
54.0\end{array}$ & $\begin{array}{l}.00234 \\
.00232 \\
.00231\end{array}$ & $\begin{array}{l}.00938 \\
.00931 \\
.00924\end{array}$ & $\begin{array}{l}.00071 \\
.00070 \\
.00070\end{array}$ & $\begin{array}{l}.00286 \\
.00283 \\
.00281\end{array}$ & $\begin{array}{l}1.018 \\
1.011 \\
1.004\end{array}$ & $\begin{array}{l}3.105 \\
3.082 \\
3.060\end{array}$ & $\begin{array}{l}3.520 \\
3.495 \\
3.469\end{array}$ \\
\hline $\begin{array}{l}24 \\
24 \\
24\end{array}$ & $\begin{array}{l}2000 \\
2000 \\
2000\end{array}$ & $\begin{array}{l}55.0 \\
60.0 \\
65.0\end{array}$ & $\begin{array}{l}.00229 \\
.00222 \\
.00215\end{array}$ & $\begin{array}{l}.00917 \\
.00888 \\
.00860\end{array}$ & $\begin{array}{l}.00069 \\
.00067 \\
.00065\end{array}$ & $\begin{array}{r}.00279 \\
.00270 \\
.00262\end{array}$ & $\begin{array}{r}.996 \\
.964 \\
.933\end{array}$ & $\begin{array}{l}3.037 \\
2.939 \\
2.846\end{array}$ & $\begin{array}{l}3.444 \\
3.332 \\
3.226\end{array}$ \\
\hline $\begin{array}{l}24 \\
24 \\
24\end{array}$ & $\begin{array}{l}2000 \\
2000 \\
2000\end{array}$ & $\begin{array}{l}70.0 \\
75.0 \\
80.0\end{array}$ & $\begin{array}{l}.00209 \\
.00205 \\
.00200\end{array}$ & $\begin{array}{l}.00839 \\
.00820 \\
.00802\end{array}$ & $\begin{array}{l}.00063 \\
.00062 \\
.00061\end{array}$ & $\begin{array}{l}.00255 \\
.00250 \\
.00244\end{array}$ & $\begin{array}{r}.911 \\
.891 \\
.871\end{array}$ & $\begin{array}{l}2.778 \\
2.716 \\
2.657\end{array}$ & $\begin{array}{l}3.150 \\
3.079 \\
3.012\end{array}$ \\
\hline $\begin{array}{l}24 \\
24 \\
24\end{array}$ & $\begin{array}{l}2000 \\
2000 \\
2000\end{array}$ & $\begin{array}{l}85.0 \\
90.0 \\
95.0\end{array}$ & $\begin{array}{l}.00196 \\
.00193 \\
.00189\end{array}$ & $\begin{array}{r}.00787 \\
.00772 \\
.00758\end{array}$ & $\begin{array}{l}.00060 \\
.00058 \\
.00057\end{array}$ & $\begin{array}{l}.00240 \\
.00235 \\
.00231\end{array}$ & $\begin{array}{r}.855 \\
.838 \\
.823\end{array}$ & $\begin{array}{l}2.606 \\
2.556 \\
2.509\end{array}$ & $\begin{array}{l}2.954 \\
2.898 \\
2.845\end{array}$ \\
\hline .24 & 2000 & $100 \cdot 0$ & .00186 & .00744 & .00056 & .00226 & .807 & $2 \cdot 462$ & 2.791 \\
\hline
\end{tabular}


TABULATION OF DATA FOR

ABSORPTION OF SOUND IN AIR VERSUS PERCENT RELATIVE HUMIDITY AT 24 DEGREES CENTIGRADE FOR A FREQUENCY OF 2500 HERTZ

\begin{tabular}{|c|c|c|c|c|c|c|c|c|c|}
\hline (1) & 121 & (3). & $(4)$ & (5) & (6) & 171 & 181 & 191 & $(10)$ \\
\hline $\begin{array}{l}\text { EMP } \\
\text { EGR } \\
\text { ENT }\end{array}$ & HERTZ & $\begin{array}{l}\text { REL } \\
\text { HUM } \\
\text { PER } \\
\text { CENT }\end{array}$ & $\begin{array}{l}\text { ATTEN } \\
\text { COEF } \\
\text { PER } \\
\text { METER }\end{array}$ & $\begin{array}{l}\text { PER } \\
\text { METER }\end{array}$ & $\begin{array}{l}\text { ATTEN } \\
\text { COEF } \\
\text { PER } \\
\text { FOOT }\end{array}$ & 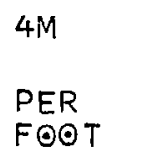 & $\begin{array}{l}\text { ATTEN } \\
\text { DB PER } \\
100 \\
\text { METER }\end{array}$ & $\begin{array}{l}\text { ATTEN } \\
\text { DB PER } \\
\text { IOOO } \\
\text { FEET }\end{array}$ & $\begin{array}{l}\text { DECAY } \\
\text { RATE } \\
\text { DB PER } \\
\text { SECOND }\end{array}$ \\
\hline $\begin{array}{l}24 \\
24 \\
24\end{array}$ & $\begin{array}{l}2500 \\
2500 \\
2500\end{array}$ & $\begin{array}{r}5.0 \\
10.0 \\
15.0\end{array}$ & $\begin{array}{l}.01687 \\
.01136 \\
.00736\end{array}$ & $\begin{array}{l}.06751 \\
.04545 \\
.02945\end{array}$ & $\begin{array}{l}.00514 \\
.00346 \\
.00224\end{array}$ & $\begin{array}{l}.02057 \\
.01385 \\
.00897\end{array}$ & $\begin{array}{l}7.330 \\
4.934 \\
3.198\end{array}$ & $\begin{array}{r}22.343 \\
15.041 \\
9.747\end{array}$ & $\begin{array}{l}25.331 \\
17.053 \\
11.051\end{array}$ \\
\hline $\begin{array}{l}24 \\
24 \\
24\end{array}$ & $\begin{array}{l}2500 \\
2500 \\
2500\end{array}$ & $\begin{array}{l}20.0 \\
25.0 \\
30.0\end{array}$ & $\begin{array}{r}.00544 \\
.00440 \\
.00389\end{array}$ & $\begin{array}{r}.02177 \\
.01763 \\
.01558\end{array}$ & $\begin{array}{l}.00165 \\
.00134 \\
.00118\end{array}$ & $\begin{array}{l}.00663 \\
.00537 \\
.00474\end{array}$ & $\begin{array}{l}2.364 \\
1.915 \\
1.691\end{array}$ & $\begin{array}{l}7.206 \\
5.837 \\
5.156\end{array}$ & $\begin{array}{l}8.170 \\
6.618 \\
5.846\end{array}$ \\
\hline $\begin{array}{l}24 \\
24 \\
24\end{array}$ & $\begin{array}{l}2500 \\
2500 \\
2500\end{array}$ & $\begin{array}{l}35.0 \\
40.0 \\
45.0\end{array}$ & $\begin{array}{l}.00360 \\
.00344 \\
.00331\end{array}$ & $\begin{array}{r}.01443 \\
.01379 \\
.01325\end{array}$ & $\begin{array}{l}.00110 \\
.00105 \\
.00101\end{array}$ & $\begin{array}{l}.00440 \\
.00420 \\
.00404\end{array}$ & $\begin{array}{l}1.567 \\
1.497 \\
1.439\end{array}$ & $\begin{array}{l}4.777 \\
4.563 \\
4.387\end{array}$ & $\begin{array}{l}5.416 \\
5.174 \\
4.974\end{array}$ \\
\hline $\begin{array}{l}24 \\
24 \\
24\end{array}$ & $\begin{array}{l}2500 \\
2500 \\
2500\end{array}$ & $\begin{array}{l}46.0 \\
47.0 \\
48.0\end{array}$ & $\begin{array}{l}.00328 \\
.00326 \\
.00324\end{array}$ & $\begin{array}{r}.01315 \\
.01305 \\
.01296\end{array}$ & $\begin{array}{l}.00100 \\
.00099 \\
.00098\end{array}$ & $\begin{array}{l}.00401 \\
.00397 \\
.00395\end{array}$ & $\begin{array}{l}1.428 \\
1.417 \\
1.407\end{array}$ & $\begin{array}{l}4 \cdot 354 \\
4 \cdot 320 \\
4 \cdot 289\end{array}$ & $\begin{array}{l}4.936 \\
4.898 \\
4.862\end{array}$ \\
\hline $\begin{array}{l}24 \\
24\end{array}$ & $\begin{array}{l}2500 \\
2500\end{array}$ & $\begin{array}{l}49.0 \\
50.0\end{array}$ & $\begin{array}{l}.00321 \\
.00319\end{array}$ & $\begin{array}{r}.01287 \\
.01279\end{array}$ & $\begin{array}{l}.00098 \\
.00097\end{array}$ & $\begin{array}{l}.00392 \\
.00389\end{array}$ & $\begin{array}{l}1.398 \\
1.388\end{array}$ & $\begin{array}{l}4.261 \\
4.233\end{array}$ & $\begin{array}{l}4.831 \\
4.799\end{array}$ \\
\hline 24 & 2500 & 51.0 & .00317 & .01270 & .00096 & .00387 & $1 \cdot 379$ & $4 \cdot 205$ & $4 \cdot 768$ \\
\hline $\begin{array}{l}24 \\
24 \\
24\end{array}$ & $\begin{array}{l}2500 \\
2500 \\
2500\end{array}$ & $\begin{array}{l}52.0 \\
53.0 \\
54.0\end{array}$ & $\begin{array}{l}.00315 \\
.00313 \\
.00311\end{array}$ & $\begin{array}{r}.01262 \\
.01254 \\
.01245\end{array}$ & $\begin{array}{l}.00096 \\
.00095 \\
.00094\end{array}$ & $\begin{array}{l}.00384 \\
.00382 \\
.00379\end{array}$ & $\begin{array}{l}1.370 \\
1.361 \\
1.352\end{array}$ & $\begin{array}{l}4 \cdot 177 \\
4 \cdot 149 \\
4 \cdot 122\end{array}$ & $\begin{array}{l}4.736 \\
4.705 \\
4.673\end{array}$ \\
\hline $\begin{array}{l}24 \\
24\end{array}$ & $\begin{array}{l}2500 \\
2500\end{array}$ & $\begin{array}{l}55.0 \\
60.0\end{array}$ & $\begin{array}{l}.00309 \\
.00300\end{array}$ & $\begin{array}{r}.01237 \\
.01200\end{array}$ & $\begin{array}{l}.00094 \\
.00091\end{array}$ & $\begin{array}{r}.00377 \\
.00365\end{array}$ & $\begin{array}{l}1.343 \\
1.303\end{array}$ & $\begin{array}{l}4.094 \\
3.972\end{array}$ & $\begin{array}{l}4.641 \\
4.503\end{array}$ \\
\hline 24 & 2500 & 65.0 & .00291 & .01164 & .00088 & .00354 & 1.264 & 3.853 & $4 \cdot 368$ \\
\hline $\begin{array}{l}24 \\
24\end{array}$ & $\begin{array}{l}2500 \\
2500\end{array}$ & $\begin{array}{l}70 \cdot 0 \\
75 \cdot 0\end{array}$ & $\begin{array}{l}.00283 \\
.00275\end{array}$ & $\begin{array}{r}.01132 \\
.01101\end{array}$ & $\begin{array}{r}.00086 \\
.00083\end{array}$ & $\begin{array}{l}.00345 \\
.00335\end{array}$ & $\begin{array}{l}1.229 \\
1.195\end{array}$ & $\begin{array}{l}3.748 \\
3.644\end{array}$ & $\begin{array}{l}4.250 \\
4.131\end{array}$ \\
\hline 24 & 2500 & 80.0 & .00269 & .01078 & .00082 & .00328 & 1.171 & 3.569 & 4.046 \\
\hline $\begin{array}{l}24 \\
24 \\
24\end{array}$ & $\begin{array}{l}2500 \\
2500 \\
2500\end{array}$ & $\begin{array}{l}85.0 \\
90.0 \\
95.0\end{array}$ & $\begin{array}{l}.00264 \\
.00259 \\
.00254\end{array}$ & $\begin{array}{r}.01057 \\
.01038 \\
.01019\end{array}$ & $\begin{array}{l}.00080 \\
.00079 \\
.00077\end{array}$ & $\begin{array}{l}.00322 \\
.00316 \\
.00310\end{array}$ & $\begin{array}{l}1.147 \\
1.127 \\
1.106\end{array}$ & $\begin{array}{l}3.498 \\
3.435 \\
3.372\end{array}$ & $\begin{array}{l}3.965 \\
3.894 \\
3.823\end{array}$ \\
\hline 2 & 2500 & $100 \cdot 0$ & 00250 & .01003 & 00076 & .00305 & 1.089 & $3 \cdot 319$ & 3.763 \\
\hline
\end{tabular}


TABULATION OF DATA FOR

ABSORPTION OF SOUND IN AIR VERSUS PERCENT RELATIVE HUMIDITY AT 24 DEGREES CENTIGRADE FOR A FREQUENCY OF 3200 HERTZ

\begin{tabular}{|c|c|c|c|c|c|c|c|c|c|}
\hline$(1)$ & (2) & (3) & $(4)$ & (5) & (6) & $(7)$ & $(8)$ & 191 & $(10)$ \\
\hline TEMP & FREQ & $\begin{array}{l}\text { REL } \\
\text { HUM }\end{array}$ & $\begin{array}{l}\text { ATTEN } \\
\text { COEF }\end{array}$ & $4 M$ & $\begin{array}{l}\text { ATTEN } \\
\text { COEF }\end{array}$ & $4 M$ & $\begin{array}{l}\text { ATTEN } \\
\text { DB PER }\end{array}$ & $\begin{array}{l}\text { ATTEN. } \\
\text { DB PER }\end{array}$ & $\begin{array}{l}\text { DECAY } \\
\text { RATE }\end{array}$ \\
\hline $\begin{array}{l}\text { DEGR } \\
\text { CENT }\end{array}$ & HERTZ & $\begin{array}{l}\text { PER } \\
\text { CENT }\end{array}$ & $\begin{array}{l}\text { PER } \\
\text { METER }\end{array}$ & $\begin{array}{l}\text { PER } \\
\text { METER }\end{array}$ & $\begin{array}{l}\text { PER } \\
\text { FOOT }\end{array}$ & $\begin{array}{l}\text { PER } \\
\text { FOOT }\end{array}$ & $\begin{array}{l}100 \\
\text { METER }\end{array}$ & $\begin{array}{l}1000 \\
\text { FEET }\end{array}$ & $\begin{array}{l}\text { DB PER } \\
\text { SECOND }\end{array}$ \\
\hline $\begin{array}{l}24 \\
24 \\
24\end{array}$ & $\begin{array}{l}3200 \\
3200 \\
3200\end{array}$ & $\begin{array}{r}5.0 \\
10.0 \\
15.0\end{array}$ & $\begin{array}{l}.02081 \\
.01737 \\
.01131\end{array}$ & $\begin{array}{l}.08324 \\
.06951 \\
.04526\end{array}$ & $\begin{array}{l}.00634 \\
.00529 \\
.00344\end{array}$ & $\begin{array}{r}.02537 \\
.02118 \\
.01379\end{array}$ & $\begin{array}{l}9.038 \\
7.547 \\
4.914\end{array}$ & $\begin{array}{l}27.550 \\
23.004 \\
14.980\end{array}$ & $\begin{array}{l}31.235 \\
26.081 \\
16.984\end{array}$ \\
\hline $\begin{array}{l}24 \\
24 \\
24\end{array}$ & $\begin{array}{l}3200 \\
3200 \\
3200\end{array}$ & $\begin{array}{l}20.0 \\
25.0 \\
30.0\end{array}$ & $\begin{array}{l}.00828 \\
.00658 \\
.00559\end{array}$ & $\begin{array}{r}.03313 \\
.02635 \\
.02239\end{array}$ & $\begin{array}{l}.00252 \\
.00200 \\
.00170\end{array}$ & $\begin{array}{l}.01010 \\
.00803 \\
.00682\end{array}$ & $\begin{array}{l}3.597 \\
2.861 \\
2.431\end{array}$ & $\begin{array}{r}10.966 \\
8.723 \\
7.411\end{array}$ & $\begin{array}{r}12.433 \\
9.890 \\
8.402\end{array}$ \\
\hline $\begin{array}{l}24 \\
24 \\
24\end{array}$ & $\begin{array}{l}3200 \\
3200 \\
3200\end{array}$ & $\begin{array}{l}35.0 \\
40.0 \\
45.0\end{array}$ & $\begin{array}{l}.00507 \\
.00474 \\
.00455\end{array}$ & $\begin{array}{l}.02030 \\
.01896 \\
.01823\end{array}$ & $\begin{array}{l}.00154 \\
.00144 \\
.00138\end{array}$ & $\begin{array}{l}.00619 \\
.00578 \\
.00555\end{array}$ & $\begin{array}{l}2.205 \\
2.059 \\
1.980\end{array}$ & $\begin{array}{l}6.721 \\
6.276 \\
6.036\end{array}$ & $\begin{array}{l}7.620 \\
7.116 \\
6.843\end{array}$ \\
\hline $\begin{array}{l}24 \\
24\end{array}$ & $\begin{array}{l}3200 \\
3200\end{array}$ & $\begin{array}{l}46.0 \\
47.0\end{array}$ & $\begin{array}{l}.00452 \\
.00449\end{array}$ & $\begin{array}{r}.01810 \\
.01796\end{array}$ & $\begin{array}{l}.00137 \\
.00136\end{array}$ & $\begin{array}{l}.00551 \\
.00547\end{array}$ & $\begin{array}{l}1.965 \\
1.950\end{array}$ & $\begin{array}{l}5.990 \\
5.944\end{array}$ & $\begin{array}{l}6.791 \\
6.739\end{array}$ \\
\hline 24 & 3200 & 48.0 & .00445 & .01782 & .00135 & .00543 & 1.935 & 5.899 & 6.687 \\
\hline $\begin{array}{l}24 \\
24\end{array}$ & $\begin{array}{l}3200 \\
3200\end{array}$ & $\begin{array}{l}49.0 \\
50.0\end{array}$ & $\begin{array}{r}.00442 \\
.00440\end{array}$ & $\begin{array}{r}.01771 \\
.01760\end{array}$ & $\begin{array}{l}.00134 \\
.00134\end{array}$ & $\begin{array}{l}.00539 \\
.00536\end{array}$ & $\begin{array}{l}1.923 \\
1.911\end{array}$ & $\begin{array}{l}5.861 \\
5.825\end{array}$ & $\begin{array}{l}6.646 \\
6.604\end{array}$ \\
\hline 24 & 3200 & 51.0 & .00437 & .01749 & .00133 & .00533 & 1.899 & $5 \cdot 788$ & 6.563 \\
\hline $\begin{array}{l}24 \\
24\end{array}$ & $\begin{array}{l}3200 \\
3200\end{array}$ & $\begin{array}{l}52.0 \\
53.0\end{array}$ & $\begin{array}{l}.00434 \\
.00431\end{array}$ & $\begin{array}{l}.01738 \\
.01727\end{array}$ & $\begin{array}{l}.00132 \\
.00131\end{array}$ & $\begin{array}{l}.00529 \\
.00526\end{array}$ & $\begin{array}{l}1.887 \\
1.875\end{array}$ & $\begin{array}{l}5.752 \\
5.715\end{array}$ & $\begin{array}{l}6.521 \\
6.480\end{array}$ \\
\hline 24 & 3200 & 54.0 & .00429 & .01716 & .00130 & .00523 & 1.863 & 5.679 & 6.438 \\
\hline $\begin{array}{l}24 \\
24\end{array}$ & $\begin{array}{l}3200 \\
3200\end{array}$ & $\begin{array}{l}55.0 \\
60.0\end{array}$ & $\begin{array}{r}.00426 \\
.00414\end{array}$ & $\begin{array}{r}.01705 \\
.01657\end{array}$ & $\begin{array}{l}.00129 \\
.00126\end{array}$ & $\begin{array}{l}.00515 \\
.00505\end{array}$ & $\begin{array}{l}1.851 \\
1.799\end{array}$ & $\begin{array}{l}5.642 \\
5.485\end{array}$ & $\begin{array}{l}6.397 \\
6.218\end{array}$ \\
\hline 24 & 3200 & 65.0 & .00402 & .01611 & .00122 & .00491 & 1.749 & 5.332 & 6.046 \\
\hline $\begin{array}{l}24 \\
24 \\
24\end{array}$ & $\begin{array}{l}3200 \\
3200 \\
3200\end{array}$ & $\begin{array}{l}70.0 \\
75.0 \\
80.0\end{array}$ & $\begin{array}{l}.00392 \\
.00383 \\
.00374\end{array}$ & $\begin{array}{r}.01571 \\
.01532 \\
.01496\end{array}$ & $\begin{array}{l}.00119 \\
.00116 \\
.00114\end{array}$ & $\begin{array}{l}.00479 \\
.00467 \\
.00456\end{array}$ & $\begin{array}{l}1.706 \\
1.663 \\
1.625\end{array}$ & $\begin{array}{l}5.201 \\
5.071 \\
4.953\end{array}$ & $\begin{array}{l}5.897 \\
5.749 \\
5.616\end{array}$ \\
\hline $\begin{array}{l}24 \\
24 \\
24\end{array}$ & $\begin{array}{l}3200 \\
3200 \\
3200\end{array}$ & $\begin{array}{l}85.0 \\
90.0 \\
95.0\end{array}$ & $\begin{array}{r}.00365 \\
.00357 \\
.00351\end{array}$ & $\begin{array}{l}.01462 \\
.01431 \\
.01407\end{array}$ & $\begin{array}{l}.00111 \\
.00109 \\
.00107\end{array}$ & $\begin{array}{l}.00445 \\
.00436 \\
.00429\end{array}$ & $\begin{array}{l}1.587 \\
1.554 \\
1.528\end{array}$ & $\begin{array}{l}4.839 \\
4.737 \\
4.658\end{array}$ & $\begin{array}{l}5.487 \\
5.370 \\
5.282\end{array}$ \\
\hline 24 & 3200 & $100 \cdot 0$ & .00346 & .01384 & .00105 & .00422 & $1 \cdot 503$ & $4 \cdot 582$ & 5.195 \\
\hline
\end{tabular}


TABULATION OF DATA FOR

ABSORPTION OF SOUND IN AIR VERSUS PERCENT RELATIVE HUMIDITY AT 24 .DEGREES CENTIGRADE FOR A FREQUENCY OF 4000 HERTZ

\begin{tabular}{|c|c|c|c|c|c|c|c|c|c|}
\hline (1) & (2) & (3) & (4) & (5) & $(6)$ & 171. & $18 !$ & $(9)$ & $(10)$ \\
\hline EMP & FREQ & $\begin{array}{l}\text { REL } \\
\text { HUM }\end{array}$ & $\begin{array}{l}\text { ATTEN } \\
\text { COEF }\end{array}$ & $4 M$ & $\begin{array}{l}\text { ATTEN } \\
\text { COEF }\end{array}$ & $4 M$ & $\begin{array}{l}\text { ATTEN } \\
\text { DB PER }\end{array}$ & $\begin{array}{l}\text { ATTEN } \\
\text { OB PER }\end{array}$ & $\begin{array}{l}\text { DECAY } \\
\text { RATE }\end{array}$ \\
\hline $\begin{array}{l}\text { DEGR } \\
\text { CENT }\end{array}$ & HERTZ & $\begin{array}{l}\text { PER } \\
\text { CENT }\end{array}$ & $\begin{array}{l}\text { PER } \\
\text { METER }\end{array}$ & $\begin{array}{l}\text { PER } \\
\text { METER }\end{array}$ & $\begin{array}{l}\text { PER } \\
\text { FOOT }\end{array}$ & $\begin{array}{l}\text { PER } \\
\text { FOOT }\end{array}$ & $\begin{array}{l}100 \\
\text { METER }\end{array}$ & $\begin{array}{l}1000 \\
\text { FEET }\end{array}$ & $\begin{array}{l}\text { DB PER } \\
\text { SECOND }\end{array}$ \\
\hline $\begin{array}{l}24 \\
24\end{array}$ & $\begin{array}{l}4000 \\
4000\end{array}$ & $\begin{array}{r}5.0 \\
10.0\end{array}$ & $\begin{array}{r}.02395 \\
.02482\end{array}$ & $\begin{array}{r}.09583 \\
.09929\end{array}$ & $\begin{array}{l}.00730 \\
.00756\end{array}$ & $\begin{array}{l}.02921 \\
.03026\end{array}$ & $\begin{array}{l}10.405 \\
10.781\end{array}$ & $\begin{array}{l}31.716 \\
32.862\end{array}$ & $\begin{array}{l}35.958 \\
37.257\end{array}$ \\
\hline 24 & 4000 & $15 \cdot 0$ & .01656 & .06624 & .00504 & .02019 & 7.192 & $21 \cdot 924$ & 24.856 \\
\hline $\begin{array}{l}24 \\
24\end{array}$ & $\begin{array}{l}4000 \\
4000\end{array}$ & $\begin{array}{l}20.0 \\
25.0\end{array}$ & $\begin{array}{l}.01225 \\
.00974\end{array}$ & $\begin{array}{l}.04902 \\
.03899\end{array}$ & $\begin{array}{l}.00373 \\
.00297\end{array}$ & $\begin{array}{r}.01494 \\
.01188\end{array}$ & $\begin{array}{l}5.322 \\
4.234\end{array}$ & $\begin{array}{l}16.224 \\
12.906\end{array}$ & $\begin{array}{l}18.394 \\
14.632\end{array}$ \\
\hline 24 & 4000 & $30 \cdot 0$ & .00810 & .03242 & .00247 & .00988 & 3.520 & 10.730 & 12.165 \\
\hline $\begin{array}{l}24 \\
24\end{array}$ & $\begin{array}{l}4000 \\
4000\end{array}$ & $\begin{array}{l}35.0 \\
40.0\end{array}$ & $\begin{array}{l}.00709 \\
.00652\end{array}$ & $\begin{array}{r}.02837 \\
.02608\end{array}$ & $\begin{array}{l}.00216 \\
.00198\end{array}$ & $\begin{array}{l}.00864 \\
.00795\end{array}$ & $\begin{array}{l}3.081 \\
2.832\end{array}$ & $\begin{array}{l}9.391 \\
8.632\end{array}$ & $\begin{array}{r}10.647 \\
9.786\end{array}$ \\
\hline 24 & 4000 & 45.0 & .00612 & .02450 & .00186 & .00747 & 2.661 & 8.111 & 9.195 \\
\hline $\begin{array}{l}24 \\
24 \\
24\end{array}$ & $\begin{array}{l}4000 \\
4000 \\
4000\end{array}$ & $\begin{array}{l}46.0 \\
47.0 \\
48.0\end{array}$ & $\begin{array}{l}.00606 \\
.00602 \\
.00598\end{array}$ & $\begin{array}{r}.02427 \\
.02409 \\
.02392\end{array}$ & $\begin{array}{l}.00184 \\
.00183 \\
.00182\end{array}$ & $\begin{array}{l}.00739 \\
.00734 \\
.00729\end{array}$ & $\begin{array}{l}2.635 \\
2.616 \\
2.597\end{array}$ & $\begin{array}{l}8.032 \\
7.974 \\
7.918\end{array}$ & $\begin{array}{l}9.106 \\
9.040 \\
8.977\end{array}$ \\
\hline $\begin{array}{l}24 \\
24 \\
24\end{array}$ & $\begin{array}{l}4000 \\
4000 \\
4000\end{array}$ & $\begin{array}{l}49.0 \\
50.0 \\
51.0\end{array}$ & $\begin{array}{l}.00594 \\
.00590 \\
.00586\end{array}$ & $\begin{array}{r}.02376 \\
.02361 \\
.02346\end{array}$ & $\begin{array}{l}.00181 \\
.00179 \\
.00178\end{array}$ & $\begin{array}{l}.00724 \\
.00719 \\
.00715\end{array}$ & $\begin{array}{l}2.580 \\
2.564 \\
2.547\end{array}$ & $\begin{array}{l}7.864 \\
7.815 \\
7.766\end{array}$ & $\begin{array}{l}8.916 \\
8.860 \\
8.804\end{array}$ \\
\hline $\begin{array}{l}24 \\
24 \\
24\end{array}$ & $\begin{array}{l}4000 \\
4000 \\
4000\end{array}$ & $\begin{array}{l}52.0 \\
53.0 \\
54.0\end{array}$ & $\begin{array}{l}.00582 \\
.00579 \\
.00575\end{array}$ & $\begin{array}{l}.02331 \\
.02316 \\
.02302\end{array}$ & $\begin{array}{l}.00177 \\
.00176 \\
.00175\end{array}$ & $\begin{array}{l}.00710 \\
.00706 \\
.00701\end{array}$ & $\begin{array}{l}2.531 \\
2.515 \\
2.499\end{array}$ & $\begin{array}{l}7.717 \\
7.667 \\
7.618\end{array}$ & $\begin{array}{l}8.749 \\
8.693 \\
8.637\end{array}$ \\
\hline $\begin{array}{l}24 \\
24 \\
24\end{array}$ & $\begin{array}{l}4000 \\
4000 \\
4000\end{array}$ & $\begin{array}{l}55.0 \\
60.0 \\
65.0\end{array}$ & $\begin{array}{l}.00571 \\
.00556 \\
.00541\end{array}$ & $\begin{array}{r}.02287 \\
.02225 \\
.02166\end{array}$ & $\begin{array}{l}.00174 \\
.00169 \\
.00165\end{array}$ & $\begin{array}{l}.00697 \\
.00678 \\
.00660\end{array}$ & $\begin{array}{l}2.483 \\
2.416 \\
2.352\end{array}$ & $\begin{array}{l}7.569 \\
7.364 \\
7.169\end{array}$ & $\begin{array}{l}8.581 \\
8.349 \\
8.128\end{array}$ \\
\hline $\begin{array}{l}24 \\
24 \\
24\end{array}$ & $\begin{array}{l}4000 \\
4000 \\
4000\end{array}$ & $\begin{array}{l}70.0 \\
75.0 \\
80.0\end{array}$ & $\begin{array}{l}.00529 \\
.00516 \\
.00505\end{array}$ & $\begin{array}{l}.02116 \\
.02067 \\
.02023\end{array}$ & $\begin{array}{l}.00161 \\
.00157 \\
.00154\end{array}$ & $\begin{array}{l}.00645 \\
.00630 \\
.00616\end{array}$ & $\begin{array}{l}2.298 \\
2.244 \\
2.197\end{array}$ & $\begin{array}{l}7.005 \\
6.841 \\
6.697\end{array}$ & $\begin{array}{l}7.942 \\
7.756 \\
7.593\end{array}$ \\
\hline $\begin{array}{l}24 \\
24 \\
24\end{array}$ & $\begin{array}{l}4000 \\
4000 \\
4000\end{array}$ & $\begin{array}{l}85.0 \\
90.0 \\
95.0\end{array}$ & $\begin{array}{l}.00495 \\
.00485 \\
.00475\end{array}$ & $\begin{array}{r}.01981 \\
.01940 \\
.01903\end{array}$ & $\begin{array}{l}.0015 .0 \\
.00147 \\
.00145\end{array}$ & $\begin{array}{l}.00603 \\
.00591 \\
.00580\end{array}$ & $\begin{array}{l}2.151 \\
2.107 \\
2.066\end{array}$ & $\begin{array}{l}6.55 .7 \\
6.423 \\
6.300\end{array}$ & $\begin{array}{l}7.434 \\
7.282 \\
7.142\end{array}$ \\
\hline$T$ & 4000 & $100 \cdot 0$ & .00466 & .01866 & .00142 & .00568 & 2.026 & 6.177 & 7.003 \\
\hline
\end{tabular}


TABULATION OF DATA FOR

ABSORPTION $\odot F$ SOUND IN AIR VERSUS PERCENT RELATIVE HUMIDITY AT 24 DEGREES CENTIGRADE FOR A FREQUENCY OF 5000 HERTZ

\begin{tabular}{|c|c|c|c|c|c|c|c|c|c|}
\hline (1) & $(2)$ & (3) & $(4)$ & (5) & $(6)$ & (7) & $(8)$ & $(9)$ & $(10)$ \\
\hline $\begin{array}{l}\text { TEMP } \\
\text { DEGR } \\
\text { CENT }\end{array}$ & HERTZ & $\begin{array}{l}\text { REL } \\
\text { HUM } \\
\text { PER } \\
\text { CENT }\end{array}$ & $\begin{array}{l}\text { ATTEN } \\
\text { COEF } \\
\text { PER } \\
\text { METER }\end{array}$ & $\begin{array}{l}4 M \\
\text { PER } \\
\text { METER }\end{array}$ & $\begin{array}{l}\text { ATTEN } \\
\text { COEF } \\
\text { PER } \\
\text { FOOT }\end{array}$ & $\begin{array}{l}4 M \\
\text { PER } \\
\text { FOOT }\end{array}$ & $\begin{array}{l}\text { ATTEN } \\
\text { DB PER } \\
100 \\
\text { METER }\end{array}$ & $\begin{array}{l}\text { ATTEN } \\
\text { DB PER } \\
1000 \\
\text { FEET }\end{array}$ & $\begin{array}{l}\text { DECAY } \\
\text { RATE } \\
\text { DB PER } \\
\text { SECOND }\end{array}$ \\
\hline $\begin{array}{l}24 \\
24 \\
24\end{array}$ & $\begin{array}{l}5000 \\
5000 \\
5000\end{array}$ & $\begin{array}{r}5.0 \\
10.0 \\
15.0\end{array}$ & $\begin{array}{r}.02643 \\
.03354 \\
.02426\end{array}$ & $\begin{array}{r}.10574 \\
.13416 \\
.09705\end{array}$ & $\begin{array}{r}.00805 \\
.01022 \\
.00739\end{array}$ & $\begin{array}{r}.03223 \\
.04089 \\
.02958\end{array}$ & $\begin{array}{l}11.481 \\
14.566 \\
10.537\end{array}$ & $\begin{array}{l}34 \cdot 995 \\
44 \cdot 399 \\
32 \cdot 118\end{array}$ & $\begin{array}{l}39.676 \\
50.338 \\
36.414\end{array}$ \\
\hline $\begin{array}{l}24 \\
24 \\
24\end{array}$ & $\begin{array}{l}5000 \\
5000 \\
5000\end{array}$ & $\begin{array}{l}20.0 \\
25.0 \\
30.0\end{array}$ & $\begin{array}{r}.01809 \\
.01423 \\
.01186\end{array}$ & $\begin{array}{r}.07239 \\
.05694 \\
.04747\end{array}$ & $\begin{array}{l}.00551 \\
.00433 \\
.00361\end{array}$ & $\begin{array}{r}.02206 \\
.01735 \\
.01446\end{array}$ & $\begin{array}{l}7.859 \\
6.182 \\
5.154\end{array}$ & $\begin{array}{l}23.956 \\
18.843 \\
15.710\end{array}$ & $\begin{array}{l}27.161 \\
21.364 \\
17.811\end{array}$ \\
\hline $\begin{array}{l}24 \\
24 \\
24\end{array}$ & $\begin{array}{l}5000 \\
5000 \\
5000\end{array}$ & $\begin{array}{l}35.0 \\
40.0 \\
45.0\end{array}$ & $\begin{array}{l}.01021 \\
.00911 \\
.00845\end{array}$ & $\begin{array}{l}.04084 \\
.03645 \\
.03381\end{array}$ & $\begin{array}{l}.00311 \\
.00277 \\
.00257\end{array}$ & $\begin{array}{r}.011244 \\
.01111 \\
.011030\end{array}$ & $\begin{array}{l}4.434 \\
3.958 \\
3.671\end{array}$ & $\begin{array}{l}13.515 \\
12.065 \\
11.189\end{array}$ & $\begin{array}{l}15.323 \\
13.679 \\
12.686\end{array}$ \\
\hline $\begin{array}{l}24 \\
24 \\
24\end{array}$ & $\begin{array}{l}5000 \\
5000 \\
5000\end{array}$ & $\begin{array}{l}46.0 \\
47.0 \\
48.0\end{array}$ & $\begin{array}{l}.00835 \\
.00826 \\
.00818\end{array}$ & $\begin{array}{l}.03340 \\
.03304 \\
.03272\end{array}$ & $\begin{array}{r}.00254 \\
.00251 \\
.00249\end{array}$ & $\begin{array}{l}.01018 \\
.01007 \\
.00997\end{array}$ & $\begin{array}{l}3.627 \\
3.588 \\
3.553\end{array}$ & $\begin{array}{l}11.056 \\
10.937 \\
10.830\end{array}$ & $\begin{array}{l}12.535 \\
12.399 \\
12.278\end{array}$ \\
\hline $\begin{array}{l}24 \\
24 \\
24\end{array}$ & $\begin{array}{l}5000 \\
5000 \\
5000\end{array}$ & $\begin{array}{l}49.0 \\
50.0 \\
51.0\end{array}$ & $\begin{array}{l}.00808 \\
.00798 \\
.00790\end{array}$ & $\begin{array}{l}.03233 \\
.03194 \\
.03162\end{array}$ & $\begin{array}{l}.00246 \\
.00243 \\
.00240\end{array}$ & $\begin{array}{l}.00985 \\
.00973 \\
.00963\end{array}$ & $\begin{array}{l}3.510 \\
3.468 \\
3.433\end{array}$ & $\begin{array}{l}10.700 \\
10.572 \\
10.465\end{array}$ & $\begin{array}{l}12.131 \\
11.986 \\
11.865\end{array}$ \\
\hline $\begin{array}{l}24 \\
24 \\
24\end{array}$ & $\begin{array}{l}5000 \\
5000 \\
5000\end{array}$ & $\begin{array}{l}52.0 \\
53.0 \\
54.0\end{array}$ & $\begin{array}{r}.00784 \\
.00777 \\
.00772\end{array}$ & $\begin{array}{l}.03136 \\
.03110 \\
.03091\end{array}$ & $\begin{array}{l}.00238 \\
.00237 \\
.00235\end{array}$ & $\begin{array}{l}.00955 \\
.00948 \\
.00942\end{array}$ & & $\begin{array}{l}10.379 \\
10.294 \\
10.230\end{array}$ & $\begin{array}{l}11.767 \\
11.671 \\
11.598\end{array}$ \\
\hline $\begin{array}{l}24 \\
24 \\
24\end{array}$ & $\begin{array}{l}5000 \\
5000 \\
5000\end{array}$ & $\begin{array}{l}55.0 \\
60.0 \\
65.0\end{array}$ & $\begin{array}{l}.00768 \\
.00747 \\
.00727\end{array}$ & $\begin{array}{l}.03072 \\
.02989 \\
.02911\end{array}$ & $\begin{array}{l}.00234 \\
.00227 \\
.00221\end{array}$ & $\begin{array}{l}.00936 \\
.00911 \\
.00887\end{array}$ & $\begin{array}{l}3.336 \\
3.246 \\
3.161\end{array}$ & $\begin{array}{r}10.169 \\
9.894 \\
9.635\end{array}$ & $\begin{array}{r}11.529 \\
11.218 \\
10.924\end{array}$ \\
\hline $\begin{array}{l}24 \\
24 \\
24\end{array}$ & $\begin{array}{l}5000 \\
5000 \\
5000\end{array}$ & $\begin{array}{l}70.0 \\
75.0 \\
80.0\end{array}$ & $\begin{array}{l}.00711 \\
.00695 \\
.00682\end{array}$ & $\begin{array}{r}.02847 \\
.02783 \\
.02729\end{array}$ & $\begin{array}{r}.00216 \\
.00212 \\
.00207\end{array}$ & $\begin{array}{r}.00867 \\
.00848 \\
.00831\end{array}$ & $\begin{array}{l}3.091 \\
3.021 \\
2.963\end{array}$ & $\begin{array}{l}9.421 \\
9.210 \\
9.032\end{array}$ & $\begin{array}{l}10.682 \\
10.442 \\
10.240\end{array}$ \\
\hline $\begin{array}{l}24 \\
24 \\
24\end{array}$ & $\begin{array}{l}5000 \\
5000 \\
5000\end{array}$ & $\begin{array}{l}85.0 \\
90.0 \\
95.0\end{array}$ & $\begin{array}{r}.00668 \\
.00656 \\
.00645\end{array}$ & $\begin{array}{r}.02675 \\
.02626 \\
.02580\end{array}$ & $\begin{array}{l}.00203 \\
.00200 \\
.00196\end{array}$ & $\begin{array}{l}.00815 \\
.00800 \\
.00786\end{array}$ & $\begin{array}{l}2.905 \\
2.851 \\
2.801\end{array}$ & $\begin{array}{l}8.855 \\
8.691 \\
8.539\end{array}$ & $\begin{array}{r}10.039 \\
9.854 \\
9.681\end{array}$ \\
\hline 24 & 5000 & 100.0 & .00633 & .02534 & .00193 & .00772 & $2 \cdot 751$ & $8 \cdot 386$ & 9.508 \\
\hline
\end{tabular}


TABULATION OF DATA FOR

ABSORPTION OF SOUND IN AIR VERSUS PERCENT RELATIVE HUMIOITY AT 24 DEGREES CENTIGRADE FOR A FREQUENCY OF 5940 HERTZ

\begin{tabular}{|c|c|c|c|c|c|c|c|c|c|}
\hline (1) & (2) & (3) & (4) & (5) & $(6)$ & $(7)$ & (8) & 191 & $(20)$ \\
\hline $\begin{array}{l}\text { EMP } \\
\text { EGR } \\
\text { ENT }\end{array}$ & HERTZ & $\begin{array}{l}\text { REL } \\
\text { HUM } \\
\text { PER } \\
\text { CENT }\end{array}$ & $\begin{array}{l}\text { ATTEN } \\
\text { COEF } \\
\text { PER } \\
\text { METER }\end{array}$ & $\begin{array}{l}4 M \\
\text { PER } \\
\text { METER }\end{array}$ & $\begin{array}{l}\text { ATTEN } \\
\text { COEF } \\
\text { PER } \\
\text { FOOT }\end{array}$ & $\begin{array}{l}\text { 4M } \\
\text { PER } \\
\text { FOOT }\end{array}$ & $\begin{array}{l}\text { ATTEN } \\
\text { DB PER } \\
1 \text { OO } \\
\text { METER }\end{array}$ & $\begin{array}{l}\text { ATTEN } \\
\text { DB PER } \\
1000 \\
\text { FEET }\end{array}$ & $\begin{array}{l}\text { DECAY } \\
\text { RATE } \\
\text { DB PER } \\
\text { SECCNO }\end{array}$ \\
\hline $\begin{array}{l}24 \\
24 \\
24\end{array}$ & $\begin{array}{l}5940 \\
5940 \\
5940\end{array}$ & $\begin{array}{r}5.0 \\
10.0 \\
15.0\end{array}$ & $\begin{array}{l}.02814 \\
.04091 \\
.03287\end{array}$ & $\begin{array}{r}.11257 \\
.16364 \\
.13149\end{array}$ & $\begin{array}{l}.00857 \\
.01247 \\
.01001\end{array}$ & $\begin{array}{l}.03431 \\
.04988 \\
.04007\end{array}$ & $\begin{array}{l}12.223 \\
17.768 \\
14.276\end{array}$ & $\begin{array}{l}37.257 \\
54 \cdot 158 \\
43.516\end{array}$ & $\begin{array}{l}42.239 \\
61.401 \\
49.336\end{array}$ \\
\hline $\begin{array}{l}24 \\
24 \\
24\end{array}$ & $\begin{array}{l}5940 \\
5940 \\
5940\end{array}$ & $\begin{array}{l}20.0 \\
25.0 \\
30.0\end{array}$ & $\begin{array}{r}.02425 \\
.01934 \\
.01599\end{array}$ & $\begin{array}{l}.09702 \\
.07739 \\
.06397\end{array}$ & $\begin{array}{r}.00739 \\
.00589 \\
.00487\end{array}$ & $\begin{array}{l}.02957 \\
.02359 \\
.01949\end{array}$ & $\begin{array}{r}10.534 \\
8.403 \\
6.945\end{array}$ & $\begin{array}{l}32.110 \\
25.614 \\
21.171\end{array}$ & $\begin{array}{l}36.404 \\
29.040 \\
24.003\end{array}$ \\
\hline $\begin{array}{l}24 \\
24 \\
24\end{array}$ & $\begin{array}{l}5940 \\
5940 \\
5940\end{array}$ & $\begin{array}{l}35.0 \\
40.0 \\
45.0\end{array}$ & $\begin{array}{r}.01374 \\
.01214 \\
.01100\end{array}$ & $\begin{array}{r}.05497 \\
.04856 \\
.04403\end{array}$ & $\begin{array}{l}.00418 \\
.00370 \\
.00335\end{array}$ & $\begin{array}{r}.01675 \\
.01480 \\
.01342\end{array}$ & $\begin{array}{l}68 \\
72 \\
80\end{array}$ & $\begin{array}{l}18.193 \\
16.070 \\
14.572\end{array}$ & $\begin{array}{l}20.626 \\
18.220 \\
16.521\end{array}$ \\
\hline $\begin{array}{l}24 \\
24 \\
24\end{array}$ & $\begin{array}{l}5940 \\
5940 \\
5940\end{array}$ & $\begin{array}{l}46.0 \\
47.0 \\
48.0\end{array}$ & $\begin{array}{l}.01083 \\
.01067 \\
.01052\end{array}$ & $\begin{array}{l}.04334 \\
.04270 \\
.04211\end{array}$ & $\begin{array}{l}.00330 \\
.00325 \\
.00320\end{array}$ & $\begin{array}{l}.01321 \\
.01301 \\
.01283\end{array}$ & & $\begin{array}{l}14 \cdot 346 \\
14.133 \\
13.936\end{array}$ & $\begin{array}{l}16.264 \\
16.024 \\
15.799\end{array}$ \\
\hline $\begin{array}{l}24 \\
24 \\
24\end{array}$ & $\begin{array}{l}5940 \\
5940 \\
5940\end{array}$ & $\begin{array}{l}49.0 \\
50.0 \\
51.0\end{array}$ & $\begin{array}{r}.01040 \\
.01029 \\
.01019\end{array}$ & $\begin{array}{l}.04162 \\
.04119 \\
.04076\end{array}$ & $\begin{array}{l}.00317 \\
.00313 \\
.00310\end{array}$ & $\begin{array}{l}.0 \\
.0 \\
.0\end{array}$ & & $\begin{array}{l}13.774 \\
13.632 \\
13.491\end{array}$ & $\begin{array}{l}15.616 \\
15.456 \\
15.295\end{array}$ \\
\hline $\begin{array}{l}24 \\
24 \\
24\end{array}$ & $\begin{array}{l}5940 \\
5940 \\
5940\end{array}$ & $\begin{array}{l}52.0 \\
53.0 \\
54.0\end{array}$ & $\begin{array}{r}.01008 \\
.00999 \\
.00991\end{array}$ & $\begin{array}{r}.04033 \\
.03999 \\
.03964\end{array}$ & $\begin{array}{l}.00307 \\
.00304 \\
.00302\end{array}$ & $\begin{array}{r}.01229 \\
.01218 \\
.01208\end{array}$ & $\begin{array}{l}4.379 \\
4.342 \\
4.304\end{array}$ & $\begin{array}{l}13.350 \\
13 \cdot 234 \\
13.119\end{array}$ & $\begin{array}{l}35 \\
04 \\
74\end{array}$ \\
\hline $\begin{array}{l}24 \\
24 \\
24\end{array}$ & $\begin{array}{l}5940 \\
5940 \\
5940\end{array}$ & $\begin{array}{l}55.0 \\
60.0 \\
65.0\end{array}$ & $\begin{array}{r}.00980 \\
.00941 \\
.00918\end{array}$ & $\begin{array}{r}.03921 \\
.03766 \\
.03674\end{array}$ & $\begin{array}{l}.00298 \\
.00287 \\
.00280\end{array}$ & $\begin{array}{r}.01195 \\
.01148 \\
.01120\end{array}$ & $\begin{array}{l}4.257 \\
4.089 \\
3.989\end{array}$ & $\begin{array}{l}12.978 \\
12.466 \\
12.160\end{array}$ & $\begin{array}{l}14.714 \\
14.133 \\
13.787\end{array}$ \\
\hline $\begin{array}{l}24 \\
24 \\
24\end{array}$ & $\begin{array}{l}5940 \\
5940 \\
5940\end{array}$ & $\begin{array}{l}70.0 \\
75.0 \\
80.0\end{array}$ & $\begin{array}{l}.00897 \\
.00878 \\
.00861\end{array}$ & $\begin{array}{r}.03589 \\
.03513 \\
.03445\end{array}$ & $\begin{array}{l}.00273 \\
.00267 \\
.00262\end{array}$ & $\begin{array}{r}.01093 \\
.01070 \\
.01050\end{array}$ & & $\begin{array}{l}11.877 \\
11.628 \\
11.401\end{array}$ & $\begin{array}{l}466 \\
183 \\
926\end{array}$ \\
\hline $\begin{array}{l}24 \\
24 \\
24\end{array}$ & $\begin{array}{l}5940 \\
5940 \\
5940\end{array}$ & $\begin{array}{l}85.0 \\
90.0 \\
95.0\end{array}$ & $\begin{array}{l}.00844 \\
.00830 \\
.00816\end{array}$ & $\begin{array}{l}.03379 \\
.03322 \\
.03265\end{array}$ & $\begin{array}{l}.00257 \\
.00253 \\
.00248\end{array}$ & $\begin{array}{l}.01030 \\
.01012 \\
.00995\end{array}$ & $\begin{array}{l}3.6 \\
3.6 \\
3.5\end{array}$ & $\begin{array}{l}.185 \\
.997 \\
.808\end{array}$ & $\begin{array}{l}12.681 \\
12.467 \\
12.254\end{array}$ \\
\hline & 940 & .0 & 0080 & 3212 & 00244 & 00979 & 0 & 0.632 & .05 \\
\hline
\end{tabular}


TABULATION OF DATA FOR

ABSORPTION OF SOUND IN AIR VERSUS PERCENT RELATIVE HUMIDITY AT 24 DEGREES CENTIGRADE FOR A FREQUENCY OF 6300 HERTZ

\begin{tabular}{|c|c|c|c|c|c|c|c|c|c|}
\hline 111 & (2) & (3) & (4) & (5) & $(6)$ & (7) & $(8)$ & 191 & $(10)$ \\
\hline $\begin{array}{l}\text { IEMP } \\
\text { JEGR } \\
\text { IENT }\end{array}$ & HERTZ & $\begin{array}{l}\text { REL } \\
\text { HUM } \\
\text { PER } \\
\text { CENT }\end{array}$ & $\begin{array}{l}\text { ATTEN } \\
\text { COEF } \\
\text { PER } \\
\text { METER }\end{array}$ & $\begin{array}{l}\text { 4M } \\
\text { PER } \\
\text { METER }\end{array}$ & $\begin{array}{l}\text { ATTEN } \\
\text { COEF } \\
\text { PER } \\
\text { FOOT }\end{array}$ & $\begin{array}{l}4 M \\
\text { PER } \\
\text { FOOT }\end{array}$ & $\begin{array}{l}\text { ATTEN } \\
\text { DB PER } \\
\text { lOO } \\
\text { METER }\end{array}$ & $\begin{array}{l}\text { ATTEN } \\
\text { DB PER } \\
\text { I OOO } \\
\text { FEET }\end{array}$ & $\begin{array}{l}\text { DECAY } \\
\text { RATE } \\
\text { DQ'PER } \\
\text { SECENO }\end{array}$ \\
\hline $\begin{array}{l}24 \\
24 \\
24\end{array}$ & $\begin{array}{l}6300 \\
6300 \\
6300\end{array}$ & $\begin{array}{r}5.0 \\
10.0 \\
15.0\end{array}$ & $\begin{array}{r}.02862 \\
.04359 \\
.03634\end{array}$ & $\begin{array}{r}11451 \\
.17437 \\
.14538\end{array}$ & $\begin{array}{r}.00872 \\
.01328 \\
.01107\end{array}$ & $\begin{array}{l}.03490 \\
.05315 \\
.04431\end{array}$ & $\begin{array}{l}12.433 \\
18.933 \\
15.785\end{array}$ & $\begin{array}{l}37.896 \\
57.709 \\
48.115\end{array}$ & $\begin{array}{l}42.965 \\
65.427 \\
54.550\end{array}$ \\
\hline $\begin{array}{l}24 \\
24 \\
24\end{array}$ & $\begin{array}{l}6300 \\
6300 \\
6300\end{array}$ & $\begin{array}{l}20.0 \\
25.0 \\
30.0\end{array}$ & $\begin{array}{l}.02692 \\
.02155 \\
.01776\end{array}$ & $\begin{array}{r}.10769 \\
.08620 \\
.07107\end{array}$ & $\begin{array}{l}.00820 \\
.00656 \\
.00541\end{array}$ & $\begin{array}{l}.03282 \\
.02627 \\
.02166\end{array}$ & $\begin{array}{r}11 \\
9 \\
7\end{array}$ & $\begin{array}{l}35.640 \\
28.529 \\
23.522\end{array}$ & $\begin{array}{l}40.406 \\
32 \cdot 345 \\
26.668\end{array}$ \\
\hline $\begin{array}{l}24 \\
24 \\
24\end{array}$ & $\begin{array}{l}6300 \\
6300 \\
6300\end{array}$ & $\begin{array}{l}35.0 \\
40.0 \\
45.0\end{array}$ & $\begin{array}{r}.01528 \\
.01344 \\
.01219\end{array}$ & $\begin{array}{l}.06115 \\
.05376 \\
.04879\end{array}$ & $\begin{array}{l}.00466 \\
.00409 \\
.00371\end{array}$ & $\begin{array}{l}.018 \\
.016 \\
.014\end{array}$ & & $\begin{array}{l}239 \\
793 \\
46\end{array}$ & $\begin{array}{l}946 \\
173 \\
308\end{array}$ \\
\hline $\begin{array}{l}24 \\
24 \\
24\end{array}$ & $\begin{array}{l}6300 \\
6300 \\
6300\end{array}$ & $\begin{array}{l}46.0 \\
47.0 \\
48.0\end{array}$ & $\begin{array}{r}.01195 \\
.01174 \\
.01157\end{array}$ & $\begin{array}{l}.04783 \\
.04699 \\
.04630\end{array}$ & $\begin{array}{l}.00364 \\
.00358 \\
.00352\end{array}$ & $\begin{array}{l}.01458 \\
.01432 \\
.01411\end{array}$ & & $\begin{array}{l}15.832 \\
15.553 \\
15.323\end{array}$ & $\begin{array}{l}949 \\
533 \\
372\end{array}$ \\
\hline $\begin{array}{l}24 \\
24 \\
24\end{array}$ & $\begin{array}{l}6300 \\
6300 \\
6300\end{array}$ & $\begin{array}{l}49.0 \\
50.0 \\
51.0\end{array}$ & $\begin{array}{l}.011141 \\
.01126 \\
.01113\end{array}$ & $\begin{array}{r}.04565 \\
.04504 \\
.04453\end{array}$ & $\begin{array}{l}.00347 \\
.00343 \\
.00339\end{array}$ & $\begin{array}{r}.01391 \\
.01372 \\
.01357\end{array}$ & & $\begin{array}{l}15.107 \\
14.906 \\
14.737\end{array}$ & $\begin{array}{l}17.128 \\
16.900 \\
16.708\end{array}$ \\
\hline $\begin{array}{l}24 \\
24 \\
24\end{array}$ & $\begin{array}{l}6300 \\
6300 \\
6300\end{array}$ & $\begin{array}{l}52.0 \\
53.0 \\
54.0\end{array}$ & $\begin{array}{r}.01102 \\
.01091 \\
.01080\end{array}$ & $\begin{array}{r}.04409 \\
.04366 \\
.04322\end{array}$ & $\begin{array}{l}.00336 \\
.00332 \\
.00329\end{array}$ & $\begin{array}{l}.01344 \\
.01330 \\
.01317\end{array}$ & $\begin{array}{l}4.787 \\
4.740 \\
4.693\end{array}$ & $\begin{array}{l}14.593 \\
14.449 \\
14.306\end{array}$ & $\begin{array}{l}16.545 \\
16.382 \\
16.219\end{array}$ \\
\hline $\begin{array}{l}24 \\
24 \\
24\end{array}$ & $\begin{array}{l}6300 \\
6300 \\
6300\end{array}$ & $\begin{array}{l}55.0 \\
60.0 \\
65.0\end{array}$ & $\begin{array}{l}.01071 \\
.01024 \\
.00995\end{array}$ & $\begin{array}{l}.04 \\
.04 \\
.03\end{array}$ & $\begin{array}{l}.00326 \\
.00312 \\
.00303\end{array}$ & $\begin{array}{l}.01 \\
.01 \\
.01\end{array}$ & & $\begin{array}{l}14 . \\
13 . \\
13 .\end{array}$ & $\begin{array}{l}978 \\
372 \\
937\end{array}$ \\
\hline $\begin{array}{l}24 \\
24 \\
24\end{array}$ & $\begin{array}{l}6300 \\
6300 \\
6300\end{array}$ & $\begin{array}{l}70 . \\
75 \\
80 .\end{array}$ & $\begin{array}{l}.00973 \\
.00951 \\
.00934\end{array}$ & $\begin{array}{l}.03893 \\
.03806 \\
.03736\end{array}$ & $\begin{array}{l}.00296 \\
.00290 \\
.00284\end{array}$ & $\begin{array}{l}.01186 \\
.01160 \\
.01138\end{array}$ & 4. & $\begin{array}{l}12.883 \\
12.596 \\
12.364\end{array}$ & $\begin{array}{l}14.607 \\
14.281 \\
14.027\end{array}$ \\
\hline $\begin{array}{l}24 \\
24\end{array}$ & $\begin{array}{l}6300 \\
6300 \\
6300\end{array}$ & $\begin{array}{l}85 . \\
90 . \\
95 .\end{array}$ & $\begin{array}{l}.00916 \\
.00900 \\
.00886\end{array}$ & $\begin{array}{l}.03666 \\
.03603 \\
.03545\end{array}$ & $\begin{array}{l}.00279 \\
.00274 \\
.00270\end{array}$ & $\begin{array}{l}.01117 \\
.01098 \\
.01080\end{array}$ & $\begin{array}{l}3 \\
3 \\
3\end{array}$ & $\begin{array}{l}12 \\
11 \\
11\end{array}$ & $\begin{array}{l}13.757 \\
13.519 \\
13.302\end{array}$ \\
\hline 2 & 6300 & $00 \cdot 0$ & .00871 & .03487 & .00265 & .01062 & 3.786 & $11 \cdot 541$ & 23.084 \\
\hline
\end{tabular}


TABULATION OF DATA FOR

ABSORPTION OF SOUND IN AIR VERSUS PERCENT RELATIVE HUMIDITY AT 24 DEGREES CENTIGRADE FOR A FREQUENCY OF 8000 HERTZ

\begin{tabular}{|c|c|c|c|c|c|c|c|c|c|}
\hline (I) & (2) & $(3)$ & (4) & (5) & $(6)$ & $(7)$ & $(8)$ & $(9)$ & $(10)$ \\
\hline $\begin{array}{l}\text { TEMP } \\
\text { DEGR } \\
\text { CENT }\end{array}$ & HERTZ & $\begin{array}{l}\text { REL } \\
\text { HUM } \\
\text { PER } \\
\text { CENT }\end{array}$ & $\begin{array}{l}\text { ATTEN } \\
\text { COEF } \\
\text { PER } \\
\text { METER }\end{array}$ & $\begin{array}{l}4 M \\
\text { PER } \\
\text { METER }\end{array}$ & $\begin{array}{l}\text { ATTEN } \\
\text { COEF } \\
\text { PER } \\
\text { FOOT }\end{array}$ & $\begin{array}{l}4 M \\
\text { PER } \\
\text { FOOT }\end{array}$ & $\begin{array}{l}\text { ATTEN } \\
\text { DB PER } \\
100 \\
\text { METER }\end{array}$ & $\begin{array}{l}\text { ATTEN } \\
\text { DB PER } \\
1000 \\
\text { FEET }\end{array}$ & $\begin{array}{l}\text { DECAY } \\
\text { RATE } \\
\text { DB PER } \\
\text { SECOND }\end{array}$ \\
\hline $\begin{array}{l}24 \\
24 \\
24\end{array}$ & $\begin{array}{l}8000 \\
8.000 \\
8000\end{array}$ & $\begin{array}{r}5.0 \\
10.0 \\
15.0\end{array}$ & $\begin{array}{r}.03178 \\
.05512 \\
.05229\end{array}$ & $\begin{array}{l}.12712 \\
.22048 \\
.20919\end{array}$ & $\begin{array}{l}.00968 \\
.01680 \\
.01594\end{array}$ & $\begin{array}{l}.03874 \\
.06720 \\
.06376\end{array}$ & $\begin{array}{l}13.802 \\
23.939 \\
22.713\end{array}$ & $\begin{array}{l}42.070 \\
72.968 \\
69.230\end{array}$ & $\begin{array}{l}47.697 \\
82.727 \\
78.489\end{array}$ \\
\hline $\begin{array}{l}24 \\
24 \\
24\end{array}$ & $\begin{array}{l}8000 \\
8000 \\
8000\end{array}$ & $\begin{array}{l}20.0 \\
25.0 \\
30.0\end{array}$ & $\begin{array}{r}.04070 \\
.03238 \\
.02703\end{array}$ & $\begin{array}{r}.16280 \\
.12955 \\
.10814 .\end{array}$ & $\begin{array}{l}.01240 \\
.00987 \\
.00824\end{array}$ & $\begin{array}{r}.04962 \\
.03948 \\
.03296\end{array}$ & $\begin{array}{l}17.676 \\
14.066 \\
11.742\end{array}$ & $\begin{array}{l}53.878 \\
42.874 \\
35.790\end{array}$ & $\begin{array}{l}61.084 \\
48.609 \\
40.576\end{array}$ \\
\hline $\begin{array}{l}24 \\
24 \\
24\end{array}$ & $\begin{array}{l}8000 \\
8000 \\
8000\end{array}$ & $\begin{array}{l}35.0 \\
40.0 \\
45.0\end{array}$ & $\begin{array}{l}.02304 \\
.02032 \\
.01811\end{array}$ & $\begin{array}{l}.09216 \\
.08129 \\
.07247\end{array}$ & $\begin{array}{l}.00702 \\
.00619 \\
.00552\end{array}$ & $\begin{array}{l}.02809 \\
.02478 \\
.02209\end{array}$ & $\begin{array}{r}10.006 \\
8.827 \\
7.869\end{array}$ & $\begin{array}{l}30.500 \\
26.905 \\
23.986\end{array}$ & $\begin{array}{l}34.580 \\
30.503 \\
27.194\end{array}$ \\
\hline $\begin{array}{l}24 \\
24 \\
24\end{array}$ & $\begin{array}{l}8000 \\
8000 \\
8000\end{array}$ & $\begin{array}{l}46.0 \\
47.0 \\
48.0\end{array}$ & $\begin{array}{r}.01777 \\
.01744 \\
.01714\end{array}$ & $\begin{array}{l}.07111 \\
.06978 \\
.06856\end{array}$ & $\begin{array}{l}.00541 \\
.00531 \\
.00522\end{array}$ & $\begin{array}{r}.02167 \\
.02127 \\
.02089\end{array}$ & $\begin{array}{l}7.721 \\
7.577 \\
7.444\end{array}$ & $\begin{array}{l}23.534 \\
23.095 \\
22.690\end{array}$ & $\begin{array}{l}26.682 \\
26.184 \\
25.725\end{array}$ \\
\hline $\begin{array}{l}24 \\
24 \\
24\end{array}$ & $\begin{array}{l}8000 \\
8000 \\
8000\end{array}$ & $\begin{array}{l}49.0 \\
50.0 \\
51.0\end{array}$ & $\begin{array}{r}.01685 \\
.01658 \\
.01634\end{array}$ & $\begin{array}{l}.06742 \\
.06634 \\
.06539\end{array}$ & $\begin{array}{l}.00513 \\
.00505 \\
.00498\end{array}$ & $\begin{array}{l}.02055 \\
.02022 \\
.01993\end{array}$ & $\begin{array}{l}7.320 \\
7.203 \\
7.100\end{array}$ & $\begin{array}{l}22.314 \\
21.955 \\
21.642\end{array}$ & $\begin{array}{l}25.299 \\
24.892 \\
24.537\end{array}$ \\
\hline $\begin{array}{l}24 \\
24 \\
24\end{array}$ & $\begin{array}{l}8000 \\
8000 \\
8000\end{array}$ & $\begin{array}{l}52.0 \\
53.0 \\
54.0\end{array}$ & $\begin{array}{l}.01611 \\
.01585 \\
.01559\end{array}$ & $\begin{array}{l}.06444 \\
.06340 \\
.06236\end{array}$ & $\begin{array}{l}.00491 \\
.00483 \\
.00475\end{array}$ & $\begin{array}{l}.01964 \\
.01932 \\
.01900\end{array}$ & $\begin{array}{l}6.997 \\
6.884 \\
6.771\end{array}$ & $\begin{array}{l}21.328 \\
20.983 \\
20.638\end{array}$ & $\begin{array}{l}24 \cdot 180 \\
23.789 \\
23.399\end{array}$ \\
\hline $\begin{array}{l}24 \\
24 \\
24\end{array}$ & $\begin{array}{l}8000 \\
8000 \\
8000\end{array}$ & $\begin{array}{l}55.0 \\
60.0 \\
65.0\end{array}$ & $\begin{array}{r}.01538 \\
.01457 \\
.01402\end{array}$ & $\begin{array}{l}.06153 \\
.05831 \\
.05610\end{array}$ & $\begin{array}{l}.00468 \\
.00444 \\
.00427\end{array}$ & $\begin{array}{l}.01875 \\
.01777 \\
.01710\end{array}$ & $\begin{array}{l}6.681 \\
6.331 \\
6.092\end{array}$ & $\begin{array}{l}20.365 \\
19.298 \\
18.569\end{array}$ & $\begin{array}{l}23.089 \\
21.879 \\
21.052\end{array}$ \\
\hline $\begin{array}{l}24 \\
24 \\
24\end{array}$ & $\begin{array}{l}8000 \\
8000 \\
8000\end{array}$ & $\begin{array}{l}70.0 \\
75.0 \\
80.0\end{array}$ & $\begin{array}{r}.01350 \\
.01318 \\
.01294\end{array}$ & $\begin{array}{r}.05403 \\
.05275 \\
.05177\end{array}$ & $\begin{array}{l}.00411 \\
.00402 \\
.00394\end{array}$ & $\begin{array}{r}.01647 \\
.01608 \\
.01578\end{array}$ & $\begin{array}{l}5.867 \\
5.727 \\
5.621\end{array}$ & $\begin{array}{l}17.883 \\
17.459 \\
17.134\end{array}$ & $\begin{array}{l}20.275 \\
19.794 \\
19.425\end{array}$ \\
\hline $\begin{array}{l}24 \\
24 \\
24\end{array}$ & $\begin{array}{l}8000 \\
8000 \\
8000\end{array}$ & $\begin{array}{l}85.0 \\
90.0 \\
95.0\end{array}$ & $\begin{array}{r}.01270 \\
.01249 \\
.01230\end{array}$ & $\begin{array}{l}.05082 \\
.04997 \\
.04921\end{array}$ & $\begin{array}{l}.00387 \\
.00380 \\
.00375\end{array}$ & $\begin{array}{l}.01549 \\
.01523 \\
.01500\end{array}$ & $\begin{array}{l}5.518 \\
5.425 \\
5.343\end{array}$ & $\begin{array}{l}16.820 \\
16.537 \\
16.286\end{array}$ & $\begin{array}{l}19.070 \\
18.749 \\
18.465\end{array}$ \\
\hline 2 & 8000 & 100.0 & .01211 & .04845 & .00369 & .01476 & 5.261 & 16.036 & 18.180 \\
\hline
\end{tabular}


TABULATION OF DATA FOR

ABSORPTION OF SOUND IN AIR VERSUS PERCENT RELATIVE HUMMIDITY AT 24 DEGREES CENTIGRADE FOR A FREQUENCY OF 10000 HERTZ

\begin{tabular}{|c|c|c|c|c|c|c|c|c|c|}
\hline (1) & $(2)$ & $(3)$ & 141 & (5) & $(6)$ & $(7)$ & $(8)$ & $(9)$ & $(10)$ \\
\hline $\begin{array}{l}\text { TEMP } \\
\text { DEGR } \\
\text { CENT }\end{array}$ & $\begin{array}{l}\text { FREQ } \\
\text { HERTZ }\end{array}$ & $\begin{array}{l}\text { REL } \\
\text { HUM } \\
\text { PER } \\
\text { CENT }\end{array}$ & $\begin{array}{l}\text { ATTEN } \\
\text { COEF } \\
\text { PER } \\
\text { METER }\end{array}$ & $\begin{array}{l}4 M \\
\text { PER } \\
\text { METER }\end{array}$ & $\begin{array}{l}\text { ATTEN } \\
\text { COEF. } \\
\text { PER } \\
\text { FOOT. }\end{array}$ & $\begin{array}{l}4 M \\
\text { PER } \\
\text { FOOT }\end{array}$ & $\begin{array}{l}\text { ATTEN } \\
\text { DB PER } \\
100 \\
\text { METER }\end{array}$ & $\begin{array}{l}\text { ATTEN } \\
\text { DB PER } \\
1000 \\
\text { FEET }\end{array}$ & $\begin{array}{l}\text { DECAY } \\
\text { RATE } \\
\text { DB PER } \\
\text { SECOND }\end{array}$ \\
\hline $\begin{array}{l}24 \\
24 \\
24\end{array}$ & $\begin{array}{l}10000 \\
10000 \\
10000\end{array}$ & $\begin{array}{r}5.0 \\
10.0 \\
15.0\end{array}$ & $\begin{array}{r}.03593 \\
.06637 \\
.06957\end{array}$ & $\begin{array}{r}.14373 \\
.26549 \\
.27828\end{array}$ & $\begin{array}{r}.01095 \\
.02023 \\
.02120\end{array}$ & $\begin{array}{l}.04381 \\
.08092 \\
.08482\end{array}$ & $\begin{array}{l}15.605 \\
28.825 \\
30.215\end{array}$ & $\begin{array}{l}47.567 \\
87.862 \\
92.097\end{array}$ & $\begin{array}{r}53.929 \\
99.613 \\
104.414\end{array}$ \\
\hline $\begin{array}{l}24 \\
24\end{array}$ & $\begin{array}{l}10000 \\
10000\end{array}$ & $\begin{array}{l}20.0 \\
25.0\end{array}$ & $\begin{array}{r}.05895 \\
.04699\end{array}$ & $\begin{array}{r}.23583 \\
.18797\end{array}$ & $\begin{array}{r}01797 \\
.01432\end{array}$ & $\begin{array}{r}.07188 \\
.05729\end{array}$ & $\begin{array}{l}25.606 \\
20.409\end{array}$ & $\begin{array}{l}78.048 \\
62.208\end{array}$ & $\begin{array}{l}88.487 \\
70.528\end{array}$ \\
\hline 24 & 10000 & 30.0 & .03942 & . 15771 & .01201 & .04807 & $17 \cdot 123$ & $52 \cdot 193$ & 59.174 \\
\hline $\begin{array}{l}24 \\
24\end{array}$ & $\begin{array}{l}10000 \\
10000\end{array}$ & $\begin{array}{l}35.0 \\
40.0\end{array}$ & $\begin{array}{r}.03377 \\
.02952\end{array}$ & $\begin{array}{l}13510 \\
11811\end{array}$ & $\begin{array}{l}.01029 \\
.00900\end{array}$ & $\begin{array}{r}.04117 \\
.03600\end{array}$ & $\begin{array}{l}14.668 \\
12.824\end{array}$ & $\begin{array}{l}44 \cdot 710 \\
39.090\end{array}$ & $\begin{array}{l}50.690 \\
44.318\end{array}$ \\
\hline 24 & 10000 & 45.0 & .02653 & .10614 & .00808 & .03235 & 11.524 & 35.128 & 39.826 \\
\hline $\begin{array}{l}24 \\
24 \\
24\end{array}$ & $\begin{array}{l}10000 \\
10000 \\
10000\end{array}$ & $\begin{array}{l}46.0 \\
47.0 \\
48.0\end{array}$ & $\begin{array}{r}.02597 \\
.02543 \\
.02492\end{array}$ & $\begin{array}{r}.10389 \\
.10173 \\
.09971\end{array}$ & $\begin{array}{l}.00791 \\
.00775 \\
.00759\end{array}$ & $\begin{array}{l}.03166 \\
.03100 \\
.03039\end{array}$ & $\begin{array}{l}11.280 \\
11.045 \\
10.826\end{array}$ & $\begin{array}{l}34 \cdot 383 \\
33.666 \\
32.999\end{array}$ & $\begin{array}{l}38.982 \\
38.169 \\
37.412\end{array}$ \\
\hline $\begin{array}{l}24 \\
24 \\
24\end{array}$ & $\begin{array}{l}10000 \\
10000 \\
10000\end{array}$ & $\begin{array}{l}49.0 \\
50.0 \\
51.0\end{array}$ & $\begin{array}{l}.02446 \\
.02399 \\
.02356\end{array}$ & $\begin{array}{l}.09784 \\
.09597 \\
.09425\end{array}$ & $\begin{array}{l}.00745 \\
.00731 \\
.00718\end{array}$ & $\begin{array}{l}.02982 \\
.02925 \\
.02872\end{array}$ & $\begin{array}{l}10.623 \\
10.420 \\
10.233\end{array}$ & $\begin{array}{l}32 \cdot 381 \\
31 \cdot 763 \\
31 \cdot 192\end{array}$ & $\begin{array}{l}36.711 \\
36.011 \\
35.364\end{array}$ \\
\hline $\begin{array}{l}24 \\
24 \\
24\end{array}$ & $\begin{array}{l}10000 \\
10000 \\
10000\end{array}$ & $\begin{array}{l}52.0 \\
53.0 \\
54.0\end{array}$ & $\begin{array}{l}.02316 \\
.02280 \\
.02244\end{array}$ & $\begin{array}{l}.09267 \\
.09122 \\
.08977\end{array}$ & $\begin{array}{l}.00706 \\
.00695 \\
.00684\end{array}$ & $\begin{array}{l}.02824 \\
.02780 \\
.02736\end{array}$ & $\begin{array}{r}10.062 \\
9.904 \\
9.747\end{array}$ & $\begin{array}{l}30.671 \\
30.190 \\
29.709\end{array}$ & $\begin{array}{l}34.773 \\
34.228 \\
33.683\end{array}$ \\
\hline $\begin{array}{l}24 \\
24 \\
24\end{array}$ & $\begin{array}{l}10000 \\
10000 \\
10000\end{array}$ & $\begin{array}{l}55.0 \\
60.0 \\
65.0\end{array}$ & $\begin{array}{r}.02212 \\
.02071 \\
.01954\end{array}$ & $\begin{array}{r}.08849 \\
.08287 \\
.07817\end{array}$ & $\begin{array}{l}.00674 \\
.00631 \\
.00595\end{array}$ & $\begin{array}{r}.02697 \\
.02525 \\
.02382\end{array}$ & $\begin{array}{l}9.608 \\
8.997 \\
8.488\end{array}$ & $\begin{array}{l}29 \cdot 285 \\
27.425 \\
25.872\end{array}$ & $\begin{array}{l}33.202 \\
31.094 \\
29.332\end{array}$ \\
\hline $\begin{array}{l}24 \\
24 \\
24\end{array}$ & $\begin{array}{l}10000 \\
10000 \\
10000\end{array}$ & $\begin{array}{l}70.0 \\
75.0 \\
80.0\end{array}$ & $\begin{array}{r}.01878 \\
.01820 \\
.01764\end{array}$ & $\begin{array}{r}.07515 \\
.07282 \\
.07056\end{array}$ & $\begin{array}{l}.00572 \\
.00554 \\
.00537\end{array}$ & $\begin{array}{l}.02290 \\
.02219 \\
.02150\end{array}$ & $\begin{array}{l}8.159 \\
7.907 \\
7.661\end{array}$ & $\begin{array}{l}24.871 \\
24.101 \\
23.351\end{array}$ & $\begin{array}{l}28 \cdot 197 \\
27.325 \\
26.474\end{array}$ \\
\hline $\begin{array}{l}24 \\
24 \\
24\end{array}$ & $\begin{array}{l}10000 \\
10000 \\
10000\end{array}$ & $\begin{array}{l}85.0 \\
90.0 \\
95.0\end{array}$ & $\begin{array}{r}.01728 \\
.01700 \\
.01674\end{array}$ & $\begin{array}{l}.06913 \\
.06803 \\
.06699\end{array}$ & $\begin{array}{l}.00526 \\
.00518 \\
.00510\end{array}$ & $\begin{array}{l}.02107 \\
.02073 \\
.02042\end{array}$ & $\begin{array}{l}7.505 \\
7.386 \\
7.274\end{array}$ & $\begin{array}{l}22.878 \\
22.514 \\
22.171\end{array}$ & $\begin{array}{l}25.938 \\
25.526 \\
25.137\end{array}$ \\
\hline 24 & 10000 & 100.0 & .01648 & .06595 & .00502 & .02010 & 7.161 & $21 \cdot 828$ & $24 \cdot 748$ \\
\hline
\end{tabular}


TABULATION OF DATA FOR

ABSORPTION OF. SOUND IN AIR VERSUS PERCENT RELATIVE HUMIDITY AT 24 DEGREES CENTIGRADE FOR A FREQUENCY OFI2500 HERTZ

\begin{tabular}{|c|c|c|c|c|c|c|c|c|c|}
\hline (1) & (2) & (3) & (4) & (5) & 161 & (7) & (8). & (9) & 1101 \\
\hline $\begin{array}{l}\text { TEMP } \\
\text { DEGR } \\
\text { CENT }\end{array}$ & HERTZ & $\begin{array}{l}\text { REL } \\
\text { HUM } \\
\text { PER } \\
\text { CENT }\end{array}$ & $\begin{array}{l}\text { A-TTEN } \\
\text { COEF } \\
\text { PER } \\
\text { METER }\end{array}$ & $\begin{array}{l}4 M \\
\text { PER } \\
\text { METER }\end{array}$ & $\begin{array}{l}\text { ATTEN } \\
\text { COEF } \\
\text { PER } \\
\text { FOOT. }\end{array}$ & $\begin{array}{l}4 M \\
\text { PER } \\
\text { FOOT }\end{array}$ & $\begin{array}{l}\text { ATTEN } \\
\text { DB PER } \\
100 \\
\text { METER }\end{array}$ & $\begin{array}{l}\text { ATTEN. } \\
\text { DB PER } \\
\text { IOOO } \\
\text { FEET }\end{array}$ & $\begin{array}{l}\text { DECAY } \\
\text { RATE } \\
\text { DB PER } \\
\text { SECOND }\end{array}$ \\
\hline $\begin{array}{l}24 \\
24 \\
24\end{array}$ & $\begin{array}{l}12500 \\
12500 \\
12500\end{array}$ & $\begin{array}{r}5.0 \\
10.0 \\
15.0\end{array}$ & $\begin{array}{r}.04094 \\
.07713 \\
.08948\end{array}$ & $\begin{array}{r}.16376 \\
.30854 \\
.35795\end{array}$ & $\begin{array}{l}.01247 \\
.02351 \\
.02727\end{array}$ & $\begin{array}{l}.04991 \\
.09404 \\
.10910\end{array}$ & $\begin{array}{l}17.780 \\
33.500 \\
38.864\end{array}$ & $\begin{array}{r}54.195 \\
102.110 \\
118.460\end{array}$ & $\begin{array}{r}61.443 \\
115.766 \\
134.304\end{array}$ \\
\hline $\begin{array}{l}24 \\
24 \\
24\end{array}$ & $\begin{array}{l}12500 \\
12500 \\
12500\end{array}$ & $\begin{array}{l}20.0 \\
25.0 \\
30.0\end{array}$ & $\begin{array}{r}.08304 \\
.06915 \\
.05728\end{array}$ & $\begin{array}{r}.33216 \\
.27661 \\
.22913\end{array}$ & $\begin{array}{l}.02531 \\
.02107 \\
.01745\end{array}$ & $\begin{array}{l}.10124 \\
.08431 \\
.06983\end{array}$ & $\begin{array}{l}36.065 \\
30.033 \\
24.878\end{array}$ & $\begin{array}{r}109.927 \\
91.542 \\
75.829\end{array}$ & $\begin{array}{r}124.630 \\
103.786 \\
85.971\end{array}$ \\
\hline $\begin{array}{l}24 \\
24 \\
24\end{array}$ & $\begin{array}{l}12500 \\
12500 \\
12500\end{array}$ & $\begin{array}{l}35.0 \\
40.0 \\
45.0\end{array}$ & $\begin{array}{r}.04968 \\
.04357 \\
.03879\end{array}$ & $\begin{array}{r}.19872 \\
.17431 \\
.15519\end{array}$ & $\begin{array}{r}.01514 \\
.01328 \\
.01182\end{array}$ & $\begin{array}{l}.06056 \\
.05312 \\
.04730\end{array}$ & $\begin{array}{l}21.576 \\
18.925 \\
16.850\end{array}$ & $\begin{array}{l}65.764 \\
57.686 \\
51.361\end{array}$ & $\begin{array}{l}74.560 \\
65.401 \\
58.231\end{array}$ \\
\hline $\begin{array}{l}24 \\
24 \\
24\end{array}$ & $\begin{array}{l}12500 \\
12500 \\
12500\end{array}$ & $\begin{array}{l}46.0 \\
47.0 \\
48.0\end{array}$ & $\begin{array}{r}.03801 \\
.03728 \\
.03658\end{array}$ & $\begin{array}{r}.15206 \\
.14912 \\
.14633\end{array}$ & $\begin{array}{l}.01158 \\
.01136 \\
.01115\end{array}$ & $\begin{array}{r}.04634 \\
.04545 \\
.04460\end{array}$ & $\begin{array}{l}16.510 \\
16.191 \\
15.887\end{array}$ & $\begin{array}{l}50.323 \\
49.351 \\
48.426\end{array}$ & $\begin{array}{l}57.054 \\
55.951 \\
54.903\end{array}$ \\
\hline $\begin{array}{l}24 \\
24 \\
24\end{array}$ & $\begin{array}{l}12500 \\
12500 \\
12500\end{array}$ & $\begin{array}{l}49.0 \\
50.0 \\
51.0\end{array}$ & $\begin{array}{l}.03591 \\
.03529 \\
.03470\end{array}$ & $\begin{array}{r}.14365 \\
.14116 \\
.13883\end{array}$ & $\begin{array}{r}.01094 \\
.01075 \\
.01057\end{array}$ & $\begin{array}{r}.04378 \\
.04302 \\
.04231\end{array}$ & $\begin{array}{l}15.597 \\
15.327 \\
15.074\end{array}$ & $\begin{array}{l}47.541 \\
46.718 \\
45.946\end{array}$ & $\begin{array}{l}53.899 \\
52.966 \\
52.091\end{array}$ \\
\hline $\begin{array}{l}24 \\
24 \\
24\end{array}$ & $\begin{array}{l}12500 \\
12500 \\
12500\end{array}$ & $\begin{array}{l}52.0 \\
53.0 \\
54.0\end{array}$ & $\begin{array}{r}.03413 \\
.03351 \\
.03293\end{array}$ & $\begin{array}{r}.13653 \\
.13404 \\
.13172\end{array}$ & $\begin{array}{r}.01040 \\
.01021 \\
.01003\end{array}$ & $\begin{array}{l}.04161 . \\
.04085 \\
.04014\end{array}$ & $\begin{array}{l}14.823 \\
14.553 \\
14.301\end{array}$ & $\begin{array}{l}45.183 \\
44.360 \\
43.591\end{array}$ & $\begin{array}{l}51.226 \\
50.293 \\
49.421\end{array}$ \\
\hline $\begin{array}{l}24 \\
24 \\
24\end{array}$ & $\begin{array}{l}12500 \\
12500 \\
12500\end{array}$ & $\begin{array}{l}55.0 \\
60.0 \\
65.0\end{array}$ & $\begin{array}{r}.03237 \\
.03001 \\
.02819\end{array}$ & $\begin{array}{r}.12951 \\
.12004 \\
.11277\end{array}$ & $\begin{array}{l}.00986 \\
.00914 \\
.00859\end{array}$ & $\begin{array}{l}.03947 \\
.03658 \\
.03437\end{array}$ & $\begin{array}{l}14.062 \\
13.033 \\
12.244\end{array}$ & $\begin{array}{l}42.861 \\
39.726 \\
37.320\end{array}$ & $\begin{array}{l}48.594 \\
45.039 \\
42.312\end{array}$ \\
\hline $\begin{array}{l}24 \\
24 \\
24\end{array}$ & $\begin{array}{l}12500 \\
12500 \\
12500\end{array}$ & $\begin{array}{l}70.0 \\
75.0 \\
80.0\end{array}$ & $\begin{array}{l}.02671 \\
.02552 \\
.02471\end{array}$ & $\begin{array}{r}.10687 \\
.10211 \\
.09884\end{array}$ & $\begin{array}{l}.00814 \\
.00778 \\
.00753\end{array}$ & $\begin{array}{l}.03257 \\
.03112 \\
.03012\end{array}$ & $\begin{array}{l}11.604 \\
11.087 \\
10.732\end{array}$ & $\begin{array}{l}35.370 \\
33.794 \\
32.713\end{array}$ & $\begin{array}{l}40.100 \\
38.314 \\
37.088\end{array}$ \\
\hline $\begin{array}{l}24 \\
24 \\
24\end{array}$ & $\begin{array}{l}12500 \\
12500 \\
12500\end{array}$ & $\begin{array}{l}85.0 \\
90.0 \\
95.0\end{array}$ & $\begin{array}{l}.02406 \\
.02341 \\
.02294\end{array}$ & $\begin{array}{l}.09626 \\
.09366 \\
.09179\end{array}$ & $\begin{array}{l}.00733 \\
.00713 \\
.00699\end{array}$ & $\begin{array}{l}.02934 \\
.02855 \\
.02797\end{array}$ & $\begin{array}{r}10.451 \\
10.170 \\
9.966\end{array}$ & $\begin{array}{l}31.857 \\
30.998 \\
30.379\end{array}$ & $\begin{array}{l}36.118 \\
35.144 \\
34.442\end{array}$ \\
\hline 24 & 12500 & 100.0 & .02262 & .09050 & .00689 & .02758 & 9.827 & 29.953 & 33.959 \\
\hline
\end{tabular}


TABULATION OF DATA FOR

ABSORPTION OF SOUND IN AIR VERSUS PERCENT RELATIVE HUMIDITY AT 25 DEGREES CENTIGRADE FOR A FREQUENCY OF 125 HERTZ

(1)

(2) (3)

(4)

(.5)

$\begin{array}{ll}\text { REL } & \text { ATTEN } \\ \text { HUM } & \text { COEF } \\ \text { PER } & \text { PER }\end{array}$

CENT. HERTZ · CENT

METER

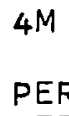

METER

.00017

.00013

.00011

.00070

.00054

.00046

$25 \quad 125 \quad 15.0$

2

2

.25

25

\section{2}

\section{2}

\section{2}

\section{2}

\section{5 \\ 2}$$
2
$$

25

$$
25
$$

(1)

25

\section{$25 \quad 125 \quad 70.0$}

$\begin{array}{lll}25 & 125 & 75.0\end{array}$

$25 \quad 125 \quad 80.0$

$\begin{array}{lll}25 & 125 & 85.0 \\ 25 & 125 & 90.0 \\ 25 & 125 & 95.0\end{array}$

.00010

.00041

.00009

.00009

.00038

.00036

(6)

(7)

(8)

(9)

$:(10)$

$\begin{array}{llll}\text { ATTEN } & 4 M & \text { ATTEN } & \text { ATTEN } \\ \text { COEF } & & \text { DE PER } & \text { DB PER } \\ \text { PER } & \text { PER } & 100 . & 1000 \\ \text { FOOT } & \text { FOOT } & \text { METER } & \text { FEET }\end{array}$

DECAY

RATE

DB PER

SECOND

\section{.00005 .00021}

.00004 .00016

.00003 .00014

$.00003: 00012$

.00002

.00002

.00011

- 00011

.00034

.00002

.00010

.00032

.00031

.00002

.00009

.00009

.00030

.00002

$\begin{array}{ll}.00002 & .00009 \\ .00002 & .00009\end{array}$

.00030

.00030

.00002

. 00009

.00002

.00030

- 00029

- 00002

.00009

.00007

.00029

.00002

.00009

.00009

.00029

.00029

.00002

.00008

.00002

.00008

.00029

.00002

.00008

.00028

.00002

.00008

.00002

.00008

.00027

.00002

.00008

.00006

.00006

.00005

.00025

.00001

.00007

.00024

.00001

.00007

.00001

.00007

.00005

.00023

.0000

.00007

.00001

.00006

00022

.00001

.00006

.00001 .00006

\begin{tabular}{|c|c|c|}
\hline $\begin{array}{r}.076 \\
.059 \\
.050\end{array}$ & $\begin{array}{l}233 \\
.179 \\
.154\end{array}$ & $\begin{array}{r}.265 \\
-204 \\
.175\end{array}$ \\
\hline $\begin{array}{l}.045 \\
.041 \\
.039\end{array}$ & $\begin{array}{r}138 \\
1127 \\
1119\end{array}$ & $\begin{array}{r}157 \\
.145 \\
\cdot 135\end{array}$ \\
\hline $\begin{array}{l}.036 \\
.035 \\
.033\end{array}$ & $\begin{array}{r}112 \\
.107 \\
.102\end{array}$ & $\begin{array}{r}128 \\
+122 \\
-116\end{array}$ \\
\hline $\begin{array}{r}.033 \\
.033 \\
.032\end{array}$ & $\begin{array}{r}102 \\
.101 \\
.100\end{array}$ & $\begin{array}{r}115 \\
.114 \\
.113\end{array}$ \\
\hline $\begin{array}{r}.032 \\
.032 \\
.032\end{array}$ & $\begin{array}{r}.099 \\
.098 \\
.098\end{array}$ & $\begin{array}{r}113 \\
.112 \\
.111\end{array}$ \\
\hline $\begin{array}{l}.031 \\
.031\end{array}$ & $\begin{array}{r}.097 \\
.096\end{array}$ & $\begin{array}{r}-110 \\
.109\end{array}$ \\
\hline .031 & .096 & .109 \\
\hline $\begin{array}{r}031 \\
.030\end{array}$ & $\begin{array}{l}095 \\
.091\end{array}$ & $\begin{array}{l}.108 \\
.104\end{array}$ \\
\hline .028 & .088 & .100 \\
\hline $\begin{array}{r}.027 \\
.026 \\
.025\end{array}$ & $\begin{array}{l}.084 \\
.081 \\
.078\end{array}$ & $\begin{array}{r}.096 \\
.092 \\
.089\end{array}$ \\
\hline .025 & .076 & .087 \\
\hline $\begin{array}{l}.024 \\
.023\end{array}$ & $\begin{array}{r}.074 \\
.072\end{array}$ & $\begin{array}{r}.084 \\
.082\end{array}$ \\
\hline .023 & .070 & .080 \\
\hline
\end{tabular}

.00005

.00021

\section{ATTEN \\ METER}

.00006 
TABULATION OF DATA FOR

ABSORPTION OF SOUND IN AIR VERSUS PERCENT RELATIVE HUMIDITY AT. 25 DEGREES CENTIGRADE FOR A FREQUENCY OF 250 HERTZ

\begin{tabular}{|c|c|c|c|c|c|c|c|c|c|}
\hline (1) & (2) & (3) & $\cdot(4)$ & (5) & (6) & 171 & $(8)$ & $(9)$ & $(10)$ \\
\hline $\begin{array}{l}\text { TEMP } \\
\text { DEGR } \\
\text { CENT }\end{array}$ & HERTZ & $\begin{array}{l}\text { REL } \\
\text { HUM } \\
\text { PER } \\
\text { CENT }\end{array}$ & $\begin{array}{l}\text { ATTEN } \\
\text { COEF } \\
\text { PER } \\
\text { METER }\end{array}$ & $\begin{array}{l}4 M \\
\text { PER } \\
\text { METER }\end{array}$ & $\begin{array}{l}\text { ATTEN } \\
\text { COEF } \\
\text { PER } \\
\text { FOOT }\end{array}$ & $\begin{array}{l}4 M \\
\text { PER } \\
\text { FOOT }\end{array}$ & $\begin{array}{l}\text { ATTEN } \\
\text { DB PER } \\
100 \\
\text { METER }\end{array}$ & $\begin{array}{l}\text { ATTEN } \\
\text { DB PER } \\
1000 \\
\text { FEET }\end{array}$ & $\begin{array}{l}\text { DECAY } \\
\text { RATE } \\
\text { DB PER } \\
\text { SECOND }\end{array}$ \\
\hline $\begin{array}{l}25 \\
25 \\
25\end{array}$ & $\begin{array}{l}250 \\
250 \\
250\end{array}$ & $\begin{array}{r}5.0 \\
10.0 \\
15.0\end{array}$ & $\begin{array}{l}.00044 \\
.00030 \\
.00026\end{array}$ & $\begin{array}{l}.00176 \\
.00122 \\
.00104\end{array}$ & $\begin{array}{l}.00013 \\
.00009 \\
.00007\end{array}$ & $\begin{array}{l}.00053 \\
.00037 \\
.00031\end{array}$ & $\begin{array}{r}.191 \\
.133 \\
.113\end{array}$ & $\begin{array}{l}.584 \\
.406 \\
.347\end{array}$ & $\begin{array}{l}.664 \\
.461 \\
.394\end{array}$ \\
\hline $\begin{array}{l}25 \\
25 \\
25\end{array}$ & $\begin{array}{l}250 \\
250 \\
250\end{array}$ & $\begin{array}{l}20.0 \\
25.0 \\
30.0\end{array}$ & $\begin{array}{l}.00023 \\
.00021 \\
.00020\end{array}$ & $\begin{array}{l}.00094 \\
.00086 \\
.00081\end{array}$ & $\begin{array}{l}.00007 \\
.00006 \\
.00006\end{array}$ & $\begin{array}{l}.00028 \\
.00026 \\
.00024\end{array}$ & $\begin{array}{l}.102 \\
.094 \\
.088\end{array}$ & $\begin{array}{l}.312 \\
.287 \\
.269\end{array}$ & $\begin{array}{l}.354 \\
.326 \\
.305\end{array}$ \\
\hline $\begin{array}{l}25 \\
25 \\
25\end{array}$ & $\begin{array}{l}250 \\
250 \\
250\end{array}$ & $\begin{array}{l}35.0 \\
40.0 \\
45.0\end{array}$ & $\begin{array}{l}.00019 \\
.00018 \\
.00017\end{array}$ & $\begin{array}{r}.00076 \\
.00073 \\
.00069\end{array}$ & $\begin{array}{r}.00005 \\
.00005 \\
.00005\end{array}$ & $\begin{array}{l}.00023 \\
.00022 \\
.00021\end{array}$ & $\begin{array}{l}.083 \\
.079 \\
.075\end{array}$ & $\begin{array}{l}.254 \\
.242 \\
.231\end{array}$ & $\begin{array}{r}.288 \\
.274 \\
.262\end{array}$ \\
\hline $\begin{array}{l}25 \\
25 \\
25\end{array}$ & $\begin{array}{l}250 \\
250 \\
250\end{array}$ & $\begin{array}{l}46.0 \\
47.0 \\
48.0\end{array}$ & $\begin{array}{l}.00017 \\
.00017 \\
.00017\end{array}$ & $\begin{array}{l}.00069 \\
.00068 \\
.00068\end{array}$ & $\begin{array}{l}.00005 \\
.00005 \\
.00005\end{array}$ & $\begin{array}{l}.00021 \\
.00020 \\
.00020\end{array}$ & $\begin{array}{l}.075 \\
.074 \\
.074\end{array}$ & $\begin{array}{l}.229 \\
.227 \\
.225\end{array}$ & $\begin{array}{l}.260 \\
.258 \\
.256\end{array}$ \\
\hline $\begin{array}{l}25 \\
25 \\
25\end{array}$ & $\begin{array}{l}250 \\
250 \\
250\end{array}$ & $\begin{array}{l}49.0 \\
50.0 \\
51.0\end{array}$ & $\begin{array}{l}.00016 \\
.00016 \\
.00016\end{array}$ & $\begin{array}{l}.00067 \\
.00067 \\
.00066\end{array}$ & $\begin{array}{l}.00005 \\
.00005 \\
.00005\end{array}$ & $\begin{array}{l}.00020 \\
.00020 \\
.00020\end{array}$ & $\begin{array}{l}.073 \\
.072 \\
.072\end{array}$ & $\begin{array}{l}.224 \\
.222 \\
.221\end{array}$ & $\begin{array}{l}.254 \\
.252 \\
.251\end{array}$ \\
\hline $\begin{array}{l}25 \\
25\end{array}$ & $\begin{array}{l}250 \\
250\end{array}$ & $\begin{array}{l}52.0 \\
53.0\end{array}$ & $\begin{array}{l}.00016 \\
.00016\end{array}$ & $\begin{array}{l}.00066 \\
.00065\end{array}$ & $\begin{array}{l}.00005 \\
.00005\end{array}$ & .00020 . & $\begin{array}{l}.072 \\
.071\end{array}$ & $\begin{array}{r}.219 \\
.218\end{array}$ & $\begin{array}{r}.249 \\
.247\end{array}$ \\
\hline 25 & 250 & $54 \cdot 0$ & .00016 & .00065 & .00004 & .00019 & .071 & .216 & .246 \\
\hline $\begin{array}{l}25 \\
25\end{array}$ & $\begin{array}{l}250 \\
250\end{array}$ & $\begin{array}{l}55.0 \\
60.0\end{array}$ & $\begin{array}{l}.00016 \\
.00015\end{array}$ & $\begin{array}{l}.00065 \\
.00062\end{array}$ & $\begin{array}{l}.00004 \\
.00004\end{array}$ & $\begin{array}{l}.00019 \\
.00019\end{array}$ & $\begin{array}{l}.070 \\
.068\end{array}$ & $\begin{array}{l}.215 \\
.208\end{array}$ & $\begin{array}{r}.244 \\
.236\end{array}$ \\
\hline 25 & 250 & 65.0 & .00015 & .00061 & .00004 & .00018 & .066 & .201 & .229 \\
\hline $\begin{array}{l}25 \\
25 \\
25\end{array}$ & $\begin{array}{l}250 \\
250 \\
250\end{array}$ & $\begin{array}{l}70.0 \\
75.0 \\
80.0\end{array}$ & $\begin{array}{l}.00014 \\
.00014 \\
.00014\end{array}$ & $\begin{array}{l}.00059 \\
.00057 \\
.00056\end{array}$ & $\begin{array}{l}.00004 \\
.00004 \\
.00004\end{array}$ & $\begin{array}{l}.00018 \\
.00017 \\
.00017\end{array}$ & $\begin{array}{l}.064 \\
.062 \\
.061\end{array}$ & $\begin{array}{r}.196 \\
.191 \\
.186\end{array}$ & $\begin{array}{r}.223 \\
.217 \\
.211\end{array}$ \\
\hline $\begin{array}{l}25 \\
25 \\
25\end{array}$ & $\begin{array}{l}250 \\
250 \\
250\end{array}$ & $\begin{array}{l}85.0 \\
90.0 \\
95.0\end{array}$ & $\begin{array}{l}.00013 \\
.00013 \\
.00012\end{array}$ & $\begin{array}{l}.00054 \\
.00053 \\
.00051\end{array}$ & $\begin{array}{l}.00004 \\
.00004 \\
.00003\end{array}$ & $\begin{array}{l}.00016 \\
.00016 \\
.00015\end{array}$ & $\begin{array}{l}.059 \\
.057 \\
.056\end{array}$ & $\begin{array}{r}.181 \\
.176 \\
.171\end{array}$ & $\begin{array}{l}.205 \\
.200 \\
.194\end{array}$ \\
\hline 25 & 250 & 100.0 & .00012 & .00050 & .00003 & .00015 & .054 & .166 & .188 \\
\hline
\end{tabular}


TABULATION OF DATA FOR

ABSORPTION OF SOUND IN AIR VERSUS PERCENT RELATIVE HUMIDITY AT 25 DEGREES CENTIGRADE FOR A FREQUENCY OF 500 HERTZ

\begin{tabular}{|c|c|c|c|c|c|c|c|c|c|}
\hline 11 & 121 & (3) & (4) & (5.) & (6) & (7) & $(8)$ & $(9)$ & $(10)$ \\
\hline $\begin{array}{l}\text { TEMP } \\
\text { DEGR } \\
\text { CENT }\end{array}$ & HERTZ & $\begin{array}{l}\text { REL } \\
\text { HUM } \\
\text { PER } \\
\text { CENT }\end{array}$ & $\begin{array}{l}\text { ATTEN } \\
\text { COEF } \\
\text { PER } \\
\text { METER }\end{array}$ & $\begin{array}{l}4 M \\
\text { PER } \\
\text { METER }\end{array}$ & $\begin{array}{l}\text { ATTEN } \\
\text { COEF } \\
\text { PER } \\
\text { FOOT }\end{array}$ & $\begin{array}{l}4 M \\
\text { PER } \\
\text { FOOT }\end{array}$ & $\begin{array}{l}\text { ATTEN } \\
\text { DB PER } \\
100 \\
\text { METER }\end{array}$ & $\begin{array}{l}\text { ATTEN } \\
\text { DB PER } \\
1000 \\
\text { FEET }\end{array}$ & $\begin{array}{l}\text { DECAY } \\
\text { RATE } \\
\text { DB PER } \\
\text { SECOND }\end{array}$ \\
\hline $\begin{array}{l}25 \\
25 \\
25\end{array}$ & $\begin{array}{l}500 \\
500 \\
500\end{array}$ & $\begin{array}{r}5.0 \\
10.0 \\
15.0\end{array}$ & $\begin{array}{l}.00130 \\
.00071 \\
.00061\end{array}$ & $\begin{array}{r}.00523 \\
.00284 \\
.00245\end{array}$ & $\begin{array}{l}.00039 \\
.00021 \\
.00018\end{array}$ & $\begin{array}{l}.00159 \\
.00086 \\
.00074\end{array}$ & $\begin{array}{l}.568 \\
.309 \\
.266\end{array}$ & $\begin{array}{r}1.731 \\
.942 \\
.812\end{array}$ & $\begin{array}{r}1.966 \\
1.070 \\
.922\end{array}$ \\
\hline $\begin{array}{l}25 \\
25 \\
25\end{array}$ & $\begin{array}{l}500 \\
500 \\
500\end{array}$ & $\begin{array}{l}20.0 \\
25.0 \\
30.0\end{array}$ & $\begin{array}{l}.00054 \\
.00050 \\
.00047\end{array}$ & $\begin{array}{l}.00219 \\
.00201 \\
.00189\end{array}$ & $\begin{array}{l}.00016 \\
.00015 \\
.00014\end{array}$ & $\begin{array}{l}.00067 \\
.00061 \\
.00057\end{array}$ & $\begin{array}{r}.238 \\
.218 \\
.205\end{array}$ & $\begin{array}{l}.727 \\
.667 \\
.625\end{array}$ & $\begin{array}{l}.826 \\
.757 \\
.710\end{array}$ \\
\hline 25 & 500 & 35.0 & .00044 & .00178 & .00013 & .00054 & .194 & .591 & .671 \\
\hline $\begin{array}{l}25 \\
25\end{array}$ & $\begin{array}{l}500 \\
500\end{array}$ & $\begin{array}{l}40 \cdot 0 \\
45.0\end{array}$ & $\begin{array}{r}.00042 \\
.00040\end{array}$ & $\begin{array}{r}.00169 \\
.00163\end{array}$ & $\begin{array}{l}.00012 \\
.00012\end{array}$ & $\begin{array}{l}.00051 \\
.00049\end{array}$ & $\begin{array}{l}.184 \\
.177\end{array}$ & $\begin{array}{l}.562 \\
.539\end{array}$ & $\begin{array}{r}.638 \\
.613\end{array}$ \\
\hline $\begin{array}{l}25 \\
25 \\
25\end{array}$ & $\begin{array}{l}500 \\
500 \\
500\end{array}$ & $\begin{array}{l}46.0 \\
47.0 \\
48.0\end{array}$ & $\begin{array}{l}.00040 \\
.00040 \\
.00039\end{array}$ & $\begin{array}{r}.00161 \\
.00160 \\
.00159\end{array}$ & $\begin{array}{l}.00012 \\
.00012 \\
.00012\end{array}$ & $\begin{array}{l}.00049 \\
.00048 \\
.00048\end{array}$ & $\begin{array}{r}.175 \\
.174 \\
.172\end{array}$ & $\begin{array}{l}.535 \\
.530 \\
.526\end{array}$ & $\begin{array}{l}.607 \\
.602 \\
.597\end{array}$ \\
\hline $\begin{array}{l}25 \\
25 \\
25\end{array}$ & $\begin{array}{l}500 \\
500 \\
500\end{array}$ & $\begin{array}{l}49.0 \\
50.0 \\
51.0\end{array}$ & $\begin{array}{l}.00039 \\
.00039 \\
.00038\end{array}$ & $\begin{array}{r}.00158 \\
.00157 \\
.00155\end{array}$ & $\begin{array}{l}.00012 \\
.00011 \\
.00011\end{array}$ & $\begin{array}{l}.00048 \\
.00047 \\
.00047\end{array}$ & $\begin{array}{r}.171 \\
.170 \\
.169\end{array}$ & $\begin{array}{r}.522 \\
.519 \\
.516\end{array}$ & $\begin{array}{r}.593 \\
.590 \\
.586\end{array}$ \\
\hline $\begin{array}{l}25 \\
25 \\
25\end{array}$ & $\begin{array}{l}500 \\
500 \\
500\end{array}$ & $\begin{array}{l}52.0 \\
53.0 \\
54.0\end{array}$ & $\begin{array}{l}.00038 \\
.00038 \\
.00038\end{array}$ & $\begin{array}{l}.00154 \\
.00153 \\
.00152\end{array}$ & $\begin{array}{l}.00011 \\
.00011 \\
.00011\end{array}$ & $\begin{array}{r}.00047 \\
.00046 \\
.00046\end{array}$ & $\begin{array}{l}.168 \\
.167 \\
166\end{array}$ & $\begin{array}{l}.512 \\
.509 \\
.506\end{array}$ & $\begin{array}{r}.582 \\
.578 \\
.574\end{array}$ \\
\hline $\begin{array}{l}25 \\
25 \\
25\end{array}$ & $\begin{array}{l}500 \\
500 \\
500\end{array}$ & $\begin{array}{l}55.0 \\
60.0 \\
65.0\end{array}$ & $\begin{array}{l}.00037 \\
.00036 \\
.00035\end{array}$ & $\begin{array}{r}.00151 \\
.00146 \\
.00142\end{array}$ & $\begin{array}{l}.00011 \\
.00011 \\
.00011\end{array}$ & $\begin{array}{l}.00046 \\
.00044 \\
.00043\end{array}$ & $\begin{array}{r}.164 \\
.159 \\
.154\end{array}$ & $\begin{array}{l}.502 \\
.485 \\
.471\end{array}$ & $\begin{array}{l}.570 \\
.551 \\
.535\end{array}$ \\
\hline $\begin{array}{l}25 \\
25\end{array}$ & $\begin{array}{l}500 \\
500\end{array}$ & $\begin{array}{l}70.0 \\
75.0\end{array}$ & $\begin{array}{l}.00034 \\
.00033\end{array}$ & $\begin{array}{r}.00138 \\
.00135\end{array}$ & $\begin{array}{l}.00010 \\
.00010\end{array}$ & $\begin{array}{r}.00042 \\
.00041\end{array}$ & $\begin{array}{l}.150 \\
.147\end{array}$ & $\begin{array}{l}.458 \\
.448\end{array}$ & $\begin{array}{l}.521 \\
.509\end{array}$ \\
\hline 25 & 500 & 80.0 & .00033 & .00132 & .00010 & .00040 & .143 & .438 & .498 \\
\hline $\begin{array}{l}25 \\
25 \\
25\end{array}$ & $\begin{array}{l}500 \\
500 \\
500\end{array}$ & $\begin{array}{l}85.0 \\
90.0 \\
95.0\end{array}$ & $\begin{array}{l}.00032 \\
.00031 \\
.00031\end{array}$ & $\begin{array}{r}.00129 \\
.00126 \\
.00124\end{array}$ & $\begin{array}{l}.00009 \\
.00009 \\
.00009\end{array}$ & $\begin{array}{l}.00039 \\
.00038 \\
.00037\end{array}$ & $\begin{array}{r}.140 \\
.137 \\
.134\end{array}$ & $\begin{array}{r}.428 \\
.419 \\
.410\end{array}$ & $\begin{array}{l}.486 \\
.476 \\
.466\end{array}$ \\
\hline 25 & 500 & 100.0 & .00030 & .00121 & .00009 & .00037 & .132 & .403 & .458 \\
\hline
\end{tabular}


TABULATION OF DATA FOR

ABSORPTION OF SOUND IN AIR VERSUS PERCENT RELATIVE HUMIDITY AT 25 DEGREES CENTIGRADE FOR A FREQUENCY OF 1000 HERTZ

\begin{tabular}{|c|c|c|c|c|c|c|c|c|c|}
\hline (1) & $(2)$ & (3) & (4) & (5) & (6) & $(7)$ & (3) & $(9)$ & $(10)$ \\
\hline $\begin{array}{l}\text { TEMP } \\
\text { DEGR } \\
\text { CENT }\end{array}$ & HERTZ & $\begin{array}{l}\text { REL } \\
\text { HUM } \\
\text { PER } \\
\text { CENT }\end{array}$ & $\begin{array}{l}\text { ATTEN } \\
\text { COEF } \\
\text { PER } \\
\text { METER }\end{array}$ & $\begin{array}{l}4 M \\
\text { PER } \\
\text { METER }\end{array}$ & $\begin{array}{l}\text { ATTEN } \\
\text { COEF } \\
\text { PER } \\
\text { FOOT }\end{array}$ & $\begin{array}{l}4 M \\
\text { PER } \\
\text { FOOT }\end{array}$ & $\begin{array}{l}\text { ATTEN } \\
\text { DB PER } \\
100 \\
\text { METER }\end{array}$ & $\begin{array}{l}\text { ATTEN } \\
\text { D. PER } \\
1000 \\
\text { FEET }\end{array}$ & $\begin{array}{l}\text { DECAY } \\
\text { RATE } \\
\text { DB PER } \\
\text { SECOND }\end{array}$ \\
\hline $\begin{array}{l}25 \\
25 \\
25\end{array}$ & $\begin{array}{l}1000 \\
1000 \\
1000\end{array}$ & $\begin{array}{r}5.0 \\
10.0 \\
15.0\end{array}$ & $\begin{array}{r}.00446 \\
.00211 \\
.00151\end{array}$ & $\begin{array}{r}.01787 \\
.00844 \\
.00604\end{array}$ & $\begin{array}{l}.00136 \\
.00064 \\
.00046\end{array}$ & $\begin{array}{l}.00544 \\
.00257 \\
.00184\end{array}$ & $\begin{array}{r}1.940 \\
.916 \\
.656\end{array}$ & $\begin{array}{l}5.914 \\
2.794 \\
2.000\end{array}$ & $\begin{array}{l}6.717 \\
3.173 \\
2.271\end{array}$ \\
\hline $\begin{array}{l}25 \\
25 \\
25\end{array}$ & $\begin{array}{l}1000 \\
1000 \\
1000\end{array}$ & $\begin{array}{l}20.0 \\
25.0 \\
30.0\end{array}$ & $\begin{array}{l}.00133 \\
.00123 \\
.00115\end{array}$ & $\begin{array}{r}.00533 \\
.00493 \\
.00461\end{array}$ & $\begin{array}{l}.00040 \\
.00037 \\
.00035\end{array}$ & $\begin{array}{l}.00162 \\
.00150 \\
.00140\end{array}$ & $\begin{array}{r}.579 \\
.535 \\
.501\end{array}$ & $\begin{array}{l}1.765 \\
1.633 \\
1.527\end{array}$ & $\begin{array}{l}2.005 \\
1.855 \\
1.734\end{array}$ \\
\hline $\begin{array}{l}25 \\
25 \\
25\end{array}$ & $\begin{array}{l}1000 \\
1000 \\
1000\end{array}$ & $\begin{array}{l}35.0 \\
40.0 \\
45.0\end{array}$ & $\begin{array}{l}.00108 \\
.00103 \\
.00099\end{array}$ & $\begin{array}{r}.00433 \\
.00412 \\
.00396\end{array}$ & $\begin{array}{l}.00033 \\
.00031 \\
.00030\end{array}$ & $\begin{array}{l}.00132 \\
.00125 \\
.00120\end{array}$ & $\begin{array}{l}.471 \\
.448 \\
.430\end{array}$ & $\begin{array}{l}1.436 \\
1.365 \\
1.311\end{array}$ & $\begin{array}{l}1.630 \\
1.550 \\
1.489\end{array}$ \\
\hline $\begin{array}{l}25 \\
25 \\
25\end{array}$ & $\begin{array}{l}1000 \\
1000 \\
1000\end{array}$ & $\begin{array}{l}46.0 \\
47.0 \\
48.0\end{array}$ & $\begin{array}{r}.00098 \\
.00097 \\
.00096\end{array}$ & $\begin{array}{r}.00393 \\
.00390 \\
.00387\end{array}$ & $\begin{array}{l}.00029 \\
.00029 \\
.00029\end{array}$ & $\begin{array}{l}.00119 \\
.00118 \\
.00118\end{array}$ & $\begin{array}{l}.426 \\
.423 \\
.420\end{array}$ & $\begin{array}{l}1.300 \\
1.290 \\
1.282\end{array}$ & $\begin{array}{l}1.477 \\
1.465 \\
1.456\end{array}$ \\
\hline $\begin{array}{l}25 \\
25 \\
25\end{array}$ & $\begin{array}{l}1000 \\
1000 \\
1000\end{array}$ & $\begin{array}{l}49.0 \\
50.0 \\
51.0\end{array}$ & $\begin{array}{l}.00096 \\
.00095 \\
.00094\end{array}$ & $\begin{array}{l}.00384 \\
.00382 \\
.00379\end{array}$ & $\begin{array}{l}.00029 \\
.00029 \\
.00028\end{array}$ & $\begin{array}{l}.00117 \\
.00116 \\
.00115\end{array}$ & $\begin{array}{r}.417 \\
.414 \\
.412\end{array}$ & $\begin{array}{l}1.273 \\
1.264 \\
1.256\end{array}$ & $\begin{array}{l}1.446 \\
1.436 \\
1.426\end{array}$ \\
\hline $\begin{array}{l}25 \\
25 \\
25\end{array}$ & $\begin{array}{l}1000 \\
1000 \\
1000\end{array}$ & $\begin{array}{l}52.0 \\
53.0 \\
54.0\end{array}$ & $\begin{array}{l}.00094 \\
.00093 \\
.00092\end{array}$ & $\begin{array}{l}.00376 \\
.00374 \\
.00371\end{array}$ & $\begin{array}{l}.00028 \\
.00028 \\
.00028\end{array}$ & $\begin{array}{l}.00114 \\
.001 .14 \\
.00113\end{array}$ & $\begin{array}{l}.409 \\
.406 \\
.403\end{array}$ & $\begin{array}{l}1 \cdot 247 \\
1 \cdot 239 . \\
1.230\end{array}$ & $\begin{array}{l}1.416 \\
1.407 \\
1.397\end{array}$ \\
\hline $\begin{array}{l}25 \\
25 \\
25\end{array}$ & $\begin{array}{l}1000 \\
1000 \\
1000\end{array}$ & $\begin{array}{l}55.0 \\
60.0 \\
65.0\end{array}$ & $\begin{array}{l}.00092 \\
.00089 \\
.00086\end{array}$ & $\begin{array}{r}.00369 \\
.00357 \\
.00347\end{array}$ & $\begin{array}{l}.00028 \\
.00027 \\
.00026\end{array}$ & $\begin{array}{l}.00112 \\
.00108 \\
.00105\end{array}$ & $\begin{array}{r}.401 \\
.388 \\
.376\end{array}$ & $\begin{array}{l}1.222 \\
1.183 \\
1.148\end{array}$ & $\begin{array}{l}1.38 .8 \\
1.343 \\
1.304\end{array}$ \\
\hline $\begin{array}{l}25 \\
25 \\
25\end{array}$ & $\begin{array}{l}1000 \\
1000 \\
1000\end{array}$ & $\begin{array}{l}70.0 \\
75.0 \\
80.0\end{array}$ & $\begin{array}{l}.00084 \\
.00082 \\
.00080\end{array}$ & $\begin{array}{l}.00338 \\
.00330 \\
.00322\end{array}$ & $\begin{array}{l}.00025 \\
.00025 \\
.00024\end{array}$ & $\begin{array}{l}.00103 \\
.00100 \\
.00098\end{array}$ & $\begin{array}{l}.367 \\
.358 \\
.350\end{array}$ & $\begin{array}{l}1.120 \\
1.093 \\
1.068\end{array}$ & $\begin{array}{l}1.272 \\
1.241 \\
1.213\end{array}$ \\
\hline 25 & 1000 & $8>\cdot 0$ & 79 & .003 & .00024 & .00096 & .343 & 1.047 & \\
\hline $\begin{array}{l}25 \\
25\end{array}$ & $\begin{array}{l}1000 \\
1000\end{array}$ & $\begin{array}{l}90.0 \\
95.0\end{array}$ & $\begin{array}{l}.00077 \\
.00075\end{array}$ & .00310 & .00023 & .00094 & $\begin{array}{r}.336 \\
.330\end{array}$ & $\begin{array}{l}1.026 \\
1.005\end{array}$ & $\begin{array}{l}1.165 \\
1.142\end{array}$ \\
\hline 2 & 0 & 100.0 & .00074 & .00298 & .00022 & .00090 & .323 & .986 & 1.120 \\
\hline
\end{tabular}


TABULATION OF DATA FOR

ABSORPTION OF SOUND IN AIR VERSUS PERCENT RELATIVE HUMIDITY AT 25 DEGREES CENTIGRADE FOR A FREQUENCY OF 2000 HERTZ

(1)

(2) (3)

(4)

(5)

161

(7)

(8)

(9)

(10)

\begin{tabular}{|c|c|c|c|c|c|c|c|c|c|}
\hline $\begin{array}{l}\text { EMP } \\
\text { EGR } \\
\text { ENT }\end{array}$ & HER TZ. & $\begin{array}{l}\text { REL } \\
\text { HUM } \\
\text { PER } \\
\text { CENT }\end{array}$ & $\begin{array}{l}\text { ATTEN } \\
\text { COEF } \\
\text { PER } \\
\text { METER }\end{array}$ & $\begin{array}{l}4 M \\
\text { PER } \\
\text { METER }\end{array}$ & $\begin{array}{l}\text { ATTEN } \\
\text { COEF } \\
\text { PER } \\
\text { FOOT }\end{array}$ & $\begin{array}{l}4 M \\
\text { PER } \\
\text { FOOT }\end{array}$ & $\begin{array}{l}\text { ATTEN } \\
\text { DB PER } \\
100 \\
\text { METER. }\end{array}$ & $\begin{array}{l}\text { ATTEN } \\
\text { DB PER } \\
1.000 \\
\text { FEET }\end{array}$ & $\begin{array}{l}\text { DECAY } \\
\text { RATE } \\
\text { DB PER } \\
\text { SECOND }\end{array}$ \\
\hline $\begin{array}{l}25 \\
25 \\
25\end{array}$ & $\begin{array}{l}2000 \\
2000 \\
2000\end{array}$ & $\begin{array}{r}5.0 \\
10.0 \\
15.0\end{array}$ & $\begin{array}{r}.01352 \\
.00739 \\
.00477\end{array}$ & $\begin{array}{r}.05410 \\
.02959 \\
.01911\end{array}$ & $\begin{array}{l}.00412 \\
.00225 \\
.00145\end{array}$ & $\begin{array}{r}.01649 \\
.00902 \\
.00582\end{array}$ & $\begin{array}{l}5.874 \\
3.213 \\
2.075\end{array}$ & $\begin{array}{r}17.906 \\
9.794 \\
6.325\end{array}$ & $\begin{array}{r}20.336 \\
11.123 \\
7.183\end{array}$ \\
\hline $\begin{array}{l}25 \\
25 \\
25\end{array}$ & $\begin{array}{l}2000 \\
2000 \\
2000\end{array}$ & $\begin{array}{l}20.0 \\
25.0 \\
30.0\end{array}$ & $\begin{array}{l}.00359 \\
.00308 \\
.00284\end{array}$ & $\begin{array}{r}.01438 \\
.01233 \\
.01136\end{array}$ & $\begin{array}{l}.00109 \\
.00094 \\
.00086\end{array}$ & $\begin{array}{l}.00438 \\
.00376 \\
.00346\end{array}$ & $\begin{array}{l}1.561 \\
1.339 \\
1.233\end{array}$ & $\begin{array}{l}4.759 \\
4.083 \\
3.761\end{array}$ & $\begin{array}{l}5.405 \\
4.637 \\
4.271\end{array}$ \\
\hline 25 & 2000 & 35.0 & .00269 & .01077 & .00082 & .00328 & 1.169 & 3.565 & 4.049 \\
\hline $\begin{array}{l}25 \\
25\end{array}$ & $\begin{array}{l}2000 \\
2000\end{array}$ & $\begin{array}{l}40.0 \\
45.0\end{array}$ & $\begin{array}{l}.00257 \\
.00246\end{array}$ & $\begin{array}{l}.01029 \\
.00986\end{array}$ & $\begin{array}{l}.00078 \\
.00075\end{array}$ & & $\begin{array}{l}1.117 \\
1.071\end{array}$ & $\begin{array}{l}3.405 \\
3.265\end{array}$ & $\begin{array}{l}3.867 \\
3.709\end{array}$ \\
\hline $\begin{array}{l}25 \\
25 \\
25\end{array}$ & $\begin{array}{l}2000 \\
2000 \\
2000\end{array}$ & $\begin{array}{l}46.0 \\
47.0 \\
48.0\end{array}$ & $\begin{array}{l}.00244 \\
.00243 \\
.00241\end{array}$ & $\begin{array}{r}.00979 \\
.00972 \\
.00964\end{array}$ & $\begin{array}{l}.00074 \\
.00074 \\
.00073\end{array}$ & $\begin{array}{r}.00298 \\
.00296 \\
.00294\end{array}$ & $\begin{array}{l}1.063 \\
1.055 \\
1.047\end{array}$ & $\begin{array}{l}3.241 \\
3.217 \\
3.193\end{array}$ & $\begin{array}{l}3.681 \\
3.654 \\
3.626\end{array}$ \\
\hline $\begin{array}{l}25 \\
25 \\
25\end{array}$ & $\begin{array}{l}2000 \\
2000 \\
2000\end{array}$ & $\begin{array}{l}49.0 \\
50.0 \\
51.0\end{array}$ & $\begin{array}{l}.00239 \\
.00237 \\
.00235\end{array}$ & $\begin{array}{l}.00957 \\
.00950 \\
.00943\end{array}$ & $\begin{array}{r}.00072 \\
.00072 \\
.00071\end{array}$ & $\begin{array}{l}.00291 \\
.00289 \\
.00287\end{array}$ & $\begin{array}{l}1.039 \\
1.032 \\
1.024\end{array}$ & $\begin{array}{l}3.169 \\
3.145 \\
3.121\end{array}$ & $\begin{array}{l}3.599 \\
3.572 \\
3.545\end{array}$ \\
\hline $\begin{array}{l}25 \\
25\end{array}$ & $\begin{array}{l}2000 \\
2000\end{array}$ & $\begin{array}{l}52.0 \\
53.0\end{array}$ & $\begin{array}{l}.00234 \\
.00232\end{array}$ & $\begin{array}{l}.00936 \\
.00929\end{array}$ & $\begin{array}{l}.00071 \\
.00070\end{array}$ & $\begin{array}{r}.00285 \\
.00283\end{array}$ & $\begin{array}{l}1.016 \\
1.008\end{array}$ & $\begin{array}{l}3.097 \\
3.074\end{array}$ & $\begin{array}{l}3.518 \\
3.492\end{array}$ \\
\hline 25 & 2000 & $54 \cdot 0$ & .00230 & .00922 & .00070 & .00281 & 1.001 & 3.053 & 3.468 \\
\hline $\begin{array}{l}25 \\
25 \\
25\end{array}$ & $\begin{array}{l}2000 \\
2000 \\
2000\end{array}$ & $\begin{array}{l}55.0 \\
60.0 \\
65.0\end{array}$ & $\begin{array}{l}.00229 \\
.00221 \\
.00215\end{array}$ & $\begin{array}{l}.00916 \\
.00884 \\
.00860\end{array}$ & $\begin{array}{l}.00069 \\
.00067 \\
.00065\end{array}$ & $\begin{array}{l}.00279 \\
.00269 \\
.00262\end{array}$ & $\begin{array}{r}.995 \\
.960 \\
.934\end{array}$ & $\begin{array}{l}3.033 \\
2.928 \\
2.848\end{array}$ & $\begin{array}{l}3 \cdot 444 \\
3 \cdot 325 \\
3.234\end{array}$ \\
\hline $\begin{array}{l}25 \\
25 \\
25\end{array}$ & $\begin{array}{l}2000 \\
2000 \\
2000\end{array}$ & $\begin{array}{l}70.0 \\
75.0 \\
80.0\end{array}$ & $\begin{array}{l}.00209 \\
.00205 \\
.00200\end{array}$ & $\begin{array}{l}.00839 \\
.00820 \\
.00803\end{array}$ & $\begin{array}{l}.00063 \\
.00062 \\
.00061\end{array}$ & $\begin{array}{l}.00255 \\
.00250 \\
.00244\end{array}$ & $\begin{array}{r}.911 \\
.890 \\
.872\end{array}$ & $\begin{array}{l}2.778 \\
2.715 \\
2.658\end{array}$ & $\begin{array}{l}3.155 \\
3.084 \\
3.019\end{array}$ \\
\hline $\begin{array}{l}25 \\
25 \\
25\end{array}$ & $\begin{array}{l}2000 \\
2000 \\
2000\end{array}$ & $\begin{array}{l}85.0 \\
90.0 \\
95.0\end{array}$ & $\begin{array}{l}.00196 \\
.00193 \\
.00189\end{array}$ & $\begin{array}{r}.00787 \\
.00772 \\
.00756\end{array}$ & $\begin{array}{l}.00060 \\
.00058 \\
.00057\end{array}$ & $\begin{array}{l}.00240 \\
.00235 \\
.00230\end{array}$ & $\begin{array}{r}.855 \\
.838 \\
.821\end{array}$ & $\begin{array}{l}2.606 \\
2.555 \\
2.505\end{array}$ & $\begin{array}{l}2.959 \\
2.902 \\
2.845\end{array}$ \\
\hline 20 & 2000 & 00.0 & .00185 & .00743 & .00056 & .00226 & .807 & 2.460 & 2.794 \\
\hline
\end{tabular}


TABULATION OF DATA FOR

ABSORPTION OF SOUND IN AIR VERSUS PERCENT RELATIVE HUMIDITY AT 25 DEGREES CENTIGRADE FOR A FREQUENCY OF 2500 HERTZ

\begin{tabular}{|c|c|c|c|c|c|c|c|c|c|}
\hline (1) & (2) & $(3)$ & $(4)$ & (5) & $(6)$ & 171 & $(8)$ & 191 & $(10)$ \\
\hline TEMP & FREQ & $\begin{array}{l}\text { REL } \\
\text { HUM }\end{array}$ & $\begin{array}{l}\text { ATTEN } \\
\text { COEF }\end{array}$ & $4 M$ & $\begin{array}{l}\text { ATTEN } \\
\text { COEF }\end{array}$ & $4 M$ & $\begin{array}{l}\text { ATTEN } \\
\text { DB PER }\end{array}$ & $\begin{array}{l}\text { ATTEN } \\
\text { DB PER. }\end{array}$ & $\begin{array}{l}\text { DECAY } \\
\text { RATE }\end{array}$ \\
\hline $\begin{array}{l}\text { DEGR } \\
\text { CENT }\end{array}$ & HERTZ & $\begin{array}{l}\text { PER } \\
\text { CENT }\end{array}$ & $\begin{array}{l}\text { PER } \\
\text { METER }\end{array}$ & $\begin{array}{l}\text { PER } \\
\text { METER }\end{array}$ & $\begin{array}{l}\text { PER } \\
\text { FOOT }\end{array}$ & $\begin{array}{l}\text { PER } \\
\text { FOOT }\end{array}$ & $\begin{array}{l}100 \\
\text { METER }\end{array}$ & $\begin{array}{l}1000 \\
\text { FEET }\end{array}$ & $\begin{array}{l}\text { DB PER } \\
\text { SECOND }\end{array}$ \\
\hline $\begin{array}{l}25 \\
25 \\
25\end{array}$ & $\begin{array}{l}2500 \\
2500 \\
2500\end{array}$ & $\begin{array}{r}5.0 \\
10.0 \\
15.0\end{array}$ & $\begin{array}{r}.01723 \\
.01082 \\
.00702\end{array}$ & $\begin{array}{l}.06895 \\
.04331 \\
.02808\end{array}$ & $\begin{array}{l}.00525 \\
.00330 \\
.00214\end{array}$ & $\begin{array}{l}.02101 \\
.01320 \\
.00856\end{array}$ & $\begin{array}{l}7.486 \\
4.703 \\
3.049\end{array}$ & $\begin{array}{r}22.819 \\
14.335 \\
9.294\end{array}$ & $\begin{array}{l}25.915 \\
16.280 \\
10.555\end{array}$ \\
\hline $\begin{array}{l}25 \\
25 \\
25\end{array}$ & $\begin{array}{l}2500 \\
2500 \\
2500\end{array}$ & $\begin{array}{l}20.0 \\
25.0 \\
30.0\end{array}$ & $\begin{array}{l}.00520 \\
.00426 \\
.00382\end{array}$ & $\begin{array}{l}.02080 \\
.01706 \\
.01530\end{array}$ & $\begin{array}{l}.00158 \\
.00130 \\
.00116\end{array}$ & $\begin{array}{l}.00634 \\
.00520 \\
.00466\end{array}$ & $\begin{array}{l}2.259 \\
1.853 \\
1.661\end{array}$ & $\begin{array}{l}6.886 \\
5.648 \\
5.063\end{array}$ & $\begin{array}{l}7.820 \\
6.415 \\
5.750\end{array}$ \\
\hline $\begin{array}{l}25 \\
25 \\
25\end{array}$ & $\begin{array}{l}2500 \\
2500 \\
2500\end{array}$ & $\begin{array}{l}35.0 \\
40.0 \\
45.0\end{array}$ & $\begin{array}{l}.00359 \\
.00343 \\
.00329\end{array}$ & $\begin{array}{l}.01438 \\
.01373 \\
.01319\end{array}$ & $\begin{array}{l}.00109 \\
.00104 \\
.00100\end{array}$ & $\begin{array}{l}.00438 \\
.00418 \\
.00402\end{array}$ & $\begin{array}{l}1.561 \\
1.491 \\
1.432\end{array}$ & $\begin{array}{l}4.759 \\
4.545 \\
4.365\end{array}$ & $\begin{array}{l}5.405 \\
5.162 \\
4.958\end{array}$ \\
\hline 25 & 2500 & 46.0 & .00327 & .01310 & .00099 & .00399 & 1.422 & $4 \cdot 335$ & 4.924 \\
\hline $\begin{array}{l}25 \\
25\end{array}$ & $\begin{array}{l}2500 \\
2500\end{array}$ & $\begin{array}{l}47 \cdot 0 \\
48 \cdot 0\end{array}$ & $\begin{array}{l}.00325 \\
.00323\end{array}$ & $\begin{array}{r}.01301 \\
.01292\end{array}$ & $\begin{array}{r}.00099 \\
.00098\end{array}$ & $\begin{array}{l}.00396 \\
.00393\end{array}$ & $\begin{array}{l}1.412 \\
1.402\end{array}$ & $\begin{array}{l}4 \cdot 305 \\
4 \cdot 275\end{array}$ & $\begin{array}{l}4.890 \\
4.856\end{array}$ \\
\hline $\begin{array}{l}25 \\
25 \\
25\end{array}$ & $\begin{array}{l}2500 \\
2500 \\
2500\end{array}$ & $\begin{array}{l}49.0 \\
50.0 \\
51.0\end{array}$ & $\begin{array}{l}.00320 \\
.00318 \\
.00316\end{array}$ & $\begin{array}{r}.01282 \\
.01273 \\
.01264\end{array}$ & $\begin{array}{l}.00097 \\
.00097 \\
.00096\end{array}$ & $\begin{array}{l}.00391 \\
.00388 \\
.00385\end{array}$ & $\begin{array}{l}1: 392 \\
1: 383 \\
1 \cdot 373\end{array}$ & $\begin{array}{l}4 \cdot 245 \\
4 \cdot 215 \\
4 \cdot 185\end{array}$ & $\begin{array}{l}4.821 \\
4.787 \\
4.753\end{array}$ \\
\hline $\begin{array}{l}25 \\
25\end{array}$ & $\begin{array}{l}2500 \\
2500\end{array}$ & $\begin{array}{l}52.0 \\
53.0\end{array}$ & $\begin{array}{l}.00313 \\
.00311\end{array}$ & $\begin{array}{r}.01255 \\
.01247\end{array}$ & $\begin{array}{l}.00095 \\
.00095\end{array}$ & $\begin{array}{l}.00382 \\
.00380\end{array}$ & $\begin{array}{l}1.363 \\
1.354\end{array}$ & $\begin{array}{l}4 \cdot 155 \\
4 \cdot 127\end{array}$ & $\begin{array}{l}4.719 \\
4.688\end{array}$ \\
\hline 25 & 2500 & $54 \cdot 0$ & .00309 & .01239 & .00094 & .00377 & $1 \cdot 345$ & 4.102 & 4.658 \\
\hline $\begin{array}{l}25 \\
25\end{array}$ & $\begin{array}{l}2500 \\
2500\end{array}$ & $\begin{array}{l}55.0 \\
60.0\end{array}$ & $\begin{array}{r}.00307 \\
.00298\end{array}$ & $\begin{array}{r}.01231 \\
.01192\end{array}$ & $\begin{array}{l}.00093 \\
.00090\end{array}$ & $\begin{array}{l}.00375 \\
.00363\end{array}$ & $\begin{array}{l}1.337 \\
1.295\end{array}$ & $\begin{array}{l}4.076 \\
3.947\end{array}$ & $\begin{array}{l}4.629 \\
4.483\end{array}$ \\
\hline 25 & 2500 & 65.0 & .00289 & .01157 & .00088 & .00 .352 & 1.256 & 3.831 & 4.351 \\
\hline $\begin{array}{l}25 \\
25 \\
25\end{array}$ & $\begin{array}{l}2500 \\
2500 \\
2500\end{array}$ & $\begin{array}{l}70.0 \\
75.0 \\
80.0\end{array}$ & $\begin{array}{l}.00280 \\
.00274 \\
.00268\end{array}$ & $\begin{array}{l}.01123 \\
.01097 \\
.01074\end{array}$ & $\begin{array}{l}.00085 \\
.00083 \\
.00081\end{array}$ & $\begin{array}{r}.00342 \\
.00334 \\
.00327\end{array}$ & $\begin{array}{l}1.220 \\
1.191 \\
1.166\end{array}$ & $\begin{array}{l}3.718 \\
3.632 \\
3.555\end{array}$ & $\begin{array}{l}4 \cdot 223 \\
4 \cdot 125 \\
4 \cdot 038\end{array}$ \\
\hline $\begin{array}{l}25 \\
25 \\
25\end{array}$ & $\begin{array}{l}2500 \\
2500 \\
2500\end{array}$ & $\begin{array}{l}85.0 \\
90.0 \\
95.0\end{array}$ & $\begin{array}{r}.00263 \\
.00258 \\
.00254\end{array}$ & $\begin{array}{l}.01054 \\
.01033 . \\
.01016\end{array}$ & $\begin{array}{r}.00080 \\
.00078 \\
.00077\end{array}$ & $\begin{array}{l}.00321 \\
.00315 \\
.00309\end{array}$ & $\begin{array}{l}1.144 \\
1 \cdot 122 \\
1.103\end{array}$ & $\begin{array}{l}3.488 \\
3.420 \\
3.364\end{array}$ & $\begin{array}{l}3.961 \\
3.884 \\
3.820\end{array}$ \\
\hline 25 & 2500 & 100.0 & .00249 & .00999 & .00076 & .00304 & 1.085 & 3.308 & 3.757 \\
\hline
\end{tabular}


TABULATION OF DATA FOR

ABSORPTION OF SOUND IN AIR VERSUS PERCENT RELATIVE HUMIDITY AT 25 DEGREES CENTIGRADE FOR. A FREQUENCY OF 3200 HERTZ

\begin{tabular}{|c|c|c|c|c|c|c|c|c|c|}
\hline (1) & (2) & $(3)$ & $(4)$ & (5) & $(6)$ & $(7)$ & $(8)$ & $19 \mid$ & $(10)$ \\
\hline TEMP & FREQ & $\begin{array}{l}\text { REL } \\
\text { HUM. }\end{array}$ & $\begin{array}{l}\text { ATTEN } \\
\text { COEF }\end{array}$ & $4 M$ & $\begin{array}{l}\text { ATTEN } \\
\text { COEF }\end{array}$ & $4 M$ & $\begin{array}{l}\text { ATTEN } \\
\text { DB PER }\end{array}$ & $\begin{array}{l}\text { ATTEN } \\
\text { DB PER }\end{array}$ & $\begin{array}{l}\text { DECAY } \\
\text { RATE }\end{array}$ \\
\hline $\begin{array}{l}\text { DEGR } \\
\text { CENT }\end{array}$ & HERTZ & $\begin{array}{l}\text { PER } \\
\text { CENT }\end{array}$ & $\begin{array}{l}\text { PER } \\
\text { METER }\end{array}$ & $\begin{array}{l}\text { PER } \\
\text { METER }\end{array}$ & $\begin{array}{l}\text { PER } \\
\text { FOOT }\end{array}$ & $\begin{array}{l}\text { PER } \\
\text { FOOT }\end{array}$ & $\begin{array}{l}100 \\
\text { METER }\end{array}$ & $\begin{array}{l}1000 \\
\text { FEET }\end{array}$ & $\begin{array}{l}\text { DB PER } \\
\text { SECOND }\end{array}$ \\
\hline $\begin{array}{l}25 \\
25 \\
25\end{array}$ & $\begin{array}{l}3200 \\
3200 \\
3200\end{array}$ & $\begin{array}{r}5.0 \\
10.0 \\
15.0\end{array}$ & $\begin{array}{r}.02157 \\
.01678 \\
.01090\end{array}$ & $\begin{array}{l}.08631 \\
.06713 \\
.04360\end{array}$ & $\begin{array}{r}.00657 \\
.00511 \\
.00332\end{array}$ & $\begin{array}{r}.02630 \\
.02046 \\
.01329\end{array}$ & $\begin{array}{l}9.37 .1 \\
7.289 \\
4.734\end{array}$ & $\begin{array}{l}28.564 \\
22.219 \\
14.430\end{array}$ & $\begin{array}{l}32 \cdot 441 \\
25 \cdot 234 \\
16 \cdot 389\end{array}$ \\
\hline $\begin{array}{l}25 \\
25 \\
25\end{array}$ & $\begin{array}{l}3200 \\
3200 \\
3200\end{array}$ & $\begin{array}{l}20.0 \\
25.0 \\
30.0\end{array}$ & $\begin{array}{l}.00800 \\
.00638 \\
.00548\end{array}$ & $\begin{array}{l}.03203 \\
.02555 \\
.02192\end{array}$ & $\begin{array}{l}.00244 \\
.00194 \\
.00167\end{array}$ & $\begin{array}{l}.00976 \\
.00779 \\
.00668\end{array}$ & $\begin{array}{l}3.477 \\
2.775 \\
2.380\end{array}$ & $\begin{array}{r}10.600 \\
8.458 \\
7.256\end{array}$ & $\begin{array}{r}12.038 \\
9.606 \\
8.241\end{array}$ \\
\hline $\begin{array}{l}25 \\
25 \\
25\end{array}$ & $\begin{array}{l}3200 \\
3200 \\
3200\end{array}$ & $\begin{array}{l}35.0 \\
40.0 \\
45.0\end{array}$ & $\begin{array}{l}.00501 \\
.00473 \\
.00455\end{array}$ & $\begin{array}{l}.02006 \\
.01893 \\
.01820\end{array}$ & $\begin{array}{r}.00152 \\
.00144 \\
.00138\end{array}$ & $\begin{array}{l}.00611 \\
.00577 \\
.00554\end{array}$ & $\begin{array}{l}2.178 \\
2.056 \\
1.976\end{array}$ & $\begin{array}{l}6.641 \\
6.267 \\
6.023\end{array}$ & $\begin{array}{l}7.543 \\
7.117 \\
6.840\end{array}$ \\
\hline $\begin{array}{l}25 \\
25 \\
25\end{array}$ & $\begin{array}{l}3200 \\
3200 \\
3200\end{array}$ & $\begin{array}{l}46.0 \\
47.0 \\
48.0\end{array}$ & $\begin{array}{l}.00451 \\
.00448 \\
.00445\end{array}$ & $\begin{array}{r}.01806 \\
.01794 \\
.01782\end{array}$ & $\begin{array}{l}.00137 \\
.00136 \\
.00135\end{array}$ & $\begin{array}{r}.00550 \\
.00546 \\
.00543\end{array}$ & $\begin{array}{l}1.960 \\
1.948 \\
1.935\end{array}$ & $\begin{array}{l}5.977 \\
5.938 \\
5.899\end{array}$ & $\begin{array}{l}6.788 \\
6.743 \\
6.699\end{array}$ \\
\hline $\begin{array}{l}25 \\
25 \\
25\end{array}$ & $\begin{array}{l}3200 \\
3200 \\
3200\end{array}$ & $\begin{array}{l}49.0 \\
50.0 \\
51.0\end{array}$ & $\begin{array}{l}.00442 \\
.00439 \\
.00436\end{array}$ & $\begin{array}{l}.01770 \\
.01758 \\
.01747\end{array}$ & $\begin{array}{l}.00134 \\
.00134 \\
.00133\end{array}$ & $\begin{array}{l}.00539 \\
.00536 \\
.00532\end{array}$ & $\begin{array}{l}1.922 \\
1.909 \\
1.896\end{array}$ & & $\begin{array}{l}6.655 \\
6.610 \\
6.566\end{array}$ \\
\hline $\begin{array}{l}25 \\
25\end{array}$ & $\begin{array}{l}3200 \\
3200\end{array}$ & $\begin{array}{l}52.0 \\
53.0\end{array}$ & $\begin{array}{l}.00433 \\
.00430\end{array}$ & . 01735 & $\begin{array}{l}.00132 \\
.00131\end{array}$ & $\begin{array}{l}.00528 \\
.00525\end{array}$ & $\begin{array}{l}1.884 \\
1.871\end{array}$ & $\begin{array}{l}5.742 \\
5.703\end{array}$ & $\begin{array}{l}6.522 \\
6.477\end{array}$ \\
\hline 25 & 3200 & 54.0 & .00428 & .01713 & .00130 & .00522 & 1.860 & 5.670 & $6 \cdot 440$ \\
\hline $\begin{array}{l}25 \\
25\end{array}$ & $\begin{array}{l}3200 \\
3200\end{array}$ & $\begin{array}{l}55.0 \\
60.0\end{array}$ & $\begin{array}{r}.00425 \\
.00413\end{array}$ & $\begin{array}{r}.01703 \\
.01654\end{array}$ & $\begin{array}{l}.00129 \\
.00126\end{array}$ & $\begin{array}{l}.00519 \\
.00504\end{array}$ & $\begin{array}{l}1.849 \\
1.796\end{array}$ & & $\begin{array}{l}6.403 \\
6.218\end{array}$ \\
\hline 25 & 3200 & 65.0 & .00402 & .01609 & .00122 & .00490 & 1.747 & $5 \cdot 326$ & 6.049 \\
\hline $\begin{array}{l}25 \\
25\end{array}$ & $\begin{array}{l}3200 \\
3200\end{array}$ & $\begin{array}{l}70.0 \\
75.0\end{array}$ & $\begin{array}{l}.00391 \\
.00382\end{array}$ & $\begin{array}{l}.01567 \\
.01528\end{array}$ & $\begin{array}{l}.00119 \\
.00116\end{array}$ & $\begin{array}{l}.00477 \\
.00465\end{array}$ & $\begin{array}{l}1.701 \\
1.659\end{array}$ & $\begin{array}{l}5.187 \\
5.057\end{array}$ & $\begin{array}{l}5.891 \\
5.743\end{array}$ \\
\hline 25 & 3200 & 80.0 & .00372 & .01491 & .00113 & .00454 & 1.619 & 4.935 & 5.604 \\
\hline $\begin{array}{l}25 \\
25 \\
25\end{array}$ & $\begin{array}{l}3200 \\
3200 \\
3200\end{array}$ & $\begin{array}{l}85.0 \\
90.0 \\
95.0\end{array}$ & $\begin{array}{r}.00364 \\
.00357 \\
.00351\end{array}$ & $\begin{array}{l}.01456 \\
.01431 \\
.01406\end{array}$ & $\begin{array}{l}.00110 \\
.00109 \\
.00107\end{array}$ & $\begin{array}{r}.00443 \\
.00436 \\
.00428\end{array}$ & $\begin{array}{l}1.581 \\
1.553 \\
1.526\end{array}$ & $\begin{array}{l}4 \cdot 820 \\
4 \cdot 736 \\
4.654\end{array}$ & $\begin{array}{l}5.474 \\
5.379 \\
5.285\end{array}$ \\
\hline 25 & 3200 & $100 \cdot 0$ & .00346 & .01384 & 00105 & .00421 & 1.502 & $4 \cdot 580$ & $5 \cdot 202$ \\
\hline
\end{tabular}


TABULATION OF DATA FOR

ABSORPTION OF SOUND IN AIR VERSUS PERCENT RELATIVE HUMIDITY AT 25 DEGREES CENTIGRADE FOR A FREQUENCY OF $4000 \mathrm{HERTZ}$

\begin{tabular}{|c|c|c|c|c|c|c|c|c|c|}
\hline (1) & $(2)$ & (3) & (4) & (5) & 161 & $(7)$ & $(8)$ & 191 & $(10)$ \\
\hline EMP & FREQ & $\begin{array}{l}\text { REL } \\
\text { HUM }\end{array}$ & $\begin{array}{l}\text { ATTEN } \\
\text { COEF }\end{array}$ & $4 M$ & $\begin{array}{l}\text { ATTEN } \\
\text { COEF }\end{array}$ & $4 M$ & $\begin{array}{l}\text { ATTEN } \\
\text { DB PER }\end{array}$ & $\begin{array}{l}\text { ATTEN } \\
\text { DB PER }\end{array}$ & $\begin{array}{l}\text { DECAY } \\
\text { RATE }\end{array}$ \\
\hline $\begin{array}{l}E G R \\
\text { ENT }\end{array}$ & HER TZ & $\begin{array}{l}\text { PER } \\
\text { CENT }\end{array}$ & $\begin{array}{l}\text { PER } \\
\text { METER }\end{array}$ & $\begin{array}{l}\text { PER } \\
\text { METER }\end{array}$ & $\begin{array}{l}\text { PER } \\
\text { FOOT }\end{array}$ & $\begin{array}{l}\text { PER } \\
\text { FOOT }\end{array}$ & $\begin{array}{l}100 \\
\text { METER }\end{array}$ & $\begin{array}{l}1000 \\
\text { FEET }\end{array}$ & $\begin{array}{l}\text { DB PER } \\
\text { SECOND }\end{array}$ \\
\hline $\begin{array}{l}25 \\
25 \\
25\end{array}$ & $\begin{array}{l}4000 \\
4000 \\
4000\end{array}$ & $\begin{array}{r}5.0 \\
10.0 \\
15.0\end{array}$ & $\begin{array}{l}.02530 \\
.02412 \\
.01588\end{array}$ & $\begin{array}{l}.10121 \\
.09651 \\
.06353\end{array}$ & $\begin{array}{l}.00771 \\
.00735 \\
.00484\end{array}$ & $\begin{array}{l}.03085 \\
.02941 \\
.01936\end{array}$ & $\begin{array}{r}10.989 \\
10.478 \\
6.898\end{array}$ & $\begin{array}{l}33.496 \\
31.939 \\
21.025\end{array}$ & $\begin{array}{l}38.042 \\
36.274 \\
23.879\end{array}$ \\
\hline $\begin{array}{l}25 \\
25\end{array}$ & $\begin{array}{l}4000 \\
4000\end{array}$ & $\begin{array}{l}20.0 \\
25.0\end{array}$ & $\begin{array}{l}.01171 \\
.00932\end{array}$ & $\begin{array}{r}.04686 \\
.03728\end{array}$ & $\begin{array}{l}.00357 \\
.00284\end{array}$ & $\begin{array}{l}.01428 \\
.01136\end{array}$ & & $\begin{array}{l}15.510 \\
12.339\end{array}$ & $\begin{array}{l}17.615 \\
14.014\end{array}$ \\
\hline 25 & 4000 & 30.0 & .00782 & .03130 & .00238 & .00954 & 3.399 & 10.361 & 11.767 \\
\hline $\begin{array}{l}25 \\
25 \\
25\end{array}$ & $\begin{array}{l}4000 \\
4000 \\
4000\end{array}$ & $\begin{array}{l}35.0 \\
40.0 \\
45.0\end{array}$ & $\begin{array}{l}.00691 \\
.00642 \\
.00608\end{array}$ & $\begin{array}{l}.02765 \\
.02570 \\
.02435\end{array}$ & $\begin{array}{l}.00210 \\
.00195 \\
.00185\end{array}$ & $\begin{array}{l}.00843 \\
.00783 \\
.00742\end{array}$ & $\begin{array}{l}3.002 \\
2.791 \\
2.644\end{array}$ & $\begin{array}{l}9.153 \\
8.508 \\
8.060\end{array}$ & $\begin{array}{r}10.395 \\
9.662 \\
9.154\end{array}$ \\
\hline $\begin{array}{l}25 \\
25 \\
25\end{array}$ & $\begin{array}{l}4000 \\
4000 \\
4000\end{array}$ & $\begin{array}{l}46.0 \\
47.0 \\
48.0\end{array}$ & $\begin{array}{l}.00604 \\
.00600 \\
.00596\end{array}$ & $\begin{array}{l}.02417 \\
.02401 \\
.02385\end{array}$ & $\begin{array}{l}.00184 \\
.00183 \\
.00181\end{array}$ & $\begin{array}{l}.00736 \\
.00732 \\
.00727\end{array}$ & $\begin{array}{l}2.624 \\
2.607 \\
2.590\end{array}$ & $\begin{array}{l}8.000 \\
7.947 \\
7.894\end{array}$ & $\begin{array}{l}9.086 \\
9.026 \\
8.966\end{array}$ \\
\hline $\begin{array}{l}25 \\
25 \\
25\end{array}$ & $\begin{array}{l}4000 \\
4000 \\
4000\end{array}$ & $\begin{array}{l}49.0 \\
50.0 \\
51.0\end{array}$ & $\begin{array}{l}.00592 \\
.00588 \\
.00584\end{array}$ & $\begin{array}{r}.02369 \\
.02353 \\
.02337\end{array}$ & $\begin{array}{l}.00180 \\
.00179 \\
.00178\end{array}$ & $\begin{array}{l}.00722 \\
.00717 \\
.00712\end{array}$ & $\begin{array}{l}2.572 \\
2.555 \\
2.538\end{array}$ & $\begin{array}{l}7 \cdot 841 \\
7 \cdot 789 \\
7 \cdot 736\end{array}$ & $\begin{array}{l}8.906 \\
8.846 \\
8.785\end{array}$ \\
\hline $\begin{array}{l}25 \\
25 \\
25\end{array}$ & $\begin{array}{l}4000 \\
4000 \\
4000\end{array}$ & $\begin{array}{l}52.0 \\
53.0 \\
54.0\end{array}$ & $\begin{array}{l}.00580 \\
.00576 \\
.00573\end{array}$ & $\begin{array}{r}.02321 \\
.02306 \\
.02293\end{array}$ & $\begin{array}{r}.00176 \\
.00175 \\
.00174\end{array}$ & $\begin{array}{l}.00707 \\
.00703 \\
.00699\end{array}$ & $\begin{array}{l}2.520 \\
2.504 \\
2.490\end{array}$ & $\begin{array}{l}7.683 \\
7.633 \\
7.590\end{array}$ & $\begin{array}{l}8.725 \\
8.669 \\
8.621\end{array}$ \\
\hline $\begin{array}{l}25 \\
25 \\
25\end{array}$ & $\begin{array}{l}4000 \\
4000 \\
4000\end{array}$ & $\begin{array}{l}55.0 \\
60.0 \\
65.0\end{array}$ & $\begin{array}{r}.00570 \\
.00554 \\
.00540\end{array}$ & $\begin{array}{l}.02280 \\
.02216 \\
.02161\end{array}$ & $\begin{array}{l}.00173 \\
.00168 \\
.00164\end{array}$ & $\begin{array}{l}.00695 \\
.00675 \\
.00658\end{array}$ & $\begin{array}{l}2.476 \\
2.407 \\
2.346\end{array}$ & $\begin{array}{l}7.548 \\
7.336 \\
7.151\end{array}$ & $\begin{array}{l}8.573 \\
8.332 \\
8.122\end{array}$ \\
\hline $\begin{array}{l}25 \\
25 \\
25\end{array}$ & $\begin{array}{l}4000 \\
4000 \\
4000\end{array}$ & $\begin{array}{l}70.0 \\
75.0 \\
80.0\end{array}$ & $\begin{array}{l}.00526 \\
.00514 \\
.00503\end{array}$ & $\begin{array}{r}.02107 \\
.02059 \\
.02014\end{array}$ & $\begin{array}{l}.00160 \\
.00156 \\
.00153\end{array}$ & $\begin{array}{l}.00642 \\
.00627 \\
.00613\end{array}$ & $\begin{array}{l}2.288 \\
2.236 \\
2.186\end{array}$ & $\begin{array}{l}6.975 \\
6.817 \\
6.665\end{array}$ & $\begin{array}{l}7.922 \\
7.742 \\
7.570\end{array}$ \\
\hline $\begin{array}{l}25 \\
25\end{array}$ & $\begin{array}{l}4000 \\
4000\end{array}$ & $\begin{array}{l}85.0 \\
90.0\end{array}$ & $\begin{array}{l}.00492 \\
.00482\end{array}$ & $\begin{array}{l}.01970 \\
.01930\end{array}$ & $\begin{array}{r}.00150 \\
.00147\end{array}$ & $\begin{array}{l}.00600 \\
.00588\end{array}$ & $\begin{array}{l}2.139 \\
2.095\end{array}$ & $\begin{array}{l}6.520 \\
6.388\end{array}$ & $\begin{array}{l}7.405 \\
7.255\end{array}$ \\
\hline - & 4000 & 95.0 & .00472 & .01890 & .00144 & .00576 & 2.052 & 6.256 & 7.105 \\
\hline 2 & 4000 & $100 \cdot 0$ & .00464 & .01858 & .00141 & .00566 & 2.017 & 6.149 & 6.984 \\
\hline
\end{tabular}


TABULATION OF DATA FOR

ABSORPTION OF SOUND IN AIR VERSUS PERCENT RELATIVE HUMIDITY

AT 25 DEGREES GENTIGRADE FOR A FREQUENCY OF 5000 HERTZ.

\begin{tabular}{|c|c|c|c|c|c|c|c|c|c|}
\hline (I) & $(2)$ & (3) & (4) & (5) & (6) & 171 & $(8)$ & (9) & $(10)$ \\
\hline $\begin{array}{l}\text { TEMP } \\
\text { DEGR } \\
\text { CENT }\end{array}$ & HERTZ & $\begin{array}{l}\text { REL } \\
\text { HUM } \\
\text { PER } \\
\text { CENT }\end{array}$ & $\begin{array}{l}\text { ATTEN } \\
\text { COEF } \\
\text { PER } \\
\text { METER }\end{array}$ & $\begin{array}{l}4 M \\
\text { PER } \\
\text { METER }\end{array}$ & $\begin{array}{l}\text { ATTEN } \\
\text { COEF } \\
\text { PER } \\
\text { FOOT }\end{array}$ & $\begin{array}{l}4 M \\
\text { PER } \\
\text { FOOT }\end{array}$ & $\begin{array}{l}\text { ATTEN } \\
\text { DB PER } \\
100 \\
\text { METER. }\end{array}$ & $\begin{array}{l}\text { ATTEN } \\
\text { DB PER } \\
1000 \\
\text { FEET }\end{array}$ & $\begin{array}{l}\text { DECAY } \\
\text { RATE } \\
\text { DB PER } \\
\text { SECOND }\end{array}$ \\
\hline $\begin{array}{l}25 \\
25 \\
25\end{array}$ & $\begin{array}{l}5000 \\
5000 \\
5000\end{array}$ & $\begin{array}{l}5.0 \\
10.0 \\
15.0\end{array}$ & $\begin{array}{l}.02818 \\
.03340 \\
.02340\end{array}$ & $\begin{array}{l}11275 \\
.13362 \\
.09362\end{array}$ & $\begin{array}{l}.00859 \\
.01018 \\
.00713\end{array}$ & $\begin{array}{r}.03436 \\
.04072 \\
.02853\end{array}$ & $\begin{array}{l}12.242 \\
14.508 \\
10.165\end{array}$ & $\begin{array}{l}37 \cdot 314 \\
44 \cdot 223 \\
30.985\end{array}$ & $\begin{array}{l}42.378 \\
50.224 \\
35.190\end{array}$ \\
\hline $\begin{array}{l}25 \\
25 \\
25\end{array}$ & $\begin{array}{l}5000 \\
5000 \\
5000\end{array}$ & $\begin{array}{l}20.0 \\
25.0 \\
30.0\end{array}$ & $\begin{array}{r}.01745 \\
.01375 \\
.01146\end{array}$ & $\begin{array}{r}.06980 \\
.05501 \\
.04585\end{array}$ & $\begin{array}{l}.00531 \\
.00419 \\
.00349\end{array}$ & $\begin{array}{r}.02127 \\
.01676 \\
.01397\end{array}$ & $\begin{array}{l}7.579 \\
5.973 \\
4.978\end{array}$ & $\begin{array}{l}23 \cdot 102 \\
18.207 \\
15.175\end{array}$ & $\begin{array}{l}26.237 \\
20.678 \\
17.234\end{array}$ \\
\hline $\begin{array}{l}25 \\
25 \\
25\end{array}$ & $\begin{array}{l}5000 \\
5000 \\
5000\end{array}$ & $\begin{array}{l}35.0 \\
40.0 \\
45.0\end{array}$ & $\begin{array}{l}.00994 \\
.00893 \\
.00836\end{array}$ & $\begin{array}{r}.03976 \\
.03573 \\
.03345\end{array}$ & $\begin{array}{l}.00303 \\
.00272 \\
.00254\end{array}$ & $\begin{array}{r}.01212 \\
.01089 \\
.01019\end{array}$ & $\begin{array}{l}4.317 \\
3.879 \\
3.632\end{array}$ & $\begin{array}{l}13.161 \\
11.824 \\
11.072\end{array}$ & $\begin{array}{l}14.0947 \\
13.429 \\
12.575\end{array}$ \\
\hline $\begin{array}{l}25 \\
25 \\
25\end{array}$ & $\begin{array}{l}5000 \\
5000 \\
5000\end{array}$ & $\begin{array}{l}46.0 \\
47.0 \\
48.0\end{array}$ & $\begin{array}{l}.00827 \\
.00816 \\
.00807\end{array}$ & $\begin{array}{r}.03309 \\
.03266 \\
.03228\end{array}$ & $\begin{array}{r}.00252 \\
.00248 \\
.00246\end{array}$ & $\begin{array}{l}.01008 \\
.00995 \\
.00984\end{array}$ & $\begin{array}{l}3.593 \\
3.546 \\
3.505\end{array}$ & $\begin{array}{l}10.953 \\
10.811 \\
10.685\end{array}$ & $\begin{array}{l}12.440 \\
12.278 \\
12.136\end{array}$ \\
\hline $\begin{array}{l}25 \\
25 \\
25\end{array}$ & $\begin{array}{l}5000 \\
5000 \\
5000\end{array}$ & $\begin{array}{l}49.0 \\
50.0 \\
51.0\end{array}$ & $\begin{array}{l}.00799 \\
.00792 \\
.00786\end{array}$ & $\begin{array}{l}.03197 \\
.03169 \\
.03145\end{array}$ & $\begin{array}{l}.00 \\
.00 \\
.00\end{array}$ & $\begin{array}{l}.00974 \\
.00966 \\
.00958\end{array}$ & $\begin{array}{l}71 \\
41 \\
15\end{array}$ & $\begin{array}{l}10.581 \\
10.490 \\
10.410\end{array}$ & $\begin{array}{l}017 \\
913 \\
823\end{array}$ \\
\hline $\begin{array}{l}25 \\
25\end{array}$ & $\begin{array}{l}5000 \\
5000\end{array}$ & $\begin{array}{l}52.0 \\
53.0\end{array}$ & $\begin{array}{l}.00781 \\
.00776\end{array}$ & $\begin{array}{l}.03126 \\
.03106\end{array}$ & $\begin{array}{l}.00238 \\
.00236\end{array}$ & $\begin{array}{l}.00952 \\
.00946\end{array}$ & $\begin{array}{l}3.394 \\
3.372\end{array}$ & $\begin{array}{l}10 \cdot 345 \\
10 \cdot 280\end{array}$ & $\begin{array}{l}11.749 \\
11.675\end{array}$ \\
\hline 25 & 5000 & $54 \cdot 0$ & .00772 & .03088 & .00235 & .00941 & 3.353 & 10.220 & 11.607 \\
\hline $\begin{array}{l}25 \\
25 \\
25\end{array}$ & $\begin{array}{l}5000 \\
5000 \\
5000\end{array}$ & $\begin{array}{l}55.0 \\
60.0 \\
65.0\end{array}$ & $\begin{array}{l}.00767 \\
.00746 \\
.00728\end{array}$ & $\begin{array}{l}.03071 \\
.02985 \\
.02912\end{array}$ & $\begin{array}{l}.00234 \\
.00227 \\
.00221\end{array}$ & $\begin{array}{l}.00936 \\
.00909 \\
.00887\end{array}$ & $\begin{array}{l}3.334 \\
3.241 \\
3.161\end{array}$ & $\begin{array}{r}10.163 \\
9.878 \\
9.637\end{array}$ & $\begin{array}{l}11.543 \\
11.219 \\
10.944\end{array}$ \\
\hline $\begin{array}{l}25 \\
25 \\
25\end{array}$ & $\begin{array}{l}5000 \\
5000 \\
5000\end{array}$ & $\begin{array}{l}70.0 \\
75.0 \\
80.0\end{array}$ & $\begin{array}{l}.00710 \\
.00695 \\
.00681\end{array}$ & $\begin{array}{r}.02843 \\
.02783 \\
.02725\end{array}$ & $\begin{array}{l}.00216 \\
.00212 \\
.00207\end{array}$ & $\begin{array}{l}.00866 \\
.00848 \\
.00830\end{array}$ & $\begin{array}{l}3.086 \\
3.021 \\
2.959\end{array}$ & $\begin{array}{l}9.409 \\
9.210 \\
9.020\end{array}$ & $\begin{array}{l}10.686 \\
10.460 \\
10.245\end{array}$ \\
\hline $\begin{array}{l}25 \\
25 \\
25\end{array}$ & $\begin{array}{l}5000 \\
5000 \\
5000\end{array}$ & $\begin{array}{l}85.0 \\
90.0 \\
95.0\end{array}$ & $\begin{array}{l}.00667 \\
.00655 \\
.00643\end{array}$ & $\begin{array}{l}.02671 \\
.02622 \\
.02573\end{array}$ & $\begin{array}{l}.00203 \\
.00199 \\
.00196\end{array}$ & $\begin{array}{r}.00814 \\
.00799 \\
.00784\end{array}$ & $\begin{array}{l}2.900 \\
2.847 \\
2.794\end{array}$ & $\begin{array}{l}8.842 \\
8.679 \\
8.516\end{array}$ & $\begin{array}{r}10.042 \\
9.857 \\
9.672\end{array}$ \\
\hline 2 & & $\cdot 0$ & .00632 & 02528 & .00192 & .00770 & 2.745 & 8.366 & 9.502 \\
\hline
\end{tabular}


TABULATION OF DATA FOR

ABSORPTION OF SOUND IN AIR VERSUS PERCENT RELATIVE HUMIDITY AT 25 DEGREES CENTIGRADE FOR A FREQUENCY OF 5940 HERTZ

\begin{tabular}{|c|c|c|c|c|c|c|c|c|c|}
\hline (1) & $(2)$ & (3) & (4.) & (5) & $(6)$ & $(7)$ & $(8)$ & $(9)$ & $(10)$ \\
\hline EMP & FREQ & $\begin{array}{l}\text { REL. } \\
\text { HUM }\end{array}$ & $\begin{array}{l}\text { ATTEN } \\
\text { COEF }\end{array}$ & $4 M$ & $\begin{array}{l}\text { ATTEN } \\
\text { COEF }\end{array}$ & $4 M$ & $\begin{array}{l}\text { ATTEN } \\
\text { DB PER }\end{array}$ & $\begin{array}{l}\text { ATTEN } \\
\text { DB PER }\end{array}$ & $\begin{array}{l}\text { DECAY } \\
\text { RATE }\end{array}$ \\
\hline $\begin{array}{l}\text { EGR } \\
\text { ENT }\end{array}$ & HER TZ & $\begin{array}{l}\text { PER } \\
\text { CENT }\end{array}$ & $\begin{array}{l}\text { PER } \\
\text { METER }\end{array}$ & $\begin{array}{l}\text { PER } \\
\text { METER }\end{array}$ & $\begin{array}{l}\text { PER } \\
\text { FOOT }\end{array}$ & $\begin{array}{l}\text { PER } \\
\text { FOOT }\end{array}$ & $\begin{array}{l}100 \\
\text { METER }\end{array}$ & $\begin{array}{l}1000 \\
\text { FEET }\end{array}$ & $\begin{array}{l}\text { DB PER } \\
\text { SECOND }\end{array}$ \\
\hline $\begin{array}{l}25 \\
25 \\
25\end{array}$ & $\begin{array}{l}5940 \\
5940 \\
5940\end{array}$ & $\begin{array}{r}5.0 \\
10.0 \\
15.0\end{array}$ & $\begin{array}{r}.02993 \\
.04133 \\
.03196\end{array}$ & $\begin{array}{r}.11972 \\
.16535 \\
.12785\end{array}$ & $\begin{array}{l}.00912 \\
.01259 \\
.00974\end{array}$ & $\begin{array}{r}.03649 \\
.05039 \\
.03897\end{array}$ & $\begin{array}{l}12.998 \\
17.953 \\
13.882\end{array}$ & $\begin{array}{l}39.621 \\
54.721 \\
42.313\end{array}$ & $\begin{array}{l}44.998 \\
62.148 \\
48.055\end{array}$ \\
\hline $\begin{array}{l}25 \\
25 \\
25\end{array}$ & $\begin{array}{l}5940 \\
5940 \\
5940\end{array}$ & $\begin{array}{l}20.0 \\
25.0 \\
30.0\end{array}$ & $\begin{array}{r}.02362 \\
.01869 \\
.01556\end{array}$ & $\begin{array}{l}.09449 \\
.07476 \\
.06227\end{array}$ & $\begin{array}{l}.00720 \\
.0 .0569 \\
.00474\end{array}$ & $\begin{array}{r}.02880 \\
.02278 \\
.01898\end{array}$ & $\begin{array}{r}10.260 \\
8.117 \\
6.761\end{array}$ & $\begin{array}{l}31.272 \\
24.743 \\
20.607\end{array}$ & $\begin{array}{l}35.517 \\
28.101 \\
23.404\end{array}$ \\
\hline $\begin{array}{l}25 \\
25 \\
25\end{array}$ & $\begin{array}{l}5940 \\
5940 \\
5940\end{array}$ & $\begin{array}{l}35.0 \\
40.0 \\
45.0\end{array}$ & $\begin{array}{r}.01335 \\
.01189 \\
.01083\end{array}$ & $\begin{array}{l}.05341 \\
.04757 \\
.04333\end{array}$ & $\begin{array}{l}.00407 \\
.00362 \\
.00330\end{array}$ & $\begin{array}{l}.01628 \\
.01450 \\
.01320\end{array}$ & & $\begin{array}{l}17.676 \\
15.745 \\
14.342\end{array}$ & $\begin{array}{l}20.075 \\
17.882 \\
16.288\end{array}$ \\
\hline $\begin{array}{l}25 \\
25 \\
25\end{array}$ & $\begin{array}{l}5940 \\
5940 \\
5940\end{array}$ & $\begin{array}{l}46.0 \\
47.0 \\
48.0\end{array}$ & $\begin{array}{l}.01067 \\
.01054 \\
.01043\end{array}$ & $\begin{array}{r}.04270 \\
.04219 \\
.04174\end{array}$ & $\begin{array}{r}.00325 \\
.00321 \\
.00318\end{array}$ & $\begin{array}{l}.01301 \\
.01286 \\
.01272\end{array}$ & $\begin{array}{l}4.636 \\
4.581 \\
4.532\end{array}$ & $\begin{array}{l}14.132 \\
13.965 \\
13.814\end{array}$ & $\begin{array}{l}16.049 \\
15.860 \\
15.689\end{array}$ \\
\hline $\begin{array}{l}25 \\
25 \\
25\end{array}$ & $\begin{array}{l}5940 \\
5940 \\
5940\end{array}$ & $\begin{array}{l}49.0 \\
50.0 \\
51.0\end{array}$ & $\begin{array}{r}.01 \\
.01 \\
.01\end{array}$ & $\begin{array}{r}.04129 \\
.04085 \\
.04048\end{array}$ & $\begin{array}{l}.01 \\
.00 \\
.01\end{array}$ & $\begin{array}{r}.01258 \\
.01245 \\
.01234\end{array}$ & & $\begin{array}{l}13 \\
13 \\
13\end{array}$ & $\begin{array}{l}19 \\
53 \\
17\end{array}$ \\
\hline $\begin{array}{l}25 \\
25 \\
25\end{array}$ & $\begin{array}{l}5940 \\
5940 \\
5940\end{array}$ & $\begin{array}{l}52.0 \\
53.0 \\
54.0\end{array}$ & $\begin{array}{r}.01002 \\
.00990 \\
.00980\end{array}$ & $\begin{array}{l}.04009 \\
.03963 \\
.03923\end{array}$ & $\begin{array}{l}.00305 \\
.00302 \\
.00298\end{array}$ & $\begin{array}{r}.01221 \\
.01208 \\
.01195\end{array}$ & $\begin{array}{l}352 \\
303 \\
260\end{array}$ & $\begin{array}{l}13.267 \\
13.117 \\
12.985\end{array}$ & $\begin{array}{l}15.068 \\
14.897 \\
14.747\end{array}$ \\
\hline $\begin{array}{l}25 \\
25 \\
25\end{array}$ & $\begin{array}{l}5940 \\
5940 \\
5940\end{array}$ & $\begin{array}{l}55.0 \\
60.0 \\
65.0\end{array}$ & $\begin{array}{r}.00972 \\
.00942 \\
.00919\end{array}$ & $\begin{array}{r}.03889 \\
.03769 \\
.03678\end{array}$ & $\begin{array}{l}.00296 \\
.00287 \\
.00280\end{array}$ & $\begin{array}{l}.01185 \\
.01148 \\
.01121\end{array}$ & $\begin{array}{l}40222 \\
4.092 \\
3.993\end{array}$ & $\begin{array}{l}871 \\
474 \\
173\end{array}$ & $\begin{array}{l}14.618 \\
14.167 \\
13.825\end{array}$ \\
\hline $\begin{array}{l}25 \\
25 \\
25\end{array}$ & $\begin{array}{l}5940 \\
5940 \\
5940\end{array}$ & $\begin{array}{l}70.0 \\
75.0 \\
80.0\end{array}$ & $\begin{array}{l}.00897 \\
.00879 \\
.00861\end{array}$ & $\begin{array}{r}.03591 \\
.03518 \\
.03446\end{array}$ & $\begin{array}{r}.00273 \\
.00268 \\
.00262\end{array}$ & $\begin{array}{r}.01094 \\
.01072 \\
.01050\end{array}$ & $\begin{array}{l}3.899 \\
3.820 \\
3.741\end{array}$ & $\begin{array}{l}11.885 \\
11.644 \\
11.404\end{array}$ & $\begin{array}{l}13.498 \\
13.225 \\
1.2 .952\end{array}$ \\
\hline $\begin{array}{l}25 \\
25 \\
25\end{array}$ & $\begin{array}{l}5940 \\
5940 \\
5940\end{array}$ & $\begin{array}{l}85.0 \\
90.0 \\
95.0\end{array}$ & $\begin{array}{l}.00846 \\
.00831 \\
.00816\end{array}$ & $\begin{array}{l}.03385 \\
.03324 \\
.03266\end{array}$ & $\begin{array}{l}.00257 \\
.00253 \\
.00248\end{array}$ & $\begin{array}{r}.01031 \\
.01013 \\
.00995\end{array}$ & $\begin{array}{l}3.675 \\
3.609 \\
3.547\end{array}$ & $\begin{array}{l}11.203 \\
11.003 \\
10.811\end{array}$ & $\begin{array}{l}12.723 \\
12.496 \\
12.279\end{array}$ \\
\hline 25 & 5940 & 100.0 & .00803 & 03215 & .00244 & .00979 & 3.490 & 0.640 & 12.084 \\
\hline
\end{tabular}


TABULATION OF DATA FOR

ABSORPTION OF SOUND IN AIR VERSUS PERCENT RELATIVE HUMIDITY AT 25 DEGREES CENTIGRADE FOR A FREQUENCY OF 6300 HERTZ

\begin{tabular}{|c|c|c|c|c|c|c|c|c|c|}
\hline (1) & $(2)$ & (3) & (4) & (5) & $(6)$ & 171 & $(8)$ & (9) & $(10)$ \\
\hline $\begin{array}{l}\text { EMP } \\
\text { EGR } \\
\text { ENT }\end{array}$ & HERTZ & $\begin{array}{l}\text { REL } \\
\text { HUM } \\
\text { PER } \\
\text { CENT }\end{array}$ & $\begin{array}{l}\text { ATTEN } \\
\text { COEF } \\
\text { PER } \\
\text { METER }\end{array}$ & $\begin{array}{l}4 M \\
\text { PER } \\
\text { METER }\end{array}$ & $\begin{array}{l}\text { ATTEN } \\
\text { COEF } \\
\text { PER } \\
\text { FOOT }\end{array}$ & $\begin{array}{l}4 M \\
\text { PER } \\
\text { FOOT }\end{array}$ & $\begin{array}{l}\text { ATTEN } \\
\text { DB PER } \\
100 \\
\text { METER }\end{array}$ & $\begin{array}{l}\text { ATTEN } \\
\text { DB PER } \\
1000 \\
\text { FEET }\end{array}$ & $\begin{array}{l}\text { DECAY } \\
\text { RATE } \\
\text { DB PER } \\
\text { SECEND }\end{array}$ \\
\hline $\begin{array}{l}25 \\
25 \\
25\end{array}$ & $\begin{array}{l}6300 \\
6300 \\
6300\end{array}$ & $\begin{array}{r}5.0 \\
10.0 \\
15.0\end{array}$ & $\begin{array}{r}.03076 \\
.04413 \\
.03514\end{array}$ & $\begin{array}{r}.12304 \\
.17654 \\
.14056\end{array}$ & $\begin{array}{r}.00937 \\
.01345 \\
.01071\end{array}$ & $\begin{array}{r}.03750 \\
.05381 \\
.04284\end{array}$ & $\begin{array}{l}13.359 \\
19.168 \\
15.261\end{array}$ & $\begin{array}{l}40.721 \\
58.425 \\
46.518\end{array}$ & $\begin{array}{l}46.248 \\
66.354 \\
52.831\end{array}$ \\
\hline $\begin{array}{l}25 \\
25 \\
25\end{array}$ & $\begin{array}{l}6300 \\
6300 \\
6300\end{array}$ & $\begin{array}{l}20.0 \\
25.0 \\
30.0\end{array}$ & $\begin{array}{r}.02593 \\
.02067 \\
.01713\end{array}$ & $\begin{array}{l}.10375 \\
.08271 \\
.06852\end{array}$ & $\begin{array}{l}.00790 \\
.00630 \\
.00522\end{array}$ & $\begin{array}{l}.03162 \\
.02521 \\
.02088\end{array}$ & $\begin{array}{r}11.265 \\
8.981 \\
7.440\end{array}$ & $\begin{array}{l}34.338 \\
27.375 \\
22.678\end{array}$ & $\begin{array}{l}38.998 \\
31.090 \\
25.755\end{array}$ \\
\hline $\begin{array}{l}25 \\
25 \\
25\end{array}$ & $\begin{array}{l}6300 \\
6300 \\
6300\end{array}$ & $\begin{array}{l}35.0 \\
40.0 \\
45.0\end{array}$ & $\begin{array}{r}.01472 \\
.01303 \\
.01184\end{array}$ & $\begin{array}{l}.05891 \\
.05215 \\
.04738\end{array}$ & $\begin{array}{l}.00448 \\
.00397 \\
.00361\end{array}$ & $\begin{array}{r}.01795 \\
.01589 \\
.01444\end{array}$ & & $\begin{array}{l}19.497 \\
17.258 \\
15.683\end{array}$ & $\begin{array}{l}22.143 \\
19.600 \\
17.811\end{array}$ \\
\hline $\begin{array}{l}25 \\
25 \\
25\end{array}$ & $\begin{array}{l}6300 \\
6300 \\
6300\end{array}$ & $\begin{array}{l}46.0 \\
47.0 \\
48.0\end{array}$ & $\begin{array}{r}.01166 \\
.01149 \\
.01134\end{array}$ & $\begin{array}{l}.04664 \\
.04599 \\
.04537\end{array}$ & $\begin{array}{l}.00355 \\
.00350 \\
.00345\end{array}$ & $\begin{array}{r}.01421 \\
.01401 \\
.01382\end{array}$ & $\begin{array}{l}5.064 \\
4.993 \\
4.926\end{array}$ & $\begin{array}{l}15.436 \\
15.220 \\
15.014\end{array}$ & $\begin{array}{l}17.531 \\
17.286 \\
17.052\end{array}$ \\
\hline $\begin{array}{l}25 \\
25 \\
25\end{array}$ & $\begin{array}{l}6300 \\
6300 \\
6300\end{array}$ & $\begin{array}{l}49.0 \\
50.0 \\
51.0\end{array}$ & $\begin{array}{r}.01122 \\
.01110 \\
.01099\end{array}$ & $\begin{array}{r}.04490 \\
.04443 \\
.04397\end{array}$ & $\begin{array}{l}.00342 \\
.00338 \\
.00335\end{array}$ & $\begin{array}{r}.01368 \\
.01354 \\
.01340\end{array}$ & $\begin{array}{l}4.875 \\
4.824 \\
4.774\end{array}$ & $\begin{array}{l}14.860 \\
14.706 \\
14.552\end{array}$ & $\begin{array}{l}16.877 \\
16.702 \\
16.527\end{array}$ \\
\hline $\begin{array}{l}25 \\
25 \\
25\end{array}$ & $\begin{array}{l}6300 \\
6300 \\
6300\end{array}$ & $\begin{array}{l}52.0 \\
53.0 \\
54.0\end{array}$ & $\begin{array}{r}.01088 \\
.01079 \\
.01068\end{array}$ & $\begin{array}{r}.04355 \\
.04317 \\
.04274\end{array}$ & $\begin{array}{l}.00331 \\
.00329 \\
.00325\end{array}$ & $\begin{array}{r}.01327 \\
.01316 \\
.01302\end{array}$ & $\begin{array}{l}4.728 \\
4.688 \\
4.641\end{array}$ & $\begin{array}{l}14 \cdot 413 \\
14 \cdot 289 \\
14 \cdot 146\end{array}$ & $\begin{array}{l}16.369 \\
16.229 \\
16.066\end{array}$ \\
\hline $\begin{array}{l}25 \\
25 \\
25\end{array}$ & $\begin{array}{l}6300 \\
6300 \\
6300\end{array}$ & $\begin{array}{l}55.0 \\
60.0 \\
65.0\end{array}$ & $\begin{array}{l}.01057 \\
.01018 \\
.00994\end{array}$ & $\begin{array}{r}.04228 \\
.04075 \\
.03976\end{array}$ & $\begin{array}{l}.00322 \\
.00310 \\
.00303\end{array}$ & $\begin{array}{r}.01288 \\
.01242 \\
.01212\end{array}$ & $\begin{array}{l}4.590 \\
4.424 \\
4.317\end{array}$ & $\begin{array}{l}13.992 \\
13.486 \\
13.159\end{array}$ & $\begin{array}{l}15.891 \\
15.316 \\
14.945\end{array}$ \\
\hline $\begin{array}{l}25 \\
25 \\
25\end{array}$ & $\begin{array}{l}6300 \\
6300 \\
6300\end{array}$ & $\begin{array}{l}70.0 \\
75.0 \\
80.0\end{array}$ & $\begin{array}{l}.00970 \\
.00950 \\
.00932\end{array}$ & $\begin{array}{r}.03883 \\
.03803 \\
.03729\end{array}$ & $\begin{array}{l}.00295 \\
.00289 \\
.00284\end{array}$ & $\begin{array}{r}.01183 \\
.01159 \\
.011136\end{array}$ & $\begin{array}{l}4.216 \\
4.130 \\
4.049\end{array}$ & $\begin{array}{l}12.851 \\
12.588 \\
12.342\end{array}$ & $\begin{array}{l}14.595 \\
14.297 \\
14.017\end{array}$ \\
\hline $\begin{array}{l}25 \\
25 \\
25\end{array}$ & $\begin{array}{l}6300 \\
6300 \\
6300\end{array}$ & $\begin{array}{l}85.0 \\
90.0 \\
95.0\end{array}$ & $\begin{array}{l}.00915 \\
.00899 \\
.00884\end{array}$ & $\begin{array}{r}.03660 \\
.03598 \\
.03536\end{array}$ & $\begin{array}{l}.00278 \\
.00274 \\
.00269\end{array}$ & $\begin{array}{r}.01115 \\
.01096 \\
.01077\end{array}$ & $\begin{array}{l}3.974 \\
3.906 \\
3.839\end{array}$ & $\begin{array}{l}12.213 \\
11.908 \\
11.702\end{array}$ & $\begin{array}{l}13.757 \\
13.524 \\
13.290\end{array}$ \\
\hline 25 & 6300 & $100 \cdot 0$ & .00870 & .03480 & .00265 & .01060 & 3.778 & 11.517 & 13.080 \\
\hline
\end{tabular}


TABULATION OF DATA FOR

ABSORPTION OF SOUND IN AIR VERSUS PERCENT RELATIVE HUMIDITY AT 25 DEGREES CENTIGRADE FOR A FREQUENCY OF 8000 HERTZ

\begin{tabular}{|c|c|c|c|c|c|c|c|c|c|}
\hline (1) & $(2)$ & (3) & $(4)$ & (5) & (6) & 171 & 181 & 191. & $(10)$ \\
\hline TEMP & FREQ & REL & ATTEN & $4 M$ & ATTEN & $4 M$ & ATTEN & ATTEN & DECAY \\
\hline DEGR & & $\begin{array}{l}\text { HUM } \\
\text { PER }\end{array}$ & $\begin{array}{l}\text { COEF } \\
\text { PER }\end{array}$ & PER & $\begin{array}{l}\text { COEF } \\
\text { PER }\end{array}$ & PER & $\begin{array}{l}\text { DB PER } \\
100\end{array}$ & $\begin{array}{l}\text { DB PER } \\
1000\end{array}$ & $\begin{array}{l}\text { RATE } \\
\text { DB PER }\end{array}$ \\
\hline CENT & HERTZ & CENT & METER & METER & F॰०T & $\mathrm{F} \odot \odot \mathrm{T}$ & METER & FEET & SECOND \\
\hline 25 & 8000 & 5.0 & .03398 & .13595 & .01035 & .04143 & 14.761 & $44 \cdot 993$ & 51.099 \\
\hline $\begin{array}{l}25 \\
25\end{array}$ & $\begin{array}{l}8000 \\
8000\end{array}$ & $\begin{array}{l}10.0 \\
15.0\end{array}$ & $\begin{array}{r}.05649 \\
.05133\end{array}$ & $\begin{array}{l}.22597 \\
.20535\end{array}$ & $\begin{array}{r}.01721 \\
.01564\end{array}$ & $\begin{array}{l}.06887 \\
.06259\end{array}$ & $\begin{array}{l}24.535 \\
22.296\end{array}$ & $\begin{array}{l}74.785 \\
67.960\end{array}$ & $\begin{array}{l}84.935 \\
77.184\end{array}$ \\
\hline 25 & 8000 & $20 \cdot 0$ & .03900 & - 15602 & .01188 & .04755 & 16.939 & 51.633 & 58.640 \\
\hline 25 & 8000 & 25.0 & .03127 & .12510 & .00953 & .03813 & 13.583 & $41 \cdot 401$ & 47.020 \\
\hline 25 & 8000 & 30.0 & .02584 & .10337 & .00787 & .03150 & $11 \cdot 223$ & 34.211 & 38.854 \\
\hline $\begin{array}{l}25 \\
25\end{array}$ & $\begin{array}{l}8000 \\
8000\end{array}$ & $\begin{array}{l}35.0 \\
40.0\end{array}$ & $\begin{array}{l}.02220 \\
.01954\end{array}$ & $\begin{array}{l}.08881 \\
.07816\end{array}$ & $\begin{array}{l}.00676 \\
.00595\end{array}$ & $\begin{array}{l}.02707 \\
.02382\end{array}$ & $\begin{array}{l}9.642 \\
8.486\end{array}$ & $\begin{array}{l}29.391 \\
25.868\end{array}$ & $\begin{array}{r}33.380 \\
29.379\end{array}$ \\
\hline 25 & 8000 & 45.0 & .01753 & .07013 & .00534 & .02137 & 7.615 & 23.212 & $26 \cdot 362$ \\
\hline $\begin{array}{l}25 \\
25\end{array}$ & $\begin{array}{l}8000 \\
8000\end{array}$ & $\begin{array}{l}46.0 \\
47.0\end{array}$ & $\begin{array}{r}.01722 \\
.01692\end{array}$ & $\begin{array}{r}.06891 \\
.06769\end{array}$ & $\begin{array}{l}.00525 \\
.00515\end{array}$ & $\begin{array}{l}.02100 \\
.02063\end{array}$ & $\begin{array}{l}7.482 \\
7.350\end{array}$ & $\begin{array}{l}22.808 \\
22.404\end{array}$ & $\begin{array}{l}25.903 \\
25.445\end{array}$ \\
\hline 25 & 8000 & 48.0 & .01666 & .06666 & .00507 & .02031 & $7 \cdot 238$ & 22.062 & 25.056 \\
\hline $\begin{array}{l}25 \\
25\end{array}$ & $\begin{array}{l}8000 \\
8000\end{array}$ & $\begin{array}{l}49.0 \\
50.0\end{array}$ & $\begin{array}{l}.01641 \\
.01613\end{array}$ & $\begin{array}{l}.06564 \\
.06455\end{array}$ & $\begin{array}{r}.00500 \\
.00491\end{array}$ & $\begin{array}{l}.02000 \\
.01967\end{array}$ & $\begin{array}{l}7.127 \\
7.008\end{array}$ & $\begin{array}{l}21.726 \\
21.363\end{array}$ & $\begin{array}{l}24.674 \\
24.262\end{array}$ \\
\hline 25 & 8000 & 51.0 & .01585 & .06343 & .00483 & .01933 & 6.887 & 20.993 & 23.842 \\
\hline $\begin{array}{l}25 \\
25\end{array}$ & $\begin{array}{l}8000 \\
8000\end{array}$ & $\begin{array}{l}52.0 \\
53.0\end{array}$ & $\begin{array}{r}.01562 \\
.01542\end{array}$ & $\begin{array}{r}.06251 \\
.06169\end{array}$ & $\begin{array}{l}.00476 \\
.00470\end{array}$ & $\begin{array}{l}.01905 \\
.01880\end{array}$ & $\begin{array}{l}6.787 \\
6.698\end{array}$ & $\begin{array}{l}20.687 \\
20.418\end{array}$ & $\begin{array}{l}23.495 \\
23.189\end{array}$ \\
\hline 25 & 8000 & 54.0 & .01523 & .06093 & .00464 & .01857 & 6.616 & 20.165 & 22.902 \\
\hline $\begin{array}{l}25 \\
25\end{array}$ & $\begin{array}{l}8000 \\
8000\end{array}$ & $\begin{array}{l}55.0 \\
60.0\end{array}$ & $\begin{array}{l}.01505 \\
.01438\end{array}$ & $\begin{array}{l}.06022 \\
.05755\end{array}$ & $\begin{array}{l}.00458 \\
.00438\end{array}$ & $\begin{array}{l}.01835 \\
.01754\end{array}$ & $\begin{array}{l}6.538 \\
6.249\end{array}$ & $\begin{array}{l}19.930 \\
19.048\end{array}$ & $\begin{array}{l}22.635 \\
21.633\end{array}$ \\
\hline 25 & 8000 & 65.0 & .01382 & .05530 & .00421 & .01685 & 6.005 & $18 \cdot 303$ & 20.787 \\
\hline $\begin{array}{l}25 \\
25\end{array}$ & $\begin{array}{l}8000 \\
8000\end{array}$ & $\begin{array}{l}70.0 \\
75.0\end{array}$ & $\begin{array}{r}.01343 \\
.01316\end{array}$ & $\begin{array}{l}.05375 \\
.05267\end{array}$ & $\begin{array}{l}.00409 \\
.00401\end{array}$ & $\begin{array}{l}.01638 \\
.01605\end{array}$ & $\begin{array}{l}5.836 \\
5.719\end{array}$ & $\begin{array}{l}17.790 \\
17.432\end{array}$ & $\begin{array}{l}20.204 \\
19.798\end{array}$ \\
\hline 25 & 8000 & 80.0 & .01291 & .05165 & .00393 & .01574 & 5.608 & 17.096 & 19.416 \\
\hline 25 & 8000 & 85.0 & .01268 & .05072 & .00386 & .01546 & 5.507 & $16 \cdot 787$ & 19.065 \\
\hline 25 & 8000 & 90.0 & .01247 & .04991 & .00380 & .015 & 5.419 & 16.518 & 18.759 \\
\hline 25 & 8000 & 95.0 & .01227 & .04909 & .00374 & .01496 & 5.330 & 16.249 & 18.454 \\
\hline 25 & 8000 & 100.0 & .01209 & .04838 & .00368 & .01474 & 5.253 & 16.013 & 18.186 \\
\hline
\end{tabular}


TABULATION OF DATA FOR

ABSORPTION OF SOUND IN AIR VERSUS PERCENT RELATIVE HUMIDITY AT 25 DEGREES CENTIGRADE FOR A FREQUENCY OF 10000 HERTZ

\begin{tabular}{|c|c|c|c|c|c|c|c|c|c|}
\hline 1) & (2) & $(3)$ & (4) & (5) & (6) & 171 & $(8)$ & $(9)$ & $(10)$ \\
\hline $\begin{array}{l}\text { EMP } \\
\text { EGR } \\
\text { ENT }\end{array}$ & HERTZ & $\begin{array}{l}\text { REL } \\
\text { HUM } \\
\text { PER } \\
\text { CENT }\end{array}$ & $\begin{array}{l}\text { ATTEN } \\
\text { COEF } \\
\text { PER } \\
\text { METER }\end{array}$ & $\begin{array}{l}4 M \\
\text { PER } \\
\text { METER }\end{array}$ & $\begin{array}{l}\text { ATTEN } \\
\text { COEF } \\
\text { PER } \\
\text { FOOT }\end{array}$ & 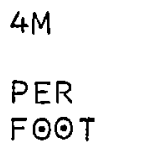 & $\begin{array}{l}\text { ATTEN } \\
\text { DB PER } \\
100 \\
\text { METER }\end{array}$ & $\begin{array}{l}\text { ATTEN } \\
\text { DB PER } \\
1000 \\
\text { FEET }\end{array}$ & $\begin{array}{l}\text { DECAY } \\
\text { RATE } \\
\text { DB PER } \\
\text { SECOND }\end{array}$ \\
\hline $\begin{array}{l}25 \\
25 \\
25\end{array}$ & $\begin{array}{l}10000 \\
10000 \\
10000\end{array}$ & $\begin{array}{r}5.0 \\
10.0 \\
15.0\end{array}$ & $\begin{array}{r}.03807 \\
.06889 \\
.06974\end{array}$ & $\begin{array}{l}15230 \\
.27557 \\
.27897\end{array}$ & $\begin{array}{l}.01160 \\
.02099 \\
.02125\end{array}$ & $\begin{array}{r}.04642 \\
.08399 \\
.08503\end{array}$ & $\begin{array}{l}16.536 \\
29.920 \\
30.289\end{array}$ & $\begin{array}{l}50.404 \\
91.197 \\
92.323\end{array}$ & $\begin{array}{r}57.244 \\
103.574 \\
104.853\end{array}$ \\
\hline $\begin{array}{l}25 \\
25 \\
25\end{array}$ & $\begin{array}{l}10000 \\
10000 \\
10000\end{array}$ & $\begin{array}{l}20.0 \\
25.0 \\
30.0\end{array}$ & $\begin{array}{l}.05733 \\
.04545 \\
.03820\end{array}$ & $\begin{array}{l}.22933 \\
.18182 \\
.15283\end{array}$ & $\begin{array}{r}.01747 \\
.01385 \\
.01164\end{array}$ & $\begin{array}{l}.06990 \\
.05542 \\
.04658\end{array}$ & $\begin{array}{l}24 \cdot 899 \\
19 \cdot 741 \\
16.594\end{array}$ & $\begin{array}{l}75.895 \\
60.173 \\
50.580\end{array}$ & $\begin{array}{l}86.195 \\
68.339 \\
57.445\end{array}$ \\
\hline $\begin{array}{l}25 \\
25 \\
25\end{array}$ & $\begin{array}{l}10000 \\
10000 \\
10000\end{array}$ & $\begin{array}{l}35.0 \\
40.0 \\
45.0\end{array}$ & $\begin{array}{l}.03261 \\
.02867 \\
.02570\end{array}$ & $\begin{array}{r}.13045 \\
.11468 \\
.10282\end{array}$ & $\begin{array}{l}.00994 \\
.00873 \\
.00783\end{array}$ & $\begin{array}{r}.03976 \\
.03495 \\
.03134\end{array}$ & $\begin{array}{l}14.163 \\
12.451 \\
11.164\end{array}$ & $\begin{array}{l}43.171 \\
37.954 \\
34.028\end{array}$ & $\begin{array}{l}49.030 \\
43.105 \\
38.646\end{array}$ \\
\hline $\begin{array}{l}25 \\
25 \\
25\end{array}$ & $\begin{array}{l}10000 \\
10000 \\
10000\end{array}$ & $\begin{array}{l}46.0 \\
47.0 \\
48.0\end{array}$ & $\begin{array}{l}.02518 \\
.02468 \\
.02418\end{array}$ & $\begin{array}{r}.10072 \\
.09873 \\
.09674\end{array}$ & $\begin{array}{l}.00767 \\
.00752 \\
.00737\end{array}$ & $\begin{array}{l}.03069 \\
.03009 \\
.02948\end{array}$ & $\begin{array}{l}10.935 \\
10.719 \\
10.504\end{array}$ & $\begin{array}{l}33.332 \\
32.673 \\
32.017\end{array}$ & $\begin{array}{l}37.856 \\
37.108 \\
36.362\end{array}$ \\
\hline $\begin{array}{l}25 \\
25 \\
25\end{array}$ & $\begin{array}{l}10000 \\
10000 \\
10000\end{array}$ & $\begin{array}{l}49.0 \\
50.0 \\
51.0\end{array}$ & $\begin{array}{l}.02374 \\
.02334 \\
.02295\end{array}$ & $\begin{array}{r}.09497 \\
.09338 \\
.09183\end{array}$ & $\begin{array}{r}.00723 \\
.00711 \\
.00699\end{array}$ & $\begin{array}{r}.02894 \\
.02846 \\
.02799\end{array}$ & $\begin{array}{r}10.312 \\
10.139 \\
9.971\end{array}$ & $\begin{array}{l}31.432 \\
30.905 \\
30.392\end{array}$ & $\begin{array}{l}35.697 \\
35.099 \\
34.517\end{array}$ \\
\hline $\begin{array}{l}25 \\
25 \\
25\end{array}$ & $\begin{array}{l}10000 \\
10000 \\
10000\end{array}$ & $\begin{array}{l}52.0 \\
53.0 \\
54.0\end{array}$ & $\begin{array}{l}.02259 \\
.02225 \\
.02192\end{array}$ & $\begin{array}{l}.09036 \\
.08903 \\
.08771\end{array}$ & $\begin{array}{l}.00688 \\
.00678 \\
.00668\end{array}$ & $\begin{array}{l}.02754 \\
.02713 \\
.02673\end{array}$ & $\begin{array}{l}9.811 \\
9.667 \\
9.523\end{array}$ & $\begin{array}{l}29.905 \\
29.466 \\
29.027\end{array}$ & $\begin{array}{l}33.964 \\
33.465 \\
32.966\end{array}$ \\
\hline $\begin{array}{l}25 \\
25 \\
25\end{array}$ & $\begin{array}{l}10000 \\
10000 \\
10000\end{array}$ & $\begin{array}{l}55.0 \\
60.0 \\
65.0\end{array}$ & $\begin{array}{l}.02164 \\
.02024 \\
.01929\end{array}$ & $\begin{array}{r}.08657 \\
.08099 \\
.07718\end{array}$ & $\begin{array}{l}.00659 \\
.00617 \\
.00588\end{array}$ & $\begin{array}{r}.02638 \\
.02468 \\
.02352\end{array}$ & $\begin{array}{l}9.400 \\
8.793 \\
8.380\end{array}$ & $\begin{array}{l}28.652 \\
26.803 \\
25.543\end{array}$ & $\begin{array}{l}32.541 \\
30.440 \\
29.010\end{array}$ \\
\hline $\begin{array}{l}25 \\
25 \\
25\end{array}$ & $\begin{array}{l}10000 \\
10000 \\
10000\end{array}$ & $\begin{array}{l}70.0 \\
75.0 \\
80.0\end{array}$ & $\begin{array}{r}.01863 \\
.01801 \\
.01759\end{array}$ & $\begin{array}{l}.07454 \\
.07206 \\
.07036\end{array}$ & $\begin{array}{l}.00568 \\
.00549 \\
.00536\end{array}$ & $\begin{array}{r}.02272 \\
.02196 \\
.02144\end{array}$ & $\begin{array}{l}8.093 \\
7.824 \\
7.639\end{array}$ & $\begin{array}{l}24.669 \\
23.848 \\
23.286\end{array}$ & $\begin{array}{l}28.017 \\
27.084 \\
26.446\end{array}$ \\
\hline $\begin{array}{l}25 \\
25 \\
25\end{array}$ & $\begin{array}{l}10000 \\
10000 \\
10000\end{array}$ & $\begin{array}{l}85.0 \\
90.0 \\
95.0\end{array}$ & $\begin{array}{l}.01729 \\
.01701 \\
.01673\end{array}$ & $\begin{array}{l}.06916 \\
.06805 \\
.06695\end{array}$ & $\begin{array}{l}.00527 \\
.00518 \\
.00510\end{array}$ & $\begin{array}{r}.02108 \\
.02074 \\
.02040\end{array}$ & $\begin{array}{l}7.509 \\
7.389 \\
7.269\end{array}$ & $\begin{array}{l}22.888 \\
22.523 \\
22.157\end{array}$ & $\begin{array}{l}25.995 \\
25.579 \\
25.164\end{array}$ \\
\hline 25 & 10000 & $100 \cdot 0$ & 01651 & $: 06605$ & .00503 & .0201 & 7.171 & 21.859 & 24.826 \\
\hline
\end{tabular}


TABULATION OF DATA FOR

ABSORPTION OF SOUND IN AIR VERSUS PERCENT RELATIVE HUMIDITY AT 25 DEGREES CENTIGRADE FOR A FREQUENCY OF 12500 HERTZ

\begin{tabular}{|c|c|c|c|c|c|c|c|c|c|}
\hline 1 & (2) & (3) & (4) & (5) & (6) & (7) & (8) & (9) & $(10)$ \\
\hline EMP & FREQ & $\begin{array}{l}\text { REL } \\
\text { HUM }\end{array}$ & $\begin{array}{l}\text { ATTEN } \\
\text { COEF }\end{array}$ & $4 M$ & $\begin{array}{l}\text { ATTEN } \\
\text { COEF }\end{array}$ & $4 M$ & $\begin{array}{l}\text { ATTEN } \\
\text { DB PER }\end{array}$ & $\begin{array}{l}\text { ATTEN } \\
\text { DB PER }\end{array}$ & $\begin{array}{l}\text { DECAY } \\
\text { RATE }\end{array}$ \\
\hline $\begin{array}{l}\text { EGR } \\
\text { ENT }\end{array}$ & HERTZ & $\begin{array}{l}\text { PER } \\
\text { CENT }\end{array}$ & $\begin{array}{l}\text { PER } \\
\text { METER }\end{array}$ & $\begin{array}{l}\text { PER } \\
\text { METER }\end{array}$ & 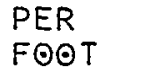 & $\begin{array}{l}\text { PER } \\
\text { FO०T }\end{array}$ & $\begin{array}{l}100 \\
\text { METER }\end{array}$ & $\begin{array}{l}1000 \\
\text { FEET }\end{array}$ & $\begin{array}{l}\text { DB PER } \\
\text { SECOND }\end{array}$ \\
\hline $\begin{array}{l}25 \\
25 \\
25\end{array}$ & $\begin{array}{l}12500 \\
12500 \\
12500\end{array}$ & $\begin{array}{r}5.0 \\
10.0 \\
15.0\end{array}$ & $\begin{array}{r}.04305 \\
.08094 \\
.09072\end{array}$ & $\begin{array}{r}.17221 \\
.32379 \\
.36290\end{array}$ & $\begin{array}{r}.01312 \\
.02467 \\
.02765\end{array}$ & $\begin{array}{r}.05248 \\
.09869 \\
.11061\end{array}$ & $\begin{array}{l}18.697 \\
35.156 \\
39.402\end{array}$ & $\begin{array}{r}56.991 \\
107.158 \\
120.100\end{array}$ & $\begin{array}{r}64.726 \\
121.700 \\
136.400\end{array}$ \\
\hline $\begin{array}{l}25 \\
25 \\
25\end{array}$ & $\begin{array}{l}12500 \\
12500 \\
12500\end{array}$ & $\begin{array}{l}20.0 \\
25.0 \\
30.0\end{array}$ & $\begin{array}{l}.08208 \\
.06714 \\
.05587\end{array}$ & $\begin{array}{l}.32832 \\
.26859 \\
.22349\end{array}$ & $\begin{array}{l}.02501 \\
.02046 \\
.01703\end{array}$ & $\begin{array}{l}.10 \\
.08 \\
.06\end{array}$ & $\begin{array}{l}35.647 \\
29.163 \\
24.265\end{array}$ & $\begin{array}{r}108.655 \\
88.890 \\
73.963\end{array}$ & $\begin{array}{r}123.401 \\
100.953 \\
84.000\end{array}$ \\
\hline $\begin{array}{l}25 \\
25 \\
25\end{array}$ & $\begin{array}{l}12500 \\
12500 \\
12500\end{array}$ & $\begin{array}{l}35.0 \\
40.0 \\
45.0\end{array}$ & $\begin{array}{r}.04844 \\
.04234 \\
.03790\end{array}$ & $\begin{array}{r}.19379 \\
.16938 \\
.15160\end{array}$ & $\begin{array}{r}.01476 \\
.01290 \\
.01155\end{array}$ & $\begin{array}{l}.05906 \\
.05162 \\
.04621\end{array}$ & & & $\begin{array}{l}72.838 \\
63.662 \\
56.983\end{array}$ \\
\hline $\begin{array}{l}25 \\
25 \\
25\end{array}$ & $\begin{array}{l}12500 \\
12500 \\
12500\end{array}$ & $\begin{array}{l}46.0 \\
47.0 \\
48.0\end{array}$ & $\begin{array}{r}.03715 \\
.03644 \\
.03578\end{array}$ & $\begin{array}{r}.14863 \\
.14579 \\
.14315\end{array}$ & $\begin{array}{l}.011132 \\
.01110 \\
.01090\end{array}$ & $\begin{array}{l}.0453 \\
.0444 \\
.0436\end{array}$ & & & $\begin{array}{l}55.863 \\
54.796 \\
53.806\end{array}$ \\
\hline $\begin{array}{l}25 \\
25 \\
25\end{array}$ & $\begin{array}{l}12500 \\
12500 \\
12500\end{array}$ & $\begin{array}{l}49.0 \\
50.0 \\
51.0\end{array}$ & $\begin{array}{r}.03517 \\
.03455 \\
.03389\end{array}$ & $\begin{array}{l}.1 \\
.1 \\
.1\end{array}$ & $\begin{array}{l}.01072 \\
.01053 \\
.01033\end{array}$ & $\begin{array}{l}.04 \\
.04 \\
.04\end{array}$ & & & $\begin{array}{l}52.881 \\
51.954 \\
50.964\end{array}$ \\
\hline $\begin{array}{l}25 \\
25 \\
25\end{array}$ & $\begin{array}{l}12500 \\
12500 \\
12500\end{array}$ & $\begin{array}{l}52.0 \\
53.0 \\
54.0\end{array}$ & $\begin{array}{r}.03329 \\
.03272 \\
.03218\end{array}$ & $\begin{array}{r}.13317 \\
.13088 \\
.12873\end{array}$ & $\begin{array}{l}.01014 \\
.00997 \\
.00980\end{array}$ & $\begin{array}{l}.04059 \\
.03989 \\
.03923\end{array}$ & $\begin{array}{l}14.459 \\
14.211 \\
13.977\end{array}$ & & $\begin{array}{l}50.053 \\
49.195 \\
48.385\end{array}$ \\
\hline 25 & 12500 & 55. & .03164 & 12 & .00964 & .03 & & & 4 \\
\hline $\begin{array}{l}25 \\
25\end{array}$ & $\begin{array}{l}12500 \\
12500\end{array}$ & $\begin{array}{l}60.0 \\
65.0\end{array}$ & & & $\begin{array}{l}.00 \\
.00\end{array}$ & $\begin{array}{l}.0 \\
.0\end{array}$ & & $\begin{array}{l}72 \\
95\end{array}$ & $\begin{array}{l}261 \\
788\end{array}$ \\
\hline 25 & 12500 & 70 & .02 & .10 & .00 & .03 & & $34 \cdot 847$ & 3 \\
\hline $\begin{array}{l}25 \\
25\end{array}$ & $\begin{array}{l}12500 \\
12500\end{array}$ & $\begin{array}{l}75 \\
80\end{array}$ & $\begin{array}{l}.02 \\
.02\end{array}$ & .10 & .00 & $\begin{array}{l}.03 \\
.02\end{array}$ & $\begin{array}{l}94 \\
76\end{array}$ & $\begin{array}{l}33.512 \\
32.541\end{array}$ & $\begin{array}{l}38.061 \\
36.957\end{array}$ \\
\hline $\begin{array}{l}25 \\
25 \\
25\end{array}$ & $\begin{array}{l}12500 \\
12500 \\
12500\end{array}$ & $\begin{array}{l}85.0 \\
90.0 \\
95.0\end{array}$ & $\begin{array}{l}.02390 \\
.02336 \\
.02299\end{array}$ & $\begin{array}{r}.09561 \\
.09345 \\
.09199\end{array}$ & $\begin{array}{r}.00728 \\
.00712 \\
.00700\end{array}$ & $\begin{array}{r}.02914 \\
.02848 \\
.02803\end{array}$ & $\begin{array}{r}10.381 \\
10.147 \\
9.988\end{array}$ & $\begin{array}{l}31.644 \\
30.928 \\
30.444\end{array}$ & $\begin{array}{l}35.939 \\
35.126 \\
34.575\end{array}$ \\
\hline 25 & S & 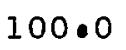 & 0 & 076 & .00691 & .02766 & 8 & .038 & 34. \\
\hline
\end{tabular}


TABULATION OF DATA FOR

ABSORPTION OF SOUND IN AIR VERSUS PERCENT RELATIVE HUMIDITY AT 30 DEGREES CENTIGRADE FOR A FREQUENCY OF 125 HERTZ

\begin{tabular}{|c|c|c|c|c|c|c|c|c|c|}
\hline (1) & (2) & (3) & (4) & (5) & $(6)$ & (7) & (8) & (9) & $(10)$ \\
\hline $\begin{array}{l}\text { TEMP } \\
\text { DEGR } \\
\text { CENT }\end{array}$ & HERTZ & $\begin{array}{l}\text { REL } \\
\text { HUM } \\
\text { PER } \\
\text { CENT }\end{array}$ & $\begin{array}{l}\text { ATTEN } \\
\text { COEF } \\
\text { PER } \\
\text { METER }\end{array}$ & $\begin{array}{l}4 M \\
\text { PER } \\
\text { METER }\end{array}$ & $\begin{array}{l}\text { ATTEN } \\
\text { COEF } \\
\text { PER } \\
\text { FOOT }\end{array}$ & $\begin{array}{l}4 M \\
\text { PER } \\
\text { FOOT }\end{array}$ & $\begin{array}{l}\text { ATTEN } \\
\text { DB PER } \\
\text { IOO } \\
\text { METER }\end{array}$ & $\begin{array}{l}\text { ATTEN } \\
\text { DB PER. } \\
\text { I OOO } \\
\text { FEET }\end{array}$ & $\begin{array}{l}\text { DECAY } \\
\text { RATE } \\
\text { DB PER } \\
\text { SECOND }\end{array}$ \\
\hline $\begin{array}{l}30 \\
30 \\
30\end{array}$ & $\begin{array}{l}125 \\
125 \\
125\end{array}$ & $\begin{array}{r}5.0 \\
10.0 \\
15.0\end{array}$ & $\begin{array}{l}.00017 \\
.00013 \\
.00011\end{array}$ & $\begin{array}{r}.00068 \\
.00052 \\
.00045\end{array}$ & $\begin{array}{l}.00005 \\
.00004 \\
.00003\end{array}$ & $\begin{array}{l}.00021 \\
.00016 \\
.00013\end{array}$ & $\begin{array}{l}.074 \\
.057 \\
.049\end{array}$ & $\begin{array}{r}.228 \\
.175 \\
.150\end{array}$ & $\begin{array}{l}.261 \\
.200 \\
.172\end{array}$ \\
\hline $\begin{array}{l}30 \\
30 \\
30\end{array}$ & $\begin{array}{l}125 \\
125 \\
125\end{array}$ & $\begin{array}{l}20.0 \\
25.0 \\
30.0\end{array}$ & $\begin{array}{l}.00010 \\
.00009 \\
.00008\end{array}$ & $\begin{array}{l}.00041 \\
.00037 \\
.00035\end{array}$ & $\begin{array}{l}.00003 \\
.00002 \\
.00002\end{array}$ & $\begin{array}{l}.00012 \\
.00011 \\
.00010\end{array}$ & $\begin{array}{l}.044 \\
.040 \\
.038\end{array}$ & $\begin{array}{l}.136 \\
.124 \\
.117\end{array}$ & $\begin{array}{l}.155 \\
.142 \\
.134\end{array}$ \\
\hline $\begin{array}{l}30 \\
30 \\
30\end{array}$ & $\begin{array}{l}125 \\
125 \\
125\end{array}$ & $\begin{array}{l}35.0 \\
40.0 \\
45.0\end{array}$ & $\begin{array}{r}.00008 \\
.00007 \\
.00007\end{array}$ & $\begin{array}{l}.00033 \\
.00031 \\
.00030\end{array}$ & $\begin{array}{r}.00002 \\
.00002 \\
.00002\end{array}$ & $\begin{array}{l}.00010 \\
.00009 \\
.00009\end{array}$ & $\begin{array}{l}.036 \\
.034 \\
.032\end{array}$ & $\begin{array}{l}.110 \\
.104 \\
.099\end{array}$ & $\begin{array}{r}.126 \\
.120 \\
.114\end{array}$ \\
\hline $\begin{array}{l}30 \\
30 \\
30\end{array}$ & $\begin{array}{l}125 \\
125 \\
125\end{array}$ & $\begin{array}{l}46 \cdot 0 \\
47 \cdot 0 \\
48 \cdot 0\end{array}$ & $\begin{array}{l}.00007 \\
.00007 \\
.00007\end{array}$ & $\begin{array}{l}.00029 \\
.00029 \\
.00029\end{array}$ & $\begin{array}{r}.00002 \\
.00002 \\
.00002\end{array}$ & $\begin{array}{l}.00009 \\
.00009 \\
.00008\end{array}$ & $\begin{array}{l}.032 \\
.032 \\
.031\end{array}$ & $\begin{array}{l}.098 \\
.097 \\
.096\end{array}$ & $\begin{array}{r}.113 \\
.112 \\
.110\end{array}$ \\
\hline $\begin{array}{l}30 \\
30 \\
30\end{array}$ & $\begin{array}{l}125 \\
125 \\
125\end{array}$ & $\begin{array}{l}49.0 \\
50.0 \\
51.0\end{array}$ & $\begin{array}{r}.00007 \\
.00007 \\
.00007\end{array}$ & $\begin{array}{r}.00028 \\
.00028 \\
.00028\end{array}$ & $\begin{array}{l}.00002 \\
.00002 \\
.00002\end{array}$ & $\begin{array}{l}.00008 \\
.00008 \\
.00008\end{array}$ & $\begin{array}{l}.031 \\
.031 \\
.030\end{array}$ & $\begin{array}{l}.095 \\
.094 \\
.0 .93\end{array}$ & $\begin{array}{l}.109 \\
.108 \\
.107\end{array}$ \\
\hline $\begin{array}{l}30 \\
30 \\
30\end{array}$ & $\begin{array}{l}125 \\
125 \\
125\end{array}$ & $\begin{array}{l}52.0 \\
53.0 \\
54.0\end{array}$ & $\begin{array}{l}.00007 \\
.00006 \\
.00006\end{array}$ & $\begin{array}{l}.00028 \\
.00027 \\
.00027\end{array}$ & $\begin{array}{l}.00002 \\
.00002 \\
.00002\end{array}$ & $\begin{array}{l}.00008 \\
.00008 \\
.00008\end{array}$ & $\begin{array}{l}.030 \\
.030 \\
.029\end{array}$ & $\begin{array}{l}.092 \\
.091 \\
.090\end{array}$ & $\begin{array}{l}.106 \\
.105 \\
.103\end{array}$ \\
\hline $\begin{array}{l}30 \\
30 \\
30\end{array}$ & $\begin{array}{l}125 \\
125 \\
125\end{array}$ & $\begin{array}{l}55.0 \\
60.0 \\
65.0\end{array}$ & $\begin{array}{l}.00006 \\
.00006 \\
.00006\end{array}$ & $\begin{array}{l}.00027 \\
.00025 \\
.00025\end{array}$ & $\begin{array}{l}.00002 \\
.00001 \\
.00001\end{array}$ & $\begin{array}{l}.00008 \\
.00007 \\
.00007\end{array}$ & $\begin{array}{l}.029 \\
.028 \\
.027\end{array}$ & $\begin{array}{l}.089 \\
.085 \\
.082\end{array}$ & $\begin{array}{l}.102 \\
.098 \\
.094\end{array}$ \\
\hline $\begin{array}{l}30 \\
30 \\
30\end{array}$ & $\begin{array}{l}125 \\
125 \\
125\end{array}$ & $\begin{array}{l}70.0 \\
75.0 \\
80.0\end{array}$ & $\begin{array}{l}.00006 \\
.00005 \\
.00005\end{array}$ & $\begin{array}{l}.00024 \\
.00023 \\
.00022\end{array}$ & $\begin{array}{l}.00001 \\
.00001 \\
.00001\end{array}$ & $\begin{array}{l}.00007 \\
.00007 \\
.00006\end{array}$ & $\begin{array}{l}.026 \\
.025 \\
.024\end{array}$ & $\begin{array}{l}.079 \\
.076 \\
.073\end{array}$ & $\begin{array}{l}.091 \\
.087 \\
.084\end{array}$ \\
\hline $\begin{array}{l}30 \\
30 \\
30\end{array}$ & $\begin{array}{l}125 \\
125 \\
125\end{array}$ & $\begin{array}{l}85.0 \\
90.0 \\
95.0\end{array}$ & $\begin{array}{r}.00005 \\
.00005 \\
.00004\end{array}$ & $\begin{array}{l}.00021 \\
.00020 \\
.00019\end{array}$ & $\begin{array}{l}.00001 \\
.00001 \\
.00001\end{array}$ & $\begin{array}{l}.00006 \\
.00006 \\
.00006\end{array}$ & $\begin{array}{l}.023 \\
.022 \\
.021\end{array}$ & $\begin{array}{l}.070 \\
.067 \\
.065\end{array}$ & $\begin{array}{r}.080 \\
.077 \\
.074\end{array}$ \\
\hline 30 & 125 & 100.0 & .00004 & .00019 & .00001 & .00005 & .020 & .063 & .072 \\
\hline
\end{tabular}


TABULATION OF DATA FOR

ABSORPTION OF SOUND IN AIR VERSUS PERCENT RELATIVE HUMIDITY AT 30 DEGREES CENTIGRADE FOR A FREQUENCY OF 250 HERTZ

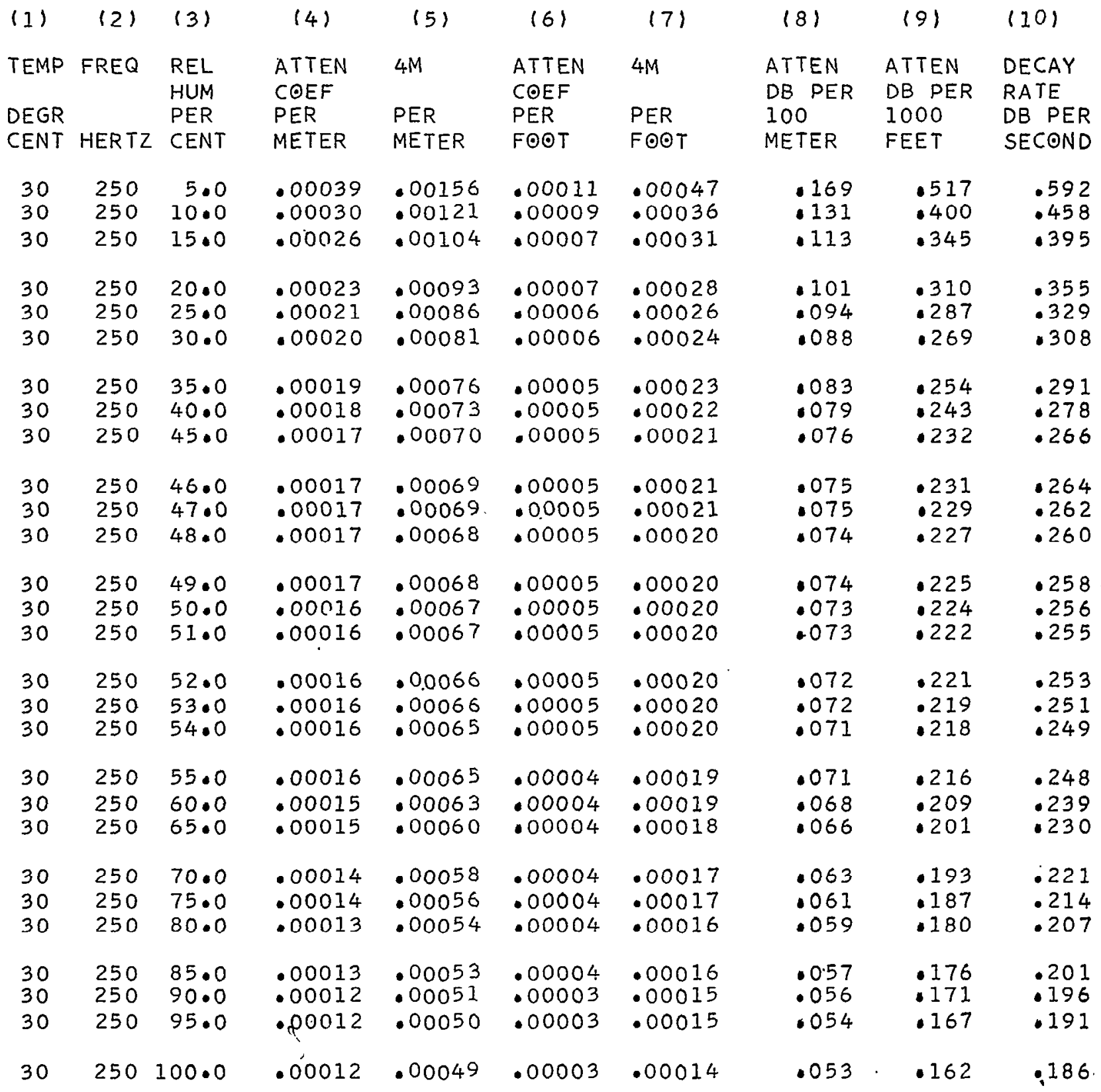


TABULATION OF DATA FOR

ABSORPTION OF SOUND IN AIR VERSUS PERCENT RELATIVE HUMIDITY AT 30 DEGREES CENTIGRADE FOR A FREQUENCY OF 500 HERTZ

\begin{tabular}{|c|c|c|c|c|c|c|c|c|c|}
\hline (1) & (2) & $(3)$ & $141 \%$ &.$(5)$ & (6) & (7) & $(8)$ & (9) & $(10)$ \\
\hline $\begin{array}{l}\text { TEMP } \\
\text { DEGR } \\
\text { CENT }\end{array}$ & HERTZ & $\begin{array}{l}\text { REL } \\
\text { HUM } \\
\text { PER } \\
\text { CENT }\end{array}$ & $\begin{array}{l}\text { ATTEN } \\
\text { COEF } \\
\text { PER } \\
\text { METER }\end{array}$ & $\begin{array}{l}4 M \\
\text { PER } \\
\text { METER }\end{array}$ & $\begin{array}{l}\text { ATTEN } \\
\text { COEF } \\
\text { PER } \\
\text { FO०T }\end{array}$ & $\begin{array}{l}4 M \\
\text { PER } \\
\text { FOOT }\end{array}$ & $\begin{array}{l}\text { ATTEN } \\
\text { DB PER } \\
100 \\
\text { METER }\end{array}$ & $\begin{array}{l}\text { ATTEN } \\
\text { DE PER } \\
\text { I0OO } \\
\text { FEET }\end{array}$ & $\begin{array}{l}\text { DECAY } \\
\text { RATE } \\
\text { DB PER } \\
\text { SECOND }\end{array}$ \\
\hline $\begin{array}{l}30 \\
30 \\
30\end{array}$ & $\begin{array}{l}500 \\
500 \\
500\end{array}$ & $\begin{array}{r}5.0 \\
10.0 \\
15.0\end{array}$ & $\begin{array}{l}.00104 \\
.00069 \\
.00059\end{array}$ & $\begin{array}{r}.00418 \\
.00278 \\
.00238\end{array}$ & $\begin{array}{l}.00031 \\
.00021 \\
.00018\end{array}$ & $\begin{array}{l}.00127 \\
.00084 \\
.00072\end{array}$ & $\begin{array}{l}.454 \\
.302 \\
.259\end{array}$ & $\begin{array}{r}1.386 \\
.920 \\
.790\end{array}$ & $\begin{array}{r}1.587 \\
1.054 \\
.905\end{array}$ \\
\hline $\begin{array}{l}30 \\
30 \\
30\end{array}$ & $\begin{array}{l}500 \\
500 \\
500\end{array}$ & $\begin{array}{l}20.0 \\
25.0 \\
30.0\end{array}$ & $\begin{array}{l}.00053 \\
.00049 \\
.00046\end{array}$ & $\begin{array}{l}.00214 \\
.00197 \\
.00184\end{array}$ & $\begin{array}{l}.00016 \\
.00015 \\
.00014\end{array}$ & $\begin{array}{l}.00065 \\
.00060 \\
.00056\end{array}$ & $\begin{array}{l}.232 \\
.214 \\
.200\end{array}$ & $\begin{array}{l}.708 \\
.654 \\
.610\end{array}$ & $\begin{array}{l}.811 \\
.749 \\
.699\end{array}$ \\
\hline $\begin{array}{l}30 \\
30\end{array}$ & $\begin{array}{l}500 \\
500\end{array}$ & $\begin{array}{l}35.0 \\
40.0\end{array}$ & .00043 & $\begin{array}{l}.00174 \\
.00166\end{array}$ & $\begin{array}{l}.00013 \\
.00012\end{array}$ & $\begin{array}{l}.00053 \\
.00050\end{array}$ & $\begin{array}{r}189 \\
.181\end{array}$ & $\begin{array}{l}.578 \\
.552\end{array}$ & $\begin{array}{l}.662 \\
.632\end{array}$ \\
\hline 30 & 500 & $45 \cdot 0$ & .00039 & .00159 & .00012 & .00048 & .173 & .527 & .604 \\
\hline $\begin{array}{l}30 \\
30\end{array}$ & $\begin{array}{l}500 \\
500\end{array}$ & $\begin{array}{l}46.0 \\
47.0\end{array}$ & $\begin{array}{r}.00039 \\
.00039\end{array}$ & $\begin{array}{r}.00158 \\
.00156\end{array}$ & .00012 & .00048 & .171 & $\begin{array}{r}.523 \\
.519 \\
\end{array}$ & $\begin{array}{r}.599 \\
.594\end{array}$ \\
\hline 30 & 500 & 48.0 & .00038 & .00155 & .00011 & .00047 & .169 & .515 & .590 \\
\hline $\begin{array}{l}30 \\
30\end{array}$ & $\begin{array}{l}500 \\
500\end{array}$ & $\begin{array}{l}49 \cdot 0 \\
50.0\end{array}$ & $\begin{array}{l}.00038 \\
.00038\end{array}$ & $\begin{array}{l}.00154 \\
.00153\end{array}$ & $\begin{array}{l}.00011 \\
.00011\end{array}$ & $\begin{array}{l}.00047 \\
.00046\end{array}$ & $\begin{array}{l}.167 \\
.166\end{array}$ & $\begin{array}{l}.511 \\
.507\end{array}$ & $\begin{array}{l}.585 \\
.580\end{array}$ \\
\hline $30^{\circ}$ & 500 & 51.0 & .00038 & .00152 & .00011 & .00046 & .165 & .503 & .576 \\
\hline $\begin{array}{l}30 \\
30 \\
30\end{array}$ & $\begin{array}{l}500 \\
500 \\
500\end{array}$ & $\begin{array}{l}52.0 \\
53.0 \\
54.0\end{array}$ & $\begin{array}{l}.00037 \\
.00037 \\
.00037\end{array}$ & $\begin{array}{r}.00151 \\
.00150 \\
.00149\end{array}$ & $\begin{array}{l}.00011 \\
.00011 \\
.00011\end{array}$ & $\begin{array}{r}.00046 \\
.00045 \\
.00045\end{array}$ & $\begin{array}{l}.163 \\
.162 \\
.161\end{array}$ & $\begin{array}{l}.499 \\
.496 \\
.493\end{array}$ & $\begin{array}{l}.572 \\
.568 \\
.564\end{array}$ \\
\hline $\begin{array}{l}30 \\
30 \\
30\end{array}$ & $\begin{array}{l}500 \\
500 \\
500\end{array}$ & $\begin{array}{l}55.0 \\
60.0 \\
65.0\end{array}$ & $\begin{array}{l}.00037 \\
.00035 \\
.00034\end{array}$ & $\begin{array}{l}.00148 \\
.00143 \\
.00139\end{array}$ & $\begin{array}{l}.00011 \\
.00010 \\
.00010\end{array}$ & $\begin{array}{l}.00045 \\
.00043 \\
.00042\end{array}$ & $\begin{array}{l}.160 \\
.156 \\
.151\end{array}$ & $\begin{array}{l}.490 \\
.476 \\
.461\end{array}$ & $\begin{array}{l}.561 \\
.545 \\
.528\end{array}$ \\
\hline $\begin{array}{l}30 \\
30 \\
30\end{array}$ & $\begin{array}{l}500 \\
500 \\
500\end{array}$ & $\begin{array}{l}70.0 \\
75.0 \\
80.0\end{array}$ & $\begin{array}{l}.00033 \\
.00033 \\
.00032\end{array}$ & $\begin{array}{r}.00135 \\
.00132 \\
.00129\end{array}$ & $\begin{array}{l}.00010 \\
.00010 \\
.00009\end{array}$ & $\begin{array}{r}.00041 \\
.00040 \\
.00039\end{array}$ & $\begin{array}{l}.147 \\
.143 \\
.140\end{array}$ & $\begin{array}{l}.449 \\
.438 \\
.427\end{array}$ & $\begin{array}{l}.514 \\
.501 \\
.490\end{array}$ \\
\hline $\begin{array}{l}30 \\
30 \\
30\end{array}$ & $\begin{array}{l}500 \\
500 \\
500\end{array}$ & $\begin{array}{l}85.0 \\
90.0 \\
95.0\end{array}$ & $\begin{array}{l}.00031 \\
.00030 \\
.00030\end{array}$ & $\begin{array}{r}.00126 \\
.00123 \\
.00120\end{array}$ & $\begin{array}{l}.00009 \\
.00009 \\
.00009\end{array}$ & $\begin{array}{l}.00038 \\
.00037 \\
.00036\end{array}$ & $\begin{array}{r}.137 \\
.133 \\
.130\end{array}$ & $\begin{array}{r}.417 \\
.407 \\
.397\end{array}$ & $\begin{array}{l}.478 \\
.466 \\
.454\end{array}$ \\
\hline 30 & 5001 & $100 \cdot 0$ & .00029 & .00116 & .00008 & .00035 & .127 & .387 & .443 \\
\hline
\end{tabular}


TABULATION OF DATA FOR

ABSORPTION OF SOUND IN AIR VERSUS PERCENT RELATIVE HUMIDITY AT 30 DEGREES CENTIGRADE FOR A FREQUENCY OF 1000 HERTZ

\begin{tabular}{|c|c|c|c|c|c|c|c|c|c|c|}
\hline 1) & 121 & (3) & 141 & (5) & (6) & (7) & (3) & 191 & $(10)$ & 1 \\
\hline TEMP & FREQ & $\begin{array}{l}\text { REL } \\
\text { HUM }\end{array}$ & $\begin{array}{l}\text { ATTEN } \\
\text { COEF }\end{array}$ & $4 M$ & $\begin{array}{l}\text { ATTEN } \\
\text { COEF }\end{array}$ & $4 M$ & $\begin{array}{l}\text { ATTEN } \\
\text { DB PER }\end{array}$ & $\begin{array}{l}\text { ATTEN } \\
\text { DB PER }\end{array}$ & $\begin{array}{l}\text { DECAY } \\
\text { RATE }\end{array}$ & $7 T$ \\
\hline $\begin{array}{l}\text { DEGR } \\
\text { CENT }\end{array}$ & HERTZ & $\begin{array}{l}\text { PER } \\
\text { CENT }\end{array}$ & $\begin{array}{l}\text { PER } \\
\text { METER }\end{array}$ & $\begin{array}{l}\text { PER } \\
\text { METER }\end{array}$ & $\begin{array}{l}\text { PER } \\
\text { FOOT }\end{array}$ & $\begin{array}{l}\text { PER } \\
\text { FO०T }\end{array}$ & $\begin{array}{l}100 \\
\text { METER }\end{array}$ & $\begin{array}{l}1000 \\
\text { FEET }\end{array}$ & $\begin{array}{l}\text { DB PER } \\
\text { SECOND }\end{array}$ & 湆: \\
\hline 30 & 1000 & 5.0 & .00367 & .01471 & .00112 & .00448 & 1.598 & $4 \cdot 871$ & 5.578 & $\varepsilon$ \\
\hline $\begin{array}{l}30 \\
30\end{array}$ & $\begin{array}{l}1000 \\
1000\end{array}$ & $\begin{array}{l}10.0 \\
15.0\end{array}$ & $\begin{array}{r}.00179 \\
.00146\end{array}$ & $\begin{array}{r}.00717 \\
.00585\end{array}$ & $\begin{array}{l}.00054 \\
.00044\end{array}$ & $\begin{array}{r}.00218 \\
.00178\end{array}$ & $\begin{array}{l}.778 \\
.635\end{array}$ & $\begin{array}{l}2.373 \\
1.937\end{array}$ & $\begin{array}{l}2.717 \\
2.218\end{array}$ & if \\
\hline $\begin{array}{l}30 \\
30 \\
30\end{array}$ & $\begin{array}{l}1000 \\
1000 \\
1000\end{array}$ & $\begin{array}{l}20.0 \\
25.0 \\
30.0\end{array}$ & $\begin{array}{l}.00132 \\
.00121 \\
.00112\end{array}$ & $\begin{array}{r}.00528 \\
.00485 \\
.00451\end{array}$ & $\begin{array}{l}.00040 \\
.00036 \\
.00034\end{array}$ & $\begin{array}{l}.00161 \\
.00147 \\
.00137\end{array}$ & $\begin{array}{l}.573 \\
.527 \\
.490\end{array}$ & $\begin{array}{l}1.749 \\
1.606 \\
1.495\end{array}$ & $\begin{array}{l}2.003 \\
1.839 \\
1.712\end{array}$ & $\begin{array}{l}0 \varepsilon \\
0 \varepsilon \\
0 \varepsilon\end{array}$ \\
\hline $\begin{array}{l}30 \\
30 \\
30\end{array}$ & $\begin{array}{l}1000 \\
1000 \\
1000\end{array}$ & $\begin{array}{l}35.0 \\
40.0 \\
45.0\end{array}$ & $\begin{array}{l}.00107 \\
.00102 \\
.00097\end{array}$ & $\begin{array}{r}.00428 \\
.00409 \\
.00391\end{array}$ & $\begin{array}{r}.00032 \\
.00031 \\
.00029\end{array}$ & $\begin{array}{l}.00130 \\
.00124 \\
.00119\end{array}$ & $\begin{array}{r}.465 \\
.444 \\
.425\end{array}$ & $\begin{array}{l}1.418 \\
1.354 \\
1.295\end{array}$ & $\begin{array}{l}1.624 \\
1.551 \\
1.483\end{array}$ & $\begin{array}{l}O E \\
0 E \\
0 \varepsilon\end{array}$ \\
\hline $\begin{array}{l}30 \\
30 \\
30\end{array}$ & $\begin{array}{l}1000 \\
1000 \\
1000\end{array}$ & $\begin{array}{l}46.0 \\
47.0 \\
48.0\end{array}$ & $\begin{array}{l}.00097 \\
.00096 \\
.00095\end{array}$ & $\begin{array}{r}.00388 \\
.00385 \\
.00382\end{array}$ & $\begin{array}{l}.00029 \\
.00029 \\
.00029\end{array}$ & $\begin{array}{l}.00118 \\
.00117 \\
.00116\end{array}$ & $\begin{array}{r}.421 \\
.418 \\
.415\end{array}$ & $\begin{array}{l}1.285 \\
1.276 \\
1.266\end{array}$ & $\begin{array}{l}1.472 \\
1.461 \\
1.450\end{array}$ & ' $\varepsilon$ \\
\hline $\begin{array}{l}30 \\
30 \\
30\end{array}$ & $\begin{array}{l}1000 \\
1000 \\
1000\end{array}$ & $\begin{array}{l}49.0 \\
50.0 \\
51.0\end{array}$ & $\begin{array}{l}.00094 \\
.00094 \\
.00093\end{array}$ & $\begin{array}{r}.00379 \\
.00376 \\
.00374\end{array}$ & $\begin{array}{l}.00028 \\
.00028 \\
.00028\end{array}$ & $\begin{array}{l}.00115 \\
.001114 \\
.00114\end{array}$ & $\begin{array}{r}.412 \\
.409 \\
.406\end{array}$ & $\begin{array}{l}1.256 \\
1.246 \\
1.238\end{array}$ & $\begin{array}{l}1.438 \\
1.427 \\
1.418\end{array}$ & $\begin{array}{l}\dot{\bar{\varepsilon}} \\
\varepsilon\end{array}$ \\
\hline $\begin{array}{l}30 \\
30 \\
30\end{array}$ & $\begin{array}{l}1000 \\
1000 \\
1000\end{array}$ & $\begin{array}{l}52.0 \\
53.0 \\
54.0\end{array}$ & $\begin{array}{l}.00092 \\
.00092 \\
.00091\end{array}$ & $\begin{array}{r}.00371 \\
.00369 \\
.00367\end{array}$ & $\begin{array}{l}.00028 \\
.00028 \\
.00027\end{array}$ & $\begin{array}{l}.00113 \\
.00112 \\
.00111\end{array}$ & $\begin{array}{l}.403 \\
.401 \\
.398\end{array}$ & $\begin{array}{l}1.231 \\
1.223 \\
1.215\end{array}$ & $\begin{array}{l}1.409 \\
1.400 \\
1.391\end{array}$ & 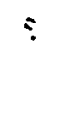 \\
\hline $\begin{array}{l}30 \\
30\end{array}$ & $\begin{array}{l}1000 \\
1000\end{array}$ & $\begin{array}{l}55.0 \\
60.0\end{array}$ & $\begin{array}{l}.00091 \\
.00088\end{array}$ & $\begin{array}{r}.00364 \\
.00353\end{array}$ & $\begin{array}{l}.00027 \\
.00026\end{array}$ & .00111 & $\begin{array}{r}.396 \\
.383\end{array}$ & $\begin{array}{l}1.207 \\
1.168\end{array}$ & $\begin{array}{l}1.382 \\
1.337\end{array}$ & \\
\hline 30 & 1000 & 65.0 & .00086 & .00344 & .00026 & .00104 & .373 & $1 \cdot 138$ & 1.304 & \\
\hline 30 & 1000 & 70.0 & .00083 & .00335 & .00025 & .00102 & .364 & 1.109 & 1.270 & \\
\hline $\begin{array}{l}30 \\
30\end{array}$ & $\begin{array}{l}1000 \\
1000\end{array}$ & $\begin{array}{l}75.0 \\
80.0\end{array}$ & $\begin{array}{l}.00081 \\
.00079\end{array}$ & $\begin{array}{l}.00326 \\
.00319\end{array}$ & $\begin{array}{l}.00024 \\
.00024\end{array}$ & $\begin{array}{r}.00099 \\
.00097\end{array}$ & $\begin{array}{l}.354 \\
.346\end{array}$ & $\begin{array}{l}1.080 \\
1.055\end{array}$ & $\begin{array}{l}1.236 \\
1.208\end{array}$ & \\
\hline $\begin{array}{l}30 \\
30 \\
30\end{array}$ & $\begin{array}{l}1000 \\
1000 \\
1000\end{array}$ & $\begin{array}{l}85.0 \\
90.0 \\
95.0\end{array}$ & $\begin{array}{l}.00077 \\
.00076 \\
.00075\end{array}$ & $\begin{array}{l}.00311 \\
.00305 \\
.00300\end{array}$ & $\begin{array}{l}.00023 \\
.00023 \\
.00022\end{array}$ & $\begin{array}{r}.00094 \\
.00093 \\
.00091\end{array}$ & $\begin{array}{r}.338 \\
.331 \\
.326\end{array}$ & $\begin{array}{r}1.031 \\
1.011 \\
.994\end{array}$ & $\begin{array}{l}1.180 \\
1.158 \\
1.138\end{array}$ & \\
\hline 30 & 1000 & 0.0 & .00073 & .00295 & .00022 & .00090 & .320 & .977 & 1.119 & \\
\hline
\end{tabular}


TABULATION OF DATA FOR

ABSORPTION OF SOUND IN AIR VERSUS PERCENT RELATIVE HUMIDITY AT 30 DEGREES CENTIGRADE FOR A FREQUENCY OF 2000 HERTZ

\begin{tabular}{|c|c|c|c|c|c|c|c|c|c|}
\hline$(1)$ & (2) & (3) & $(4)$ & (5) & $(6)$ & $(7)$ & 181 & $(9)$ & $(10)$ \\
\hline 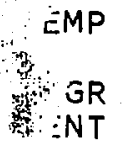 & HERTZ & $\begin{array}{l}\text { REL } \\
\text { HUM } \\
\text { PER } \\
\text { CENT }\end{array}$ & $\begin{array}{l}\text { ATTEN } \\
\text { COEF } \\
\text { PER } \\
\text { METER }\end{array}$ & $\begin{array}{l}4 M \\
\text { PER } \\
\text { METER }\end{array}$ & $\begin{array}{l}\text { ATTEN } \\
\text { COEF } \\
\text { PER ! } \\
\text { FOOT }\end{array}$ & $\begin{array}{l}4 M \\
\text { PER } \\
\text { FOOT }\end{array}$ & $\begin{array}{l}\text { ATTEN } \\
\text { DB PER } \\
100 \\
\text { METER }\end{array}$ & $\begin{array}{l}\text { ATTEN } \\
\text { DB PER } \\
1000 \\
\text { FEET }\end{array}$ & $\begin{array}{l}\text { DECAY } \\
\text { RATE } \\
\text { DB PER } \\
\text { SECOND }\end{array}$ \\
\hline 0 & 2000 & 5.0 & .01227 & .04909 & .00374 & .01496 & 5.330 & $16 \cdot 247$ & 18.605 \\
\hline & $\begin{array}{l}2000 \\
2000\end{array}$ & $\begin{array}{l}10.0 \\
15.0\end{array}$ & $\begin{array}{r}.00580 \\
.00387\end{array}$ & $\begin{array}{r}.02322 \\
.01549\end{array}$ & $\begin{array}{r}.00176 \\
.00118\end{array}$ & $\begin{array}{l}.00707 \\
.00472\end{array}$ & $\begin{array}{l}2.521 \\
1.682\end{array}$ & $\begin{array}{l}7.685 \\
5.127\end{array}$ & $\begin{array}{l}8.801 \\
5.872\end{array}$ \\
\hline$\therefore$ & $\begin{array}{l}2000 \\
2000 \\
2000\end{array}$ & $\begin{array}{l}20.0 \\
25.0 \\
30.0\end{array}$ & $\begin{array}{r}.00321 \\
.00296 \\
.00278\end{array}$ & $\begin{array}{r}.01284 \\
.01184 \\
.01113\end{array}$ & $\begin{array}{l}.00097 \\
.00090 \\
.00084\end{array}$ & $\begin{array}{l}.00391 \\
.00360 \\
.00339\end{array}$ & $\begin{array}{l}1.394 \\
1.285 \\
1.208\end{array}$ & $\begin{array}{l}4.250 \\
3.919 \\
3.684\end{array}$ & $\begin{array}{l}4.867 \\
4.488 \\
4.218\end{array}$ \\
\hline 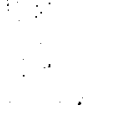 & $\begin{array}{l}2000 \\
2000 \\
2000\end{array}$ & $\begin{array}{l}35.0 \\
40.0 \\
45.0\end{array}$ & $\begin{array}{l}.00263 \\
.00250 \\
.00238\end{array}$ & $\begin{array}{l}.01054 \\
.01002 \\
.00955\end{array}$ & $\begin{array}{l}.00080 \\
.00076 \\
.00072\end{array}$ & $\begin{array}{l}.00321 \\
.00305 \\
.00291\end{array}$ & $\begin{array}{l}1.144 \\
1.088 \\
1.037\end{array}$ & $\begin{array}{l}3.488 \\
3.316 \\
3.163\end{array}$ & $\begin{array}{l}3.994 \\
3.798 \\
3.622\end{array}$ \\
\hline $\begin{array}{l}0 \\
30 \\
30\end{array}$ & $\begin{array}{l}2000 \\
2000 \\
2000\end{array}$ & $\begin{array}{l}46.0 \\
47.0 \\
48.0\end{array}$ & $\begin{array}{l}.00236 \\
.00235 \\
.00233\end{array}$ & $\begin{array}{l}.00947 \\
.00941 \\
.00935\end{array}$ & $\begin{array}{r}.00072 \\
.00071 \\
.00071\end{array}$ & $\begin{array}{l}.00288 \\
.00286 \\
.00285\end{array}$ & $\begin{array}{l}1.029 \\
1.022 \\
1.015\end{array}$ & $\begin{array}{l}3.136 \\
3.115 \\
3.094\end{array}$ & $\begin{array}{l}3.592 \\
3.568 \\
3.544\end{array}$ \\
\hline $\begin{array}{l}30 \\
0 \\
0\end{array}$ & $\begin{array}{l}2000 \\
2000 \\
2000\end{array}$ & $\begin{array}{l}49.0 \\
50.0 \\
51.0\end{array}$ & $\begin{array}{l}.00232 \\
.00230 \\
.00229\end{array}$ & $\begin{array}{l}.00928 \\
.00922 \\
.00916\end{array}$ & $\begin{array}{l}.00070 \\
.00070 \\
.00069\end{array}$ & $\begin{array}{l}.00283 \\
.00281 \\
.00279\end{array}$ & $\begin{array}{r}1.008 \\
1.001 \\
.994\end{array}$ & $\begin{array}{l}3.073 \\
3.052 \\
3.031\end{array}$ & $\begin{array}{l}3.520 \\
3.496 \\
3.472\end{array}$ \\
\hline $\begin{array}{l}30 \\
30\end{array}$ & $\begin{array}{l}2000 \\
2000\end{array}$ & $\begin{array}{l}52.0 \\
53.0\end{array}$ & $\begin{array}{l}.00227 \\
.00226\end{array}$ & $\begin{array}{l}.00910 \\
.00904\end{array}$ & $\begin{array}{l}.00069 \\
.00068\end{array}$ & $\begin{array}{l}.00277 \\
.00275\end{array}$ & $\begin{array}{r}.988 \\
.982\end{array}$ & & $\begin{array}{l}3.450 \\
3.429\end{array}$ \\
\hline 30 & 2000 & 54.0 & .00224 & .00899 & .00068 & .00274 & .976 & 2.976 & 3.408 \\
\hline $\begin{array}{l}30 \\
30 \\
30\end{array}$ & $\begin{array}{l}2000 \\
2000 \\
2000\end{array}$ & $\begin{array}{l}55.0 \\
60.0 \\
65.0\end{array}$ & $\begin{array}{l}.00223 \\
.00217 \\
.00211\end{array}$ & $\begin{array}{r}.00893 \\
.00868 \\
.00845\end{array}$ & $\begin{array}{l}.00068 \\
.00066 \\
.00064\end{array}$ & $\begin{array}{l}.00272 \\
.00264 \\
.00257\end{array}$ & $\begin{array}{r}.970 \\
.942 \\
.917\end{array}$ & $\begin{array}{l}2.957 \\
2.873 \\
2.797\end{array}$ & $\begin{array}{l}3.387 \\
3.29 \mathrm{C} \\
3.203\end{array}$ \\
\hline $\begin{array}{l}30 \\
30 \\
30\end{array}$ & $\begin{array}{l}2000 \\
2000 \\
2000\end{array}$ & $\begin{array}{l}70.0 \\
75.0 \\
80.0\end{array}$ & $\begin{array}{l}.00205 \\
.00200 \\
.00196\end{array}$ & $\begin{array}{r}.00823 \\
.00803 \\
.00784\end{array}$ & $\begin{array}{l}.00062 \\
.00061 \\
.00059\end{array}$ & $\begin{array}{l}.00250 \\
.00244 \\
.00239\end{array}$ & $\begin{array}{r}.893 \\
.872 \\
.851\end{array}$ & $\begin{array}{l}2.724 \\
2.657 \\
2.596\end{array}$ & $\begin{array}{l}3.119 \\
3.043 \\
2.973\end{array}$ \\
\hline $\begin{array}{l}30 \\
30 \\
30\end{array}$ & $\begin{array}{l}2000 \\
2000 \\
2000\end{array}$ & $\begin{array}{l}85.0 \\
90.0 \\
95.0\end{array}$ & $\begin{array}{l}.00192 \\
.00188 \\
.00185\end{array}$ & $\begin{array}{l}.00769 \\
.00755 \\
.00740\end{array}$ & $\begin{array}{l}.00058 \\
.00057 \\
.00056\end{array}$ & $\begin{array}{l}.00234 \\
.00230 \\
.00225\end{array}$ & $\begin{array}{r}.835 \\
.819 \\
.803\end{array}$ & $\begin{array}{l}2.547 \\
2 \cdot 498 \\
2.449\end{array}$ & $\begin{array}{l}2.917 \\
2.861 \\
2.805\end{array}$ \\
\hline 30 & 2000 & $100 \cdot 0$ & .00182 & .00728 & .00055 & .00222 & .790 & 2.410 & 2.760 \\
\hline
\end{tabular}


TABULATION OF DATA FOR

ABSORPTION OF SOUND IN AIR VERSUS PERCENT RELATIVE HUMIDITY AT 30 DEGREES CENTIGRADE FOR A FREQUENCY OF 2500 HERTZ

\begin{tabular}{|c|c|c|c|c|c|c|c|c|c|}
\hline 111 & (2) & (3) & (4) & (5) & $(6)$ & $(7)$ & 181 & $(9)$ & $(10)$ \\
\hline $\begin{array}{l}\text { EMP } \\
\text { EGR } \\
\text { ENT }\end{array}$ & $\begin{array}{l}\text { FREQ } \\
\text { HERTZ }\end{array}$ & $\begin{array}{l}\text { REL } \\
\text { HUM } \\
\text { PER } \\
\text { CENT }\end{array}$ & $\begin{array}{l}\text { ATTEN } \\
\text { COEF } \\
\text { PER } \\
\text { METER }\end{array}$ & $\begin{array}{l}4 M \\
\text { PER } \\
\text { METER }\end{array}$ & $\begin{array}{l}\text { ATTEN } \\
\text { COEF } \\
\text { PER } \\
\text { FOOT }\end{array}$ & $\begin{array}{l}4 M \\
\text { PER } \\
\text { FOOT }\end{array}$ & $\begin{array}{l}\text { ATTEN } \\
\text { DB PER } \\
100 \\
\text { METER }\end{array}$ & $\begin{array}{l}\text { ATTEN } \\
\text { DB PER } \\
1000 \\
\text { FEET }\end{array}$ & $\begin{array}{l}\text { DECAY } \\
\text { RATE } \\
\text { DB PER } \\
\text { SECOND }\end{array}$ \\
\hline $\begin{array}{l}30 \\
30 \\
30\end{array}$ & $\begin{array}{l}2500 \\
2500 \\
2500\end{array}$ & $\begin{array}{r}5.0 \\
10.0 \\
15.0\end{array}$ & $\begin{array}{r}.01757 \\
.00884 \\
.00572\end{array}$ & $\begin{array}{l}.07029 \\
.03536 \\
.02289\end{array}$ & $\begin{array}{l}.00535 \\
.00269 \\
.00174\end{array}$ & $\begin{array}{l}.02142 \\
.01077 \\
.00697\end{array}$ & $\begin{array}{l}7.631 \\
3.839 \\
2.485\end{array}$ & $\begin{array}{r}23.262 \\
11.702 \\
7.577\end{array}$ & $\begin{array}{r}26.638 \\
13.401 \\
8.676\end{array}$ \\
\hline $\begin{array}{l}30 \\
30 \\
30\end{array}$ & $\begin{array}{l}2500 \\
2500 \\
2500\end{array}$ & $\begin{array}{l}20.0 \\
25.0 \\
30.0\end{array}$ & $\begin{array}{l}.00446 \\
.00398 \\
.00373\end{array}$ & $\begin{array}{r}.01787 \\
.01592 \\
.01495\end{array}$ & $\begin{array}{l}.00136 \\
.00121 \\
.00113\end{array}$ & $\begin{array}{l}.00544 \\
.00485 \\
.00455\end{array}$ & $\begin{array}{l}1.941 \\
1.728 \\
1.623\end{array}$ & $\begin{array}{l}5.916 \\
5.268 \\
4.947\end{array}$ & $\begin{array}{l}6.775 \\
6.033 \\
5.665\end{array}$ \\
\hline $\begin{array}{l}30 \\
30\end{array}$ & $\begin{array}{l}2500 \\
2500\end{array}$ & $\begin{array}{l}35.0 \\
40.0\end{array}$ & $\begin{array}{l}.00354 \\
.00338\end{array}$ & $\begin{array}{r}.01419 \\
.01354\end{array}$ & $\begin{array}{l}.00108 \\
.00103\end{array}$ & $\begin{array}{l}.00432 \\
.00412\end{array}$ & $\begin{array}{l}1.540 \\
1.470\end{array}$ & $\begin{array}{l}4.696 \\
4.482\end{array}$ & $\begin{array}{l}5.378 \\
5.133\end{array}$ \\
\hline 30 & 2500 & $45 \cdot 0$ & .00324 & .01298 & .00098 & .00395 & 1.410 & $4 \cdot 298$ & 4.922 \\
\hline $\begin{array}{l}30 \\
30\end{array}$ & $\begin{array}{l}2500 \\
2500\end{array}$ & $\begin{array}{l}46.0 \\
47.0\end{array}$ & $\begin{array}{l}.00321 \\
.00319\end{array}$ & $\begin{array}{r}.01287 \\
.01277\end{array}$ & $\begin{array}{l}.00098 \\
.00097\end{array}$ & $\begin{array}{l}.00392 \\
.00389\end{array}$ & $\begin{array}{l}1.398 \\
1.386\end{array}$ & $\begin{array}{l}4 \cdot 261 \\
4 \cdot 226\end{array}$ & $\begin{array}{l}4.880 \\
4.840\end{array}$ \\
\hline 30 & 2500 & 48.0 & .00316 & .01267 & .00096 & .00386 & $1 \cdot 376$ & $4 \cdot 194$ & 4.803 \\
\hline $\begin{array}{l}30 \\
30\end{array}$ & $\begin{array}{l}2500 \\
2500\end{array}$ & $\begin{array}{l}49.0 \\
50.0\end{array}$ & $\begin{array}{l}.00314 \\
.00312\end{array}$ & $\begin{array}{r}.01257 \\
.01248\end{array}$ & $\begin{array}{l}.00095 \\
.00095\end{array}$ & $\begin{array}{l}.00383 \\
.00380\end{array}$ & $\begin{array}{l}1.365 \\
1.355\end{array}$ & $\begin{array}{l}4.162 \\
4.130\end{array}$ & $\begin{array}{l}4 \cdot 766 \\
4 \cdot 729\end{array}$ \\
\hline 30 & 2500 & 51.0 & .00309 & .01238 & .00094 & .00377 & 1.344 & 4.097 & 4.692 \\
\hline $\begin{array}{l}30 \\
30 \\
30\end{array}$ & $\begin{array}{l}2500 \\
2500 \\
.2500\end{array}$ & $\begin{array}{l}52.0 \\
53.0 \\
54.0\end{array}$ & $\begin{array}{l}.00307 \\
.00304 \\
.00302\end{array}$ & $\begin{array}{r}.01228 \\
.01218 \\
.01209\end{array}$ & $\begin{array}{r}.00093 \\
.00092 \\
.00092\end{array}$ & $\begin{array}{l}.00374 \\
.00371 \\
.00368\end{array}$ & $\begin{array}{l}1.333 \\
1.323 \\
1.312\end{array}$ & $\begin{array}{l}4.065 \\
4.033 \\
4.001\end{array}$ & $\begin{array}{l}4.655 \\
4.619 \\
4.582\end{array}$ \\
\hline $\begin{array}{l}30 \\
30 \\
30\end{array}$ & $\begin{array}{l}2500 \\
2500 \\
2500\end{array}$ & $\begin{array}{l}55.0 \\
60.0 \\
65.0\end{array}$ & $\begin{array}{r}.00300 \\
.00292 \\
.00284\end{array}$ & $\begin{array}{r}.01201 \\
.01168 \\
.01138\end{array}$ & $\begin{array}{l}.00091 \\
.00089 \\
.00086\end{array}$ & $\begin{array}{l}.00366 \\
.00356 \\
.00347\end{array}$ & $\begin{array}{l}1.304 \\
1.268 \\
1.236\end{array}$ & $\begin{array}{l}3.977 \\
3.867 \\
3.768\end{array}$ & $\begin{array}{l}4.555 \\
4.428 \\
4.315\end{array}$ \\
\hline $\begin{array}{l}30 \\
30 \\
30\end{array}$ & $\begin{array}{l}2500 \\
2500 \\
2500\end{array}$ & $\begin{array}{l}70.0 \\
75.0 \\
80.0\end{array}$ & $\begin{array}{l}.00277 \\
.00271 \\
.00265\end{array}$ & $\begin{array}{r}.01111 \\
.01086 \\
.01063\end{array}$ & $\begin{array}{l}.00084 \\
.00082 \\
.00081\end{array}$ & $\begin{array}{l}.00338 \\
.00331 \\
.00324\end{array}$ & $\begin{array}{l}1.206 \\
1.179 \\
1.154\end{array}$ & $\begin{array}{l}3.676 \\
3.596 \\
3.518\end{array}$ & $\begin{array}{l}4.210 \\
4.118 \\
4.028\end{array}$ \\
\hline $\begin{array}{l}30 \\
30 \\
30\end{array}$ & $\begin{array}{l}2500 \\
2500 \\
2500\end{array}$ & $\begin{array}{l}85.0 \\
90.0 \\
95.0\end{array}$ & $\begin{array}{l}.00259 \\
.00254 \\
.00250\end{array}$ & $\begin{array}{r}.01039 \\
.01019 \\
.01000\end{array}$ & $\begin{array}{l}.00079 \\
.00077 \\
.00076\end{array}$ & $\begin{array}{l}.00316 \\
.00310 \\
.00304\end{array}$ & $\begin{array}{l}1.128 \\
1.107 \\
1.086\end{array}$ & $\begin{array}{l}3.440 \\
3.375 \\
3.310\end{array}$ & $\begin{array}{l}3.940 \\
3.865 \\
3.791\end{array}$ \\
\hline 30 & 2500 & $100 \cdot 0$ & .00246 & .00984 & .00075 & .00300 & 1.069 & $3 \cdot 259$ & 3.732 \\
\hline
\end{tabular}


TABULATION OF DATA FOR

ABSORPTION OF SOUND IN AIR VERSUS PERCENT RELATIVE HUMIDITY AT 30 DEGREES CENTIGRADE FOR A FREQUENCY OF 3200 HERTZ

\begin{tabular}{|c|c|c|c|c|c|c|c|c|c|}
\hline$(1)$ & (2) & $(3)$ & (4) & (5) & $(6)$ & $(7)$ & $(8)$ & $|9|$ & $(10)$ \\
\hline $\begin{array}{l}\text { TEMP } \\
\text { DEGR } \\
\text { CENT }\end{array}$ & HERTZ & $\begin{array}{l}\text { REL } \\
\text { HUM } \\
\text { PER } \\
\text { CENT }\end{array}$ & $\begin{array}{l}\text { ATTEN } \\
\text { COEF } \\
\text { PER } \\
\text { METER }\end{array}$ & $\begin{array}{l}4 M \\
\text { PER } \\
\text { METER }\end{array}$ & $\begin{array}{l}\text { ATTEN } \\
\text { COEF } \\
\text { PER } \\
\text { FOOT }\end{array}$ & $\begin{array}{l}4 M \\
\text { PER } \\
\text { FOOT }\end{array}$ & $\begin{array}{l}\text { ATTEN } \\
\text { DB. PER } \\
100 \\
\text { METER }\end{array}$ & $\begin{array}{l}\text { ATTEN } \\
\text { DB PER } \\
1000 \\
\text { FEET }\end{array}$ & $\begin{array}{l}\text { DECAY } \\
\text { RATE } \\
\text { DB PER } \\
\text { SECOND }\end{array}$ \\
\hline $\begin{array}{l}30 \\
30 \\
30\end{array}$ & $\begin{array}{l}3200 \\
3200 \\
3200\end{array}$ & $\begin{array}{r}5.0 \\
10.0 \\
15.0\end{array}$ & $\begin{array}{r}.02379 \\
.01331 \\
.00864\end{array}$ & $\begin{array}{l}.09519 \\
.05327 \\
.03456\end{array}$ & $\begin{array}{l}.00725 \\
.00405 \\
.00263\end{array}$ & $\begin{array}{l}.02901 \\
.01623 \\
.01053\end{array}$ & $\begin{array}{r}10.335 \\
5.783 \\
3.753\end{array}$ & $\begin{array}{l}31.504 \\
17.629 \\
11.439\end{array}$ & $\begin{array}{l}36.077 \\
20.188 \\
13.100\end{array}$ \\
\hline $\begin{array}{l}30 \\
30 \\
30\end{array}$ & $\begin{array}{l}3200 \\
3200 \\
3200\end{array}$ & $\begin{array}{l}20.0 \\
25.0 \\
30.0\end{array}$ & $\begin{array}{l}.00652 \\
.00556 \\
.00511\end{array}$ & $\begin{array}{r}.02610 \\
.02227 \\
.02047\end{array}$ & $\begin{array}{l}.00198 \\
.00169 \\
.00156\end{array}$ & $\begin{array}{l}.00795 \\
.00679 \\
.00624\end{array}$ & $\begin{array}{l}2.834 \\
2.418 \\
2.223\end{array}$ & $\begin{array}{l}8.638 \\
7.373 \\
6.776\end{array}$ & $\begin{array}{l}9.892 \\
8.443 \\
7.759\end{array}$ \\
\hline $\begin{array}{l}30 \\
30 \\
30\end{array}$ & $\begin{array}{l}3200 \\
3200 \\
3200\end{array}$ & $\begin{array}{l}35.0 \\
40.0 \\
45.0\end{array}$ & $\begin{array}{l}.00485 \\
.00464 \\
.00446\end{array}$ & $\begin{array}{r}.01943 \\
.01858 \\
.01787\end{array}$ & $\begin{array}{l}.00148 \\
.00141 \\
.00136\end{array}$ & $\begin{array}{l}.00592 \\
.00566 \\
.00544\end{array}$ & $\begin{array}{l}2.110 \\
2.018 \\
1.940\end{array}$ & & $\begin{array}{l}7.366 \\
7.044 \\
6.772\end{array}$ \\
\hline 30 & 3200 & 46.0 & .00443 & .01772 & .00135 & .00540 & 1.924 & 5.866 & 6.718 \\
\hline $\begin{array}{l}30 \\
30\end{array}$ & $\begin{array}{l}3200 \\
3200\end{array}$ & $\begin{array}{l}47.0 \\
48.0\end{array}$ & $\begin{array}{r}.00439 \\
.00436\end{array}$ & $\begin{array}{r}.01759 \\
.01747\end{array}$ & $\begin{array}{r}.00134 \\
.00133\end{array}$ & $\begin{array}{l}.00536 \\
.00532\end{array}$ & $\begin{array}{l}1.910 \\
1.897\end{array}$ & & $\begin{array}{l}6.670 \\
6.623\end{array}$ \\
\hline $\begin{array}{l}30 \\
30 \\
30\end{array}$ & $\begin{array}{l}3200 \\
3200 \\
3200\end{array}$ & $\begin{array}{l}49.0 \\
50.0 \\
51.0\end{array}$ & $\begin{array}{l}.00433 \\
.00430 \\
.00427\end{array}$ & $\begin{array}{l}.01735 \\
.01723 \\
.01710\end{array}$ & $\begin{array}{l}.00132 \\
.00131 \\
.00130\end{array}$ & $\begin{array}{l}.00528 \\
.00525 \\
.00521\end{array}$ & $\begin{array}{l}1.884 \\
1.870 \\
1.857\end{array}$ & $\begin{array}{l}5.743 \\
5.702 \\
5.661\end{array}$ & $\begin{array}{l}6.576 \\
6.530 \\
6.483\end{array}$ \\
\hline $\begin{array}{l}30 \\
30 \\
30\end{array}$ & $\begin{array}{l}3200 \\
3200 \\
3200\end{array}$ & $\begin{array}{l}52.0 \\
53.0 \\
54.0\end{array}$ & $\begin{array}{r}.00424 \\
.00421 \\
.00418\end{array}$ & $\begin{array}{r}.01698 \\
.01686 \\
.01674\end{array}$ & $\begin{array}{l}.00129 \\
.00128 \\
.00127\end{array}$ & $\begin{array}{l}.00517 \\
.00513 \\
.00510\end{array}$ & $\begin{array}{l}1.844 \\
1.830 \\
1.817\end{array}$ & $\begin{array}{l}5.621 \\
5.580 \\
5.540\end{array}$ & $\begin{array}{l}6.436 \\
6.390 \\
6.344\end{array}$ \\
\hline $\begin{array}{l}30 \\
30 \\
30\end{array}$ & $\begin{array}{l}3200 \\
3200 \\
3200\end{array}$ & $\begin{array}{l}55.0 \\
60.0 \\
65.0\end{array}$ & $\begin{array}{l}.00415 \\
.00402 \\
.00390\end{array}$ & $\begin{array}{l}.01663 \\
.01609 \\
.01563\end{array}$ & $\begin{array}{l}.00126 \\
.00122 \\
.001119\end{array}$ & $\begin{array}{l}.00506 \\
.00490 \\
.00476\end{array}$ & $\begin{array}{l}i .805 \\
1.747 \\
1.697\end{array}$ & $\begin{array}{l}5.504 \\
5.326 \\
5.174\end{array}$ & $\begin{array}{l}6.303 \\
6.099 \\
5.925\end{array}$ \\
\hline $\begin{array}{l}30 \\
30 \\
30\end{array}$ & $\begin{array}{l}3200 \\
3200 \\
3200\end{array}$ & $\begin{array}{l}70.0 \\
75.0 \\
80.0\end{array}$ & $\begin{array}{l}.00381 \\
.00373 \\
.00365\end{array}$ & $\begin{array}{r}.01526 \\
.01493 \\
.01462\end{array}$ & $\begin{array}{l}.00116 \\
.00113 \\
.00111\end{array}$ & $\begin{array}{l}.00465 \\
.00455 \\
.00445\end{array}$ & $\begin{array}{l}1.657 \\
1.621 \\
1.587\end{array}$ & $\begin{array}{l}5.051 \\
4.943 \\
4.839\end{array}$ & $\begin{array}{l}5.785 \\
5.661 \\
5.542\end{array}$ \\
\hline $\begin{array}{l}30 \\
30 \\
30\end{array}$ & $\begin{array}{l}3200 \\
3200 \\
3200\end{array}$ & $\begin{array}{l}85.0 \\
90.0 \\
95.0\end{array}$ & $\begin{array}{l}.00358 \\
.00352 \\
.00345\end{array}$ & $\begin{array}{r}.01435 \\
.01409 \\
.01383\end{array}$ & $\begin{array}{l}.00109 \\
.00107 \\
.00105\end{array}$ & $\begin{array}{l}.00437 \\
.00429 \\
.00421\end{array}$ & $\begin{array}{l}1.558 \\
1.529 \\
1.501\end{array}$ & $\begin{array}{l}4.750 \\
4.663 \\
4.577\end{array}$ & $\begin{array}{l}5.440 \\
5.340 \\
5.242\end{array}$ \\
\hline 30 & 3200 & $100 \cdot 0$ & .00339 & .01358 & .00103 & .00413 & 1.474 & $4 \cdot 494$ & 5.147 \\
\hline
\end{tabular}


TABULATION OF DATA FOR

ABSORPTION OF SOUND IN AIR VERSUS PERCENT RELATIVE HUMIDITY AT 30 DEGREES CENTIGRADE FOR A FREQUENCY OF 4000 HERTZ

\begin{tabular}{|c|c|c|c|c|c|c|c|c|c|}
\hline 1) & $(2)$ & (3) & (4) & (5) & 161 & 171 & (8) & (9) & 1101 \\
\hline EMP & FREQ & $\begin{array}{l}\text { REL } \\
\text { HUM }\end{array}$ & $\begin{array}{l}\text { ATTEN } \\
\text { COEF }\end{array}$ & $4 M$ & $\begin{array}{l}\text { ATTEN } \\
\text { COEF }\end{array}$ & $4 M$ & $\begin{array}{l}\text { ATTEN } \\
\text { DB PER }\end{array}$ & $\begin{array}{l}\text { ATTEN } \\
\text { DB PER }\end{array}$ & $\begin{array}{l}\text { DECAY } \\
\text { RATE }\end{array}$ \\
\hline $\begin{array}{l}\text { EGR } \\
\text { ENT }\end{array}$ & HERTZ & $\begin{array}{l}\text { PER } \\
\text { CENT }\end{array}$ & $\begin{array}{l}\text { PER } \\
\text { METER }\end{array}$ & $\begin{array}{l}\text { PER } \\
\text { METER }\end{array}$ & 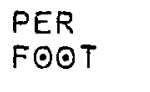 & 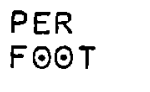 & $\begin{array}{l}100 \\
\text { METER }\end{array}$ & $\begin{array}{l}1000 \\
\text { FEET }\end{array}$ & $\begin{array}{l}\text { DB PER } \\
\text { SECOND }\end{array}$ \\
\hline $\begin{array}{l}30 \\
30 \\
30\end{array}$ & $\begin{array}{l}4000 \\
4000 \\
4000\end{array}$ & $\begin{array}{r}5.0 \\
10.0 \\
15.0\end{array}$ & $\begin{array}{l}.03013 \\
.01990 \\
.01298\end{array}$ & $\begin{array}{r}.12053 \\
.07962 \\
.05192\end{array}$ & $\begin{array}{l}.00918 \\
.00606 \\
.00395\end{array}$ & $\begin{array}{l}.03673 \\
.02427 \\
.01582\end{array}$ & $\begin{array}{r}13.086 \\
8.645 \\
5.637\end{array}$ & $\begin{array}{l}39.888 \\
26.352 \\
17.183\end{array}$ & $\begin{array}{l}45.679 \\
30.177 \\
19.678\end{array}$ \\
\hline $\begin{array}{l}30 \\
30 \\
30\end{array}$ & $\begin{array}{l}4000 \\
4000 \\
4000\end{array}$ & $\begin{array}{l}20.0 \\
25.0 \\
30.0\end{array}$ & $\begin{array}{l}.00964 \\
.00786 \\
.00703\end{array}$ & $\begin{array}{r}.03858 \\
.03146 \\
.02814\end{array}$ & $\begin{array}{r}.00294 \\
.00239 \\
.00214\end{array}$ & $\begin{array}{l}.01176 \\
.00959 \\
.00857\end{array}$ & $\begin{array}{l}4.189 \\
3.416 \\
3.055\end{array}$ & $\begin{array}{r}12.770 \\
10.413 \\
9.313\end{array}$ & $\begin{array}{l}14.624 \\
11.925 \\
10.665\end{array}$ \\
\hline $\begin{array}{l}30 \\
30 \\
30\end{array}$ & $\begin{array}{l}4000 \\
4000 \\
4000\end{array}$ & $\begin{array}{l}35.0 \\
40.0 \\
45.0\end{array}$ & $\begin{array}{l}.00655 \\
.00626 \\
.00603\end{array}$ & $\begin{array}{l}.02620 \\
.02506 \\
.02413\end{array}$ & $\begin{array}{l}.00199 \\
.00190 \\
.00183\end{array}$ & $\begin{array}{l}.00798 \\
.00763 \\
.00735\end{array}$ & $\begin{array}{l}2.844 \\
2.720 \\
2.620\end{array}$ & $\begin{array}{l}8.671 \\
8.293 \\
7.987\end{array}$ & $\begin{array}{l}9.930 \\
9.497 \\
9.147\end{array}$ \\
\hline $\begin{array}{l}30 \\
30 \\
30\end{array}$ & $\begin{array}{l}4000 \\
4000 \\
4000\end{array}$ & $\begin{array}{l}46.0 \\
47.0 \\
48.0\end{array}$ & $\begin{array}{l}.00598 \\
.00594 \\
.00590\end{array}$ & $\begin{array}{l}.02395 \\
.02377 \\
.02362\end{array}$ & $\begin{array}{l}.00182 \\
.00181 \\
.00180\end{array}$ & $\begin{array}{l}.00730 \\
.00724 \\
.00720\end{array}$ & $\begin{array}{l}2.600 \\
2.581 \\
2.565\end{array}$ & $\begin{array}{l}7.927 \\
7.868 \\
7.818\end{array}$ & $\begin{array}{l}9.078 \\
9.011 \\
8.953\end{array}$ \\
\hline $\begin{array}{l}30 \\
30 \\
30\end{array}$ & $\begin{array}{l}4000 \\
4000 \\
4000\end{array}$ & $\begin{array}{l}49.0 \\
50.0 \\
51.0\end{array}$ & $\begin{array}{r}.00586 \\
.00583 \\
.00579\end{array}$ & $\begin{array}{r}.02347 \\
.02332 \\
.02317\end{array}$ & $\begin{array}{l}.00178 \\
.00177 \\
.00176\end{array}$ & $\begin{array}{l}.00715 \\
.00710 \\
.00706\end{array}$ & $\begin{array}{l}2.548 \\
2.532 \\
2.515\end{array}$ & $\begin{array}{l}7.768 \\
7.718 \\
7.668\end{array}$ & $\begin{array}{l}8.896 \\
8.838 \\
8.781\end{array}$ \\
\hline $\begin{array}{l}30 \\
30 \\
30\end{array}$ & $\begin{array}{l}4000 \\
4000 \\
4000\end{array}$ & $\begin{array}{l}52.0 \\
53.0 \\
54.0\end{array}$ & $\begin{array}{r}.00575 \\
.00571 \\
.00567\end{array}$ & $\begin{array}{l}.02301 \\
.02286 \\
.02271\end{array}$ & $\begin{array}{l}.00175 \\
.00174 \\
.00173\end{array}$ & $\begin{array}{l}.00701 \\
.00696 \\
.00692\end{array}$ & $\begin{array}{l}2.499 \\
2.482 \\
2.466\end{array}$ & $\begin{array}{l}7.617 \\
7.567 \\
7.517\end{array}$ & $\begin{array}{l}8.723 \\
8.666 \\
8.608\end{array}$ \\
\hline $\begin{array}{l}30 \\
30 \\
30\end{array}$ & $\begin{array}{l}4000 \\
4000 \\
4000\end{array}$ & $\begin{array}{l}55.0 \\
60.0 \\
65.0\end{array}$ & $\begin{array}{l}.00564 \\
.00548 \\
.00532\end{array}$ & $\begin{array}{r}.02257 \\
.02192 \\
.02129\end{array}$ & $\begin{array}{r}.00171 \\
.00167 \\
.00162\end{array}$ & $\begin{array}{l}.00687 \\
.00668 \\
.00648\end{array}$ & $\begin{array}{l}2.450 \\
2.380 \\
2.311\end{array}$ & $\begin{array}{l}7.469 \\
7.254 \\
7.046\end{array}$ & $\begin{array}{l}8.554 \\
8.307 \\
8.069\end{array}$ \\
\hline 30 & 4000 & 70.0 & .00518 & .02072 & .00157 & .00631 & 2 & 58 & 7.853 \\
\hline $\begin{array}{l}30 \\
30\end{array}$ & $\begin{array}{l}4000 \\
4000\end{array}$ & $\begin{array}{l}75.0 \\
80.0\end{array}$ & $\begin{array}{l}.00504 \\
.00494\end{array}$ & $\begin{array}{r}.02018 \\
.01979\end{array}$ & $\begin{array}{l}.00153 \\
.00150\end{array}$ & .006 & $\begin{array}{l}2.191 \\
2.149\end{array}$ & & $\begin{array}{l}7.650 \\
7.502\end{array}$ \\
\hline 30 & 4000 & 85.0 & .00485 & .01942 & .00148 & .00592 & & $6 \cdot 427$ & 7.360 \\
\hline 30 & $\begin{array}{l}4000 \\
4000\end{array}$ & $\begin{array}{l}90.0 \\
95.0\end{array}$ & $\begin{array}{l}.00477 \\
.00468\end{array}$ & $\begin{array}{r}.01908 \\
.01875\end{array}$ & $\begin{array}{l}.00145 \\
.00142\end{array}$ & $\begin{array}{l}.00581 \\
.00571\end{array}$ & $\begin{array}{l}2.071 \\
2.036\end{array}$ & $\begin{array}{l}6.314 \\
6.206\end{array}$ & $\begin{array}{l}7.231 \\
7.107\end{array}$ \\
\hline 30 & 4000 & 100.0 & 00461 & .01847 & .00140 & 00562 & .005 & $6 \cdot 112$ & 7.000 \\
\hline
\end{tabular}


TABULATION OF DATA FOR

ABSORPTION OF SOUND IN AIR VERSUS PERCENT RELATIVE HUMIDITY AT 30 DEGREES CENTIGRADE FOR A FREQUENCY OF. 5000 HERTZ

\begin{tabular}{|c|c|c|c|c|c|c|c|c|c|}
\hline (1) & (2) & $(3)$ & $(4)$ & (5) & $16 !$ & 171 & $(8)$ & $(9)$ & $(10)$ \\
\hline $\begin{array}{l}\text { I EMP } \\
\text { JEGR } \\
\text { IENT }\end{array}$ & HERTZ & $\begin{array}{l}\text { REL } \\
\text { HUM } \\
\text { PER } \\
\text { CENT }\end{array}$ & $\begin{array}{l}\text { ATTEN } \\
\text { COEF } \\
\text { PER } \\
\text { METER }\end{array}$ & $\begin{array}{l}4 M \\
\text { PER } \\
\text { METER }\end{array}$ & $\begin{array}{l}\text { ATTEN } \\
\text { COEF } \\
\text { PER } \\
\text { FOOT }\end{array}$ & $\begin{array}{l}4 M \\
\text { PER } \\
\text { FOOT }\end{array}$ & $\begin{array}{l}\text { ATTEN } \\
\text { DB PER } \\
100 \\
\text { METER }\end{array}$ & $\begin{array}{l}\text { ATTEN } \\
\text { DB PER } \\
1000 \\
\text { FEET }\end{array}$ & $\begin{array}{l}\text { DECAY } \\
\text { RATE } \\
\text { DB PER } \\
\text { SECOND }\end{array}$ \\
\hline $\begin{array}{l}30 \\
30 \\
30\end{array}$ & $\begin{array}{l}5000 \\
5000 \\
5000\end{array}$ & $\begin{array}{r}5.0 \\
10.0 \\
15.0\end{array}$ & $\begin{array}{r}.03700 \\
.02885 \\
.01884\end{array}$ & $\begin{array}{r}.14801 \\
.11541 \\
.07539\end{array}$ & $\begin{array}{l}.01127 \\
.00879 \\
.00574\end{array}$ & $\begin{array}{l}.04511 \\
.03517 \\
.02298\end{array}$ & $\begin{array}{r}16.071 \\
12.531 \\
8.286\end{array}$ & $\begin{array}{l}48.985 \\
38.196 \\
24.952\end{array}$ & $\begin{array}{l}56.096 \\
43.740 \\
28.574\end{array}$ \\
\hline $\begin{array}{l}30 \\
30\end{array}$ & $\begin{array}{l}5000 \\
5000\end{array}$ & $\begin{array}{l}20.0 \\
25.0\end{array}$ & $\begin{array}{l}.01392 \\
.01117\end{array}$ & $\begin{array}{l}.05571 \\
.04471\end{array}$ & $\begin{array}{l}.00424 \\
.00340\end{array}$ & $\begin{array}{r}.01698 \\
01362\end{array}$ & & $\begin{array}{l}18.439 \\
14.797\end{array}$ & \\
\hline 30 & 5000 & 30.0 & .00963 & .03853 & .00293 & .01174 & 4.184 & 12.753 & 14.604 \\
\hline $\begin{array}{l}30 \\
30\end{array}$ & $\begin{array}{l}5000 \\
5000\end{array}$ & $\begin{array}{l}35.0 \\
40.0\end{array}$ & $\begin{array}{l}.00884 \\
.00836\end{array}$ & $\begin{array}{l}.03537 \\
.03345\end{array}$ & $\begin{array}{l}.00269 \\
.00254\end{array}$ & $\begin{array}{r}.01078 \\
.01019\end{array}$ & & $\begin{array}{l}11: 708 \\
11: 071\end{array}$ & $\begin{array}{l}13.407 \\
12.679\end{array}$ \\
\hline 30 & 5000 & $45 \cdot 0$ & .00805 & .03220 & .00245 & .00981 & 3.496 & 10.656 & 12.203 \\
\hline $\begin{array}{l}30 \\
30\end{array}$ & $\begin{array}{l}5000 \\
5000\end{array}$ & $\begin{array}{l}46.0 \\
47.0\end{array}$ & $\begin{array}{l}.00799 \\
.00794\end{array}$ & $\begin{array}{r}.03196 \\
.03176\end{array}$ & $\begin{array}{r}.00243 \\
.00242\end{array}$ & $\begin{array}{r}.00974 \\
.00968\end{array}$ & $\begin{array}{l}3.470 \\
3.448\end{array}$ & $\begin{array}{l}10.578 \\
10.512\end{array}$ & $\begin{array}{l}12.114 \\
12.038\end{array}$ \\
\hline 30 & 5000 & $48 \cdot 0$ & .00789 & .03156 & .00240 & .00962 & $3 \cdot 427$ & $10 \cdot 445$ & 11.961 \\
\hline $\begin{array}{l}30 \\
30\end{array}$ & $\begin{array}{l}5000 \\
5000\end{array}$ & $\begin{array}{l}49.0 \\
50.0\end{array}$ & $\begin{array}{r}.00784 \\
.00779\end{array}$ & $\begin{array}{l}.03136 \\
.03116\end{array}$ & $\begin{array}{l}.00238 \\
.00237\end{array}$ & $\begin{array}{l}.00955 \\
.00949\end{array}$ & & $\begin{array}{l}10 \cdot 379 \\
10 \cdot 312\end{array}$ & $\begin{array}{l}11.885 \\
11.809\end{array}$ \\
\hline 30 & 5000 & 51.0 & .00774 & .03096 & .00235 & .00943 & 3.361 & 10.246 & 11.733 \\
\hline $\begin{array}{l}30 \\
30 \\
30\end{array}$ & $\begin{array}{l}5000 \\
5000 \\
5000\end{array}$ & $\begin{array}{l}52.0 \\
53.0 \\
54.0\end{array}$ & $\begin{array}{l}.00769 \\
.00764 \\
.00759\end{array}$ & $\begin{array}{r}.03076 \\
.03056 \\
.03038\end{array}$ & $\begin{array}{l}.00234 \\
.00232 \\
.00231\end{array}$ & $\begin{array}{l}.00937 \\
.00931 \\
.00926\end{array}$ & & $\begin{array}{l}10.179 \\
10.113 \\
10.057\end{array}$ & $\begin{array}{l}11.657 \\
11.581 \\
11.517\end{array}$ \\
\hline $\begin{array}{l}30 \\
30 \\
30\end{array}$ & $\begin{array}{l}5000 \\
5000 \\
5000\end{array}$ & $\begin{array}{l}55.0 \\
60.0 \\
65.0\end{array}$ & $\begin{array}{l}.00755 \\
.00734 \\
.00715\end{array}$ & $\begin{array}{l}.03022 \\
.02938 \\
.02862\end{array}$ & $\begin{array}{l}.00230 \\
.00223 \\
.00218\end{array}$ & $\begin{array}{l}.00921 \\
.00895 \\
.00872\end{array}$ & $\begin{array}{l}3.281 \\
3.190 \\
3.107\end{array}$ & $\begin{array}{r}10.001 \\
9.725 \\
9.472\end{array}$ & $\begin{array}{l}11.453 \\
11.136 \\
10.847\end{array}$ \\
\hline $\begin{array}{l}30 \\
30 \\
30\end{array}$ & $\begin{array}{l}5000 \\
5000 \\
5000\end{array}$ & $\begin{array}{l}70.0 \\
75.0 \\
80.0\end{array}$ & $\begin{array}{l}.00697 \\
.00680 \\
.00665\end{array}$ & $\begin{array}{l}.02790 \\
.02723 \\
.02661\end{array}$ & $\begin{array}{l}.00212 \\
.00207 \\
.00202\end{array}$ & $\begin{array}{l}.00850 \\
.00830 \\
.00811\end{array}$ & $\begin{array}{l}3.029 \\
2.957 \\
2.889\end{array}$ & $\begin{array}{l}9.235 \\
9.014 \\
8.806\end{array}$ & $\begin{array}{l}10.575 \\
10.322 \\
10.085\end{array}$ \\
\hline $\begin{array}{l}30 \\
30 \\
30\end{array}$ & $\begin{array}{l}5000 \\
5000 \\
5000\end{array}$ & $\begin{array}{l}85.0 \\
90.0 \\
95.0\end{array}$ & $\begin{array}{l}.00650 \\
.00639 \\
.00629\end{array}$ & $\begin{array}{r}.02602 \\
.02559 \\
.02516\end{array}$ & $\begin{array}{l}.00198 \\
.00194 \\
.00191\end{array}$ & $\begin{array}{l}.00793 \\
.00779 \\
.00767\end{array}$ & $\begin{array}{l}2.825 \\
2.778 \\
2.732\end{array}$ & $\begin{array}{l}8.611 \\
8.468 \\
8.328\end{array}$ & $\begin{array}{l}9.861 \\
9.698 \\
9.537\end{array}$ \\
\hline 30 & 5000 & 100.0 & .00619 & .02479 & .00188 & .00755 & 2.691 & 3.204 & 9.395 \\
\hline
\end{tabular}


TABULATION OF DATA FOR

ABSORPTION OF SOUND IN AIR VERSUS PERCENT RELATIVE HUMIDITY AT 30 DEGREES CENTIGRADE FOR A FREQUENCY OF 5940 HERTZ

\begin{tabular}{|c|c|c|c|c|c|c|c|c|c|}
\hline$(1)$ & (2) & $(3)$ & $(4)$ & (5) & $(6)$ & 171 & $(8)$ & 191 & $(10)$ \\
\hline $\begin{array}{l}\text { TEMP } \\
\text { DEGR } \\
\text { CENT }\end{array}$ & HERTZ & $\begin{array}{l}\text { REL } \\
\text { HUM } \\
\text { PER } \\
\text { CENT }\end{array}$ & $\begin{array}{l}\text { ATTEN } \\
\text { COEF } \\
\text { PER } \\
\text { METER }\end{array}$ & $\begin{array}{l}4 M \\
\text { PER } \\
\text { METER }\end{array}$ & $\begin{array}{l}\text { ATTEN } \\
\text { COEF } \\
\text { PER } \\
\text { FOOT }\end{array}$ & $\begin{array}{l}4 M \\
\text { PER } \\
\text { FOOT }\end{array}$ & $\begin{array}{l}\text { ATTEN } \\
\text { DB PER } \\
100 \\
\text { METER }\end{array}$ & $\begin{array}{l}\text { ATTEN } \\
\text { DB PER } \\
1000 \\
\text { FEET }\end{array}$ & $\begin{array}{l}\text { DECAY } \\
\text { RATE } \\
\text { DB PER } \\
\text { SECOND }\end{array}$ \\
\hline $\begin{array}{l}30 \\
30 \\
30\end{array}$ & $\begin{array}{l}5940 \\
5940 \\
5940\end{array}$ & $\begin{array}{r}5.0 \\
10.0 \\
15.0\end{array}$ & $\begin{array}{r}.04168 \\
.03890 \\
.02571\end{array}$ & $\begin{array}{r}.16672 \\
.15561 \\
.10285\end{array}$ & $\begin{array}{r}.01270 \\
.01185 \\
.00783\end{array}$ & $\begin{array}{l}.05081 \\
.04743 \\
.03135\end{array}$ & $\begin{array}{l}18.102 \\
16.895 \\
11.167\end{array}$ & $\begin{array}{l}55.175 \\
51.497 \\
34.039\end{array}$ & $\begin{array}{l}63.185 \\
58.973 \\
38.980\end{array}$ \\
\hline $\begin{array}{l}30 \\
30 \\
30\end{array}$ & $\begin{array}{l}5940 \\
5940 \\
5940\end{array}$ & $\begin{array}{l}20.0 \\
25.0 \\
30.0\end{array}$ & $\begin{array}{r}.01901 \\
.01523 \\
.01289\end{array}$ & $\begin{array}{r}.07607 \\
.06092 \\
.05159\end{array}$ & $\begin{array}{l}.00579 \\
.00464 \\
.00393\end{array}$ & $\begin{array}{r}.02318 \\
.01856 \\
.01572\end{array}$ & $\begin{array}{l}8.260 \\
6.614 \\
5.601\end{array}$ & $\begin{array}{l}25.177 \\
20.160 \\
17.073\end{array}$ & $\begin{array}{l}28.831 \\
23.087 \\
19.551\end{array}$ \\
\hline $\begin{array}{l}30 \\
30 \\
30\end{array}$ & $\begin{array}{l}5940 \\
5940 \\
5940\end{array}$ & $\begin{array}{l}35.0 \\
40.0 \\
45.0\end{array}$ & $\begin{array}{r}.01145 \\
.01068 \\
.01018\end{array}$ & $\begin{array}{l}.04583 \\
.04273 \\
.04073\end{array}$ & $\begin{array}{l}.00349 \\
.00325 \\
.00310\end{array}$ & $\begin{array}{r}.01397 \\
.01302 \\
.01241\end{array}$ & $\begin{array}{r}4.976 \\
4.639 \\
4.422\end{array}$ & $\begin{array}{l}15.169 \\
14.142 \\
13.481\end{array}$ & $\begin{array}{l}17.371 \\
16.195 \\
15.438\end{array}$ \\
\hline $\begin{array}{l}30 \\
30 \\
30\end{array}$ & $\begin{array}{l}5940 \\
5940 . \\
5940\end{array}$ & $\begin{array}{l}46.0 \\
47.0 \\
48.0\end{array}$ & $\begin{array}{r}.01011 \\
.01005 \\
.00998\end{array}$ & $\begin{array}{r}.04046 \\
.04020 \\
.03994\end{array}$ & $\begin{array}{l}.00308 \\
.00306 \\
.00304\end{array}$ & $\begin{array}{r}.01233 \\
.01225 \\
.01217\end{array}$ & $\begin{array}{l}4.393 \\
4.365 \\
4.336\end{array}$ & $\begin{array}{l}13 \cdot 391 \\
13 \cdot 304 \\
13 \cdot 217\end{array}$ & $\begin{array}{l}15.335 \\
15.236 \\
15.136\end{array}$ \\
\hline $\begin{array}{l}30 \\
30 \\
30\end{array}$ & $\begin{array}{l}5940 \\
5940 \\
5940\end{array}$ & $\begin{array}{l}49.0 \\
50.0 \\
51.0\end{array}$ & $\begin{array}{r}.00991 \\
.00985 \\
.00978\end{array}$ & $\begin{array}{l}.03967 \\
.03941 \\
.03915\end{array}$ & $\begin{array}{r}.00302 \\
.00300 \\
.00298\end{array}$ & $\begin{array}{r}.01209 \\
.01201 \\
.01193\end{array}$ & $\begin{array}{l}4.308 \\
4.279 \\
4.251\end{array}$ & $\begin{array}{l}13 \cdot 131 \\
13 \cdot 044 \\
12.957\end{array}$ & $\begin{array}{l}15.037 \\
14.937 \\
14.838\end{array}$ \\
\hline $\begin{array}{l}30 \\
30 \\
30\end{array}$ & $\begin{array}{l}5940 \\
5940 \\
5940\end{array}$ & $\begin{array}{l}52.0 \\
53.0 \\
54.0\end{array}$ & $\begin{array}{r}.00972 \\
.00967 \\
.00961\end{array}$ & $\begin{array}{r}.03889 \\
.03868 \\
.03847\end{array}$ & $\begin{array}{l}.00296 \\
.00294 \\
.00293\end{array}$ & $\begin{array}{l}.01185 \\
.01179 \\
.01172\end{array}$ & $\begin{array}{l}4 \cdot 223 \\
4 \cdot 200 \\
4.177\end{array}$ & $\begin{array}{l}12.871 \\
12.802 \\
12.732\end{array}$ & $\begin{array}{l}14.740 \\
14.660 \\
14.581\end{array}$ \\
\hline $\begin{array}{l}30 \\
30 \\
30\end{array}$ & $\begin{array}{l}5940 \\
5940 \\
5940\end{array}$ & $\begin{array}{l}55.0 \\
60.0 \\
65.0\end{array}$ & $\begin{array}{l}.00956 \\
.00930 \\
.00908\end{array}$ & $\begin{array}{l}.03826 \\
.03721 \\
.03633\end{array}$ & $\begin{array}{l}.00291 \\
.00283 \\
.00276\end{array}$ & $\begin{array}{l}.01166 \\
.01134 \\
.01107\end{array}$ & $\begin{array}{l}4.154 \\
4.040 \\
3.944\end{array}$ & $\begin{array}{l}12.663 \\
12.316 \\
12.023\end{array}$ & $\begin{array}{l}14.501 \\
14.103 \\
13.768\end{array}$ \\
\hline $\begin{array}{l}30 \\
30 \\
30\end{array}$ & $\begin{array}{l}5940 \\
5940 \\
5940\end{array}$ & $\begin{array}{l}70.0 \\
75.0 \\
80.0\end{array}$ & $\begin{array}{l}.00886 \\
.00867 \\
.00848\end{array}$ & $\begin{array}{r}.03545 \\
.03469 \\
.03394\end{array}$ & $\begin{array}{l}.00270 \\
.00264 \\
.00258\end{array}$ & $\begin{array}{r}.01080 \\
.01057 \\
.01034\end{array}$ & $\begin{array}{l}3.849 \\
3.767 \\
3.685\end{array}$ & $\begin{array}{l}11.733 \\
11.482 \\
11.234\end{array}$ & $\begin{array}{l}13.436 \\
13.149 \\
12.865\end{array}$ \\
\hline $\begin{array}{l}30 \\
30 \\
30\end{array}$ & $\begin{array}{l}5940 \\
5940 \\
5940\end{array}$ & $\begin{array}{l}85.0 \\
90.0 \\
95.0\end{array}$ & $\begin{array}{l}.00831 \\
.00814 \\
.00798\end{array}$ & $\begin{array}{l}.03325 \\
.03259 \\
.03193\end{array}$ & $\begin{array}{r}.00253 \\
.00248 \\
.00243\end{array}$ & $\begin{array}{l}.01013 \\
.00993 \\
.00973\end{array}$ & $\begin{array}{l}3.610 \\
3.539 \\
3.467\end{array}$ & $\begin{array}{l}11.004 \\
10.787 \\
10.570\end{array}$ & $\begin{array}{l}12.601 \\
12.353 \\
12.104\end{array}$ \\
\hline 30 & 5940 & 100.0 & .00786 & .03147 & .00239 & .00959 & 3.416 & $10 \cdot 414$ & 11.926 \\
\hline
\end{tabular}


TABULATIEN OF OATA FOR

ABSORPTION OF SOUND IN AIR VERSUS PERCENT RELATIVE HUMIDITY AT 30 DEGREES CENTIGRADE FOR A FREQUENCY OF 6300 HERTZ

\begin{tabular}{|c|c|c|c|c|c|c|c|c|c|}
\hline (1) & (2) & 131 & (4) & (5) & (6) & 171 & $(8)$ & $(9)$ & $(10)$ \\
\hline $\begin{array}{l}\text { EMP } \\
\text { EGR } \\
\text { ENT }\end{array}$ & FREQ & $\begin{array}{l}\text { REL } \\
\text { HUM } \\
\text { PER } \\
\text { CENT }\end{array}$ & $\begin{array}{l}\text { ATTEN } \\
\text { COEF } \\
\text { PER } \\
\text { METER }\end{array}$ & $\begin{array}{l}\text { 4M } \\
\text { PER } \\
\text { METER }\end{array}$ & $\begin{array}{l}\text { ATTEN } \\
\text { COEF } \\
\text { PER } \\
\text { FOOT }\end{array}$ & $\begin{array}{l}4 M \\
\text { PER } \\
\text { FCOT }\end{array}$ & $\begin{array}{l}\text { ATTEN } \\
\text { DB PER } \\
\text { IOO } \\
\text { HETER }\end{array}$ & $\begin{array}{l}\text { ATTEN } \\
\text { DB PER } \\
\text { IOOO } \\
\text { FEET }\end{array}$ & $\begin{array}{l}\text { DECAY } \\
\text { RATE } \\
\text { DS PER } \\
\text { SECEMD }\end{array}$ \\
\hline $\begin{array}{l}30 \\
30 \\
30\end{array}$ & $\begin{array}{l}6300 \\
6300 \\
6300\end{array}$ & $\begin{array}{r}5.0 \\
10.0 \\
15.0\end{array}$ & $\begin{array}{l}.04346 \\
.04235 \\
.02813\end{array}$ & $\begin{array}{r}17387 \\
.16942 \\
.11254\end{array}$ & $\begin{array}{l}.01324 \\
.01291 \\
.00857\end{array}$ & $\begin{array}{l}.05299 \\
.05164 \\
.03430\end{array}$ & $\begin{array}{l}18 \cdot 878 \\
28 \cdot 395 \\
12 \cdot 219\end{array}$ & $\begin{array}{l}57.542 \\
56.071 \\
37.244\end{array}$ & $\begin{array}{l}65 \\
64 \\
42\end{array}$ \\
\hline $\begin{array}{l}30 \\
30 \\
30\end{array}$ & $\begin{array}{l}6300 \\
6300 \\
6300\end{array}$ & $\begin{array}{l}20.0 \\
25 \bullet 0 \\
30.0\end{array}$ & $\begin{array}{r}.02090 \\
.01673 \\
.01409\end{array}$ & $\begin{array}{l}.08362 \\
.06692 \\
.05637\end{array}$ & $\begin{array}{l}.00637 \\
.00509 \\
.00429\end{array}$ & $\begin{array}{l}.02548 \\
.02039 \\
.01718\end{array}$ & $\begin{array}{l}9.079 \\
7.266 \\
6.121\end{array}$ & $\begin{array}{l}27.674 \\
22.148 \\
18.658\end{array}$ & $\begin{array}{l}31.692 \\
25.363 \\
21.366\end{array}$ \\
\hline $\begin{array}{l}30 \\
30 \\
30\end{array}$ & $\begin{array}{l}6300 \\
6300 \\
6300\end{array}$ & $\begin{array}{l}35.0 \\
40.0 \\
45.0\end{array}$ & $\begin{array}{l}.01247 \\
.01162 \\
.01099\end{array}$ & $\begin{array}{r}.04991 \\
.04648 \\
.04397\end{array}$ & $\begin{array}{r}.00380 \\
.00354 \\
.00335\end{array}$ & $\begin{array}{l}.01521 \\
.01416 \\
.01340\end{array}$ & $\begin{array}{l}5.419 \\
5.046 \\
4.774\end{array}$ & $\begin{array}{l}18 \\
82 \\
52\end{array}$ & $\begin{array}{l}116 \\
515 \\
365\end{array}$ \\
\hline $\begin{array}{l}30 \\
30 \\
30\end{array}$ & $\begin{array}{l}6300 \\
6300 \\
6300\end{array}$ & $\begin{array}{l}46.0 \\
47.0 \\
48.0\end{array}$ & $\begin{array}{r}.01091 \\
.01084 \\
.01077\end{array}$ & $\begin{array}{r}.04366 \\
.04337 \\
.04310\end{array}$ & $\begin{array}{l}.00332 \\
.00330 \\
.00328\end{array}$ & $\begin{array}{l}.01330 \\
.01322 \\
.01313\end{array}$ & $\begin{array}{l}4.740 \\
4.709 \\
4.679\end{array}$ & $\begin{array}{l}14.450 \\
14.353 \\
14.264\end{array}$ & $\begin{array}{l}348 \\
637 \\
395\end{array}$ \\
\hline $\begin{array}{l}30 \\
30 \\
30\end{array}$ & $\begin{array}{l}6300 \\
6300 \\
6300\end{array}$ & $\begin{array}{l}49.0 \\
50.0 \\
51.0\end{array}$ & $\begin{array}{r}.01070 \\
.01064 \\
.01057\end{array}$ & $\begin{array}{l}.04283 \\
.04256 \\
.04229\end{array}$ & $\begin{array}{l}.00326 \\
.00324 \\
.00322\end{array}$ & $\begin{array}{l}.01305 \\
.01297 \\
.01289\end{array}$ & & $\begin{array}{l}14 \\
14 \\
13\end{array}$ & $\begin{array}{r}32 \\
130 \\
227\end{array}$ \\
\hline $\begin{array}{l}30 \\
30 \\
30\end{array}$ & $\begin{array}{l}6300 \\
6300 \\
6300\end{array}$ & $\begin{array}{l}52.0 \\
53.0 \\
54.0\end{array}$ & $\begin{array}{r}.01050 \\
.01043 \\
.01037\end{array}$ & $\begin{array}{l}.04202 \\
.04175 \\
.04150\end{array}$ & $\begin{array}{l}.00320 \\
.00318 \\
.00326\end{array}$ & $\begin{array}{l}.01280 \\
.01272 \\
.01265\end{array}$ & $\begin{array}{l}62 \\
33 \\
06\end{array}$ & $\begin{array}{l}13.906 \\
13.817 \\
13.737\end{array}$ & $\begin{array}{l}15.925 \\
15.823 \\
15.731\end{array}$ \\
\hline $\begin{array}{l}30 \\
30 \\
30\end{array}$ & $\begin{array}{l}6300 \\
6300 \\
6300\end{array}$ & $\begin{array}{l}55.0 \\
60.0 \\
65.0\end{array}$ & $\begin{array}{l}.01032 \\
.01005 \\
.00980\end{array}$ & $\begin{array}{r}.04129 \\
.04021 \\
.03923\end{array}$ & $\begin{array}{r}.00314 \\
.00306 \\
.00298\end{array}$ & $\begin{array}{l}.01258 \\
.01225 \\
.01195\end{array}$ & & $\begin{array}{l}365 \\
07 \\
84\end{array}$ & $\begin{array}{l}15.649 \\
15.239 \\
14.869\end{array}$ \\
\hline $\begin{array}{l}30 \\
30 \\
30\end{array}$ & $\begin{array}{l}6300 \\
6300 \\
6300\end{array}$ & $\begin{array}{l}70.0 \\
75.0 \\
80.0\end{array}$ & $\begin{array}{l}.00958 \\
.00937 \\
.00918\end{array}$ & $\begin{array}{r}.03833 \\
.03749 \\
.03672\end{array}$ & $\begin{array}{l}.00292 \\
.00285 \\
.00279\end{array}$ & $\begin{array}{l}.01 \\
.01 \\
.01\end{array}$ & $\begin{array}{l}2 \\
1 \\
7\end{array}$ & $\begin{array}{l}12.6 \\
12.4 \\
12.1\end{array}$ & $\begin{array}{l}14 \\
13\end{array}$ \\
\hline $\begin{array}{l}30 \\
30 \\
30\end{array}$ & $\begin{array}{l}6300 \\
6300 \\
6300\end{array}$ & $\begin{array}{l}85.0 \\
90.0 \\
95.0\end{array}$ & $\begin{array}{l}.00899 \\
.00882 \\
.00865\end{array}$ & $\begin{array}{r}.03596 \\
.03528 \\
.03461\end{array}$ & $\begin{array}{r}.00274 \\
.00268 \\
.00263\end{array}$ & $\begin{array}{l}.01096 \\
.01075 \\
.01055\end{array}$ & $\frac{4}{8}$ & $\begin{array}{l}11.902 \\
11.678 \\
11.454\end{array}$ & $\begin{array}{l}13.629 \\
13.373 \\
13.127\end{array}$ \\
\hline & 6300 & 0. & .00850 & 03400 & .00259 & .01036 & 3.692 & $11 \cdot 254$ & 12.888 \\
\hline
\end{tabular}


TABULATION OF DATA FOR

ABSORPTION OF SOUND IN AIR VERSUS PERCENT RELATIVE HUMIDITY AT 30 DEGREES CENTIGRADE FOR A FREQUENCY OF 8000 . HERTZ

\begin{tabular}{|c|c|c|c|c|c|c|c|c|c|}
\hline (1) & (2) & (3) & 141 & (5) & (6) & (7) & $(8)$ & $(9)$ & $(10)$ \\
\hline TEMP & FREQ & $\begin{array}{l}\text { REL } \\
\text { HUM }\end{array}$ & $\begin{array}{l}\text { ATTEN } \\
\text { COEF }\end{array}$ & $4 M$ & $\begin{array}{l}\text { ATTEN } \\
\text { COEF }\end{array}$ & $4 M$ & $\begin{array}{l}\text { ATTEN } \\
\text { DB PER }\end{array}$ & $\begin{array}{l}\text { ATTEN } \\
\text { DB PER }\end{array}$ & $\begin{array}{l}\text { DECAY } \\
\text { RATE }\end{array}$ \\
\hline $\begin{array}{l}\text { DEGR } \\
\text { CENT }\end{array}$ & HERTZ & $\begin{array}{l}\text { PER } \\
\text { CENT }\end{array}$ & $\begin{array}{l}\text { PER } \\
\text { METER }\end{array}$ & $\begin{array}{l}\text { PER } \\
\text { METER }\end{array}$ & 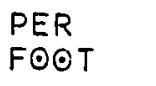 & $\begin{array}{l}\text { PER } \\
\text { FOOT }\end{array}$ & $\begin{array}{l}100 \\
\text { METER }\end{array}$ & $\begin{array}{l}1000 \\
\text { FEET }\end{array}$ & $\begin{array}{l}\text { DB PER } \\
\text { SECOND }\end{array}$ \\
\hline $\begin{array}{l}30 \\
30\end{array}$ & $\begin{array}{l}8000 \\
8000\end{array}$ & $\begin{array}{r}5.0 \\
10.0\end{array}$ & $\begin{array}{l}.04873 \\
.05961\end{array}$ & $\begin{array}{r}.19493 \\
.23845\end{array}$ & $\begin{array}{r}.01485 \\
.01817\end{array}$ & $\begin{array}{l}.05941 \\
.07268\end{array}$ & $\begin{array}{l}21.164 \\
25: 890\end{array}$ & $\begin{array}{l}64.510 \\
78.915\end{array}$ & $\begin{array}{l}73.874 \\
90.370\end{array}$ \\
\hline 30 & 8000 & 15.0 & .04270 & .17080 & .01301 & .05206 & 18.545 & 56.527 & 64.733 \\
\hline $\begin{array}{l}30 \\
30 \\
30\end{array}$ & $\begin{array}{l}8000 \\
8000 \\
8000\end{array}$ & $\begin{array}{l}20.0 \\
25.0 \\
30.0\end{array}$ & $\begin{array}{l}.03203 \\
.02539 \\
.02129\end{array}$ & $\begin{array}{l}.12814 \\
.10157 \\
.08518\end{array}$ & $\begin{array}{l}.00976 \\
.00774 \\
.00649\end{array}$ & $\begin{array}{l}.03905 \\
.03096 \\
.02596\end{array}$ & $\begin{array}{r}13.913 \\
11.028 \\
9.248\end{array}$ & $\begin{array}{l}42.408 \\
33.616 \\
28.190\end{array}$ & $\begin{array}{l}48.565 \\
38.496 \\
32.282\end{array}$ \\
\hline $\begin{array}{l}30 \\
30 \\
30\end{array}$ & $\begin{array}{l}8000 \\
8000 \\
8000\end{array}$ & $\begin{array}{l}35.0 \\
40.0 \\
45.0\end{array}$ & $\begin{array}{r}.01851 \\
.01666 \\
.01557\end{array}$ & $\begin{array}{l}.07407 \\
.06666 \\
.06231\end{array}$ & $\begin{array}{l}.00564 \\
.00507 \\
.00474\end{array}$ & $\begin{array}{l}.02257 \\
.02031 \\
.01899\end{array}$ & $\begin{array}{l}8.042 \\
7.238 \\
6.766\end{array}$ & $\begin{array}{l}24.513 \\
22.062 \\
20.623\end{array}$ & $\begin{array}{l}28.071 \\
25.265 \\
23.617\end{array}$ \\
\hline $\begin{array}{l}30 \\
30 \\
30\end{array}$ & $\begin{array}{l}8000 \\
8000 \\
8000\end{array}$ & $\begin{array}{l}46.0 \\
47.0 \\
48.0\end{array}$ & $\begin{array}{r}.01542 \\
.01527 \\
.01509\end{array}$ & $\begin{array}{l}.06168 \\
.06109 \\
.06036\end{array}$ & $\begin{array}{l}.00470 \\
.00465 \\
.00459\end{array}$ & $\begin{array}{l}.01880 \\
.01862 \\
.01839\end{array}$ & $\begin{array}{l}6.697 \\
6.633 \\
6.553\end{array}$ & $\begin{array}{l}20.413 \\
20.218 \\
19.976\end{array}$ & $\begin{array}{l}23.376 \\
23.153 \\
22.876\end{array}$ \\
\hline $\begin{array}{l}30 \\
30 \\
30\end{array}$ & $\begin{array}{l}8000 \\
8000 \\
8000\end{array}$ & $\begin{array}{l}49.0 \\
50.0 \\
51.0\end{array}$ & $\begin{array}{r}.01492 \\
.01478 \\
.01466\end{array}$ & $\begin{array}{l}.05969 \\
.05912 \\
.05866\end{array}$ & $\begin{array}{l}.00454 \\
.00450 \\
.00446\end{array}$ & $\begin{array}{r}.01819 \\
.01802 \\
.01787\end{array}$ & $\begin{array}{l}6.480 \\
6.419 \\
6.369\end{array}$ & $\begin{array}{l}19.754 \\
19.567 \\
19.413\end{array}$ & $\begin{array}{l}22.621 \\
22.408 \\
22.231\end{array}$ \\
\hline $\begin{array}{l}30 \\
30 \\
30\end{array}$ & $\begin{array}{l}8000 \\
8000 \\
8000\end{array}$ & $\begin{array}{l}52.0 \\
53.0 \\
54.0\end{array}$ & $\begin{array}{r}.01455 \\
.01446 \\
.011438\end{array}$ & $\begin{array}{r}.05821 \\
.05787 \\
.05754\end{array}$ & $\begin{array}{l}.00443 \\
.00441 \\
.00438\end{array}$ & $\begin{array}{l}.01774 \\
.01764 \\
.017553\end{array}$ & $\begin{array}{l}6.320 \\
6.284 \\
6.248\end{array}$ & $\begin{array}{l}19.265 \\
19.154 \\
19.044\end{array}$ & $\begin{array}{l}22.061 \\
21.935 \\
21.808\end{array}$ \\
\hline $\begin{array}{l}30 \\
30 \\
30\end{array}$ & $\begin{array}{l}8000 \\
8000 \\
8000\end{array}$ & $\begin{array}{l}55.0 \\
60.0 \\
65.0\end{array}$ & $\begin{array}{l}.01430 \\
.01394 \\
.01360\end{array}$ & $\begin{array}{r}.05722 \\
.05576 \\
.05443\end{array}$ & $\begin{array}{l}.00436 \\
.00424 \\
.00414\end{array}$ & $\begin{array}{r}.01744 \\
.01699 \\
.01659\end{array}$ & $\begin{array}{l}6.213 \\
6.054 \\
5.909\end{array}$ & $\begin{array}{l}18.938 \\
18.454 \\
18.013\end{array}$ & $\begin{array}{l}21.687 \\
21.133 \\
20.628\end{array}$ \\
\hline $\begin{array}{l}30 \\
30 \\
30\end{array}$ & $\begin{array}{l}8000 \\
8000 \\
8000\end{array}$ & $\begin{array}{l}70.0 \\
75.0 \\
80.0\end{array}$ & $\begin{array}{r}.01331 \\
.01304 \\
.01280\end{array}$ & $\begin{array}{r}.05326 \\
.05217 \\
.05120\end{array}$ & $\begin{array}{l}.00405 \\
.00397 \\
.00390\end{array}$ & $\begin{array}{r}.01623 \\
.01590 \\
.01560\end{array}$ & $\begin{array}{l}5.782 \\
5.664 \\
5.559\end{array}$ & $\begin{array}{l}17.626 \\
17.266 \\
16.944\end{array}$ & $\begin{array}{l}20.185 \\
19.773 \\
19.403\end{array}$ \\
\hline $\begin{array}{l}30 \\
30 \\
30\end{array}$ & $\begin{array}{l}8000 \\
8000 \\
8000\end{array}$ & $\begin{array}{l}85.0 \\
90.0 \\
95.0\end{array}$ & $\begin{array}{r}.01255 \\
.01234 \\
.01113\end{array}$ & $\begin{array}{r}.05022 \\
.04938 \\
.04855\end{array}$ & $\begin{array}{l}.00382 \\
.00376 \\
.00369\end{array}$ & $\begin{array}{l}.01530 \\
.01505 \\
.01479\end{array}$ & $\begin{array}{l}5.453 \\
5.362 \\
5.271\end{array}$ & $\begin{array}{l}16.621 \\
16.344 \\
16.068\end{array}$ & $\begin{array}{l}19.034 \\
18.717 \\
18.400\end{array}$ \\
\hline 30 & 8000 & 100.0 & .01193 & 04773 & .00363 & .01454 & $5 \cdot 182$ & 15.797 & 18.090 \\
\hline
\end{tabular}


TABULATION OF DATA FOR

ABSORPTION OF SOUND. IN AIR VERSUS PERCENT RELATIVE HUMIDITY AT 30 DEGREES CENTIGRADE FOR A FREQUENCY OF 10000 HERTZ

\begin{tabular}{|c|c|c|c|c|c|c|c|c|c|}
\hline (1) & (2) & (3) & $(4)$ & (5) & (6) & (7) & (8) & 191 & $(10)$ \\
\hline $\begin{array}{l}\text { EMP } \\
\text { EGR } \\
\text { ENT }\end{array}$ & HERTZ & $\begin{array}{l}\text { REL } \\
\text { HUM } \\
\text { PER } \\
\text { CENT }\end{array}$ & $\begin{array}{l}\text { ATTEN } \\
\text { COEF } \\
\text { PER } \\
\text { METER }\end{array}$ & $\begin{array}{l}4 M \\
\text { PER } \\
\text { METER }\end{array}$ & $\begin{array}{l}\text { ATTEN } \\
\text { COEF } \\
\text { PER } \\
\text { FOOT }\end{array}$ & $\begin{array}{l}4 M \\
\text { PER } \\
\text { F@OT. }\end{array}$ & $\begin{array}{l}\text { ATTEN } \\
\text { DB PER } \\
100 \\
\text { METER }\end{array}$ & $\begin{array}{l}\text { ATTEN } \\
\text { DB PER } \\
1000 \\
\text { FEET }\end{array}$ & $\begin{array}{l}\text { DECAY } \\
\text { RATE } \\
\text { DB PER } \\
\text { SECOND }\end{array}$ \\
\hline $\begin{array}{l}30 \\
30 \\
30\end{array}$ & $\begin{array}{l}10000 \\
10000 \\
10000\end{array}$ & $\begin{array}{r}5.0 \\
10.0 \\
15.0\end{array}$ & $\begin{array}{r}.05382 \\
.07760 \\
.06263\end{array}$ & $\begin{array}{r}.21530 \\
.31040 \\
.25052\end{array}$ & $\begin{array}{r}.01640 \\
.02365 \\
.01909\end{array}$ & $\begin{array}{l}.06562 \\
.09461 \\
.07636\end{array}$ & $\begin{array}{l}23.377 \\
33.702 \\
27.201\end{array}$ & $\begin{array}{r}71.254 \\
102.727 \\
82.910\end{array}$ & $\begin{array}{r}81.597 \\
117.639 \\
94.945\end{array}$ \\
\hline $\begin{array}{l}30 \\
30 \\
30\end{array}$ & $\begin{array}{l}10000 \\
10000 \\
10000\end{array}$ & $\begin{array}{l}20.0 \\
25.0 \\
30.0\end{array}$ & $\begin{array}{l}.04658 \\
.03744 \\
.03119\end{array}$ & $\begin{array}{r}1 \\
.18634 \\
.14979 \\
.12479\end{array}$ & $\begin{array}{r}.01419 \\
.01141 \\
.00950\end{array}$ & $\begin{array}{l}.05679 \\
.04565 \\
.03803\end{array}$ & $\begin{array}{l}20.232 \\
16.264 \\
13.549\end{array}$ & $\begin{array}{l}61.670 \\
49.573 \\
41.299\end{array}$ & $\begin{array}{l}70.622 \\
56.769 \\
47.295\end{array}$ \\
\hline $\begin{array}{l}30 \\
30 \\
30\end{array}$ & $\begin{array}{l}10000 \\
10000 \\
10000\end{array}$ & $\begin{array}{l}35.0 \\
40.0 \\
45.0\end{array}$ & $\begin{array}{r}.02700 \\
.02402 \\
.02191\end{array}$ & $\begin{array}{l}.10803 \\
.09609 \\
.08766\end{array}$ & $\begin{array}{l}.00823 \\
.00732 \\
.00667\end{array}$ & $\begin{array}{r}.03292 \\
.02928 \\
.02671\end{array}$ & $\begin{array}{r}11.730 \\
10.433 \\
9.517\end{array}$ & $\begin{array}{l}35.754 \\
31.801 \\
29.010\end{array}$ & $\begin{array}{l}40.944 \\
36.417 \\
33.222\end{array}$ \\
\hline $\begin{array}{l}30 \\
30 \\
30\end{array}$ & $\begin{array}{l}10000 \\
10000 \\
10000\end{array}$ & $\begin{array}{l}46.0 \\
47.0 \\
48.0\end{array}$ & $\begin{array}{l}.02159 \\
.02129 \\
.02102\end{array}$ & $\begin{array}{r}.08638 \\
.08519 \\
.08408\end{array}$ & $\begin{array}{l}.00658 \\
.00649 \\
.00640\end{array}$ & $\begin{array}{l}.02633 \\
.02596 \\
.02562\end{array}$ & $\begin{array}{l}9.379 \\
9.250 \\
9.129\end{array}$ & $\begin{array}{l}28.589 \\
28.194 \\
27.825\end{array}$ & $\begin{array}{l}32.739 \\
32.287 \\
31.865\end{array}$ \\
\hline $\begin{array}{l}30 \\
30 \\
30\end{array}$ & $\begin{array}{l}10000 \\
10000 \\
10000\end{array}$ & $\begin{array}{l}49.0 \\
50.0 \\
51.0\end{array}$ & $\begin{array}{l}.02079 \\
.02059 \\
.02039\end{array}$ & $\begin{array}{r}.08317 \\
.08237 \\
.08157\end{array}$ & $\begin{array}{r}.00633 \\
.00627 \\
.00621\end{array}$ & $\begin{array}{r}.02535 \\
.02510 \\
.02486\end{array}$ & $\begin{array}{l}9.030 \\
8.943 \\
8.857\end{array}$ & $\begin{array}{l}27 \cdot 524 \\
27 \cdot 261 \\
26.997\end{array}$ & $\begin{array}{l}31.520 \\
31.218 \\
30.917\end{array}$ \\
\hline $\begin{array}{l}30 \\
30 \\
30\end{array}$ & $\begin{array}{l}10000 \\
10000 \\
10000\end{array}$ & $\begin{array}{l}52.0 \\
53.0 \\
54.0\end{array}$ & $\begin{array}{l}.02019 \\
.02003 \\
.01987\end{array}$ & $\begin{array}{r}.08078 \\
.08013 \\
.07948\end{array}$ & $\begin{array}{l}.00615 \\
.00610 \\
.00605\end{array}$ & $\begin{array}{l}.02462 \\
.02442 \\
.02422\end{array}$ & $\begin{array}{l}771 \\
700 \\
630\end{array}$ & $\begin{array}{l}734 \\
519 \\
305\end{array}$ & $\begin{array}{l}30.615 \\
30.369 \\
30.124\end{array}$ \\
\hline $\begin{array}{l}30 \\
30 \\
30\end{array}$ & $\begin{array}{l}10000 \\
10000 \\
10000\end{array}$ & $\begin{array}{l}55.0 \\
60.0 \\
65.0\end{array}$ & $\begin{array}{r}.01967 \\
.01895 \\
.01852\end{array}$ & $\begin{array}{l}.07869 \\
.07580 \\
.07409\end{array}$ & $\begin{array}{l}.00599 \\
.00577 \\
.00564\end{array}$ & $\begin{array}{l}.02398 \\
.02310 \\
.02258\end{array}$ & $\begin{array}{l}8.544 \\
8.231 \\
8.044\end{array}$ & $\begin{array}{l}26.042 \\
25.088 \\
24.520\end{array}$ & $\begin{array}{l}.822 \\
.730 \\
.079\end{array}$ \\
\hline $\begin{array}{l}30 \\
30 \\
30\end{array}$ & $\begin{array}{l}10000 \\
10000 \\
10000\end{array}$ & $\begin{array}{l}70.0 \\
75.0 \\
80.0\end{array}$ & $\begin{array}{r}.01812 \\
.01777 \\
.01745\end{array}$ & $\begin{array}{l}.07250 \\
.07109 \\
.06982\end{array}$ & $\begin{array}{l}.00552 \\
.00541 \\
.00532\end{array}$ & $\begin{array}{r}.02209 \\
.02166 \\
.02128\end{array}$ & $\begin{array}{l}7.871 \\
7.719 \\
7.580\end{array}$ & $\begin{array}{l}23.993 \\
23.528 \\
23.106\end{array}$ & $\begin{array}{l}27.476 \\
26.943 \\
26.461\end{array}$ \\
\hline $\begin{array}{l}30 \\
30 \\
30\end{array}$ & $\begin{array}{l}10000 \\
10000 \\
10000\end{array}$ & $\begin{array}{l}85.0 \\
90.0 \\
95.0\end{array}$ & $\begin{array}{r}.01715 \\
.01688 \\
.01662\end{array}$ & $\begin{array}{l}.06860 \\
.06754 \\
.06648\end{array}$ & $\begin{array}{l}.00522 \\
.00514 \\
.00506\end{array}$ & $\begin{array}{l}.02091 \\
.02058 \\
.02026\end{array}$ & $\begin{array}{r}7.448 \\
7.333 \\
7.218\end{array}$ & $\begin{array}{l}22.703 \\
22.352 \\
22.001\end{array}$ & $\begin{array}{l}25.999 \\
25.597 \\
25.195\end{array}$ \\
\hline 30 & 0000 & 0.0 & .01637 & 06549 & 00499 & 01 & 7.110 & 1.674 & 24.820 \\
\hline
\end{tabular}


TABULATION OF DATA FOR

ABSORPTION OF SOUND IN AIR VERSUS PERCENT RELATIVE HUMIDITY AT 30 DEGREES CENTIGRADE FOR A FREQUENCY OF 12500 HERTZ

\begin{tabular}{|c|c|c|c|c|c|c|c|c|c|}
\hline 11 & (2) & (3) & $(4)$ & $(5)$ & (6) & $17 !$ & (8) & $(9)$ & 1201 \\
\hline $\begin{array}{l}\text { EMP } \\
\text { EGR } \\
\text { ENT }\end{array}$ & $\begin{array}{l}\text { FREQ } \\
\text { HERTZ }\end{array}$ & $\begin{array}{l}\text { REL } \\
\text { HUM } \\
\text { PER } \\
\text { CENT }\end{array}$ & $\begin{array}{l}\text { ATTEN } \\
\text { COEF } \\
\text { PER } \\
\text { METER }\end{array}$ & $\begin{array}{l}4 M \\
\text { PER } \\
\text { METER }\end{array}$ & $\begin{array}{l}\text { ATTEN } \\
\text { COEF } \\
\text { PER } \\
\text { FOOT }\end{array}$ & $\begin{array}{l}4 M \\
\text { PER } \\
\text { FOOT }\end{array}$ & $\begin{array}{l}\text { ATTEN } \\
\text { DB PER } \\
1.00 \\
\text { METER }\end{array}$ & $\begin{array}{l}\text { ATTEN } \\
\text { DB PER } \\
1000 \\
\text { FEET }\end{array}$ & $\begin{array}{l}\text { DECAY } \\
\text { RATE } \\
\text { DB PER } \\
\text { SECOND }\end{array}$ \\
\hline $\begin{array}{l}30 \\
30 \\
30\end{array}$ & $\begin{array}{l}12500 \\
12500 \\
12500\end{array}$ & $\begin{array}{r}5.0 \\
10.0 \\
15.0\end{array}$ & $\begin{array}{l}.06 \\
.09 \\
.08\end{array}$ & $\begin{array}{r}.24113 \\
.39257 \\
.35460\end{array}$ & $\begin{array}{r}.01837 \\
.02991 \\
.02702\end{array}$ & $\begin{array}{l}.07349 \\
.11965 \\
.10808\end{array}$ & & $\begin{array}{r}79.801 \\
129.920 \\
117.354\end{array}$ & $\begin{array}{r}91.386 \\
148.779 \\
134.389\end{array}$ \\
\hline $\begin{array}{l}30 \\
30 \\
30\end{array}$ & $\begin{array}{l}12500 \\
12500 \\
12500\end{array}$ & $\begin{array}{l}20.0 \\
25.0 \\
30.0\end{array}$ & $\begin{array}{l}.06 \\
.05 \\
.04\end{array}$ & $\begin{array}{r}.27055 \\
.21863 \\
.18208\end{array}$ & $\begin{array}{l}.02 \\
.02 \\
.0\end{array}$ & $\begin{array}{l}.08246 \\
.06664 \\
.05549\end{array}$ & & $\begin{array}{l}89.537 \\
72.355 \\
60.258\end{array}$ & $\begin{array}{r}102.535 \\
82.858 \\
69.005\end{array}$ \\
\hline $\begin{array}{l}30 \\
30 \\
30\end{array}$ & $\begin{array}{l}12500 \\
12500 \\
12500\end{array}$ & $\begin{array}{l}35.0 \\
40.0 \\
45.0\end{array}$ & $\begin{array}{l}.0 \\
.02 \\
.03\end{array}$ & $\begin{array}{r}.15752 \\
.13960 \\
.12625\end{array}$ & $\begin{array}{l}.01 \\
.01 \\
.00\end{array}$ & & & & $\begin{array}{l}69.8 \\
90.9 \\
848\end{array}$ \\
\hline $\begin{array}{l}3.0 \\
30 \\
30\end{array}$ & $\begin{array}{l}12500 \\
12500 \\
12500\end{array}$ & $\begin{array}{l}46.0 \\
47.0 \\
48.0\end{array}$ & $\begin{array}{l}.03 \\
.03 \\
.03\end{array}$ & $\begin{array}{l}.12416 \\
.12219 \\
.12044\end{array}$ & $\begin{array}{l}.00 \\
.00 \\
.00\end{array}$ & $\begin{array}{l}784 \\
724 \\
671\end{array}$ & $\begin{array}{l}480 \\
266 \\
077\end{array}$ & $\begin{array}{l}89 \\
38 \\
60\end{array}$ & $\begin{array}{l}54 \\
308 \\
46\end{array}$ \\
\hline $\begin{array}{l}30 \\
30 \\
30\end{array}$ & $\begin{array}{l}12500 \\
12500 \\
12500\end{array}$ & $\begin{array}{l}49.0 \\
50.0 \\
51.0\end{array}$ & $\begin{array}{l}.02966 \\
.02918 \\
.02872\end{array}$ & $\begin{array}{r}.11865 \\
.11673 \\
.11488\end{array}$ & $\begin{array}{l}.00904 \\
.00889 \\
.00875\end{array}$ & $\begin{array}{l}.03616 \\
.03558 \\
.03501\end{array}$ & $\begin{array}{l}12.883 \\
12.074 \\
12.473\end{array}$ & & $\begin{array}{l}44.969 \\
44.242 \\
43.538\end{array}$ \\
\hline $\begin{array}{l}30 \\
30 \\
30\end{array}$ & $\begin{array}{l}12500 \\
12500 \\
12500\end{array}$ & $\begin{array}{l}52.0 \\
53.0 \\
54.0\end{array}$ & $\begin{array}{l}.0 \\
.0 \\
.0\end{array}$ & $\begin{array}{r}.11348 \\
.11209 \\
.111086\end{array}$ & $\begin{array}{l}.00 \\
.00\end{array}$ & & $\begin{array}{l}21 \\
70 \\
37\end{array}$ & & $\begin{array}{l}109 \\
79 \\
16\end{array}$ \\
\hline $\begin{array}{l}30 \\
30 \\
30\end{array}$ & $\begin{array}{l}12500 \\
12500 \\
12500\end{array}$ & $\begin{array}{l}55.0 \\
60.0 \\
65.0\end{array}$ & $\begin{array}{l}.02741 \\
.02633 \\
.02537\end{array}$ & $\begin{array}{r}.10964 \\
.10535 \\
.10149\end{array}$ & $\begin{array}{l}.00835 \\
.00802 \\
.00773\end{array}$ & $\begin{array}{l}.03342 \\
.03211 \\
.03093\end{array}$ & $\begin{array}{l}04 \\
38 \\
19\end{array}$ & $\begin{array}{l}286 \\
865 \\
588\end{array}$ & $\begin{array}{l}41.553 \\
39.926 \\
38.463\end{array}$ \\
\hline $\begin{array}{l}30 \\
30\end{array}$ & $\begin{array}{l}12500 \\
12500\end{array}$ & $\begin{array}{l}70 \\
75\end{array}$ & $\begin{array}{l}.02 \\
.02\end{array}$ & $\begin{array}{l}.05 \\
.05\end{array}$ & .00 & & & $\begin{array}{l}769 \\
166\end{array}$ & $\begin{array}{l}326 \\
335\end{array}$ \\
\hline 30 & 12500 & 80.0 & .02386 & .09545 & .00727 & .02909 & 363 & 31.588 & 36.173 \\
\hline $\begin{array}{l}30 \\
30 \\
30\end{array}$ & $\begin{array}{l}12500 \\
12500 \\
12500\end{array}$ & $\begin{array}{l}85.0 \\
90.0 \\
95.0\end{array}$ & $\begin{array}{r}.02347 \\
.02312 \\
.02277\end{array}$ & $\begin{array}{r}.09390 \\
.09250 \\
.09111\end{array}$ & $\begin{array}{l}.00715 \\
.00704 \\
.00694\end{array}$ & $\begin{array}{l}.02862 \\
.02819 \\
.02777\end{array}$ & $\begin{array}{r}10.195 \\
10.044 \\
9.892\end{array}$ & $\begin{array}{l}31.076 \\
30.614 \\
30.152\end{array}$ & $\begin{array}{l}35.587 \\
35.058 \\
34.0529\end{array}$ \\
\hline 30 & & & .02248 & 08992 & .00685 & .02741 & 9.764 & 9.761 & 34.081 \\
\hline
\end{tabular}

